\title{
1995 Annual Water Monitoring Report LEHR Environmental Restoration University of California at Davis
}
D. L. Stewart
T. L. Liikala
R. M. Smith
J. K. Merz
D. R. Sauer
D. J. Bates
R. Schalla
D. C. Lanigan
F. A. Spane
G. V. Last
C. D. Taylor
W. D. Webber

March 1996

Prepared for

the Oakland Operations Office of the U.S. Department of Energy under Contract DE-AC06-76RLO 1830

Pacific Northwest National Laboratory

Richland, Washington 99352

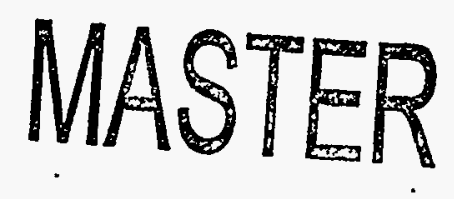

DISTAIBUTION OF THAS DOCUMENT IS LALLMUTED 


\section{DISCLAIMER}

This report was prepared as an account of work sponsored by an agency of the United States Government. Neither the United States Government nor any agency thereof, nor Battelle Memorial Institute, nor any of their employees, makes any warranty, express or implied, or assumes any legal liability or responsibility for the accuracy, completeness, or usefulness of any information, apparatus, product, or process disclosed, or represents that its use would not infringe privately owned rights. Reference herein to any specific commercial product, process, or service by trade name, trademark, manufacturer, or otherwise does not necessarily constitute or imply its endorsement, recommendation, or favoring by the United States Government or any agency thereof, or Battelle Memorial Institute. The views and opinions of authors expressed herein do not necessarily state or reflect those of the United States Government or any agency thereof.

\section{PACIFIC NORTHWEST NATIONAL LABORATORY operated by \\ BATTELLE \\ for the \\ UNITED STATES DEPARTMENT OF ENERGY} under Contract DE-AC06-76RLO 1830

Printed in the United States of America

Available to DOE and DOE contractors from the

Office of Scientific and Technical Information, P.O. Box 62, Oak Ridge, TN 37831; prices available from (615) 576-8401.

Available to the public from the National Technical Information Service, U.S. Department of Commerce, 5285 Port Royal Rd., Springfield, VA 22161 


\section{DISCLAIMER}

Portions of this document may be illegible in electronic image products. Images are produced from the best available original document. 


\section{DISCLAIMER}

This report was prepared as an account of work sponsored by an agency of the United States Government. Neither the United States Government nor any agency thereof, nor any of their employees, make any warranty, express or implied, or assumes any legal liability or responsibility for the accuracy, completeness, or usefulness of any information, apparatus, product, or process disclosed, or represents that its use would not infringe privately owned rights. Reference herein to any specific commercial product, process, or service by trade name, trademark, manufacturer, or otherwise does not necessarily constitute or imply its endorsement, recommendation, or favoring by the United States Government or any agency thereof. The views and opinions of authors expressed herein do not necessarily state or reflect those of the United States Government or any agency thereof. 



\section{Executive Summary}

This 1995 Annual Water Monitoring Report presents analytical data collected between January and December 1995 at the Laboratory for Energy-Related Health Research (LEHR) located at the University of California (UC), Davis. This report has been prepared by Pacific Northwest National Laboratory ${ }^{(a)}$ in compliance with the Water Monitoring Plan for the LEHR site, which contains the sample collection, analysis, and quality assurance/quality control procedures and reporting requirements. Water monitoring during 1995 was conducted in conjunction with the Remedial Investigation/Feasibility Study currently being implemented at the LEHR site as part of a U.S. Department of Energy (DOE)-sponsored environmental restoration program. The Water Monitoring Plan and this report are intended to comply with DOE Order 5400.1, General Environmental Protection Program.

The 1995 water monitoring program continued the basic program from 1994 with two major changes. First, the sampling and analytical schedule for the 18 monitoring wells in the program was modified; second, five new monitoring wells were constructed and added to the monitoring program.

Based on a review of historical groundwater monitoring data compiled since the fall of 1990 , the list of analytes included in the program was reduced and the schedule for analyzing the remaining analytes was revised. The revision was implemented for the first time in the summer monitoring period. Analytes eliminated from the program were those that were 1) important for establishing baseline groundwater chemistry (alkalinity, anions, Eh, total organic carbon, and chemical oxygen demand); 2) important for establishing sources of contamination (ammonia); 3) not detected in water samples or not from the LEHR site (formaldehyde, ammonia, total kjeldahl nitrogen); and 4) duplicates of another measurement (laboratory turbidity). Reductions in the analytical schedule were based on the monitoring history for each well; the resultant constituents of concern list was developed for individual wells. Depending on its importance in a well, each analyte was analyzed quarterly, semi-annually, or annually.

Because specific wells are not monitored for every analyte every quarter, monitoring results must be evaluated quickly to determine whether the current quarter's sampling results follow historical trends. If not, the sampling schedule must be adjusted to ensure the specific well and analyte are included in the next sampling period (if they are not automatically included).

Groundwater elevation data were assessed by compiling groundwater elevation hydrographs and elevation contour plots to establish generalized flow conditions. This analysis supports previous conclusions that the uppermost water-bearing zone can be

(a) Pacific Northwest National Laboratory is operated for the U.S. Department of Energy by Battelle under Contract DE-AC06-76RLO 1830. 
separated into two hydrostratigraphic units (HSUs). Groundwater flow directions were similar to those reported previously, but water levels were much higher in 1995 than in 1994. During 1995, the lateral gradient in HSU-1 was toward the east with a slight northerly component. A water table mound appeared in the winter of 1995 , but it dissipated quickly. The lateral gradient in HSU-2 continues to be consistently toward the east, with a slight northerly component.' The vertical gradient between HSU-1 and HSU-2 reversed direction, as reported in previous years. Groundwater flow was downward during the winter through summer period as the water table was falling; this gradient switched to upward following the summer irrigation season.

The geology in the vicinity of the LEHR site was characterized during the Phase II Site Characterization. The lithologic data collected as part of this effort are consistent with the hydrogeologic model developed during the Phase II Characterization.

For this report, approximately 14,000 analytical values were reviewed with respect to data quality objectives stated in the Water Monitoring Plan for the LEHR site. The data were reviewed using validation procedures described in this plan. After evaluation for quality, the data were accepted if they met the precision, accuracy, representativeness, and completeness goals.

A statistical analysis was conducted on analytical results from 1995 groundwater samples (Section 7.0). The purpose of this analysis was to compare data from downgradient wells with data from background wells to evaluate impacts to groundwater in the vicinity of the LEHR site. Data for HSU-1 and HSU-2 were evaluated separately. Based on the results of the statistical analyses, concentrations of nitrate, total chromium, hexavalent chromium, and barium were concluded to be significantly higher at the $95 \%$ confidence level in most downgradient wells than established background concentrations for both HSUs. Other constituents, including arsenic, selenium, chloroform (and several other volatile organic compounds), carbon-14, tritium, and the pesticide dieldrin, were found at concentrations exceeding background concentrations at the $95 \%$ confidence level in only a few wells. These parameters appear to be located beneath a small or localized portion of the site; results are similar to those reported for 1994.

Chloroform, acetone, and methylene chloride were reported in surface water samples collected at the LEHR site in 1995. The remaining volatile organic compounds were reported only sporadically in surface water. . Acetone and methylene chloride are routinely found in laboratory samples as a result of laboratory contamination, but methylene chloride has never been detected in upstream samples and is regularly detected in STPO samples. The only semivolatile organic compounds detected in 1995 surface water or storm water sampling were phthalate compounds attributed to laboratory contamination. Several metals were reported above maximum concentration levels in surface water and storm water samples. No radionuclides were reported in downstream surface water in concentrations exceeding upstream levels. Ammonia and nitrate were found in STPO samples at concentrations well above background, causing downstream samples to have elevated levels of these constituents also. 
An assessment of the groundwater monitoring network was conducted based on groundwater data presented in this 1995 Annual Water Monitoring Report. The revised monitoring program was considered sufficient to monitor environmental hazards from LEHR. Although the extent of constituents in the upper aquifer is laterally defined using hydropunch sampling and groundwater monitoring data, the current monitoring network should be supplemented to the east and northeast of the site to further evaluate the downgradient extent of site constituents.

As reported in the past, the lateral extent of site constituents is greater in HSU-2 than in HSU-1. As chloroform migrates downward from HSU-1 into HSU-2, it migrates. laterally from the apparent source area north of Landfill 2 downgradient to the east, northeast beyond the UC Davis property boundary. Tritium appears to be migrating downgradient of well UCD1-14, where it is reported regularly. Nitrate decreases rapidly downgradient of site source areas to generally regional concentrations at the UC Davis property boundary. Based on this analysis, the monitoring network should be supplemented to further evaluate downgradient migration of, at a minimum, chloroform, hexavalent chromium, and tritium in HSU-2.

The new wells added to the network indicate that site contamination is not migrating to the south and is staying in HSU-I and the upper half of HSU-2. Contamination is not present or is present at low concentrations in the lower part of the upper aquifer. 


\section{Contents}

Executive Summary $\ldots \ldots \ldots \ldots \ldots \ldots \ldots \ldots \ldots \ldots \ldots \ldots \ldots$ iii

Acronyms and Abbreviations $\ldots \ldots \ldots \ldots \ldots \ldots \ldots \ldots \ldots \ldots$

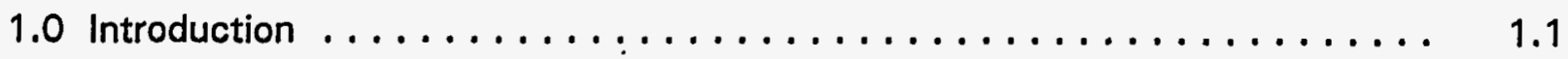

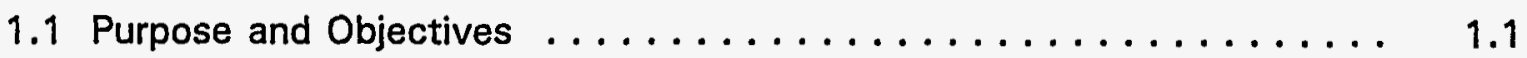

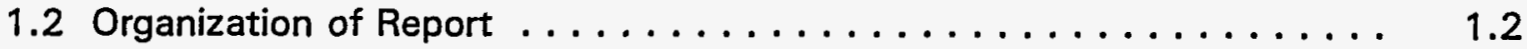

1.3 Project Overview ........................ 1.3

2.01995 Water Monitoring Program . . . . . . . . . . . . . . 2.1

2.1 Groundwater Monitoring . . . . . . . . . . . . . . . . . 2.1

2.2 Surface Water and Storm Water Monitoring . . . . . . . . . . 2.2

2.3 Analytical Program . . . . . . . . . . . . . . . . . . 2.3

2.4 Quality Assurance/Quality Control Program .............. 2.3

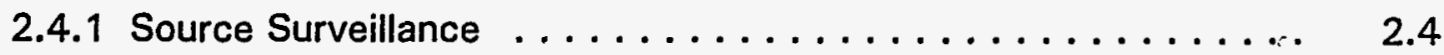

2.4.2 Laboratory Audit ...................... 2.4

3.0 Quality Assurance and Data Validation Summary . . . . . . . . . . . 3.1

3.1 Quality Assurance Criteria.$\ldots \ldots \ldots \ldots \ldots \ldots \ldots \ldots \ldots \ldots \ldots$

3.1 .1 Precision $\ldots \ldots \ldots \ldots \ldots \ldots \ldots \ldots \ldots \ldots \ldots \ldots \ldots \ldots \ldots$

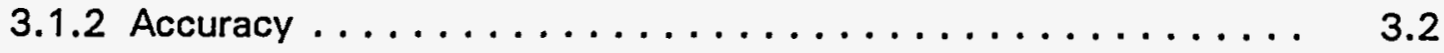

3.1.3 Representativeness . . . . . . . . . . . . . . . . 3.3

3.1 .4 Completeness $\ldots \ldots \ldots \ldots \ldots \ldots \ldots \ldots \ldots \ldots \ldots . \ldots \ldots$

3.1 .5 Comparability . . . . . . . . . . . . . . . . . 3.4

3.2 Data Validation Methods . . . . . . . . . . . . . . . . 3.4

3.3 Summary of Data Validation Results $\ldots \ldots \ldots \ldots \ldots \ldots \ldots \ldots$

3.4 Data Validation Results by Chemical Group . . . . . . . . . . . . . 3.6

3.4.1 Volatile Organic Compounds . . ............... 3.6

3.4.2 Semivolatile Organic Compounds . . . . . . . . . . . . 3.8

3.4.3 Pesticides/Polychlorinated Biphenyls . . . . . . . . . . 3.9

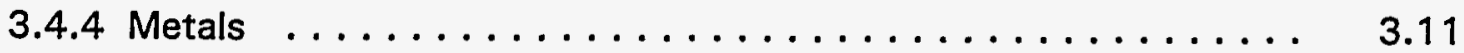

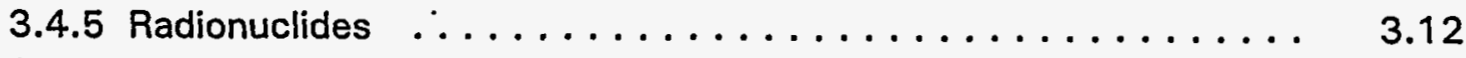

3.4.6 General Chemicals .................... 3.13 


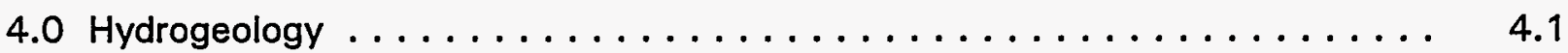

4.1 Regional Setting $\ldots \ldots \ldots \ldots \ldots \ldots \ldots \ldots \ldots \ldots \ldots \ldots \ldots \ldots \ldots$

4.2 LEHR Site Hydrogeology . . . . . . . . . . . . . . . . 4.2

4.3 Groundwater Elevations and Gradients . . . . . . . . . . . . . . 4.5

4.3.1 Seasonal/Annual Groundwater Elevations . . . . . . . . 4.5

4.3.2 Groundwater Elevations During $1995 \ldots \ldots \ldots \ldots \ldots \ldots \ldots . .6 .6$

4.3.3 Horizontal Groundwater Gradients During $1995 \ldots \ldots \ldots \ldots \ldots .4 .7$

4.3.4 Vertical Groundwater Gradients During $1995 \ldots \ldots \ldots \ldots .4 .8$

5.0 Groundwater Analytical Results $\ldots \ldots \ldots \ldots \ldots \ldots \ldots \ldots \ldots \ldots .1$

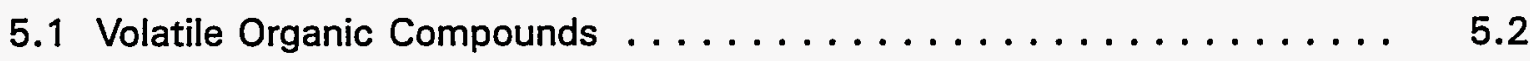

5.2 Semivolatile Organic Compounds $\ldots \ldots \ldots \ldots \ldots \ldots \ldots \ldots \ldots .4$

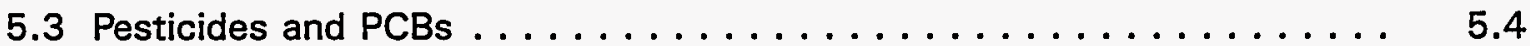

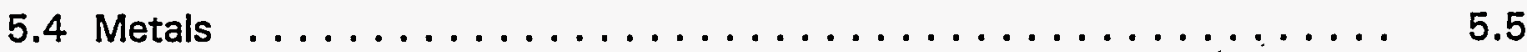

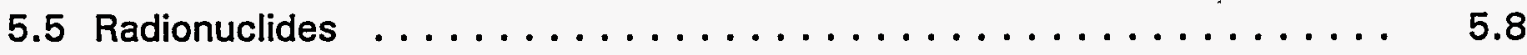

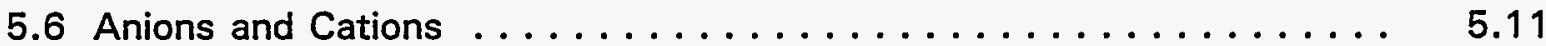

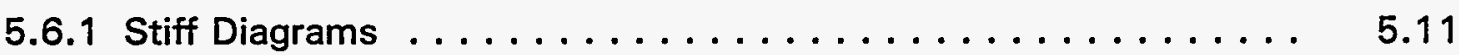

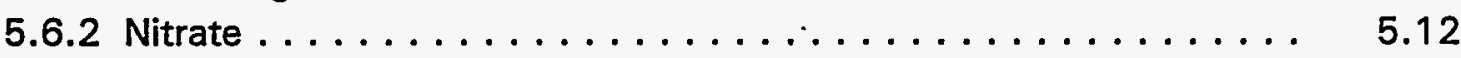

6.0 Surface Water and Storm Water Analytical Results . . . . . . . . . . . 6.1

6.1 Volatile Organic Compounds . ................... 6.1

6.2 Semivolatile Organic Compounds $\ldots \ldots \ldots \ldots \ldots \ldots \ldots \ldots \ldots, 6.2$

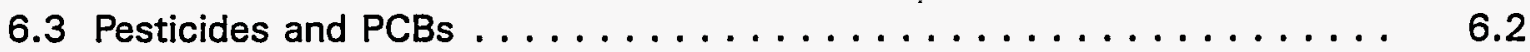

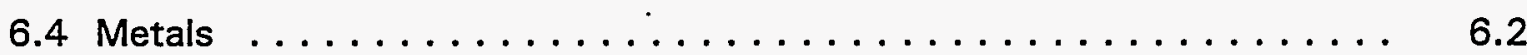

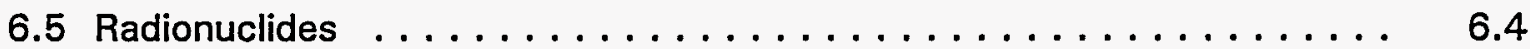

6.6 Anions and Cations $\ldots \ldots \ldots \ldots \ldots \ldots \ldots \ldots \ldots \ldots \ldots \ldots \ldots \ldots \ldots$

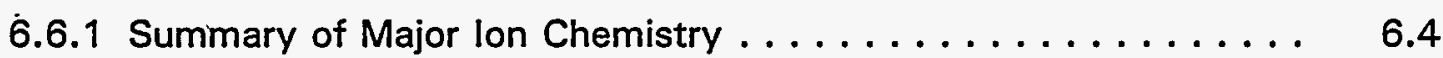

6.6 .2 Stiff Diagrams . . . . . . . . . . . . . .

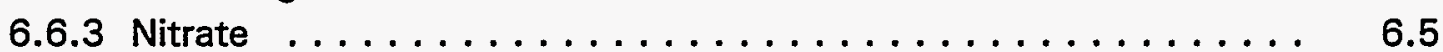

7.0 Statistical Analysis $\ldots \ldots \ldots \ldots \ldots \ldots \ldots \ldots \ldots \ldots \ldots \ldots \ldots .1$

7.1 Comparison Strategy . . . . . . . . . . . . . . . . 7.2

7.2 Parameters with No Results Above Detection Limit in 1995

Downgradient Samples (Class I) 
7.3 Parameters with Results Above Detection Limit in 1995 Downgradient

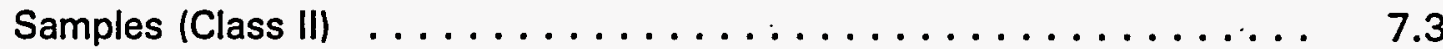

7.4 Results of Statistical Comparison of Downgradient to Background ......................... 7.4

7.4.1 Upper Tolerance Limit Comparison Results . . . . . . . . . . 7.4

7.4.2 Fisher's Exact Test Comparison Results . . . . . . . . . 7.5

7.5 Discussion of Statistical Analysis Results . . . . . . . . . . . 7.5

8.0 Evaluation of Water Monitoring Program $\ldots \ldots \ldots \ldots \ldots$

8.1 Analyte List $\ldots \ldots \ldots \ldots \ldots \ldots \ldots \ldots \ldots \ldots \ldots \ldots \ldots \ldots$

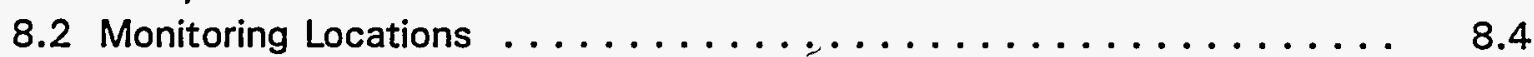

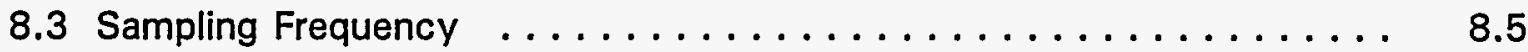

8.4 Analytical Techniques $\ldots \ldots \ldots \ldots \ldots \ldots \ldots \ldots \ldots \ldots \ldots$

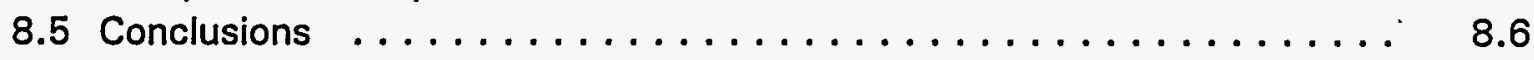

9.0 References $\ldots \ldots \ldots \ldots \ldots \ldots \ldots \ldots \ldots \ldots \ldots \ldots \ldots \ldots \ldots \ldots$

Appendix A - Groundwater and Trip Blank Analytical Results . . . . . . . . . A.1

Appendix B - Surface Water and Storm Water Analytical Results . . . . . . . . B.1

Appendix C - Time Series Tables . . . . . . . . . . . . . . . . C.1

Appendix D - Fall Quarter 1995 Water Monitoring $\ldots \ldots \ldots \ldots$. . . . . . .

Appendix E - Groundwater Elevation Data $\ldots \ldots \ldots \ldots \ldots \ldots \ldots$. . . . . . . . . 


\section{Figures}

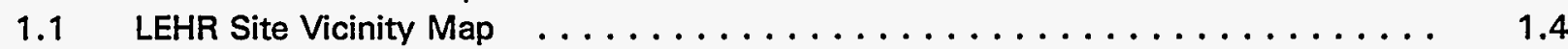

2.1 Location Map - Water Monitoring Points $\ldots \ldots \ldots \ldots \ldots \ldots \ldots \ldots \ldots$

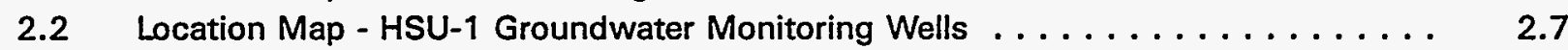

2.3 Location Map - HSU-2 Groundwater Monitoring Wells . . . . . . . . . . . . . . 2.9

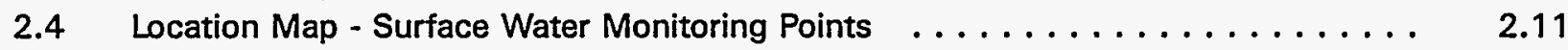

2.5 Location Map - Storm Water Runoff Monitoring Points $\ldots \ldots \ldots \ldots \ldots \ldots$

4.1 Average Elevation of HSU-1 and HSU-2 and Their Relationship to Regional

Stratigraphy and Hydrostratigraphy . . . . . . . . . . . . . 4.10

4.2 Location of West to East and North to South Cross-Sections of

Across the LEHR Site . . . . . . . . . . . . . . . . . . 4.11

4.3 West to East Cross-Section Across the LEHR Site . . . . . . . . . . . . . . . 4.13

4.4 North to South Cross-Section Across the LEHR Site . . . . . . . . . . . . . . 4.15

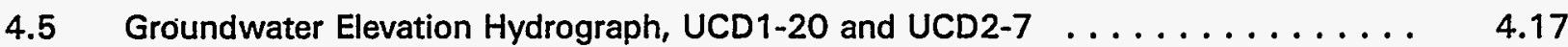

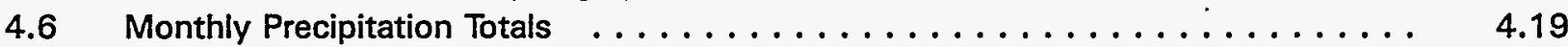

4.7 Groundwater Elevation Hydrograph, Selected HSU-1 Wells $\ldots \ldots \ldots \ldots \ldots \ldots$. . . . 4.21

4.8 Groundwater Elevation Hydrograph, Selected HSU-2 Wells . . . . . . . . . . . 4.23

4.9 Groundwater Elevation Hydrograph, UCD1-25, -34, and UCD2-2, -35 . . . . . . 4.25

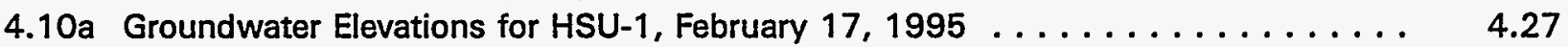

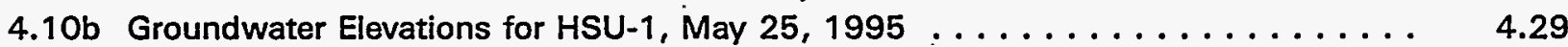

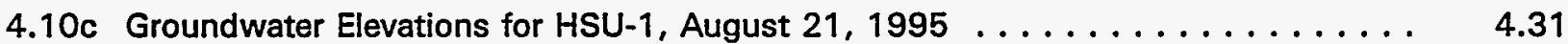

4.10d Groundwater Elevations for HSU-1, November 27, $1995 \ldots \ldots \ldots \ldots \ldots$. . . . . . 4.33

4.11a Groundwater Elevations for HSU-2, February 17, $1995 \ldots \ldots \ldots \ldots \ldots$

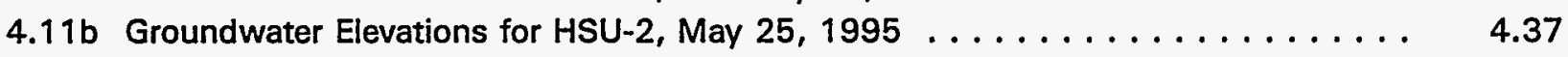

4.11c Groundwater Elevations for the HSU-2, August 31, $1995 \ldots \ldots \ldots$. . . . . . . . 4.39

4.11d Groundwater Elevations for HSU-2, November 27, $1995 \ldots \ldots \ldots \ldots \ldots$. . . . . . 4.41

4.12 Groundwater Elevation Hydrograph UCD1-1 and UCD2-16 . . . . . . . . . 4.43

4.13 Groundwater Elevation Hydrograph UCD1-3, -20, and UCD2-7 $\ldots \ldots \ldots \ldots .4 .45$

4.14 Groundwater Elevation Hydrograph UCD1-4 and UCD2-15 . . . . . . . . . . . 4.47

4.15 Groundwater Elevation Hydrograph UCD1-8, -13, and UCD2-14 . . . . . . . . . 4.49

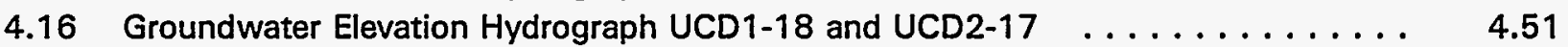

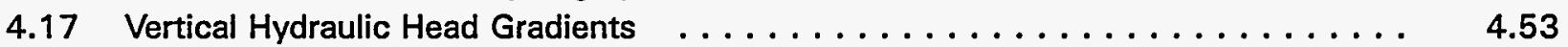

5.1 Chloroform Concentrations Detected in HSU-1 Monitoring Wells, $1995 \ldots \ldots .14$

5.2 Chloroform Concentrations Detected in HSU-2 Monitoring Wells, $1995 \ldots \ldots .14$

$5.3 \quad$ Nitrate Concentrations Detected HSU-1 Monitoring Wells, $1995 \ldots \ldots \ldots$

5.4 Nitrate Concentrations Detected in HSU-2 Monitoring Wells, $1995 \ldots \ldots \ldots . . \ldots 5$

5.5 Chromium Concentrations Detected in HSU-1 Monitoring Wells, 1995 . . . . . 5.16

5.6 Chromium Concentrations Detected in HSU-2 Monitoring Wells, 1995 . . . . . . 5.16

5.7 Tritium Concentrations Detected in HSU-1 Monitoring Wells, $1995 \ldots \ldots \ldots$

5.8 Tritium Concentrations Detected in HSU-2 Monitoring Wells, $1995 \ldots \ldots . \ldots .17$

5.9 Carbon-14 Concentrations Detected in HSU-1 Monitoring Wells, 1995 . . . . . 5.18

5.10 Carbon-14 Concentrations Detected in HSU-2 Monitoring Wells, $1995 \ldots \ldots . . .5$

5.11 Concentration Contour Plot - Chloroform in HSU-1, $1995 \ldots \ldots \ldots \ldots . \ldots . \ldots$

5.12 Concentration Contour' Plot - Chloroform in Upper Portion of HSU-2, $1995 \ldots \ldots .21$

5.13 Concentration Contour Plot - Chloroform in Middle Portion of HSU-2, $1995 \ldots 5.23$

5.14 Concentration Contour Plot - Nitrate in HSU-1, $1995 \ldots \ldots \ldots \ldots \ldots \ldots$ 
5.15 Concentration Contour Plot - Nitrate in Upper Portion of HSU-2, $1995 \ldots \ldots .27$

5.16 Concentration Contour Plot - Nitrate in Middle Portion of HSU-2, $1995 \ldots \ldots .29$

5.17 Concentration Contour Plot - Hexavalent Chromium in HSU-1, $1995 \ldots \ldots . \ldots . . .51$

5.18 Concentration Contour Plot - Hexavalent Chromium in Upper Portion of

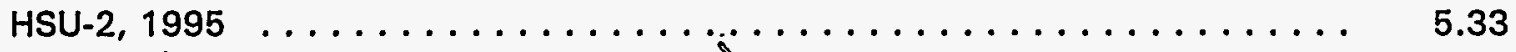

5.19 Concentration Contour Plot - Hexavalent Chromium in Middle Portion of

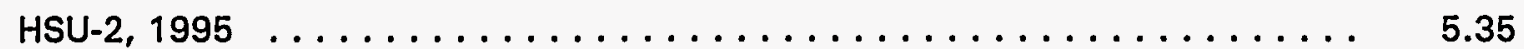

5.20 Concentration Contour Plot - Tritium in HSU-1, $1995 \ldots \ldots \ldots \ldots \ldots . \ldots \ldots$

5.21 Concentration Contour Plot - Tritium in Upper Portion of HSU-2, 1995 . . . . . . . 5.39

5.22 Concentration Contour Plot - Tritium in Middle Portion of HSU-2, $1995 \ldots \ldots .41$

5.23 Stiff Diagram Map - Groundwater Chemistry at Monitoring Wells . . . . . . . 5.43

5.24 Stiff Diagrams - HSU-1 Wells, Mean $1990-1995 \ldots \ldots \ldots \ldots \ldots \ldots \ldots \ldots . \ldots . \ldots . \ldots$

5.25 Stiff Diagrams - HSU-2 Wells, Mean $1990-1995 \ldots \ldots \ldots \ldots \ldots \ldots \ldots \ldots$

6.1 Chloroform Concentrations in Surface Water Samples, $1995 \ldots \ldots \ldots$

6.2 Total Trihalomethane Concentrations in Surface Water Samples, $1995 \ldots \ldots . \ldots$

6.3 Tritium Concentrations in Surface Water Samples, $1995 \ldots \ldots \ldots \ldots \ldots \ldots . \ldots .6 . \ldots$

6.4 Nitrate Concentrations in Surface Water Samples, $1995 \ldots \ldots \ldots \ldots \ldots$

6.5 Total Dissolved Solids in Surface Water Samples, $1995 \ldots \ldots \ldots \ldots \ldots . \ldots . \ldots 6$

6.6 Surface Water Monitoring Locations Mean $1990-1995 \ldots \ldots \ldots \ldots \ldots . \ldots$

7.1 Statistical Test Selection Flow Chart $\ldots \ldots \ldots \ldots \ldots \ldots \ldots \ldots \ldots$

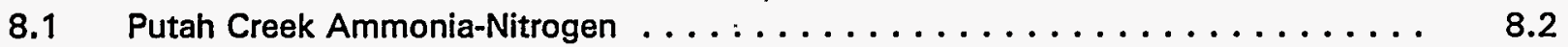

8.2 Concentrations of Nitrogen-Containing Compounds in UC Davis Wastewater Treatment Plant Outfall ....................... 8.3 


\section{Tables}

2.1 Groundwater Monitoring Parameters and Schedule for LEHR Site

Beginning Summer $1995 \ldots \ldots \ldots \ldots \ldots \ldots \ldots \ldots \ldots \ldots$

2.2 Summary of Water Monitoring - February through December $1995 \ldots \ldots$. . . .

2.18

Analytical Constituents for Groundwater Samples . . . . . . . . . . . . .

2.19

2.4 Analytical Constituents for Surface Water $\ldots \ldots \ldots \ldots \ldots \ldots \ldots \ldots \ldots \ldots$

2.5 Analytical Constituents for Storm Water Runoff Samples . . . . . . . . . .

2.20

2.6 Water Monitoring Constituents and Analytical Methods $\ldots \ldots \ldots \ldots \ldots \ldots \ldots$

2.21

3.2 Relative Percent Difference for Field Duplicates, Groundwater,

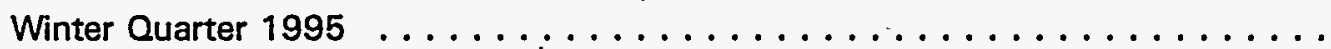

3.3 Relative Percent Difference for Field Duplicates, Groundwater,

Spring Quarter $1995 \ldots \ldots \ldots \ldots \ldots \ldots \ldots \ldots \ldots \ldots \ldots$

3.4 Relative Percent Difference for Field Duplicates, Groundwater,

Summer Quarter $1995 \ldots \ldots \ldots \ldots \ldots \ldots \ldots$. . . . . . . . . . . .

3.5 Relative Percent Difference for Field Duplicates, Groundwater,

Fall Quarter 1995 . . . . . . . . . . . . . . . . . . . . . . . . . . . . . . .

3.6 Relative Percent Difference for Field Duplicates, Groundwater, Surface Water,

Winter Quarter $1995 \ldots \ldots \ldots \ldots \ldots \ldots \ldots \ldots \ldots$

3.7 Relative Percent Difference for Field Duplicates, Groundwater, Surface Water, Spring Quarter $1995 \ldots \ldots \ldots \ldots \ldots \ldots \ldots \ldots \ldots$

3.8 Relative Percent Difference for Field Duplicates, Groundwater, Surface Water, Summer Quarter 1995 . . . . . . . . . . . . . . . . . . . . .

3.9 Relative Percent Difference for Field Duplicates, Groundwater, Surface Water,

Fall Quarter $1995 \ldots \ldots \ldots \ldots \ldots \ldots$. . . . . . . . . . . . . . . . . . .

3.10 Relative Percent Difference for Field Duplicate, Storm Water Runoff,

Winter Quarter $1995 \ldots \ldots \ldots \ldots \ldots \ldots \ldots \ldots$

3.24

3.11 Samples Matrix Spike and Duplicate Results Ranges of Accuracy and Precision ..

3.12 Analytical Laboratory Control Sample Spike Results Ranges of Accuracy . . . . .

3.13 Summary of Detections in Trip Blanks . . . . . . . . . . . . . . .

4.1 Hydrogeologic Contracts in the LEHR Site Wells . . . . . . . . . . . .

4.2 Comparison of HSU-1 Lateral Hydraulic Head Gradient and Groundwater

Flow Direction Determinations at the LEHR Site for Four 1995

Water-Level Measurement Surveys . . . . . . . . . . . . . . .

4.55

4.3 Comparison of HSU-2 Lateral Hydraulic Head Gradient and Groundwater

Flow Direction Determinations at the LEHR Site for Four 1995 Water-Level

Measurement Surveys . . . . . . . . . . . . . . . . .

4.4 Screened Intervals for Well Pairs . . . . . . . . . . . . . . . . . .

5.1 Numbers of Detections, Positive Results, and Maximum Values in

Groundwater, $1995 \ldots \ldots \ldots \ldots \ldots \ldots \ldots \ldots \ldots$

5.2 Summary of Detected Constituents in Groundwater, 1995 -

Volatile Organics . . . . . . . . . . . . . . . . . . . . .

5.3 Summary of Detected Constituents in Groundwater, 1995 -

Semivolatile Organic Compound's . . . . . . . . . . . . . . . 
5.4 Summary of Detected Constituents in Groundwater, 1995 -

Pesticides and PCBS . . . . . . . . . . . . . . . . . . .

5.5 Summary of Detected Constituents in Groundwater,

1995 - Metals . . . . . . . . . . . . . . . . . . . . . . . .

5.6 Summary of Detected Constituents in Groundwater, 1995 -

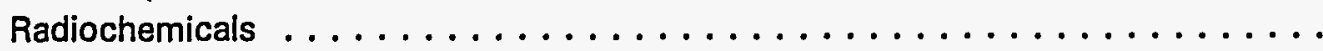

5.7 Summary of Detected Constituents in Groundwater, 1995 -

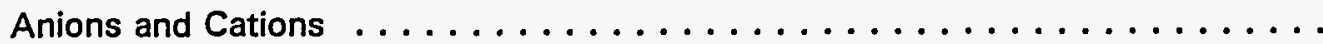

5.8 Summary of Detected Constituents in Groundwater, 1995 -

Other Parameters . . . . . . . . . . . . . . . . . . . . .

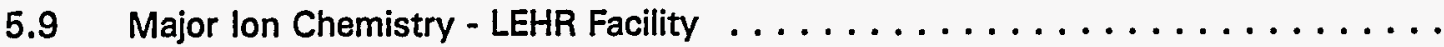

5.125

5.10 Major Ion Chemistry - LEHR Facility ....................

6.1 Number of Detections, Positive Results, and Maximum Values in

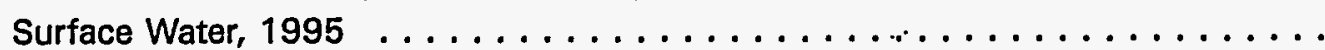

6.2 Summary of Detected Constituents in Surface Water and Storm Water,

1995, Volatile Organics . . . . . . . . . . . . . . . . . . . . . . . .

6.3 Summary of Detected Constituents in Surface Water and Storm Water, 1995, Semivolatile Organic Compounds . . . . . . . . . . . . . .

6.4 Summary of Detected Constituents in Surface Water and Storm Water, 1995, Pesticides and PCBs . . . . . . . . . . . . . . . . . . . .

6.5 Summary of Detected Constituents in Surface Water and Storm Water, 1995, Metals . . . . . . . . . . . . . . . . . . . . . . . . .

6.6 Summary of Detected Constituents in Surface Water and Storm Water, 1995, Radiochemicals . . . . . . . . . . . . . . . . . . .

6.7 Summary of Detected Constituents in Surface Water and Storm Water, 1995, Anions and Cations . . . . . . . . . . . . . . . . . . .

6.8 Summary of Detected Constituents in Surface Water and Storm Water, 1995, Other Parameters . . . . . . . . . . . . . . . . . .

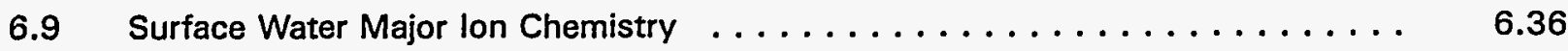

6.10 Surface Water Change Balance . . . . . . . . . . . . . . . . . 6.37

7.1 Summary of Samples and Detects for Two-Year Background and 1995 Downgradient Samples . . . . . . . . . . . . . . . . . . . . .

7.2 Parameters with at Least One Downgradient Result Above the Detection Limit . . . . . . . . . . . . . . . . . . . . .

7.3a Percent of Results Exceeding UTL in Downgradient HSU-1 Wells

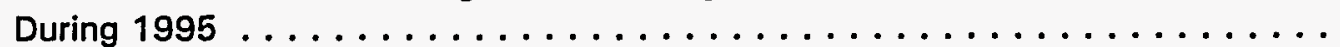

7.3b Percent of Results Exceeding UTL in Downgradient HSU-2 Wells

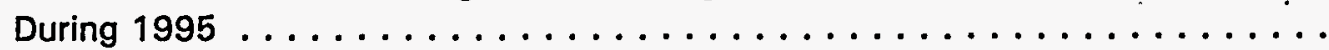

7.4a Probability the Results in Downgradient HSU-1 Wells During 1995 Could Be from Background . . . . . . . . . . . . . . . . .

7.4b Probability the Results in Downgradient HSU-2 Wells During 1995

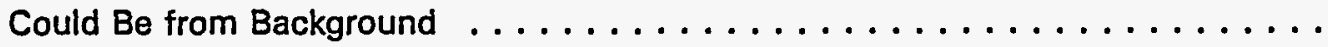

7.5 Parameters Above Detection Limit and Significantly Different

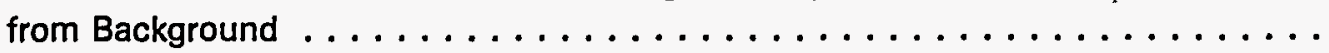

7.6 Regulatory Significance of Parameters That Exceeded Background 


\title{
List of Acronyms and Abbreviations
}

\author{
as $P$ \\ as phosphorus \\ as $\mathrm{N}$ \\ as nitrogen \\ bgs \\ CLP \\ below ground surface \\ (U.S. Environmental Protection Agency) Contract Laboratory Program \\ CLP SOW OLC \\ CLP SOW OLM \\ USEPA Contract Laboratory Program Statement of Work for Low \\ Concentrations for Organic Analysis \\ USEPA Contract Laboratory Program Statement of Work for Organic Analysis \\ Multi-media, Multi-concentration \\ CPT \\ cone penetrometer test \\ COA \\ Coordinator of Quality \\ CRDL \\ contract required detection limit \\ DOE \\ (U.S.) Department of Energy \\ DQO \\ data quality objectives \\ DWR \\ (State of California) Department of Water Resoürces \\ EC \\ Electrical Conductivity \\ Eh Redox Potential \\ FS \\ GC \\ GC/MS \\ HSU \\ Feasibility Study \\ gas chromatograph \\ gas chromatograph/mass spectrometer \\ hydrostratigraphic unit \\ IDL \\ instrument detection limit \\ ILC SOW \\ Statement of Work for Low Concentration Water Inorganics (USEPA Contract \\ Laboratory Program) \\ LAS \\ LEHR \\ Lockheed Analytical Services (Standard Operating Procedure) \\ $M C L$ \\ Laboratory for Energy-Related Health Research \\ Maximum Contaminant Level \\ MDA \\ $\mathrm{mg} / \mathrm{L}$ \\ $\mathrm{NIOSH}$ \\ NTU \\ PARCC \\ PCB \\ PCD \\ $\mathrm{pCi} / \mathrm{L}$ \\ PCU \\ Minimum Detectable Activity \\ milligrams per liter \\ National Institute for Occupational Safety and Health \\ nephelometric turbidity unit \\ precision, accuracy, representativeness, completeness, and comparability \\ polychlorinated biphenyls \\ Putah Creek Downstream \\ picocurie per liter \\ Putah Creek Upstream \\ PNL \\ Pacific Northwest Laboratory \\ PQL practical quantitation limit
}




$\begin{array}{ll}\text { QA } & \text { quality assurance } \\ \text { QAPJP } & \text { Quality Assurance Project Plan } \\ \text { QAPP } & \text { Quality Assurance Program Plan } \\ \text { QC } & \text { quality control } \\ \text { RI/FS } & \text { Remedial Investigation/Feasibility Study } \\ \text { RPD } & \text { relative percent difference } \\ \text { SOW } & \text { Statement of Work } \\ \text { STPO } & \text { Wastewater treatment plan outfall } \\ \text { SVOC } & \text { semivolatile organic compound } \\ \text { SWAT } & \text { Solid Waste Assessment Test } \\ \text { TDS } & \text { total dissolved solids } \\ \text { TEM } & \text { transient electromagnetics } \\ \text { TIC } & \text { tentatively identified constituents } \\ \text { TKN } & \text { Total Kjeldahl Nitrogen } \\ \text { UC } & \text { University of California } \\ \text { USEPA } & \text { United States Environmental Protection Agency } \\ \text { USGS } & \text { United States Geological Survey. } \\ \text { UTL } & \text { upper tolerance limit } \\ \mu \text { g/L } & \text { micrograms per liter }\end{array}$




\subsection{Introduction}

This 1995 Annual Water Monitoring Report presents analytical data collected between January 1995 and December 1995 at the Laboratory for Energy-Related Health Research (LEHR) located at the University of California (UC), Davis (Figure 1.1). This report has been prepared in compliance with the Water Monitoring Plan (PNL 1994) as revised for the LEHR site. The Water Monitoring Plan presents the sample collection, analysis, and quality assurance/quality control $(\mathrm{QA} / \mathrm{QC})$ procedures and reporting requirements for the water monitoring program. Water monitoring during 1995 was conducted in conjunction with the Remedial Investigation/Feasibility Study (RI/FS) currently being implemented at the LEHR site as part of a U.S. Department of Energy (DOE)-sponsored environmental restoration program. A detailed description of the RI/FS program being conducted at the LEHR site is presented in the Final Draft RI/FS Work Plan (DOE 1994a).

The Water Monitoring Plan (PNL 1994 and revisions) and this report are intended to comply with the DOE General Environmental Protection Program presented in DOE Order 5400.1. This Order specifies the purpose, scope, policy, and responsibilities for environmental protection and includes environmental monitoring and surveillance programs at all DOE facilities. This Order also specifies that water monitoring programs conducted by DOE will conform with federal, state, and local regulations.

\subsection{Purpose and Objectives}

The purpose of the water monitoring program at the LEHR site is to monitor the presence or absence of constituents of concern in groundwater and surface water and changes in their concentrations (or movement) with time. As stated in the Water Monitoring Plan (PNL 1994), the purpose of this 1995 Annual Water Monitoring Report is to present a more detailed analysis of the results contained in the 1995 quarterly data transmittals (PNL 1995a, 1995b) and a statistical evaluation of the data. The specific objectives of this report are to

- present an evaluation and discussion of water monitoring data for groundwater and surface water samples collected during 1995

- discuss changes in the hydrogeological conceptual model

- present data validation methods and results in relation to QA criteria

- identify data gaps and potential deficiencies in the water monitoring program

- evaluate the presence of constituents of concern in downgradient wells in relation to background wells 
- evaluate the presence of constituents of concern at surface water and storm water sampling locations.

This report is based on water monitoring data collected during the winter, spring, summer, and fall quarters of 1995.

\subsection{Organization of Report}

The organization of this report was developed based on the following documents:

- the Water Monitoring Plan (PNL 1994 and revisions)

- the 1994 Annual Water Monitoring Report for the LEHR site (DOE 1995)

- the document entitled "Long Term Ground Water Monitoring Program Guidance" (California Base Closure Environmental Committee 1994).

Groundwater investigation background material, including the history, objectives, and results of site investigations and water monitoring, is described in the remainder of Section 1. Section 2 describes the development of groundwater, surface water, analytical, and QA/OC programs conducted during 1995. Data validation methods and results are presented and discussed in relation to QA criteria in Section 3. Section 4 presents a discussion of the hydrogeologic setting and includes an update of the LEHR site conceptual hydrogeologic model. Section 4 also discusses horizontal and vertical groundwater gradients observed during 1995, including water level elevation contour maps and hydrographs of water level elevations and vertical gradients for well pairs.

Section 5 contains an evaluation and discussion of water monitoring data for groundwater samples collected during 1995 using summary tables, graphical illustrations, and Stiff diagrams for major anions and cations. In addition, summary tables of all historical measurements are provided for selected constituents. Similar tabular and graphical presentations support the discussion of surface water and storm water monitoring results in Section 6. Section 7 presents an evaluation of the constituents of concern in downgradient wells in relation to background wells based on applicable statistical comparisons. Section 8 presents an evaluation of data gaps and potential deficiencies in the monitoring program. References cited are listed in Section 9.

Five appendixes accompany this report. Appendix A contains tables of analytical data for groundwater samples collected during 1995. Appendix B presents tables of 1995 surface water and storm water analytical data. Appendix $\mathrm{C}$ provides historical data tables for each well and each analyte that has been regularly detected in at least one well. New wells are not included in Appendix $C$ because they have been sampled only once. Appendix $D$ presents the groundwater, surface water, analytical, and $Q A / Q C$ programs conducted during fall quarter 1995; groundwater elevation data, presented as a cumulative table, hydrographs, and contour maps, are included in Appendix $E$. 


\subsection{Project Overview}

DOE funded research on laboratory animals at the LEHR site from 1960 until 1985 . As a result of these studies, a variety of laboratory wastes, including radioactive, organic, and inorganic materials, were generated and disposed onsite. Portions of the site were previously used as the UC Davis landfill, which included two disposal units. A third disposal unit is located approximately 600 feet east of the site. These disposal units total approximately six acres (Dames \& Moore 1990a).

Additional waste management areas at the LEHR site include DOE and UC Davis disposal trenches, outdoor dog pens, domestic septic tanks, and radiologic effluent treatment systems. Impacts from these disposal and chemical dispensing areas have been evaluated during previous investigations or will be evaluated during the RI/FS program. Detailed descriptions of known waste-generating and disposal processes are included in the Environmental Survey Preliminary Report (DOE 1988). A discussion of potential environmental impacts at the LEHR site is presented in the Phase II Site Characterization Report (Dames \& Moore 1993) and the Draft RI/FS Work Plan (DOE 1994a).

The DOE and UC Davis have sponsored 11 environmental evaluations at the LEHR site and adjacent landfills. Two surveys, the initial Assessment Survey (Rockwell 1984) and the Environmental Survey Preliminary Report (DOE 1988), documented the potential environmental impacts from past operations at the LEHR site. The Phase I investigation (Wahler 1988, 1989) was an initial investigation of environmental impacts from past LEHR activities.

Two additional studies, the Solid Waste Assessment Test (SWAT) investigation (Dames \& Moore 1990a) and the Old UC Davis Landfill Additional Characterization (Dames \& Moore 1991a), addressed potential impacts from the former UC Davis landfill. A Contaminant Pathway Analysis was conducted to assess health risk exposure from the LEHR site on the main UC Davis campus (Dames \& Moore 1990b). Other environmental evaluations include the following: Evaluation of Potential Nitrate and Hexavalent Chromium Sources (Dames \& Moore 1990c); Waste Burial Trench Investigation (Dames \& Moore 1991b); Sediment and Surface Water Sampling in the South Fork of Putah Creek (Dames \& Moore 1990d); and CEQA Preliminary Study for Characterization (Dames \& Moore 1991c), which provided a summary of the previous investigations and presented results of additional soil and groundwater investigations at the LEHR site. A more comprehensive study for the LEHR site was presented in the Phase // Site Characterization (Dames \& Moore 1993).

The purpose of the RI/FS being conducted at the LEHR site is to develop the appropriate amount of information to support an informed risk management decision regarding the most appropriate remedial actions. This information includes data obtained from the Water Monitoring Program. 


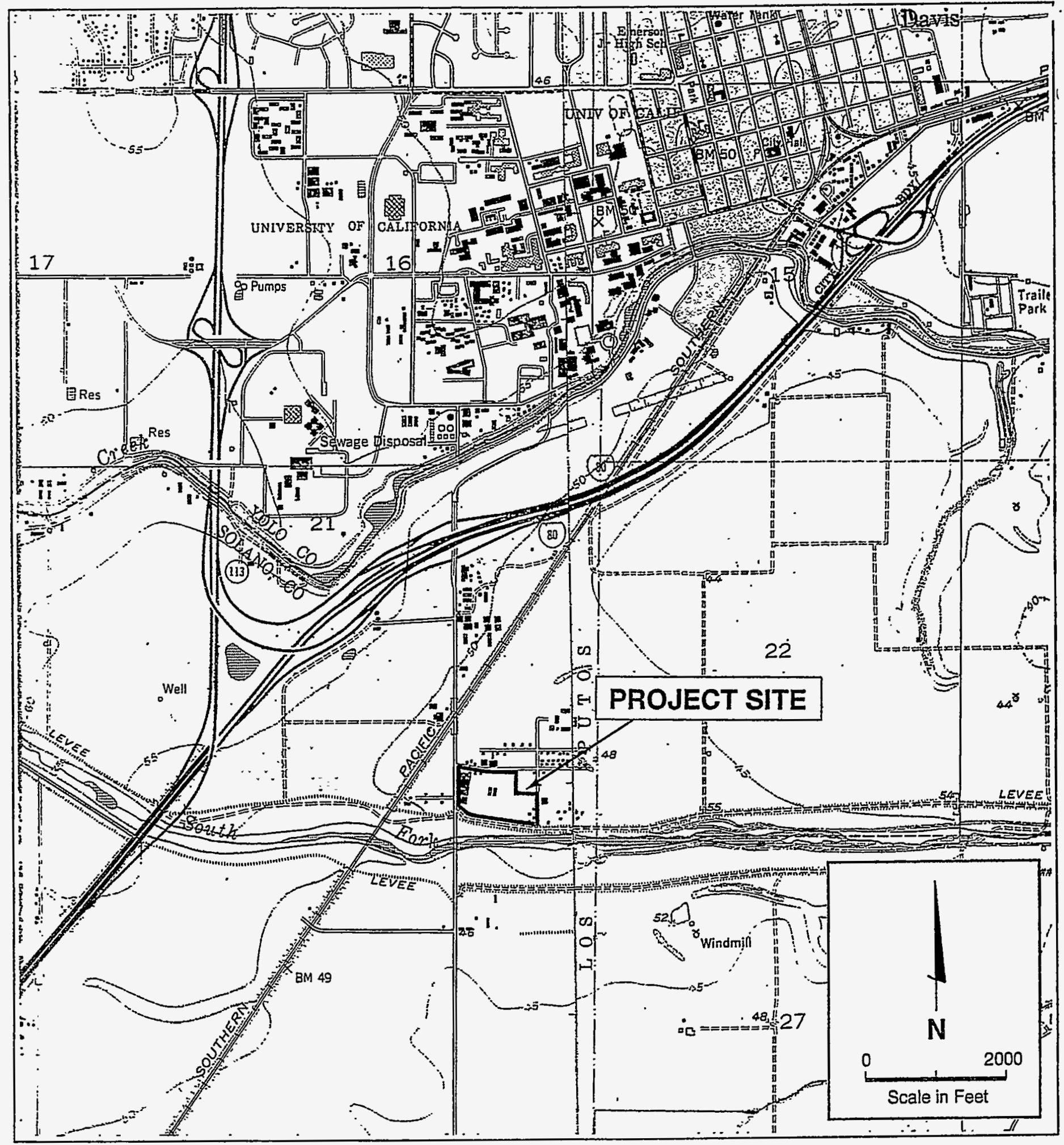

REFERENCE: USGS 7.5 Minute Series Quadrangles; Merritt, CA, 1981 and Davis, $C_{2} 1982$

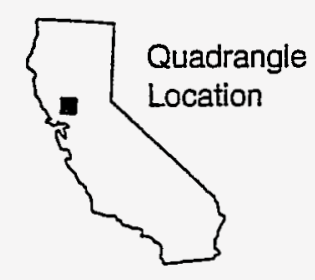

Figure 1.1. LEHR Site Vicinity Map 


\subsection{Water Monitoring Program}

Groundwater and surface water monitoring at the LEHR site began under the Phase II Site Characterization in November 1990 and, until 1995, consisted of quarterly sampling and analysis of groundwater and surface water and weekly groundwater level sounding. The Phase II water monitoring program, developed initially by UC Davis and DOE, was modified in the second half of 1995 so that a specific list of analytes and their sampling frequency was identified for each well. As a result, some analytes were eliminated from the program, while others were measured in specific wells in selected quarters. In addition, water levels were monitored monthly, and five new wells were drilled and added to the monitoring system. Since November 1990, monitoring has been conducted for DOE under the management of Pacific Northwest National Laboratory (PNNL). ${ }^{\text {(a) }}$

The following sections describe water monitoring activities conducted during the winter through fall quarters of 1995. Water monitoring locations are shown in Figure 2.1: Section 2.1 presents a brief description of the groundwater monitoring program; the surface water monitoring program, including storm water monitoring, is described in Section 2.2; and Section 2.3 presents a summary of the analytical program. A detailed description of groundwater and surface water monitoring procedures can be found in the Water Monitoring Plan (PNL 1994).

\subsection{Groundwater Monitoring}

The 1995 water monitoring program at LEHR was the same as that of 1994 for the first half of the year; groundwater samples were collected, field measurements were made, and samples were analyzed in the laboratory. All wells in the monitoring program were sampled each time, and samples from each well were analyzed for a complete set of analytes. In June 1995, DOE, UC Davis, and regulatory personnel agreed to modify the program to include fewer analytes and to sample wells at a reduced frequency, depending on the history of each well. The revised program applied to the 18 wells in the monitoring network and was implemented in the summer quarter. The modified sampling program is presented in Table 2.1, at the end of Section 2. During the summer and early fall, five additional monitoring wells were drilled. These.wells were sampled for the first time as part of the monitoring program during the fall quarter 1995.

- As described in previous annual reports, the wells are all completed within the uppermost aquifer beneath the LEHR site. The upper aquifer consists of 1) a fine-grained sequence called hydrostratigraphic unit one (HSU-1), which extends from the water table to approximately 80 feet below ground surface (bgs); and 2) a coarse-grained sequence that extends from 80 feet to about 135 feet bgs, called HSU-2. The upper aquifer is

(a) Pacific Northwest National Laboratory is operated by Battelle for the U.S. Department of Energy under Contract DE-AC06-76RLO 1830. 
bounded below by a thick clay sequence that acts as an aquitard between the upper and the second aquifers. Sediments in HSU-1 were deposited as discontinuous bands with alternating fine and coarse-grained materials. Because of the lateral discontinuity of the layers, HSU-1 is characterized by a low transmissivity and poor water-supply production capability. HSU-2, on the other hand, has a much higher transmissivity than HSU-1. The upper boundary of the first aquifer is the water table, which has fluctuated seasonally in the past from about 35 to 70 feet bgs. During 1995, the water table was high because of the high amount of precipitation in the winter and spring and fluctuated from a spring high of about 28 feet bgs to a summer low of about 48 feet bgs (based on UCD1-20). The hydrogeology of the site is discussed in more detail in Section 4.0.

At the start of 1995, the monitoring network included 13 wells completed in HSU-1 and five wells completed in HSU-2. Five other existing wells (UCD1-3, UCD1-5, UCD1-6, UCD1-8, and UCD1-91, are generally dry and are not included in the sampling network. They are used to measure water levels if the water table is sufficiently high to reach the screened interval in the wells. Well specifications and the 1995 sampling history for each well are presented in Table 2.2. Well UCD1/2-27 was completed with a Westbay monitoring system in which seven different screened zones can be sampled. This well was constructed to determine the nature of groundwater chemistry as a function of vertical position in the upper aquifer. Three of the seven sample zones are in HSU-1 and four are in HSU-2, as indicated by the number designation in the well name. Wells with a "1" after the UCD designation (e.g., UCD 1-20) are completed in HSU-1, and wells with a " 2 " are completed in HSU-2 (e.g., UCD2-14). Figures 2.2 and 2.3 show these well locations. Two wells, UCD1-18 and UCD2-17, are located upgradient of the LEHR site and are considered background wells for HSU-1 and HSU-2, respectively. New wells UCD1-34 and UCD2-35 are located immediately south of the LEHR site and are also considered upgradient wells, but have not been designated background wells.

\subsection{Surface Water and Storm Water Monitoring}

The existing surface water monitoring program at the LEHR site began in November 1990 and is conducted at three locations along the South Fork of Putah Creek (see Figure 2.41. Surface water samples are collected quarterly in conjunction with groundwater monitoring from three sampling points: point PCU, located upstream of the LEHR site; point PCD, located downstream of the LEHR site and UC Davis property; and point STPO, located at the outfall of the UC Davis wastewater treatment plant, which discharges into the South Fork of Putah Creek between points PCU and PCD, upstream of the LEHR site. The outfall is located approximately one mile from the UC Davis wastewater treatment plant on the main campus. A listing of surface water samples collected during 1995 is provided in Table 2.2.

Storm water runoff monitoring at the LEHR site began in the fall of 1994 . Storm water samples are scheduled to be collected twice each year-once at the beginning of the rainy season (fall or early winter) and once near the end of the rainy season (late winter or early spring). During 1995, storm water samples were collected only during the winter. Grab 
samples were collected from two locations onsite during each event (Figure 2.5). One location, SWL-1, is the lift station located on the western edge of the site; the second location, SWL-2, is a storm drain located west of the western dog pens.

Storm water runoff at the site is collected in storm drains on the western portion of the site only. The runoff is directed to the lift station where it is pumped across Old Davis Road and channeled to Putah Creek. Rain water that lands on the remaining portions of the site either ponds and infiltrates or is directed to the UC Davis wastewater treatment plant.

\subsection{Analytical Program}

Tables 2.3, 2.4, and 2.5 summarize the requested chemical and radiological analyses of groundwater, surface water, and storm water runoff samples (respectively) collected during 1995 from the water monitoring locations discussed. Methods for each analysis are listed in Table 2.6. Water samples were collected according to procedures described in the Water Monitoring Plan (PNL 1994). Samples were shipped daily via Federal Express for overnight delivery to Lockheed Analytical Services (Lockheed) in Las Vegas, Nevada. Immediately upon receipt of the sample shipment, Lockheed documented the condition of the sample containers on the chain-of-custody form and faxed a copy to the task manager. If the condition of the sample was acceptable, Lockheed was given approval to begin analyses.

\subsection{Quality Assurance/Quality Control Program}

The overall quality assurance (QA) objective is to sample and analyze water from the LEHR site in a manner that ensures that technical data generated during the monitoring program are accurate and representative, able to withstand scientific and legal scrutiny, and useful for evaluating site conditions and remedial alternatives. The criteria commonly used to specify QA goals are precision, accuracy, representativeness, completeness, and comparability (PARCC). Precision is determined by analyzing and evaluating sample duplicates. Accuracy is determined by analyzing spiked samples. Representativeness is determined by evaluating the results of trip blanks, laboratory method blanks, blind duplicate samples, and laboratory duplicate samples. Comparability is achieved through standard sampling procedures, analytical methods, and units of measurement. Completeness is defined as the percentage of valid data relative to the total number of analytes.

An evaluation of the PARCC parameters is accomplished through data validation. The purpose of the data validation is to verify that data meet data quality objectives (DQOs) and $Q A$ criteria required for the water monitoring program, risk assessment, and the objectives set forth in the Quality Assurance Project Plan (QAPjP). The QAPjP is presented as 
an appendix in both the Water Monitoring Plan (PNL 1994) and the Final Draft RI/FS Work Plan (DOE 1994a). A summary of data validation results is presented in Section 3.

One source surveillance of field activities and one laboratory audit were conducted by the LEHR QA Representative and a LEHR Technical Representative to verify compliance to the appropriate requirements during the reporting period. Both of these activities were performed in accordance with PNNL Quality Instruction QP-05, Revision 0, "Supplier and Hanford Contractor Audits." Details of each of these verification activities are described in the following paragraphs.

\subsubsection{Source Surveillance}

The source surveillance of field activities in support of the LEHR Groundwater Monitoring Program was performed December 4-5, 1995. The surveillance was based on the requirements delineated in the Amendment to Water Monitoring Plan as revised in August 1995.

During this source surveillance, three findings and two observations were identified. The two observations identified during the surveillance precipitated revisions to the Water Monitoring Plan. IT Corporation provided timely and appropriate corrective action responses to these findings and observations. Following verification of implementation of the corrective action through review of field memoranda by PNNL $Q A$, the source surveillance was closed.

\subsubsection{Laboratory Audit}

An audit was conducted on December 6-7, 1995, to verify that Lockheed Analytical Services (LAS) has effectively implemented the PNNL QA requirements specified in the Novated Laboratory Services Agreement, Contract No. 241303-A-18 for the LEHR project, and the $Q A$ requirements specified within the Lockheed Quality Assurance Management Plan, Revision 3, and applicable administrative and technical procedures.

Seven findings and three observations were identified during the audit. Lockheed Analytical Services provided timely corrective action responses including evidence of completion in many cases. Following review of the corrective action responses and the evidence of completion submitted by Lockheed, the audit was closed. 

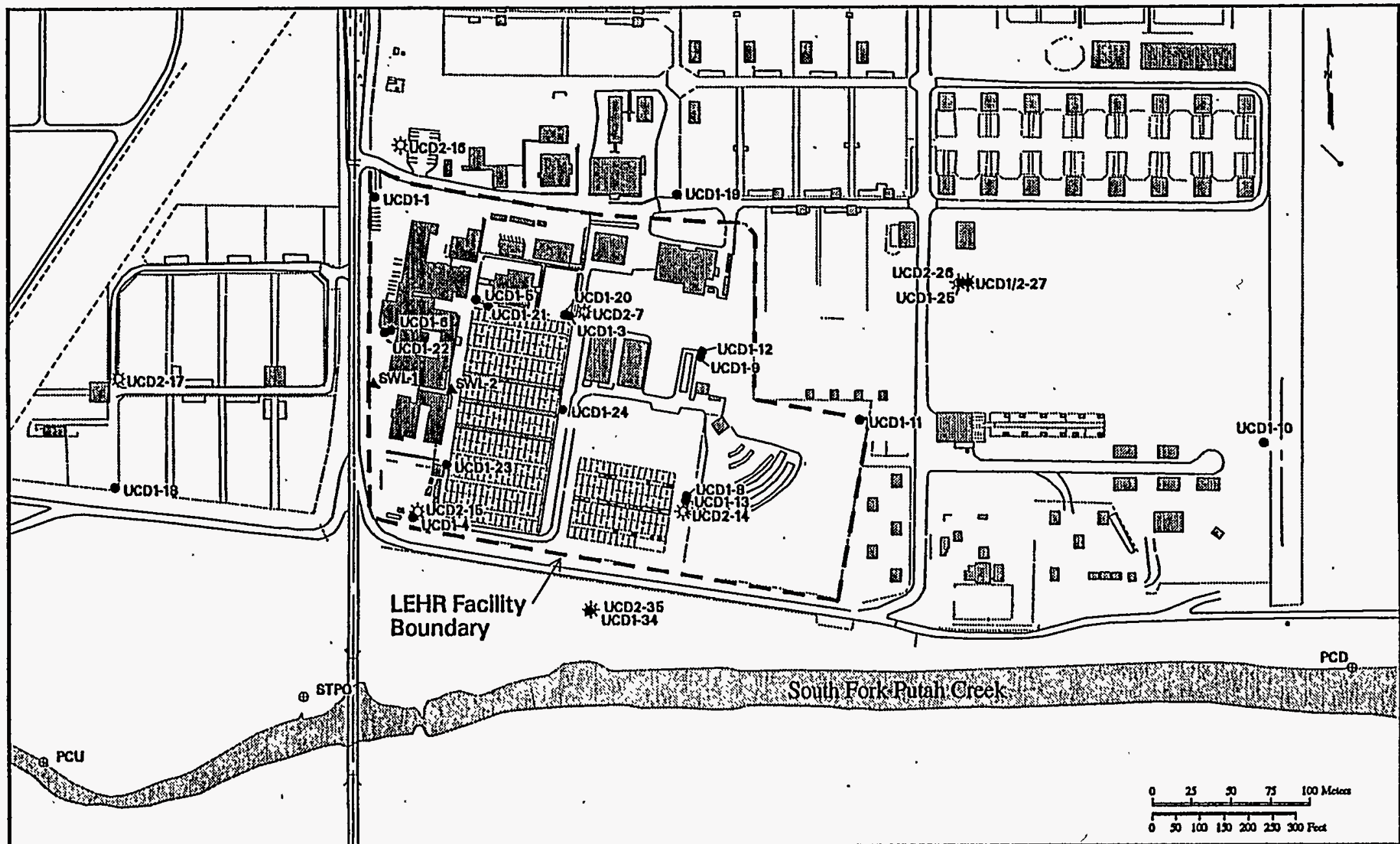

\section{EXPLANATION}

- HSU-1 Monitoring Well

HSU-2 Monitoring Well

- Surface Water Monitoring Point

\ Storm Water Runoff Monitoring Point

Figure 2.1. Location Map - Water Monitoring Points 


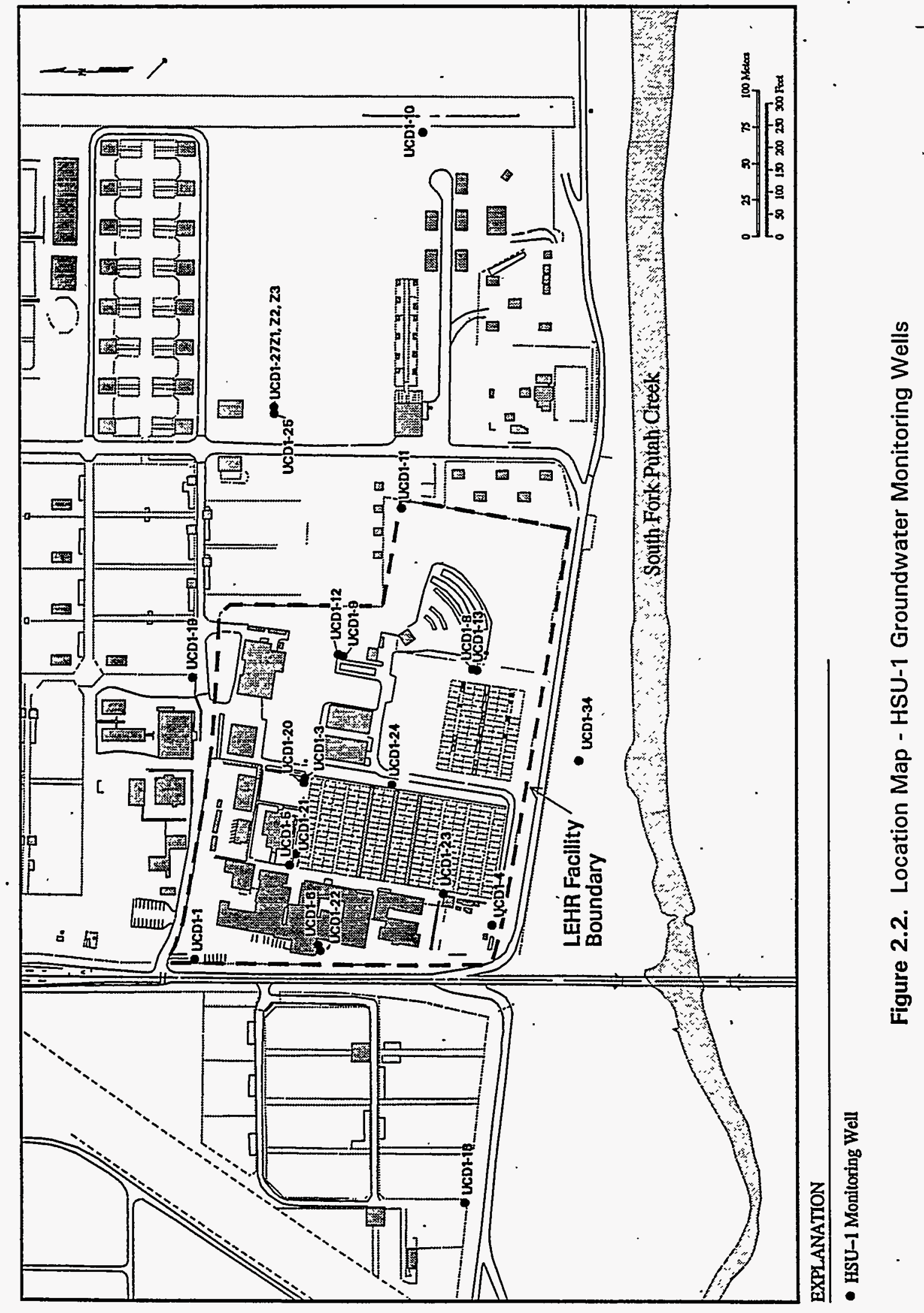




\section{.}




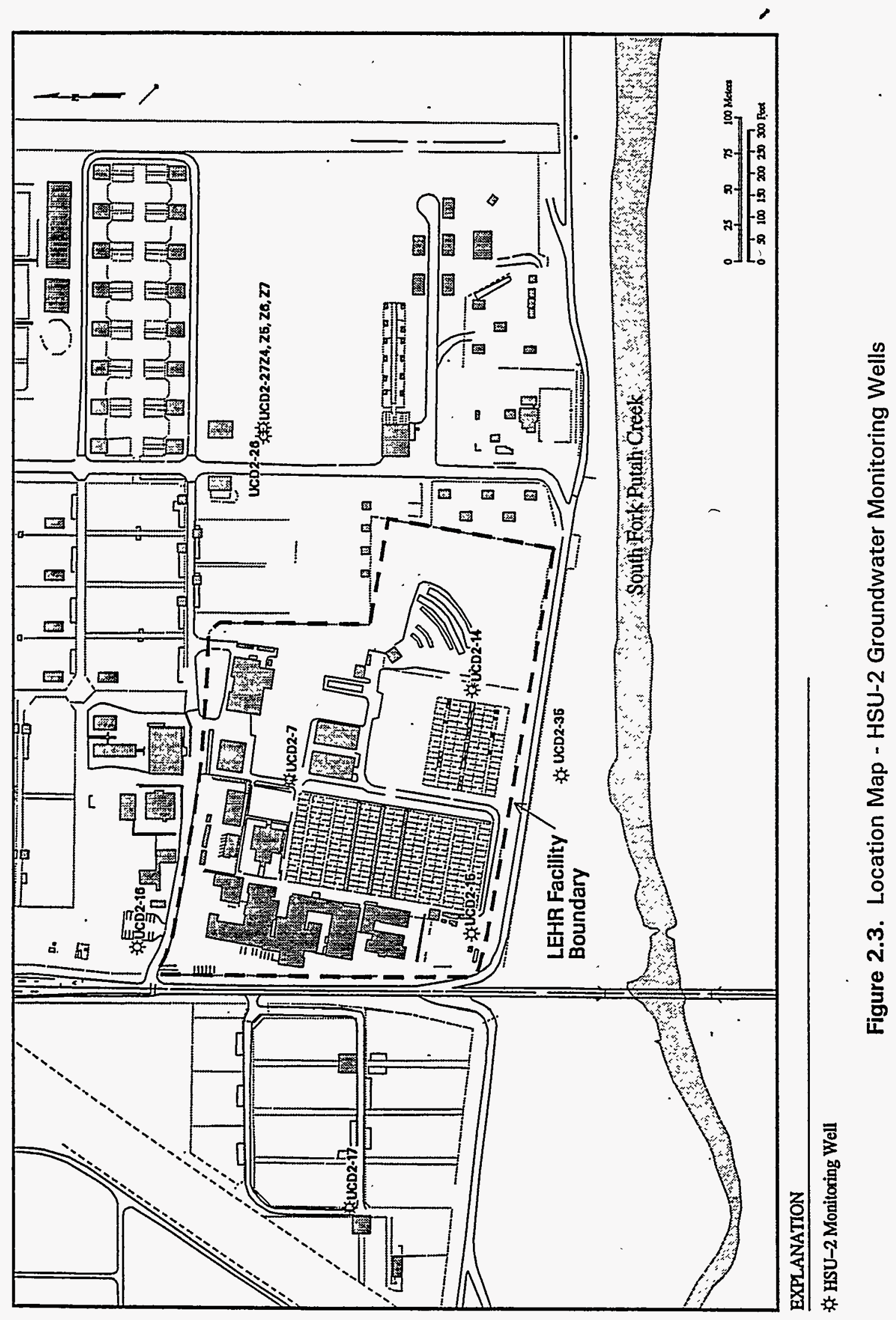




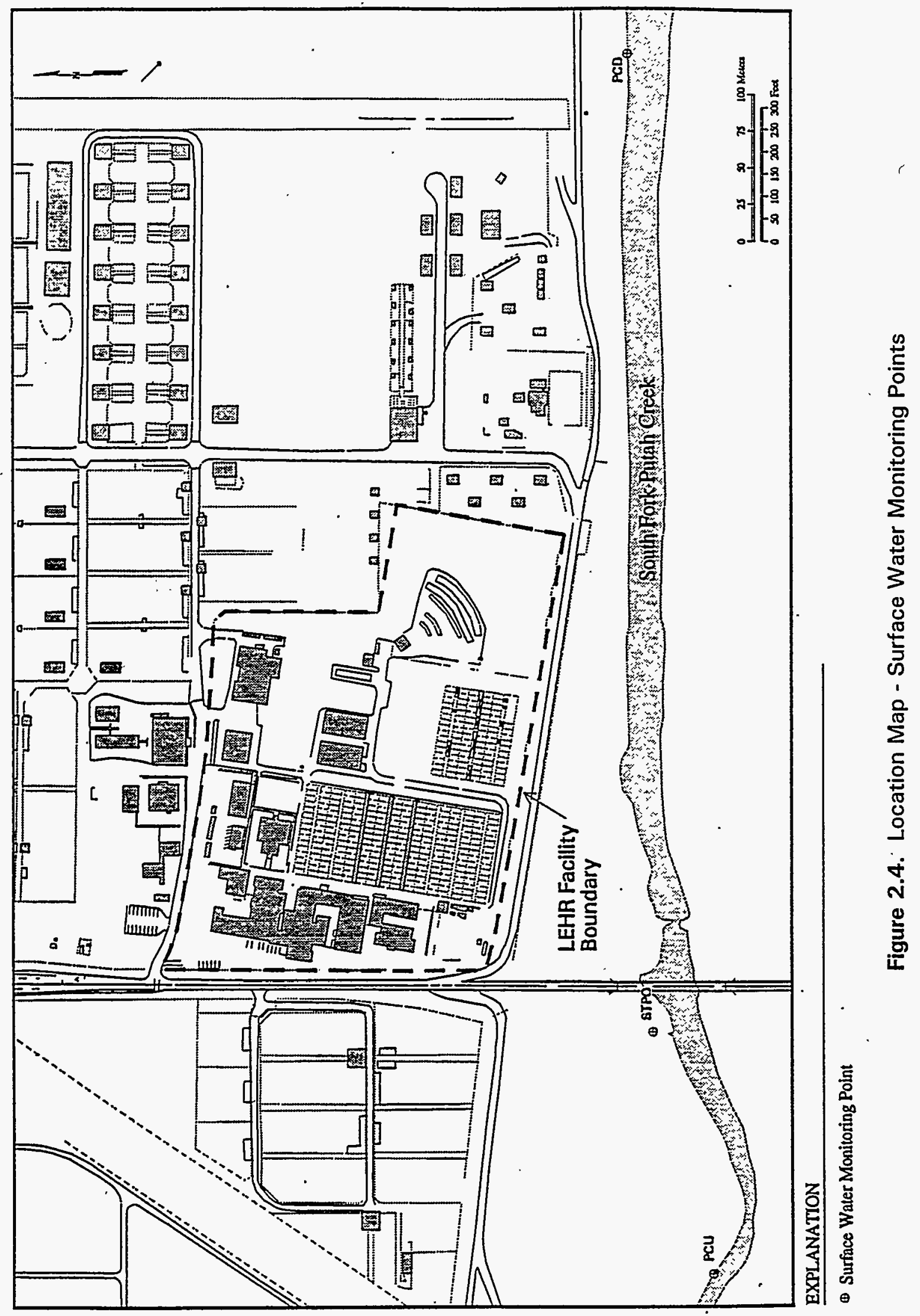





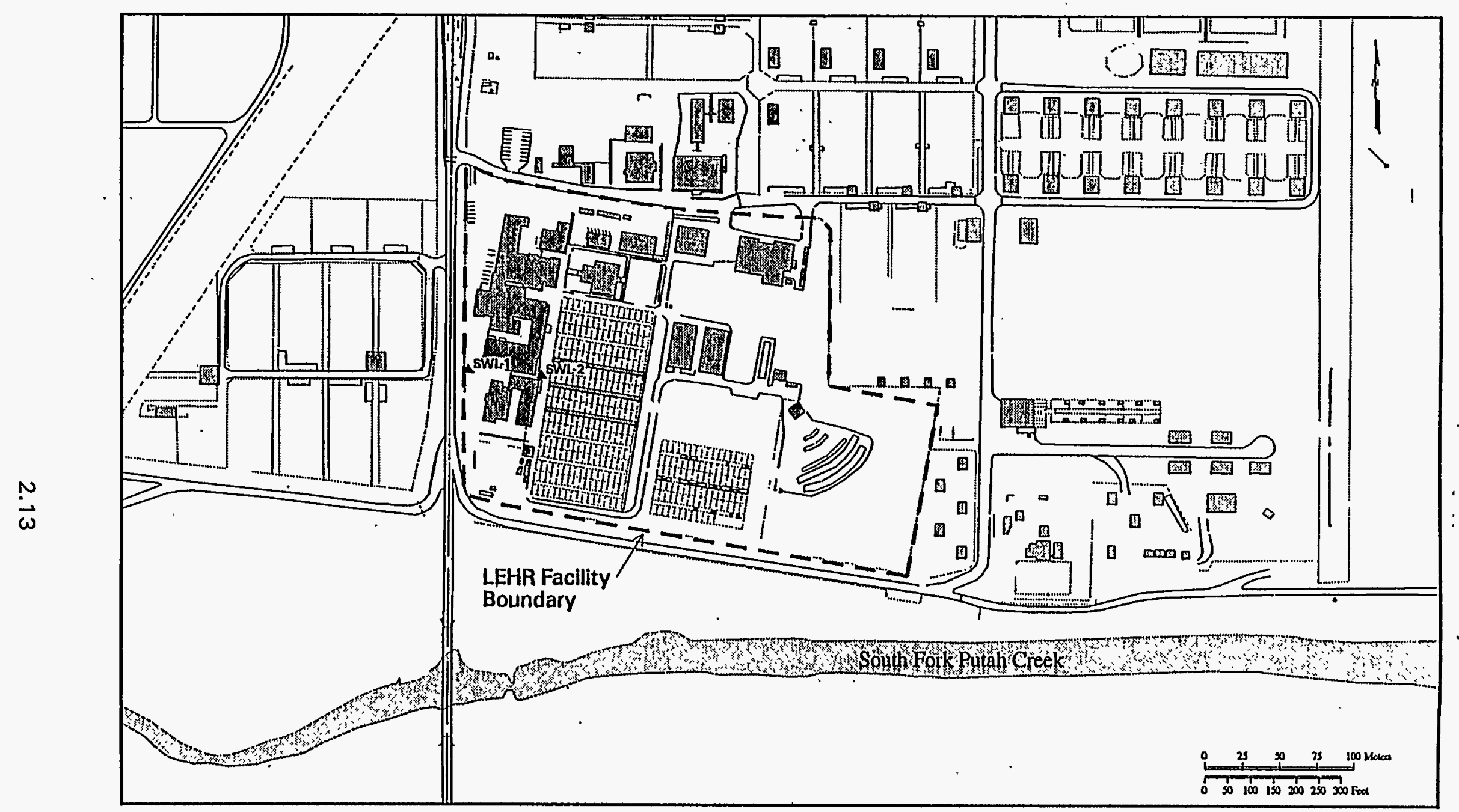

EXPLANATION

- Storm Water Runoff Monitoring Point

Figure 2.5. Location Map - Storm Water Runoff Monitoring Points 
Table 2.1. Groundwater Monitoring Parameters and Schedule for LEHR Site Beginning Summer $1995^{(\mathrm{a})}$

\begin{tabular}{|c|c|c|c|c|c|c|c|c|c|c|c|c|c|c|c|c|}
\hline \multirow[b]{3}{*}{ Well } & \multicolumn{16}{|c|}{ Analyte/Analysis } \\
\hline & \multicolumn{9}{|c|}{ Radiological Analytes } & \multirow[b]{2}{*}{ vocs ${ }^{(b)}$} & \multirow[b]{2}{*}{ SVOCs ${ }^{(0)}$} & \multirow[b]{2}{*}{ Metals } & \multirow[b]{2}{*}{ Nitrate } & \multirow[b]{2}{*}{$\begin{array}{c}\text { Chromium } \\
\text { (hexavalent) }\end{array}$} & \multirow[b]{2}{*}{ TDS } & \multirow[b]{2}{*}{$\begin{array}{r}\text { Pesticides } \\
\text { \& PCBs }\end{array}$} \\
\hline & Gamma & Tritium & C-14 & Sr-90 & Ra-226 & Pu-241 & Am-241 & $\begin{array}{l}\text { Gross } \\
\text { beta }\end{array}$ & $\begin{array}{l}\text { Gross } \\
\text { alpha }\end{array}$ & & & & & & & \\
\hline $\begin{array}{l}\text { UCD1-18 } \\
\text { UCD1-1 } \\
\text { UCD1-4 } \\
\text { UCD1-10 } \\
\text { UCD1-11 } \\
\text { UCD1-12 } \\
\text { UCD1-13 } \\
\text { UCD1-19 } \\
\text { UCD1-20 } \\
\text { UCD1-21 } \\
\text { UCD1-22 } \\
\text { UCD1-23 } \\
\text { UCD1-24 } \\
\text { UCD1-25 } \\
\text { UCD1-34 }\end{array}$ & $\begin{array}{l}A \\
\cdot \\
0 \\
A \\
A \\
0 \\
0 \\
A \\
S \\
A \\
S \\
S \\
A \\
Q \\
0\end{array}$ & $\begin{array}{l}\text { A } \\
. \\
0 \\
A \\
A \\
0 \\
0 \\
A \\
S \\
A \\
S \\
S \\
A \\
0 \\
0\end{array}$ & $\begin{array}{l}\text { A } \\
: \\
\vdots \\
\vdots \\
0 \\
0 \\
\vdots \\
\text { S } \\
\text { A } \\
\vdots \\
\text { S } \\
\text { A } \\
0 \\
0\end{array}$ & 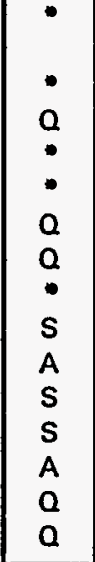 & $\begin{array}{l}: \\
: \\
0 \\
: \\
0 \\
0 \\
\vdots \\
\text { S } \\
\text { A } \\
\text { S } \\
\text { S } \\
\text { A } \\
0 \\
0\end{array}$ & $\begin{array}{l}: \\
: \\
: \\
: \\
: \\
: \\
: \\
\mathrm{A} \\
\mathrm{S} \\
: \\
\mathrm{a} \\
\mathrm{a}\end{array}$ & $\begin{array}{l}: \\
: \\
: \\
: \\
: \\
: \\
: \\
\mathrm{A} \\
\mathrm{S} \\
: \\
\mathrm{Q} \\
\mathrm{Q}\end{array}$ & $\begin{array}{l}A \\
- \\
0 \\
A \\
A \\
0 \\
0 \\
A \\
S \\
A \\
S \\
S \\
A \\
Q \\
0\end{array}$ & $\begin{array}{l}A \\
- \\
0 \\
A \\
A \\
0 \\
0 \\
A \\
S \\
A \\
S \\
S \\
A \\
0 \\
0\end{array}$ & $\begin{array}{l}\text { A } \\
. \\
0 \\
0 \\
A \\
0 \\
0 \\
A \\
A \\
A \\
* \\
A \\
A \\
0 \\
\dot{0}\end{array}$ & $\begin{array}{l}\text { A } \\
: \\
\text { A } \\
\text { A } \\
\text { A } \\
\text { A } \\
: \\
\text { A } \\
: \\
: \\
: \\
0 \\
0\end{array}$ & 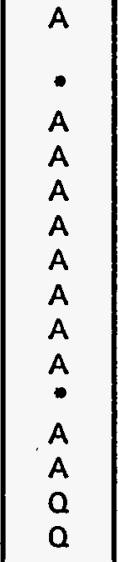 & $\begin{array}{l}S \\
S \\
Q \\
0 \\
S \\
0 \\
A \\
A \\
A \\
A \\
: \\
: \\
A \\
Q \\
Q\end{array}$ & $\begin{array}{l}\text { S } \\
\text { s } \\
0 \\
0 \\
\text { S } \\
0 \\
0 \\
0 \\
\text { S } \\
A \\
: \\
A \\
A \\
0 \\
0\end{array}$ & $\begin{array}{l}\text { A } \\
: \\
0 \\
0 \\
A \\
0 \\
0 \\
A \\
A \\
A \\
S \\
A \\
A \\
\text { Q } \\
0\end{array}$ & $\begin{array}{l}\text { A } \\
: \\
0 \\
A \\
A \\
0 \\
0 \\
A \\
A \\
: \\
\vdots \\
A \\
0 \\
0\end{array}$ \\
\hline $\begin{array}{l}\text { UCD2-17 } \\
\text { UCD2-7 } \\
\text { UCD2-14 } \\
\text { UCD2-15 } \\
\text { UCD2-16 } \\
\text { UCD2-35 } \\
\text { UCD2-26 } \\
\text { UCD1/2-27(d) }\end{array}$ & $\begin{array}{l}\text { A } \\
\text { s } \\
a \\
0 \\
S \\
0 \\
0 \\
a\end{array}$ & $\begin{array}{l}A \\
s \\
a \\
0 \\
S \\
0 \\
0 \\
a\end{array}$ & $\begin{array}{l}\text { A } \\
\text { S } \\
0 \\
\vdots \\
0 \\
0 \\
0\end{array}$ & $\begin{array}{l}- \\
s \\
0 \\
0 \\
s \\
0 \\
0 \\
0\end{array}$ & $\begin{array}{l}- \\
s \\
0 \\
0 \\
s \\
0 \\
0 \\
0\end{array}$ & $\begin{array}{l}: \\
: \\
0 \\
0 \\
0 \\
0 \\
0\end{array}$ & $\begin{array}{l}: \\
: \\
0 \\
: \\
0 \\
0 \\
0\end{array}$ & $\begin{array}{l}\text { A } \\
\text { S } \\
0 \\
0 \\
S \\
0 \\
0 \\
0\end{array}$ & $\begin{array}{l}A \\
\text { s } \\
0 \\
0 \\
S \\
0 \\
0 \\
0\end{array}$ & $\begin{array}{l}A \\
A \\
0 \\
0 \\
S \\
0 \\
0 \\
0\end{array}$ & $\begin{array}{l}\text { A } \\
* \\
\text { A } \\
\text { A } \\
\text { a } \\
0 \\
0\end{array}$ & $\begin{array}{l}\text { A } \\
\text { A } \\
0 \\
A \\
0 \\
Q \\
Q \\
Q\end{array}$ & $\begin{array}{l}S \\
s \\
0 \\
0 \\
S \\
0 \\
0 \\
0\end{array}$ & $\begin{array}{l}s \\
s \\
Q \\
Q \\
Q \\
0 \\
0 \\
0\end{array}$ & $\begin{array}{l}A \\
A \\
0 \\
0 \\
0 \\
0 \\
0 \\
0\end{array}$ & $\begin{array}{l}A \\
A \\
0 \\
Q \\
* \\
0 \\
0 \\
0\end{array}$ \\
\hline \multicolumn{17}{|c|}{$\begin{array}{l}\mathrm{A}=\text { annual sampling (February); } \mathrm{S}=\text { semi-annual sampling (February and August); } \mathrm{Q}=\text { quarterly sampling (Feb., May, Aug., and Nov.,); }{ }^{\bullet}=\text { sample not } \\
\text { analyzed for constituent }\end{array}$} \\
\hline
\end{tabular}


Table 2.2. Summary of Water Monitoring - February through December 1995

\begin{tabular}{|c|c|c|c|c|c|c|c|c|}
\hline $\begin{array}{l}\text { Well or } \\
\text { Sample } \\
\text { Location }\end{array}$ & $\begin{array}{c}\text { Total } \\
\text { Depth } \\
\text { (feet bgs) }\end{array}$ & $\begin{array}{l}\text { Screened } \\
\text { Interval } \\
\text { (feet bgs) }\end{array}$ & HSU & $\begin{array}{c}\text { Date } \\
\text { Completed }\end{array}$ & $\begin{array}{c}\text { Winter } \\
1995 \\
2 / 14-2 / 23\end{array}$ & $\begin{array}{c}\text { Spring } \\
1995 \\
5 / 16-5 / 25\end{array}$ & $\begin{array}{c}\text { Summer } \\
1995 \\
8 / 23-8 / 31\end{array}$ & $\begin{array}{c}\text { Fall } \\
1995 \\
11 / 28-12 / 12\end{array}$ \\
\hline UCD1-1 & 56.5 & $46.5-56.5$ & 1 & $10 / 09 / 87$ & $\mathbf{s}$ & $\mathrm{S}^{\left({ }^{(a)}\right.}$ & $R$ & $\bar{R}$ \\
\hline UCD1-4 & 57.2 & $45-55$ & 1 & $10 / 14 / 87$ & S & $\mathrm{s}$ & $R$ & $\bar{R}$ \\
\hline UCD2-7 & 90 & $80-90$ & 2 & $11 / 05 / 87$ & $\mathrm{~S}^{(\mathrm{a})}$ & S & $\bar{R}$ & - \\
\hline UCD1-10 & 70.0 & $54-69$ & 1 & $10 / 11 / 89$ & $\mathrm{~s}$ & $S$ & $\bar{R}$ & $\bar{R}$ \\
\hline UCD1-11 & 66.5 & $50-65$ & 1 & $10 / 17 / 89$ & $\mathrm{~s}$ & 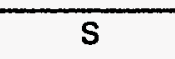 & $\mathbf{R}$ & - \\
\hline UCD1-12 & 65.0 & $49.5-64.5$ & 1 & $10 / 19 / 89$ & $\mathrm{~s}$ & 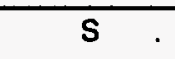 & $\mathrm{R}^{(\mathrm{a})}$ & $R^{(\mathrm{d})}$ \\
\hline UCD1-13 & 65.0 & $50-65$ & 1 & $10 / 26 / 89$ & $\mathrm{~s}$ & s & $R$ & $\mathrm{R}^{(\mathrm{a})}$ \\
\hline UCD2-14 & 85 & $75-85$ & 2 & $11 / 15 / 89$ & $\bar{s}$ & $\mathrm{~s}$ & $\mathrm{R}^{(\mathrm{a})}$ & $\mathbf{R}$ \\
\hline UCD2-15 & 120.5 & $91-116$ & 2 & $03 / 28 / 90$ & $\mathrm{~s}$ & $\bar{s}$ & $\bar{R}$ & 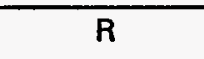 \\
\hline UCD2-16 & 122 & $92-117$ & 2 & $04 / 04 / 90$ & $\mathbf{s}$ & $\mathrm{s}$ & $\bar{R}$ & $\bar{R}$ \\
\hline UCD2-17 & 143 & $88-113$ & 2 & $04 / 10 / 90$ & $\mathrm{~s}$ & $\mathrm{~s}$ & $\bar{R}$ & - \\
\hline UCD1-18 & 70 & $54-69$ & 1 & $10 / 04 / 90$ & $S^{(8)}$ & $\mathrm{s}$ & $R$ & - \\
\hline UCD1-19 & 74.5 & $56.5-71.5$ & 1 & $10 / 01 / 90$ & $\mathbf{S}$ & $\mathrm{S}^{(\mathrm{a})}$ & $R$ & $R$ \\
\hline UCD1-20 & 73 & $57-72$ & 1 & $10 / 09 / 90$ & $\mathrm{~s}$ & $\bar{s}$ & $\bar{R}$ & $\bar{R}$ \\
\hline UCD1-21 & 73 & $57-72$ & 1 & $10 / 11 / 90$ & s & s & - & - \\
\hline UCD1-22 & 73 & $57-72$ & 1 & $10 / 25 / 90$ & s & $\mathrm{s}$ & $\bar{R}$ & - \\
\hline UCD1-23 & 73 & $56.5-71.5$ & 1 & $10 / 17 / 90$ & 5 & $\mathrm{~s}$ & $\bar{R}$ & - \\
\hline UCD1-24 & 73 & $57-72$ & 1 & $10 / 22 / 90$ & $\mathrm{~s}$ & $s$ & - & - \\
\hline UCD1-25 & 75 & $60-75$ & 1 & $10 / 6 / 95$ & - & - & - & $R$ \\
\hline UCD2-26 & 102 & $87-102$ & 2 & $10 / 6 / 95$ & - & - & - & $\bar{R}$ \\
\hline
\end{tabular}


Table 2.2. (contd)

\begin{tabular}{|c|c|c|c|c|c|c|c|c|}
\hline $\begin{array}{l}\text { Well or } \\
\text { Sample } \\
\text { Location }\end{array}$ & $\begin{array}{c}\text { Total } \\
\text { Depth } \\
\text { (feet bgs) }\end{array}$ & $\begin{array}{l}\text { Screened } \\
\text { Interval } \\
\text { (feet bgs) }\end{array}$ & HSU & $\begin{array}{c}\text { Date } \\
\text { Completed }\end{array}$ & $\begin{array}{c}\text { Winter } \\
1995 \\
2 / 14-2 / 23\end{array}$ & $\begin{array}{c}\text { Spring } \\
1995 \\
5 / 16-5 / 25\end{array}$ & $\begin{array}{c}\text { Summer } \\
1995 \\
8 / 23-8 / 31\end{array}$ & $\begin{array}{c}\text { Fall } \\
1995 \\
11 / 28-12 / 12\end{array}$ \\
\hline UCD1-27Z1 & 136 & 53-55 & 1 & $10 / 6 / 95$ & - & - & - & B \\
\hline UCD1-27Z2 & 136 & $63-65$ & 1 & $10 / 6 / 95$ & - & - & - & $B$ \\
\hline UCD1-27Z3 & 136 & $73-75$ & 1 & $10 / 6 / 95$ & - & - & - & $B$ \\
\hline UCD2-27Z4 & 136 & $86-88$ & 2 & $10 / 6 / 95$ & - & - & - & B \\
\hline UCD2-27Z5 & 136 & $96-98$ & 2 & 10/6/95 & - & - & - & B \\
\hline UCD2-27Z6 & 136 & $106-108$ & 2 & $10 / 6 / 95$ & - & - & - & $B$ \\
\hline UCD2-27Z7 & 136 & $116-118$ & 2 & $10 / 6 / 95$ & - & - & - & B \\
\hline UCD1-34 & 80 & $61-76$ & 1 & $10 / 6 / 95$ & - & - & - & $\bar{R}$ \\
\hline UCD2-35 & 130 & $107-122$ & 2 & $10 / 6 / 95$ & - & - & - & $\bar{R}$ \\
\hline $\mathrm{PCU}$ & - & - & - & - & $\mathbf{G}$ & $G^{(a)}$ & $\mathrm{G}^{(\mathrm{a})}$ & $\bar{G}$ \\
\hline PCD & - & - & - & - & $\mathbf{G}$ & $\mathbf{G}$ & $\mathbf{G}$ & $\mathrm{G}^{\left(\mathbf{a}^{\prime}\right.}$ \\
\hline STPO & - & - & - & - & $G^{(a)}$ & G & $G$ & $\mathbf{G}$ \\
\hline SWL-1 & - & - & - & - & - & $G^{(a, b)}$ & - & - \\
\hline SWL-2 & - & - & - & - & - & $G^{(b)}$ & - & - \\
\hline \multicolumn{9}{|c|}{$\begin{array}{ll}\text { (a) Field duplicate sample collected. } \\
\text { (b) Storm water runoff samples collected. } \\
\text { B Sampled collected with a Westbay bailer. A revised suite of samples was obtained. } \\
\text { G } & \text { Grab surface water or storm water sample. } \\
\text { R } & \text { Well purged and sampled with a dedicated submersible pump. A revised suite of samples collected. } \\
\text { S } & \text { Well purged and sampled with dedicated submersible pump. A full suite of samples was obtained. } \\
& \text { Not applicable. } \\
\text { Note: } & \text { Wells UCD1-3, UCD1-5, UCD1-6, UCD1-8, UCD1-9 are not sampled as part of the quarterly monitoring program. } \\
\end{array}$} \\
\hline
\end{tabular}


Table 2.3. Analytical Constituents for Groundwater Samples ${ }^{(a)}$

\begin{tabular}{|c|c|c|}
\hline Field & \multicolumn{2}{|c|}{ Laboratory } \\
\hline $\begin{array}{l}\mathrm{pH} \\
\text { Electrical conductivity } \\
\text { Temperature } \\
\text { Turbidity } \\
\text { [Eh] } \\
\text { Water level }\end{array}$ & $\begin{array}{l}\text { Volatile organic } \\
\text { compounds } \\
\text { Semivolatile organic } \\
\text { compounds } \\
\text { Radiologic analyses } \\
\text { tritium, strontium-90, } \\
\text { gross alpha, gross beta, } \\
\text { gamma emitters, } \\
\text { carbon-14, radium-226, } \\
\text { americium-241, and } \\
\text { plutonium-241. } \\
\text { Pesticides and PCBs } \\
\text { Metals } \\
\text { antimony, arsenic, } \\
\text { barium, beryllium, cadmium, } \\
\text { cobalt, copper, iron, } \\
\text { lead, mercury, } \\
\text { molybdenum, nickel, } \\
\text { selenium, silver, } \\
\text { thallium, vanadium } \\
\text { zinc }\end{array}$ & $\begin{array}{l}\text { total chromium } \\
\text { hexavalent chromium } \\
\text { nitrate } \\
\text { [ammonia] } \\
\text { [TKN] } \\
\text { [alkalinity] } \\
\text { cations } \\
\text { [anions] } \\
\text { total dissolved solids } \\
\text { [turbidity] } \\
\text { [total organic carbon] } \\
\text { [chemical oxygen } \\
\quad \text { demand] } \\
\text { [formaldehyde] }\end{array}$ \\
\hline \multicolumn{3}{|c|}{$\begin{array}{l}\text { (a) UCD1-1, UCD1-4, UCD2-7, UCD1-10, UCD1-11, UCD1-12, UCD1-13, UCD2-14, } \\
\text { UCD2-15, UCD2-16, UCD2-17, UCD1-18, UCD1-19, UCD1-20, UCD1-21, UCD1-22, } \\
\text { UCD1-23, UCD1-24, UCD1-25, UCD2-26, UCD1/2-27, UCD1-34, UCD2-35. } \\
\text { [ ] Parameter eliminated from the monitoring program starting summer quarter } 1995 .\end{array}$} \\
\hline
\end{tabular}


Table 2.4. Analytical Constituents for Surface Water ${ }^{(a)}$

\begin{tabular}{||l|ll||}
\hline \multicolumn{1}{|c|}{ Field. } & \multicolumn{2}{|c||}{ Laboratory } \\
\hline \hline $\mathrm{pH}$ & Volatile organic & total chromium \\
Electrical conductivity & compounds & hexavalent chromium \\
Temperature & Semivolatile organic & nitrate \\
Turbidity & compounds & [ammonia] \\
[Eh] & Pesticides and PCBs & [TKN] \\
Approximate flow rate & Radiologic analyses & [alkalinity] \\
lobtained from Solano County & tritium, strontium-90, & cations \\
Irrigation District) & gross alpha, gross beta, & [anions] \\
& gamma emitters, & total dissolved solids \\
& carbon-14, radium-226, & [turbidity] \\
& americium-241, & [total organic carbon] \\
& plutonium-241 & [chemical oxygen \\
& Metals & demand] \\
& antimony, arsenic, & \\
& barium, beryllium, cadmium, & \\
& cobalt, copper, iron, & \\
& lead, mercury, & \\
& molybdenum, nickel, & \\
& selenium, silver, & \\
\hline \hline la) Collected at locations PCD & PCU, and STPO. & \\
[ ] Parameter eliminated from & the monitoring program starting summer quarter 1995. \\
\hline \hline
\end{tabular}


Table 2.5. Analytical Constituents for Storm Water Runoff Samples

\begin{tabular}{|c|c|c|}
\hline Field & \multicolumn{2}{|c|}{ Laboratory - Each Storm Event } \\
\hline $\begin{array}{l}\text { To be collected during two } \\
\text { storm events per year from } \\
\text { two locations }\end{array}$ & \multicolumn{2}{|c|}{$\begin{array}{l}\text { To be collected during two storm events per year from two } \\
\text { locations }^{(b)}\end{array}$} \\
\hline $\begin{array}{l}\mathrm{pH} \\
\text { Electrical conductivity } \\
\text { Temperature } \\
\text { Turbidity } \\
\text { Eh }\end{array}$ & $\begin{array}{l}\text { Volatile organic compounds } \\
\text { Semivolatile organic compounds } \\
\text { Pesticides and PCBs } \\
\text { Radiologic analyses } \\
\text { tritium, strontium-90, } \\
\text { gross alpha, gross beta, } \\
\text { gamma emitters, } \\
\text { carbon-14, radium-226, } \\
\text { americium-241, } \\
\text { plutonium-241 } \\
\text { Metals } \\
\text { antimony, arsenic, barium, } \\
\text { beryllium, cadmium, cobalt, } \\
\text { copper, iron, lead, mercury, } \\
\text { molybdenum, nickel, } \\
\text { selenium, silver, } \\
\text { thallium, vanadium, } \\
\text { zinc }\end{array}$ & $\begin{array}{l}\text { total chromium } \\
\text { hexavalent chromium } \\
\text { nitrate } \\
\text { ammonia } \\
\text { alkalinity } \\
\text { cations } \\
\text { anions } \\
\text { turbidity } \\
\text { total suspended solids } \\
\text { total dissolved solids } \\
\text { total organic carbon } \\
\text { total oil and grease } \\
\text { formaldehyde }\end{array}$ \\
\hline $\begin{array}{l}S W L-1 \text { and } S W L-2 \\
\text { After two years of } m\end{array}$ & 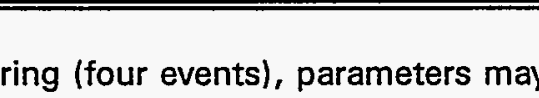 & nodified. \\
\hline
\end{tabular}


Table 2.6. Water Monitoring Constituents and Analytical Methods

\begin{tabular}{|c|c|c|}
\hline $\begin{array}{l}\text { Analyte } \\
\text { Fraction }\end{array}$ & $\begin{array}{l}\text { Analytical } \\
\text { Parameter }\end{array}$ & $\begin{array}{c}\text { Method } \\
\text { Reference }\end{array}$ \\
\hline VOCs & Target Compound List & CLP SOW OLC 02.0 \\
\hline SVOCs & Target Compound List & CLP SOW OLM 01.8 \\
\hline Pesticides/PCBs & Target Compound List & CLP SOW OLM 01.8 \\
\hline Metals & $\begin{array}{l}\text { antimony } \\
\text { arsenic } \\
\text { barium } \\
\text { beryllium } \\
\text { cadmium } \\
\text { chromium (total) } \\
\text { chromium }{ }^{6+} \text { (hexavalent) } \\
\text { cobalt } \\
\text { copper } \\
\text { iron } \\
\text { lead } \\
\text { mercury } \\
\text { molybdenum } \\
\text { nickel } \\
\text { selenium } \\
\text { silver } \\
\text { thallium } \\
\text { vanadium } \\
\text { zinc }\end{array}$ & $\begin{array}{l}\text { ILC SOW } 2.0 \\
\text { ILC SOW } 2.0 \\
\text { ILC SOW } 2.0 \\
\text { ILC SOW } 2.0 \\
\text { ILC SOW } 2.0 \\
\text { ILC SOW } 2.0 \\
\text { USEPA } 7196 \\
\text { ILC SOW } 2.0 \\
\text { ILC SOW } 2.0 \\
\text { ILC SOW } 2.0 \\
\text { ILC SOW } 2.0 \\
\text { ILC SOW } 2.0 \\
\text { ILC SOW } 2.0 \\
\text { ILC SOW } 2.0 \\
\text { ILC SOW } 2.0 \\
\text { ILC SOW } 2.0 \\
\text { ILC SOW } 2.0 \\
\text { ILC SOW } 2.0 \\
\text { ILC SOW } 2.0\end{array}$ \\
\hline Radiological & $\begin{array}{l}\text { tritium } \\
\text { strontium-90 } \\
\text { gamma spectral analysis } \\
\text { gross alpha } \\
\text { gross beta } \\
\text { carbon-14 } \\
\text { radium-226 } \\
\text { americium-241 } \\
\text { plutonium-241 }\end{array}$ & $\begin{array}{l}\text { USEPA } 906.0 \\
\text { USEPA } 905.0 \\
\text { USEPA } 901.1 \\
\text { USEPA } 9310 \\
\text { USEPA } 9310 \\
\text { USEPA C-01 M } \\
\text { USEPA } 903.1 \\
\text { LAS } 108 \\
\text { LAS } 178\end{array}$ \\
\hline $\begin{array}{l}\text { Miscellaneous } \\
\text { organics }\end{array}$ & $\begin{array}{l}\text { formaldehyde } \\
\text { total organic carbon } \\
\text { total oil and grease }\end{array}$ & $\begin{array}{c}\text { Modified NIOSH } 3500= \\
415.2(9060) \\
413.2\end{array}$ \\
\hline
\end{tabular}


Table 2.6. (contd)

\begin{tabular}{|c|c|c|}
\hline $\begin{array}{l}\text { Analyte } \\
\text { Fraction }\end{array}$ & $\begin{array}{l}\text { Analytical } \\
\text { Parameter }\end{array}$ & $\begin{array}{l}\text { Method } \\
\text { Reference }\end{array}$ \\
\hline $\begin{array}{l}\text { Miscellaneous } \\
\text { inorganics }\end{array}$ & $\begin{array}{l}\text { alkalinity, as } \mathrm{CaCO}_{3} \\
\text { ammonia-nitrogen } \\
\text { anions } \\
\text { chloride } \\
\text { nitrate, as } \mathrm{N} \\
\text { phosphate, total (as P) } \\
\text { sulfate } \\
\text { cations } \\
\text { calcium } \\
\text { magnesium } \\
\text { sodium } \\
\text { potassium } \\
\text { chemical oxygen demand } \\
\text { total dissolved solids } \\
\text { total suspended solids } \\
\text { total kjeldahl nitrogen } \\
\text { (TKN) } \\
\text { turbidity }\end{array}$ & $\begin{array}{l}\text { USEPA } 310.1 \\
\text { USEPA } 350.1 \text { (350.2) } \\
\text { USEPA } 300.0 \\
\text { USEPA } 300.0 \\
\text { USEPA } 365.2 \\
\text { USEPA } 300.0 \\
\text { USEPA } 6010 \\
\text { USEPA } 6010 \\
\text { USEPA } 6010 \\
\text { USEPA } 6010 \\
\text { USEPA } 410.1 \\
\text { USEPA } 160.1 \\
\text { USEPA } 160.2 \\
\text { USEPA } 351.2 \\
\text { USEPA } 180.1\end{array}$ \\
\hline Field parameters & $\begin{array}{l}\mathrm{pH} \\
\text { turbidity } \\
\text { temperature } \\
\text { electrical conductivity (EC) } \\
\text { redox potential (Eh) }\end{array}$ & $\begin{array}{l}\text { SOP } 2.0 \\
\text { SOP } 2.0 \\
\text { SOP } 2.0 \\
\text { SOP } 2.0 \\
\text { SOP } 2.0\end{array}$ \\
\hline \multicolumn{3}{|c|}{$\begin{array}{ll}\text { VOCs } & \text { - volatile organic compounds } \\
\text { SVOCs } & \text { - semivolatile organic compound } \\
\text { PCBs } & \text { - polychlorinated biphenyls }\end{array}$} \\
\hline
\end{tabular}




\subsection{Quality Assurance and Data Validation Summary}

Data validation was performed on analytical results received from Lockheed Analytical Services in Las Vegas, Nevada, for 1995 groundwater, surface water, and storm water runoff samples. As stated in Section 2, the purpose of the data validation is to verify that the data meet DQOs and $Q A$ criteria required for the water monitoring program and risk assessment as set forth in the QAPjP.

This section describes the methods and presents the results of the data validation. Section 3.1 presents the criteria used to review the DQOs for each PARCC parameter. Section 3.2 summarizes the data validation procedures. Section 3.3 presents an overall summary of the data validation results; data validation results for each analysis in relation to the PARCC parameters are discussed in Section 3.4.

\subsection{Quality Assurance Criteria}

Samples collected during the 1995 water monitoring program were analyzed and validated according to criteria established in the program's QAPjP. Included in the QAPjP are quality control sample collection requirements and specific $Q A$ goals for the measurement data. These QA goals, or DQOs, are quantitative and qualitative statements that specify the quality of data used to support project decisions. They are expressed in terms of precision, accuracy, representativeness, completeness, and comparability (PARCC) (see Table 3.1).

\subsubsection{Precision}

Precision measures the ability to reproduce measurements under a given set of conditions. Precision can be expressed as the relative percent difference (RPD):

$$
R P D=\frac{|A-B|}{\left[\frac{|A+B|}{2}\right]} \times 100
$$

where $A=$ the measured concentration of the analyte in a sample

$B=$ the measured concentration of the analyte in a duplicate sample.

\section{Sampling (field) Precision}

Blind duplicate samples are collected and analyzed to evaluate the precision of the measurement systems and estimate the variability in the sampling process. The RPD has been calculated for those analyte pairs with at least one detection above the reporting limit. If the RPD. between the duplicate sample results is greater than the $100 \%$ acceptance criteria as defined in the QAPjP, the system is considered to be out of 
statistical control, and further investigation is initiated. As outlined in the QAPjP, one blind duplicate is collected for every 10 samples or matrix type. Thirteen blind duplicate samples were collected during 1995 from groundwater, surface water, and storm water runoff locations and analyzed for the various constituents that were outlined in Tables 2.3, 2.4, and 2.5. A summary of the field duplicate results is presented in Tables 3.2 through 3.10 at the end of Section 3.

\section{Analytical Precision}

Following recommended methodologies, sample duplicate and matrix spike duplicates are performed to estimate the precision in the analytical process. The required frequency for duplicate analysis is set forth in the analytical methods. Both sample and matrix spike duplicates assess matrix effects and analytical variability.

Specific acceptance criteria for each standard method and parameter measured have been established and outlined in the QAPjP. If the RPD between duplicate results falls outside the acceptance criteria, the analytical system is considered to be out of control, and other data quality results are reviewed to establish the validity of the data. Laboratory duplicates were prepared and analyzed for the various constituents that were outlined in Tables 2.3 through 2.5. A summary of the sample duplicate and matrix spike duplicate results is presented in Table 3.11 .

\subsubsection{Accuracy}

Accuracy is a measure of the bias of a method or the level or agreement of a measurement with a known true value. Accuracy can be assessed using percent recovery, which is calculated using the following equation:

$$
\frac{A-B}{C} \times 100
$$

where $A=$ the measured concentration of the spiked analyte in a spiked sample

$B=$ the measured concentration of the spiked analyte in an unspiked sample

$\mathrm{C}=$ the concentration of the analyte. used for spiking.

Analysis of matrix or surrogate spikes and laboratory control spike samples are used to evaluate analytical accuracy. A matrix spike is a solution of method analytes (at known concentrations) that is added ("spiked") into a field sample before the sample is prepared for analysis. Laboratory control spike analyses have the same function as matrix spike analyses and differ only in that the spike solution is added to a laboratory blank sample rather than a field sample. The results of these spike sample analyses are used to measure the percent recovery of each spiked compound. This percent recovery is a measure of the accuracy of the method. Specific acceptance criteria have been established for each standard method and parameter measured and are outlined in the QAPjP. 
Table 3.1. Quality Control Sample Evaluation Criteria

\begin{tabular}{|c|c|c|c|}
\hline Objective. & & QC Program & Evaluation Criteria \\
\hline Precision & $\begin{array}{l}- \\
- \\
-\end{array}$ & $\begin{array}{l}\text { Field duplicate pairs } \\
\text { Matrix spike } \\
\text { Matrix spike duplicate }\end{array}$ & Relative percent difference \\
\hline Accuracy & $\begin{array}{l}- \\
- \\
-\end{array}$ & $\begin{array}{l}\text { Laboratory control spikes } \\
\text { Matrix spikes } \\
\text { Surrogate spikes }\end{array}$ & Percent recovery \\
\hline Representativeness & $\begin{array}{l}- \\
- \\
-\end{array}$ & $\begin{array}{l}\text { Trip blanks } \\
\text { Field duplicates } \\
\text { Method blanks }\end{array}$ & $\begin{array}{l}\text { Qualitative degree of } \\
\text { confidence. }\end{array}$ \\
\hline Completeness & & $\begin{array}{l}\text { Holding time } \\
\text { Valid data }\end{array}$ & Percent valid data \\
\hline Comparability & - & $\begin{array}{l}\text { Analytical methods } \\
\text { Field duplicates }\end{array}$ & $\begin{array}{l}\text { Qualitative degree of } \\
\text { confidence }\end{array}$ \\
\hline
\end{tabular}

Surrogate'spikes are compounds other than method analytes that have been selected for each organic compound analysis. The percent recovery is monitored to ensure adequate performance on a measurement-by-measurement basis. Surrogate spike recoveries are summarized for each sample analysis in the laboratory data packages. These recoveries are compared with specific acceptance criteria, which are outlined in the analytical methods. High surrogate recoveries indicate that reported results are higher than the actual concentrations of analytes in field locations; low surrogate recoveries may indicate that reported results are lower than actual concentrations.

The results of the sample matrix and surrogate recoveries and laboratory control spike samples are reviewed as part of the validation process. Results for sample matrix and laboratory control spike analyses are presented in Tables 3.11 and 3.12, respectively. The results are compared with the acceptable ranges established in the QAPjP to provide an indication of laboratory analytical performance.

\subsubsection{Representativeness}

Representativeness is a qualitative parameter that evaluates how the data represent the actual environmental conditions. Representativeness is determined by evaluating the results of trip blanks, laboratory method blanks, and blind duplicate samples.

Travel blanks were used to identify volatile organic compounds (VOCs) that might have been introduced during sample transit or storage at the laboratory. The blanks were supplied by Lockheed and returned each sampling day in the cooler used to transport samples for volatile analyses. Thirty two trip blank samples were collected and analyzed during the 1995 water monitoring program. The results of the trip blank samples are presented in Table 3.13 and discussed in Section 3.4.1. 
Laboratory method blanks are used to demonstrate that all glassware and reagents used in the analytical procedure are free of interferences and compounds of primary interest. Each method blank is subjected to all laboratory procedures from sample preparation to quantitation. If an analyte is detected in a method blank, either an interference or contamination in the laboratory process is indicated. 'The required frequency for analyzing method blanks is specified in the standard operation procedure for each analytical method, at least one per day for each method/instrument and/or per sample preparation set. Laboratory method blanks are evaluated as part of the validation process. Identification of these compounds at similar concentrations in primary samples results in questionable data because of biases introduced by the analytical process.

Blind duplicate samples collected and analyzed for this program are evaluated to determine if results are similar in concentration to the primary sample data. Analyses of blind duplicate samples are also used to estimate precision in the sampling and analytical process (Section 3.1.1).

\subsubsection{Completeness}

Completeness is defined as the percentage of valid data compared with the total number of samples collected for analysis. Completeness is determined after precision and accuracy are calculated, and the results are compared with the DQOs. As outlined in the QAPjP, the objective for completeness for all measurements is $95 \%$. The percentage of valid data is determined using the following equation:

$$
\text { Percent Valid Data }=\frac{\text { number of valid data points }}{\text { total number of measurements }} \times 100
$$

Valid data are determined by comparing analytical results with a set of guidelines designed to establish defensibility and reliability of a given data result. Data that fall outside these criteria are labeled, or qualified, as rejected. Data that are determined to have limited usefulness, or that are indicative of bias, are qualified as estimated. Data that have been qualified as estimated are considered valid.

\subsubsection{Comparability}

Comparability is an expression of the confidence with which one data set can be compared with another. Comparability is achieved through standard sampling procedures, analytical methods, and units of measurement. Reported methodologies and quantitation limits are compared with those outlined in the QAPjP.

\subsection{Data Validation Methods}

Table 3.1 presents the criteria used to review DQOs for each PARCC parameter. These criteria include 1 ) a review of quality control (OC) samples collected in the field, 2) laboratory QC samples, and 3) analytical method performance. The field QC samples and analytical data reports were reviewed in accordance with validation procedures established in the QAPjP. Data validation procedures presented in the Water Monitoring Plan (PNL 1994) were applied to data collected during the water monitoring activities. 
QC samples included field duplicates, trip blanks, laboratory method blanks, and control spikes. One field duplicate was collected for every 10 primary samples and evaluated to identify sources of error affecting the quality of the data. The locations of field duplicate samples were randomly selected during the planning stage for quarterly monitoring activities. Trip blanks were used to identify VOCs that might have been introduced during sample transit (to and from the field) or laboratory storage. One set of trip blanks was included in each shipment cooler containing samples for volatiles analysis. In addition, the laboratory analyzed a method blank and a method blank spike for each analytical batch to detect reagent contamination and proper instrument performance.

The three primary objectives of validation are 1) a review of sampling, analytical; and data reduction protocols for correctness, 2) a quantitative assessment of the measurement data validity, and 3) an assessment of data completeness. The project data validation procedures were designed to review each data set and identify biases inherent to the data, including assessment of laboratory performance, overall precision and accuracy, representativeness, and completeness.

A U.S. Environmental Protection Agency Contract Laboratory Program (CLP) or CLP-quivalent data package was obtained for samples collected during 1995. A CLP data package includes raw data generated during the analytical process along with the sample results. At a minimum, $10 \%$ of the laboratory data were randomly subjected to validation in accordance with the USEPA National Functional Guidelines for Laboratory Data Review, Organics (EPA 1991) and Inorganics (EPA 1989a). This level of validation included a detailed review of sample data and was performed along with a complete review of QC summary information provided by the laboratory. The remaining $90 \%$ of the laboratory data were reviewed using a subset of the National Functional Guidelines validation criteria described in the Water Monitoring Plan (PNL 1994). This review included an assessment of holding time violations, blank contamination, calibration, precision, and accuracy.

\subsection{Summary of Data Validation Results}

Samples collected during the 1995 water monitoring program were analyzed and validated according to criteria established in the program QAPjP. Included in the QAPjP are quality control sample collection requirements and specific DQOs for the measurement data. Data are evaluated and compared with these quality goals through data validation.

Validation discrepancies identified during the 1995 water monitoring program included equipment calibration failure, surrogate recovery problems, matrix biases, and holding time

violations. The majority of the data associated with these anomalies have been flagged as estimated or not detected. Generally, such flags do not render the data unusable for their intended purpose.

There were few qualifications identified in the quality control data. Approximately $98 \%$ of the data were valid, satisfying the DQOs established for this program (see table below). The data collected during the 1995 rounds of groundwater, surface water, and storm water runoff sampling are considered valid as qualified and representative of actual conditions. 
Summary of Data Validation of Scheduled Analyses

\begin{tabular}{||l|c|c|c|c|c||}
\hline & $\begin{array}{c}\text { Number of } \\
\text { Field Samples } \\
\text { Collected }\end{array}$ & $\begin{array}{c}\text { Number of } \\
\text { Constituents } \\
\text { Requested }\end{array}$ & $\begin{array}{c}\text { Number of } \\
\text { Constituents } \\
\text { Qualified }\end{array}$ & $\begin{array}{c}\text { Number of } \\
\text { Constituents } \\
\text { Rejected }\end{array}$ & $\begin{array}{c}\text { Percent of } \\
\text { Valid } \\
\text { Results }\end{array}$ \\
\hline \hline $\begin{array}{c}\text { Volatile Organic } \\
\text { Compounds }\end{array}$ & 87 & 3,472 & 291 & 232 & 93 \\
\hline $\begin{array}{c}\text { Semivolatile } \\
\text { Organic } \\
\text { Compounds }\end{array}$ & 62 & 3,968 & 200 & 0 & 100 \\
\hline $\begin{array}{l}\text { Pesticides/ } \\
\text { Polychlorinated } \\
\text { Biphenyl }\end{array}$ & 82 & 2,296 & 495 & 0 & 100 \\
\hline Metals & 94 & 1,441 & 43 & 0 & 100 \\
\hline Radionuclides & 87 & 1,762 & 115 & 0 & 100 \\
\hline General Chemicals & 188 & 1,389 & 62 & 45 & 97 \\
\hline
\end{tabular}

The following sections present a summary of data validation results and their impact on the established DQOs in relation to the PARCC parameters. Analytical results for groundwater, surface water, and storm water samples collected during the 1995 water monitoring program are tabulated in Appendixes A and B. Data validation flags have been applied to those sample results that fell outside specified tolerance limits and therefore did not meet the program's DQOs. An explanation of the data flags is provided as a key to Appendixes $A$ and $B$.

\subsection{Data Validation Results by Chemical Group}

\subsubsection{Volatile Organic Compounds}

The following sections discuss the results of the VOC analyses (CLP SOW OLC2.0) and provide an evaluation of the PARCC goals.

\section{Completeness of Scheduled Analyses}

Eighty-seven field samples were collected and submitted to the laboratory for VOC analyses, and data results were received for all samples scheduled for analyses. More than $93 \%$ of the results reported for VOC analyses are valid. There were few qualifications identified in the quality control data for VOC analyses. Out of approximately 3,472 individual analytical results (both detected and nondetected), 291 results were qualified; of those, only 232 results were qualified as rejected. The results for VOC analyses for this program (93\%) were thus slightly below the data quality objective for completeness (95\%). 


\section{Precision}

Blind duplicate samples were collected from 13 locations during the annual water monitoring program. All of the blind duplicates were analyzed for VOCs and yielded six duplicate pairs containing at least one positive analyte detection. These sample locations include STPO, UCD1-18, and UCD2-7, collected in the winter quarter; PCU, UCD1-1, and UCD1-19 collected in the spring quarter; UCD1-12, UCD2-14, and PCD collected in the summer quarter; UCD1-13, UCD1-12, and STPO collected in the fall quarter; and storm water runoff location SWL-1 collected in spring 1995 . For the 16 analyte pairs reported with positive detections, all had RPDs of less than $100 \%$ (with the exception of UCD1-1 and UCD1-12), satisfying the DQOs. As indicated in the quarterly report for winter and spring 1995, it was concluded that the duplicate VOC sample from UCD1-1 was likely collected from UCD1-12. Again, results from the duplicate samples collected from UCD1-12 in the summer were variable. The repeated analyses yielded results which were more consistent with the historical trend and duplicate sample results even though the sample was outside the holding time. The results of the blind duplicate analyses are shown in Tables 3.2 through 3.10 .

\section{Accuracy}

Laboratory control spikes were performed at the required frequency using the concentrations and conditions specified for the analytical method for VOCs. Method EPA CLP SOW OLC2.0 also specifies the recovery criteria for each spiked compound.

Table 3.12 presents a summary of laboratory control spike samples. The percent recovery of each spiking compound was calculated and compared with the laboratory QC limits. The results for laboratory control spikes show the laboratory analytical system to be in control.

Surrogate spikes were performed for compounds analyzed for VOCs in accordance with the method. All recoveries were within the QC acceptance criteria. This evaluation indicates acceptable laboratory performance.

\section{Representativeness}

Thirty-two trip blank samples were collected and analyzed during the annual water monitoring program. Twenty-five of these 32 blanks were found to be free of analyte contamination. Xylene was reported at a concentration of $1.3 \mu \mathrm{g} / \mathrm{L}$ in a single trip blank sample (TBGW0016) collected during the winter quarter. Xylene was not reported in samples directly associated with this trip blank. Reported detections of acetone in TBGW0023, TBGW0051, TBGW0113, TBGW0114, TBGW0125, and TBGWO290 collected in the spring, summer, and fall quarters of 1995 were qualified with a " $\mathrm{J}$ " because of instrument calibration responses and method blank contamination. This occurrence impacted all samples in the given analytical sequence reported below the detection limit. The analytical results for the travel blank samples are presented in Table 3.13.

Laboratory method blanks were analyzed at the required frequency for VOCs. Acetone, 1,2 dibromo-3 chloropropane, and 2 butanone are solvents commonly used in laboratory environments during extraction of water, soil, or solid waste from organic compounds. As 
such, these compounds can be detected in the VOC analyses. No corrective action is taken if these contaminants are detected in reagent blanks at less than five times the detection limit.

Low levels of acetone were reported in some method blanks and identified as a laboratory contaminant. Data associated with these blanks have been appropriately qualified as not detected (U) to indicate bias introduced by the analytical process (see Tables A.1 and B.1).

\section{Comparability}

The analyses for VOCs were conducted in accordance with the procedures outlined in the QAPjP. Laboratory reporting limits met the guidelines established in the workplan for those parameters not detected in the samples.

\subsubsection{Semivolatile Organic Compounds}

The following sections discuss the results of the semivolatile organic compounds (SVOC) analyses (CLP SOW OLM1.8) and provide an evaluation of the PARCC goals.

\section{Completeness of Scheduled Analyses}

Sixty-two field samples were collected and submitted to the laboratory for SVOC analyses, and data results were received for all samples scheduled for analyses. Onehundred percent of the results reported for SVOC analyses are valid. There were few qualifications identified in the quality control data. Out of a total of approximately 3,968 individual analytical results (both detected and nondetected), 200 results were qualified; of those, none was qualified as rejected. The completeness objectives for SVOC analyses for this program were achieved.

\section{Precision}

Detectable levels of SVOCs were reported in the seven duplicate samples collected and analyzed by method EPA CLP OLM1.8. The remainder of the duplicate samples that were collected were not analyzed for SVOCs. RPDs were calculated for compounds that were detected in the duplicate samples; these results are shown in Tables 3.2, 3.6, and 3.7. Of the seven analyte pairs, only four met the DQO requirement of $100 \%$. All other SVOCs detected were also present in the method blank.

\section{Accuracy}

Sample matrix spikes were performed for the fall quarter sampling using concentrations and conditions specified for the analytical method for SVOCs. Method EPA CLP SOW OLM1.8 also specifies the recovery criteria for each spiked compound.

Table 3.11 presents a summary of matrix spike samples. The percent recovery of each spiking compound was calculated and compared with the acceptance limits outlined in the QAPjP. In addition, the RPD between recoveries was calculated. Evaluation of the results for sample matrix spikes show the laboratory analytical system to be in control. 
Laboratory control spikes were performed at the required frequency using the concentrations and conditions specified for the analytical method for SVOCs. Method EPA CLP SOW OLM1.8 also specifies the recovery criteria for each spiked compound.

Table 3.12 presents a summary of laboratory control spike samples. The percent recovery of each spiking compound was calculated and compared with the laboratory $\mathrm{OC}$ limits. Evaluation of the results for laboratory control spikes show the laboratory analytical system to be in control, with the exception of 1,4 dichlorobenzene, which is slightly below the acceptable range.

Surrogate spikes were performed for compounds analyzed for SVOCs in accordance with the analytical method. All recoveries were within the QC acceptance criteria. This evaluation indicates acceptable laboratory performance.

\section{Representativeness}

Laboratory method blanks were analyzed at the required frequency for the analytical method for SVOCs. Common laboratory SVOCs include bis(2-ethylhexyl)phthalate and di-n-butylphthalate. No corrective action is taken if these contaminants are detected in reagent blanks at less than five times the detection limit.

Random occurrences of contamination associated with these blanks were identified; however, concentrations reported were below the contract required quantitation limit (CROL). These detections could result from a number of factors including laboratory glassware, sample preparation procedures, or carryover during analyses. Sample results associated with these blank contaminants have been flagged "not detected" (U) to indicate bias introduced by the analytical process (see Tables A.2 and B.2).

\section{Comparability}

The analyses for SVOCs were conducted in accordance with the procedures outlined in the QAPjP. Laboratory reporting limits met the guidelines established in the workplan for those parameters not detected in the samples.

\subsubsection{Pesticides/Polychlorinated Biphenyl}

The following sections discuss the results of the pesticides and polychlorinated biphenyl (PCBs) analyses (CLP SOW OLM1.8) and provide an evaluation of the PARCC goals.

\section{Completeness of Scheduled Analyses}

Eighty-two field samples were collected and submitted to the laboratory for pesticide and PCB analyses. Data results were received from the laboratory for all samples scheduled for analyses. One-hundred percent of the results reported for pesticide and PCB analyses are valid. Out of approximately 2,296 individual analytical results (both detected and nondetected), 495 were qualified (see "Accuracy," below, for further explanation). Of those data qualified, none were qualified as rejected. The completeness objectives for pesticides and PCBs for this program were achieved. 


\section{Precision}

No detectable levels of pesticides or PCBs were reported in 10 of the 11 field duplicate samples analyzed by method EPA CLP OLM1.8. RPDs were calculated for UCD1-13. Only duplicate samples from UCD1-13, collected in fall 1995, showed detectable levels but are qualified with either a J, P, or JP, indicating that the values were either estimated or the RPD between columns was greater than $25 \%$. The results of this blind duplicate are shown in Table 3.5.

\section{Accuracy}

Sample matrix spikes were performed using concentrations and conditions specified for the analytical method for pesticides and PCBs. Method EPA CLP SOW OLM1.8 also specifies the recovery criteria for each spiked compound.

Table 3.11 presents a summary of matrix spike samples. The percent recovery of each spiking compound was calculated and compared with the acceptance limits outlined in the QAPjP. The results of the percent recovery for each compound are expressed as a range. Evaluation of the results for sample matrix spikes show the laboratory analytical system to be in control, with the exception of aldrin and endrin, which were biased low. Results associated with these batches have been flagged appropriately.

Laboratory control spikes were performed at the required frequency using the concentrations and conditions specified for the analytical method for pesticides and PCBs. Method EPA CLP SOW OLM1.8 also specifies the recovery criteria for each spiked compound.

Table 3.12 presents a summary of laboratory control spike samples. The percent recovery of each spiking compound was calculated and compared with the laboratory QC limits. Evaluation of the results for laboratory control spikes shows the laboratory analytical system to be in control.

Surrogate spikes were performed for compounds analyzed for pesticides and PCBs in accordance with the appropriate method. Some pesticide and PCB data were qualified as estimated because of low surrogate recovery in some samples. However, acceptance crieria for pesticide surrogates are advisory only and resulted in no impact on the technical quality of the data. Surrogate failures were limited to only one of two surrogates applicable to this method. These results are consistent with historical data. This evaluation indicates acceptable laboratory performance.

\section{Representativeness}

Laboratory method blanks were prepared and analyzed using reagents, technique, and instrumentation identical to those used in field samples. Laboratory method blanks were reviewed as part of the validation process. No target analytes were reported above the CROL for the laboratory method blanks. 


\section{Comparability}

The analyses for pesticides and PCBs were conducted in accordance with the procedures outlined in the QAPjP. Laboratory reporting limits met the guidelines established in the workplan for those parameters not detected in the samples.

\subsubsection{Metals (EPA CLP ILC2.0 and SW846 6010)}

The following sections discuss the results of the metals analyses (CLP SOW ILC2.0 and SW846 6010) and provide an evaluation of the PARCC goals.

\section{Completeness of Scheduled Analyses}

Ninety-four field samples were collected and submitted to the laboratory for metal analyses. Data results were received from the laboratory for all samples scheduled for analyses. One-hundred percent of the results reported for metal analyses are valid. Out of approximately 1,441 individual analytical results (both detected and nondetected), 43 were qualified; of those, none was qualified as rejected. The completeness objectives for metal analyses for this program were achieved.

\section{Precision}

For metal analyses by methods EPA CLP ILC2.0, all 11 duplicate pairs were reported with positive detections of metal analytes, yielding 82 analyte pairs with at least one detection above the reporting limit. All pairs except hexavalent chromium for UCD1-19, collected in the spring quarter, had RPDs of less than the DQO of $100 \%$. A summary of blind duplicates is presented in Tables 3.2 through 3.10 .

\section{Accuracy}

Sample matrix spikes were performed at the required frequency for all sampling events using the concentrations and conditions specified in the analytical method for metals. The recovery criteria for each spiked compound are specified in the QAPJP.

Table 3.11 presents a summary of matrix spike samples. The percent recovery of each spiking compound was calculated and compared with the acceptance limits outlined in the QAPjP. An evaluation of the results for sample matrix spikes shows the laboratory analytical system to be in control.

Laboratory control spikes were performed at the required frequency using the concentrations and conditions specified in the analytical method for metals. Method EPA CLP SOW ILC2.0 also specifies the recovery criteria for each spiked compound.

Table 3.12 presents a summary of laboratory control spike samples. The percent recovery of each spiking compound was calculated and compared with the laboratory QC limits. In addition, the RPD between recoveries was calculated. An evaluation of the results for laboratory control spikes shows the laboratory analytical system to be in control. 


\section{Representativeness}

Laboratory method blanks were analyzed at the required frequency in the analytical methods for metals. No corrective action is taken if these constituents are detected in reagent blanks at less than five times the detection limit.

Random occurrences of contamination were identified associated with these blanks; however, concentrations reported were below the CROL. These detections, which were associated frequently with nickel, chromium, iron, and zinc, could result from a number of factors including laboratory glassware, sample preparation procedures, or carryover during analyses. Sample results associated with these blank contaminants have been flagged not detected $(U)$ to indicate bias introduced by the analytical process (see Tables A.4 and B.4).

\section{Comparability}

The analyses for metals were conducted in accordance with the procedures outlined in the QAPjP. Laboratory reporting limits met the guidelines established in the workplan for those parameters not detected in the samples.

\subsubsection{Radionuclides}

The following sections discuss the results of the radionuclides analyses and provide an evaluation of the PARCC goals. Various methodologies are used to perform these analyses. These methods are summarized in Table 2.6.

\section{Completeness of Scheduled Analyses}

Eighty-seven field samples were collected and submitted to the laboratory for radionuclide analyses, and data results were received for all samples scheduled for analyses. One-hundred percent of the results reported for radionuclides analyses are valid. Out of approximately 1,762 individual analytical results (both detected and nondetected), 115 were qualified; of those, no results were qualified as rejected. The completeness objectives for this program were achieved.

\section{Precision}

For radionuclide analyses, all 10 duplicate pairs were reported with positive detections of various radionuclides, yielding 18 analyte pairs with at least one detection above the associated uncertainty. The RPDs associated with radionuclides analyses have been calculated for samples with detected values greater than the minimum detectable activity (MDA) and the uncertainty (error). The results of the blind duplicate analyses are shown in Tables 3.2 through 3.10; all RPDs met the DQO requirement.

\section{Accuracy}

Sample matrix spikes were performed at the required frequency for all sampling events using the concentrations and conditions specified for the analytical method for radionuclides. The recovery criteria for each spiked compound are specified in the QAPjP. 
Table 3.11 presents a summary of matrix spike samples. The percent recovery of each spiking compound was calculated and compared with the acceptance limits outlined in the QAPjP. An evaluation of the results for sample matrix spikes shows the laboratory analytical system to be in control for most radionuclides. Results of matrix spikes for selected radionuclides, carbon-14, americium-241, and tritium, are below the acceptance limits, and positive results have been flagged with "J."

Laboratory control spikes were performed at the required frequency using the concentrations and conditions specified for the analytical methods for radionuclides. Analytical methods also specify the recovery criteria for each spiked compound.

Table 3.12 presents a summary of laboratory control spike samples. The percent recovery of each spiking compound was calculated and compared with the laboratory QC limits. An evaluation of the results for laboratory control spikes shows the laboratory analytical system to be in control.

\section{Representativeness}

Laboratory method blanks were analyzed at the required frequency for the analytical method for radionuclides. No corrective action is taken if these contaminants are detected in reagent blanks at less than five times the detection limit.

\section{Comparability}

The analyses for radionuclides were conducted in accordance with the procedures outlined in the QAPjP. Laboratory reporting limits met the guidelines established in the workplan for those parameters not detected in the samples.

\subsubsection{General Chemicals}

The following sections discuss the results of the analyses for general chemicals and provide an evaluation of the PARCC goals. The various methodologies used to perform these analyses are outlined in Table 2.6.

\section{Completeness of Scheduled Analyses}

One-hundred-eighty-eight field samples were collected and submitted to the laboratory for general chemical analyses. Data results were received from the laboratory for all samples scheduled for analyses. Ninety-seven percent of the results reported for general chemical analyses are valid. Sample results that were qualified as estimated (J) are described below.

There were few qualifications identified in the quality control data. Out of approximately 1,389 individual analytical results (both detected and nondetected), 55 were qualified; of those, none of the analytical laboratory results was qualified as rejected. Also included under general are the results for the field parameters. Forthy five results for specific conductivity for summer and fall have been rejected due to lack of $Q A$ 
documentation for calibration of the instruments and incorrect recording of the results (i.e., multiplier was not used). The completeness objectives for general chemical analyses for this program were achieved.

\section{Precision}

For general chemical analyses, 11 out of 12 duplicate pairs were reported with positive detections of various constituents, yielding 87 analyte pairs with at least one detection above the reporting limit. Four duplicate analyte pairs had calculated RPDs outside the acceptance criteria.

\section{Accuracy}

Sample matrix spikes were performed at the required frequency for all sampling events using the concentrations and conditions specified in the analytical method for general chemicals. The recovery criteria for each spiked compound are specified in the QAPjP.

Table 3.11 presents a summary of. matrix spike samples. The percent recovery of each spiking compound was calculated and compared with the acceptance limits outlined in the QAPjP. Evaluation of the results for sample matrix spikes shows the laboratory analytical system to be in control.

Laboratory control spikes were performed at the required frequency using the concentrations and conditions specified for the analytical method for general chemicals. These methods also specify the recovery criteria for each spiked compound.

Table 3.12 presents a summary of laboratory control spike samples. The percent recovery of each spiking compound was calculated and compared with the laboratory QC limits. In addition, the RPD between recoveries was calculated. An evaluation of the results for laboratory control spikes shows the laboratory analytical system to be in control.

\section{Representativeness}

Laboratory method blanks were prepared and analyzed using reagents, techniques, and instruments identical to those used for the field samples. Laboratory method blanks were reviewed as part of the validation process. No target analytes were reported above the contract detection limits for the laboratory method blanks.

\section{Comparability}

The analyses for general chemicals were conducted in accordance with the procedures outlined in the QAPjP. Laboratory reporting limits met the guidelines established in the workplan for those parameters not detected in the samples. 
TABLE 3.2

\section{RELATIVE PERCENT DIFFERENCE FOR FIELD DUPLICATES, GROUNDWATER WINTER QUARTER 1995 \\ LEHR ENVIRONMENTAL RESTORATION}

\begin{tabular}{|c|c|c|c|c|c|c|}
\hline \multirow{2}{*}{\begin{tabular}{|l} 
Location \\
UCD1-18
\end{tabular}} & \multirow{2}{*}{$\frac{\mid \text { Parameter }}{\text { Arsenic }}$} & \multirow{2}{*}{$\begin{array}{l}\text { Unit } \\
\text { ug/L }\end{array}$} & \multicolumn{2}{|c|}{ Result } & Duplicate & \multirow{2}{*}{$\begin{array}{c}\text { RPD\% (1) } \\
25.29\end{array}$} \\
\hline & & & 4.9 & Up I & $3.8 \quad$ Up I & \\
\hline & Barium & $\mathrm{ug} / \mathrm{L}$ & 152 & & 152 & 0.00 \\
\hline & Bismuth-214 & $\mathrm{pCi} / \mathrm{L}$ & 30 & & 9 & 107.69 \\
\hline & Calcium & $\mathrm{mg} / \mathrm{L}$ & 39.5 & & 40.2 & 1.76 \\
\hline & Chloride & $\mathrm{mg} / \mathrm{L}$ & 43 & & 44 & 2.30 \\
\hline & Chromium & $\mathrm{ug} / \mathrm{L}$ & 14.6 & & 14.7 & 0.68 \\
\hline & Cobalt & $\mathrm{ug} / \mathrm{L}$ & 1.5 & IB & 1.5 & 0.00 \\
\hline & Copper & $\mathrm{ug} / \mathrm{L}$ & 1.4 & IB & 1.5 & 6.90 \\
\hline & Di-n-Butylphthalate & $\mathrm{ug} / \mathrm{L}$ & $<12$ & IU & 2 & 142.86 \\
\hline & Magnesium & $\mathbf{m g} / \mathbf{L}$ & 96.9 & & 97.7 & 0.82 \\
\hline & Nickel & $\mathrm{ug} / \mathrm{L}$ & 65.1 & & 66 & 1.37 \\
\hline & Nitrogen, Nitrate (as N) & $\mathrm{mg} / \mathrm{L}$ & 14 & & 14 & 0.00 \\
\hline & Radium-226 & $\mathrm{pCi} / \mathrm{L}$ & 0.33 & & 0.4 & 19.18 \\
\hline & Sodium & $\mathrm{mg} / \mathrm{L}$ & 39.7 & & 39.9 & 0.50 \\
\hline & Sulfate & $\mathrm{mg} / \mathrm{L}$ & 39 & & 39 & 0.00 \\
\hline & Total Dissolved Solids & $\mathrm{mg} / \mathrm{L}$ & 610 & & 620 & 1.63 \\
\hline & Turbidity & NTU & 1.7 & & 1.7 & 0.00 \\
\hline & Vanadium & ug/L & 10.6 & & 10.6 & 0.00 \\
\hline & Zine & $\mathrm{ug} / \mathrm{L}$ & 1.8 & IB & 2.6 & 36.36 \\
\hline \multirow[t]{22}{*}{ UCD2-7 } & Ammonia-Nitrogen & $\mathrm{mg} / \mathbf{L}$ & $<0.05$ & & 0.26 & 135.48 \\
\hline & Arsenic & $\mathrm{ug} / \mathrm{L}$ & 3.1 & Jk I & Jk I & 0.00 \\
\hline & Barium & ug/L & 167 & & 160 & 4.28 \\
\hline & Bis(2-Ethylhexyl)phthalate & $u g / L$ & 6 & Jc IJ & 2 & 100.00 \\
\hline & Calcium . & $\mathbf{m g / L}$ & 36.4 & & 36.2 & 0.55 \\
\hline & Chloride & $\mathrm{mg} / \mathrm{L}$ & 21 & & 21 & 0.00 \\
\hline & Chromium & $\mathrm{ug} / \mathrm{L}$ & 45.6 & & 44.6 & 2.22 \\
\hline & Chromium, Hexavalent (+6) & ug/L & 43 & & 42 & 2.35 \\
\hline & Copper & ug/L & 1.1 & IB & IB & 9.52 \\
\hline & Magnesium & $\mathrm{mg} / \mathrm{L}$ & 78.7 & & 77.8 & 1.15 \\
\hline & Mercury & ug/L & 0.68 & & $<0.2$ & 109.09 \\
\hline & \begin{tabular}{|l} 
Molybdenum \\
\end{tabular} & ug/L & 1.3 & IB & 1.1 & 16.67 \\
\hline & Nitrogen, Nitrate (as N) & $\mathbf{m g} / \mathbf{L}$ & 12 & & 10 & 18.18 \\
\hline & Potassium & $\mathrm{mg} / \mathrm{L}$ & 0.698 & IB & 0.867 & 21.60 \\
\hline & Radium-226 & $\mathrm{pCi} / \mathrm{L}$ & $<0.24$ & & 0.24 & 0.00 \\
\hline & Selenium & $\mathbf{u g} / \mathbf{L}$ & $<3$ & IU & JQ IB & 23.53 \\
\hline & Sodium & $\mathbf{m g} / \mathbf{L}$ & 47.1 & & 46.1 & 2.15 \\
\hline & Sulfate & $\mathbf{m g} / \mathbf{L}$ & 41 & & 40 & 2.47 \\
\hline & Total Dissolved Solids & $\mathrm{mg} / \mathrm{L}$ & 550 & & 530 & 3.70 \\
\hline & Tritium & $\mathrm{pCi} / \mathrm{L}$ & $<310$ & & 360 & 14.93 \\
\hline & \begin{tabular}{|l} 
Vanadium \\
\end{tabular} & $\mathrm{ug} / \mathrm{L}$ & 7.7 & IB & 7.5 & 2.63 \\
\hline & Zine & $\mathrm{ug} / \mathrm{L}$ & 5.3 & IB & 5.2 & 1.90 \\
\hline
\end{tabular}

See tables in Appendix A for data qualifier explanations

(1)Relative Percent Difference (RPD) = ABS(100*(Result-Duplicate)/((Result+Duplicate)/2))

Analytes included with at least one detection above reporting limit.

$<=$ Not reported above the reporting limit.

(If results were below the reporting limit, then the reporting limit was used for RPD calculations.) 
TABLE 3.3

RELATIVE PERCENT DIFFERENCE FOR FIELD DUPLICATES, GROUNDWATER SPRING QUARTER 1995

LEHR ENVIRONMENTAL RESTORATION

\begin{tabular}{|c|c|c|c|c|c|c|}
\hline Location & Parameter & Unit & \multicolumn{2}{|c|}{ Result } & Duplicate & RPD\% (1) \\
\hline \multirow[t]{26}{*}{ UCD1-1 } & 1,1-Dichloroethane & $\mathrm{ug} / \mathrm{L}$ & $<1$ & 10 & $6.8 \mathrm{a}$ & 148.72 \\
\hline & 1,1-Dichloroethene & ug/L & $<1$ & Jf IU & $13 a$ & 171.43 \\
\hline & 1,2-Dichloroethane & ug/L & $<1$ & Jf IU & $3.3 \mathrm{a}$ & 106.98 \\
\hline & 1,2-Dichloropropane & ug/L & $<1$ & $\mathrm{IU}$ & $0.51 \mathrm{a}$ & 64.90 \\
\hline & Acetone & $\mathrm{ug} / \mathrm{L}$ & $<5$ & Re IU & UJc IBJ & 6.19 \\
\hline & Barium & ug $\sqrt{L}$ & 237 & & 248 & 4.54 \\
\hline & Bismuth-214 & $\mathrm{pCi} / \mathrm{L}$ & 12.8 & & 9 & 34.86 \\
\hline & Bromodichloromethane & $\mathrm{ug} / \mathrm{L}$ & $<1$ & IU & $0.7 \mathbf{a}$ & 35.29 \\
\hline & Calcium & $\mathrm{mg} / \mathbf{L}$ & 62.5 & & 62.7 & 0.32 \\
\hline & Chloride & $\mathrm{mg} / \mathrm{L}$ & 7.8 & & 7.7 & 1.29 \\
\hline & Chloroform & $\mathrm{ug} / \mathrm{L}$ & $<1$ & Jf IU & $5800 a$ & 199.93 \\
\hline & Chromium & $\mathrm{ug} / \mathrm{L}$ & 29.2 & & 30.1 & 3.04 \\
\hline & Chromium, Hexavalent (+6) & ug/L & 18 & & 19 & 5.41 \\
\hline & Iron & $\mathrm{mg} / \mathrm{L}$ & 0.018 & IB & 0.015 & 17.97 \\
\hline & Lead-214 & $\mathrm{pCi} / \mathrm{L}$ & 12.2 & & 16 & 26.95 \\
\hline & Magnesium & $\mathrm{mg} / \mathrm{L}$ & 153 & & 154 & 0.65 \\
\hline & Molybdenum & ug/L & 2.2 & IB & 1.9 & 14.63 \\
\hline & $\begin{array}{ll}\text { Nickel } \\
\end{array}$ & ug/L & 1.7 & IB & 1.8 & 5.71 \\
\hline & Nitrogen, Nitrate (as N) & $\mathrm{mg} / \mathbf{L}$ & 16 & & 16 & 0.00 \\
\hline & Radium-226 & $\mathrm{pCi} / \mathrm{L}$ & 0.3 & & 0.15 & 66.67 \\
\hline & Sodium & $\mathrm{mg} / \mathrm{L}$ & 33.5 & & 33.7 & 0.60 \\
\hline & Strontium-90 & $\mathrm{pCi/L}$ & 0.66 & & 0.7 & 5.88 \\
\hline & \begin{tabular}{|l} 
Sulfate \\
\end{tabular} & $\mathrm{mg} / \mathrm{L}$ & 27 & & 27 & 0.00 \\
\hline & Total Dissolved Solids & $\mathrm{mg} / \mathrm{L}$ & 830 & & 810 & 2.44 \\
\hline & Turbidity & NTU & 0.62 & & 0.59 & 4.96 \\
\hline & Vanadium & ug/L & 4.9 & IB & 4.6 & 6.32 \\
\hline \multirow[t]{22}{*}{ UCD1-19 } & Barium & $\mathrm{ug} / \mathrm{L}$ & $\overline{88.6}$ & & 81.5 & 8.35 \\
\hline & \begin{tabular}{|l|} 
Bismuth-214 \\
\end{tabular} & $\mathrm{pCi} / \mathrm{L}$ & 21.9 & & 19 & 14.18 \\
\hline & \begin{tabular}{|l} 
Calcium \\
\end{tabular} & $\mathrm{mg} / \mathrm{L}$ & 60.1 & $\dot{-}$ & 60 & 0.17 \\
\hline & \begin{tabular}{|l|} 
Chloride \\
\end{tabular} & $\mathrm{mg} / \mathrm{L}$ & 19 & & 19 & 0.00 \\
\hline & \begin{tabular}{|l|} 
Chromium \\
\end{tabular} & $\mathrm{ug} / \mathrm{L}$ & 170 & & 171 & 0.59 \\
\hline & Chromium, Hexavalent (+6) & $\mathrm{ug} / \mathrm{L}$ & $<10$ & & 54 & 137.50 \\
\hline & Gross Alpha & $\mathrm{pCi} / \mathrm{L}$ & 7.9 & & 11 & 32.80 \\
\hline & Iron & $\mathrm{ug} / \mathrm{L}$ & 22 & IB & $<100$ & 125.73 \\
\hline & \begin{tabular}{|l|} 
Lead-214 \\
\end{tabular} & $\mathrm{pCi} / \mathrm{L}$ & 24 & & 19.5 & 20.69 \\
\hline & Magnesium & $\mathrm{mg} / \mathrm{L}$ & 170 & & 166 & 2.38 \\
\hline & Molybdenum & $\mathrm{ug} / \mathrm{L}$ & 1.3 & IB & 1.2 & 8.00 \\
\hline & \begin{tabular}{|l} 
Nickel \\
\end{tabular} & ug/ & 1.4 & IB & 1.4 & 0.00 \\
\hline & Nitrogen, Nitrate (as $\mathrm{N}$ ) & $\mathrm{mg} / \mathrm{L}$ & 19 & & 19 & 0.00 \\
\hline & \begin{tabular}{|l|} 
Plutonium-241 \\
\end{tabular} & $\mathrm{pCi} / \mathrm{L}$ & $<1.8$ & & 2.7 & 40.00 \\
\hline & \begin{tabular}{|l} 
Potassium \\
\end{tabular} & $\mathrm{mg} / \mathrm{L}$ & 0.73 & IB & 1.13 & 43.01 \\
\hline & Radium-226 & $\mathrm{pCi} / \mathrm{L}$ & 0.069 & & 0.112 & 47.51 \\
\hline & Selenium & $\mathrm{ug} / \mathrm{L}$ & 9.3 & & 8.4 & 10.17 \\
\hline & \begin{tabular}{|l} 
Sodium \\
\end{tabular} & $\mathrm{mg} / \mathrm{L}$ & 74.1 & & 74 & 0.14 \\
\hline & Sulfate & $\mathrm{mg} / \mathrm{L}$ & 59 & & 59 & 0.00 \\
\hline & Total Dissolved Solids & $\mathrm{mg} / \mathbf{L}$ & 950 & & 940 & 1.06 \\
\hline & Turbidity & NTU & $<0.1$ & & 0.69 & 149.37 \\
\hline & Vanadium & ug/L & 6.4 & IB & IB & 24.56 \\
\hline
\end{tabular}

See tables in Appendix A for data qualifier explanations

(1)Relative Percent Difference $($ RPD $)=$ ABS(100*(Result-Duplicate) $/(($ Result+Duplicate)/2))

Analytes included with at least one detection above reporting limit.

$<=$ Not reported above the reporting limit.

(If results were below the reporting limit, then the reporting limit was used for RPD calculations.)

$\mathrm{a}=$ The duplicate VOC sample from UCD1-1 was determined to be likely collected from UCD1-12. 
TABLE 3:4

RELATIVE PERCENT DIFFERENCE FOR FIELD DUPLICATES, GROUNDWATER SUMMER QUARTER 1995

LEHR ENVIRONMENTAL RESTORATION

\begin{tabular}{|c|c|c|c|c|c|c|}
\hline Location & Parameter & Units & & & Duplicate & RPD\%(1) \\
\hline$\overline{\text { UCD1-12 }}$ & 1,1,2-Trichloroethane & $\mathrm{ug} / \mathrm{L}$ & 2.8 & $\mathbf{I H}$ & $3.5 \quad$ Jf I & 22.22 \\
\hline & 1,1-Dichloroethane & $\mathrm{ug} / \mathrm{L}$ & 4.1 & IH & Jf I & 76.69 \\
\hline & 1,1-Dichloroethene & $\mathrm{ug} / \mathrm{L}$ & 1.2 & $\mathbf{I H}$ & Jf I & 175.00 \\
\hline & 1,2-Dichloroethane & $\mathrm{ug} / \mathrm{L}$ & 4.7 & $\bar{H}$ & Jf I & 22.64 \\
\hline & 1,2-Dichloropropane & $\mathbf{u g} / \mathbf{L}$ & $<1$ & IU & 0.72 & 32.56 \\
\hline & Acetone & ug/L & 2.4 & $\mathrm{IJH}$ & $<2.5$ & 4.08 \\
\hline & Bromodichloromethane & $\mathrm{ug} / \mathrm{L}$ & 0.82 & $\mathrm{IJH}$ & 2.1 & 87.67 \\
\hline & Chloroform & $\mathrm{ug} / \mathrm{L}$ & 5700 & $\mathrm{IDH}$ & 9100 & 45.95 \\
\hline & Methylene Chloride & $\mathrm{ug} / \mathrm{L}$ & 1 & IJH & $\leq 1$ & 0.00 \\
\hline $\mathrm{UCD2-14}$ & Arsenic & ug/L & 2.1 & & $<2$ & 4.88 \\
\hline & Barium & $\mathrm{ug} / \mathrm{L}$ & 244 & & 252 & 3.23 \\
\hline & Calcium & $\mathrm{mg} / \mathrm{L}$ & 58.5 & & 60.1 & 2.70 \\
\hline & Chromium & $\mathrm{ug} / \mathrm{L}$ & 43.1 & & 44.9 & 4.09 \\
\hline & Chromium, Hexavalent $(+6)$ & ug/L & 37 & & 37 & 0.00 \\
\hline & Cobalt & $\mathbf{u g} / \mathbf{L}$ & 6.5 & IB & 6.9 & 5.97 \\
\hline & Copper & ug/L & 2.2 & IB & 2.5 & 12.77 \\
\hline . & Iron & ug/L & 103 & & 85.8 & 18.22 \\
\hline 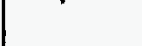 & Magnesium & $\mathrm{mg} / \mathrm{L}$ & 137 & & 142 & 3.58 \\
\hline & Molybdenum & ug/L & 1.7 & IB & 1.4 & 19.35 \\
\hline & Nickel & ug/L & 11.2 & IB & 9.5 & 16.43 \\
\hline & Nitrogen, Nitrate (as N) & $\mathbf{m g} / \mathbf{L}$ & 11 & & 11 & 0.00 \\
\hline & \begin{tabular}{|l|} 
Potassium \\
\end{tabular} & $\mathrm{mg} / \mathrm{L}$ & 0.918 & IB & $<2$ & 74.16 \\
\hline & Sodium & $\mathrm{ug} / \mathrm{L}$ & 45000 & & 47100 & 4.56 \\
\hline & Total Dissolved Solids & $\mathrm{mg} / \mathrm{L}$ & 860 & & 850 & 1.17 \\
\hline & Vanadium & $\mathrm{ug} / \mathrm{L}$ & 9.2 & IB & 9.5 & 3.21 \\
\hline & Zinc & $\mathrm{ug} / \mathrm{L}$ & 5.5 & IB & 4.6 & 17.82 \\
\hline
\end{tabular}

See tables in Appendix A for data qualifier explanations

(1)Relative Percent Difference (RPD) = ABS(100*(Result-Duplicate) $/(($ Result+Duplicate $) / 2)$ )

Analytes included with at least one detection above reporting limit.

$<=$ Not reported above the reporting limit.

(If results were below the reporting limit, then the reporting limit was used for RPD calculations.) 
TABLE 3.5

RELATIVE PERCENT DIFFERENCE FOR FIELD DUPLICATES, GROUNDWATER

FALL QUARTER 1995

LEHR ENVIRONMENTAL RESTORATION

\begin{tabular}{|c|c|c|c|c|c|}
\hline Location & Parameter & Units & Result & Duplicate & RPD\%(1) \\
\hline \multirow[t]{6}{*}{ UCD1-12 } & 1,1,2-Trichloroethane & $\mathrm{ug} / \mathrm{L}$ & 1.1 & (1) & 58.06 \\
\hline & 1,1-Dichloroethane & $\mathrm{ug} / \mathrm{L}$ & 5.8 & 6.8 & 15.87 \\
\hline & 1,1-Dichloroethene & $\mathrm{ug} / \mathrm{L}$ & 11 & 13 & 16.67 \\
\hline & 1,2-Dichloroethane & $\mathrm{ug} / \mathrm{L}$ & 3.2 & 3.8 & 17.14 \\
\hline & Bromodichloromethane & $\mathbf{u g} / \mathrm{L}$ & 0.63 & 0.74 & 16.06 \\
\hline & Chloroform & $\mathrm{ug} / \mathrm{L}$ & 5800 & 6400 & 9.84 \\
\hline \multirow[t]{11}{*}{ UCD1-13 } & Alpha-Chlordane & $\mathrm{ug} / \mathrm{L}$ & 0.0057 & 0.0059 & 3.45 \\
\hline & Bismuth-214 & $\mathrm{pCi} / \mathrm{L}$ & 2.1 & 8.2 & 118.45 \\
\hline & Carbon-14 & $\mathrm{pCi} / \mathrm{L}$ & 1900 & 1770 & 7.08 \\
\hline & Chloroform & $\mathbf{u g} / \mathbf{L}$ & $\mathrm{Jz} \mathrm{I}$ & $\mathrm{JzI}$ & 5.71 \\
\hline & Chromium, Hexavalent (+6) & $\mathrm{ug} / \mathrm{L}$ & 76 & 75 & 1.32 \\
\hline & Dieldrin & $\mathbf{u g} / \mathbf{L}$ & 0.028 & 0.024 & 15.38 \\
\hline & Endrin & ug/L & 0.0085 & $<0.02$ & 80.70 \\
\hline & Gamma-Chlordane & $\mathbf{u g} / \mathbf{L}$ & 0.0049 & 0.0071 & 36.67 \\
\hline & Radium-226 & $\mathrm{pCi} / \mathrm{L}$ & 0.25 & 0.012 & 181.68 \\
\hline & Total Dissolved Solids & $\mathbf{m g} / \mathbf{L}$ & 1100 & 1000 & 9.52 \\
\hline & Tritium & $\mathrm{pCi} / \mathrm{L}$ & 16480 & 15190 & 8.15 \\
\hline
\end{tabular}

See tables in Appendix A for data qualifier explanations

(1)Relative Percent Difference (RPD) = ABS(100*(Result-Duplicate)/((Result+Duplicate)/2))

Analytes included with at least one detection above reporting limit.

$<=$ Not reported above the reporting limit.

(If results were below the reporting limit, then the reporting limit was used for RPD calculations.) 
TABLE 3.6

RELATIVE PERCENT DIFFERENCE FOR FIELD DUPLICATES, SURFACE WATER WINTER QUARTER 1995

LEHR ENVIRONMENTAL RESTORATION

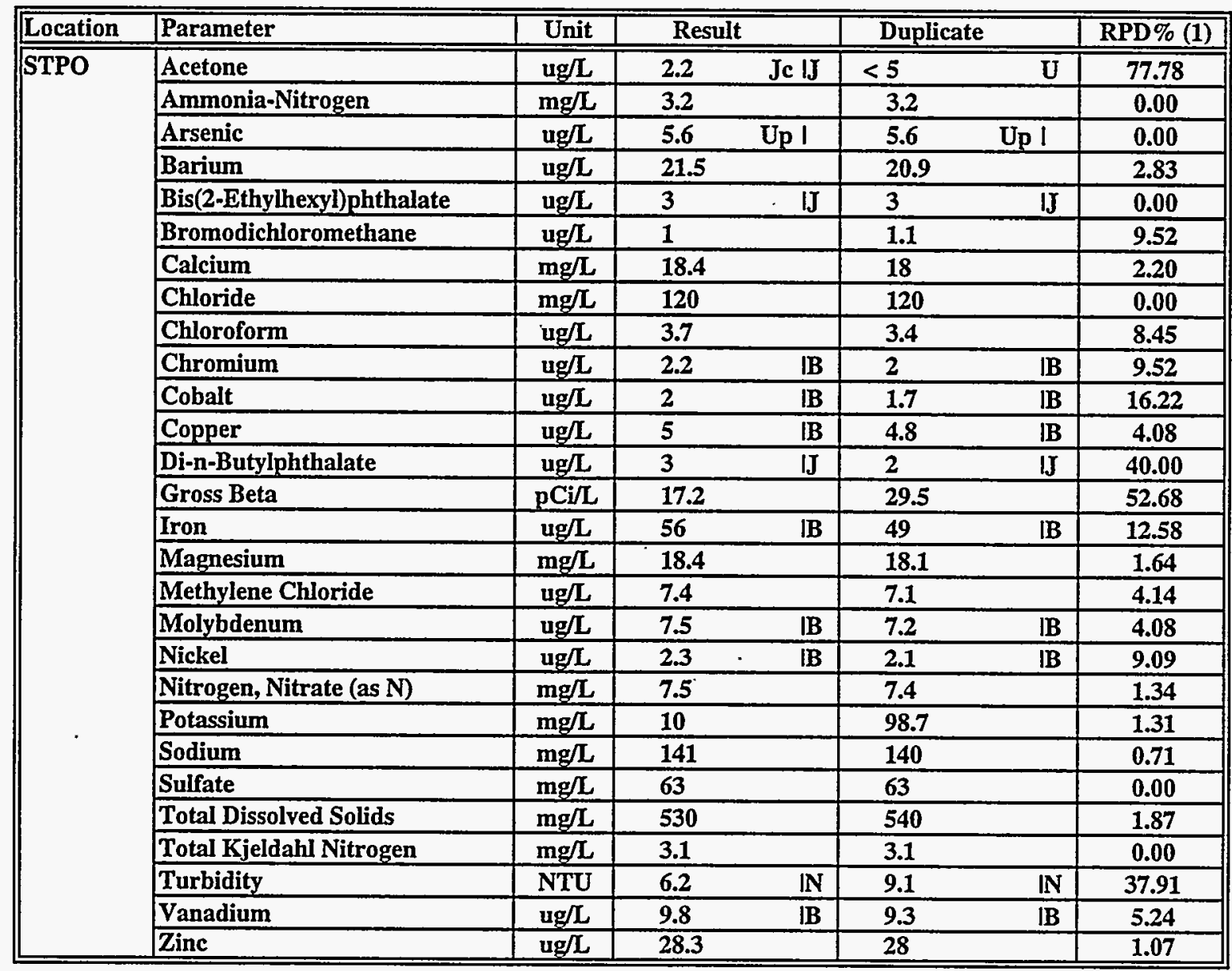

See tables in Appendix A for data qualifier explanations

(1)Relative Percent Difference $(R P D)=A B S(100 *$ (Result-Duplicate $) /(($ Result+Duplicate $) / 2))$

Analytes included with at least one detection above reporting limit.

$<=$ Not reported above the reporting limit.

(If results were below the reporting limit, then the reporting limit was used for RPD calculations.) 
TABLE 3.7

RELATIVE PERCENT DIFFERENCE FOR FIELD DUPLICATES, SURFACE WATER SPRING QUARTER 1995

LEHR ENVIRONMENTAL RESTORATION

\begin{tabular}{|c|c|c|c|c|c|c|c|}
\hline \multirow{2}{*}{$\begin{array}{l}\text { Location } \\
\text { PCU }\end{array}$} & \multirow{2}{*}{$\begin{array}{l}\text { Parameter } \\
\text { Acetone }\end{array}$} & \multirow{2}{*}{$\begin{array}{l}\text { Unit } \\
\text { ug/L }\end{array}$} & \multicolumn{2}{|c|}{ Result } & \multicolumn{2}{|c|}{ Duplicate } & \multirow{2}{*}{$\frac{\text { RPD\% (1) }}{4.65}$} \\
\hline & & & 2.1 & $\mathrm{Uz} \mathbf{I B J}$ & 2.2 & $\mathrm{Uz} \mid \mathrm{BJ}$ & \\
\hline & Barium & $\mathrm{ug} / \mathrm{L}$ & 113 & $\quad \mathbf{I B}$ & 114 & IB & 0.88 \\
\hline & Bis(2-Ethylhexyl)phthalate & ug/L & 2 & $\mathrm{Uz} \mid \mathbf{B J}$ & 10 & $\mathbf{I B J}$ & 133.33 \\
\hline & Bismuth-214 & $\mathrm{pCi} / \mathrm{L}$ & 21 & & $<8.8$ & & 81.88 \\
\hline & Calcium & $\mathrm{mg} / \mathrm{L}$ & 41.3 & & 41 & & 0.73 \\
\hline & Chloride & $\mathrm{mg} / \mathrm{L}$ & 26 & & 26 & & 0.00 \\
\hline & Chromium & $\mathrm{ug} / \mathrm{L}$ & 4.3 & IB & 4 & IB & 7.23 \\
\hline & Di-n-Butylphthalate & $\mathrm{ug} / \mathrm{L}$ & 130 & Jf $\mid \mathbf{B}$ & 38 & Jf IB & 109.52 \\
\hline & DiethylPhthalate & $\mathbf{u g} / \mathbf{L}$ & 2 & IJ & 1 & $\mathrm{IJ}$ & 66.67 \\
\hline & Iron & $\mathrm{ug} / \mathrm{L}$ & 39 & IB & 39 & IB & 0.00 \\
\hline & Magnesium & $\mathrm{mg} / \mathrm{L}$ & 46.5 & & 46 & & 1.08 \\
\hline & \begin{tabular}{|l|} 
Nickel \\
\end{tabular} & $\mathrm{ug} / \mathbf{L}$ & 1.2 & IB & 1.1 & IB & 8.70 \\
\hline & Nitrogen, Nitrate (as N) & $\mathrm{mg} / \mathrm{L}$ & 2.6 & $\cdot$ & 2.6 & & 0.00 \\
\hline & Potassium & $\mathbf{m g} / \mathbf{L}$ & 1.52 & IB & 1.71 & IB & 11.76 \\
\hline & Sodium & $\mathrm{mg} / \mathrm{L}$ & 29.5 & & 28.9 & & 2.05 \\
\hline & Sulfate & $\mathrm{mg} / \mathrm{L}$ & 34 & & 34 & & 0.00 \\
\hline & Total Dissolved Solids & $\mathrm{mg} / \mathrm{L}$ & 370 & IC & 370 & IC & 0.00 \\
\hline & Turbidity & NTU & 62 & & 55 & & 11.97 \\
\hline & Zinc & $\mathrm{ug} / \mathrm{L}$ & 3.7 & IB & 3.2 & IB & 14.49 \\
\hline
\end{tabular}

See tables in Appendix A for data qualifier explanations

(1)Relative Percent Difference (RPD) $=$ ABS $(100 *$ (Result-Duplicate $) /(($ Result+Duplicate $) / 2))$

Analytes included with at least one detection above reporting limit.

$<=$ Not reported above the reporting limit.

(If results were below the reporting limit, then the reporting limit was used for RPD calculations.) 
TABLE 3.8

RELATTVE PERCENT DIFFERENCE FOR FIELD DUPLICATES, SURFACE WATER SUMMER QUARTER 1995 LEHR ENVIRONMENTAL RESTORATION

\begin{tabular}{|c|c|c|c|c|c|c|c|}
\hline Location & Parameter & Units & \multicolumn{2}{|c|}{ Result } & \multicolumn{2}{|c|}{ Duplicate } & RPD\%(1) \\
\hline \multirow[t]{14}{*}{ PCU } & Arsenic & ug/L & 2.5 & & 2.6 & & 3.92 \\
\hline & Barium & ug/L & 113 & & 112 & & 0.89 \\
\hline & Calcium & $\mathrm{mg} / \mathrm{L}$ & 35.2 & & 33.1 & & 6.15 \\
\hline & Chromium & $\mathrm{ug} / \mathrm{L}$ & 3.3 & IB & 4.3 & IB & 26.32 \\
\hline & Iron & $\mathrm{ug} / \mathrm{L}$ & 58.6 & IB & 67.8 & IB & 14.56 \\
\hline & Magnesium & $\mathrm{mg} / \mathrm{L}$ & 41.3 & & 38.5 & & 7.02 \\
\hline & Molybdenum & ug/L & 1.1 & IB & 1.1 & IB & 0.00 \\
\hline & Nickel & ug/L & 4.6 & IB & 4.5 & IB & 2.20 \\
\hline & Nitrogen, Nitrate (as N) & $\mathrm{mg} / \mathrm{L}$ & 1.5 & & 1.5 & & 0.00 \\
\hline & Potassium & $\mathbf{m g} / \mathbf{L}$ & 1.67 & IB & 1.92 & IB & 13.93 \\
\hline & Sodium & $\mathrm{mg} / \mathrm{L}$ & 30.5 & & 28.1 & & 8.19 \\
\hline & Total Dissolved Solids & $\mathrm{mg} / \mathrm{L}$ & 290 & & 300 & & 3.39 \\
\hline & Vanadium & $\mathrm{ug} / \mathrm{L}$ & 7.6 & IB & 4.9 & IB & 43.20 \\
\hline & Zinc & $\mathbf{u g} / \mathrm{L}$ & 6 & IB & 9.2 & IB & 42.11 \\
\hline
\end{tabular}

See tables in Appendix A for data qualifier explanations

(1)Relative Percent Difference (RPD) $=$ ABS(100*(Result-Duplicate) $/(($ Result+Duplicate)/2))

Analytes included with at least one detection above reporting limit.

$<=$ Not reported above the reporting limit.

(If results were below the reporting limit, then the reporting limit was used for RPD calculations.) 
TABLE 3.9

RELATIVE PERCENT DIFFERENCE FOR FIELD DUPLICATES, SURFACE WATER

S FALL QUARTER 1995

LEHR ENVIRONMENTAL RESTORATION

\begin{tabular}{|c|c|c|c|c|c|c|c|}
\hline \multirow{2}{*}{$\frac{\text { Location }}{\text { PCD }}$} & \multirow{2}{*}{\begin{tabular}{|l} 
Parameter \\
Acetone \\
\end{tabular}} & \multirow{2}{*}{$\begin{array}{l}\text { Units } \\
\text { ug/L }\end{array}$} & \multicolumn{2}{|c|}{ Result } & \multicolumn{2}{|c|}{ Duplicate } & \multirow{2}{*}{$\frac{\text { RPD\%(1) }}{10.17}$} \\
\hline & & & 3.1 & Jz IJB & 2.8 & $\mathrm{Jz}$ IJB & \\
\hline & Americium-241 & $\mathrm{pCi} / \mathrm{L}$ & 0.035 & & 0.056 & & 46.15 \\
\hline & Antimony & $\mathrm{ug} / \mathrm{L}$ & $<5$ & IU & 4.7 & IB & 6.19 \\
\hline & Arsenic & ug/L & 2.7 & & 2 & & 29.79 \\
\hline & Barium & ug/L & 73.6 & & 73 & & 0.82 \\
\hline & Bismuth-214 & $\mathrm{pCi} / \mathrm{L}$ & 6.2 & & 9.8 & & 45.00 \\
\hline & Calcium & $\mathbf{m g} / \mathbf{L}$ & 23.2 & & 22.9 & & 1.30 \\
\hline & Chloroform & ug/L & 1.9 & Jy 1 & $<1$ & IU & 62.07 \\
\hline & Chromium & ug/L & 4.5 & IB & 4.7 & IB & 4.35 \\
\hline & Copper & $\mathrm{ug} / \mathrm{L}$ & $<10$ & $\mathrm{IU}$ & 10 & IB & 0.00 \\
\hline & Gross Beta & $\mathrm{pCi} / \mathrm{L}$ & 2.8 & & 3.4 & & 19.35 \\
\hline & Iron & ug/L & 244 & & 269 & & 9.75 \\
\hline & Magnesium & mgL & 29.2 & & 29 & & 0.69 \\
\hline & Nickel & ug/L & 4.4 & IB & 5.2 & IB & 16.67 \\
\hline & Nitrogen, Nitrate (as N) & $\mathrm{mg} / \mathrm{L}$ & 1.8 & & 1.9 & & 5.41 \\
\hline & Potassium & $\mathbf{m g} / \mathbf{L}$ & 2.87 & IB & 3.3 & IB & 13.94 \\
\hline & Radium-226 & $\mathrm{pCi} / \mathrm{L}$ & 0.12 & & 0.86 & & 151.02 \\
\hline & Sodium & $\mathrm{mg} / \mathrm{L}$ & 19.1 & & 19.1 & & 0.00 \\
\hline & Strontium-90 & $\mathrm{pCi} / \mathrm{L}$ & 1.17 & & 1.39 & & 17.19 \\
\hline & Total Dissolved Solids & $\mathbf{m g} / \mathbf{L}$ & 300 & & 260 & & 14.29 \\
\hline & Zinc & $\mathrm{ug} / \mathrm{L}$ & 8.2 & Jm IENB & 9 & Jm IENB & 9.30 \\
\hline
\end{tabular}

See tables in Appendix A for data qualifier explanations

(1)Relative Percent Difference (RPD) $=$ ABS(100*(Result-Duplicate) $/(($ Result+Duplicate $) / 2))$

Analytes included with at least one detection above reporting limit.

$<=$ Not reported above the reporting limit.

(If results were below the reporting limit, then the reporting limit was used for RPD calculations.) 
TABLE 3.10

RELATIVE PERCENT DIFFERENCE FOR FIELD DUPLICATES, STORM WATER RUNOFF WINTER QUARTER 1995 LEHR ENVIRONMENTAL RESTORATION

\begin{tabular}{|c|c|c|c|c|c|c|c|}
\hline \multirow{2}{*}{\begin{tabular}{|l} 
LOCATION \\
SWL00002
\end{tabular}} & \multirow{2}{*}{$\begin{array}{l}\text { PARAMETER } \\
\text { Calcium }\end{array}$} & \multirow{2}{*}{$\begin{array}{l}\text { UNITS } \\
\text { mg/L }\end{array}$} & \multicolumn{2}{|c|}{ RESULTS } & \multicolumn{2}{|c|}{ DUPLICATE } & \multirow{2}{*}{$\begin{array}{c}\overline{R P D}(\mathbf{1}) \\
2.9\end{array}$} \\
\hline & & & 0.57 & |B & 0.598 & [B & \\
\hline & Magnesium & $\mathrm{mg} / \mathrm{L}$ & 0.13 & |B & 0.128 & [B & 1.1 \\
\hline & Nitrogen, Nitrate (as N) & $\mathrm{mg} / \mathrm{L}$ & 0.13 & & $<0.1$ & & 16.7 \\
\hline & Potassium & $\mathrm{mg} / \mathrm{L}$ & 0.89 & |B & $<2$ & jU & $\overline{58.9}$ \\
\hline & Sodium & $\mathrm{mg} / \mathrm{L}$ & 0.29 & [B & 0.266 & |B & 4.8 \\
\hline & Alkalinity Total(as CaCO3) & $\mathrm{mg} / \mathrm{L}$ & 14 & & 14 & & 0.0 \\
\hline & Total Organic Carbon & $\mathrm{mg} / \mathrm{L}$ & 2.5 & & 2.5 & & 0.0 \\
\hline & Turbidity & NTU & 3.1 & & 3.1 & & 0.0 \\
\hline & Antimony & $\mathrm{ug} / \mathrm{L}$ & 156 & & 137 & & 8.5 \\
\hline & Barium & $\mathrm{ug} / \mathrm{L}$ & 3.3 & |B & 3 & |B & 6.3 \\
\hline & Copper ${ }^{\circ}$ & $\mathrm{ug} / \mathrm{L}$ & 2.2 & |B & 1.9 & |B & 9.5 \\
\hline & Lead & ug/L & 38.4 & & 35.9 & & 4.4 \\
\hline & Zinc & ug/L & 94.1 & & 112 & & 11.9 \\
\hline & Bis(2-Ethylhexyl)phthalate & $\mathrm{ug} / \mathrm{L}$ & $<10$ & $\mathbf{U}$. & 0.5 & $\mathbf{J}$ & 92.7 \\
\hline & Di-n-Butylphthalate & $\mathrm{ug} / \mathrm{L}$ & 8 & $\mathbf{U z}[\mathbf{B J}$ & 5 & $\mathrm{Uz}[\mathrm{BJ}$ & 28.6 \\
\hline & Acetone & $u g / L$ & 4.6 & UJe $\mid \mathbf{B J}$ & 8.8 & Jc l & 46.7 \\
\hline
\end{tabular}

See tables in Appendix A for data qualifier explanations

(1)Relative Percent Difference $(\mathrm{RPD})=\mathrm{ABS}(100 *($ Result - Duplicate $) /(($ Result + Duplicate $) / 2))$

Analytes included with at least one detection above reporting limit.

$<=$ Not reported above the detection limit .

If results were below reporting limit then the limit was used for RPD calculations. 
Table 3.11. Sample Matrix Spike and Duplicate Results Ranges of Accuracy and Precision

\begin{tabular}{|c|c|c|c|c|c|}
\hline \multirow[b]{2}{*}{ Parameter } & \multicolumn{3}{|c|}{$\begin{array}{l}\text { Percent Recovery } \\
\text { Reported Range }\end{array}$} & \multicolumn{2}{|c|}{ RPD $^{(b)}$} \\
\hline & Matrix Spike & $\begin{array}{c}\text { Duplicate } \\
\text { Matrix Spike }\end{array}$ & $\begin{array}{l}\text { Control } \\
\text { Range }^{(a)}\end{array}$ & $\begin{array}{l}\text { Range of } \\
\text { Results }^{(c)}\end{array}$ & Limit \\
\hline \multicolumn{6}{|c|}{ Semivolatile Organic Compounds } \\
\hline Phenol & $56-74$ & $56-70$ & $12-89$ & $2-9$ & 42 \\
\hline 2-Chlorophenol & $64-73$ & $58-71$ & $27-123$ & $3-11$ & 40 \\
\hline 1,4-Dichlorobenzene & $44-53$ & $43-48$ & $36-97$ & $2-11$ & 28 \\
\hline N-Nitroso-di-n-propylamine & $65-85$ & $60-74$ & $41-116$ & $8-25$ & 38 \\
\hline 1,2,4-Trichlorobenzene & $54-63$ & $51-53$ & $39-98$ & $6-12$ & 28 \\
\hline 4-Chloro-3-methylphenol & $86-92$ & $74-85$ & $23-97$ & $7-17$ & 42 \\
\hline Acenaphthene & $72-77$ & $63-66$ & $46-118$ & $12-15$ & 31 \\
\hline 4-Nitrophenol & $66-83$ & $63-69$ & $10-80$ & $5-23$ & 50 \\
\hline 2,4-Dinitrotoluene & $80-90$ & $70-81$ & $24-96$ & $11-13$ & 38 \\
\hline Pentachlorophenol & $63-124$ & $52-110$ & $9-103$ & $4-19$ & 50 \\
\hline Pyrene & $73-94$ & $70-91$ & $26-127$ & $3-18$ & 31 \\
\hline \multicolumn{6}{|l|}{ Pesticides/PCBs } \\
\hline gamma-BHC (Lindane) & $60-100$ & $95-95$ & $56-123$ & $0-45$ & 15 \\
\hline Heptachlor & $48-100$ & $48-95$ & $40-131$ & $0-34$ & 20 \\
\hline Aldrin & $45-115$ & $39-100$ & $40-120$ & $12-52$ & 18 \\
\hline Dieldrin & $58-100$ & $92-96$ & $52-126$ & $0-47$ & 18 \\
\hline Endrin & $54-90$ & $87-90$ & $56-121$ & $3-48$ & 21 \\
\hline 4,4'-DDT & $56-85$ & $82-87$ & $38-127$ & $2-42$ & 27 \\
\hline \multicolumn{6}{|l|}{ Metals } \\
\hline Antimony & $92-101$ & - & $80-120$ & $0-2$ & 25 \\
\hline Arsenic & $86-105$ & - & $80-120$ & $0-7$ & 25 \\
\hline Barium & $90-109$ & - & $80-120$ & $0-4$ & 25 \\
\hline Beryllium & $88-110$ & - & $80-120$ & 0 & 25 \\
\hline Cadmium & $92-103$ & - & $80-120$ & 0 & $\cdot 25$ \\
\hline Chromium & $93-104$ & - & $80-120$ & $0-3$ & 25 \\
\hline Hexavalent Chromium & $90-110$ & - & $80-120$ & $0-5$ & 25 \\
\hline
\end{tabular}


Table 3.11. (contd)

\begin{tabular}{|c|c|c|c|c|c|}
\hline \multirow[b]{2}{*}{ Parameter } & \multicolumn{3}{|c|}{$\begin{array}{c}\text { Percent Recovery } \\
\text { Reported Range }\end{array}$} & \multicolumn{2}{|c|}{$\mathrm{RPD}^{(\mathrm{b})}$} \\
\hline & Matrix Spike & $\begin{array}{c}\text { Duplicate } \\
\text { Matrix Spike }\end{array}$ & $\begin{array}{l}\text { Control } \\
\text { Range }^{(a)}\end{array}$ & $\begin{array}{l}\text { Range of } \\
\text { Results } \\
(c)\end{array}$ & Limit \\
\hline Cobalt & $92-108$ & $\therefore$ & $80-120$ & 0 & 25 \\
\hline Copper & $91-99$ & - & $80-120$ & 0 & 25 \\
\hline Iron & $80-101$ & - & $80-120$ & $1-5$ & 25 \\
\hline Lead & $95-102$ & - & $80-120$ & $0-3$ & 25 \\
\hline Mercury & $90-119$ & - & $80-120$ & 0 & 25 \\
\hline Molybdenum & $94-99$ & - & $80-120$ & 0 & 25 \\
\hline Nickel & $89-101$ & - & $80-120$ & $0-1$ & 25 \\
\hline Selenium & $88-105$ & - & $80-120$ & $2-7$ & 25 \\
\hline Silver & $89-98$ & - & $80-120$ & 0 & 25 \\
\hline Thallium & $92-100$ & - & $80-120$ & 0 & 25 \\
\hline Vanadium & $91-104$ & - & $80-120$ & $0-6$ & 25 \\
\hline Zinc & $80-132$ & - & $80-120$ & $1-2$ & 25 \\
\hline \multicolumn{6}{|c|}{ General Water Quality Parameters } \\
\hline Alkalinity & - & - & - & $0-2$ & 25 \\
\hline Chemical Oxygen Demand & $91-118$ & - & $80-120$ & 0 & 25 \\
\hline Formaldehyde & $95-118$ & - & $80-120$ & 0 & 25 \\
\hline Total Kjeldahl Nitrogen & $43-88$ & - & $80-120$ & 0 & 25 \\
\hline Total Dissolved Solids & - & - & - & $0-36$ & 25 \\
\hline Total ,Organic Carbon & $91-105$ & - & $80-120$ & $0-9$ & 25 \\
\hline Turbidity & $96-128$ & - & $80-120$ & $0-4$ & 25 \\
\hline
\end{tabular}


Table 3.11. (contd)

\begin{tabular}{|c|c|c|c|c|c|}
\hline \multirow[b]{2}{*}{ Parameter } & \multicolumn{3}{|c|}{$\begin{array}{l}\text { Percent Recovery } \\
\text { Reported Range }\end{array}$} & \multicolumn{2}{|c|}{ RPD $^{(b)}$} \\
\hline & Matrix Spike & $\begin{array}{c}\text { Duplicate } \\
\text { Matrix Spike }\end{array}$ & $\begin{array}{l}\text { Control } \\
\text { Range }^{(\mathrm{a})}\end{array}$ & $\begin{array}{l}\text { Range of } \\
\text { Results }^{(c)}\end{array}$ & Limit \\
\hline \multicolumn{6}{|l|}{ Radionuclides } \\
\hline Radium-226 & $75-105$ & - & $80-120$ & - & - \\
\hline Strontium-90 & $83-126$ & - & $75-125$ & - & - \\
\hline Carbon-14 & $51-100$ & - & $75-125$ & - & - \\
\hline Gross Alpha & $65-146$ & - & $70-130$ & - & - \\
\hline Gross Beta & $86-130$ & - & $70-130$ & - & - \\
\hline Americium-241 & $61-92$ & - & $80-120$ & - & - \\
\hline Plutonium-241 & $81-111$ & - & $80-120$ & - & - \\
\hline Tritium & $60-117$ & - & $80-120$ & - & - \\
\hline \multicolumn{6}{|l|}{ Anions \& Cations } \\
\hline Ammonia-Nitrogen & $106-115$ & - & $80-120$ & 0 & 25 \\
\hline Calcium & $98-104$ & - & $80-120$ & $0-2$ & 25 \\
\hline Chloride & $94-111$ & - & $80-120$ & $0-12$ & 25 \\
\hline Magnesium & $93-104$ & - & $80-120$ & $0-3$ & 25 \\
\hline Nitrate as Nitrogen & $83-118$ & - & $80-120$ & $0-7$ & 25 \\
\hline Potassium & $99-102$ & - & $80-120$ & 0 & 25 \\
\hline Sodium & $101-101$ & - & $80-120$ & $0-3$ & 25 \\
\hline Sulfate & $96-114$ & - & $80-120$ & 0 & 25 \\
\hline Total Phosphorus & $95-100$ & - & $80-120$ & 0 & 25 \\
\hline \multicolumn{6}{|c|}{$\begin{array}{l}\text { (a) Control range for spike recovery and RPD duplicate precision as defined in the QAPjP. } \\
\text { (b) Relative percent difference. } \\
\text { (c) For semivolatiles and pesticides/PCBs, A RPD range was calculated and is presented for } \\
\text { each set of matrix spike and matrix spike duplicates. For metals, anions and cations, and } \\
\text { general water quality parameters, RPDs have been calculated for laboratory-prepared sample } \\
\text { duplicates in lieu of spike duplicates. } \\
\text { - Analysis not performed in accordance with methodology. }\end{array}$} \\
\hline
\end{tabular}


Table 3.12. Analytical Laboratory Control Sample Spike Results Range of Accuracy

\begin{tabular}{|c|c|c|c|}
\hline \multirow[b]{2}{*}{ Method } & \multirow[b]{2}{*}{ Chemical } & \multicolumn{2}{|c|}{ Accuracy $^{(\mathrm{a})}$} \\
\hline & & $\begin{array}{l}\text { Laboratory LCS } \\
\text { Percent Recovery } \\
\text { Reported Range }^{(b)}\end{array}$ & $\begin{array}{c}\text { Acceptable } \\
\text { Range }^{(c)}\end{array}$ \\
\hline \multirow{12}{*}{$\begin{array}{l}\text { Volatile Organic } \\
\text { Compounds }\end{array}$} & Vinyl chloride & $78-140$ & $60-140$ \\
\hline & 1,2-Dichloroethane & $82-139$ & $60-140$ \\
\hline & Carbon tetrachloride & $78-118$ & $60-140$ \\
\hline & 1,2-Dichloropropane & $80-120$ & $60-140$ \\
\hline & cis-1,3-Dichloropropene & $76-124$ & $60-14 Q$ \\
\hline & Trichloroethene & $80-120$ & $60-140$ \\
\hline & 1,1,2-Trichloroethane & $78-132$ & $60-140$ \\
\hline & Benzene & $82-120$ & $60-140$ \\
\hline & Bromoform & $72-118$ & $60-140$ \\
\hline & Tetrachloroethene & $82-122$ & $60-140$ \\
\hline & 1,2-Dibromomethane & $76-132$ & $60-140$ \\
\hline & 1,4-Dichlorobenzene & $76-118$ & $60-140$ \\
\hline \multirow{11}{*}{$\begin{array}{l}\text { Semivolatile Organic } \\
\text { Compounds }\end{array}$} & 4-Nitrophenol & $57-95$ & $10-80$ \\
\hline & 2,4-Dinitrotoluene & $59-92$ & $24-96$ \\
\hline & Pentachlorophenol & $41-133$ & $9-103$ \\
\hline & Pyrene & $44-100$ & $26-127$ \\
\hline & Phenol & $52-84$ & $12-89$ \\
\hline & 2-Chlorophenol & $61-93$ & $27-123$ \\
\hline & 1,4-Dichlorobenzene & $32-65$ & $36-97$ \\
\hline & N-Nitroso-di-n-propylamine & $58-84$ & $41-116$ \\
\hline & 1,2,4-Trichlorobenzene & $40-72$ & $39-98$ \\
\hline & 4-Chloro-3-methylphenol & $56-95$ & $23-97$ \\
\hline & Acenaphthene & $53-88$ & $46-118$ \\
\hline
\end{tabular}


Table 3.12. (contd)

\begin{tabular}{|c|c|c|c|}
\hline \multirow[b]{2}{*}{ Method } & \multirow[b]{2}{*}{ Chemical } & \multicolumn{2}{|c|}{ Accuracy $^{(a)}$} \\
\hline & & $\begin{array}{l}\text { Laboratory LCS } \\
\text { Percent Recovery } \\
\text { Reported Range }{ }^{(b)}\end{array}$ & $\begin{array}{c}\text { Acceptable } \\
\text { Range }^{(c)}\end{array}$ \\
\hline \multirow[t]{6}{*}{ Pesticides/PCBs } & gamma-BHC & $65-110$ & $56-123$ \\
\hline & Heptachlor & $47-95$ & $40-131$ \\
\hline & Dieldrin & $75-105$ & $40-120$ \\
\hline & Endrin & $57-100$ & $52-126$ \\
\hline & 4,4'-DDT & $70-97$ & $56-121$ \\
\hline & Aldrin & $38-95$ & $38-127$ \\
\hline \multirow[t]{9}{*}{ Radionuclides } & Gross Alpha & $85-135$ & $70-130$ \\
\hline & Gross Beta & $94-122$ & $70-130$ \\
\hline & Carbon-14 & $86-106$ & $75-125$ \\
\hline & Radium-226 & $77-94$ & $80-120$ \\
\hline & Strontium-90 & $76-121$ & $75-127$ \\
\hline & Tritium & $89-103$ & $80-120$ \\
\hline & Americium-241 & $75-95$ & $80-120$ \\
\hline & Plutonium-241 & $83-104$ & $80-120$ \\
\hline & $\begin{array}{l}\text { Gamma Analysis } \\
\text { Cesium-137 } \\
\text { Cobalt-60 } \\
\end{array}$ & $\begin{array}{l}90-108 \\
92-105 \\
\end{array}$ & $\begin{array}{l}80-120 \\
80-120 \\
\end{array}$ \\
\hline \multirow[t]{7}{*}{ Metals } & Antimony & $93-111$ & $75-125$ \\
\hline & Arsenic & $87-111$ & $75-125$ \\
\hline & Barium & $89-112$ & $75-125$ \\
\hline & Beryllium & $93-113$ & $75-125$ \\
\hline & Cadmium & $97-120$ & $75-125$ \\
\hline & Chromium & $93-110$ & $75-125$ \\
\hline & Hexavalent Chromium & $90-104$ & $75-125$ \\
\hline
\end{tabular}


Table 3.12. (contd)

\begin{tabular}{|c|c|c|c|}
\hline \multirow[b]{2}{*}{ Method } & \multirow[b]{2}{*}{ Chemical } & \multicolumn{2}{|c|}{ Accuracy ${ }^{(a)}$} \\
\hline & & $\begin{array}{l}\text { Laboratory LCS } \\
\text { Percent Recovery } \\
\text { Reported Range }^{(b)}\end{array}$ & $\begin{array}{c}\text { Acceptable } \\
\text { Range }^{(c)}\end{array}$ \\
\hline \multirow[t]{12}{*}{ Metals } & Cobalt & $94-110$ & $75-125$ \\
\hline & Copper & $95-113$ & $75-125$ \\
\hline & Iron & $84-111$ & $75-125$ \\
\hline & Lead & $91-112$ & $75-125$ \\
\hline & Mercury & $86-116$ & $75-125$ \\
\hline & Molybdenum & $89-109$ & $75-125$ \\
\hline & Nickel & $95-113$ & $75-125$ \\
\hline & Selenium & $97-120$ & $75-125$ \\
\hline & Silver & $94-111$ & $75-125$ \\
\hline & Thallium & $89-109$ & $75-125$ \\
\hline & Vanadium & $92-107$ & $75-125$ \\
\hline & Zinc & $97-120$ & $75-125$ \\
\hline \multirow[t]{9}{*}{ Anions and Cations } & Ammonia as Nitrogen & $104-109$ & $75-125$ \\
\hline & Calcium & $100-105$ & $75=125$ \\
\hline & Chloride & $97-107$ & $75-125$ \\
\hline & Magnesium & $99-106$ & $75-125$ \\
\hline & Nitrate & $95-110$ & $75-125$ \\
\hline & Potassium & $97-104$ & $75-125$ \\
\hline & Sodium & $98-104$ & $75-125$ \\
\hline & Sulfate & $95-109$ & $75-125$ \\
\hline & Total Phosphorous & $99-99$ & $75-125$ \\
\hline
\end{tabular}


Table 3.12. (contd)

\begin{tabular}{|c|c|c|c|}
\hline \multirow[b]{2}{*}{ Method } & \multirow[b]{2}{*}{ Chemical } & \multicolumn{2}{|c|}{ Accuracy ${ }^{(a)}$} \\
\hline & & $\begin{array}{l}\text { Laboratory LCS } \\
\text { Percent Recovery } \\
\text { Reported Range }\end{array}$ & $\begin{array}{c}\text { Acceptable } \\
\text { Range }^{(c)}\end{array}$ \\
\hline \multirow{7}{*}{$\begin{array}{l}\text { Groundwater Quality } \\
\text { Parameters }\end{array}$} & Alkalinity & $99-100$ & $75-125$ \\
\hline & Chemical Oxygen Demand & $90-114$ & $75-125$ \\
\hline & Formaldehyde & $111-119$ & $75-125$ \\
\hline & Total Kjeldahl Nitrogen & $97-102$ & $75-125$ \\
\hline & Total Dissolved Solids & $97-107$ & $75-125$ \\
\hline & Total Organic Carbon & $95-104$. & $75-125$ \\
\hline & Turbidity & $94-109$ & $75-125$ \\
\hline \multicolumn{4}{|c|}{$\begin{array}{l}\text { (a) Accuracy expressed as percent recovery. } \\
\text { (b) Range of Laboratory Control Sample (LCS) spike recoveries from all laboratory } \\
\text { reports associated with this annual monitoring. } \\
\text { (c) Acceptable range for percent recovery as defined by the QAPjP. }\end{array}$} \\
\hline
\end{tabular}


TABLE 3.13

SUMIMARY OF DETECTIONS IN TRIP BLANKS

LEHR ENVIRONMENTAL RESTORATION

\begin{tabular}{|c|c|c|c|c|c|c|}
\hline Quarter & Blank & Method & Parameter & Results & & Units \\
\hline WINTER & TBGW0016 & CLPVOA & Xylenes (Total) & 1.3 & & $\mathrm{ug} / \mathrm{L}$ \\
\hline WINTER & TBGW0017 & CLPVOA & All Analytes & ND & & \\
\hline WINTER & TBGW0018 & CLPVOA & All Analytes & ND & & \\
\hline WINTER & TBGW0020 & CLPVOA & All Analytes & ND & & \\
\hline WINTER & TBSW0002 & CLPYOA & All Analytes & ND & & \\
\hline WINTER & TBSW0003 & CLPVOA & All Analytes & ND & & \\
\hline SPRING & TBGW0023 & CLPVOA & Acetone & 2.3 & $\mathrm{Jc}|\mathrm{BJ}|$ & $\mathrm{ug} / \mathrm{L}$ \\
\hline SPRING & TBGW0024 & CLPVOA & All Analytes & ND & & \\
\hline SPRING & TBGW0025 & CLPVOA & All Analytes & ND & & \\
\hline SPRING & TBGW0026 & CLPVOA & All Analytes & ND & & \\
\hline SPRING & TBGW0027 & CLPVOA & All Analytes & ND & & \\
\hline SPRING & TBSW0004 & CLPVOA & All Analytes & ND. & & \\
\hline SUMMER & TBGW0100 & CLPVOA & All Analytes & ND & & \\
\hline SUMMER & TBGW0101 & CLPVOA & All Analytes & ND & & \\
\hline SUMMER & TBGW0102 & CLPVOA & All Analytes & ND & & \\
\hline SUMMER & TBGW0103 & CLPVOA & All Analytes & ND & & \\
\hline SUMMER & TBGW0104 & CLPVOA & All Analytes & ND & & \\
\hline SUMMER & TBGW0105 & CLPVOA & All Analytes & ND & & \\
\hline SUMMER & TBSW0051 & CLPVOA & Acetone & 2.3 & $\mathrm{Jc} \mid \mathrm{J}$ & $\mathrm{ug} / \mathrm{L}$ \\
\hline FALL & TBGW0113 & CLPVOA & Acetone & 2.3 & $\mathrm{Jc} \mid \mathrm{J}$ & $\mathrm{ug} / \mathrm{L}$ \\
\hline FALL & TBGW0114 & CLPVOA & Acetone & 2.5 & $\mathrm{Jc}|\mathrm{J}|$ & $\mathrm{ug} / \mathrm{L}$ \\
\hline FALL & TBGW0115 & CLPVOA & All Analytes & ND & & \\
\hline FALL & TBGW0122 & CLPYOA & All Analytes & ND & & \\
\hline FALL & TBGW0123 & CLPVOA & All Analytes & ND & & \\
\hline FALL & TBGW0124 & CLPVOA & All Analytes & ND & & \\
\hline FALL & TBGW0125 & CLPVOA & Acetone & 2.1 & $\mathrm{Jc} \mid \mathrm{JB}$ & $\mathrm{ug} / \mathrm{L}$ \\
\hline FALL & TBGW0126 & CLPVOA & All Analytes & ND & & \\
\hline FALL & TBGW0127 & CLPVOA & All Analytes & ND & & \\
\hline FALL. & TBGW0128 & CLPVOA & All Analytes & ND & & \\
\hline FALL & TBGW0129 & CLPVÖA & All Analytes & ND & & \\
\hline FALL & TBGW0130 & CLPVOA & All Analytes & ND & & \\
\hline FALL & TBGW0290 & CLPVOA & Acetone & 2.2 & $\mathrm{Jz} \mid \mathrm{JB}$ & $\mathrm{ug} / \mathrm{L}$ \\
\hline
\end{tabular}

ND $=$ Not Detected

See Appendix A for data explanations 



\subsection{Hydrogeology}

The hydrogeology in the vicinity of the LEHR site was initially characterized during the Phase II Site Characterization (DOE 1993). In 1995, five new monitoring wells were installed and five additional cone penetrometer tests (CPT) were performed as part of the RI/FS program. This section summarizes the regional setting and the hydrogeology of the LEHR site. A refined site hydrogeologic conceptual model is presented, based primarily on interpretation of logs from the recently completed wells and, to a lesser extent, on the CPT logs. In addition, a discussion is provided on groundwater elevations and flow directions and horizontal and vertical hydraulic gradients observed during 1995.

\subsection{Regional Setting}

The LEHR site is located in the southern portion of the Sacramento Valley on the relatively flat-lying Putah Plain (DWR 1978). Average elevation at the site is approximately 50 feet above mean sea level (msl). Relief across the site is approximately two feet, with the lowest point near the cobalt-60 field. The land surface slopes gently east/northeast toward the Sacramento River at approximately five feet per mile.

The Sacramento Valley is a large, asymmetrical physiographic basin bordered by the Coast Ranges to the west, the Klamath Mountains and Cascade Range to the north, and the Sierra Nevada Mountains to the east. Marine and continental sedimentary deposits occur within the basin, ranging in age from Jurassic to Recent. Norris and Webb (1976) suggest the sediments are over 30,000 feet thick near the valley axis. Deformation of these deposits during uplift of the surrounding mountains resulted in a regional dip of the sediments from the sides of the valley toward its axis.

The LEHR site is located east of the valley axis on the geomorphic unit termed "low alluvial plains and fans" or Putah Plain (DWR 1978). This surface represents distal portions of alluvial fan deposits associated with Putah Creek, referred to as the Putah Creek Fan (Mann 1992). Sediments from within alluvial fan deposits consist primarily of silts and clays, with coarse-grained sediments occurring locally. During geologic time, Putah Creek changed course many times throughout this area, resulting in ribbon-like strips of coarse, main-channel deposits flanked by overbank silts and clays. The ages of these deposits range from late Pleistocene to Recent. Thickness of the alluvial fan deposits is reported to be between 140 feet (Mann 1992) and 180 feet (DWR 1978).

Underlying the Putah Creek Fan is the Plio-Pleistocene age Tehama Formation. The Tehama Formation consists of fine-grained sands and silts with discontinuous lenses of coarse sand and gravel. It is the principal water-bearing formation on the west side of the Sacramento Valley. This unit is thought to be coeval with the Laguna Formation on the east side of the Sacramento Valley (DWR 1978; Olmsted and Davis 1961). 
Locally, both unconfined and confined aquifers occur in the upper 3,000 feet of sediments in the Sacramento Valley. No regionally continuous confining units exist (DWR 1978). Groundwater generally flows toward the Sacramento River from the west and east sides. At various depths beneath the valley floor, saline water is found, trapped there during deposition of the marine sediments. The depth to the base of the fresh water varies from as little as 400 feet to over 3,000 feet.

The major groundwater sources for public and private water supplies in the Sacramento Valley are the unconsolidated sediments of Pliocene and Pliestocene ages and the older alluvium. The younger alluvium and stream channel deposits are less frequently tapped. Groundwater is recharged through leakage from streams and rivers and direct infiltration from precipitation and irrigation.

\subsection{LEHR Site Hydrogeology}

This section focuses on the principal hydrogeologic units beneath the LEHR site. For the purposes of this report, a hydrogeologic unit is defined as a laterally continuous geologic unit with hydrologic significance. Hydrologic significance is indicated by hydraulic conductivity or hydrochemistry contrasts between units. Hydrogeologic units depicted in this report correspond to hydrostratigraphic units referenced in previous reports (DOE $1993,1994,1995)$. In a strict sense, a hydrostratigraphic unit as proposed by Maxey $(1964)$ is defined as a body of rock having considerable lateral extent and composing a geologic framework for a reasonably distinct hydrologic system.

Hydrogeologic unit interpretation was based primarily on physical sample descriptions from two of the five new monitoring wells, hydraulic characterization results, and geophysical logging (DOE 1993). Physical sample descriptions included texture, grading, color, consolidation, and gross mineralogy. Natural gamma, porosity, and bulk density logs were used to corroborate the sample descriptions and identify permeable zones for screening in one well. Daily borehole logs for the new wells and CPT logs from 1995 will be presented in a subsequent report (Liikala et al. 1996). Boring logs for existing monitoring wells and CPT logs completed during previous investigations are presented in DOE (1993).

The five new monitoring wells were installed in August and September 1995 using the air rotary drilling method. The wells are located in two clusters (Figure 2.1). The first cluster is located east of the LEHR site, approximately 60 feet south of Building U-1, and contains three wells, UCD1-25, UCD2-26, and UCD1/2-27. Well UCD1-25 is completed near the bottom of HSU-1; well UCD2-26 is completed near the top of HSU-2. Well UCD227 monitors seven intervals, three within HSU-1 and four within HSU-2. These wells were installed to determine the nature and extent of contamination downgradient of all source areas with the exception of Landfill 3. They were also designed to provide information on groundwater flow direction, groundwater velocity, vertical anisotropy, effective porosity, and horizontal hydraulic conductivity.

The second cluster is located south of the LEHR site and north of Putah Creek. This cluster contains two wells, UCD1-34 and UCD2-35. Well UCD1-34 is completed near the 
bottom of HSU-1, and well UCD2-35 is completed near the bottom of HSU-2. These wells were installed upgradient of all suspected onsite source areas to determine background concentrations for contaminants of concern, and downgradient of Putah Creek, which receives flow from the UC Davis outfall. They were also designed to provide information on groundwater flow direction and vertical variation in near well horizontal hydraulic conductivity.

Four hydrogeologic units were encountered during drilling operations in 1995 at the LEHR site. They are, in descending order, the vadose zone, HSU-1, HSU-2, and the unnamed aquitard beneath HSU-2. Figure 4.1 is a generalized hydrogeologic column showing the order and average elevations of these units and their relationships to the regional stratigraphy and hydrostratigraphy. Table 4.1 lists the unit depths and thicknesses in the LEHR site wells. Figure 4.2 shows the location of two cross-sections, one west to east and one north to south. The cross-sections are presented in Figures 4.3 and 4.4 and incorporate portions of previously presented cross-sections in DOE (1993). Detailed descriptions for each of the hydrogeologic units follow.

The vadose zone comprises primarily unsaturated clays and silts with sands and gravels intermixed, indicative of a relatively low-energy depositional environment. Immature caliche and organic matter were also noted. The fine-grained materials varied with depth, having been described as dark grey to brown and dry to very moist. The clays were further described as mottled and having low to high plasticity and slight to high toughness. Poorly to well-graded sands and angular to subrounded gravels were observed.

Recent measurements indicate the vadose zone is approximately 42 feet thick near well UCD1-25 and 47 feet thick near well UCD1-34. However, thicknesses across the site vary with seasonal water table fluctuations. It is also important to note that wells UCD1-34 and UCD2-35 are located on an artificially constructed levy that places the ground surface at these wells approximately 7 feet higher than wells UCD1-25, UCD2-26, and UCD1/2-27.

HSU-1 is lithologically similar to the vadose zone in texture, grading, toughness, and plasticity. Calcareous cement and caliche nodules were noted at several locations within this unit. Colors included olive brown, dark yellowish brown, and greenish to dark grey. Well-defined laminations were observed in well UCD1/2-27. The contact between HSU-1 and HSU-2 ranges from approximately 76 to 88 feet bgs across the LEHR site. Thickness of HSU-1 was approximately 40 and 29 feet in wells UCD1/2-27 and UCD2-35, respectively.

HSU $=2$ extends to a depth of 126 feet bgs in well UCD1/2-27 for a thickness of 44 feet, and a depth of 130 feet bgs in well UCD2-35 for a thickness of 54 feet. A minimum thickness of 31 feet was measured in well UCD2-16. This unit is composed primarily of sands in the upper portion of the unit and gravels in the middle to lower portions of the unit, suggesting a relatively higher-energy depositional environment. Samples were described as well-graded, unconsolidated, and wet, consisting of approximately 70 to $90 \%$ quartz and 10 to $30 \%$ mafic minerals. The sands ranged from very fine 
to very coarse; the gravels were very fine to medium pebble-sized, and angular to rounded. Flowing sand conditions were encountered in the upper portion of HSU-2.

Collectively, sediments from the vadose zone, HSU-1, and HSU-2 make up the Putah Creek Fan beneath the LEHR site. Thicknesses range from 118 feet (UCD2-15) to 138 feet (UCD2-17). DOE (1993) further subdivided the Putah Creek Fan into two lithofacies: an upper lithofacies consisting of the interbedded clays and silts, with some sand and gravel deposits (i.e., the vadose zone and HSU-1); and a lower lithofacies consisting primarily of the sands and gravels (i.e., HSU-2).

The unnamed aquitard beneath HSU-2 was encountered in wells UCD1/2-27 and UCD2-35 at 126 and 130 feet bgs, respectively. Depth of penetration into this aquitard in all wells ranged from 2.5 feet (UCD2-15) to 10 feet (UCD1/2-27). Cuttings were described as light brown silty clay to clay with some caliche. These materials displayed high plasticity and toughness. At no location did the drill depth advance through the unnamed aquitard; the maximum depth penetrated in all of the investigations was 143 feet bgs in well UCD2-17. The unnamed aquitard is thought to be approximately 90 feet thick in the vicinity of the LEHR site (DOE 1994) and corresponds to the Tehama Formation (DOE 1993).

Regionally, HSU-2 is referred to as the first aquifer. Wells completed in HSU-2 are reported to have high specific capacities. At greater depths, Mann (1992) indicates that there are sand and gravel aquifers within the Tehama Formation jointly referred to as the second aquifer. Regional data from drillers' logs show the presence of a gravel unit below the unnamed aquitard. This gravel unit is identified as HSU-3 in DOE (1994). These sediments are reported to be less permeable than HSU-2 (DOE 1995). In addition, wells completed in these sediments have lower specific capacities than wells completed in HSU-2.

Slug testing was conducted during October and November 1995 at existing and new well installations to provide information.on the range and possible spatial variation of hydraulic properties for HSU-1 and HSU-2 at the LEHR site. In total, 11 existing HSU-1 wells (UCD1-10, -11, -12,-13,-18,-19, -20, -21, -22, -23, -24) and five existing HSU-2 wells (UCD2-7, $-14,-15,-16,-17$ ) were characterized using the slug test method. Slug testing was also conducted at the five new wells.

Analytical methods used in the analysis of the slug tests exhibiting overdamped responses (i.e., exponential decay pattern) include the type-curve matching method for unconfined and confined aquifers, as presented in Hyder et al.' (1994), Hyder and Butler (1995), Spane and Wurstner (1993), and Spane (1994). Because these analytical methods can use all or any part of the slug test response in the analysis procedure, they are particularly useful in the analysis of unconfined aquifer tests (e.g., HSU-1 wells). They also do not have any of the inherent analytical weaknesses of the commonly used Bouwer and Rice method (described in Bouwer and Rice [1976] and Bouwer [1989]) for unconfined aquifer slug tests, which are discussed in Hyder and Butler (1995) and Brown et al. 
(1985). For HSU-2 slug tests exhibiting underdamped oscillatory test responses, the analytical method described in Van der Kamp $(1976,1984)$ was used.

Final analysis of all slug tests conducted during October and November 1995 have not been completed. Preliminary results from the analysis of selected slug tests indicate the following ranges for hydraulic conductivity for HSU-1 and HSU-2.

\begin{tabular}{||l|c|c||}
\hline \multirow{2}{*}{ Hydrogeologic Unit } & \multicolumn{2}{|c|}{ Hydraulic Conductivity } \\
\cline { 2 - 3 } & feet/day & feet/second \\
\hline \hline HSU-1 & $1.9-11$ & $2.2 \times 10^{-5}-1.3 \times 10^{-4}$ \\
\hline HSU-2 ${ }^{\text {(a) }}$ & $120-620$ & $1.4 \times 10^{-3}-7.2 \times 10^{-3}$ \\
\hline $\begin{array}{l}\text { (a) Preliminary estimates listed for HSU-2 are based only on } \\
\text { analysis of oscillatory slug test responses. }\end{array}$ \\
\hline
\end{tabular}

These preliminary values are comparable to the hydraulic conductivity range reported for HSU-1 of $7.1 \times 10^{-6}$ to $8.3 \times 10^{-5}$ feet/second and generally higher than the range cited for HSU-2 of $3.1 \times 10^{-4}$ to $9.4 \times 10^{-4}$ feet/second in DOE (1993). Final analytical results of the slug tests conducted during October and November 1995 will be presented in a subsequent report.

\subsection{Groundwater Elevations and Gradients}

This section provides a discussion of groundwater elevations and flow directions observed during 1995. Groundwater elevations measured during 1995 are discussed in terms of seasonal/annual fluctuations and horizontal and vertical gradients. Data sources include monthly electrical water-level indicator (E-tape) measurements collected by both International Technology Corporation (IT Corp.) and UC Davis, and limited continuous pressure transducer readings collected by PNNL. Additional water-level data, not presented in this report, were collected by the State of California Water Resources Control Board.

\subsubsection{Seasonal/Annual Groundwater Elevations}

Figure 4.5 illustrates the seasonal/annual variations in groundwater elevations during the period November 1990 through December 1995. This figure contains hydrographs for wells UCD1-20 (screened in HSU-1) and UCD2-7 (screened in HSU-2). These hydrographs are typical of seasonal/annual fluctuations observed across the LEHR site. 
Cumulative groundwater elevation data through 1995 are presented in tabular form by well in Appendix E. Hydrographs of these data are shown as Figures E.1 through E.13. Monitoring well screen intervals are shown in Table 2.2. Based on a review of the hydrographs, these observations can be made:

- The pattern of groundwater elevation fluctuation in both HSU-1 and HSU-2 is similar in shape and magnitude of change.

- The lowest groundwater elevations typically occur in July or August and the maximums in March.

- Seasonal/annual fluctuations since 1990 range from a maximum of approximately 40 feet between August 1994 and March 1995 to a minimum of 15 feet between August 1993 and March 1994.

- There appears to be an overall increase of approximately 13-14 feet in average groundwater elevations between November 1990 and December 1995.

The observed pattern of water-level fluctuations indicates that groundwater withdrawal during the irrigation season is the dominant factor influencing the seasonal pattern. The amount by which water levels recover during the winter rainy season probably depends on the amount of annual precipitation. Figure 4.6 shows the monthly precipitation data for the Davis area. This graph was constructed using monthly data from January 1993 through December 1995 from a station at UC Davis. The maximum rate of water-level recovery (i.e., steepest slope) is observed during September and is most likely due to the cessation of major groundwater withdrawals following the irrigation season.

\subsubsection{Groundwater Elevations During 1995}

Figures 4.7 and 4.8 show composite hydrographs for selected monitoring wells screened in HSU-1 and HSU-2, respectively. These two hydrographs illustrate the fluctuations in the potentiometric surfaces for HSU-1 and HSU-2 during 1995 compared with the cumulative precipitation. The timing of each quarterly sampling period is also noted. Based on a review of these hydrographs, the following observations can be made:

- Seasonal/annual fluctuations in 1995 groundwater levels ranged from nearly 21 feet in HSU-1 to just over 17 feet in HSU-2.

- The lowest levels during 1995 were observed in both January and August and the highest levels in March.

- The most rapid decline of water levels during 1995 was observed during May and June, and the maximum rate of water level recovery-was observed during January and March. 
A review of continuously monitored water levels from two well clusters and the precipitation data (Figure 4.9) reveals a strong correlation between the increased precipitation and a rise in the water levels at both well clusters. The rapid response of water levels (within a day after the precipitation event) is believed to be in response to increased recharge from Putah Creek resulting from an increase in river stage caused by surface runoff from the heavy local precipitation event. Figure 4.9 illustrates that the water level in HSU-1 near Putah Creek (UCD1-34) dramatically rises in response to the precipitation event. HSU-2 (UCD2-35) responds in concert with HSU-1 but shows a dampened increase in water level. Note that the downward vertical gradient between HSU-1 and HSU-2 is strongest soon after the HSU-1 water level rises, then dissipates with time as HSU-2 responds.

Away from Putah Creek (near UCD1-25 and UCD2-26) the water levels also respond to the precipitation event; here, however, it is HSU-2. which responds first, creating a stronger upward vertical gradient that slowly dissipates with time. These observations are consistent with low horizontal hydraulic conductivities in HSU-1 and high hydraulic conductivities in HSU-2. That is, water is transported vertically through HSU-1 to HSU-2 near its source of recharge (i.e., Putah Creek), then transported quickly through HSU-2, and finally back to HSU-1, as the vertical gradient becomes upward.

\subsubsection{Horizontal Groundwater Gradients During 1995}

DOE $(1993,1994 a)$ indicates that, on average, the hydraulic gradients of both HSU-1 and HSU-2 are on the order of $10^{-3}$ feet/foot, with a general northeast groundwater flow direction. However, the hydraulic gradient is highly variable and depends on the time of year. This variability is associated with both the natural and anthropogenic (man-induced) stresses affecting the aquifer, as discussed in the previous sections.

Changes in hydraulic gradient and groundwater flow direction throughout 1995 can be observed in Figures 4.10 and 4.11. These figures illustrate quarterly equipotential maps for HSU-1 and HSU-2, respectively. The four quarterly maps for each HSU represent times during the year that range from natural, non-stressed conditions (February), to seasonal periods representative of anthropogenically stressed conditions (August).

HSU-1 is present at depths ranging from 30 to 70 feet bgs and consists of fine-grained sediments, primarily silts and clays, with infrequent, discontinuous sand and gravel lenses. During February 1995, the horizontal groundwater gradient appears to be fairly steep near the southwest corner of the site, and radiating outward towards the north and northeast. In May and August (Figure 4.10), the gradient in HSU-1 is toward the northeast. In November, the gradient appears to swing even further eastward, except locally around wells UCD1-5 and UCD1-20. Rapid water level recovery during this period produces a more irregular potentiometric surface that may be attributed to local variations in hydraulic conductivity. 
HSU-2 ranges from approximately 80 to 135 feet bgs and consists of coarse grained material. The horizontal groundwater gradient in HSU-2 during 1995 is more consistently toward the east, with a very slight northerly component.

To quantitatively assess lateral hydraulic gradients and groundwater flow directions, the commercially available WATER-VEL (In-Situ 1991) software program was used. WATER-VEL calculates hydraulic gradients and groundwater flow direction by fitting a linear, two-dimensional trend surface (least squares) to the hydraulic head data. The technique is accurate as long as the 2-D, linear approximation is applicable (i.e., no significant vertical groundwater gradient exists within the aquifer). Tables 4.2 and 4.3 list the analytical results of the four quarterly measurement surveys for HSU-1 and HSU-2, respectively. The average groundwater flow directions calculated using WATER-VEL are consistent with those discussed above, indicating a more northerly groundwater flow component for HSU-1 with a wider variation in groundwater flow direction than exhibited by HSU-2.

A comparison of these results indicates an overall similarity in lateral gradients for both HSUs during 1995. Average horizontal groundwater gradients in HSU-1 during 1995 ranged from approximately $4 \times 10^{-4}$ feet/foot (February 1995) to $1.6 \times 10^{-3}$ feet/foot (August 1995). Average horizontal groundwater gradients in HSU-2 during 1995 ranged from approximately $3.9 \times 10^{-4}$ feet/foot (February 1995) to $1.5 \times 10^{-3}$ feet/foot (May 1995). Tables 4.2 and 4.3 indicate that lower hydraulic gradients occurred during the winter and fall, while higher hydraulic gradients occurred during the spring and summer (during periods of significant offsite groundwater withdrawals).

\subsubsection{Vertical Groundwater Gradients During 1995}

Seven well pairs, consisting of a monitoring well completed in HSU-1 adjacent to a monitoring well completed in HSU-2, exist at the LEHR site. These well pairs are listed in Table 4.4. This table includes the screened intervals, mid-screen elevation, and the elevation differential between mid-screen elevations for each well pair. In addition, a Westbay well was installed near one of these well pairs and consists of seven isolated monitoring intervals (Table 4.5). Table 4.5 also lists the water level elevations for each zone (computed from pressure transducer readings collected at $2400 \mathrm{hrs}$ on October 28, 1995) and the differential between adjacent zones. Additional pressure transducer readings were collected continuously over a two-week period but have not yet been fully analyzed. A review of the data in Table 4.5 suggests that there was an upward vertical gradient in both HSU-1 and HSU-2 at the time of these measurements, except between zones 6 and 7 where the vertical gradient appears to be downward. This evaluation, however, does not account for the variable response of different hydrogeologic zones to barometric pressure changes (resulting from their different barometric efficiencies). Thus, the actual vertical gradients may differ from those of the apparent gradients suggested by differences in water level elevation. Similar limitations should be noted when examining the information presented throughout the remainder of this section. 
Figures 4.9 and $4.12-4.16$ illustrate the 1995 hydrographs for each well pair. Based on a review of these hydrographs (see also Appendix E), the water levels appear to match fairly closely during the winter months of January, February, and early March. At the onset of the irrigation season in March, the water level decline in HSU-1 appears to lag behind the steep decline shown on the hydrographs for HSU-2 wells. As water levels in the HSU-2 wells begin to rise in the fall at the cessation of the irrigation season, HSU-1 water levels also begin to rise, and again appear to lag somewhat behind the HSU-2 levels.

Figure 4.17 illustrates the apparent vertical gradient between each well pair. The vertical gradient is expressed here as the difference in water levels between the wells in a pair (HSU-2 water level minus HSU-1 water level), divided by the vertical differential in feet between the mid points of the respective screened intervals. For a downward vertical gradient (i.e., HSU-1 water level is greater than HSU-2 water level) the result will be a negative number, and for an upward vertical gradient the result will be a positive number. Again, it should be noted that these data have not been corrected to account for the barometric responses of the hydrogeologic units, thus the actual vertical gradients may vary. It should also be noted that well UCD2-14 is not solely representative of HSU-2, since it was completed across the contact between HSU-1 and HSU-2.

Figure 4.17 suggests that apparent vertical gradients are highly variable between January and March, while water levels are recovering from the previous irrigation season. Significant negative (downward) vertical gradients occur in all well pairs from April to September (the primary irrigation season) as water is withdrawn from HSU-2. The apparent negative vertical gradients reach a maximum in the May and June, and vary from -0.15 feet/foot in the UCD1-13/UCD2-14 well pair, to -0.01 feet/foot in the UCD1-1/UCD2-16 well pair. The apparent negative vertical gradients then decrease and reverse to apparent positive vertical gradients in nearly all well pairs from September through November, as water levels in HSU-2 wells begin to recover.

This pattern of seasonal changes in apparent vertical gradients between well pairs suggests that groundwater moves downward from HSU-1 into HSU-2 during water level declines in spring and summer. In the fall and early winter, as water levels begin to recover, groundwater moves vertically from HSU-2 into HSU-1. The apparent lag in water level change between the HSU-1 and HSU-2 reflects the low vertical hydraulic diffusivity $\left(\mathrm{K}_{\mathrm{v}} / \mathrm{S}_{\mathrm{s}}\right)$ of HSU-1 relative to HSU-2. 


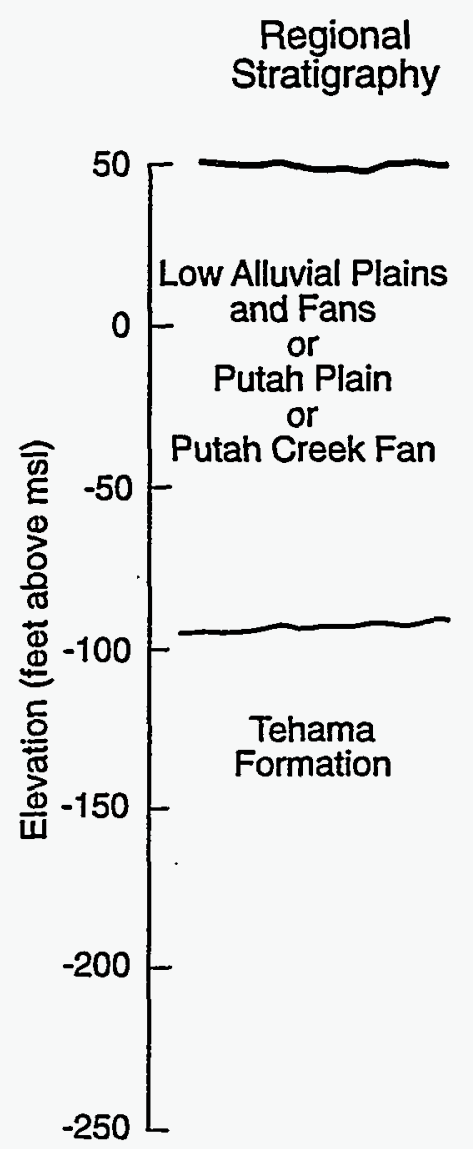

LEHR Site Hydrogeology

Regional

Hydrostratigraphy
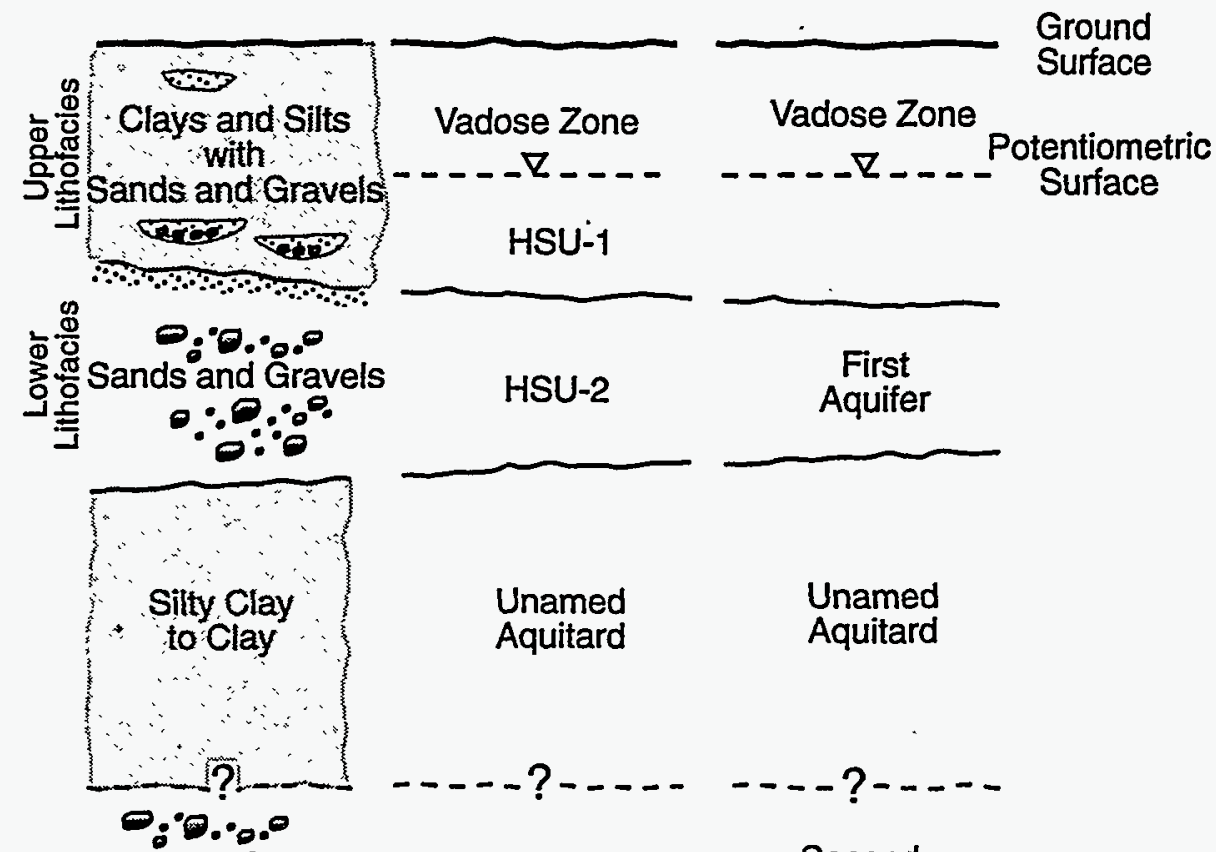

Sands and Gravels $\because \because 0$

HSU-3

Unamed Unamed

Aquitard Aquitard

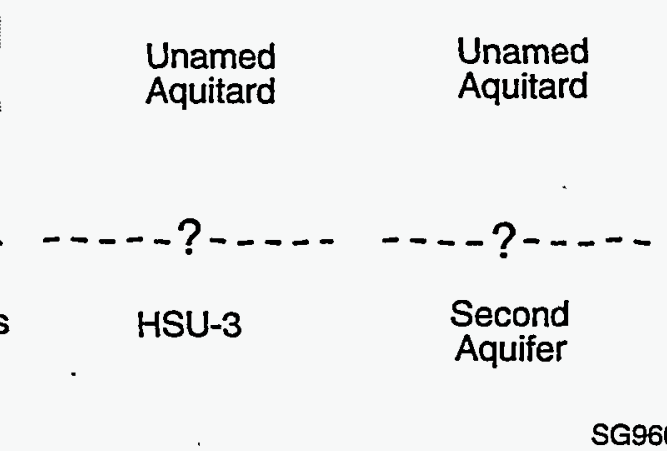

SG96020380.1

Figure 4.1. Average Elevations of HSU-1 and HSU-2 and Their Relationship to Regional Stratigraphy and Hydrostratigraphy 


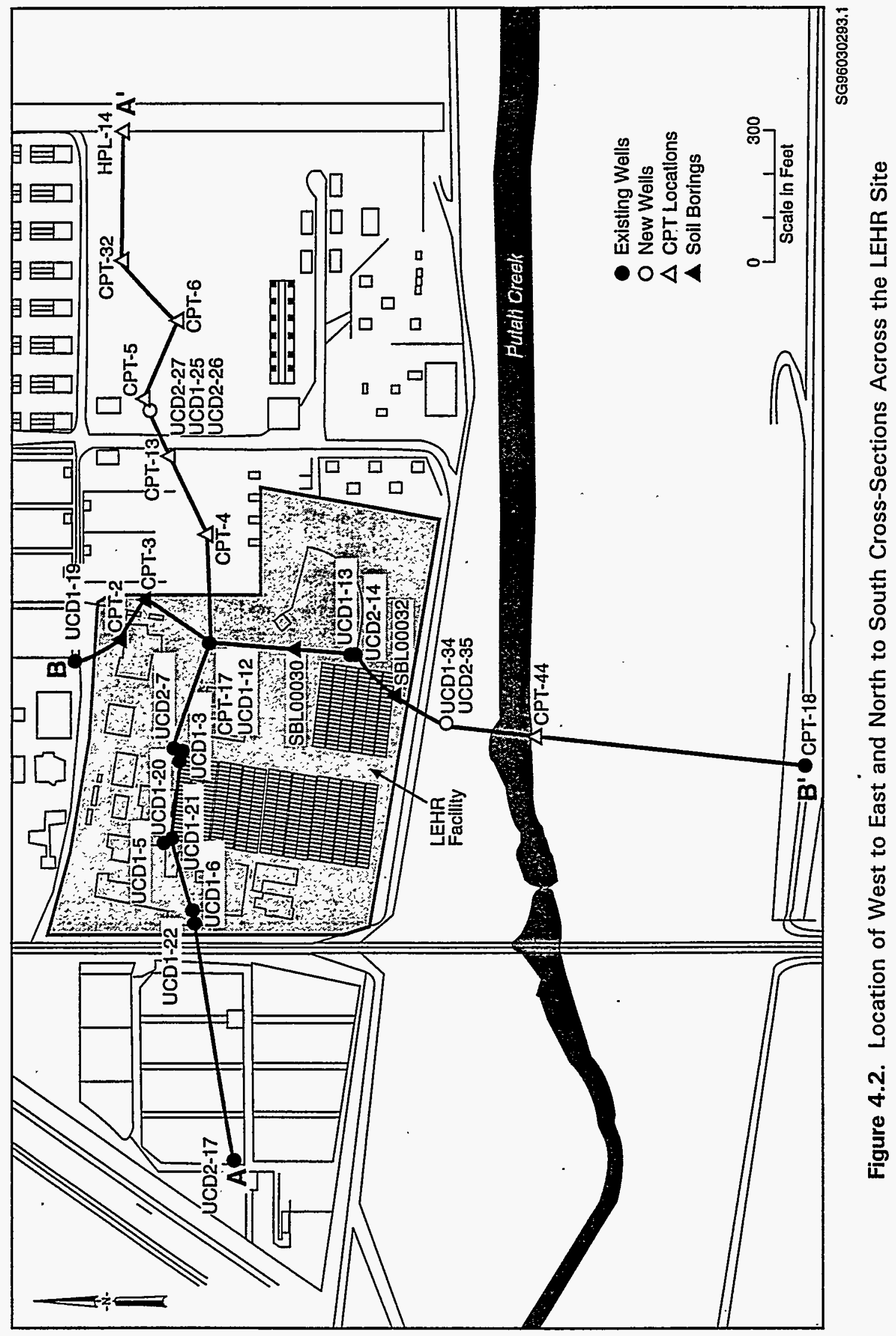




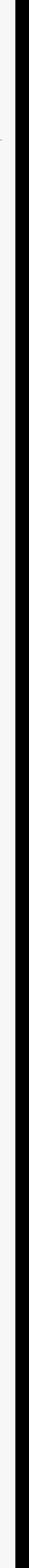




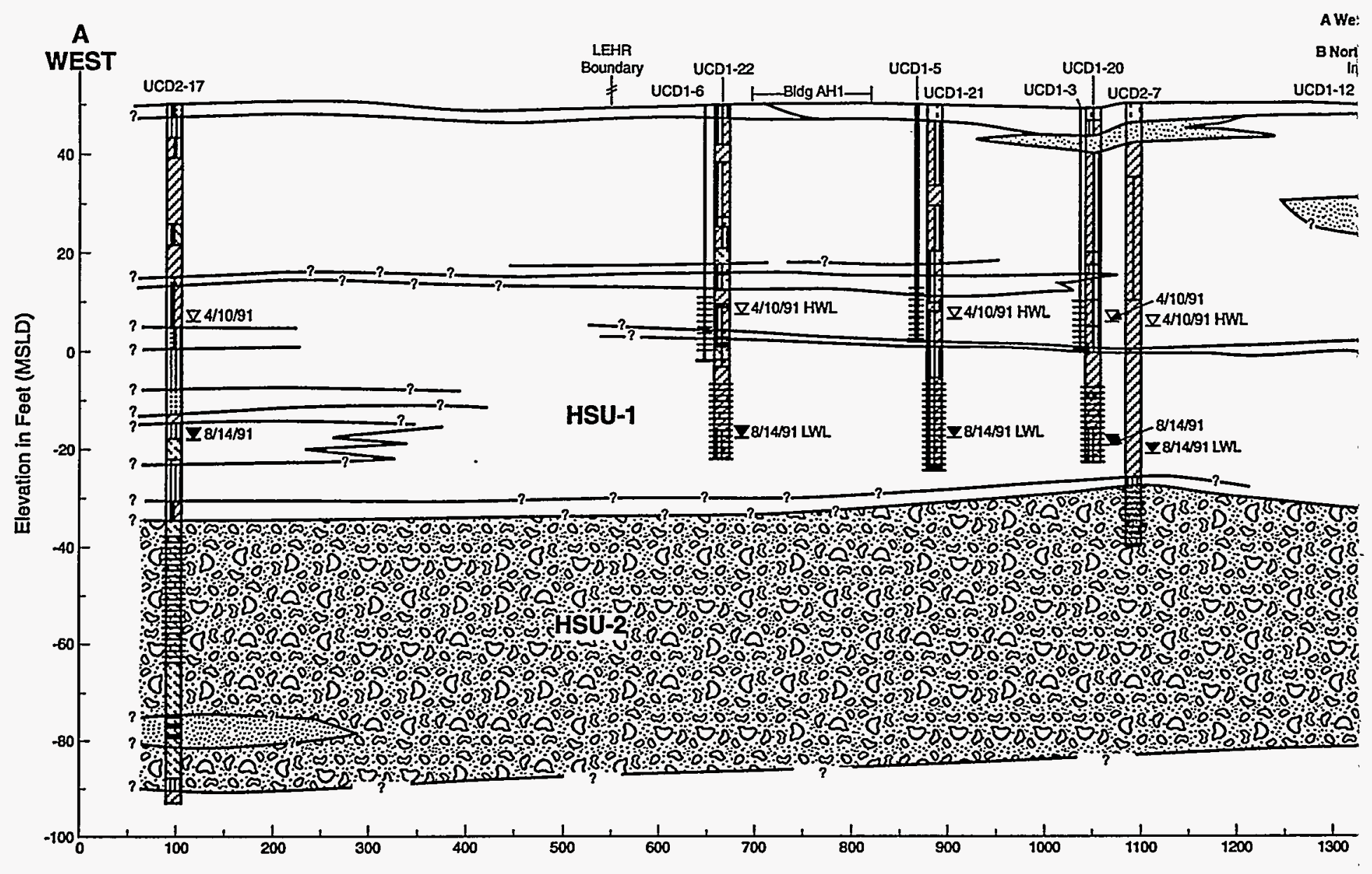

\section{Explanation}

Z 4/10/91 HWL: High Groundwater Level

7 8/14/91 LWL: Low Groundwater Level

\section{圭 Screened Interval}

\section{Stratigraphic Lithology}

Interbedded Clay, Silty Clay and Silt

Fine-Grained Sand and Silty Sand

Govg. Well-Rounded Cobbles and Gravel with Some Sand

\section{Boring Log Lithology}

Silty Clay, Sandy C Clayed Silt, Sandy! Silty Sand, Sand wi Clayey Sand, Sand Clayey Gravel, Gra Silty Gravel, Gravel Sandy Gravel, Gral Gravelly Sand, San

Figure 4.3. West to East Cross-? 
' 31 - A' East

and

h-B'South LEHR

tersect Boundary

$=$

CPT-04

CPT-13 UCD1-25 UCD2-26 CPT-05
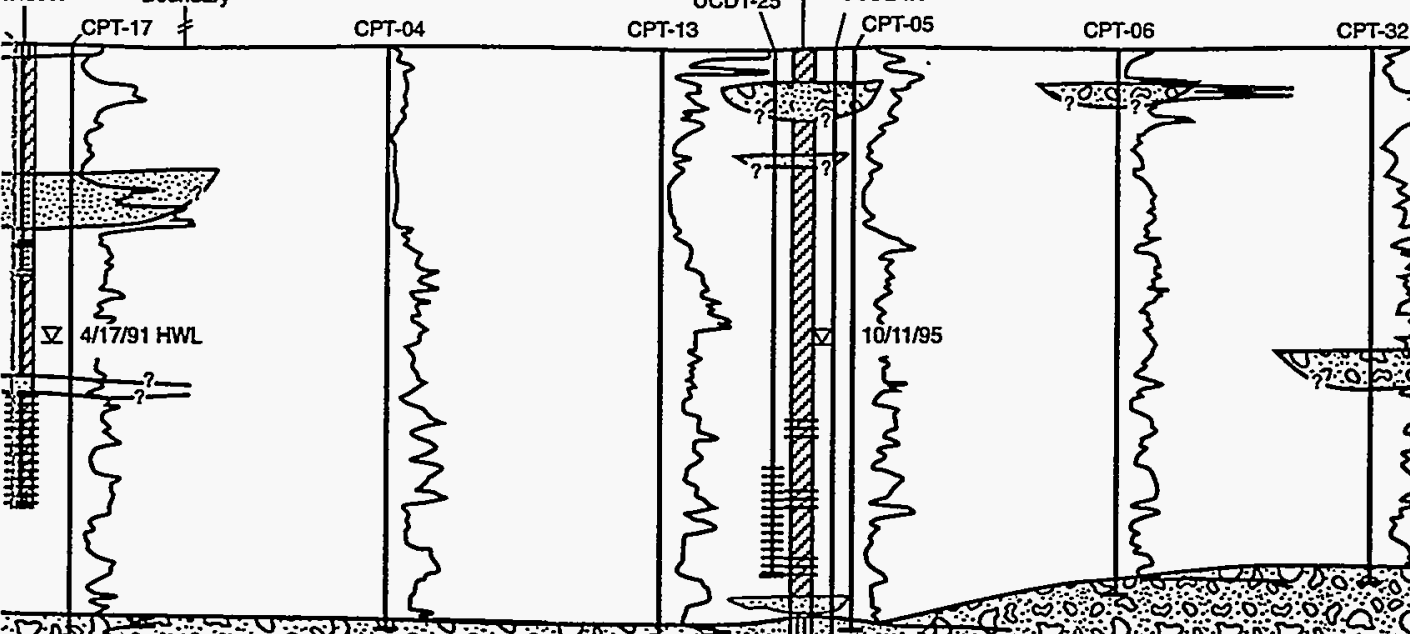

HPL-14

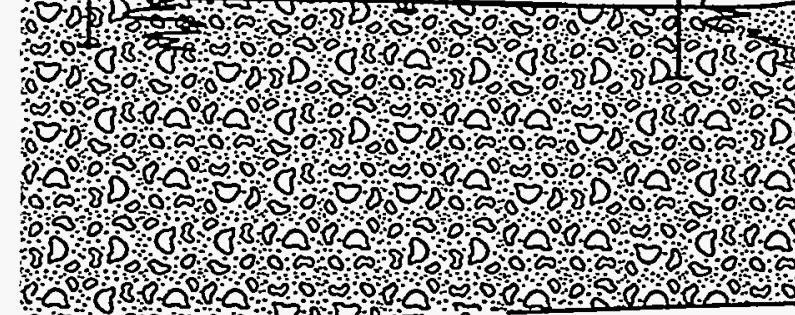

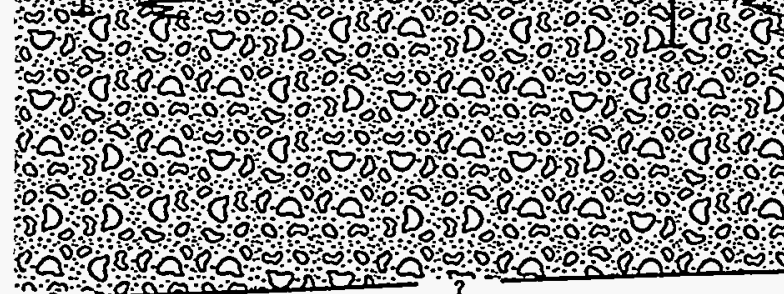
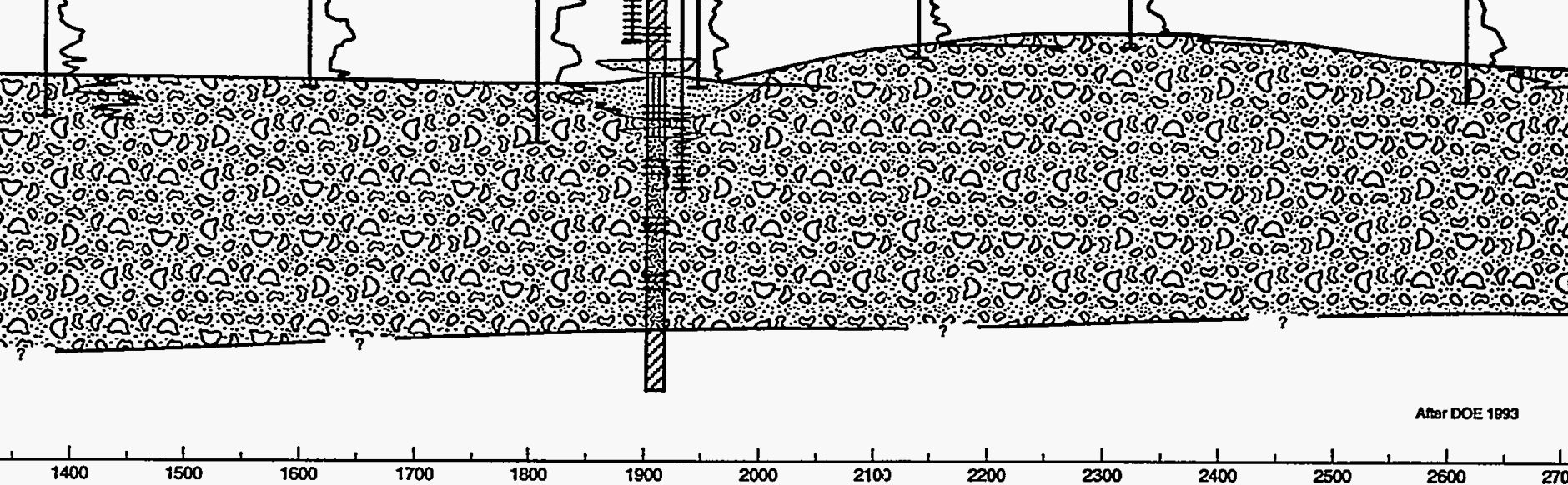

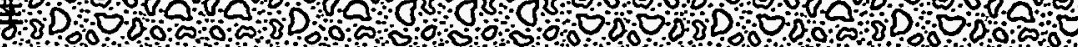

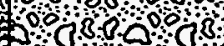

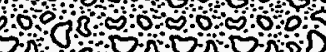

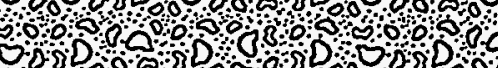

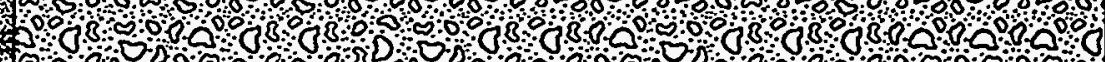

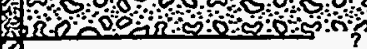

View Baseline in Feet

Aftor DOE 1993

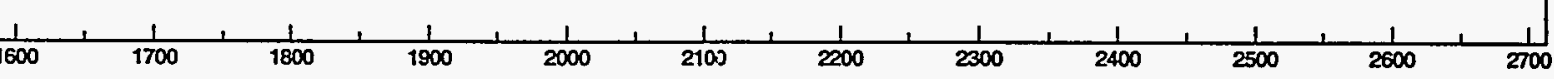

scoocosesas

\section{CPT Tip Resistance}

lay, Gravelly Clay

Silt, Gravelly Silt

th Silt

with Clay

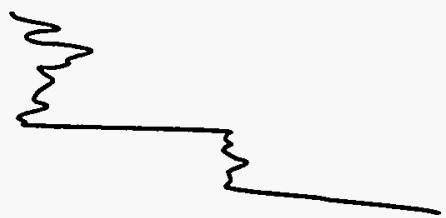

vel with Clay

with Silt

rel with Sand

d with Gravel

Section Across the LEHR Site 


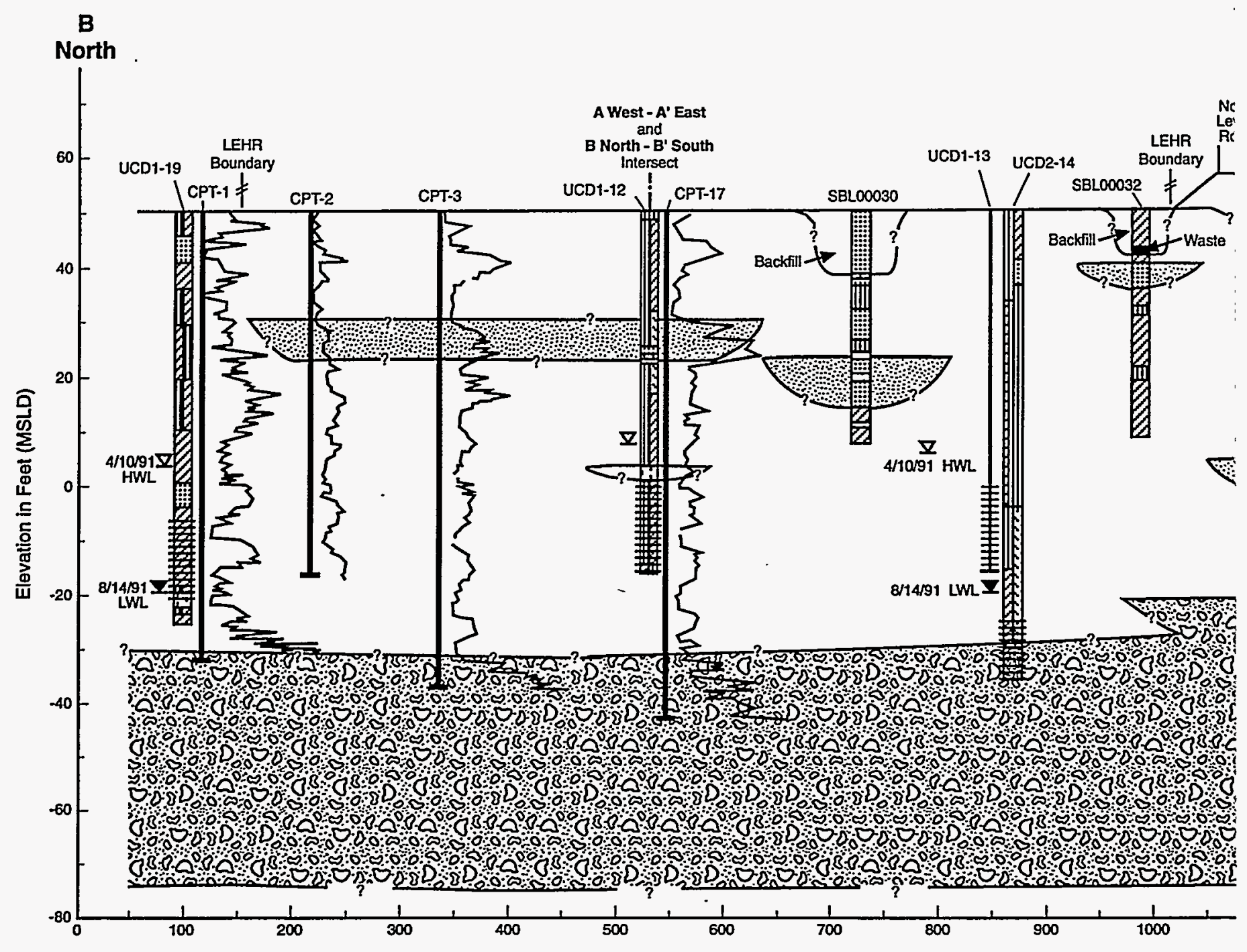

Explanation

Z 4/10/91 HWL: High Groundwater Level

Boring Lo .

₹ 8/14/91 LWL: Low Groundwater Level

圭 Screened Interval

DIII

Stratigraphic Lithology

Interbedded Clay, Silty Clay and Silt

Fine-Grained Sand and Silty Sand

Well-Rounded Cobbles and Gravel

[In⿴囗十) Cla

$\mathrm{Sil}^{\circ}$

Sinstis $\mathrm{Cl}$

$\mathrm{Cl}$

Sil

$\mathrm{Sa}$

Gris with Some Sand

Figure 4.4. North to South 

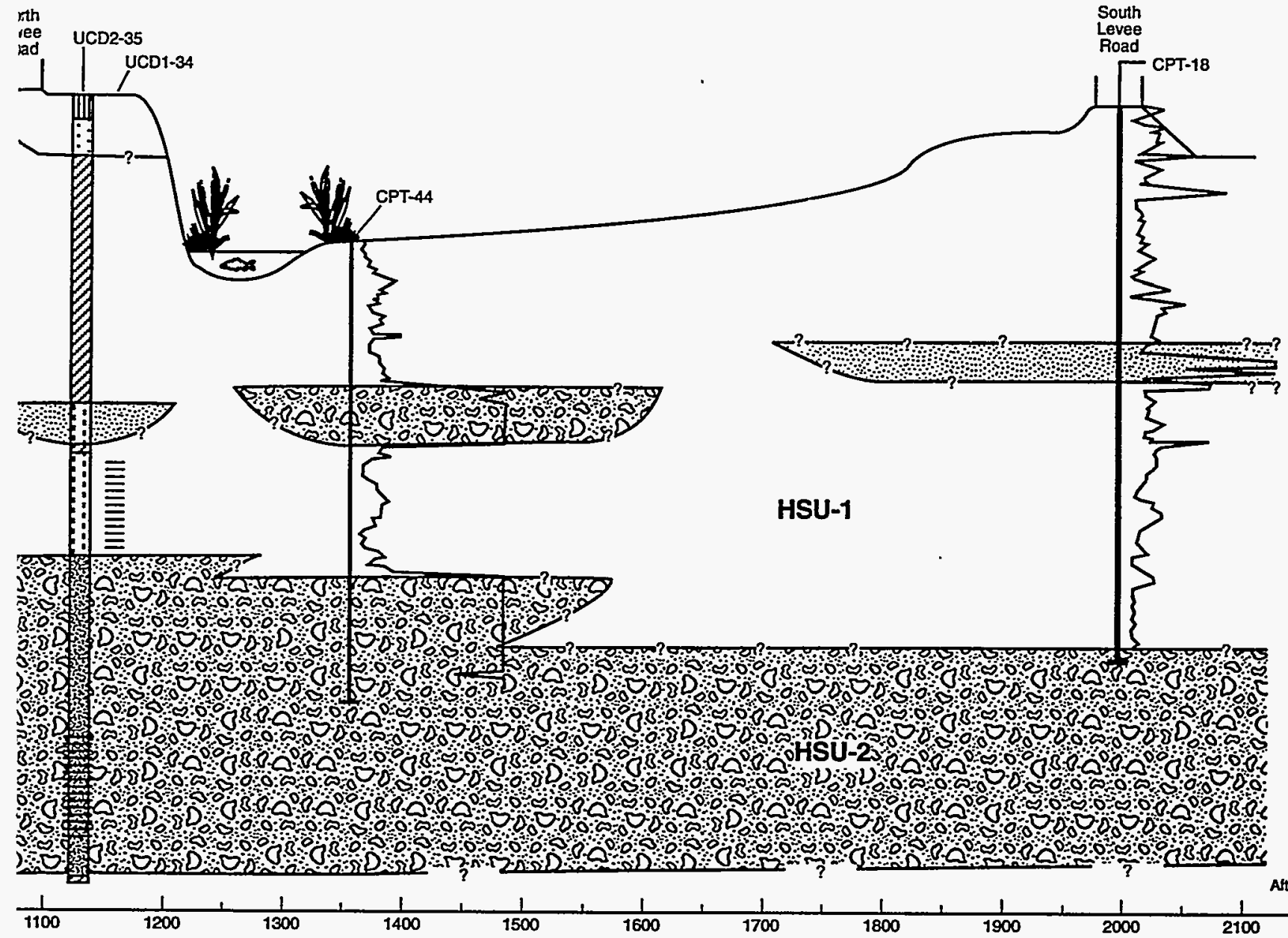

After DOE 1993

View Baseline in Feet

\section{g Lithology}

iy Clay, Sandy Clay, Gravelly Clay ıyed Silt, Sandy Silt, Gravelly Silt ly Sand, Sand with Silt lyey Sand, Sand with Clay lyey Gravel, Gravel with Clay y Gravel, Gravel with Silt ndy Gravel, Gravel with Sand avelly Sand, Sand with Gravel

\section{CPT Tip Resistance}

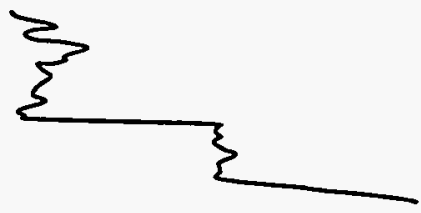

\section{Cross-Section Across the LEHR Site}




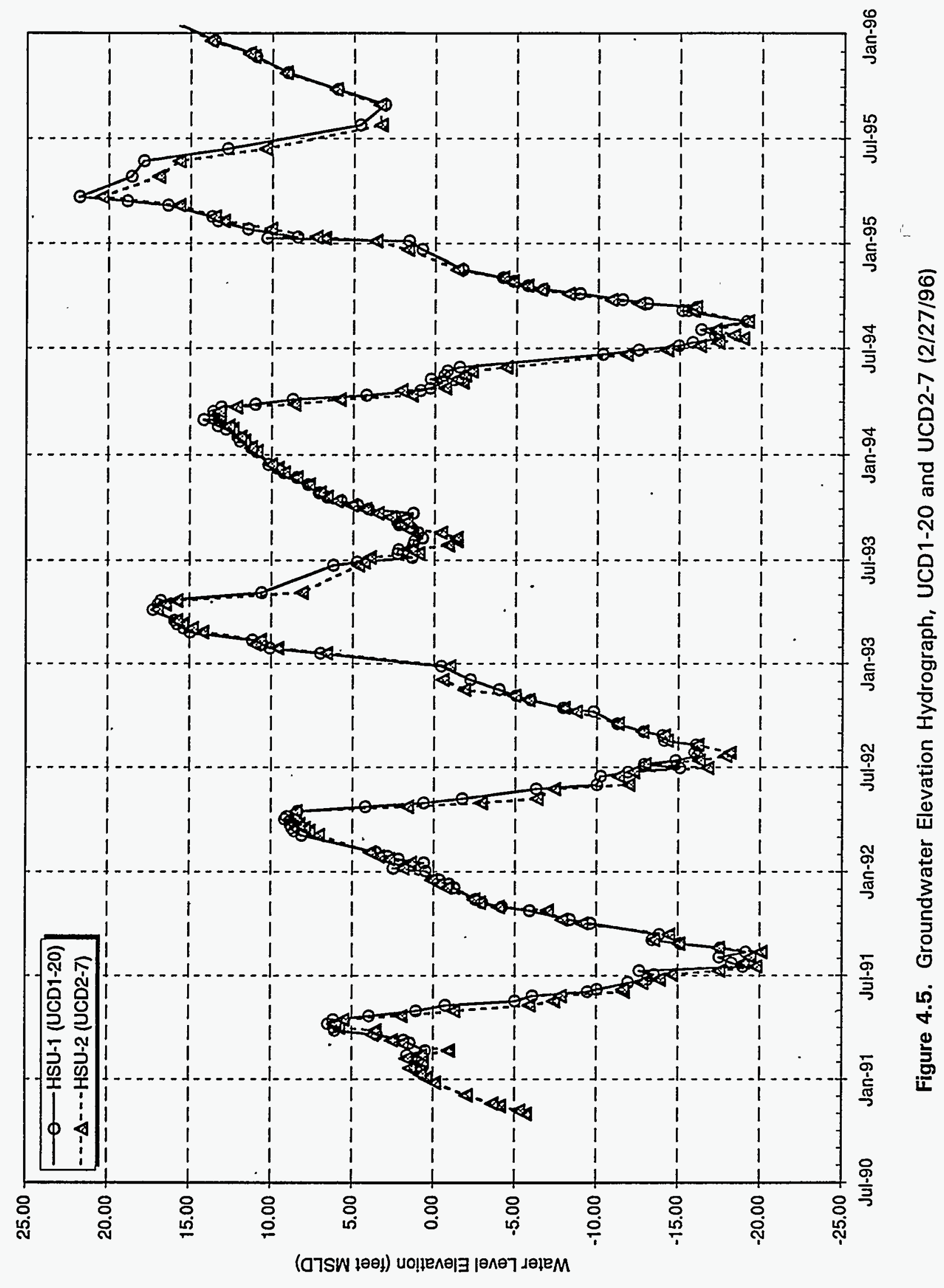




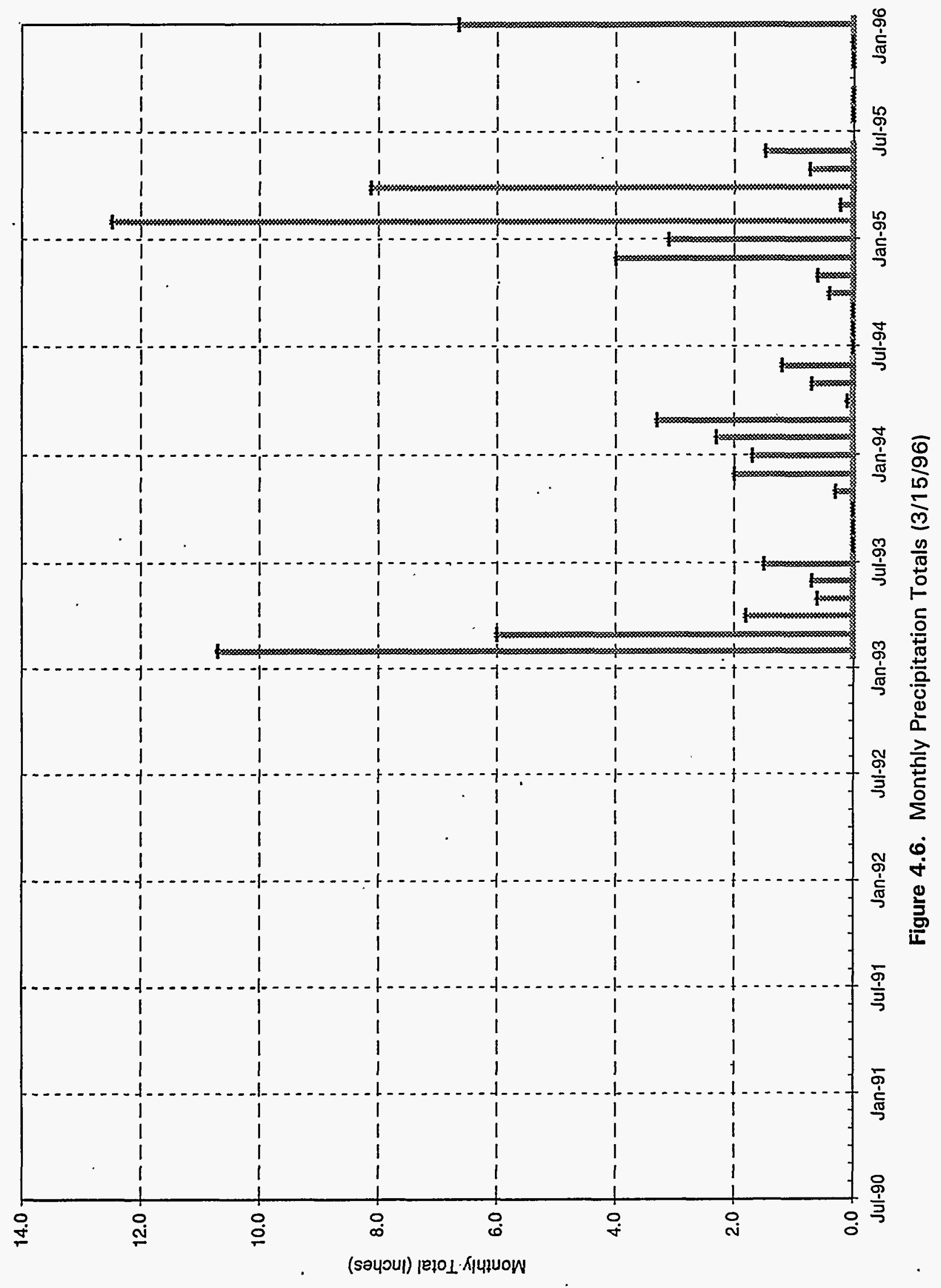




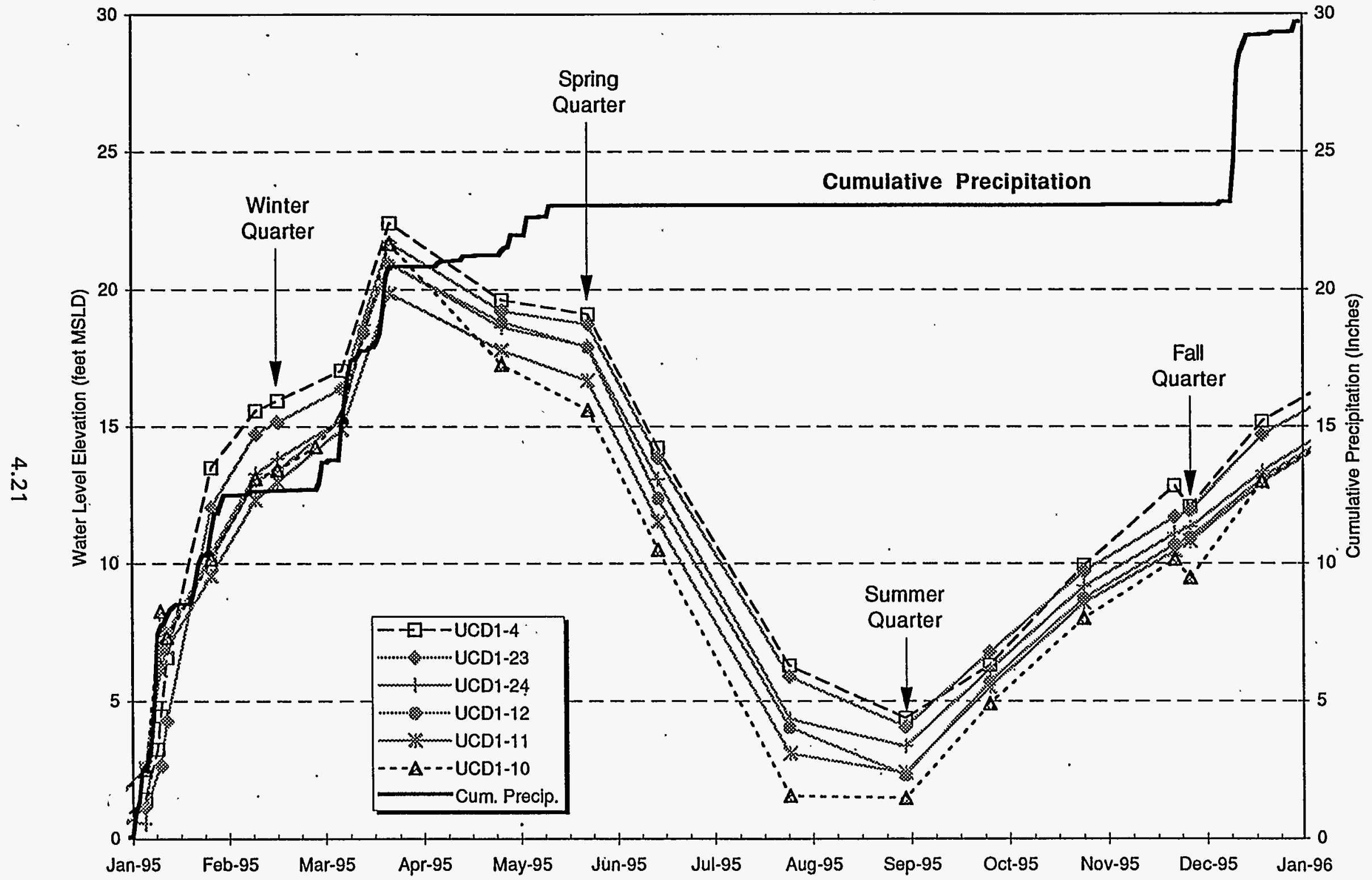

Figure 4.7. Groundwater Elevation Hydrograph, Selected HSU-1 Wells (2/26/96) 


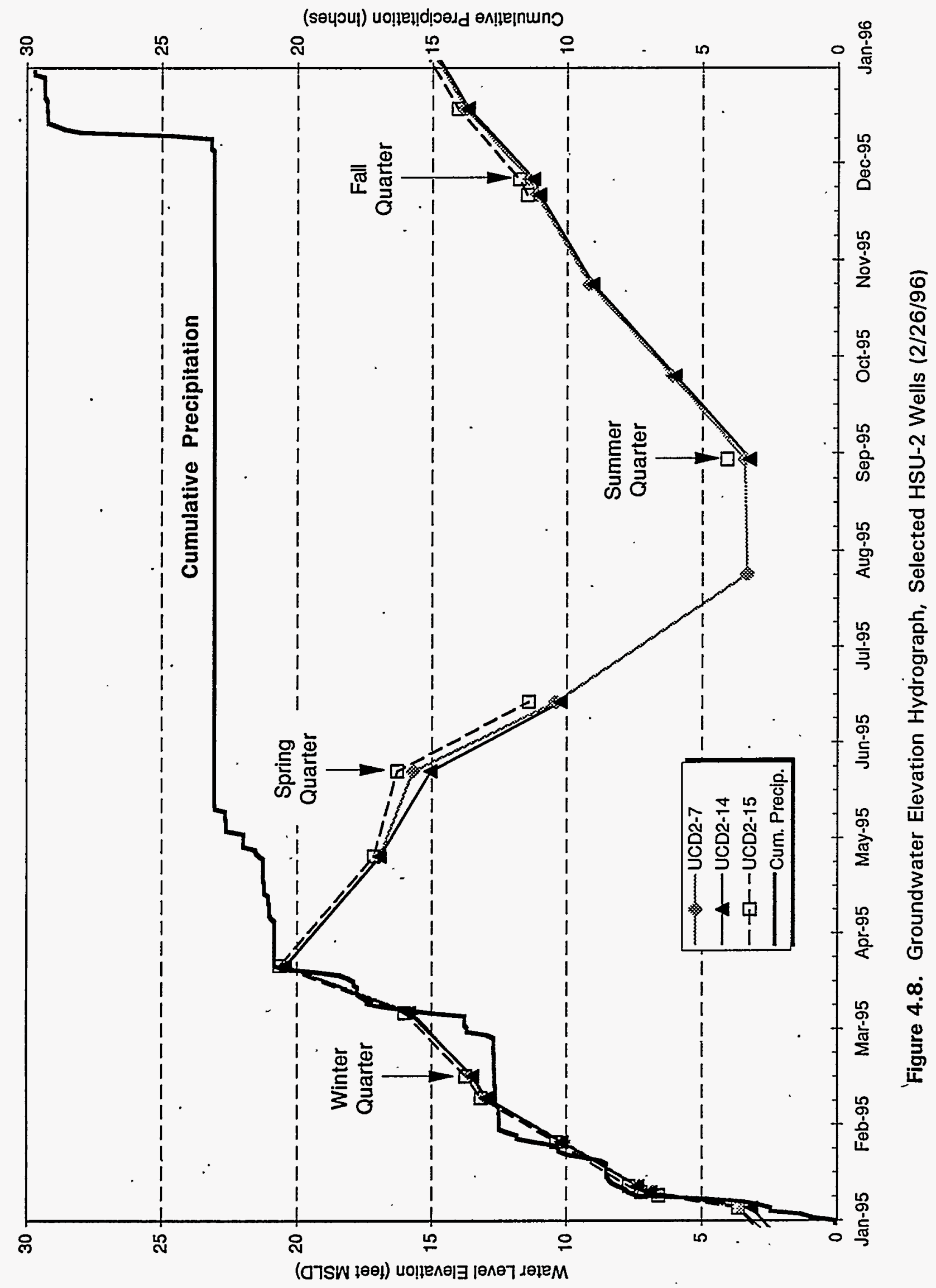




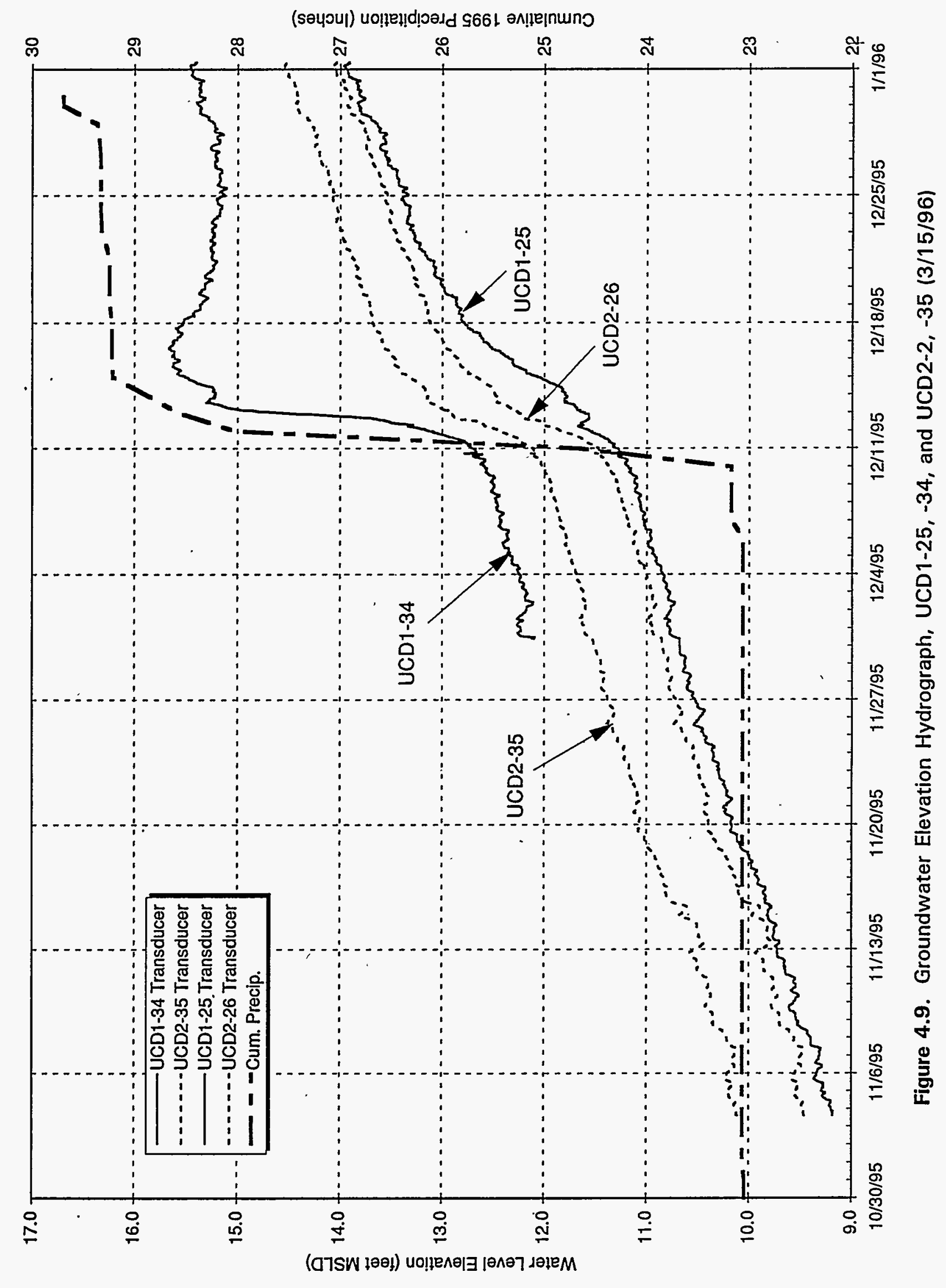




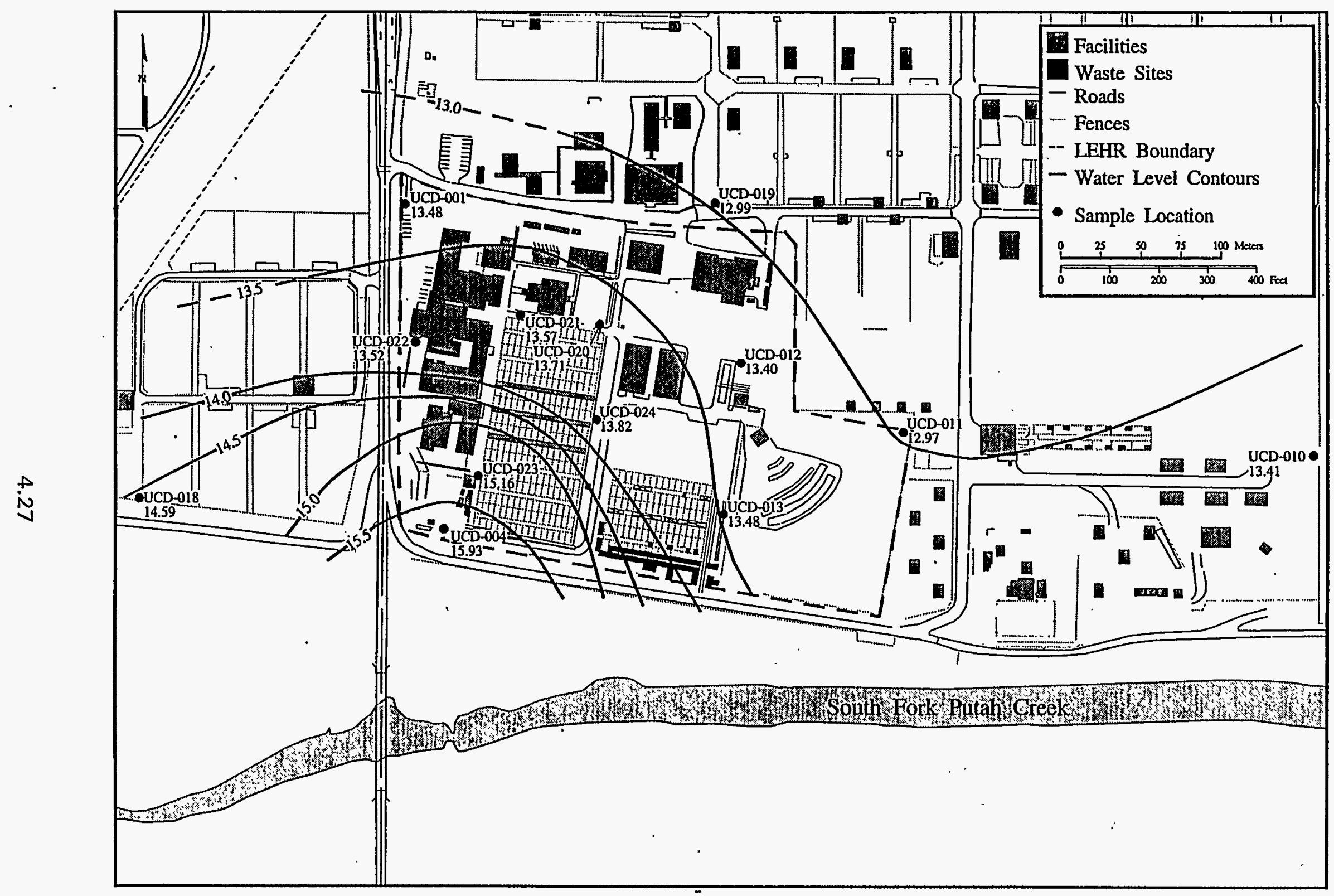

Figure 4.10a. Groundwater Elevations for HSU-1, February 17, 1995 


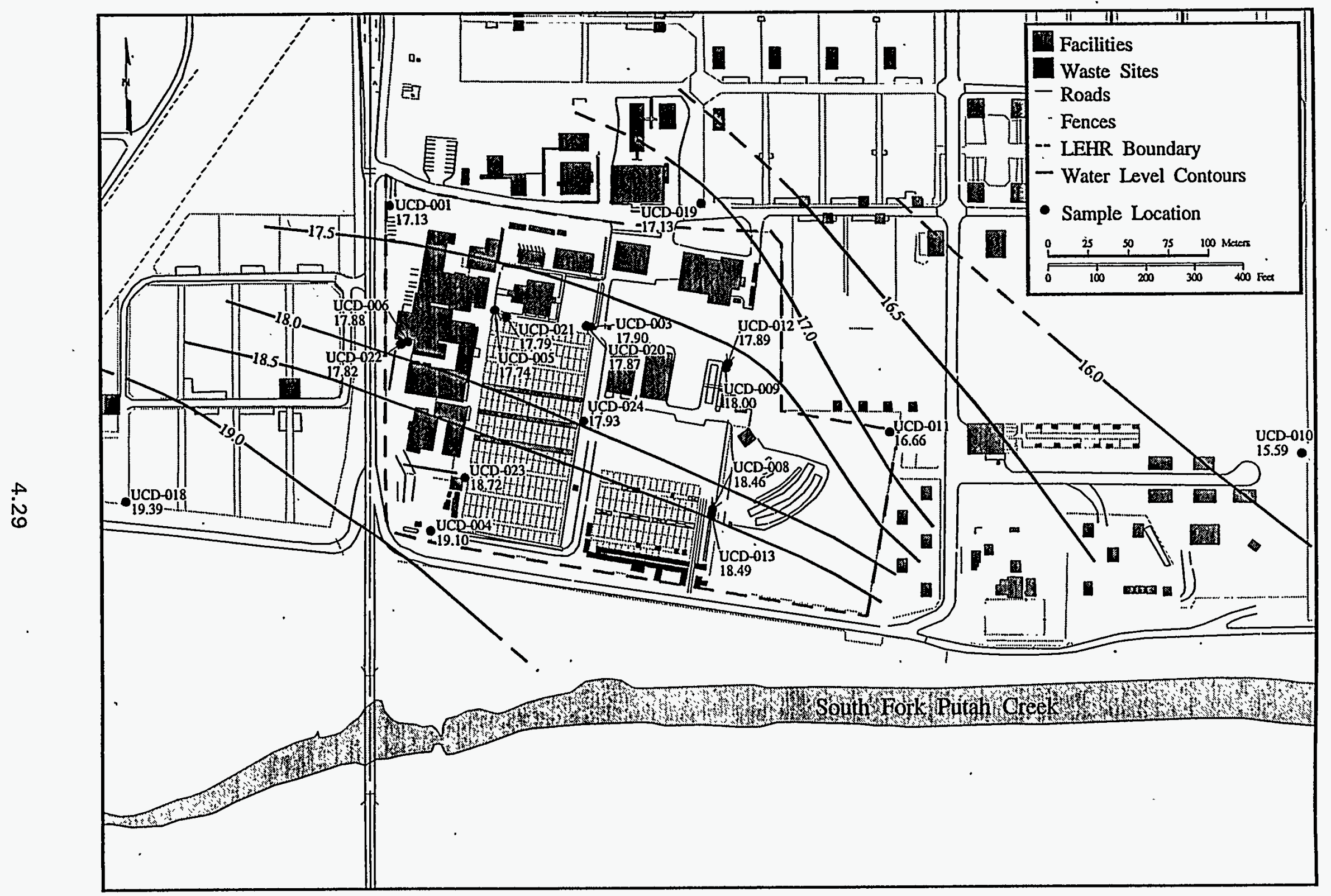

Figure 4.10b. Groundwater Elevations for HSU-1, May 25, 1995 


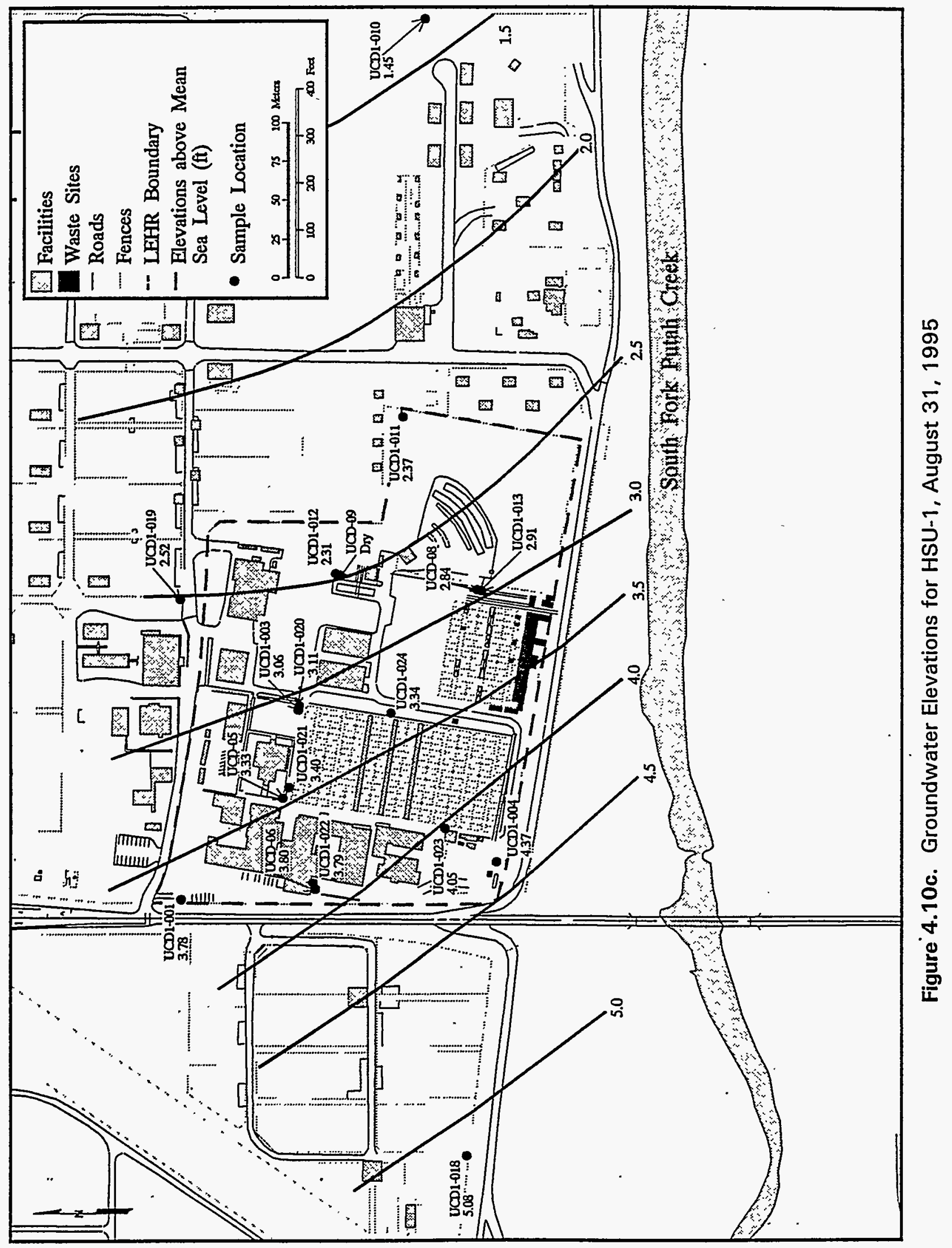




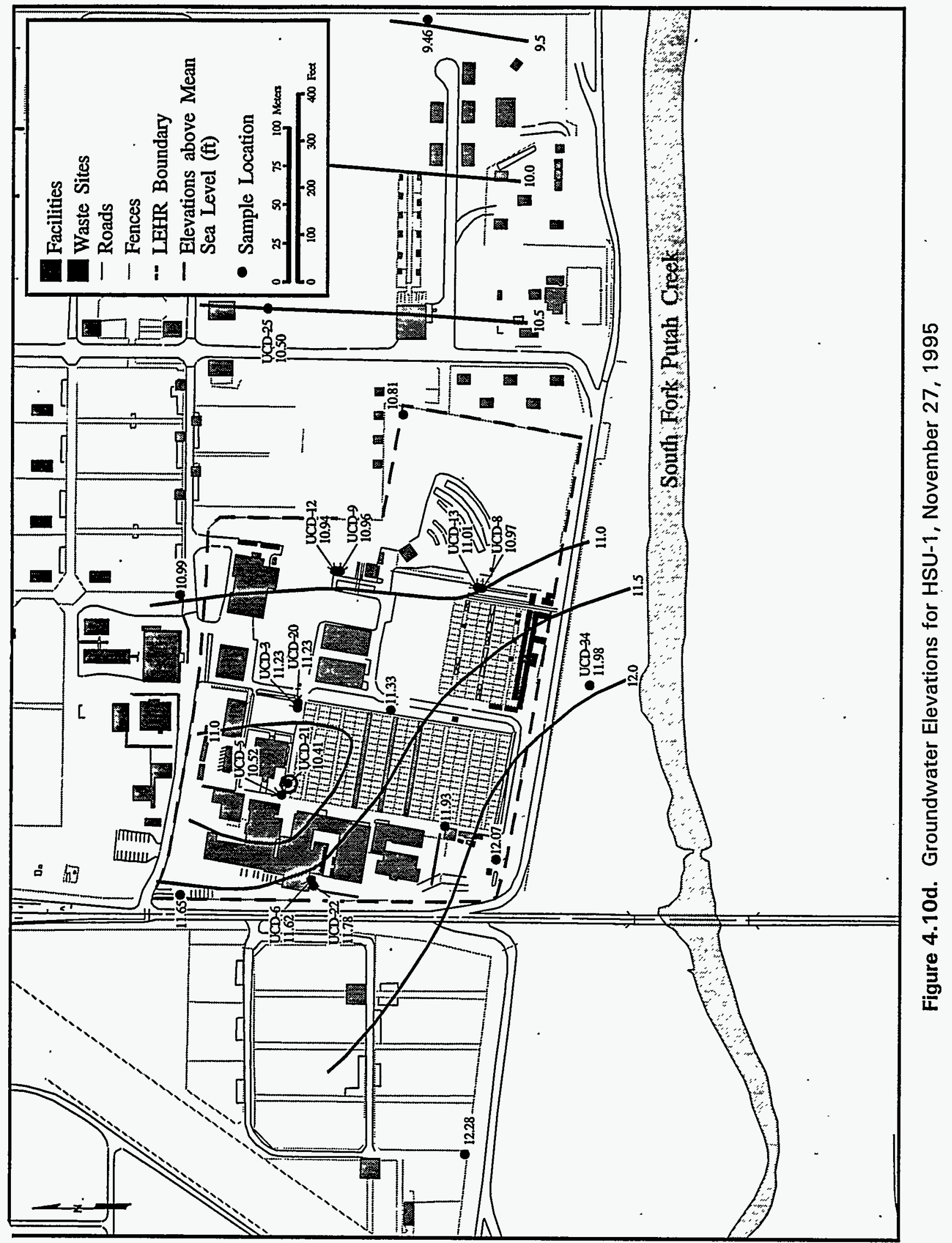




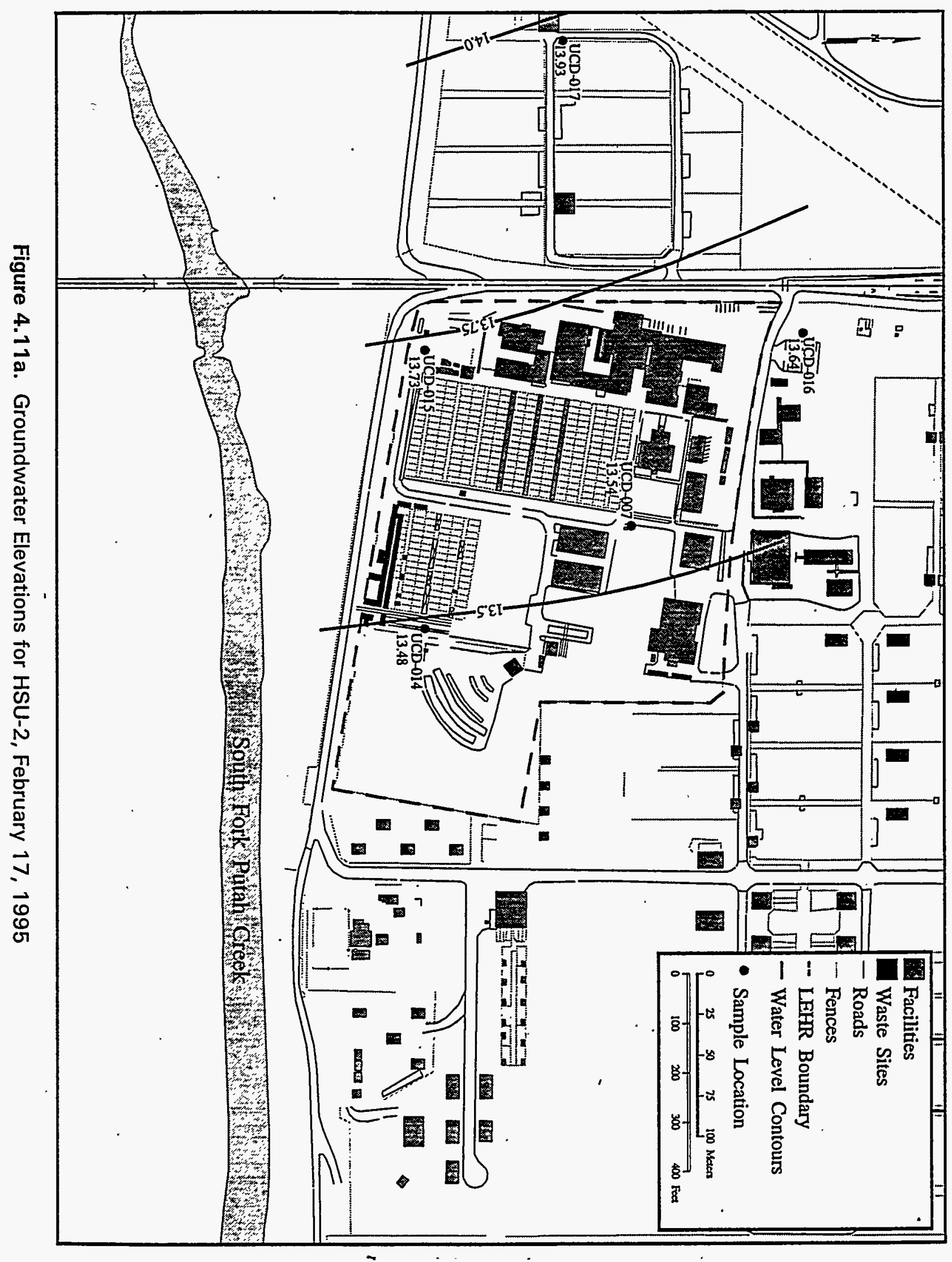




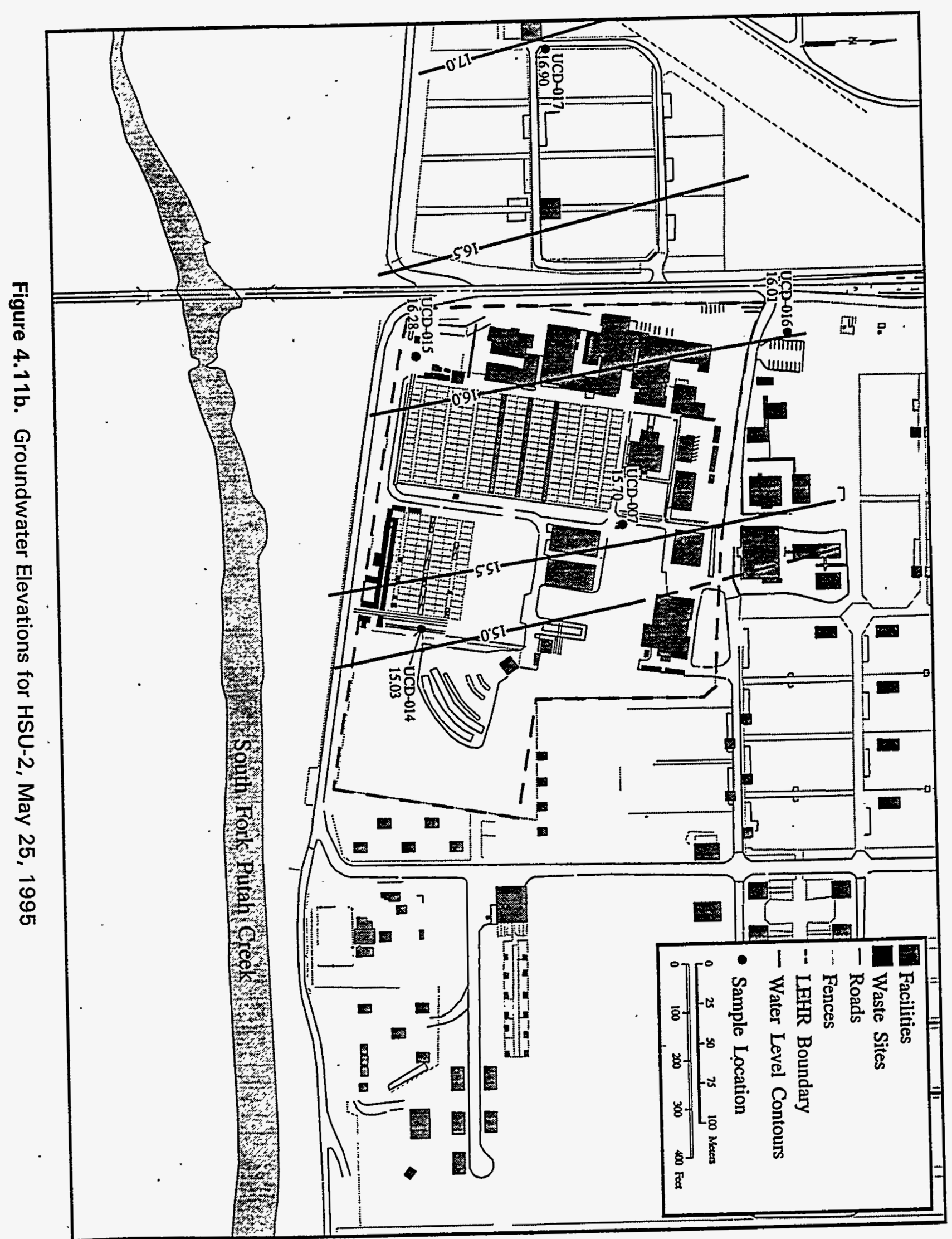




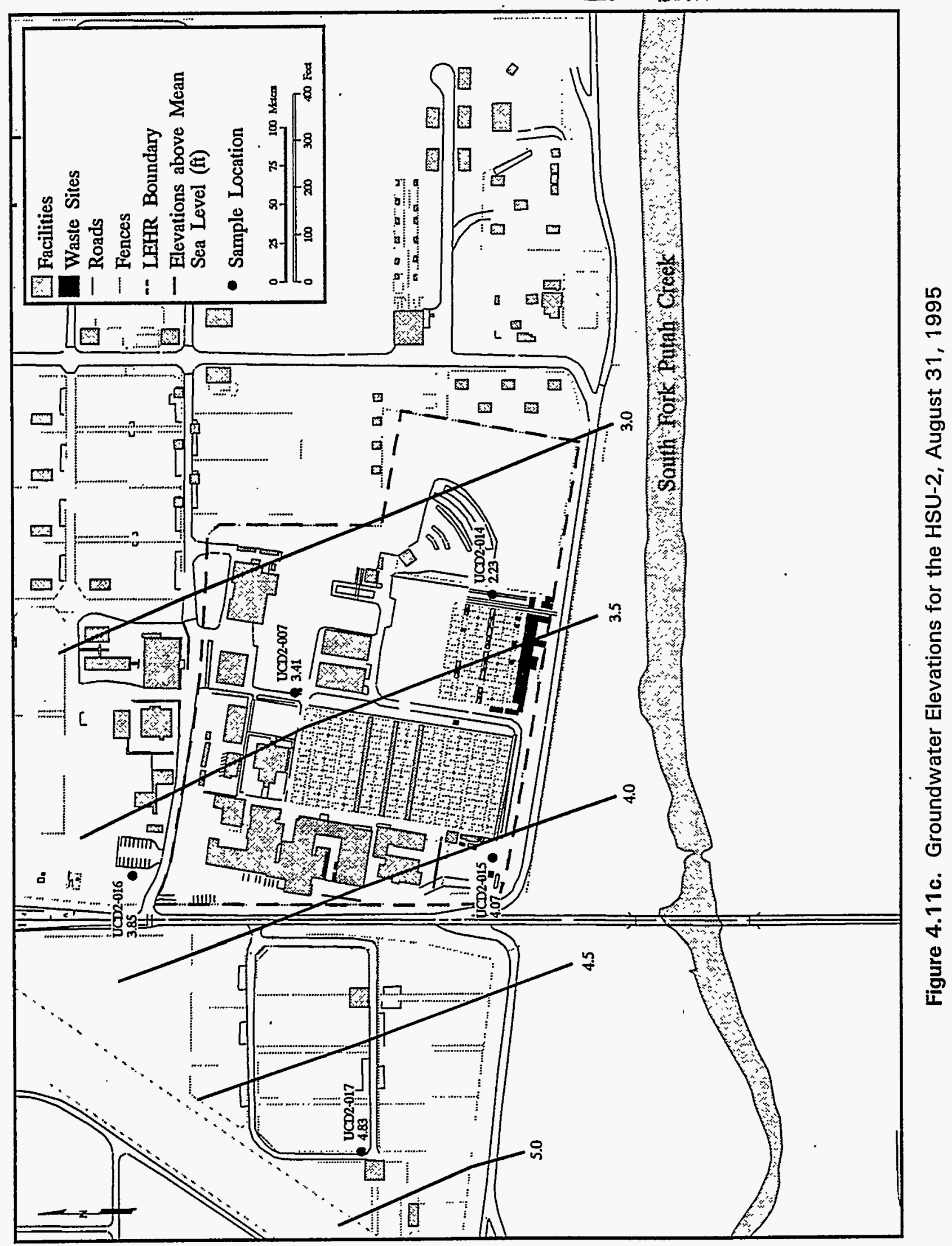




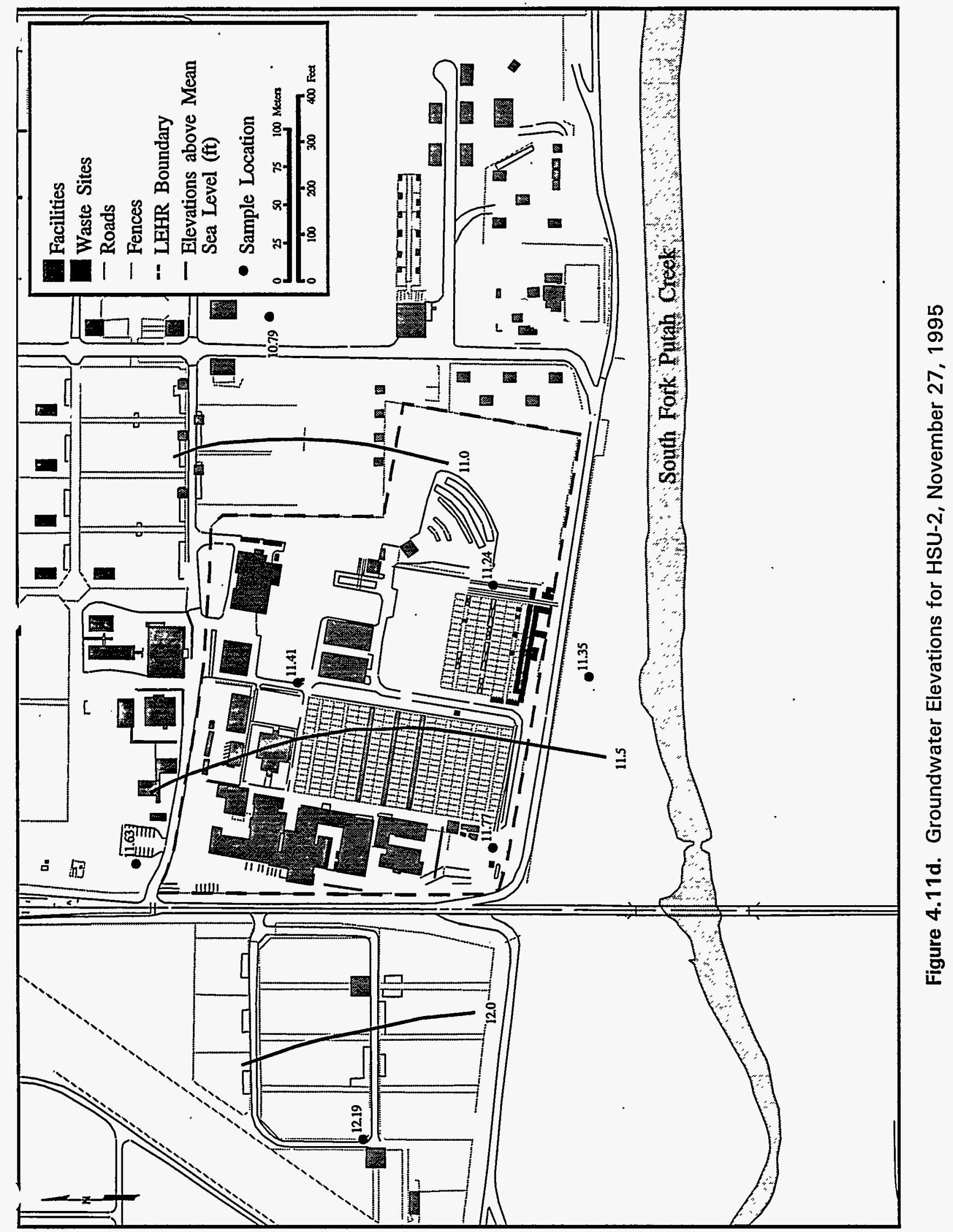




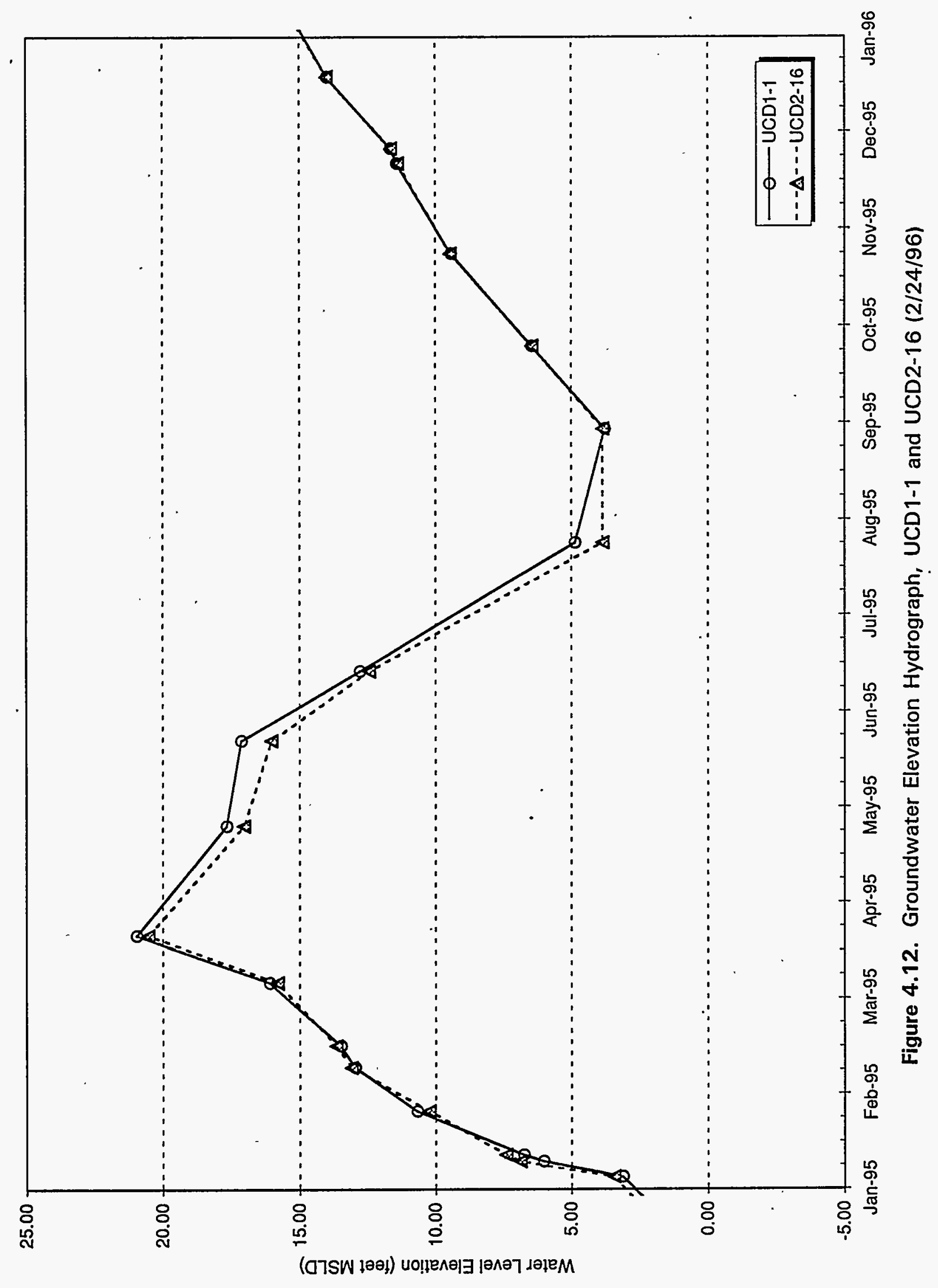

4.43 


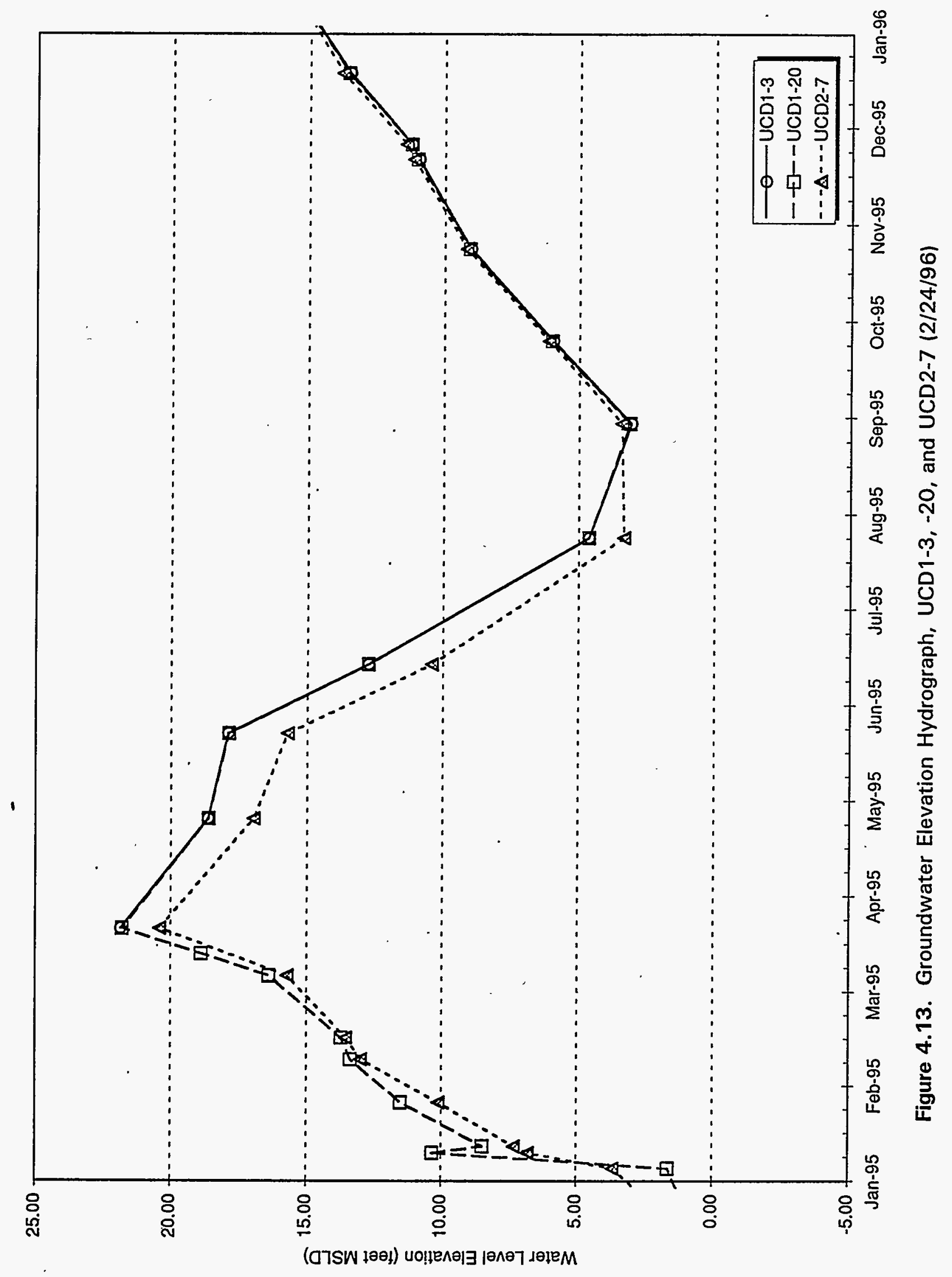




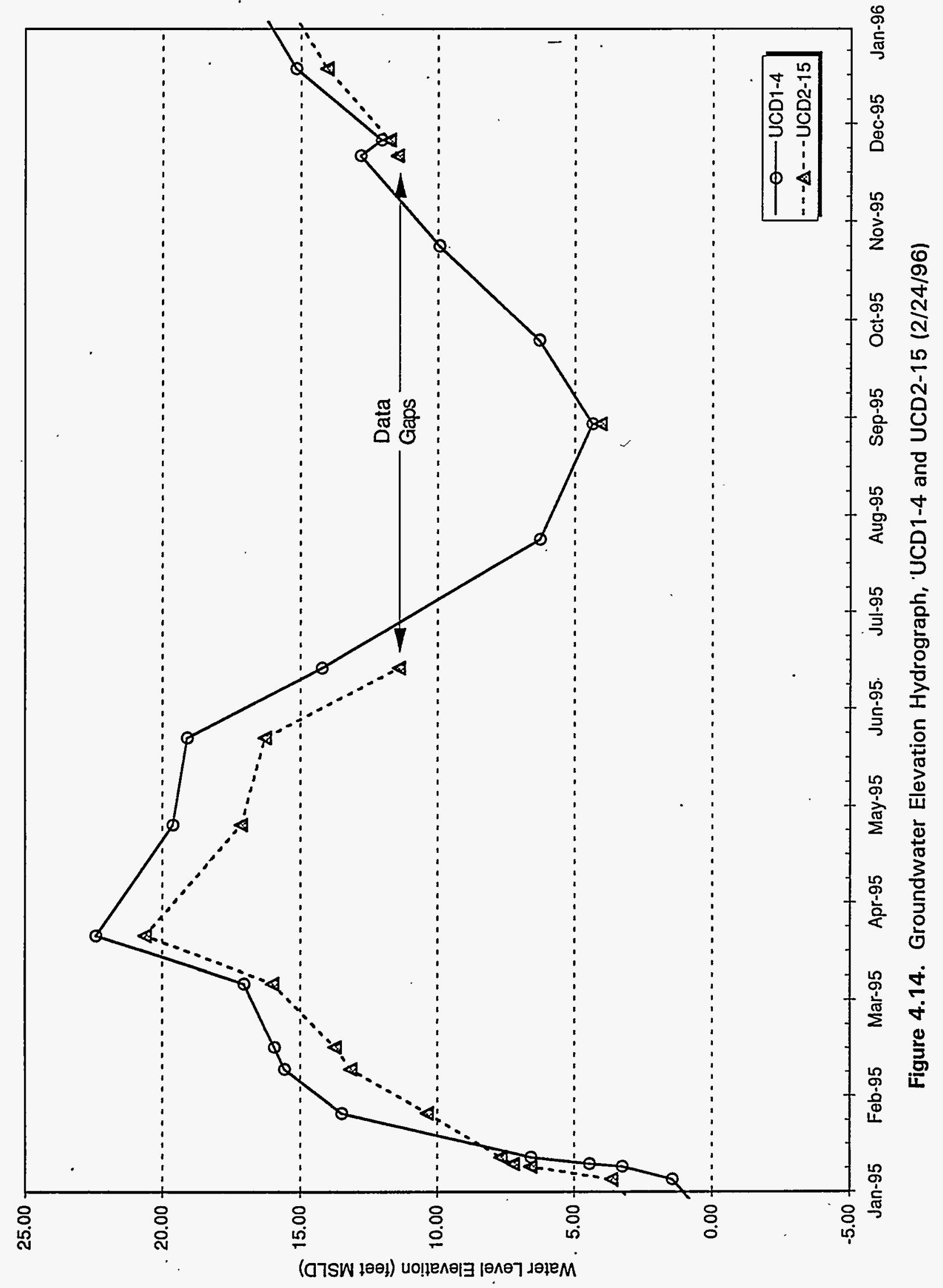




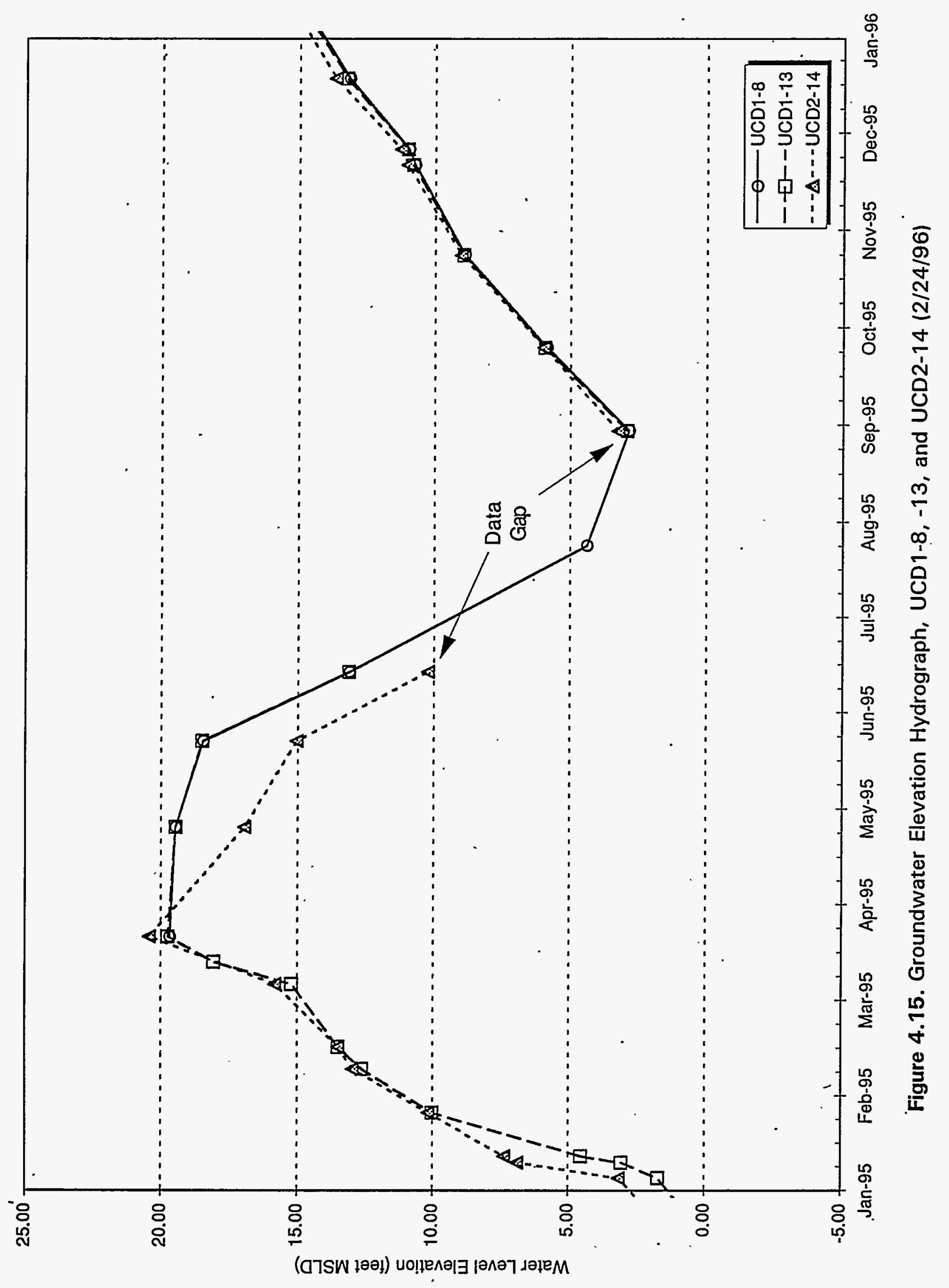




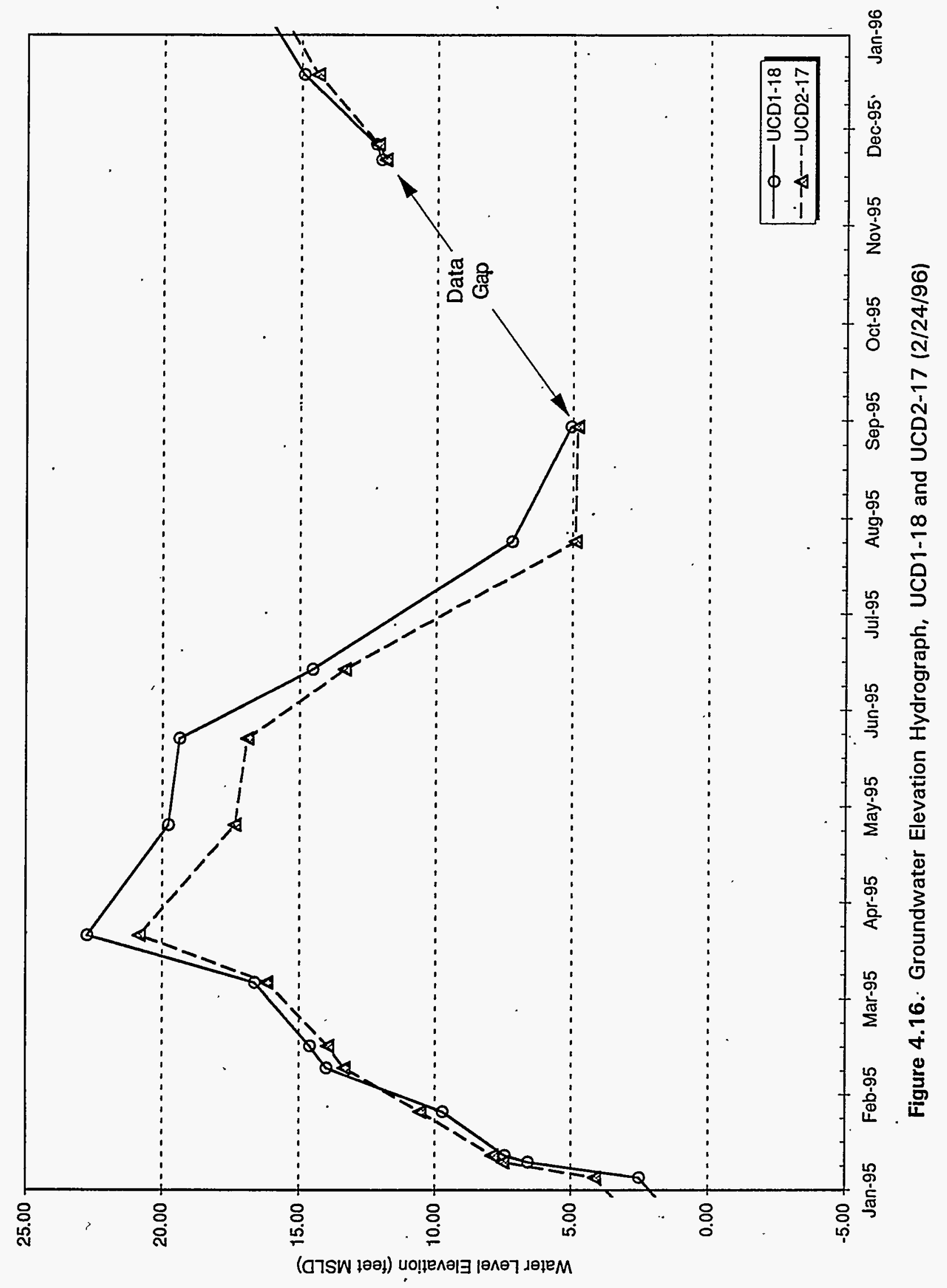




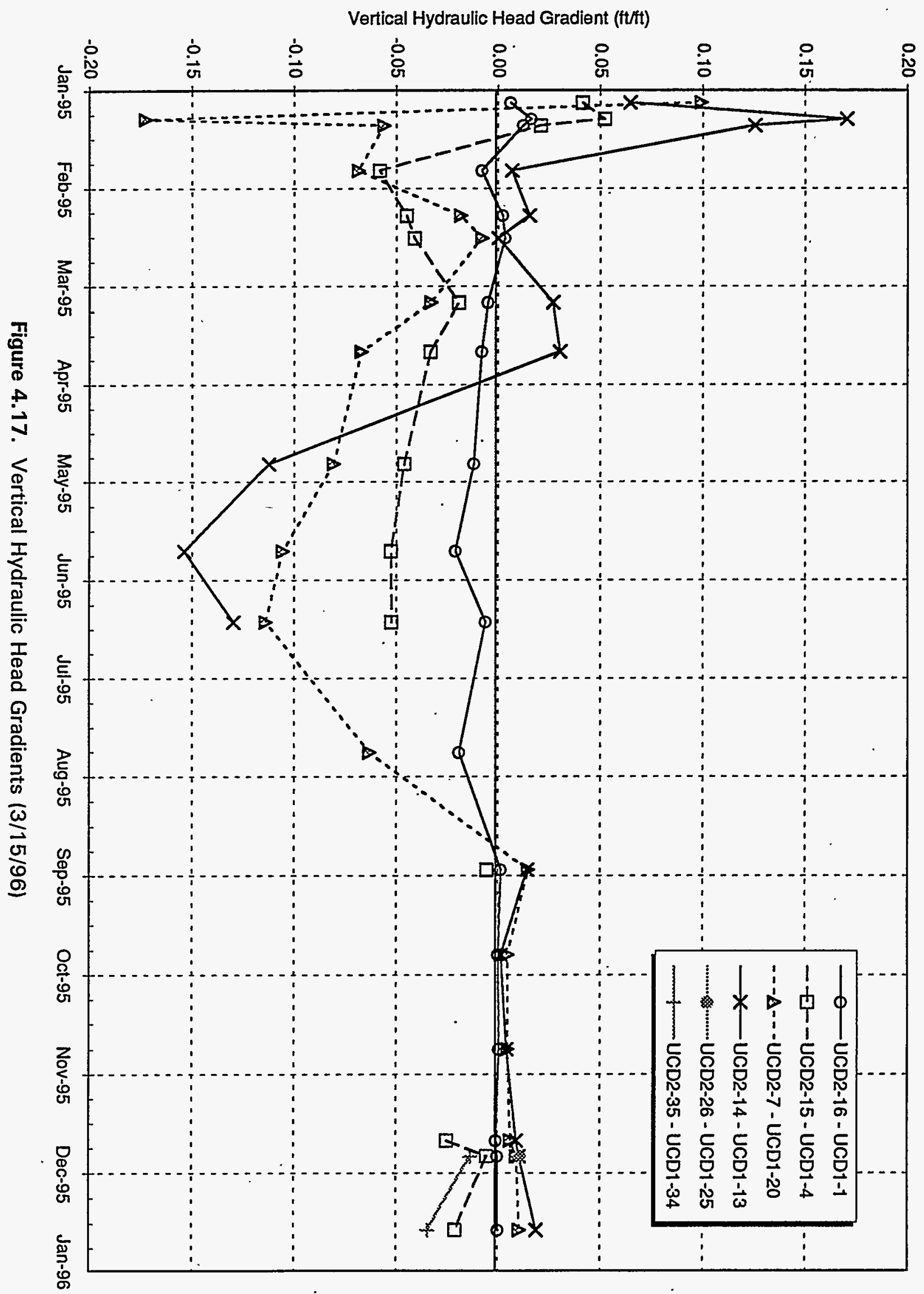


Table 4.1. Hydrogeologic Contacts in the LEHR Site Wells

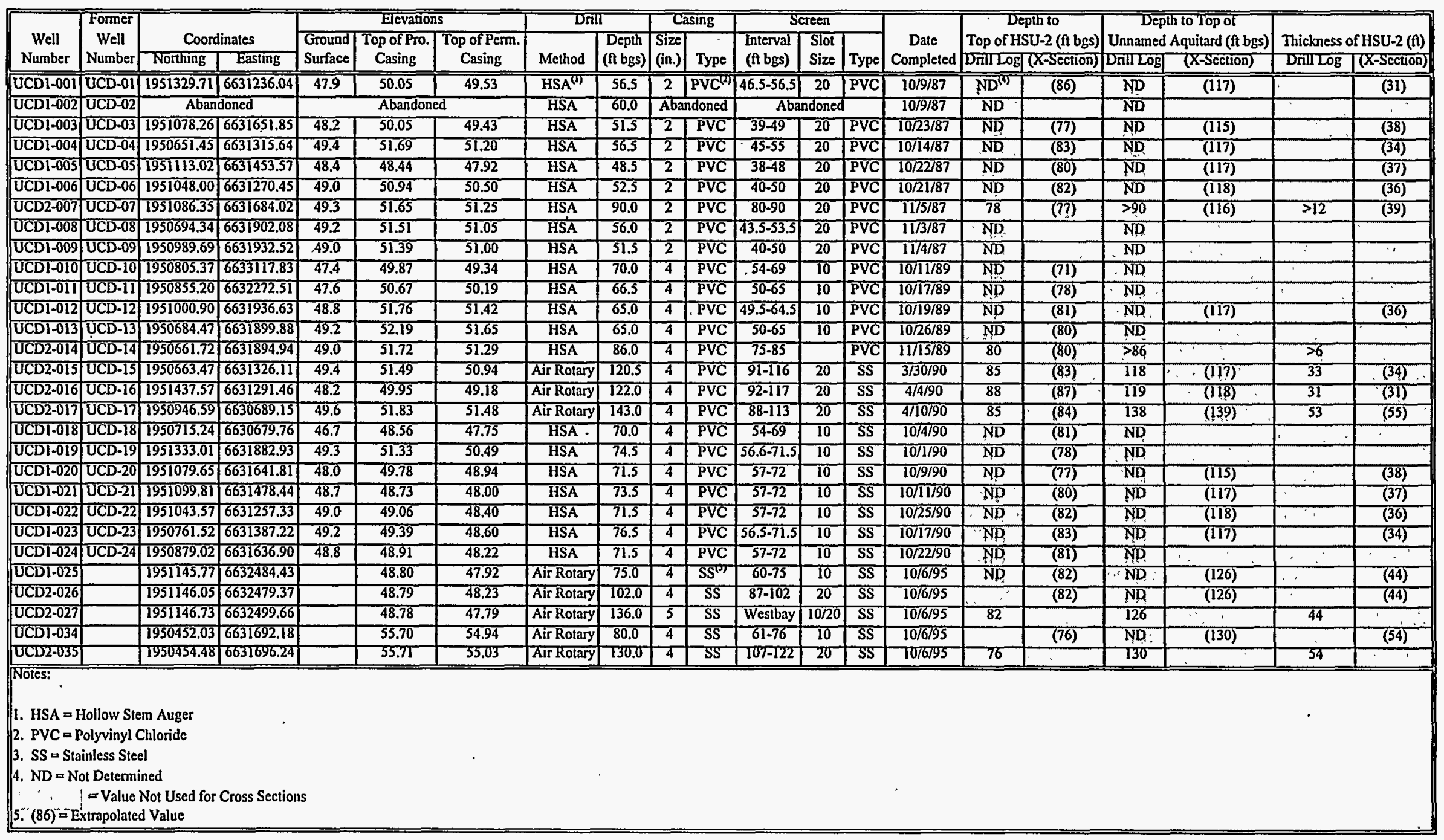


Table 4.4. Screened Intervals for Well Pairs

\begin{tabular}{|l|c|c|c||}
\hline $\begin{array}{c}\text { Monitoring } \\
\text { Well Pairs }\end{array}$ & $\begin{array}{c}\text { Screened } \\
\text { Interval } \\
\text { (ft bgs) }\end{array}$ & $\begin{array}{c}\text { Midscreen } \\
\text { Depth } \\
\text { (ft bgs) }\end{array}$ & $\begin{array}{c}\text { Differential } \\
\text { (ft bgs) }\end{array}$ \\
\hline \hline UCD1-4 & $45-55$ & 50 & - \\
\hline UCD2-15 & $91-116$ & 103.5 & 53.5 \\
\hline \hline UCD1-1 & $46.5-56.5$ & 51.5 & - \\
\hline UCD2-16 & $92-117$ & 104.5 & 53 \\
\hline \hline UCD1-20 & $57-72$ & 64.5 & - \\
\hline UCD2-7 & $80-90$ & 85 & 20.5 \\
\hline \hline UCD1-13 & $50-65$ & 57.5 & - \\
\hline UCD2-14 & $75-85$ & 80 & 22.5 \\
\hline \hline UCD1-18 & $54-69$ & 61.5 & - \\
\hline UCD2-17 & $88-113$ & 100.5 & 39 \\
\hline \hline UCD1-25 & $60-75$ & 67.5 & - \\
\hline UCD2-26 & $87-102$ & 94.5 & 27 \\
\hline \hline UCD1-34 & $61-76$ & 68.5 & - \\
\hline UCD2-35 & $107-122$ & 114.5 & 46 \\
\hline
\end{tabular}

Table 4.5. Screened Intervals for the Westbay Well (UCD2-27)

\begin{tabular}{|c|c|c|c|c|c||}
\hline $\begin{array}{c}\text { Monitoring } \\
\text { Interval }\end{array}$ & $\begin{array}{c}\text { Screened } \\
\text { Interval } \\
\text { (ft bgs) }\end{array}$ & $\begin{array}{c}\text { Midscreen } \\
\text { Depth } \\
\text { (ft bgs) }\end{array}$ & $\begin{array}{c}\text { Differential } \\
\text { (ft bgs) }\end{array}$ & $\begin{array}{c}\text { Water Level } \\
\text { Elevation (ft msl) } \\
2400 \text { hrs } \\
10 / 28 / 95\end{array}$ & $\begin{array}{c}\text { Water Level } \\
\text { Differential } \\
\text { (feet) }\end{array}$ \\
\hline \hline Zone 1 & $53-55$ & 54 & - & 8.73 & - \\
\hline Zone 2 & $63-65$ & 64 & 10 & 8.76 & 0.03 \\
\hline Zone 3 & $73-75$ & 74 & 10 & 8.89 & 0.13 \\
\hline Zone 4 & $86-88$ & 87 & 13 & 8.89 & 0.00 \\
\hline Zone 5 & $96-98$ & 97 & 10 & 8.93 & 0.04 \\
\hline Zone 6 & $106-108$ & 107 & 10 & 9.00 & 0.07 \\
\hline Zone 7 & $116-118$ & 117 & 10 & 8.89 & -0.11 \\
\hline
\end{tabular}




\subsection{Groundwater Analytical Results}

This section summarizes the analytical results from the 1995 groundwater monitoring program. Groundwater data are presented in a series of tables and figures and discussed in detail. Groundwater data are organized into summary tables of detected site constituents and complete data tables containing all analyses. Tables 5.1 through 5.8 present constituents that were detected in groundwater during the four quarters of the 1995 monitoring program, with results presented by well and by quarter. The entire groundwater monitoring results for 1995 , with both detections and nondetections for each of the analytical groups, are presented in Appendix A.

Groundwater analytical results for selected parameters are also presented in a series of figures that illustrate the distribution of various parameters across the site and over time. Figures 5.1 through 5.10 are bar graphs showing the concentrations of the five major constituents detected.

Figures 5.11 through 5.22 are concentration contour plots of chloroform, hexavalent chromium, nitrate, and tritium. These plots use mean data from the 1995 monitoring program where possible; hydropunch data collected in 1995 and 1994 are also used to fill gaps where no 1995 monitoring data are available. The concentration contour plots are drawn for those depth intervals corresponding to HSU-1, upper HSU-2, and middle HSU-2.

Appendix $C$ presents summary tables of constituent concentrations for key parameters measured at LEHR. These tables include data collected from fall quarter 1990 through fall quarter 1995 and illustrate the variation of results over time.

The analytical results of major cations and anions for groundwater samples are presented in Tables 5.9 and 5.10 and in Figures 5.23 - 5.25 (Stiff diagrams). These diagrams are plotted on a map of the site to show areal changes in general water chemistry. Stiff diagrams plot the concentrations of major cations and anions in milliequivalents (meq) per liter. Cations (positively charged ions) are plotted on the left side of the diagram, and anions (negatively charged ions) are plotted on the right side. Data compiled in Table 5.9 consist of mean values using all measurements for each sampling point and constituent since 1990. The concentrations presented in Table 5.9 were converted to equivalent concentrations and reported in Table 5.10 with a presentation of charge balance information. These data will be discussed further in Section 5.6.

The following sections discuss the 1995 groundwater monitoring results using these tables and figures. A discussion is presented for each analytical group that includes the location and magnitude of detections, a comparison with regulatory MCLs, and a review of overall trends. 
A summary of volatile organic compounds (VOCs) detected in 1995 groundwater samples is presented in Table 5.2. Each 1995 quarterly groundwater sample was analyzed for the target compound list identified in the table. Fourteen VOCs were reported in groundwater samples collected during 1995: chloroform, 1,1,2-trichloroethane, 1,1-dichloroethane, 1,1-dichloroethene, 1,2-dichloroethane, 1,2-dichloropropane, 2-butanone, acetone, bromodichloromethane, bromoform, carbon disulfide, methylene chloride, tetrachloroethene, and toluene.

Chloroform was the most frequently reported compound found in several wells that included the following: UCD1-11 (two of two quarters), UCD1-12 (four of four quarters), UCD1-13 (four of four quarters), UCD1-21 (one of two quarters), UCD1-23 (two of two quarters), UCD1-24 (two of two quarters), and UCD2-14 (four of four quarters). Of the remaining VOCs reported during 1995 1,1,2-trichloroethane, 1,1-dichloroethane, 1,1-dichloroethene, 1,2-dichloroethane, and bromodichloromethane were fourid only in well UCD1-12.

2-butanone, also known as methylethyl ketone (MEK), a common solvent, was found only in UCD1-27Z1 at $27 \mu / \mathrm{L}$. This level was confirmed on reanalysis of the sample. The EPA Region 9 preliminary remediation goal (PRG) for MEK in tap water is $1,900 \mu \mathrm{g} / \mathrm{L}$.

Acetone was reported in one of four quarters in UCD1-12 and in six of seven sample zones in UCD1/2-27. All detected acetone concentrations were estimated below the contract required quantification limit (CROL) of $5 \mu \mathrm{g} / \mathrm{L}$, and all were qualified with a " $c$ " during data validation, indicating poor calibration. The EPA Region 9 PRG for acetone in tap water is $610 \mu \mathrm{g} / \mathrm{L}$. Bromoform was reported only in UCD2-27Z6 at just below the CRQL of $1 \mu \mathrm{g} / \mathrm{L}$.

Carbon disulfide was detected in four of seven sampling zones in UCD1/2-27, when the well was sampled as part of the monitoring network in the fall quarter of 1995 . The highest reported concentration was $2.4 \mu \mathrm{g} / \mathrm{L}$ in UCD1-27Z3. Carbon disulfide was detected in three of the seven sample zones in October 1995, when the well was informally sampled to determine how well it had been developed. Carbon disulfide had never been detected in water samples from LEHR before. The source for this constituent is unknown, but its presence in the well will be monitored in the future. The EPA Region 9 PRG for carbon disulfide in tap water is $21 \mu \mathrm{g} / \mathrm{L}$.

Methylene chloride was detected in one of four quarters in UCD1-12 and UCD2-27Z4. Both analyses were $1 \mu \mathrm{g} / \mathrm{L}$, and a duplicate analysis of UCD1-12 was reported as less than the CROL of $2 \mu \mathrm{g} / \mathrm{L}$. The National Primary Drinking Water Standard for methylene chloride is $5 \mu \mathrm{g} / \mathrm{L}$.

Tetrachlorethane, also known as perchloroethene (PCE) was detected in one of two quarters in UCD1-24. PCE has been detected only in UCD1-24 and on only two occasions in 1994. The maximum concentration detected has been $0.84 \mu \mathrm{g} / \mathrm{L}$, well below the $\mathrm{MCL}$ of $5 \mu \mathrm{g} / \mathrm{L}$. 
Tetrachlorethane, also known as perchloroethene (PCE) was detected in one of two quarters in UCD1-24. PCE has been detected only in UCD1-24 and on only two occasions in 1994. The maximum concentration detected has been $0.84 \mu \mathrm{g} / \mathrm{L}$, well below the $\mathrm{MCL}$ of $5 \mu \mathrm{g} / \mathrm{L}$.

The highest concentrations of VOCs were consistently reported in samples analyzed from well UCD1-12. Detections of chloroform (ranging from 1,000 $\mu \mathrm{g} / \mathrm{L}$ to $5,800 \mu \mathrm{g} / \mathrm{L}$ ), 1,1-dichloroethane (ranging from $0.51 \mu \mathrm{g} / \mathrm{L}$ to $9.2 \mu \mathrm{g} / \mathrm{L}$ ), 1,1-dichloroethene (ranging from $1.1 \mu \mathrm{g} / \mathrm{L}$ to $18 \mu \mathrm{g} / \mathrm{L}$ ), and 1,2-dichloroethane (ranging from $<1 \mu \mathrm{g} / \mathrm{L}$ to $5.9 \mu \mathrm{g} / \mathrm{L}$ ), exceeded their respective MCLs in samples from well UCD1-12. The MCLs for each of these parameters are $100 \mu \mathrm{g} / \mathrm{L}$ for total trihalomethanes (chloroform is the major component); $5 \mu \mathrm{g} / \mathrm{L}$ for 1,1-dichloroethane; $6 \mu \mathrm{g} / \mathrm{L}$ for 1,1-dichloroethene; and $0.5 \mu \mathrm{g} / \mathrm{L}$ for 1,2-dichloroethane. These data are consistent with those reported for 1994.

Chloroform concentrations in groundwater are depicted in Figures 5.1, 5.2, 5.11, 5.12, and 5.13. Figure 5.1 is a bar graph of reported chloroform values in HSU-1 groundwater . samples. As shown in this figure, the highest reported chloroform values for 1995 are consistently reported in samples from UCD1-12 (up to $5,800 \mu \mathrm{g} / \mathrm{L}$ ). The elevated levels of chloroform in UCD1-12 require sample dilution prior to analysis. Due to analytical limitations, sample dilutions were not always adequate; the chloroform values reported for UCD1-12 in these cases are estimated concentrations. Figure 5.2 is a bar graph of reported chloroform values in HSU-2 groundwater samples. As shown in this figure, chloroform was reported only in samples collected from UCD2-14 (up to $4.1 \mu \mathrm{g} / \mathrm{L}$ ).

The chloroform distribution beneath LEHR is presented in Figures 5.11, 5.12 and 5.13. Four major observations can be made from these figures.

1. At all three depths in the aquifer, the highest chloroform concentrations are in an area located on the eastern side of LEHR in the vicinity of Landfill 2. In general, the highest concentrations in this likely source area are in HSU-1 and decrease with depth in the aquifer.

2. Chloroform has migrated downgradient to the east from the source area identified in the first observation.

3. Chloroform plumes in the upper and middle layers of HSU-2 extend farther to the east and have dispersed in a wider pattern than the plume in HSU-1.

4. Chloroform concentrations are higher in the middle and upper portions of HSU-2 than in HSU-1 in areas more than 100 feet downgradient (east) of the source area. This is opposite to the trend noted in the first observation.

Based on these observations, it is apparent that the source of chloroform at LEHR is in the vicinity of Landfill 2 and that the chloroform was disposed at or near the ground surface such that HSU-1 is contaminated with the highest concentrations of chloroform. The 
downward transport of chloroform from HSU-1 to HSU-2 is accompanied by a significant groundwater velocity contrast. As discussed in Section 4.0, horizontal groundwater gradients for HSU-1 and HSU-2 are about the same, approximately 0.001 . Hydraulic conductivities range from 10 to 1,000 times higher in $\mathrm{HSU}-2$ than $\mathrm{HSU}-1$. Therefore, horizontal groundwater flow velocities are 10 to 1,000 times faster in HSU-2 than in HSU-1, which explains why plumes in HSU-2 have spread farther. The velocity contrast would also result in considerable dilution and dispersion of chloroform as it moves from HSU-1 into HSU-2 as evidenced by lower chloroform concentrations near the source in HSU-2. Because of large seasonal fluctuations in groundwater withdrawals and recharge, fluctuating vertical hydraulic gradients result in groundwater flow reversals between HSU-1 and HSU-2. During the fall, winter, and spring, when the water table is rising, HSU-1 receives much of its recharging water from below, in HSU-2. In areas where chloroform has been transported downgradient in HSU-2, HSU-1 is recharged with chloroform-contaminated water from HSU-2. This appears to be the primary reason why chloroform has spread so far downgradient in HSU-1 despite its much lower groundwater velocity.

A summary table for chloroform is presented in Appendix C. These data show that chloroform has been reported in several wells across the site and confirm that the highest concentrations and most consistent detections are in samples from well UCD1-12. Chloroform has also been consistently reported in wells UCD1-13 and UCD1-24.

\subsection{Semivolatile Organic Compounds}

A summary of semivolatile organic compounds (SVOCs) detected in 1995 groundwater samples is presented in Table 5.3. Each 1995 quarterly groundwater sample was analyzed for the target compound list identified in Table 2.5. Six SVOCs were reported in 1995 groundwater samples; these compounds and their maximum concentrations are bis(2-ethylhexyl)phthalate $(29 \mu \mathrm{g} / \mathrm{L})$, di-n-butylphthalate $(180 \mu \mathrm{g} / \mathrm{L})$, diethyl phthalate $(2.0 \mu \mathrm{g} / \mathrm{L})$, dimethyl phthalate $(6.3 \mu \mathrm{g} / \mathrm{L}), \mathrm{N}$-nitrosodiphenylamine $(0.64 \mu \mathrm{g} / \mathrm{L})$, and phenol $(230 \mu \mathrm{g} / \mathrm{L})$. Of these, only bis(2-ethylhexyl) phthalate exceeded its EPA Region 9 PRG for tap water of $4.8 \mu \mathrm{g} / \mathrm{L}$. Bis(2-ethylhexyl)phthalate is commonly the result of sample contamination during the analytical process and has been attributed to laboratory contamination in previous LEHR samples. Bis(2-ethylhexyl)phthalate has been reported sporadically in groundwater samples throughout the water monitoring program. These reported detections were not confirmed upon subsequent sampling and are, therefore, the likely result of laboratory contamination. In addition, method blanks contained bis(2-ethylhexyl)phthalate at concentrations up to $6.0 \mu \mathrm{g} / \mathrm{L}$. These findings are similar to those reported for 1994.

\subsection{Pesticides and PCBs}

A summary of pesticides and polychloride biphenyis (PCBs) detected in 1995 groundwater samples is presented in Table 5.4. 1995 quarterly groundwater samples were analyzed for the target compound list identified in Table 2.3. Four pesticide compounds were reported above detection limits in 1995 groundwater samples (Table 5.4); alpha 
chlordane, gamma chlordane, dieldrin, and endrin and were reported only in well UCD1-13. These results are the same as reported for 1994. Alpha and gamma chlordane were detected twice at levels below the CROL of $0.01 \mu \mathrm{g} / \mathrm{L}$, and endrin was detected two times below its CRQL of $0.02 \mu \mathrm{g} / \mathrm{L}$. Dieldrin was detected twice slightly above its CRQL at $0.029 \mu \mathrm{g} / \mathrm{L}$ and $0.028 \mu \mathrm{g} / \mathrm{L}$. No reported pesticide value exceeded an MCL, but dieldrin exceeded the EPA Region 9 PRG of $0.0042 \mu \mathrm{g} / \mathrm{L}$ for tap water. No PCB compounds were reported in 1995 groundwater samples.

\subsection{Metals}

A summary of metals detected in 1995 groundwater samples is presented in Table 5.5. Each 1995 quarterly groundwater sample was analyzed for 17 metals (see Table 2.3) and hexavalent chromium. The metals beryllium, cadmium, lead, silver, and thallium were not detected in any 1995 groundwater samples. The remaining metals analyzed were detected above detection limits in several wells during the year as discussed in the following:

- Antimony was reported above the method detection limit (MDL) but below the CRQL in six wells in 1995. Based on data presented in the summary table in Appendix $C_{r}$ antimony appears and disappears in random fashion throughout the monitoring network in both background and downgradient wells. Antimony concentrations ranged from $1.0 \mu \mathrm{g} / \mathrm{L}$ in UCD2-15 to $2.3 \mu \mathrm{g} / \mathrm{L}$ in UCD2-16 and UCD2-27Z4. The MCL for antimony is $6 \mu \mathrm{g} / \mathrm{L}$.

- Arsenic was reported at least one time above the detection limit in all 18 original wells during 1995 (Table 5.5). Arsenic was also reported above the detection limit for all seven zones of UCD1/2-27 and UCD1-34 but was not detected in new wells UCD1-25, UCD2-26, and UCD2-35. Reported concentrations range from an estimated $2.0 \mu \mathrm{g} / \mathrm{L}$ to $8.6 \mu \mathrm{g} / \mathrm{L}$. These findings are similar to those reported for 1994 . No arsenic values were reported above the MCL of $50 \mu \mathrm{g} / \mathrm{L}$.

Arsenic has only been reported above detection limits since the summer quarter 1993, when the analytical method was changed and the detection limit was lowered to $2.0 \mu \mathrm{g} / \mathrm{L}$. Based on an interpretation of reported results, arsenic values are consistent among wells and occur at concentrations near the detection limit (Appendix C).

- Barium was reported above the detection limit in every 1995 LEHR groundwater sample except the sample from UCD2-26 (Table 5.5). Reported concentrations range from $25.7 \mu \mathrm{g} / \mathrm{L}$ to $355 \mu \mathrm{g} / \mathrm{L}$. In general, the highest concentrations of barium are reported in wells UCD1-4, UCD1-11, UCD1-12, and UCD1-13 in HSU-1; and in UCD2-14 in HSU-2 (barium summary table, Appendix $\mathrm{C}$ ). The lowest values for barium are consistently reported for well UCD1-10. No barium concentrations were reported above the MCL of $1,000 \mu \mathrm{g} / \mathrm{L}$.

- Total chromium was reported above the detection limit in every 1995 LEHR groundwater sample, including all new wells (see Table 5.5). Reported concentrations range 
from 3.0 to $290 \mu \mathrm{g} / \mathrm{L}$. The highest total chromium values are consistently reported for UCD1-11, UCD1-12, and UCD1-19. The lowest values are generally reported for upgradient wells UCD1-18 and UCD2-17 and downgradient wells UCD 1-22 and UCD2-15. Values that exceed the chromium MCL of $50 \mu \mathrm{g} / \mathrm{L}$ were reported for six wells: UCD1-10, UCD1-11, UCD1-12, UCD1-13, UCD1-19, and UCD1-21. These trends are the same as identified in 1994. No samples from HSU-2 had a reported concentration of chromium that exceeded the MCL. Section 7.0 provides a statistical evaluation and analysis of the significance of the chromium results.

- Hexavalent chromium was reported above the detection limit at least once in 16 of 18 original monitoring wells during 1995 (Table 5.5). Reported concentrations range from 6 to $310 \mu \mathrm{g} / \mathrm{L}$. In general, hexavalent chromium results are similar to total chromium results because $80 \%$ or more of the chromium detected in the groundwater is in hexavalent form. This is consistent with the chemistry of chromium, in which the two common valence states of chromium $(+3$ and +6$)$ have distinctly different aqueous speciation. The +3 form is immobile, and the +6 (hexavalent) form is mobile. Hexavalent chromium concentrations for 1995 are shown in Figures 5.5 and 5.6. Distributions of hexavalent chromium are shown in Figures $5.17,5.18$, and 5.19. The highest concentrations of hexavalent chromium are typically reported for wells UCD1-11, UCD1-12, and UCD1-19, as reported for 1994 and as shown in Figure 5.5. There is no specific MCL for hexavalent chromium; however, values from seven wells exceeded the MCL of $50 \mu \mathrm{g} / \mathrm{L}$ for total chromium during 1995. The data presented in the summary tables in Appendix $C$ indicate that, of the seven wells in which chromium exceeded the MCL, three wells, UCD1-11, UCD1-12, and UCD1-19, exceeded the MCL by greater than a factor of 3 .

Bar graphs for hexavalent chromium (Figures 5.5 and 5.6) show that 1995 results are consistent within each well throughout the year and that the highest values were typically reported for downgradient wells. Values for chromium and hexavalent chromium show similar trends across the site, with values generally consistent over time (see time series tables in Appendix C). The correlation between chromium and hexavalent chromium is consistent with past conclusions from this monitoring program that most chromium in solution in groundwater is in the hexavalent form (Dames \& Moore 1993; DOE 1994a). Section 7 provides a statistical evaluation and analysis of the significance of the hexavalent chromium results.

The hexavalent chromium distribution beneath LEHR is presented in Figures 5.17, 5.18, and 5.19. As with chloroform and nitrate, chromium concentrations decrease with depth in the aquifer. Two lobes of hexavalent chromium-contaminated groundwater are present; one is centered north of Landfill 2, and the other is located northeast of Landfill 1. Both lobes contain hexavalent chromium above the total chromium MCL of $50 \mu \mathrm{g} / \mathrm{L}$. The pattern can be seen in HSU-1, but only a few detections of these lobes can be seen in the upper portion of HSU-2. Only two locations near the center of the western lobe are above the MCL. Hexavalent chromium was not detected above the MCL in the middle portion of HSU-2. 
The chromium distribution is distinctly different from the chloroform and nitrate distributions. The narrow zone of water with low chromium concentration between the two lobes is located in the same area where the highest chloroform and nitrate concentrations are located (see Figures 5.14 and 5.17). It is unclear why chromium is distributed in this manner, but two explanations are most probable. The two lobes could represent two distinct sources of hexavalent chromium that are different from the chloroform and nitrate sources; or the sources of chloroform and nitrate could contain no hexavalent chromium and might displace groundwater that contains high levels of chromium.

- Cobalt was reported above the detection limit in four original HSU-1 wells and one original HSU-2 well. In addition, cobalt was detected in all new HSU-1 wells and new well UCD2-35 in HSU-2. All reported detections were less than the CROL of $10 \mu \mathrm{g} / \mathrm{L}$ and at levels similar to that found in background well UCD1-18. There is no regulatory limit for cobalt in water.

- Copper was reported above the detection limit in six original HSU-1 wells and two original HSU-2 wells. In addition, it was detected in new wells UCD1-25 and UCD2-26. All reported detections were less than the CROL of $10 \mu \mathrm{g} / \mathrm{L}$ and at levels similar to those in background well UCD1-18. The national secondary drinking water standard for copper is $1,000 \mu \mathrm{g} / \mathrm{L}$.

- Mercury was reported above the detection limit in five original wells, three in HSU-1 and two in HSU-2 in 1995. Reported concentrations ranged from 0.23 to $0.68 \mu \mathrm{g} / \mathrm{L}$. No mercury concentrations were reported above the MCL of $2 \mu \mathrm{g} / \mathrm{L}$.

- Molybdenum was reported above the detection limit in 12 original wells, eight in HSU-1 and four in HSU-2. In addition, molybdenum was detected in most of the new monitoring wells except UCD1-25, UCD2-26, and UCD2-27Z6. Reported concentrations ranged from 1.1 to $5.5 \mu \mathrm{g} / \mathrm{L}$. No molybdenum concentrations were reported above the EPA Region 9 PRG of $180 \mu \mathrm{g} / \mathrm{L}$ for tap water.

- Nickel was reported above the detection limit in 15 original wells and all new wells except UCD2-26 during 1995. Reported concentrations range from 1.0 to $65.1 \mu \mathrm{g} / \mathrm{L}$. No nickel concentrations exceeded the MCL of $100 \mu \mathrm{g} / \mathrm{L}$.

Nickel detections in wells have been sporadic and at concentrations near the detection limit throughout the monitoring program, except for wells UCD1-18 and UCD1-23. Nickel has been reported frequently in these two wells but at variable concentrations. These are the same as results presented in 1994. Section 7 provides a statistical evaluation and analysis of the significance of the nickel results.

- Selenium was reported above the detection limit at least one time in 10 wells during 1995. Selenium has historically been detected at the highest concentrations in wells UCD1-10, UCD1-11, UCD1-12, and UCD1-19. Reported concentrations range from 
3.2 to $23.0 \mu \mathrm{g} / \mathrm{L}$. At least one reported value from each of the wells UCD1-10, UCD1-11, and UCD1-12, exceeded the MCL of $10 \mu \mathrm{g} / \mathrm{L}$ at least one time during 1995.

The time series table (Appendix $C$ ) for selenium shows that frequent detections in wells UCD1-10, UCD1-11, UCD1-12, and UCD1-19 are consistent with monitoring results from previous years. Section 7 provides a statistical evaluation and analysis of the significance of the selenium results.

- Vanadium was reported above the detection limit in all 18 original wells during 1995. In addition, it was detected in six of 11 new sample locations. Reported concentrations during 1995 ranged from 4.0 to $13.4 \mu \mathrm{g} / \mathrm{L}$. There is no $\mathrm{MCL}$ for vanadium, but the EPA Region 9 PRG for tap water is $260 \mu \mathrm{g} / \mathrm{L}$.

- Zinc was reported above the detection limit in every well sampled, both original and new, during 1995. Zinc concentrations ranged from 1.2 to $559 \mu \mathrm{g} / \mathrm{L}$. All zinc levels were greater than the CROL of $20 \mu \mathrm{g} / \mathrm{L}$ except in samples collected in wells UCD1-25, UCD1-27 (two of three zones), UCD1-34, and UCD2-27Z5. The highest concentrations are from UCD1/2-27 and may be associated with the Westbay system. This situation will continue to be monitored. The EPA secondary drinking water standard for zinc is $5,000 \mu \mathrm{g} / \mathrm{L}$.

\subsection{Radionuclides}

This section presents the unique format required for presentation of radionuclide data and the summary of analytical results. A summary of radionuclides detected in 1995 groundwater samples is presented in Table 5.6. The analyses identified in Table 2.3 were performed for the 1995 groundwater monitoring program.

Radionuclide analyses are reported with the calculated activity of the sample, the minimum detectable activity (MDA), and the counting error. The MDA describes quantitatively the sensitivity of the analytical procedures used and represents a minimum detectable radionuclide activity in a sample medium at the time of analysis. Variables include decay rate, sample counting time, sample volume and density, chemical recovery, background counts, and detector efficiency, all affect the resulting MDA for a given sample. The counting error represents the total statistical uncertainty resulting from measuring all random processes involved in the analytical procedures used. Individual errors associated with these processes are propagated to estimate the total uncertainty.

The uncertainty value is reported as a two-sigma (two standard deviations) error for each sample analysis result. This uncertainty, therefore, approximates a $95 \%$ confidence interval about the reported value. Non-random systematic errors will not be reported since they cannot be accurately quantified. Good laboratory practices, $\mathrm{QA} / \mathrm{OC}$ programs/ procedures, and frequent instrument calibrations minimize any incurred systematic errors. 
Radionuclide results are reported as the calculated value (V) followed by the total uncertainty $(T)$. The format follows the convention $(V \pm T)$. The significance of the radionuclide results can be evaluated by comparing the reported value with the reported uncertainty that is calculated for each sample. Although gross alpha and gross beta analyses are not performed for specific radionuclides. (i.e., they measure total alpha or beta energy released in a sample), they are included in the radionuclide analytical results tables for consistency in reporting radionuclide characteristics.

Radionuclide concentrations reported in groundwater that exceed the corresponding uncertainty or MDA are highlighted in Table 5.6. These values represent a $95 \%$ level of confidence that measurable radioactivity above zero is present for a given radionuclide, and the result is referred to as a "positive value." It should be emphasized, however, that despite being statistically detectable on the basis of total uncertainty, a given sample result may not be significant in terms of health risk, presence of site contamination, or regional background concentrations. A more detailed discussion of radiation measurement at or near the detection limit is presented by Currie (1968).

The 1995 radionuclide results indicate that, for groundwater sampling locations, the major observable impact due to radionuclides was located in wells UCD1-13 and UCD2-14 for tritium and carbon-14. Carbon-14 was also detected at just above the MDA in wells UCD1-12 and UCD1-23. Americium-241 was reported in UCD1-10 at just above the MDA. No other spatial or temporal trends were noted for radionuclides.

A summary of reported analytical results for detected radionuclide constituents in groundwater samples is presented in Table 5.6. Complete analytical results for groundwater samples, including data validation flags and reason codes, are presented in Appendix A, Table A.5.

Uranium-238 and thorium-232 are naturally occurring radionuclides found in most soils, including soils at the LEHR site. Uranium-238 decays into daughter products (progeny) such as thorium-234, radium-226, radon-222, bismuth-214, and lead-210. Thorium-232 progeny include radium-228, radon-220, lead-212, bismuth-212, and lead-208. Because these parent radionuclides are found in soils and groundwater, their progeny are also expected to be found in soil and groundwater analyses results.

Significant radionuclide concentrations were reported in groundwater during 1995 for tritium (UCD1-13 and UCD2-14) and carbon-14 (UCD1-12, UCD1-13, UCD1-23, and $U C D 2-14)$. All other radionuclide concentrations were below background well results (UCD2-17 and UCD1-18) or below detection limits as determined by associated counting uncertainties with the exception of miscellaneous "positive values" for plutonium-241, americium-241, and strontium-90.

Plutonium-241 was reported at levels greater than the MDA or counting error in two wells, UCD1-19 and UCD1-22. The two reported positive values were the first since the summer of 1994, when plutonium analyses were first performed. In the case of UCD1-19, 
the plutonium activity of $2.7( \pm 1.9) \mathrm{pCi} / \mathrm{L}$ was reported for a duplicate sample for which the primary sample contained no detectable plutonium-241. In the case of well UCD1-22, the plutonium-241 activity of $3.2( \pm 2.3) \mathrm{pCi} / \mathrm{L}$ was slightly greater than the MDA of $3.0 \mathrm{pCi} / \mathrm{L}$. In both cases; plutonium is considered not to be present because of past trends (Appendix $\mathrm{C}$ ) and the fact that the values are just above the error or MDA.

Americium-241 was reported at levels greater than the MDA or counting error in five wells during 1995, UCD1-10, UCD1-24, UCD1-27Z2, UCD2-14, and UCD2-27Z7. Reported activities range from 0.029 to $0.106 \mathrm{pCi} / \mathrm{L}$. The reported activities were slightly greater than the counting errors or MDA and are not considered positive detections, but these wells will be monitored to determine whether these are random occurrences, as expected. Another factor that indicates that these results are not positive detections is that americium-241 would be present because of the later decay of plutonium-241. Plutonium has not been reported as a detection in the same wells that americium-241 has been reported in. Only well UCD1-10 has had a similar activity reported, in one sample prior to 1995.

Strontium-90 was reported at a level greater than the MDA or counting error only in well UCD2-14 during 1995. The reported activity was $1.06 \mathrm{pCi} / \mathrm{L}$, slightly greater than the MDA or counting error and not considered a positive detection. Strontium-90 was reported above the MDA in UCD2-14 in the summer, but was not detected in the fall. Strontium-90 has been detected randomly throughout the monitoring network over time, in 14 of 18 wells, including background well UCD1-18 (Appendix C). Because of variations in counting errors and the fact that the reported value is close to these limits, the detection of strontium-90 is probably not real. The reported value did not exceed the MCL of $8 \mathrm{pCi} / \mathrm{L}$.

Both gross alpha and gross beta results at all sampling locations and times were low enough to meet federal and state drinking water standards except for background well UCD2-17, in which the gross alpha activity in the winter quarter was $46.8 \mathrm{pCi} / \mathrm{L}$, exceeding the $\mathrm{MCL}$ of $15 \mathrm{pCi} / \mathrm{L}$. No spatial or temporal trends were observed for any radionuclides reported in groundwater during 1995. All gross beta detections reported were less than the $\mathrm{MCL}(50 \mathrm{pCi} / \mathrm{L}$ ), with a maximum detection of $16.2 \pm 3.4 \mathrm{pCi} / \mathrm{L}$ (well UCD2-17, winter quarter). The anomalously high gross alpha and beta values for background well UCD2-17 in the winter quarter indicate that the analyses were likely in error.

The tritium (Figures 5.7 and 5.8) and carbon-14 (Figures 5.9 and 5.10) detections reported are predominantly localized in wells UCD1-13 and UCD2-14. Low levels lless than $300 \mathrm{pCi} / \mathrm{L}$ ) of carbon-14 were also reported in wells UCD1-12 and UCD1-23. The maximum reported detections occurred in well UCD1-13 for both tritium $119,600 \pm$ $1,200 \mathrm{pCi} / \mathrm{L})$ and carbon-14 (1,900 $\pm 180 \mathrm{pCi} / \mathrm{L})$. The $\mathrm{MCL}$ for tritium is $20,000 \mathrm{pCi} / \mathrm{L}$, and carbon-14 has no MCL. Tritium distributions at LEHR are presented in Figures 5.20, 5.21 and 5.22. These figures show that tritium is located at and slightly downgradient of its source in the disposal pits south of Landfill 2. In addition, tritium concentrations decreased with depth in the aquifer just like the other major contaminants. One isolated 
value from a 1994 hydropunch located in the extreme northeast of Figure 5.20 is believed to be suspect and unrelated to the tritium plume. No parts of the tritium plumes exceeded the MCL of $20,000 \mathrm{pCi} / \mathrm{L}$.

\subsection{Anions and Cations}

This section presents a summary of analytical results for anions and cations in groundwater in both HSU-1 and HSU-2. Analytical results for anion and cation analyses for groundwater are summarized in Table 5.7. Complete analytical results for anions and cations reported in groundwater are presented in Appendix A, Table A.6. Groundwater data for anions and cations are also presented in several figures and graphs that have been used to compare general water quality trends over time, by well or by location. As discussed in the introduction to this section, nitrate has been consistently reported within monitoring wells at the LEHR site at levels above background. Therefore, additional figures and graphs depicting nitrate (as nitrogen) have also been included to illustrate relationships between upgradient and downgradient wells for this parameter.

\subsubsection{Stiff Diagrams}

Stiff diagrams (Figures 5.23, 5.24, and 5.25) were prepared for all and HSU-1 and HSU-2 wells to evaluate and compare the general water types present at the LEHR site. Stiff diagrams were prepared from well analyses averaged over the entire sampling history of the wells. Mean values were used for two reasons: 1) changes in the monitoring program eliminated the required anion data in the second half of the year; and 2) major ion chemistry has remained stable within each well over the lifetime of the monitoring system. The summary data are presented in Tables 5.9 and 5.10 .

In general, most downgradient HSU-1 wells consistently show similar patterns of major cation and anion concentrations compared with samples from background well UCD1-18, but the solute concentrations are higher beneath LEHR than in the background. Apparent differences consistently occur in wells UCD1-10, with greater sodium and sulfate, and UCD1-12, with higher magnesium and chloride concentrations.

Stiff diagrams for HSU-2 wells show that HSU-2 waters are similar in composition to HSU-1 except that solute concentrations are lower. Stiff diagrams exhibit less variability for HSU-2 wells than for HSU-1 wells. HSU-2 wells consistently show similar patterns of major cation and anion concentrations compared with upgradient well UCD2-17.

In general, groundwater samples from both HSU-1 and HSU-2 are predominantly of the magnesium-bicarbonate type, which is typical for this area. Groundwater samples from HSU-1 wells generally exhibit higher concentrations of magnesium and bicarbonate than HSU-2 wells. The magnesium bicarbonate water type in the vicinity of the LEHR site has been documented in several studies. A report by the USGS (Evenson 1985) identified magnesium bicarbonate groundwater in the area and suggested that recharge of Putah Creek surface water, which is a magnesium bicarbonate type, influences water quality 
adjacent to Putah Creek and south of Davis. Work by Davisson (Davisson and Criss 1993) also identifies magnesium bicarbonate groundwater in the Davis vicinity and provides data based on naturally occurring stable isotopes of oxygen, which further suggests the influence of Putah Creek recharge on groundwater quality in an area adjacent to Putah Creek and south of Davis that includes the LEHR site (Davisson et al. 1993).

\subsubsection{Nitrate}

Reported nitrate concentrations show a great deal of variability across the site. Bar graphs showing nitrate concentrations in each well in 1995 are presented in Figures 5.3 and 5.4, and nitrate distributions are presented in Figures 5.14, 5.15, and 5.16. Reported concentrations in HSU-1 samples from several wells are consistently above those reported for background well UCD1-18. The highest nitrate concentrations are consistently reported in HSU-1 wells UCD1-10, UCD1-11, UCD1-12, UCD1-21, and UCD1-24, and HSU-2 well UCD2-7. Reported nitrate concentrations in several of the onsite monitoring wells and in background well UCD1-18 exceed the MCL for nitrate (as nitrogen) of $10 \mathrm{mg} / \mathrm{L}$ during one or more quarters during 1995. Nitrate concentrations ranged from $0.16 \mathrm{mg} / \mathrm{L}$ in new well UCD1-34 to $72 \mathrm{mg} / \mathrm{L}$ in UCD1-12.

Reported nitrate concentrations in HSU-2 were consistently higher in downgradient wells UCD2-7 and UCD2-14 than in upgradient well UCD2-17 throughout 1995. Although nitrate concentrations appear to be elevated in several HSU-2 wells with respect to the upgradient well, nitrate was consistently detected at concentrations above the MCL of $10 \mathrm{mg} / \mathrm{L}$ in only one, UCD2-7. Nitrate concentrations greater than the $\mathrm{MCL}$ were reported in every quarter for UCD2-7 and during the summer quarter for UCD2-14. Higher nitrate concentrations in UCD2-14 may be due to the fact that the well has been completed partially in HSU-1, where nitrate concentrations are higher. Nitrate concentrations reported in each HSU-2 well have been consistent through time.

The nitrate distribution beneath LEHR is presented in Figures 5.14, 5.15, and 5.16. Two major observations can be made from those figures:

1. Much of the groundwater within HSU-1 beneath LEHR and surrounding property contains nitrate at concentrations above the MCL of $10 \mathrm{mg} / \mathrm{L}$. The upper portion of $\mathrm{HSU}-2$ also contains nitrate in excess of the MCL.

2. The nitrate plume located just north of Landfill 2 appears to follow a pattern similar to the chloroform plume. The highest concentrations are centered near the eastern boundary of LEHR and decrease with depth in the aquifer. The plume has migrated downgradient to the east. 
Based on the distribution of nitrate, it appears that a nitrate source exists at LEHR in an area just northeast of Landfill 2 in the same area as the source of chloroform. The plume migrating eastward from this area has merged with the regionally high nitrate levels that are above the MCL. The very low concentrations along the southern boundary of LEHR are the result of recharge from Putah Creek. 


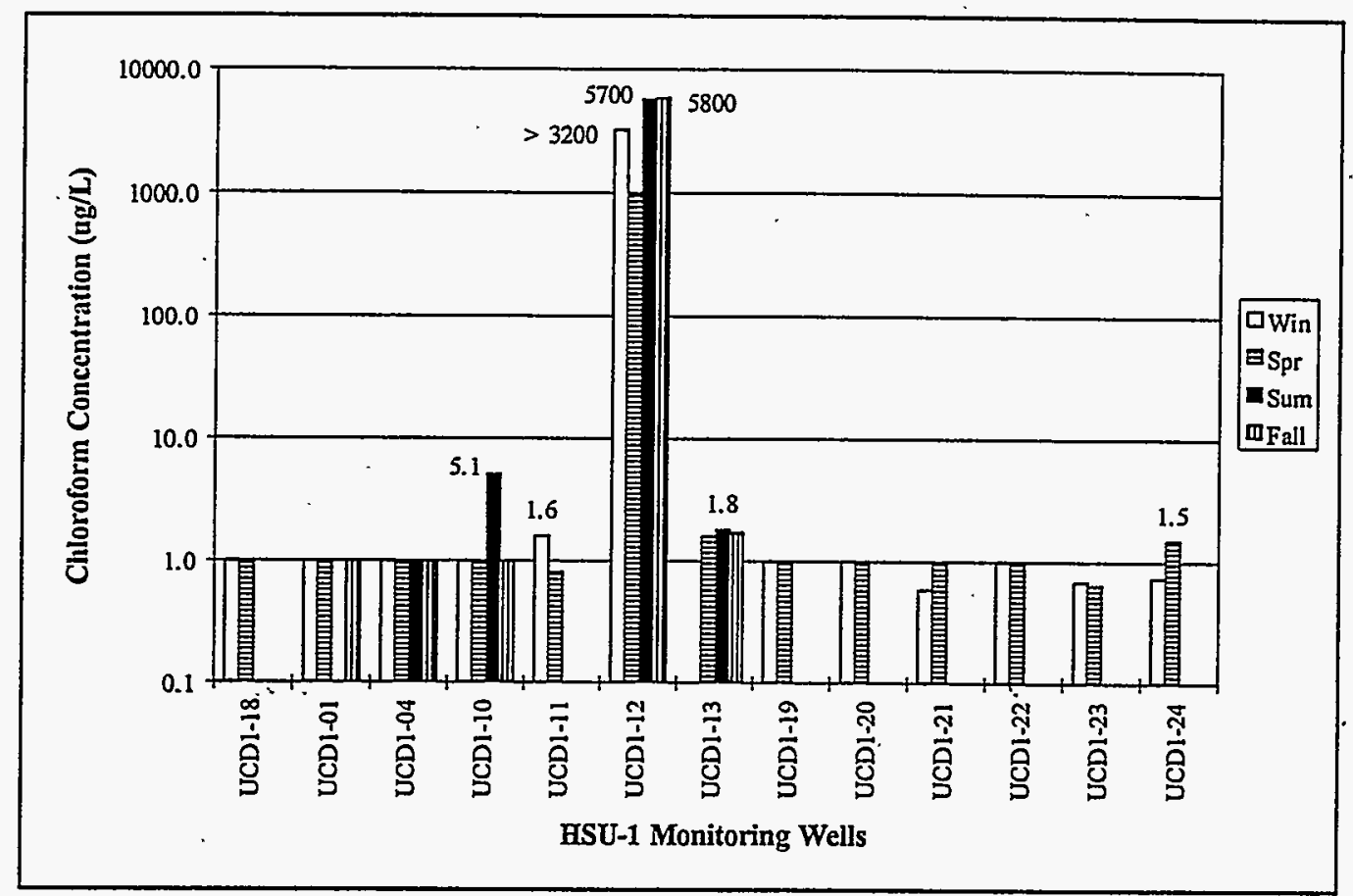

Figure 5.1 Chloroform Concentrations Detected in HSU-1 Monitoring Wells, 1995

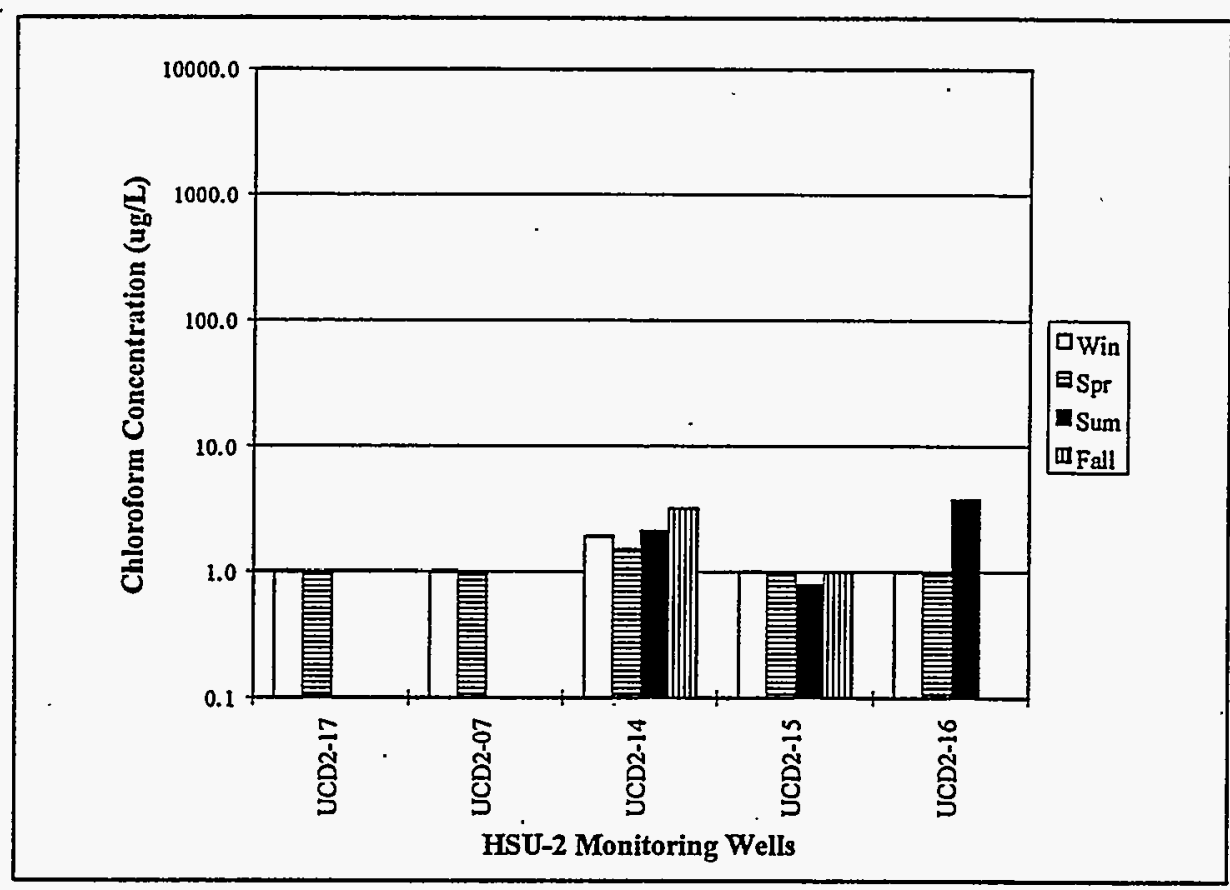

Figure 5.2. Chloroform Concentrations Detected in HSU-2 Monitoring Wells, 1995 


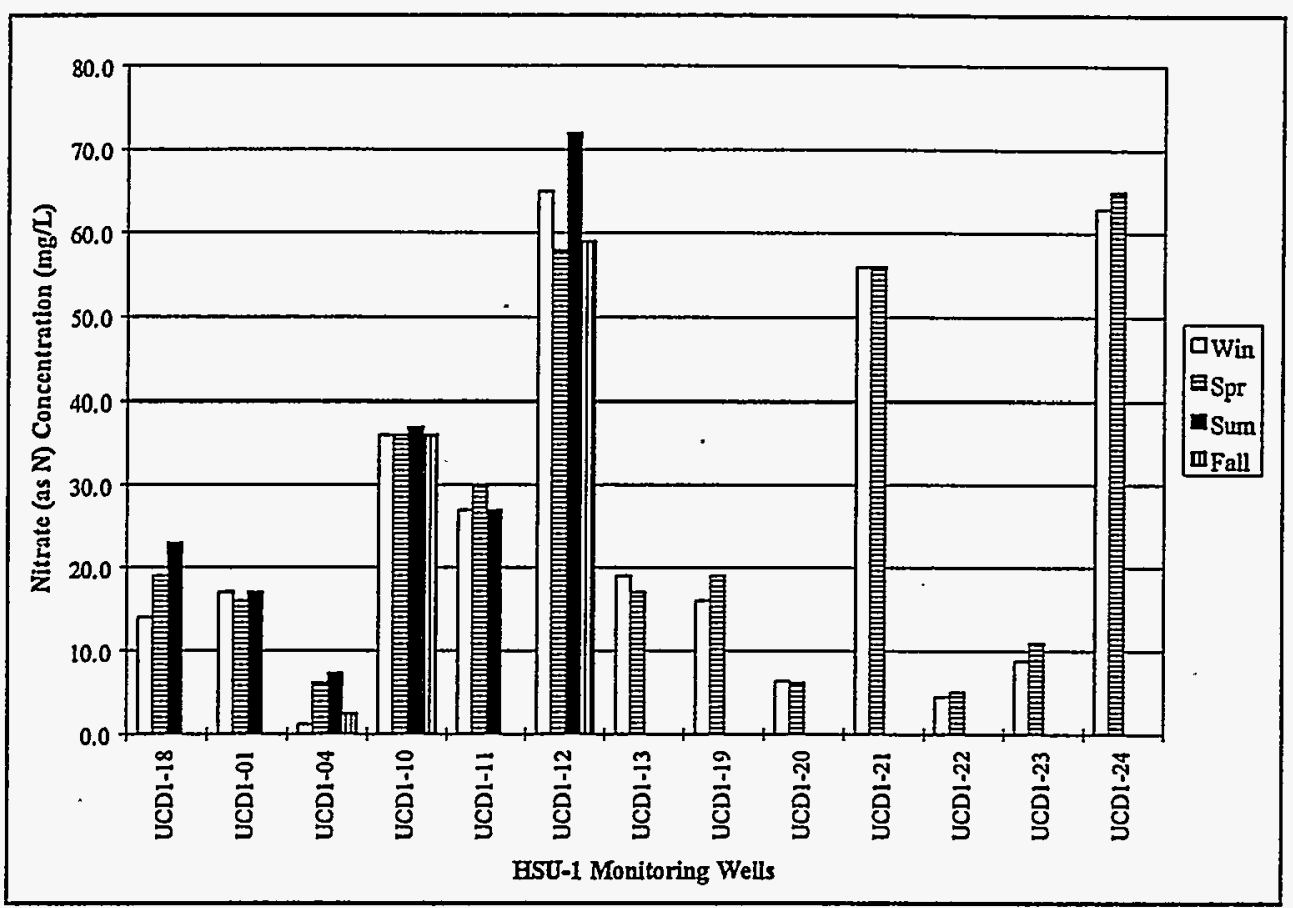

Figure 5.3. Nitrate Concentrations Detected HSU-1 Monitoring Wells, 1995

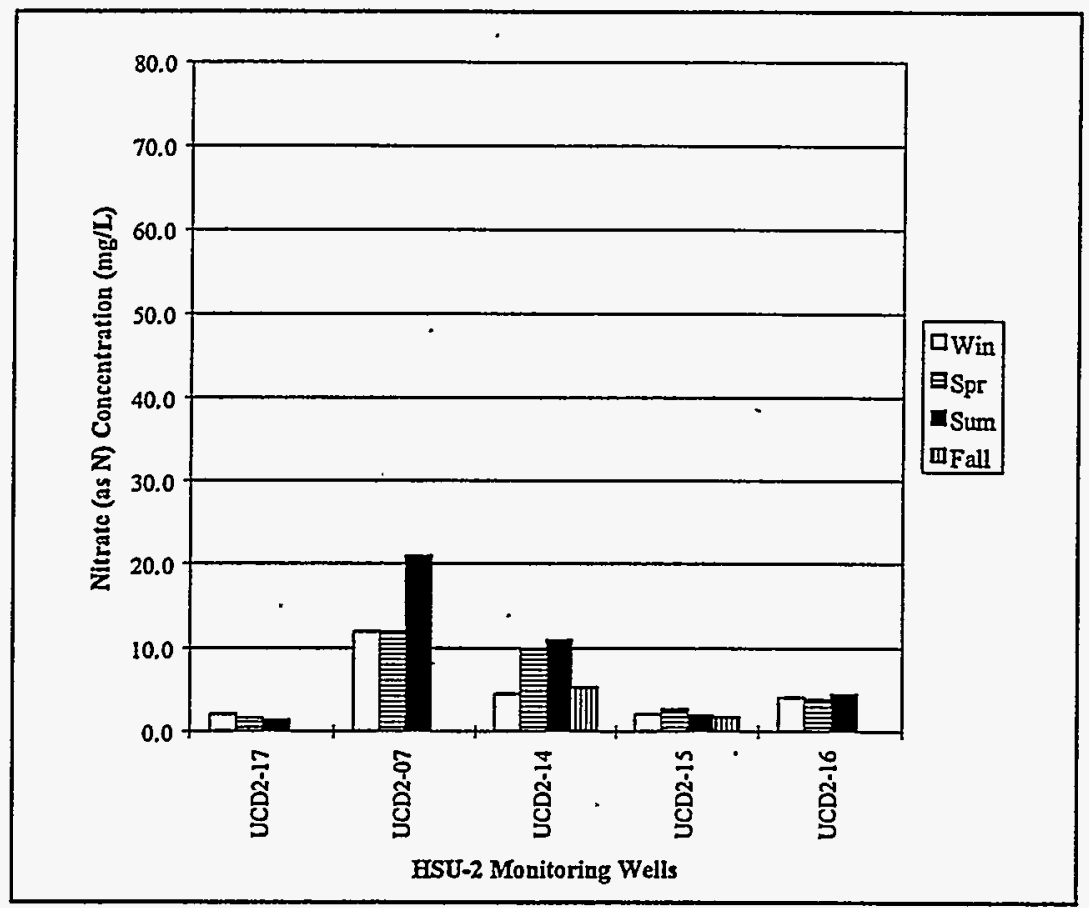

Figure 5.4. Nitrate Concentrations Detected in HSU-2 Monitoring Wells, 1995 


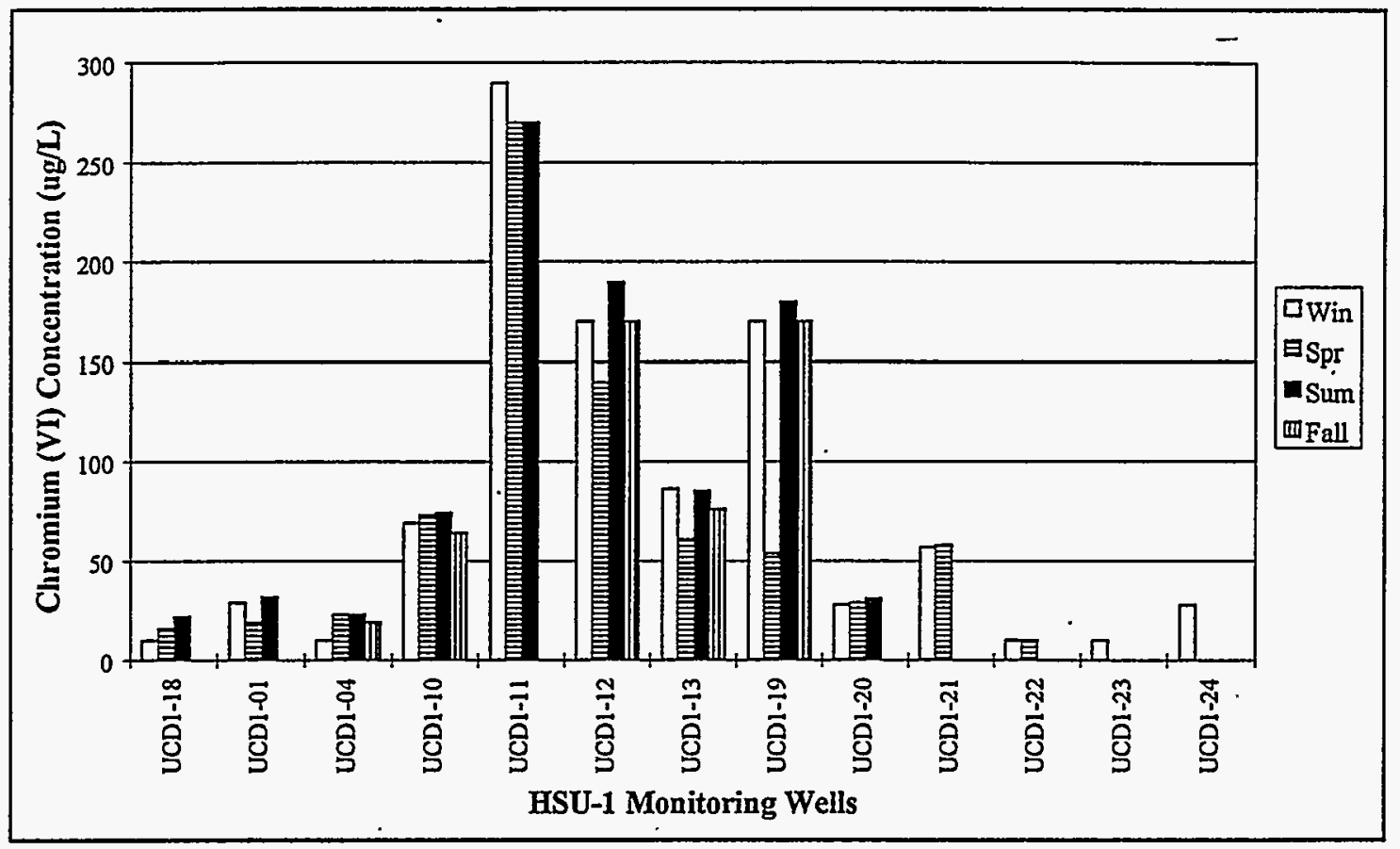

Figure 5.5. Chromium Concentrations Detected in HSU-1 Monitoring Wells, 1995

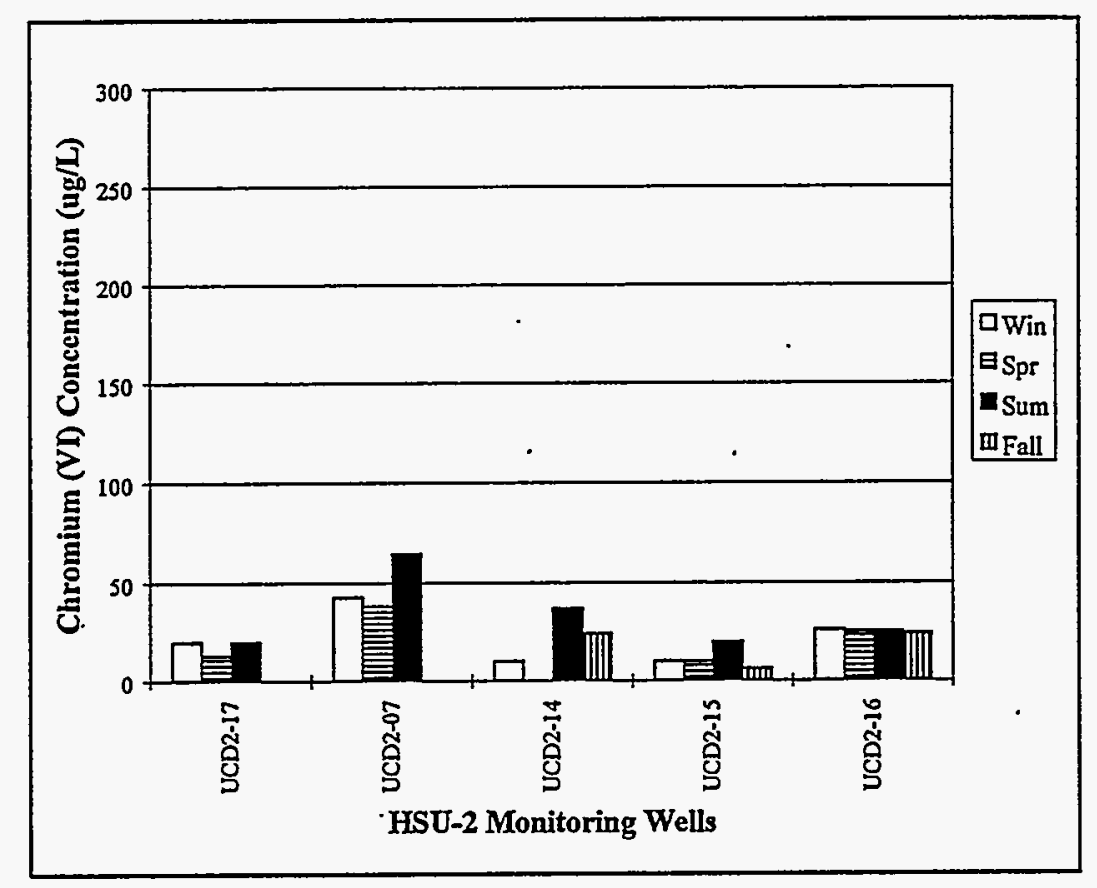

Figure 5.6. Chromium Concentrations Detected in HSU-2 Monitoring Wells, 1995 


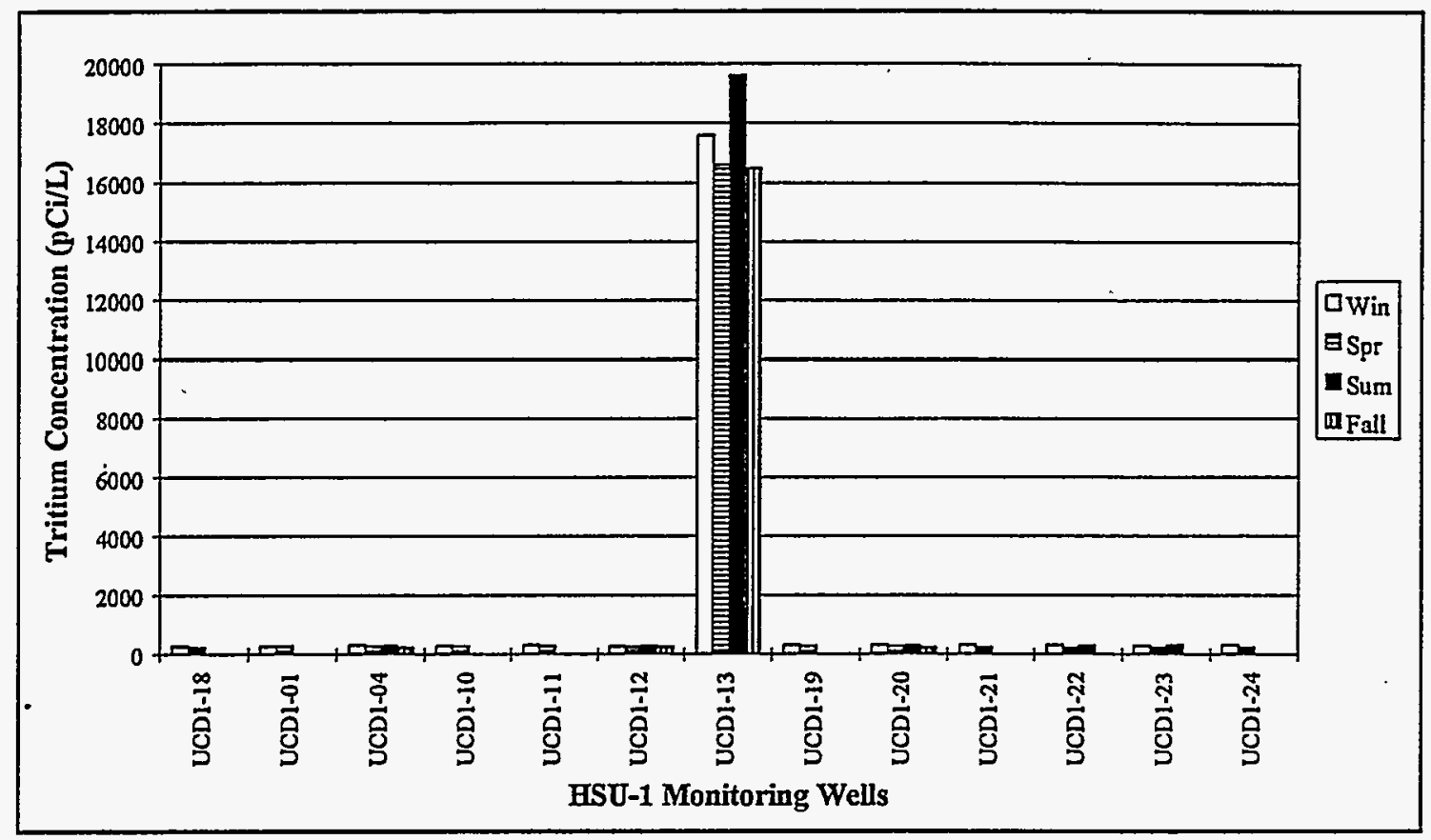

Figure 5.7. Tritium Concentrations Detected in HȘU-1 Monitoring Wells, 1995 .

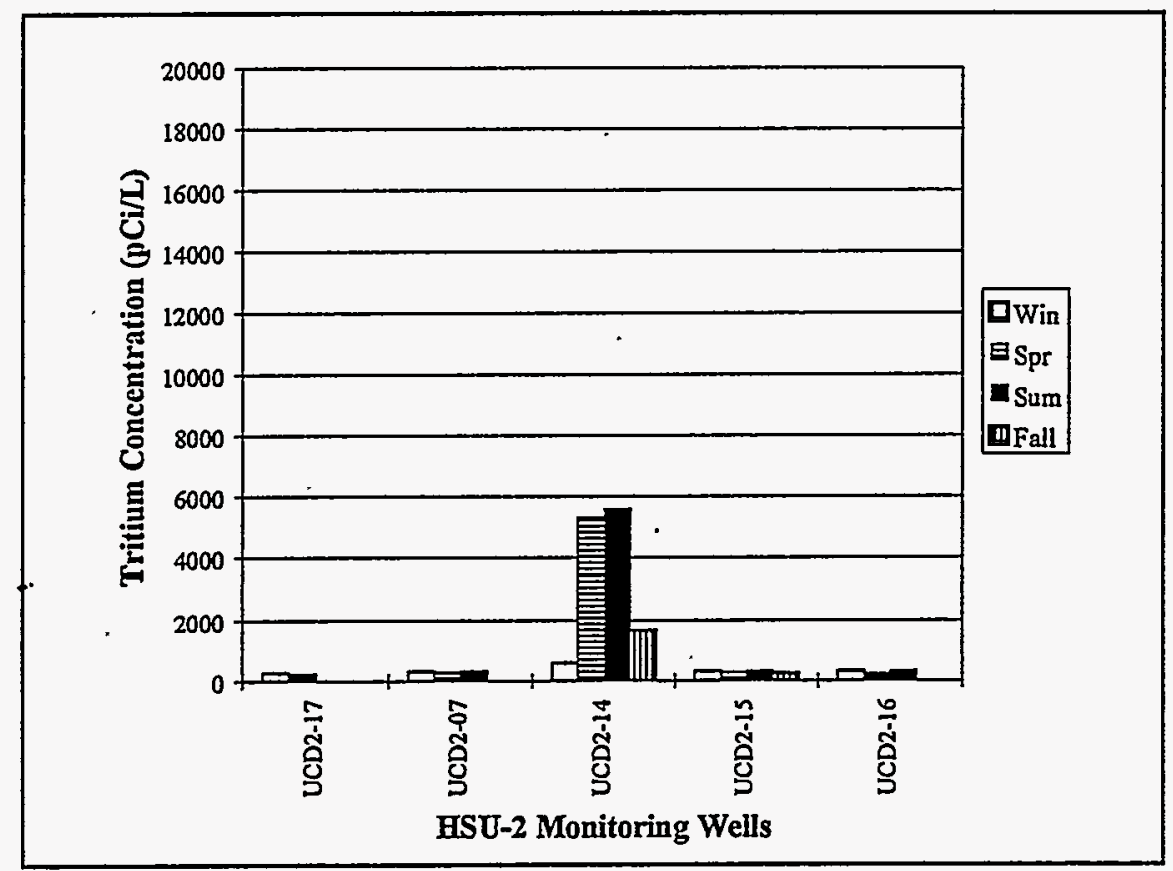

Figure 5.8. Tritium Concentrations Detected in HSU-2 Monitoring Wells, 1995 


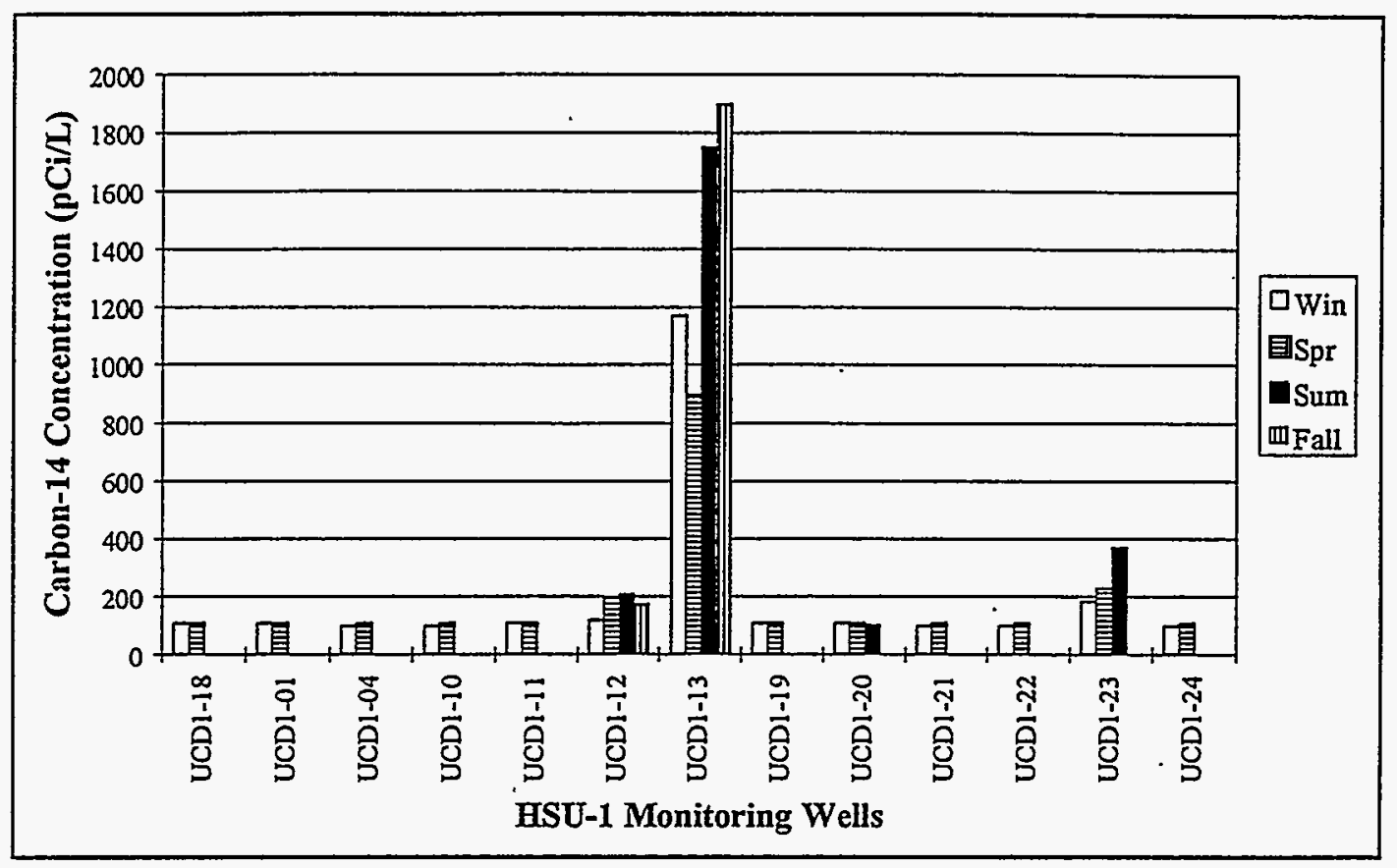

Figure 5.9. Carbon-14 Concentrations. Detected in HSU-1 Monitoring Wells, 1995

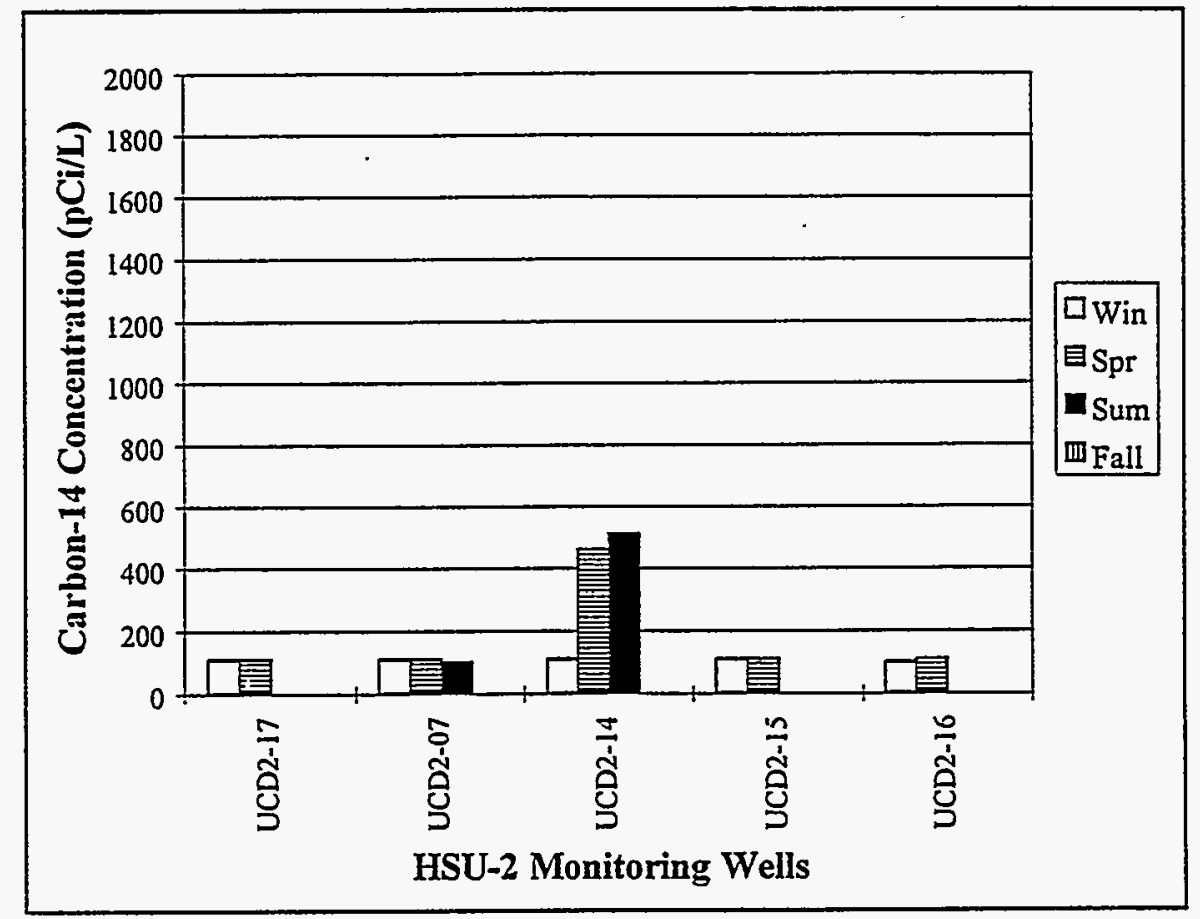

Figure 5.10. Carbon-14 Concentrations Detected in HSU-2 Monitoring Wells, 1995 


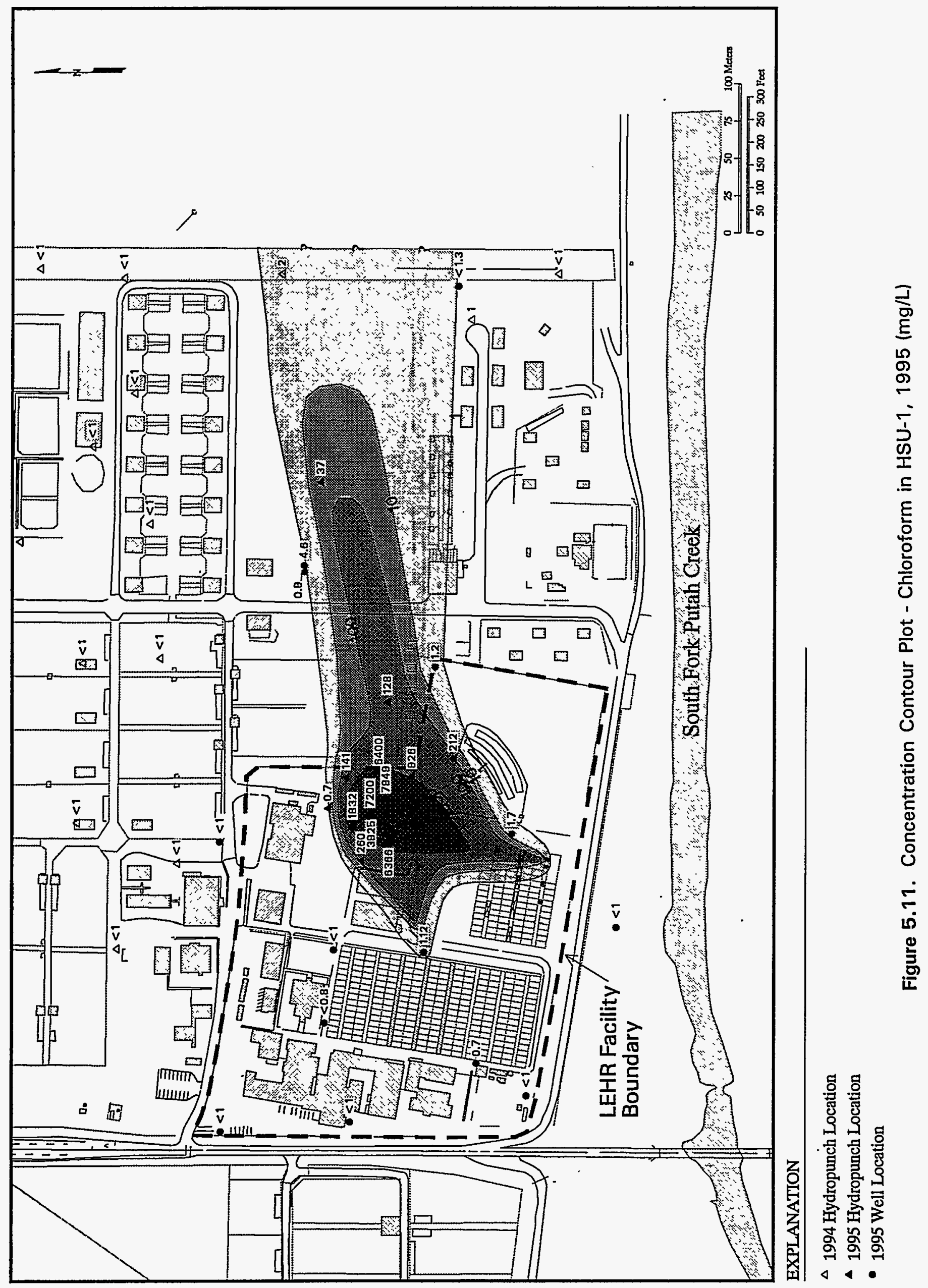




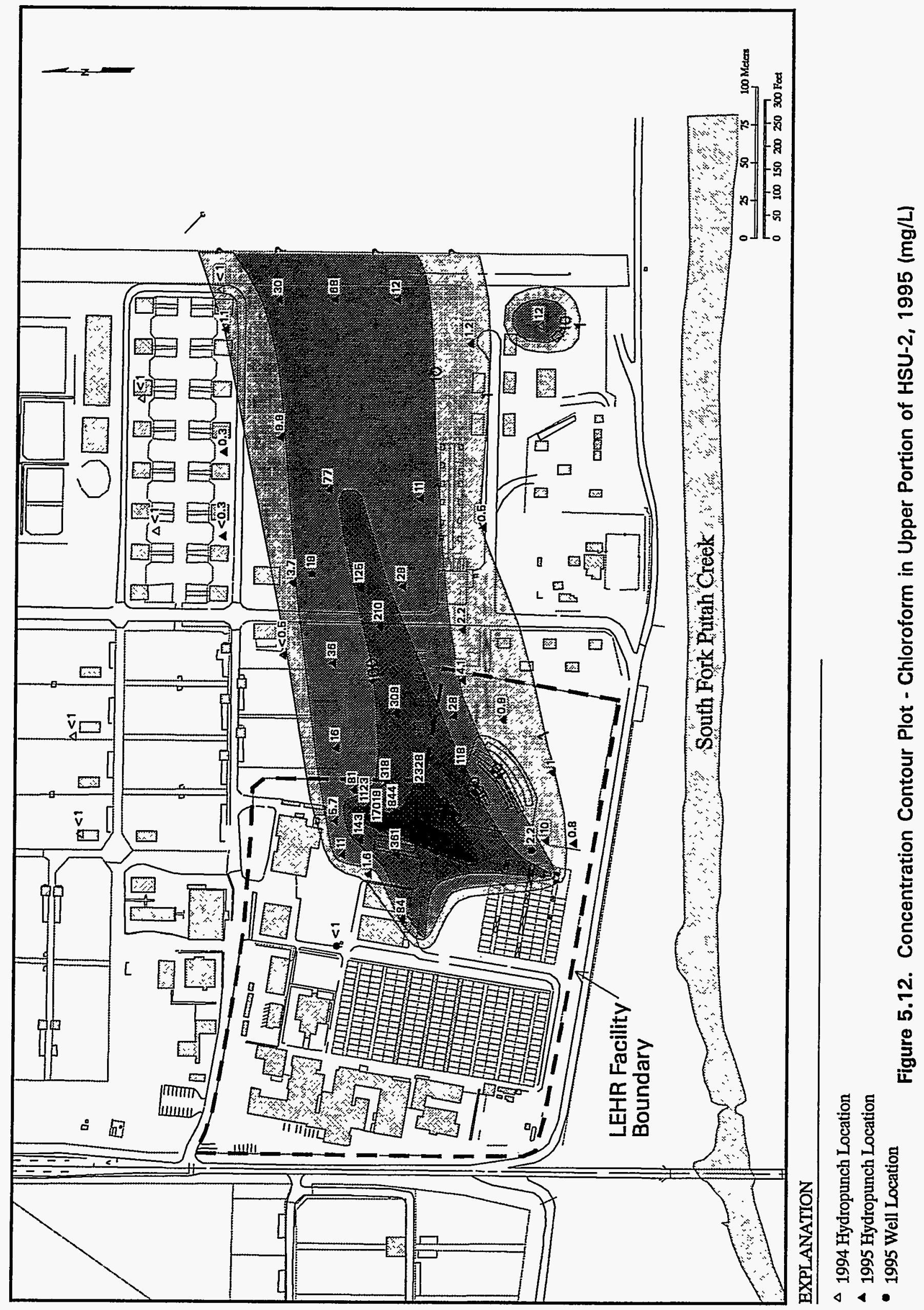




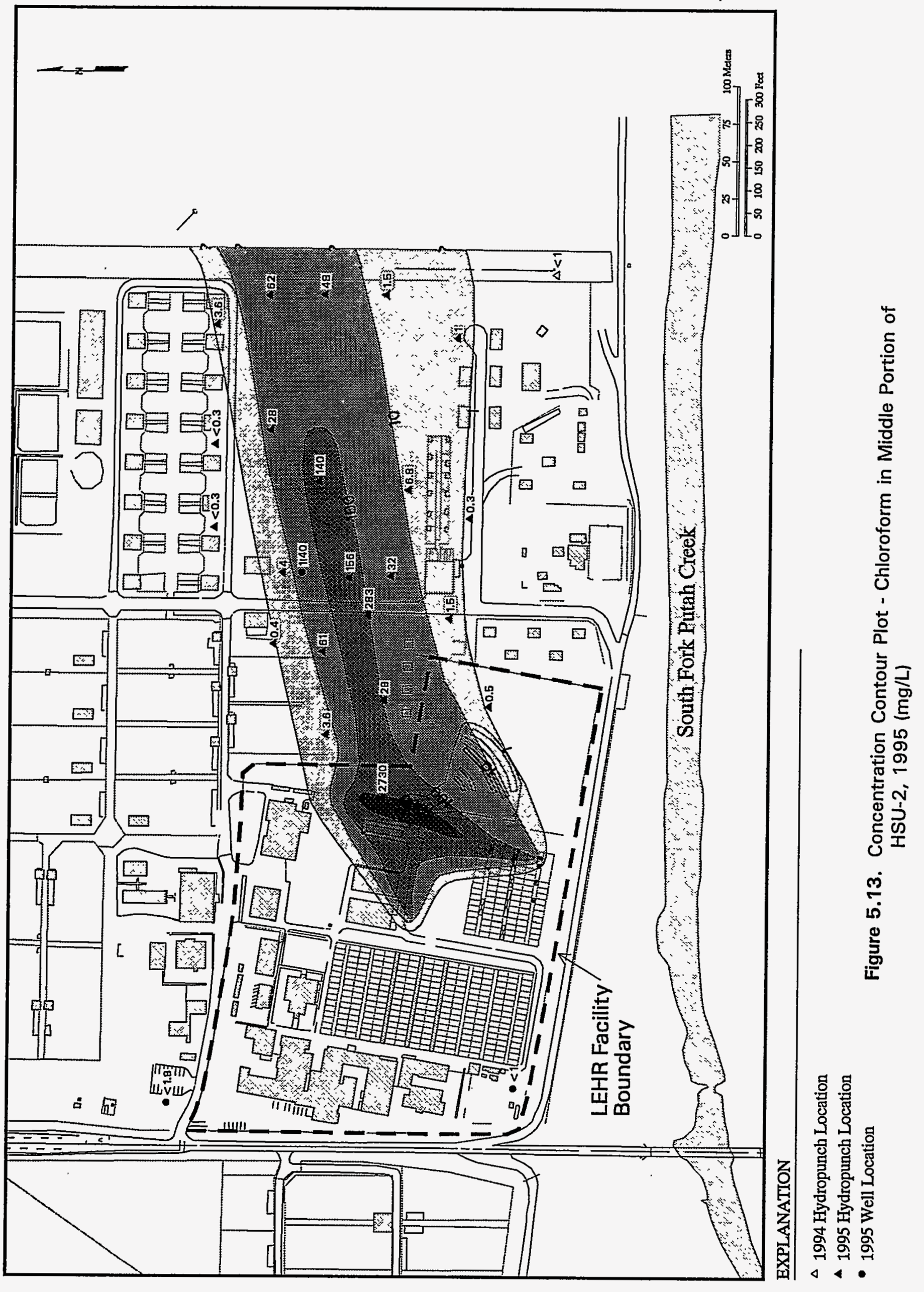




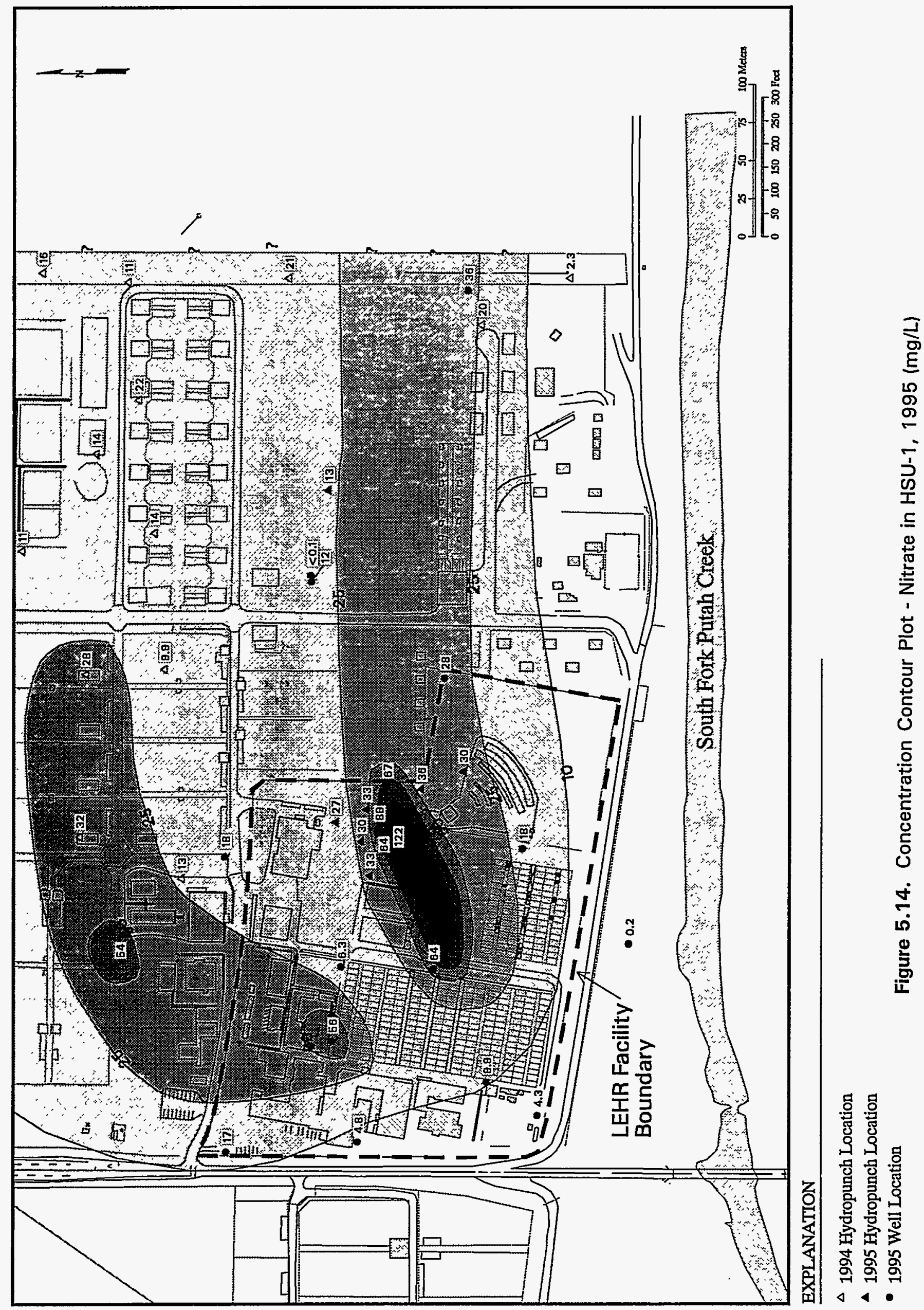




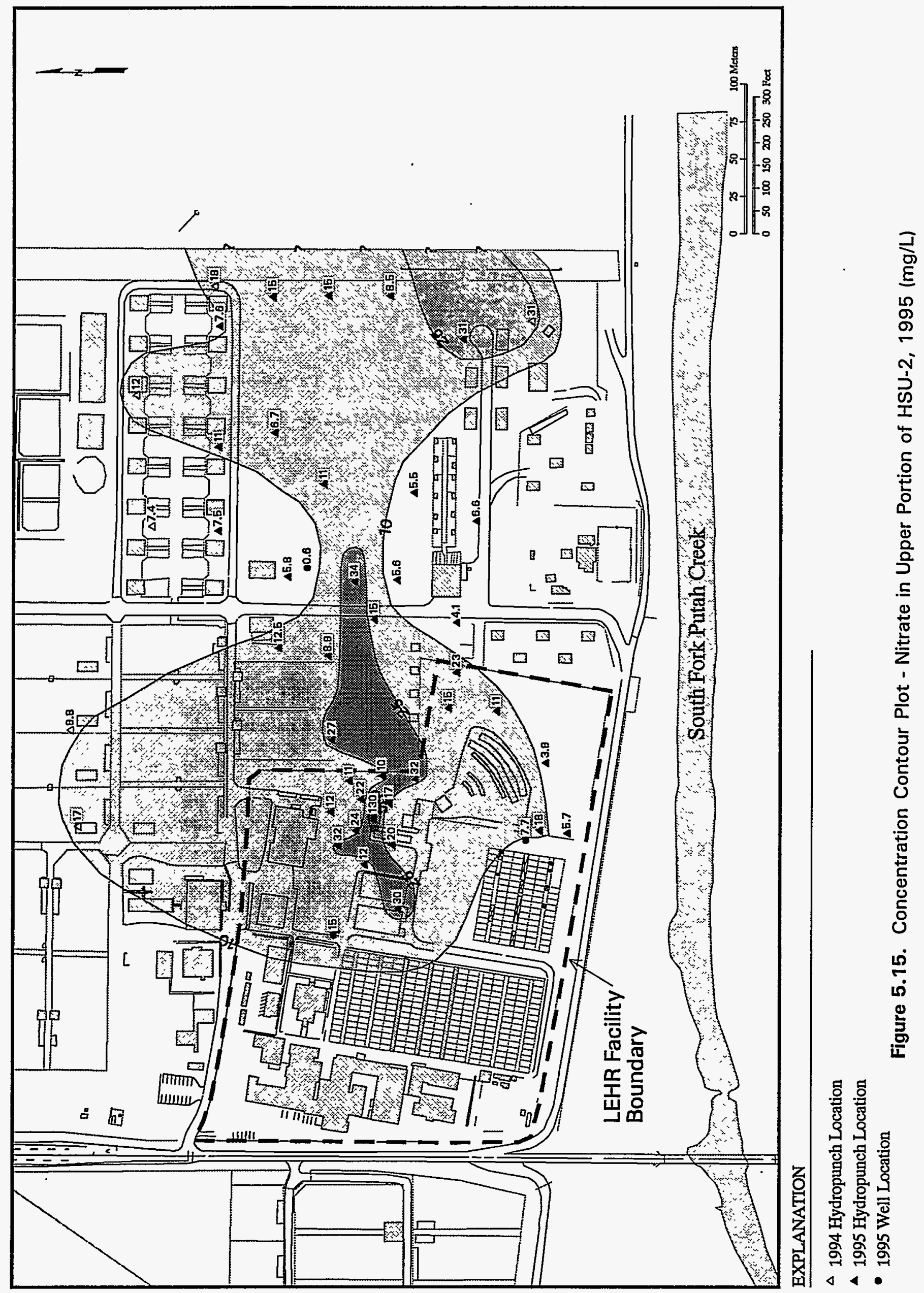




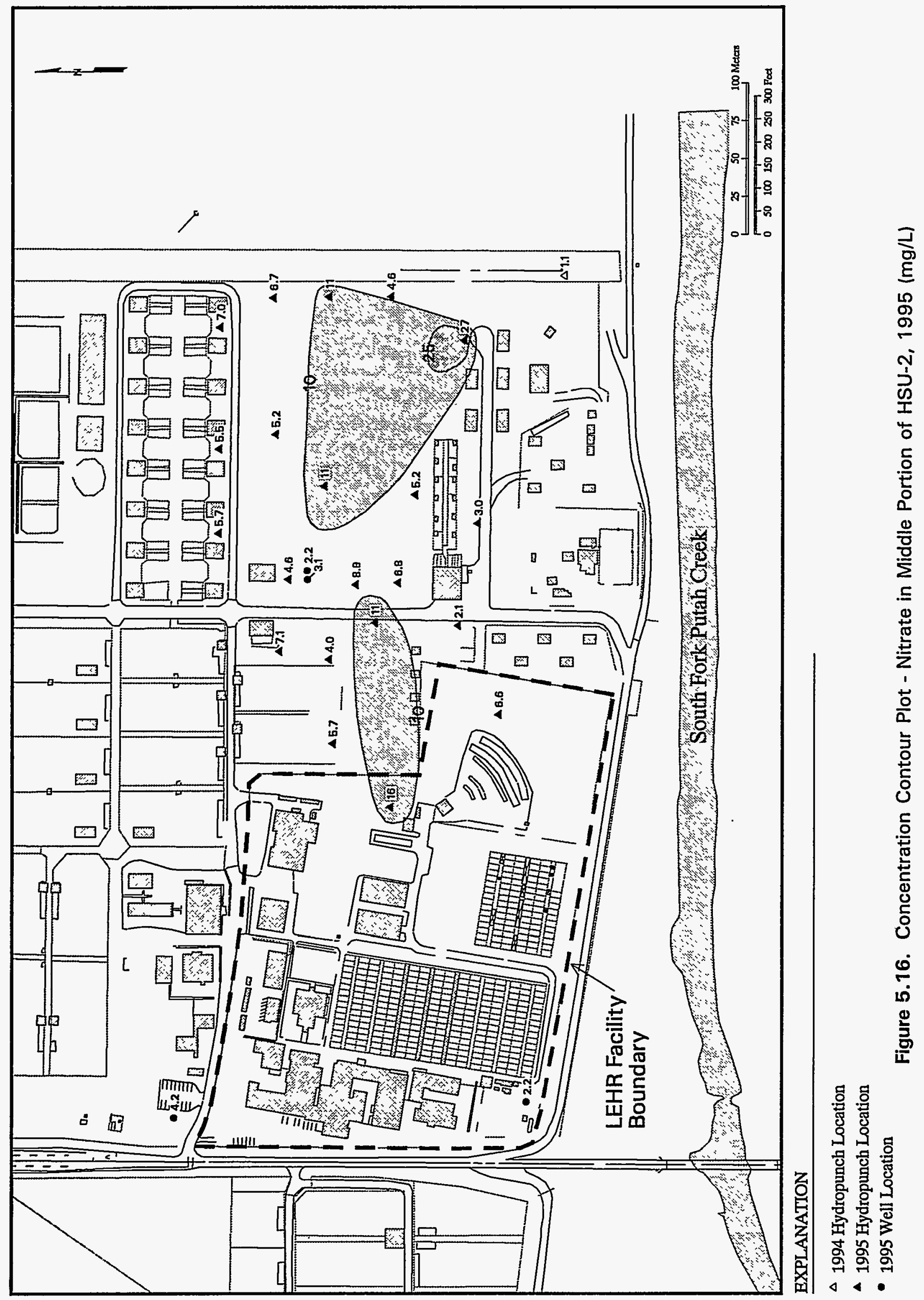




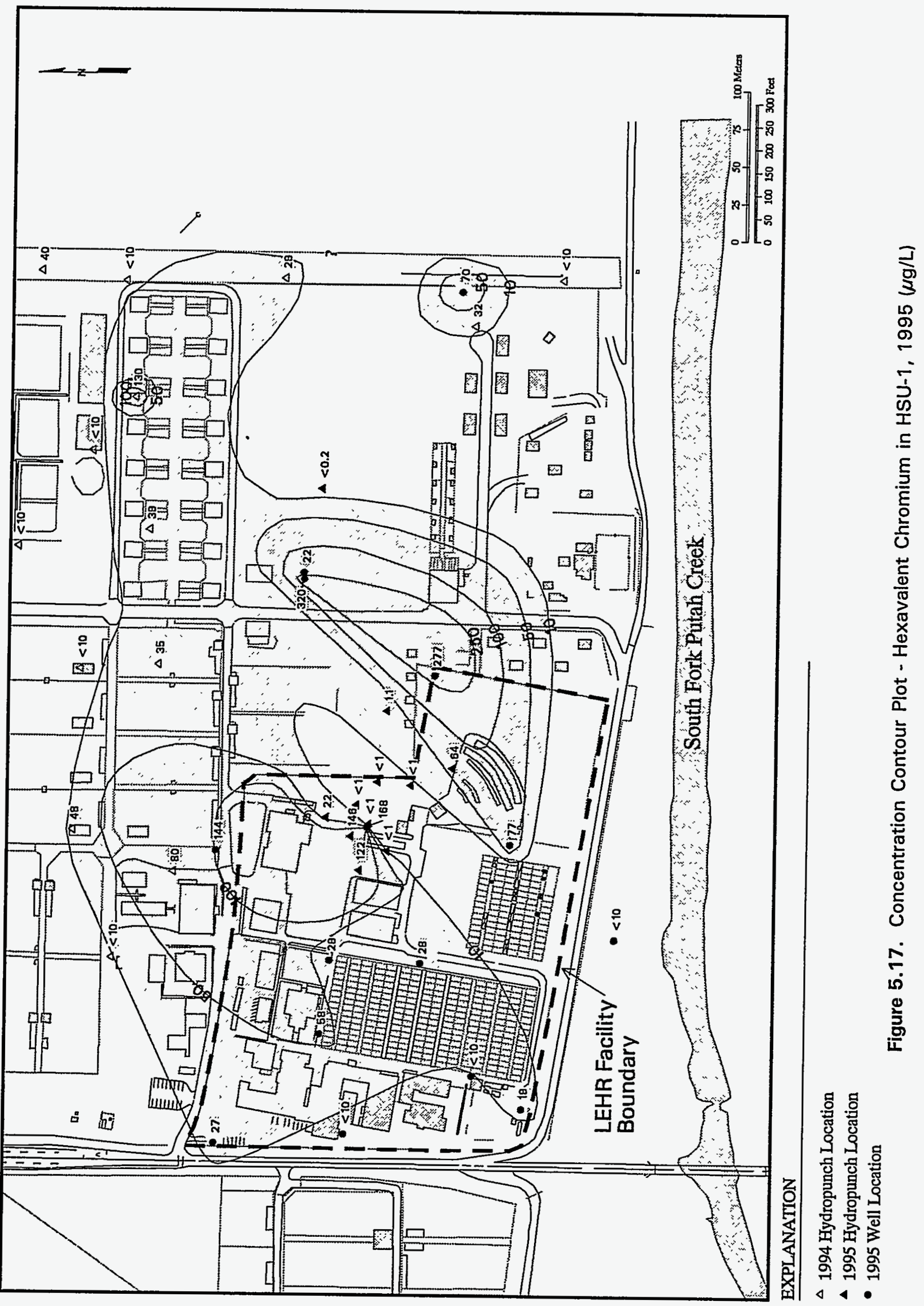




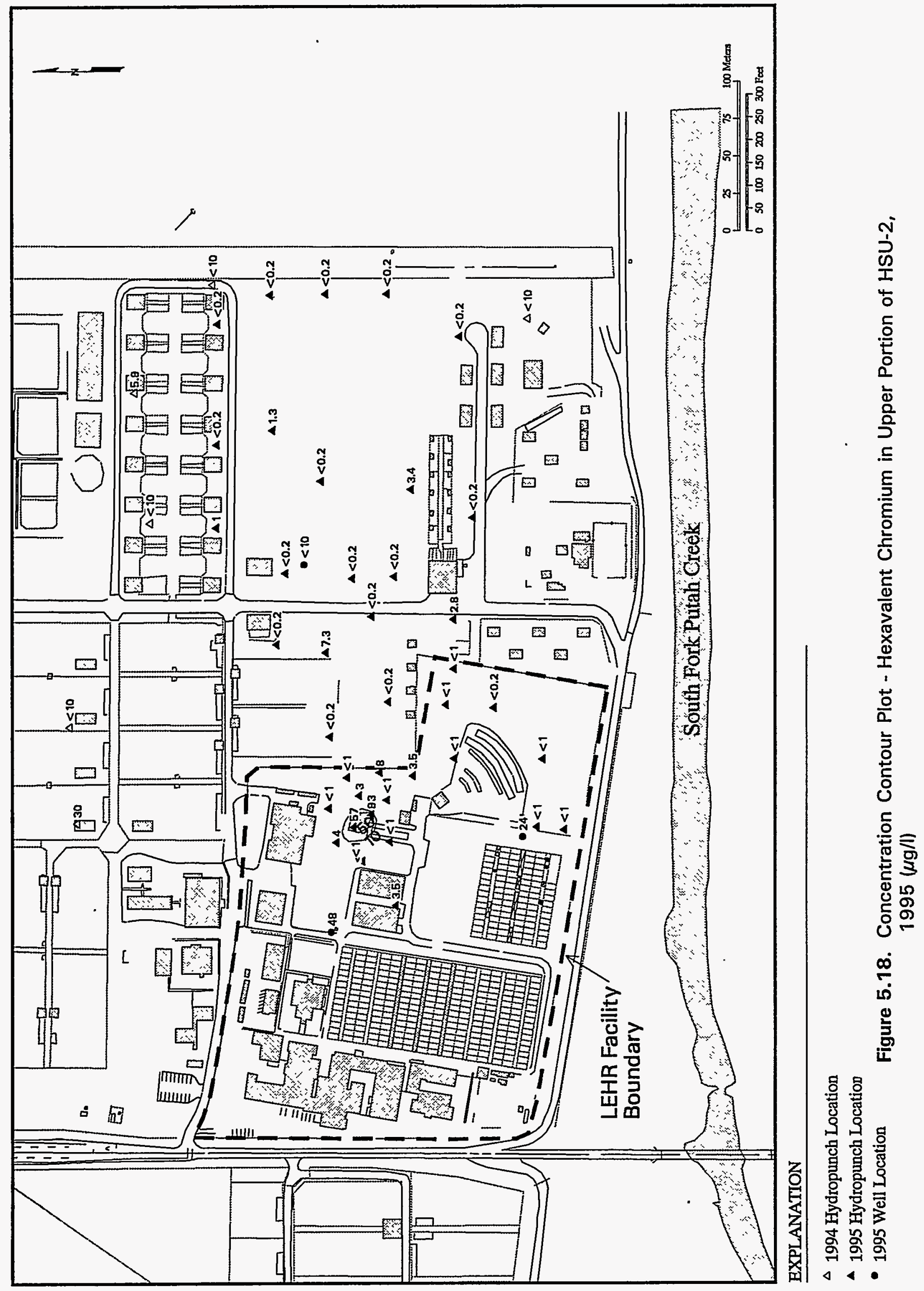




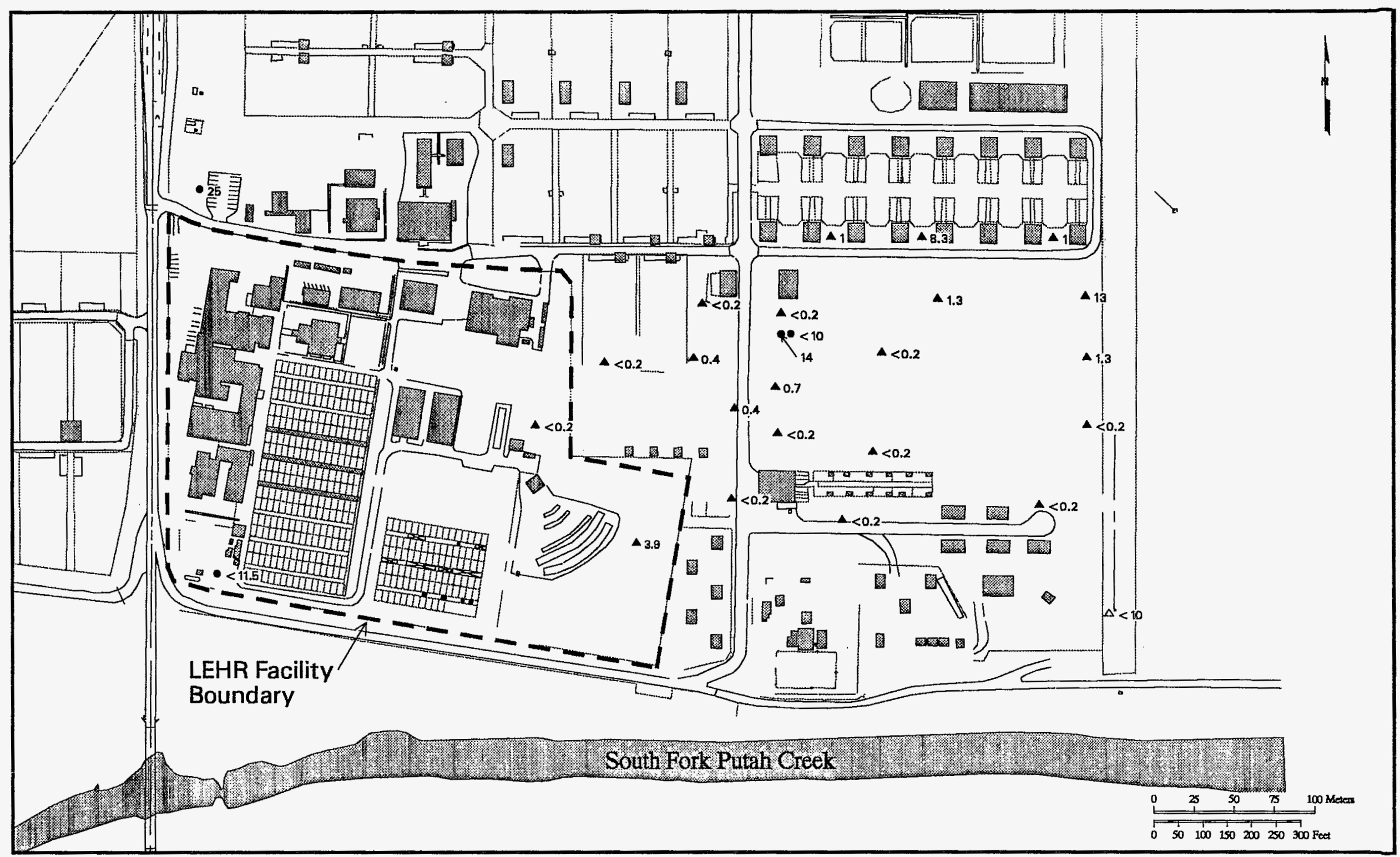

EXPLANATION

$\triangle 1994$ Hydropunch Location

- 1995 Hydropunch Location

- 1995 Well Location

Figure 5.19. Concentration Contour Plot - Hexavalent Chromium in Middle Portion of HSU-2, $1995(\mu \mathrm{g} / \mathrm{L})$ 


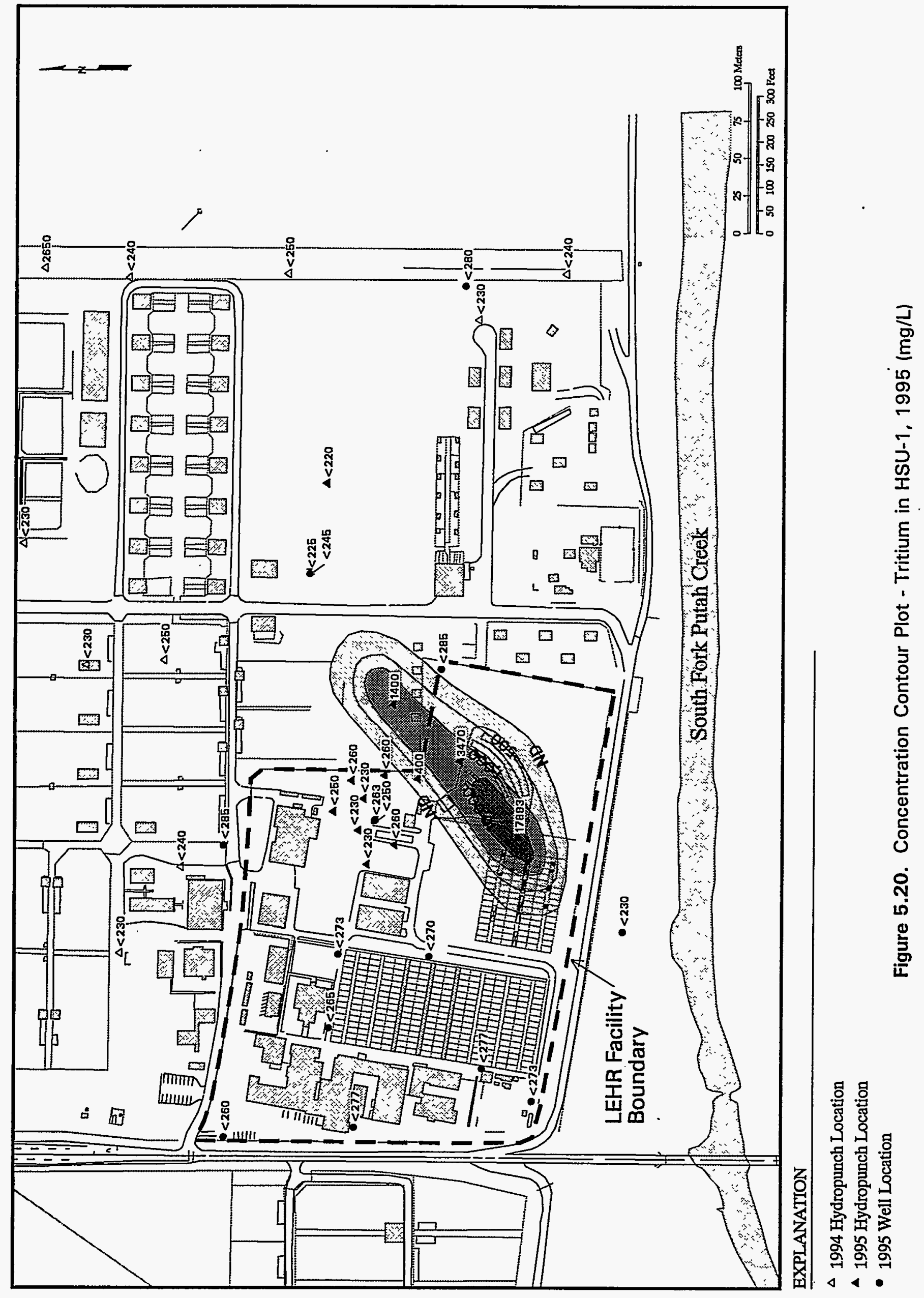


NOILFNVTdX'

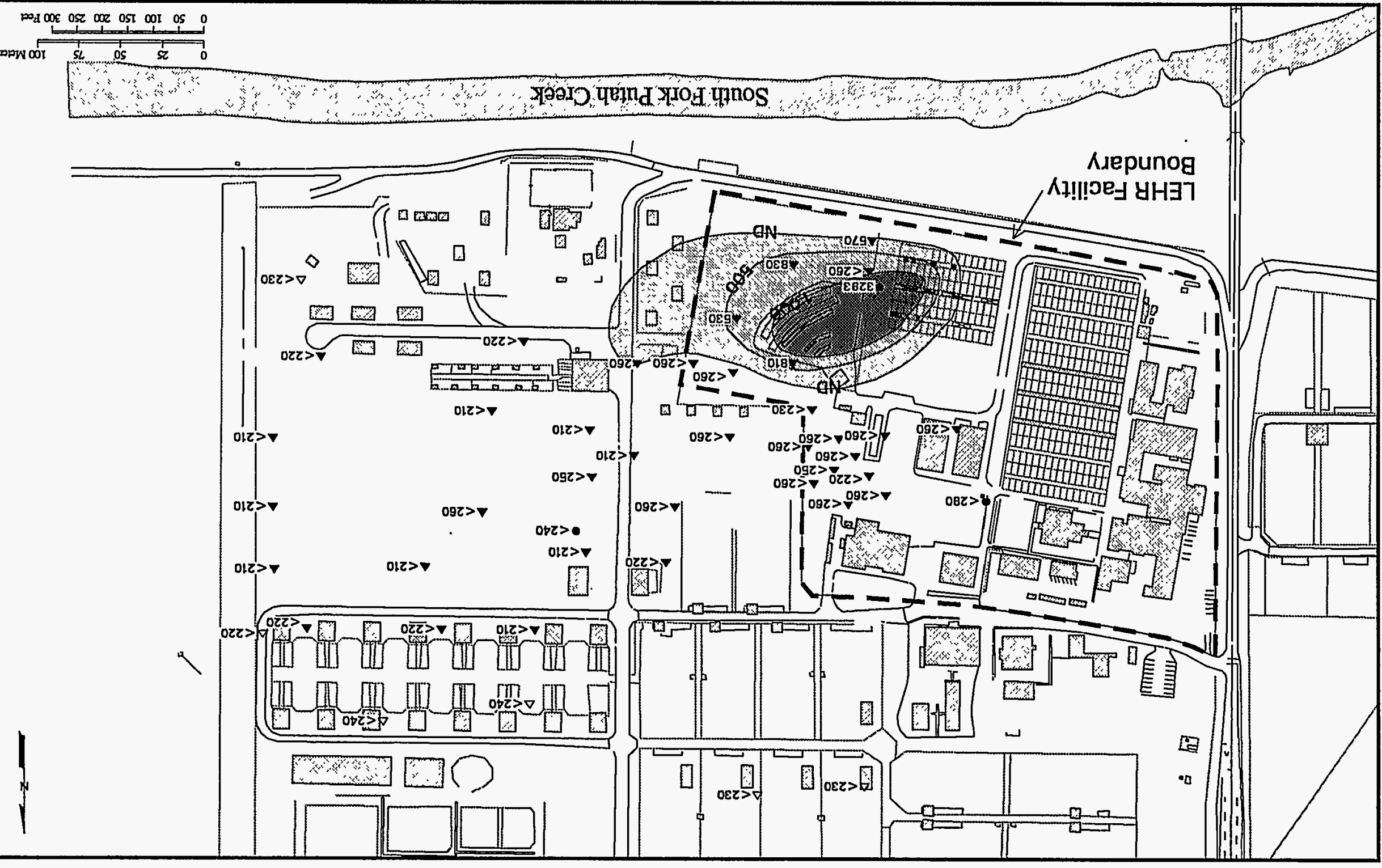




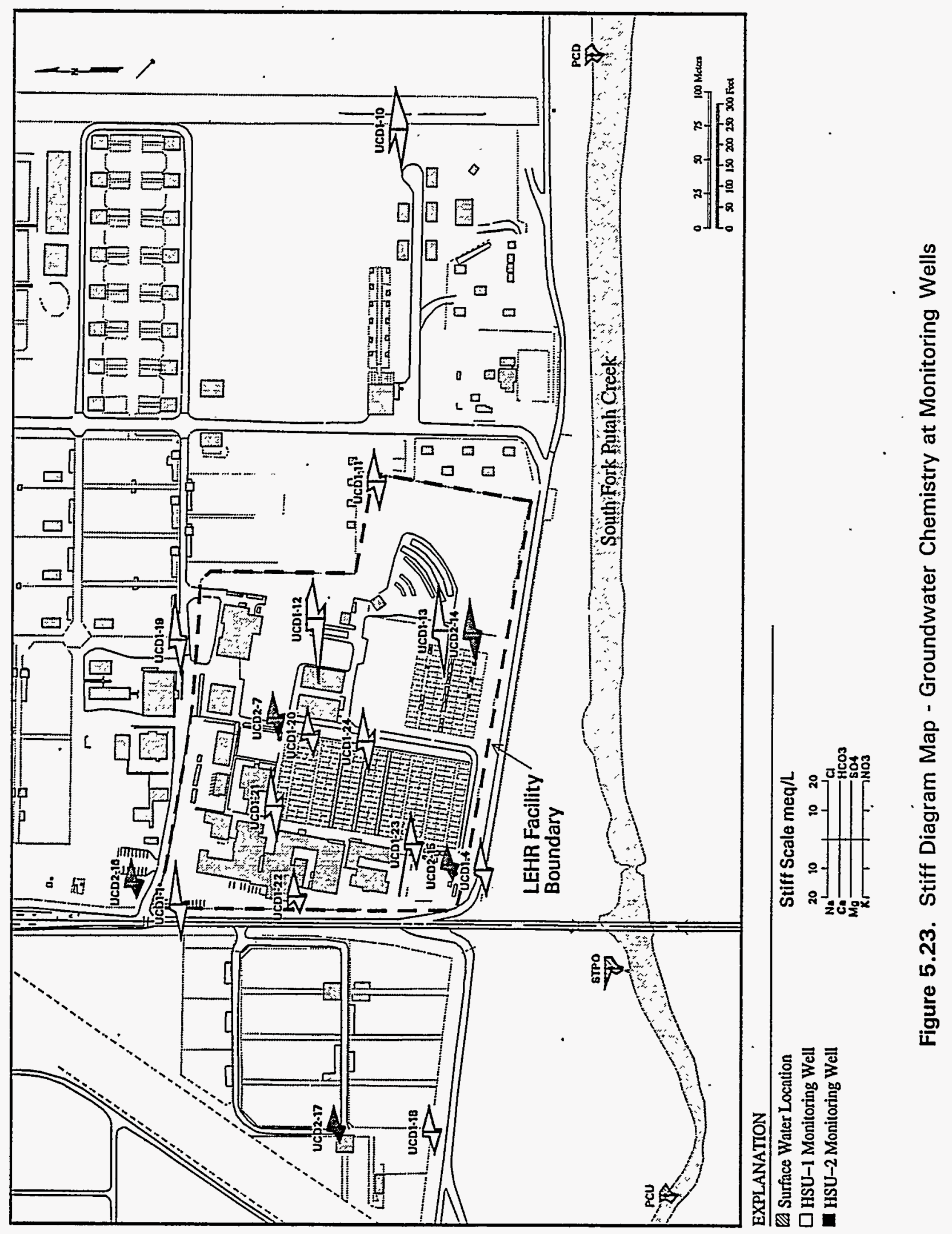




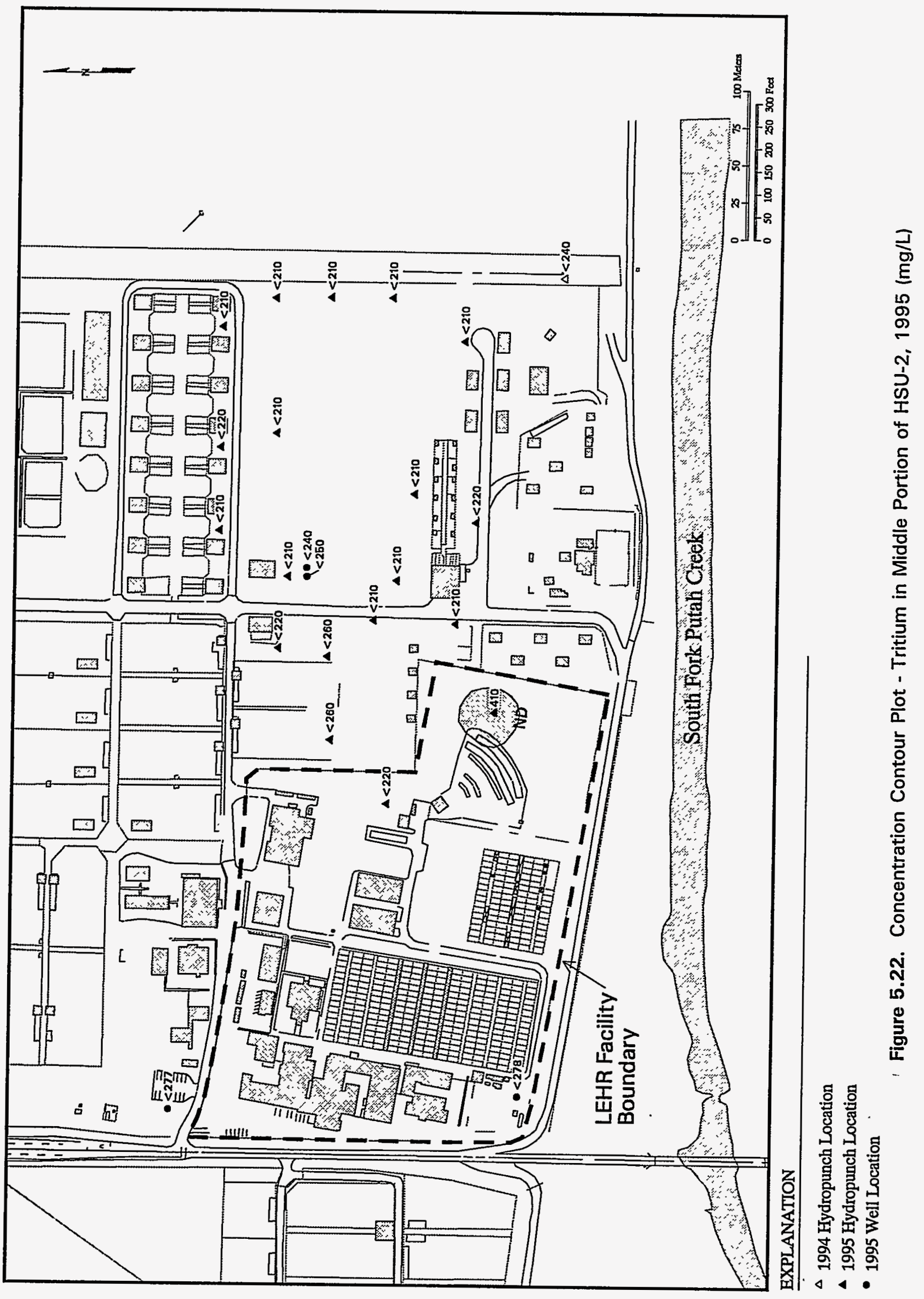




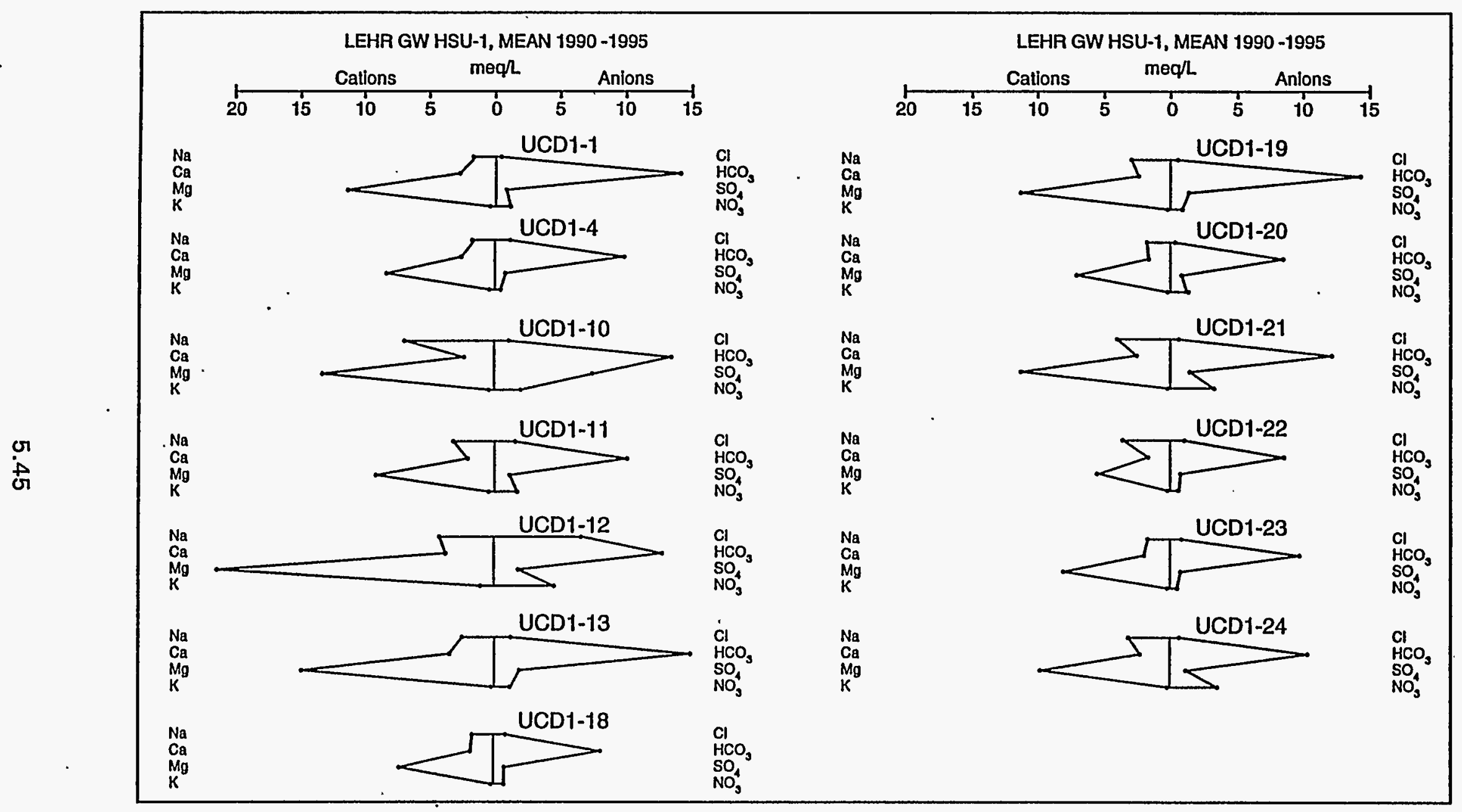

Figure 5.24. Stiff Diagrams - HSU-1 Wells, Mean 1990-1995 


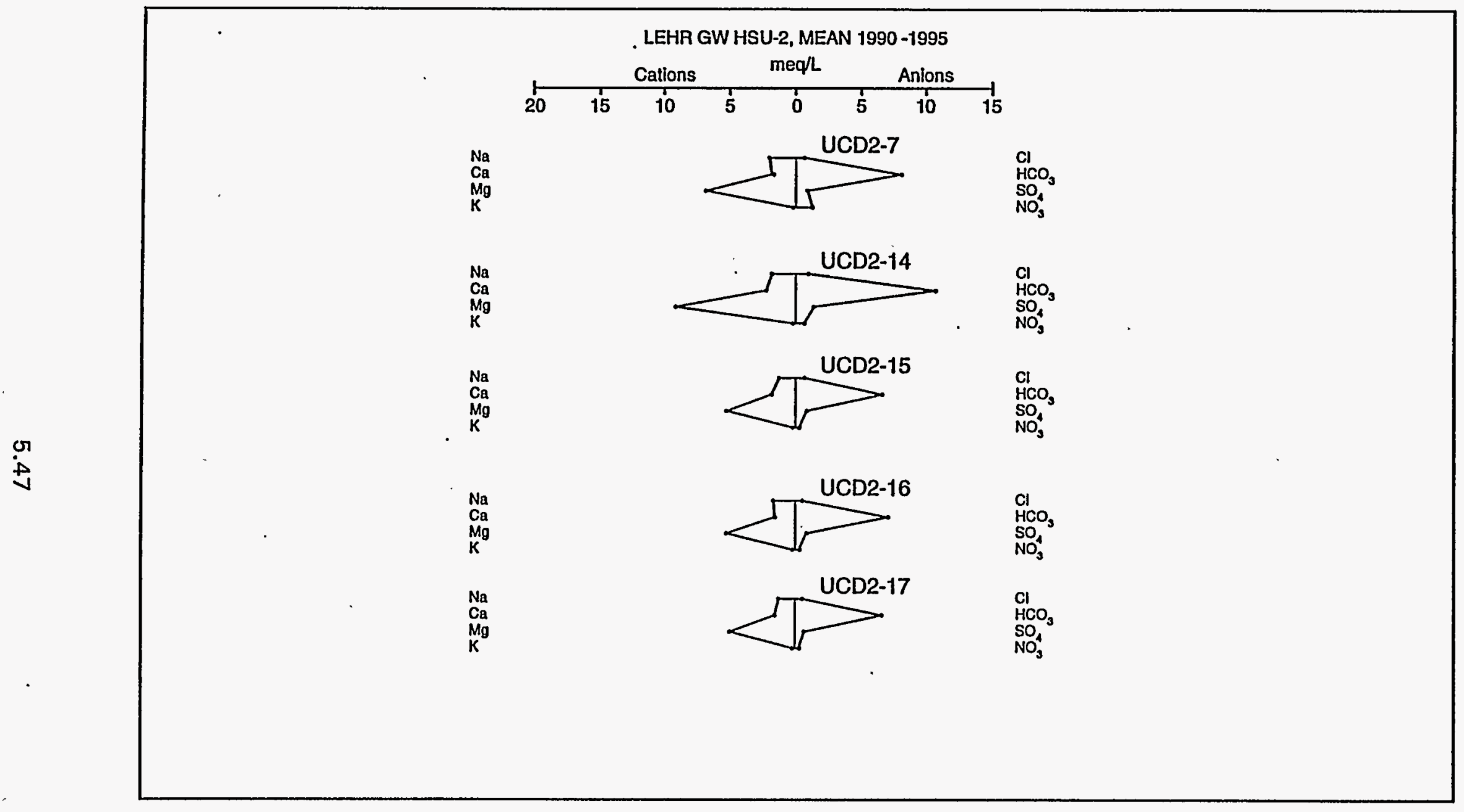

Figure 5.25. Stiff Diagrams - HSU-2 Wells, Mean 1990-1995 
TABLE 5.1

NUMBER OF DETECTIONS, POSITIVE RESULTS AND MAXIMUM VALUES IN GROUNDWATER, 1995 1995 ANNUAL WATER MONITORING REPORT

LEHR ENVIRONMENTAL RESTORATION, DAVIS, CALIFORNIA

\begin{tabular}{|c|c|c|c|c|c|c|c|c|c|c|c|c|c|c|}
\hline & & \multicolumn{6}{|c|}{ UPGRADIENT WELLS } & \multicolumn{6}{|c|}{ DOWNGRADIENT WELLS } \\
\hline & PARAMETER & MCL & $\begin{array}{c}\text { Number of } \\
\text { Results }\end{array}$ & $\begin{array}{l}\text { Number of } \\
\text { Detections }\end{array}$ & $\begin{array}{l}\text { Number } \\
>\text { MCL }\end{array}$ & $\begin{array}{l}\text { Maximu } \\
\text { m Value }\end{array}$ & $\begin{array}{c}\text { Date of } \\
\text { Maximum }\end{array}$ & $\begin{array}{c}\text { Location of } \\
\text { Maximum }\end{array}$ & $\begin{array}{c}\text { Number of } \\
\text { Results }\end{array}$ & $\begin{array}{l}\text { Number of } \\
\text { Detections }\end{array}$ & $\begin{array}{l}\text { Number } \\
>\mathrm{MCL}\end{array}$ & $\begin{array}{c}\text { Maximum } \\
\text { Value }\end{array}$ & $\begin{array}{c}\text { Date of } \\
\text { Maximum }\end{array}$ & $\begin{array}{l}\text { Location of } \\
\text { Maximum }\end{array}$ \\
\hline Cations \& Anions & Ammonia-Nitrogen & - & 5 & 0 & 0 & - & - & - & 35 & 4 & 0 & 4.4 & 23-Feb-95 & UCD1-13 \\
\hline $\mathrm{mg} / \mathrm{L}$ & Calcium & - & 5 & 5 & $\mathbf{0}$ & 46.1 & 23-May-95 & UCD1-18 & 51 & 51 & 0 & 75.4 & 17-May-95 & UCD1-13 \\
\hline & Chloride & $250 \mathrm{~S}$ & 5 & 5 & 0 & 44 & 22-Feb-95 & UCD1-18 & 35 & 35 & 0 & 220 & 23-Feb-95 & UCD1-12 \\
\hline & Magnesium & & 5 & 5 & $\mathbf{0}$ & 112 & 23-May-95 & UCD1-18 & 51 & 51 & 0 & 227 & 23-Feb-95 & UCD1-12 \\
\hline & Nitrogen, Nitrate (as N) & $10 \#$ & 7 & 7 & 4 & 23 & 30-Aug-95 & UCD1-18 & 61 & 58 & 31 & 72 & 28-Aug-95 & UCD1-12 \\
\hline & Potassium & & 5 & 1 & 0 & 0.942 & 23-Мау-95 & UCD1-18 & 51 & 35 & $\mathbf{0}$ & 2.25 & 05-Dec-95 & UCD1-27Z1 \\
\hline & Sodium & - & 5 & 5 & o & 42.3 & 23-May-95 & UCD1-18 & 51 & 51 & 0 & 191 & 05-Dec-95 & UCD1-27Z1 \\
\hline & Sulfate & $250 \mathrm{~S}$ & 5 & 5 & $\mathbf{0}$ & 39 & 22-Feb-95 & UCD1-18 & 35 & 35 & 2 & 290 & $16-F \mathrm{cb}-95$ & UCD1-10. \\
\hline & Alkalinity Total(as $\mathrm{CaCO})(\mathrm{mg} / \mathrm{L})$ & - & 5 & 5 & 0 & 460 & 23-May-95 & UCD1-18 & 35 & 35 & o & 870 & 18-May-95 & UCD1-19 \\
\hline General Chemical & Total Dissolved Solids (mgíl) & $500 \mathrm{~S}$ & 5 & 5 & 3 & 660 & 23-May-95 & UCD1-18 & 63 & 63 & 43 & 1700 & 28-Aug-95 & UCD1-12 \\
\hline Parameters & Total Organic Carbon (mg/L) & - & 5 & 2 & o & 1 & 22-Feb-95 & UCD1-18 & 35 & 18 & $\mathbf{0}$ & 2.8 & 23-Feb-95 & UCD1-12 \\
\hline & Turbidity (NTU) & - & 5 & 3 & o & 1.7 & 22-Feb-95 & UCD1-18 & 35 & 23 & 0 & 7.9 & 16-Feb-95 & UCD1-4 \\
\hline Metals & Antimony & 6 & 5 & 1 & o & 1.8 & 23-May-95 & UCD2-17 & 51 & 6 & 0 & 2.3 & 07-Dec-95 & UCD2-27Z4 \\
\hline lug/L & Arsenic & 50 & 5 & 4 & 0 & 4.9 & 22-Feb-95 & UCD1-18 & 51 & 30 & $\mathbf{0}$ & 8.6 & 05-Dec-95 & UCD1-27Z1 \\
\hline & Barium & 1000 & 5 & 5 & $\mathbf{0}$ & 188 & 23-May-95 & UCD1-18 & 51 & 50 & $\mathbf{0}$ & 355 & 17-May-95 & UCD1-12 \\
\hline & Chromium & 50 & 5 & 5 & 0 & 21.3 & 23-May-95 & UCD1-18 & 51 & 50 & 14 & 290 & 29-Nov-95 & UCD1-25 \\
\hline & Chromium, Iexavalent (+6) & 0.05 & 7 & 3 & 3 & 22 & 30-Aug-95 & UCD1-18 & 65 & 49 & 49 & 310 & 29-Nov-95 & UCD1-25 \\
\hline & Cobalt & - & 5 & 2 & 0 & 1.5 & 22-Fcb-95 & UCD1-18 & 51 . & 12 & 0 & 6.9 & 30-Aug-95 & UCD2-14 \\
\hline & Copper & $1000 \mathrm{~S}$ & 5 & 2 & o & 1.5 & 22-Feb-95 & UCD1-18 & 51 & 12 & $\mathbf{0}$ & 10.2 & 04-Dec-95 & UCD2-26 \\
\hline & Iron & $300 \mathrm{~s}$ & 5 & 2 & 0 & 17.3 & 23-May-95 & UCD2-17 & 51 & 31 & 7 & 3970 & 05-Dec-95 & UCD1-27Z1 \\
\hline & Mercury & & 5 & 0 & 0 & - & - & - & 51 & 5 & $\mathbf{0}$ & 0.68 & 16-Feb-95 & UCD2-7 \\
\hline & Molybdenum & - & 5 & $\mathbf{0}$ & 0 & - & - & - & 51 & 27 & $\mathbf{0}$ & 5.5 & 05-Dec-95 & UCD1-27Z1 \\
\hline & Nickel & 100 & 5 & 4 & o & 66 & 22-Feb-95 & UCD1-18 & 51 & 38 & 0 & 39 & 15-reb-95 & UCD1-23 \\
\hline & Selenium & 5 & 5 & $\mathbf{0}$ & 0 & - & - & - & 51 & 19 & 10 & 23 & 16- reb-95 & UCD1-10 \\
\hline & Vanadium & - & 5 & 5 & 0 & 10.6 & 22-Feb-95 & UCD1-18 & 51 & 44 & 0 & 13.4 & 29-Nov-95 & UCD2-14 \\
\hline & Zinc & $5000 \mathrm{~S}$ & 5 & 5 & o. & 4.9 & 23-May-95 & UCD2-17 & 51 & 38 & $\mathbf{0}$ & 559 & 05-Dec-95 & UCD1-27Z1 \\
\hline Pesticides \& PCB's & Alpha-Chlordane & - & 5 & 0 & 0 & - & - & - & 58 & 3 & 0 & 0.0059 & 30-Nov-95 & UCD1-13 \\
\hline $\mathrm{ug} / \mathrm{L}$ & Dieldrin & - & 5 & 0 & o & - & - & - & 58 & 3 & 0 & 0.029 & 23-Feb-95 & UCD1-13 \\
\hline & Endrin & 0.2 & 5 & 0 & 0 & - & - & - & 58 & 2 & 0 & 0.0 & 23-Fcb-95 & UCD1-13 \\
\hline & Gamma-Chlordane & - & 5 & 0 & 0 & - & - & - & 58 & 3 & 0 & 0.0071 & 30-Nov-95 & UCD1-13 \\
\hline Radionuclides & Actinium-228 & - & 5 & $\mathbf{0}$ & $\mathbf{0}$ & - & - & - & 62 & 1 & $\mathbf{0}$ & 29 & 24-May-95 & UCD2-14 \\
\hline $\mathrm{pCi} / \mathrm{L}$ & Americium-241 & - & 5 & $\mathbf{0}$ & 0 & - & - & - & 49 & 5 & $\mathbf{0}$ & 0.106 & 16-May-95 & UCD1-10 \\
\hline & Bismuth-214 & - & 5 & 2 & 0 & 30 & 22-Feb-95 & UCD1-18 & 62 & 28 & $\mathbf{0}$ & 48 & 22-Feb-95 & UCD1-1 \\
\hline & Carbon-14. & - & 5 & o & o & - & - & - & 56 & 16 & $\mathbf{0}$ & 1900 & 30-Nov-95 & UCD1-13 \\
\hline & Gross Alpha & 15 & 5 & 1 & 1 & 46.8 & 22-Feb-95 & UCD2-17 & 62 & 2 & $\mathbf{0}$ & 8.1 & 30-Nov-95 & UCD1-34 \\
\hline & Gross Beta & 50 & 5 & 1 & $\mathbf{0}$ & 16.2 & 22-Fcb-95 & UCD2-17 & 62 & 5 & $\mathbf{0}$ & 15 & 23-Feb-95 & UCD1-13 \\
\hline & Lead-212 & - & 5 & 0 & 0 & - & - & - & 62 & 2 & 0 & 9 & 14-Feb-95 & UCD1-19 \\
\hline & Lead-214 & - & 5 & 1 & $\mathbf{0}$ & 9.1 & 23-May-95 & UCD2-17 & 62 & 26 & 0 & 35 & 16-May-95 & UCD2-7 \\
\hline & Plutonium-241 & - & 5 & $\mathbf{0}$ & 0 & - & - & 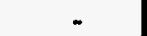 & 49 & 2 & $\mathbf{0}$ & 3.2 & 23-May-95 & UCD1-22 \\
\hline & Radium-226 & 5 & 5 & 3 & 0 & 0.4 & 22-Feb-95 & UCD1-18 & 62 & 23 & 0 & 1.05 & 11-Dec-95 & UCD2-27Z7 \\
\hline & Strontium-90 & 8 & 5 & 0 & 0 & - & - & - & 62 & 1 & 0 & 1.06 & 30-Aug-95 & UCD2-14 \\
\hline & Tritium & $20000 *$ & 5 & o & $\mathbf{0}$ & - & - & - & 61 & 9 & 0 & 19600 & 30-Aug-95 & UCD1-13 \\
\hline & Uranium-235 & & J & 1 & 0 & 22 & 23-May-95 & UCD2-17 & 62 & 0 & 0 & - & - & - \\
\hline
\end{tabular}


TABLE 5.1

NUMBER OF DETECTIONS, POSITIVE RESULTS AND MAXIMUM VALUES IN.GROUNDWATER, 1995 1995 ANNUAL WATER MONITORING REPORT LEHR ENVIRONMENTAL RESTORATION, DAVIS, CALIFORNIA

\begin{tabular}{|c|c|c|c|c|c|c|c|c|c|c|c|c|c|c|}
\hline & \multirow[b]{2}{*}{ PARAMETER } & \multirow[b]{2}{*}{ MCL } & \multicolumn{6}{|c|}{ UPGRADYENT WELLS } & \multicolumn{6}{|c|}{ DOWNGRADIENT WELLS } \\
\hline & & & $\begin{array}{c}\begin{array}{c}\text { Number of } \\
\text { Results }\end{array} \\
\end{array}$ & $\begin{array}{l}\text { Number of } \\
\text { Detections }\end{array}$ & $\begin{array}{l}\text { Number } \\
>\mathrm{MCL}\end{array}$ & $\begin{array}{l}\text { Maximu } \\
\text { m Value }\end{array}$ & $\begin{array}{c}\text { Date of } \\
\text { Maximum }\end{array}$ & $\begin{array}{c}\text { Location of } \\
\text { Maximum }\end{array}$ & $\begin{array}{c}\begin{array}{c}\text { Number of } \\
\text { Results }\end{array} \\
\end{array}$ & $\begin{array}{l}\text { Number of } \\
\text { Detections }\end{array}$ & $\begin{array}{c}\text { Number } \\
>\text { MCL }\end{array}$ & $\begin{array}{c}\text { Maximum } \\
\text { Value } \\
\end{array}$ & $\begin{array}{c}\text { Date of } \\
\text { Maximum }\end{array}$ & $\begin{array}{l}\text { Location of } \\
\text { Maximum }\end{array}$ \\
\hline \multirow{7}{*}{$\begin{array}{l}\text { Semivolatile Organic } \\
\mathrm{ug} / \mathrm{L}\end{array}$} & 4-Methylphenol & - & 5 & 0 & 0 & - & - & - & 46 & 4 & 0 & 96 & 05-Dec-95 & UCD1-27Z1 \\
\hline & Bis(2-Ethylhexyl)phthalate & 4 & 5 & 2 & 1 & 7 & 23-May-95 & UCD1-18 & 46 & 17 & 6 & 29 & 07-Dec-95 & UCD2-27Z4 \\
\hline & Di-n-Butylphthalate & - & $\mathbf{5}$ & 4 & 0 & 50 & 23-May-95 & UCD1-18 & 46 & 7 & $\mathbf{0}$ & 180 & 16-May-95 & UCD1-10 \\
\hline & Dicthyl Phthalate & - & 5 & 2 & $\mathbf{0}$ & 1 & 23-May-95 & UCD1-18 & 46 & 3 & $\mathbf{0}$ & 2 & 23-May-95 & UCD1-22 \\
\hline & Dimethyl Phthalate & - & 5 & $\mathbf{0}$ & $\mathbf{0}$ & - & - & - & 46 & 1 & $\mathbf{0}$ & 6.3 & 05-Dec-95 & UCD1-27Z1 \\
\hline & N-Nitrosodiphenylamine & - & $\mathbf{5}$ & $\mathbf{0}$ & $\mathbf{0}$ & - & - & - & 46 & 1 & $\mathbf{0}$ & 0.64 & 06-Dec-95 & UCD1-27Z2 \\
\hline & Phenol & - & 5 & $\mathbf{0}$ & $\mathbf{0}$ & - & - & - & 46 & 6 & $\mathbf{0}$ & 230 & 05-Dec-95 & UCD1-27Z1 \\
\hline \multirow{15}{*}{$\begin{array}{l}\text { Volatile Organics } \\
\mathrm{ug} / \mathrm{L}\end{array}$} & 1,1,2-Trichlorocthane & & 5 & $\mathbf{0}$ & 0 & - & - & - & 63 & 5 & $\mathbf{0}$ & 3.5 & 28-Aug-95 & UCD1-12 \\
\hline & 1,1-Dichloroethane & & $\mathbf{5}$ & $\mathbf{0}$ & 0 & - & - & - & 62 & 6 & 4 & 9.2 & 28-Aug-95 & UCD1-12 \\
\hline & 1,1-Dichloroethene & 6 & 5 & $\mathbf{0}$ & $\mathbf{0}$ & - & - & - & 62 & 6 & 4 & 18 & 28-Aug-95 & UCD1-12 \\
\hline & 1,2-Dichloroethane & 0.5 & 5 & $\mathbf{0}$ & $\mathbf{0}$ & - & - & - & 62 & 5 & 5 & 5.9 & 28-Aug-95 & UCD1-12 \\
\hline & 1,2-Dichloropropane & 5 & 5 & $\mathbf{0}$ & $\mathbf{0}$ & - & - & - & 62 & 1 & $\mathbf{0}$ & 0.72 & 28-Aug-95 & UCD1-12 \\
\hline & 2-Butanone & - & 5 & $\mathbf{0}$ & 0 & - & - & - & 63 & 1 & 0 & 27 & 05-Dec-95 & UCD1-27Z1 \\
\hline & 4-Methyl-2-Pentanone & - & 5 & $\mathbf{0}$ & 0 & - & - & - & 63 & 1 & 0 & $\mathbf{5}$ & 05-Dec-95 & UCD1-27Z1 \\
\hline & Acetone & - & 5 & $\mathbf{0}$ & $\mathbf{0}$ & - & - & - & 62 & 7 & $\mathbf{0}$ & 4.7 & 05-Dec-95 & UCD1-27Z1 \\
\hline & Bromodichloromethane & & 5 & 0 & $\mathbf{0}$ & - & - & - & 62 & 5 & $\mathbf{0}$ & 2.1 & 28-Aug-95 & UCD1-12 \\
\hline & Bromoform & a & 5 & 0 & 0 & - & - & - & 63 & 1 & $\mathbf{0}$ & 0.95 & 08-Dec-95 & UCD2-27Z6 \\
\hline & Carbon Disulfide & - & 5 & $\mathbf{0}$ & $\mathbf{0}$ & - & - & - & 63 & 4 & $\mathbf{0}$ & 2.4 & 07-Dec-95 & UCD1-27Z3 \\
\hline & Chloroform & & 5 & $\mathbf{0}$ & $\mathbf{0}$ & - & - & - & 61 & 32 & 6 & 9100 & 28-Aug-95 & UCD1-12 \\
\hline & Methylene Chloride & 5 & 5 & $\mathbf{0}$ & $\mathbf{0}$ & - & - & - & 63 & 2 & $\mathbf{0}$ & 1 & 07-Dec-95 & UCD2-27Z4 \\
\hline & | Tetrachloroethene & 5 & 5 & 0 & $\mathbf{0}$ & - & - & - & 63 & 1 & $\mathbf{0}$ & 0.84 & 24-May-95 & UCD1-24 \\
\hline & Toluene & 1000 & 5 & $\mathbf{0}$ & $\mathbf{0}$ & - & - & - & 63 & 2 & $\mathbf{0}$ & 1.3 & 05-Dec-95 & UCD1-27Z1 \\
\hline
\end{tabular}

- = Not Applicable

MCL = Maximum Contamination Level (primary), Title 22, Division 4, Chapter 15.

$a=$ MCL for total trihalomethanes is $100 \mathrm{ug} / \mathrm{L}$. Chloroform is the predominant trihalomethane detected at LEHR.

$\#=$ USEPA MCL.

$s=$ Secondary Drinking Water Standard

$*=$ Proposed MCL for Tritium is $60,000 \mathrm{pCi} / \mathrm{L}$. 
TABLE 5.2

SUMMARY OF DETECTED CONSTITUENTS IN GROUNDWATER, 1995

VOLATILE ORGANICS

1995 ANNUAL WATER MONITORING REPORT

LEHR ENVIRONMENTAL RESTORATION, DAVIS, CALIFORNIA

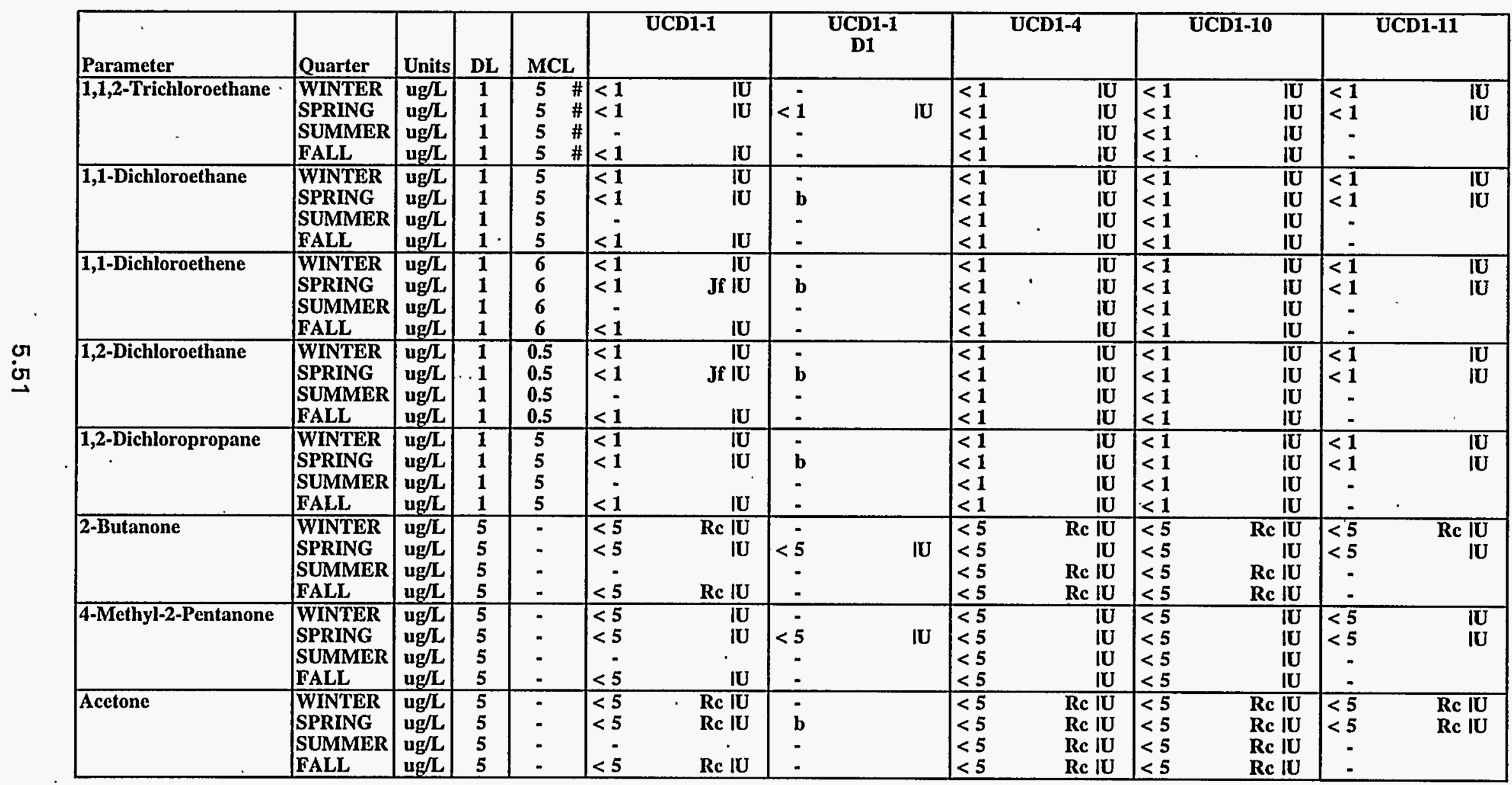

Page 1 of 17 
TABLE 5.2

SUMMARY OF DETECTED CONSTITUENTS IN GROUNDWATER, 1995

VOLATILE ORGANICS

1995 ANNUAL WATER MONITORING REPORT

LEHR ENVIRONMENTAL RESTORATION, DAVIS, CALIFORNIA

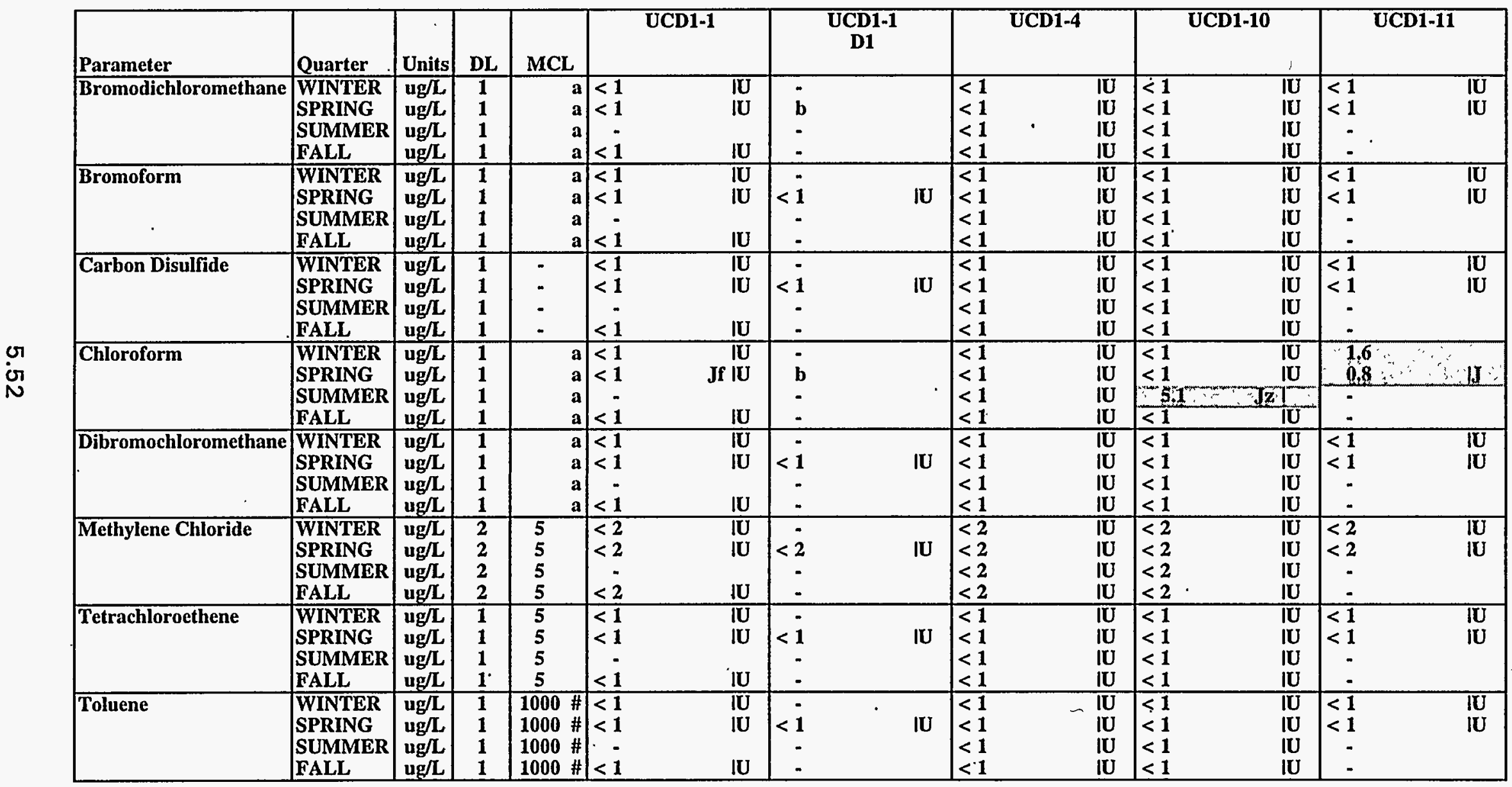


TABLE 5.2

SUMMARY OF DETECTED CONSTITUENTS IN GROUNDWATER, 1995

VOLATILE ORGANICS

1995 ANNUAL WATER MONITORING REPORT

LEHR ENVIRONMENTAL RESTORATION, DAVIS, CALIFORNIA

\begin{tabular}{|c|c|c|c|c|c|c|c|c|c|c|c|c|}
\hline \multirow{2}{*}{\begin{tabular}{|l} 
Parameter \\
$1,1,2-$ Trichloroethane
\end{tabular}} & \multirow[b]{2}{*}{\begin{tabular}{|l} 
Quarter \\
WINTER \\
SPRING \\
SUMMER \\
FALL \\
\end{tabular}} & \multirow[b]{2}{*}{\begin{tabular}{|l|} 
Units \\
$\mathrm{ug} / \mathrm{L}$ \\
$\mathrm{ug} / \mathrm{L}$ \\
$\mathrm{ug} / \mathrm{L}$ \\
$\mathrm{ug} / \mathrm{L}$ \\
\end{tabular}} & \multirow[b]{2}{*}{$\begin{array}{l}\frac{D L}{1} \\
1 \\
1 \\
1\end{array}$} & \multirow{2}{*}{\begin{tabular}{cc}
\multicolumn{2}{c}{ MCL } \\
$\mathbf{5}$ & $\#$ \\
5 & $\#$ \\
5 & $\#$ \\
5 & $\#$ \\
\end{tabular}} & \multicolumn{2}{|c|}{ UCD1-12 } & \multicolumn{2}{|c|}{$\begin{array}{l}\text { UCD1-12 } \\
\text { D1 }\end{array}$} & \multicolumn{2}{|c|}{ UCD1-13 } & \multicolumn{2}{|c|}{$\begin{array}{c}\text { UCD1-13 } \\
\text { D1 }\end{array}$} \\
\hline & & & & & \begin{tabular}{|c|}
0.9 \\
$<1$ \\
2.8 \\
1.1 \\
\end{tabular} & $\frac{\mathrm{IJ}}{\mathrm{IU}}$ & $\begin{array}{l}- \\
3.5 \\
2\end{array}$ & Jf I & $\begin{array}{l}<1 \\
<1 \\
<1 \\
<1\end{array}$ & $\begin{array}{l}\text { IU } \\
\mathbf{I U} \\
\mathbf{I U} \\
\mathbf{U}\end{array}$ & $\begin{array}{r}- \\
< \\
<1\end{array}$ & IU \\
\hline 1,1-Dichloroethane & $\begin{array}{l}\text { WINTER } \\
\text { SPRING } \\
\text { SUMMER } \\
\text { FALL } \\
\end{array}$ & $\begin{array}{l}\mathrm{ug} / \mathrm{L} \\
\mathrm{ug} / \mathrm{L} \\
\mathrm{ug} / \mathrm{L} \\
\mathrm{ug} / \mathrm{L} \\
\end{array}$ & $\begin{array}{l}1 \\
1 \\
1 \\
1 \\
\end{array}$ & $\begin{array}{l}5 \\
5 \\
5 \\
5 \\
\end{array}$ & $\begin{array}{l}6 \\
0.51 \\
4.1 \\
5.8 \\
\end{array}$ & 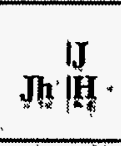 & $\begin{array}{l}- \\
-92 \\
2.8 \\
6.8\end{array}$ & $\mathrm{Jf} \mathrm{T}^{-}$ & $\begin{array}{l}<1 \\
<1 \\
<1 \\
<1\end{array}$ & $\begin{array}{l}\mathbf{I U} \\
\mathbf{I U} \\
\mathbf{I U} \\
\mathbf{I U} \\
\end{array}$ & $\begin{array}{r}- \\
< \\
<1 \\
\end{array}$ & $\mathbf{I U}$ \\
\hline 1,1-Dichloroethene & $\begin{array}{l}\text { WINTER } \\
\text { SPRING } \\
\text { SUMMER } \\
\text { FALL } \\
\end{array}$ & $\begin{array}{l}\mathrm{ug} / \mathrm{L} \\
\mathrm{ug} / \mathrm{L} \\
\mathrm{ug} / \mathrm{L} \\
\mathrm{ug} / \mathrm{L} \\
\end{array}$ & $\begin{array}{l}1 \\
1 \\
1 \\
1 \\
\end{array}$ & $\begin{array}{l}6 \\
6 \\
6 \\
6 \\
\end{array}$ & $\begin{array}{l}9.3 \\
1.1 \\
1.2 \\
11 \\
\end{array}$ & J̦h & $\begin{array}{l}- \\
18 \\
13\end{array}$ & IfT & $\begin{array}{l}<1 \\
<1 \\
<1 \\
<1\end{array}$ & $\begin{array}{l}\mathrm{IU} \\
\mathrm{IU} \\
\mathrm{IU} \\
\mathrm{IU}\end{array}$ & $\begin{array}{l}: \\
<1\end{array}$ & $\mathbf{I U}$ \\
\hline 1,2-Dichloroethane & \begin{tabular}{|l} 
WINTER \\
SPRING \\
SUMMER \\
FALL \\
\end{tabular} & \begin{tabular}{|l|}
$\mathrm{ug} / \mathrm{L}$ \\
$\mathrm{ug} / \mathrm{L}$ \\
$\mathrm{ug} / \mathrm{L}$ \\
$\mathrm{ug} / \mathrm{L}$ \\
\end{tabular} & $\begin{array}{l}\mathbf{1} \\
\mathbf{1} \\
\mathbf{1} \\
\mathbf{1}\end{array}$ & $\begin{array}{l}0.5 \\
0.5 \\
0.5 \\
0.5 \\
\end{array}$ & $\begin{array}{c}4 \\
<1 \\
4.7 \\
3.2 \\
\end{array}$ & Jhו" & $\begin{array}{l}- \\
- \\
5,9 \\
3,8\end{array}$ & Jf & $\begin{array}{l}<1 \\
<1 \\
<1 \\
<1\end{array}$ & $\begin{array}{l}\text { IU } \\
\text { IU } \\
\text { IU } \\
\text { IU }\end{array}$ & $\begin{array}{r}: \\
<1\end{array}$ & $\mathbf{I U}$ \\
\hline 1,2-Dichloropropane & $\begin{array}{l}\text { WINTER } \\
\text { SPRING } \\
\text { SUMMER } \\
\text { FALL } \\
\end{array}$ & $\begin{array}{l}\mathrm{ug} / \mathrm{L} \\
\mathrm{ug} / \mathrm{L} \\
\mathrm{ug} / \mathrm{L} \\
\mathrm{ug} / \mathrm{L} \\
\end{array}$ & $\begin{array}{l}1 \\
1 \\
1 \\
1\end{array}$ & $\begin{array}{l}5 \\
5 \\
5 \\
5\end{array}$ & $\begin{array}{l}<1 \\
<1 \\
<1 \\
<1 \\
\end{array}$ & $\begin{array}{r}\mathbf{I U} \\
\mathbf{I U} \\
\mathbf{R h} \mid \mathbf{I U} \\
\mathbf{I U} \\
\end{array}$ & $\begin{array}{l}- \\
0.72 \\
1\end{array}$ & $\frac{\mathrm{J}}{\mathrm{IU}}$ & $\begin{array}{l}<1 \\
<1 \\
<1 \\
<1\end{array}$ & $\begin{array}{l}\mathrm{IU} \\
\mathrm{IU} \\
\mathrm{IU} \\
\mathrm{IU}\end{array}$ & $\begin{array}{r}- \\
- \\
<1\end{array}$ & IU \\
\hline 2-Butanone & \begin{tabular}{|l} 
WINTER \\
SPRING \\
SUMMER \\
FALL \\
\end{tabular} & $\begin{array}{l}\mathrm{ug} / \mathrm{L} \\
\mathrm{ug} / \mathrm{h} \\
\mathrm{ug} / \mathrm{L} \\
\mathrm{ug} / \mathrm{L}\end{array}$ & $\begin{array}{l}5 \\
5 \\
5 \\
5 \\
\end{array}$ & 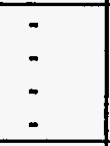 & $\begin{array}{l}<5 \\
<5 \\
<5 \\
<5 \\
\end{array}$ & $\begin{array}{l}\text { Rc } \mathbf{I U} \\
\mathbf{I U} \\
\text { Re } \mathbf{I U} \\
\text { Rc } \mathbf{I U} \\
\end{array}$ & $\begin{array}{r}\quad \\
<5 \\
<5 \\
\end{array}$ & $\begin{array}{l}\operatorname{Rc} \mid \mathbf{U} \\
\operatorname{Re} \mid \mathbf{U}\end{array}$ & $\begin{array}{l}<5 \\
<5 \\
<5 \\
<5\end{array}$ & $\begin{array}{l}\text { Re IU } \\
\text { IU } \\
\operatorname{Rc} \text { IU } \\
\operatorname{Rc} \text { IU }\end{array}$ & $\begin{array}{r}- \\
- \\
<5\end{array}$ & Re IU \\
\hline 4-Methyl-2-Pentanone & \begin{tabular}{|l|} 
WINTER \\
SPRING \\
SUMMER \\
FALL \\
\end{tabular} & $\begin{array}{l}\mathrm{ug} / \mathrm{L} \\
\mathrm{ug} / \mathrm{L} \\
\mathrm{ug} / \mathrm{L} \\
\mathrm{ug} / \mathrm{L} \\
\end{array}$ & $\begin{array}{l}5 \\
5 \\
5 \\
5 \\
\end{array}$ & $\begin{array}{l}- \\
- \\
\end{array}$ & $\begin{array}{l}<5 \\
<5 \\
<5 \\
<5 \\
\end{array}$ & Rh $\underset{\text { IU }}{\text { IU }}$ & $\begin{array}{r}\quad \\
<5 \\
<5 \\
<5\end{array}$ & $\underset{\mathbf{I U}}{\mathbf{I U}}$ & $\begin{array}{l}<5 \\
<5 \\
<5 \\
<5\end{array}$ & $\begin{array}{l}\mathbf{I U} \\
\mathbf{I U} \\
\mathbf{I U} \\
\mathbf{I U}\end{array}$ & $\begin{array}{r}- \\
- \\
<5\end{array}$ & $\mathbf{I U}$ \\
\hline Acetone & $\begin{array}{l}\text { WINTER } \\
\text { SPRING } \\
\text { SUMMER } \\
\text { FALL } \\
\end{array}$ & $\begin{array}{l}\mathrm{ug} / \mathrm{L} \\
\mathrm{ug} / \mathrm{L} \\
\mathrm{ug} / \mathrm{L} \\
\mathrm{ug} / \mathrm{L} \\
\end{array}$ & $\begin{array}{l}5 \\
5 \\
5 \\
5 \\
\end{array}$ & $\begin{array}{l}- \\
- \\
-\end{array}$ & $\begin{array}{l}<5 \\
<5 \\
2,4 \\
<5 \\
\end{array}$ & $\begin{array}{l}\mathrm{Rc} \text { IU } \\
\mathrm{Rc} \text { IU } \\
\mathrm{Je} \mid \mathrm{JH} \\
\mathrm{Rc} \text { IU } \\
\end{array}$ & $\begin{array}{r}\quad \\
<5 \\
<5 \\
\end{array}$ & $\begin{array}{l}\mathbf{R e} \mid \mathbf{U} \\
\mathbf{R e} \mid \mathbf{U} \\
\end{array}$ & $\begin{array}{l}<5 \\
<5 \\
<5 \\
<5 \\
\end{array}$ & $\begin{array}{l}\operatorname{Rc} \text { IU } \\
\text { Rc IU } \\
\text { Rc IU } \\
\text { Rc IU } \\
\end{array}$ & $\begin{array}{r}- \\
- \\
<5\end{array}$ & Re IU \\
\hline
\end{tabular}

Page 3 of 17 
TABLE 5.2

SUMMARY OF DETECTED CONSTITUENTS IN GROUNDWATER, 1995 VOLATILE ORGANICS

1995 ANNUAL WATER MONITORING REPORT

LEHR ENVIRONMENTAL RESTORATION, DAVIS, CALIFORNIA

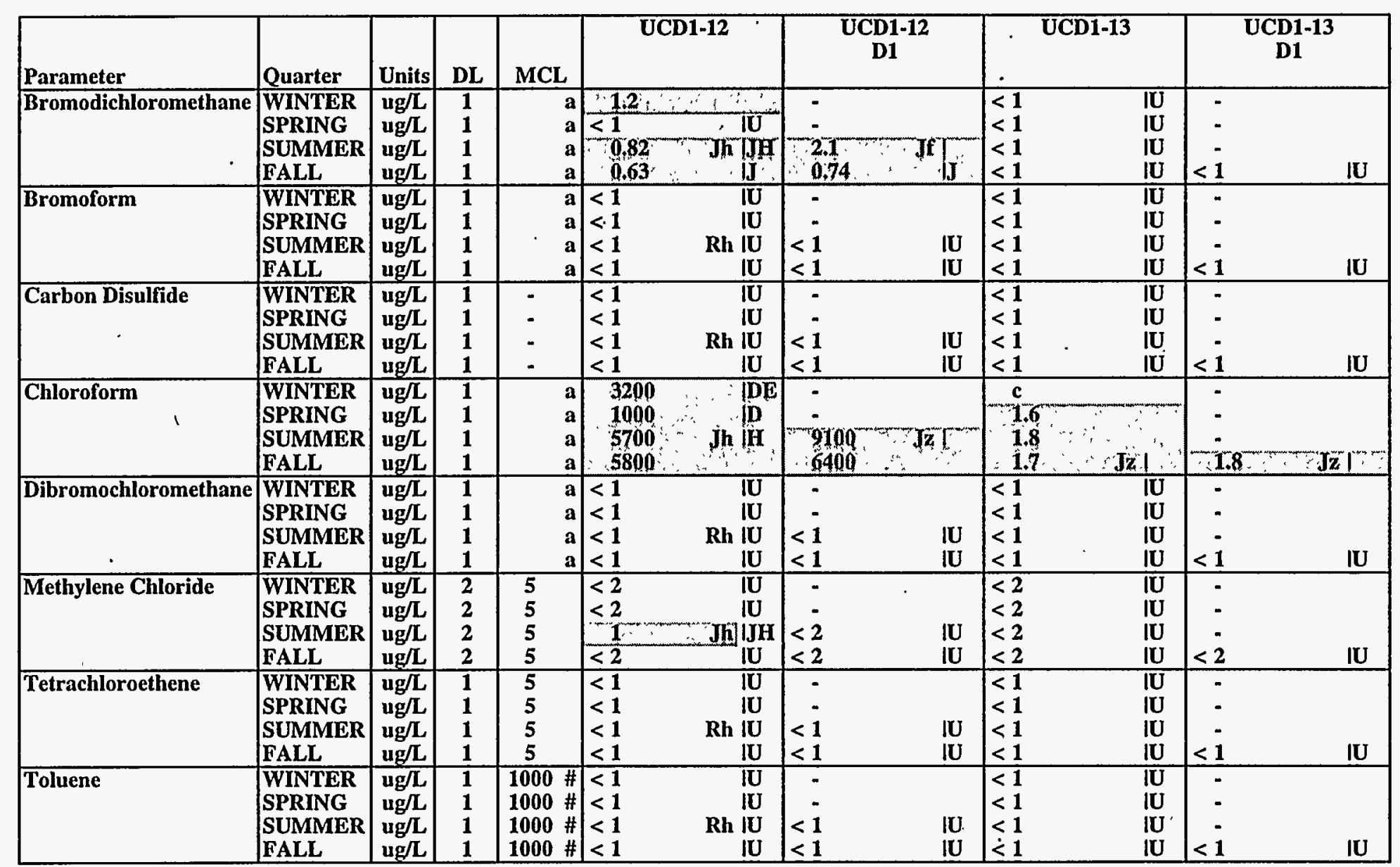


TABLE 5.2

SUMMARY OF DETECTED CONSTITUENTS IN GROUNDWATER, 1995

VOLATILE ORGANICS

1995 ANNUAL WATER MONITORING REPORT

LEHR ENVIRONMENTAL RESTORATION, DAVIS, CALIFORNIA

\begin{tabular}{|c|c|c|c|c|c|c|c|c|c|c|c|c|c|c|}
\hline \multirow{2}{*}{$\begin{array}{l}\text { Parameter } \\
1,1,2-\text { Trichloroethane }\end{array}$} & \multirow[b]{2}{*}{\begin{tabular}{|l} 
Quarter \\
WINTER \\
SPRING \\
SUMMER \\
FALL \\
\end{tabular}} & \multirow[b]{2}{*}{\begin{tabular}{|l|} 
Units \\
$\mathrm{ug} / \mathrm{L}$ \\
$\mathrm{ug} / \mathrm{L}$ \\
$\mathrm{ug} / \mathrm{L}$ \\
$\mathrm{ug} / \mathrm{L}$ \\
\end{tabular}} & \multirow[b]{2}{*}{$\begin{array}{c}\text { DL } \\
1 \\
1 \\
1 \\
1\end{array}$} & \multirow[b]{2}{*}{\begin{tabular}{cc|} 
MCL \\
5 & $\#$ \\
5 & $\#$ \\
5 & $\#$ \\
5 & $\#$
\end{tabular}} & \multicolumn{2}{|c|}{ UCD1-18 } & \multicolumn{2}{|r|}{$\begin{array}{c}\text { UCD1-18 } \\
\text { D1 }\end{array}$} & \multicolumn{2}{|c|}{ UCD1-19 } & \multicolumn{2}{|c|}{$\begin{array}{c}\text { UCD1-19 } \\
\text { D1 }\end{array}$} & \multicolumn{2}{|c|}{ UCD1-20 } \\
\hline & & & & & $\begin{array}{r}<1 \\
<1 \\
- \\
- \\
\end{array}$ & $\underset{\mathbf{I U}}{\mathbf{I U}}$ & $\begin{array}{r}1 \\
- \\
- \\
- \\
\end{array}$ & IU & $\begin{array}{r}<1 \\
<1 \\
- \\
\end{array}$ & IU & $\begin{array}{r}<1 \\
-\end{array}$ & IU & $\begin{array}{r}<1 \\
<1 \\
-\end{array}$ & $\underset{\mathbf{I U}}{\mathbf{I U}}$ \\
\hline 1,1-Dichloroethane & \begin{tabular}{|l|} 
WINTER \\
SPRING \\
SUMMER \\
FALL
\end{tabular} & $\begin{array}{l}\mathrm{ug} / \mathrm{L} \\
\mathrm{ug} / \mathrm{L} \\
\mathrm{ug} / \mathrm{L} \\
\mathrm{ug} / \mathrm{L}\end{array}$ & $\begin{array}{l}1 \\
1 \\
1 \\
1\end{array}$ & \begin{tabular}{l|}
5 \\
5 \\
5 \\
5
\end{tabular} & $\begin{array}{l}<1 \\
<1 \\
-\end{array}$ & $\begin{array}{l}\text { IU } \\
\text { IU }\end{array}$ & $\begin{array}{l}<1 \\
\vdots \\
-\end{array}$ & IU & $\begin{array}{l}<1 \\
<1 \\
:\end{array}$ & $\begin{array}{l}\text { IU } \\
\text { IU }\end{array}$ & $\begin{array}{c}<1 \\
:\end{array}$ & IU & $\begin{array}{r}<1 \\
<1 \\
\vdots\end{array}$ & $\begin{array}{l}\mathbf{I U} \\
\mathbf{I U}\end{array}$ \\
\hline 1,1-Dichloroethene & $\begin{array}{l}\text { WINTER } \\
\text { SPRING } \\
\text { SUMMER } \\
\text { FALL } \\
\end{array}$ & $\begin{array}{l}\mathrm{ug} / \mathrm{L} \\
\mathrm{ug} / \mathrm{L} \\
\mathrm{ug} / \mathrm{L} \\
\mathrm{ug} / \mathrm{L}\end{array}$ & $\begin{array}{l}1 \\
1 \\
1 \\
1\end{array}$ & $\begin{array}{l}6 \\
6 \\
6 \\
6 \\
\end{array}$ & $\begin{array}{r}<1 \\
<1 \\
- \\
-\end{array}$ & IU & $\begin{array}{c}<1 \\
: \\
-\end{array}$ & IU & $\begin{array}{r}<1 \\
<1 \\
- \\
\end{array}$ & $\begin{array}{l}\text { IU } \\
\text { IU }\end{array}$ & $\begin{array}{r}<1 \\
\vdots\end{array}$ & IU & $\begin{array}{r}<1 \\
<1 \\
\vdots \\
\end{array}$ & $\begin{array}{l}\text { IU } \\
\mathbf{I U}\end{array}$ \\
\hline 1,2-Dichloroethane & $\begin{array}{l}\text { WINTER } \\
\text { SPRING } \\
\text { SUMMER } \\
\text { FALL } \\
\end{array}$ & $\begin{array}{l}\mathrm{ug} / \mathrm{L} \\
\mathrm{ug} / \mathrm{L} \\
\mathrm{ug} / \mathrm{L} \\
\mathrm{ug} / \mathrm{L} \\
\end{array}$ & $\begin{array}{l}1 \\
1 \\
1 \\
1 \\
\end{array}$ & $\begin{array}{l}0.5 \\
0.5 \\
0.5 \\
0.5 \\
\end{array}$ & $\begin{array}{r}<1 \\
<1 \\
- \\
- \\
\end{array}$ & $\begin{array}{l}\text { IU } \\
\text { IU }\end{array}$ & $\begin{array}{c}<1 \\
: \\
- \\
\end{array}$ & IU & $\begin{array}{r}<1 \\
<1 \\
- \\
-\end{array}$ & $\begin{array}{l}\text { IU } \\
\text { IU }\end{array}$ & $\begin{array}{r}<1 \\
- \\
-\end{array}$ & $\mathbf{I U}$ & $\begin{array}{r}<1 \\
<1 \\
- \\
\end{array}$ & $\begin{array}{l}\text { IU } \\
\mathbf{I U}\end{array}$ \\
\hline 1,2-Dichloropropane & $\begin{array}{l}\text { WINTER } \\
\text { SPRING } \\
\text { SUMMER } \\
\text { FALL } \\
\end{array}$ & $\begin{array}{l}\mathrm{ug} / \mathrm{L} \\
\mathrm{ug} / \mathrm{L} \\
\mathrm{ug} / \mathrm{L} \\
\mathrm{ug} / \mathrm{L}\end{array}$ & $\begin{array}{l}1 \\
1 \\
1 \\
1 \\
\end{array}$ & $\begin{array}{l}5 \\
5 \\
5 \\
5 \\
\end{array}$ & $\begin{array}{r}<1 \\
<1 \\
- \\
- \\
\end{array}$ & IU & $\begin{array}{r}<1 \\
\vdots \\
- \\
\end{array}$ & $\begin{array}{r}\mathbf{I U} \\
- \\
\end{array}$ & $\begin{array}{r}<1 \\
<1 \\
! \\
-\end{array}$ & $\begin{array}{l}\text { IU } \\
\mathbf{I U}\end{array}$ & $\begin{array}{r}<1 \\
\vdots\end{array}$ & & $\begin{array}{r}<1 \\
<1 \\
\quad \\
-\end{array}$ & . \\
\hline 2-Butanone & $\begin{array}{l}\text { WINTER } \\
\text { SPRING } \\
\text { SUMMER } \\
\text { FALL }\end{array}$ & $\begin{array}{l}\mathrm{ug} / \mathrm{L} \\
\mathrm{ug} / \mathrm{L} \\
\mathrm{ug} / \mathrm{L} \\
\mathrm{ug} / \mathrm{L}\end{array}$ & $\begin{array}{l}5 \\
5 \\
5 \\
5\end{array}$ & $:$ & $\begin{array}{r}<5 \\
<5 \\
- \\
-\end{array}$ & $\begin{array}{l}\operatorname{Rc} \mid \mathrm{U} \\
\operatorname{Rc} \mathbf{I U}\end{array}$ & $\begin{array}{l}<5 \\
\vdots\end{array}$ & Re IU & $\begin{array}{l}<5 \\
<5 \\
-\end{array}$ & $\begin{array}{r}\mathbf{R e} \\
\boldsymbol{I U} \\
\mathbf{I U}\end{array}$ & $\begin{array}{r}<5 \\
-\end{array}$ & $\mathbf{I U}$ & $\begin{array}{r}<5 \\
<5 \\
\vdots \\
-\end{array}$ & Rc $\underset{\mathbb{I U}}{\mathbb{I U}}$ \\
\hline 4-Methyl-2-Pentanone & $\begin{array}{l}\text { WINTER } \\
\text { SPRING } \\
\text { SUMMER } \\
\text { FALL } \\
\end{array}$ & $\begin{array}{l}\mathrm{ug} / \mathrm{L} \\
\mathrm{ug} / \mathrm{L} \\
\mathrm{ug} / \mathrm{L} \\
\mathrm{ug} / \mathrm{L} \\
\end{array}$ & $\begin{array}{l}5 \\
5 \\
5 \\
5 \\
\end{array}$ & $\begin{array}{l}- \\
- \\
- \\
-\end{array}$ & $\begin{array}{r}<5 \\
<5 \\
- \\
- \\
\end{array}$ & $\begin{array}{l}\text { IU } \\
\text { IU }\end{array}$ & $\begin{array}{r}<5 \\
\vdots \\
\vdots\end{array}$ & & $\begin{array}{r}<5 \\
<5 \\
- \\
- \\
\end{array}$ & IU & $\begin{array}{r}<5 \\
- \\
\end{array}$ & IU & $\begin{array}{r}<5 \\
<5 \\
\quad \\
- \\
\end{array}$ & $\begin{array}{l}\text { IU } \\
\mathbf{I U}\end{array}$ \\
\hline Acetone & $\begin{array}{l}\text { WINTER } \\
\text { SPRING } \\
\text { SUMMER } \\
\text { FALL } \\
\end{array}$ & $\begin{array}{l}\mathrm{ug} / \mathrm{L} \\
\mathrm{ug} / \mathrm{L} \\
\mathrm{ug} / \mathrm{L} \\
\mathrm{ug} / \mathrm{L}\end{array}$ & $\begin{array}{l}5 \\
5 \\
5 \\
5 \\
\end{array}$ & $\begin{array}{l}\ddot{-} \\
- \\
\end{array}$ & $\begin{array}{r}<5 \\
<5 \\
\vdots \\
-\end{array}$ & $\begin{array}{l}\text { Rc IU } \\
\text { Rc IU }\end{array}$ & $\begin{array}{l}<5 \\
: \\
-\end{array}$ & Rc'lU & $\begin{array}{r}<5 \\
<5 \\
-\end{array}$ & $\begin{array}{l}\text { Rc IU } \\
\text { Re IU }\end{array}$ & $\begin{array}{c}<5 \\
\vdots \\
-\end{array}$ & Re IU & $\begin{array}{r}<5 \\
<5 \\
-\end{array}$ & $\begin{array}{l}\operatorname{Re} I U \\
\operatorname{Re} \mathbb{U}\end{array}$ \\
\hline
\end{tabular}


TABLE 5.2

SUMMARY OF DETECTED CONSTITUENTS IN GROUNDWATER, 1995

VOLATILE ORGANICS

1995 ANNUAL WATER MONITORING REPORT

LEHR ENVIRONMENTAL RESTORATION, DAVIS, CALIFORNIA

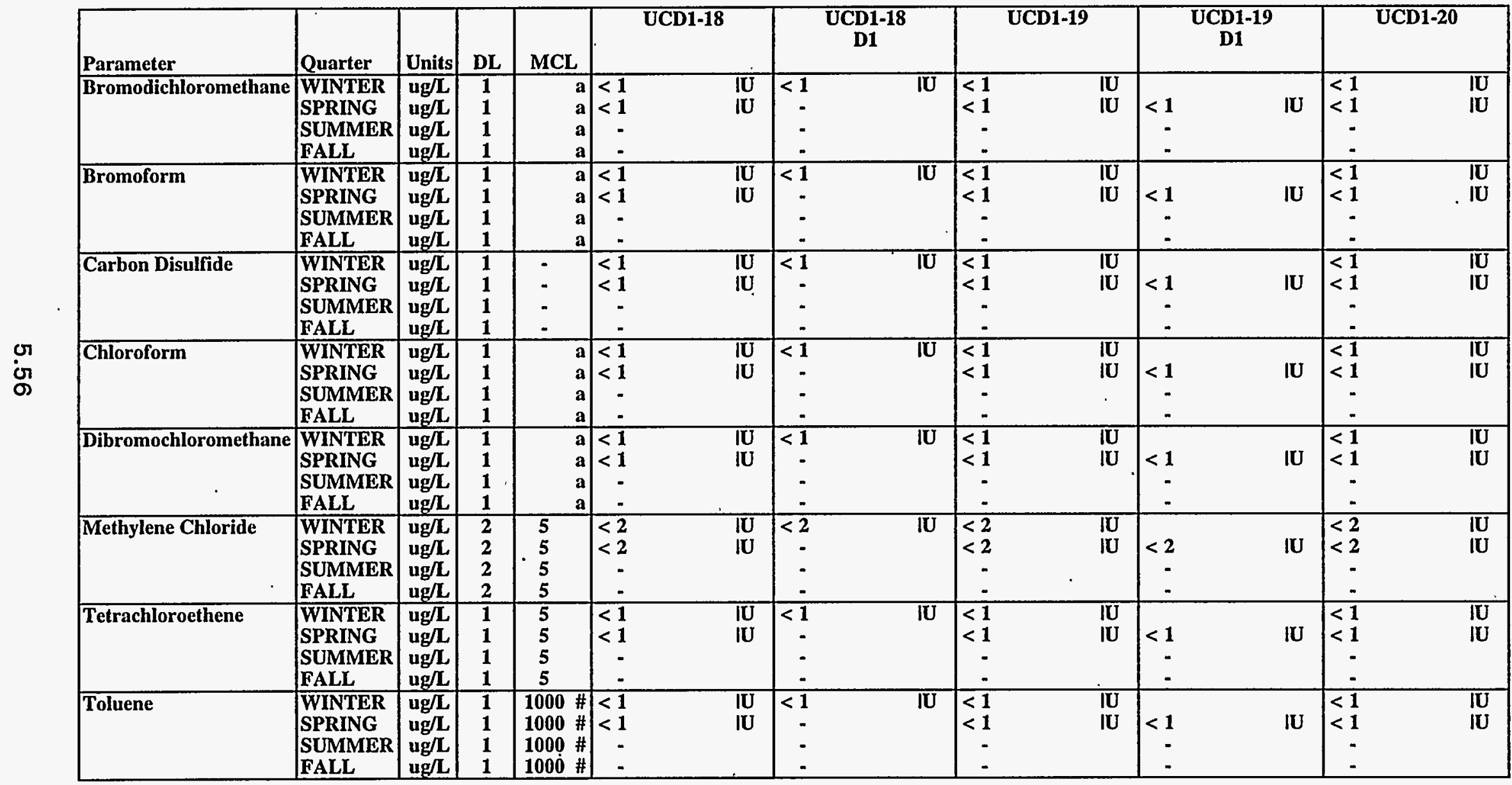


TABLE 5.2

SUMMARY OF DETECTED CONSTITUENTS IN GROUNDWATER, 1995

VOLATILE ORGANICS

1995 ANNUAL WATER MONITORING REPORT

LEHR ENVIRONMENTAL RESTORATION, DAVIS, CALIFORNIA

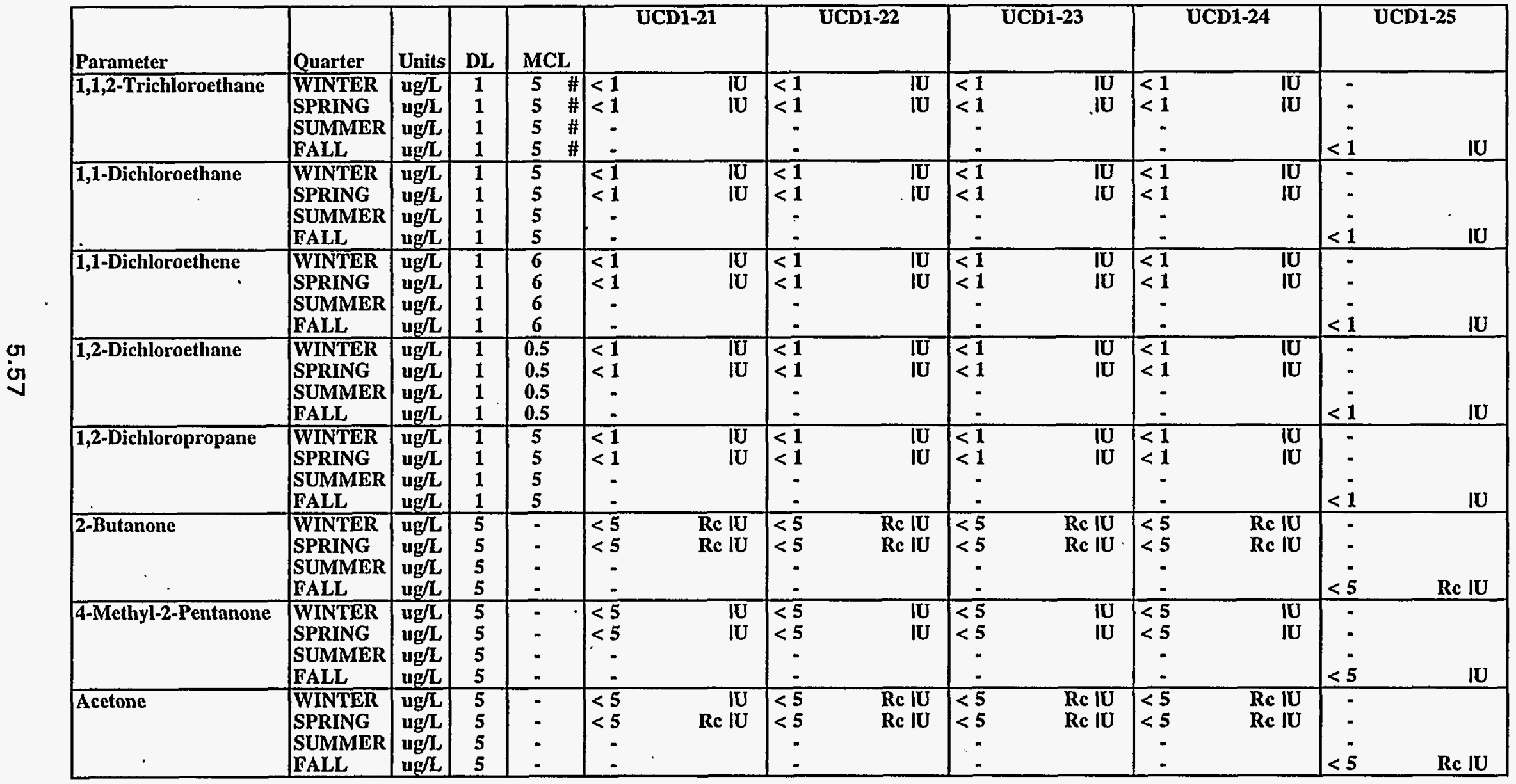


TABLE 5.2

SUMMARY OF DETECTED CONSTITUENTS IN GROUNDWATER, 1995

VOLATILE ORGANICS

1995 ANNUAL WATER MONITORING REPORT

LEHR ENVIRONMENTAL RESTORATION, DAVIS, CALIFORNIA

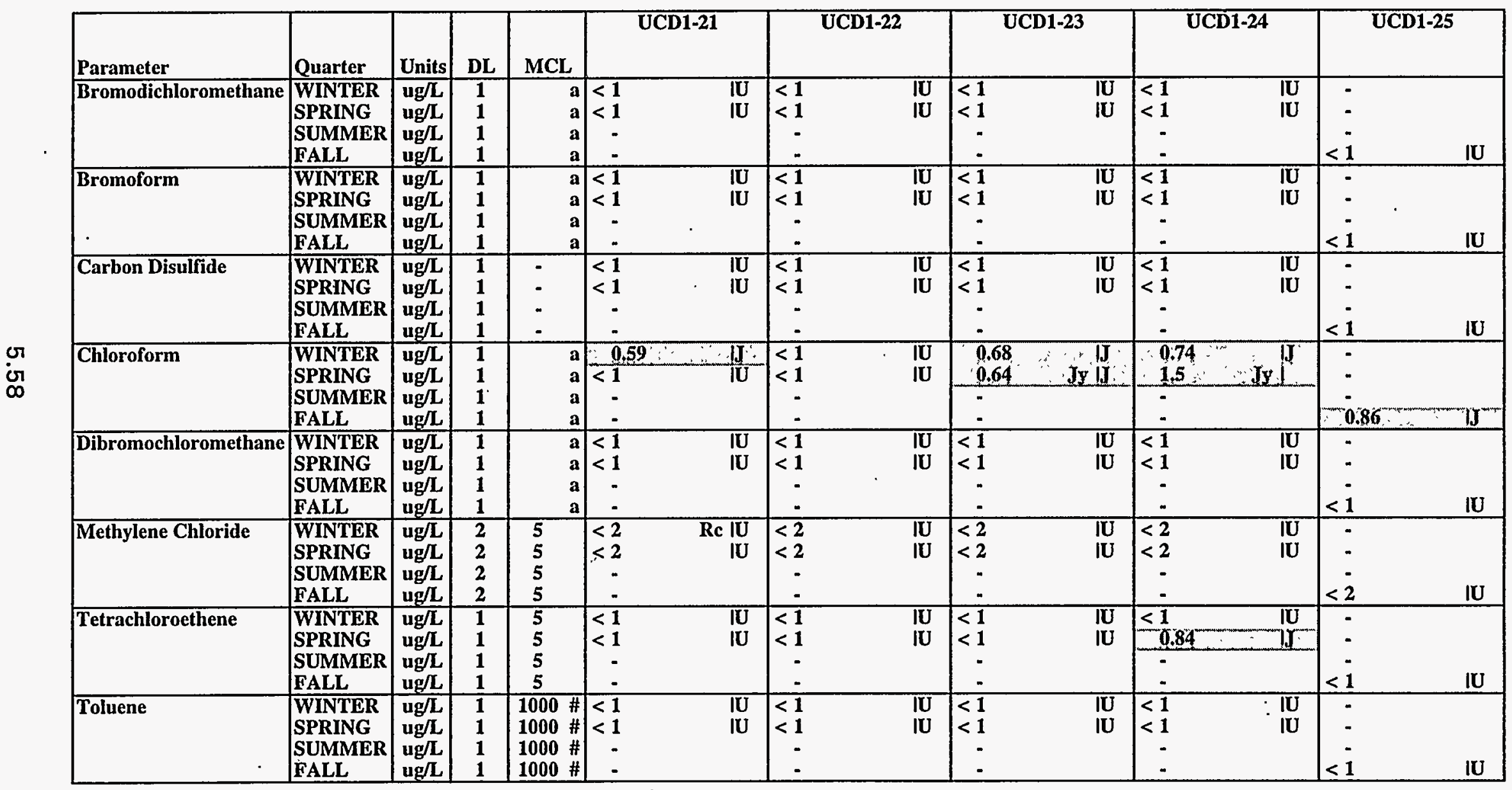


TABLE 5.2

SUMMARY OF DETECTED CONSTITUENTS IN GROUNDWATER, 1995

VOLATILE ORGANICS

1995 ANNUAL WATER MONITORING REPORT

LEHR ENVIRONMENTAL RESTORATION, DAVIS, CALIFORNIA

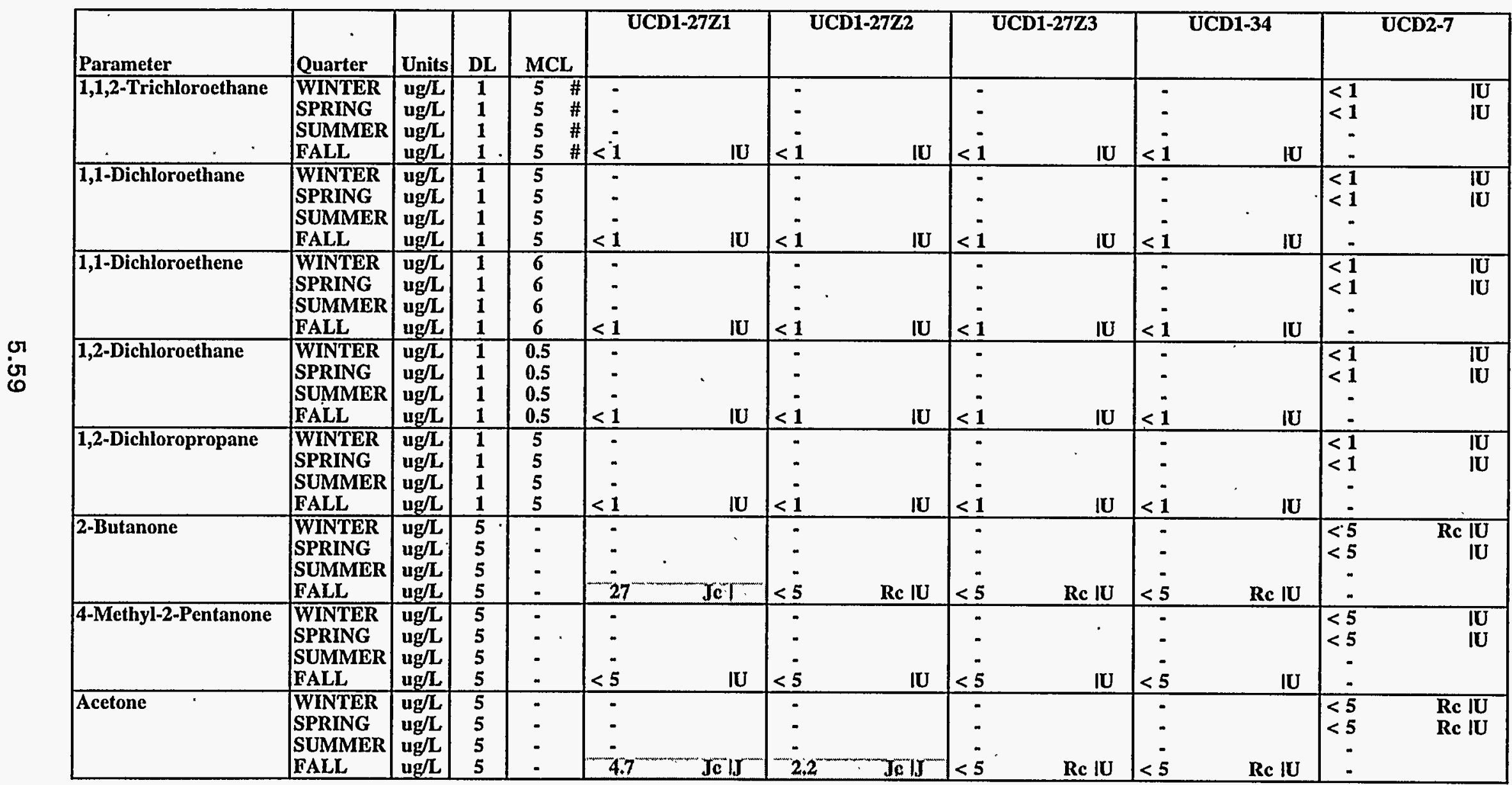

Page 9 of 17 
TABLE 5.2

SUMMARY OF DETECTED CONSTITUENTS IN GROUNDWATER, 1995

VOLATILE ORGANICS

1995 ANNUAL WATER MONITORING REPORT

LEHR ENVIRONMENTAL RESTORATION, DAVIS, CALIFORNIA

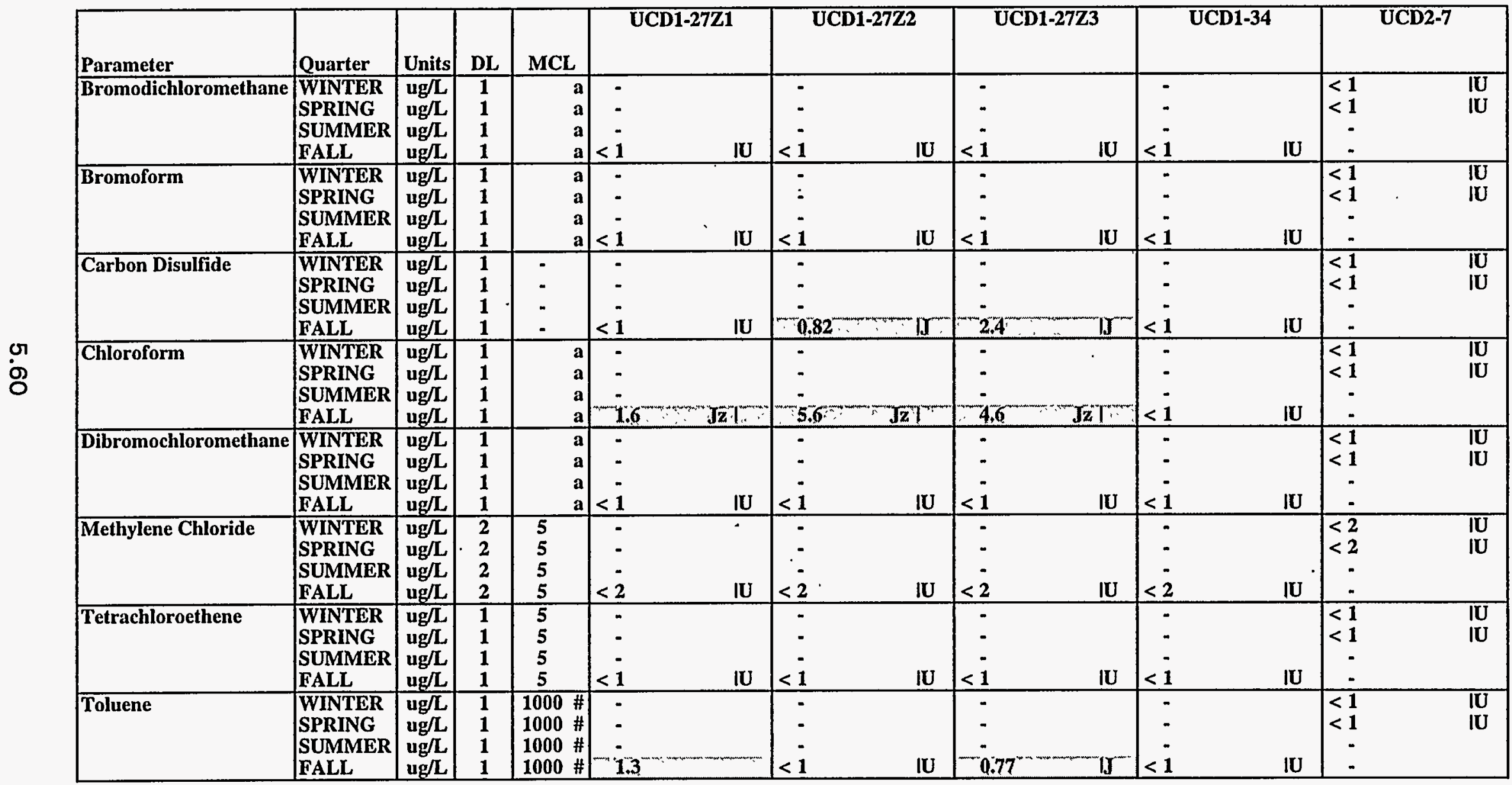


TABLE 5.2

SUMMARY OF DETECTED CONSTITUENTS IN GROUNDWATER, 1995

VOLATILE ORGANICS

1995 ANNUAL WATER MONITORING REPORT

LEHR ENVIRONMENTAL RESTORATION, DAVIS, CALIFORNIA

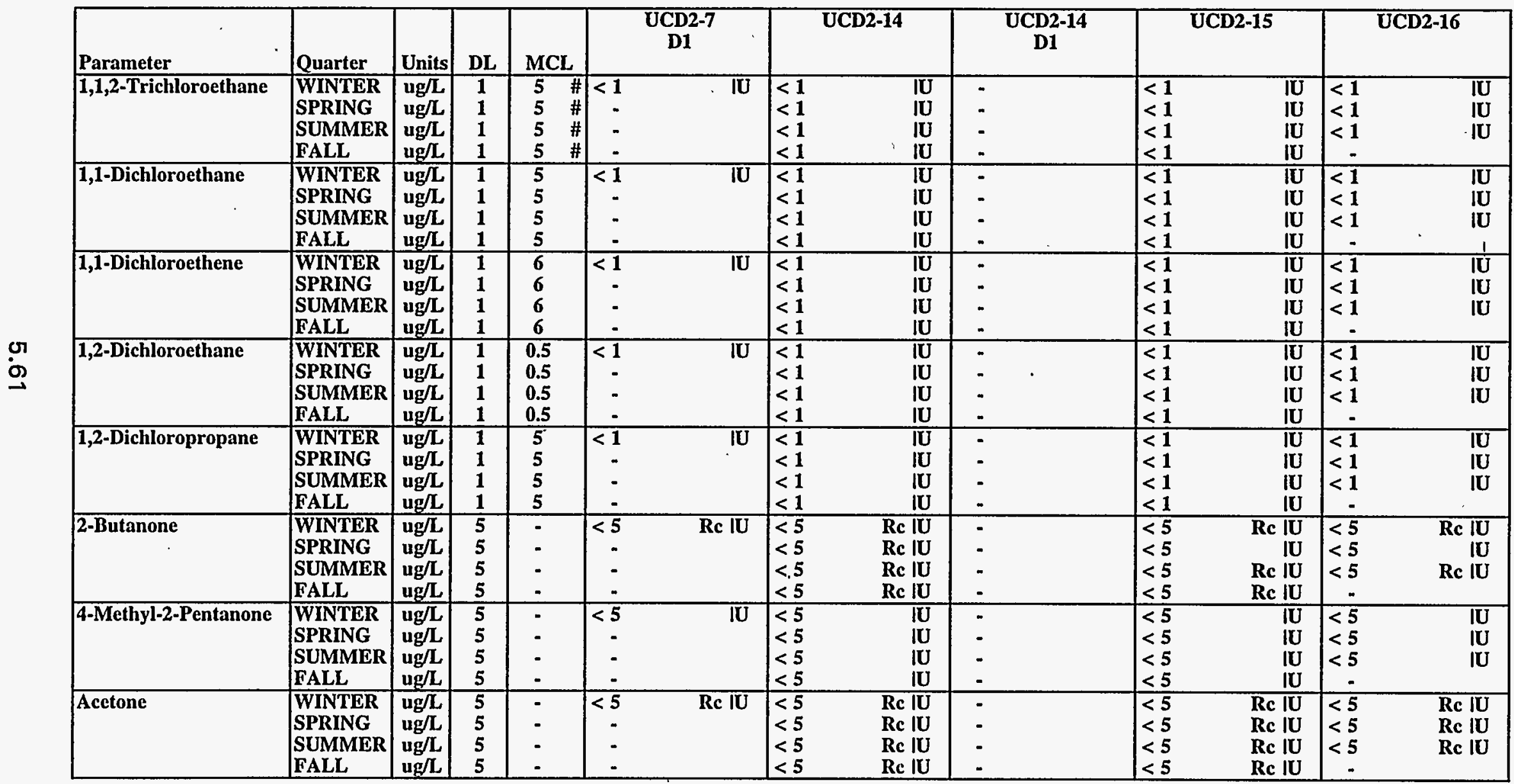

Page 11 of 17 
TABLE 5.2

SUMMARY OF DETECTED CONSTITUENTS IN GROUNDWATER, 1995

VOLATILE ORGANICS

1995 ANNUAL WATER MONITORING REPORT

LEHR ENVIRONMENTAL RESTORATION, DAVIS, CALIFORNIA

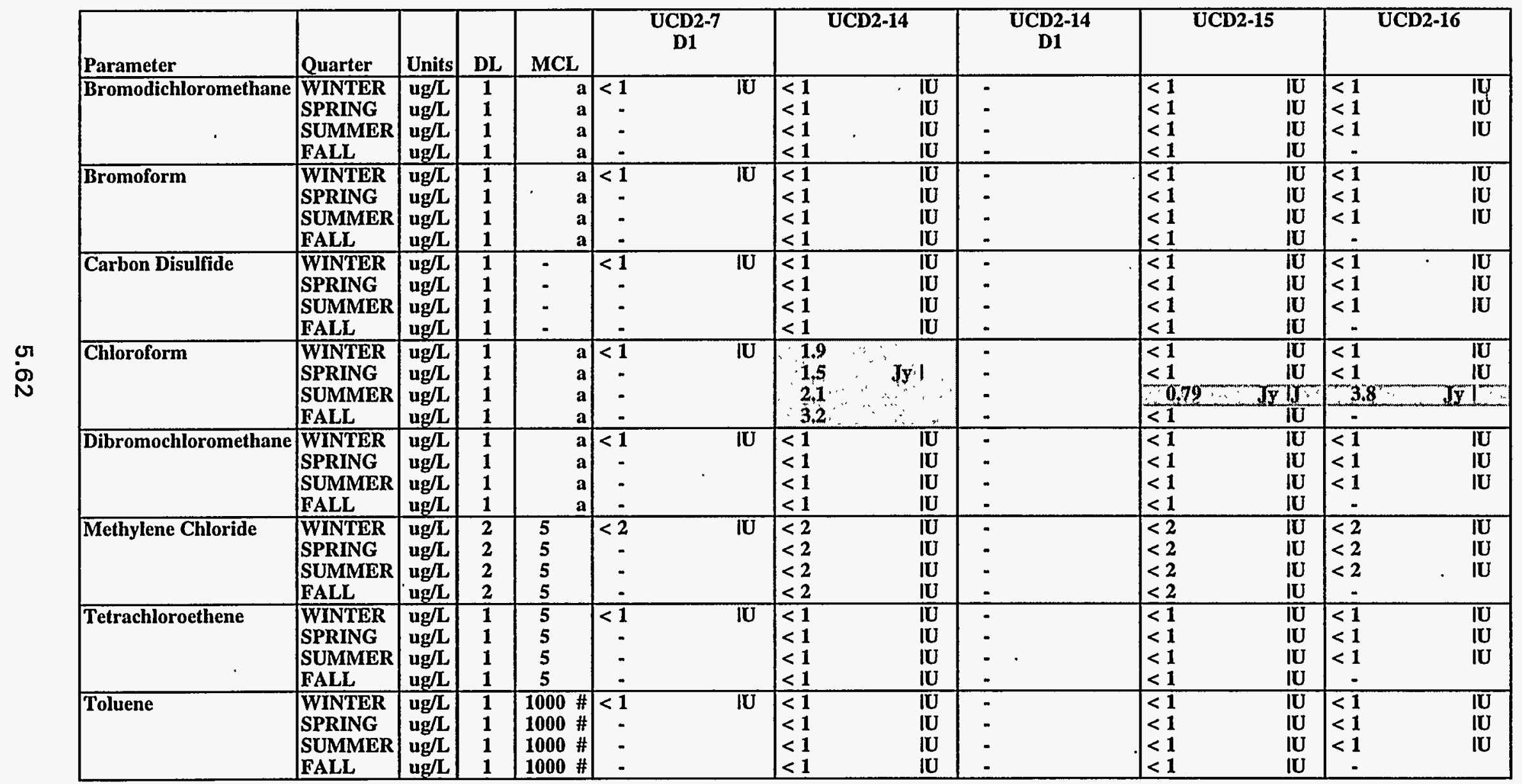


TABLE 5.2

SUMMARY OF DETECTED CONSTITUENTS IN GROUNDWATER, 1995

VOLATILE ORGANICS

1995 ANNUAL WATER MONITORING REPORT

LEHR ENVIRONMENTAL RESTORATION, DAVIS, CALIFORNIA

\begin{tabular}{|c|c|c|c|c|c|c|c|c|c|c|c|c|c|c|}
\hline \multirow{2}{*}{\begin{tabular}{|l} 
Parameter \\
$1,1,2-$ Trichloroethane
\end{tabular}} & \multirow[b]{2}{*}{\begin{tabular}{|l} 
Quarter \\
WINTER \\
SPRING \\
SUMMER \\
FALL \\
\end{tabular}} & \multirow[b]{2}{*}{$\begin{array}{l}\text { Units } \\
\text { ug/L } \\
\text { ug/L } \\
\text { ug/L } \\
\text { ug/L }\end{array}$} & \multirow[b]{2}{*}{$\begin{array}{c}\text { DL } \\
1 \\
1 \\
1 \\
1 \\
\end{array}$} & MCL & \multicolumn{2}{|c|}{ UCD2-17 } & \multicolumn{2}{|c|}{$\overline{\mathrm{UCD} 2-26}$} & \multicolumn{2}{|c|}{ UCD2-27Z4 } & \multicolumn{2}{|c|}{ UCD2-27Z5 } & \multicolumn{2}{|c|}{ UCD2-27Z6 } \\
\hline & & & & \begin{tabular}{cc|}
5 & $\#$ \\
5 & $\#$ \\
5 & $\#$ \\
5 & $\#$
\end{tabular} & $\begin{array}{l}<1 \\
<1 \\
\vdots \\
-\end{array}$ & $\begin{array}{l}\mathbf{I U} \\
\mathbf{I U}\end{array}$ & $\begin{array}{r}- \\
<1\end{array}$ & $\mathbf{I U}$ & $\begin{array}{r}- \\
<1\end{array}$ & $\mathbf{I U}$ & $\begin{array}{r}- \\
\end{array}$ & $\mathbf{I U}$ & 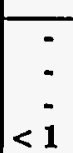 & $\mathbf{I U}$ \\
\hline 1,1-Dichloroethane & $\begin{array}{l}\text { WINTER } \\
\text { SPRING } \\
\text { SUMMER } \\
\text { FALL } \\
\end{array}$ & $\begin{array}{l}\mathrm{ug} / \mathrm{L} \\
\mathrm{ug} / \mathrm{L} \\
\mathrm{ug} / \mathrm{L} \\
\mathrm{ug} / \mathrm{L}\end{array}$ & $\begin{array}{l}1 \\
1 \\
1 \\
1\end{array}$ & \begin{tabular}{l|}
5 \\
5 \\
5 \\
5 \\
\end{tabular} & $\begin{array}{r}<1 \\
<1 \\
- \\
-\end{array}$ & $\mathbf{I U}$ & $\begin{array}{r}- \\
- \\
<1 \\
\end{array}$ & $\mathbf{I U}$ & $\begin{array}{r}- \\
< \\
\end{array}$ & 10 & $\begin{array}{r}- \\
< \\
<1 \\
\end{array}$ & 10 & $\begin{array}{r}: \\
< \\
<1\end{array}$ & $\mathbf{I U}$ \\
\hline 1,1-Dichloroethene & \begin{tabular}{|l|} 
WINTER \\
SPRING \\
SUMMER \\
FALL
\end{tabular} & $\begin{array}{l}\text { ug/L } \\
\text { ug/L } \\
\text { ug/L } \\
\text { ug/L }\end{array}$ & $\begin{array}{l}1 \\
1 \\
1 \\
1\end{array}$ & $\begin{array}{l}6 \\
6 \\
6 \\
6\end{array}$ & $\begin{array}{r}<1 \\
<1 \\
-\end{array}$ & $\begin{array}{l}\mathbf{I U} \\
\mathbf{I U}\end{array}$ & $\begin{array}{r}- \\
\\
<1\end{array}$ & IU & $\begin{array}{r}- \\
<1\end{array}$ & $\mathbf{I U}$ & $\begin{array}{r}- \\
<i\end{array}$ & $\mathbf{I U}$ & $\begin{array}{r}- \\
\\
<1\end{array}$ & IU \\
\hline 1,2-Dichloroethane & $\begin{array}{l}\text { WINTER } \\
\text { SPRING } \\
\text { SUMMER } \\
\text { FALL } \\
\end{array}$ & $\begin{array}{l}\mathrm{ug} / \mathrm{L} \\
\mathrm{ug} / \mathrm{L} \\
\mathrm{ug} / \mathrm{L} \\
\mathrm{ug} / \mathrm{L}\end{array}$ & $\begin{array}{l}1 \\
1 \\
1 \\
1\end{array}$ & $\begin{array}{l}0.5 \\
0.5 \\
0.5 \\
0.5 \\
\end{array}$ & $\begin{array}{r}<1 \\
<1 \\
- \\
-\end{array}$ & $\begin{array}{l}\mathbf{I U} \\
\mathbf{I U}\end{array}$ & $\begin{array}{r}- \\
< \\
<1 \\
\end{array}$ & $\mathbf{I U}$ & $\begin{array}{r}: \\
< \\
<1 \\
\end{array}$ & $\mathbf{U}$ & $\begin{array}{r}- \\
- \\
<1 \\
\end{array}$ & $\mathbf{I U}$ & $\begin{array}{r}- \\
<1 \\
\end{array}$ & $\mathbf{I U}$ \\
\hline 1,2-Dichloropropane & $\begin{array}{l}\text { WINTER } \\
\text { SPRING } \\
\text { SUMMER } \\
\text { FALL }\end{array}$ & $\begin{array}{l}\mathrm{ug} / \mathrm{L} \\
\mathrm{ug} / \mathrm{L} \\
\mathrm{ug} / \mathrm{L} \\
\mathrm{ug} / \mathrm{L}\end{array}$ & $\begin{array}{l}1 \\
1 \\
1 \\
1\end{array}$ & $\begin{array}{l}5 \\
5 \\
5 \\
5\end{array}$ & $\begin{array}{r}<1 \\
<1 \\
- \\
-\end{array}$ & $\begin{array}{l}\mathbf{I U} \\
\mathbf{I U}\end{array}$ & $\begin{array}{r}- \\
- \\
<1\end{array}$ & IU & $\begin{array}{r}- \\
<1\end{array}$ & IU & $\begin{array}{r}- \\
- \\
<1\end{array}$ & $\mathbf{I U}$ & $\begin{array}{r}- \\
<1\end{array}$ & IU \\
\hline 2-Butanone & $\begin{array}{l}\text { WINTER } \\
\text { SPRING } \\
\text { SUMMER } \\
\text { FALL } \\
\end{array}$ & $\begin{array}{l}\mathrm{ug} / \mathrm{L} \\
\mathrm{ug} / \mathrm{L} \\
\mathrm{ug} / \mathrm{L} \\
\mathrm{ug} / \mathrm{L} \\
\end{array}$ & $\begin{array}{l}5 \\
5 \\
5 \\
5 \\
\end{array}$ & $\begin{array}{l}- \\
- \\
-\end{array}$ & $\begin{array}{r}<5 \\
<5 \\
\vdots \\
\end{array}$ & $\begin{array}{l}\text { Rc IU } \\
\text { Re IU }\end{array}$ & $\begin{array}{r}- \\
- \\
<5 \\
\end{array}$ & $\operatorname{Re} \mid \mathbf{U}$ & $\begin{array}{r}- \\
<5 \\
\end{array}$ & Re IU & $\begin{array}{r}- \\
- \\
<5 \\
\end{array}$ & Re IU & $\begin{array}{r}\vdots \\
<5 \\
\end{array}$ & $\mathbf{J} \mathbf{I U}$ \\
\hline 4-Methyl-2-Pentanone & $\begin{array}{l}\text { WINTER } \\
\text { SPRING } \\
\text { SUMMER } \\
\text { FALL }\end{array}$ & $\begin{array}{l}\mathrm{ugg} / \mathrm{L} \\
\mathrm{ug} / \mathrm{L} \\
\mathrm{ug} / \mathrm{L} \\
\mathrm{ug} / \mathrm{L}\end{array}$ & $\begin{array}{l}5 \\
5 \\
5 \\
5\end{array}$ & :- & $\begin{array}{r}<5 \\
<5 \\
: \\
\end{array}$ & $\begin{array}{l}\mathbf{I U} \\
\mathbf{I U}\end{array}$ & $\begin{array}{l}- \\
<5\end{array}$ & IU & $\begin{array}{l}- \\
<5\end{array}$ & IU & $\begin{array}{r}- \\
- \\
<5\end{array}$ & $2 \pi$ & $\begin{array}{l}-5 \\
<5\end{array}$ & 310 \\
\hline Acetone & $\begin{array}{l}\text { WINTER } \\
\text { SPRING } \\
\text { SUMMER } \\
\text { FALL }\end{array}$ & $\begin{array}{l}\text { ug/h } \\
\text { ugh/ } \\
\text { ugh } \\
\text { ugh }\end{array}$ & $\begin{array}{l}5 \\
5 \\
5 \\
5\end{array}$ & $:$ & $\begin{array}{r}<5 \\
<5 \\
5\end{array}$ & $\begin{array}{l}\operatorname{Rc} \text { IU } \\
\operatorname{Re} \text { IU }\end{array}$ & $\begin{array}{l}- \\
< \\
<5\end{array}$ & $\operatorname{Re} \mid \mathbf{U}$ & $\begin{array}{l}- \\
- \\
3 .\end{array}$ & $\mathrm{Jc} \mid \mathbf{J B}$ & $\begin{array}{c}- \\
- \\
- \\
-2.2\end{array}$ & Ten & 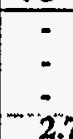 & $\mathrm{Tctor}$ \\
\hline
\end{tabular}

Page 13 of 17 
TABLE 5.2

SUMMARY OF DETECTED CONSTITUENTS IN GROUNDWATER, 1995

VOLATILE ORGANICS

1995 ANNUAL WATER MONITORING REPORT

LEHR ENVIRONMENTAL RESTORATION, DAVIS, CALIFORNIA

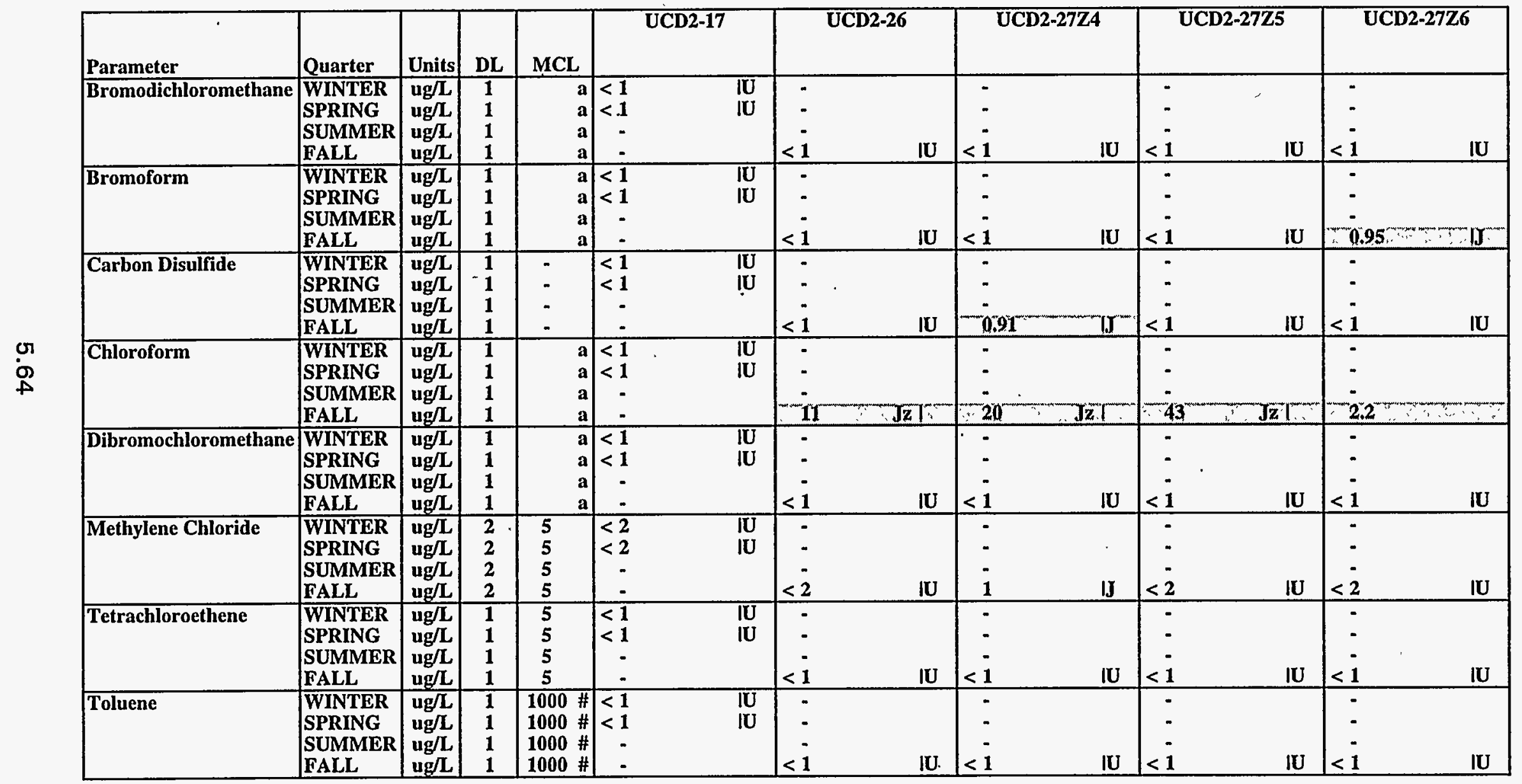


TABLE 5.2

SUMMARY OF DETECTED CONSTITUENTS IN GROUNDWATER, 1995

VOLATILE ORGANICS

1995 ANNUAL WATER MONITORING REPORT

LEHR ENVIRONMENTAL RESTORATION, DAVIS, CALIFORNIA

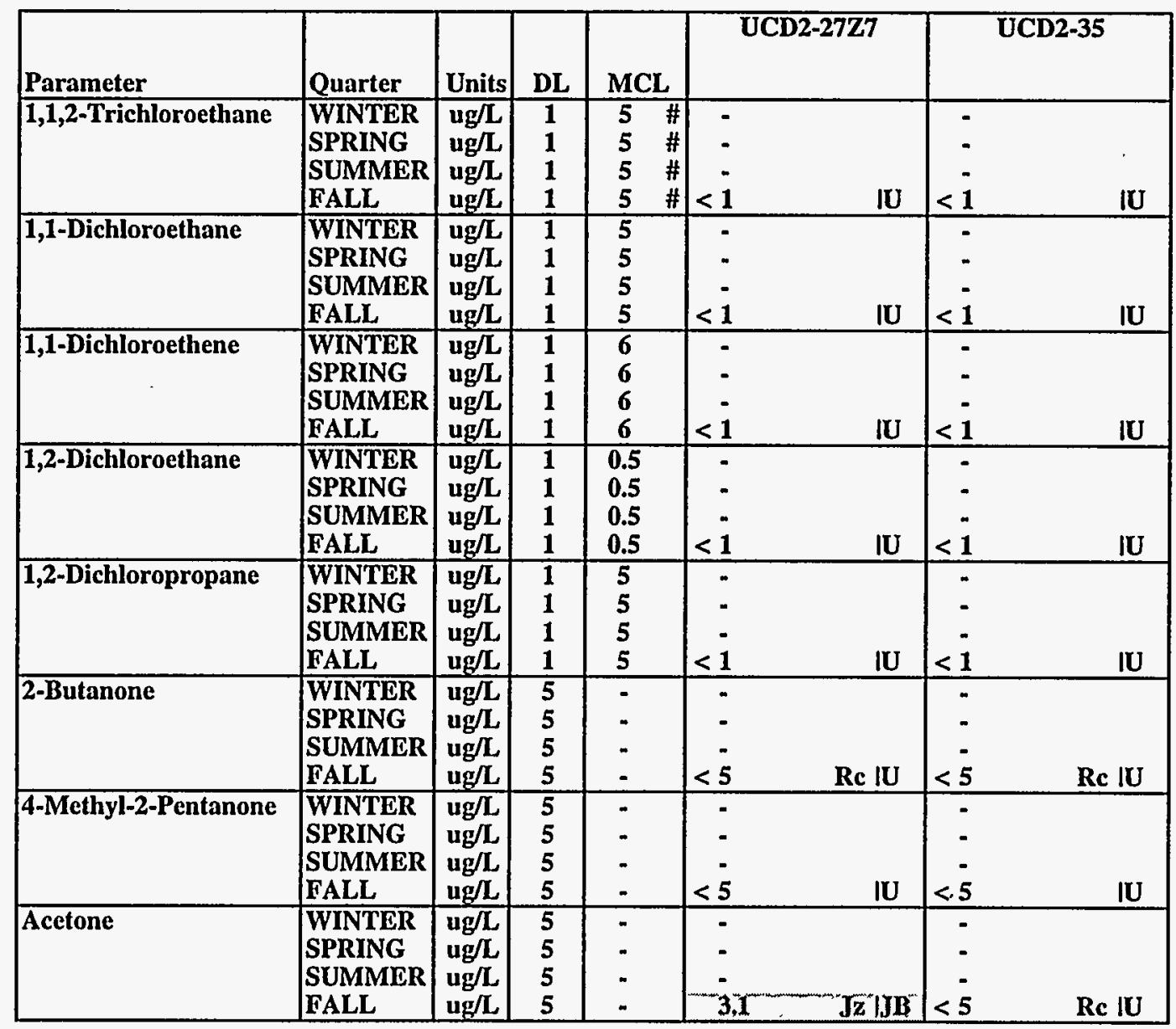

Page 15 of 17 
TABLE 5.2

SUMMARY OF DETECTED CONSTITUENTS IN GROUNDWATER, 1995

VOLATILE ORGANICS

1995 ANNUAL WATER MONITORING REPORT

LEHR ENVIRONMENTAL RESTORATION, DAVIS, CALIFORNIA

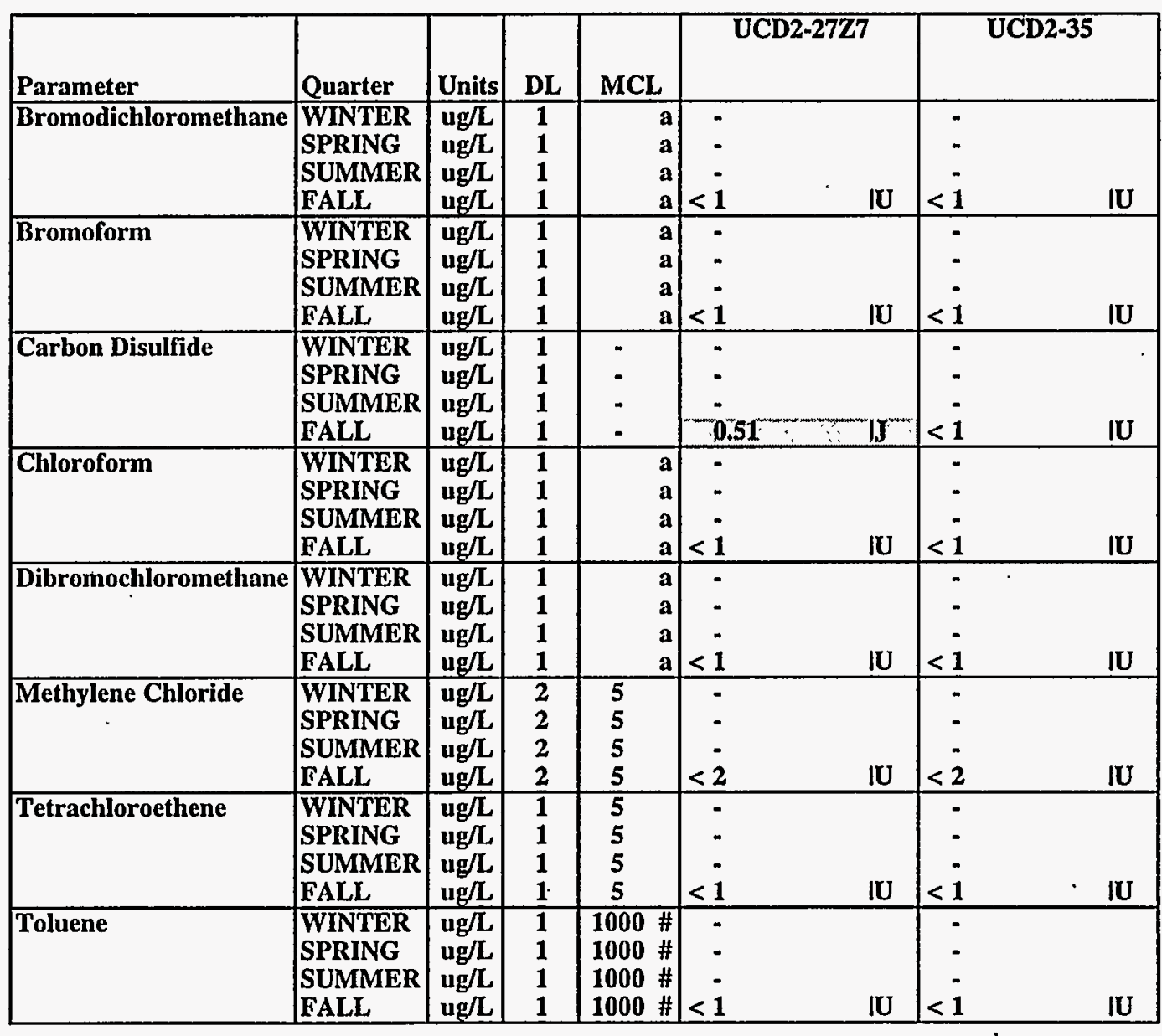

Page 16 of 17 
TABLE 5.2

SUMMARY OF DETECTED CONSTITUENTS IN GROUNDWATER, 1995

VOLATILE ORGANICS

1995 ANNUAL WATER MONITORING REPORT

LEHR ENVIRONMENTAL RESTORATION, DAVIS, CALIFORNIA

D1 = Duplicate sample.

- = Parameter not analyzed or no MCL.

DL = Detection limit.

$<=$ Constituent below detection limit. Detection limits may vary depending on interference by other sample constituents.

MCL = Maximum Contaminant Level (primary), Title 22 Division 4 Chapter 15.

\# = USEPA MCL

$a=$ MCL for total trihalomethanes is $100 \mathrm{ug} / \mathrm{L}$. Chloroform is the predominant trihalomethane detected at LEHR.

$b=$ Volatile organic sample likely collected from UCD1-12. Results reported (ug/L) by the laboratory are as follows:

1,1-Dichloroethane $=6.8 ; 1,1$-Dichloroethene $=13 ; 1,2-$ Dichloroethane $=3.3 ; 1,2$-Dichloropropane $=0.51$;

Acetone $=4.7$; Bromodichloromethane $=0.7$; Chloroform $=5,800$.

$c=$ Laboratory reported chloroform concentration of $140 \mathrm{ug} / \mathrm{L}$ was determined to be laboratory error.

Highlighted values indicate positive results.

See Appendix A for explanation of data qualifiers. 
TABLE 5.3

SUMMARY OF DETECTED CONSTITUENTS IN GROUNDWATER, 1995 SEMIVOLATILE ORGANIC COMPOUNDS

1995 ANNUAL WATER MONITORING REPORT

LEHR ENVIRONMENTAL RESTORATION, DAVIS, CALIFORNIA

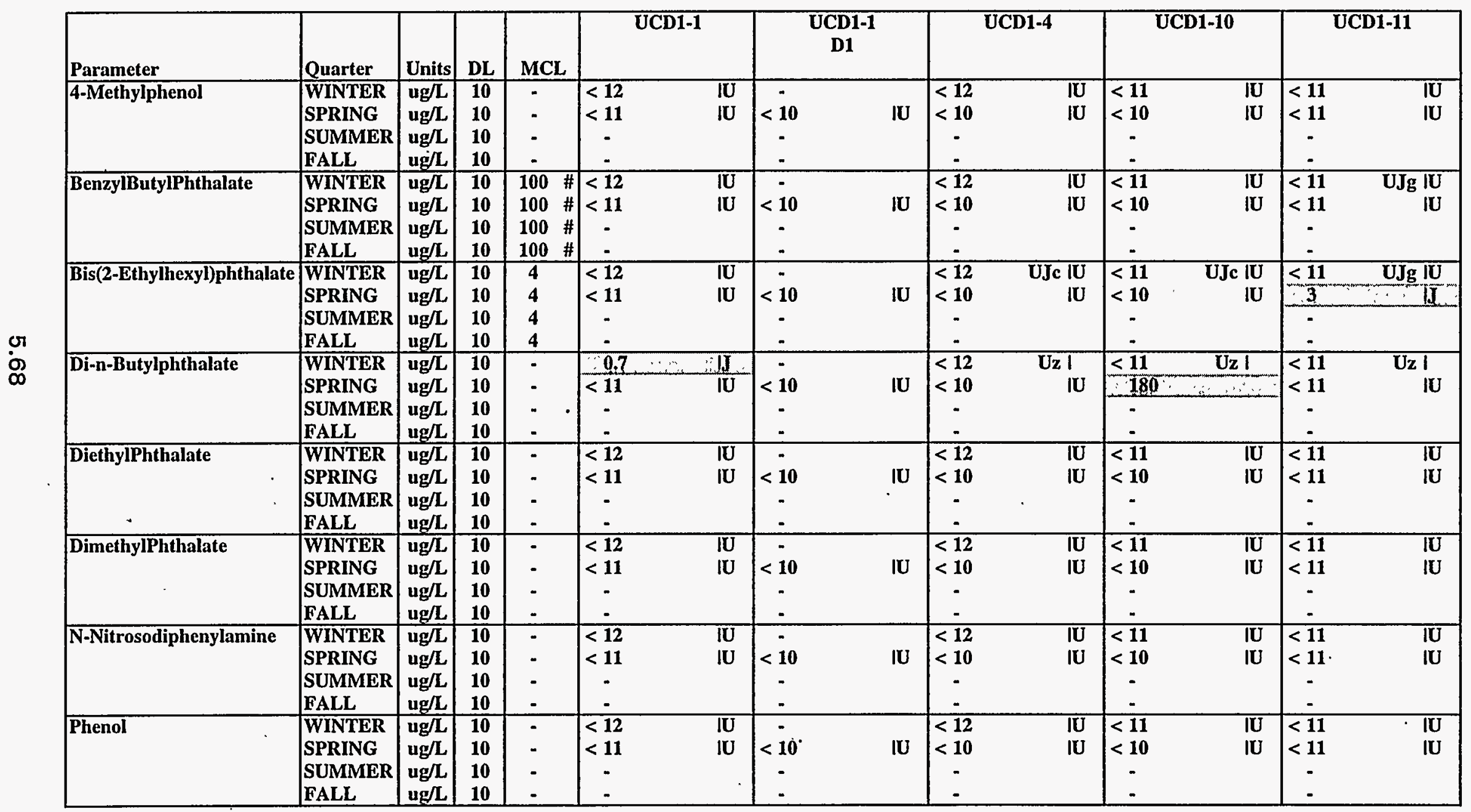


TABLE 5.3

SUMMARY OF DETECTED CONSTITUENTS IN GROUNDWATER, 1995

SEMIVOLATILE ORGANIC COMPOUNDS

1995 ANNUAL WATER MONITORING REPORT

LEHR ENVIRONMENTAL RESTORATION, DAVIS, CALIFORNIA

\begin{tabular}{|c|c|c|c|c|c|c|c|c|c|c|c|}
\hline Parameter & Quarter & Units & DL & MCL & & & $\begin{array}{l}\text { UCD1-12 } \\
\text { D1 }\end{array}$ & $\begin{array}{l}\text { UCD1-12 } \\
\text { D2 }\end{array}$ & & & $\begin{array}{c}\text { UCD1-13 } \\
\text { D1 }\end{array}$ \\
\hline 4-Methylphenol & \begin{tabular}{|l|} 
WINTER \\
SPRING \\
SUMMER \\
FALL \\
\end{tabular} & $\begin{array}{l}\mathrm{ug} / \mathrm{L} \\
\mathrm{ug} / \mathrm{L} \\
\mathrm{ug} / \mathrm{L} \\
\mathrm{ug} / \mathrm{L} \\
\end{array}$ & $\begin{array}{l}10 \\
10 \\
10 \\
10 \\
\end{array}$ & \begin{tabular}{l|}
- \\
- \\
- \\
- \\
\end{tabular} & $\begin{array}{c}<12 \\
<11 \\
- \\
- \\
\end{array}$ & $\begin{array}{l}\text { IU } \\
\text { IU }\end{array}$ & $\begin{array}{l}- \\
- \\
- \\
\end{array}$ & $\begin{array}{l}- \\
. \\
. \\
\end{array}$ & $\begin{array}{c}<12 \\
<11 \\
- \\
- \\
\end{array}$ & $\begin{array}{l}\text { IU } \\
\text { IU }\end{array}$ & $\begin{array}{l}- \\
- \\
- \\
\end{array}$ \\
\hline BenzylButylPhthalate & \begin{tabular}{|l|} 
WINTER \\
SPRING \\
SUMMER \\
FALL \\
\end{tabular} & $\begin{array}{l}\mathrm{ug} / \mathrm{L} \\
\mathrm{ug} / \mathrm{L} \\
\mathrm{ug} / \mathrm{L} \\
\mathrm{ug} / \mathrm{L}\end{array}$ & $\begin{array}{l}10 \\
10 \\
10 \\
10 \\
\end{array}$ & \begin{tabular}{ll|}
100 & $\#$ \\
100 & $\#$ \\
100 & $\#$ \\
100 & $\#$ \\
\end{tabular} & $\begin{array}{c}<12 \\
<11 \\
- \\
-\end{array}$ & $\begin{array}{l}\text { IU } \\
\text { IU }\end{array}$ & 5 & 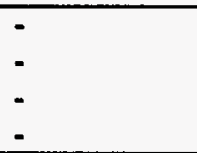 & $\begin{array}{c}<12 \\
<11 \\
\quad ! \\
.\end{array}$ & $\begin{array}{l}\text { IU } \\
\text { IU }\end{array}$ & $\begin{array}{l}- \\
- \\
-\end{array}$ \\
\hline $\begin{array}{c}\text { Bis(2-Ethylhexyl)phthalate } \\
\text {. }\end{array}$ & \begin{tabular}{|l|} 
WINTER \\
SPRING \\
SUMMER \\
FALL \\
\end{tabular} & $\begin{array}{l}\mathrm{ug} / \mathrm{L} \\
\mathrm{ug} / \mathrm{L} \\
\mathrm{ug} / \mathrm{L} \\
\mathrm{ug} / \mathrm{L}\end{array}$ & $\begin{array}{l}10 \\
10 \\
10 \\
10 \\
\end{array}$ & \begin{tabular}{l|}
4 \\
4 \\
4 \\
4 \\
\end{tabular} & $\begin{array}{c}<12 \\
<11 \\
- \\
- \\
\end{array}$ & $\begin{array}{l}\mathrm{IU} \\
\mathrm{IU}\end{array}$ & $\begin{array}{l}- \\
- \\
- \\
\end{array}$ & $\begin{array}{l}- \\
- \\
- \\
\end{array}$ & $\begin{array}{c}<12 \\
<11 \\
- \\
- \\
\end{array}$ & $\begin{array}{l}\text { IU } \\
\text { IU }\end{array}$ & $\begin{array}{l}- \\
- \\
-\end{array}$ \\
\hline Di-n-Butylphthalate & $\begin{array}{l}\text { WINTER } \\
\text { SPRING } \\
\text { SUMMER } \\
\text { FALL } \\
\end{array}$ & $\begin{array}{l}\mathrm{ug} / \mathrm{L} \\
\mathrm{ug} / \mathrm{L} \\
\mathrm{ug} / \mathrm{L} \\
\mathrm{ug} / \mathrm{L} \\
\end{array}$ & $\begin{array}{l}10 \\
10 \\
10 \\
10 \\
\end{array}$ & $\begin{array}{l}- \\
- \\
- \\
\end{array}$ & $\begin{array}{c}2 \\
<11 \\
- \\
- \\
\end{array}$ & $\frac{\mathrm{IJ}}{\mathrm{IU}}$ & $\begin{array}{l}- \\
- \\
- \\
\end{array}$ & $\begin{array}{l}- \\
- \\
- \\
\end{array}$ & $\begin{array}{c}\frac{2}{<11} \\
\\
- \\
\end{array}$ & $\frac{\mathrm{II}}{\mathrm{IU}}$ & $\begin{array}{l}- \\
- \\
- \\
-\end{array}$ \\
\hline DiethylPhthalate & \begin{tabular}{|l|} 
WINTER \\
SPRING \\
SUMMER \\
FALL \\
\end{tabular} & $\begin{array}{l}\mathrm{ug} / \mathrm{L} \\
\mathrm{ug} / \mathrm{L} \\
\mathrm{ug} / \mathrm{L} \\
\mathrm{ug} / \mathrm{L} \\
\end{array}$ & $\begin{array}{l}10 \\
10 \\
10 \\
10 \\
\end{array}$ & $\begin{array}{l}. \\
- \\
.\end{array}$ & $\begin{array}{c}<12 \\
<11 \\
- \\
- \\
\end{array}$ & $\begin{array}{l}\text { IU } \\
\text { IU }\end{array}$ & $\begin{array}{l}- \\
- \\
-\end{array}$ & $\begin{array}{l}- \\
- \\
- \\
-\end{array}$ & $\begin{array}{c}<12 \\
<11 \\
- \\
-\end{array}$ & $\begin{array}{l}\text { IU } \\
\text { IU }\end{array}$ & $\begin{array}{l}- \\
- \\
- \\
-\end{array}$ \\
\hline DimethylPhthalate & \begin{tabular}{l|} 
WINTER \\
SPRING \\
SUMMER \\
FALL \\
\end{tabular} & $\begin{array}{l}\mathrm{ug} / \mathrm{L} \\
\mathrm{ug} / \mathrm{L} \\
\mathrm{ug} / \mathrm{L} \\
\mathrm{ug} / \mathrm{L}\end{array}$ & $\begin{array}{l}10 \\
10 \\
10 \\
10 \\
\end{array}$ & $\begin{array}{l}- \\
- \\
- \\
\end{array}$ & $\begin{array}{c}<12 \\
<11 \\
- \\
- \\
\end{array}$ & $\begin{array}{l}\text { IU } \\
\text { IU }\end{array}$ & $\begin{array}{l}i \\
. \\
-\end{array}$ & $\begin{array}{l}. \\
. \\
.\end{array}$ & $\begin{array}{c}<12 \\
<11 \\
- \\
-\end{array}$ & $\begin{array}{l}\text { IU } \\
\text { IU }\end{array}$ & - \\
\hline N-Nitrosodiphenylamine & $\begin{array}{l}\text { WINTER } \\
\text { SPRING } \\
\text { SUMMER } \\
\text { FALL } \\
\end{array}$ & $\begin{array}{l}\mathrm{ug} / \mathrm{L} \\
\mathrm{ug} / \mathrm{L} \\
\mathrm{ug} / \mathrm{L} \\
\mathrm{ug} / \mathrm{L} \\
\end{array}$ & $\begin{array}{l}10 \\
10 \\
10 \\
10 \\
\end{array}$ & $\begin{array}{l}- \\
- \\
-\end{array}$ & $\begin{array}{c}<12 \\
<11 \\
- \\
- \\
\end{array}$ & IU & $\begin{array}{l}. \\
. \\
.\end{array}$ & - & $\begin{array}{c}<12 \\
<11 \\
- \\
-\end{array}$ & $\begin{array}{l}\text { IU } \\
\text { IU }\end{array}$ & $\begin{array}{l}- \\
- \\
-\end{array}$ \\
\hline Phenol & $\begin{array}{l}\text { WINTER } \\
\text { SPRING } \\
\text { SUMMER } \\
\text { FALL } \\
\end{array}$ & $\begin{array}{l}\mathrm{ug} / \mathrm{L} \\
\mathrm{ug} / \mathrm{L} \\
\mathrm{ug} / \mathrm{L} \\
\mathrm{ug} / \mathrm{L}\end{array}$ & $\begin{array}{l}10 \\
10 \\
10 \\
10 \\
\end{array}$ & $\begin{array}{l}- \\
- \\
-\end{array}$ & $\begin{array}{c}<12 \\
<11 \\
. \\
. \\
\end{array}$ & $\begin{array}{l}\text { IU } \\
\text { IU }\end{array}$ & $\begin{array}{l}- \\
- \\
- \\
-\end{array}$ & $\begin{array}{l}- \\
- \\
- \\
-\end{array}$ & $\begin{array}{c}<12 \\
<11 \\
- \\
-\end{array}$ & $\begin{array}{l}\text { IU } \\
\text { IU }\end{array}$ & $\begin{array}{l}- \\
- \\
-\end{array}$ \\
\hline
\end{tabular}


TABLE 5.3

SUMMARY OF DETECTED CONSTITUENTS IN GROUNDWATER, 1995

SEMIVOLATILE ORGANIC COMPOUNDS

1995 ANNUAL WATER MONITORING REPORT

LEHR ENVIRONMENTAL RESTORATION, DAVIS, CALIFORNIA

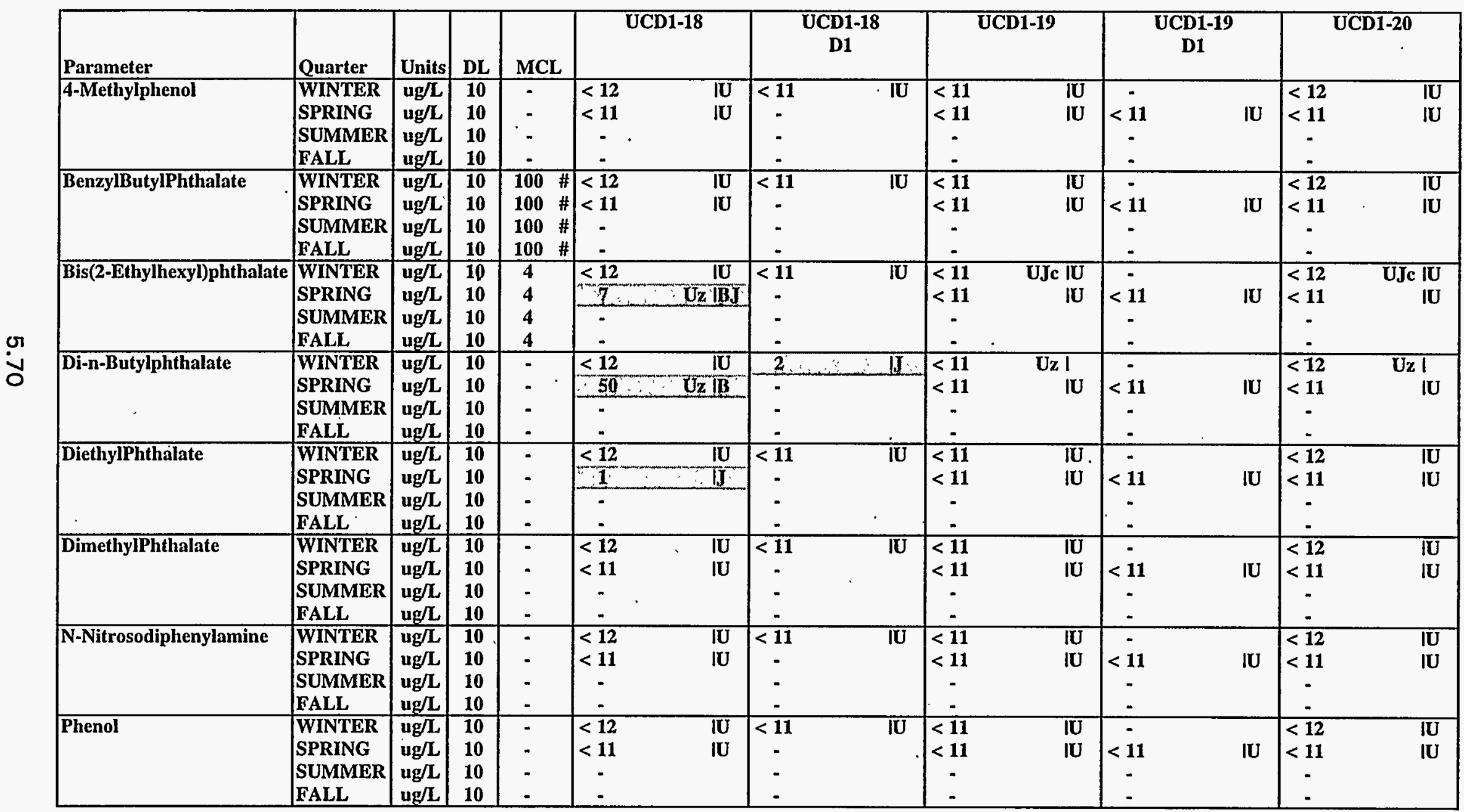


TABLE 5.3

SUMMARY OF DETECTED CONSTITUENTS IN GROUNDWATER, 1995

SEMIVOLATILE ORGANIC COMPOUNDS

1995 ANNUAL WATER MONITORING REPORT

LEHR ENVIRONMENTAL RESTORATION, DAVIS, CALIFORNIA

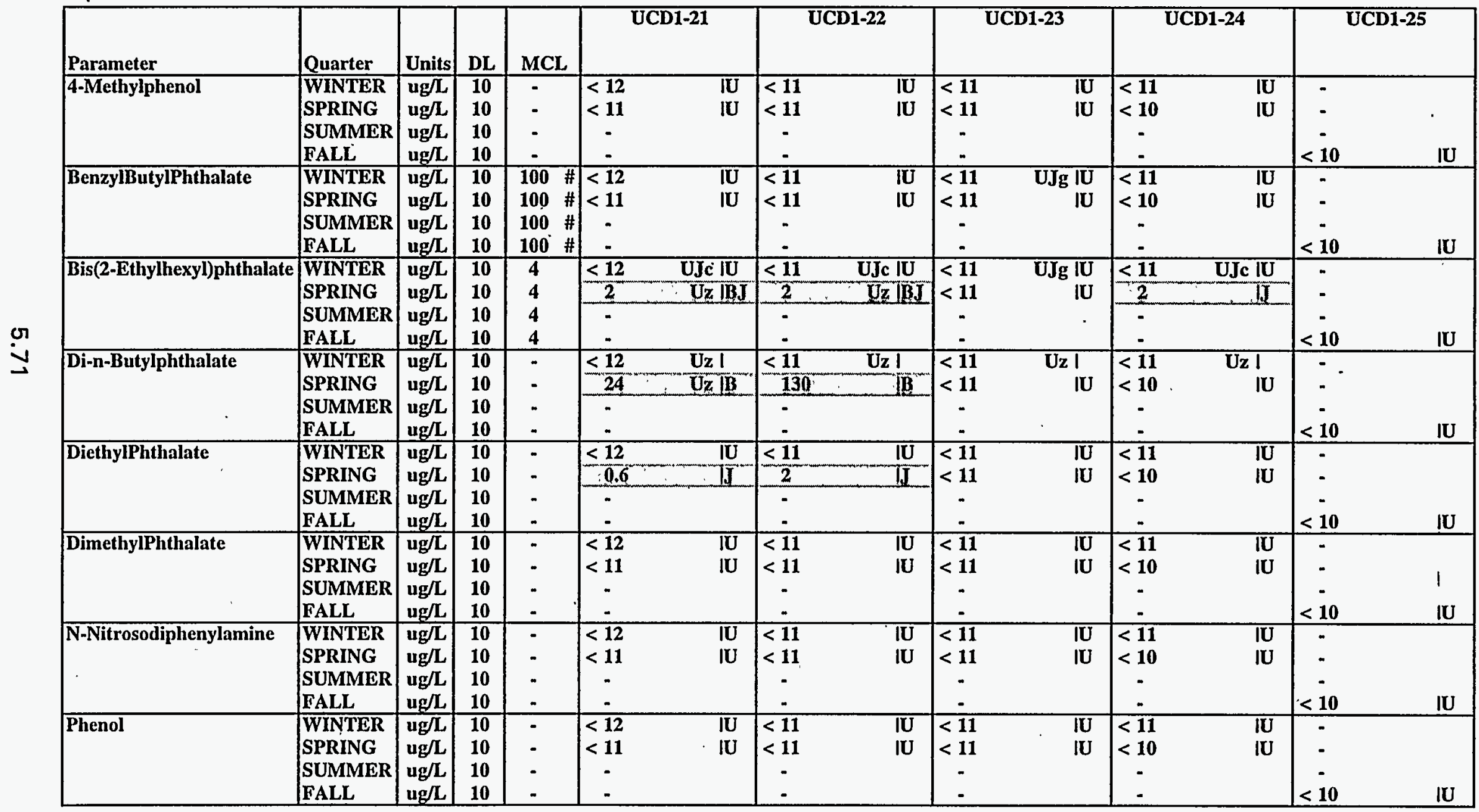


TABLE 5.3

SUMMARY OF DETECTED CONSTITUENTS IN GROUNDWATER, 1995

SEMIVOLATILE ORGANIC COMPOUNDS

1995 ANNUAL WATER MONITORING REPORT

LEHR ENVIRONMENTAL RESTORATION, DAVIS, CALIFORNIA

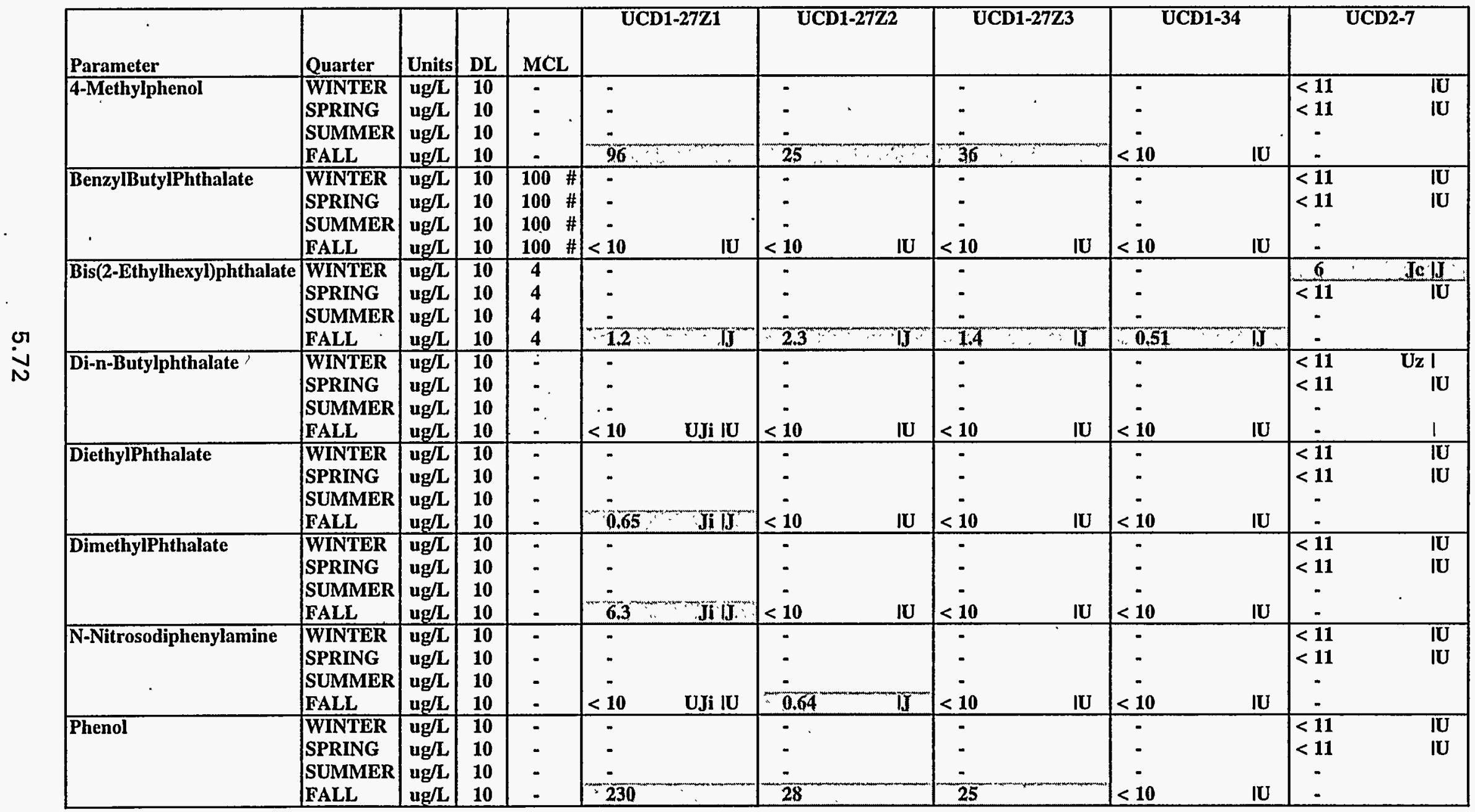


TABLE 5.3

SUMMARY OF DETECTED CONSTITUENTS IN GROUNDWATER, 1995

SEMIVOLATILE ORGANIC COMPOUNDS

1995 ANNUAL WATER MONITORING REPORT

LEHR ENVIRONMENTAL RESTORATION, DAVIS, CALIFORNIA

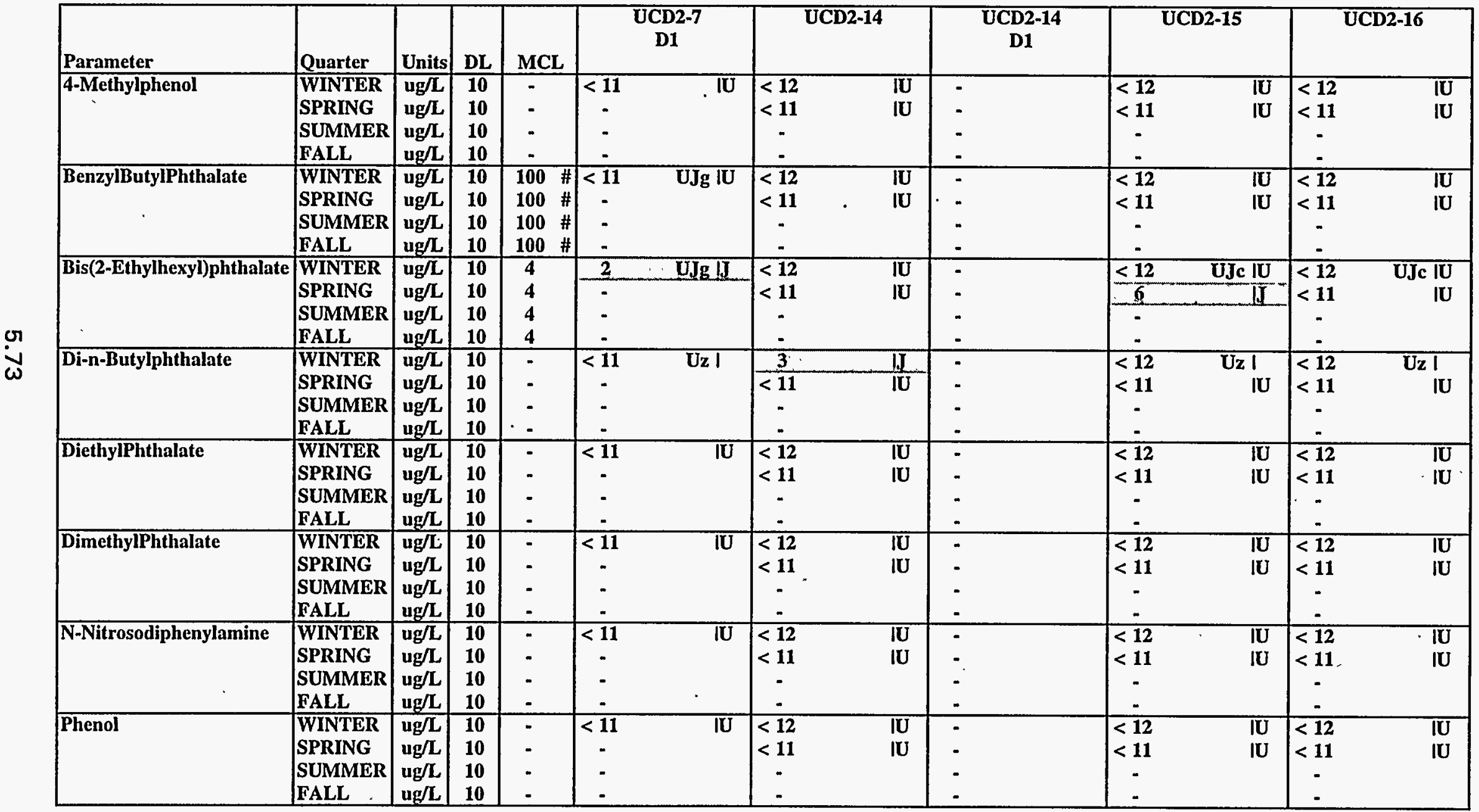


TABLE 5.3

SUMMARY OF DETECTED CONSTITUENTS IN GROUNDWATER, 1995

SEMIVOLATILE ORGANIC COMPOUNDS

1995 ANNUAL WATER MONITORING REPORT

LEHR ENVIRONMENTAL RESTORATION, DAVIS, CALIFORNIA

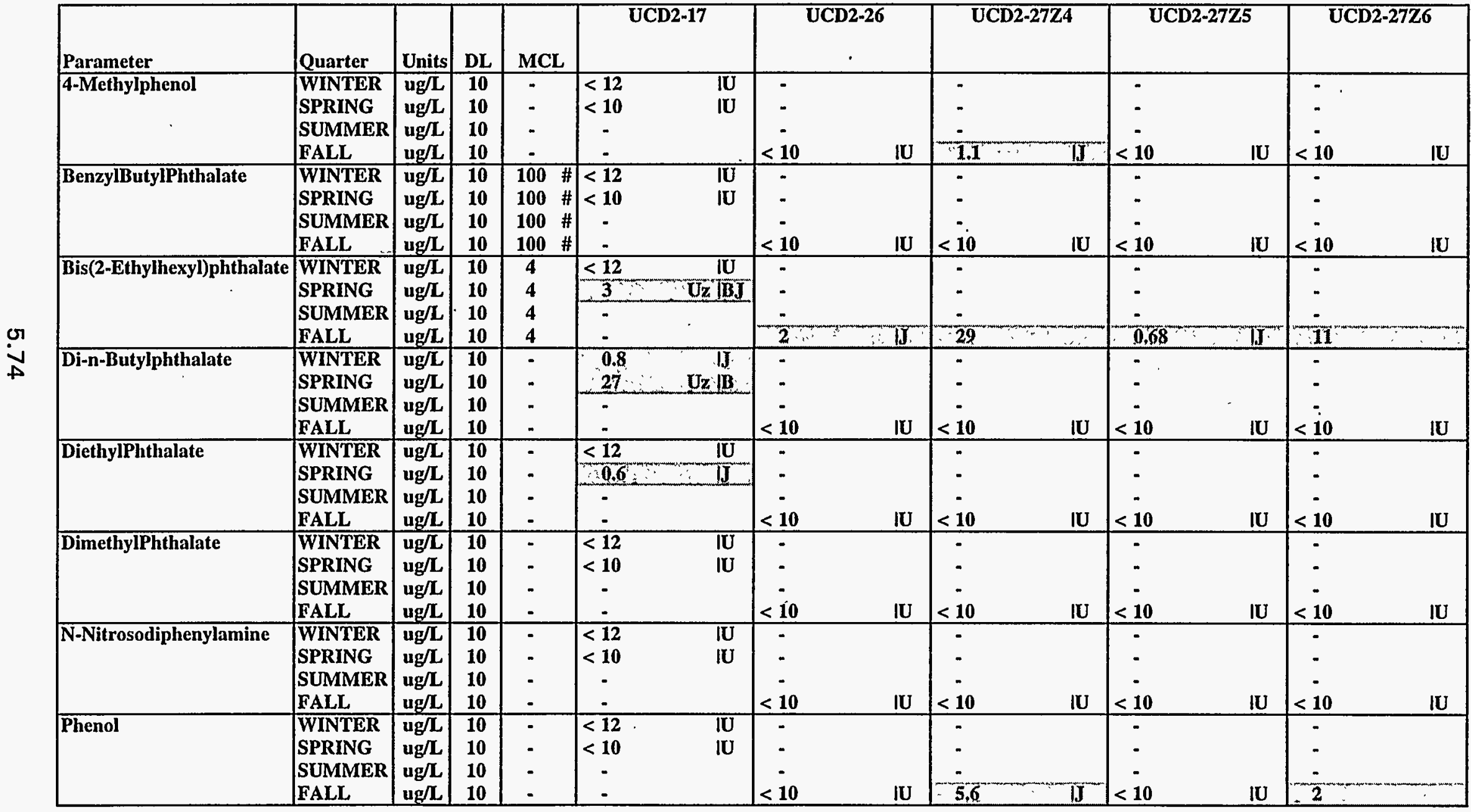


TABLE 5.3

SUMMARY OF DETECTED CONSTITUENTS IN GROUNDWATER, 1995

SEMIVOLATILE ORGANIC COMPOUNDS

1995 ANNUAL WATER MONITORING REPORT

LEHR ENVIRONMENTAL RESTORATION, DAVIS, CALIFORNIA

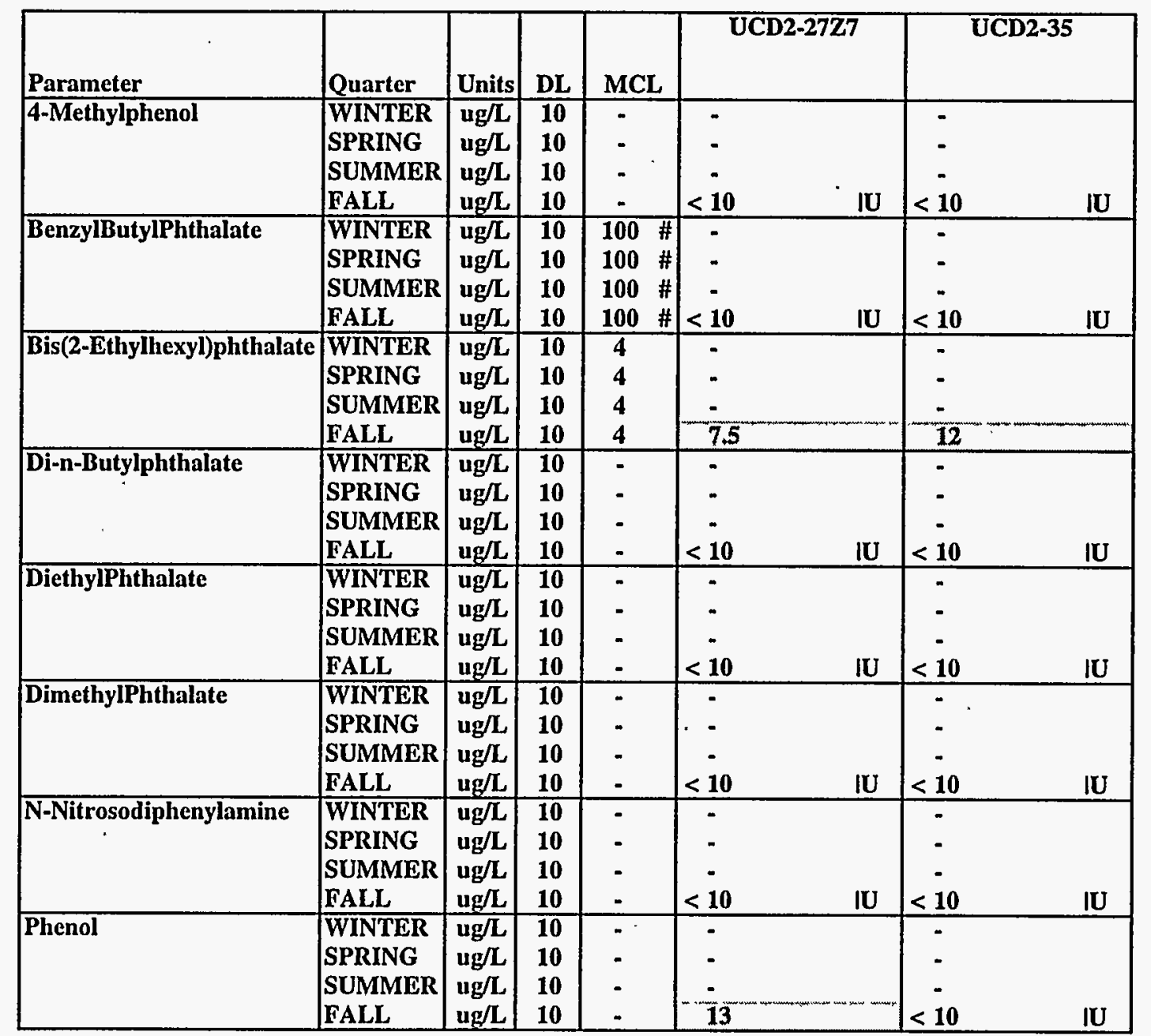


TABLE 5.3

SUMMARY OF DETECTED CONSTITUENTS IN GROUNDWATER, 1995

SEMIVOLATILE ORGANIC COMPOUNDS

1995 ANNUAL WATER MONITORING REPORT

LEHR ENVIRONMENTAL RESTORATION, DAVIS, CALIFORNIA

D1 = Duplicate sample.

- = Parameter not analyzed or no MCL.

DL $=$ Detection limit.

$<=$ Constituent below detection limit. Detection limits may vary depending on interference by other sample constituents.

MCL = Maximum Contaminant Level (primary), Title 22 Division 4 Chapter 15.

\# = USEPA MCL

Highlighted values indicate positive results.

See Appendix A for explanation of data qualifiers. 
TABLE 5.4

SUMMARY OF DETECTED CONSTITUENTS IN GROUNDWATER, 1995

PESTICIDES AND PCBS

1995 ANNUAL WATER MONITORING REPORT

LEHR ENVIRONMENTAL RESTORATION, DAVIS, CALIFORNIA

\begin{tabular}{|c|c|c|c|c|c|c|c|c|c|c|c|c|c|c|c|}
\hline Parameter & Quarter & Units & DL & MCL & UCD1-1 & & $\begin{array}{c}\text { UCD1-1 } \\
\text { D1 }\end{array}$ & UCD1-4 & & UCD1-10 & & UCD1-11 & & UCD1-12 & \\
\hline Alpha-Chlordane & $\begin{array}{l}\text { WINTER } \\
\text { SPRING } \\
\text { SUMMER } \\
\text { FALL }\end{array}$ & \begin{tabular}{|l|}
$\operatorname{ugg} / \mathrm{L}$ \\
$\mathrm{ug} / \mathrm{L}$ \\
$\mathrm{ug} / \mathrm{L}$ \\
$\mathrm{ug} / \mathrm{L}$
\end{tabular} & $\begin{array}{l}0.01 \\
0.01 \\
0.01 \\
0.01\end{array}$ & $:$ & $\begin{array}{l}<0.012 \\
<0.01 \\
- \\
-\end{array}$ & $\begin{array}{l}\mathbf{I U} \\
\mathbf{I U}\end{array}$ & $\begin{array}{ll}0.01 & \text { IU } \\
- & \end{array}$ & $\begin{array}{l}<0.012 \\
<0.011 \\
<0.01 \\
<0.01\end{array}$ & | & $\begin{array}{l}<0.011 \\
<0.011 \\
-\end{array}$ & $\begin{array}{l}\mathbf{I U} \\
\mathbf{I U}\end{array}$ & $\begin{array}{l}<0.011 \\
<0.011 \\
- \\
-\end{array}$ & $\begin{array}{l}\text { IU } \\
\text { IU } \\
\end{array}$ & $\begin{array}{l}<0.011 \\
<0.01 \\
<0.01 \\
<0.01\end{array}$ & $\begin{array}{l}\mathrm{IU} \\
\mathrm{IU} \\
\mathrm{IU} \\
\mathrm{IU}\end{array}$ \\
\hline Dieldrin & $\begin{array}{l}\text { WINTER } \\
\text { SPRING } \\
\text { SUMMER } \\
\text { FALL } \\
\end{array}$ & $\begin{array}{l}\mathrm{ug} / \mathrm{L} \\
\mathrm{ug} / \mathrm{L} \\
\mathrm{ug} / \mathrm{L} \\
\mathrm{ug} / \mathrm{L}\end{array}$ & $\begin{array}{l}0.02 \\
0.02 \\
0.02 \\
0.02 \\
\end{array}$ & 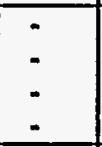 & $\begin{array}{c}<0.024 \\
<0.021 \\
- \\
- \\
\end{array}$ & $\begin{array}{l}\mathrm{IU} \\
\mathrm{IU}\end{array}$ & $\begin{array}{l}<0.021 \\
- \\
\end{array}$ & $\begin{array}{l}<0.025 \\
<0.021 \\
<0.02 \\
<0.02 \\
\end{array}$ & $\begin{array}{l}\text { IU } \\
\text { IU } \\
\text { IU } \\
\text { IU } \\
\end{array}$ & $\begin{array}{c}<0.022 \\
<0.022 \\
\vdots \\
-\end{array}$ & IU & $\begin{array}{c}<0.022 \\
<0.021 \\
- \\
-\end{array}$ & $\begin{array}{l}\text { IU } \\
\text { IU } \\
\end{array}$ & $\begin{array}{l}<0.022 \\
<0.021 \\
<0.02 \\
<0.02\end{array}$ & $\begin{array}{l}\text { IU } \\
\text { IU } \\
\text { IU } \\
\text { IU }\end{array}$ \\
\hline Endrin & $\begin{array}{l}\text { WINTER } \\
\text { SPRING } \\
\text { SUMMER } \\
\text { FALL } \\
\end{array}$ & $\begin{array}{l}\mathrm{ug} / \mathrm{h} \\
\mathrm{ug} / \mathrm{L} \\
\mathrm{ug} / \mathrm{L} \\
\mathrm{ug} / \mathrm{L}\end{array}$ & $\begin{array}{l}0.02 \\
0.02 \\
0.02 \\
0.02 \\
\end{array}$ & $\begin{array}{l}0.2 \\
0.2 \\
0.2 \\
0.2 \\
\end{array}$ & $\begin{array}{c}<0.024 \\
<0.021 \\
- \\
- \\
\end{array}$ & $\begin{array}{l}\mathbf{I U} \\
\mathbf{I U}\end{array}$ & $\begin{array}{l}<0.021 \\
- \\
- \\
\end{array}$ & $\begin{array}{l}<0.025 \\
<0.021 \\
<0.02 \\
<0.02\end{array}$ & \begin{tabular}{|l|}
$\mathbf{I U}$ \\
$\mathrm{IU}$ \\
$\mathrm{IU}$ \\
$\mathrm{IU}$ \\
\end{tabular} & $\begin{array}{l}<0.022 \\
<0.022 \\
-\end{array}$ & $\begin{array}{l}\mathbf{I U} \\
\mathbf{I U} \\
\mathbf{I}\end{array}$ & $\begin{array}{c}<0.022 \\
<0.021 \\
: \\
\end{array}$ & $\begin{array}{l}\text { IV } \\
\text { IU }\end{array}$ & $\begin{array}{l}<0.022 \\
<0.021 \\
<0.02 \\
<0.02\end{array}$ & $\begin{array}{l}\text { IU } \\
\text { IU } \\
\text { IU } \\
\text { IU }\end{array}$ \\
\hline Gamma-Chlordane & $\begin{array}{l}\text { WINTER } \\
\text { SPRING } \\
\text { SUMMER } \\
\text { FALL } \\
\end{array}$ & \begin{tabular}{|l|}
$\mathrm{ug} / \mathrm{L}$ \\
$\mathrm{ug} / \mathrm{L}$ \\
$\mathrm{ug} / \mathrm{L}$ \\
$\mathrm{ug} / \mathrm{L}$ \\
\end{tabular} & \begin{tabular}{l|}
0.01 \\
0.01 \\
0.01 \\
0.01 \\
\end{tabular} & $\begin{array}{l}- \\
- \\
-\end{array}$ & $\begin{array}{c}<0.012 \\
<0.01 \\
- \\
\end{array}$ & $\begin{array}{l}\text { IU } \\
\text { IU }\end{array}$ & $\begin{array}{c}<0.01 \\
- \\
\end{array}$ & $\begin{array}{l}<0.012 \\
<0.011 \\
<0.01 \\
<0.01 \\
\end{array}$ & \begin{tabular}{|l|} 
IU \\
IU \\
IU \\
\end{tabular} & $\begin{array}{c}<0.011 \\
<0.011 \\
- \\
- \\
\end{array}$ & IU & $\begin{array}{c}<0.011 \\
<0.011 \\
\vdots \\
- \\
\end{array}$ & \begin{tabular}{|l|} 
IU \\
IU \\
\end{tabular} & $\begin{array}{l}<0.011 \\
<0.01 \\
<0.01 \\
<0.01 \\
\end{array}$ & $\begin{array}{l}\mathrm{IU} \\
\mathrm{IU} \\
\mathrm{IU} \\
\mathrm{IU} \\
\end{array}$ \\
\hline Parameter & Quarter & Units & DL & MCL & $\begin{array}{c}\text { UCD1-12 } \\
\text { D1 }\end{array}$ & & $\begin{array}{c}\text { UCD1-12 } \\
\text { D2 }\end{array}$ & UCD1-13 & & $\begin{array}{c}\text { UCD1-13 } \\
\text { D1 }\end{array}$ & & UCD1-18 & & $\begin{array}{c}\text { UCD1-18 } \\
\text { D1 }\end{array}$ & \\
\hline Alpha-Chlordane & $\begin{array}{l}\text { WINTER } \\
\text { SPRING } \\
\text { SUMMER } \\
\text { FALL } \\
\end{array}$ & $\begin{array}{l}\mathrm{ug} / \mathrm{L} \\
\mathrm{ug} / \mathrm{L} \\
\mathrm{ug} / \mathrm{L} \\
\mathrm{ug} / \mathrm{L} \\
\end{array}$ & $\begin{array}{l}0.01 \\
0.01 \\
0.01 \\
0.01\end{array}$ & $\begin{array}{l} \\
: \\
\end{array}$ & $\begin{array}{c}- \\
<0.01\end{array}$ & $\mathbf{I U}$ & 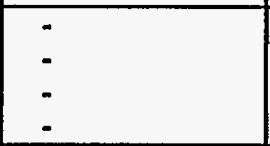 & $\begin{array}{l}0.0054 \\
<0.01 \\
<0.01 \\
0.0057\end{array}$ & IJ & $\begin{array}{l}- \\
0.0059\end{array}$ & & $\begin{array}{l}<0.012 \\
<0.011 \\
-\end{array}$ & $\begin{array}{l}\mathrm{IU} \\
\mathrm{IU}\end{array}$ & $\begin{array}{c}<0.012 \\
: \\
\vdots\end{array}$ & IU \\
\hline Dieldrin & $\begin{array}{l}\text { WINTER } \\
\text { SPRING } \\
\text { SUMMER } \\
\text { FALL } \\
\end{array}$ & $\begin{array}{l}\mathrm{ug} / \mathrm{L} \\
\mathrm{ug} / \mathrm{L} \\
\mathrm{ug} / \mathrm{L} \\
\mathrm{ug} / \mathrm{L} \\
\end{array}$ & \begin{tabular}{l|l|}
0.02 \\
0.02 \\
0.02 \\
0.02 \\
\end{tabular} & $\begin{array}{l}- \\
: \\
\end{array}$ & $<\dot{-}$ & $\mathbf{I U}$ & $\begin{array}{l}- \\
- \\
-\end{array}$ & $\begin{array}{l}0.029 \\
<0.021 \\
<0.02 \\
0.028\end{array}$ & $\mid$ & $\begin{array}{l}- \\
- \\
0.024\end{array}$ & & $\begin{array}{c}<0.024 \\
<0.022 \\
- \\
-\end{array}$ & $\begin{array}{l}\mathrm{IU} \\
\mathbf{I U}\end{array}$ & $\begin{array}{c}<0.024 \\
- \\
-\end{array}$ & $\mathbf{I U}$ \\
\hline Endrin & \begin{tabular}{|l|} 
WINTER \\
SPRING \\
SUMMER \\
FALL \\
\end{tabular} & \begin{tabular}{|l|}
$\mathrm{ug} / \mathrm{L}$ \\
$\mathrm{ug} / \mathrm{L}$ \\
$\mathrm{ug} / \mathrm{L}$ \\
$\mathrm{ug} / \mathrm{L}$ \\
\end{tabular} & \begin{tabular}{l|}
0.02 \\
0.02 \\
0.02 \\
0.02 \\
\end{tabular} & $\begin{array}{l}0.2 \\
0.2 \\
0.2 \\
0.2 \\
\end{array}$ & $<0.0$ & $\mathrm{IU}$ & $\begin{array}{l}\text { - } \\
- \\
-\end{array}$ & $\begin{array}{c}0.012 \\
<0.021 \\
<0.02 \\
0.00085 \\
\end{array}$ & \begin{tabular}{|l|}
$\mathrm{IJ}$ \\
$\mathrm{IU}$ \\
$\mathrm{IU}$ \\
$\mathrm{IJP}$ \\
\end{tabular} & $\begin{array}{c}- \\
\dot{-} \\
<0.02 \\
\end{array}$ & IU & $\begin{array}{l}<0.024 \\
<0.022 \\
\vdots \\
\end{array}$ & $\begin{array}{l}\text { IU } \\
\text { IU }\end{array}$ & $\begin{array}{c}<0.024 \\
- \\
-\end{array}$ & IU \\
\hline Gamma-Chlordane & $\begin{array}{l}\text { WINTER } \\
\text { SPRING } \\
\text { SUMMER } \\
\text { FALL } \\
\end{array}$ & \begin{tabular}{|l|}
$\mathrm{ug} / \mathrm{L}$ \\
$\mathrm{ug} / \mathrm{L}$ \\
$\mathrm{ug} / \mathrm{L}$ \\
$\mathrm{ug} / \mathrm{L}$ \\
\end{tabular} & $\begin{array}{l}0.01 \\
0.01 \\
0.01 \\
0.01\end{array}$ & 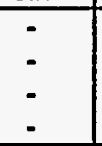 & $<-\dot{0.01}$ & IU & $\begin{array}{l}- \\
- \\
-\end{array}$ & $\begin{array}{l}\quad 0.0051 \\
<0.01 \\
\leq 0.01 \\
0.0049\end{array}$ & IU & $\begin{array}{c}- \\
\vdots \\
0.0071\end{array}$ & TyP & $\begin{array}{l}<0.012 \\
<0.011 \\
- \\
-\end{array}$ & $\begin{array}{l}\mid \mathbf{I U} \\
\mathbf{I U}\end{array}$ & $\begin{array}{c}<0.012 \\
:\end{array}$ & IU \\
\hline
\end{tabular}

Page 1 of 4 
TABLE 5.4

SUMMARY OF DETECTED CONSTITUENTS IN GROUNDWATER, 1995

PESTICIDES AND PCBS

1995 ANNUAL WATER MONITORING REPORT

LEHR ENVIRONMENTAL RESTORATION, DAVIS, CALIFORNIA

\begin{tabular}{|c|c|c|c|c|c|c|c|c|c|c|c|c|c|c|c|c|}
\hline \multirow{2}{*}{\begin{tabular}{|l} 
Parameter \\
Alpha-Chlordane
\end{tabular}} & \multirow{2}{*}{\begin{tabular}{|l} 
Quarter \\
WINTER \\
SPRING \\
SUMMER \\
FALL \\
\end{tabular}} & \multirow[b]{2}{*}{$\begin{array}{l}\text { Units } \\
\mathrm{ug} / \mathrm{L} \\
\mathrm{ug} / \mathrm{L} \\
\mathrm{ug} / \mathrm{L} \\
\mathrm{ug} / \mathrm{L}\end{array}$} & \multirow{2}{*}{\begin{tabular}{|c|} 
DL \\
0.01 \\
0.01 \\
0.01 \\
0.01 \\
\end{tabular}} & \multirow[b]{2}{*}{$\begin{array}{l}\text { MCL } \\
\vdots \\
\vdots \\
\end{array}$} & \multicolumn{2}{|l|}{ UCD1-19 } & \multicolumn{2}{|l|}{$\begin{array}{c}\text { UCD1-19 } \\
\text { D1 }\end{array}$} & \multicolumn{2}{|c|}{ UCD1-20 } & \multicolumn{2}{|c|}{ UCD1-21 } & \multicolumn{2}{|c|}{ UCD1-22 } & \multicolumn{2}{|c|}{ UCD1-23 } \\
\hline & & & & & $\begin{array}{c}<0.01 \\
<0.01 \\
- \\
\end{array}$ & IU & $\begin{array}{l}<0.01 \\
\vdots\end{array}$ & IV & $\begin{array}{l}<0.012 \\
<0.01 \\
\vdots \\
-\end{array}$ & $\begin{array}{l}\mathbf{I U} \\
\mathbf{I U}\end{array}$ & $\begin{array}{l}<0.014 \\
<0.011 \\
-\end{array}$ & $\begin{array}{l}\mathbf{I U} \\
\mathbf{I U}\end{array}$ & $\begin{array}{l}<0.011 \\
<0.011 \\
\vdots\end{array}$ & IU & $\begin{array}{l}<0.01 \\
<0.011 \\
\vdots\end{array}$ & $\begin{array}{l}\text { IU } \\
\text { IU }\end{array}$ \\
\hline Dieldrin & \begin{tabular}{|l} 
WINTER \\
SPRING \\
SUMMER \\
FALL
\end{tabular} & $\begin{array}{l}\text { ug/L } \\
\text { ug/L } \\
\text { ug/L } \\
\text { ug/L }\end{array}$ & \begin{tabular}{|l|}
0.02 \\
0.02 \\
0.02 \\
0.02
\end{tabular} & $\begin{array}{l} \\
: \\
\end{array}$ & $\begin{array}{l}<0.02 \\
<0.021 \\
:\end{array}$ & $\begin{array}{l}\mathrm{IU} \\
\mathrm{IU} \\
\end{array}$ & $<0.021$ & IU & $\begin{array}{l}<0.025 \\
<0.021 \\
\vdots\end{array}$ & $\begin{array}{l}\mathbf{I U} \\
\mathbf{I U}\end{array}$ & $\begin{array}{l}<0.027 \\
<0.021 \\
:\end{array}$ & IU & $\begin{array}{l}<0.023 \\
<0.022 \\
\vdots\end{array}$ & $\begin{array}{l}\mathbf{I U} \\
\mathbf{I U}\end{array}$ & $\begin{array}{l}<0.02 \\
<0.021 \\
\vdots\end{array}$ & $\begin{array}{l}\text { IU } \\
\text { IU }\end{array}$ \\
\hline Endrin & $\begin{array}{l}\text { WINTER } \\
\text { SPRING } \\
\text { SUMMER } \\
\text { FALL } \\
\end{array}$ & $\begin{array}{l}\text { ug/L } \\
\text { ug/L } \\
\text { ug/L } \\
\text { ug/L } \\
\end{array}$ & $\begin{array}{l}0.02 \\
0.02 \\
0.02 \\
0.02 \\
\end{array}$ & $\begin{array}{l}0.2 \\
0.2 \\
0.2 \\
0.2 \\
\end{array}$ & $\begin{array}{l}<0.02 \\
<0.021 \\
- \\
- \\
\end{array}$ & $\begin{array}{l}\mathrm{IU} \\
\mathrm{IU}\end{array}$ & $\begin{array}{c}<0.021 \\
\vdots \\
\end{array}$ & $\mathbf{I U}$ & $\begin{array}{l}<0.025 \\
<0.021 \\
- \\
- \\
\end{array}$ & IU & $\begin{array}{l}<0.027 \\
<0.021 \\
- \\
- \\
\end{array}$ & $\begin{array}{l}\mathbf{I U} \\
\mathbf{I U}\end{array}$ & \begin{tabular}{|c|}
$<0.023$ \\
$<0.022$ \\
- \\
- \\
\end{tabular} & $\begin{array}{l}\mathbf{I U} \\
\mathbf{I U}\end{array}$ & \begin{tabular}{|c|}
$<0.02$ \\
$<0.021$ \\
- \\
-
\end{tabular} & $\begin{array}{l}\text { IU } \\
\text { IU }\end{array}$ \\
\hline Gamma-Chlordane & $\begin{array}{l}\text { WINTER } \\
\text { SPRING } \\
\text { SUMMER } \\
\text { FALL } \\
\end{array}$ & $\begin{array}{l}\text { ug/L } \\
\text { ug/L } \\
\text { ug/L } \\
\text { ug/L } \\
\end{array}$ & \begin{tabular}{l|l}
0.01 \\
0.01 \\
0.01 \\
0.01 \\
\end{tabular} & $\begin{array}{l}- \\
- \\
-\end{array}$ & $\begin{array}{c}<0.01 \\
<0.01 \\
- \\
\end{array}$ & $\begin{array}{l}\text { IU } \\
\text { IU } \\
\text { | }\end{array}$ & $\begin{array}{c}<0.01 \\
- \\
- \\
\end{array}$ & $\mathbf{I U}$ & \begin{tabular}{|l|}
$<0.012$ \\
$<0.01$ \\
- \\
-
\end{tabular} & $\begin{array}{l}\text { IU } \\
\text { IU }\end{array}$ & \begin{tabular}{|l}
$<0.014$ \\
$<0.011$ \\
- \\
-
\end{tabular} & $\begin{array}{l}\mathrm{IU} \\
\mathrm{IU} \\
\mathbf{T}\end{array}$ & \begin{tabular}{|c|}
$<0.011$ \\
$<0.011$ \\
- \\
-
\end{tabular} & IU & \begin{tabular}{|l}
$<0.01$ \\
$<0.011$ \\
- \\
-
\end{tabular} & $\begin{array}{l}\text { IU } \\
\text { IU }\end{array}$ \\
\hline Parameter & Quarter & Units & DL & MCL & UCD1-24 & & UCD1-25 & & UCD1 & $27 \mathrm{Z1}$ & UCD1. & $\overline{27 Z 2}$ & UCD1 & $27 \mathbf{Z 3}$ & UC & \\
\hline Alpha-Chlordane & $\begin{array}{l}\text { WINTER } \\
\text { SPRING } \\
\text { SUMMER } \\
\text { FALL } \\
\end{array}$ & \begin{tabular}{|l|}
$\mathrm{ug} / \mathrm{L}$ \\
$\mathrm{ug} / \mathrm{L}$ \\
$\mathrm{ug} / \mathrm{L}$ \\
$\mathrm{ug} / \mathrm{L}$ \\
\end{tabular} & \begin{tabular}{l|l|}
0.01 \\
0.01 \\
0.01 \\
0.01 \\
\end{tabular} & $\begin{array}{l} \\
\vdots \\
\end{array}$ & \begin{tabular}{|l}
$<0.011$ \\
$<0.01$ \\
- \\
-
\end{tabular} & $\begin{array}{l}\mathbf{I U} \\
\mathbf{I U} \\
\end{array}$ & $\begin{array}{c}\dot{-} \\
\vdots \\
<0.01 \\
\end{array}$ & $\mathbf{U}$ & $\begin{array}{c}- \\
\vdots \\
<0.01 \\
\end{array}$ & $\mathbf{U J s} \mid \mathbf{U}$ & $\begin{array}{c}- \\
\vdots \\
<0.01 \\
\end{array}$ & UJs IU & $\begin{array}{c}- \\
\vdots \\
<0.01 \\
\end{array}$ & UJs IU & $\begin{array}{c}- \\
- \\
<0.01\end{array}$ & $\mathbf{I U}$ \\
\hline Dieldrin & $\begin{array}{l}\text { WINTER } \\
\text { SPRING } \\
\text { SUMMER } \\
\text { FÄLL }\end{array}$ & \begin{tabular}{|l|}
$\mathrm{ug} / \mathrm{L}$ \\
$\mathrm{ug} / \mathrm{L}$ \\
$\mathrm{ug} / \mathrm{L}$ \\
$\mathrm{ug} / \mathrm{L}$ \\
\end{tabular} & $\begin{array}{l}0.02 \\
0.02 \\
0.02 \\
0.02 \\
\end{array}$ & $\begin{array}{l}- \\
: \\
\end{array}$ & $\begin{array}{c}<0.021 \\
<0.021 \\
- \\
- \\
\end{array}$ & $\begin{array}{l}\text { TU } \\
\text { IU }\end{array}$ & $\begin{array}{c}- \\
- \\
- \\
<0.02 \\
\end{array}$ & U & $\begin{array}{c}- \\
- \\
<0.02\end{array}$ & UJs IU & $\begin{array}{c}- \\
- \\
<0.02\end{array}$ & UJs IU & $\begin{array}{c}- \\
- \\
<0.02\end{array}$ & UJs IU & $\begin{array}{c}- \\
<0.02\end{array}$ & IU \\
\hline Endrin & $\begin{array}{l}\text { WINTER } \\
\text { SPRING } \\
\text { SUMMER } \\
\text { FALL } \\
\end{array}$ & $\begin{array}{l}\mathrm{ug} / \mathrm{L} \\
\mathrm{ug} / \mathrm{L} \\
\mathrm{ug} / \mathrm{L} \\
\mathrm{ug} / \mathrm{L}\end{array}$ & \begin{tabular}{l|l|}
0.02 \\
0.02 \\
0.02 \\
0.02 \\
\end{tabular} & $\begin{array}{l}0.2 \\
0.2 \\
0.2 \\
0.2 \\
\end{array}$ & $\begin{array}{c}<0.021 \\
<0.021 \\
- \\
-\end{array}$ & $\begin{array}{l}\text { IU } \\
\text { IU }\end{array}$ & $\begin{array}{c}- \\
- \\
<0.02 \\
0\end{array}$ & $\mathbf{U}$ & $\begin{array}{c}- \\
- \\
<0.02\end{array}$ & UJs IU & $\begin{array}{c}- \\
- \\
<0.02 \\
\end{array}$ & UJs IU & $\begin{array}{c}- \\
- \\
<0.02\end{array}$ & UJs IU & $\begin{array}{c}- \\
\vdots \\
<0.02 \\
\end{array}$ & $\mathbf{I U}$ \\
\hline Gamma-Chlordane & $\begin{array}{l}\text { WINTER } \\
\text { SPRING } \\
\text { SUMMER } \\
\text { FALL } \\
\end{array}$ & \begin{tabular}{|l|}
$\mathrm{ug} / \mathrm{L}$ \\
$\mathrm{ug} / \mathrm{L}$ \\
$\mathrm{ug} / \mathrm{L}$ \\
$\mathrm{ug} / \mathrm{L}$
\end{tabular} & \begin{tabular}{l|}
0.01 \\
0.01 \\
0.01 \\
0.01 \\
\end{tabular} & $\begin{array}{l} \\
- \\
- \\
-\end{array}$ & $\begin{array}{c}<0.011 \\
<0.01 \\
- \\
-\end{array}$ & $\begin{array}{l}\text { IU } \\
\text { IU }\end{array}$ & $\begin{array}{c} \\
\\
<0.01 \\
\end{array}$ & $\mathrm{IU}$ & $\begin{array}{c}- \\
- \\
<0.01\end{array}$ & UJs IU & $\begin{array}{c}- \\
\\
<0.01 \\
\end{array}$ & UJs IU & $\begin{array}{c}- \\
- \\
<0.01\end{array}$ & UJs IU & $\begin{array}{c}- \\
- \\
<0.01\end{array}$ & IU \\
\hline
\end{tabular}


TABLE 5.4

SUMMARY OF DETECTED CONSTITUENTS IN GROUNDWATER, 1995

PESTICIDES AND PCBS

1995 ANNUAL WATER MONITORING REPORT

LEHR ENVIRONMENTAL RESTORATION, DAVIS, CALIFORNIA

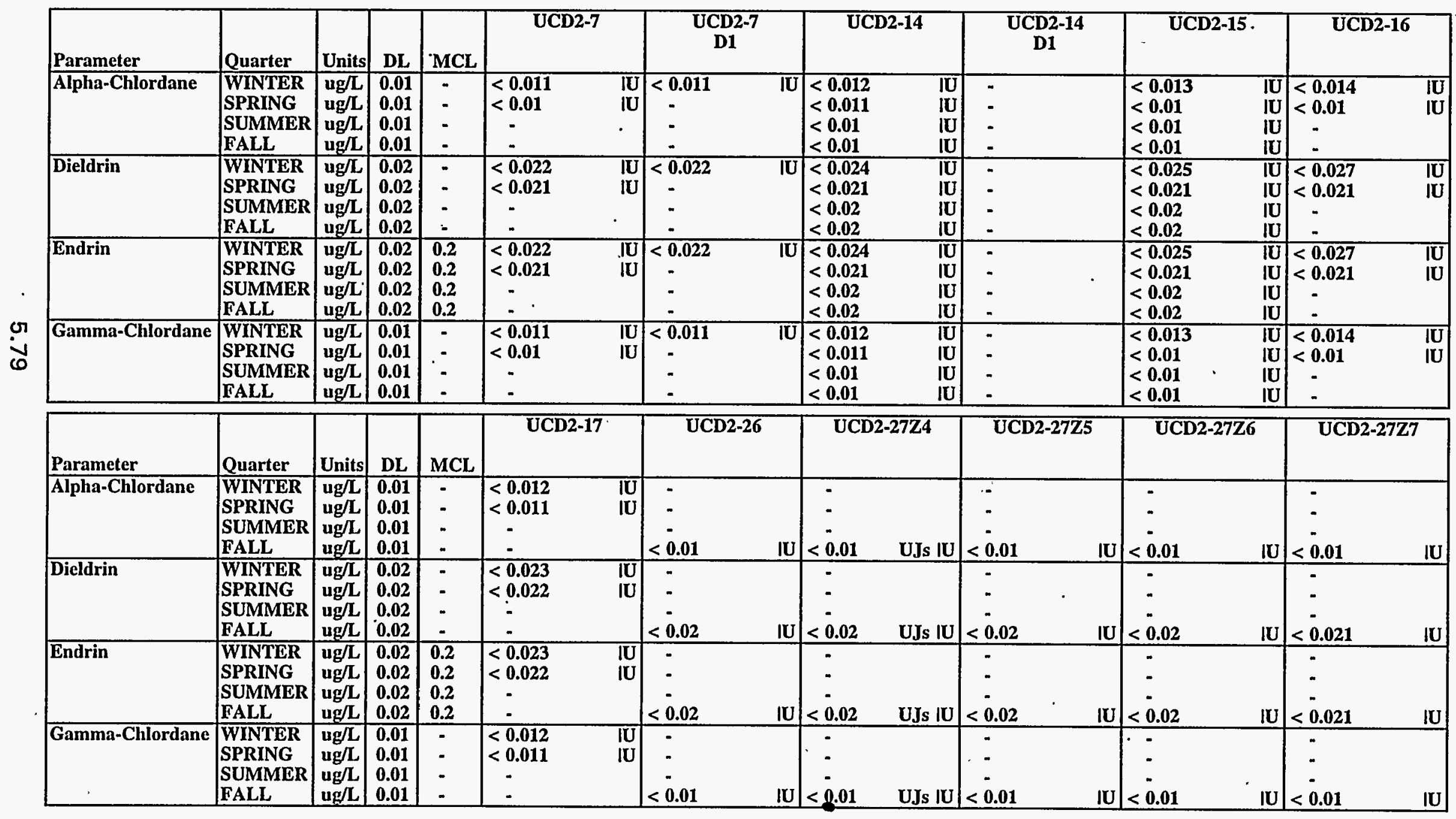

Page 3 of 4 


\section{TABLE 5.4}

SUMMARY OF DETECTED CONSTITUENTS IN GROUNDWATER, 1995

PESTICIDES AND PCBS

1995 ANNUAL WATER MONITORING REPORT LEHR ENVIRONMENTAL RESTORATION, DAVIS, CALIFORNIA

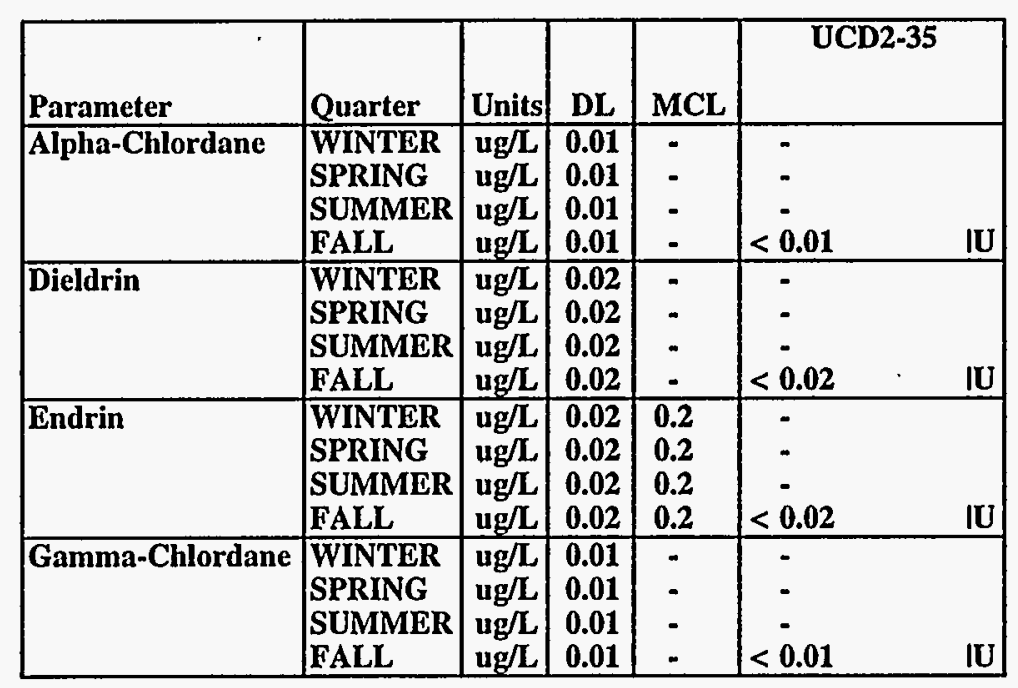

D1 = Duplicate sample.

- = Parameter not analyzed or no MCL.

DL $=$ Detection limit.

$<=$ Constituent below detection limit. Detection limits may vary depending on interference by other sample constituents. MCL = Maximum Contaminant Level (primary), Title 22 Division 4 Chapter 15.

Highlighted values indicate positive results.

See Appendix A for explanation of data qualifiers. 
TABLE 5.5

SUMMARY OF DETECTED CONSTITUENTS IN GROUNDWATER, 1995

METALS

1995 ANNUAL WATER MONITORING REPORT

LEHR ENVIRONMENTAL RESTORATION, DAVIS, CALIFORNIA

\begin{tabular}{|c|c|c|c|c|c|c|c|c|c|c|c|c|c|c|}
\hline \multirow{2}{*}{\begin{tabular}{|l} 
Parameter \\
Antimony
\end{tabular}} & \multirow[b]{2}{*}{\begin{tabular}{|l} 
Quarter \\
WINTER \\
SPRING \\
SUMMER \\
FALL
\end{tabular}} & \multirow[b]{2}{*}{\begin{tabular}{|l|} 
Units \\
ug/L \\
ug/L \\
ug/L \\
ug/L
\end{tabular}} & \multirow[b]{2}{*}{$\begin{array}{c}\text { DL } \\
5 \\
5 \\
5 \\
5\end{array}$} & \multirow[b]{2}{*}{$\begin{array}{l}\text { MCL } \\
6 \\
6 \\
6 \\
6\end{array}$} & \multicolumn{2}{|c|}{ UCD1-1 } & \multicolumn{2}{|c|}{$\begin{array}{l}\text { UCD1-1 } \\
\text { D1 }\end{array}$} & \multicolumn{2}{|c|}{ UCD1-4 } & \multicolumn{2}{|c|}{ UCD1-10 } & \multicolumn{2}{|c|}{ UCD1-11 } \\
\hline & & & & & $\begin{array}{c}<5 \\
<5 \\
- \\
-\end{array}$ & $\begin{array}{l}\text { IU } \\
\text { IU }\end{array}$ & $\begin{array}{l}<5 \\
:\end{array}$ & IU & $\begin{array}{l}<5 \\
<5 \\
-\end{array}$ & $\begin{array}{l}\text { IU } \\
\text { IU }\end{array}$ & $\begin{array}{r}<5 \\
<5 \\
-\end{array}$ & $\mathbf{I U}$ & $\begin{array}{r}<5 \\
<5 \\
- \\
-\end{array}$ & $\begin{array}{l}\mathrm{IU} \\
\mathrm{IU}\end{array}$ \\
\hline \multirow[t]{2}{*}{ Arsenic } & \multirow{2}{*}{$\begin{array}{l}\text { WINTER } \\
\text { SPRING } \\
\text { SUMMER } \\
\text { FALL }\end{array}$} & \multirow{2}{*}{\begin{tabular}{|l|}
$\mathrm{ug} / \mathrm{L}$ \\
$\mathrm{ug} / \mathrm{L}$ \\
$\mathrm{ug} / \mathrm{L}$ \\
$\mathrm{ug} / \mathrm{L}$
\end{tabular}} & \multirow{2}{*}{$\begin{array}{l}2 \\
2 \\
2 \\
2\end{array}$} & \multirow{2}{*}{$\begin{array}{l}50 \\
50 \\
50 \\
50\end{array}$} & \multirow{2}{*}{$\begin{array}{r}4 \\
<2 \\
-\end{array}$} & \multirow[t]{2}{*}{$\frac{\mathrm{Up} \text { IU }}{\mathrm{IU}}$} & \multirow{2}{*}{$<2$} & \multirow[t]{2}{*}{$\mathbf{I U}$} & \multirow{2}{*}{$\begin{array}{c}2,8 \\
<2 \\
-\end{array}$} & \multirow[t]{2}{*}{$\overline{I K} \frac{1}{\mid U}$} & $\begin{array}{l}3.7 \\
2.5 \\
\end{array}$ & \multirow[t]{2}{*}{$\overline{\mathrm{Jk}} \mid$} & $\begin{array}{l}3.9 \\
2.8 \\
\end{array}$ & \multirow[t]{2}{*}{ Jk I } \\
\hline & & & & & & & & & & & $\therefore$ & & - & \\
\hline \multirow[t]{2}{*}{ Barium } & \multirow{2}{*}{$\begin{array}{l}\text { WINTER } \\
\text { SPRING } \\
\text { SUMMER } \\
\text { FALL }\end{array}$} & \multirow{2}{*}{\begin{tabular}{|l|}
$\operatorname{ugg} / \mathrm{L}$ \\
$\operatorname{ug} / \mathrm{L}$ \\
$\operatorname{ug} / \mathrm{L}$ \\
$\operatorname{ug} / \mathrm{L}$
\end{tabular}} & \multirow{2}{*}{$\begin{array}{l}20 \\
20 \\
20 \\
20\end{array}$} & \multirow{2}{*}{$\begin{array}{l}1000 \\
1000 \\
1000 \\
1000\end{array}$} & \multicolumn{2}{|l|}{$\begin{array}{r}219 \\
237 \\
\end{array}$} & \multicolumn{2}{|l|}{-248} & \multicolumn{2}{|l|}{$\begin{array}{l}202 \\
310\end{array}$} & \multicolumn{2}{|l|}{$\begin{array}{l}25.7 \\
30.1\end{array}$} & \multicolumn{2}{|l|}{$\begin{array}{l}.262 \\
325\end{array}$} \\
\hline & & & & & \multicolumn{2}{|l|}{$:$} & \multicolumn{2}{|l|}{ 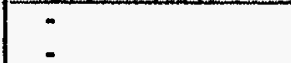 } & - & & 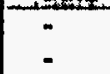 & & - & \\
\hline Chromium & $\begin{array}{l}\text { WINTER } \\
\text { SPRING }\end{array}$ & $\begin{array}{l}\text { ug/h } \\
\text { ug/L }\end{array}$ & $\begin{array}{l}10 \\
10\end{array}$ & $\begin{array}{l}50 \\
50\end{array}$ & $\begin{array}{r}32.8 \\
29.2 \\
\end{array}$ & & 30.1 & & $\begin{array}{l}9.5 \\
26.9 \\
\end{array}$ & T⿱一⿻上丨冖巾 & $\begin{array}{r}67,2 \\
74,9 \\
\end{array}$ & & $\begin{array}{l}266 \\
279 \\
\end{array}$ & \\
\hline & $\begin{array}{l}\text { SUMMER } \\
\text { FALL }\end{array}$ & $\begin{array}{l}\text { ug/L } \\
\text { ug/L }\end{array}$ & $\begin{array}{l}10 \\
10\end{array}$ & $\begin{array}{l}\mathbf{5 0} \\
\mathbf{5 0}\end{array}$ & - & & $:$ & & 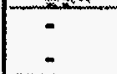 & & - & & - & \\
\hline Chromium, Hexavalent (+6) & $\begin{array}{l}\text { WINTER } \\
\text { SPRING } \\
\text { SUMMER } \\
\text { FALL }\end{array}$ & $\begin{array}{l}\mathrm{ug} / \mathrm{L} \\
\mathrm{ug} / \mathrm{L} \\
\mathrm{ug} / \mathrm{L} \\
\mathrm{ug} / \mathrm{L}\end{array}$ & $\begin{array}{l}10 \\
10 \\
10 \\
10\end{array}$ & $\begin{array}{l}50 \\
50 \\
50 \\
50\end{array}$ & $\begin{array}{l}29 \\
18 \\
32 \\
\end{array}$ & 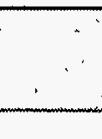 & $\frac{-19}{-}$ & & $\begin{aligned}<10 \\
233 \\
23 \\
19\end{aligned}$ & & $\begin{array}{l}69 \\
73 \\
74 \\
64\end{array}$ & " & $\begin{array}{l}290 \\
270 \\
270 \\
\end{array}$ & \\
\hline Cobalt & $\begin{array}{l}\text { WINTER } \\
\text { SPRING } \\
\text { SUMMER } \\
\text { FALL }\end{array}$ & $\begin{array}{l}\mathrm{ug} / \mathrm{L} \\
\mathrm{ug} / \mathrm{L} \\
\mathrm{ug} / \mathrm{L} \\
\mathrm{ug} / \mathrm{L}\end{array}$ & $\begin{array}{l}10 \\
10 \\
10 \\
10 \\
10\end{array}$ & : & $\begin{array}{c}1.4 \\
<10 \\
-\end{array}$ & $\frac{\mathbb{I B}}{\mathrm{IU}}$ & $<10$ & IU & $\begin{array}{c}<10 \\
<10 \\
<10 \\
- \\
-\end{array}$ & $\begin{array}{l}\text { IU } \\
\text { IU }\end{array}$ & $\begin{array}{c}\quad 04 \\
<10 \\
<10 \\
-\end{array}$ & $\begin{array}{l}\mathbf{I U} \\
\mathbf{I U}\end{array}$ & $\begin{array}{c}- \\
<10 \\
<10 \\
\vdots\end{array}$ & $\begin{array}{l}\mathrm{TU} \\
\mathbf{I U}\end{array}$ \\
\hline Copper & $\begin{array}{l}\text { WINTER } \\
\text { SPRING } \\
\text { SUMMER } \\
\text { FALL } \\
\end{array}$ & $\begin{array}{l}\text { ug/L } \\
\text { ug/L } \\
\text { ug/L } \\
\text { ug/L }\end{array}$ & \begin{tabular}{l|}
10 \\
10 \\
10 \\
10 \\
\end{tabular} & $\begin{array}{ll}1000 & S \\
1000 & S \\
1000 & S \\
1000 & S \\
\end{array}$ & $\begin{array}{c}1.9 \\
<10 \\
- \\
\end{array}$ & $\frac{\overline{I B}}{\mathbf{I U}}$ & $\begin{array}{c}<10 \\
-\end{array}$ & IU & $\begin{array}{l}<10 \\
<10 \\
\vdots \\
\end{array}$ & $\begin{array}{l}\text { IU } \\
\text { IU }\end{array}$ & $\begin{array}{c}2.6 \\
<10 \\
- \\
-\end{array}$ & $\frac{\mid \mathbf{B}}{\mathbf{I U}}$ & $\frac{1}{<10}$ & $\frac{\overline{\mid B}}{\frac{\mathbf{B}}{\mid \mathbf{U}}}$ \\
\hline Iron & \begin{tabular}{|l|} 
WINTER \\
SPRING
\end{tabular} & $\begin{array}{l}\text { ug/L } \\
\text { ug/L }\end{array}$ & $\begin{array}{l}100 \\
100 \\
100\end{array}$ & $\begin{array}{ll}300 & S \\
300 & S \\
\end{array}$ & $\begin{array}{l}<100 \\
18,8\end{array}$ & $\begin{array}{l}\text { IU } \\
\mathbf{B} \\
\end{array}$ & 15.7 & IB & $\begin{array}{l}19,3 \\
93,9 \\
\end{array}$ & $\begin{array}{l}\not \mathbf{B} \\
\mathbf{B}\end{array}$ & $\begin{array}{l}24.9 \\
67.3 \\
\end{array}$ & $\begin{array}{l}\boldsymbol{B} \\
\mathbf{B} \\
\end{array}$ & $\begin{array}{l}20 \\
34.8 \\
\end{array}$ & $\begin{array}{l}\mathbf{B} \\
\mathbf{B} \\
\end{array}$ \\
\hline & $\begin{array}{l}\text { SUMMER } \\
\text { FALL }\end{array}$ & $\begin{array}{l}\text { ug/L } \\
\text { ug/L }\end{array}$ & $\begin{array}{l}100 \\
100 \\
\end{array}$ & $\begin{array}{ll}300 & S \\
300 & S \\
\end{array}$ & - & & - & & $\therefore$ & & - & & 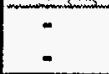 & \\
\hline
\end{tabular}


TABLE 5.5

SUMMARY OF DETECTED CONSTITUENTS IN GROUNDWATER, 1995

METALS

1995 ANNUAL WATER MONITORING REPORT

LEHR ENVIRONMENTAL RESTORATION, DAVIS, CALIFORNIA

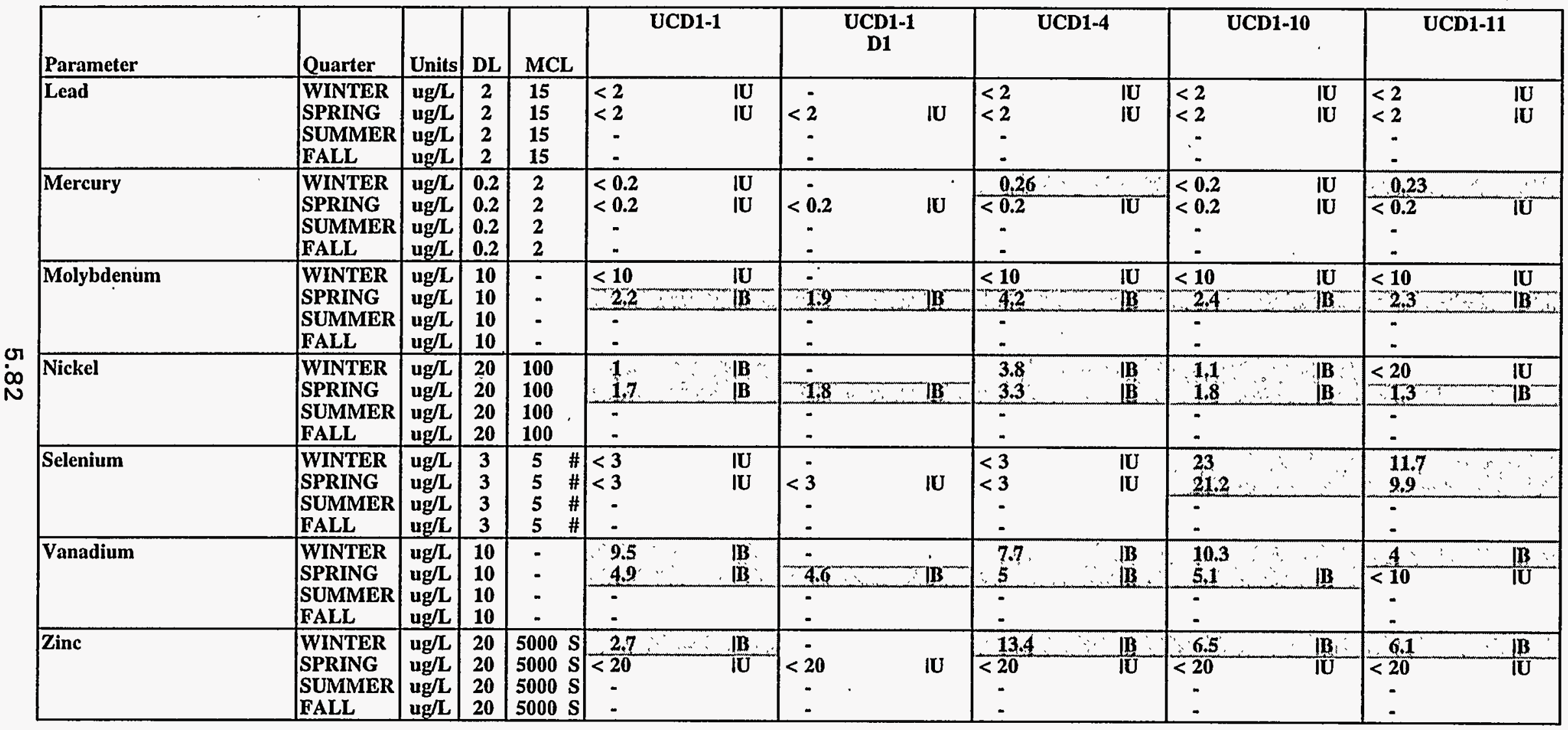


TABLE 5.5

SUMMARY OF DETECTED CONSTITUENTS IN GROUNDWATER, 1995

METALS

1995 ANNUAL WATER MONITORING REPORT

LEHR ENVIRONMENTAL RESTORATION, DAVIS, CALIFORNIA

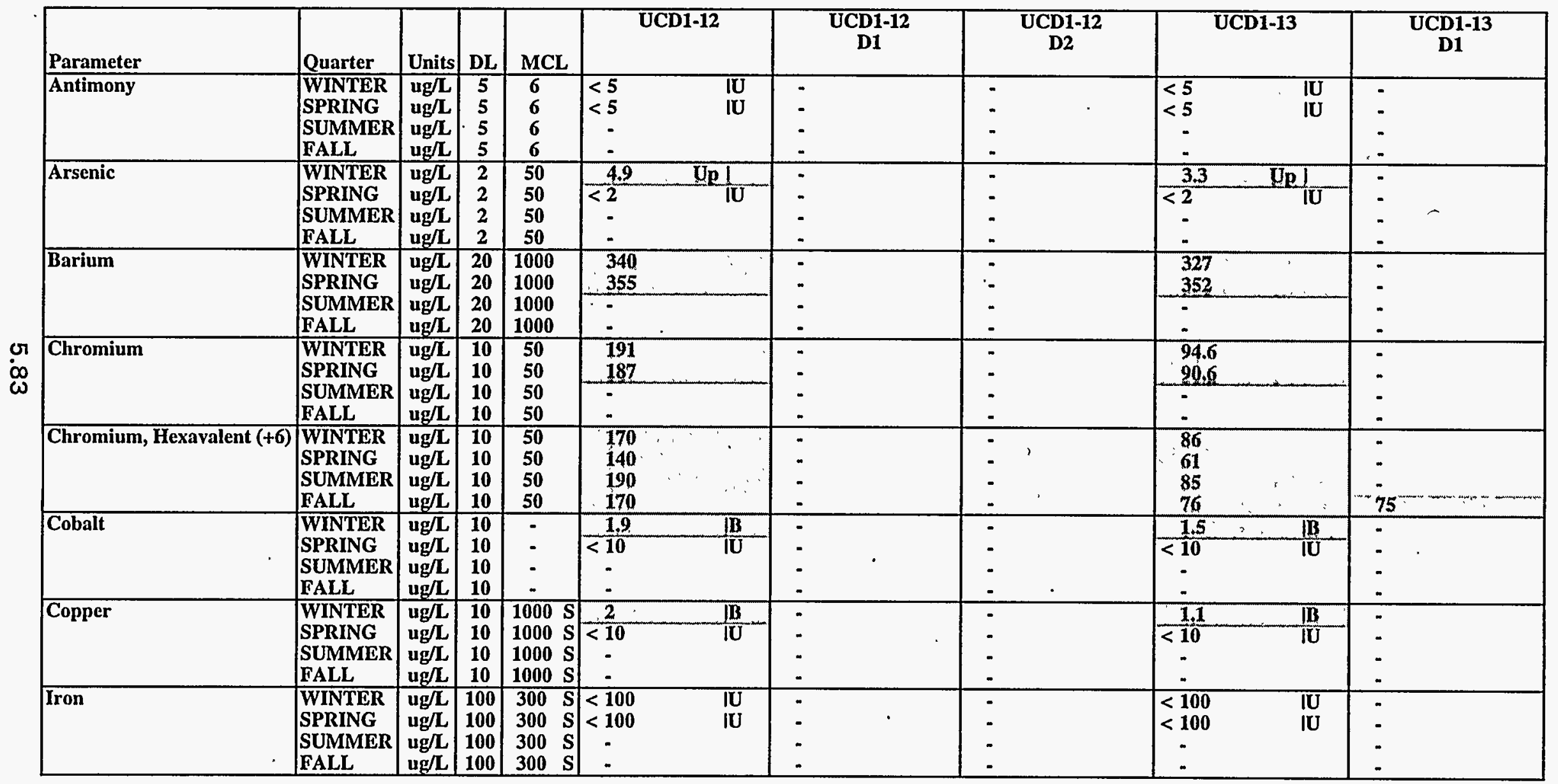


TABLE 5.5

SUMMARY OF DETECTED CONSTITUENTS IN GROUNDWATER, 1995

METALS

1995 ANNUAL WATER MONITORING REPORT

LEHR ENVIRONMENTAL RESTORATION, DAVIS, CALIFORNIA

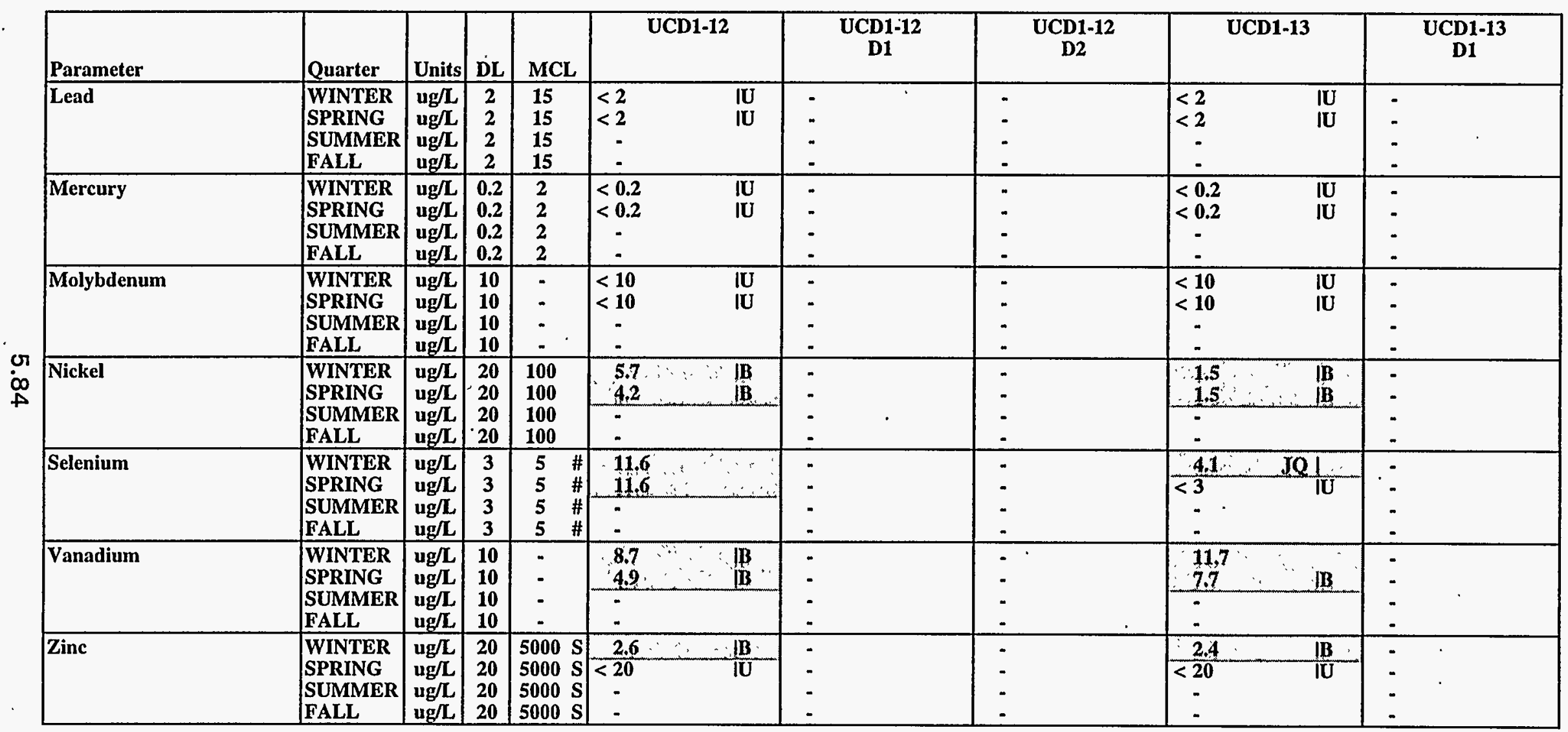


TABLE 5.5

SUMMARY OF DETECTED CONSTITUENTS IN GROUNDWATER, 1995

METALS

1995 ANNUAL WATER MONITORING REPORT

LEHR ENVIRONMENTAL RESTORATION, DAVIS, CALIFORNIA

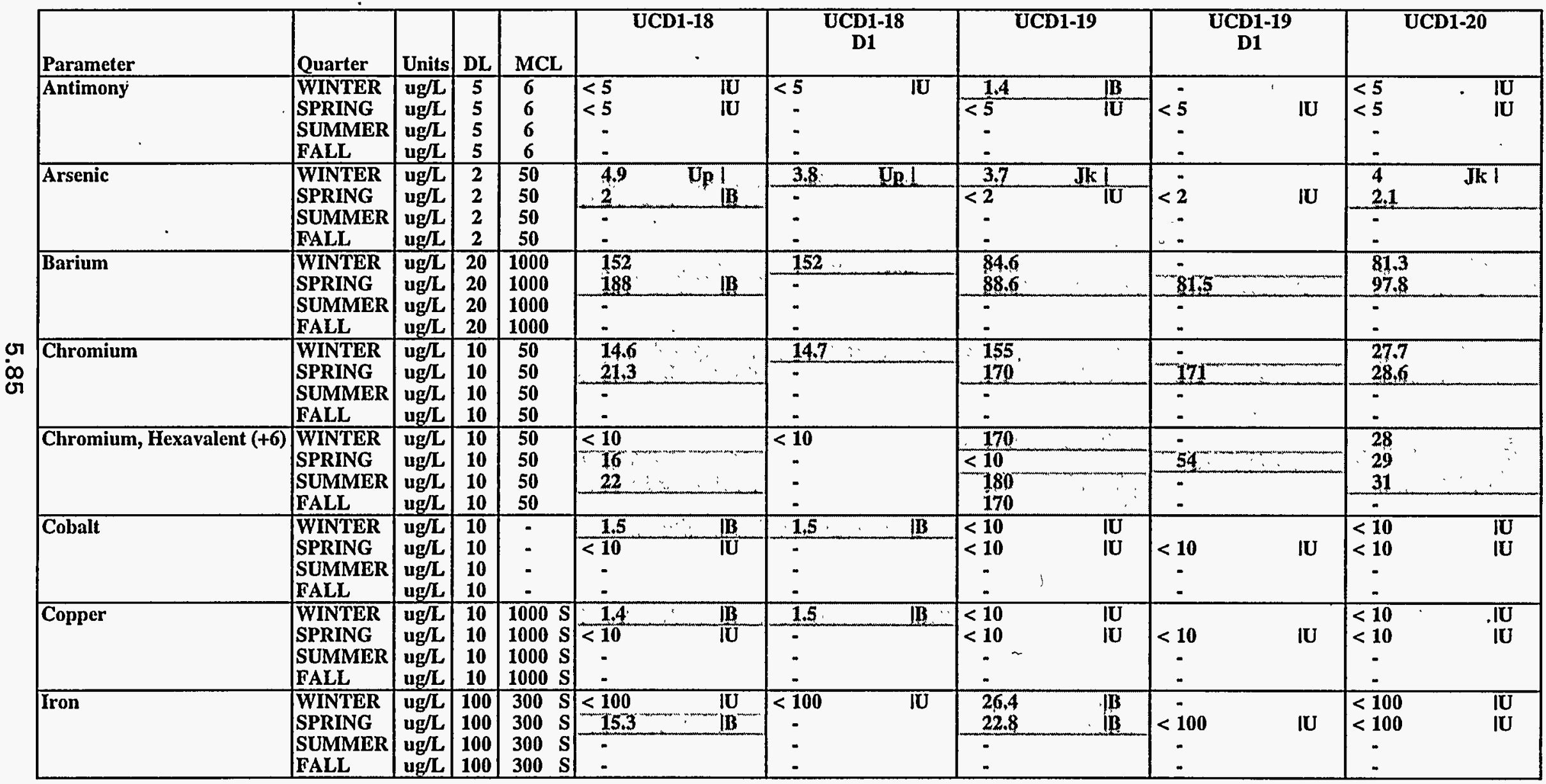


TABLE 5.5

SUMMARY OF DETECTED CONSTITUENTS IN GROUNDWATER, 1995

METALS

1995 ANNUAL WATER MONITORING REPORT

LEHR ENVIRONMENTAL RESTORATION, DAVIS, CALIFORNIA

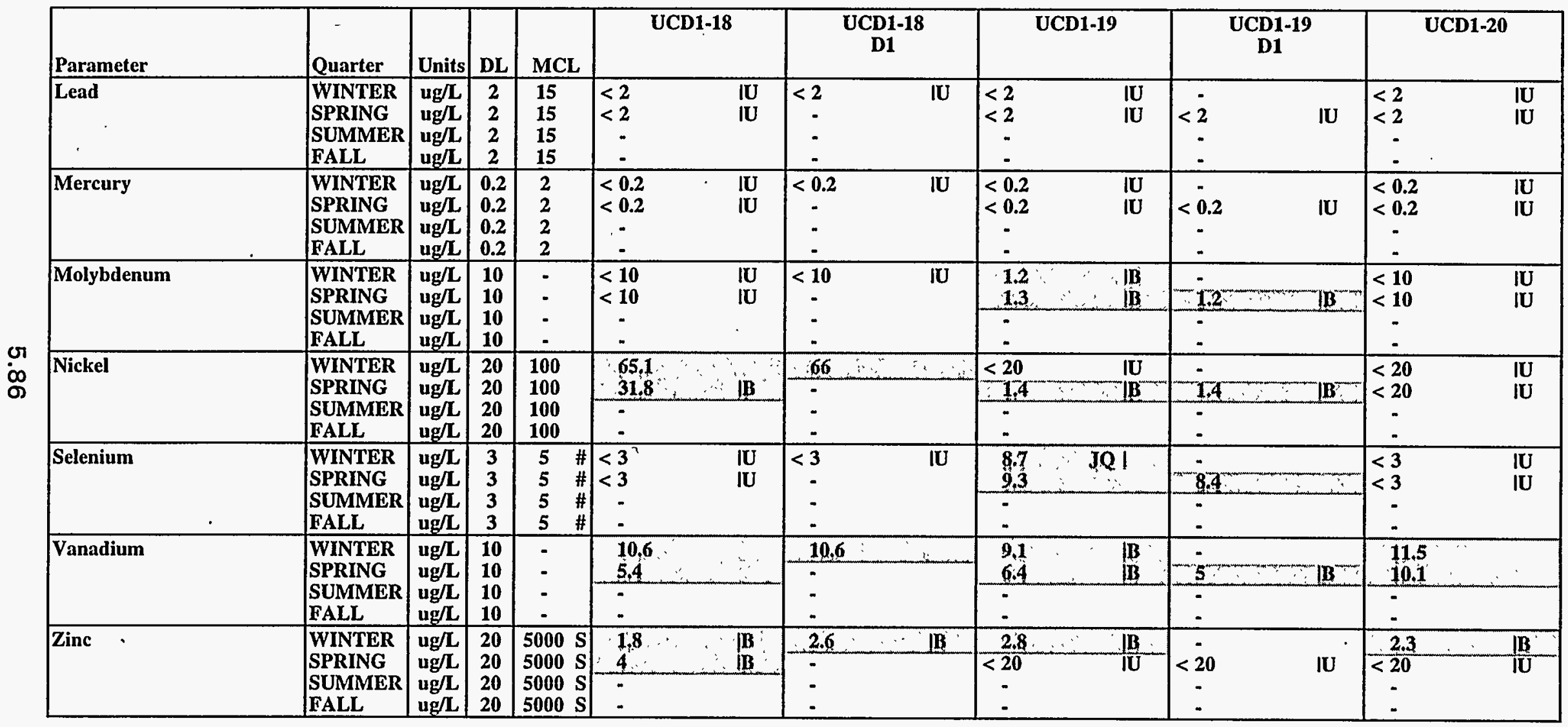


TABLE 5.5

SUMMARY OF DETECTED CONSTITUENTS IN GROUNDWATER, 1995

METALS

1995 ANNUAL WATER MONITORING REPORT

LEHR ENVIRONMENTAL RESTORATION, DAVIS, CALIFORNIA

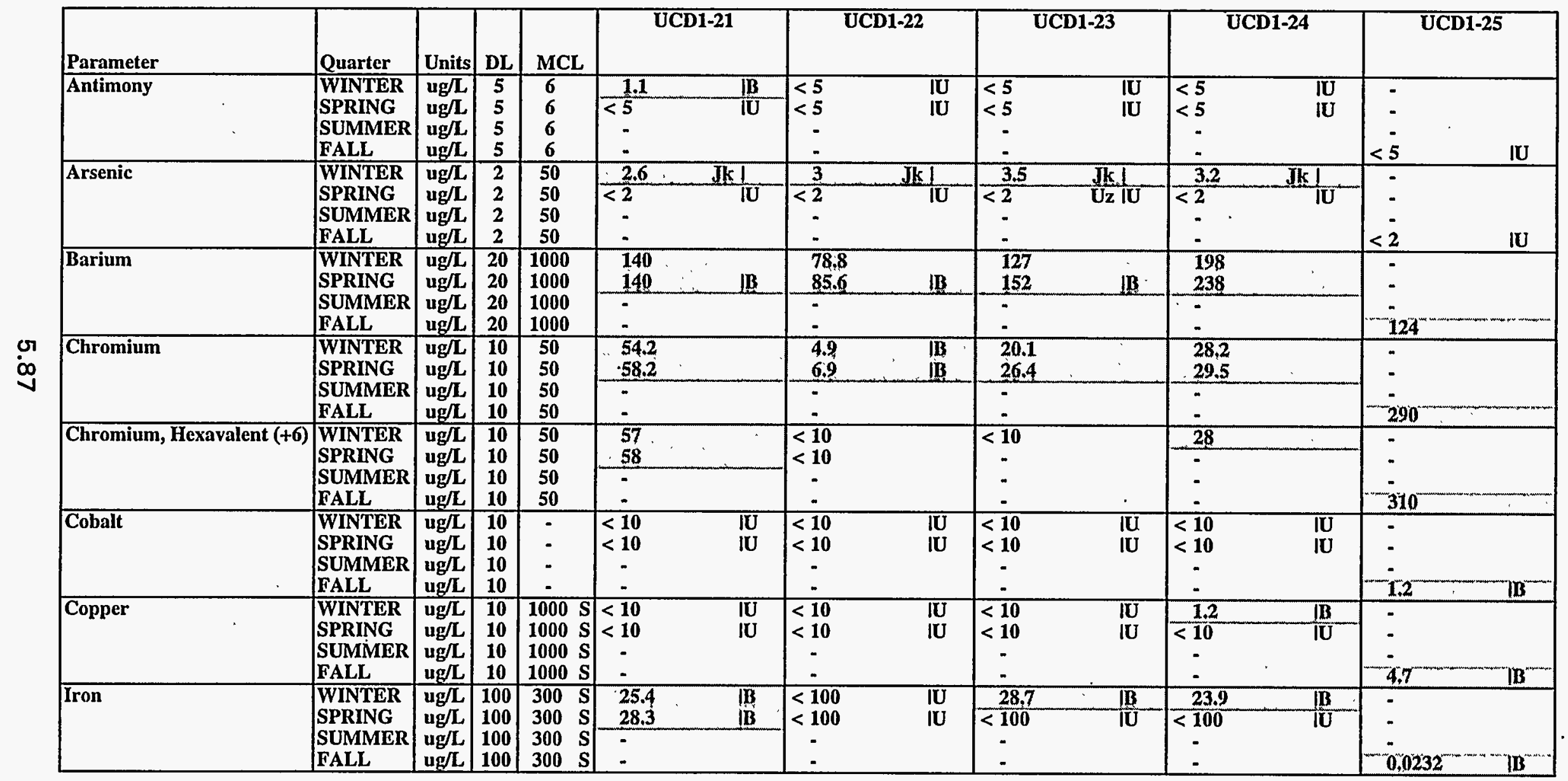

Page 7 of 17 
TABLE 5.5

SUMMARY OF DETECTED CONSTITUENTS IN GROUNDWATER, 1995

METALS

1995 ANNUAL WATER MONITORING REPORT

LEHR ENVIRONMENTAL RESTORATION, DAVIS, CALIFORNIA

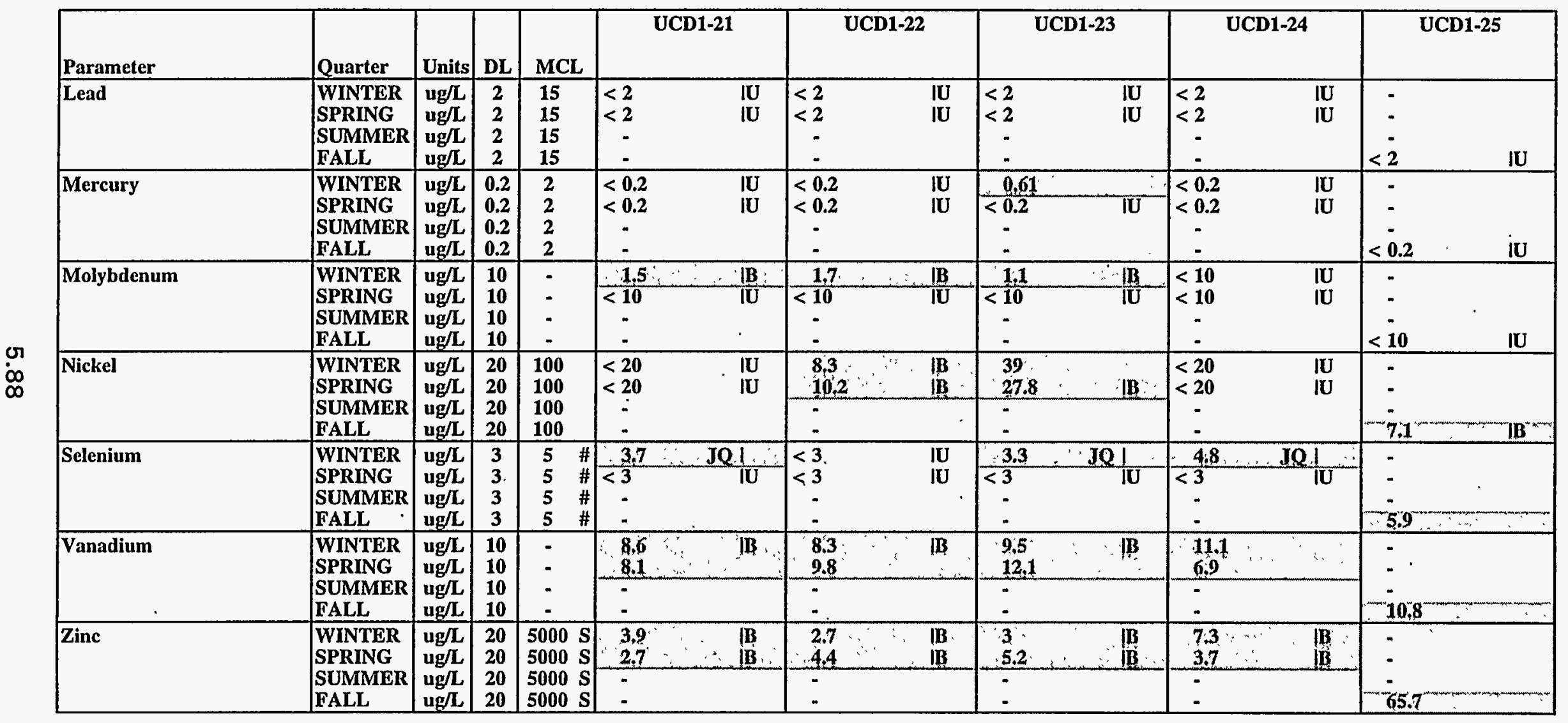


TABLE 5.5

SUMMARY OF DETECTED CONSTITUENTS IN GROUNDWATER, 1995

METALS

1995 ANNUAL WATER MONITORING REPORT

LEHR ENVIRONMENTAL RESTORATION, DAVIS, CALIFORNIA

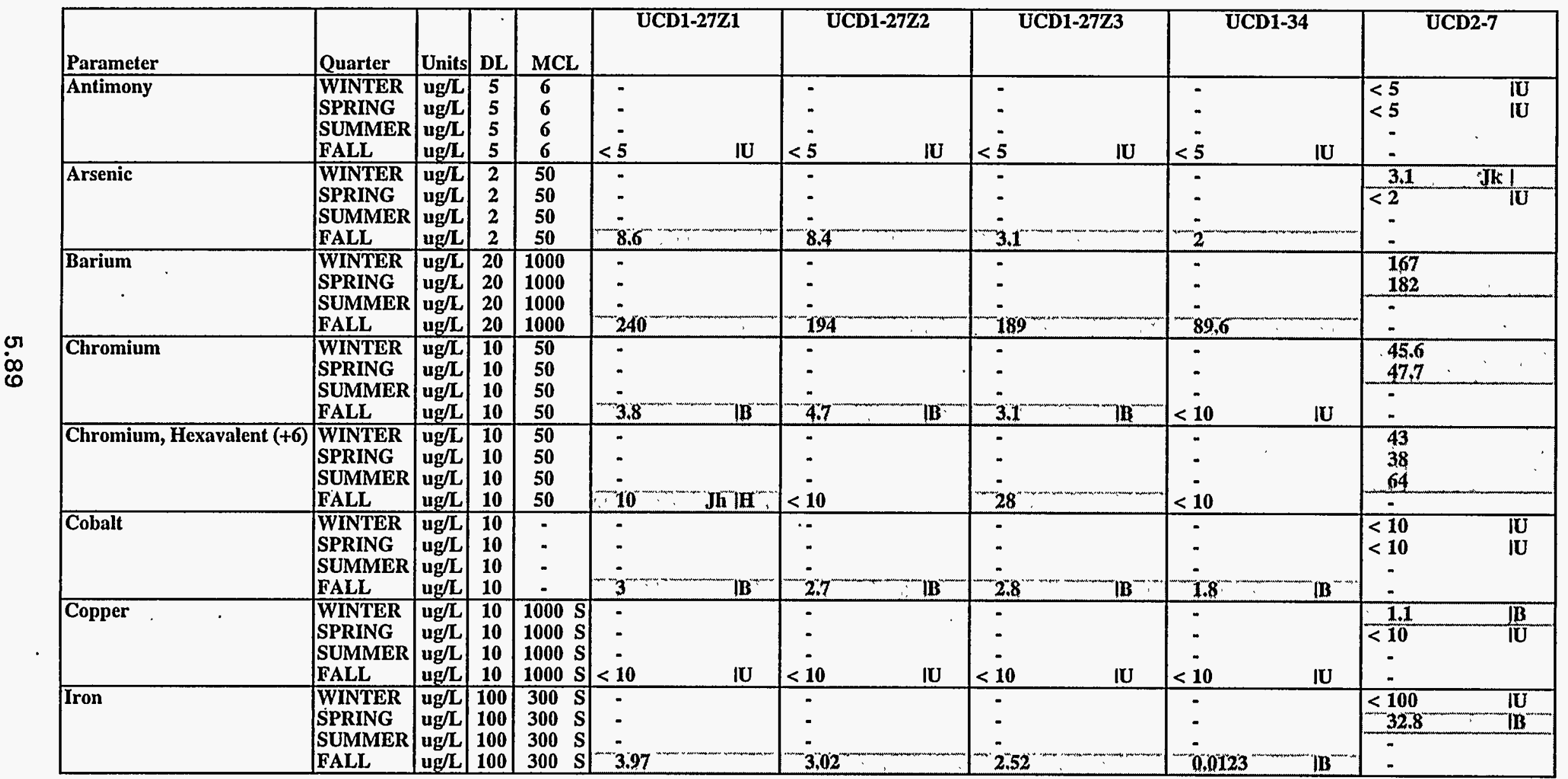


TABLE 5.5

SUMMARY OF DETECTED CONSTITUENTS IN GROUNDWATER, 1995

METALS

1995 ANNUAL WATER MONITORING REPORT

LEHR ENVIRONMENTAL RESTORATION, DAVIS, CALIFORNIA

\begin{tabular}{|c|c|c|c|c|c|c|c|c|c|}
\hline & & & & & UCD1-27Z1 & UCD1-27Z2 & UCD1-27Z3 & UCD1-34 & UCD2-7 \\
\hline Lead & $\begin{array}{l}\text { WINTER } \\
\text { SPRING } \\
\text { SUMMER } \\
\text { FALL } \\
\end{array}$ & $\begin{array}{l}\mathrm{ug} / \mathrm{L} \\
\mathrm{ug} / \mathrm{L} \\
\mathrm{ug} / \mathrm{L} \\
\mathrm{ug} / \mathrm{L}\end{array}$ & \begin{tabular}{|l}
2 \\
2 \\
2 \\
2 \\
\end{tabular} & $\begin{array}{l}15 \\
15 \\
15 \\
15\end{array}$ & $\begin{array}{r}- \\
<2 \\
\end{array}$ & $\begin{array}{r}- \\
- \\
<2 \\
\end{array}$ & $\begin{array}{r}- \\
\\
<2 \\
\end{array}$ & $\begin{array}{r}- \\
- \\
<2 \\
\end{array}$ & $\begin{array}{|cc|}<2 & \text { IU } \\
<2 & \text { IU } \\
\vdots & \\
\end{array}$ \\
\hline Mercury & $\begin{array}{l}\text { WINTER } \\
\text { SPRING } \\
\text { SUMMER } \\
\text { FALL } \\
\end{array}$ & $\begin{array}{l}\text { ug/L } \\
\text { ug } / \mathrm{L} \\
\mathrm{ug} / \mathrm{L} \\
\mathrm{ug} / \mathrm{L}\end{array}$ & $\begin{array}{l}0.2 \\
0.2 \\
0.2 \\
0.2 \\
\end{array}$ & $\begin{array}{l}2 \\
2 \\
2 \\
2 \\
\end{array}$ & $\begin{array}{c}- \\
- \\
<0.2 \\
\end{array}$ & $\begin{array}{c}- \\
- \\
<0.2 \\
\end{array}$ & $\begin{array}{r}- \\
- \\
<0.2 \\
\end{array}$ & $\begin{array}{l}-\dot{-} \\
<0.2 \\
\end{array}$ & $\begin{array}{l}0.68 \\
-0.2 \\
\vdots\end{array}$ \\
\hline Molybdenum & $\begin{array}{l}\text { WINTER } \\
\text { SPRING } \\
\text { SUMMER }\end{array}$ & $\begin{array}{l}\text { ug/L } \\
\text { ug/L } \\
\text { ug/L }\end{array}$ & $\begin{array}{l}10 \\
10 \\
10\end{array}$ & 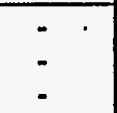 & $\div$ & $:$ & 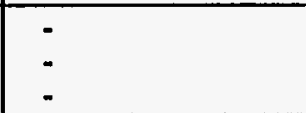 & 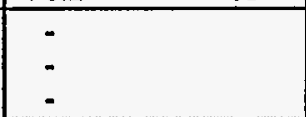 & $\begin{array}{ll}1.3 & (\mathbf{B} \\
3.1 & {[\mathbf{B}} \\
-\end{array}$ \\
\hline & FALL & $\mathrm{ug} / \mathrm{L}$ & 10 & - & 5,5 & 5.2 & 2.1 & IB & . \\
\hline Nickel & $\begin{array}{l}\text { WINTER } \\
\text { SPRING } \\
\text { SUMMER } \\
\text { FALL }\end{array}$ & $\begin{array}{l}\mathrm{ug} / \mathrm{L} \\
\mathrm{ug} / \mathrm{L} \\
\mathrm{ug} / \mathrm{L} \\
\mathrm{ug} / \mathrm{L}\end{array}$ & $\begin{array}{l}20 \\
20 \\
20 \\
20\end{array}$ & $\begin{array}{l}100 \\
100 \\
100 \\
100\end{array}$ & $\begin{array}{l}- \\
- \\
32.5\end{array}$ & $\begin{array}{l}- \\
i 1.6\end{array}$ & $\begin{array}{l}- \\
- \\
6.9\end{array}$ & $\begin{array}{l}- \\
- \\
- \\
13.1\end{array}$ & \begin{tabular}{|l|l}
$<20$ & IU \\
1,5 & IB \\
- & \\
\end{tabular} \\
\hline Selenium & $\begin{array}{l}\text { WINTER } \\
\text { SPRING } \\
\text { SUMMER } \\
\text { FALL } \\
\end{array}$ & $\begin{array}{l}\mathrm{ug} / \mathrm{L} \\
\mathrm{ug} / \mathrm{L} \\
\mathrm{ug} / \mathrm{L} \\
\mathrm{ug} / \mathrm{L}\end{array}$ & $\begin{array}{l}\mathbf{3} \\
\mathbf{3} \\
\mathbf{3} \\
\mathbf{3}\end{array}$ & $\begin{array}{ll}\mathbf{5} & \# \\
\mathbf{5} & \# \\
\mathbf{5} & \# \\
\mathbf{5} & \#\end{array}$ & $\begin{array}{l}\vdots \\
<3\end{array}$ & $\begin{array}{r}- \\
- \\
<3\end{array}$ & $\begin{array}{r}- \\
<3 \\
\end{array}$ & $\begin{array}{r}-\vdots \\
<3 \\
\end{array}$ & $\begin{array}{ll}<3 & \text { IU } \\
3.4 & \\
& \end{array}$ \\
\hline \begin{tabular}{|l|} 
Vanadium \\
\end{tabular} & $\begin{array}{l}\text { WINTER } \\
\text { SPRING } \\
\text { SUMMER } \\
\text { FALL }\end{array}$ & \begin{tabular}{|l|} 
ug/L \\
ug/L \\
ug/L \\
ug/L
\end{tabular} & $\begin{array}{l}10 \\
10 \\
10 \\
10\end{array}$ & : & $\begin{array}{l}- \\
< \\
<10\end{array}$ & $\begin{array}{c}- \\
- \\
<10\end{array}$ & $\begin{array}{c}- \\
- \\
<10\end{array}$ & $\begin{array}{l}- \\
- \\
-8.2 \\
\end{array}$ & $\begin{array}{lll}7.7 & \mathbf{B} \\
5 & 1 \mathrm{~B} \\
- & \\
\end{array}$ \\
\hline$\overline{\text { Zinc }}$ & $\begin{array}{l}\text { WINTER } \\
\text { SPRING } \\
\text { SUMMER } \\
\text { FALL } \\
\end{array}$ & $\begin{array}{l}\operatorname{ug} / \mathrm{L} \\
\operatorname{ug} / \mathrm{L} \\
\mathrm{ug} / \mathrm{L} \\
\mathrm{ug} / \mathrm{L}\end{array}$ & $\begin{array}{l}20 \\
20 \\
20 \\
20 \\
\end{array}$ & $\begin{array}{ll}5000 & S \\
5000 & S \\
5000 & S \\
5000 & S \\
\end{array}$ & $\begin{array}{l}- \\
- \\
- \\
559 \\
\end{array}$ & $\begin{array}{l}- \\
- \\
218 \\
\end{array}$ & Jm|ENBi & $\begin{array}{c}- \\
- \\
-36,3 \\
\end{array}$ & $\begin{array}{c}5.3 \\
<20 \\
- \\
\end{array}$ \\
\hline
\end{tabular}


TABLE 5.5

SUMMARY OF DETECTED CONSTITUENTS IN GROUNDWATER, 1995

METALS

1995 ANNUAL WATER MONITORING REPORT

LEHR ENVIRONMENTAL RESTORATION, DAVIS, CALIFORNIA

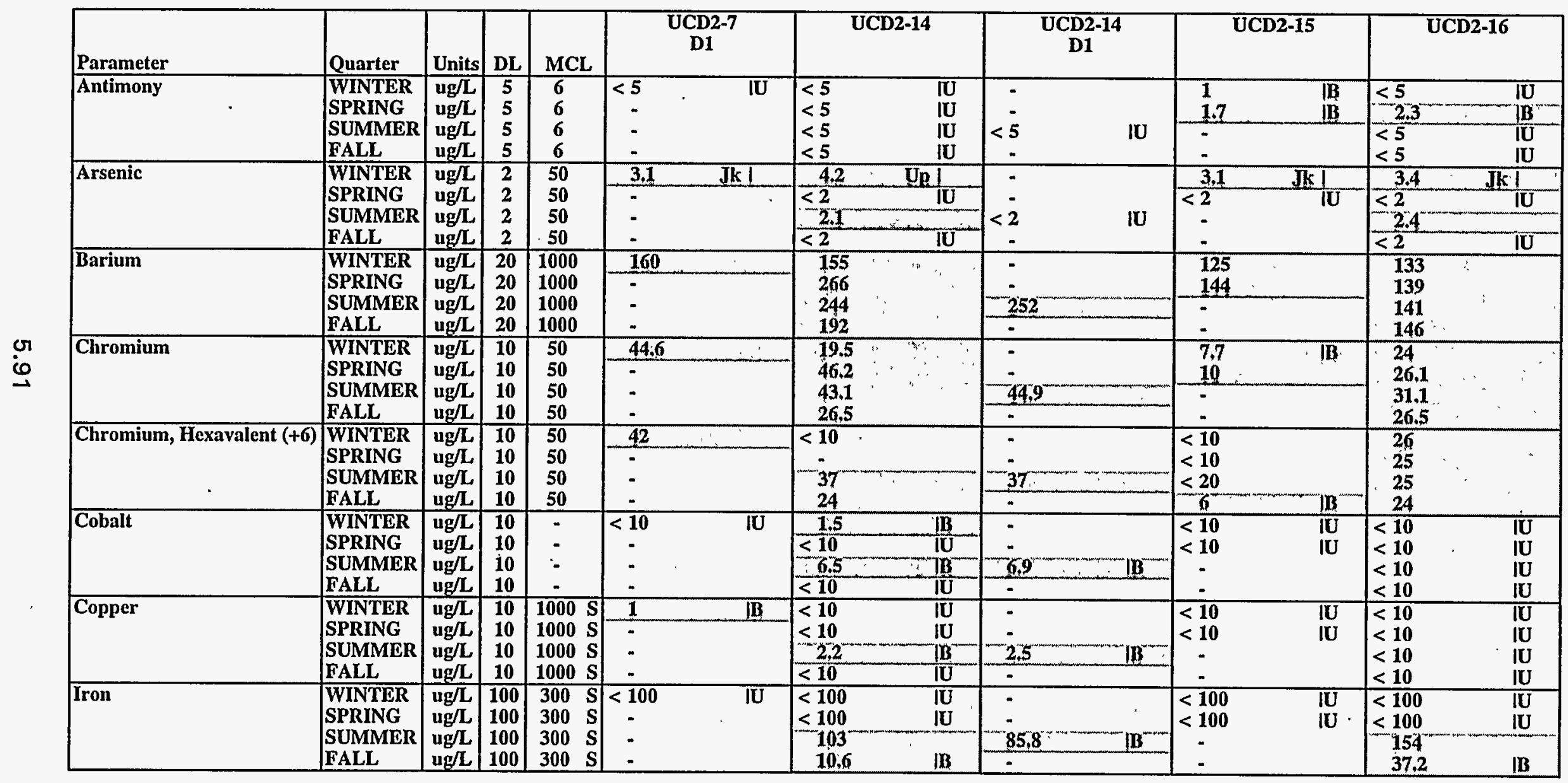

Page 11 of 17 
TABLE 5.5

SUMMARY OF DETECTED CONSTITUENTS IN GROUNDWATER, 1995

METALS

1995 ANNUAL WATER MONITORING REPORT

LEHR ENVIRONMENTAL RESTORATION, DAVIS, CALIFORNIA

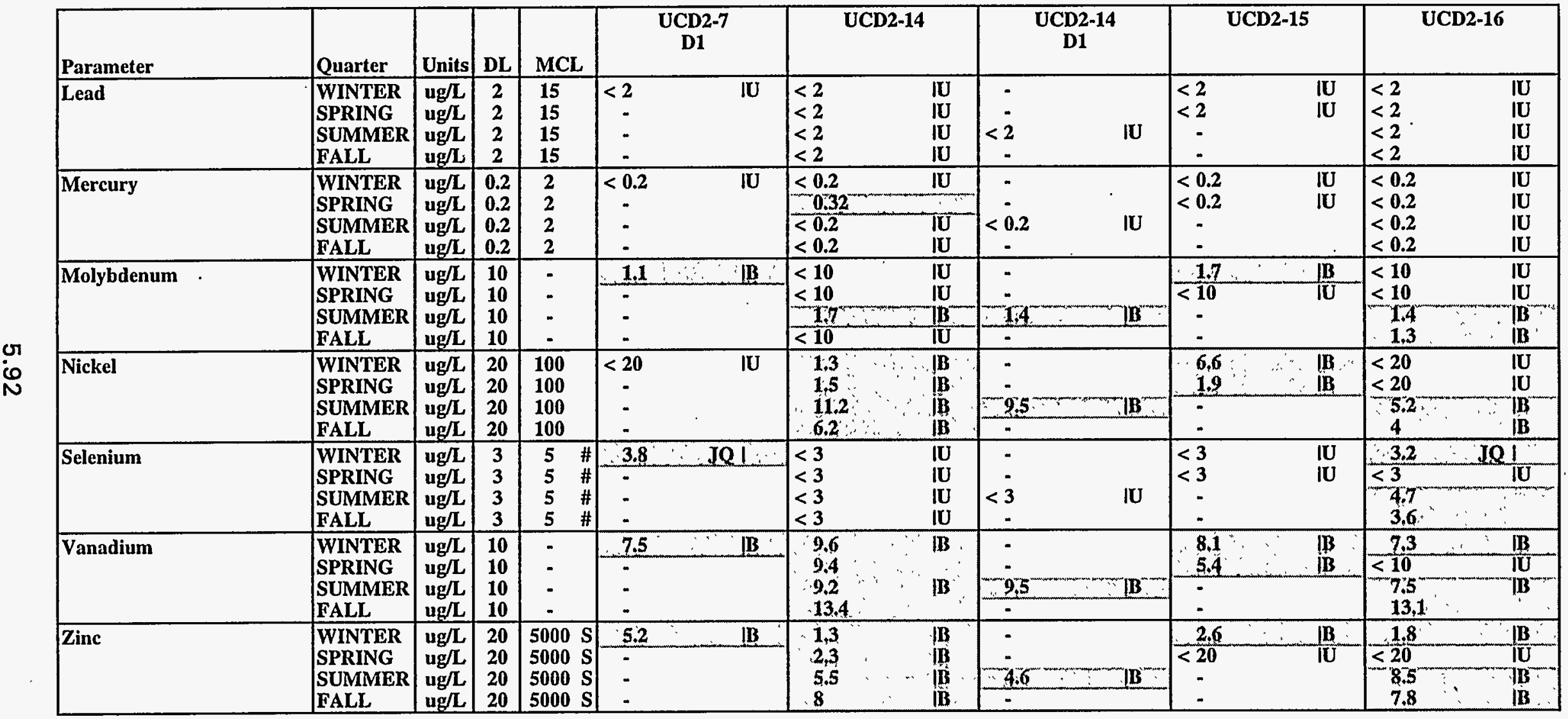


TABLE 5.5

SUMMARY OF DETECTED CONSTITUENTS IN GROUNDWATER, 1995

METALS

1995 ANNUAL WATER MONITORING REPORT

LEHR ENVIRONMENTAL RESTORATION, DAVIS, CALIFORNIA

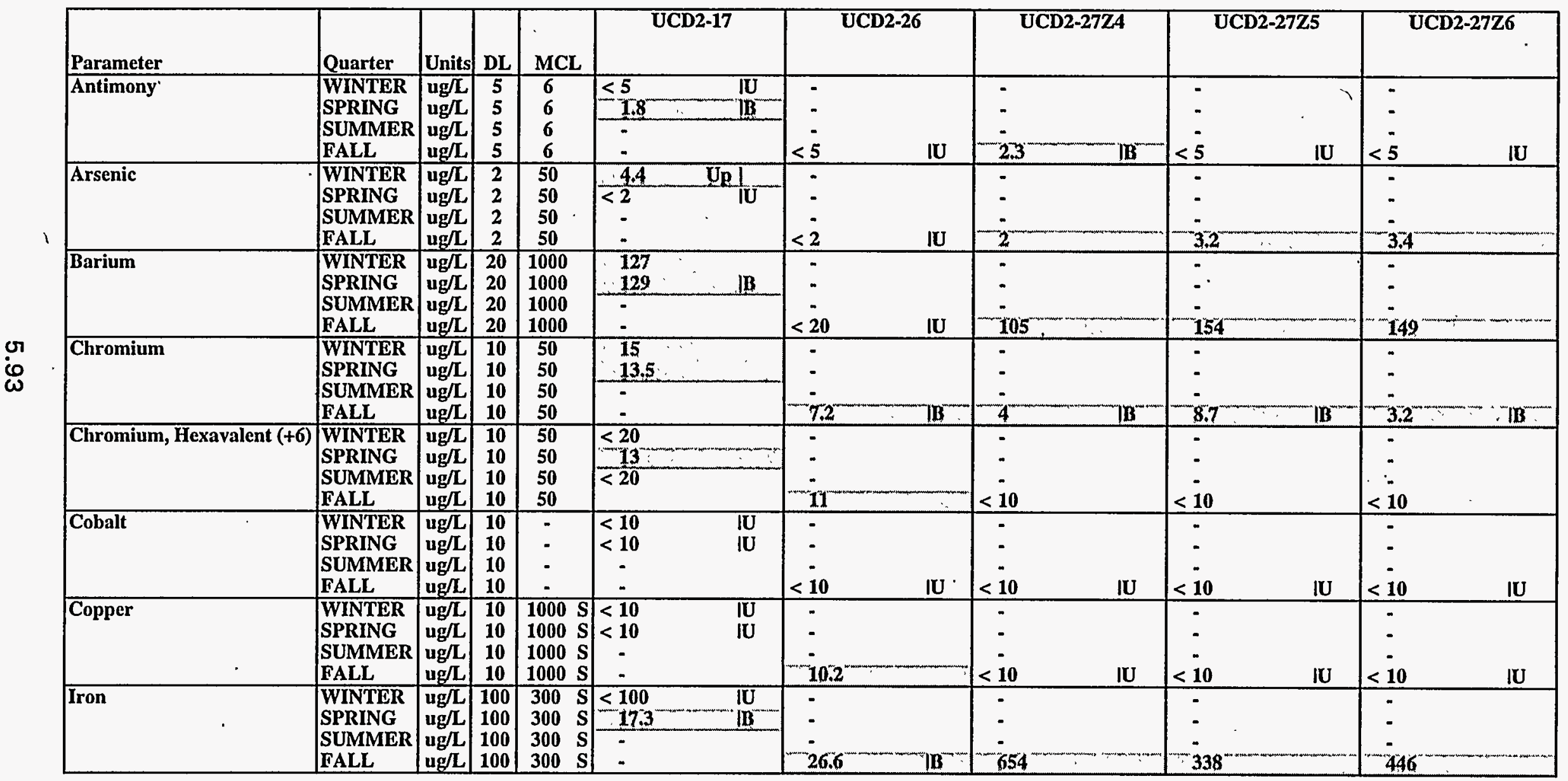


TABLE 5.5

SUMMARY OF DETECTED CONSTITUENTS IN GROUNDWATER, 1995

METALS

1995 ANNUAL WATER MONITORING REPORT

LEHR ENVIRONMENTAL RESTORATION, DAVIS, CALIFORNIA

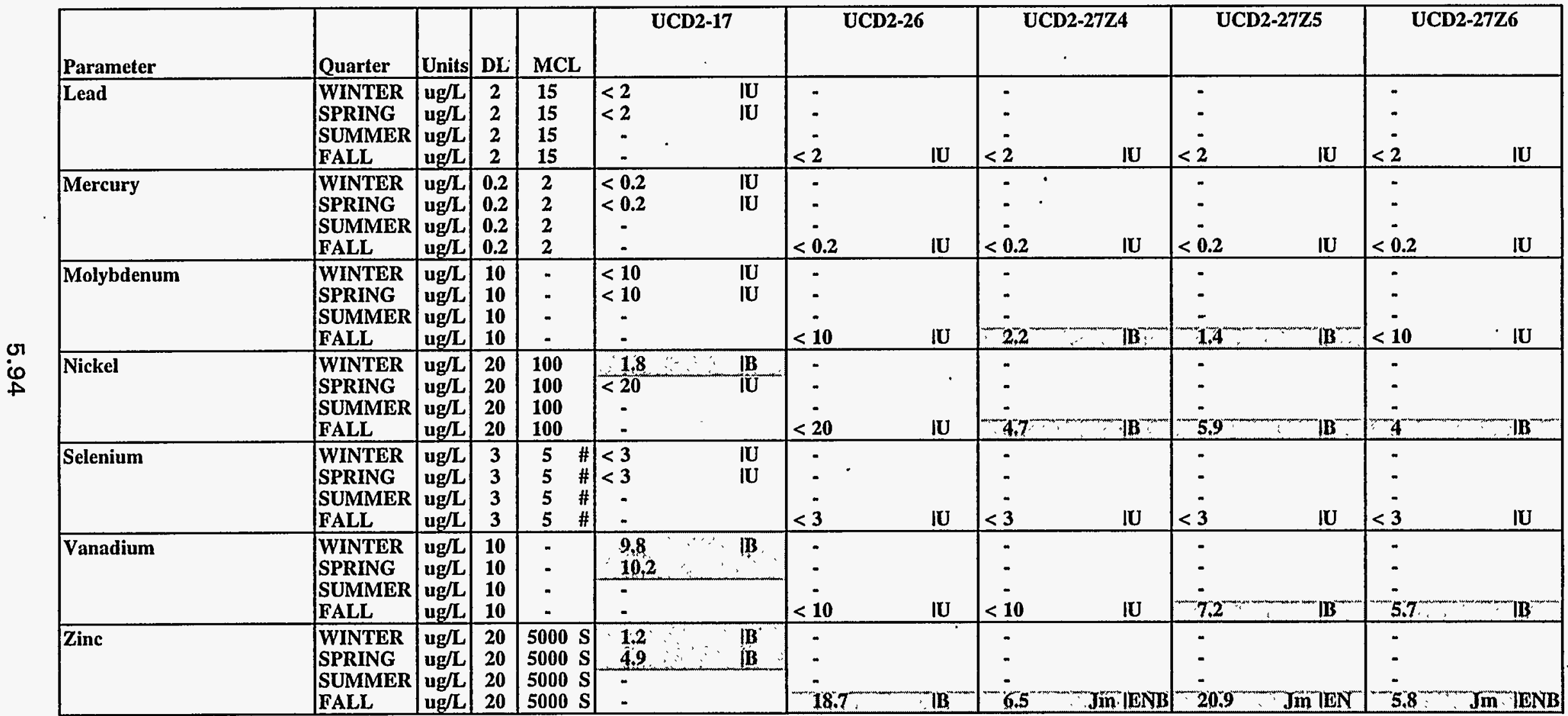


TABLE 5.5

SUMMARY OF DETECTED CONSTITUENTS IN GROUNDWATER, 1995

METALS

1995 ANNUAL WATER MONITORING REPORT

LEHR ENVIRONMENTAL RESTORATION, DAVIS, CALIFORNIA

\begin{tabular}{|c|c|c|c|c|c|c|}
\hline & & & & & UCD2-27Z7 & UCD2-35 \\
\hline Antimony & $\begin{array}{l}\text { WINTER } \\
\text { SPRING } \\
\text { SUMMER } \\
\text { FALL }\end{array}$ & $\begin{array}{l}\text { ug/L } \\
\text { ug/L } \\
\text { ug/L } \\
\text { ug/L }\end{array}$ & $\begin{array}{l}5 \\
5 \\
5 \\
5\end{array}$ & $\begin{array}{l}6 \\
6 \\
6 \\
6\end{array}$ & $\begin{array}{r}-\dot{ } \\
<\dot{5}\end{array}$ & $\begin{array}{r}- \\
- \\
<5\end{array}$ \\
\hline Arsenic & $\begin{array}{l}\text { WINTER } \\
\text { SPRING } \\
\text { SUMMER } \\
\text { FALL }\end{array}$ & $\begin{array}{l}\text { ug/L } \\
\text { ug/L } \\
\text { ug/L } \\
\text { ug/L }\end{array}$ & $\begin{array}{l}2 \\
2 \\
2 \\
2\end{array}$ & $\begin{array}{l}\mathbf{5 0} \\
\mathbf{5 0} \\
\mathbf{5 0} \\
\mathbf{5 0}\end{array}$ & $\dot{-}$ & $\begin{array}{r}- \\
- \\
<2\end{array}$ \\
\hline Barium & \begin{tabular}{|l|} 
WINTER \\
SPRING \\
SUMMER \\
FALL
\end{tabular} & $\begin{array}{l}\text { ug/L } \\
\text { ug/L } \\
\text { ug/L } \\
\text { ug/L }\end{array}$ & $\begin{array}{l}20 \\
20 \\
20 \\
20\end{array}$ & $\begin{array}{l}1000 \\
1000 \\
1000 \\
1000\end{array}$ & $\begin{array}{l}- \\
- \\
- \\
133\end{array}$ & $\begin{array}{c}- \\
- \\
- \\
99.1\end{array}$ \\
\hline Chromium & $\begin{array}{l}\text { WINTER } \\
\text { SPRING } \\
\text { SUMMER } \\
\text { FALL }\end{array}$ & $\begin{array}{l}u g / L \\
u g / L \\
u g / L \\
u g / L\end{array}$ & $\begin{array}{l}10 \\
10 \\
10 \\
10\end{array}$ & $\begin{array}{l}\mathbf{5 0} \\
\mathbf{5 0} \\
\mathbf{5 0} \\
\mathbf{5 0}\end{array}$ & $\begin{array}{l}- \\
- \\
- \\
3\end{array}$ & $\begin{array}{l}- \\
- \\
- \\
3,6\end{array}$ \\
\hline Chromium, Hexavalent $(+6)$ & $\begin{array}{l}\text { WINTER } \\
\text { SPRING } \\
\text { SUMMER } \\
\text { FALL } \\
\end{array}$ & $\begin{array}{l}\text { ug/L } \\
\text { ug/L } \\
\text { ug/L } \\
\text { ug/L }\end{array}$ & $\begin{array}{l}10 \\
10 \\
10 \\
10\end{array}$ & $\begin{array}{l}50 \\
50 \\
50 \\
50\end{array}$ & $\begin{array}{c}- \\
- \\
<10\end{array}$ & $\begin{array}{l}- \\
- \\
- \\
<10\end{array}$ \\
\hline Cobalt & $\begin{array}{l}\text { WINTER } \\
\text { SPRING } \\
\text { SUMMER } \\
\text { FALL }\end{array}$ & $\begin{array}{l}u g / L \\
u g / L \\
u g / L \\
u g / L\end{array}$ & $\begin{array}{l}10 \\
10 \\
10 \\
10\end{array}$ & ". & $\begin{array}{c}- \\
- \\
<10\end{array}$ & $\begin{array}{l}- \\
- \\
1.4\end{array}$ \\
\hline Copper & $\begin{array}{l}\text { WINTER } \\
\text { SPRING } \\
\text { SUMMER } \\
\text { FALL }\end{array}$ & $\begin{array}{l}\text { ug/L } \\
\text { ug/L } \\
\text { ug/L } \\
\text { ug/L }\end{array}$ & $\begin{array}{l}10 \\
10 \\
10 \\
10\end{array}$ & $\begin{array}{ll}1000 & \mathrm{~S} \\
1000 & \mathrm{~S} \\
1000 & \mathrm{~S} \\
1000 & \mathrm{~S}\end{array}$ & $\begin{array}{c}- \\
- \\
- \\
<10\end{array}$ & $\begin{array}{c}- \\
- \\
- \\
<10\end{array}$ \\
\hline Iron & $\begin{array}{l}\text { WINTER } \\
\text { SPRING } \\
\text { SUMMER } \\
\text { FALL }\end{array}$ & $\begin{array}{l}\text { ug/L } \\
\text { ug/L } \\
\text { ug/L } \\
\text { ug/L }\end{array}$ & $\begin{array}{l}100 \\
100 \\
100 \\
100\end{array}$ & $\begin{array}{ll}300 & S \\
300 & \mathbf{S} \\
\mathbf{3 0 0} & \mathbf{S} \\
\mathbf{3 0 0} & \mathbf{S} \\
\end{array}$ & $\begin{array}{l}- \\
- \\
- \\
762^{+}\end{array}$ & $\begin{array}{l}- \\
- \\
- \\
14,5\end{array}$ \\
\hline
\end{tabular}

Page 15 of 17 
TABLE 5.5

SUMMARY OF DETECTED CONSTITUENTS IN GROUNDWATER, 1995 METALS

1995 ANNUAL WATER MONITORING REPORT

LEHR ENVIRONMENTAL RESTORATION, DAVIS, CALIFORNIA

\begin{tabular}{|c|c|c|c|c|c|c|}
\hline Parameter & Quarter & Units & DL & MCL & UCD2-27Z7 & UCD2-35 \\
\hline Lead & $\begin{array}{l}\text { WINTER } \\
\text { SPRING } \\
\text { SUMMER } \\
\text { FALL }\end{array}$ & $\begin{array}{l}\mathrm{ug} / \mathrm{L} \\
\mathrm{ug} / \mathrm{L} \\
\mathrm{ug} / \mathrm{L} \\
\mathrm{ug} / \mathrm{L}\end{array}$ & $\begin{array}{l}2 \\
2 \\
2 \\
2\end{array}$ & $\begin{array}{l}15 \\
15 \\
15 \\
15\end{array}$ & $\begin{array}{r}\dot{ } \\
<\dot{2}\end{array}$ & $\begin{array}{r}: \\
<2\end{array}$ \\
\hline Mercury & $\begin{array}{l}\text { WINTER } \\
\text { SPRING } \\
\text { SUMMER } \\
\text { FALL } \\
\end{array}$ & $\begin{array}{l}\mathrm{ug} / \mathrm{L} \\
\mathrm{ug} / \mathrm{L} \\
\mathrm{ug} / \mathrm{L} \\
\mathrm{ug} / \mathrm{L}\end{array}$ & $\begin{array}{l}0.2 \\
0.2 \\
0.2 \\
0.2 \\
\end{array}$ & $\begin{array}{l}2 \\
2 \\
2 \\
2\end{array}$ & $\begin{array}{c}\dot{-} \\
<\overline{0.2} \\
\end{array}$ & $\begin{array}{c}\vdots \\
\vdots \\
<0.2\end{array}$ \\
\hline Molybdenum & $\begin{array}{l}\text { WINTER } \\
\text { SPRING } \\
\text { SUMMER } \\
\text { FALL } \\
\end{array}$ & $\begin{array}{l}\mathrm{ug} / \mathrm{L} \\
\mathrm{ug} / \mathrm{L} \\
\mathrm{ug} / \mathrm{L} \\
\mathrm{ug} / \mathrm{L}\end{array}$ & $\begin{array}{l}10 \\
10 \\
10 \\
10 \\
\end{array}$ & $\begin{array}{l}- \\
- \\
-\end{array}$ & $\begin{array}{l}- \\
\vdots \\
1.4\end{array}$ & $\begin{array}{l}- \\
- \\
2 \\
\end{array}$ \\
\hline Nickel & $\begin{array}{l}\text { WINTER } \\
\text { SPRING } \\
\text { SUMMER } \\
\text { FALL } \\
\end{array}$ & $\begin{array}{l}\mathrm{ug} / \mathrm{L} \\
\mathrm{ug} / \mathrm{L} \\
\mathrm{ug} / \mathrm{L} \\
\mathrm{ug} / \mathrm{L}\end{array}$ & $\begin{array}{l}20 \\
20 \\
20 \\
20 \\
\end{array}$ & \begin{tabular}{l|}
100 \\
100 \\
100 \\
100 \\
\end{tabular} & $\begin{array}{l}- \\
- \\
- \\
4.7\end{array}$ & $\begin{array}{l}: \\
: \\
92\end{array}$ \\
\hline Selenium & $\begin{array}{l}\text { WINTER } \\
\text { SPRING } \\
\text { SUMMER } \\
\text { FALL }\end{array}$ & $\begin{array}{l}\mathrm{ug} / \mathrm{L} \\
\mathrm{ug} / \mathrm{L} \\
\mathrm{ug} / \mathrm{L} \\
\mathrm{ug} / \mathrm{L}\end{array}$ & $\begin{array}{l}3 \\
3 \\
3 \\
3\end{array}$ & $\begin{array}{ll}\mathbf{5} & \# \\
\mathbf{5} & \# \\
\mathbf{5} & \# \\
\mathbf{5} & \#\end{array}$ & $\begin{array}{r}\vdots \\
<3 \\
\end{array}$ & $\begin{array}{r}-\dot{-} \\
<3\end{array}$ \\
\hline Vanadium & $\begin{array}{l}\text { WINTER } \\
\text { SPRING } \\
\text { SUMMER } \\
\text { FALL } \\
\end{array}$ & $\begin{array}{l}\mathrm{ug} / \mathrm{L} \\
\mathrm{ug} / \mathrm{L} \\
\mathrm{ug} / \mathrm{L} \\
\mathrm{ug} / \mathrm{L}\end{array}$ & $\begin{array}{l}10 \\
10 \\
10 \\
10 \\
\end{array}$ & $\begin{array}{l}: \\
: \\
\end{array}$ & $\begin{array}{l}- \\
- \\
5 \\
\end{array}$ & $\begin{array}{l}- \\
- \\
9.6\end{array}$ \\
\hline Zinc & \begin{tabular}{|l|} 
WINTER \\
SPRING \\
SUMMER \\
FALL \\
\end{tabular} & $\begin{array}{l}\text { ug/L } \\
\text { ug } / L \\
\text { ug/L } \\
\text { ug } / L\end{array}$ & \begin{tabular}{l|}
20 \\
20 \\
20 \\
20
\end{tabular} & $\begin{array}{ll}5000 & S \\
5000 & S \\
5000 & S \\
5000 & S \\
\end{array}$ & $\begin{array}{l}- \\
- \\
7.4\end{array}$ & $\begin{array}{l}- \\
- \\
14\end{array}$ \\
\hline
\end{tabular}


TABLE 5.5

SUMMARY OF DETECTED CONSTITUENTS IN GROUNDWATER, 1995

METALS

1995 ANNUAL WATER MONITORING REPORT

LEHR ENVIRONMENTAL RESTORATION, DAVIS, CALIFORNIA

D1 = Duplicate sample.

- = Parameter not analyzed or no MCL.

DL $=$ Detection limit.

$<=$ Constituent below detection limit. Detection limits may vary depending on interference by other sample constituents.

MCL = Maximum Contaminant Level (primary), Title 22 Division 4 Chapter 15.

\# = USEPA MCL

$\mathrm{S}=$ Secondary Drinking Water Standard

Highlighted values indicate positive results.

See Appendix A for explanation of data qualifiers. 
TABLE 5.6

SUMMARY OF DETECTED CONSTITUENTS IN GROUNDWATER, 1995

RADIOCHEMICALS

1995 ANNUAL WATER MONITORING REPORT

LEHR ENVIRONMENTAL RESTORATION, DAVIS, CALIFORNIA

\begin{tabular}{|c|c|c|c|c|c|c|c|c|c|}
\hline Parameter & Quarter & Units & MCL & Method & UCD1-1 & $\begin{array}{c}\text { UCD1-1 } \\
\text { D1 } \\
\end{array}$ & UCD1-4 & UCD1-10 & UCD1-11 \\
\hline Gross Alpha & $\begin{array}{l}\text { WINTER } \\
\text { SPRING } \\
\text { SUMMER } \\
\text { FALL } \\
\end{array}$ & $\begin{array}{l}\mathrm{pCi} / \mathrm{L} \\
\mathrm{pCi} / \mathrm{L} \\
\mathrm{pCi} / \mathrm{L} \\
\mathrm{pCi} / \mathrm{L}\end{array}$ & $\begin{array}{l}15 \\
15 \\
15 \\
15 \\
\end{array}$ & $\begin{array}{l}9310 \\
9310 \\
9310 \\
9310 \\
\end{array}$ & $\begin{array}{rr}1.1 \pm 2.5 & \mid C \\
0.1 \pm 3.8 & \mid C \\
- & \\
- & \end{array}$ & \begin{tabular}{rr|}
- & \\
$0.4 \pm 3.8$ & $\mathrm{IC}$ \\
- & \\
- & \\
\end{tabular} & \begin{tabular}{rr|}
$-1.2 \pm 2.1$ & $\mid C$ \\
$6.5 \pm 4.9$ & $\mid C$ \\
$1.9 \pm 4.3$ & $\mid C$ \\
$1 \pm 3$ & $|C|$ \\
\end{tabular} & $\begin{array}{rr}1.9 \pm 5.8 & \mid C \\
0 \pm 6 & \mid C \\
- & \\
- & \\
\end{array}$ & $\begin{array}{rr}2.7 \pm 5.2 & \mid \mathrm{C} \\
0.8 \pm 3.7 & \mid \mathrm{C} \\
- & \\
- & \\
\end{array}$ \\
\hline Gross Beta & $\begin{array}{l}\text { WINTER } \\
\text { SPRING } \\
\text { SUMMER } \\
\text { FALL }\end{array}$ & $\begin{array}{l}\mathrm{pCi} / \mathrm{L} \\
\mathrm{pCi} / \mathrm{L} \\
\mathrm{pCi} / \mathrm{L} \\
\mathrm{pCl} / \mathrm{L}\end{array}$ & $\begin{array}{l}\mathbf{5 0} \\
\mathbf{5 0} \\
\mathbf{5 0} \\
\mathbf{5 0}\end{array}$ & $\begin{array}{l}9310 \\
9310 \\
9310 \\
9310\end{array}$ & \begin{tabular}{cc|}
$2.2 \pm 5.4$ & $\mid \mathrm{C}$ \\
- & \\
- &
\end{tabular} & \begin{tabular}{rr|}
$-3.1 \pm 5 . \overline{1}$ & $\mid \mathrm{C}$ \\
- & \\
- &
\end{tabular} & $\begin{array}{r}0.7 \pm 3.1 \\
2.9 \pm 4.7 \\
2 \pm 4 \\
1.7 \pm 2\end{array}$ & $\begin{array}{r}-2.8 \pm 6.9 \\
1.1 \pm 6.8 \\
- \\
-\end{array}$ & $\begin{array}{r}1.4 \pm 4.6 \\
4.6 \pm 5.3 \\
- \\
-\end{array}$ \\
\hline Actinlum-228 & \begin{tabular}{|l|} 
WINTER \\
SPRING \\
SUMMER \\
FALL \\
\end{tabular} & $\begin{array}{l}\mathrm{pCi} / \mathrm{L} \\
\mathrm{pCl} / \mathrm{L} \\
\mathrm{pCi} / \mathrm{L} \\
\mathrm{pCi} / \mathrm{L}\end{array}$ & $\begin{array}{l}- \\
- \\
- \\
\end{array}$ & \begin{tabular}{|l|}
901.1 \\
901.1 \\
901.1 \\
901.1 \\
\end{tabular} & $\begin{array}{r}18 \pm 22 \\
-6.1 \pm 6.3 \\
- \\
-\end{array}$ & $\begin{array}{r}20 \pm 22 \\
- \\
-\end{array}$ & $\begin{array}{r}0.4 \pm 7.7 \\
-1.7 \pm 7.6 \\
0.9 \pm 7.4 \\
-0.1 \pm 7.2 \\
\end{array}$ & $\begin{array}{r}1 \pm 21 \\
2 \pm 21 \\
- \\
-\end{array}$ & $\begin{array}{r}6 \pm 21 \\
-9 \pm 12 \\
- \\
-\end{array}$ \\
\hline Americium-241 & $\begin{array}{l}\text { WINTER } \\
\text { SPRING } \\
\text { SUMMER } \\
\text { FALL } \\
\end{array}$ & $\begin{array}{l}\mathrm{pCi} / \mathrm{L} \\
\mathrm{pCi} / \mathrm{L} \\
\mathrm{pCi} / \mathrm{L} \\
\mathrm{pCl} / \mathrm{L}\end{array}$ & $\begin{array}{l}- \\
- \\
- \\
-\end{array}$ & $\begin{array}{l}\text { LAS108 } \\
\text { LAS108 } \\
\text { LAS108 } \\
\text { LAS108 } \\
\end{array}$ & $\begin{array}{c}-0.001 \pm 0.029 \mathrm{Jm} \mid \\
-0.006 \pm 0.044 \\
-\end{array}$ & $\begin{array}{r}-0.049 \pm 0.066 \\
- \\
\end{array}$ & $\begin{array}{c}-0.033 \pm 0.04 \mathrm{Jm} \mid \\
-0.005 \pm 0.04 \\
- \\
- \\
\end{array}$ & $\begin{array}{c}-0.002 \pm 0.03 \mathrm{Jm} \\
- \\
- \\
\end{array}$ & $\begin{array}{c}0.006 \pm 0.058 \mathrm{Jm} \mid \\
-0.004 \pm 0.053 \\
- \\
- \\
\end{array}$ \\
\hline Bismuth-214 & $\begin{array}{l}\text { WINTER } \\
\text { SPRING } \\
\text { SUMMER } \\
\text { FALL } \\
\end{array}$ & $\begin{array}{l}\mathrm{pCi} / \mathrm{L} \\
\mathrm{pCi} / \mathrm{L} \\
\mathrm{pCi} / \mathrm{L} \\
\mathrm{pCi} / \mathrm{L}\end{array}$ & $\begin{array}{l}- \\
- \\
-\end{array}$ & $\begin{array}{l}901.1 \\
901.1 \\
901.1 \\
901.1 \\
\end{array}$ & 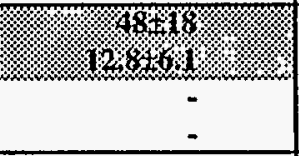 & $\begin{array}{r}- \\
9 \pm 14 \\
- \\
\end{array}$ & 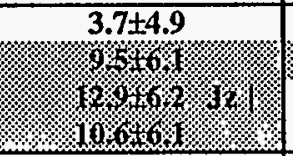 & $\begin{array}{r}8 \pm 13 \\
\% \text { Kat } \\
- \\
-\end{array}$ & $\begin{array}{r}-2 \pm 13 \\
6 \\
\times- \\
- \\
-\end{array}$ \\
\hline Carbon-14 & $\begin{array}{l}\text { WINTER } \\
\text { SPRING } \\
\text { SUMMER } \\
\text { FALL } \\
\end{array}$ & $\begin{array}{l}\mathrm{pCi} / \mathrm{L} \\
\mathrm{pCi} / \mathrm{L} \\
\mathrm{pC} \mathrm{L} / \mathrm{L} \\
\mathrm{pCi} / \mathrm{L}\end{array}$ & $\begin{array}{l}. \\
. \\
.\end{array}$ & $\begin{array}{l}\text { LSC } \\
\text { LSC } \\
\text { LSC } \\
\text { LSC }\end{array}$ & $\begin{array}{r}-9 \pm 82 \\
3 \pm 83 \\
- \\
-\end{array}$ & $\begin{array}{r}-19 \pm 81 \\
- \\
-\end{array}$ & $\begin{array}{r}-34 \pm 77 \\
43 \pm 86 \\
- \\
-\end{array}$ & $\begin{array}{r}-14 \pm 78 \\
-5 \pm 82 \\
- \\
-\end{array}$ & $\begin{array}{r}2 \pm 80 \\
14 \pm 84 \\
- \\
-\end{array}$ \\
\hline Lead-212 & \begin{tabular}{|l|} 
WINTER \\
SPRING \\
SUMMER \\
FALL \\
\end{tabular} & $\begin{array}{l}\mathrm{pCi} / \mathrm{L} \\
\mathrm{pCi} / \mathrm{L} \\
\mathrm{pCi} / \mathrm{L} \\
\mathrm{pCi} / \mathrm{L}\end{array}$ & $\begin{array}{l}- \\
- \\
-\end{array}$ & $\begin{array}{l}901.1 \\
901.1 \\
901.1 \\
901.1\end{array}$ & $\begin{array}{r}11 \pm 10 \\
-4.5 \pm 5 \\
- \\
-\end{array}$ & $\begin{array}{r}- \\
4.4 \pm 9.8 \\
-\end{array}$ & $\begin{array}{l}2.1 \pm 4.9 \\
0.6 \pm 5.1 \\
0.8 \pm 5.4 \\
1.2 \pm 4.9\end{array}$ & $\begin{array}{r}0.7 \pm 9.5 \\
6.2 \pm 9.7 \\
- \\
-\end{array}$ & $\begin{array}{r}7 \pm 9.3 \\
3.7 \pm 9.8 \\
- \\
\end{array}$ \\
\hline Lead-214 & $\begin{array}{l}\text { WINTER } \\
\text { SPRING } \\
\text { SUMMER } \\
\text { FALL } \\
\end{array}$ & $\begin{array}{l}\mathrm{pCi} / \mathrm{L} \\
\mathrm{pCi} / \mathrm{L} \\
\mathrm{pCi} / \mathrm{L} \\
\mathrm{pCi} / \mathrm{L}\end{array}$ & $\begin{array}{l}- \\
- \\
-\end{array}$ & $\begin{array}{l}901.1 \\
901.1 \\
901.1 \\
901.1\end{array}$ & 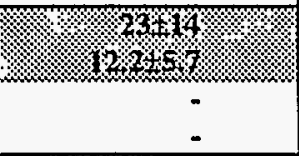 & $\begin{array}{r}- \\
16 \pm 12 \\
-\end{array}$ & $\begin{array}{c}2.2 \pm 4.8 \\
10.3 \\
1.6 \\
7.5 \pm 5.3\end{array}$ & $\begin{array}{r}1+10 \\
2 \times 12 \\
- \\
-\end{array}$ & $\begin{array}{c}-11.4 \pm 8.2 \\
1 \times \\
-\end{array}$ \\
\hline
\end{tabular}


TABLE 5.6

SUMMARY OF DETECTED CONSTITUENTS IN GROUNDWATER, 1995

RADIOCHEMICALS

1995 ANNUAL WATER MONITORING REPORT

LEHR ENVIRONMENTAL RESTORATION, DAVIS, CALIFORNIA

\begin{tabular}{|c|c|c|c|c|c|c|c|c|c|}
\hline Parameter & Quarter & Units & MCL & Method & UCD1-1 & $\begin{array}{c}\text { UCD1-1 } \\
\text { D1 }\end{array}$ & UCD1-4 & - & UCD1-11 \\
\hline Plutonium-241 & $\begin{array}{l}\text { WINTER } \\
\text { SPRING } \\
\text { SUMMER } \\
\text { FALL } \\
\end{array}$ & $\begin{array}{l}\mathrm{pCi} / \mathrm{L} \\
\mathrm{pCi} / \mathrm{L} \\
\mathrm{pCi} / \mathrm{L} \\
\mathrm{pCi} / \mathrm{L}\end{array}$ & $\begin{array}{l}- \\
- \\
-\end{array}$ & $\begin{array}{l}\text { LAS178 } \\
\text { LAS178 } \\
\text { LAS178 } \\
\text { LAS178 }\end{array}$ & $\begin{array}{c}-0.1 \pm 1.3 \\
-0.5 \pm 1.8 \mathrm{Jz} \mid \\
- \\
-\end{array}$ & $\begin{array}{c}-3 \pm 1.8 \mathrm{Jz} \mid \\
- \\
-\end{array}$ & $\begin{array}{c}-0.2 \pm 1.5 \\
-0.5 \pm 1.8 \mathrm{Jz} \mid \\
- \\
-\end{array}$ & $\begin{array}{r}-0.2 \pm 1.5 \\
1 \pm 1.8 \mathrm{Jz} \mid \\
-\end{array}$ & $\begin{array}{r}-0.9 \pm 1.6 \\
0 \pm 1.8 \mathrm{Jz} \mid \\
- \\
-\end{array}$ \\
\hline Radium-226 & \begin{tabular}{|l|} 
WINTER \\
SPRING \\
SUMMER \\
FALL \\
WINTER \\
SPRING \\
SUMMER \\
FALL \\
\end{tabular} & $\begin{array}{l}\mathrm{pCl} / \mathrm{L} \\
\mathrm{pCl} / \mathrm{L} \\
- \\
\mathrm{pCi} / \mathrm{L} \\
\mathrm{pCl} / \mathrm{L} \\
\mathrm{pCl} / \mathrm{L} \\
\mathrm{pCl} / \mathrm{L} \\
\mathrm{pCi} / \mathrm{L}\end{array}$ & $\begin{array}{l}\mathbf{5} \\
5 \\
- \\
5 \\
5 \\
5 \\
5 \\
5\end{array}$ & $\begin{array}{c}901.1 \\
901.1 \\
901.1 \\
901.1 \\
903.1 \mathrm{M} \\
903.1 \mathrm{M} \\
903.1 \mathrm{M} \\
903.1 \mathrm{M} \\
\end{array}$ & $\begin{array}{r}-490 \pm 130 \\
43 \pm 60 \\
- \\
- \\
0.17 \pm 0.18 \\
-0.80 .63 \\
-\end{array}$ & $\begin{array}{r}-70 \pm 120 \\
- \\
- \\
- \\
1540 \\
-\end{array}$ & $\begin{array}{r}-40 \pm 56 \\
-38 \pm 55 \\
- \\
-4 \pm 54 \\
0.04 \pm 0.15 \\
0.1 \pm 0.12 \\
0.18 \pm 0.14 \\
0.133 \pm 0.1\end{array}$ & $\begin{array}{r}-110 \pm 110 \\
40 \pm 120 \\
- \\
- \\
0.019 \pm 0.083 \\
0.00 \\
-\end{array}$ & $\begin{array}{r}-280 \pm 120 \\
80 \pm 120 \\
- \\
- \\
0.19 \pm 0.18 \\
0.093 \pm 0.086 \\
- \\
-\end{array}$ \\
\hline Strontium-90 & \begin{tabular}{|l|} 
WINTER \\
SPRING \\
SUMMER \\
FALL \\
\end{tabular} & $\begin{array}{l}\mathrm{pCl} / \mathrm{L} \\
\mathrm{pCl} / \mathrm{L} \\
\mathrm{pCl} / \mathrm{L} \\
\mathrm{pCl} / \mathrm{L}\end{array}$ & $\begin{array}{l}8 \\
8 \\
8 \\
8\end{array}$ & $\begin{array}{l}905 \\
905 \\
905 \\
905\end{array}$ & $\begin{array}{r}-0.3 \pm 1.1 \\
0.66 \pm 0.51 \\
- \\
-\end{array}$ & $\begin{array}{r}0.7 \pm 0.47 \\
-\end{array}$ & $\begin{array}{r}-0.2 \pm 3 \\
0.67 \pm 0.46 \\
-0.16 \pm 0.25 \\
0.06 \pm 0.66\end{array}$ & $\begin{array}{r}-0.7 \pm 3 \\
0.7 \pm 0.47 \\
- \\
-\end{array}$ & 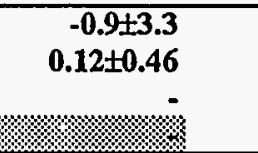 \\
\hline Tritium & \begin{tabular}{|l|} 
WINTER \\
SPRING \\
SUMMER \\
FALL \\
\end{tabular} & $\begin{array}{l}\mathrm{pCl} / \mathrm{L} \\
\mathrm{pCl} / \mathrm{L} \\
\mathrm{pCl} / \mathrm{L} \\
\mathrm{pCl} / \mathrm{L}\end{array}$ & $\begin{array}{l}20000 * \\
20000 * \\
20000 * \\
20000 *\end{array}$ & $\begin{array}{l}906 \\
906 \\
906 \\
906\end{array}$ & $\begin{array}{r}-220 \pm 150 \\
-10 \pm 220 \\
- \\
-\end{array}$ & $\begin{array}{r}-50 \pm 210 \\
-\end{array}$ & $\begin{array}{r}20 \pm 220 \\
-80 \pm 210 \\
-110 \pm 220 \\
-30 \pm 160\end{array}$ & $\begin{array}{r}0 \pm 220 \\
30 \pm 220 \\
- \\
-\end{array}$ & $\begin{array}{r}-140 \pm 200 \\
-40 \pm 220 \\
- \\
-\end{array}$ \\
\hline Uranium-235 & $\begin{array}{l}\text { WINTER } \\
\text { SPRING } \\
\text { SUMMER } \\
\text { FALL } \\
\end{array}$ & $\begin{array}{l}\mathrm{pCi} / \mathrm{L} \\
\mathrm{pCi} / \mathrm{L} \\
\mathrm{pCi} / \mathrm{L} \\
\mathrm{pCi} / \mathrm{L}\end{array}$ & $\begin{array}{l}- \\
- \\
- \\
-\end{array}$ & $\begin{array}{l}901.1 \\
901.1 \\
901.1 \\
901.1\end{array}$ & $\begin{array}{r}-13 \pm 15 \\
-5 \pm 13 \\
- \\
-\end{array}$ & $\begin{array}{r}-4 \pm 27 \\
- \\
-\end{array}$ & $\begin{array}{r}2 \pm 15 \\
2 \pm 15 \\
7 \pm 15 \\
-3 \pm 15 \\
\end{array}$ & $\begin{array}{r}-2 \pm 27 \\
-8 \pm 27 \\
- \\
-\end{array}$ & $\begin{array}{r}26 \pm 26 \\
-4 \pm 26 \\
- \\
-\end{array}$ \\
\hline
\end{tabular}


TABLE 5.6

SUMMARY OF DETECTED CONSTITUENTS IN GROUNDWATER, 1995

RADIOCHEMICALS

1995 ANNUAL WATER MONITORING REPORT

LEHR ENVIRONMENTAL RESTORATION, DAVIS, CALIFORNIA

\begin{tabular}{|c|c|c|c|c|c|c|c|c|c|}
\hline Parameter & Quarter & Units & MCL & Method & UCD1-12 & UCD1-13 & $\begin{array}{r}\text { UCD1-13 } \\
\text { D1 }\end{array}$ & UCD1-18 & $\begin{array}{r}\text { UCD1-18 } \\
\text { D1 }\end{array}$ \\
\hline Gross Alpha & \begin{tabular}{|l|} 
WINTER \\
SPRING \\
SUMMER \\
FALL \\
\end{tabular} & $\begin{array}{l}\mathrm{pCi} / \mathrm{L} \\
\mathrm{pCi} / \mathrm{L} \\
\mathrm{pCi} / \mathrm{L} \\
\mathrm{pCi} / \mathrm{L}\end{array}$ & $\begin{array}{l}15 \\
15 \\
15 \\
15 \\
\end{array}$ & $\begin{array}{l}9310 \\
9310 \\
9310 \\
9310 \\
\end{array}$ & $\begin{array}{rr}2.4 \pm 8.4 & \mid C \\
-2.8 \pm 4.9 & \mid C \\
11 \pm 11 & \mid C \\
6 \pm 10 & \mid C \\
\end{array}$ & $\begin{array}{ll}2.7 \pm 5.7 & \mid C \\
3.4 \pm 5.4 & \mid C \\
4.4 \pm 7.6 & |C| \\
0.8 \pm 5.3 & |C|\end{array}$ & $\begin{array}{r}-\overline{-} \\
3.6 \pm 6 . \overline{6} \\
\end{array}$ & $\begin{array}{c}0.1 \pm 3.3 \quad \mid C \\
2.1 \pm 3.1 \mathrm{Jm} / \mathrm{C} \\
- \\
- \\
\end{array}$ & $\begin{array}{rr}-1 \pm 2.7 & \mid \mathrm{C} \\
- & \\
- & \\
- & \\
\end{array}$ \\
\hline Gross Beta & \begin{tabular}{|l|} 
WINTER \\
SPRING \\
SUMMER \\
FALL \\
\end{tabular} & $\begin{array}{l}\mathrm{pCi} / \mathrm{L} \\
\mathrm{pCi} / \mathrm{L} \\
\mathrm{pCi} / \mathrm{L} \\
\mathrm{pCi} / \mathrm{L}\end{array}$ & $\begin{array}{l}50 \\
50 \\
50 \\
50 \\
\end{array}$ & $\begin{array}{l}9310 \\
9310 \\
9310 \\
9310 \\
\end{array}$ & 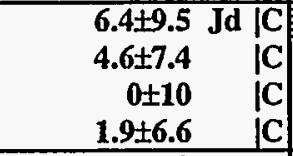 & 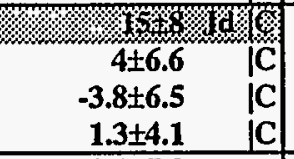 & $\begin{array}{r}- \\
- \\
- \\
.8 \pm 5\end{array}$ & 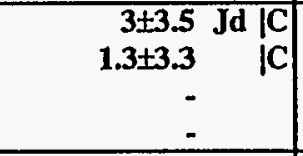 & $\begin{array}{c}0.9 \pm 3.5 \mathrm{Jd} \mid \mathrm{C} \\
- \\
- \\
-\end{array}$ \\
\hline Actinium-228 & \begin{tabular}{|l|} 
WINTER \\
SPRING \\
SUMMER \\
FALL \\
\end{tabular} & $\begin{array}{l}\mathrm{pCi} / \mathrm{L} \\
\mathrm{pCi} / \mathrm{L} \\
\mathrm{pCi} / \mathrm{L} \\
\mathrm{pCi} / \mathrm{L}\end{array}$ & $\begin{array}{l}- \\
- \\
- \\
-\end{array}$ & \begin{tabular}{|l|}
901.1 \\
901.1 \\
901.1 \\
901.1 \\
\end{tabular} & $\begin{array}{r}4.9 \pm 8 \\
7.2 \pm 7.6 \\
-15 \pm 12 \\
7.9 \pm 7.6 \\
\end{array}$ & $\begin{array}{r}0.1 \pm 7.8 \\
0.1 \pm 7.7 \\
-4 \pm 21 \\
-1.1 \pm 7.2 \\
\end{array}$ & $\begin{array}{r}- \\
- \\
4.7 \pm 7.4 \\
\end{array}$ & $\begin{array}{r}-19 \pm 10 \\
6 \pm 21 \\
- \\
- \\
\end{array}$ & $\begin{array}{r}-9.2 \pm 6.7 \\
- \\
- \\
- \\
\end{array}$ \\
\hline Americium-241 & $\begin{array}{l}\text { WINTER } \\
\text { SPRING } \\
\text { SUMMER } \\
\text { FALL } \\
\end{array}$ & $\begin{array}{l}\mathrm{pCi} / \mathrm{L} \\
\mathrm{pC} / \mathrm{L} \\
\mathrm{pCi} / \mathrm{L} \\
\mathrm{pCi} / \mathrm{L}\end{array}$ & $\begin{array}{l}- \\
- \\
-\end{array}$ & $\begin{array}{l}\text { LAS108 } \\
\text { LAS108 } \\
\text { LAS108 } \\
\text { LAS108 } \\
\end{array}$ & $\begin{array}{c}0.007 \pm 0.039 \mathrm{Jm} \mid \\
-0.014 \pm 0.049 \\
- \\
-\end{array}$ & $\begin{array}{c}-0.026 \pm 0.03 \mathrm{Jm} \mid \\
0.032 \pm 0.058 \\
-\end{array}$ & - & $\begin{array}{c}0.015 \pm 0.084 \mathrm{Jm} \text { I } \\
0.004 \pm 0.014 \\
- \\
-\end{array}$ & $\begin{array}{c}0.002 \pm 0.052 \mathrm{Jm} \mid \\
- \\
- \\
- \\
\end{array}$ \\
\hline Bismuth-214 & \begin{tabular}{|l|} 
WINTER \\
SPRING \\
SUMMER \\
FALL \\
\end{tabular} & $\begin{array}{l}\mathrm{pCi} / \mathrm{L} \\
\mathrm{pCi} / \mathrm{L} \\
\mathrm{pCi} / \mathrm{L} \\
\mathrm{pCi} / \mathrm{L}\end{array}$ & $\begin{array}{l}- \\
- \\
-\end{array}$ & $\begin{array}{l}901.1 \\
901.1 \\
901.1 \\
901.1 \\
\end{array}$ & 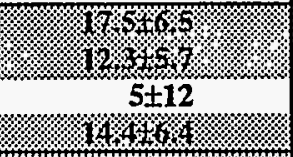 & $\begin{array}{c}8 \% \\
142 x \\
4 \pm 13 \\
2.1 \pm 5.2\end{array}$ & $\begin{array}{r}- \\
- \\
-\end{array}$ & $\begin{array}{r}110 \% \\
11 \pm 13 \\
- \\
-\end{array}$ & $\begin{array}{r}9 \pm 14 \\
- \\
- \\
-\end{array}$ \\
\hline Carbon-14 & \begin{tabular}{|l|} 
WINTER \\
SPRING \\
SUMMER \\
FALL \\
\end{tabular} & $\begin{array}{l}\mathrm{pCi} / \mathrm{L} \\
\mathrm{pCi} / \mathrm{L} \\
\mathrm{pCi} / \mathrm{L} \\
\mathrm{pCi} / \mathrm{L}\end{array}$ & $\begin{array}{l}- \\
- \\
- \\
\end{array}$ & $\begin{array}{l}\text { LSC } \\
\text { LSC } \\
\text { LSC } \\
\text { LSC } \\
\end{array}$ & 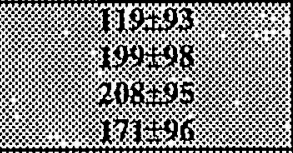 & \%onowlok & - & $\begin{array}{r}42 \pm 87 \\
-1 \pm 84 \\
- \\
-\end{array}$ & $\begin{array}{r}-1 \pm 83 \\
- \\
- \\
\end{array}$ \\
\hline Lead-212 & \begin{tabular}{|l|} 
WINTER \\
SPRING \\
SUMMER \\
FALL \\
\end{tabular} & $\begin{array}{l}\mathrm{pCi} / \mathrm{L} \\
\mathrm{pC} \mathrm{C} / \mathrm{L} \\
\mathrm{pCi} / \mathrm{L} \\
\mathrm{pCi} / \mathrm{L}\end{array}$ & $\begin{array}{l}- \\
- \\
- \\
\end{array}$ & $\begin{array}{l}901.1 \\
901.1 \\
901.1 \\
901.1 \\
\end{array}$ & $\begin{array}{r}-1.2 \pm 5.1 \\
3 \pm 9.7 \\
6.2 \pm 5.4 \\
\end{array}$ & $\begin{array}{r}0.6 \pm 5.1 \\
5.5 \pm 5.4 \\
3 \pm 10 \\
1.4 \pm 4.8 \\
\end{array}$ & $\begin{array}{r}- \\
- \\
0.2 \pm 5.2\end{array}$ & $\begin{array}{r}2 \pm 10 \\
1.8 \pm 9.6 \\
- \\
-\end{array}$ & $\begin{array}{r}9 \pm 10 \\
. \\
- \\
\end{array}$ \\
\hline Lead-214 & \begin{tabular}{|l|} 
WINTER \\
SPRING \\
SUMMER \\
FALL \\
\end{tabular} & $\begin{array}{l}\mathrm{pCi} / \mathrm{L} \\
\mathrm{pCi} / \mathrm{L} \\
\mathrm{pCi} / \mathrm{L} \\
\mathrm{pCi} / \mathrm{L}\end{array}$ & $\begin{array}{l}- \\
- \\
-\end{array}$ & $\begin{array}{l}901.1 \\
901.1 \\
901.1 \\
901.1 \\
\end{array}$ & 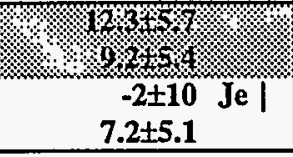 & 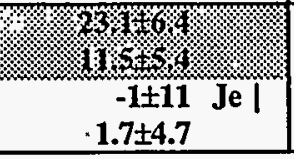 & $\begin{array}{r}- \\
5.8 \pm 5.3 \\
\end{array}$ & $\begin{array}{r}18 \pm 13 \\
5 \pm 12 \\
- \\
\end{array}$ & $\begin{array}{r}12 \pm 12 \\
- \\
- \\
\end{array}$ \\
\hline
\end{tabular}


TABLE 5.6

SUMMARY OF DETECTED CONSTITUENTS IN GROUNDWATER, 1995

RADIOCHEMICALS

1995 ANNUAL WATER MONITORING REPORT

LEHR ENVIRONMENTAL RESTORATION, DAVIS, CALIFORNIA

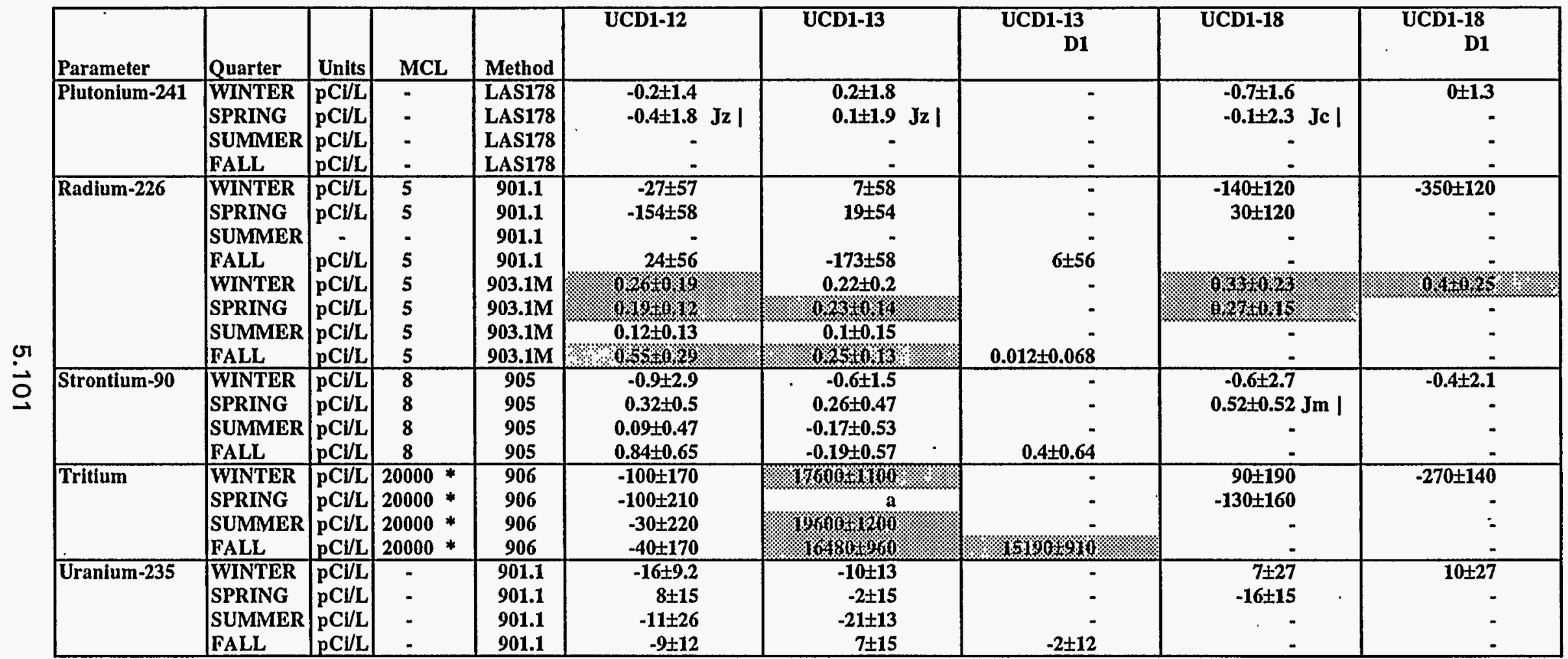


TABLE 5.6

SUMMARY OF DETECTED CONSTITUENTS IN GROUNDWATER, 1995

RADIOCHEMICALS

1995 ANNUAL WATER MONITORING REPORT

LEHR ENVIRONMENTAL RESTORATION, DAVIS, CALIFORNIA

\begin{tabular}{|c|c|c|c|c|c|c|c|c|c|}
\hline Parameter & Quarter & Units & MCL & Method & UCD1-19 & $\begin{array}{c}\text { UCD1-19 } \\
\text { D1 }\end{array}$ & UCD1-20 & UCD1-21 & UCD1-22 \\
\hline Gross Alpha & $\begin{array}{l}\text { WINTER } \\
\text { SPRING } \\
\text { SUMMER } \\
\text { FALL }\end{array}$ & $\begin{array}{l}\mathrm{pCi} / \mathrm{L} \\
\mathrm{pCi} / \mathrm{L} \\
\mathrm{pCi} / \mathrm{L} \\
\mathrm{pCi} / \mathrm{L}\end{array}$ & $\begin{array}{l}15 \\
15 \\
15 \\
15\end{array}$ & $\begin{array}{l}9310 \\
9310 \\
9310 \\
9310 \\
\end{array}$ & $\begin{array}{rr}5.7 \pm 6.9 & \mid C \\
7.9 \pm 6 & \mid C \\
- & \\
- & \\
\end{array}$ & \begin{tabular}{rr|}
- & \\
$0 \pm 5$ & $|C|$ \\
- & \\
\end{tabular} & $\begin{array}{rr}1.5 \pm 2.3 & \mid C \\
1 \pm 2.3 & \mid C \\
0.6 \pm 2.1 & \mid C \\
- & \end{array}$ & 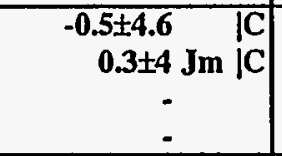 & \begin{tabular}{ll|}
$0.9 \pm 2.9$ & $\mid \mathrm{C}$ \\
$1.2 \pm 3.3 \mathrm{Jm}$ & $\mid \mathrm{C}$ \\
$3.5 \pm 4.5$ & $\mid \mathrm{C}$ \\
\multicolumn{1}{|c}{} & \\
\end{tabular} \\
\hline Gross Beta & $\begin{array}{l}\text { WINTER } \\
\text { SPRING } \\
\text { SUMMER } \\
\text { FALL }\end{array}$ & $\begin{array}{l}\mathrm{pCi} / \mathrm{L} \\
\mathrm{pCi} / \mathrm{L} \\
\mathrm{pCi} / \mathrm{L} \\
\mathrm{pCi} / \mathrm{L}\end{array}$ & $\begin{array}{l}\mathbf{5 0} \\
\mathbf{5 0} \\
\mathbf{5 0} \\
\mathbf{5 0}\end{array}$ & $\begin{array}{l}9310 \\
9310 \\
9310 \\
9310\end{array}$ & $\begin{array}{rr}4.3 \pm 6.8 & \mid C \\
3 \pm 5.9 & \mid C \\
- & \\
- & \end{array}$ & $\begin{array}{r}0 \pm 6.1 \\
- \\
-\end{array}$ & $\begin{array}{r}0.8 \pm 2.3 \\
1.8 \pm 2.6 \\
0.7 \pm 2.2 \\
-\end{array}$ & \begin{tabular}{rr|}
$3.9 \pm 6$ & $\mid C$ \\
$-1.5 \pm 4.9$ & $|C|$ \\
- & \\
- &
\end{tabular} & $\begin{array}{rr}0.4 \pm 3.6 & \mid C \\
2.2 \pm 3.2 & \mid C \\
-0.7 \pm 3.8 & \mid C \\
- & \end{array}$ \\
\hline Actinium-228 & $\begin{array}{l}\text { WINTER } \\
\text { SPRING } \\
\text { SUMMER } \\
\text { FALL }\end{array}$ & $\begin{array}{l}\mathrm{pCi} / \mathrm{L} \\
\mathrm{pCi} / \mathrm{L} \\
\mathrm{pC} \mathrm{L} / \mathrm{L} \\
\mathrm{pCi} / \mathrm{L}\end{array}$ & - & \begin{tabular}{|l|}
901.1 \\
901.1 \\
901.1 \\
901.1 \\
\end{tabular} & $\begin{array}{r}-2.2 \pm 7.6 \\
5.9 \pm 7.7 \\
- \\
-\end{array}$ & $\begin{array}{r}-3.2 \pm 7.5 \\
- \\
-\end{array}$ & $\begin{array}{r}-20 \pm 130 \\
7 \pm 22 \\
2 \pm 21 \\
-\end{array}$ & $\begin{array}{r}-45 \pm 46 \\
0 \pm 17 \\
- \\
- \\
\end{array}$ & $\begin{array}{r}-88 \pm 84 \\
5 \pm 20 \\
-10.6 \pm 5.3 \\
- \\
\end{array}$ \\
\hline Americium-241 & \begin{tabular}{|l|} 
WINTER \\
SPRING \\
SUMMER \\
FALL
\end{tabular} & $\begin{array}{l}\mathrm{pCi} / \mathrm{L} \\
\mathrm{pCi} / \mathrm{L} \\
\mathrm{pCi} / \mathrm{L} \\
\mathrm{pCi} / \mathrm{L}\end{array}$ & $\begin{array}{l}- \\
- \\
-\end{array}$ & $\begin{array}{l}\text { LAS108 } \\
\text { LAS108 } \\
\text { LAS108 } \\
\text { LAS108 }\end{array}$ & $\begin{array}{c}-0.019 \pm 0.05 \mathrm{Jm} \mid \\
0.01 \pm 0.051 \\
- \\
-\end{array}$ & $\begin{array}{r}0.019 \pm 0.053 \\
- \\
-\end{array}$ & $\begin{array}{c}0.018 \pm 0.082 \mathrm{Jm} \mid \\
-0.027 \pm 0.041 \\
- \\
-\end{array}$ & $\begin{array}{c}-0.09 \pm 0.23 \mathrm{Jm} \mid \\
0.013 \pm 0.029 \\
- \\
-\end{array}$ & $\begin{array}{c}-0.013 \pm 0.07 \mathrm{Jm} \mid \\
0.015 \pm 0.02 \\
0.016 \pm 0.021 \\
-\end{array}$ \\
\hline Bismuth-214 & $\begin{array}{l}\text { WINTER } \\
\text { SPRING }\end{array}$ & $\begin{array}{l}\mathrm{pCi} / \mathrm{L} \\
\mathrm{pCi} / \mathrm{L}\end{array}$ & - & $\begin{array}{l}901.1 \\
901.1\end{array}$ & $\begin{array}{l}0.2 \pm 5.2 \\
2, \% \text {. }\end{array}$ & \% 6 - & $\frac{-59 \pm 54}{2616}$ & $\begin{array}{r}3 \pm 78 \\
615\end{array}$ & $\begin{array}{r}11 \pm 80 \\
8 \pm 13\end{array}$ \\
\hline & $\begin{array}{l}\text { SUMMER } \\
\text { FALL }\end{array}$ & $\begin{array}{l}\mathrm{pCi} / \mathrm{L} \\
\mathrm{pCi} / \mathrm{L}\end{array}$ & $\because$ & $\begin{array}{l}901.1 \\
901.1\end{array}$ & $\cdot \cdot$ & $\begin{array}{l}- \\
-\end{array}$ & $\begin{array}{c}3 \pm 14 \mathrm{Jz} \mid \\
-\end{array}$ & - & 20 \\
\hline Carbon-14 & $\begin{array}{l}\text { WINTER } \\
\text { SPRING } \\
\text { SUMMER } \\
\text { FALL }\end{array}$ & $\begin{array}{l}\mathrm{pCi} / \mathrm{L} \\
\mathrm{pCi} / \mathrm{L} \\
\mathrm{pCi} / \mathrm{L} \\
\mathrm{pCi} / \mathrm{L}\end{array}$ & $\begin{array}{l}\text { - } \\
- \\
.\end{array}$ & $\begin{array}{l}\text { LSC } \\
\text { LSC } \\
\text { LSC } \\
\text { LSC }\end{array}$ & $\begin{array}{r}-41 \pm 77 \\
-8 \pm 82 \\
- \\
-\end{array}$ & $\begin{array}{r}-12 \pm 81 \\
- \\
-\end{array}$ & $\begin{array}{r}2 \pm 80 \\
-12 \pm 81 \\
102 \% \%\end{array}$ & $\begin{array}{r}-34 \pm 77 \\
35 \pm 87 \\
- \\
-\end{array}$ & $\begin{array}{r}-4 \pm 79 \\
-26 \pm 82 \\
- \\
-\end{array}$ \\
\hline Lead-212 & $\begin{array}{l}\text { WINTER } \\
\text { SPRING } \\
\text { SUMMER } \\
\text { FALL }\end{array}$ & $\begin{array}{l}\mathrm{pCi} / \mathrm{L} \\
\mathrm{pCi} / \mathrm{L} \\
\mathrm{pCi} / \mathrm{L} \\
\mathrm{pCi} / \mathrm{L}\end{array}$ & $\begin{array}{l}- \\
- \\
-\end{array}$ & $\begin{array}{l}901.1 \\
901.1 \\
901.1 \\
901.1\end{array}$ & $\begin{array}{r}1.2 \pm 5.1 \\
- \\
-\end{array}$ & $\begin{array}{r}-4.4 \pm 5 . \overline{2} \\
- \\
-\end{array}$ & $\begin{array}{r}-14 \pm 54 \\
-12 \pm 9.5 \\
2 \pm 10 \\
-\end{array}$ & $\begin{array}{r}-5 \pm 56 \\
5 \pm 10 \\
- \\
-\end{array}$ & $\begin{array}{r}15 \pm 54 \\
-3.4 \pm 9.4 \\
0.2 \pm 5.4 \\
-\end{array}$ \\
\hline Lead-214 & $\begin{array}{l}\text { WINTER } \\
\text { SPRING }\end{array}$ & $\begin{array}{l}\mathrm{pCl} / \mathrm{L} \\
\mathrm{pCl} / \mathrm{L}\end{array}$ & $\ddot{-}$ & $\begin{array}{l}901.1 \\
901.1\end{array}$ & $2.3 \pm 4.7$ & \%o & $\frac{-67 \pm 46}{314 x}$ & $\begin{array}{r}-14 \pm 63 \\
7 \pm 12\end{array}$ & $\begin{array}{r}-27 \pm 62 \\
3 \pm 11\end{array}$ \\
\hline & $\begin{array}{l}\text { SUMMER } \\
\text { FALL }\end{array}$ & $\mid \begin{array}{l}\mathrm{pCi} / \mathrm{L} \\
\mathrm{pCi} / \mathrm{L}\end{array}$ & - & $\begin{array}{l}901.1 \\
901.1\end{array}$ & - & - $\quad-$ & $2 \pm 11 \mathrm{Jz} \mid$ & - & $\frac{1}{-}$ \\
\hline
\end{tabular}


TABLE 5.6

SUMMARY OF DETECTED CONSTITUENTS IN GROUNDWATER, 1995

RADIOCHEMICALS

1995 ANNUAL WATER MONITORING REPORT

LEHR ENVIRONMENTAL RESTORATION, DAVIS, CALIFORNIA

\begin{tabular}{|c|c|c|c|c|c|c|c|c|c|}
\hline Parameter & Quarter & Units & MCL & Method & UCD1-19 & $\begin{array}{r}\text { UCD1-19 } \\
\text { D1 }\end{array}$ & UCD1-20 & UCD1-21 & UCD1-22 \\
\hline Plutonium-241 & $\begin{array}{l}\text { WINTER } \\
\text { SPRING } \\
\text { SUMMER } \\
\text { FALL } \\
\end{array}$ & $\begin{array}{l}\mathrm{pCi} / \mathrm{L} \\
\mathrm{pCi} / \mathrm{L} \\
\mathrm{pCi} / \mathrm{L} \\
\mathrm{pCi} / \mathrm{L}\end{array}$ & $\begin{array}{l}- \\
- \\
- \\
-\end{array}$ & $\begin{array}{l}\text { LAS178 } \\
\text { LAS178 } \\
\text { LAS178 } \\
\text { LAS178 }\end{array}$ & $\begin{array}{r}0.1 \pm 1.6 \\
0 \pm 1.8 \mathrm{Jz} \mid \\
- \\
-\end{array}$ & 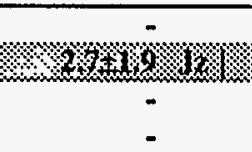 & $\begin{array}{c}-0.1 \pm 1.6 \\
-0.3 \pm 1.9 \mathrm{Jz} \mid \\
- \\
-\end{array}$ & $\begin{array}{c}0.2 \pm 1.8 \\
1.7 \pm 1.9 \mathrm{Jc} \mid \\
- \\
-\end{array}$ & $\begin{array}{c}-1.1 \pm 1.5 \\
1.5 \pm 2.9 \mathrm{Jc} \\
-\end{array}$ \\
\hline Radium-226 & \begin{tabular}{|l|} 
WINTER \\
SPRING \\
SUMMER \\
FALL \\
WINTER \\
SPRING \\
SUMMER \\
FALL \\
\end{tabular} & $\begin{array}{l}\mathrm{pCi} / \mathrm{L} \\
\mathrm{pCi} / \mathrm{L} \\
- \\
\mathrm{pCi} / \mathrm{L} \\
\mathrm{pCi} / \mathrm{L} \\
\mathrm{pCi} / \mathrm{L} \\
\mathrm{pCi} / \mathrm{L} \\
\mathrm{pCi} / \mathrm{L}\end{array}$ & $\begin{array}{l}5 \\
5 \\
5 \\
5 \\
5 \\
5 \\
5 \\
5\end{array}$ & $\begin{array}{c}901.1 \\
901.1 \\
901.1 \\
901.1 \\
903.1 \mathrm{M} \\
903.1 \mathrm{M} \\
903.1 \mathrm{M} \\
903.1 \mathrm{M} \\
\end{array}$ & \begin{tabular}{|c|}
$-133 \pm 57$ \\
$0 \pm 57$ \\
- \\
- \\
$0.069 \pm 0.1$ \\
- \\
-
\end{tabular} & $\begin{array}{r}54 \pm 57 \\
- \\
- \\
- \\
0.112 \pm 0.1 \\
- \\
-\end{array}$ & $\begin{array}{c}-580 \pm 630 \\
-270 \pm 120 \\
- \\
- \\
0.103 \pm 0.087 \\
0.093 \pm 0.086 \\
-\end{array}$ & $\begin{array}{r}-100 \pm 650 \\
-290 \pm 120 \\
- \\
- \\
0.14 \pm 0.15 \\
0.063 \pm 0.092 \\
- \\
-\end{array}$ & $\begin{array}{r}-1770 \pm 680 \\
0 \pm 110 \\
- \\
- \\
0.048 \pm 0.082 \\
-\end{array}$ \\
\hline Strontium-90 & $\begin{array}{l}\text { WINTER } \\
\text { SPRING } \\
\text { SUMMER } \\
\text { FALL } \\
\end{array}$ & $\begin{array}{l}\mathrm{pCl} / \mathrm{L} \\
\mathrm{pCi} / \mathrm{L} \\
\mathrm{pCi} / \mathrm{L} \\
\mathrm{pCl} / \mathrm{L}\end{array}$ & \begin{tabular}{|l|}
8 \\
8 \\
8 \\
8 \\
\end{tabular} & $\begin{array}{l}905 \\
905 \\
905 \\
905 \\
\end{array}$ & $\begin{array}{r}-0.8 \pm 3.5 \\
0.75 \pm 0.5 \\
- \\
-\end{array}$ & $\begin{array}{r}0.27 \pm 0.46 \\
- \\
\end{array}$ & $\begin{array}{r}-0.6 \pm 3 \\
0.5 \pm 0.48 \\
-0.15 \pm 0.26 \\
-\end{array}$ & $\begin{array}{c}-1 \pm 3.3 \\
0.37 \pm 0.5 \mathrm{Jm} \mid \\
- \\
-\end{array}$ & $\begin{array}{c}-0.6 \pm 3.8 \\
0.31 \pm 0.59 \mathrm{Jm} \mid \\
0.25 \pm 0.37 \\
\end{array}$ \\
\hline Tritium & $\begin{array}{l}\text { WINTER } \\
\text { SPRING } \\
\text { SUMMER } \\
\text { FALL } \\
\end{array}$ & $\begin{array}{l}\mathrm{pCi} / \mathrm{L} \\
\mathrm{pCi} / \mathrm{L} \\
\mathrm{pC} / \mathrm{L} \\
\mathrm{pCi} / \mathrm{L}\end{array}$ & $\begin{array}{l}20000 * \\
20000 * \\
20000 * \\
20000 *\end{array}$ & $\begin{array}{l}906 \\
906 \\
906 \\
906\end{array}$ & $\begin{array}{r}-110 \pm 200 \\
-30 \pm 220 \\
- \\
-\end{array}$ & $\begin{array}{r}20 \pm 220 \\
-\end{array}$ & $\begin{array}{r}-30 \pm 220 \\
b \\
-50 \pm 220 \\
70 \pm 170\end{array}$ & $\begin{array}{r}-100 \pm 210 \\
50 \pm 180 \\
- \\
-\end{array}$ & $\begin{array}{r}-190 \pm 190 \\
80 \pm 180 \\
100 \pm 240 \\
-\end{array}$ \\
\hline Uranium-235 & $\begin{array}{l}\text { WINTER } \\
\text { SPRING } \\
\text { SUMMER } \\
\text { FALL }\end{array}$ & $\begin{array}{l}\mathrm{pCi} / \mathrm{L} \\
\mathrm{pCi} / \mathrm{L} \\
\mathrm{pCi} / \mathrm{L} \\
\mathrm{pCi} / \mathrm{L}\end{array}$ & $\begin{array}{l}- \\
- \\
-\end{array}$ & $\begin{array}{l}901.1 \\
901.1 \\
901.1 \\
901.1\end{array}$ & $\begin{array}{r}11 \pm 15 \\
0 \pm 16 \\
-\end{array}$ & $\begin{array}{r}- \\
-3 \pm 15 \\
-\end{array}$ & $\begin{array}{r}40 \pm 150 \\
14 \pm 26 \\
9 \pm 27 \\
\end{array}$ & $\begin{array}{r}-60 \pm 150 \\
15 \pm 27 \\
-\end{array}$ & $\begin{array}{r}40 \pm 150 \\
13 \pm 26 \\
-5 \pm 13\end{array}$ \\
\hline
\end{tabular}


TABLE 5.6

SUMMARY OF DETECTED CONSTITUENTS IN GROUNDWATER, 1995

RADIOCHEMICALS

1995 ANNUAL WATER MONITORING REPORT

LEHR ENVIRONMENTAL RESTORATION, DAVIS, CALIFORNIA

\begin{tabular}{|c|c|c|c|c|c|c|c|c|c|}
\hline Parameter & Quarter & Units & MCL & Method & UCD1-23 & UCD1-24 & UCD1-25 & UCD1-27Z1 & UCD1-27Z2 \\
\hline Gross Alpha & \begin{tabular}{|l|} 
WINTER \\
SPRING \\
SUMMER \\
FALL \\
\end{tabular} & $\begin{array}{l}\mathrm{pCi} / \mathrm{L} \\
\mathrm{pCl} / \mathrm{L} \\
\mathrm{pCl} / \mathrm{L} \\
\mathrm{pCl} / \mathrm{L}\end{array}$ & $\begin{array}{l}15 \\
15 \\
15 \\
15 \\
\end{array}$ & $\begin{array}{l}9310 \\
9310 \\
9310 \\
9310 \\
\end{array}$ & $\begin{array}{cc}3.2 \pm 5 & \mid C \\
0.1 \pm 2.7 \mathrm{Jm} & \mid \mathrm{C} \\
1.2 \pm 4 & \mid \mathrm{C} \\
- & \\
\end{array}$ & 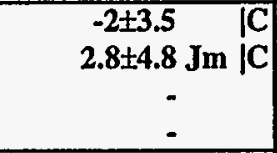 & $\begin{array}{r}- \\
- \\
-4.1 \pm 6.3 \\
\end{array}$ & $\begin{array}{r}- \\
i \\
-2.3+3.3\end{array}$ & $\begin{array}{r}-\overline{-} \\
- \\
-0.5 \pm 2.5\end{array}$ \\
\hline Gross Beta & \begin{tabular}{|l|} 
WINTER \\
SPRING \\
SUMMER \\
FALL \\
\end{tabular} & $\begin{array}{l}\mathrm{pCl} / \mathrm{L} \\
\mathrm{pCl} / \mathrm{L} \\
\mathrm{pCl} / \mathrm{L} \\
\mathrm{pCC} / \mathrm{L}\end{array}$ & $\begin{array}{l}50 \\
50 \\
50 \\
50 \\
\end{array}$ & $\begin{array}{l}9310 \\
9310 \\
9310 \\
9310 \\
\end{array}$ & $\begin{array}{rr}-0.5 \pm 4.8 & \mid \mathrm{C} \\
0.3 \pm 3.2 & \mid \mathrm{C} \\
1.1 \pm 4 & \mid \mathrm{C} \\
& - \\
\end{array}$ & $\begin{array}{rr}2.2 \pm 5.4 & \text { CC } \\
0 \pm 4.6 & \text { IC } \\
- & \\
- & \\
\end{array}$ & $\begin{array}{r}- \\
- \\
0.4 \pm 10\end{array}$ & $\begin{array}{r}-\overline{-} \\
0 \pm 2.9 \\
\end{array}$ & $\begin{array}{r}- \\
- \\
2 \pm 2 \\
\end{array}$ \\
\hline Actinium-228 & \begin{tabular}{|l|} 
WINTER \\
SPRING \\
SUMMER \\
FALL \\
\end{tabular} & $\begin{array}{l}\mathrm{pCi} / \mathrm{L} \\
\mathrm{pCi} / \mathrm{L} \\
\mathrm{pC} / \mathrm{L} \\
\mathrm{pCi} / \mathrm{L}\end{array}$ & $\begin{array}{l}- \\
- \\
-\end{array}$ & $\begin{array}{l}901.1 \\
901.1 \\
901.1 \\
901.1 \\
\end{array}$ & $\begin{array}{r}-13 \pm 10 \\
0.6 \pm 7.7 \\
-3 \pm 24\end{array}$ & $\begin{array}{r}-5 \pm 22 \\
-11 \pm 5 \\
- \\
-\end{array}$ & $\begin{array}{r}- \\
- \\
0.7 \pm 7.6 \\
\end{array}$ & $\begin{array}{r}- \\
- \\
-6 \pm 13 \\
\end{array}$ & $\begin{array}{r}- \\
- \\
3 \pm 13\end{array}$ \\
\hline Americium-241 & \begin{tabular}{|l|} 
WINTER \\
SPRING \\
SUMMER \\
FALL \\
\end{tabular} & $\begin{array}{l}\mathrm{pCi} / \mathrm{L} \\
\mathrm{pCi} / \mathrm{L} \\
\mathrm{pCi} / \mathrm{L} \\
\mathrm{pC} \mathrm{L} / \mathrm{L}\end{array}$ & :- & $\begin{array}{l}\text { LAS108 } \\
\text { LAS108 } \\
\text { LAS108 } \\
\text { LAS108 } \\
\end{array}$ & $\begin{array}{c}0.008 \pm 0.05 \mathrm{Jm} . \mathrm{I} \\
0.006 \pm 0.016 \\
- \\
-\end{array}$ & $\begin{array}{c}0 \pm 0.048 \mathrm{Jm} \\
0.04 \\
0 \\
0\end{array}$ & $\begin{array}{r}- \\
\\
0.035 \pm 0.037 \\
\end{array}$ & $\begin{array}{r}- \\
- \\
- \\
0.01 \pm 0.022 \\
\end{array}$ & $\begin{array}{r}- \\
- \\
0\end{array}$ \\
\hline Bismuth-214 & \begin{tabular}{|l|} 
WINTER \\
SPRING \\
SUMMER \\
FALL \\
\end{tabular} & $\begin{array}{l}\mathrm{pCi} / \mathrm{L} \\
\mathrm{pCi} / \mathrm{L} \\
\mathrm{pCi} / \mathrm{L} \\
\mathrm{pCi} / \mathrm{L}\end{array}$ & : & $\begin{array}{l}901.1 \\
901.1 \\
901.1 \\
901.1 \\
\end{array}$ & $\begin{array}{r}15 \pm 15 \\
0.7 \pm 5.1 \\
11 \pm 14 \mathrm{Jz} \mid \\
\end{array}$ & $\begin{array}{c}-1 \pm 13 \\
6 \\
- \\
-\end{array}$ & $\begin{array}{r}- \\
- \\
-\end{array}$ & $\begin{array}{r}- \\
- \\
18 \times 468\end{array}$ & $\begin{array}{r}- \\
- \\
2 \pm 7.5\end{array}$ \\
\hline Carbon-14 & \begin{tabular}{|l|} 
WINTER \\
SPRING \\
SUMMER \\
FALL \\
\end{tabular} & $\begin{array}{l}\mathrm{pC} / \mathrm{L} \\
\mathrm{pCi} / \mathrm{L} \\
\mathrm{pC} \mathrm{L} / \mathrm{L} \\
\mathrm{pC} / \mathrm{L}\end{array}$ & : & $\begin{array}{l}\text { LSC } \\
\text { LSC } \\
\text { LSC } \\
\text { LSC } \\
\end{array}$ & $\begin{array}{c}230.100 \\
370 \ldots 10\end{array}$ & $\begin{array}{r}45 \pm 83 \\
18 \pm 86 \\
- \\
\end{array}$ & $\begin{array}{r}- \\
19 \pm 74 \\
\end{array}$ & $\begin{array}{r}- \\
- \\
30 \pm 85 \\
\end{array}$ & $\begin{array}{r}- \\
19 \pm 84\end{array}$ \\
\hline Lead-212 & \begin{tabular}{|l|} 
WINTER \\
SPRING \\
SUMMER \\
FALL \\
\end{tabular} & $\begin{array}{l}\mathrm{pCi} / \mathrm{L} \\
\mathrm{pCi} / \mathrm{L} \\
\mathrm{pCi} / \mathrm{L} \\
\mathrm{pCi} / \mathrm{L}\end{array}$ & $\begin{array}{l}- \\
- \\
-\end{array}$ & $\begin{array}{l}901.1 \\
901.1 \\
901.1 \\
901.1 \\
\end{array}$ & $\begin{array}{r}2 \pm 11 \\
-2 \pm 5.1 \\
-1.1 \pm 9.4 \\
-\end{array}$ & $\begin{array}{r}0 \pm 10 \\
-2.8 \pm 5 \\
- \\
- \\
\end{array}$ & $\begin{array}{r}- \\
- \\
4.8 \pm 5.3 \\
\end{array}$ & $\begin{array}{r}- \\
- \\
2.8 \pm 5.6 \\
\end{array}$ & $\begin{array}{r}- \\
- \\
- \\
-10.7 \pm 5.1 \\
\end{array}$ \\
\hline Lead-214 & \begin{tabular}{|l|} 
WINTER \\
SPRING \\
SUMMER \\
FALL \\
\end{tabular} & $\begin{array}{l}\mathrm{pCl} / \mathrm{L} \\
\mathrm{pCl} / \mathrm{L} \\
\mathrm{pCl} / \mathrm{L} \\
\mathrm{pCl} / \mathrm{L}\end{array}$ & $\begin{array}{l}- \\
- \\
-\end{array}$ & $\begin{array}{l}901.1 \\
901.1 \\
901.1 \\
901.1 \\
\end{array}$ & $\begin{array}{r}3 \pm 13 \\
-0.2 \pm 4.8 \\
26112 \\
- \\
\end{array}$ & $\begin{array}{r}4.8 \pm 8.7 \\
2.9 \pm 4.9 \\
- \\
- \\
\end{array}$ & $\begin{array}{c}- \\
- \\
- \\
1253\end{array}$ & $\begin{array}{r}i \\
6.7 \pm 6.6 \\
\end{array}$ & $\begin{array}{r}- \\
- \\
5.6 \pm 6.5 \\
\end{array}$ \\
\hline
\end{tabular}

Page 7 of 15 
TABLE 5.6

SUMMARY OF DETECTED CONSTITUENTS IN GROUNDWATER, 1995

RADIOCHEMICALS

1995 ANNUAL WATER MONITORING REPORT

LEHR ENVIRONMENTAL RESTORATION, DAVIS, CALIFORNIA

\begin{tabular}{|c|c|c|c|c|c|c|c|c|c|}
\hline Parameter & Quarter & Units & MCL & Method & UCD1-23 & UCD1-24 & UCD1-25 & UCD1-27Z1 & UCD1-27Z2 \\
\hline Plutonium-241 & \begin{tabular}{|l|} 
WINTER \\
SPRING \\
SUMMER \\
FALL \\
\end{tabular} & $\begin{array}{l}\mathrm{pCi} / \mathrm{L} \\
\mathrm{pCi} / \mathrm{L} \\
\mathrm{pCi} / \mathrm{L} \\
\mathrm{pCi} / \mathrm{L}\end{array}$ & $\begin{array}{l}- \\
- \\
- \\
\end{array}$ & $\begin{array}{l}\text { LAS178 } \\
\text { LAS178 } \\
\text { LAS178 } \\
\text { LAS178 } \\
\end{array}$ & $\begin{array}{c}-0.4 \pm 1.5 \\
-0.5 \pm 1.5 \mathrm{Jc} \mathrm{I} \\
- \\
- \\
\end{array}$ & $\begin{array}{r}0 \pm 1.5 \\
-1.1 \pm 1.5 \mathrm{Jc} \mid \\
- \\
- \\
\end{array}$ & $\begin{array}{c}- \\
- \\
- \\
0.5 \pm 3.5 \mathrm{Jl} 1\end{array}$ & $\begin{array}{r}- \\
- \\
0.2+2.2 \\
\end{array}$ & $\begin{array}{r}- \\
- \\
-0.6 \pm 2.2 \\
\end{array}$ \\
\hline Radium-226 & \begin{tabular}{|l|} 
WINTER \\
SPRING \\
SUMMER \\
FALL \\
WINTER \\
SPRING \\
SUMMER \\
FALL \\
\end{tabular} & \begin{tabular}{|c|}
$\mathrm{pCi} / \mathrm{L}$ \\
$\mathrm{pCi} / \mathrm{L}$ \\
- \\
$\mathrm{pCi} / \mathrm{L}$ \\
$\mathrm{pCi} / \mathrm{L}$ \\
$\mathrm{pCi} / \mathrm{L}$ \\
$\mathrm{pCi} / \mathrm{L}$ \\
$\mathrm{pCi} / \mathrm{L}$
\end{tabular} & $\begin{array}{l}5 \\
5 \\
- \\
5 \\
5 \\
5 \\
5 \\
5 \\
\end{array}$ & $\begin{array}{c}901.1 \\
901.1 \\
901.1 \\
901.1 \\
903.1 \mathrm{M} \\
903.1 \mathrm{M} \\
903.1 \mathrm{M} \\
903.1 \mathrm{M} \\
\end{array}$ & $\begin{array}{c}-10 \pm 130 \\
-188 \pm 59 \\
- \\
- \\
0.15 \pm 0.14 \\
0.15 \pm 0.1 \\
0.016 \pm 0.1 \\
-\end{array}$ & $\begin{array}{r}-110 \pm 110 \\
-55 \pm 56 \\
- \\
- \\
0.09 \pm 0.12 \\
0190 \% \\
-- \\
-\end{array}$ & $\begin{array}{r}- \\
- \\
-4 \pm 56 \\
- \\
- \\
- \\
0.14 \pm 0.11 \\
\end{array}$ & $\begin{array}{r}- \\
-14 \pm 67 \\
- \\
- \\
0.085 \pm 0.091 \\
\end{array}$ & $\begin{array}{r}- \\
- \\
-173 \pm 68 \\
- \\
- \\
0\end{array}$ \\
\hline Strontium-90 & \begin{tabular}{|l|} 
WINTER \\
SPRING \\
SUMMER \\
FALL \\
\end{tabular} & $\begin{array}{l}\mathrm{pCi} / \mathrm{L} \\
\mathrm{pCi} / \mathrm{L} \\
\mathrm{pCi} / \mathrm{L} \\
\mathrm{pCi} / \mathrm{L}\end{array}$ & \begin{tabular}{|l|}
8 \\
8 \\
8 \\
8 \\
\end{tabular} & $\begin{array}{l}905 \\
905 \\
905 \\
905 \\
\end{array}$ & $\begin{array}{c}-0.6 \pm 3.2 \\
-0.15 \pm 0.51 \mathrm{Jm} \mid \\
-0.04 \pm 0.25 \\
-\end{array}$ & $\begin{array}{c}-0.6 \pm 3.1 \\
0.36 \pm 0.49 \mathrm{Jm} \mid \\
- \\
- \\
\end{array}$ & $\begin{array}{r}- \\
- \\
0.38 \pm 0.66 \\
\end{array}$ & $\begin{array}{r}- \\
0.15 \pm 0.68 \\
\end{array}$ & $0.32 \pm 0.6$ \\
\hline Tritium & \begin{tabular}{|l|} 
WINTER \\
SPRING \\
SUMMER \\
FALL \\
\end{tabular} & $\begin{array}{l}\mathrm{pCi} / \mathrm{L} \\
\mathrm{pCi} / \mathrm{L} \\
\mathrm{pC} / \mathrm{L} \\
\mathrm{pCl} / \mathrm{L}\end{array}$ & $\begin{array}{l}20000 * \\
20000 * \\
20000 * \\
20000 * \\
\end{array}$ & $\begin{array}{l}906 \\
906 \\
906 \\
906 \\
\end{array}$ & $\begin{array}{r}-250 \pm 180 \\
100 \pm 180 \\
120 \pm 250 \\
-\end{array}$ & $\begin{array}{r}30 \pm 220 \\
20 \pm 170 \\
- \\
-\end{array}$ & $\begin{array}{r} \\
- \\
-80 \pm 150\end{array}$ & $\begin{array}{r}- \\
- \\
-10 \pm 170\end{array}$ & $\begin{array}{r}- \\
- \\
-90+160\end{array}$ \\
\hline Uranium-235 & \begin{tabular}{|l|} 
WINTER \\
SPRING \\
SUMMER \\
FALL \\
\end{tabular} & $\begin{array}{l}\mathrm{pCi} / \mathrm{L} \\
\mathrm{pCi} / \mathrm{L} \\
\mathrm{pC} / \mathrm{L} \\
\mathrm{pCi} / \mathrm{L}\end{array}$ & $\begin{array}{l}- \\
- \\
-\end{array}$ & $\begin{array}{l}901.1 \\
901.1 \\
901.1 \\
901.1\end{array}$ & $\begin{array}{r}-23 \pm 22 \\
3 \pm 14 \\
4 \pm 27 \\
-\quad-\end{array}$ & $\begin{array}{r}-2 \pm 27 \\
1 \pm 12 \\
-\end{array}$ & $\begin{array}{r}- \\
-10.8 \pm 7.8\end{array}$ & - & - \\
\hline
\end{tabular}


TABLE 5.6

SUMMARY OF DETECTED CONSTITUENTS IN GROUNDWATER, 1995

RADIOCHEMICALS

1995 ANNUAL WATER MONITORING REPORT

LEHR ENVIRONMENTAL RESTORATION, DAVIS, CALIFORNIA

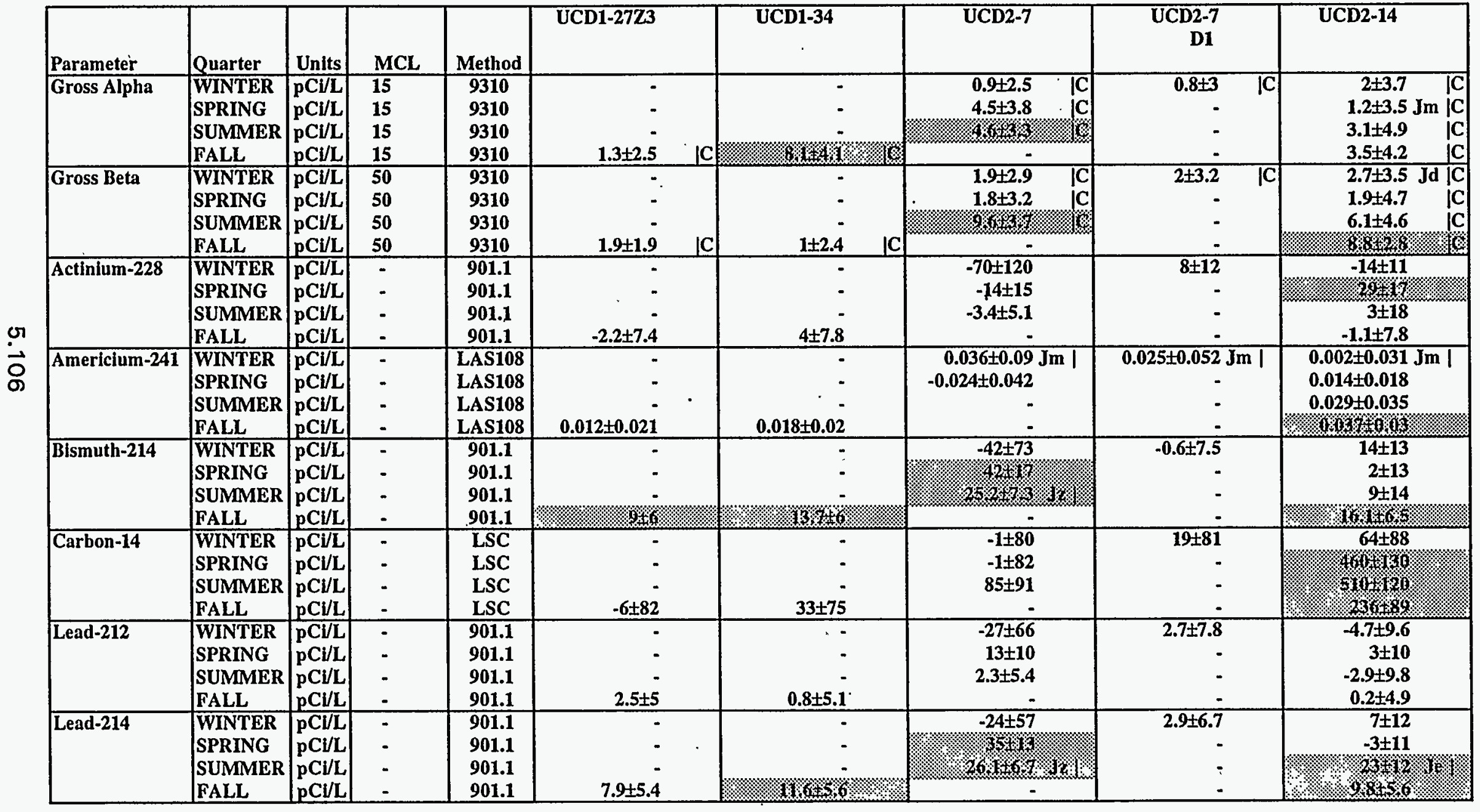


TABLE 5.6

SUMMARY OF DETECTED CONSTITUENTS IN GROUNDWATER, 1995

RADIOCHEMICALS

1995 ANNUAL WATER MONITORING REPORT

LEHR ENVIRONMENTAL RESTORATION, DAVIS, CALIFORNIA

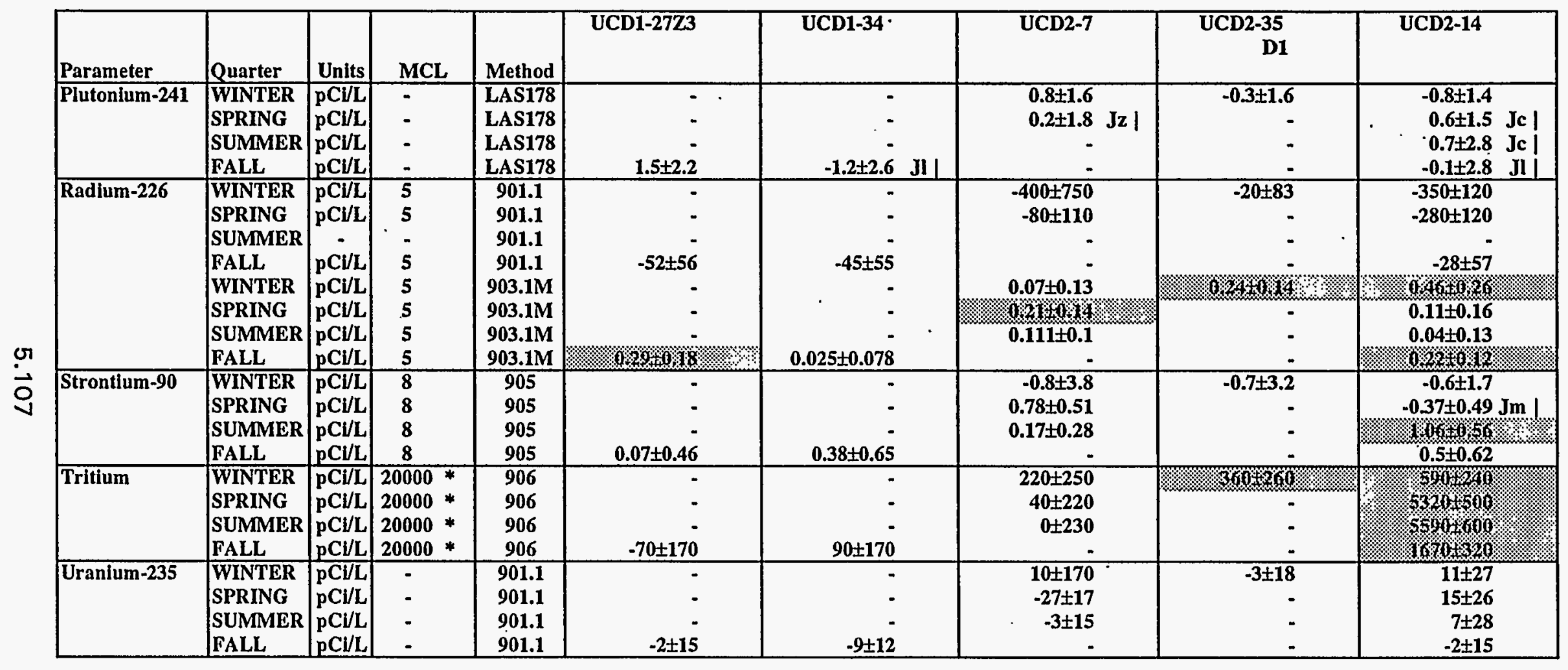


TABLE 5.6

SUMMARY OF DETECTED CONSTITUENTS IN GROUNDWATER, 1995

RADIOCHEMICALS

1995 ANNUAL WATER MONITORING REPORT

LEHR ENVIRONMENTAL RESTORATION, DAVIS, CALIFORNIA

\begin{tabular}{|c|c|c|c|c|c|c|c|c|c|}
\hline Parameter & Quarter & Units & MCL & Method & UCD2-15 & UCD2-16 & UCD2-17 & UCD2-26 & UCD2-27ZA \\
\hline Gross Alpha & $\begin{array}{l}\text { WINTER } \\
\text { SPRING } \\
\text { SUMMER } \\
\text { FALL }\end{array}$ & $\left|\begin{array}{l}\mathrm{pCi} / \mathrm{L} \\
\mathrm{pCi} / \mathrm{L} \\
\mathrm{pCi} / \mathrm{L} \\
\mathrm{pC} / \mathrm{L}\end{array}\right|$ & $\begin{array}{l}15 \\
15 \\
15 \\
15\end{array}$ & $\begin{array}{l}9310 \\
9310 \\
9310 \\
9310\end{array}$ & $\begin{array}{ll}1.2 \pm 2.8 & \mid C \\
1.9 \pm 2.8 & \text { |C } \\
0.6 \pm 1.8 & \text { |C } \\
0.5 \pm 2.7 & \mid C\end{array}$ & $\begin{array}{rr}2.1 \pm 3.1 & \mid C \\
2.4 \pm 2.6 & \text { |C } \\
2.5 \pm 2.9 & \text { IC } \\
- & \end{array}$ & $\begin{array}{c}1.2 \pm 2.2 \mathrm{Jm} \mid \mathrm{C} \\
- \\
-\end{array}$ & $\begin{array}{r}- \\
1.2 \pm 2.7\end{array}$ & $\begin{array}{r}- \\
-0.5 \pm 2.2\end{array}$ \\
\hline Gross Beta & $\begin{array}{l}\text { WINTER } \\
\text { SPRING } \\
\text { SUMMER } \\
\text { FALL } \\
\end{array}$ & $\begin{array}{l}\mathrm{pCi} / \mathrm{L} \\
\mathrm{pCi} / \mathrm{L} \\
\mathrm{pCi} / \mathrm{L} \\
\mathrm{pCi} / \mathrm{L}\end{array}$ & $\begin{array}{l}50 \\
50 \\
50 \\
50\end{array}$ & $\begin{array}{l}9310 \\
9310 \\
9310 \\
9310 \\
\end{array}$ & \begin{tabular}{r|r}
$1 \pm 2.5$ & $\mid \mathrm{C}$ \\
$0.3 \pm 2.5$ & $\mathrm{j}$ \\
$0.1 \times 2.6$ & \\
$0.6 \pm 2$ & $\mathrm{C}$ \\
\end{tabular} & $\begin{array}{rr}0.9 \pm 2.9 & \mid \mathrm{C} \\
1.4 \pm 2.7 & \mid \mathrm{C} \\
0.5 \pm 2.5 & \mid \mathrm{C} \\
- & \\
\end{array}$ & $\begin{array}{c}2.7 \pm 2 \\
- \\
-\end{array}$ & $\begin{array}{r}- \\
- \\
3.2 \pm 2.4\end{array}$ & $\begin{array}{r}\dot{y} \\
\\
0.3 \pm 1.7 \\
\end{array}$ \\
\hline Actinium-228 & $\begin{array}{l}\text { WINTER } \\
\text { SPRING: } \\
\text { SUMMER } \\
\text { FALL } \\
\end{array}$ & $\begin{array}{l}\mathrm{pCi} / \mathrm{L} \\
\mathrm{pCi} / \mathrm{L} \\
\mathrm{pCi} / \mathrm{L} \\
\mathrm{pCi} / \mathrm{L}\end{array}$ & $\begin{array}{l}- \\
- \\
-\end{array}$ & $\begin{array}{l}901.1 \\
901.1 \\
901.1 \\
901.1 \\
\end{array}$ & $\begin{array}{r}100 \pm 130 \\
12 \pm 22 \\
-31 \pm 17 \\
-6 \pm 12 \\
\end{array}$ & $\begin{array}{r}5 \pm 21 \\
-5.1 \pm 7.8 \\
4 \pm 20 \\
- \\
\end{array}$ & $\begin{array}{r}-92 \pm 56 \\
-3.1 \pm 7.8 \\
\vdots \\
\end{array}$ & $\begin{array}{r}- \\
- \\
19 \pm 28 \\
\end{array}$ & $\begin{array}{r}- \\
5.4 \pm 6.5\end{array}$ \\
\hline Americium-241 & \begin{tabular}{|l|} 
WINTER \\
SPRING \\
SUMMER \\
FALL \\
\end{tabular} & $\begin{array}{l}\mathrm{pCi} / \mathrm{L} \\
\mathrm{pCi} / \mathrm{L} \\
\mathrm{pC} / \mathrm{L} \\
\mathrm{pCi} / \mathrm{L}\end{array}$ & :- & $\begin{array}{l}\text { LAS108 } \\
\text { LAS108 } \\
\text { LAS108 } \\
\text { LAS108 } \\
\end{array}$ & $\begin{array}{c}0.009 \pm 0.058 \mathrm{Jm} \mathrm{I} \\
0.005 \pm 0.049 \\
- \\
- \\
\end{array}$ & $\begin{array}{c}-0.029 \pm 0.07 \mathrm{Jm} \mid \\
0.036 \pm 0.057 \\
- \\
- \\
\end{array}$ & $\begin{array}{c}-0.062 \pm 0.06 \mathrm{Jm}] \\
0.023 \pm 0.026 \\
- \\
- \\
\end{array}$ & $\begin{array}{r}-\overline{-} \\
- \\
0.014 \pm 0.02 \\
\end{array}$ & $\begin{array}{r}- \\
- \\
0.017 \pm 0.025 \\
\end{array}$ \\
\hline Bismuth-214 & $\begin{array}{l}\text { WINTER } \\
\text { SPRING } \\
\text { SUMMER } \\
\text { FALL }\end{array}$ & $\begin{array}{l}\mathrm{pCi} / \mathrm{L} \\
\mathrm{pCi} / \mathrm{L} \\
\mathrm{pCi} / \mathrm{L} \\
\mathrm{pC} / \mathrm{L}\end{array}$ & $\begin{array}{l}- \\
- \\
-\end{array}$ & \begin{tabular}{|l|}
901.1 \\
901.1 \\
901.1 \\
901.1 \\
\end{tabular} & $\begin{array}{r}-17 \pm 77 \\
18 \pm 15 \\
6 \pm 14 \quad \mathrm{Jz} \mid \\
5.4 \pm 7.1 \\
\end{array}$ & $\begin{array}{r}-11 \pm 11 \\
7.5 \pm 5.7 \\
-2 \pm 12 \quad \mathrm{Jz} \mid \\
-\end{array}$ & $\begin{array}{r}-108+30 \\
10 \\
- \\
-\end{array}$ & $\begin{array}{r}- \\
- \\
-7 \pm 20\end{array}$ & $\begin{array}{r}- \\
5.7 \pm 5 \\
\end{array}$ \\
\hline Carbon-14 & $\begin{array}{l}\text { WINTER } \\
\text { SPRING } \\
\text { SUMMER } \\
\text { FALL }\end{array}$ & $\begin{array}{l}\mathrm{pCl} / \mathrm{L} \\
\mathrm{pCi} / \mathrm{L} \\
\mathrm{pCl} / \mathrm{L} \\
\mathrm{pCl} / \mathrm{L}\end{array}$ & $\begin{array}{l}\text { - } \\
- \\
-\end{array}$ & $\begin{array}{l}\text { LSC } \\
\text { LSC } \\
\text { LSC } \\
\text { LSC }\end{array}$ & $\begin{array}{r}2 \pm 80 \\
7 \pm 83 \\
- \\
-\end{array}$ & $\begin{array}{r}-4 \pm 79 \\
35 \pm 85 \\
- \\
-\end{array}$ & $\begin{array}{r}-19 \pm 81 \\
3 \pm 84 \\
- \\
-\end{array}$ & $\begin{array}{r}- \\
- \\
4 \pm 83\end{array}$ & $\begin{array}{r}-\overline{-} \\
-3 \pm 82\end{array}$ \\
\hline Lead-212 & $\begin{array}{l}\text { WINTER } \\
\text { SPRING } \\
\text { SUMMER } \\
\text { FALL }\end{array}$ & $\begin{array}{l}\mathrm{pCi} / \mathrm{L} \\
\mathrm{pCi} / \mathrm{L} \\
\mathrm{pCi} / \mathrm{L} \\
\mathrm{pCi} / \mathrm{L}\end{array}$ & - & $\begin{array}{l}901.1 \\
901.1 \\
901.1 \\
901.1\end{array}$ & $\begin{array}{r}42 \pm 53 \\
-6.1 \pm 9.8 \\
1 \pm 9.5 \\
0.7 \pm 5.3 \\
\end{array}$ & $\begin{array}{r}3.2 \pm 9.9 \\
-2.9 \pm 4.7 \\
-1 \pm 8.9 \\
-\end{array}$ & $\begin{array}{r}31 \pm 56 \\
3.7 \pm 5.2 \\
- \\
-\end{array}$ & $\begin{array}{r}- \\
- \\
-7 \pm 18\end{array}$ & $\begin{array}{r}-\overline{-} \\
0.5 \pm 4.4\end{array}$ \\
\hline Lead-214 & $\begin{array}{l}\text { WINTER } \\
\text { SPRING } \\
\text { SUMMER } \\
\text { FALL } \\
\end{array}$ & $\begin{array}{l}\mathrm{pCi} / \mathrm{L} \\
\mathrm{pCi} / \mathrm{L} \\
\mathrm{pCi} / \mathrm{L} \\
\mathrm{pCi} / \mathrm{L}\end{array}$ & $\begin{array}{l}- \\
- \\
-\end{array}$ & \begin{tabular}{|l|}
901.1 \\
901.1 \\
901.1 \\
901.1 \\
\end{tabular} & $\begin{array}{c}-23 \pm 64 \\
3 \pm 643 \\
3 \pm 11 \\
2.5 \pm 6.1 \\
\end{array}$ & $\begin{array}{c}3 \pm 11 \\
8 . \mathrm{J}= \\
8 \pm 11 \mathrm{Jz} \\
- \\
\end{array}$ & $\begin{array}{c}-22 \pm 62 \\
921+62.4 \\
- \\
- \\
\end{array}$ & $\begin{array}{r}- \\
- \\
-6 \pm 18 \\
\end{array}$ & $\begin{array}{c}- \\
- \\
-\end{array}$ \\
\hline
\end{tabular}


TABLE 5.6

SUMMARY OF DETECTED CONSTITUENTS IN GROUNDWATER, 1995

\section{RADIOCHEMICALS}

1995 ANNUAL WATER MONITORING REPORT

LEHR ENVIRONMENTAL RESTORATION, DAVIS, CALIFORNIA

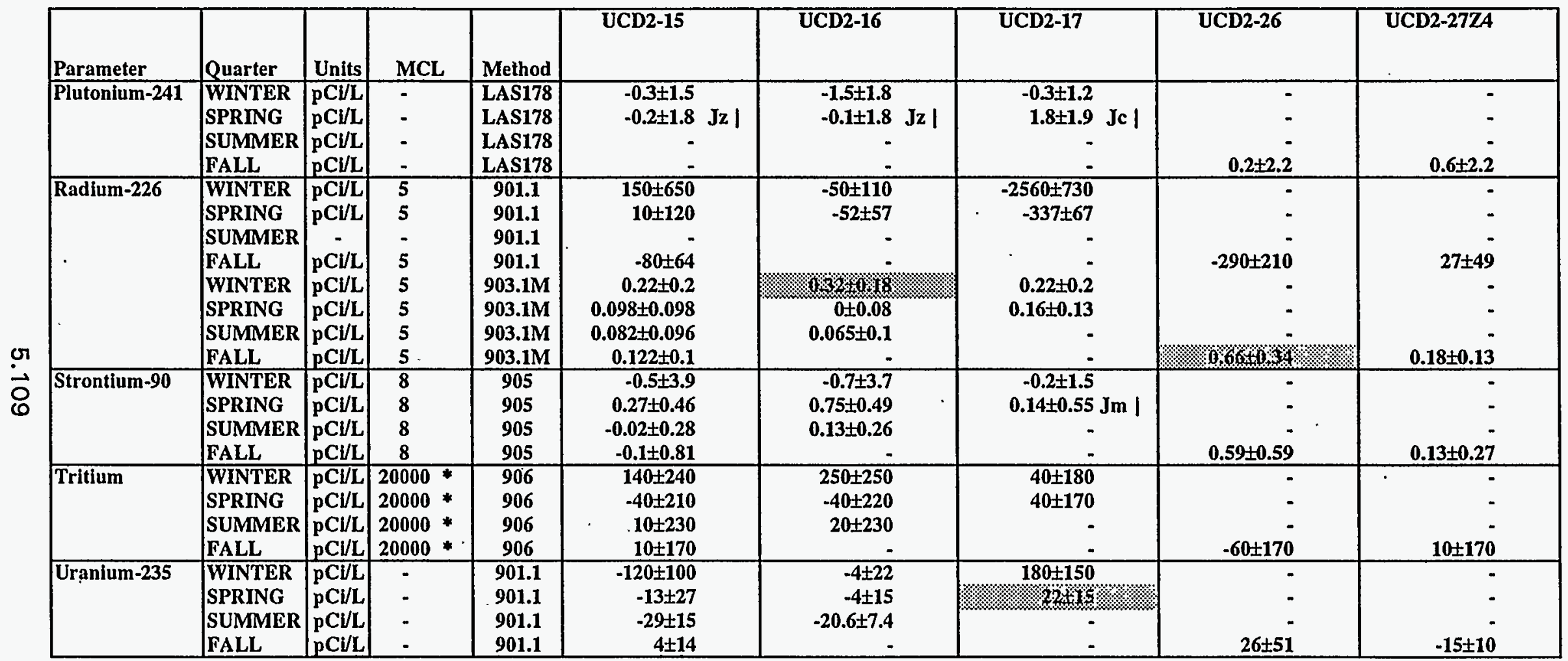


TABLE 5.6

SUMMARY OF DETECTED CONSTITUENTS IN GROUNDWATER, 1995 RADIOCHEMICALS

1995 ANNUAL WATER MONITORING REPORT

LEHR ENVIRONMENTAL RESTORATION, DAVIS, CALIFORNIA

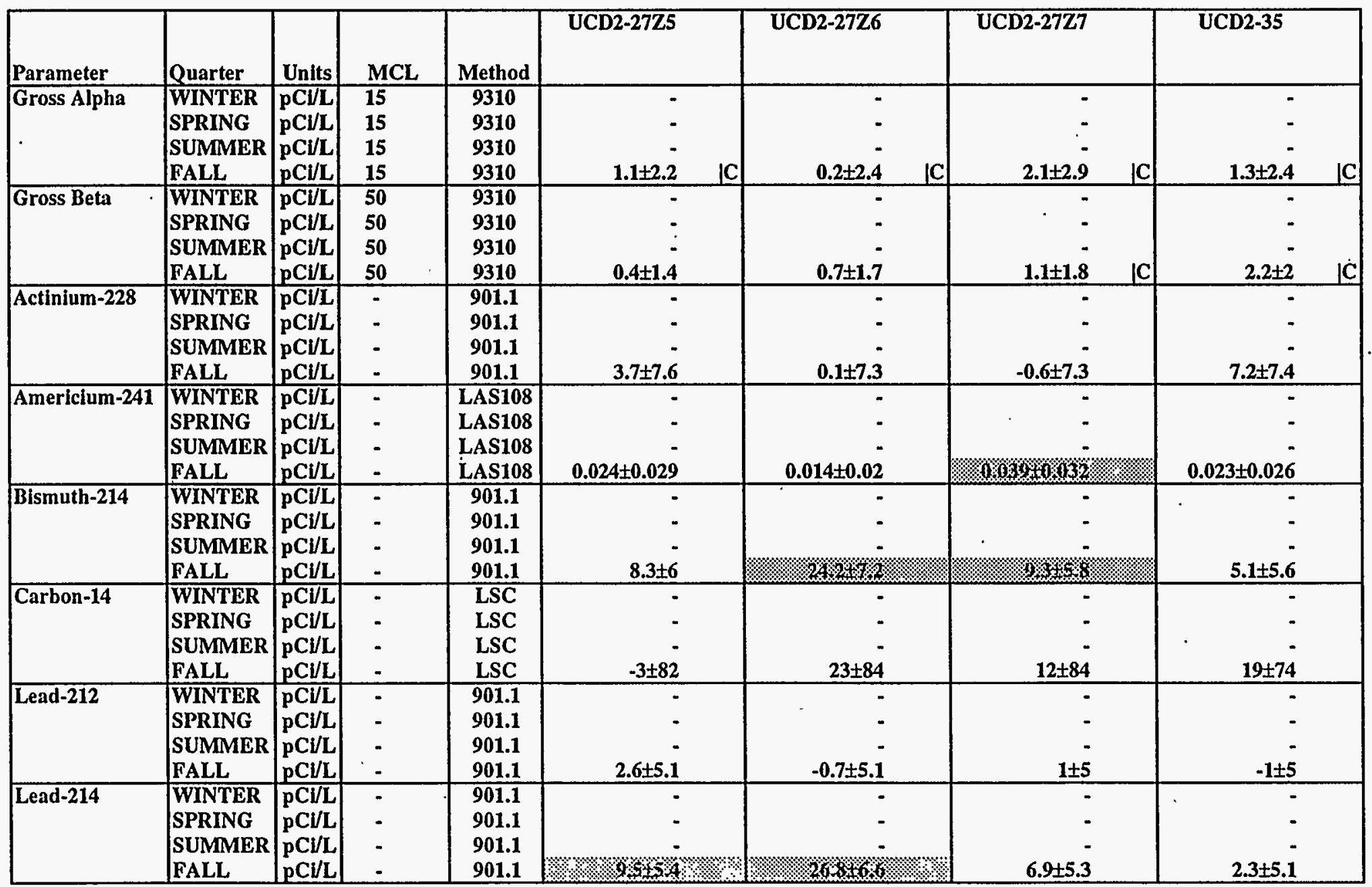

Page 13 of 15 
TABLE 5.6

SUMMARY OF DETECTED CONSTITUENTS IN GROUNDWATER, 1995

RADIOCHEMICALS

1995 ANNUAL WATER MONITORING REPORT

LEHR ENVIRONMENTAL RESTORATION, DAVIS, CALIFORNIA

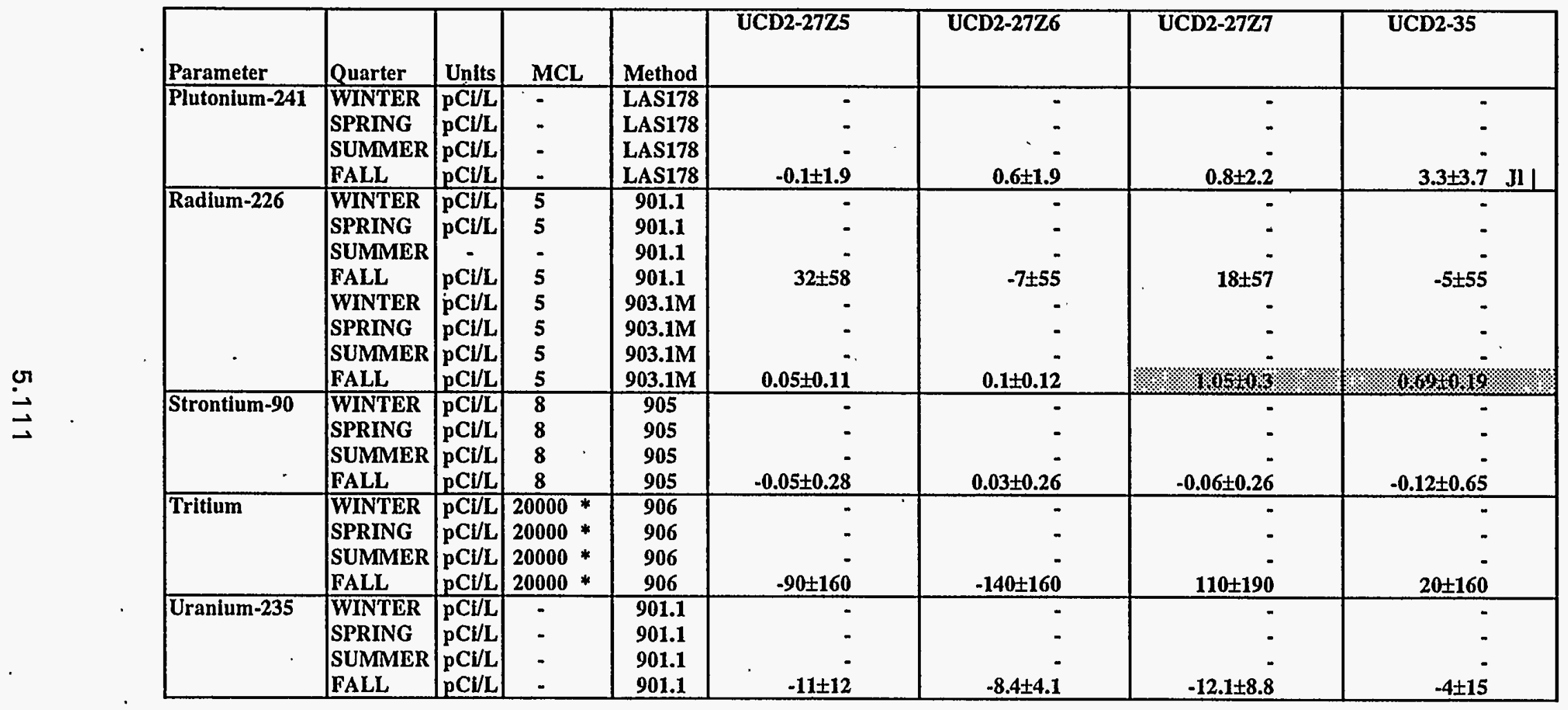


TABLE 5.6

SUMMARY OF DETECTED CONSTITUENTS IN GROUNDWATER, 1995

RADIOCHEMICALS

1995 ANNUAL WATER MONITORING REPORT

LEHR ENVIRONMENTAL RESTORATION, DAVIS, CALIFORNIA

$\begin{aligned} \text { D1 } & =\text { Duplicate sample. } \\ - & =\text { Not analyzed. }\end{aligned}$

MCL = Maximum Contaminant Level (primary), Title 22, Division 4, Chapter 15.

* = Proposed MCL for Tritlum is $60000 \mathrm{pC} / \mathrm{L}$.

$\mathrm{a}=$ Reported tritium actlvity of $50 \mathrm{pCi} / \mathrm{L}$ is suspected to belong to UCD1-20. The tritium activity for UCD1-20 was reported as $16,600 \mathrm{pC} / \mathrm{L}$, whicl more consistent with the historical trend for UCD1-13.

$b=$ Reported tritium activity of $16,600 \mathrm{pCi} / \mathrm{L}$ is suspected to belong to UCD1-13. The tritium activity for UCD1-13 was reported as $50 \mathrm{pCi} / \mathrm{L}$, whicl more consistent with the historical trend for UCD1-20.

Highilghted values indicate results where the value is greater than the calculated uncertainty.

Note: Radionuclide results are reported as calculated value, $V$, followed by the total uncertainty, $T$, in the format $V \pm T$. A calculated value that equal the uncertainty ("positive value") represents $95 \%$ level of confidence that measurable radioactivity above zero is present for a given radionucli

See Appendix A for explanation of data qualifiers. 
TABLE 5.7

SUMMARY OF DETECTED CONSTITUENTS IN GROUNDWATER, 1995

ANIONS AND CATIONS

1995 ANNUAL WATER MONITORING REPORT

LEHR ENVIRONMENTAL RESTORATION, DAVIS, CALIFORNIA

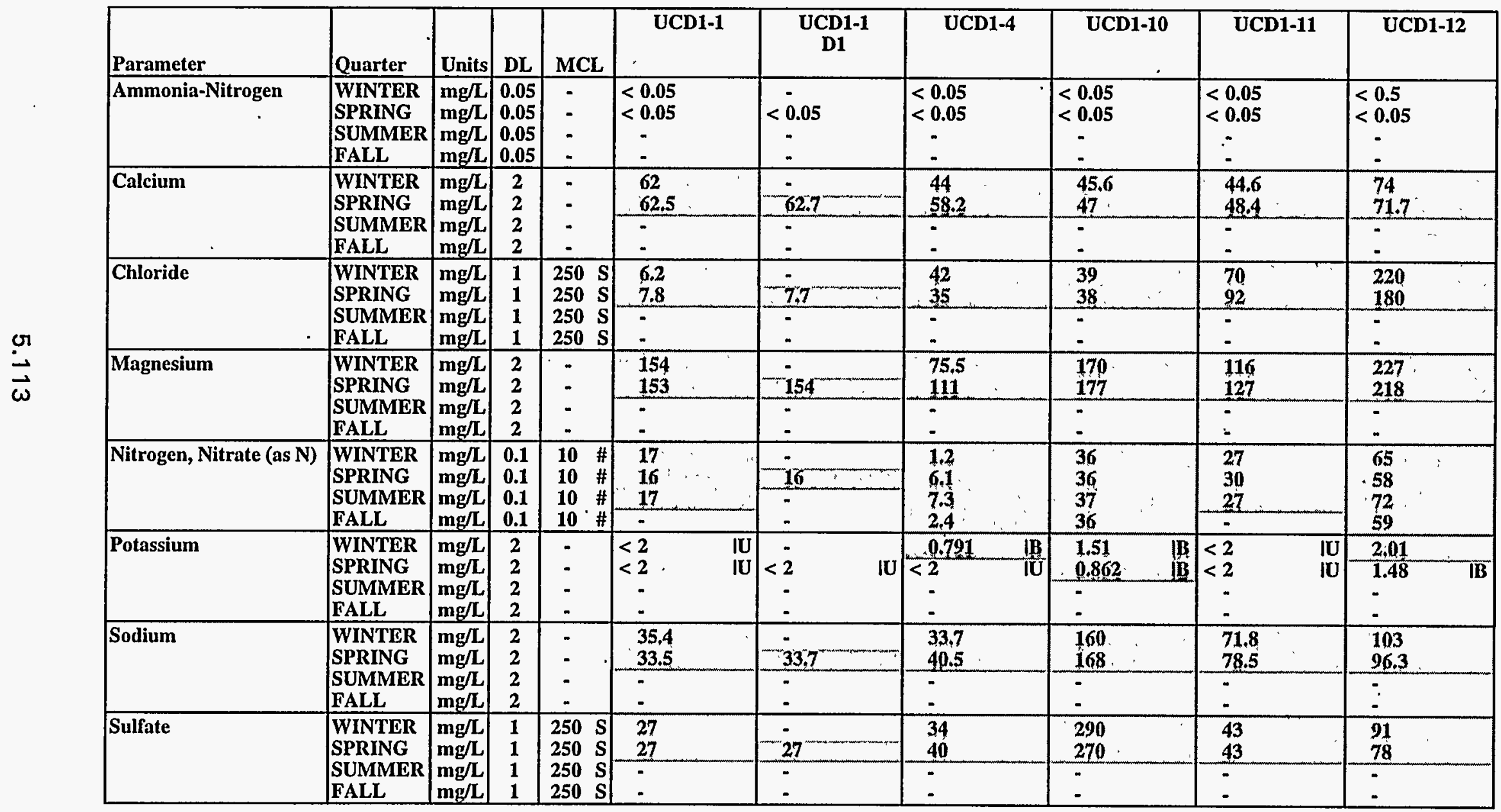


TABLE 5.7

SUMMARY OF DETECTED CONSTITUENTS IN GROUNDWATER, 1995 ANIONS AND CATIONS

1995 ANNUAL WATER MONITORING REPORT LEHR ENVIRONMENTAL RESTORATION, DAVIS, CALIFORNIA

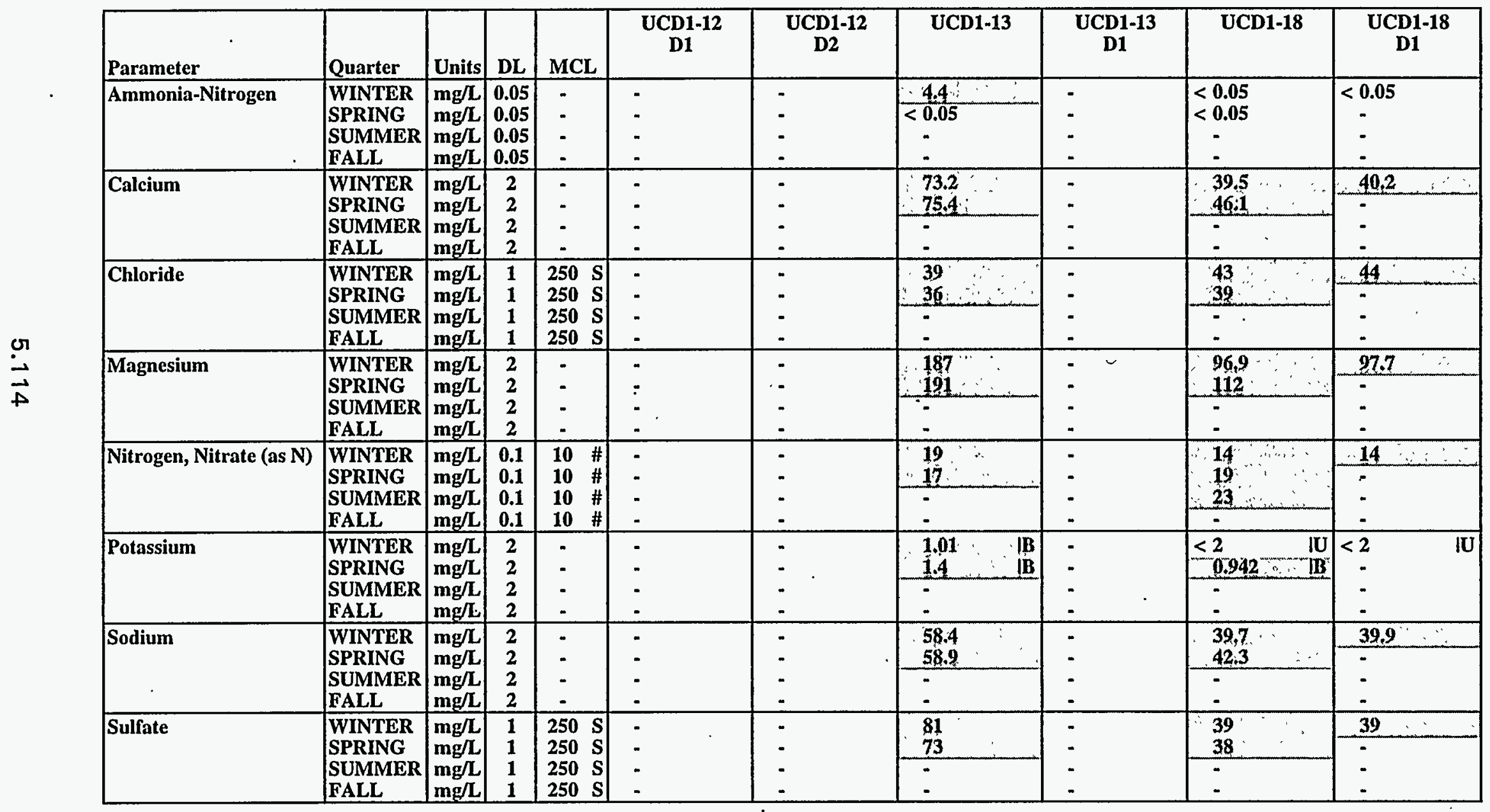


TABLE 5.7

SUMMARY OF DETECTED CONSTITUENTS IN GROUNDWATER, 1995

ANIONS AND CATIONS

1995 ANNUAL WATER MONITORING REPORT

LEHR ENVIRONMENTAL RESTORATION, DAVIS, CALIFORNIA

\begin{tabular}{|c|c|c|c|c|c|c|c|c|c|c|}
\hline Parameter & Quarter & Units & DL & MCL & UCD1-19 & $\begin{array}{c}\text { UCD1-19 } \\
\text { D1 }\end{array}$ & UCD1-20 & UCD1-21 & UCD1-22 & UCD1-23 \\
\hline Ammonia-Nitrogen & $\begin{array}{l}\text { WINTER } \\
\text { SPRING } \\
\text { SUMMER } \\
\text { FALL }\end{array}$ & $\begin{array}{l}\mathrm{mg} / \mathrm{L} \\
\mathrm{mg} / \mathrm{L} \\
\mathrm{mg} / \mathrm{L} \\
\mathrm{mg} / \mathrm{L}\end{array}$ & $\begin{array}{l}0.05 \\
0.05 \\
0.05 \\
0.05\end{array}$ & $\begin{array}{l}- \\
: \\
-\end{array}$ & $\begin{array}{c}0.1 \\
<0.05 \\
\vdots \\
\end{array}$ & $\begin{array}{l}<0.05 \\
\vdots\end{array}$ & $\begin{array}{c}<0.05 \\
<0.05 \\
- \\
-\end{array}$ & $\begin{array}{c}<0.05 \\
<0.05 \\
\vdots \\
-\end{array}$ & $\begin{array}{c}<0.05 \\
<0.05 \\
- \\
-\end{array}$ & $\begin{array}{l}<0.05 \\
<0.05 \\
- \\
-\end{array}$ \\
\hline \multirow[t]{2}{*}{ Calcium } & \multirow{2}{*}{\begin{tabular}{|l|} 
WINTER \\
SPRING \\
SUMMER \\
FALL
\end{tabular}} & \multirow{2}{*}{\begin{tabular}{|l|}
$\mathrm{mg} / \mathrm{L}$ \\
$\mathrm{mg} / \mathrm{L}$ \\
$\mathrm{mg} / \mathrm{L}$ \\
$\mathrm{mg} / \mathrm{L}$ \\
\end{tabular}} & \multirow{2}{*}{\begin{tabular}{|l|}
2 \\
2 \\
2 \\
2 \\
\end{tabular}} & \multirow{2}{*}{ ". } & $\begin{array}{l}57.9 \\
60.1 \\
\end{array}$ & $-\overline{60}$ & $\begin{array}{l}25.2 \\
25.4\end{array}$ & $\begin{array}{l}54.9 \\
56.6\end{array}$ & $\begin{array}{l}36.5 \\
38.8\end{array}$ & $\begin{array}{l}46.3 \\
51.7 \\
\end{array}$ \\
\hline & & & & & 5 & 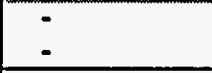 & $:$ & $:$ & $:$ & - \\
\hline Chloride & $\begin{array}{l}\text { SUMMER } \\
\text { FALL }\end{array}$ & $\begin{array}{l}\mathrm{mg} / \mathrm{L} \\
\mathrm{mg} / \mathrm{L}\end{array}$ & $\begin{array}{l}1 \\
1\end{array}$ & $\begin{array}{l}250 \mathrm{~S} \\
250 \mathrm{~S} \\
\end{array}$ & - & $\div$ & $\because$ & - & - & - \\
\hline \multirow[t]{2}{*}{ Magnesium } & \multirow{2}{*}{$\begin{array}{l}\text { WINTER } \\
\text { SPRING } \\
\text { SUMMER } \\
\text { FALL } \\
\end{array}$} & \multirow{2}{*}{\begin{tabular}{|l|}
$\mathrm{mg} / \mathrm{L}$ \\
$\mathrm{mg} / \mathrm{L}$ \\
$\mathrm{mg} / \mathrm{L}$ \\
$\mathrm{mg} / \mathrm{L}$
\end{tabular}} & \multirow{2}{*}{$\begin{array}{l}2 \\
2 \\
2 \\
2 \\
\end{array}$} & \multirow{2}{*}{$\begin{array}{l} \\
: \\
- \\
\end{array}$} & $\begin{array}{l}164 \\
170 \\
\end{array}$ & $-i 66$ & 62.2 & $\begin{array}{l}136 \\
140\end{array}$ & $\begin{array}{l}72,3 \\
77,1\end{array}$ & $\begin{array}{l}110 \\
122\end{array}$ \\
\hline & & & & & - & $\vdots$ & $:$ & - & - & 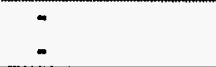 \\
\hline Nitrogen, Nitrate (as N) & $\begin{array}{l}\text { WINTER } \\
\text { SPRING }\end{array}$ & $\begin{array}{l}\mathrm{mg} / \mathrm{L} \\
\mathrm{mg} / \mathrm{L}\end{array}$ & \begin{tabular}{l|}
0.1 \\
0.1
\end{tabular} & $\begin{array}{ll}10 & \# \\
10 & \#\end{array}$ & $\begin{array}{l}16 \\
19 \\
\end{array}$ & -19 & 6.4 & $\begin{array}{l}56 \\
56\end{array}$ & $\begin{array}{l}4.5 \\
5\end{array}$ & 8.8 \\
\hline \multirow[t]{2}{*}{ Sodium } & $\begin{array}{l}\text { WINTER } \\
\text { SPRING }\end{array}$ & $\begin{array}{l}\mathrm{mg} / \mathrm{L} \\
\mathrm{mg} / \mathrm{L}\end{array}$ & $\begin{array}{l}2 \\
2\end{array}$ & - & $\begin{array}{l}67.5 \\
74.1\end{array}$ & 74 & $\begin{array}{l}36.8 \\
36.7\end{array}$ & $\begin{array}{l}92,9 \\
96,5 \\
\end{array}$ & $\begin{array}{l}88.7 \\
97.6\end{array}$ & $\begin{array}{l}42.7 \\
45.2\end{array}$ \\
\hline & $\begin{array}{l}\text { SUMMER } \\
\text { FALL }\end{array}$ & $\begin{array}{l}\mathrm{mg} / \mathrm{L} \\
\mathrm{mg} / \mathrm{L}\end{array}$ & $\begin{array}{l}2 \\
2\end{array}$ & - & - & $:$ & $:$ & $\vdots$ & : & 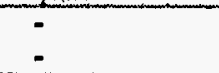 \\
\hline Sulfate & $\begin{array}{l}\text { WINTER } \\
\text { SPRING }\end{array}$ & \begin{tabular}{l|}
$\mathrm{mg} / \mathrm{h}$ \\
$\mathrm{mg} / \mathrm{h}$
\end{tabular} & $\begin{array}{l}1 \\
1\end{array}$ & $\begin{array}{ll}250 \mathrm{~S} \\
250 \mathrm{~S}\end{array}$ & $\begin{array}{l}57 \\
59 \\
\end{array}$ & 59 & $\begin{array}{l}24 \\
27 \\
\end{array}$ & $\begin{array}{l}58 \\
61 \\
\end{array}$ & $\begin{array}{l}33 \\
32 \\
\end{array}$ & $\begin{array}{r}30 \\
30 \\
\end{array}$ \\
\hline & $\begin{array}{l}\text { SUMMER } \\
\text { FALL }\end{array}$ & $\left|\begin{array}{l}\mathrm{mg} / \mathrm{L} \\
\mathrm{mg} / \mathrm{L}\end{array}\right|$ & $\begin{array}{l}1 \\
1\end{array}$ & $\begin{array}{ll}250 & S \\
250 & S\end{array}$ & $:$ & - & $:$ & - & 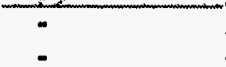 & $\vdots$ \\
\hline
\end{tabular}

Page 3 of 8 
TABLE 5.7

SUMMARY OF DETECTED CONSTITUENTS IN GROUNDWATER, 1995

ANIONS AND CATIONS

1995 ANNUAL WATER MONITORING REPORT

LEHR ENVIRONMENTAL RESTORATION, DAVIS, CALIFORNIA

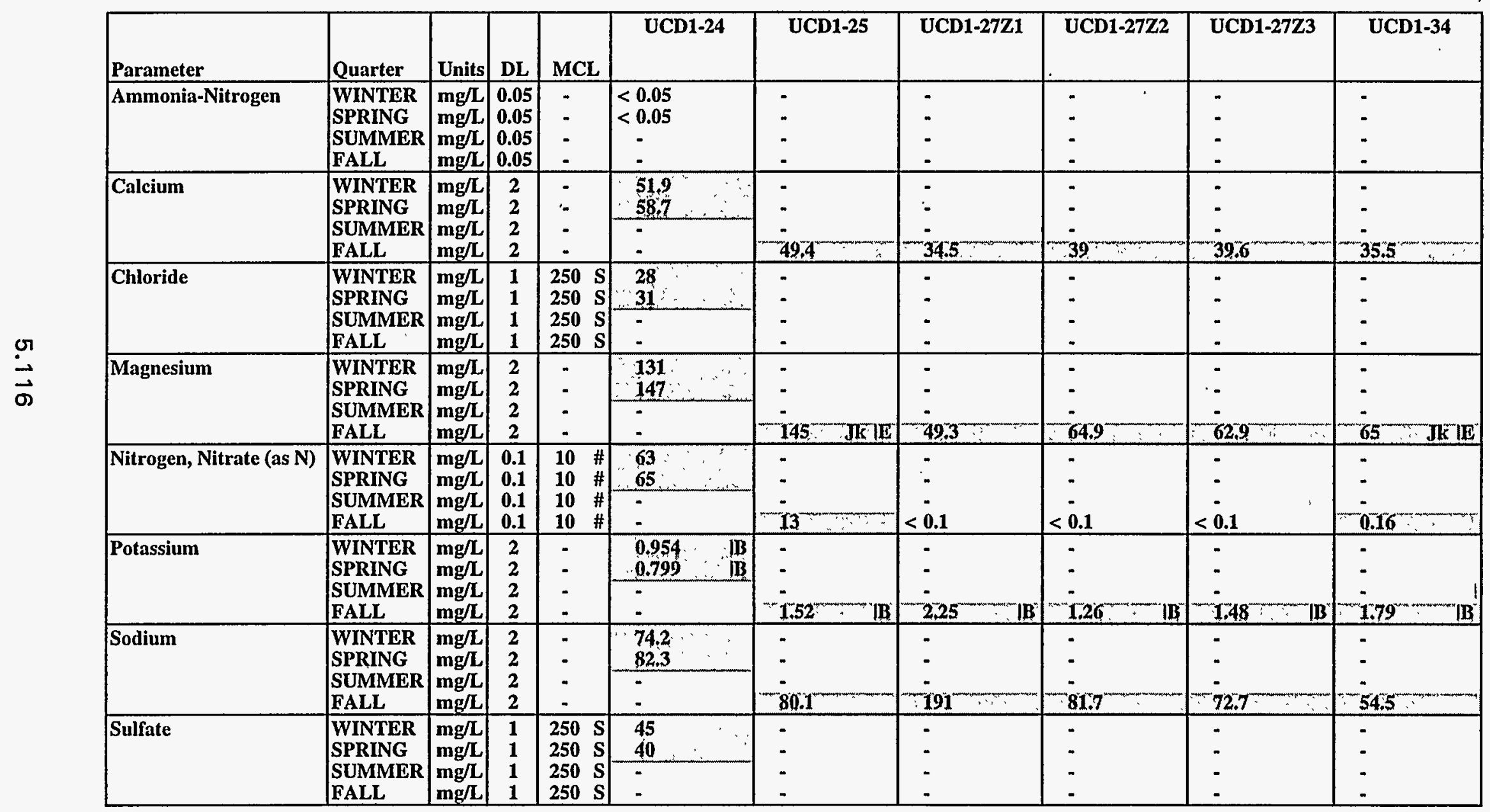


TABLE 5.7

SUMMARY OF DETECTED CONSTITUENTS IN GROUNDWATER, 1995

ANIONS AND CATIONS

1995 ANNUAL WATER MONITORING REPORT

LEHR ENVIRONMENTAL RESTORATION, DAVIS, CALIFORNIA

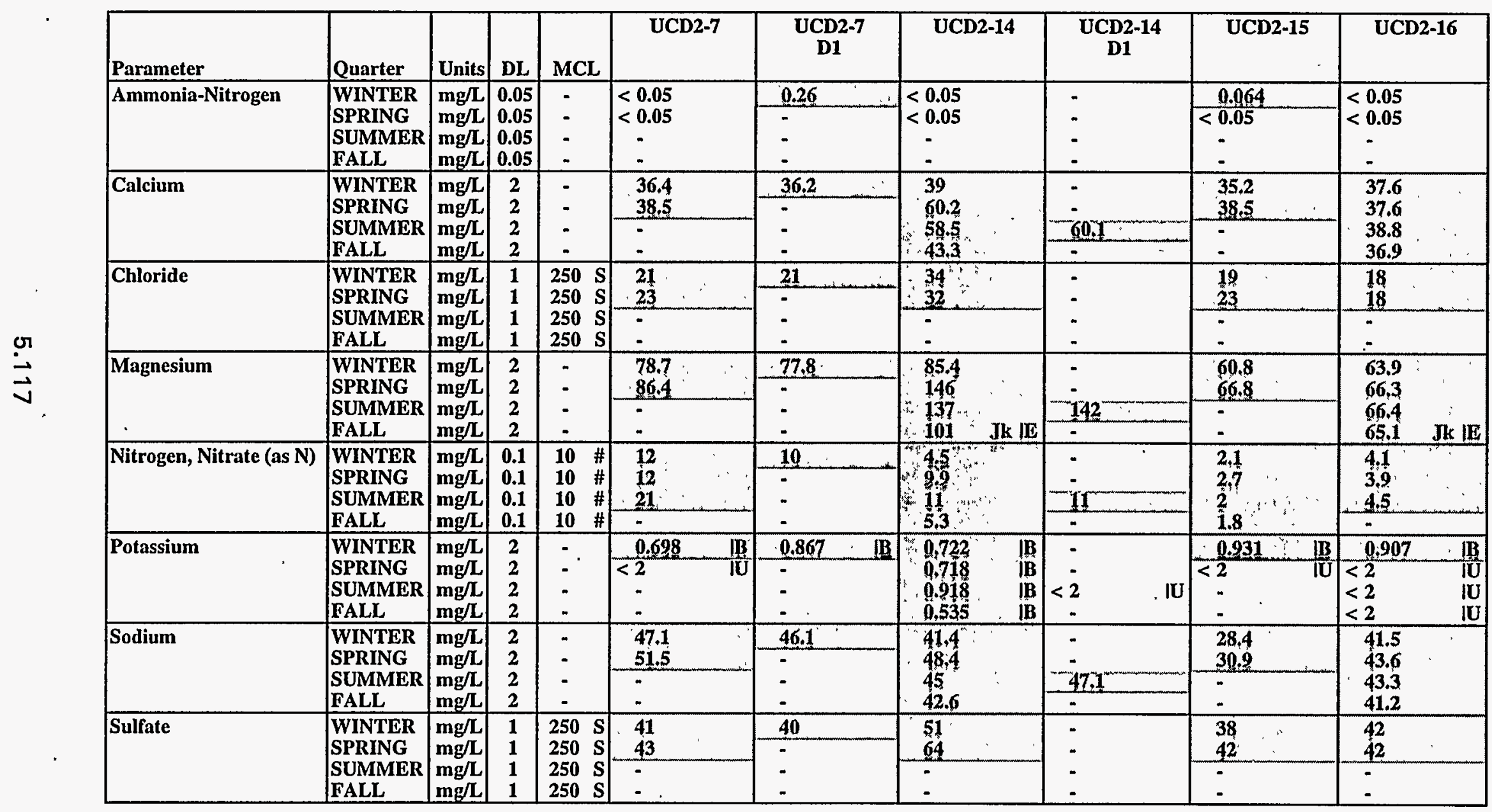


TABLE 5.7

SUMMARY OF DETECTED CONSTITUENTS IN GROUNDWATER, 1995 ANIONS AND CATIONS

1995 ANNUAL WATER MONITORING REPORT

LEHR ENVIRONMENTAL RESTORATION, DAVIS, CALIFORNIA

\begin{tabular}{|c|c|c|c|c|c|c|c|c|c|c|}
\hline Parameter & Quarter & Units & DL & MCL & UCD2-17 & UCD2-26 & UCD2-27Z4 & UCD2-27Z5 & UCD2-27Z6 & UCD2-27Z7 \\
\hline Ammonia-Nitrogen & \begin{tabular}{|l|} 
WINTER \\
SPRING \\
SUMMER \\
FALL \\
\end{tabular} & \begin{tabular}{l|}
$\mathrm{mg} / \mathrm{h}$ \\
$\mathrm{mg} / \mathrm{h}$ \\
$\mathrm{mg} / \mathrm{L}$ \\
$\mathrm{mg} / \mathrm{L}$
\end{tabular} & \begin{tabular}{|l|}
0.05 \\
0.05 \\
0.05 \\
0.05 \\
\end{tabular} & $\begin{array}{l}- \\
: \\
- \\
\end{array}$ & $\begin{array}{c}<0.05 \\
<0.05 \\
- \\
-\end{array}$ & $\begin{array}{l}: \\
: \\
\end{array}$ & $:$ & $:$ & $\begin{array}{l}\vdots \\
: \\
\end{array}$ & $\begin{array}{l}- \\
- \\
-\end{array}$ \\
\hline Calcium & $\begin{array}{l}\text { WINTER } \\
\text { SPRING } \\
\text { SUMMER } \\
\text { FALL } \\
\end{array}$ & $\begin{array}{l}\mathrm{mg} / \mathrm{L} \\
\mathrm{mg} / \mathrm{h} \\
\mathrm{mg} / \mathrm{h} \\
\mathrm{mg} / \mathrm{L}\end{array}$ & $\begin{array}{l}2 \\
2 \\
2 \\
2 \\
\end{array}$ & $\begin{array}{l}- \\
- \\
-\end{array}$ & $\begin{array}{l}36.4 \\
37.3 \\
- \\
-\end{array}$ & $\begin{array}{l}- \\
- \\
- \\
36.5\end{array}$ & $\begin{array}{l}- \\
30,2\end{array}$ & $\begin{array}{l}- \\
- \\
35.3\end{array}$ & $\begin{array}{l}- \\
- \\
35.2\end{array}$ & $\begin{array}{l}- \\
- \\
33.8\end{array}$ \\
\hline Magnesium & \begin{tabular}{|l|} 
WINTER \\
SPRING \\
SUMMER \\
FALL \\
\end{tabular} & \begin{tabular}{|l|}
$\mathrm{mg} / \mathrm{h}$ \\
$\mathrm{mg} / \mathrm{h}$ \\
$\mathrm{mg} / \mathrm{h}$ \\
$\mathrm{mg} / \mathrm{L}$ \\
\end{tabular} & $\begin{array}{l}2 \\
2 \\
2 \\
2 \\
\end{array}$ & $\begin{array}{l}- \\
: \\
- \\
\end{array}$ & $\begin{array}{r}61,1 \\
62.3 \\
- \\
\end{array}$ & $\begin{array}{l}- \\
- \\
66.5 \mathrm{JK} \mathrm{IE}\end{array}$ & $\begin{array}{l}- \\
- \\
64,2\end{array}$ & $\begin{array}{l}- \\
- \\
67 \\
67\end{array}$ & $\begin{array}{l}: \\
61 \\
0\end{array}$ & $\begin{array}{l}- \\
- \\
58.1 \\
5\end{array}$ \\
\hline Nitrogen, Nitrate (as N) & \begin{tabular}{l|} 
WINTER \\
SPRING \\
SUMMER \\
FALL \\
\end{tabular} & $\begin{array}{l}\mathrm{mg} / \mathrm{L} \\
\mathrm{mg} / \mathrm{L} \\
\mathrm{mg} / \mathrm{L} \\
\mathrm{mg} / \mathrm{L}\end{array}$ & $\begin{array}{l}0.1 \\
0.1 \\
0.1 \\
0.1\end{array}$ & \begin{tabular}{ll|}
10 & $\#$ \\
10 & $\#$ \\
10 & $\#$ \\
10 & $\#$ \\
\end{tabular} & $\begin{array}{l}2,1 \\
1.6 \\
1.4 \\
0\end{array}$ & $\begin{array}{l} \\
\vdots \\
3,1\end{array}$ & $\begin{array}{l}- \\
- \\
0,6\end{array}$ & $\begin{array}{l}- \\
- \\
2.4 !\end{array}$ & $\begin{array}{l}- \\
\vdots \\
144\end{array}$ & $\begin{array}{l}- \\
- \\
122\end{array}$ \\
\hline Sodium & \begin{tabular}{l|} 
WINTER \\
SPRING \\
SUMMER \\
FALL \\
\end{tabular} & \begin{tabular}{|l|}
$\mathrm{mg} / \mathrm{L}$ \\
$\mathrm{mg} / \mathrm{L}$ \\
$\mathrm{mg} / \mathrm{L}$ \\
$\mathrm{mg} / \mathrm{L}$
\end{tabular} & $\begin{array}{l}2 \\
2 \\
2 \\
2 \\
\end{array}$ & $\begin{array}{l}- \\
: \\
- \\
\end{array}$ & $\begin{array}{l}30.7 \\
30.3 \\
- \\
\end{array}$ & $\begin{array}{l}- \\
- \\
- \\
367 \\
\end{array}$ & $\begin{array}{l}- \\
: \\
39.4 \\
\end{array}$ & $\begin{array}{l}- \\
- \\
34.6 \\
\end{array}$ & $\begin{array}{l}- \\
- \\
41,8 \\
\end{array}$ & $\begin{array}{l}- \\
- \\
51.9 \\
\end{array}$ \\
\hline Sulfate & \begin{tabular}{|l|} 
WINTER \\
SPRING \\
SUMMER \\
FALL \\
\end{tabular} & \begin{tabular}{|l|}
$\mathrm{mg} / \mathrm{h}$ \\
$\mathrm{mg} / \mathrm{h}$ \\
$\mathrm{mg} / \mathrm{L}$ \\
$\mathrm{mg} / \mathrm{L}$
\end{tabular} & $\begin{array}{l}1 \\
1 \\
1 \\
1 \\
\end{array}$ & $\begin{array}{ll}250 & S \\
250 & S \\
250 & S \\
250 & S\end{array}$ & $\begin{array}{l}39 \\
34 \\
:\end{array}$ & $\begin{array}{l}- \\
: \\
- \\
\end{array}$ & $\begin{array}{l}- \\
: \\
- \\
\end{array}$ & 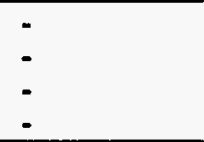 & $\begin{array}{l}- \\
: \\
- \\
\end{array}$ & $\begin{array}{l}- \\
- \\
- \\
\end{array}$ \\
\hline
\end{tabular}


TABLE 5.7

SUMMARY OF DETECTED CONSTITUENTS IN GROUNDWATER, 1995 ANIONS AND CATIONS

1995 ANNUAL WATER MONITORING REPORT LEHR ENVIRONMENTAL RESTORATION, DAVIS, CALIFORNIA

\begin{tabular}{|c|c|c|c|c|c|}
\hline Parameter & Quarter & Units & DL & MCL & UCD2-35 \\
\hline Ammonia-Nitrogen & $\begin{array}{l}\text { WINTER } \\
\text { SPRING } \\
\text { SUMMER } \\
\text { FALL }\end{array}$ & $\begin{array}{l}\mathrm{mg} / \mathrm{L} \\
\mathrm{mg} / \mathrm{L} \\
\mathrm{mg} / \mathrm{L} \\
\mathrm{mg} / \mathrm{L}\end{array}$ & $\begin{array}{l}0.05 \\
0.05 \\
0.05 \\
0.05 \\
\end{array}$ & $\begin{array}{l}: \\
:\end{array}$ & $:$ \\
\hline Calcium & \begin{tabular}{|l|} 
WINTER \\
SPRING \\
SUMMER \\
FALL \\
\end{tabular} & $\begin{array}{l}\mathrm{mg} / \mathrm{L} \\
\mathrm{mg} / \mathrm{L} \\
\mathrm{mg} / \mathrm{L} \\
\mathrm{mg} / \mathrm{L}\end{array}$ & \begin{tabular}{|l}
2 \\
2 \\
2 \\
2 \\
\end{tabular} & $\begin{array}{l}- \\
- \\
- \\
\end{array}$ & $\begin{array}{l}- \\
\vdots \\
37.2\end{array}$ \\
\hline Chloride & $\begin{array}{l}\text { WINTER } \\
\text { SPRING } \\
\text { SUMMER } \\
\text { FALL }\end{array}$ & $\begin{array}{l}\mathrm{mg} / \mathrm{L} \\
\mathrm{mg} / \mathrm{L} \\
\mathrm{mg} / \mathrm{L} \\
\mathrm{mg} / \mathrm{L}\end{array}$ & $\begin{array}{l}1 \\
1 \\
1 \\
1\end{array}$ & $\begin{array}{ll}250 & S \\
250 & S \\
250 & S \\
250 & S\end{array}$ & $:$ \\
\hline Magnesium & \begin{tabular}{|l|} 
WINTER \\
SPRING \\
SUMMER \\
FALL \\
\end{tabular} & $\begin{array}{l}\mathrm{mg} / \mathrm{L} \\
\mathrm{mg} / \mathrm{L} \\
\mathrm{mg} / \mathrm{L} \\
\mathrm{mg} / \mathrm{L}\end{array}$ & $\begin{array}{l}2 \\
2 \\
2 \\
2\end{array}$ & $\begin{array}{l}- \\
\vdots \\
\vdots\end{array}$ & $\begin{array}{ll}- & \\
- & \\
63.8 & \mathrm{JK} / \mathrm{E}\end{array}$ \\
\hline Nitrogen, Nitrate (as N) & \begin{tabular}{|l|} 
WINTER \\
SPRING \\
SUMMER \\
FALL
\end{tabular} & $\begin{array}{l}\mathrm{mg} / \mathrm{L} \\
\mathrm{mg} / \mathrm{L} \\
\mathrm{mg} / \mathrm{L} \\
\mathrm{mg} / \mathrm{L}\end{array}$ & $\begin{array}{l}0.1 \\
0.1 \\
0.1 \\
0.1\end{array}$ & \begin{tabular}{ll|}
10 & $\#$ \\
10 & $\#$ \\
10 & $\#$ \\
10 & $\#$ \\
\end{tabular} & $\begin{array}{l}- \\
\vdots \\
1.2\end{array}$ \\
\hline Potassium & $\begin{array}{l}\text { WINTER } \\
\text { SPRING } \\
\text { SUMMER } \\
\text { FALL }\end{array}$ & $\begin{array}{l}\mathrm{mg} / \mathrm{L} \\
\mathrm{mg} / \mathrm{L} \\
\mathrm{mg} / \mathrm{L} \\
\mathrm{mg} / \mathrm{L}\end{array}$ & $\begin{array}{l}2 \\
2 \\
2 \\
2 \\
\end{array}$ & \begin{tabular}{l|} 
\\
$:$ \\
\end{tabular} & $\begin{array}{l}: \\
\vdots \\
0.982\end{array}$ \\
\hline Sodium & $\begin{array}{l}\text { WINTER } \\
\text { SPRING } \\
\text { SUMMER } \\
\text { FALL }\end{array}$ & $\begin{array}{l}\mathrm{mg} / \mathrm{L} \\
\mathrm{mg} / \mathrm{L} \\
\mathrm{mg} / \mathrm{L} \\
\mathrm{mg} / \mathrm{L}\end{array}$ & $\begin{array}{l}2 \\
2 \\
2 \\
2\end{array}$ & $\begin{array}{l}: \\
: \\
\end{array}$ & $\begin{array}{l}- \\
- \\
- \\
33,3\end{array}$ \\
\hline Sulfate & $\begin{array}{l}\text { WINTER } \\
\text { SPRING } \\
\text { SUMMER } \\
\text { FALL }\end{array}$ & $\begin{array}{l}\mathrm{mg} / \mathrm{L} \\
\mathrm{mg} / \mathrm{L} \\
\mathrm{mg} / \mathrm{L} \\
\mathrm{mg} / \mathrm{L}\end{array}$ & $\begin{array}{l}- \\
1 \\
1 \\
1 \\
1\end{array}$ & \begin{tabular}{ll|}
250 & $S$ \\
250 & $S$ \\
250 & $S$ \\
250 & $S$
\end{tabular} & 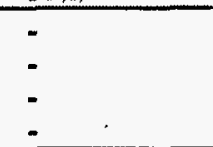 \\
\hline
\end{tabular}

Page 7 of 8 
TABLE 5.7

SUMMARY OF DETECTED CONSTITUENTS IN GROUNDWATER, 1995

ANIONS AND CATIONS

1995 ANNUAL WATER MONITORING REPORT

LEHR ENVIRONMENTAL RESTORATION, DAVIS, CALIFORNIA

D1, D2 = Duplicate sample.

- = Parameter not analyzed or no MCL.

DL = Detection limit.

$<=$ Constituent below detection limit. Detection limits may vary depending on interference by other sample constituents.

MCL = Maximum Contaminant Level (primary), Title 22 Division 4 Chapter 15.

\# = USEPA MCL

$S=$ Secondary drinking water standard.

Highlighted values indicate positive results.

See Appendix A for explanation of data qualifiers. 
TABLE 5.8

SUMMARY OF DETECTED CONSTITUENTS IN GROUNDWATER, 1995

OTHER PARAMETERS

1995 ANNUAL WATER MONITORING REPORT

LEHR ENVIRONMENTAL RESTORATION, DAVIS, CALIFORNIA

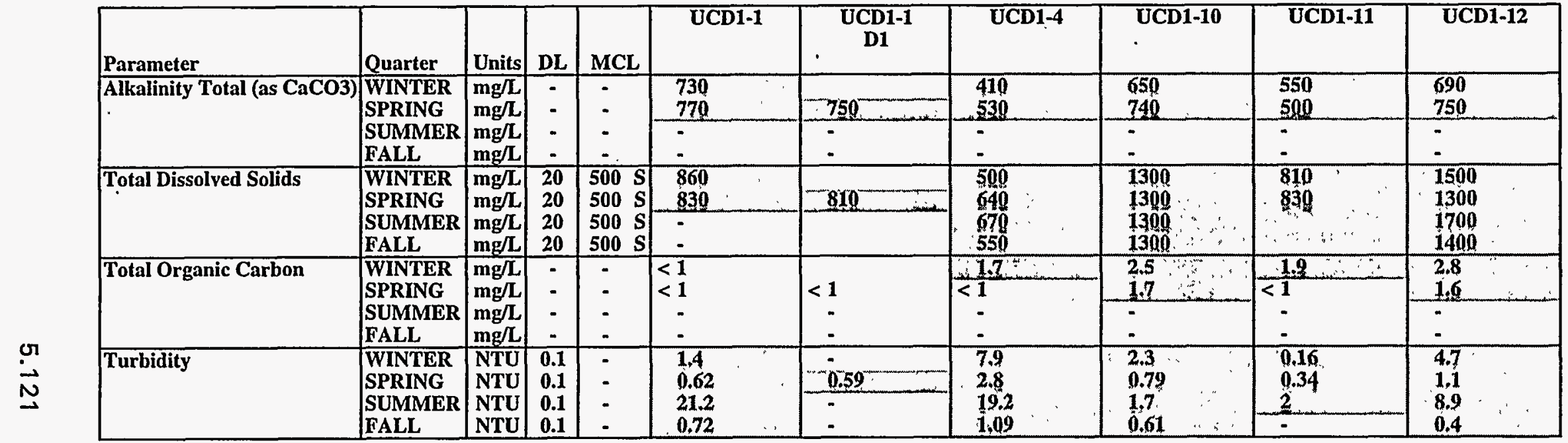

\begin{tabular}{|c|c|c|c|c|c|c|c|c|c|c|}
\hline Parameter & Quarter & Units & DL & MCL & $\begin{array}{c}\text { UCD1-12 } \\
\text { D1 }\end{array}$ & $\begin{array}{l}\text { UCD1-12 } \\
\text { D2 }\end{array}$ & UCD1-13 & $\begin{array}{c}\text { UCD1-13 } \\
\text { D1 }\end{array}$ & UCD1-18 & $\begin{array}{l}\text { UCD1-18 } \\
\cdot \text { D1 }\end{array}$ \\
\hline \multirow[t]{2}{*}{ Alkalinity Total (as CaCO3) } & $\begin{array}{l}\text { WINTER } \\
\text { SPRING }\end{array}$ & $\begin{array}{l}\mathrm{mg/h} \\
\mathrm{mg} / \mathrm{L}\end{array}$ & $\because$ & - & : & $:$ & $\begin{array}{l}460 \\
860\end{array}$ & \multirow{2}{*}{$\begin{array}{l}- \\
-\end{array}$} & $\begin{array}{l}400 \\
460\end{array}$ & \multirow{2}{*}{$\begin{array}{l}\text { sou } \\
- \\
- \\
-\end{array}$} \\
\hline & $\begin{array}{l}\text { SUMMER } \\
\text { FALC }\end{array}$ & $\begin{array}{l}\mathrm{mg} / \mathrm{L} \\
\mathrm{mg} / \mathrm{L}\end{array}$ & $\because$ & - & $\dot{-}$ & 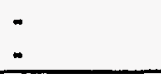 & - & & & \\
\hline \multirow[t]{3}{*}{ Total Dissolved Solids } & WINTER & $\mathrm{mg} / \mathrm{L}$ & 20 & $500 \mathrm{~s}$ & - & - & 1100 & \multirow{3}{*}{ 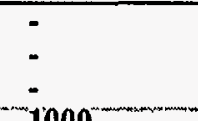 } & 610 & 620 \\
\hline & SPRING & $\mathrm{mg} / \mathrm{L}$ & 20 & $500 \mathrm{~s}$ & - & - & 1000 & & 660 & - \\
\hline & SUMMER & $\mathbf{m g} / \mathbf{L}$ & 20 & $500 S$ & : & - & 1200 & & 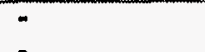 & - \\
\hline \multirow[t]{4}{*}{ Total Organic Carbon } & \begin{tabular}{|l} 
WINTER \\
WINT
\end{tabular} & $\frac{m g / L}{m g / L}$ & 20 & 400 & - & - & 1.1 & \multirow{4}{*}{$\begin{array}{l}: \\
\vdots \\
\end{array}$} & 1 & 1 \\
\hline & SPRING & $\mathrm{mg} / \mathrm{L}$ & . & - & - & - & 6 & & $=$ & - \\
\hline & SUMMER & $\mathrm{mg} / \mathrm{L}$ & - & - & - & - & - & & - & - \\
\hline & FALL & $\mathrm{mg} / \mathrm{L}$ & - & - & - & - & & & $\pi$ & \\
\hline \multirow[t]{3}{*}{ Turbidity } & WINTER & NTU & 0.1 & - & - & - & 0.52 & \multirow{3}{*}{$=$} & 1.7 & 1.7 \\
\hline & SPRING & $\mid \begin{array}{l}\text { NTU } \\
\text { NTr }\end{array}$ & 0.1 & - & -89 & : & 0.24 & & $\begin{array}{l}0.89 \\
0.47\end{array}$ & \multirow{2}{*}{$:$} \\
\hline & FALL & NTU & 0.1 & - & - & - & $\begin{array}{l}0.43 \\
0.7\end{array}$ & & -2.4 & \\
\hline
\end{tabular}


TABLE 5.8

SUMMARY OF DETECTED CONSTITUENTS IN GROUNDWATER, 1995

OTHER PARAMETERS

1995 ANNUAL WATER MONITORING REPORT

LEHR ENVIRONMENTAL RESTORATION, DAVIS, CALIFORNIA

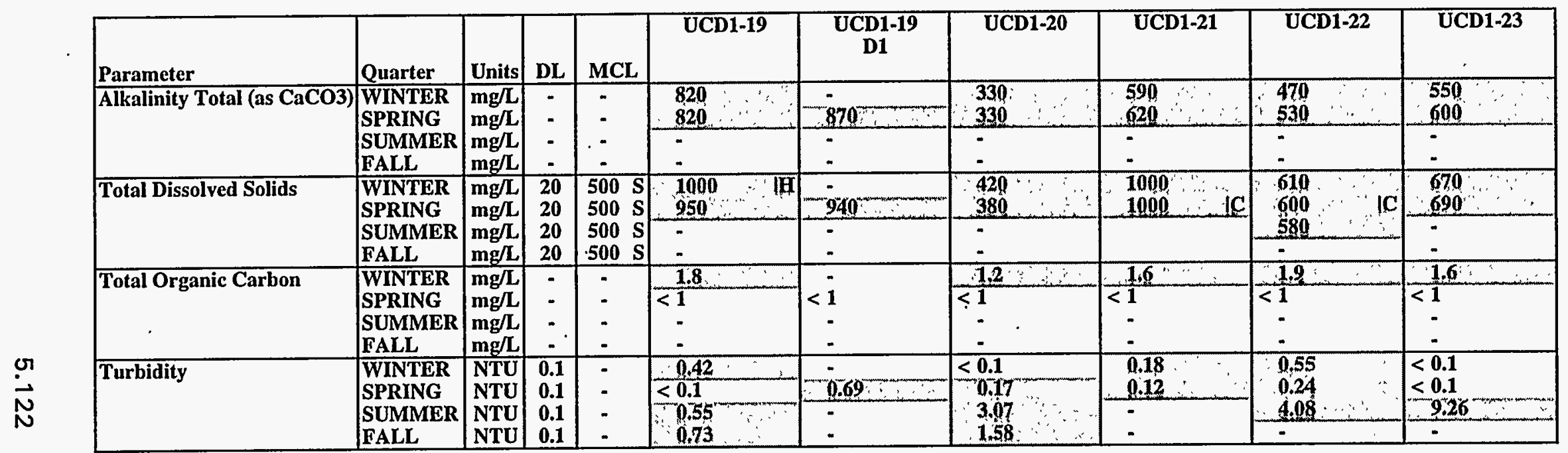

\begin{tabular}{|c|c|c|c|c|c|c|c|c|c|c|}
\hline Parameter & Quarter & Units & DL & MCL & UCD1-24 & UCD1-25 & UCD1-27Z1 & UCD1-27Z2 & UCD1-27Z3 & UCD1-34 \\
\hline Alkalinity Total (as CaCO3) & $\begin{array}{l}\text { WINTER } \\
\text { SPRING } \\
\text { SUMMER } \\
\text { FALL } \\
\end{array}$ & \begin{tabular}{|l|}
$\mathrm{mg} / \mathrm{h}$ \\
$\mathrm{mg} / \mathrm{L}$ \\
$\mathrm{mg} / \mathrm{L}$ \\
$\mathrm{mg} / \mathrm{L}$
\end{tabular} & $\begin{array}{l}: \\
: \\
- \\
\end{array}$ & $:$ & $\begin{array}{l}510 \\
560 \\
- \\
\end{array}$ & - & - & - & - & : \\
\hline Total Dissolved Solids & $\begin{array}{l}\text { WINTER } \\
\text { SPRING } \\
\text { SUMMER } \\
\text { FALL }\end{array}$ & $\begin{array}{l}\mathrm{mg} / \mathrm{L} \\
\mathrm{mg} / \mathrm{h} \\
\mathrm{mg} / \mathrm{L} \\
\mathrm{mg} / \mathrm{L}\end{array}$ & $\begin{array}{l}20 \\
20 \\
20 \\
20\end{array}$ & $\begin{array}{ll}500 & S \\
500 & S \\
500 & S \\
500 & S\end{array}$ & $\begin{array}{c}970 \\
1100 \\
\end{array}$ & 860 & 940 & 460 & $490 \div-\cdots$ & $470 \div$ \\
\hline Total Organic Carbon & $\begin{array}{l}\text { WINTER } \\
\text { SPRING } \\
\text { SUMMER } \\
\text { FALL }\end{array}$ & $\mid$\begin{tabular}{l|}
$\mathrm{mg} / \mathrm{L}$ \\
$\mathrm{mg} / \mathrm{L}$ \\
$\mathrm{mg} / \mathrm{L}$ \\
$\mathrm{mg} / \mathrm{L}$
\end{tabular} & $\div$ & $\begin{array}{l}500 \\
- \\
-\end{array}$ & $\frac{1.5}{<1}$ & - & : & - & P. & - \\
\hline Turbidity & $\begin{array}{l}\text { WINTER } \\
\text { SPRING } \\
\text { SUMMER } \\
\text { FALL }\end{array}$ & \begin{tabular}{|l|} 
NTU \\
NTU \\
NTU \\
NTU
\end{tabular} & $\begin{array}{l}0.1 \\
0.1 \\
0.1 \\
0.1\end{array}$ & $:$ & $\begin{array}{l}<0.1 \\
<0.1\end{array}$ & 10.85 & 11.23 & 1.6 & 9.81 & 20.1 \\
\hline
\end{tabular}

Page 2 of 4 
TABLE 5.8

SUMMARY OF DETECTED CONSTITUENTS IN GROUNDWATER, 1995

OTHER PARAMETERS

1995 ANNUAL WATER MONITORING REPORT

LEHR ENVIRONMENTAL RESTORATION, DAVIS, CALIFORNIA

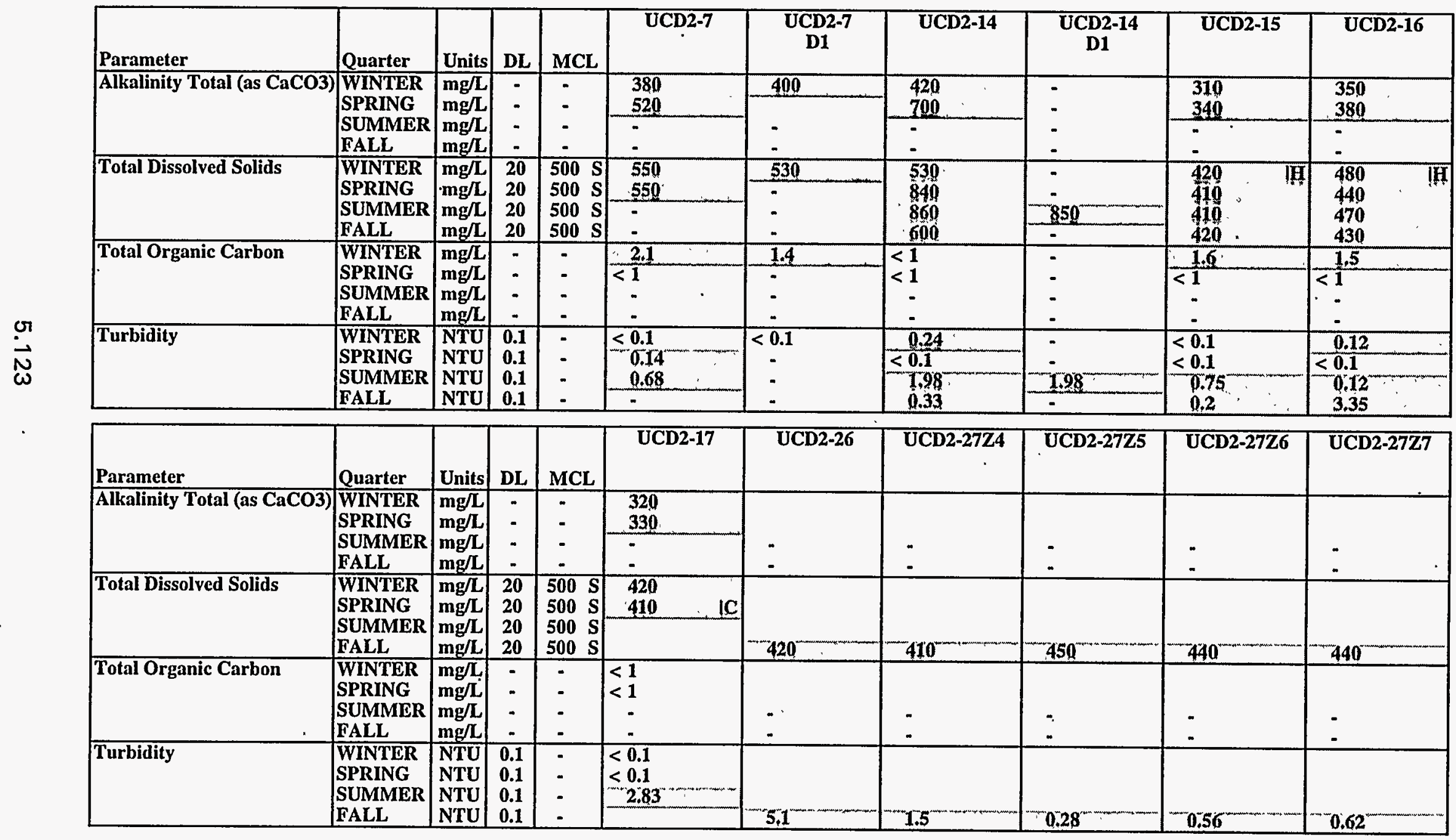

Page 3 of 4 
TABLE 5.8

SUMMARY OF DETECTED CONSTITUENTS IN GROUNDWATER, 1995

OTHER PARAMETERS

1995 ANNUAL WATER MONITORING REPORT

LEHR ENVIRONMENTAL RESTORATION, DAVIS, CALIFORNIA

\begin{tabular}{|c|c|c|c|c|c|}
\hline Parameter & Quarter & Units & DL & MCL & UCD2-35 \\
\hline Alkalinity Total (as CaCO3) & $\begin{array}{l}\text { WINTER } \\
\text { SPRING } \\
\text { SUMMER } \\
\text { FALL }\end{array}$ & $\begin{array}{l}\mathrm{mg} / \mathrm{L} \\
\mathrm{mg} / \mathrm{L} \\
\mathrm{mg} / \mathrm{L} \\
\mathrm{mg} / \mathrm{L}\end{array}$ & $\begin{array}{l}\ddot{-} \\
- \\
-\end{array}$ & $\begin{array}{l}- \\
: \\
\cdot \\
\cdot\end{array}$ & $\begin{array}{l}- \\
- \\
-\end{array}$ \\
\hline Total Dissolved Solids & $\begin{array}{l}\text { WINTER } \\
\text { SPRING } \\
\text { SUMMER } \\
\text { FALL }\end{array}$ & $\begin{array}{l}\mathrm{mg} / \mathrm{L} \\
\mathrm{mg} / \mathrm{L} \\
\mathrm{mg} / \mathrm{L} \\
\mathrm{mg} / \mathrm{L}\end{array}$ & $\begin{array}{l}20 \\
20 \\
20 \\
20\end{array}$ & $\begin{array}{ll}\mathbf{5 0 0} & \mathrm{S} \\
\mathbf{5 0 0} & \mathbf{S} \\
\mathbf{5 0 0} & \mathrm{S} \\
\mathbf{5 0 0} & \mathrm{S}\end{array}$ & $\begin{array}{l}- \\
- \\
- \\
460\end{array}$ \\
\hline Total Organic Carbon & $\begin{array}{l}\text { WINTER } \\
\text { SPRING } \\
\text { SUMMER } \\
\text { FALL }\end{array}$ & $\begin{array}{l}\mathrm{mg} / \mathrm{L} \\
\mathrm{mg} / \mathrm{L} \\
\mathrm{mg} / \mathrm{h} \\
\mathrm{mg} / \mathrm{L}\end{array}$ & $\begin{array}{l}- \\
- \\
-\end{array}$ & $\begin{array}{l}- \\
: \\
- \\
\end{array}$ & $\begin{array}{l}- \\
- \\
-\end{array}$ \\
\hline Turbidity & $\begin{array}{l}\text { WINTER } \\
\text { SPRING } \\
\text { SUMMER } \\
\text { FALL }\end{array}$ & $\begin{array}{l}\text { NTU } \\
\text { NTU } \\
\text { NTU } \\
\text { NTU }\end{array}$ & $\begin{array}{l}0.1 \\
0.1 \\
0.1 \\
0.1\end{array}$ & $\begin{array}{l}\overline{-} \\
\dot{-} \\
.\end{array}$ & $\begin{array}{l}- \\
- \\
-19.9\end{array}$ \\
\hline
\end{tabular}

D1 = Duplicate sample.

- = Parameter not analyzed or no MCL.

DL $=$ Detection limit.

$<=$ Constituent below detection limit. Detection limits may vary depending on interference by other sample constituents.

MCL = Maximum Contaminant Level (primary), Title 22 Division 4 Chapter 15.

$\mathrm{S}=$ Secondary drinking water standard.

$E]$ Highlighted values indicate positive results.

See Appendix A for explanation of data qualifiers. 
Table 5.9. Major Ion Groundwater Chemistry (weight basis) - LEHR Facility

\begin{tabular}{|c|c|c|c|c|c|c|c|c|c|c|}
\hline \multicolumn{2}{|c|}{ Site ID } & $\begin{array}{c}\text { Calcium } \\
\text { mg/L }\end{array}$ & $\begin{array}{c}\text { Magnesium } \\
\mathrm{mg} / \mathrm{L}\end{array}$ & $\begin{array}{l}\text { Sodium } \\
\mathrm{mg} / \mathrm{L}\end{array}$ & $\begin{array}{c}\text { Potassium } \\
\text { mg/L }\end{array}$ & $\begin{array}{c}\text { Alkalinity } \\
\text { as } \mathrm{CaCO}_{3} \\
\mathrm{mg} / \mathrm{L}\end{array}$ & $\begin{array}{l}\text { Sulfate } \\
\text { mg/L. }\end{array}$ & $\begin{array}{l}\text { Chlorido } \\
\text { mg/L }\end{array}$ & $\begin{array}{l}\text { Nitrate } \\
\text { as } \mathrm{N} \\
\mathrm{mg} / \mathrm{L}\end{array}$ & $\begin{array}{c}\text { Phosphate } \\
\text { as P } \\
\text { mg/L }\end{array}$ \\
\hline $\begin{array}{l}\text { Upper } \\
\text { HSU } \\
\text { (HSU-1) }\end{array}$ & $\begin{array}{l}\text { UCD1-18 } \\
\text { UCD1-01 } \\
\text { UCD1-04 } \\
\text { UCD1-10 } \\
\text { UCD1-11 } \\
\text { UCD1-12 } \\
\text { UCD 1-13 } \\
\text { UCD 1-19 } \\
\text { UCD 1-20 } \\
\text { UCD 1-21 } \\
\text { UCD 1-22 } \\
\text { UCD 1-23 } \\
\text { UCD 1-24 }\end{array}$ & $\begin{array}{l}35.9 \\
56.5 \\
51.3 \\
45.5 \\
41.6 \\
78.0 \\
69.1 \\
48.9 \\
33.1 \\
50.3 \\
31.9 \\
40.0 \\
46.2\end{array}$ & $\begin{array}{l}89.1 \\
139 \\
101 \\
161 \\
110 \\
258 \\
181 \\
136 \\
85.5 \\
136 \\
66.4 \\
97.2 \\
119 \\
\end{array}$ & $\begin{array}{c}38.0 \\
35.2 \\
36.3 \\
159.7 \\
72.7 \\
94.9 \\
56.5 \\
66.6 \\
43.2 \\
92.5 \\
83.0 \\
41.1 \\
72.2\end{array}$ & $\begin{array}{l}1.26 \\
1.15 \\
1.10 \\
1.37 \\
1.27 \\
3.88 \\
2.95 \\
1.49 \\
1.58 \\
1.50 \\
1.60 \\
1.08 \\
1.36 \\
\end{array}$ & $\begin{array}{l}413 \\
707 \\
497 \\
680 \\
507 \\
644 \\
745 \\
721 \\
433 \\
613 \\
433 \\
492 \\
520 \\
\end{array}$ & $\begin{array}{l}37.8 \\
36.6 \\
40.7 \\
369 \\
56.5 \\
89.7 \\
94.1 \\
64.8 \\
40.7 \\
67.3 \\
35.4 \\
30.9 \\
52.8 \\
\end{array}$ & $\begin{array}{c}33.4 \\
10.8 \\
40.4 \\
38.0 \\
59.7 \\
240 \\
37.7 \\
16.9 \\
8.1 \\
20.5 \\
35.5 \\
30.4 \\
25.8 \\
\end{array}$ & $\begin{array}{c}11.6 \\
14.8 \\
4.5 \\
29.0 \\
24.5 \\
64.3 \\
16.5 \\
13.1 \\
18.1 \\
46.4 \\
9.4 \\
7.0 \\
49.4\end{array}$ & $\begin{array}{l}0.56 \\
0.65 \\
0.68 \\
0.63 \\
0.67 \\
0.62 \\
0.66 \\
0.61 \\
0.61 \\
0.60 \\
0.55 \\
0.60 \\
0.61 .\end{array}$ \\
\hline $\begin{array}{l}\text { Lower } \\
\text { HSU } \\
\text { (HSU-2) }\end{array}$ & $\begin{array}{l}U C D 2-17 \\
U C D 2-07 \\
U C D 2-14 \\
U C D 2-15 \\
U C D 2-16\end{array}$ & $\begin{array}{l}32.8 \\
36.5 \\
47.1 \\
35.9 \\
33.8\end{array}$ & $\begin{array}{c}60.3 \\
83.0 \\
112 \\
62.8 \\
62.9\end{array}$ & $\begin{array}{l}26.8 \\
48.0 \\
43.6 \\
28.6 \\
41.5\end{array}$ & $\begin{array}{l}0.80 \\
1.00 \\
0.90 \\
0.93 \\
1.24\end{array}$ & $\begin{array}{l}336 \\
404 \\
535 \\
332 \\
358\end{array}$ & $\begin{array}{l}35.3 \\
40.3 \\
64.5 \\
34.7 \\
43.2\end{array}$ & $\begin{array}{l}17.6 \\
23.4 \\
34.8 \\
20.2 \\
18.5\end{array}$ & $\begin{array}{c}2.0 \\
18.7 \\
7.6 \\
2.6 \\
3.5\end{array}$ & $\begin{array}{l}0.51 \\
0.54 \\
0.62 \\
0.52 \\
0.51\end{array}$ \\
\hline
\end{tabular}


Table 5.10. Major Ion Groundwater Chemistry (equivalent basis) - LEHR Facility

\begin{tabular}{|c|c|c|c|c|c|c|c|c|c|c|c|c|c|}
\hline \multicolumn{2}{|c|}{ Site ID } & \begin{tabular}{|c|}
$\begin{array}{c}\text { Calcium } \\
\text { meq/L. }\end{array}$ \\
1.79 \\
2.82
\end{tabular} & $\begin{array}{c}\begin{array}{c}\text { Magnesium } \\
\text { meq/L }\end{array} \\
7.33 \\
11.40\end{array}$ & \begin{tabular}{|c}
$\begin{array}{c}\text { Sodium } \\
\text { meq/L }\end{array}$ \\
1.65 \\
1.53
\end{tabular} & $\begin{array}{c}\begin{array}{c}\text { Potassium } \\
\text { meq/L }\end{array} \\
0.03 \\
0.03\end{array}$ & \begin{tabular}{|c|}
$\begin{array}{c}\text { Carbonate } \\
\text { meq/L }\end{array}$ \\
8.25 \\
14.12 \\
\end{tabular} & $\begin{array}{c}\begin{array}{c}\text { Sulfate } \\
\text { meq/L }\end{array} \\
0.79 \\
0.76\end{array}$ & $\begin{array}{c}\begin{array}{c}\text { Chloride } \\
\text { meq/L }\end{array} \\
0.94 \\
0.31\end{array}$ & $\begin{array}{c}\text { Nitrate } \\
\text { meq/L }\end{array}$ & \begin{tabular}{|c}
$\begin{array}{c}\text { Phosphate } \\
\text { meq/L }\end{array}$ \\
0.05 \\
0.06
\end{tabular} & \begin{tabular}{|c|}
$\begin{array}{c}\text { Sum } \\
\text { Cations }\end{array}$ \\
10.80 \\
15.78
\end{tabular} & $\begin{array}{c}\begin{array}{c}\text { Sum } \\
\text { Anions }\end{array} \\
10.86 \\
16.31\end{array}$ & $\begin{array}{l}\text { Relative \% } \\
\text { Difference }\end{array}$ \\
\hline $\begin{array}{l}\text { Upper } \\
\text { HSU } \\
(H S U-1)\end{array}$ & $\begin{array}{l}\text { UCD1-18 } \\
\text { UCD1-01 } \\
\text { UCD1-04 } \\
\text { UCD1-10 } \\
\text { UCD 1-11 } \\
\text { UCD1-12 } \\
\text { UCD1-13 } \\
\text { UCD1-19 } \\
\text { UCD1-20 } \\
\text { UCD1-21 } \\
\text { UCD1-22 } \\
\text { UCD1-23 } \\
\text { UCD1-24 }\end{array}$ & $\begin{array}{l}1.79 \\
2.82 \\
2.56 \\
2.27 \\
2.07 \\
3.89 \\
3.45 \\
2.44 \\
1.65 \\
2.51 \\
1.59 \\
2.00 \\
2.30\end{array}$ & $\begin{array}{c}7.33 \\
11.40 \\
8.34 \\
13.21 \\
9.01 \\
21.25 \\
14.86 \\
11.19 \\
7.04 \\
11.18 \\
5.46 \\
8.00 \\
9.81\end{array}$ & $\begin{array}{l}1.65 \\
1.53 \\
1.58 \\
6.95 \\
3.16 \\
4.13 \\
2.46 \\
2.90 \\
1.88 \\
4.02 \\
3.61 \\
1.79 \\
3.14\end{array}$ & $\begin{array}{l}0.03 \\
0.03 \\
0.03 \\
0.04 \\
0.03 \\
0.10 \\
0.08 \\
0.04 \\
0.04 \\
0.04 \\
0.04 \\
0.03 \\
0.03\end{array}$ & \begin{tabular}{|c|}
8.25 \\
14.12 \\
9.92 \\
13.58 \\
10.12 \\
12.87 \\
14.89 \\
14.41 \\
8.66 \\
12.25 \\
8.66 \\
9.82 \\
10.40 \\
\end{tabular} & $\begin{array}{l}0.79 \\
0.76 \\
0.85 \\
7.69 \\
1.18 \\
1.87 \\
1.96 \\
1.35 \\
0.85 \\
1.40 \\
0.74 \\
0.64 \\
1.10\end{array}$ & $\begin{array}{l}0.94 \\
0.31 \\
1.14 \\
1.07 \\
1.69 \\
6.76 \\
1.06 \\
0.48 \\
0.23 \\
0.58 \\
1.00 \\
0.86 \\
0.73\end{array}$ & $\begin{array}{l}0.83 \\
1.05 \\
0.32 \\
2.07 \\
1.75 \\
4.59 \\
1.18 \\
0.93 \\
1.29 \\
3.31 \\
0.67 \\
0.50 \\
3.53\end{array}$ & $\begin{array}{l}0.05 \\
0.06 \\
0.07 \\
0.06 \\
0.07 \\
0.06 \\
0.06 \\
0.06 \\
0.06 \\
0.06 \\
0.05 \\
0.06 \\
0.06\end{array}$ & \begin{tabular}{|l}
10.80 \\
15.78 \\
12.51 \\
22.46 \\
14.28 \\
29.37 \\
20.84 \\
16.56 \\
10.61 \\
17.76 \\
10.70 \\
11.81 \\
15.30 \\
\end{tabular} & $\begin{array}{l}10.86 \\
16.31 \\
12.29 \\
24.47 \\
14.80 \\
26.14 \\
19.16 \\
17.23 \\
11.08 \\
17.60 \\
11.12 \\
11.88 \\
15.81 \\
\end{array}$ & $\begin{array}{c}-0.5 \\
-3.3 \\
1.7 \\
-8.6 \\
-3.6 \\
11.6 \\
8.4 \\
-4.0 \\
-4.4 \\
0.8 \\
-3.8 \\
-0.6 \\
-3.3 \\
\end{array}$ \\
\hline $\begin{array}{l}\text { Lower } \\
\text { HSU } \\
(\text { HSU-2) }\end{array}$ & $\begin{array}{l}\text { UCD2-17 } \\
\text { UCD2-07 } \\
\text { UCD2-14 } \\
\text { UCD2-15 } \\
\text { UCD2-16 }\end{array}$ & $\begin{array}{l}1.64 \\
1.82 \\
2.35 \\
1.79 \\
1.69\end{array}$ & $\begin{array}{l}4.96 \\
6.83 \\
9.22 \\
5.16 \\
5.18\end{array}$ & $\begin{array}{l}1.17 \\
2.09 \\
1.89 \\
1.25 \\
1.81\end{array}$ & $\begin{array}{l}0.02 \\
0.03 \\
0.02 \\
0.02 \\
0.03\end{array}$ & $\begin{array}{c}6.71 \\
8.08 \\
10.70 \\
6.64 \\
7.15\end{array}$ & $\begin{array}{l}0.74 \\
0.84 \\
1.34 \\
0.72 \\
0.90\end{array}$ & $\begin{array}{l}0.50 \\
0.66 \\
0.98 \\
0.57 \\
0.52\end{array}$ & $\begin{array}{l}0.15 \\
1.34 \\
0.54 \\
0.19 \\
0.25\end{array}$ & $\begin{array}{l}0.05 \\
0.05 \\
0.06 \\
0.05 \\
0.05\end{array}$ & $\begin{array}{c}7.79 \\
10.76 \\
13.49 \\
8.22 \\
8.70\end{array}$ & $\begin{array}{c}8.14 \\
10.96 \\
13.63 \\
8.17 \\
8.87\end{array}$ & $\begin{array}{r}-4.5 \\
-1.9 \\
-1.0 \\
0.7 \\
-1.9\end{array}$ \\
\hline $\begin{array}{ll}\text { Notes: } \\
\text { 1. } & \mathrm{Eac}^{2} \\
& \mathrm{Ca}^{2} \\
& \mathrm{Mg}^{2} \\
& \mathrm{Na}^{+} \\
\mathrm{K}^{+} \\
\mathrm{Alk}^{-} \\
\mathrm{SO}^{\circ} \\
\mathrm{Cl}^{-} \\
\mathrm{NO}_{3} \\
\mathrm{PO}_{4}\end{array}$ & $\begin{array}{l}\text { hentry cons } \\
+ \text { (mg/L)/20 } \\
{ }^{2}+(\mathrm{mg} / \mathrm{L}) / 12 \\
(\mathrm{mg} / \mathrm{L}) / 22 . \\
(\mathrm{mg} / \mathrm{L}) / 39.1 \\
\text { alinity }(\mathrm{mg} C \\
2 \mathrm{mg} / \mathrm{L}) / 48 \\
(\mathrm{mg} / \mathrm{L}) / 35.4 \\
(\mathrm{mg} \mathrm{N} / \mathrm{L}) / 1 \\
3(\mathrm{mg} \mathrm{P/L}) / 1\end{array}$ & $\begin{array}{l}04=\mathrm{Ca}( \\
156=\mathrm{M} \\
9=\mathrm{Na}(\mathrm{r} \\
2=\mathrm{K}(\mathrm{m} \\
\left.\mathrm{aCO}_{3} / \mathrm{L}\right) / 5 \mathrm{C} \\
0308=\mathrm{S} \\
3=\mathrm{Cl}^{\circ}(\mathrm{n} \\
.007=\mathrm{N} \\
0.325=\mathrm{P}\end{array}$ & $\begin{array}{l}\text { equivalent co } \\
\text { meq/L). } \\
\text { (meq/L). } \\
\text { neq/L). } \\
\text { eq/L). } \\
.044=\mathrm{CO}_{3}^{-2} \\
O_{4}^{-2} \text { (meq/L). } \\
\text { leq/L). } \\
O_{3}^{\prime}(\mathrm{meq} / \mathrm{L}) \text {. } \\
O_{4}^{-3} \text { (meq/L). }\end{array}$ & neq/L). & of yalues & esented in & to 6 & accordin & & & & & \\
\hline
\end{tabular}




\subsection{Surface Water and Storm Water Analytical Results}

This section summarizes the analytical results from the 1995 surface water and storm water monitoring programs. Surface water samples were collected quarterly from three locations along Putah Creek: upstream of the site (PCU), downstream of the site (PCD), and from the UC Davis wastewater treatment plant outfall (STPO) (Figure 2.4). During the 1995 water monitoring program, grab samples of storm water were collected once from two onsite locations (SWL-1 and SWL-2) during March (Figure 2.5).

Surface water and storm water data are presented in a series of tables and figures similar to those for groundwater analytical results in Section 5. Tables 6.2 through 6.8 present constituents that were detected in surface water and storm water during the 1995 monitoring program, with results presented by location and quarter. Appendix $B$ presents data tables that include the entire 1995 surface water and storm water results, including detections and nondetections.

Surface water data have also been presented in a series of figures that include bar graphs of selected parameters in Figures 6.1 through 6.5. Stiff diagrams are shown in Figure 6.6. Appendix $C$ presents time series tables for parameters detected in surface water. These tables include data from fall 1990 through fall 1995 . No figures are presented for storm water results, because samples were only collected during one storm event in 1995.

Sections 6.2 through 6.6 discuss the 1995 surface water and storm water results according to analytical group. Each discussion presents the location and magnitude of detections for the analytical group, a comparison with regulatory maximum contamination levels (MCLs), and a review of overall trends.

\subsection{Volatile Organic Compounds}

A summary of VOCs detected in 1995 surface water and storm water samples is presented in Table 6.2. The 1995 quarterly surface water and storm water samples were analyzed for the target compounds identified in Tables 2.4 and 2.5. Seven VOCs were reported in surface water samples collected during 1995: acetone, bromodichloromethane, bromoform, chloroform, dibromochloromethane, methylene chloride, and toluene. Acetone was the most frequently reported compound in samples from PCU (two of four quarters), PCD (two of four quarters), STPO (three of four quarters), and storm water (two of two samples). While acetone was detected at low concentrations at all of the surface water locations (maximum value of $8.8 \mu \mathrm{g} / \mathrm{L}$ ), most of the analyses received data qualifiers to indicate that blank sample analyses were also reported to contain acetone (maximum value of $5.0 \mu \mathrm{g} / \mathrm{L}$ ). Therefore, the acetone appears to be laboratory contamination. Acetone is a commonly reported laboratory contaminant, as is methylene chloride. One or more of the trihalomethanes, chloroform, bromodichloromethane, dibromochloromethane, and 
bromoform were detected mainly in STPO (all four quarters) and in PCD (three of four quarters). The use of chlorine disinfectants in the UC Davis wastewater treatment plant is the apparent source of these constituents. According to UC Davis, effluent from the waste water goes through a dechlorination process such that the effluent meets the $100 \mu \mathrm{g} / \mathrm{L}$ of residual chlorine requirement. The trihalomethane concentrations detected are well below

the $100 \mu \mathrm{g} / \mathrm{L} \mathrm{MCL}$. Reported detections of methylene chloride at STPO exceed the MCL of $5 \mu \mathrm{g} / \mathrm{L}$ for two of four quarters. While methylene chloride is a common laboratory contaminant and has been found in blank samples, it is generally detected in STPO samples and never in PCU samples. Therefore, the methylene chloride appears to be an actual constituent of STPO. No other VOCs exceed an MCL in 1995 for surface water or storm water samples.

\subsection{Semivolatile Organic Compounds}

A summary of SVOCs detected in 1995 surface water and storm water samples is presented in Table 6.3. The 1995 quarterly surface water and storm water samples were analyzed for the target compound list identified in Tables 2.4 and 2.5. The only SVOCs detected during the 1995 sampling were four phthalate compounds that were detected equally in all samples. The random detection in all sample locations and the facts that many of the sample analyses were qualified with blank contamination and that phthalates are common laboratory contaminants indicate that these constituents are not naturally present in the surface water and storm water samples.

\subsection{Pesticides and PCBs}

A summary of pesticides and PCBs detected in 1995 surface water and storm water samples is presented in Table 6.4. Each 1995 quarterly surface water and storm water sample was analyzed for the target compound list identified in Tables 2.4 and 2.5. Two pesticide compounds, alpha- and gamma-chlordane, were the only reported compounds above detection limits in 1995 surface water and storm water samples. The compounds were detected only in a sample from SWL- 1 at 0.013 and $0.015 \mu \mathrm{g} / \mathrm{L}$. Because these compounds are isomers of chlordane, the MCL for chlordane, $2 \mu \mathrm{g} / \mathrm{L}$, applies to the sum of the two concentrations. The MCL is not exceeded. No PCB compounds were detected in 1995 surface water and storm water samples.

\subsection{Metals}

A summary of metals reported in 1995 surface water samples is presented in Table 6.5. Each quarterly surface water sample was analyzed for 16 metals (Tables 2.4 and 2.5) and hexavalent chromium. Beryllium, cadmium, hexavalent chromium, mercury, silver, and thallium were not reported in any 1995 surface water samples. Of the metals detected in 1995, three, antimony, lead, and selenium, exceeded their respective MCLs. Antimony was detected in both storm water sample locations at concentrations above its MCL of $6 \mu \mathrm{g} / \mathrm{L}$. Lead was reported only in storm water samples SWL-1 and SWL-2 and 
was reported above its MCL of $15 \mu \mathrm{g} / \mathrm{L}$ at SWL-2, as was the case in 1994. Selenium was detected only in STPO, and the fall sample exceeded the MCL of $5 \mu \mathrm{g} / \mathrm{L}$. The metals group is discussed in more detail below.

- Antimony was reported twice below the detection limit and twice above the detection limit. Reported detections were $4.7 \mu \mathrm{g} / \mathrm{L}$ in PCD, $1.2 \mu \mathrm{g} / \mathrm{L}$ in STPO, $74.8 \mu \mathrm{g} / \mathrm{L}$ in $\mathrm{SWL}-1$, and $156 \mu \mathrm{g} / \mathrm{L}$ in SWL-2. The storm water detections were reported above the $\mathrm{MCL}$ of $6 \mu \mathrm{g} / \mathrm{L}$, and their concentrations are consistent with levels reported in 1994 .

- Arsenic was reported above detection limits in samples from PCD, PCU, and STPO during 1995 but was not detected in the storm water sampling. Reported detections ranged in concentration from $2.5 \mu \mathrm{g} / \mathrm{L}$ (PCU) to $5.6 \mu \mathrm{g} / \mathrm{L}$ (STPO). No arsenic values were reported above the MCL of $50 \mu \mathrm{g} / \mathrm{L}$.

Arsenic has only been reported above detection limits since the summer quarter of 1993, when the analytical method was changed and the detection limit lowered to $2.0 \mu \mathrm{g} / \mathrm{L}$. In general, reported arsenic values occur at concentrations near the detection limit.

- Barium was detected in every 1995 surface water and storm water sample collected. Reported concentrations range from $3.3 \mu \mathrm{g} / \mathrm{L}$ (SWL-2) to $113 \mu \mathrm{g} / \mathrm{L}$ (PCU and PCD). No barium concentrations were reported above. the MCL of $1000 \mu \mathrm{g} / \mathrm{L}$.

Barium concentrations are typically highest in samples from PCU and lowest in STPO. Concentrations in samples from all three locations tend to be consistent over time:

- Lead was detected only in storm water samples during 1995. The sample collected at SWL-2 contained lead at $38.4 \mu \mathrm{g} / \mathrm{L}$, above the MCL of $15 \mu \mathrm{g} / \mathrm{L}$.

- Selenium was detected only twice in STPO samples. The highest value, $7.4 \mu \mathrm{g} / \mathrm{L}$, exceeded the MCL of $5 \mu \mathrm{g} / \mathrm{L}$. This is the only time the selenium MCL has been exceeded since the detection limit was lowered to $3 \mu \mathrm{g} / \mathrm{L}$ in the summer quarter of 1993.

- Vanadium was reported above the detection limit nine times in 1995 surface water samples from all locations except SWL-2. Surface water detections ranged in concentration from $1.0 \mu \mathrm{g} / \mathrm{L}$ (SWL-1) to $10.5 \mu \mathrm{g} / \mathrm{L}$ (STPO). There is no MCL for vanadium.

- Zinc was detected in every 1995 surface water and storm water sample collected. Reported zinc concentrations ranged from a low of $2.1 \mu \mathrm{g} / \mathrm{L}$ at PCD to a high of 94.1. at SWL-2. No samples exceeded the national secondary drinking water standard of $5,000 \mu \mathrm{g} / \mathrm{L}$. 


\subsection{Radionuclides}

A summary of analytical results for reported radionuclide constituents in surface water and storm water runoff samples is presented in Table 6.6. Complete analytical results for surface water and storm water runoff samples are presented in Appendix B. 'The presentation of radionuclide data for these analyses continues with the format used in Section 5.5.

There were no radionuclides reported in downstream surface water samples at concentrations exceeding upstream levels. There was no detectable concentration of radionuclides in storm water samples. Tritium was reported at both STPO and the downgradient PCD sample locations at less than the detection limit for all surface water and storm water samples in 1995. Americium-241 was detected at PCU and PCD at slightly above the MDA. These data reflect the variations in background counts and are not considered detectable concentrations.

All gross beta detections were reported less than the $\mathrm{MCL}(50 \mathrm{pCi} / \mathrm{L})$ with a maximum detection of $29.5 \pm 4.1 \mathrm{pCi} / \mathrm{L}$ (STPO, winter quarter 1995, duplicate sample). These data are at concentrations similar to those found in 1994. Gross alpha analyses were less than the detection limit for all samples collected in 1995.

\subsection{Anions and Cations}

This section presents a summary of analytical results reported for anions and cations in surface water and storm water. Analytical results for anions and cations are summarized in Table 6.7. Complete analytical results for anions and cations in surface water and storm water samples are presented in Appendix B, Table B.6. Results reported for the major anions and cations are described using summary tables and Stiff diagrams.

\subsubsection{Summary of Major Ion Chemistry}

The major ion chemistry for Putah Creek is presented in Table 6.9. For direct comparison purposes, data from Table 6.9 have been "normalized" to an equivalents basis in Table 6.10. In addition, this calculation must be performed to draw the Stiff diagrams that will be discussed in the next section.

The sums of cations and anions and their RPDs are shown in Table 6.10. Normally, this comparison is done on individual sample analyses, not on mean values, but it is done here to summarize the data. A negative charge balance (relative percent difference) indicates an excess of negatively charged anions, and a positive charge balance indicates an excess of positively charged cations. 
A number of observations can be made from the data presented in Tables 6.9 and 6.10:

- Putah Creek waters are classified as magnesium/sodium-carbonate-dominated waters.

- The outfall from the UC Davis wastewater treatment plant contains significantly more sodium and chloride than Putah Creek.

- The increased sodium and chloride content of STPO results in an overall higher total dissolved solids content in STPO than in Putah Creek.

\subsubsection{Stiff Diagrams}

Stiff diagrams were prepared for surface water sampling locations using the data in Table 6.9 to evaluate and compare the general water types present at these sampling locations. These stiff diagrams are presented in Figures 6.6 and 5.23.

As presented in the 1994 annual report and in Section 6.6.1, samples from STPO have greater reported concentrations of sodium and chloride than samples from PCU and PCD. The STPO input is reflected in higher concentrations of sodium and chloride in downstream (PCD) samples than upstream (PCU) samples.

\subsubsection{Nitrate}

Nitrate concentrations reported for surface water samples during 1995 are presented in Table 6.7 and Figure 6.4. Nitrate concentrations in surface water samples did not exceed the MCL of $10 \mathrm{mg} / \mathrm{L}$ (for nitrate as nitrogen) in any of the 1995 samples. Reported concentrations of nitrate in STPO were higher than in the upstream and downstream samples with the exception of the summer quarter 1995, when they were similar to concentrations in PCU and PCD (Figure 6.4). This is the same trend noted in 1994. As shown in the time series data presented in Appendix $C$, concentrations of nitrate in surface water samples are variable over time but appear to stay within the same general ranges. 


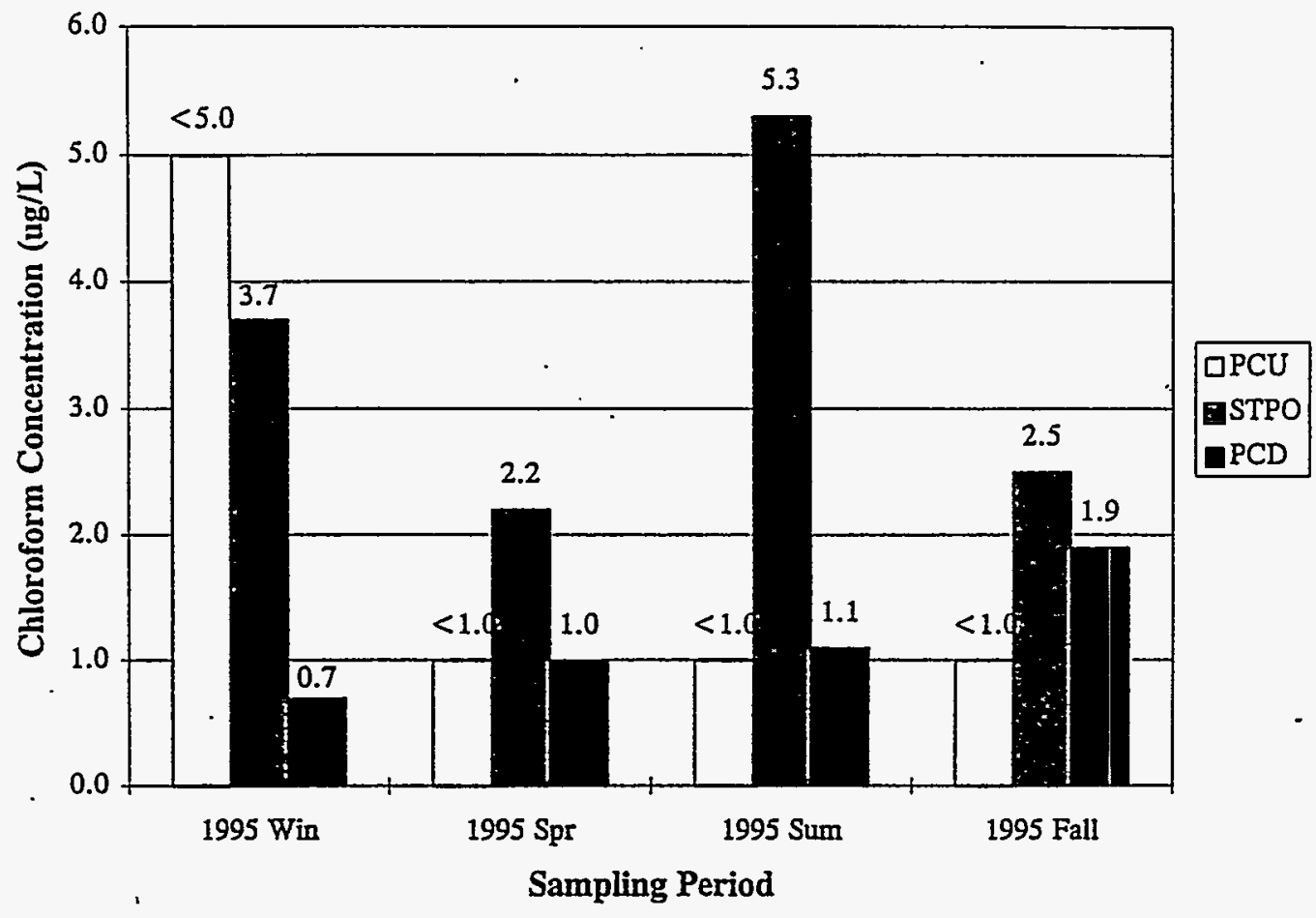

Figure 6.1. Chloroform Concentrations in Surface Water Samples, 1995

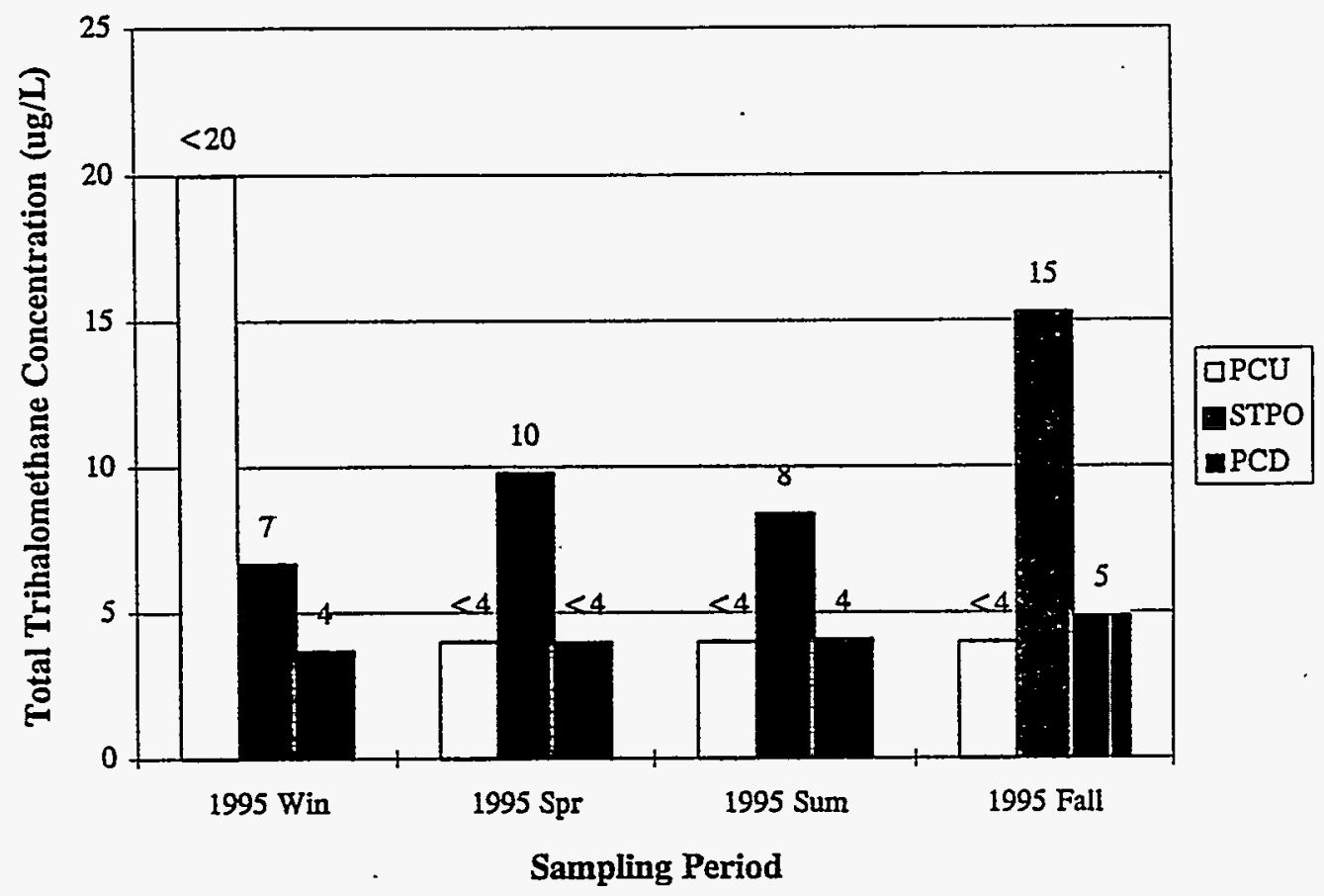

Figure 6.2. Total Trihalomethane Concentrations in Surface Water Samples, 1995 


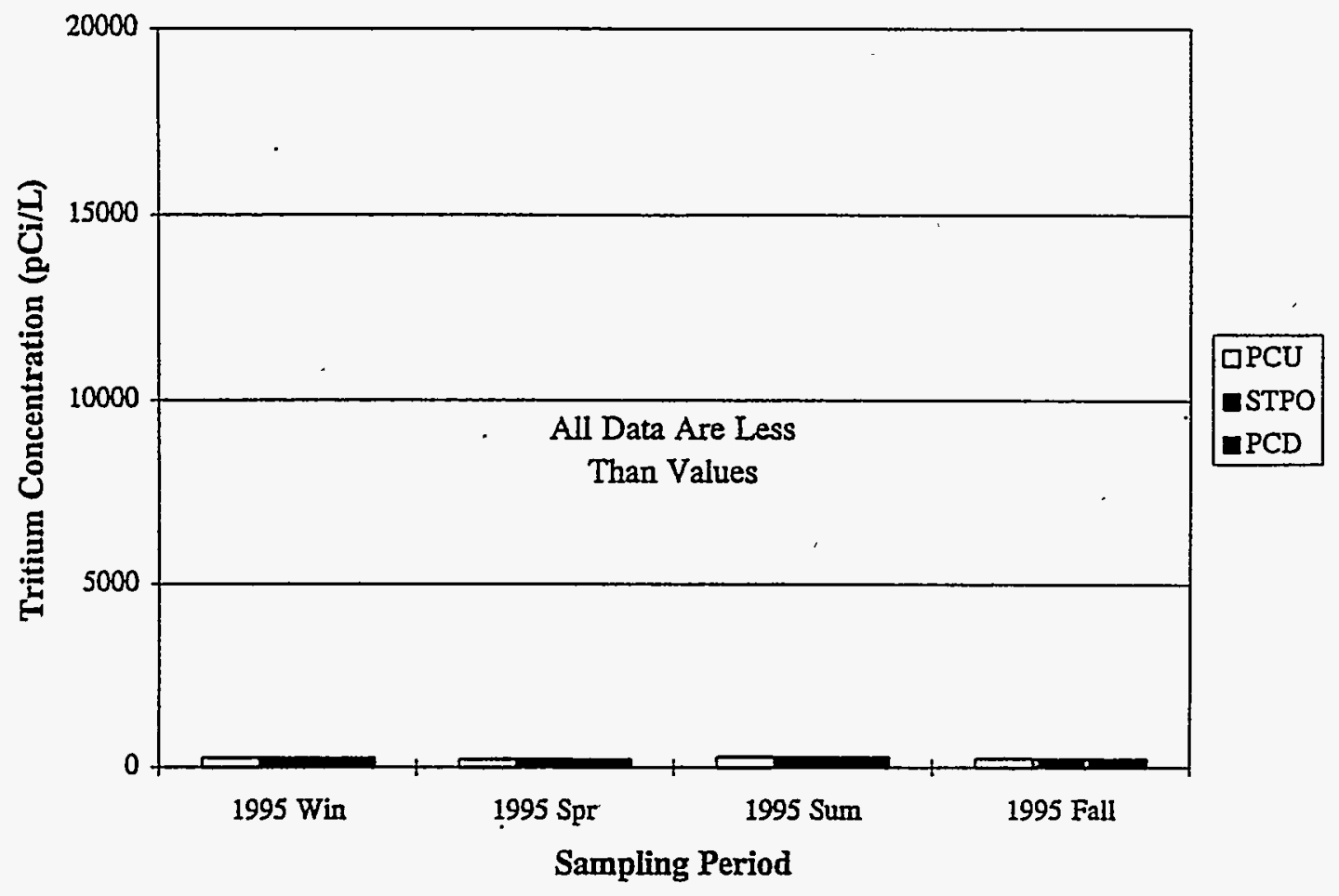

Figure 6.3. Tritium Concentrations in Surface Water Samples, 1995

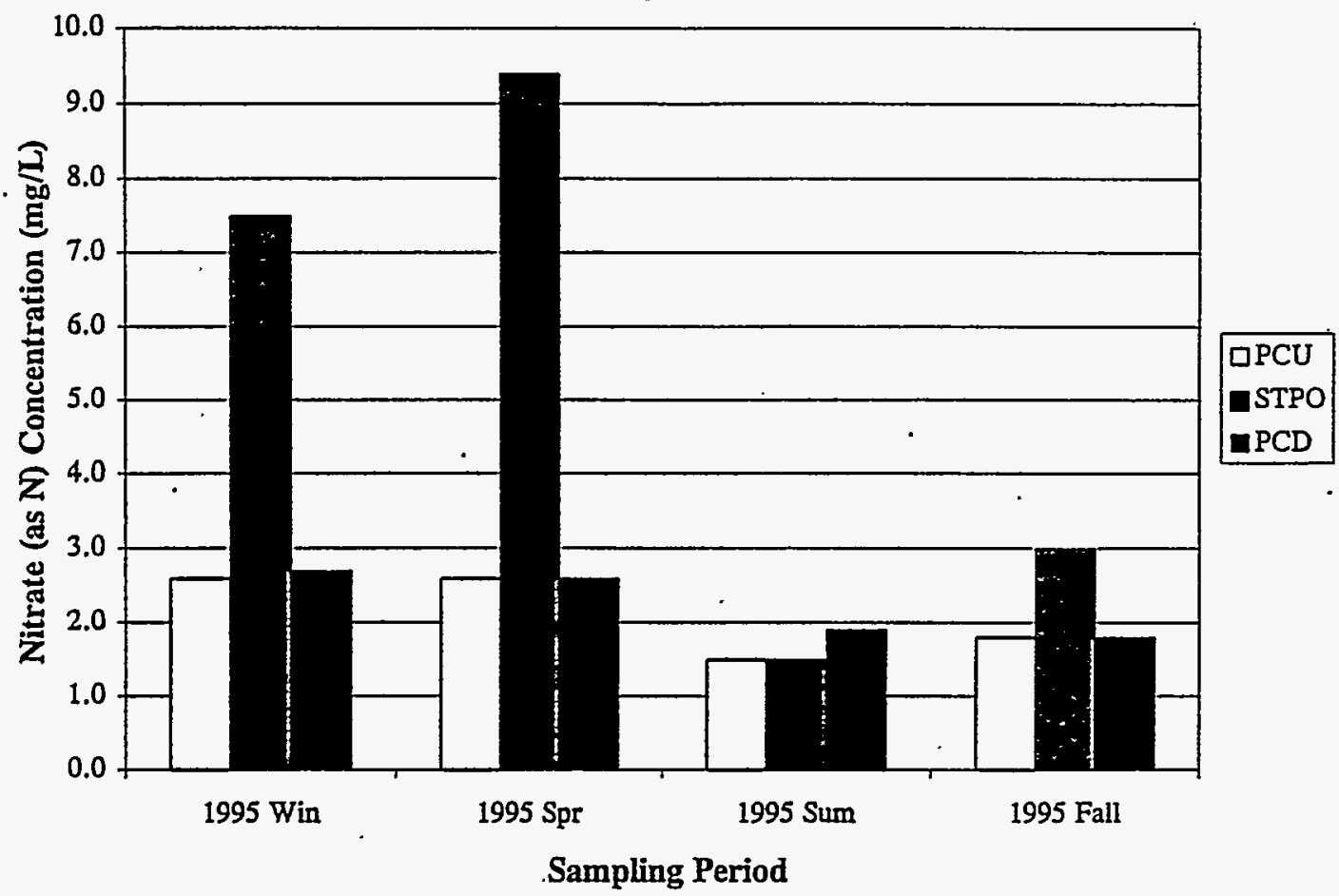

Figure 6.4. Nitrate Concentrations in Surface Water Samples, 1995 


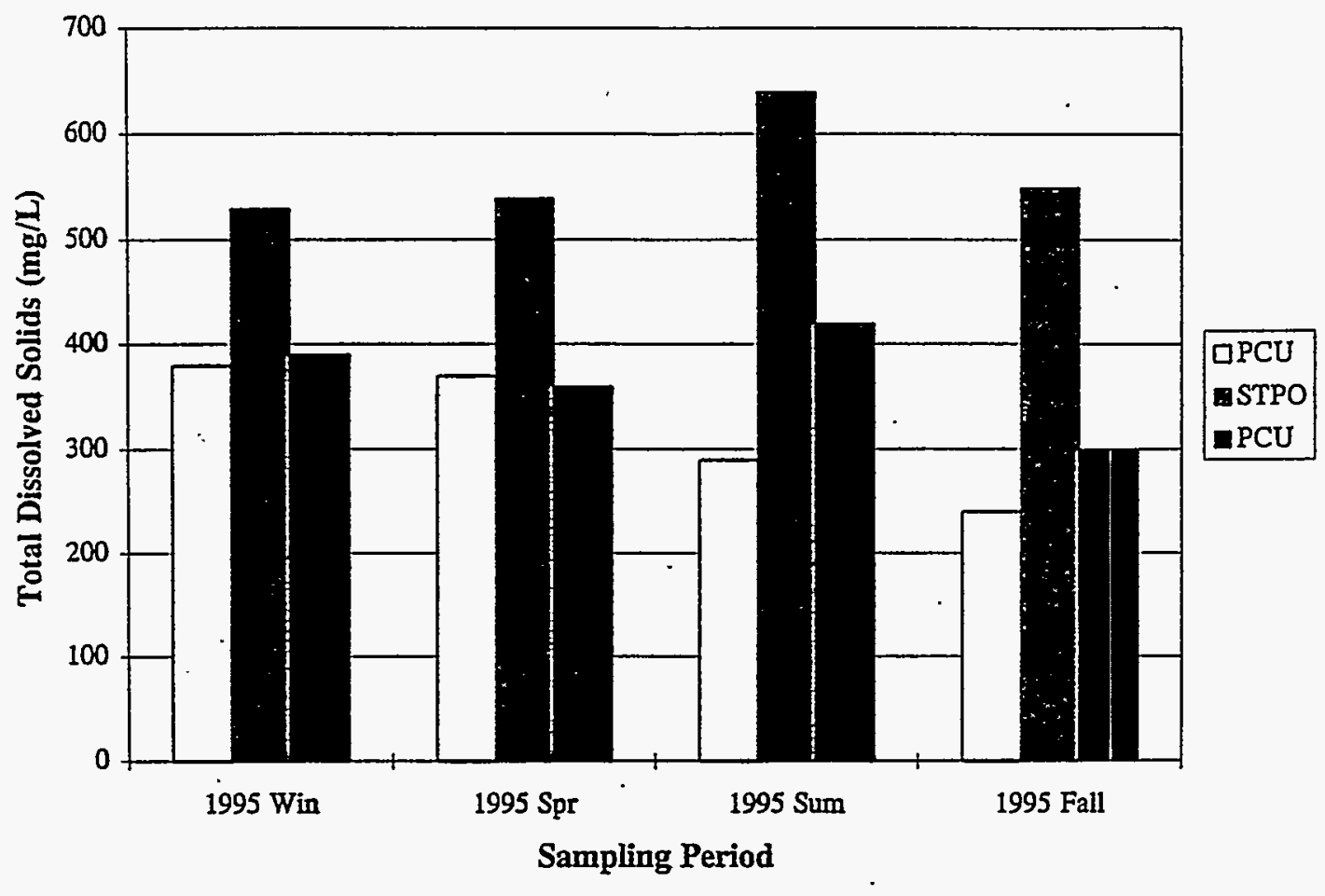

Figure 6.5. Total Dissolved Solids in Surface Water Samples, 1995 


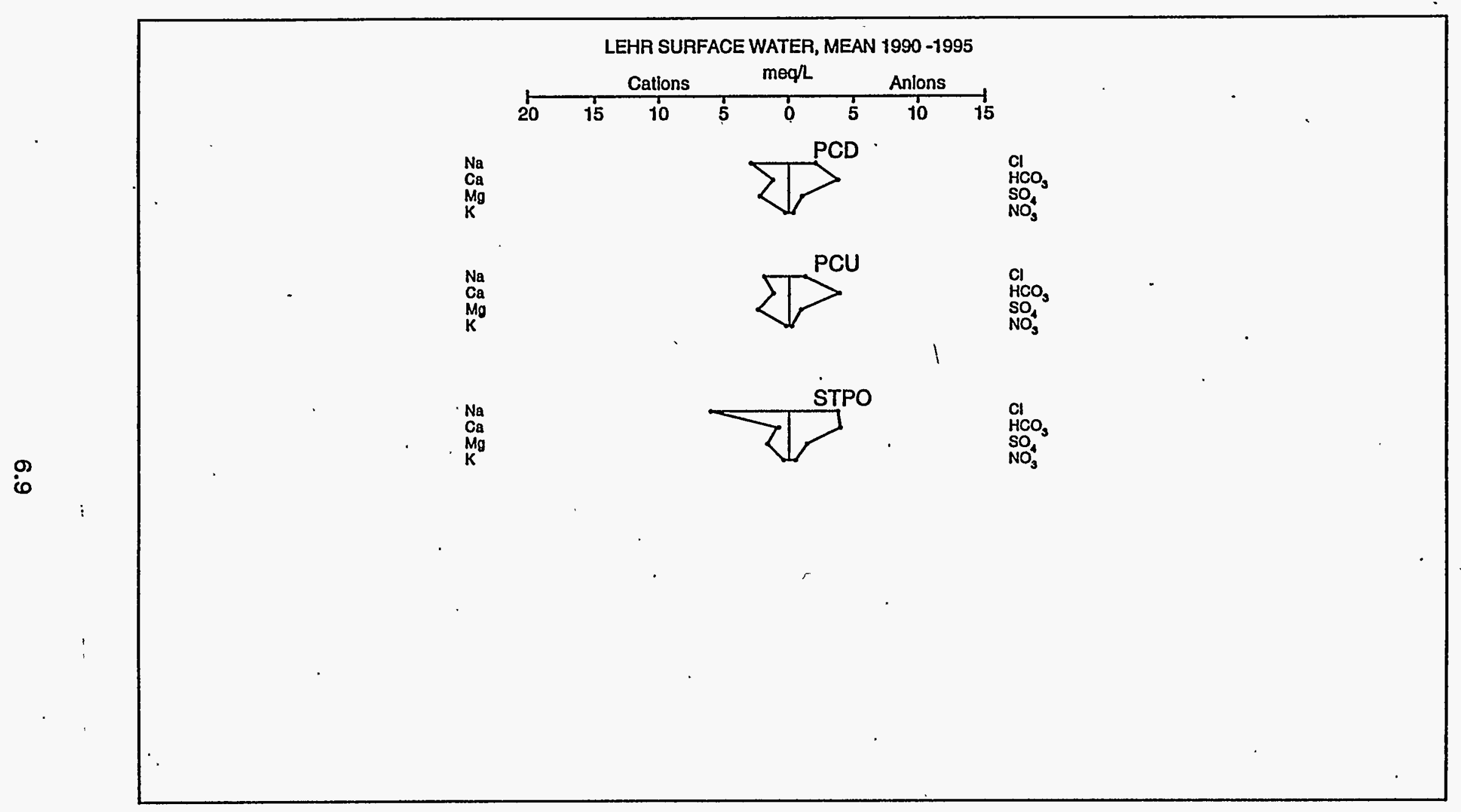

Figure 6.6. Surface Water Monitoring Locations, Mean 1990-1995 
TABLE 6.1

NUMBER OF DETECTIONS, POSITIVE RESULTS AND MAXIMUM VALUES IN SURFACE WATER, 1995

1995 ANNUAL WATER MONITORING REPORT

LEHR ENVIRONMENTAL RESTORATION, DAVIS, CALIFORNIA

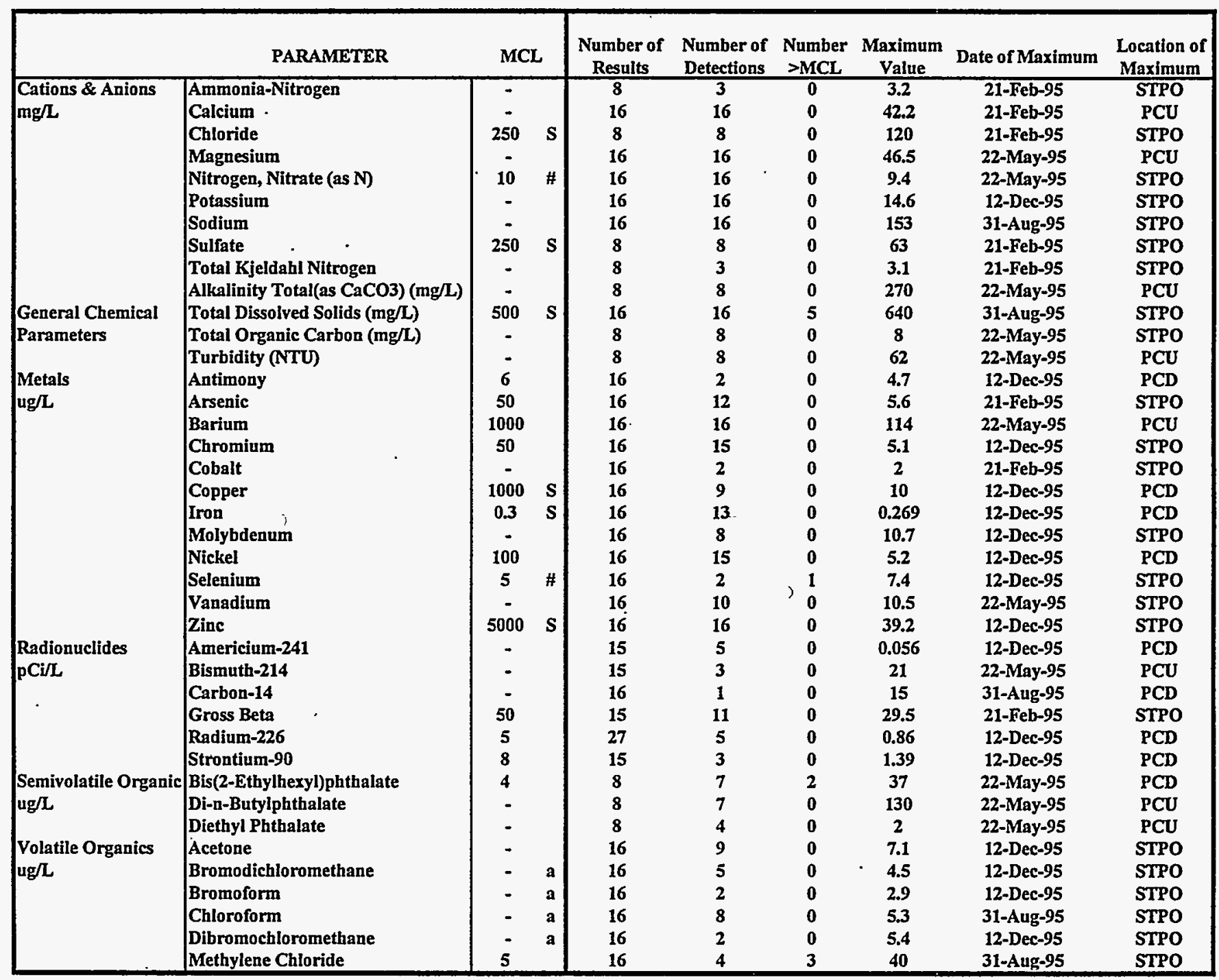

- = Not Applicable

$\mathrm{MCL}=$ Maximum Contamination Level (primary), Title 22, Division 4, Chapter 15.

$a=$ MCL for total trihalomethanes is $100 \mathrm{ug} / \mathrm{L}$. Chloroform is the predominant trihalomethane detected at LEHR.

\# = USEPA MCL.

$s=$ Secondary Drinking Water Standard

- = Proposed MCL for Tritium is $60,000 \mathrm{pCi} / \mathrm{L}$. 
TABLE 6.2

SUMMARY OF DETECTED CONSTITUENTS IN SURFACE WATER AND STORM WATER, 1995

VOLATILE ORGANICS

1995 ANNUAL WATER MONITORING REPORT

LEHR ENVIRONMENTAL RESTORATION, DAVIS, CALIFORNIA

\begin{tabular}{|c|c|c|c|c|c|c|c|c|c|c|c|c|}
\hline \multirow{2}{*}{\begin{tabular}{|l} 
Parameter \\
$1,1,2-$ Trichloroethane
\end{tabular}} & \multirow{2}{*}{\begin{tabular}{|l} 
Quarter \\
WINTER \\
SPRING \\
SUMMER \\
FALL \\
\end{tabular}} & \multirow[b]{2}{*}{$\begin{array}{l}\text { Units } \\
\mathrm{ug} / \mathrm{L} \\
\mathrm{ug} / \mathrm{L} \\
\mathrm{ug} / \mathrm{L} \\
\mathrm{ug} / \mathrm{L}\end{array}$} & \multirow[b]{2}{*}{$\begin{array}{c}\text { DL } \\
1 \\
1 \\
1 \\
1 \\
\end{array}$} & MCL & \multicolumn{2}{|c|}{ PCD } & \multicolumn{2}{|c|}{$\begin{array}{c}\text { PCD } \\
\text { D1 }\end{array}$} & \multicolumn{2}{|c|}{ PCU } & \multicolumn{2}{|c|}{$\begin{array}{c}\text { PCU } \\
\text { D1 }\end{array}$} \\
\hline & & & & $\begin{array}{ll}\mathbf{5} & \# \\
\mathbf{5} & \# \\
\mathbf{5} & \# \\
\mathbf{5} & \# \\
\end{array}$ & $\begin{array}{l}<1 \\
<1 \\
<1 \\
<1\end{array}$ & $\begin{array}{l}\text { IU } \\
\text { IU } \\
\text { IU } \\
\text { IU }\end{array}$ & $\begin{array}{r}- \\
- \\
<1 \\
\end{array}$ & $\mathbf{I U}$ & $\begin{array}{l}<1 \\
<1 \\
<1 \\
<1\end{array}$ & $\begin{array}{l}\mathbf{I U} \\
\mathbf{I U} \\
\mathbf{I U} \\
\mathbf{I U}\end{array}$ & $\begin{array}{r}- \\
<1 \\
<1 \\
-\end{array}$ & $\begin{array}{l}\text { IU } \\
\text { IU }\end{array}$ \\
\hline 1,1-Dichloroethane & $\begin{array}{l}\text { WINTER } \\
\text { SPRING } \\
\text { SUMMER } \\
\text { FALL } \\
\end{array}$ & $\begin{array}{l}\mathrm{ug} / \mathrm{L} \\
\mathrm{ug} / \mathrm{L} \\
\mathrm{ug} / \mathrm{L} \\
\mathrm{ug} / \mathrm{L}\end{array}$ & $\begin{array}{l}1 \\
1 \\
1 \\
1 \\
\end{array}$ & $\begin{array}{l}5 \\
5 \\
5 \\
5 \\
\end{array}$ & $\begin{array}{l}<1 \\
<1 \\
<1 \\
<1\end{array}$ & $\begin{array}{l}\text { IU } \\
\text { IU } \\
\text { IU } \\
\text { IU }\end{array}$ & $\begin{array}{r}- \\
- \\
\end{array}$ & $\mathbf{I U}$ & $\begin{array}{l}<1 \\
<1 \\
<1 \\
<1\end{array}$ & $\begin{array}{l}\text { IU } \\
\text { IU } \\
\text { IU } \\
\text { IU }\end{array}$ & $\begin{array}{r}- \\
<1 \\
<1 \\
- \\
\end{array}$ & $\begin{array}{l}\text { IU } \\
\text { IU }\end{array}$ \\
\hline 1,1-Dichloroethene & \begin{tabular}{|l|} 
WINTER \\
SPRING \\
SUMMER \\
FALL \\
\end{tabular} & $\begin{array}{l}\mathrm{ug} / \mathrm{L} \\
\mathrm{ug} / \mathrm{L} \\
\mathrm{ug} / \mathrm{L} \\
\mathrm{ug} / \mathrm{L}\end{array}$ & $\begin{array}{l}1 \\
1 \\
1 \\
1\end{array}$ & $\begin{array}{l}6 \\
6 \\
6 \\
6 \\
\end{array}$ & $\begin{array}{l}<1 \\
<1 \\
<1 \\
<1\end{array}$ & $\begin{array}{l}\text { IU } \\
\text { IU } \\
\text { IU } \\
\text { IU }\end{array}$ & $\begin{array}{r}- \\
- \\
<1\end{array}$ & $\mathbf{I U}$ & $\begin{array}{l}<1 \\
<1 \\
<1 \\
<1\end{array}$ & $\begin{array}{l}\mathbf{I U} \\
\mathbf{I U} \\
\mathbf{I U} \\
\mathbf{I U}\end{array}$ & $\begin{array}{r}- \\
<1 \\
<1 \\
\end{array}$ & $\begin{array}{l}\mathbf{I U} \\
\mathbf{I U}\end{array}$ \\
\hline 1,2-Dichlorocthane & $\begin{array}{l}\text { WINTER } \\
\text { SPRING } \\
\text { SUMMER } \\
\text { FALL } \\
\end{array}$ & $\begin{array}{l}\mathrm{ug} / \mathrm{L} \\
\mathrm{ug} / \mathrm{L} \\
\mathrm{ug} / \mathrm{L} \\
\mathrm{ug} / \mathrm{L}\end{array}$ & $\begin{array}{l}1 \\
1 \\
1 \\
1\end{array}$ & $\begin{array}{l}0.5 \\
0.5 \\
0.5 \\
0.5\end{array}$ & $\begin{array}{l}<1 \\
<1 \\
<1 \\
<1\end{array}$ & $\begin{array}{l}\text { IU } \\
\text { IU } \\
\text { IU } \\
\mathbf{I U}\end{array}$ & $\begin{array}{r}-\dot{-} \\
<1\end{array}$ & IU & $\begin{array}{l}<1 \\
<1 \\
<1 \\
<1\end{array}$ & $\begin{array}{l}\mathbf{I U} \\
\mathbf{I U} \\
\mathbf{I U} \\
\mathbf{I U}\end{array}$ & $\begin{array}{r}- \\
<1 \\
<1 \\
-\end{array}$ & $\begin{array}{l}\text { IU } \\
\text { IU }\end{array}$ \\
\hline 1,2-Dichloropropane & $\begin{array}{l}\text { WINTER } \\
\text { SPRING } \\
\text { SUMMER } \\
\text { FALL } \\
\end{array}$ & $\begin{array}{l}u g / L \\
u g / L \\
u g / L \\
u g / L\end{array}$ & $\begin{array}{l}1 \\
1 \\
1 \\
1\end{array}$ & $\begin{array}{l}5 \\
5 \\
5 \\
5\end{array}$ & $\begin{array}{l}<1 \\
<1 \\
<1 \\
<1\end{array}$ & $\begin{array}{l}\text { IU } \\
\text { IU } \\
\mathbf{I U} \\
\mathbf{I U}\end{array}$ & $\begin{array}{r}- \\
- \\
<1\end{array}$ & $\mathbf{I U}$ & $\begin{array}{l}<1 \\
<1 \\
<1 \\
<1\end{array}$ & $\begin{array}{l}\mathbf{I U} \\
\mathbf{I U} \\
\mathbf{I U} \\
\mathbf{I U}\end{array}$ & $\begin{array}{r}- \\
<1 \\
<1 \\
-\end{array}$ & $\begin{array}{l}\text { IU } \\
\text { IU }\end{array}$ \\
\hline 2-Butanone & \begin{tabular}{|l|} 
WINTER \\
SPRING \\
SUMMER \\
FALL \\
\end{tabular} & $\begin{array}{l}\mathrm{ug} / \mathrm{L} \\
\mathrm{ug} / \mathrm{L} \\
\mathrm{ug} / \mathrm{L} \\
\mathrm{ug} / \mathrm{L}\end{array}$ & $\begin{array}{l}5 \\
5 \\
5 \\
5\end{array}$ & $\begin{array}{l}\ddot{-} \\
.\end{array}$ & $\begin{array}{l}<5 \\
<5 \\
<5 \\
<5\end{array}$ & $\begin{array}{l}\text { Re IU } \\
\text { Re IU } \\
\text { Re IU } \\
\text { Re IU }\end{array}$ & $\begin{array}{r}- \\
- \\
<5\end{array}$ & Re IU & $\begin{array}{l}<5 \\
<5 \\
<5 \\
<5\end{array}$ & $\begin{array}{l}\operatorname{Rc} \mid \mathbf{U} \\
\operatorname{Re} \mid \mathbf{U} \\
\operatorname{Re} \mid \mathbf{U} \\
\operatorname{Rc} \mid \mathbf{U}\end{array}$ & $\begin{array}{r}<5 \\
<5 \\
-\end{array}$ & $\begin{array}{l}\text { Re IU } \\
\operatorname{Re} \mid \mathbf{U}\end{array}$ \\
\hline 4-Methyl-2-Pentanone & $\begin{array}{l}\text { WINTER } \\
\text { SPRING } \\
\text { SUMMER } \\
\text { FALL } \\
\end{array}$ & $\begin{array}{l}\mathrm{ug} / \mathrm{L} \\
\mathrm{ug} / \mathrm{L} \\
\mathrm{ug} / \mathrm{L} \\
\mathrm{ug} / \mathrm{L}\end{array}$ & $\begin{array}{l}\mathbf{5} \\
\mathbf{5} \\
\mathbf{5} \\
\mathbf{5}\end{array}$ & $\begin{array}{l}. \\
. \\
.\end{array}$ & $\begin{array}{l}<5 \\
<5 \\
<5 \\
<5\end{array}$ & $\begin{array}{l}\mathbf{I U} \\
\mathbf{I U} \\
\mathbf{I U} \\
\mathbf{I U}\end{array}$ & $\begin{array}{r}- \\
- \\
<5\end{array}$ & IU & $\begin{array}{l}<5 \\
<5 \\
<5 \\
<5\end{array}$ & $\begin{array}{l}\mathbf{I U} \\
\mathbf{I U} \\
\mathbf{I U} \\
\mathbf{I U}\end{array}$ & $\begin{array}{r}- \\
<5 \\
<5 \\
-\end{array}$ & $\begin{array}{l}\text { IU } \\
\text { IU }\end{array}$ \\
\hline Acetone & $\begin{array}{l}\text { WINTER } \\
\text { SPRING } \\
\text { SUMMER } \\
\text { FALL } \\
\end{array}$ & $\begin{array}{l}\mathrm{ug} / \mathrm{L} \\
\mathrm{ug} / \mathrm{L} \\
\mathrm{ug} / \mathrm{L} \\
\mathrm{ug} / \mathrm{L}\end{array}$ & $\begin{array}{l}5 \\
5 \\
5 \\
5\end{array}$ & $\begin{array}{l}- \\
- \\
- \\
\end{array}$ & $\frac{<\frac{5}{2.2}}{\frac{<5}{3.1}}$ & 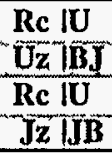 & $\begin{array}{c}- \\
- \\
- \\
2.8\end{array}$ & Jz TIB & $\frac{<\frac{5}{2.1}}{\frac{<5}{2.8}}$ & 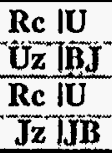 & $\begin{array}{c}-\frac{-}{2,2} \\
<5 \\
-\end{array}$ & $\frac{\mathrm{Uz} \backslash \mathrm{BJ}}{\mathrm{Rc} \mid \mathrm{U}}$ \\
\hline
\end{tabular}

Page 1 of 5 
TABLE 6.2

SUMMARY OF DETECTED CONSTITUENTS IN SURFACE WATER AND STORM WATER, 1995

VOLATILE ORGANICS

1995 ANNUAL WATER MONITORING REPORT

LEHR ENVIRONMENTAL RESTORATION, DAVIS, CALIFORNIA

\begin{tabular}{|c|c|c|c|c|c|c|c|c|c|c|c|c|}
\hline \multirow{2}{*}{\begin{tabular}{|l} 
Parameter \\
Bromodichloromethane \\
\end{tabular}} & \multirow{2}{*}{\begin{tabular}{|l|} 
Quarter \\
WINTER \\
SPRING \\
SUMMER \\
FALL \\
\end{tabular}} & \multirow[b]{2}{*}{\begin{tabular}{|l|} 
Units \\
ug/L \\
ug/L \\
ug/L \\
ug/L \\
\end{tabular}} & \multirow[b]{2}{*}{$\begin{array}{c}\text { DL } \\
1 \\
1 \\
1 \\
1 \\
\end{array}$} & MCL & \multicolumn{2}{|c|}{ PCD } & \multicolumn{2}{|c|}{$\begin{array}{c}\text { PCD } \\
\text { D1 }\end{array}$} & \multicolumn{2}{|c|}{ PCU } & \multicolumn{2}{|c|}{$\begin{array}{l}\text { PCU } \\
\text { D1 }\end{array}$} \\
\hline & & & & $\begin{array}{r}\mathbf{a} \\
\mathbf{a} \\
\mathbf{a} \\
\mathbf{a} \\
\end{array}$ & $\begin{array}{l}<1 \\
<1 \\
<1 \\
<1 \\
\end{array}$ & $\begin{array}{l}\text { IU } \\
\text { IU } \\
\text { IU } \\
\text { IU }\end{array}$ & $\begin{array}{r}: \\
<1 \\
\end{array}$ & IU & $\begin{array}{l}<1 \\
<1 \\
<1 \\
<1\end{array}$ & $\begin{array}{l}\text { IU } \\
\text { IU } \\
\text { IU } \\
\text { IU }\end{array}$ & $\begin{array}{r}< \\
<1 \\
<1 \\
\end{array}$ & $\begin{array}{l}\text { IU } \\
\text { IU }\end{array}$ \\
\hline Bromoform & $\begin{array}{l}\text { WINTER } \\
\text { SPRING } \\
\text { SUMMER } \\
\text { FALL } \\
\end{array}$ & \begin{tabular}{|l|}
$\mathrm{ug} / \mathrm{L}$ \\
$\mathrm{ug} / \mathrm{L}$ \\
$\mathrm{ug} / \mathrm{L}$ \\
$\mathrm{ug} / \mathrm{L}$ \\
\end{tabular} & $\begin{array}{l}1 \\
1 \\
1 \\
1 \\
\end{array}$ & $\begin{array}{l}\mathbf{a} \\
\mathbf{a} \\
\mathbf{a} \\
\mathbf{a}\end{array}$ & $\begin{array}{l}<1 \\
<1 \\
<1 \\
<1\end{array}$ & $\begin{array}{l}\text { IU } \\
\text { IU } \\
\text { IU } \\
\text { IU } \\
\end{array}$ & $\begin{array}{r}- \\
- \\
<1 \\
\end{array}$ & IU & $\begin{array}{l}<1 \\
<1 \\
<1 \\
<1\end{array}$ & $\begin{array}{l}\text { IU } \\
\text { IU } \\
\text { IU } \\
\text { IU }\end{array}$ & $\begin{array}{l}<i \\
<1 \\
\end{array}$ & $\begin{array}{l}\text { IU } \\
\text { IU }\end{array}$ \\
\hline Carbon Disulfide & $\begin{array}{l}\text { WINTER } \\
\text { SPRING } \\
\text { SUMMER } \\
\text { FALL } \\
\end{array}$ & \begin{tabular}{|l|}
$\mathrm{ug} / \mathrm{L}$ \\
$\mathrm{ug} / \mathrm{L}$ \\
$\mathrm{ug} / \mathrm{L}$ \\
$\mathrm{ug} / \mathrm{L}$ \\
\end{tabular} & $\begin{array}{l}1 \\
1 \\
1 \\
1 \\
\end{array}$ & $\begin{array}{l}- \\
- \\
-\end{array}$ & \begin{tabular}{|l|}
$<1$ \\
$<1$ \\
$<1$ \\
$<1$ \\
\end{tabular} & $\begin{array}{l}\mathrm{IU} \\
\mathrm{IU} \\
\mathrm{IU} \\
\mathrm{IU} \\
\end{array}$ & $\begin{array}{r}- \\
- \\
<1 \\
\end{array}$ & $\mathbf{I U}$ & $\begin{array}{l}<1 \\
<1 \\
<1 \\
<1\end{array}$ & $\begin{array}{l}\text { IU } \\
\text { IU } \\
\text { IU } \\
\text { IU } \\
\end{array}$ & $\begin{array}{r}- \\
<1 \\
<1 \\
\end{array}$ & $\begin{array}{l}\text { IU } \\
\text { IU }\end{array}$ \\
\hline Chloroform & $\begin{array}{l}\text { WINTER } \\
\text { SPRING } \\
\text { SUMMER } \\
\text { FALL } \\
\end{array}$ & \begin{tabular}{|l|}
$\mathrm{ug} / \mathrm{L}$ \\
$\mathrm{ug} / \mathrm{L}$ \\
$\mathrm{ug} / \mathrm{L}$ \\
$\mathrm{ug} / \mathrm{L}$ \\
\end{tabular} & $\begin{array}{l}1 \\
1 \\
1 \\
1 \\
\end{array}$ & $\begin{array}{l}\mathbf{a} \\
\mathbf{a} \\
\mathbf{a} \\
\mathbf{a}\end{array}$ & $\begin{array}{c}0,7 \\
<1 \\
1,1 \\
1,9 \\
\end{array}$ & $\frac{1 \mathrm{~J}}{\mathrm{IU}}$ & $\begin{array}{r}- \\
- \\
<1 \\
\end{array}$ & $\mathbf{I U}$ & $\begin{array}{l}<1 \\
<1 \\
<1 \\
<1\end{array}$ & $\begin{array}{l}\text { IU } \\
\text { IU } \\
\text { IU } \\
\text { IU } \\
\end{array}$ & $\begin{array}{r}- \\
<1 \\
<1 \\
-\end{array}$ & $\begin{array}{l}\text { IU } \\
\text { IU }\end{array}$ \\
\hline Dibromochloromethane & $\begin{array}{l}\text { WINTER } \\
\text { SPRING } \\
\text { SUMMER } \\
\text { FALL } \\
\end{array}$ & \begin{tabular}{|l|}
$\mathrm{ug} / \mathrm{L}$ \\
$\mathrm{ug} / \mathrm{L}$ \\
$\mathrm{ug} / \mathrm{L}$ \\
$\mathrm{ug} / \mathrm{L}$ \\
\end{tabular} & $\begin{array}{l}1 \\
1 \\
1 \\
1 \\
\end{array}$ & $\begin{array}{l}\mathbf{a} \\
\mathbf{a} \\
\mathbf{a} \\
\mathbf{a}\end{array}$ & $\begin{array}{l}<1 \\
<1 \\
<1 \\
<1 \\
\end{array}$ & $\begin{array}{l}\mathbf{I U} \\
\mathbf{I U} \\
\mathbf{I U} \\
\mathbf{I U} \\
\end{array}$ & $\begin{array}{r}- \\
- \\
<1 \\
\end{array}$ & $\mathbf{I U}$ & $\begin{array}{l}<1 \\
<1 \\
<1 \\
<1\end{array}$ & $\begin{array}{l}\mathbf{I U} \\
\mathbf{I U} \\
\mathbf{I U} \\
\mathbf{I U}\end{array}$ & $\begin{array}{l}< \\
<1 \\
<1 \\
-\end{array}$ & $\begin{array}{l}\text { IU } \\
\text { IU }\end{array}$ \\
\hline Methylene Chloride & $\begin{array}{l}\text { WINTER } \\
\text { SPRING } \\
\text { SUMMER } \\
\text { FALL } \\
\end{array}$ & $\begin{array}{l}\mathrm{ug} / \mathrm{L} \\
\mathrm{ug} / \mathrm{L} \\
\mathrm{ug} / \mathrm{L} \\
\mathrm{ug} / \mathrm{L}\end{array}$ & $\begin{array}{l}2 \\
2 \\
2 \\
2 \\
\end{array}$ & $\begin{array}{l}5 \\
5 \\
5 \\
5 \\
\end{array}$ & $\begin{array}{l}<2 \\
<2 \\
<2 \\
<2\end{array}$ & $\begin{array}{l}\text { IU } \\
\text { IU } \\
\text { IU } \\
\text { IU }\end{array}$ & $\begin{array}{c}\vdots \\
\vdots \\
<2\end{array}$ & $\mathbf{I U}$ & $\begin{array}{l}<2 \\
<2 \\
<2 \\
<2\end{array}$ & $\begin{array}{l}\mathbf{I U} \\
\mathbf{I U} \\
\mathrm{IU} \\
\mathbf{I U}\end{array}$ & $\begin{array}{l}< \\
<2 \\
<2 \\
-\end{array}$ & $\underset{\mathbf{I U}}{\mathbf{I U}}$ \\
\hline Tetrachloroethene & $\begin{array}{l}\text { WINTER } \\
\text { SPRING } \\
\text { SUMMER } \\
\text { FALL } \\
\end{array}$ & $\begin{array}{l}\mathrm{ug} / \mathrm{L} \\
\mathrm{ug} / \mathrm{L} \\
\mathrm{ug} / \mathrm{L} \\
\mathrm{ug} / \mathrm{L} \\
\end{array}$ & $\begin{array}{l}1 \\
1 \\
1 \\
1 \\
\end{array}$ & \begin{tabular}{l|}
5 \\
5 \\
5 \\
5 \\
\end{tabular} & $\begin{array}{l}<1 \\
<1 \\
<1 \\
<1 \\
\end{array}$ & $\begin{array}{l}\text { IU } \\
\text { IU } \\
\text { IU } \\
\text { IU } \\
\end{array}$ & $\begin{array}{r}- \\
- \\
<1 \\
\end{array}$ & $\mathbf{I U}$ & $\begin{array}{l}<1 \\
<1 \\
<1 \\
<1 \\
\end{array}$ & $\begin{array}{l}\mathrm{IU} \\
\mathrm{IU} \\
\mathrm{IU} \\
\mathrm{IU}\end{array}$ & $\begin{array}{l}< \\
<1 \\
<1 \\
\end{array}$ & $\begin{array}{l}\mathbf{I U} \\
\mathbf{I U}\end{array}$ \\
\hline Toluene & $\begin{array}{l}\text { WINTER } \\
\text { SPRING } \\
\text { SUMMER } \\
\text { FALL } \\
\end{array}$ & $\begin{array}{l}\mathrm{ug} / \mathrm{L} \\
\mathrm{ug} / \mathrm{L} \\
\mathrm{ug} / \mathrm{L} \\
\mathrm{ug} / \mathrm{L} \\
\end{array}$ & $\begin{array}{l}1 \\
1 \\
1 \\
1 \\
\end{array}$ & $\begin{array}{ll}1000 & \# \\
1000 & \# \\
1000 & \# \\
1000 & \#\end{array}$ & $\begin{array}{l}<1 \\
<1 \\
<1 \\
<1\end{array}$ & $\begin{array}{l}\text { IU } \\
\text { IU } \\
\text { IU } \\
\text { IU } \\
\end{array}$ & $\begin{array}{r}- \\
- \\
<1\end{array}$ & IU & $\begin{array}{l}<1 \\
<1 \\
<1 \\
<1\end{array}$ & $\begin{array}{l}\mathrm{IU} \\
\mathrm{IU} \\
\mathrm{IU} \\
\mathrm{IU} \\
\end{array}$ & $\begin{array}{l}< \\
<1 \\
< \\
-\end{array}$ & $\begin{array}{l}\mathbf{I U} \\
\mathbf{I U}\end{array}$ \\
\hline
\end{tabular}


TABLE 6.2

SUMMARY OF DETECTED CONSTITUENTS IN SURFACE WATER AND STORM WATER, 1995

VOLATILE ORGANICS

1995 ANNUAL WATER MONITORING REPORT

LEHR ENVIRONMENTAL RESTORATION, DAVIS, CALIFORNIA

\begin{tabular}{|c|c|c|c|c|c|c|c|c|c|c|c|c|}
\hline \multirow{2}{*}{$\begin{array}{l}\text { Parameter } \\
\text { 1,1,2-Trichloroethane }\end{array}$} & \multirow[b]{2}{*}{\begin{tabular}{|l|} 
Quarter \\
WINTER \\
SPRING \\
SUMMER \\
FALL \\
\end{tabular}} & \multirow[b]{2}{*}{\begin{tabular}{|l|} 
Units \\
ug/L \\
ug/L \\
ug/L \\
ug/L
\end{tabular}} & \multirow[b]{2}{*}{$\begin{array}{l}\text { DL } \\
1 \\
1 \\
1 \\
1\end{array}$} & \multirow[b]{2}{*}{\begin{tabular}{cc|} 
MCL \\
$\mathbf{5}$ & $\#$ \\
5 & $\#$ \\
5 & $\#$ \\
5 & $\#$
\end{tabular}} & \multicolumn{2}{|c|}{ STPO } & \multicolumn{2}{|c|}{$\begin{array}{l}\text { STPO } \\
\text { D1 }\end{array}$} & \multicolumn{2}{|c|}{ SWL-1 } & SWL-2 & $\begin{array}{l}\text { SWL-2 } \\
\text { D1 }\end{array}$ \\
\hline & & & & & $\begin{array}{l}<1 \\
<1 \\
<1 \\
<1\end{array}$ & $\begin{array}{l}\text { IU } \\
\text { IU } \\
\text { IU } \\
\text { IU } \\
\end{array}$ & $\begin{array}{c}<1 \\
\vdots \\
- \\
-\end{array}$ & $\mathbf{I U}$ & $\begin{array}{c}<1 \\
- \\
- \\
-\end{array}$ & IU & $\begin{array}{cc}<1 & \text { IU } \\
- & \\
- & \\
\end{array}$ & $\begin{array}{rr}<1 & \text { IU } \\
\vdots & \\
- & \\
\end{array}$ \\
\hline 1,1-Dichloroethane & \begin{tabular}{|l|} 
WINTER \\
SPRING \\
SUMMER \\
FALL \\
\end{tabular} & \begin{tabular}{|l|}
$\mathrm{ug} / \mathrm{L}$ \\
$\mathrm{ug} / \mathrm{L}$ \\
$\mathrm{ug} / \mathrm{L}$ \\
$\mathrm{ug} / \mathrm{L}$ \\
\end{tabular} & $\begin{array}{l}1 \\
1 \\
1 \\
1 \\
\end{array}$ & \begin{tabular}{l|l}
5 \\
5 \\
5 \\
5
\end{tabular} & $\begin{array}{l}<1 \\
<1 \\
<1 \\
<1 \\
\end{array}$ & $\begin{array}{l}\text { IU } \\
\text { IU } \\
\text { IU } \\
\text { IU } \\
\end{array}$ & $\begin{array}{c}<1 \\
\vdots \\
\vdots \\
\end{array}$ & IU & $\begin{array}{r}<1 \\
- \\
- \\
\end{array}$ & IU & $\begin{array}{cc}<1 & \text { IU } \\
: & \\
: & \\
\end{array}$ & $\begin{array}{c}<1 \\
: \\
: \\
\end{array}$ \\
\hline 1,1-Dichloroethene & $\begin{array}{l}\text { WINTER } \\
\text { SPRING } \\
\text { SUMMER } \\
\text { FALL } \\
\end{array}$ & $\begin{array}{l}\mathrm{ug} / \mathrm{L} \\
\mathrm{ug} / \mathrm{L} \\
\mathrm{ug} / \mathrm{L} \\
\mathrm{ug} / \mathrm{L} \\
\end{array}$ & $\begin{array}{l}1 \\
1 \\
1 \\
1\end{array}$ & $\begin{array}{l}6 \\
6 \\
6 \\
6 \\
\end{array}$ & $\begin{array}{l}<1 \\
<1 \\
<1 \\
<1 \\
\end{array}$ & $\begin{array}{l}\text { IU } \\
\text { IU } \\
\text { IU } \\
\text { IU } \\
\end{array}$ & $\begin{array}{r}<1 \\
\vdots \\
\vdots \\
\end{array}$ & IU & $\begin{array}{c}<1 \\
- \\
- \\
-\end{array}$ & IU & 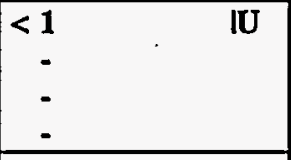 & $\begin{array}{c}1 \\
- \\
- \\
\end{array}$ \\
\hline 1,2-Dichloroethane & $\begin{array}{l}\text { WINTER } \\
\text { SPRING } \\
\text { SUMMER } \\
\text { FALL }\end{array}$ & $\begin{array}{l}\mathrm{ug} / \mathrm{L} \\
\mathrm{ug} / \mathrm{L} \\
\mathrm{ug} / \mathrm{L} \\
\mathrm{ug} / \mathrm{L}\end{array}$ & $\begin{array}{l}1 \\
1 \\
1 \\
1\end{array}$ & $\begin{array}{l}0.5 \\
0.5 \\
0.5 \\
0.5\end{array}$ & $\begin{array}{l}<1 \\
<1 \\
<1 \\
<1\end{array}$ & $\begin{array}{l}\mathrm{IU} \\
\mathrm{IU} \\
\mathrm{IU} \\
\mathrm{IU}\end{array}$ & $\begin{array}{l}<1 \\
\vdots \\
-\end{array}$ & $\mathbf{I U}$ & $\begin{array}{c}<1 \\
- \\
-\end{array}$ & IU & $\begin{array}{ll}<1 & \text { IU } \\
\vdots & \\
\vdots & \end{array}$ & $\begin{array}{c}<1 \\
- \\
- \\
-\end{array}$ \\
\hline 1,2-Dichloropropane & $\begin{array}{l}\text { WINTER } \\
\text { SPRING } \\
\text { SUMMER } \\
\text { FALL }\end{array}$ & $\begin{array}{l}\mathrm{ug} / \mathrm{L} \\
\mathrm{ug} / \mathrm{L} \\
\mathrm{ug} / \mathrm{L} \\
\mathrm{ug} / \mathrm{L}\end{array}$ & $\begin{array}{l}1 \\
1 \\
1 \\
1\end{array}$ & $\begin{array}{l}5 \\
5 \\
5 \\
5 \\
\end{array}$ & $\begin{array}{l}<1 \\
<1 \\
<1 \\
<1\end{array}$ & $\begin{array}{l}\mathrm{IU} \\
\mathrm{IU} \\
\mathrm{IU} \\
\mathrm{IU} \\
\end{array}$ & $\begin{array}{l}<1 \\
\vdots \\
\end{array}$ & IU & $\begin{array}{c}<1 \\
- \\
- \\
-\end{array}$ & IU & $\begin{array}{c}<1 \\
\vdots \\
- \\
\end{array}$ & $\begin{array}{c}<1 \\
- \\
- \\
\end{array}$ \\
\hline 2-Butanone & $\begin{array}{l}\text { WINTER } \\
\text { SPRING } \\
\text { SUMMER } \\
\text { FALL } \\
\end{array}$ & \begin{tabular}{|l|}
$\mathrm{ug} / \mathrm{L}$ \\
$\mathrm{ug} / \mathrm{L}$ \\
$\mathrm{ug} / \mathrm{L}$ \\
$\mathrm{ug} / \mathrm{L}$ \\
\end{tabular} & $\begin{array}{l}5 \\
5 \\
5 \\
5 \\
\end{array}$ & $\begin{array}{l}- \\
- \\
-\end{array}$ & $\begin{array}{l}<5 \\
<5 \\
<5 \\
<5 \\
\end{array}$ & $\begin{array}{l}\text { Rc IU } \\
\text { Rc IU } \\
\text { Rc IU } \\
\text { Rc IU } \\
\end{array}$ & $\begin{array}{c}<5 \\
- \\
- \\
-\end{array}$ & Re IU & $\begin{array}{r}<5 \\
- \\
- \\
-\end{array}$ & $\mathbf{R c}$ IU & $\begin{array}{c}<5 \\
- \\
- \\
- \\
\end{array}$ & Rc IU \\
\hline 4-Methyl-2-Pentanone & $\begin{array}{l}\text { WINTER } \\
\text { SPRING } \\
\text { SUMMER } \\
\text { FALL }\end{array}$ & $\begin{array}{l}\mathrm{ug} / \mathrm{L} \\
\mathrm{ug} / \mathrm{L} \\
\mathrm{ug} / \mathrm{L} \\
\mathrm{ug} / \mathrm{L}\end{array}$ & $\begin{array}{l}5 \\
5 \\
5 \\
5\end{array}$ & $\begin{array}{l}: \\
: \\
:\end{array}$ & $\begin{array}{l}<5 \\
<5 \\
<5 \\
<5\end{array}$ & $\begin{array}{l}\mathbf{I U} \\
\mathbf{I U} \\
\mathbf{I U} \\
\mathbf{U U}\end{array}$ & $\begin{array}{c}<5 \\
\vdots \\
\vdots\end{array}$ & & $\begin{array}{r}<5 \\
- \\
- \\
-\end{array}$ & & $\begin{array}{c}<5 \\
: \\
- \\
-\end{array}$ & $\begin{array}{c}<5 \\
- \\
- \\
-\end{array}$ \\
\hline \multirow[t]{2}{*}{ Acetone } & \multirow{2}{*}{$\begin{array}{l}\text { WINTER } \\
\text { SPRING } \\
\text { SUMMER } \\
\text { FALL }\end{array}$} & \multirow{2}{*}{$\begin{array}{l}\text { ug/L } \\
\text { ug/L } \\
\text { ug/L } \\
\text { ug/L }\end{array}$} & \multirow{2}{*}{$\begin{array}{l}5 \\
5 \\
5 \\
5\end{array}$} & \multirow{2}{*}{ 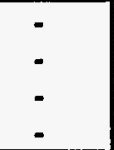 } & \multirow{2}{*}{ 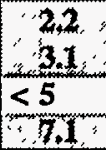 } & \multirow{2}{*}{ 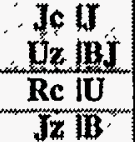 } & \multirow{2}{*}{$\begin{array}{c}<5 \\
: \\
-\end{array}$} & \multirow[t]{2}{*}{$\operatorname{Re} \mathbf{I U}$} & 6 & Ie & $4.6^{3}: \mathrm{OJCLJ}$ & $8.8 \mathrm{Je}$ \\
\hline & & & & & & & & & - & & $\begin{array}{l}- \\
- \\
-\end{array}$ & $\begin{array}{l}- \\
- \\
-\end{array}$ \\
\hline
\end{tabular}

Page 3 of 5 
TABLE 6.2

SUMMARY OF DETECTED CONSTITUENTS IN SURFACE WATER AND STORM WATER, 1995

VOLATILE ORGANICS

1995 ANNUAL WATER MONITORING REPORT

LEHR ENVIRONMENTAL RESTORATION, DAVIS, CALIFORNIA

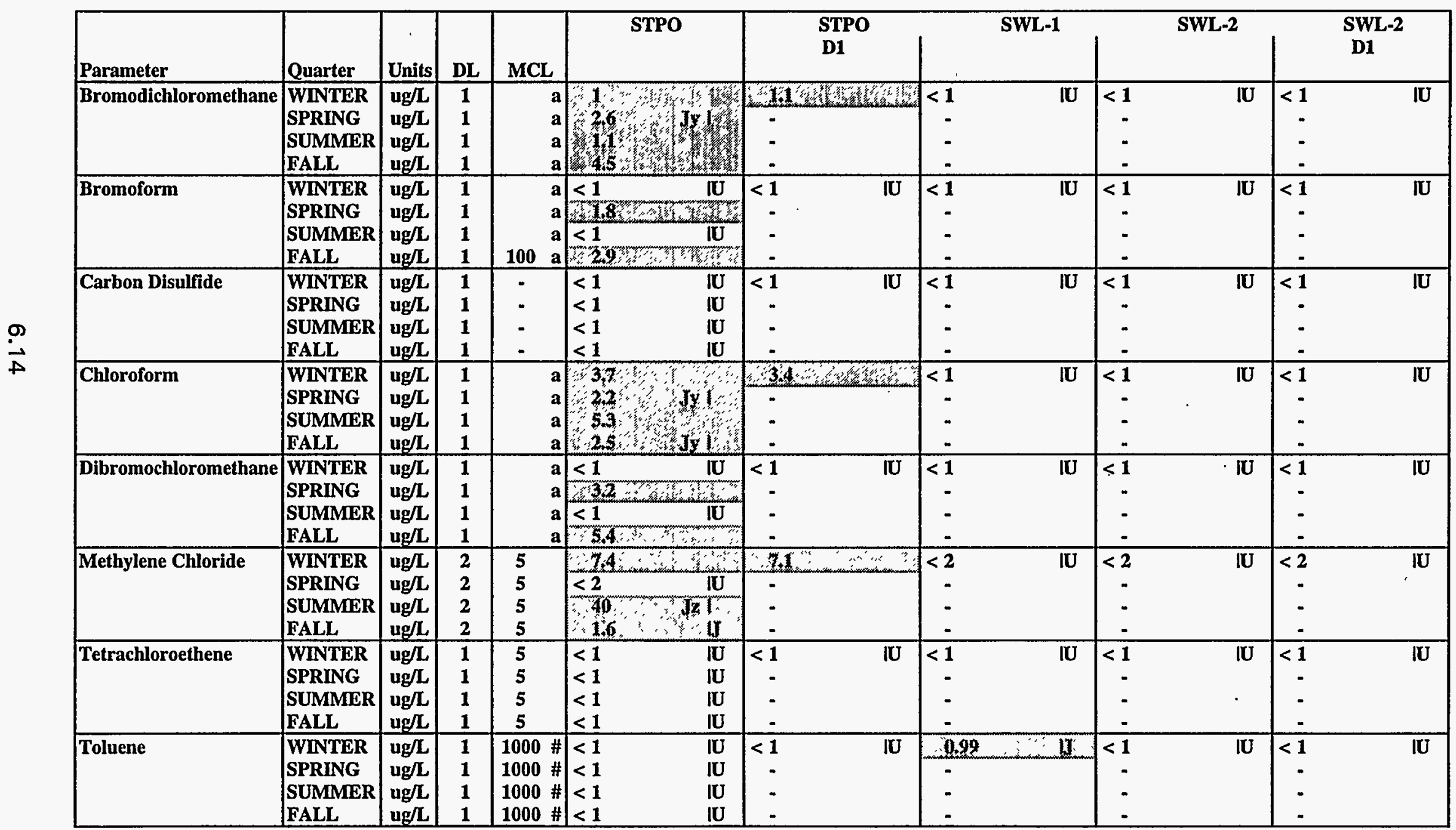

Page 4 of 5 


\section{TABLE 6.2}

SUMMARY OF DETECTED CONSTITUENTS IN SURFACE WATER AND STORM WATER, 1995

VOLATILE ORGANICS

1995 ANNUAL WATER MONITORING REPORT

LEHR ENVIRONMENTAL RESTORATION, DAVIS, CALIFORNIA

D1 = Duplicate sample.

- = Parameter not analyzed or no MCL.

DL = Detection limit.

$<=$ Constituent below detection limit. Detection limits may vary depending on interference by other sample constituents. MCL = Maximum Contaminant Level (primary), Title 22 Division 4 Chapter 15.

\# = USEPA MCL

$\mathrm{a}=$ MCL for total trihalomethanes is $100 \mathrm{ug} / \mathrm{L}$. Chloroform is the predominant trihalomethane detected at LEHR.

[.] Highlighted values indicate positive results.

See Appendix A for explanation of data qualifiers. 
TABLE 6.3

SUMMARY OF DETECTED CONSTITUENTS IN SURFACE WATER AND STORM WATER, 1995

SEMIVOLATILE ORGANIC COMPOUNDS

1995 ANNUAL WATER MONITORING REPORT

LEHR ENVIRONMENTAL RESTORATION, DAVIS, CALIFORNIA

\begin{tabular}{|c|c|c|c|c|c|c|c|c|c|c|c|}
\hline \multirow{2}{*}{\begin{tabular}{|l} 
Parameter \\
4-Methylphenol
\end{tabular}} & \multirow[b]{2}{*}{\begin{tabular}{|l} 
Quarter \\
WINTER \\
SPRING \\
SUMMER \\
FALL
\end{tabular}} & \multirow[b]{2}{*}{$\begin{array}{l}\text { Units } \\
\text { ug/L } \\
\text { ug/L } \\
\text { ug/L } \\
\text { ug/L }\end{array}$} & \multirow[b]{2}{*}{$\begin{array}{l}\text { DL } \\
10 \\
10 \\
10 \\
10\end{array}$} & MCL & \multicolumn{2}{|c|}{ PCD } & \multirow[t]{2}{*}{$\begin{array}{c}\text { PCD } \\
\text { D1 }\end{array}$} & \multicolumn{2}{|c|}{ PCU } & \multicolumn{2}{|c|}{$\begin{array}{c}\text { PCU } \\
\text { D1 }\end{array}$} \\
\hline & & & & $\begin{array}{l}: \\
: \\
:\end{array}$ & $\begin{array}{c}<11 \\
<10 \\
- \\
-\end{array}$ & $\begin{array}{l}\mathrm{IU} \\
\mathrm{IU}\end{array}$ & & $\begin{array}{c}<12 \\
<11 \\
- \\
-\end{array}$ & $\mathbf{I U}$ & $\begin{array}{c}- \\
- \\
-\end{array}$ & $\mathbf{I U}$ \\
\hline BenzylButylPhthalate & $\begin{array}{l}\text { WINTER } \\
\text { SPRING } \\
\text { SUMMER } \\
\text { FALL }\end{array}$ & $\begin{array}{l}\mathrm{ug} / \mathrm{L} \\
\mathrm{ug} / \mathrm{L} \\
\mathrm{ug} / \mathrm{L} \\
\mathrm{ug} / \mathrm{L}\end{array}$ & $\begin{array}{l}10 \\
10 \\
10 \\
10\end{array}$ & $\begin{array}{ll}100 & \# \\
100 & \# \\
100 & \# \\
100 & \#\end{array}$ & $\begin{array}{c}<11 \\
<10 \\
- \\
-\end{array}$ & $\begin{array}{l}\mathrm{IU} \\
\mathrm{IU}\end{array}$ & $\begin{array}{l}\overline{-} \\
- \\
-\end{array}$ & $\mid \begin{array}{c}<12 \\
<11 \\
- \\
-\end{array}$ & $\underset{\mathbf{I U}}{\mathbf{I U}}$ & $<+$ & $\mathbf{I U}$ \\
\hline Bis(2-Ethylhexyl)phthalate & $\begin{array}{l}\text { WINTER } \\
\text { SPRING }\end{array}$ & $\begin{array}{l}\text { ug/L } \\
\text { ug/L }\end{array}$ & $\begin{array}{l}10 \\
10\end{array}$ & $\begin{array}{l}4 \\
4\end{array}$ & $\begin{array}{r}<11 \\
37\end{array}$ & IU & - & $\begin{array}{l}0.7 \\
2\end{array}$ & 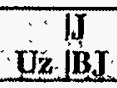 & $\therefore$ & $\mathrm{Z} \cdot \mathrm{BI}$ \\
\hline & $\begin{array}{l}\text { SUMMER } \\
\text { FALL }\end{array}$ & $\begin{array}{l}\mathrm{ug} / \mathrm{L} \\
\mathrm{ug} / \mathrm{L} \\
\end{array}$ & $\begin{array}{l}10 \\
10 \\
\end{array}$ & $\begin{array}{l}4 \\
4 \\
\end{array}$ & $\div$ & & - & - & & $\therefore$ & \\
\hline Di-n-Butylphthalate & $\begin{array}{l}\text { WINTER } \\
\text { SPRING }\end{array}$ & $\begin{array}{l}\text { ug/L } \\
\text { ug/L }\end{array}$ & $\begin{array}{l}10 \\
10\end{array}$ & 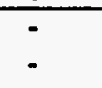 & $\begin{array}{r}3 \\
59 \\
\end{array}$ & II & - & $\begin{array}{l}<12 \\
130 \\
\end{array}$ & $\begin{array}{l}\mathrm{IO} \\
\mathrm{JI} \mid \mathrm{B} \\
\end{array}$ & $\frac{-}{38}$ & fiB \\
\hline & $\begin{array}{l}\text { SUMMER } \\
\text { FALL }\end{array}$ & $\begin{array}{l}\mathrm{ug} / \mathrm{L} \\
\mathrm{ug} / \mathrm{L}\end{array}$ & $\begin{array}{l}10 \\
10\end{array}$ & $\therefore$ & - & & - & 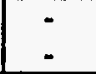 & & - & \\
\hline DiethylPhthalate & $\begin{array}{l}\text { WINTER } \\
\text { SPRING }\end{array}$ & \begin{tabular}{|l|}
$\mathrm{ug} / \mathrm{L}$ \\
$\mathrm{ug} / \mathrm{L}$
\end{tabular} & 10 & - & $\leq 11$ & IU & $\therefore$ & $\frac{<12}{2}$ & $\mathrm{IU}$ & $i$ & $\pi$ \\
\hline & $\begin{array}{l}\text { SUMMER } \\
\text { FALL }\end{array}$ & $\begin{array}{l}\mathrm{ug} / \mathrm{L} \\
\mathrm{ug} / \mathrm{L}\end{array}$ & $\begin{array}{l}10 \\
10\end{array}$ & 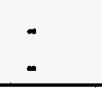 & - & & - & - & & - & \\
\hline DimethylPhthalate & \begin{tabular}{|l|} 
WINTER \\
SPRING \\
SUMMER \\
FALL \\
\end{tabular} & \begin{tabular}{|l|}
$\mathrm{ug} / \mathrm{L}$ \\
$\mathrm{ug} / \mathrm{L}$ \\
$\mathrm{ug} / \mathrm{L}$ \\
$\mathrm{ug} / \mathrm{L}$ \\
\end{tabular} & \begin{tabular}{l|}
10 \\
10 \\
10 \\
10 \\
\end{tabular} & $\begin{array}{l}- \\
- \\
-\end{array}$ & $\begin{array}{c}<11 \\
<10 \\
. \\
.\end{array}$ & $\begin{array}{l}\text { IU } \\
\text { IU }\end{array}$ & $\begin{array}{l}- \\
- \\
- \\
\end{array}$ & $\begin{array}{c}<12 \\
<11 \\
- \\
- \\
\end{array}$ & $\begin{array}{l}\mathbf{I U} \\
\mathbf{I U}\end{array}$ & $\begin{array}{c}- \\
-11 \\
-\end{array}$ & $\mathbf{I U}$ \\
\hline N-Nitrosodiphenylamine & $\begin{array}{l}\text { WINTER } \\
\text { SPRING } \\
\text { SUMMER } \\
\text { FALL } \\
\end{array}$ & $\begin{array}{l}\mathrm{ug} / \mathrm{L} \\
\mathrm{ug} / \mathrm{L} \\
\mathrm{ug} / \mathrm{L} \\
\mathrm{ug} / \mathrm{L}\end{array}$ & $\begin{array}{l}10 \\
10 \\
10 \\
10\end{array}$ & $\begin{array}{l}- \\
- \\
- \\
\end{array}$ & $\begin{array}{c}<11 \\
<10 \\
\cdot \\
. \\
\end{array}$ & $\begin{array}{l}\text { IU } \\
\text { IU }\end{array}$ & $\begin{array}{l}- \\
- \\
-\end{array}$ & $\begin{array}{c}<12 \\
<11 \\
- \\
-\end{array}$ & $\begin{array}{l}\mathrm{IU} \\
\mathrm{IU}\end{array}$ & $\begin{array}{c}- \\
- \\
-\end{array}$ & \\
\hline Phenol & $\begin{array}{l}\text { WINTER } \\
\text { SPRING } \\
\text { SUMMER } \\
\text { FALL } \\
\end{array}$ & $\begin{array}{l}\mathrm{ug} / \mathrm{L} \\
\mathrm{ug} / \mathrm{L} \\
\mathrm{ug} / \mathrm{L} \\
\mathrm{ug} / \mathrm{L}\end{array}$ & $\begin{array}{l}10 \\
10 \\
10 \\
10 \\
\end{array}$ & $\begin{array}{l}- \\
- \\
- \\
-\end{array}$ & $\begin{array}{c}<11 \\
<10 \\
- \\
-\end{array}$ & $\begin{array}{l}\text { IU } \\
\text { IU }\end{array}$ & $\begin{array}{l}. \\
- \\
-\end{array}$ & $\begin{array}{c}<12 \\
<11 \\
- \\
-\end{array}$ & $\begin{array}{l}\mathbf{I U} \\
\mathbf{I U}\end{array}$ & $\begin{array}{c}- \\
-11 \\
-\end{array}$ & \\
\hline
\end{tabular}


TABLE 6.3

SUMMARY OF DETECTED CONSTITUENTS IN SURFACE WATER AND STORM WATER, 1995

SEMTVOLATILE ORGANIC COMPOUNDS

1995 ANNUAL WATER MONITORING REPORT

LEHR ENVIRONMENTAL RESTORATION, DAVIS, CALIFORNIA

\begin{tabular}{|c|c|c|c|c|c|c|c|c|c|}
\hline Parameter & Quarter & Units & DL & MCL & STPO & $\begin{array}{c}\text { STPO } \\
\text { D1 }\end{array}$ & SWL-1 & SWL-2 & $\begin{array}{c}\text { SWL-2 } \\
\text { D1. }\end{array}$ \\
\hline 4-Methylphenol & $\begin{array}{l}\text { WINTER } \\
\text { SPRING } \\
\text { SUMMER } \\
\text { FALL } \\
\end{array}$ & $\begin{array}{l}\mathrm{ug} / \mathrm{L} \\
\mathrm{ug} / \mathrm{L} \\
\mathrm{ug} / \mathrm{L} \\
\mathrm{ug} / \mathrm{L}\end{array}$ & $\begin{array}{l}10 \\
10 \\
10 \\
10\end{array}$ & $\begin{array}{l}\dot{-} \\
\dot{-} \\
-\end{array}$ & $\begin{array}{c}<12 \\
<10 \\
- \\
-\end{array}$ & $\begin{array}{ll}<\mathbf{1 2} & \text { IU } \\
- & \\
- & \\
- & \end{array}$ & $\begin{array}{cc}<\mathbf{1 0} & \text { IU } \\
- & \\
- & \\
- & \end{array}$ & $\begin{array}{cc}<10 & \text { IU } \\
- & \\
- & \\
- & \end{array}$ & $\begin{array}{ll}\mathbf{1 0} & \text { IU } \\
- & \\
- & \\
- & \end{array}$ \\
\hline BenzylButylPhthalate & $\begin{array}{l}\text { WINTER } \\
\text { SPRING } \\
\text { SUMMER } \\
\text { FALL } \\
\end{array}$ & $\begin{array}{l}\mathrm{ug} / \mathrm{L} \\
\mathrm{ug} / \mathrm{L} \\
\mathrm{ug} / \mathrm{L} \\
\mathrm{ug} / \mathrm{L}\end{array}$ & $\begin{array}{l}10 \\
10 \\
10 \\
10 \\
\end{array}$ & $\begin{array}{ll}100 & \# \\
100 & \# \\
100 & \# \\
100 & \# \\
\end{array}$ & $\begin{array}{c}<12 \\
<10 \\
- \\
- \\
\end{array}$ & $\begin{array}{ll}<12 & \text { IU } \\
\vdots & \\
\vdots & \\
\end{array}$ & 年, & $\begin{array}{c}<10 \\
- \\
- \\
-\end{array}$ & $\begin{array}{c}<10 \\
- \\
- \\
-\end{array}$ \\
\hline Bis(2-Ethylhexyl)phthalate & \begin{tabular}{|l|} 
WINTER \\
SPRING \\
SUMMER \\
FALL \\
\end{tabular} & $\begin{array}{l}\text { ug/L } \\
\text { ug/L } \\
\text { ug/L } \\
\text { ug/L }\end{array}$ & $\begin{array}{l}10 \\
10 \\
10 \\
10 \\
\end{array}$ & \begin{tabular}{l|}
4 \\
4 \\
4 \\
4 \\
\end{tabular} & 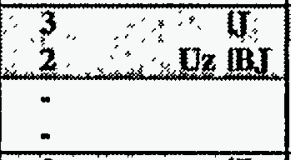 & 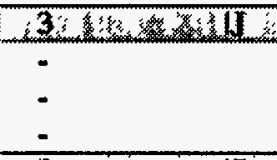 & $\begin{array}{l}1 \\
- \\
- \\
\end{array}$ & \begin{tabular}{cc|}
$\mathbf{1 0}$ & IU \\
- & \\
- & \\
\end{tabular} & $\begin{array}{l}0.5 \\
: \\
- \\
\end{array}$ \\
\hline Di-n-Butylphthalate & $\begin{array}{l}\text { WINTER } \\
\text { SPRING } \\
\text { SUMMER } \\
\text { FALL } \\
\end{array}$ & $\begin{array}{l}\mathrm{ug} / \mathrm{L} \\
\mathrm{ug} / \mathrm{L} \\
\mathrm{ug} / \mathrm{L} \\
\mathrm{ug} / \mathrm{L}\end{array}$ & $\begin{array}{l}10 \\
10 \\
10 \\
10\end{array}$ & $\begin{array}{l}i \\
- \\
-\end{array}$ & 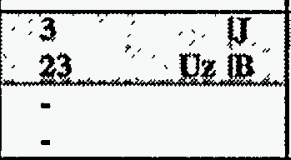 & 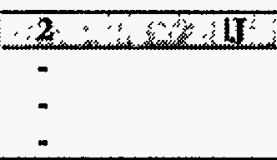 & $\begin{array}{l}6 \cdot 1-\text { UaIDI } \\
: \\
-\end{array}$ & $\frac{8}{-}$ & $\mathrm{Ux} \cdot \mathrm{BJ}$ \\
\hline DiethylPhthalate & \begin{tabular}{|l|} 
WINTER \\
SPRING \\
SUMMER \\
FALL
\end{tabular} & $\begin{array}{l}\mathrm{ug} / \mathrm{L} \\
\mathrm{ug} / \mathrm{L} \\
\mathrm{ug} / \mathrm{L} \\
\mathrm{ug} / \mathrm{L}\end{array}$ & $\begin{array}{l}10 \\
10 \\
10 \\
10\end{array}$ & $\begin{array}{l} \\
\\
.\end{array}$ & $\begin{array}{l}\frac{<12}{12} \\
\frac{1}{-} \\
-\end{array}$ & $\begin{array}{c}<12 \\
- \\
- \\
-\end{array}$ & $\begin{array}{c}<10 \\
- \\
- \\
-\end{array}$ & $\begin{array}{c}<10 \\
- \\
-\end{array}$ & $\begin{array}{c}<10 \\
: \\
!\end{array}$ \\
\hline DimethylPhthalate & $\begin{array}{l}\text { WINTER } \\
\text { SPRING } \\
\text { SUMMER } \\
\text { FALL }\end{array}$ & $\begin{array}{l}\mathrm{ug} / \mathrm{L} \\
\mathrm{ug} / \mathrm{L} \\
\mathrm{ug} / \mathrm{L} \\
\mathrm{ug} / \mathrm{L}\end{array}$ & $\begin{array}{l}10 \\
10 \\
10 \\
10\end{array}$ & $\dot{.}$ & $\begin{array}{c}<12 \\
<10 \\
! \\
\cdot\end{array}$ & $\begin{array}{c}<12 \\
- \\
- \\
-\end{array}$ & $\begin{array}{c}<10 \\
- \\
- \\
-\end{array}$ & $\begin{array}{c}<10 \\
: \\
:\end{array}$ & $\begin{array}{c}\mathbf{1 0} \\
\vdots \\
\cdot\end{array}$ \\
\hline N-Nitrosodiphenylamine & $\begin{array}{l}\text { WINTER } \\
\text { SPRING } \\
\text { SUMMER } \\
\text { FALL } \\
\end{array}$ & $\begin{array}{l}\mathrm{ug} / \mathrm{L} \\
\mathrm{ug} / \mathrm{L} \\
\mathrm{ug} / \mathrm{L} \\
\mathrm{ug} / \mathrm{L}\end{array}$ & $\begin{array}{l}10 \\
10 \\
10 \\
10\end{array}$ & $\begin{array}{l}- \\
- \\
-\end{array}$ & $\begin{array}{c}<12 \\
<10 \\
- \\
- \\
\end{array}$ & $\begin{array}{c}<12 \\
: \\
\vdots \\
\end{array}$ & $\begin{array}{c}<10 \\
\vdots \\
\vdots \\
\end{array}$ & $\begin{array}{c}<10 \\
- \\
- \\
\end{array}$ & $\begin{array}{c}<10 \\
- \\
-\end{array}$ \\
\hline Phenol & \begin{tabular}{|l|} 
WINTER \\
SPRING \\
SUMMER \\
FALL \\
\end{tabular} & $\begin{array}{l}\mathrm{ug} / \mathrm{L} \\
\mathrm{ug} / \mathrm{L} \\
\mathrm{ug} / \mathrm{L} \\
\mathrm{ug} / \mathrm{L}\end{array}$ & $\begin{array}{l}10 \\
10 \\
10 \\
10\end{array}$ & - & $\begin{array}{c}<12 \\
<10 \\
- \\
-\end{array}$ & $\begin{array}{l}<12 \\
\vdots \\
\vdots\end{array}$ & $\begin{array}{c}\mathbf{1 0} \\
- \\
- \\
-\end{array}$ & $\begin{array}{c}<10 \\
\vdots \\
- \\
-\end{array}$ & $\begin{array}{c}<\mathbf{1 0} \\
- \\
- \\
-\end{array}$ \\
\hline
\end{tabular}


TABLE 6.3

SUMMARY OF DETECTED CONSTITUENTS IN SURFACE WATER AND STORM WATER, 1995

SEMIVOLATILE ORGANIC COMPOUNDS

1995 ANNUAL WATER MONITORING REPORT

LEHR ENVIRONMENTAL RESTORATION, DAVIS, CALIFORNIA

D1 = Duplicate sample.

- = Parameter not analyzed or no MCL.

DL $=$ Detection limit.

$<$ = Constituent below detection limit. Detection limits may vary depending on interference by other sample constituents.

MCL = Maximum Contaminant Level (primary), Title 22 Division 4 Chapter 15.

\# = USEPA MCL

Highlighted values indicate positive results.

See Appendix A for explanation of data qualifiers. 
TABLE 6.4

SUMMARY OF DETECTED CONSTITUENTS IN SURFACE WATER AND STORM WATER, 1995 PESTICIDES AND PCBS

1995 ANNUAL WATER MONITORING REPORT

LEHR ENVIRONMENTAL RESTORATION, DAVIS, CALIFORNIA

\begin{tabular}{|c|c|c|c|c|c|c|c|c|c|c|c|c|}
\hline \multirow{2}{*}{\begin{tabular}{|l} 
Parameter \\
Alpha-Chlordane
\end{tabular}} & \multirow[b]{2}{*}{\begin{tabular}{|l|} 
Quarter \\
WINTER \\
SPRING \\
SUMMER \\
FALL \\
\end{tabular}} & \multirow[b]{2}{*}{$\begin{array}{l}\text { Units } \\
\text { ug/L } \\
\text { ug/L } \\
\text { ug/L } \\
\text { ug/L }\end{array}$} & \multirow[b]{2}{*}{$\begin{array}{l}\text { DL } \\
0.01 \\
0.01 \\
0.01 \\
0.01\end{array}$} & \multirow[b]{2}{*}{$\begin{array}{c}\text { MCL } \\
- \\
- \\
-\end{array}$} & \multicolumn{2}{|c|}{ PCD } & \multicolumn{2}{|c|}{$\begin{array}{c}\text { PCD } \\
\text { D1 }\end{array}$} & \multicolumn{2}{|c|}{ PCU } & \multicolumn{2}{|c|}{$\begin{array}{c}\text { PCU } \\
\text { D1 }\end{array}$} \\
\hline & & & & & $\begin{array}{l}<0.011 \\
<0.011 \\
<0.01 \\
<0.01\end{array}$ & $\begin{array}{ll} & \text { IU } \\
\text { UJs } & \mathbb{I U} \\
\text { UJs } & \text { IU } \\
\text { UJs } & \text { IU }\end{array}$ & $\begin{array}{c}- \\
\dot{-} \\
<\overline{0.01}\end{array}$ & UJs IU & $\begin{array}{l}<0.012 \\
<0.011 \\
<0.01 \\
<0.01\end{array}$ & 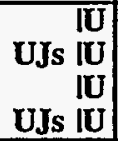 & $\begin{array}{l}<\overline{0.01} \\
<0.01 \\
\end{array}$ & $\underset{I U}{\text { IU }}$ \\
\hline Dieldrin & $\begin{array}{l}\text { WINTER } \\
\text { SPRING } \\
\text { SUMMER } \\
\text { FALL } \\
\end{array}$ & $\begin{array}{l}\mathrm{ug} / \mathrm{L} \\
\mathrm{ug} / \mathrm{L} \\
\mathrm{ug} / \mathrm{L} \\
\mathrm{ug} / \mathrm{L}\end{array}$ & $\begin{array}{l}0.02 \\
0.02 \\
0.02 \\
0.02 \\
\end{array}$ & $\begin{array}{l}- \\
- \\
- \\
-\end{array}$ & $\begin{array}{l}<0.022 \\
<0.022 \\
<0.02 \\
<0.02\end{array}$ & \begin{tabular}{ll|} 
& $\mathbb{I U}$ \\
UJs & $\mathbb{I U}$ \\
UJs & $\mathbb{U}$ \\
UJs & $\mathbb{I U}$
\end{tabular} & $\begin{array}{c}- \\
- \\
<0.02\end{array}$ & UJs IU & $\begin{array}{l}<0.024 \\
<0.022 \\
<0.02 \\
<0.02\end{array}$ & 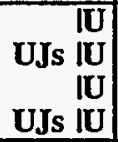 & $\begin{array}{l}<\overline{0.021} \\
<0.02 \\
-\end{array}$ & $\underset{\mathbf{I U}}{\mathbf{I U}}$ \\
\hline Endrin & $\begin{array}{l}\text { WINTER } \\
\text { SPRING } \\
\text { SUMMER } \\
\text { FALL }\end{array}$ & $\begin{array}{l}\mathrm{ug} / \mathrm{L} \\
\mathrm{ug} / \mathrm{L} \\
\mathrm{ug} / \mathrm{L} \\
\mathrm{ug} / \mathrm{L}\end{array}$ & $\begin{array}{l}0.02 \\
0.02 \\
0.02 \\
0.02 \\
\end{array}$ & $\begin{array}{l}0.2 \\
0.2 \\
0.2 \\
0.2 \\
\end{array}$ & $\begin{array}{l}<0.022 \\
<0.022 \\
<0.02 \\
<0.02\end{array}$ & $\begin{array}{l}\text { IU } \\
\text { UJs IU } \\
\text { UJs IU } \\
\text { UJs IU } \\
\end{array}$ & $\begin{array}{c}- \\
- \\
<\overline{0.02}\end{array}$ & UJs IU & $\begin{array}{l}<0.024 \\
<0.022 \\
<0.02 \\
<0.02\end{array}$ & $\begin{array}{cc}\text { UJs } & \begin{array}{l}\text { IU } \\
\text { IU } \\
\text { UJs }\end{array} \\
\end{array}$ & $\begin{array}{l}<\overline{0.021} \\
<0.02 \\
-\end{array}$ & $\begin{array}{l}\text { IU } \\
\mathbf{I U}\end{array}$ \\
\hline Gamma-Chlordane & $\begin{array}{l}\text { WINTER } \\
\text { SPRING } \\
\text { SUMMER } \\
\text { FALL } \\
\end{array}$ & $\begin{array}{l}\mathrm{ug} / \mathrm{L} \\
\mathrm{ug} / \mathrm{L} \\
\mathrm{ug} / \mathrm{L} \\
\mathrm{ug} / \mathrm{L}\end{array}$ & $\begin{array}{l}0.01 \\
0.01 \\
0.01 \\
0.01 \\
\end{array}$ & $\begin{array}{l}- \\
- \\
- \\
\end{array}$ & $\begin{array}{l}<0.011 \\
<0.011 \\
<0.01 \\
<0.01\end{array}$ & \begin{tabular}{ll|} 
& $\mathbf{I U}$ \\
UJs & $\mathbf{I U}$ \\
UJs & $\mathbf{I U}$ \\
UJs & $\mathbb{I U}$ \\
\end{tabular} & $\begin{array}{c}- \\
- \\
<\overline{0.01} \\
\end{array}$ & UJs IU & $\begin{array}{l}<0.012 \\
<0.011 \\
<0.01 \\
<0.01\end{array}$ & $\begin{array}{cc}\mathbf{I U} \\
\text { UJs } & \mathbf{I U} \\
& \mathbf{I U} \\
\text { UJs } & \mathbf{I U} \\
\end{array}$ & $\begin{array}{l}<\dot{0.01} \\
<0.01 \\
-\end{array}$ & $\begin{array}{l}\mathbf{I U} \\
\mathbf{I U}\end{array}$ \\
\hline
\end{tabular}

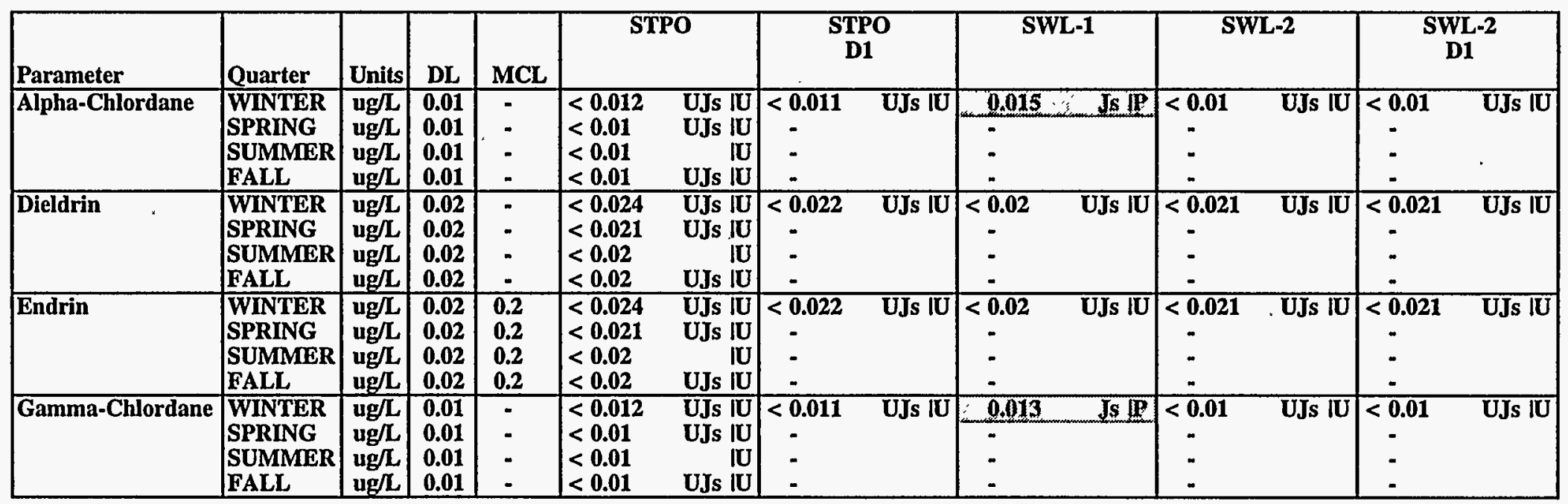


TABLE 6.4

SUMMARY OF DETECTED CONSTITUENTS IN SURFACE WATER AND STORM WATER, 1995

PESTICIDES AND PCBS

1995 ANNUAL WATER MONITORING REPORT

LEHR ENVIRONMENTAL RESTORATION, DAVIS, CALIFORNIA

D1 = Duplicate sample.

- = Parameter not analyzed or no MCL.

DL = Detection limit.

$<=$ Constituent below detection limit. Detection limits may vary depending on interference by other sample constituents. MCL = Maximum Contaminant Level (primary), Title 22 Division 4 Chapter 15.

Highlighted values indicate positive results.

See Appendix A for explanation of data qualifiers. 
TABLE 6.5

SUMMARY OF DETECTED CONSTITUENTS IN SURFACE WATER AND STORM WATER, 1995

METALS

1995 ANNUAL WATER MONITORING REPORT

LEHR ENVIRONMENTAL RESTORATION, DAVIS, CALIFORNIA

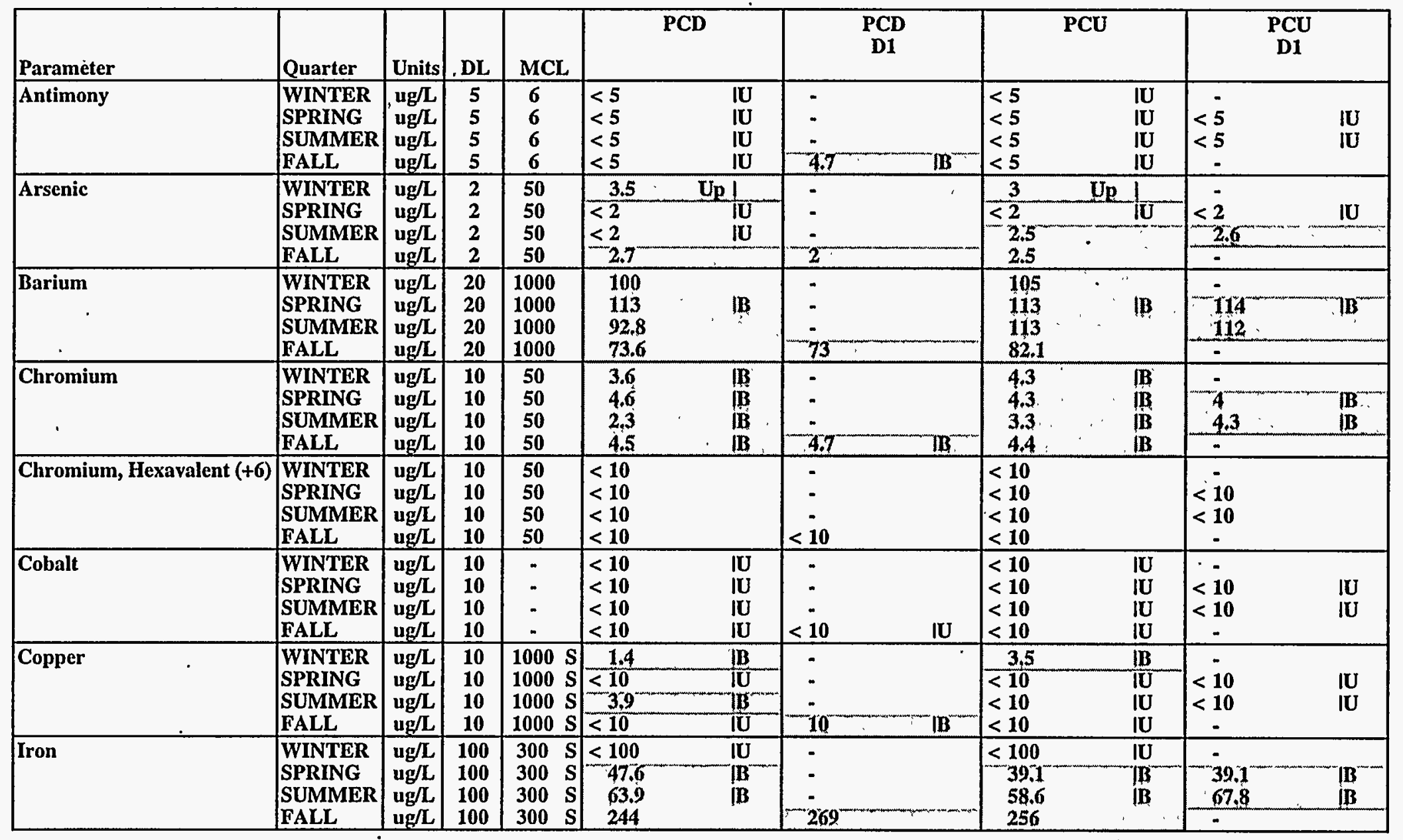


TABLE 6.5

SUMMARY OF DETECTED CONSTITUENTS IN SURFACE WATER AND STORM WATER, 1995

METALS

1995 ANNUAL WATER MONITORING REPORT

LEHR ENVIRONMENTAL RESTORATION, DAVIS, CALIFORNIA

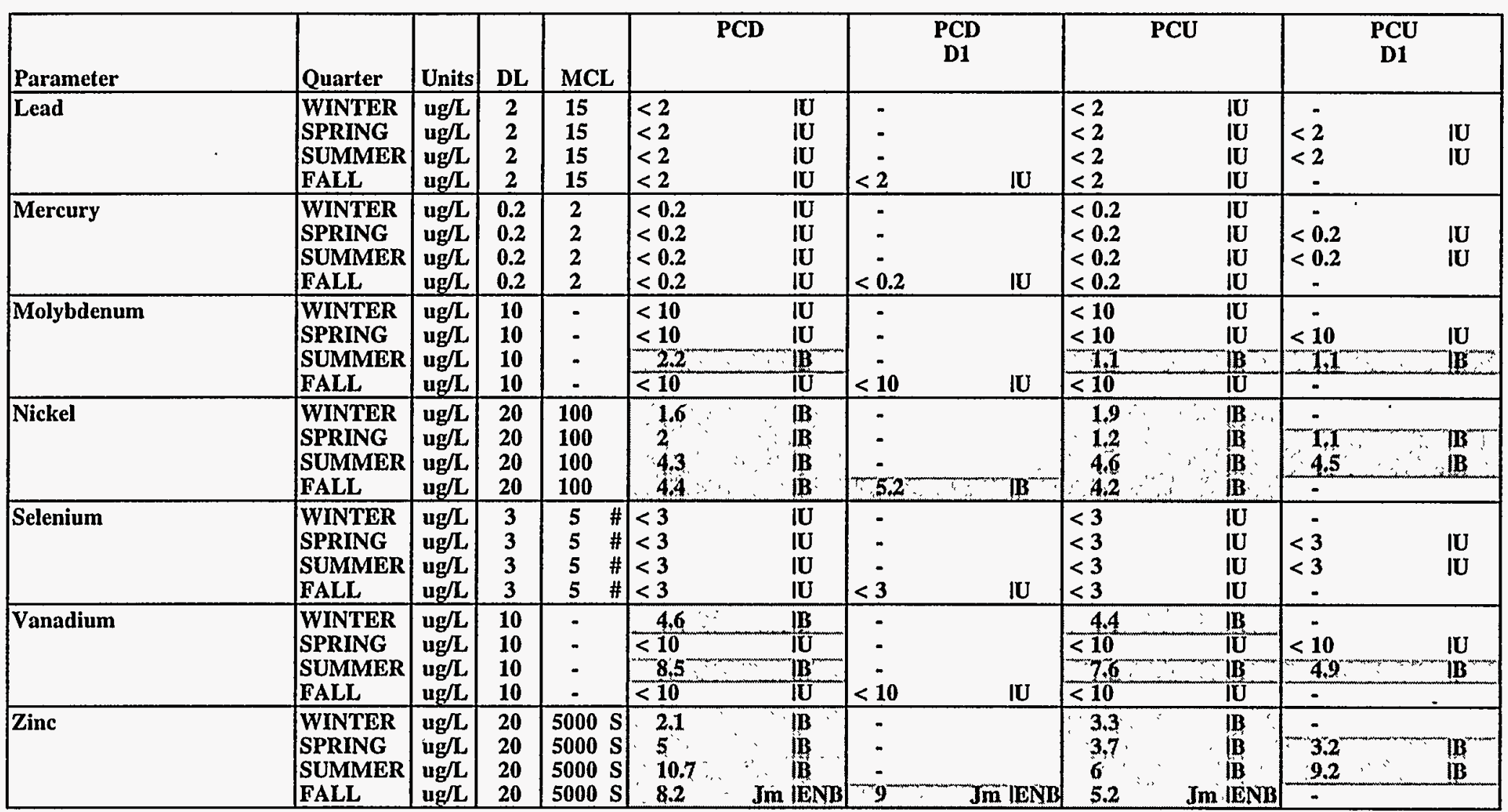


TABLE 6.5

SUMMARY OF DETECTED CONSTITUENTS IN SURFACE WATER AND STORM WATER, 1995

METALS

1995 ANNUAL WATER MONITORING REPORT

LEHR ENVIRONMENTAL RESTORATION, DAVIS, CALIFORNIA

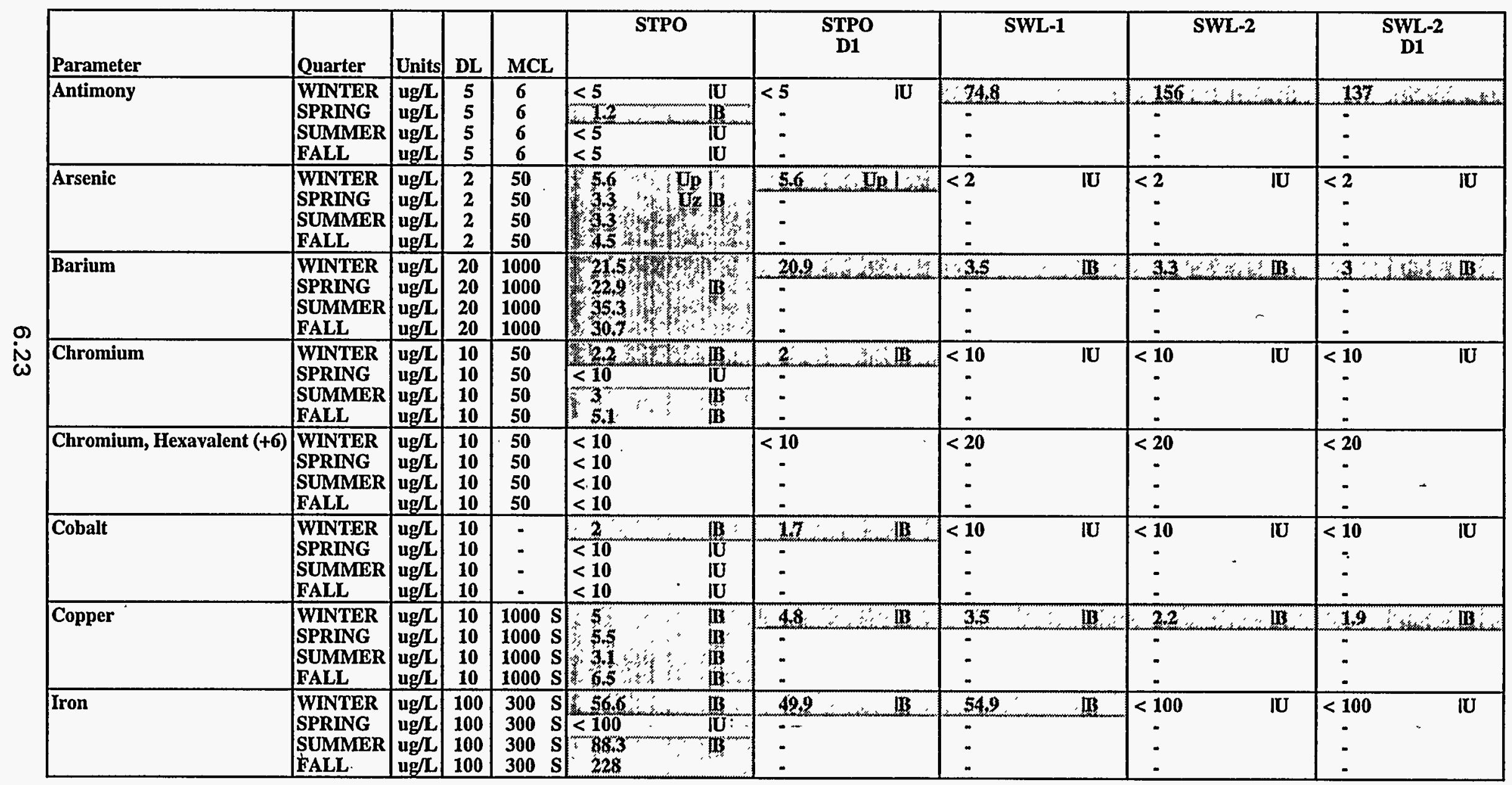


TABLE 6.5

SUMMARY OF DETECTED CONSTITUENTS IN SURFACE WATER AND STORM WATER, 1995

METALS

1995 ANNUAL WATER MONITORING REPORT

LEHR ENVIRONMENTAL RESTORATION, DAVIS, CALIFORNIA

\begin{tabular}{|c|c|c|c|c|c|c|c|c|c|}
\hline Parameter & Quarter & Units & DL & MCL & STPO & $\underset{\text { D1 }}{\text { STPO }}$ & SWL-1 & SWL-2 & $\begin{array}{c}\text { SWL-2 } \\
\text { D1 }\end{array}$ \\
\hline \multirow[t]{2}{*}{ Lead } & \multirow{2}{*}{$\begin{array}{l}\text { WINTER } \\
\text { SPRING } \\
\text { SUMMER } \\
\text { FALL }\end{array}$} & \multirow{2}{*}{$\begin{array}{l}\text { ug/L } \\
\text { ug/L } \\
\text { ug/L } \\
\text { ug/L }\end{array}$} & \multirow{2}{*}{$\begin{array}{l}2 \\
2 \\
2 \\
2 \\
2\end{array}$} & \multirow{2}{*}{$\begin{array}{l}15 \\
15 \\
15 \\
15\end{array}$} & \multirow{2}{*}{$\begin{array}{ll}<2 & \text { IU } \\
<2 & \text { IU } \\
<2 & \text { IU } \\
<2 & \text { IU }\end{array}$} & \multirow{2}{*}{$<2 \quad$ IU } & $2104, \cdots=3$ & $384,4=$ & $\therefore 35.9,8 \%$ \\
\hline & & & & & & & $\begin{array}{l}- \\
- \\
-\end{array}$ & - & 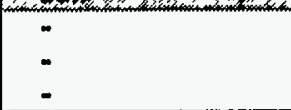 \\
\hline Mercury & $\begin{array}{l}\text { WINTER } \\
\text { SPRING } \\
\text { SUMMER } \\
\text { FALL }\end{array}$ & $\begin{array}{l}\operatorname{ug} / \mathbf{L} \\
\mathrm{ug} / \mathrm{L} \\
\mathrm{ug} / \mathrm{L} \\
\mathrm{ug} / \mathrm{L}\end{array}$ & $\begin{array}{l}0.2 \\
0.2 \\
0.2 \\
0.2\end{array}$ & $\begin{array}{l}2 \\
2 \\
2 \\
2\end{array}$ & \begin{tabular}{|ll}
$<0.2$ & IU \\
$<0.2$ & IU \\
$<0.2$ & IU \\
$<0.2$ & IU
\end{tabular} & $\begin{array}{c}<0.2 \\
- \\
-\end{array}$ & 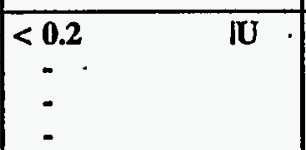 & 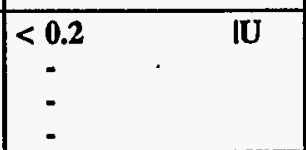 & $\begin{array}{c}<0.2 \\
\vdots \\
-\end{array}$ \\
\hline Molybdenum & $\begin{array}{l}\text { WINTER } \\
\text { SPRING } \\
\text { SUMMER } \\
\text { FALL } \\
\end{array}$ & $\begin{array}{l}\operatorname{ug} / \mathbf{L} \\
\mathrm{ug} / \mathrm{L} \\
\mathrm{ug} / \mathrm{L} \\
\mathrm{ug} / \mathrm{L}\end{array}$ & $\begin{array}{l}10 \\
10 \\
10 \\
10\end{array}$ & - & $\begin{array}{r}75 \\
6 \\
73 \\
10.7\end{array}$ & $\begin{array}{l}72 \\
- \\
- \\
-\end{array}$ & $\begin{array}{|cc|}<10 & \text { IU } \\
\vdots & \\
\end{array}$ & $\begin{array}{c}10 \\
\vdots \\
\\
\end{array}$ & $\begin{array}{c}<10 \\
- \\
- \\
\end{array}$ \\
\hline Nickel & $\begin{array}{l}\text { WINTER } \\
\text { SPRING } \\
\text { SUMMER } \\
\text { FALL } \\
\end{array}$ & \begin{tabular}{|l|}
$\mathrm{ug} / \mathrm{L}$ \\
$\mathrm{ug} / \mathrm{L}$ \\
$\mathrm{ug} / \mathrm{L}$ \\
$\mathrm{ug} / \mathrm{L}$
\end{tabular} & $\begin{array}{l}20 \\
20 \\
20 \\
20\end{array}$ & $\begin{array}{l}100 \\
100 \\
100 \\
100 \\
\end{array}$ & 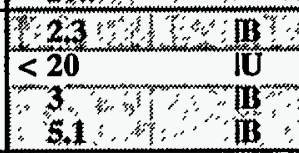 & $\begin{array}{l}21-11-13 \\
- \\
-\end{array}$ & $\begin{array}{l}1.6 \\
- \\
-\end{array}$ & $\begin{array}{c}<20 \\
: \\
-\end{array}$ & $\begin{array}{c}<20 \\
- \\
- \\
- \\
\end{array}$ \\
\hline Selenium & $\begin{array}{l}\text { WINTER } \\
\text { SPRING } \\
\text { SUMMER } \\
\text { FALL } \\
\end{array}$ & $\begin{array}{l}\text { ug/L } \\
\text { ug/L } \\
\text { ug/L } \\
\text { ug/L }\end{array}$ & $\begin{array}{l}3 \\
3 \\
3 \\
3 \\
\end{array}$ & $\begin{array}{ll}\mathbf{5} & \# \\
\mathbf{5} & \# \\
\mathbf{5} & \# \\
\mathbf{5} & \# \\
\end{array}$ & 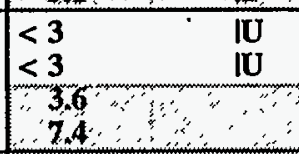 & $\begin{array}{cc}<3 & \text { IU } \\
- & \\
- & \\
\end{array}$ & $\begin{array}{r}<3 \\
: \\
:\end{array}$ & $\begin{array}{c}<3 \\
- \\
- \\
\end{array}$ & $\begin{array}{c}<3 \\
\vdots \\
- \\
\end{array}$ \\
\hline Vanadium & $\begin{array}{l}\text { WINTER } \\
\text { SPRING } \\
\text { SUMMER } \\
\text { FALL }\end{array}$ & \begin{tabular}{|l|}
$\operatorname{ug} / \mathbf{L}$ \\
$\operatorname{ug} / \mathbf{L}$ \\
$\operatorname{ug} / \mathbf{L}$ \\
$\operatorname{ug} / \mathrm{L}$
\end{tabular} & $\begin{array}{l}10 \\
10 \\
10 \\
10\end{array}$ & $:$ & 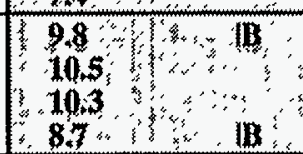 & $\begin{array}{l}93-x-1 \mathrm{~B}^{6} \\
- \\
-\end{array}$ & $\because \cdots \cdots$ & $\begin{array}{c}<10 \\
: \\
- \\
\end{array}$ & $\begin{array}{c}<10 \\
- \\
- \\
\end{array}$ \\
\hline Zinc & WINTER & & 20 & $5000 \mathrm{~S}$ & 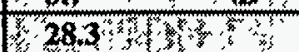 & $x^{2}+3$ & $60.8 \cdots$ & $\therefore 941$ & $112 \quad 3$ \\
\hline & $\begin{array}{l}\text { SPRING } \\
\text { SUMMER } \\
\text { FALL }\end{array}$ & $\begin{array}{l}\mathrm{ug} / \mathrm{L} \\
\mathrm{ug} / \mathrm{L} \\
\mathrm{ug} / \mathrm{L}\end{array}$ & $\begin{array}{l}20 \\
20 \\
20\end{array}$ & $\begin{array}{l}\mathbf{5 0 0 0} \mathrm{S} \\
\mathbf{5 0 0 0} \mathrm{S} \\
\mathbf{5 0 0 0} \mathrm{S}\end{array}$ & $\begin{array}{r}33,6 \\
39,2 \\
39,2\end{array}$ & 5 & 5 & $:$ & 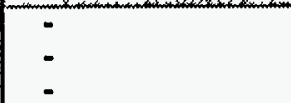 \\
\hline
\end{tabular}


TABLE 6.5

SUMMARY OF DETECTED CONSTITUENTS IN SURFACE WATER AND STORM WATER, 1995

METALS

1995 ANNUAL WATER MONITORING REPORT

LEHR ENVIRONMENTAL RESTORATION, DAVIS, CALIFORNIA

D1 = Duplicate sample.

- = Parameter not analyzed or no MCL.

DL = Detection limit.

$<=$ Constituent below detection limit. Detection limits may vary depending on interference by other sample constituents

MCL = Maximum Contaminant Level (primary), Title 22 Division 4 Chapter 15.

\# = USEPA MCL.

$\mathrm{S}=$ Secondary Drinking Water Standard.

Highlighted values indicate positive results.

See Appendix A for explanation of data qualifiers.

(1)

N 
TABLE 6.6

SUMMARY OF DETECTED CONSTITUENTS IN SURFACE WATER AND STORM WATER, 1995 RADIOCHEMICALS

1995 ANNUAL WATER MONITORING REPORT

LEHR ENVIRONMENTAL RESTORATION, DAVIS, CALIFORNIA

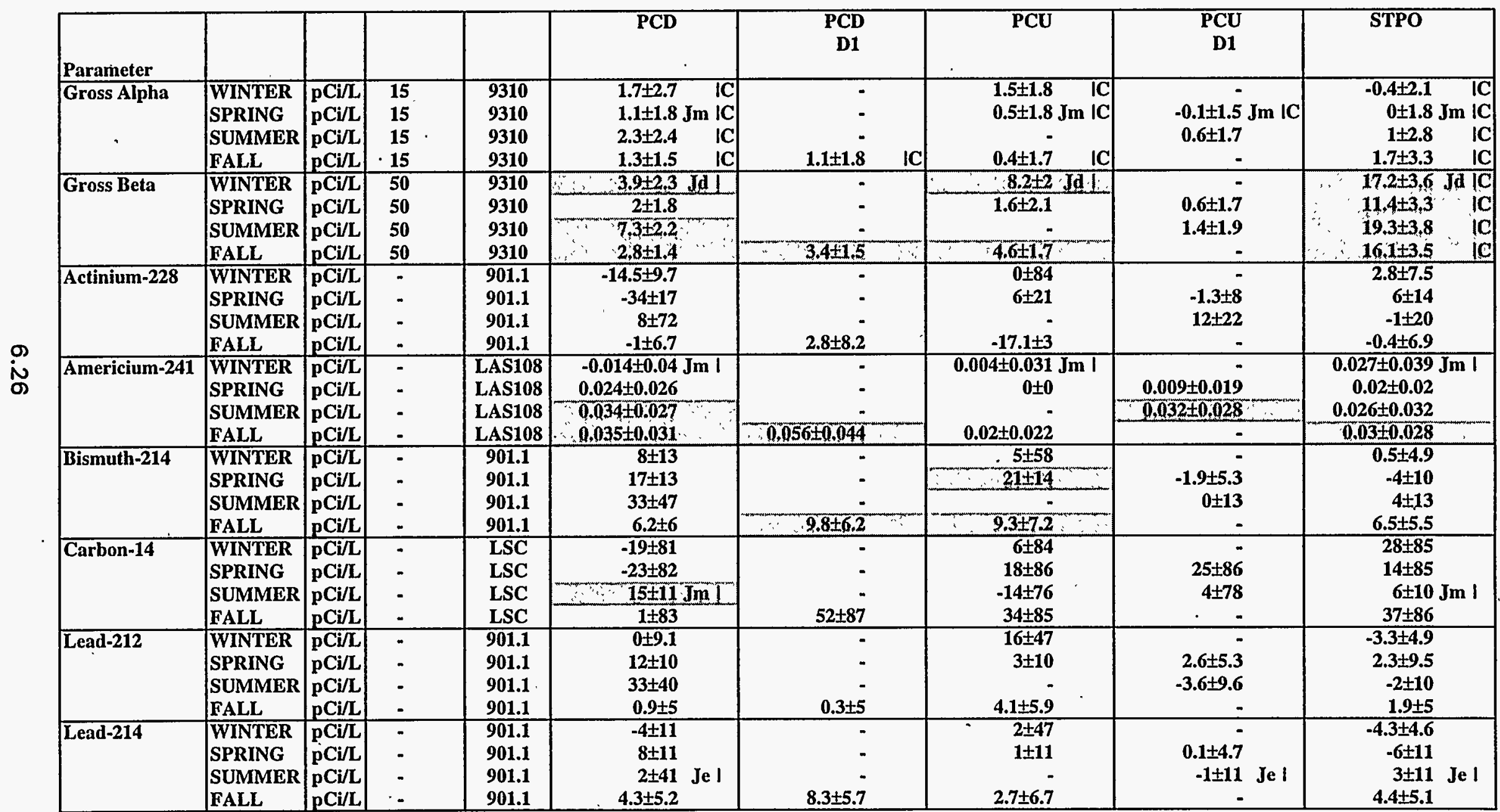


TABLE 6.6

SUMMARY OF DETECTED CONSTITUENTS IN SURFACE WATER AND STORM WATER, 1995

RADIOCHEMICALS

1995 ANNUAL WATER MONITORING REPORT

LEHR ENVIRONMENTAL RESTORATION, DAVIS, CALIFORNIA

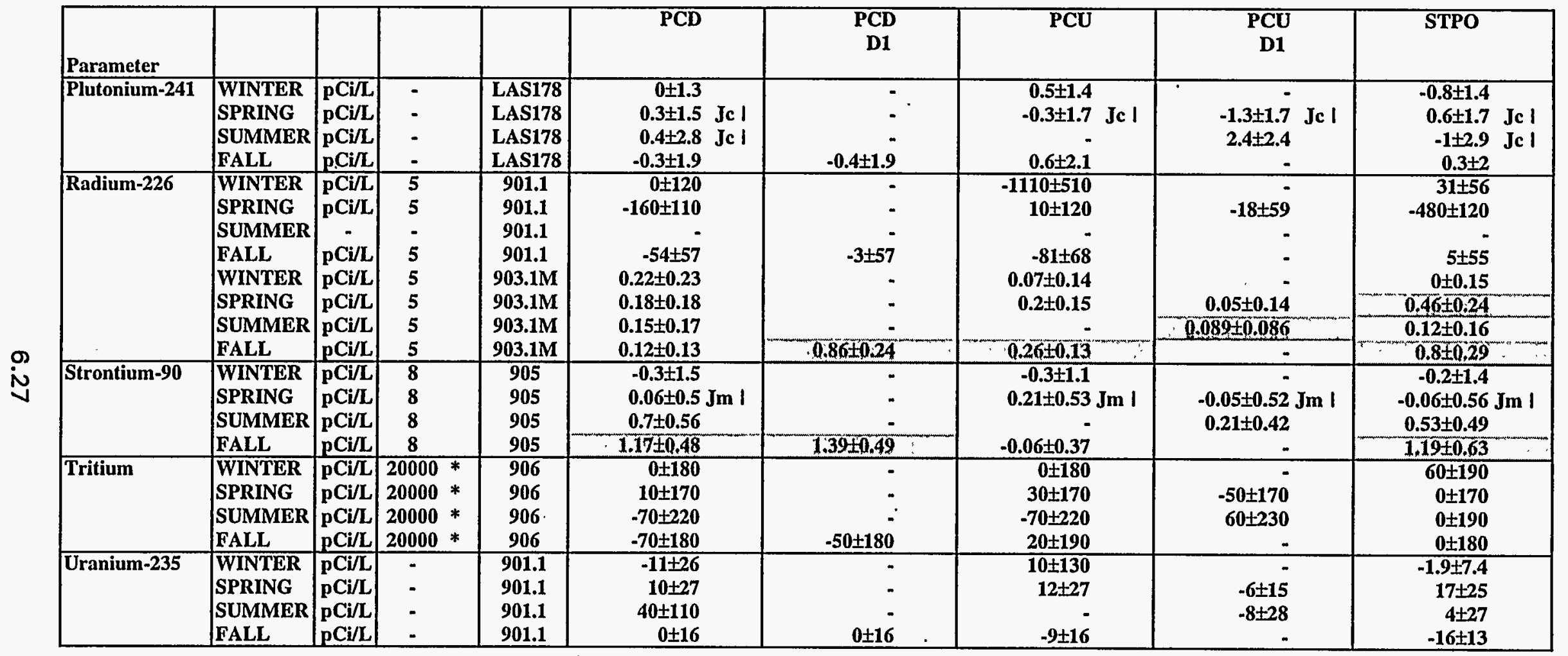


TABLE 6.6

SUMMARY OF DETECTED CONSTITUENTS IN SURFACE WATER AND STORM WATER, 1995 RADIOCHEMICALS

1995 ANNUAL WATER MONITORING REPORT

LEHR ENVIRONMENTAL RESTORATION, DAVIS, CALIFORNIA

\begin{tabular}{|c|c|c|c|c|c|c|c|c|}
\hline Parameter & & & & & $\begin{array}{r}\text { STPO } \\
\text { D1 }\end{array}$ & SWL-1 & SWL-2 & $\begin{array}{r}\text { SWL-2 } \\
\text { D1 }\end{array}$ \\
\hline Gross Alpha' & \begin{tabular}{|l|} 
WINTER \\
SPRING \\
SUMMER \\
FALL \\
\end{tabular} & $\begin{array}{l}\mathrm{pCi} / \mathrm{L} \\
\mathrm{pCi} / \mathrm{L} \\
\mathrm{pCi} / \mathrm{L} \\
\mathrm{pCi} / \mathrm{L}\end{array}$ & $\begin{array}{l}15 \\
15 \\
15 \\
15\end{array}$ & $\begin{array}{l}9310 \\
9310 \\
9310 \\
9310\end{array}$ & $\begin{array}{rr}3.2 \pm 3 & \mathbf{I C} \\
- & \\
- & \\
- & \end{array}$ & $\begin{array}{r}0.14 \pm 0.56 \\
- \\
- \\
-\end{array}$ & $\begin{array}{r}0.29 \pm 0.54 \\
- \\
-\end{array}$ & $\begin{array}{r}-0.06 \pm 0.46 \\
- \\
-\end{array}$ \\
\hline Gross Beta & $\begin{array}{l}\text { WINTER } \\
\text { SPRING } \\
\text { SUMMER } \\
\text { FALL }\end{array}$ & $\begin{array}{l}\text { pCi/L } \\
\text { pCi/L } \\
\text { pCi/L } \\
\text { pCi/L }\end{array}$ & $\begin{array}{l}\mathbf{5 0} \\
\mathbf{5 0} \\
\mathbf{5 0} \\
\mathbf{5 0}\end{array}$ & $\begin{array}{l}9310 \\
9310 \\
9310 \\
9310\end{array}$ & $\begin{array}{c}=1,29.544 \text { IJIC } \\
- \\
- \\
-\end{array}$ & $\begin{array}{r}1.7 \pm 1.3 \\
\because \\
-\end{array}$ & $\begin{array}{r}0.5 \pm 1.2 \\
- \\
- \\
-\end{array}$ & $\begin{array}{r}0.7 \pm 1.2 \\
- \\
- \\
\end{array}$ \\
\hline Actinium-228 & $\begin{array}{l}\text { WINTER } \\
\text { SPRING } \\
\text { SUMMER } \\
\text { FALL } \\
\end{array}$ & $\begin{array}{l}\mathrm{pCi} / \mathrm{L} \\
\mathrm{pCi} / \mathrm{L} \\
\mathrm{pCi} / \mathrm{L} \\
\mathrm{pCi} / \mathrm{L}\end{array}$ & 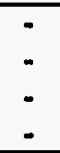 & $\begin{array}{l}901.1 \\
901.1 \\
901.1 \\
901.1\end{array}$ & $\begin{array}{r}3.8 \pm 7.4 \\
- \\
- \\
\end{array}$ & $\begin{array}{r}-1.2 \pm 7.4 \\
- \\
-\end{array}$ & $\begin{array}{r}2+21 \\
- \\
-\end{array}$ & $\begin{array}{r}0.7 \pm 7.7 \\
- \\
-\end{array}$ \\
\hline Americium-241 & $\begin{array}{l}\text { WINTER } \\
\text { SPRING } \\
\text { SUMMER } \\
\text { FALL } \\
\end{array}$ & $\begin{array}{l}\mathrm{pCi} / \mathrm{L} \\
\mathrm{pCi} / \mathrm{L} \\
\mathrm{pCi} / \mathrm{L} \\
\mathrm{pCi} / \mathrm{L}\end{array}$ & $\begin{array}{l}- \\
- \\
-\end{array}$ & $\begin{array}{l}\text { LAS108 } \\
\text { LAS108 } \\
\text { LAS108 } \\
\text { LAS108 }\end{array}$ & $\begin{array}{c}0.097 \pm 0.075 \text { Jm IY } \\
- \\
- \\
-\end{array}$ & $\begin{array}{r}0.022 \pm 0.024 \\
- \\
-\end{array}$ & $\begin{array}{r}0.012 \pm 0.031 \\
- \\
-\end{array}$ & $\begin{array}{r}0.011 \pm 0.021 \\
- \\
-\end{array}$ \\
\hline Bismuth-214 & $\begin{array}{l}\text { WINTER } \\
\text { SPRING } \\
\text { SUMMER } \\
\text { FALL }\end{array}$ & $\begin{array}{l}\mathrm{pCi} / \mathrm{L} \\
\mathrm{pCi} / \mathrm{L} \\
\mathrm{pCi} / \mathrm{L} \\
\mathrm{pCi} / \mathrm{L}\end{array}$ & $\begin{array}{l}- \\
.\end{array}$ & $\begin{array}{l}901.1 \\
901.1 \\
901.1 \\
901.1\end{array}$ & $\begin{array}{r}6.4 \pm 5.3 \\
. \\
.\end{array}$ & $\begin{array}{r}0.5 \pm 5.3 \\
- \\
-\end{array}$ & $\begin{array}{r}-6 \pm 10 \\
. \\
-\end{array}$ & $\begin{array}{r}-1.4 \pm 5.3 \\
- \\
-\end{array}$ \\
\hline Carbon-14 & $\begin{array}{l}\text { WINTER } \\
\text { SPRING } \\
\text { SUMMER } \\
\text { FALL } \\
\end{array}$ & $\begin{array}{l}\mathrm{pCi} / \mathrm{L} \\
\mathrm{pCi} / \mathrm{L} \\
\mathrm{pCi} / \mathrm{L} \\
\mathrm{pCi} / \mathrm{L}\end{array}$ & $\begin{array}{l}- \\
- \\
.\end{array}$ & $\begin{array}{l}\text { LSC } \\
\text { LSC } \\
\text { LSC } \\
\text { LSC }\end{array}$ & $\begin{array}{r}-16 \pm 82 \\
- \\
- \\
-\end{array}$ & $\begin{array}{r}16 \pm 83 \\
- \\
- \\
-\end{array}$ & $\begin{array}{r}-3 \pm 81 \\
- \\
- \\
-\end{array}$ & $\begin{array}{r}-57 \pm 76 \\
- \\
-\end{array}$ \\
\hline Lead-212 & $\begin{array}{l}\text { WINTER } \\
\text { SPRING } \\
\text { SUMMER } \\
\text { FALL } \\
\end{array}$ & $\begin{array}{l}\mathrm{pCi} / \mathrm{L} \\
\mathrm{pCi} / \mathrm{L} \\
\mathrm{pCi} / \mathrm{L} \\
\mathrm{pCi} / \mathrm{L}\end{array}$ & $\begin{array}{l}\text { - } \\
. \\
.\end{array}$ & $\begin{array}{l}901.1 \\
901.1 \\
901.1 \\
901.1\end{array}$ & $\begin{array}{r}2.7 \pm 5.1 \\
- \\
-\end{array}$ & $\begin{array}{r}2.3 \pm 5.1 \\
- \\
- \\
\end{array}$ & $\begin{array}{r}2 \pm 10 \\
- \\
-\end{array}$ & $\begin{array}{r}-3.1 \pm 5.1 \\
- \\
-\end{array}$ \\
\hline Lead-214 & $\begin{array}{l}\text { WINTER } \\
\text { SPRING } \\
\text { SUMMER } \\
\text { FALL } \\
\end{array}$ & $\begin{array}{l}\mathrm{pCi} / \mathrm{L} \\
\mathrm{pCi} / \mathrm{L} \\
\mathrm{pCi} / \mathrm{L} \\
\mathrm{pCi} / \mathrm{L}\end{array}$ & $\begin{array}{l}- \\
. \\
.\end{array}$ & $\begin{array}{l}901.1 \\
901.1 \\
901.1 \\
901.1\end{array}$ & $\begin{array}{r}4.3 \pm 5 \\
- \\
- \\
-\end{array}$ & $\begin{array}{r}-1.9 \pm 4.9 \\
- \\
-\end{array}$ & $\begin{array}{r}0 \pm 11 \\
- \\
- \\
-\end{array}$ & $\begin{array}{r}-3.8 \pm 3.9 \\
- \\
-\end{array}$ \\
\hline
\end{tabular}


TABLE 6.6

SUMMARY OF DETECTED CONSTITUENTS IN SURFACE WATER AND STORM WATER, 1995

RADIOCHEMICALS

1995 ANNUAL WATER MONITORING REPORT

LEHR ENVIRONMENTAL RESTORATION, DAVIS, CALIFORNIA

\begin{tabular}{|c|c|c|c|c|c|c|c|c|}
\hline Parameter & & & & & $\begin{array}{r}\text { STPO } \\
\text { D1 }\end{array}$ & SWL-1 & SWL-2 & $\begin{array}{r}\text { SWL-2 } \\
\text { D1 }\end{array}$ \\
\hline Plutonium-241 & $\begin{array}{l}\text { WINTER } \\
\text { SPRING } \\
\text { SUMMER } \\
\text { FALL } \\
\end{array}$ & $\begin{array}{l}\mathrm{pCi} / \mathrm{L} \\
\mathrm{pCi} / \mathrm{L} \\
\mathrm{pCi} / \mathrm{L} \\
\mathrm{pCi} / \mathrm{L} \\
\end{array}$ & $\begin{array}{l}- \\
- \\
-\end{array}$ & $\begin{array}{l}\text { LAS178 } \\
\text { LAS178 } \\
\text { LAS178 } \\
\text { LAS178 } \\
\end{array}$ & $\begin{array}{r}-0.4 \pm 1.2 \\
- \\
- \\
-\end{array}$ & $\begin{array}{r}-0.5 \pm 1.4 \\
: \\
- \\
-\end{array}$ & $\begin{array}{r}0.3 \pm 1.6 \\
- \\
-\end{array}$ & $\begin{array}{r}-1.1+2.4 \\
- \\
- \\
\end{array}$ \\
\hline Radium-226 & \begin{tabular}{|l} 
WINTER \\
SPRING \\
SUMMER \\
FALL \\
WINTER \\
SPRING \\
SUMMER \\
FALL
\end{tabular} & $\begin{array}{l}\mathrm{pCi} / \mathrm{L} \\
\mathrm{pCi} / \mathrm{L} \\
- \\
\mathrm{pCi} / \mathrm{L} \\
\mathrm{pCi} / \mathrm{L} \\
\mathrm{pCi} / \mathrm{L} \\
\mathrm{pCi} / \mathrm{L} \\
\mathrm{pCi} / \mathrm{L}\end{array}$ & $\begin{array}{l}5 \\
5 \\
- \\
5 \\
5 \\
5 \\
5 \\
5\end{array}$ & \begin{tabular}{|c|}
901.1 \\
901.1 \\
901.1 \\
901.1 \\
$903.1 \mathrm{M}$ \\
$903.1 \mathrm{M}$ \\
$903.1 \mathrm{M}$ \\
$903.1 \mathrm{M}$ \\
\end{tabular} & $\begin{array}{r}-34 \pm 54 \\
- \\
- \\
0.17 \pm 0.17 \\
- \\
-\end{array}$ & $\begin{array}{r}-55 \pm 55 \\
- \\
0 \pm 0.13 \\
- \\
- \\
-\end{array}$ & $\begin{array}{r}-80 \pm 110 \\
- \\
-0.039 \pm 0.093 \\
- \\
-\end{array}$ & $\begin{array}{r}-68 \pm 57 \\
- \\
- \\
0.2 \pm 0.17 \\
- \\
- \\
\end{array}$ \\
\hline Strontium-90 & $\begin{array}{l}\text { WINTER } \\
\text { SPRING } \\
\text { SUMMER } \\
\text { FALL }\end{array}$ & $\begin{array}{l}\mathrm{pCi} / \mathrm{L} \\
\mathrm{pCi} / \mathrm{L} \\
\mathrm{pCi} / \mathrm{L} \\
\mathrm{pCi} / \mathrm{L}\end{array}$ & \begin{tabular}{|l|}
8 \\
8 \\
8 \\
8 \\
\end{tabular} & $\begin{array}{l}905 \\
905 \\
905 \\
905\end{array}$ & $\begin{array}{r}-0.5 \pm 8.7 \\
: \\
-\end{array}$ & $\begin{array}{r}-0.03 \pm 0.77 \\
- \\
- \\
\end{array}$ & $\begin{array}{r}0.06 \pm 0.77 \\
- \\
- \\
-\end{array}$ & $\begin{array}{r}-0.17 \pm 0.81 \\
- \\
- \\
-\end{array}$ \\
\hline Tritium & $\begin{array}{l}\text { WINTER } \\
\text { SPRING } \\
\text { SUMMER } \\
\text { FALL }\end{array}$ & $\begin{array}{l}\mathrm{pCi} / \mathrm{L} \\
\mathrm{pCi} / \mathrm{L} \\
\mathrm{pCi} / \mathrm{L} \\
\mathrm{pCi} / \mathrm{L}\end{array}$ & $\begin{array}{l}20000 * \\
20000 * \\
20000 * \\
20000 *\end{array}$ & $\begin{array}{l}906 \\
906 \\
906 \\
906\end{array}$ & $\begin{array}{r}50 \pm 190 \\
- \\
- \\
-\end{array}$ & $\begin{array}{c}-40 \pm 200 \mathrm{Jm} \mathrm{I} \\
- \\
-\end{array}$ & $\begin{array}{c}-60 \pm 200 \mathrm{Jm} \mathrm{I} \\
\vdots \\
-\end{array}$ & $\begin{array}{c}150 \pm 220 \mathrm{Jm} \mathrm{I} \\
- \\
-\end{array}$ \\
\hline Uranium-235 & $\begin{array}{l}\text { WINTER } \\
\text { SPRING } \\
\text { SUMMER } \\
\text { FALL }\end{array}$ & $\begin{array}{l}\mathrm{pCi} / \mathrm{L} \\
\mathrm{pCi} / \mathrm{L} \\
\mathrm{pCi} / \mathrm{L} \\
\mathrm{pCi} / \mathrm{L}\end{array}$ & $\begin{array}{l}- \\
- \\
-\end{array}$ & $\begin{array}{l}901.1 \\
901.1 \\
901.1 \\
901.1\end{array}$ & $\begin{array}{r}-3 \pm 14 \\
: \\
:\end{array}$ & $\begin{array}{r}6 \pm 14 \\
- \\
-\end{array}$ & $\begin{array}{r}-6 \pm 28 \\
: \\
-\end{array}$ & $\begin{array}{r}0 \pm 14 \\
- \\
-\end{array}$ \\
\hline
\end{tabular}




\section{TABLE 6.6 \\ SUMMARY OF DETECTED CONSTITUENTS IN SURFACE WATER AND STORM WATER, 1995 \\ RADIOCHEMICALS \\ 1995 ANNUAL WATER MONITORING REPORT \\ LEHR ENVIRONMENTAL RESTORATION, DAVIS, CALIFORNIA}

\section{D1 = Duplicate sample.}

- = Not analyzed.

MCL = Maximum Contaminant Level (primary்), Title 22, Division 4, Chapter 15.

* = Proposed MCL for Tritium is $60000 \mathrm{pCi} / \mathrm{L}$.

$\mathrm{a}=$ Reported tritium activity of $50 \mathrm{pCi} / \mathrm{L}$ is suspected to belong to $\mathrm{UCD1-20}$. The tritium activity for $\mathrm{UCD1-20}$ was reported as $16,600 \mathrm{pCi} / \mathrm{L}$, which is more consistent with the historical trend for UCD1-13.

$b=$ Reported tritium activity of $16,600 \mathrm{pCi} / \mathrm{L}$ is suspected to belong to $\mathrm{UCD1}-13$. The tritium activity for $\mathrm{UCD1}-13$ was $\mathrm{reported}$ as $50 \mathrm{pCi} / \mathrm{L}$, which is more consistent with the historical trend for UCD1-20.

Highlighted values indicate results where the value is greater than the calculated uncertainty.

Note: Radionuclide results are reported as calculated value, $V$, followed by the total uncertainty, $T$, in the format $V \pm T$. A calculated value that equals or exceeds the uncertainty ("positive value") represents $95 \%$ level of confidence that measurable radioactivity above zero is present for a given radionuclide.

See Appendix A for explanation of data qualifiers. 
TABLE 6.7

SUMMARY OF DETECTED CONSTITUENTS IN SURFACE WATER AND STORM WATER, 1995

ANIONS AND CATIONS

1995 ANNUAL WATER MONITORING REPORT

LEHR ENVIRONMENTAL RESTORATION, DAVIS, CALIFORNIA

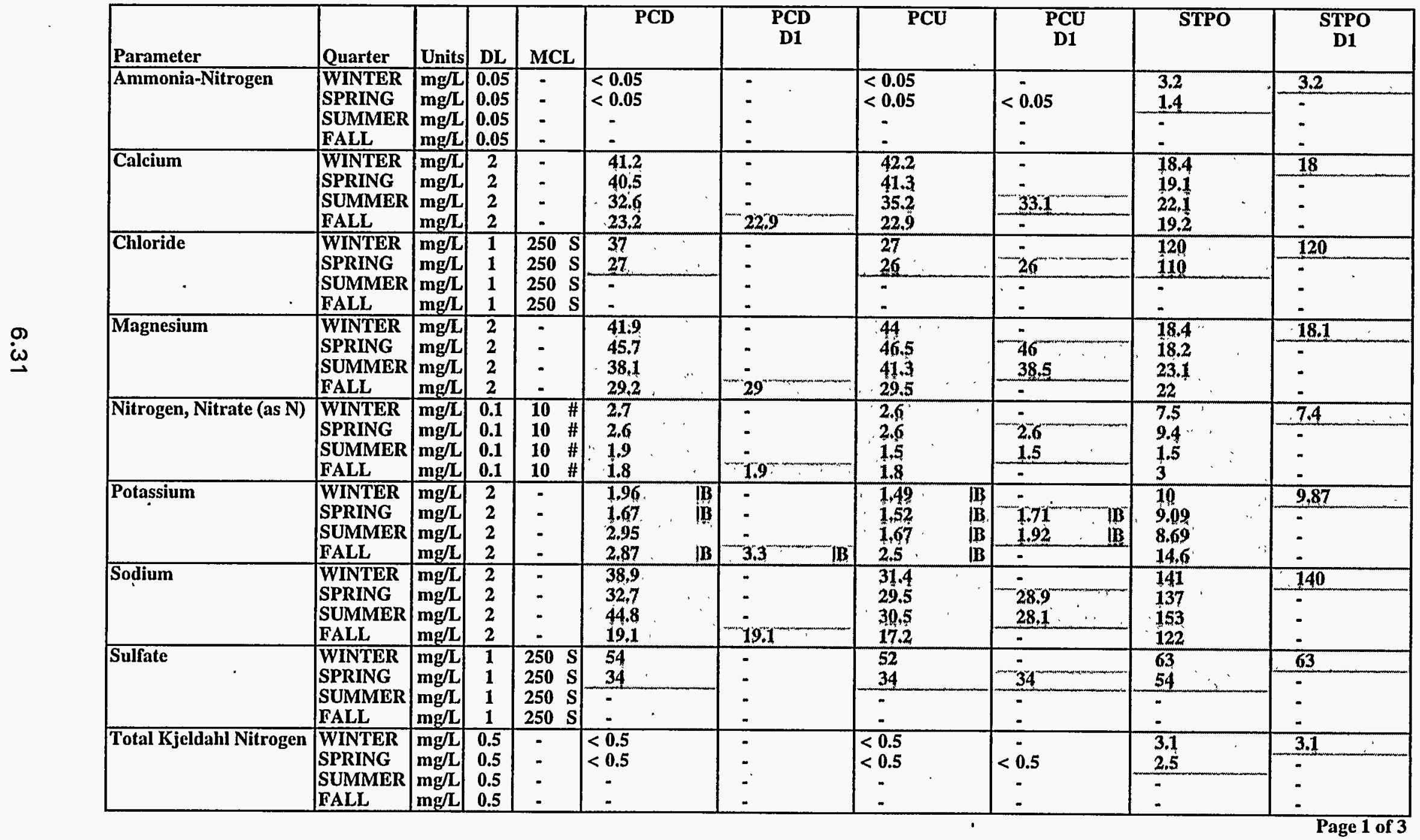


TABLE 6.7

SUMMARY OF DETECTED CONSTITUENTS IN SURFACE WATER AND STORM WATER, 1995 ANIONS AND CATIONS

1995 ANNUAL WATER MONITORING REPORT

LEHR ENVIRONMENTAL RESTORATION, DAVIS, CALIFORNIA

\begin{tabular}{|c|c|c|c|c|c|c|c|}
\hline Parameter & Quarter & Units & DL & MCL & SWL-1 & SWL-2 & $\begin{array}{c}\text { SWL-2 } \\
\text { D1 }\end{array}$ \\
\hline \multirow[t]{3}{*}{ Ammonia-Nitrogen } & WINTER & $\mathrm{mg} / \mathrm{L}$ & 0.05 & - & - & - & - \\
\hline & $\begin{array}{l}\text { SPRING } \\
\text { SUMMTER }\end{array}$ & $\mathrm{mg} / \mathrm{L}$ & $\begin{array}{l}0.05 \\
0.05\end{array}$ & 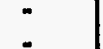 & 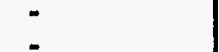 & : & : \\
\hline & FALL & $\mathrm{mg} / \mathrm{L}$ & 0.05 & - & - & & \\
\hline \multirow[t]{3}{*}{ Calcium } & \multirow{3}{*}{$\begin{array}{l}\text { WINTER } \\
\text { SPRING } \\
\text { SUMMER } \\
\text { FALL }\end{array}$} & \multirow{3}{*}{\begin{tabular}{l|}
$\mathrm{mg} / \mathrm{L}$ \\
$\mathrm{mg} / \mathrm{L}$ \\
$\mathrm{mg} / \mathrm{L}$ \\
$\mathrm{mg} / \mathrm{L}$
\end{tabular}} & \multirow{3}{*}{$\begin{array}{l}2 \\
2 \\
2 \\
2\end{array}$} & \multirow[t]{3}{*}{ - } & 135 & 0.573 & 20598 ; 18 \\
\hline & & & & & - & - & - \\
\hline & & & & & 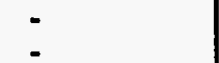 & $:$ & $:$ \\
\hline \multirow[t]{4}{*}{ Chloride } & WINTER & $\mathrm{mg} / \mathbf{L}$ & $\overline{1}$ & $250 \mathrm{~s}$ & 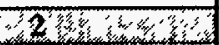 & $<1$ & $<1$ \\
\hline & SPRING & $\mathrm{mg} / \mathrm{L}$ & 1 & $250 \mathrm{~s}$ & - & - & - \\
\hline & SUMMER & $\mathrm{mg} / \mathrm{L}$ & 1 & $250 \mathrm{~s}$ & - & - & - \\
\hline & FALL & $\mathrm{mg} / \mathrm{L}$ & 1 & $250 \mathrm{~s}$ & - & & \\
\hline \multirow[t]{3}{*}{ Magnesium } & \multirow{3}{*}{$\begin{array}{l}\text { WINTER } \\
\text { SPRING } \\
\text { SUMMER } \\
\text { FALL }\end{array}$} & \multirow{3}{*}{$\begin{array}{l}\mathrm{mg} / \mathrm{L} \\
\mathrm{mg} / \mathrm{L} \\
\mathrm{mg} / \mathrm{L} \\
\mathrm{mg} / \mathrm{L}\end{array}$} & \multirow{3}{*}{$\begin{array}{l}2 \\
2 \\
2 \\
2\end{array}$} & \multirow{3}{*}{ 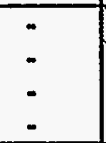 } & $0,455 \leqslant 313$ & D.126 $\div \mathrm{BB}$ & $1-0128$ \\
\hline & & & & & - & - & \\
\hline & & & & & :- & 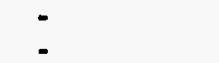 & : \\
\hline \multirow[t]{4}{*}{ Nitrogen, Nitrate (as N) } & \multirow{4}{*}{\begin{tabular}{|l|} 
WINTER \\
SPRING \\
SUMMER \\
FALL
\end{tabular}} & \multirow{4}{*}{$\begin{array}{l}\mathrm{mg} / \mathrm{L} \\
\mathrm{mg} / \mathrm{L} \\
\mathrm{mg} / \mathrm{h} \\
\mathrm{mg} / \mathrm{L}\end{array}$} & 0.1 & \multirow{4}{*}{$\begin{array}{ll}10 & \# \\
10 & \# \\
10 & \# \\
10 & \#\end{array}$} & 0.24 & $\mathbf{0 . 1 3}$ & \multirow{4}{*}{$\begin{array}{c}<0.1 \\
- \\
-\end{array}$} \\
\hline & & & 0.1 & & - & - & \\
\hline & & & 0.1 & & - & - & \\
\hline & & & 0.1 & & - & & \\
\hline \multirow[t]{3}{*}{ Potassium } & \multirow{3}{*}{$\begin{array}{l}\text { WINTER } \\
\text { SPRING } \\
\text { SUMMER } \\
\text { FALL } \\
\end{array}$} & \multirow{3}{*}{$\begin{array}{l}\mathrm{mg} / \mathrm{L} \\
\mathrm{mg} / \mathrm{L} \\
\mathrm{mg} / \mathrm{L} \\
\mathrm{mg} / \mathrm{L}\end{array}$} & \multirow{3}{*}{$\begin{array}{l}2 \\
2 \\
2 \\
2 \\
\end{array}$} & \multirow[t]{3}{*}{ " } & 1.3 & $0.888+78$ & \multirow{3}{*}{$<2$} \\
\hline & & & & & - & $-\infty$ & \\
\hline & & & & & - & - & \\
\hline \multirow[t]{3}{*}{ Sodium } & \multirow{4}{*}{$\begin{array}{l}\text { WINTER } \\
\text { SPRING } \\
\text { SUMMER } \\
\text { FALL } \\
\end{array}$} & \multirow{3}{*}{\begin{tabular}{l|}
$\mathrm{mg} / \mathrm{L}$ \\
$\mathrm{mg} / \mathrm{L}$ \\
$\mathrm{mg} / \mathrm{L}$ \\
$\mathrm{mg} / \mathrm{L}$
\end{tabular}} & \multirow{3}{*}{$\begin{array}{l}2 \\
2 \\
2 \\
2\end{array}$} & \multirow{3}{*}{. } & 3,66 & $0.286: \mathbb{B}$ & $20.266=2 B$ \\
\hline & & & & & & & \\
\hline & & & & & - & - & - \\
\hline \multirow{4}{*}{ Sulfate } & & $\mid \frac{\mathrm{mg} / \mathrm{L}}{\mathrm{mg} / \mathrm{L}}$ & $\frac{2}{1}$ & \multirow{4}{*}{$\begin{array}{|ll|}250 & \mathrm{~S} \\
250 & \mathrm{~S} \\
250 & \mathrm{~S} \\
250 & \mathrm{~S} \\
\end{array}$} & 12 & $<i$ & $<\dot{1}$ \\
\hline & SPRING & $\mathrm{mg} / \mathrm{L}$ & 1 & & & - & $\because$ \\
\hline & SUMMER & $\mathrm{mg} / \mathrm{L}$ & 1 & & - & - & - \\
\hline & & $\mathrm{mg} / \mathrm{L}$ & 1 & & $=$ & - & - \\
\hline Total Kjeldahl Nitrogen & WINTER & $\mathrm{mg} / \mathrm{L}$ & 0.5 & - & - & - & - \\
\hline & SPRIN & $\mathrm{mg} / \mathrm{L}$ & 0.5 & - & - & - & - \\
\hline & FALL & $\begin{array}{l}\mathrm{mg} / \mathrm{L} \\
\mathrm{mg} / \mathrm{L}\end{array}$ & 0.5 & - & - & : & \\
\hline
\end{tabular}


TABLE 6.7

SUMMARY OF DETECTED CONSTITUENTS IN SURFACE WATER AND STORM WATER, 1995

ANIONS AND CATIONS

1995 ANNUAL WATER MONITORING REPORT

LEHR ENVIRONMENTAL RESTORATION, DAVIS, CALIFORNIA

D1 = Duplicate sample.

- = Parameter not analyzed or no MCL.

DL $=$ Detection limit.

$<=$ Constituent below detection limit. Detection limits may vary depending on interference by other sample constituents.

MCL = Maximum Contaminant Level (primary), Title 22 Division 4 Chapter 15.

\# = USEPA MCL.

$S=$ Secondary drinking water standard.

Highlighted values indicate positive results.

See Appendix A for explanation of data qualifiers. 
TABLE 6.8

SUMMARY OF DETECTED CONSTITUENTS IN SURFACE WATER AND STORM WATER, 1995 OTHER PARAMETERS

1995 ANNUAL WATER MONITORING REPORT

LEHR ENVIRONMENTAL RESTORATION, DAVIS, CALIFORNIA

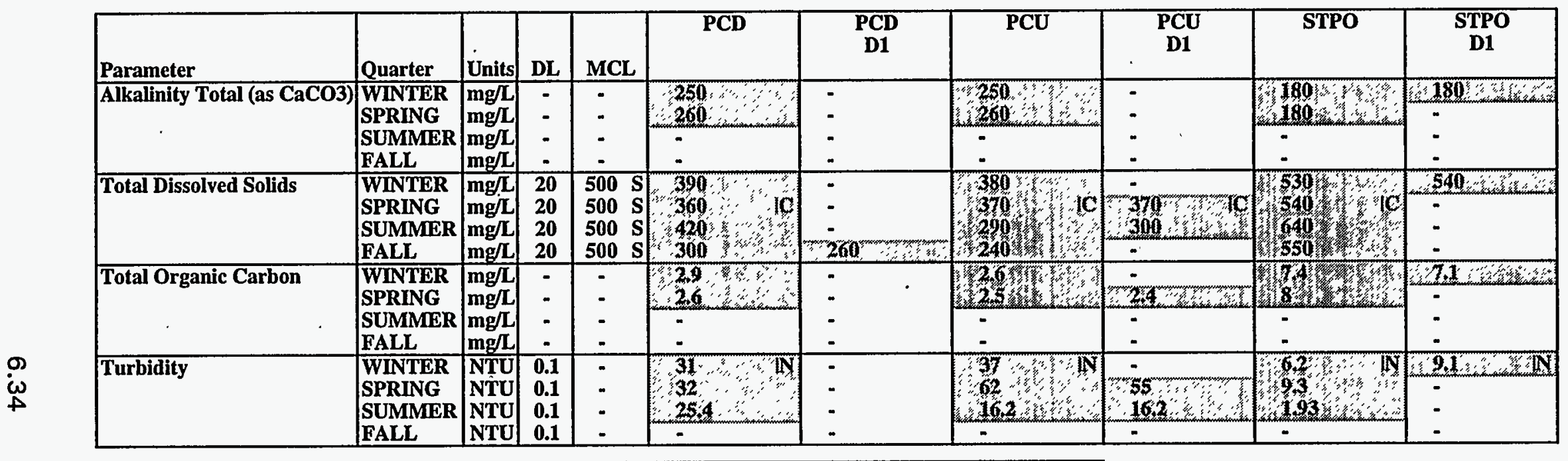

\begin{tabular}{|c|c|c|c|c|c|c|c|}
\hline Parameter & Quarter & Units & DL & MCL & SWL-1 & SWL-2 & $\begin{array}{c}\text { SWL-2 } \\
\text { D1 }\end{array}$ \\
\hline \multirow[t]{2}{*}{ Alkalinity Total (as CaCO3) } & \multirow{2}{*}{$\begin{array}{l}\text { WINTER } \\
\text { SPRING } \\
\text { SUMMER } \\
\text { FALL } \\
\end{array}$} & \multirow{2}{*}{$\begin{array}{l}\mathrm{mg} / \mathrm{L} \\
\mathrm{mg} / \mathrm{L} \\
\mathrm{mg} / \mathrm{L} \\
\mathrm{mg} / \mathrm{L}\end{array}$} & \multirow{2}{*}{$\begin{array}{l}- \\
- \\
-\end{array}$} & \multirow{2}{*}{$\begin{array}{l}- \\
- \\
- \\
-\end{array}$} & 15 & 14 & $14 \div$ \\
\hline & & & & & $\begin{array}{l}- \\
- \\
- \\
\end{array}$ & $\begin{array}{l}- \\
- \\
-\end{array}$ & $\begin{array}{l}- \\
- \\
- \\
\end{array}$ \\
\hline Total Dissolved Solids & \begin{tabular}{l|} 
WINTER \\
SPRING \\
SUMMER \\
FALL \\
\end{tabular} & $\begin{array}{l}\mathrm{mg} / \mathrm{L} \\
\mathrm{mg} / \mathrm{L} \\
\mathrm{mg} / \mathrm{L} \\
\mathrm{mg} / \mathrm{L}\end{array}$ & $\begin{array}{l}20 \\
20 \\
20 \\
20 \\
\end{array}$ & $\begin{array}{ll}500 & S \\
500 & S \\
500 & S \\
500 & S \\
\end{array}$ & $\begin{array}{l}25 \\
- \\
- \\
-\end{array}$ & $\begin{array}{c}<20 \\
- \\
- \\
-\end{array}$ & $\begin{array}{c}<20 \\
- \\
- \\
-\end{array}$ \\
\hline \multirow[t]{2}{*}{ Total Organic Carbon } & \multirow{2}{*}{$\begin{array}{l}\text { WINTER } \\
\text { SPRING } \\
\text { SUMMER } \\
\text { FALL }\end{array}$} & \multirow{2}{*}{$\begin{array}{l}\mathrm{mg} / \mathrm{L} \\
\mathrm{mg} / \mathrm{L} \\
\mathrm{mg} / \mathrm{L} \\
\mathrm{mg} / \mathrm{L}\end{array}$} & \multirow{2}{*}{$\begin{array}{l}- \\
- \\
-\end{array}$} & \multirow{2}{*}{$\begin{array}{l}- \\
\end{array}$} & 6.8 & 25 & 25 \\
\hline & & & & & $\begin{array}{l}- \\
- \\
\end{array}$ & $\begin{array}{l}- \\
- \\
- \\
\end{array}$ & $\begin{array}{l}- \\
- \\
-\end{array}$ \\
\hline \multirow[t]{2}{*}{ Turbidity } & \multirow{2}{*}{$\begin{array}{l}\text { WINTER } \\
\text { SPRING } \\
\text { SUMMER } \\
\text { FALL } \\
\end{array}$} & \multirow{2}{*}{\begin{tabular}{|l|} 
NTU \\
NTU \\
NTU \\
NTU
\end{tabular}} & \multirow{2}{*}{$\begin{array}{l}0.1 \\
0.1 \\
0.1 \\
0.1 \\
\end{array}$} & \multirow{2}{*}{ - } & 17 & 3.1 & 31 \\
\hline & & & & & $\begin{array}{l}- \\
- \\
-\end{array}$ & - & - \\
\hline
\end{tabular}


TABLE 6.8

SUMMARY OF DETECTED CONSTITUENTS IN SURFACE WATER AND STORM WATER, 1995

OTHER PARAMETERS

1995 ANNUAL WATER MONITORING REPOR'

\section{LEHR ENVIRONMENTAL RESTORATION, DAVIS, CALIFORNIA}

D1 = Duplicate sample.

- Parameter not analyzed or no MCL.

DL $=$ Detection limit.

$<=$ Constituent below detection limit. Detection limits may vary depending on interference by other sample constituents. MCL = Maximum Contaminant Level (primary), Title 22 Division 4 Chapter 15.

$\mathrm{S}=$ Secondary drinking water standard.

Highlighted values indicate positive results.

See Appendix A for explanation of data qualifiers.

a 



\subsection{Statistical Analysis}

Comparisons were made between parameter results from downgradient wells and results from a background well to identify possible impacts of the LEHR site on groundwater quality. These impacts may be a direct result of adding a parameter to the groundwater, especially those that do not naturally occur, such as pesticides, volatiles, and semivolatiles. The impacts may also be indirect due to ensuing changes in geochemistry or hydrochemistry caused by adding other parameters. Parameters likely to be impacted in this manner are anions, some metals, and other parameters. For this report comparisons were conducted on analytical results from downgradient wells for the 1995 groundwater samples.

Similar analyses were not conducted for surface water results because the source of water for the upstream sample, PCU, varies during the year. As discussed in Section 6, the source for PCU during periods of high flow in Putah Creek is upstream Putah Creek, but during the dry summer months, when there is low flow in Putah Creek, discharge water from STPO backs up into the reach where the PCU station is located. When this happens, the PCU analysis is not representative of background (upstream) conditions. Because of this, it would not be appropriate to conduct analyses of these data. A qualitative discussion of surface water analytical results is presented in Section 6.

Data from both groundwater HSUs were included in the analysis. However, the two HSUs were evaluated separately because of their different physical and hydraulic properties (see Section 4). Analytical results from background well UCD1-18 were used for background characterization of HSU-1, and results for background well UCD2-17 were used for background characterization of HSU-2. These are the western-most wells in the LEHR site monitoring network and are background to the LEHR site and all other monitoring wells. The HSU-1 downgradient wells included in this analysis are UCD1-1, UCD1-4, UCD1-10, UCD1-11, UCD1-12, UCD1-13, UCD1-19, UCD1-20, UCD1-21, UCD1-22, UCD1-23, and UCD1-24. Also analyzed were the following HSU-1 wells sampled for the first time in the fourth quarter of 1995: UCD1-25,UCD1-34, UCD1-27Z1, UCD1-27Z2, and UCD1-27Z3. Downgradient wells from HSU-2 included in this analysis are UCD2-7, UCD2-14, UCD2-15, UCD2-16, and the following wells sampled for the first time in the fourth quarter of 1995: UCD2-26, UCD2-35, UCD2-27Z4, UCD2-27Z5, UCD2-27Z6, and UCD2-27Z7. Although wells UCD1-34 and UCD2-35 are also candidates for upgradient wells, they have only been sampled once and were treated as possibly downgradient for this year's comparisons. 
For this report, data from the third quarter of 1993 through the second quarter of 1995 were used to characterize background groundwater conditions. This two-year background characterization data set includes results from the most recent eight quarters of full sampling and analysis and was chosen for the following reasons:

- The EPA (1989b) recommends using the most recent six to eight data points from background monitoring wells to evaluate background groundwater quality. This results in a reduction of potential historical bias when comparing the most recent sample events of 1995.

- Using two years of data for the background evaluation is consistent with the evaluation presented in the 1994 Annual Water Monitoring Report (PNL 1995).

\subsection{Comparison Strategy}

In general, a numerical result is not reported if the analysis is below the detection limit. Occasionally, a numerical result will be reported even when the value is less than the detection limit. The comparisons to background were divided into two general classes:

I Parameters with no results reported greater than the detection limit in any 1995 downgradient well sampling. Parameters falling into this category are considered to demonstrate no LEHR site impact on the groundwater and warrant no further comparison in this section.

II Parameters with results reported greater than the detection limit in one or more 1995 downgradient well samples. These parameters are considered to show a possible LEHR site impact on groundwater. This class was evaluated by statistical methods to determine whether the downgradient results were similar to background or were statistically different than background, indicating a possible effect of the LEHR site on groundwater quality.

Table 7.1 (at the end of Section 7) summarizes the number of samples taken and the number of results above the detection limit for the two-year background characterization data and the 1995 downgradient samples for HSU-1 and HSU-2. It also designates which class a parameter fell into for each HSU. Radionuclides, which are not arbitrarily censored at a detection limit, were labeled "detected" if their reported value exceeded their reported detection limit. 


\subsection{Parameters with No Results Above Detection Limit in 1995 Downgradient Samples (Class I)}

Table 7.1 shows that the following parameters had no results reported above the detection limit in downgradient samples during 1995 (Class I) and do not need to be compared with background:

$\begin{array}{ll}\text { Volatiles } & \text { Most volatiles, except } 10 \text { in HSU-1 and one in HSU-2. } \\ \text { Semivolatiles } & \text { All semivolatiles in both HSUs with the exception of a few } \\ & \text { sporadic phthalate and phenol compounds. } \\ \text { Pesticides } & \text { Pesticides in both HSUs with the exception of dieldrin in } \\ & \text { HSU-1. } \\ \text { Metals } & \text { Antimony, beryllium, cadmium, cobalt, lead, molybdenum, } \\ & \text { silver, and thallium in both HSUs; copper in HSU-1, and nickel } \\ \text { and potassium in HSU-2. } & \text { Bismuth-212, cesium-134, cesium-137, cobalt-57, } \\ \text { Radionuclides } & \text { cobalt-60, lead-210, potassium-40, radium-223, } \\ & \text { thallium-208, thorium-234, and uranium-235 in both HSUs; } \\ & \text { actinium-228 in HSU-1 and lead-212 and plutonium-241 in } \\ & \text { HSU-2. } \\ \text { Phosphate in both HSUs } & \text { Chemical oxygen demand, formaldehyde, and total kjeldahl } \\ \text { Anions } & \text { nitrogen in both HSUs. } \\ \text { Other Parameters } & \end{array}$

\subsection{Parameters with Results Above Detection Limit in 1995 Downgradient Samples (Class II)}

Table 7.1 also presents a summary of those parameters with results above the detection limit in one or more 1995 downgradient samples (Class II). These parameters will be further evaluated by statistical methods to determine whether the downgradient results could have come from the background or were statistically different than background, indicating a possible effect of the LEHR site on groundwater quality. Guidance for the statistical approach for the Class II parameters is based on Statistical Analysis of Groundwater Monitoring Data at RCRA Facilities (EPA 1989b) as described in the 1994 Annual Water Monitoring Report (PNL 1995). Figure 7.1, redrawn and modified from that document, presents the types of analyses conducted based on the characteristics of the data. The process shown in Figure 7.1 was used to select the appropriate statistical method to evaluate each parameter. This process differs slightly from that used in the 1994 Annual Report due to replacement of the approximate test of proportions with Fisher's Exact test. The selection of statistical method is similar to last year but was based solely on how well the background could be characterized. If fewer than $50 \%$ of the background values for a parameter were above the detection limit, a Fisher's Exact test was used to compare the background with each downgradient well that had values above the detection limit. If $50 \%$ or more of the background values for a parameter were above 
the detection limit, an upper $95 \%$ tolerance limit (UTL) with $95 \%$ confidence was calculated and used to compare with downgradient results.

The Fisher's Exact test replaces the approximate test of proportions previously used because the Fisher's Exact test can be used regardless of the percentage of nondetects. The Fisher's Exact test is similar to the test of proportions and compares the number of detects and nondetects in a well with the number of detects and nondetects in the background and determines if the downgradient well results are likely to have been from the background.

When greater than $50 \%$ detections are present for an analytical parameter in the background well, more detailed statistics are possible. For this report, parameters that satisfied this criterion were evaluated by calculating a $95 \%$ UTL. The UTL calculation provides an upper limit concentration for the parameter in the background well. If the percentage of detections in the background well is less than $85 \%$ but greater than $50 \%$, the mean and standard deviations must first be adjusted using Cohen's method (Cohen 1959) before calculating the UTL. If the percentage of detections is greater than $85 \%$, the UTL can be calculated using values of one-half the detection limit for nondetections. Once this UTL concentration is established for the sample results of the background well, each downgradient well result is compared with the UTL value.

A list of all Class II parameters in both HSUs is given in Table 7.2 along with the selected statistical method for each (note, no parameters fell into the percentage of detections for using one-half the detection limit for UTL calculations). In general, anions, field, and radionuclides are analyzed using the UTL method; pesticides, volatiles, and semivolatiles are generally analyzed using the Fisher's Exact test. Other parameters and metals show a mixture of the two methods. Radionuclides were analyzed using the UTL method because their results are not arbitrarily censored at a detection limit, and a reported result is always available for statistical analysis.

\subsection{Results of Statistical Comparisons of Downgradient to Background}

This section presents the results of the statistical comparison of background and downgradient groundwater data. Tables $7.3 \mathrm{a}$ and $7.3 \mathrm{~b}$ present a summary of the results of the statistical analysis using UTL comparisons for HSU-1 and HSU-2, respectively. Tables 7.4a and $7.4 \mathrm{~b}$ present a summary of the results of the statistical analysis using Fisher's Exact test for HSU-1 and HSU-2, respectively. A discussion of the results of the statistical analysis is presented below.

\subsubsection{Upper Tolerance Limit Comparison Results}

Tables 7.3a and 7.3b present a summary of the statistical analysis for parameters that were evaluated by a background UTL calculation for HSU-1 and HSU-2, respectively. There were parameters from each of the analytical groups except pesticides, volatiles, and semivolatiles. 
After calculating the background UTL, analytical results from the downgradient wells were compared with the UTL value. A downgradient result that exceeds the UTL is considered to be elevated with respect to background at a $95 \%$ confidence level and may indicate an effect of the LEHR site on groundwater quality. Results that are below the UTL value are considered to be within likely background levels and indicate no LEHR site impact. Tables 7.3a and 7.3b list the percentage of 1995 samples in each well that exceeded a UTL. These percentages must be interpreted with some caution since many parameters were only sampled once or twice in many wells during 1995. A dash $(-)$ in the tables indicates none of the results exceeded the UTL in a well; a period (.) indicates that no samples were taken in a well.

\subsubsection{Fisher's Exact Test Comparison Results}

Tables 7.4a and 7.4b present a summary of the results for parameters evaluated by the Fisher's Exact test. There were parameters from each of the analytical groups except anions and field.

The Fisher's Exact test reports the probability that the number of detects and nondetects in a well could have resulted from a well with the number of detects and nondetects observed in the background data. Tables $7.4 \mathrm{a}$ and $7.4 \mathrm{~b}$ list the probabilities calculated for downgradient wells that had at least one reported result above the detection limit. Small probability values $(<0.05)$ indicate that it is unlikely the results represent background but are a likely LEHR site impact. Larger probability values indicate that it is possible the results could have come from background and may not represent LEHR site impact.

\subsection{Significance of Statistical Analysis Results}

The 1995 water monitoring program included the analysis of 196 parameters. Sixtyfive of the 196 parameters were reported as detected in at least one sample (Table 5.1). Statistical analyses of the 65 detected parameters indicated that 29 parameters were reported at concentrations significantly different from concentrations found in background wells UCD 1-18 or UCD2-17. These results are summarized in Table 7.5. The purpose of this section is to discuss the significance of the findings reported in Table 7.5.

Table 7.5 presents the parameters that were detected above background levels, the HSU in which the parameter was detected, and the type of parameter effect. A direct parameter effect results from constituents that were disposed of at LEHR and indirect effects are secondary effects resulting from direct actions. An example of a direct effect is the disposal of chloroform in an area on the north side of Landfill 2. An indirect effect would be the appearance of elevated magnesium concentrations in groundwaters; magnesium was not discharged to the ground at LEHR, but natural magnesium in the soil may be mobilized because of actions taken on the site. The number of wells in which each parameter was detected above background is also reported to indicated the significance of the finding. 
While the results presented in Table 7.5 represent significant differences between onsite and background data, not all of the differences are significant from a hazard standpoint. A parameter is considered significant from a hazard or use standpoint if the maximum concentration exceeded a regulatory limit such as an MCL. Table 7.6 provides the maximum concentrations reported for each of the parameters in 1995 and regulatory limits for reference. A parameter is judged to be significant if its maximum concentration was greater than the regulatory limit. Such parameters can be identified in Table 7.6 by a number entry in the "Significant" column. A number entry corresponds to the number of sample locations in which the parameter exceeded the stated regulatory limit for at least one quarter during 1995 . Of the 29 parameters that were significantly different from background, 13 have exceeded a regulatory limit. The remainder of this section will focus on these 13 parameters.

Sulfate, total dissolved solids (TDS), and $\mathrm{pH}$ were identified as significant because they exceeded a secondary drinking water limit. While not hazardous parameters, their elevated concentrations indicate a secondary effect of actions on LEHR. Sulfate was detected above the regulatory limit only in well UCD1-10 which is consistent with historical trends (Table C.5). The regulatory limit for TDS was exceeded in all but one HSU-1 wells, including the background, but eight sampling locations in HSU-1 and two in HSU-2 were significantly greater than background. The regulatory limit for $\mathrm{pH}$ was exceeded in UCD2-15 for one quarter and is not considered significant because the mean $\mathrm{pH}$ for the well is less than 8.

Nitrate concentrations at five HSU-1 and two HSU-2 sampling locations exceeded the regulatory limit and background levels. Ten wells in HSU-1 exceeded the nitrate regulatory limit, but only five wells exceeded background because the nitrate concentration in background well UCD1-18 also exceeded the limit. While groundwater nitrate concentrations in the region surrounding LEHR are elevated above the drinking water limit, five HSU-1 wells exceeded the background level, indicating an effect from LEHR. The nitrate plumes were discussed in Section 5.0. The highest nitrate levels are located at the north end of Landfill 2.

Total and hexavalent chromium exceeded regulatory limits and background concentrations in seven HSU-1 wells scattered across the site. Most of the total chromium measured is in hexavalent form and their concentrations are closely related, therefore, they will be discussed without distinguishing either form. The MCL for chromium was exceeded only in UCD2-7 in HSU-2. This well has historically contained chromium at 50 to $60 \mu \mathrm{g} / \mathrm{L}$. These chromium data are consistent with the long-term monitoring trends and what was reported in 1994. These data indicate that elevated chromium levels in groundwaters beneath LEHR are the result of direct disposal of chromium containing solutions at LEHR.

Selenium exceeded the MCL of $5 \mu \mathrm{g} / \mathrm{L}$ in four wells, UCD1-10, UCD1-11, UCD1-12, and UCD1-19, during 1995. These results are consistent with historical trends (Table C.3). There is no know direct release of selenium from LEHR, therefore, these elevated levels appear to be the indirect result of some unknown activity at LEHR. 
Americium-241 was reported above background in one well, UCD1-10, for one quarter in 1995. The value exceeded the background level, but just slightly above the MDA. It is likely that this "detection" is due to a random fluctuation in background counting, but will continue to be monitored to establish a trend. Americium-241 was detected one other time in UCD1-10 in 1994.

Carbon-14 was detected at levels exceeding background in four HSU-1 (UCD1-12, UCD1-13, UCD1-20, and UCD1-23) and 1 HSU-2 (UCD2-14) wells in 1995. These findings are consistent with historical trends (Table C.4) except for the detection in UCD120. The one reported detection was just above the MDA and attributed to fluctuations in background counting. These data indicate that carbon-14 contamination is likely from three isolated sources, near UCD1-12 north of Landfill 2, in the vicinity of the southwest trenches (UCD1-23), and in the vicinity of the southern half of Landfill 2 (UCD1-13 and UCD2-14). Carbon-14 is not detected in other wells, indicating that a continuous plume is not present at the site.

Of the volatile constituents that exceeded background, only those detected in well UCD1-12 exceeded MCLs. Chloroform concentrations in UCD1-12 were 3 to 4 orders of magnitude higher than in any other well in the monitoring system and 1,1-Dichloroethane, 1,1-Dichloroethene, 1,2-Dichloroethane were only detected in UCD1-12. Clearly, the source of these constituents is in the vicinity of the north end of Landfill 2. At this time, the source is unknown, but as presented in Section 5.0, the extent of the chloroform plume and its distribution on LEHR is well delineated.

Based on the results in this section the list of parameters that indicate a potential effect of the LEHR site on groundwater can be reduced to those shown in Table 7.6. Of these, nitrate, chromium, carbon-14, tritium, and the volatiles could be directly attributable to LEHR site activities. Most of the remaining parameters are likely an indirect effect caused by one of the direct groundwater contaminants. 


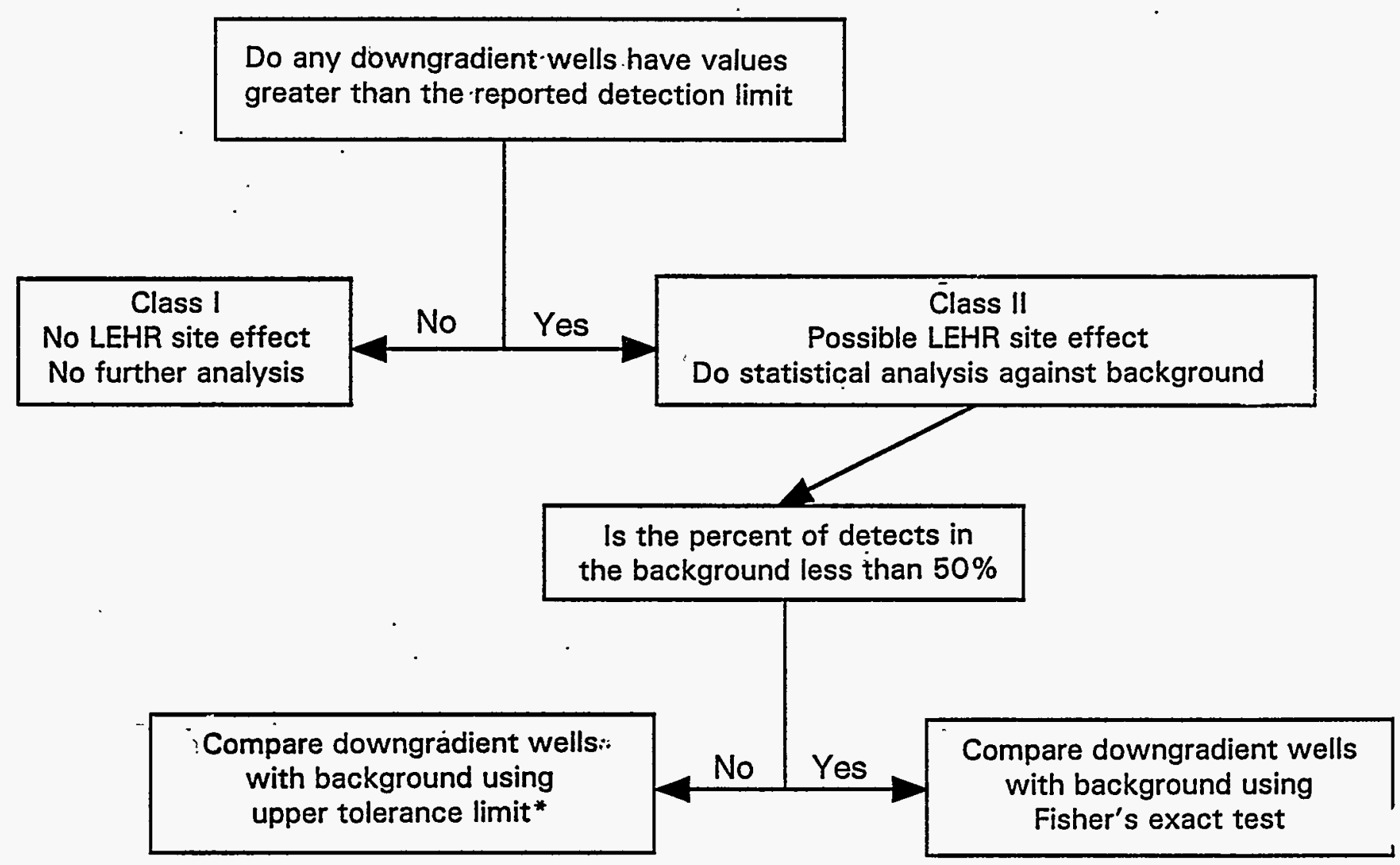

* If percent detects in background is $100 \%$ between 85 and $100 \%$ between 50 and $85 \%$
Calculate mean and standard deviation using raw data

DL values replaced with $1 / 2 \mathrm{DL}$

Cohen's method

Figure 7.1. Statistical Test Selection Flow Chart 
Table 7.1. Summary of Samples and Detects for Two-Year Background and 1995 Downgradient Samples

\begin{tabular}{|c|c|c|c|c|c|c|c|c|c|c|c|}
\hline \multirow[b]{3}{*}{$\begin{array}{c}\text { Analytical } \\
\text { Group }\end{array}$} & \multirow[b]{3}{*}{ Parameter Name } & \multicolumn{5}{|c|}{ HSU-1 } & \multicolumn{5}{|c|}{$\mathrm{HSU}-2$} \\
\hline & & \multicolumn{2}{|c|}{ Background } & \multicolumn{2}{|c|}{ Downgradient } & \multirow[b]{2}{*}{ Class } & \multicolumn{2}{|c|}{ Background } & \multicolumn{2}{|c|}{. Downgradient. } & \multirow[b]{2}{*}{ Class } \\
\hline & & Samples & Detects & Samples & Detects & & Samples & Detects & Samples & Detects & \\
\hline \multirow[t]{4}{*}{ Anions } & Chloride & 9 & 9 & 26 & 26 & II & 10 & 10 & 9 & 9 & II \\
\hline & Nitrogen, Nitrate (as N) & 9 & 9 & 39 & 36 & II & 10 & 10 & 22 & 22 & II \\
\hline & Phosphate, Total (as P) & 9 & - & 26 & - & $\mathbf{I}$ & 10 & - & 9 & - & 1 \\
\hline & Sulfate & 9 & 9 & 26 & 26 & II & 10 & 10 & 9 & 9 & 11 \\
\hline \multirow[t]{5}{*}{ Field } & $E H^{\prime} F$ & 8 & 8 & 26 & 26 & ॥ & 7 & 7 & 9 & 9 & II \\
\hline & Specific Conductance (EC) & 9 & 9 & 37 & 37 & II & 10 & 10 & 14 & 14 & II \\
\hline & Temperature & 9 & 9 & 37 & 37 & II & 10 & 10 & 14 & 14 & II \\
\hline & Turbidity & 8 & 8 & 37 & 37 & II & 10 & 10 & 13 & 13 & 11 \\
\hline & $\mathrm{pH}$ & 9 & 9 & $37^{\circ}$ & 37 & II & 10 & 10 & 14 & 14 & II \\
\hline \multirow[t]{8}{*}{ General } & Alkalinity, Total(as $\mathrm{CaCO}$ ) & 9 & 9 & 26 & 26 & II & 10 & 10 & 9 & 9 & II \\
\hline & Ammonia-Nitrogen & 9 & - & 26 & 2 & II & 10 & 2 & 9 & 2 & II \\
\hline & Chemical Oxygen Demand & 4 & - & 26 & - & 1 & 4 & 1 & 9 & - & 1 \\
\hline & Formaldehyde & 8 & 1 & 12 & - & 1 & 9 & - & 5 & - & 1 \\
\hline & Total Dissolved Solids & 9 & 9 & 41 & 41 & II & 10 & 10 & 22 & 22 & II \\
\hline & Total Kjeldahl Nitrogen & 10 & - & 26 & - & 1 & 10 & 1 & 9 & - & 1 \\
\hline & Total Organic Carbon & 9 & 7 & 26 & 14 & II & 10 & 3 & 9 & 4 & II \\
\hline & Turbidity & 9 & 9 & 26 & 20 & II & 10 & 8 & 9 & 3 & II \\
\hline \multirow[t]{9}{*}{ Metals } & Antimony & 9 & - & 31 & - & 1 & 10 & - & 20 & - & 1 \\
\hline & Arsenic & 9 & 7 & 31 & 19 & II & 10 & 6 & 20 & 11 & II \\
\hline & Barium & 9 & 9 & 31 & 31 & 11 & 10 & 10 & 20 & 19 & II \\
\hline & Beryllium & 9 & 1 & 31 & - & 1 & 10 & - & 20 & - & 1 \\
\hline & Cadmium & 9 & - & 31 & - & 1 & 10 & - & 20 & - & 1 \\
\hline & Calcium & 9 & 9 & 31 & 31 & II & 10 & 10 & 20 & 20 & 11 \\
\hline & Chromium & 9 & 9 & 31 & 24 & II & 10 & 8 & 20 & 13 & II \\
\hline & Chromium, Hexavalent $(+6)$ & 9 & 5 & 43 & 36 & II & 10 & 4 & 22 & 12 & II \\
\hline & Cobalt & 9 & - & 31 & - & 1 & 10 & - & 20 & - & 1 \\
\hline
\end{tabular}


Table 7.1. (contd)

\begin{tabular}{|c|c|c|c|c|c|c|c|c|c|c|c|}
\hline \multirow[b]{3}{*}{$\begin{array}{l}\text { Analytical } \\
\text { Group }\end{array}$} & \multirow[b]{3}{*}{1 Parameter Name } & \multicolumn{5}{|c|}{ HSU-1 } & \multicolumn{5}{|c|}{$\mathrm{HSU}-2$} \\
\hline & & \multicolumn{2}{|c|}{ Background } & \multicolumn{2}{|c|}{ Downgradient } & \multirow[b]{2}{*}{ Class } & \multicolumn{2}{|c|}{ Background } & \multicolumn{2}{|c|}{ Downgradient } & \multirow[b]{2}{*}{ Class } \\
\hline & & Samples & Dotects & Samples & Detects & & Samples & Detects & Samples & Detects & \\
\hline \multirow[t]{14}{*}{ Metals (contd) } & Copper & 9 & - & 31 & - & 1 & 10 & - & 20 & 1 & II \\
\hline & Iron & 9 & $3^{-}$ & 31 & 3 & II & 10 & 3 & 20 & 6 & II \\
\hline & Lead & 9 & - & 31 & . - & 1 & 10 & 1 & 20 & - & 1 \\
\hline & Magnesium & 9 & 9 & 31 & 31 & II & 10 & 10 & 20 & 20 & II \\
\hline & Mercury & 9 & 1 & 31 & 3 & II & 10 & - & 20 & 2 & 11 \\
\hline & Molybdenum & 9 & - & 31 & - & 1 & 10 & - & 20 & . - & 1 \\
\hline & Nickel & 9 & 7 & 31 & 3 & iI & 10 & - & 20 & - & 1 \\
\hline & Potassium & 9 & 3 & 31 & 1 & II & 10 & 3 & 20 & - & 1 \\
\hline & Selenium & 9 & 1 & 31 & 14 & II & 10 & 2 & 20 & 5 & II \\
\hline & Silver & 9 & - & 31 & - & 1 & 10 & - & 20 & - & 1 \\
\hline & Sodium & 9 & 9 & 31. & 31 & II & 10 & 10 & 20 & 20 & II \\
\hline & Thallium & 9 & - & 31 & - & 1 & 10 & - & 20 & - & 1 \\
\hline & Vanadium & 9 & 5 & 31 & 7 & II & 10 & 1 & 20 & 2 & II \\
\hline & Zinc & 9 & 1 & 31 & 4 & II & 10 & 1 & 20 & 1 & II \\
\hline \multirow[t]{12}{*}{ Pesticides } & $4,4^{\prime}-\mathrm{DDD}$ & 9 & - & 39 & - & 1 & 10 & - & 19 & - & 1 \\
\hline & $4,4^{\prime}-\mathrm{DDE}$ & 9 & - & 39 & - & 1 & 10 & - & 19 & - & 1 \\
\hline & $4,4^{\prime}-\mathrm{DDT}$ & 9 & - & 39 & - & $T$ & 10 & - & 19 & - & 1 \\
\hline & Aldrin & 9 & - & 39 & - & 1 & 10 & - & 19 & - & 1 \\
\hline & Alpha-BHC & 9 & - & 39 & - & .1 & 10 & - & 19 & - & 1 \\
\hline & Alpha-Chlordano & 9 & - & 39 & - & 1 & 10 & - & 19 & - & 1 \\
\hline & Aroclor-1016 & 9 & - & 39 & - & 1 & 10 & - & 19 & - & 1 \\
\hline & Aroclor-1221 & 9 & - & 39 & - & 1 & 10 & - & 19 & - & 1 \\
\hline & Aroclor- 1232 & 9 & - & 39 & - & 1 & 10 & - & 19 & - & 1 \\
\hline & Aroclor-1242 & 9 & - & 39 & - & 1 & 10 & - & 19 & - & 1 \\
\hline & Aroclor-1248 & 9 & - & 39 & - & 1 & 10 & - & 19 & - & 1 \\
\hline & Aroclor-1254 & 9 & - & 39 & - & 1 & 10 & - & 19 & - & 1 \\
\hline
\end{tabular}


Table 7.1. (contd)

\begin{tabular}{|c|c|c|c|c|c|c|c|c|c|c|c|}
\hline \multirow[b]{3}{*}{$\begin{array}{l}\text { Analytical } \\
\text { Group }\end{array}$} & \multirow[b]{3}{*}{ Parameter Name } & \multicolumn{5}{|c|}{ HSU-1 } & \multicolumn{5}{|c|}{ HSU-2 } \\
\hline & & \multicolumn{2}{|c|}{ Background } & \multicolumn{2}{|c|}{ Downgradient } & \multirow[b]{2}{*}{ Class } & \multicolumn{2}{|c|}{ Background } & \multicolumn{2}{|c|}{ Downgradient } & \multirow{2}{*}{ Class } \\
\hline & & Samples & Detects & Samples & Detects & & Samples & Datects & Samples & Detects & \\
\hline \multirow{16}{*}{$\begin{array}{l}\text { Pesticides } \\
\text { (contd) }\end{array}$} & Aroclor- 1260 & 9 & - & 39 & - & 1 & 10 & - & 19 & - & 1 \\
\hline & Beta-BHC & 9 & - & 39 & - & $\mathbf{I}$ & 10 & - & 19 & - & 1 \\
\hline & Delta-BHC & 9 & - & 39 & - & 1 & 10 & - & 19 & - & 1 \\
\hline & Dieldrin & 9 & - & 39 & 3 & II & 10 & - & 19 & - & 1 \\
\hline & Endosulfan I & 9 & - & 39 & - & 1 & 10 & - & 19 & - & 1 \\
\hline & Endosulfan II & 9 & - & 39 & - & 1. & 10 & - & 19 & - & $\mathbf{I}$ \\
\hline & Endosulfan Sulfate & 9 & - & 39 & - & 1 & 10 & - & 19 & - & 1 \\
\hline & Endrin & 9 & - & 39 & - & 1 & 10 & - & 19 & - & 1 \\
\hline & Endrin Aldehyde & 9 & - & 39 & - & 1 & 10 & - & 19 & - & 1 \\
\hline & Endrin Ketone & 9 & - & 39 & - & 1 & 10 & - & 19 & - & 1 \\
\hline & Gamma-BHC & 9 & - & 39 & - & 1 & 10 & - & 19 & - & 1 \\
\hline & Gamma-Chlordane & 9 & - & 39 & - & 1 & 10 & - & 19 & $\cdot-$ & 1 \\
\hline & Heptachlor & 9 & - & 39 & - & 1 & 10 & - & 19 & - & I \\
\hline & Heptachlor Epoxide & 9 & - & 39 & - & 1 & 10 & - & 19 & - & 1 \\
\hline & Methoxychlor & 9 & - & 39 & - & 1 & 10 & - & 19 & - & 1 \\
\hline & Toxaphene & 9 & - & 39 & - & 1 & 10 & - & 19 & - & 1 \\
\hline \multirow[t]{10}{*}{ Radionuclides } & Actinium-228 & 7 & - & 41 & - & 1 & 7 & - & 21 & 1 & II \\
\hline & Americium-241 & 4 & - & 32 & 4 & \|I & 5 & 1 & 17 & 2 & II \\
\hline & Bismuth-212 & 5 & - & 35 & - & 1 & 4 & - & 17 & - & 1 \\
\hline & Bismuth-214 & 7 & 3 & 41 & 23 & II & 7 & 2 & 21 & 5 & II \\
\hline & Carbon-14 & 9 & - & 38 & 13 & II & 10 & 2 & 18 & 3 & 11 \\
\hline & Cesium-134 & . & . & 6 & - & I & . & . & 4 & - & 1 \\
\hline & Cesium-137 & 7 & 1 & 41 & - & 1 & 7 & - & 21 & - & 1 \\
\hline & Cobalt- 67 & . & . & 6 & - & 1 & . & . & 4 & - & 1 \\
\hline & Cobalt -60 & 7 & - & 41 & - & 1 & 7 & - & 21 & - & 1 \\
\hline & Gross Alpha & 9 & - & 41 & 1 & 11 & 10 & 1 & 21 & 1 & II \\
\hline
\end{tabular}




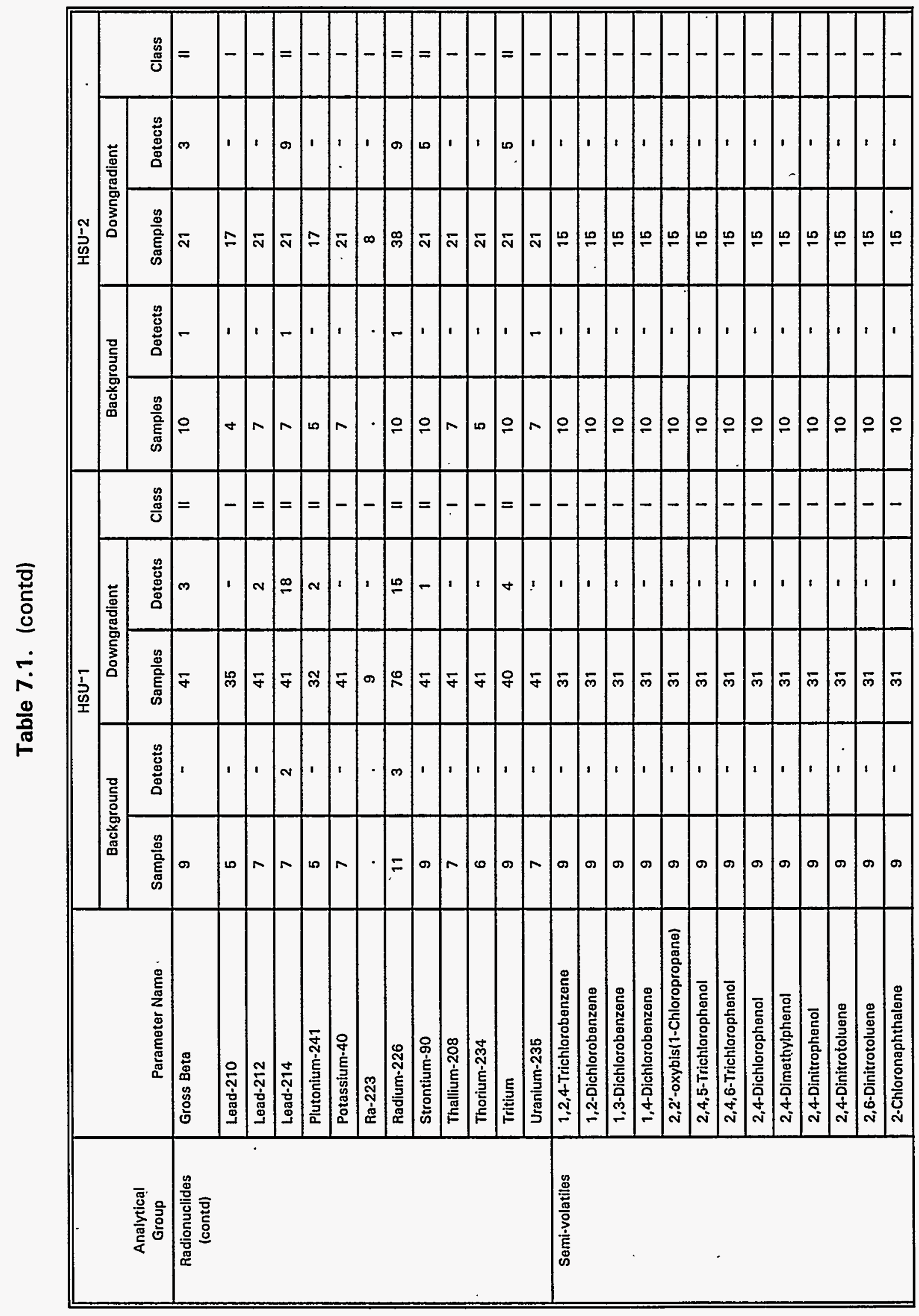


Table 7.1. (contd)

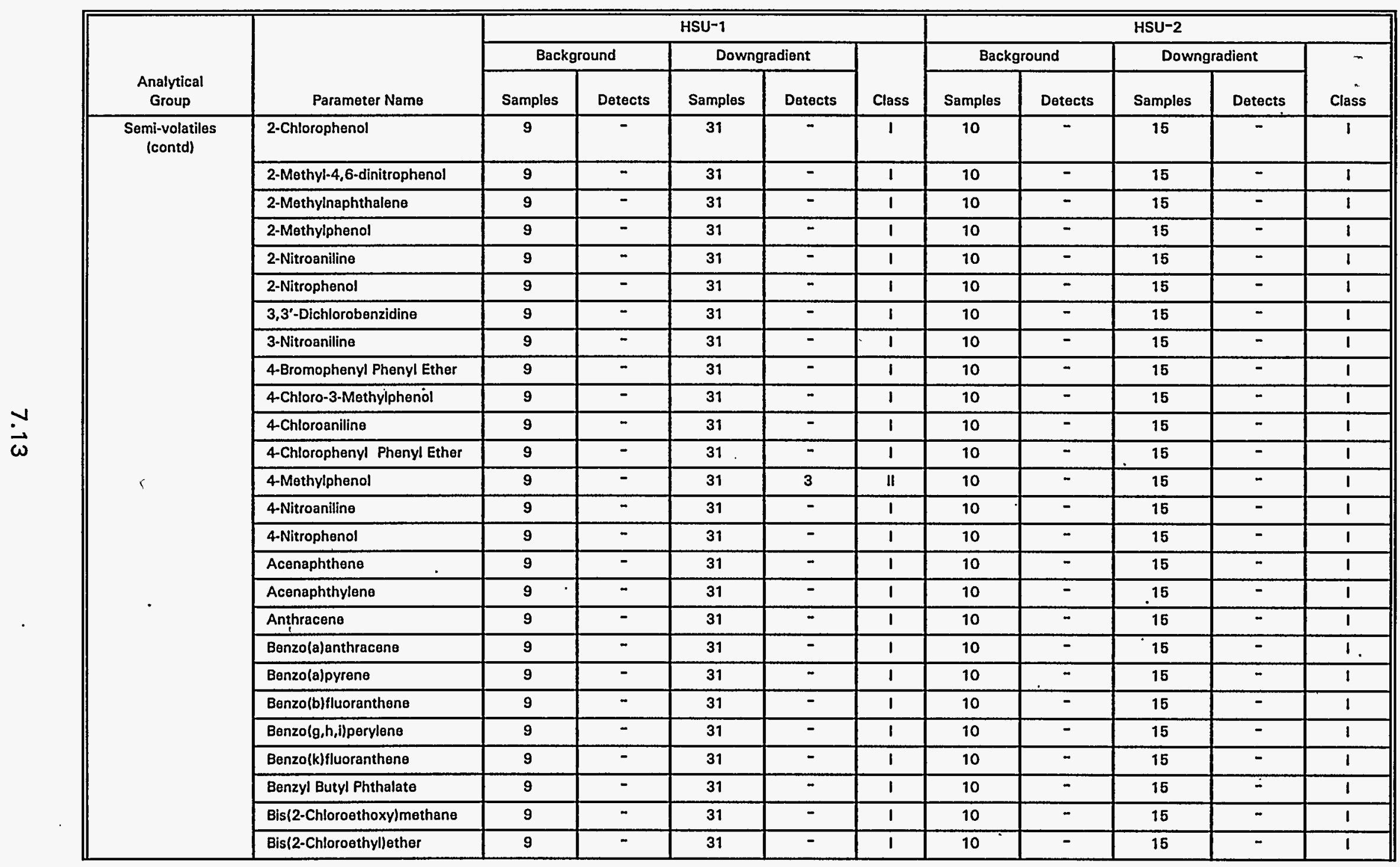


Table 7.1. (contd)

\begin{tabular}{|c|c|c|c|c|c|c|c|c|c|c|c|}
\hline \multirow[b]{3}{*}{$\begin{array}{l}\text { Analytical } \\
\text { Group }\end{array}$} & \multirow[b]{3}{*}{ Parameter Name } & \multicolumn{5}{|c|}{ HSU-1 } & \multicolumn{5}{|c|}{$\mathrm{HSU}-2$} \\
\hline & & \multicolumn{2}{|c|}{ Background } & \multicolumn{2}{|c|}{ Downgradient } & \multirow[b]{2}{*}{ Class } & \multicolumn{2}{|c|}{ Background } & \multicolumn{2}{|c|}{ Downgradient } & \multirow[b]{2}{*}{ Class } \\
\hline & & Samples & Detects & Samples & Detects & & Samples & Detects & Samples & Detects & \\
\hline \multirow{25}{*}{$\begin{array}{l}\text { Semi-volatiles } \\
\text { (contd) }\end{array}$} & Bis(2-Ethylhexyl)phthalate & 9 & - & 31 & - & 1 & 10 & - & 15 & 3 & II \\
\hline & Carbazole & 9 & - & 31 & - & 1 & 10 & - & 15 & - & 1 \\
\hline & Chrysene & 9 & - & 31 & - & 1 & 10 & - & 15 & - & 1 \\
\hline & Di-n-Butylphthalate & 9 & 1 & 31 & 3 & II & 10 & 1 & 15 & - & 1 \\
\hline & Di-n-Octylphthalate & 9 & - & 31 & - & 1 & 10 & - & 15 & - & 1 \\
\hline & Dibenzo(a,h)anthracene & 9 & - & 31 & - & 1 & 10 & - & 15 & $\overline{-}$ & 1 \\
\hline & Dibenzofuran & 9 & - & 31 & - & 1 & 10 & - & 15 & - & 1 \\
\hline & Diethyl Phthalate & 9 & - & 31 & - & 1 & 10 & - & 15 & $=$ & 1 \\
\hline & Dimethyl Phthalate & 9 & - & 31 & - & 1 & 10 & - & 15 & - & 1 \\
\hline & Fluoranthene & 9 & - & 31 & - & 1 & 10 & - & 15 & - & 1 \\
\hline & Fluorene & 9 & - & 31 & - & 1 & 10 & - & 15 & - & 1 \\
\hline & Hexachlorobenzene & 9 & - & 31 & - & 1 & 10 & - & 15 & - & 1 \\
\hline & Hexachlorobutadiene & 9 & - & 31 & - & 1 & 10 & - & 15 & - & $\overline{1}$ \\
\hline & Hexachlorocyclopentadiene & 9 & - & 31 & - & T & 10 & - & 15 & - & 1 \\
\hline & Hexachloroethane & 9 & - & 31 & - & 1 & 10 & - & 15 & - & 1 \\
\hline & Indeno(1,2,3-cd)pyreñe & 9 & - & 31 & - & 1 & 10 & - & 15 & - & 1 \\
\hline & Isophorone & 9 & - & 31 & - & 1 & 10 & - & 15 & - & 1 \\
\hline & N-Nitrosodi-n-propylamine & 9 & - & 31 & - & 1 & 10 & - & $\overline{15}$ & - & 1 \\
\hline & N-Nitrosodiphenylamine & 9 &.- & 31 & - & 1 & 10 & - & 15 & - & 1 \\
\hline & Naphthalene & 9 & - & 31 & - & 1 & 10 & - & 15 & - & 1 \\
\hline & Nitrobenzene & 9 & - & 31 & - & 1 & 10 & - & 15 & - & $T$ \\
\hline & Pentachlorophenol & 9 & - & 31 & - & 1 & 10 & - & 15 & - & 1 \\
\hline & Phenanthrene & 9 & - & 31 & - & 1 & 10 & - & 15 & - & 1 \\
\hline & Phenol & 9 & - & 31 & 3 & II & 10 & - & 16 & 1 & II \\
\hline & Pyrene & 9 & - & 31 & - & 1 & 10 & - & 15 & - & 1 \\
\hline
\end{tabular}


Table 7.1. (contd)

\begin{tabular}{|c|c|c|c|c|c|c|c|c|c|c|c|}
\hline \multirow{3}{*}{$\begin{array}{l}\text { Analytical } \\
\text { Group }\end{array}$} & \multirow[b]{3}{*}{ Parameter Name } & \multicolumn{5}{|c|}{ HSU-1 } & \multicolumn{5}{|c|}{ HSU-2 } \\
\hline & & \multicolumn{2}{|c|}{ Background } & \multicolumn{2}{|c|}{ Downgradient } & \multirow[b]{2}{*}{ Class } & \multicolumn{2}{|c|}{ Background } & \multicolumn{2}{|c|}{ Downgradient } & \multirow[b]{2}{*}{ Class } \\
\hline & & Samples & Detects & Samples & Detects & & Samples & Detects & Samples & Detects & \\
\hline \multirow[t]{26}{*}{ Volatiles } & 1,1,1-Trichloroethane & 9 & - & 43 & - & 1 & 10 & - & 20 & - & 1 \\
\hline & 1,1,2,2-Tetrachloroethane & 9 & - & 43 & - & $\mathbf{I}$ & 10 & - & 20 & - & 1 \\
\hline & 1,1,2-Trichloroethane & 9 & - & 43 & 5 & II & 10 & - & 20 & - & 1 \\
\hline & 1,1-Dichloroethane & 9 & - & 42 & 5 & II & 10 & - & 20 & - & 1 \\
\hline & 1,1-Dichloroethene & 9 & - & 42 & 6 & 11 & 10 & - & 20 & - & 1 \\
\hline & 1,2-Dibromo-3-chloropropane & 9 & - & 43 & - & 1 & 10 & - & 20 & - & 1 \\
\hline & 1,2-Dibromoethane & 9 & - & 43 & - & 1 & 10 & - & 20 & - & 1 \\
\hline & 1,2-Dichlorobenzene & 9 & - & 43 & - & $\mathbf{I}$ & 10 & - & 20 & - & 1 \\
\hline & 1,2-Dichloroethane & 9 & - & 42 & 5 & II & 10 & - & 20 & - & 1 \\
\hline & 1,2-Dichloropropane & 9 & - & 42 & - & 1 & 10 & - & 20 & - & 1 \\
\hline & 1,3-Dichlorobenzene & 9 & - & 43 & - & 1 & 10 & - & 20 & - & 1 \\
\hline & 1,4-Dichlorobenzene & 9 & - & 43 & - & 1 & 10 & - & 20 & - & 1 \\
\hline & 2-Butanone & 9 & - & 43 & 1 & II & 10 & - & 20 & - & 1 \\
\hline & 2-Hexanone & 9 & - & 43 & - & 1 & 10 & - & 20 & - & 1 \\
\hline & 4-Methyl-2-Pentanone & 9 & - & 43 & - & 1 & 10 & - & 20 & - & 1 \\
\hline & Acetone & 9 & - & 42 & - & 1 & 10 & - & 20 & - & 1 \\
\hline & Benzeno & 9 & - & 43 & - & 1 & 10 & - & 20 & - & 1 \\
\hline & Bromochloromethane & 9 & - & 43 & - & 1 & 10 & - & 20 & - & 1 \\
\hline & Bromodichloromethane & 9 & - & 44 & 4 & II & 10 & - & 20 & - & $\mathrm{I}$ \\
\hline & Bromoform & 9 & - & 43 & - & 1 & 10 & - & 20 & - & 1 \\
\hline & Bromomethane & 9 & - & 43 & - & 1 & 10 & - & 20 & - & 1 \\
\hline & Carbon Disulfide & 9 & - & 43 & 1 & II & 10 & - & 20 & - & 1 \\
\hline & Carbon Tetrachloride & 9 & - & 43 & - & 1 & 10 & - & 20 & - & 1 \\
\hline & Chlorobenzene & 9 & - & 43 & - & 1 & 10 & - & 20 & - & 1 \\
\hline & Chloroethane & 9 & - & 43 & - & 1 & 10 & - & 20 & - & 1 \\
\hline & Chloroform & 9 & - & 44 & 18 & II & 10 & - & 20 & 9 & II \\
\hline
\end{tabular}


Table 7.1. (contd)

\begin{tabular}{|c|c|c|c|c|c|c|c|c|c|c|c|}
\hline \multirow[b]{3}{*}{$\begin{array}{l}\text { Analytical } \\
\text { Group }\end{array}$} & \multirow[b]{3}{*}{ Parameter Name } & \multicolumn{5}{|c|}{ HSU-1 } & \multicolumn{5}{|c|}{ HSU-2 } \\
\hline & & \multicolumn{2}{|c|}{ Background } & \multicolumn{2}{|c|}{ Downgradient } & \multirow[b]{2}{*}{ Class } & \multicolumn{2}{|c|}{ Background } & \multicolumn{2}{|c|}{ Downgradient } & \multirow[b]{2}{*}{ Class } \\
\hline & & Samples & Detects & Samples & Detects & & Samples & Detects & Samples & Detects & \\
\hline \multirow{9}{*}{$\begin{array}{c}\text { Volatiles } \\
\text { (contd) }\end{array}$} & Dibromochloromethane & 9 & - & 43 & - & 1 & 10 & - & 20 & - & 1 \\
\hline & Ethyl Benzene & 9 & - & 43 & - & $T$ & 10 & - & 20 & - & $T$ \\
\hline & Methylene Chloride & 9 & - & 43 & - & $T$ & 10 & - & 20 & - & $T$ \\
\hline & Tetrachloroethene & 9 & - & 43 & - & $T$ & 10 & - & 20 & - & $T$ \\
\hline & Toluene & 9 & - & 44 & 2 & II & 10 & - & 20 & - & 1 \\
\hline & Trichloroothene & 9 & - & 43 & - & 1 & 10 & - & 20 & - & $\mathrm{I}$ \\
\hline & Vinyl Chloride & 9 & - & 43 & - & $T$ & 10 & - & 20 & - & $T$ \\
\hline & Xylenes (Total) & 9 & - & 44 & 1 & II & 10 & - & 20 & - & $T$ \\
\hline & cis-1,2-Dichloroethene & 9 & - & 43 & - & 1 & 10 & - & 20 & - & 1 \\
\hline
\end{tabular}


Table 7.2. Parameters With at Least One Downgradient Result Above the Detection Limit

\begin{tabular}{|c|c|c|c|c|c|c|c|c|c|}
\hline \multirow[b]{2}{*}{ Analytical Group } & \multirow[b]{2}{*}{ Parameter Name } & \multicolumn{4}{|c|}{ HSU-1 Background } & \multicolumn{4}{|c|}{ HSU-2 Background } \\
\hline & & Samples & Detects & $\%$ Detects & Method & Samples & Detects & $\%$ Detects & Method \\
\hline \multirow[t]{3}{*}{ Anions } & Chloride & 9 & 9 & 100 & UTL & 10 & 10 & 100 & UTL \\
\hline & Nitrogen, Nitrate (as N) & 9 & 9 & 100 & UTL & 10 & 10 & 100 & UTL \\
\hline & Sulfate & 9 & 9 & 100 & UTL & 10 & 10 & 100 & UTL \\
\hline \multirow[t]{5}{*}{ Field } & $E H-F$ & 8 & 8 & 100 & UTL & 7 & 7 & 100 & UTL \\
\hline & Specific Conductance (EC) & 9 & 9 & 100 & UTL & 10 & 10 & 100 & UTL \\
\hline & Temperature & 9 & 9 & 100 & UTL & 10 & 10 & 100 & UTL \\
\hline & Turbidity & 8 & 8 & 100 & UTL & 10 & 10 & 100 & UTL \\
\hline & $\mathrm{pH}$ & 9 & 9 & 100 & UTL & 10 & 10 & 100 & UTL \\
\hline \multirow[t]{5}{*}{ General } & Alkalinity, Total(as CaCO3) & 9 & 9 & 100 & UTL & 10 & 10 & 100 & UTL \\
\hline & Ammonia-Nitrogen & 9 & - & 0 & Fisher & 10 & 2 & 20 & Fisher \\
\hline & Total Dissolved Solids & 9 & 9 & 100 & UTL & 10 & 10 & 100 & UTL \\
\hline & Total Organic Carbon & 9 & 7 & 78 & UTL-Cohen & 10 & 3 & 30 & Fisher \\
\hline & Turbidity & 9 & 9 & 100 & UTL & 10 & 8 & 80 & UTL-Cohen \\
\hline \multirow[t]{15}{*}{ Metals } & Arsenic & 9 & 7 & 78 & UTL-Cohen & 10 & 6 & 60 & UTL-Cohen \\
\hline & Barium & 9 & 9 & 100 & UTL & 10 & 10 & 100 & UTL \\
\hline & Calcium & 9 & 9 & 100 & UTL & 10 & 10 & 100 & UTL \\
\hline & Chromium & 9 & 9 & 100 & UTL & 10 & 8 & 80 & UTL \\
\hline & Chromium, Hexavalent $(+6)$ & 9 & 5 & 56 & UTL-Cohen & 10 & 4 & 40 & Fisher \\
\hline & Copper & . & . & . & & 10 & - & 0 & Fisher \\
\hline & Iron & 9 & 3 & 33 & Fisher & 10 & 3 & 30 & Fisher \\
\hline & Magnesium & 9 & 9 & 100 & UTL & 10 & 10 & 100 & UTL \\
\hline & Mercury & 9 & 1 & 11 & Fisher & 10 & - & 0 & Fisher \\
\hline & Nickel & 9 & 7 & 78 & UTL & . & . & . & \\
\hline & Potassium & 9 & 3 & 33 & Fisher & . & . & . & \\
\hline & Selenium & 9 & 1 & 11 & Fisher & 10 & 2 & 20 & Fisher \\
\hline & Sodium & 9 & 9 & 100 & UTL & 10 & 10 & 100 & UTL \\
\hline & Vanadium & 9 & 5 & 56 & UTL & 10 & 1 & 10 & Fisher \\
\hline & Zinc & 9 & 1 & 11 & Fisher & 10 & 1 & 10 & Fisher \\
\hline Pesticides & Dieldrin & 9 & - & 0 & Fisher & . & - & . & \\
\hline
\end{tabular}


Table 7.2. (contd)

\begin{tabular}{|c|c|c|c|c|c|c|c|c|c|}
\hline \multirow[b]{2}{*}{ Analytical Group } & \multirow[b]{2}{*}{ Parameter Name } & \multicolumn{4}{|c|}{ HSU-1 Background } & \multicolumn{4}{|c|}{ HSU-2 Background } \\
\hline & & Samples & Detects & $\%$ Detects & Method & \begin{tabular}{|l|} 
Samples \\
\end{tabular} & Detects & $\%$ Detects & Method \\
\hline \multirow[t]{12}{*}{ Radionuclides } & Actinium-228 &. & $\cdot$ &. & & 7 & - & 0 & UTL \\
\hline & Americium-241 & 4 & - & 이 & UTL & 5 & 1 & 20 & UTL \\
\hline & Bismuth-214 & 7 & 3 & 43 & UTL & 7 & 2 & 29 & $\overline{U T L}$ \\
\hline & Carbon-14 & 9 & - & 0 & UTL & 10 & 2 & 20 & $\overline{U T L}$ \\
\hline & Gross Alpha & 9 & - & of & UTL & 10 & 1 & 10 & $\overline{\text { UTL }}$ \\
\hline & Gross Betạ & 9 & - & 0 & UTL & 10 & 1 & 10 & $\overline{\text { UTL }}$ \\
\hline & Lead-212 & 7 & - & 0 & $\overline{\text { UTL }}$ & . & . & . & \\
\hline & Lead-214 & 7 & 2 & 29 & UTL & 7 & 1 & 14 & UTL \\
\hline & Plutonium-241 & 5 & $-\cdots$ & 0 & UTL & . & . & . & \\
\hline & Radium-226 & 11 & 3 & 27 & UTL & 10 & 1 & 10 & UTL \\
\hline & Strontium-90 & 9 & - & 0 & $\overline{\text { UTL }}$ & 10 & - & 0 & UTL \\
\hline & Tritium & 9 & - & 0 & UTL & 10 & - & 이 & UTL \\
\hline \multirow[t]{4}{*}{ Semi-volatiles } & 4-Methylphenol & 9 & - & 0 & Fisher & . & . & . & \\
\hline & Bis(2-Ethylhexyl)phthalate & . & . & . & & 10 & - & 0 & Fisher \\
\hline & Di-n-Butylphthalate & 9 & 1 & 11 & Fisher &. & . & . & \\
\hline & Phenol & $\overline{9}$ & - & 0 & Fisher & 10 & - & 0 & Fisher \\
\hline \multirow[t]{10}{*}{ Volatiles } & 1,1,2-Trichloroethane & 9 & - & 0 & Fisher &. & . & . & \\
\hline & 1,1-Dichloroethane & 9 & - & 0 & Fisher & . & . & . & \\
\hline & 1,1-Dichloroethene & 9 & - & 0 & Fisher & . & . & . & \\
\hline & 1.2-Dichloroethane & $\overline{9}$ & - & 0 & Fisher & . & . & . & \\
\hline & 2-Butanone & 9 & - & 0 & Fisher & . & . & . & \\
\hline & Bromodichloromethane & $\overline{9}$ & - & o & Fisher & . & . & . & \\
\hline & Carbon Disulfide & 9 & - & of & Fisher & . &. & . & \\
\hline & Chloroform & 9 & - & 0 & Fisher & 10 & - & 0 & Fisher \\
\hline & Toluene & 9 & - & 0 & Fisher & . & . & . & \\
\hline & Xylenes (Total) & 9 & - & 0 & Fisher & . & . & . & \\
\hline
\end{tabular}




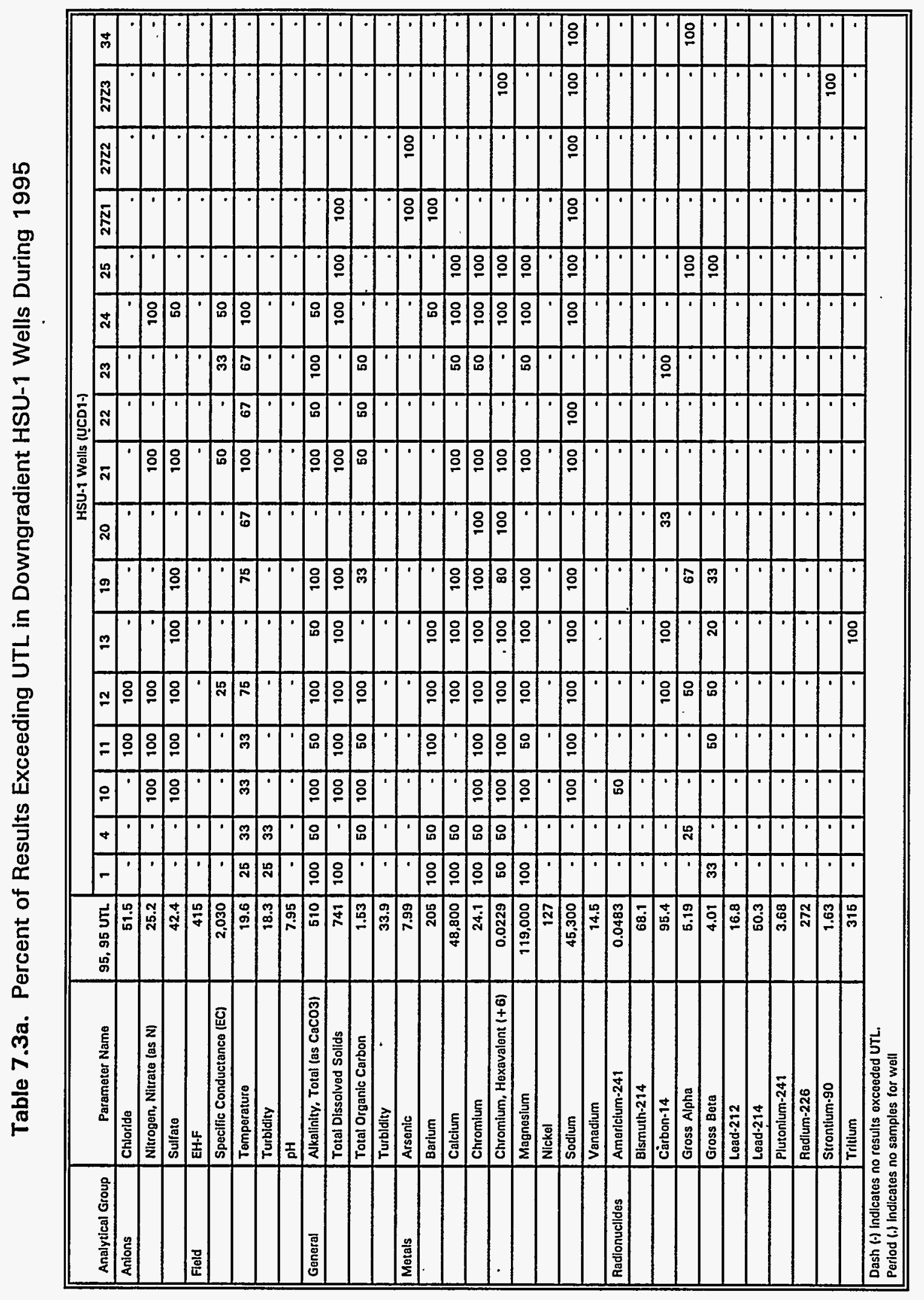


Table 7.3b. Percent of Results Exceeding UTL in Downgradient HSU-2 Wells During 1995

\begin{tabular}{|c|c|c|c|c|c|c|c|c|c|c|c|c|}
\hline \multirow[b]{2}{*}{ Analytical Group } & \multirow[b]{2}{*}{ Paramoter Name } & \multirow[b]{2}{*}{ 95, 95 UTL } & \multicolumn{10}{|c|}{ HSU-2 Wells (UCD2-) } \\
\hline & & & 7 & 14 & 16 & 16 & 26 & 2724 & 2725 & 2726 & $27 z 7$ & 35 \\
\hline \multirow[t]{3}{*}{ Anions } & Chlorido & 20.6 & 100 & 100 & 50 & - & & . & & & & \\
\hline & Nitrogen, Nitrate (as N) & 3.79 & 100 & 100 & - & 100 & & - & $\cdot$ & - & $\cdot$ & \\
\hline & \begin{tabular}{|l|} 
Sulfate \\
\end{tabular} & 41.0 & 67 & 100 & 50 & 100 & & & & & & \\
\hline \multirow[t]{5}{*}{ Field } & EH.F & 362 & $\dot{-}$ & - & - & & & $\dot{5}$ & & & & \\
\hline & Spocfific Conductanco (EC) & 1,330 & $\cdot$ & 25 & 33 & 67 & & . & & . & & \\
\hline & \begin{tabular}{|l} 
Temperature \\
\end{tabular} & 19.3 & 25 & 25 & 33 & 33 & &. & & & & \\
\hline & \begin{tabular}{|l|} 
Turbidity \\
\end{tabular} & 0.740 & $\cdot$ & 50 & 50 & - & & $\dot{5}$ & & & & \\
\hline & $\mathrm{pH}$ & 8.07 & $\cdot$ &. & 33 & $\cdot$ & & . & & . & & \\
\hline \multirow[t]{3}{*}{ General } & Alkalinity, Total (as CaCO3) & 361 & 100 & 100 & $\dot{-}$ & 50 & & & & & & \\
\hline & \begin{tabular}{|l|} 
Total Dissolved Solids \\
\end{tabular} & 438 & 100 & 100 & - & 75 & & - & 100 & 100 & 100 & 100 \\
\hline & \begin{tabular}{|l|} 
Turbidity \\
\end{tabular} & 2.37 & - & $\cdot$ & $=$ & - & & $\cdot$ & . & 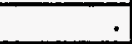 & . & \\
\hline \multirow[t]{6}{*}{ Motals } & - Arsenic & 5.63 & & & - & - & & - & $\cdot$ & - & - & \\
\hline & Barlum & 145 & 100 & 100 & - & 25 & - & $\dot{-}$ & 100 & 100 & - & \\
\hline & \begin{tabular}{|l|} 
Calclum \\
\end{tabular} & 39,500 & & 80 & - & - & $\cdot$ & - & $\cdot$ & $\cdot$ & - & - \\
\hline & Chromium & 24.8 & 100 & 80 & $\cdot$ & 76 & 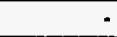 & - & - & $\cdot$ & - & - \\
\hline & Magnasium & 65,000 & 100 & 100 & 50 & 75 & 100 & - & 100 & -1 & - & \\
\hline & Sodium & 32,200 & 100 & 100 & - & 100 & 100 & 100 & 100 & 100 & 100 & 100 \\
\hline \multirow[t]{10}{*}{ Radionuclides } & \begin{tabular}{|l|l|l} 
Actinium-228 \\
\end{tabular} & 112 & & -1 & $\therefore$ & - & - & - & $\dot{0}$ & - & 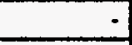 & - \\
\hline & \begin{tabular}{|l|} 
Americlium-241 \\
\end{tabular} & 0.125 & & & 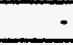 & - & & - & $\cdot$ & - & - & - \\
\hline & \begin{tabular}{|l|l|l|l|} 
Blsmuth-214 \\
\end{tabular} & 161 & $\cdot$ & $\therefore$ & - & - & F. & - & $\cdot$ & 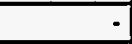 & - & \\
\hline & Carbon-14 & 190 & & 75 & - & - & - & 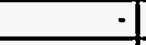 & $\cdot$ & $\cdot$ & - & \\
\hline & Gross Alpha & 47.8 & $\dot{-}$ & $\cdot$ & - & - & - & $\dot{-}$ & - & - & - & $\overline{-}$ \\
\hline & \begin{tabular}{|l|} 
Gross Beta \\
\end{tabular} & 16.9 & 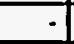 & - & - & - & - & - & - & - & - & \\
\hline & Lead-214 & 39.3 & - & $\dot{1}$ & $\cdot$ & - & - & -1 & $\therefore$ & $\dot{-}$ & 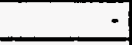 & - \\
\hline & \begin{tabular}{|l|} 
Radium-226 \\
\end{tabular} & 2,050 & & $\dot{-}$ & $\div$ & - & $\cdot$ & - & - & - & - & $\div$ \\
\hline & \begin{tabular}{|l|} 
Strontlum-90 \\
\end{tabular} & 1.26 & $\cdot$ & $\cdot$ & - & - & & - & 100 & - & 100 & \\
\hline & \begin{tabular}{|l|} 
Tritlum \\
\end{tabular} & 292 & 25 & 75 & 25 & - & & $\therefore$ & 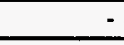 & - & $\cdot$ & . \\
\hline
\end{tabular}


Table 7.4a. Probability that Results in Downgradient HSU-1 Wells During 1995 Could Be from Background

\begin{tabular}{|c|c|c|c|c|c|c|c|c|c|c|c|c|c|c|c|c|c|c|}
\hline \multirow[b]{2}{*}{ Analytical Group } & \multirow[b]{2}{*}{ Paramoter Name } & \multicolumn{17}{|c|}{ HSU-1 Wells (UCD1-) } \\
\hline & & 1 & 4 & 10 & 11 & 12 & 13 & 19 & 20 & 21 & 22 & 23 & 24 & 25 & 2721 & 2722 & 2723 & 34 \\
\hline General & Ammonia-Nitrogen & - & - & - & - & - & 0.18 & 0.25 & - & - & - & - & - & . & & . & . & - \\
\hline \multirow[t]{5}{*}{ Metals } & Iron & - & - & - & - & - & - & $\cdot$ & - & - & - & - & - & - & 0.4 & 0.4 & 0.4 & - \\
\hline & Mercury & - & 0.35 & - & 0.35 & - & - & - & - & - & - & 0.35 & - & - & - & - & - & - \\
\hline & Potassium & - & - & - & - & 0.62 & - & - & - & - & - & - & - & - & - & - & - & - \\
\hline & Selenium & - & - & $60 \%$ & 00 of & 0008 & 0.35 & 6.82 & - & 0.35 & - & 0.35 & 0.35 & 0.2 & - & - & - & \\
\hline & Zine & - & - & $\cdot$ & - & - & - & - & - & - & - & - & - & 0.2 & 0.2 & 0.2 & - & 0.2 \\
\hline Pesticides & Dieldrin & - & . & - & - & - & .0 .93 & - & - & - & - & - & - & - & - & - & - & - \\
\hline \multirow[t]{3}{*}{ Semi-volatiles } & 4-Methylphenol & - & - & -1 & - & - & - & - & - & - & - & - & - & - & 0.1 & 0.1 & 0.1 & - \\
\hline & Di-n-Butylphthalate & - & - & 0.35 & - & - & - & - & - & 0.35 & 0.35 & - & - & - & - & - & - & - \\
\hline & Phenol & - & - & - & - & & - & - & - & - & - & - & - & - & 0.1 & 0.1 & 0.1 & - \\
\hline \multirow[t]{10}{*}{ Volatiles } & 1,1,2-Trichloroethane & - & - & - & - & $800 \%$ & - & $\cdot$ & - & - & - & - & - & - & - & - & - & - \\
\hline & 1,1-Dichloroethane & - & - & - & - & ;:0: & - & - & - & - & - & - & - & - & - & - & - & - \\
\hline & 1,1-Dichloroethene & - & - & - & - & 26004 & - & - & - & - & - & - & - & - & - & - & - & - \\
\hline & 1,2-Dichloroethane & - & - & - & - & 40.09 & - & - & - & - & - & - & - & - & - & - & - & - \\
\hline & 2-Butanone & - & - & - & - & - & - & - & - & - & - & - & - & - & 0.1 & - & - & - \\
\hline & Bromodichloromethane & - & $\cdot$ & - & - & 0.14 & - & $\cdot$ & - & - & - & - & - & - & 0.18 & 0.18 & - & - \\
\hline & Carbon Disulfide & - & - & - & - & & - & $\cdot$ & - & - & - & - & - & - & - & - & 0.1 & - \\
\hline & Chloroform & - & - & 0.31 & 0.18 & 80001 & .0 .91 & - & - & - & - & - & 0.18 & - & 002 & 1004 & 0.1 & - \\
\hline & Toluene & - & - & $\cdot$ & - & - & - & $\cdot$ & - & - & - & $\cdot$ & - & - & 0.1 & 0.18 & - & - \\
\hline & Xylenes (Total) & 0.36 & - & - & - & - & - & $\cdot$ & -1 & - & - & - & - & - & - & - & - & - \\
\hline
\end{tabular}


Table 7.4b. Probability that Results in Downgradient HSU-2 Wells During 1995 Could Be from Background

\begin{tabular}{|c|c|c|c|c|c|c|c|c|c|c|c|}
\hline \multirow[b]{2}{*}{ Analytical Group } & \multirow[b]{2}{*}{ Parameter Name } & \multicolumn{10}{|c|}{ HSU-2 Wells (UCD2-) } \\
\hline & & 7 & 14 & 15 & 16 & 26 & $27 Z 4$ & 2725 & 2726 & 2727 & 35 \\
\hline \multirow[t]{2}{*}{ General } & Ammonia-Nitrogen & 0.58 & & 0.45 & - & & . & . & . & . & \\
\hline & Total Organic Carbon & 0.31 & - & 0.58 & 0.58 & . & . & . & . & . & \\
\hline \multirow[t]{7}{*}{ Metals } & Chromium, Hexavalent $(+6)$ & 0.07 & 0.28 & - & 0.07 & 0.45 & - & - & - & - & . \\
\hline & Copper & - & - & - & - & 0.09 & - & - & - & - & . \\
\hline & Iron & - & 0.85 & - & 0.79 & - & 0.36 & 0.36 & 0.36 & 0.36 & \\
\hline & Mercury & 0.23 & 0.33 & - & - & - & - & - & - & - & \\
\hline & Selenium & 0.2 & & - & 0.09 & - & - & - & - & - & \\
\hline & Vanadium & - & 0.57 & - & 0.51 & - & - & - & $\therefore$ & - & . \\
\hline & Zinc & - & - & - & - & - & - & 0.18 & - & - & . \\
\hline \multirow[t]{2}{*}{ Semi-volatiles } & Bis(2-Ethylhexyl)phthalate & - & & - & - & - & 0.09 & - & 0.09 & - & 0.09 \\
\hline & Phenol & - & & - & - & - & - & - & - & 0.09 & . \\
\hline Volatiles & Chloroform & - & \% & - & 0.23 & 0.09 & 0.09 & 0.09 & 0.09 & - & . \\
\hline
\end{tabular}


Table 7.5. Parameters Above Detection Limit and Significantly Different from Background

\begin{tabular}{|c|c|c|c|c|}
\hline Analytical Group & Parameter Name & Effect & HSU-1(a) & $\mathrm{HSU}-2^{(\mathrm{al})}$ \\
\hline \multirow[t]{3}{*}{ Anions } & Chloride & Indirect & 2 & 3 \\
\hline & Nitrogen, Nitrate (as N) & Direct & 5 & 3 \\
\hline & Sulfate & Indirect & 7 & 4 \\
\hline \multirow[t]{4}{*}{ Field } & Specific Conductance (EC) & Indirect & 4 & 3 \\
\hline & Temperature & Indirect & 11 & 4 \\
\hline & Turbidity & Indirect & 2 & 2 \\
\hline & $\mathrm{pH}$ & Indirect. & 0 & 1 \\
\hline \multirow[t]{3}{*}{ General } & Alkalinity Total (as $\mathrm{CaCO}$ ) & Indirect & 11 & 3 \\
\hline & Total Dissolved Solids & Indirect & 10 & 7 \\
\hline & Total Organic Carbon & Indirect & 8 & 0 \\
\hline \multirow[t]{8}{*}{ Metals } & Arsenic & Indirect & 2 & 0 \\
\hline & Barium & Indirect & 7 & 5 \\
\hline & Calcium & Indirect & 9 & 1 \\
\hline & Chromium & Direct & 12 & 3 \\
\hline & Chromium, Hexavalent $(+6)$ & Direct & 12 & 0 \\
\hline & Magnesium & Indirect & 10 & 6 \\
\hline & Selenium & Indirect & 4 & 0 \\
\hline & Sodium & Indirect & 13 & 9 \\
\hline Pesticides & Dieldrin & Direct & 1 & 0 \\
\hline \multirow[t]{5}{*}{ Radionuclides } & Americium-241 & Indirect & 1 & 0 \\
\hline & Carbon-14 & Direct & 4 & 1 \\
\hline & Gross Alpha & Indirect & 5 & 0 \\
\hline & Gross Beta & Indirect & 6 & 0 \\
\hline & Tritium & Direct & 1 & 3 \\
\hline \multirow[t]{5}{*}{ Volatiles } & 1,1,2-Trichloroethane & Direct & 1 & 0 \\
\hline & 1,1-Dichloroethane & Direct & 1 & 0 \\
\hline & 1,1-Dichloroethene & Direct & 1 & 0 \\
\hline & 1,2-Dichloroethane & Direct & 1 & 0 \\
\hline & Chloroform & Direct & 4 & 1 \\
\hline
\end{tabular}


Table 7.6. Regulatory Significance of Parameters That Exceeded Background

\begin{tabular}{|c|c|c|c|c|c|}
\hline \multirow{2}{*}{$\begin{array}{l}\text { Analytical } \\
\text { Group }\end{array}$} & \multirow[b]{2}{*}{ Parameter Name } & \multirow{2}{*}{$\begin{array}{c}\text { Maximum } \\
\text { Concentration }\end{array}$} & \multirow{2}{*}{$\begin{array}{c}\text { Regulatory } \\
\text { Limit }\end{array}$} & \multicolumn{2}{|c|}{ Significant } \\
\hline & & & & HSU-1 & HSU-2 \\
\hline \multirow[t]{3}{*}{ Anions } & Chloride & $220 \mathrm{mg} / \mathrm{L}$ & $250^{(2)}$ & No & No \\
\hline & Nitrogen, Nitrate (as N) & $72 \mathrm{mg} / \mathrm{L}$ & 10 & $5^{\text {(b) }}$ & 2 \\
\hline & Sulfate & $290 \mathrm{mg} / \mathrm{L}$ & $250^{(3)}$ & 1 & No \\
\hline \multirow[t]{3}{*}{ Field } & Specific Conductance (EC) & 2120 umhos/cm & None & No & No \\
\hline & Temperature & $23.9^{\circ} \mathrm{F}$ & None & No & No \\
\hline & Turbidity & $19.2 \mathrm{NTU}$ & None & No & No \\
\hline \multirow[t]{4}{*}{ General } & Alkalinity Total(as $\mathrm{CaCO} 3$ ) & $870 \mathrm{mg} / \mathrm{L}$ & None & No & No \\
\hline & Total Dissolved Solids & $1700 \mathrm{mg} / \mathrm{L}$ & $500^{(2)}$ & $8^{(b)}$ & 2 \\
\hline & Total Organic Carbon & $2.8 \mathrm{mg} / \mathrm{L}$ & None & No & No \\
\hline & pH & $8.7 \mathrm{mg} / \mathrm{L}$ & $6.5-8.5$ & No & 1 \\
\hline \multirow[t]{8}{*}{ Metals } & Arsenic & $8.6 \mu \mathrm{g} / \mathrm{L}$ & 50 & No & No \\
\hline & Barium & $355 \mu \mathrm{g} / \mathrm{L}$ & 1,000 & No & No \\
\hline & Calcium & $75.4 \mathrm{mg} / \mathrm{L}$ & None & No & No \\
\hline & Chromium & $290 \mu \mathrm{g} / \mathrm{L}$ & 50 & 7 & No \\
\hline & Chromium, Hexavalent $(+6)$ & $310 \mu \mathrm{g} / \mathrm{L}$ & 50 & 7 & 1 \\
\hline & Magnesium & $227 \mathrm{mg} / \mathrm{L}$ & None & No & No \\
\hline & Selenium & $23 \mu \mathrm{g} / \mathrm{L}$ & 5 & 4 & No \\
\hline & Sodium & $191 \mathrm{mg} / \mathrm{L}$ & None & No & No \\
\hline Pesticides & Dieldrin & $0.029 \mu \mathrm{g} / \mathrm{L}$ & None & No & No \\
\hline \multirow[t]{5}{*}{ Radionuclides } & Americium-241 & 0.106 & None & 1 & No \\
\hline & Carbon-14 & 1,900 & None & 4 & 1 \\
\hline & Gross Alpha & $8.1 \mathrm{pCi} / \mathrm{L}$ & 15 & No & No \\
\hline & Gross Beta & $15 \mathrm{pCi} / \mathrm{L}$ & 50 & No & No \\
\hline & Tritium & $19,600 \mathrm{pCi} / \mathrm{L}$ & 20,000 & No & No \\
\hline \multirow[t]{5}{*}{ Volatiles } & 1,1,2-Trichloroethane & $3.5 \mu \mathrm{g} / \mathrm{L}$ & 5 & No & No \\
\hline & 1,1-Dichloroethane & $9.2 \mu \mathrm{g} / \mathrm{L}$ & 5 & 1 & No \\
\hline & 1,1-Dichloroethene & $18 \mu \mathrm{g} / \mathrm{L}$ & 6 & 1 & No \\
\hline & 1,2-Dichloroethane & $5.9 \mu \mathrm{g} / \mathrm{L}$ & 0.5 & 1 & No \\
\hline & Chloroform & $9,100 \mu \mathrm{g} / \mathrm{L}$ & (c) & 1 & No \\
\hline
\end{tabular}

(a) Indicates that the regulatory limit reported is a secondary drinking water regulation and based on the aesthetic quality of the water not a health hazard.

(b) Indicates that the background concentration exceeded the regulatory limit. A number indicates how many sampling locations exceeded the regulatory limit for at least one quarter during 1995.

(c) MCL for total trihalomethanes is $100 \mu \mathrm{g} / \mathrm{L}$. Chioroform is the predominant trihalomethane detected at LEHR. 


\subsection{Evaluation of Water Monitoring Program}

Guidance for Long-Term Groundwater Monitoring (California Base Closure Environmental Committee 1994) recommends that data gaps and potential deficiencies in the monitoring network be identified based on data presented in the annual report. This section presents an assessment of the groundwater monitoring network using analytical results from the 1995 monitoring program and historical trends from previous monitoring and from three hydropunch groundwater sampling campaigns. This evaluation of the monitoring program focused on four major questions:

- Is the analyte list sufficient?

- Are the monitoring locations adequate?

- Is the sampling frequency appropriate for site conditions?

- Are the appropriate analytical techniques used?

The analyte list is evaluated from the standpoint of 1) the constituents of concern as they relate to direct hazard and 2) other analytes that further our understanding of how those constituents of concern behave in the environment. The adequacy of monitoring well locations is judged by how well the monitoring data can be used to identify configuration changes in contaminant plumes that were delineated in greațer detail by onetime sampling at many locations, e.g., hydropunch. Sampling frequency is judged by how well temporal variations are detected and judged to be significant. We determine how appropriate an analytical technique is by comparing the detection limit with either the background concentration or some regulatory limit, such as an MCL or a PRG.

During the summer of 1995, DOE, UC Davis, and California and federal regulators agreed to revise the groundwater monitoring program. Program revisions included changes in the analyte list and sampling frequency. The group also agreed to the installation of five new monitoring wells and groundwater sampling using the hydropunch technique. The following sections present discussions of each of these topics and the four questions identified above.

\subsection{Analyte List}

The analyte list is composed of hazardous constituents that are known or suspected to have been disposed at LEHR; parameters that are used as gross indicators of the presence of contamination, and general parameters that are used to characterize the general chemistry of the water. 
Three analyte lists were presented in Tables 2.3, 2.4, and 2.5 for groundwater, surface water, and storm water runoff samples, respectively. The analyte lists for groundwater and surface water were modified starting in the summer quarter of 1995 . The analyte list for storm water runoff was not modified in 1995. Changes to the groundwater and surface water analyte lists eliminated the following analytes:

- Field - Eh

- Laboratory - ammonia, TKN, alkalinity, anions, turbidity, total organic carbon, chemical oxygen demand, and formaldehyde

Eh had been measured for the previous seven quarters and was well established within each well (Table C.6). In general, all of the groundwaters were found to be oxidizing with average Eh values in the range of +112 to $+177 \mathrm{mV}$. The same results were determined for surface waters.

Ammonia was eliminated from the groundwater monitoring program because over the previous eight quarters it was generally found to be less than the detection limit of $50 \mu \mathrm{g} / \mathrm{L}$. Ammonia was detected sporadically across the monitoring network and sampling periods but never more than twice in any single well. It was always detected in the UC Davis wastewater treatment plant outfall (STPO) at concentrations in excess of $1,000 \mu \mathrm{g} / \mathrm{L}$, which caused an increase in the downstream monitoring station (PCD) over the normal Putah Creek background of slightly above the detection limit. These trends can be seen in Figure 8.1. It is clear from Figure 8.1 that the UC Davis wastewater treatment

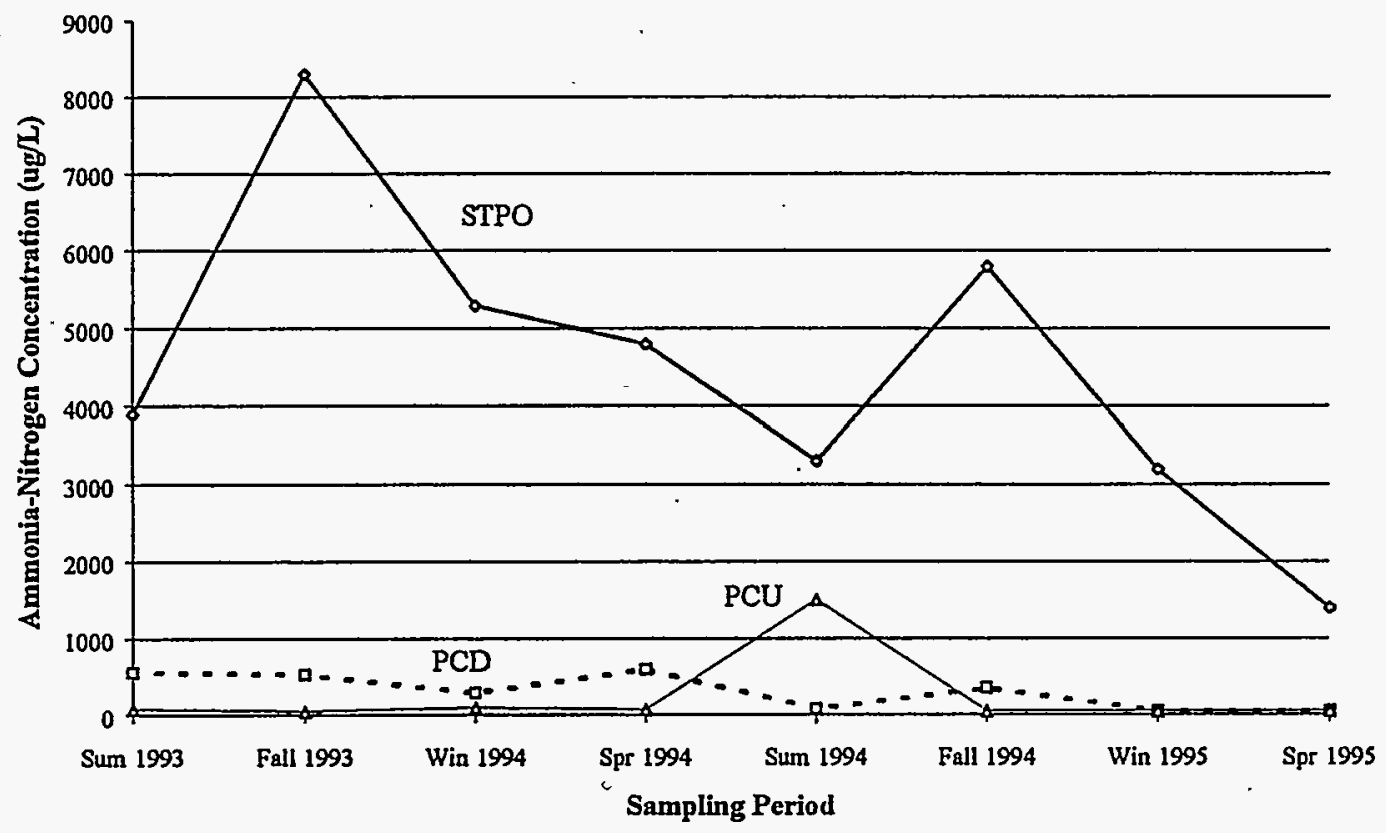

Figure 8.1. Putah Creek Ammonia-Nitrogen 
plant has routinely discharged ammonia at levels in excess of those found upstream of the outfall. The elevated levels have affected the concentrations of ammonia in Putah Creek, as indicated by the fact that PCD is generally higher than PCU. This, while interesting, has no bearing on LEHR. Clearly the ammonia source is the UC Davis wastewater plant outfall, which is controlled by an NPDES permit.

TKN was eliminated from the groundwater monitoring system because it had been analyzed for the previous eight quarters and reported above the detection limit of $500 \mathrm{mg} / \mathrm{L}$ nitrogen only twice. In surface water, TKN was reported above the detection limit for all samples collected at STPO, all but two samples at PCD, and only one sample at PCU. TKN, ammonia, and ammonia plus nitrate results for STPO are shown in Figure 8.2. These data show that TKN and ammonia nitrogen results are closely correlated, indicating that most of the TKN detected is from ammonia. Organic compounds may provide some additional TKN that has been detected. As indicated in Figure 8.2, TOC is generally at concentrations greater than TKN. Nitrate is not a significant part of the TKN, as indicated by the fact that the nitrate plus ammonia trend does not follow the TKN trend. These data indicate that TKN is generally sensing ammonia, which is from the UC Davis wastewater treatment plant and not significant to LEHR. Therefore, TKN was eliminated from the analyte list.

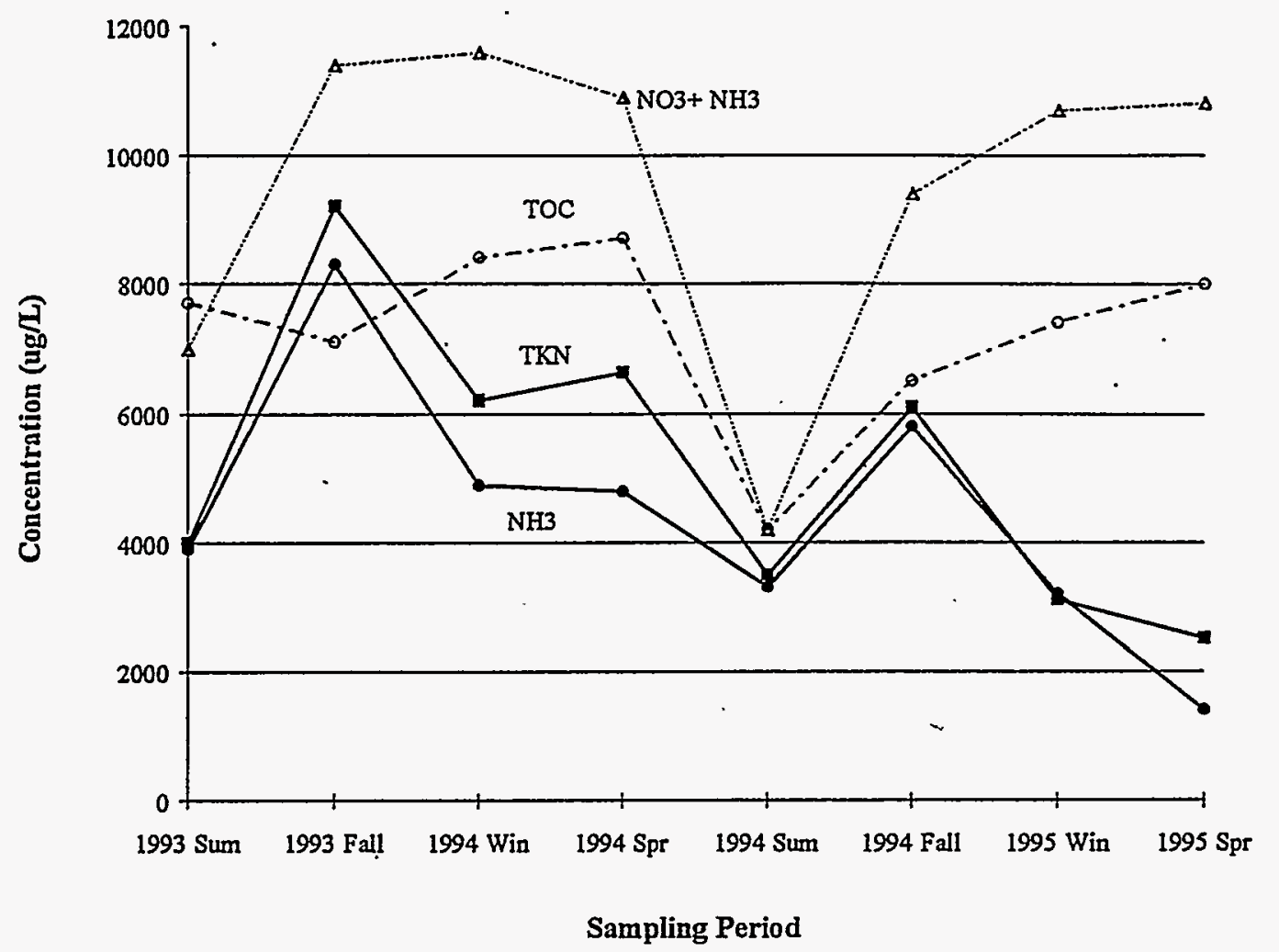

Figure 8.2. Concentrations of Nitrogen-Containing Compounds in UC Davis Wastewater Treatment Plant Outfall (STPO) 
Alkalinity and anions, except nitrate, were eliminated because they are important chiefly to determine the general chemistry of the groundwater and surface water and not because they are constituents of concern that were disposed of in hazardous quantities. Because the database contains considerable general chemistry data, and those data indicate fairly stable conditions over time, the anion group was eliminated from the program.

Laboratory turbidity was eliminated from the program because field turbidity is measured at the time of sampling. There is no reason to duplicate this measurement, especially after the laboratory sample has been stored for a period of time.

Total organic carbon (TOC) and chemical oxygen demand (COD) are parameters that provide an indication of the chemical conditions at the site and are not hazardous constituents. Once a baseline concentration has been established for each parameter, there is little need to continue monitoring their levels. TOC has been analyzed for eight quarters and COD has been analyzed for 14 quarters, and baseline concentrations have been established for each. Therefore, TOC and COD were eliminated from further analysis.

The last analyte eliminated from the monitoring program is formaldehyde, which has been analyzed in every sampling event since the program began in the fall of 1990 . Formaldehyde has been detected sporadically in 16 of the 18 original monitoring wells, including both background wells, ranging from 0.26 to $3.5 \mathrm{mg} / \mathrm{L}$ in UCD1-21. There is no drinking water standard for formaldehyde, but the EPA Region 9 PRG for formaldehyde in tap water (concentration that produces a hazard index of 1 ) is $5.5 \mathrm{mg} / \mathrm{L}$. Formaldehyde has been detected four times in surface water samples at a maximum concentration of $2.1 \mathrm{mg} / \mathrm{L}$. Therefore, formaldehyde has never been detected above a regulatory limit, and it has generally been reported as less than the detection limit. For these reasons, formaldehyde was eliminated from the monitoring program.

\subsection{Monitoring Locations}

The adequacy of the monitoring network is judged on the basis of whether contaminant plumes can be mapped using the data collected. Figures 5.11 through 5.21 show the distributions of the major contaminants in LEHR groundwater. Based on these figures, it appears that the plumes can be mapped. The figures were drawn from composite database consisting of the following in order of priority:

- 1995 monitoring data

- 1995 hydropunch data

- 1994 hydropunch data

Complete plume diagrams could be drawn only by combining these data. If a plume is to be monitored every year to determine how it is changing in size and concentration, permanent monitoring wells need to be constructed in critical areas or hydropunch samples must be collected every year. Data collected from the new monitoring well, UCD1/2-27, 
showed that contamination in HSU-2 was concentrated in the top half of the unit and did not extend to the bottom of the aquifer. Based on these results, it is important to monitor the upper half of HSU-2 only.

Five new wells were drilled in 1995; three (UCD1-25, UCD2-26, and UCD1/2-27) were located directly east of LEHR, on the northern edge of the chloroform plume. Two wells, UCD1-34 and UCD2-35, were located south of Landfill 2 between the southern boundary of LEHR and Putah Creek. While these wells have only been sampled once as part of the monitoring program, they are already providing valuable information in those areas.

While drawing the plume diagrams for Section 5 , we identified the following areas that need additional monitoring wells:

- south and east of new well UCD1-25, wells need to be completed along the centerline of the chloroform plume in both HSU-1 and HSU-2.

- in the vicinity of the Nishi well, east of UC Davis property.

Chloroform-contaminated groundwater has been detected in these areas, but its extent and distribution are unknown. Additional monitoring wells will allow these plumes to be monitored.

Three surface water monitoring stations are located along the reach of Putah Creek that flows past LEHR. One station is upgradient of LEHR (PCU), one in at the discharge of the UC Davis wastewater treatment plant (STPO), and one is downgradient of all UC Davis and LEHR property (PCD). In previous reports, the location of PCD has been reported to be at a location just upstream of the storm runoff ditch on the eastern boundary of UC Davis property. This reported location was incorrect; the actual sampling location has always been 150 feet downgradient of the ditch discharge point. The true location of PCD is shown on all figures in which it is noted in this report.

\subsection{Sampling Frequency}

The sampling frequency was changed from quarterly sampling for every well and analyte in the monitoring program to a mix of schedules for each well and each analyte in a well. This change was predicated on an understanding of groundwater characteristics that had been developed over the five years of quarterly monitoring. Three sampling frequencies, quarterly, semiannual, and annual, were implemented in the summer quarter of 1995. All wells except UCD1-21 and UCD1-24 are sampled at least semiannually; UCD1-21 and UCD1-24 are sampled annually. Eight wells, UCD1-1, UCD1-11, UCD1-18, UCD1-20, UCD1-22, UCD1-23, UCD2-7, and UCD2-17, are sampled semiannually; the remaining eight wells are sampled quarterly. All new wells are sampled quarterly for the first year, after which the specific monitoring program for each well will be reviewed and modified as appropriate. 
While a specific well may be monitored in accordance with one of the three sampling frequencies, specific analytes may not be analyzed as frequently. For example, as shown in Table 2.1, UCD1-18 is sampled semiannually but only for nitrate and chromium; all other constituents included are analyzed on an annual basis. Some constituents, such as strontium-90, radium-226, plutonium-241, and americium-241, have been eliminated from the sampling program for UCD1-18. The reduced sampling and analysis frequencies were based on the concept that a stable baseline has been established for a specific constituent in a specific well.

For the two sampling periods in 1995 when the revised monitoring schedule was implemented, only one issue has been identified. In several wells, chloroform results did not correspond to historical trends. Normally, the lab would be asked to re-analyze the sample, but in the case of VOC analyses, all three sample viais are used if the sample contained a sufficient amount of chloroform to require dilution. Therefore, no sample remains to perform a re-analysis. This issue existed before the program revision, but the data could be checked in the following quarterly sampling period. In the current plan, the monitoring program must be modified to collect a sample from the well in the next quarterly sampling event, if it is not already scheduled. No other issues have been identified, and the program revision appears to be adequate.

\subsection{Analytical Techniques}

The analytical techniques used in the program are generally the specified techniques for EPA's Certified Laboratory Program (CLP). The detection limits for each technique are lower in most cases than a regulatory or human health risk limit. The major limitation of the analytical program is that there are no analytical techniques to detect the bioaccumulating constituents of concern (e.g., chlordane) in water at the concentrations at which they are hazardous via the food chain. For example, the MCL for chlordane is $2 \mu \mathrm{g} / \mathrm{L}$; the concentration in water that would yield a $1 \mathrm{E}-6$ excess cancer risk in humans based on direct exposure is $0.052 \mu \mathrm{g} / \mathrm{L}$; and the detection limit is $0.01 \mu \mathrm{g} / \mathrm{L}$. The detection limit is sufficiently low for these two criteria.

\subsection{Conclusions}

The water monitoring program at LEHR was reviewed and revised in the second half of 1995. The revision included a reduction in the numbers of analytes as well as their sampling frequency. In addition, five new monitoring wells were constructed at two locations. Based on these changes, the following conclusions can be made:

- The reduced sampling list is sufficient to monitor environmental hazards from LEHR

- The sampling frequency, while more complex than the previous monitoring program, is sufficient to monitor environmental hazards. Modifications to the program need to be made manually to ensure that followup sampling is conducted where analytical problems arise. 
- The analytical techniques used are EPA approved and the laboratory is certified by the State of California.

- Current monitoring locations provide good coverage for evaluating groundwater contamination on the LEHR facility but are insufficient to monitor contaminant plumes that extend downgradient to the east of LEHR and UC Davis property. Monitoring locations on Putah Creek are sufficient for monitoring impacts of LEHR on that water body. Although not plotted correctly on maps in the previous reports, the downgradient sampling station has always been located downstream of all UC Davis and LEHR property and possible water discharges associated with that property. 


\subsection{References}

Bouwer, H. 1989. "The Bouwer and Rice Slug Test - An Update." Ground Water, 27(3):304-309.

Bouwer, $\mathrm{H}$, and RC Rice. 1976. "A Slug Test for Determining Hydraulic Conductivity of Unconfined Aquifers With Completely or Partially Penetrating Wells." Water Resources Research, 1.2:423-428.

Brown, DL, TN Narasimhan, and Z Demir. 1985. "An Evaluation of the Bouwer and Rice Method of Slug Test Analysis." Water Resources Research, 31(5):1239-1246.

California Base Closure Environmental Committee. 1994. Long-Term Ground Water Monitoring Program Guidance. Site Characterization Process Action Team for the California Base Closure Environmental Committee.

California Department of Water Resources (DWR). 1978. Evaluation of Groundwater Resources: Sacramento Valley. Bulletin 118-6.

Cohen, AC Jr. 1959. "Simplified Estimators for the Normal Distribution When Samples Are Singly Censored or Truncated." Biometrics 1(3):217-237.

Currie, LA. "Limits for Qualitative Detection and Quantitative Determination." Analytical Chemistry, 40(3):586-593.

Dames \& Moore. 1990a. Final SWAT Report, Old UC Davis Landfill, UC Davis.

Dames \& Moore. 1990b Contaminant Pathway Analysis for the UC Davis Campus from the LEHR Study Site, UC Davis.

Dames \& Moore. 1990c. Evaluation of Potential Nitrate and Hexavalent Chromium Sources in the Vicinity of the UC Davis LEHR Facility for the UC Davis.

Dames \& Moore. 1990d. Putah Creek Sediment and Water Sampling for the UC Davis.

Dames \& Moore. 1991a. Old UCD Landfill Additional Characterization - CPT and Hydropinch Investigation - UC Davis South Campus Inactive Disposal Sites.

Dames \& Moore. 1991b. Waste Burial-Trench Investigation LEHR Facility, UC Davis.

Dames \& Moore. 1991a CEQA Preliminary Environmental Study for Site Characterization Work at the Inactive UC Davis Landfill. 
Dames \& Moore. 1993. Phase // Characterization Report for the LEHR Environmental Restoration, UC Davis.

Davisson, ML, and RE Criss. 1993. "Stable Isotope Imaging of a Dynamic Groundwater System in the Southwestern Sacramento Valley, California, USA." J. Hydrol., $144: 213-246$.

Davisson, ML, RE Criss, and KR Campbell. November 1993. Preliminary Report on the Stable Isotope Imaging and Characterization of Surface and Ground Water Resources in the Southern Sacramento Valley. UCRL-ID-115393, Lawrence Livermore National Laboratory, Berkley, California.

Evenson, KD. January 1985. Chemical Quality of Ground Water in Yolo and Solano Counties, California. USGS Water Resources Investigations Report 84-4244.

Hyder, Z, JJ Butler Jr, CD McElwee, and W Liu. 1994. "Slug Tests in Partíally Penetrating Wells." Water Resources Research, 31(5):1239-1246.

Hyder, Z, and JJ Butler Jr. 1995. "Slug Tests in Unconfined Formations: An Assessment of the Bouwer and Rice Technique." Ground Water, 33(1):16-22.

In-Situ, Inc. 1991. WATER-VELTM Groundwater Velocity. ISI-GWV-2.21-1, In-Situ, Inc., Laramie, Wyoming.

Liikala, TL, DC Lanigan, GV Last, RE Lewis, DR Newcomer, FA Spane, DL Stewart, SS Teel, VR Vermeul, and WC Schaal. 1996. "Groundwater Characterization Field Activities for 1995-96." PNNL-11092, Pacific Northwest National Laboratory, Richland, Washington.

Mann, JT. January 17, 1992. "Hydrogeology of Lower Putah Creek - Preliminary Discussion." Solano County Water Agency.

Maxey, GB. 1964. "Hydrostratigraphic Units." Journal of Hydrology, vol. 2, pp.124-129.

Norris, RM, and RW Webb. 1976. Geology of California. John Wiley \& Sons, New York. .

Olmsted, FH, and GH Davis. 1961. Geologic Features and Groundwater Storage Capacity of the Sacramento Valley, California. USGS Water Supply Paper 1497.

Pacific Northwest Laboratory. January 1994. Water Monitoring Plan, LEHR Environmental Restoration, Davis California. 
Pacific Northwest National Laboratory. 1995a. Quarterly Groundwater and Surface Water Monitoring Results, Winter and Spring Quarters 1995. DOE Phase II Characterization, LEHR Facility, Davis California. PNNL-11083, PNNL, Richland, Washington.

Pacific Northwest National Laboratory. 1995b Quarterly Groundwater and Surface Water Monitoring Results, Summer Quarter 1995. DOE Phase II Characterization, LEHR Facility, Davis, California. PNNL-1 1084, PNNL, Richland, Washington.

Rockwell International Corporation. 1984. Initial Assessment Survey of the DOE LEHR Site of UC Davis.

Spane, FA Jr. 1994. "Applicability of Slug Interference Tests for Hydraulic . Characterization of Unconfined Aquifers: (1) Analytical Assessment." PNL-SA-24283, Pacific Northwest Laboratory, Richland, Washington (accepted for publication in Ground Water in the January-February 1996 Issue)

Spane, FA Jr., and SK Wurstner. 1993. "DERIV: A Program for Calculating Pressure Derivatives for Use in Hydraulic Test Analysis." Ground.Water, 31(5):814-822.

U.S. Department of Energy (DOE). 1988. Environmental Survey Preliminary Report Laboratory for Energy-Related Health Research, Davis, California.

U.S. Department of Energy (DOE). 1993. Phase // Characterization Report for the LEHR Environmental Restoration, UC Davis. DOE San Francisco Field Office.

U.S. Department of Energy (DȮE). 1994a. Final Draft RI/FS Work Plan, LEHR Environmental Restoration, UC Davis. Oakland Operations Office.

U.S. Department of Energy (DOE). 1994b 1993 Annual Water Monitoring Report for the LEHR Environmental Restoration, Davis, California. Oakland Operations Office.

U.S. Department of Energy (DOE). 1995. 1994 Annual Water Monitoring Report, LEHR Environmental Restoration, UC Davis. Oakland Operations Office.

U.S. Environmental Protection Agency (EPA). 1989a. USEPA National Functional Guidelines for Laboratory Data Review, Inorganics. EPA, Washington, D.C.

U.S. Environmental Protection Agency (EPA). 1989b Statistical Analysis of Groundwater Monitoring Data at RCRA Facilities. EPA, Washington, D.C.

U.S. Environmental Protection Agency (EPA). 1991. USEPA National Functional Guidelines for Laboratory Data Review, Organics. EPA, Washington, D.C.

U.S. Geological Survey (USGS). 1995. WATSTOR Groundwater Database Search for Analytical and Well Completion Data in Davis, California Area. 
Van der Kamp, G. 1976. "Determining Aquifer Transmissivity by Means of Well Response Tests: The Underdamped Case." Water Resources Research, 12(1):71-77.

Van der Kamp, G. 1984. "Underdamped Well Response Considering Friction in the Well Tubing." Saskatchewan Research Council, SRC Pub. No. R0844-2-c-84. Prepared for Rockwell Hanford Operations.

Wahler Associates. 1988. Groundwater and Soils Investigation, U.C. Davis LEHR Facility. Davis, California.

Wahler Associates. 1989. Groundwater and Soils Investigation, U.C. Davis LEHR Facility. Davis, California. 
Appendix A

Groundwater and Trip Blank Analytical Results 



\section{Appendix A}

\section{Groundwater and Trip Blank Analytical Results}

\section{How to Read Appendix A Data Qualifiers}

The key below defines the relationship between a given result and the associated data qualifiers, as presented in the tables of analytical results in Appendix A.

\begin{tabular}{||l|c|c|c|c||}
\hline \multicolumn{1}{|c|}{ Key $\rightarrow$} & A & B & C & D \\
\hline \hline Example Data $\rightarrow$ & $<$ & 5.200 & Uz & JB \\
\hline \hline A $\quad$ < Signifies that analyte level is less than indicated value. \\
B Indicated value in units as noted in table footnote. \\
C Data qualifier(s) ${ }^{*}$ assigned by Consultant's data review team. \\
D Data qualifier(s) ${ }^{*}$ assigned by reporting laboratory. \\
\hline
\end{tabular}

In the example above, the chemical was analyzed for, but was qualified as not detected due to the bias introduced by contamination in the method blank associated with this analysis. Presence of the analyte in the method blank is noted by the laboratory qualifier, "B." The Consultants qualifier, "Uz," indicates that the analyte concentration is considered to be below the adjusted detection limit (quantitation limit) due to the level of contamination in the method blank. 


\section{Data Validation Qualifiers \\ Assigned by Consultants Data Review Team}

The following definitions provide brief explanations of the qualifiers assigned in the data review process. Validation reason codes for GC/MS organics, GC organics, and metals are recorded by the data reviewers and retained in review support documentation for the data review process.

\begin{tabular}{|c|c|}
\hline Flag & Data Qualifier Definition \\
\hline $\mathrm{U}$ & $\begin{array}{l}\text { The analyte was analyzed for, but was not detected above the reported sample } \\
\text { quantitation limit. }\end{array}$ \\
\hline $\mathrm{J}$ & $\begin{array}{l}\text { The analyte was positively identified; the associated numerical value is the approximate } \\
\text { concentration of the analyte in the sample. }\end{array}$ \\
\hline $\mathbf{N}$ & $\begin{array}{l}\text { The analysis indicates the presence of an analyte for which there is presumptive } \\
\text { evidence to make a "tentative identification." }\end{array}$ \\
\hline $\mathrm{NJ}$ & $\begin{array}{l}\text { The analysis indicates the presence of an analyte that has been "tentatively identified" } \\
\text { and the associated numerical value represents its approximate concentration. }\end{array}$ \\
\hline UJ & $\begin{array}{l}\text { The analyte was not detected above the reported sample quantitation limit. However, } \\
\text { the reported quantitation limit is approximate and may or may not represent the actual } \\
\text { limit of quantitation necessary to accurately and precisely measure the analyte in the } \\
\text { sample. }\end{array}$ \\
\hline $\mathbf{R}$ & $\begin{array}{l}\text { The sample results are rejected due to serious deficiencies in the ability to analyze the } \\
\text { sample and meet quality control criteria. The presence or absence of the analyte cannot } \\
\text { be verified. }\end{array}$ \\
\hline c & Calibration failure; poor or unstable response. \\
\hline d & Laboratory duplicate imprecision. \\
\hline e & Laboratory duplicate control sample imprecision. \\
\hline$f$ & Field duplicate imprecision. \\
\hline $\bar{h}$ & Holding time violation. \\
\hline $\mathbf{k}$ & Serial dilution imprecision. \\
\hline 1 & Laboratory control sample recovery failure. \\
\hline $\mathbf{m}$ & Matrix spike/matrix spike duplicate recovery failure. \\
\hline $\mathbf{0}$ & Calibration blank contamination (metals/inorganics only). \\
\hline $\mathbf{p}$ & Preparation blank contamination (metals/inorganics only). \\
\hline $\mathbf{r}$ & Linearity failure in initial calibration. \\
\hline $\mathbf{s}$ & Surrogate spike recovery failure (GC organics and GC/MS organics only). \\
\hline $\mathbf{z}$ & Method blank contamination. \\
\hline
\end{tabular}




\section{Lockheed Analytical Services \\ Data Qualifiers for Organic Analyses \\ (Revised 01/19/1994)}

\begin{tabular}{|c|c|}
\hline \multicolumn{2}{|r|}{ For Use On the Analytical Data Reporting Forms } \\
\hline A & For CLP analyses only - The TIC is a suspected aldol-condensation product. \\
\hline B & $\begin{array}{l}\text { Any constituent that was also detected in the associated blank whose } \\
\text { concentration was greater than the practical or reporting detection limit (PQL or } \\
\text { RDL). }\end{array}$ \\
\hline C & Constituent confirmed by GS/MS analysis [pesticide/PCB analyses only]. \\
\hline $\mathbf{D}$ & $\begin{array}{l}\text { Constituent detected in the diluted sample. It also indicates that an accurate } \\
\text { quantitation is not possible due to surrogates being diluted out of the samples } \\
\text { during the course of the analysis. }\end{array}$ \\
\hline E & Constituent concentration exceeded the calibration range. \\
\hline G & $\begin{array}{l}\text { The quantitation is not gasoline or diesel but believed to be some other } \\
\text { combination of hydrocarbons. }\end{array}$ \\
\hline $\mathbf{H}$ & $\begin{array}{l}\text { Sample analysis performed outside of method- or client-specified maximum } \\
\text { holding time requirement. }\end{array}$ \\
\hline$J$ & $\begin{array}{l}\text { Estimated value - (1) constituent detected at a level less than the RDL or PQL } \\
\text { and greater than or equal to the MDL; (2) estimated concentration for TICs (for } \\
\text { CLP reporting on/y) }\end{array}$ \\
\hline $\mathbf{N}$ & $\begin{array}{l}\text { For CLP reporting only - Tentatively identified constituents (TICs) identified } \\
\text { based on mass spectral library search. }\end{array}$ \\
\hline $\mathbf{P}$ & $\begin{array}{l}\text { For } C L P \text { reporting only - The percent difference between the concentrations } \\
\text { detected on both GC columns was greater than } 25 \text { percent [pesticide/PCB } \\
\text { analyses only]. }\end{array}$ \\
\hline $\mathbf{U}$ & $\begin{array}{l}\text { For CLP reporting only - Constituent was analyzed for but not detected (sample } \\
\text { quantitation must be corrected for dilution and percent moisture). }\end{array}$ \\
\hline$X, Y$, or $Z$ & Analyst-defined qualifier. \\
\hline $\begin{array}{c}\text { N/A } \\
\text { (\% Moisture) }\end{array}$ & $\begin{array}{l}\text { N/A in the \% moisture cell indicates that data are reported on an "as received" } \\
\text { basis. A value in the \% moisture cell indicates that data are reported based on a } \\
\text { "dry weight" basis. For non-CLP work, RDLs are not adjusted for \% moisture } \\
\text { even when data are reported on a "dry weight" basis. }\end{array}$ \\
\hline \multicolumn{2}{|r|}{ For Use On the QC Data Reporting Forms } \\
\hline * & $\begin{array}{l}\text { QC data (i.e., percent recovery data for matrix spike, matrix spike duplicate, } \\
\text { laboratory control standard, or surrogates; and RPD for matrix spike duplicate or } \\
\text { unspiked duplicate) exceeded acceptance limits. }\end{array}$ \\
\hline
\end{tabular}




\section{Lockheed Analytical Services \\ Data Qualifiers for Organic Analyses (contd)}

\begin{tabular}{||l|l||}
\hline$a^{1}$ & $\begin{array}{l}\text { The spike recovery and/or RPD for matrix spike and matrix spike duplicates } \\
\text { cannot be evaluated due to insufficient spiking level compared to the elevated } \\
\text { sample analyte concentration. }\end{array}$ \\
\hline$b^{1}$ & $\begin{array}{l}\text { The RPD cannot be computed because the sample and/or duplicate concentration } \\
\text { was below the RDL. }\end{array}$ \\
\hline
\end{tabular}

A. 4 
Lockheed Analytical Services

Data Qualifiers for Inorganic Analyses

\begin{tabular}{|c|c|}
\hline \multicolumn{2}{|r|}{ For Use On the Analytical Data Reporting Forms } \\
\hline B & $\begin{array}{l}\text { For CLP analyses only - Reported value is less than the contract required } \\
\text { detection limit (CRDL) but greater than or equal to the instrument detection limit } \\
\text { (IDL). }\end{array}$ \\
\hline C & $\begin{array}{l}\text { For Routine, Non-CLP analyses only - Any constituent that was also detected in } \\
\text { the associated blank whose concentration was greater than the reporting } \\
\text { detection limit (RDL). }\end{array}$ \\
\hline $\mathbf{D}$ & $\begin{array}{l}\text { Presence of high levels of interfering constituents required dilution of sample } \\
\text { which increased the RDL by the dilution factor. }\end{array}$ \\
\hline $\mathbf{E}$ & Estimated value due to presence of interference. \\
\hline H & $\begin{array}{l}\text { Sample analysis performed outside of method- or client-specified maximum } \\
\text { holding time requirement. }\end{array}$ \\
\hline $\mathbf{M}$ & For CLP analyses only - Duplicate injection precision criterion was not met. \\
\hline $\mathbf{N}$ & Matrix spike recovery exceeded acceptance limits. \\
\hline $\mathbf{S}$ & Reported value was determined from the method of standard addition. \\
\hline $\mathbf{U}$ & $\begin{array}{l}\text { For CLP reporting only - Constituent was analyzed for but not detected (sample } \\
\text { quantitation must be corrected for dilution and percent moisture). }\end{array}$ \\
\hline $\mathbf{W}$ & $\begin{array}{l}\text { For AAS only - Post-digestion spike for Furnace AAS did not meet acceptance } \\
\text { criteria and sample absorbance is less than } 50 \% \text { of spike absorbance. }\end{array}$ \\
\hline$X, Y$, or $Z$ & Analyst-defined qualifier. \\
\hline * & $\begin{array}{l}\text { Relative Percent difference (RPD) for duplicate analysis exceeded acceptance } \\
\text { limits. }\end{array}$ \\
\hline+ & Correlation coefficient (r) for the MSA is less than 0.995 . \\
\hline \multicolumn{2}{|r|}{ For Use On the QC Data. Reporting Forms. } \\
\hline$a^{1}$ & $\begin{array}{l}\text { The spike recovery and/or RPD for matrix spike and matrix spike duplicates } \\
\text { cannot be evaluated due to insufficient spiking level compared to the elevated } \\
\text { sample analyte concentration. }\end{array}$ \\
\hline $\mathbf{b}^{1}$ & $\begin{array}{l}\text { The RPD cannot be computed because the sample and/or duplicate concentration } \\
\text { was below the RDL. }\end{array}$ \\
\hline
\end{tabular}


Lockheed Analytical Services

Data Qualifiers for Radiochemical Analyses

\begin{tabular}{|c|l||}
\hline \multicolumn{1}{|c|}{ For Use On the Analytical Data Reporting Forms } \\
\hline \hline B & $\begin{array}{l}\text { Any constituent that was also detected in the associated blank whose } \\
\text { concentration was greater than the reporting detection limit (RDL) and/or } \\
\text { minimum detectable activity (MDA). }\end{array}$ \\
\hline C & $\begin{array}{l}\text { Presence of high TDS in sample required reduction of sample size which increased } \\
\text { the MDA. }\end{array}$ \\
\hline D & Constituent detected in the diluted sample. \\
\hline E & Constituent concentration exceeded the calibration or attenuation curve range. \\
\hline F & For Alpha Spectrometry Only - FWHM exceed acceptance limits. \\
\hline H & $\begin{array}{l}\text { Sample analysis-performed outside of method-specified maximum holding time } \\
\text { requirement. }\end{array}$ \\
\hline Y & Chemical yield exceeded acceptance limits. \\
\hline$*$ & $\begin{array}{l}\text { QC data (i.e., percent recovery data for laboratory control standard and matrix } \\
\text { spike; and RPD for replicate analyses) exceeded acceptance limits. }\end{array}$ \\
\hline $\mathbf{a}^{1}$ & $\begin{array}{l}\text { The spike recovery and/or RPD for matrix spike and duplicates cannot be } \\
\text { evaluated due to insufficient spiking level compared to the elevated sample } \\
\text { analyte concentration. }\end{array}$ \\
\hline $\mathbf{b}^{1}$ & $\begin{array}{l}\text { The RPD cannot be computed because the sample and/or duplicate concentration } \\
\text { was below the MDA. }\end{array}$ \\
\hline
\end{tabular}


TABLE A.1

ANALYTICAL RESULTS FOR GROUNDWATER 1995

VOLATILE ORGANIC COMPOUNDS

1995 ANNUAL WATER MONITORING REPORT

LEHR ENVIRONMENTAL RESTORATION, DAVIS, CALIFORNIA

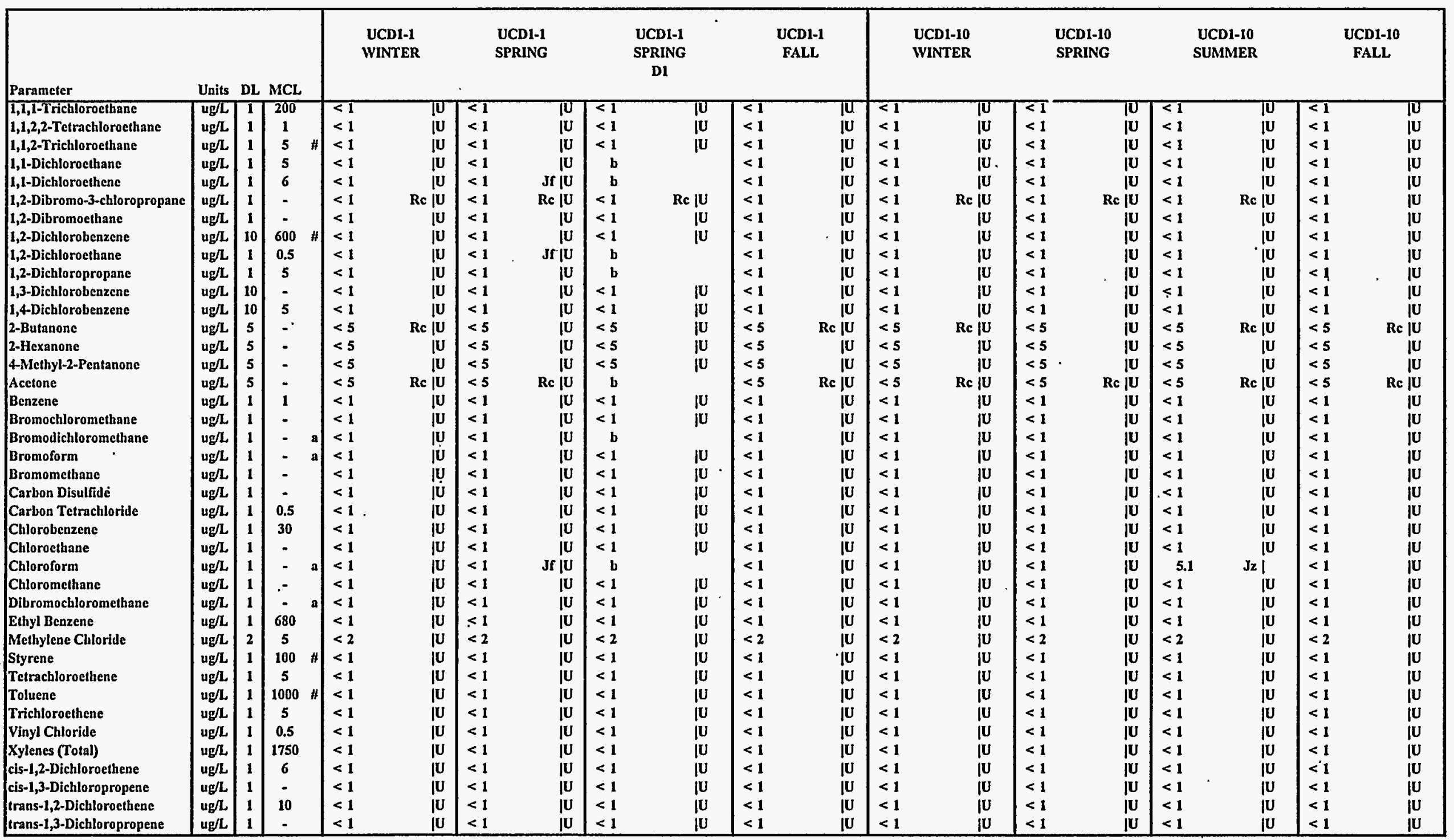


TABLE A.1

ANALYTICAL RESULTS FOR GROUNDWATER 1995

VOLATILE ORGANIC COMPOUNDS

1995 ANNUAL WATER MONITORING REPORT

LEHR ENVIRONMENTAL RESTORATION, DAVIS, CALIFORNIA

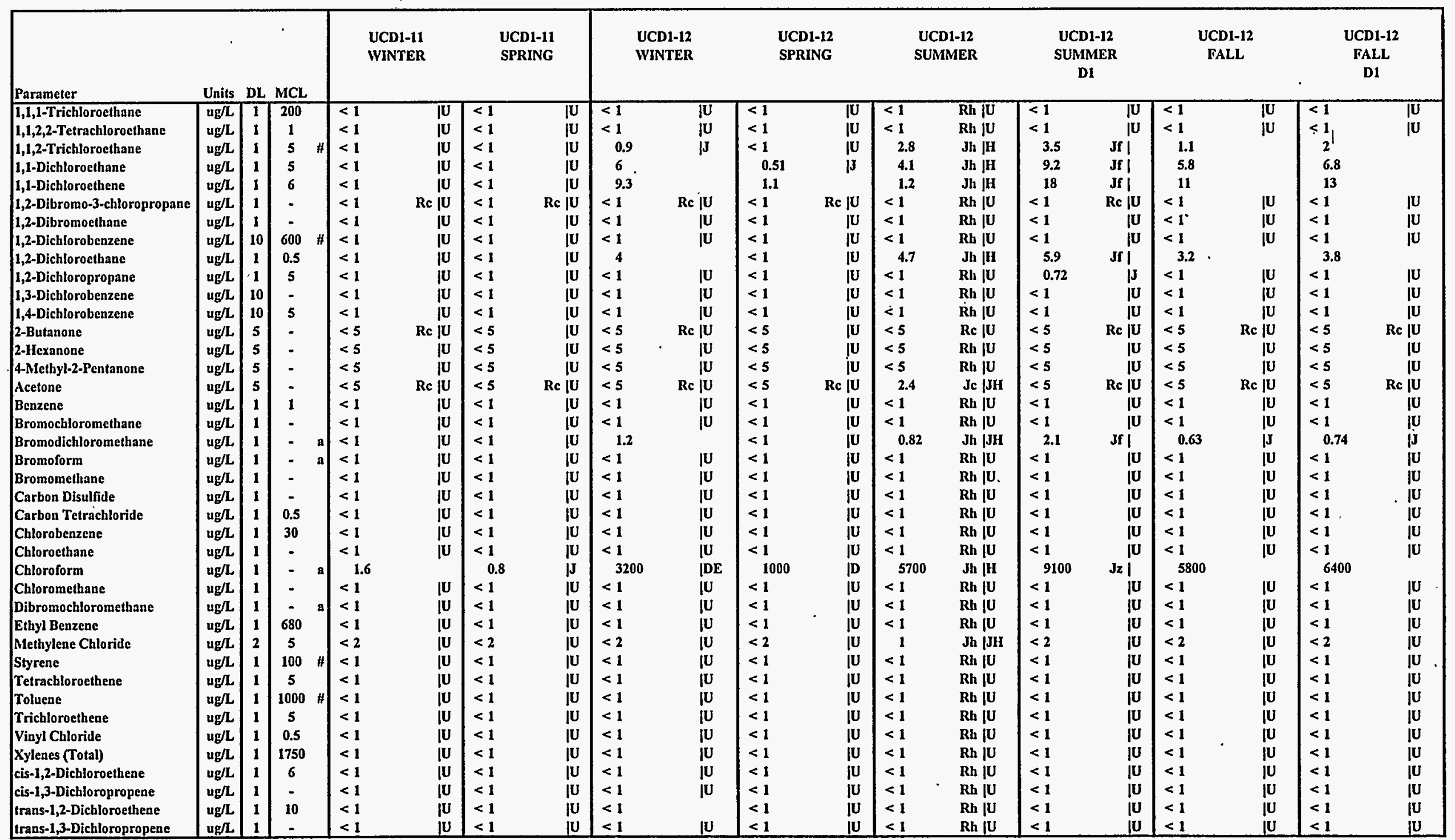


TABLE A.1

ANALYTICAL RESULTS FOR GROUNDWATER 1995

VOLATILE ORGANIC COMPOUNDS

1995 ANNUAL WATER MONITORING REPORT

LEHR ENVIRONMENTAL RESTORATION, DAVIS, CALIFORNIA

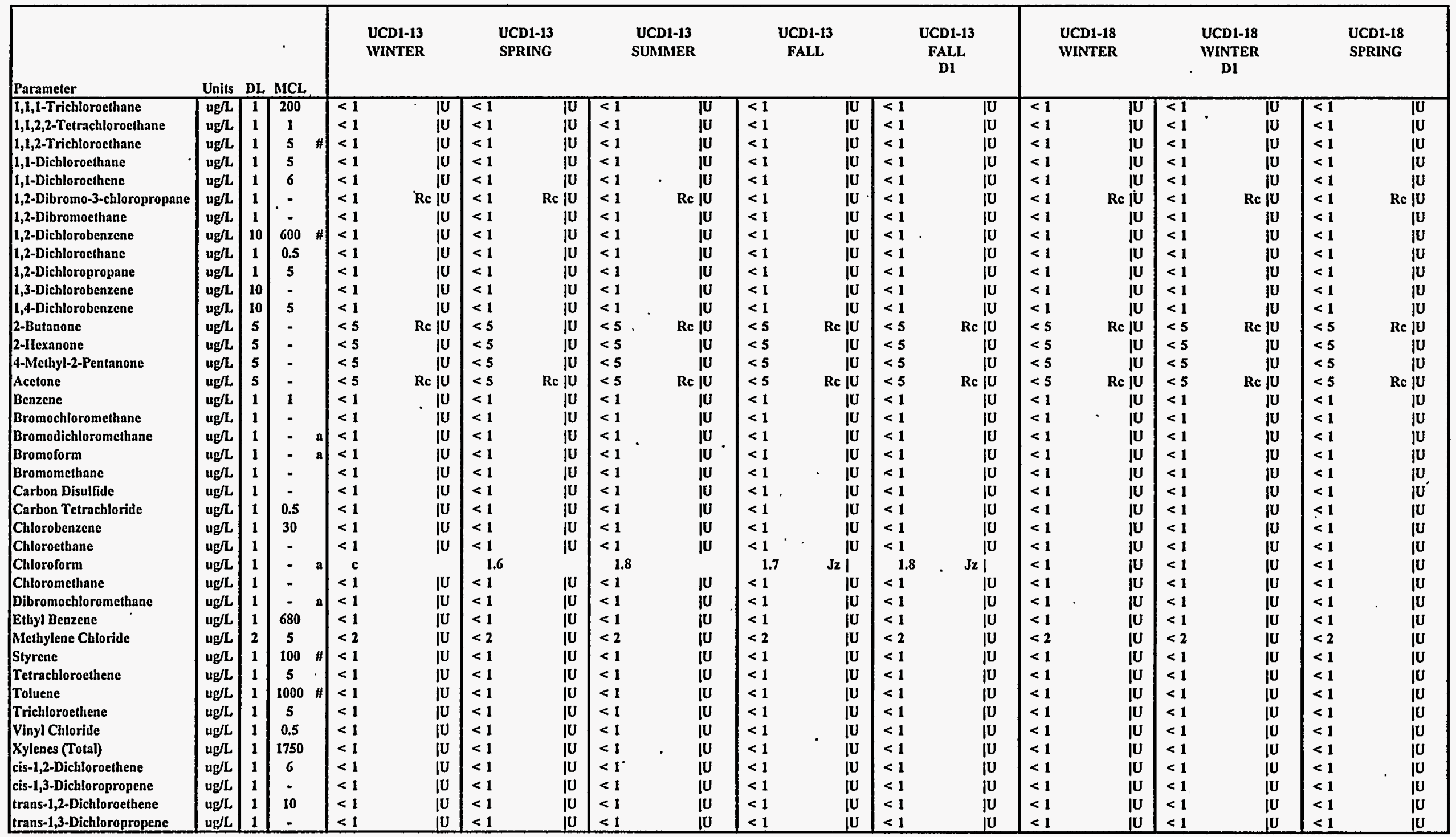




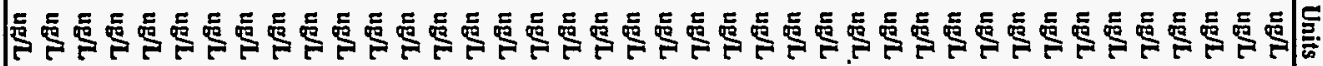

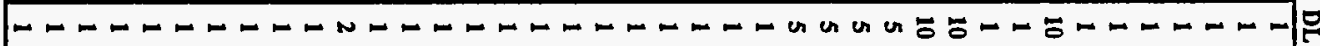

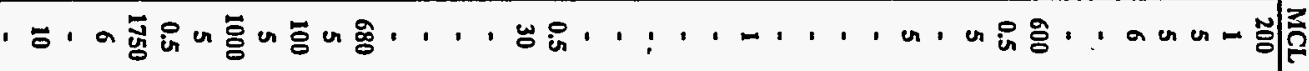

$\wedge \wedge \wedge \wedge \wedge \wedge \wedge \wedge \wedge \wedge \wedge \wedge \wedge \wedge \wedge \wedge \wedge \wedge \wedge \wedge \wedge \wedge \wedge \wedge \wedge \wedge \wedge \wedge \wedge \wedge \wedge \wedge \wedge \wedge \wedge \wedge \wedge \wedge \wedge \wedge$

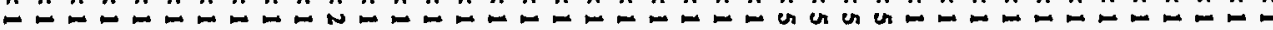

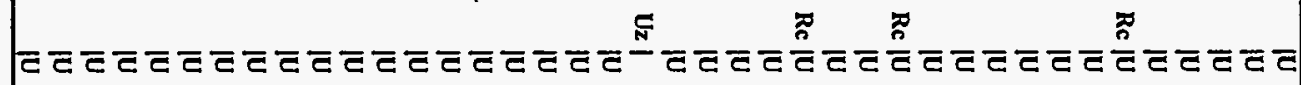
$\wedge \wedge \wedge \wedge \wedge \wedge \wedge \wedge \wedge \wedge \wedge \wedge \wedge \wedge \wedge \wedge \wedge \wedge \wedge \wedge \wedge \wedge \wedge \wedge \wedge \wedge \wedge \wedge \wedge \wedge \wedge \wedge \wedge \wedge \wedge \wedge \wedge \wedge \wedge \wedge$

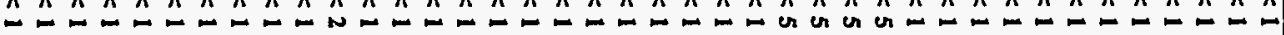

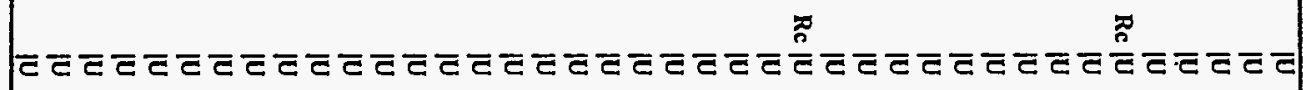

$\wedge \wedge \wedge \wedge \wedge \wedge \wedge \wedge \wedge \wedge \wedge \wedge \wedge \wedge \wedge \wedge \wedge \wedge \wedge \wedge \wedge \wedge \wedge \wedge \wedge \wedge \wedge \wedge \wedge \wedge \wedge \wedge \wedge \wedge \wedge \wedge \wedge \wedge \wedge \wedge$

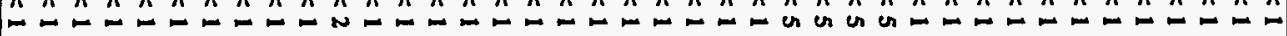

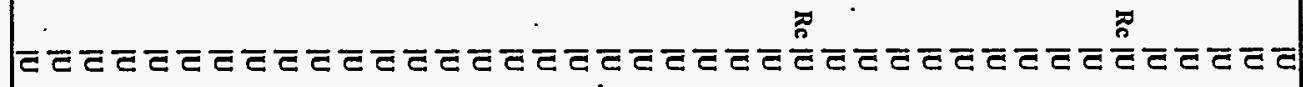

$\wedge \wedge \wedge \wedge \wedge \wedge \wedge \wedge \wedge \wedge \wedge \wedge \wedge \wedge \wedge \wedge \wedge \wedge \wedge \wedge \wedge \wedge \wedge \wedge \wedge \wedge \wedge \wedge \wedge \wedge \wedge \wedge \wedge \wedge \wedge \wedge \wedge \wedge \wedge \wedge$

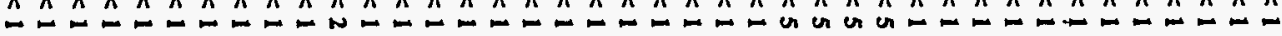

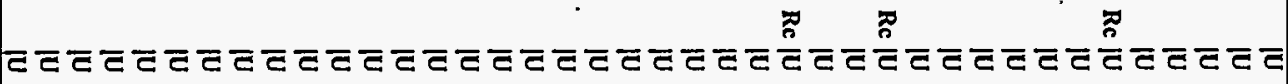

$\wedge \wedge \wedge \wedge \wedge \wedge \wedge \wedge \wedge \wedge \wedge \wedge \wedge \wedge \wedge \wedge \wedge \wedge \wedge \wedge \wedge \wedge \wedge \wedge \wedge \wedge \wedge \wedge \wedge \wedge \wedge \wedge \wedge \wedge \wedge \wedge \wedge \wedge \wedge \wedge$

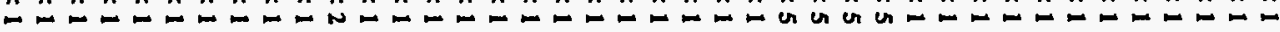

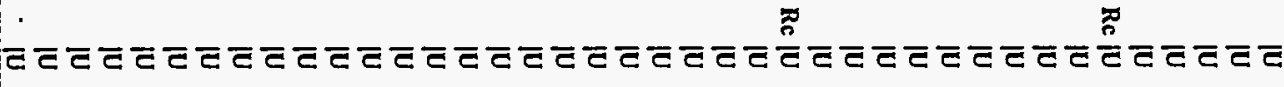

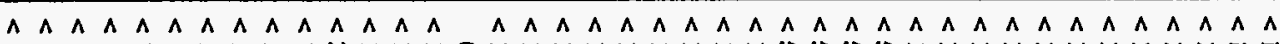

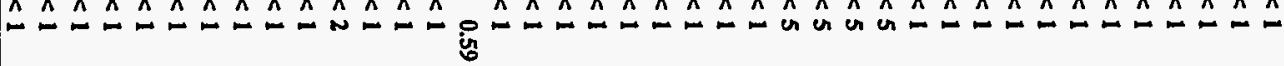

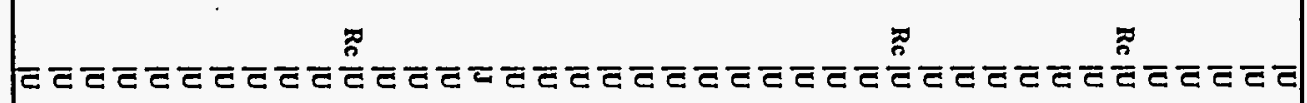

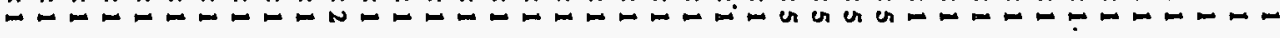

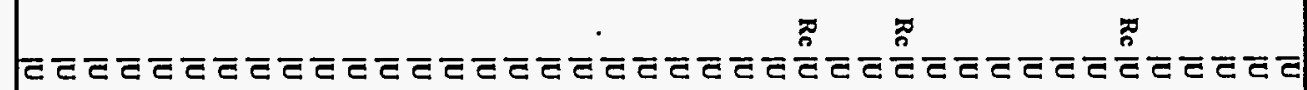

$\hat{\wedge} \wedge \wedge \wedge \wedge \wedge \wedge \wedge \wedge \wedge \wedge \wedge \wedge \wedge \wedge \wedge \wedge \wedge \wedge \wedge \wedge \wedge \wedge \wedge \wedge \wedge \wedge \wedge \wedge \wedge \wedge \wedge \wedge \wedge \wedge \wedge \wedge \wedge \wedge$ 


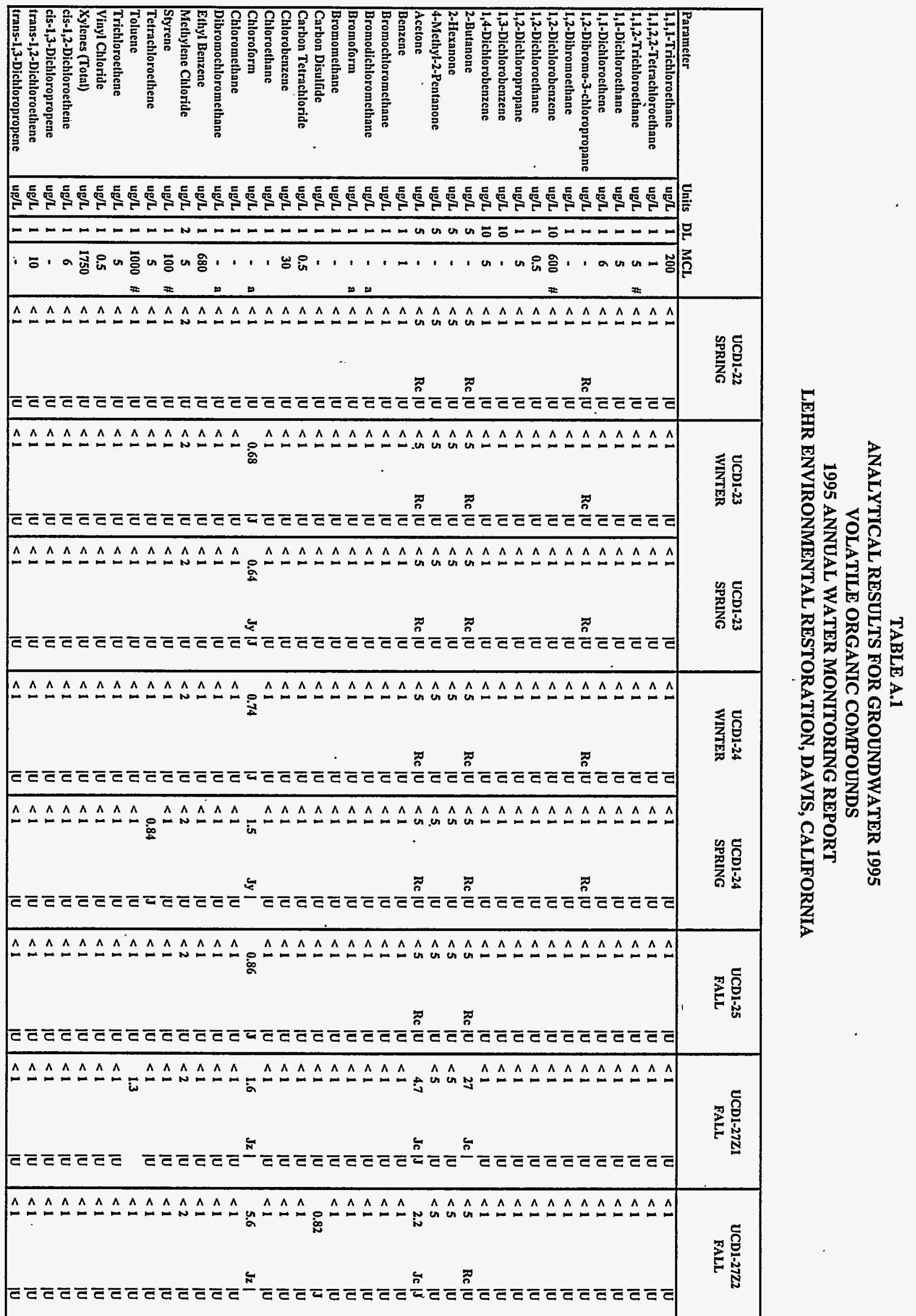




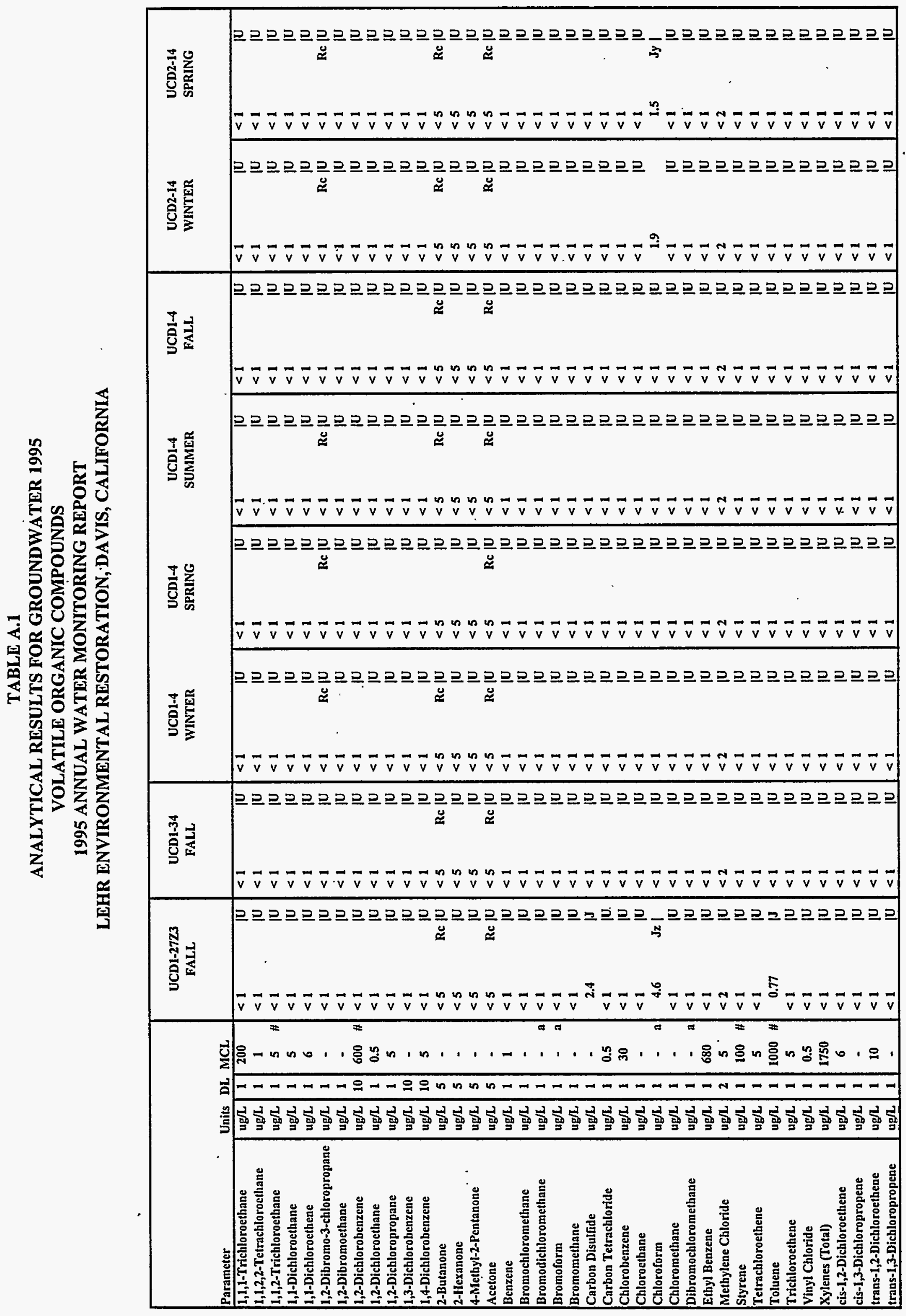


TABLE A.1

ANALYTICAL RESULTS FOR GROUNDWATER 1995

VOLATILE ORGANIC COMPOUNDS

1995 ANNUAL WATER MONITORING REPORT

LEHR ENVIRONMENTAL RESTORATION, DAVIS, CALIFORNIA

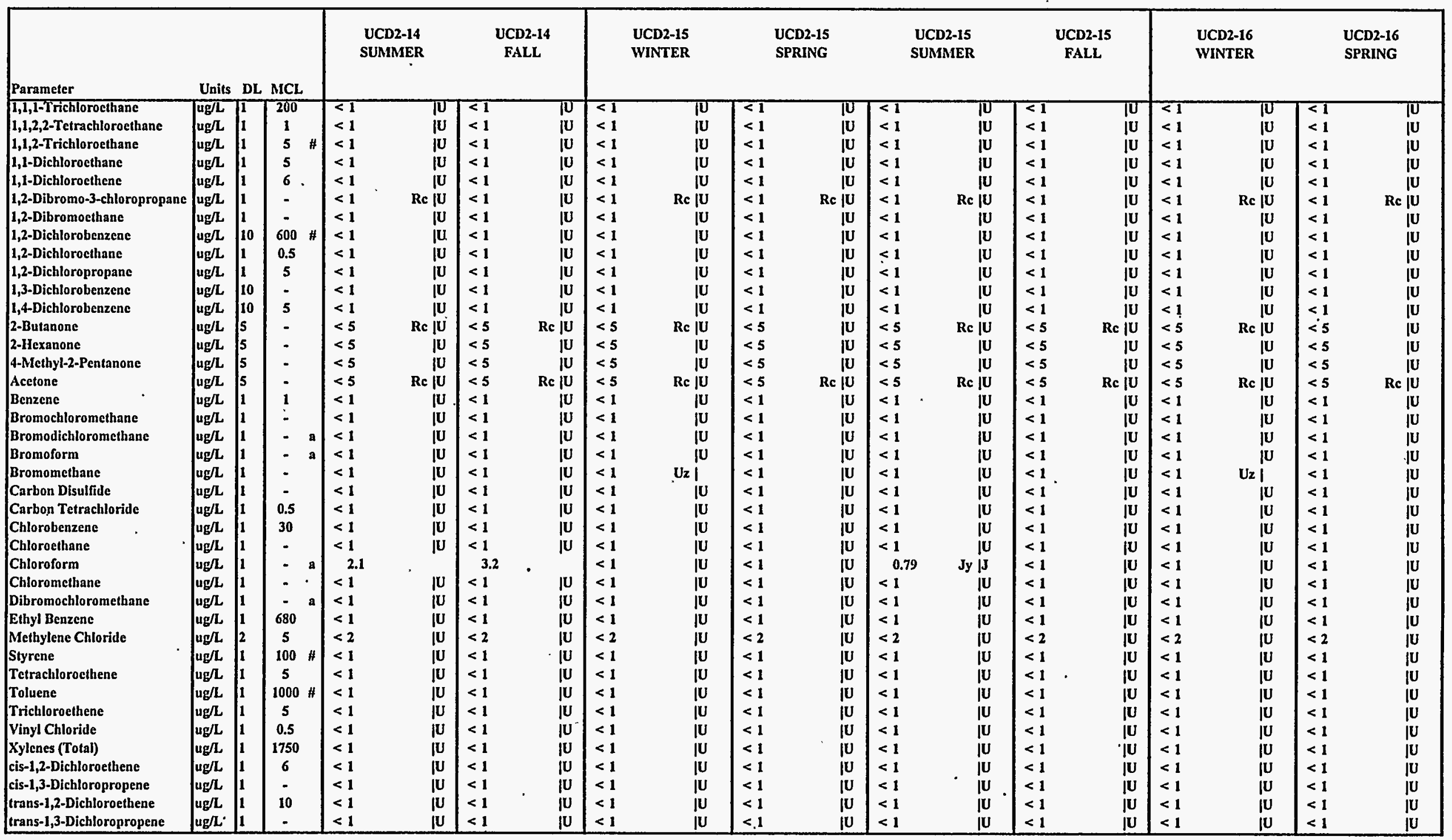


TABLE A.1

ANALYTICAL RESULTS FOR GROUNDWATER 1995

VOLATILE ORGANIC COMPOUNDS

1995 ANNUAL WATER MONITORING REPORT

LEHR ENVIRONMENTAL RESTORATION, DAVIS, CALIFORNIA

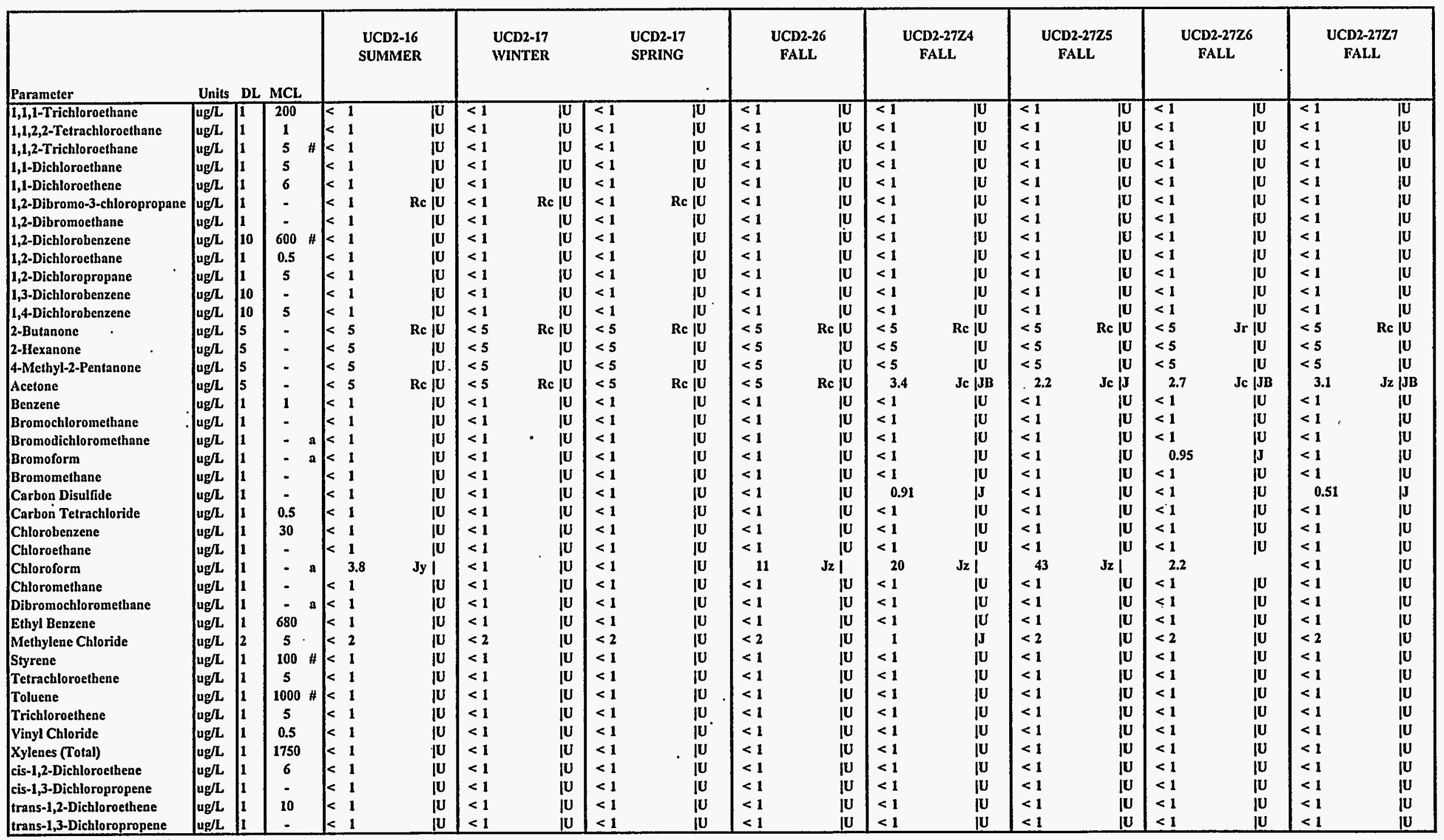


TABLE A.1

ANALYTICAL RESULTS FOR GROUNDWATER 1995

VOLATILE ORGANIC COMPOUNDS

1995 ANNUAL WATER MONITORING REPORT

LEHR ENVIRONMENTAL RESTORATION, DAVIS, CALIFORNIA

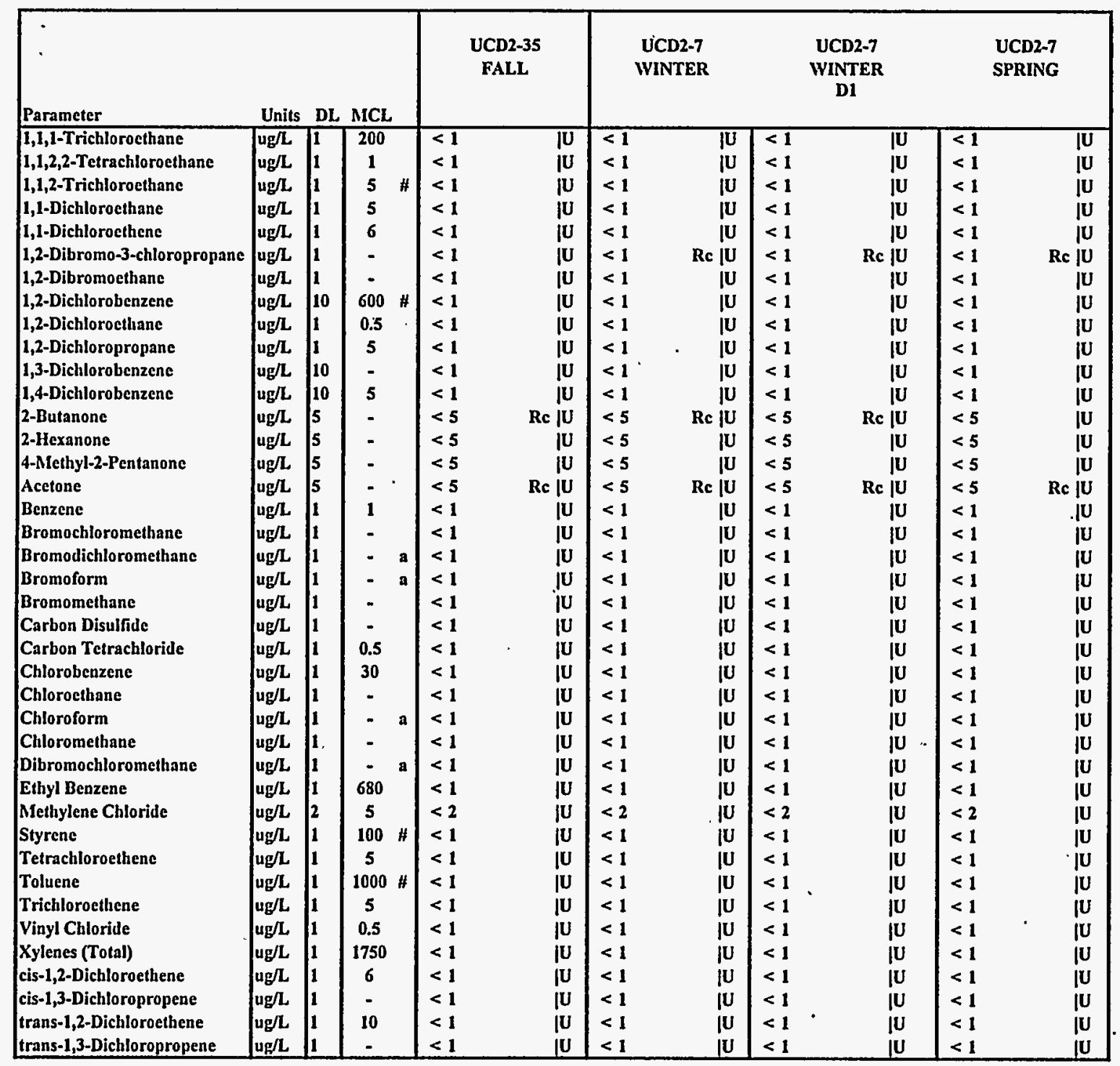




\section{TABLE A.1 \\ ANALYTICAL RESULTS FOR GROUNDWATER 1995 \\ VOLATILE ORGANIC COMPOUNDS \\ 1995 ANNUAL WATER MONITORING REPORT \\ LEHR ENVIRONMENTAL RESTORATION, DAVIS, CALIFORNIA}

D1 = Duplicate Sample

- = Pạrameter not analyzed or no MCL

DL = Detection Limit

$<$ = Constituent below detection limit. Detection limits may vary depending on interference by other sample constituents.

MCL = Maximum Contaminant Level (primary), Title 22 Division 4 Chapter 15.

\# = USEPA MCL

a = MCL for total trihalomethanes is $100 \mathrm{ug} / \mathrm{L}$. Chloroform is the predominant trihalomethane detected at LEHR.

b = Volatile Organic Sample likely collected from UCD1-12. Results reported (ug/L) by the laboratory are as follows 1,1-Dichlorethane=6.8, 1,1-Dichloroethene=13, 1,2-Dichloroethane $=3.3,1,2$-Dichloropropane $=0.51$, Acetone $=4.7$, Bromodichloromethane $=0.7$, Chloroform $=5,800$.

$c=$ Laboratory reported chloroform concentration of $140 \mathrm{ug} / \mathrm{L}$ was determined to be laboratory error.

Sce-Appendix A for explanation of data qualifiers. 


\begin{tabular}{|c|c|}
\hline 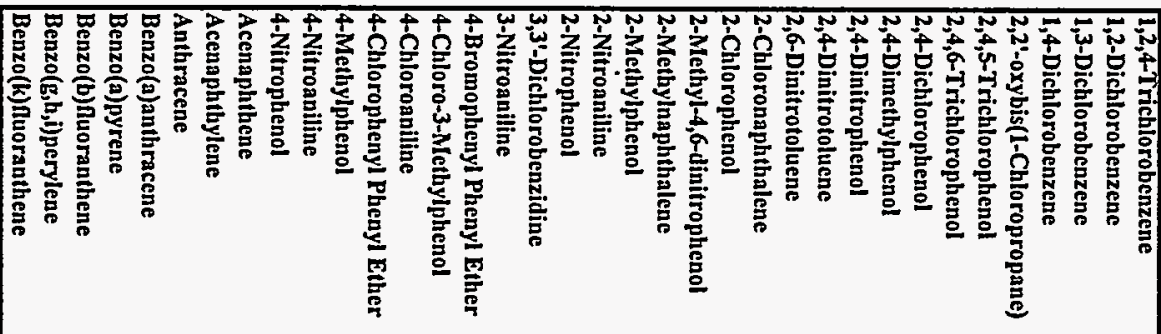 & $\frac{1}{4}$ \\
\hline 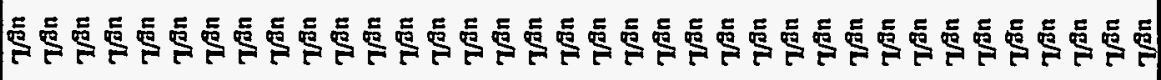 & 突 \\
\hline 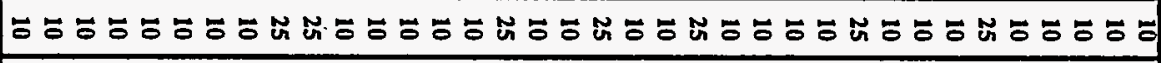 & 을 \\
\hline 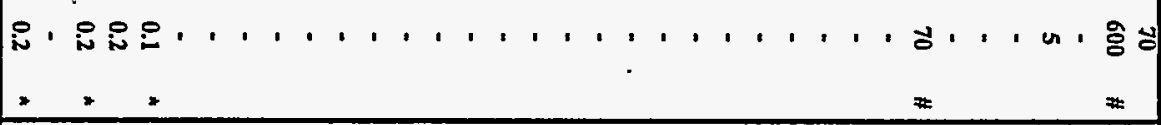 & ลิ \\
\hline 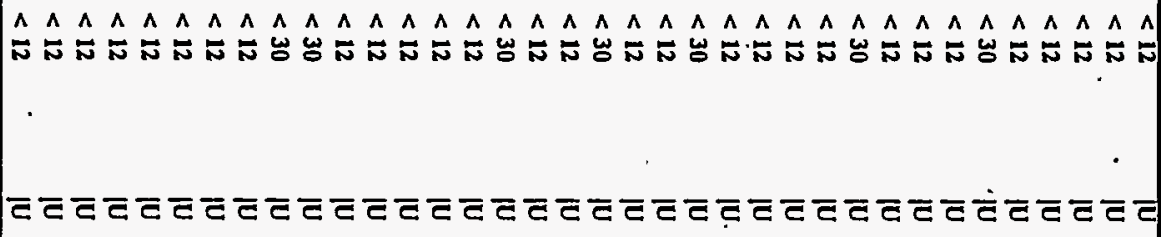 & 焉是 \\
\hline 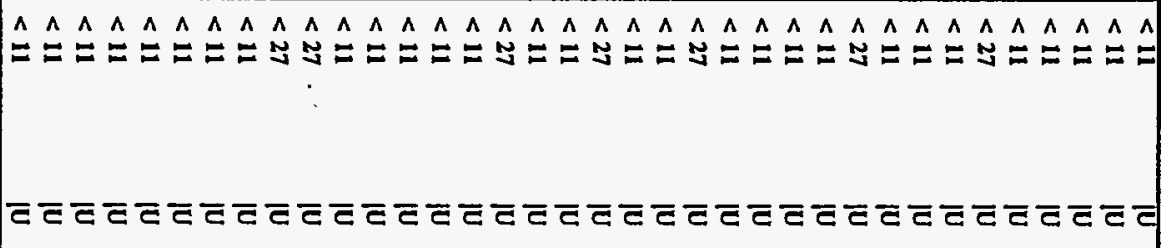 & 胥 \\
\hline 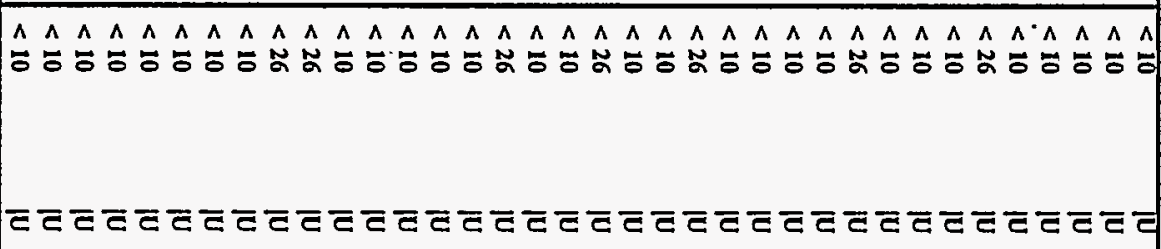 & 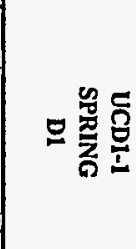 \\
\hline 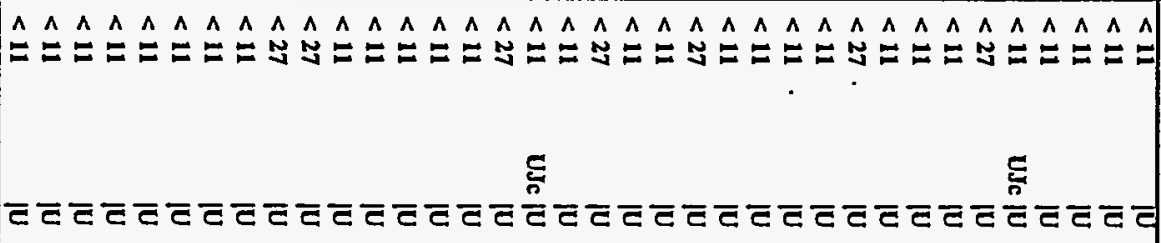 & 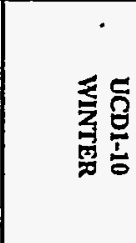 \\
\hline 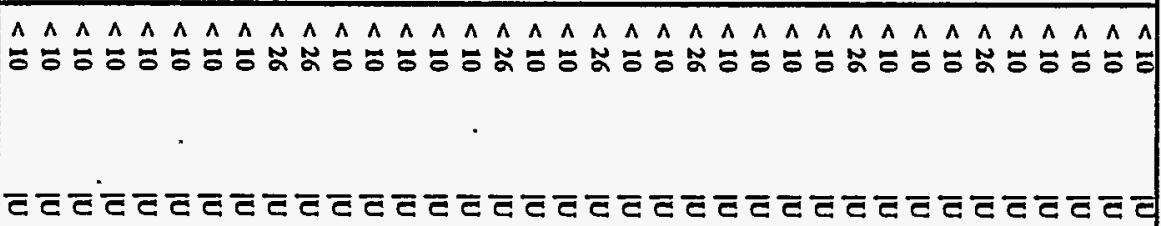 & 萦气 \\
\hline 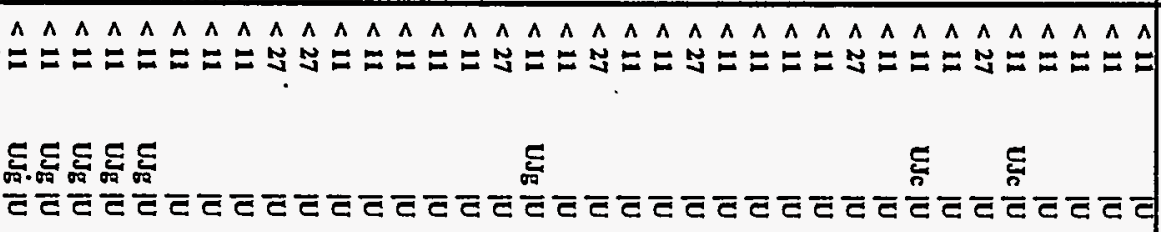 & 蓄实 \\
\hline 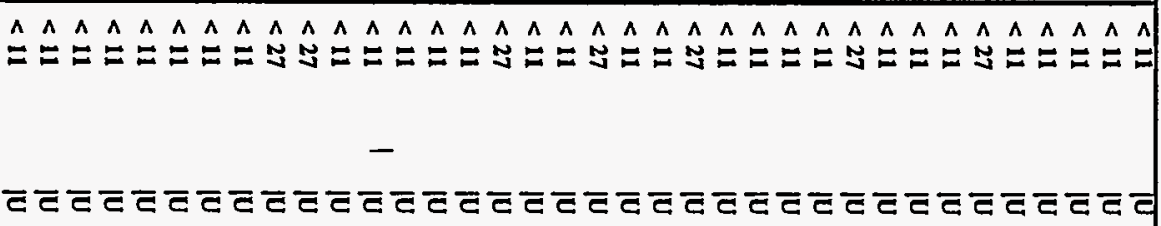 & 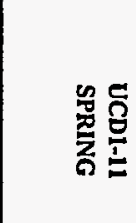 \\
\hline
\end{tabular}




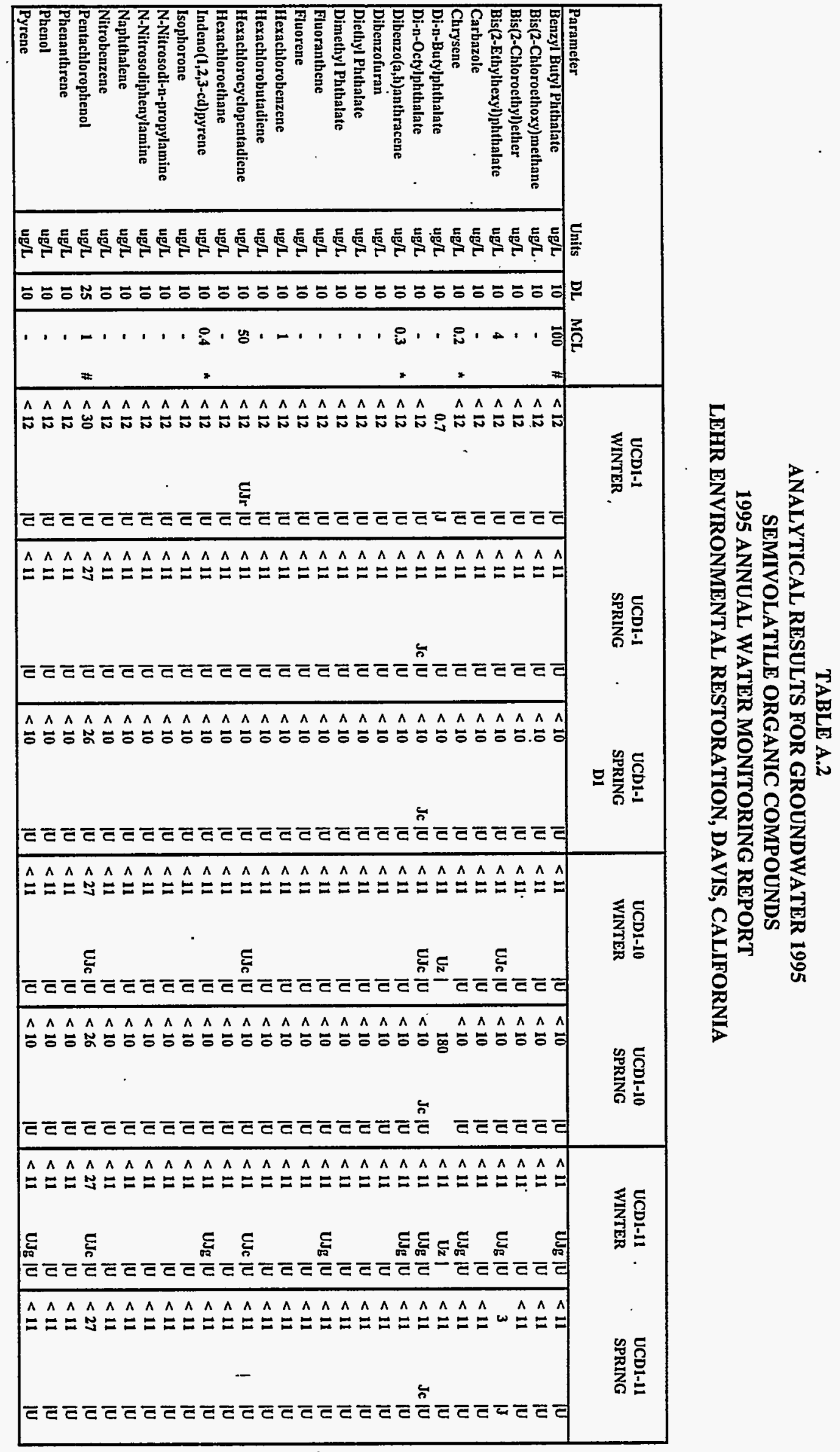




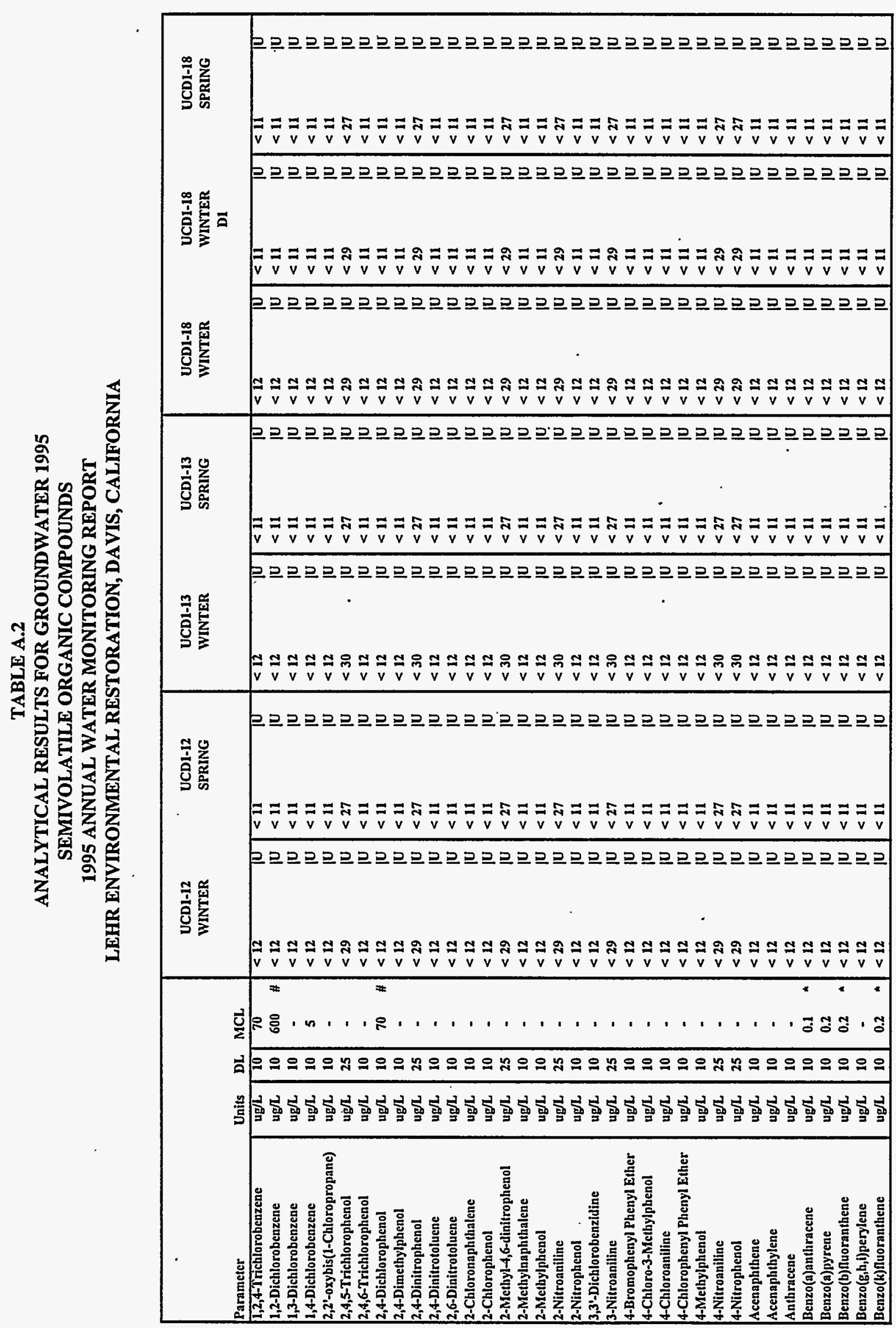




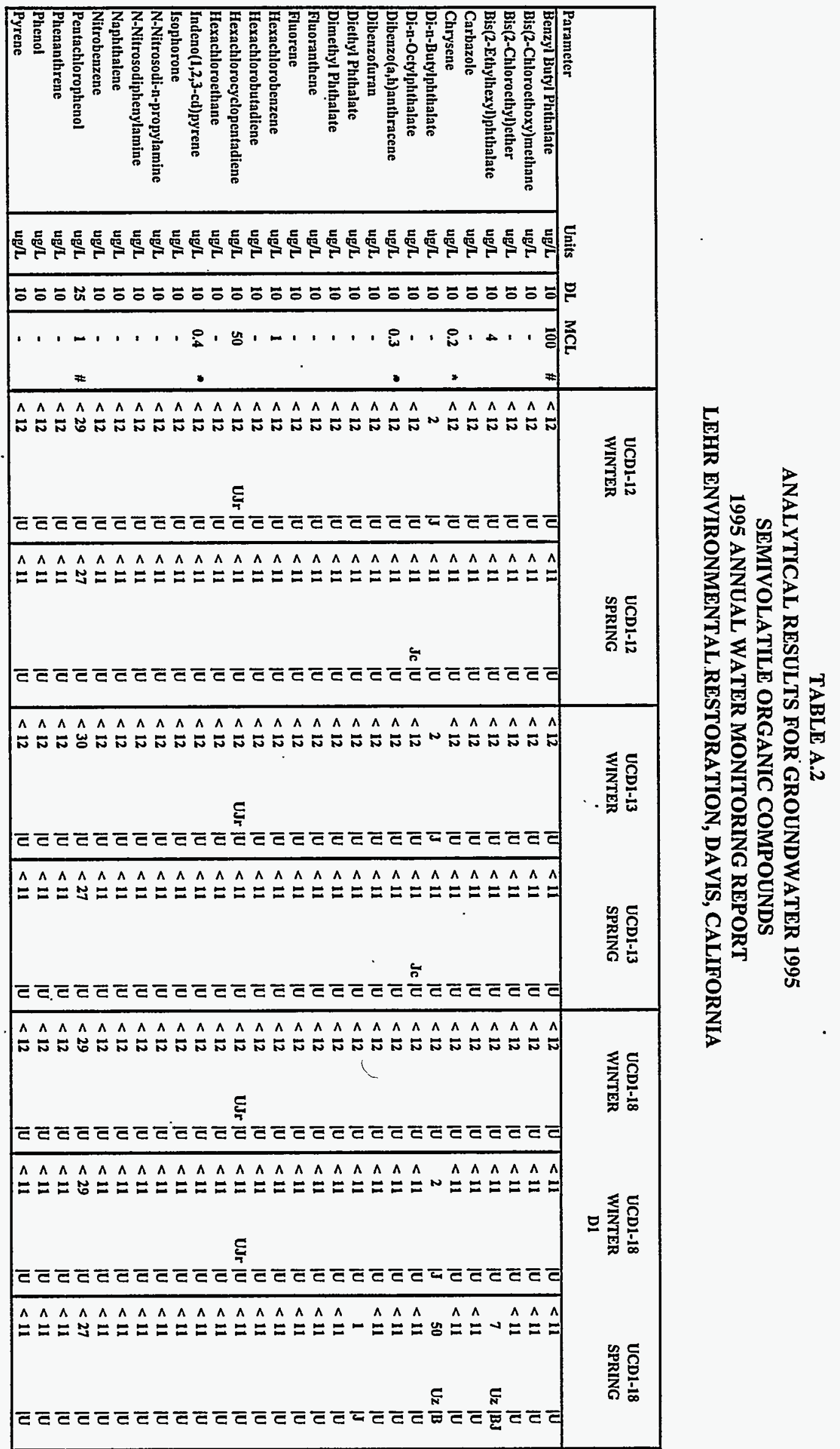




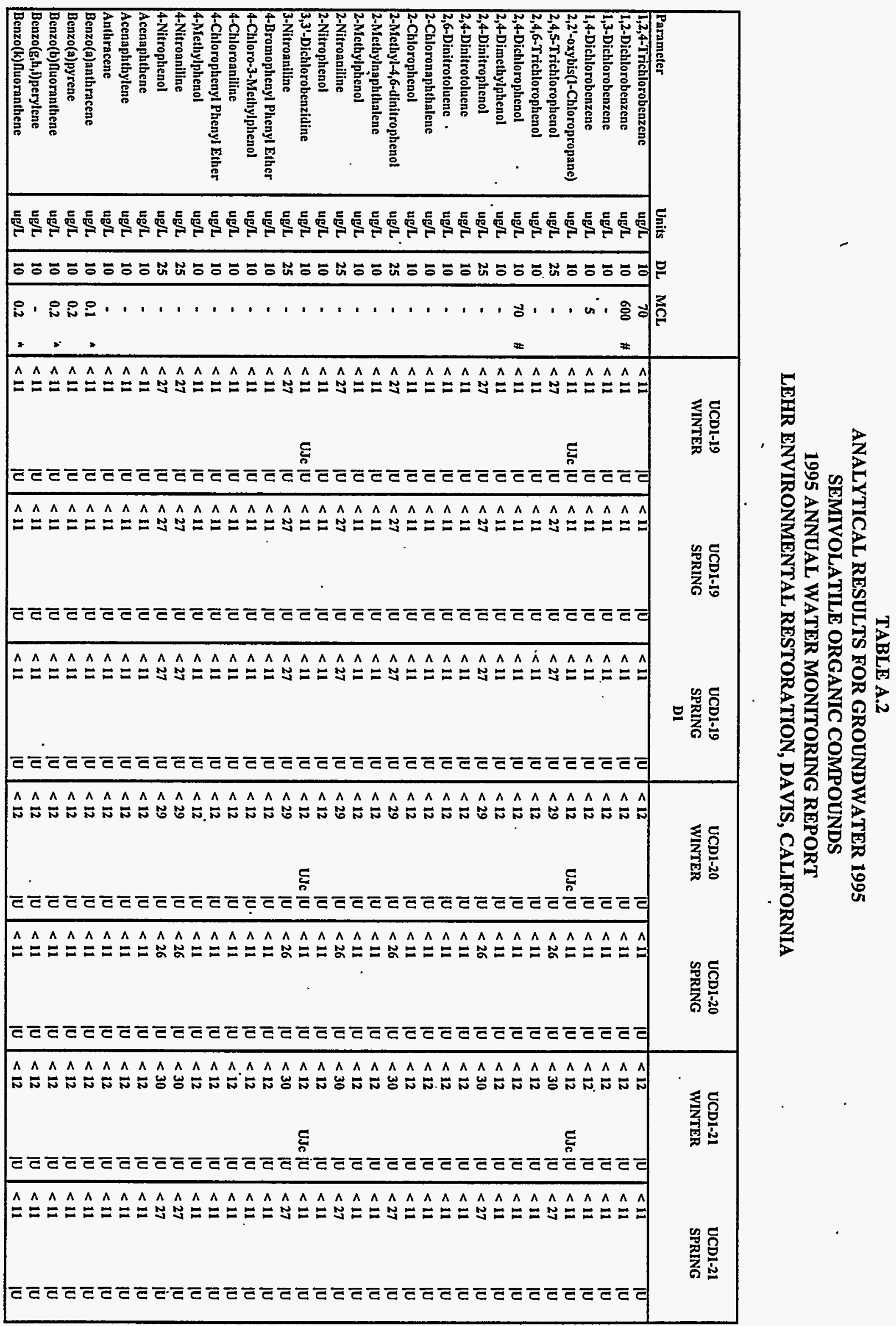




\begin{tabular}{|c|c|}
\hline 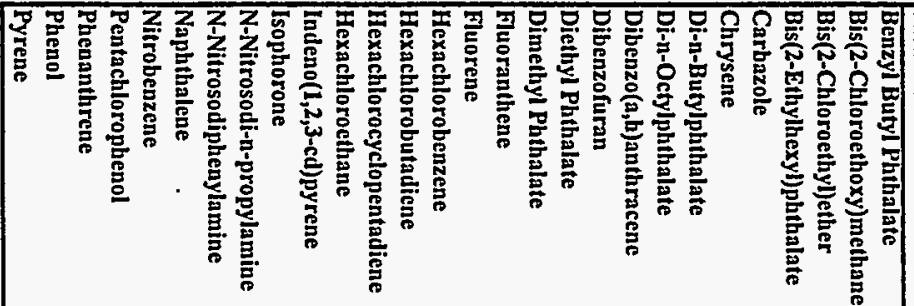 & $\frac{1}{20}$ \\
\hline 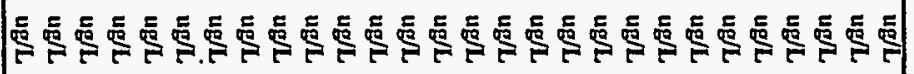 & 䍃 \\
\hline 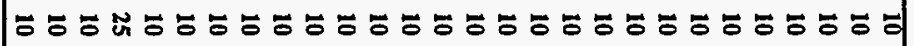 & 焉 \\
\hline 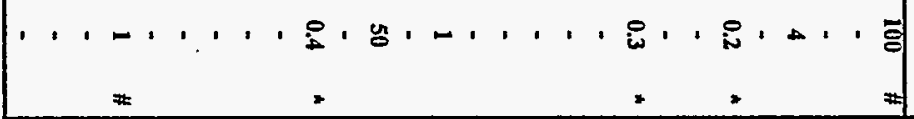 & ลี \\
\hline 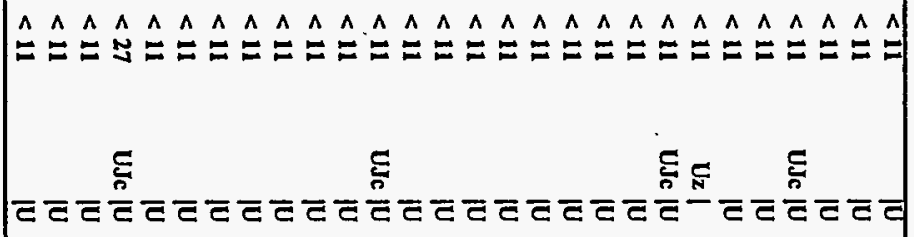 & 焉导 \\
\hline 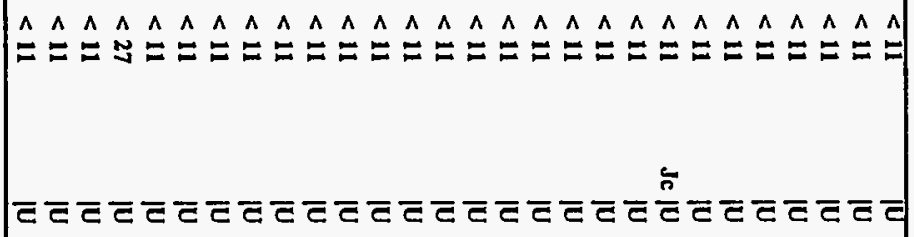 & 象 \\
\hline 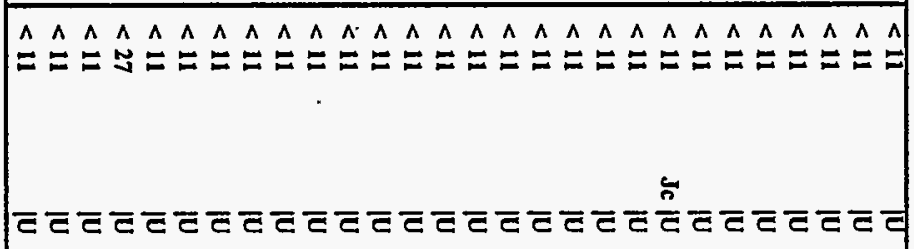 & 봃 宽 \\
\hline 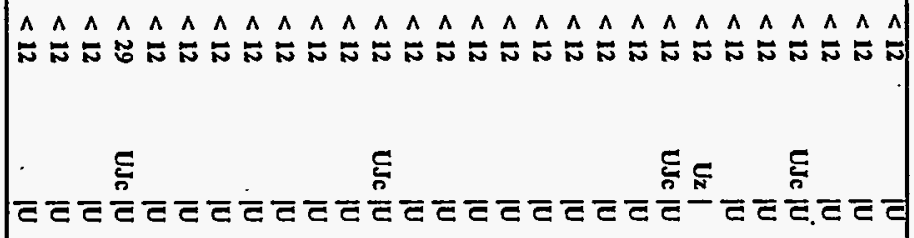 & 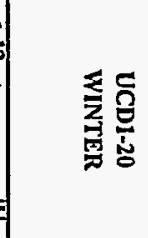 \\
\hline 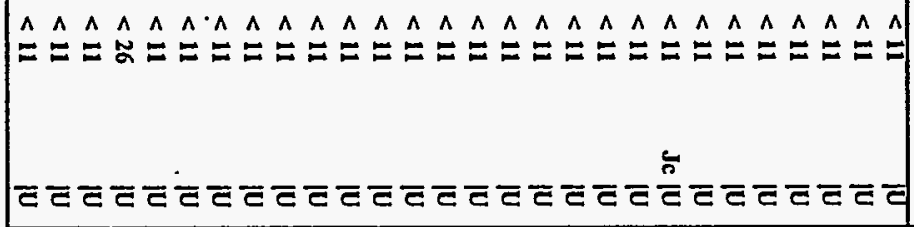 & 羿号 \\
\hline 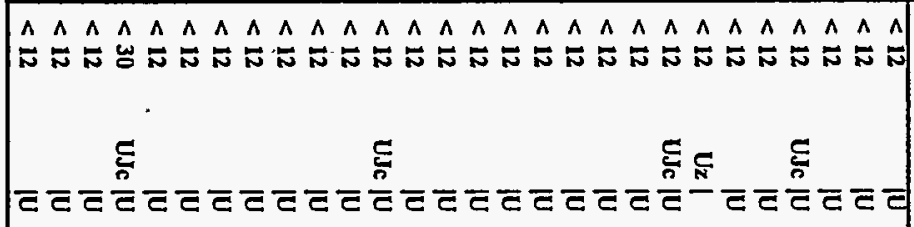 & 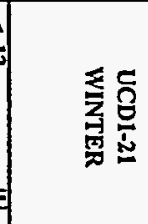 \\
\hline 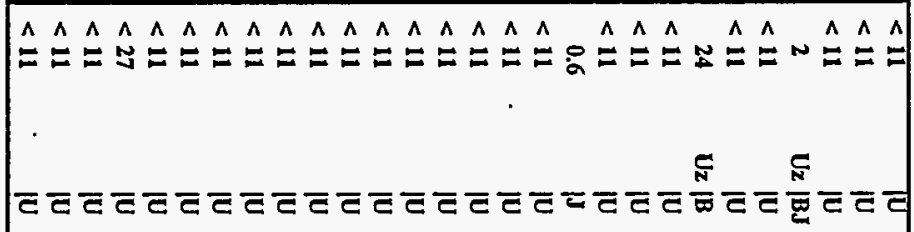 & 第实 \\
\hline
\end{tabular}




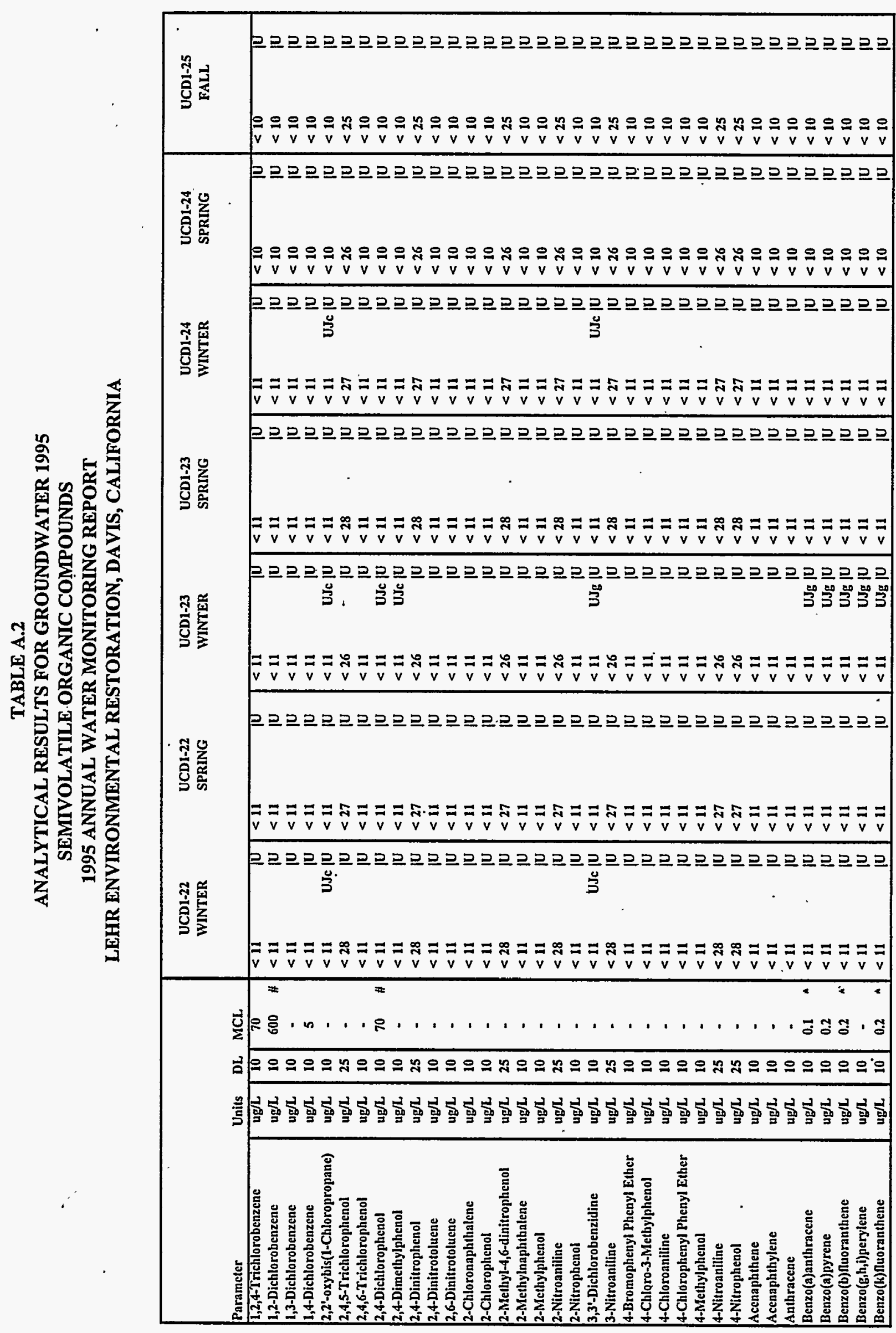


TABLE A.2

ANALYTICAL RESULTS FOR GROUNDWATER 1995

SEMIVOLATILE ORGANIC COMPOUNDS

1995 ANNUAL WATER MONITORING REPORT

LEHR ENVIRONMENTAL RESTORATION, DAVIS, CALIFORNIA

\begin{tabular}{|c|c|c|c|c|c|c|c|c|c|c|c|c|c|c|c|c|c|c|}
\hline \multirow{2}{*}{ Parameter } & \multirow{2}{*}{ Units } & \multirow{2}{*}{ DL } & \multicolumn{2}{|l|}{ MCL } & \multicolumn{2}{|c|}{$\begin{array}{l}\text { UCD1-22 } \\
\text { WINTER }\end{array}$} & \multicolumn{2}{|c|}{$\begin{array}{l}\text { UCD1-22 } \\
\text { SPRING }\end{array}$} & \multicolumn{2}{|c|}{$\begin{array}{l}\text { UCD1-23 } \\
\text { WINTER }\end{array}$} & \multicolumn{2}{|c|}{$\begin{array}{l}\text { UCDI-23 } \\
\text { SPRING }\end{array}$} & \multicolumn{2}{|c|}{$\begin{array}{l}\text { UCDI-24 } \\
\text { WINTER }\end{array}$} & \multicolumn{2}{|c|}{$\begin{array}{l}\text { UCD1-24 } \\
\text { SPRING }\end{array}$} & \multicolumn{2}{|c|}{$\begin{array}{l}\text { UCD1-25 } \\
\text { FALL }\end{array}$} \\
\hline & & & & $\#$ & & & $<11$ & & & & & & & & 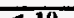 & & & \\
\hline $\begin{array}{l}\text { Benzyl Butyl Phthalate } \\
\text { Bis(2-Chloroethoxy)methane }\end{array}$ & ug/L. & & &. & $<11$ & & $<11$ & & $2 \pi$ & $00 \mathrm{~g}$ & -11 & 0 & & ju & $<10$ & (1) & $<10$ & [U \\
\hline Bis(2-Chlorocthoxy)methane & ug/L & 10 & & & $<11$ & |0 & $<11$ & ju & $<11$ & 10 & $<\mathbf{1 1}$ & |U & $<11$ & |U & $<10$ & |U & $<10$ & |U \\
\hline Bis(2-Chloroethyl)ether & ug/L & 10 & - & & $<11$ & $\mathbf{j} \mathbf{U}$ & $<11$ & $\{\mathbf{U}$ & $<11$ & JU & $<11$ & |U & $<11$ & $\mathbf{| U}$ & $<10$ & U & $<10$ & |U \\
\hline Bis(2-Ethylhexyl)phthalate & ug/L & 10 & 4 & & $<11$ & UJc ju & 2 & $\mathbf{U z}$ |BJ & $<11$ & $\mathbf{U J g} \mathbf{j} \mathbf{U}$ & $<11$ & jU & $<11$ & UJc ju & 2 & jJ & $<10$ & ju \\
\hline Carbazole & ug/L & 10 & - & & $<11$ & ju & $<11$ & $\mathbf{j} \mathbf{U}$ & $<\mathbf{1 1}$ & jU & $<11$ & ju & $<11$ & ju & $<10$ & ju & $<10$ & ju \\
\hline Chrysene & ug/L & 10 & 0.2 & * & $<11$ & ju & $<11$ & ju & $<11$ & $\mathbf{U} \mathbf{J}_{\mathbf{g}} \mid \mathbf{U}$ & $<11$ & U & $<11$ & ju & $<10$ & ju & $<10$ & ju \\
\hline Di-n-Butylphthalate & $\mathrm{ug} / \mathrm{L}$ & 10 & - & & $<\mathbf{1 1}$ & $\mathrm{Uz} \mid$ & 130 & B & $<11$ & $\mathbf{U z}$ & $<11$ & $\mathrm{U}$ & $<11$ & $\mathbf{U z}$ & $<10$ & ן & $<10$ & ju \\
\hline Di-n-Octylphthalate & ug/L & 10 & - & & $<11$ & UJe $\mathbf{U}$ & $<11$ & ju & $<11$ & UJg $\mid \mathbf{U}$ & $<11$ & |U & $<11$ & UJc ju & $<10$ & ju & $<10$ & jU \\
\hline Dibenzo(a,h)an thracene & ug/L & 10 & & * & $<11$ & [U & $<11$ & jU & $<11$ & $\mathbf{U J g} \mid \mathbf{U}$ & $<11$ & |U & $<\mathbf{1 1}$ & $\mathbf{j}$ & $<10$ & ju & $<10$ & ju \\
\hline Dibenzofuran & ug/L & 10 & - & & $<11$ & ju & $<11$ & ju & $<11$ & IU & $<11$ & |U & $<11$ & ju & $<10$ & ju & $<10$ & ju \\
\hline Diethyl Phthalate & $\mathrm{ug} / \mathrm{L}$ & 10 & - & & $<11$ & jU & 2 & jJ & $<11$ & ju & $<11$ & ju & $<11$ & jU & $<10$ & ju & $<10$ & ju \\
\hline Dimethyl Phthalate & $u g / L$ & 10 & - & & $<11$ & ju & $<11$ & ן & $<11$ & ju & $<11$ & jU & $<11$ & ןU & $<10$ & ju & $<10$ & ju \\
\hline Fluoranthene & ug/L & 10 & - & & $<11$ & |U & $<11$ & ju & $<11$ & $\mathbf{U J g} \mid \mathbf{U}$ & $<11$ & jU & $<11$ & ju & $<10$ & ju & $<10$ & ju \\
\hline Fluorene & ug/L & 10 & - & & $<11$ & ju & $<11$ & ju & $<11$ & jU & $<11$ & ju & $<11$ & ju & $<10$ & ju & $<10$ & ju \\
\hline Hexachlorobenzene & ug/L & 10 & 1 & & $<11$ & ju & $<11$ & ןU & $<11$ & jU & $<11$ & ju & $<11$ & jU & $<10$ & ju & $<10$ & ju \\
\hline Hexachlorobutadiene & ug/L & 10 & - & & $<11$ & ju & $<11$ & ju & $<11$ & $\mathbf{U} \mathbf{J} \boldsymbol{j} \mathbf{U}$ & $<11$ & jU & $<11$ & UU & $<10$ & ju & $<10$ & ju \\
\hline Hexachlorocyclopentadiene & ug/L & 10 & 50 & & $<11$ & UJc ju & $<11$ & ju & $<11$ & $\boldsymbol{j} \mathbf{U}$ & $<11$ & jU & $<11$ & $\mathbf{U J c} \mid \mathbf{U}$ & $<10$ & ju & $<10$ & ju \\
\hline Hexachloroethane & ug/L & 10 & - & & $<11$ & ju & $<11$ & ju & $<11$ & $\mathbf{j u}$ & $<11$ & |U & $<\mathbf{1 1}$ & IU & $<10$ & ju & $<10$ & ju \\
\hline Indeno(1,2,3-cd)pyrene & ug/L & 10 & & * & $<11$ & ןU & $<11$ & ןU & $<11$ & UJg $\mathbf{U}$ & $<11$ & jU & $<11$ & |U & $<10$ & ju & $<10$ & ju \\
\hline Isophorone & ug/L & 10 & - & & $<11$ & |U & $<11$ & ju & $<11$ & ju & $<11$ & jU & $<11$ & IU & $<10$ & ju & $<10$ & ju \\
\hline N-Nitrosodi-n-propylamine & ug/L & 10 & - & & $<11$ & ju & $<11$ & ju & $<11$ & ju & $<11$ & |U & $<11$ & jU & $<10$ & ju & $<10$ & ju \\
\hline N-Nitrosodiphenylamine & ug/L & 10 & - & & $<11$ & ןU & $<11$ & ju & $<11$ & ju & $<11$ & jU & $<11$ & ju & $<10$ & ju & $<10$ & ju \\
\hline Naphthalene & ug/L & 10 & - & & $<11$ & ju & $<11$ & ju & $<11$ & ju & $<11$ & ju & $<11$ & ju & $<10$ & ju & $<10$ & ju \\
\hline Nitrobenzenc & $\mathrm{ug} / \mathrm{L}$ & 10 & - & & $<11$ & ju & $<11$ & ju & $<11$ & $\boldsymbol{U} \mathbf{U}$ & $<11$ & ju & $<11$ & $\mathbf{j u}$ & $<10$ & ju & $<10$ & ju \\
\hline Pentachlorophenol & ug/L & 25 & & $\#$ & $<28$ & UJc $\mathbf{j}$ & $<27$ & |U & $<.26$ & UJc $\mid \mathbf{U}$ & $<28$ & |U & $<27$ & $\mathbf{U J c} \mid \mathbf{U}$ & $<26$ & ju & $<25$ & ju \\
\hline Phenanthrene & ug $/ \mathrm{L}$ & 10 & - & & $<11$ & ju & $<11$ & UU & $<11$ & ju & $<11$ & [U & $<11$ & IU & $<10$ & U & $<10$ & ju \\
\hline Phenol & ug/L & 10 & - & & $<11$ & ju & $<11$ & ן & $<11$ & & $<11$ & jU & $<11$ & [U & $<10$ & ju & $<10$ & ju \\
\hline Pyrene & ug/L & 10 & - & & $<11$ & $\mathrm{ju}$ & $<11$ & ju & $<11$ & $U J_{g} j \mathrm{U}$ & $<11$ & $\mathbf{U}$ & $<11$ & jU & $<10$ & jU & $<10$ & jU \\
\hline
\end{tabular}




\begin{tabular}{|c|c|}
\hline 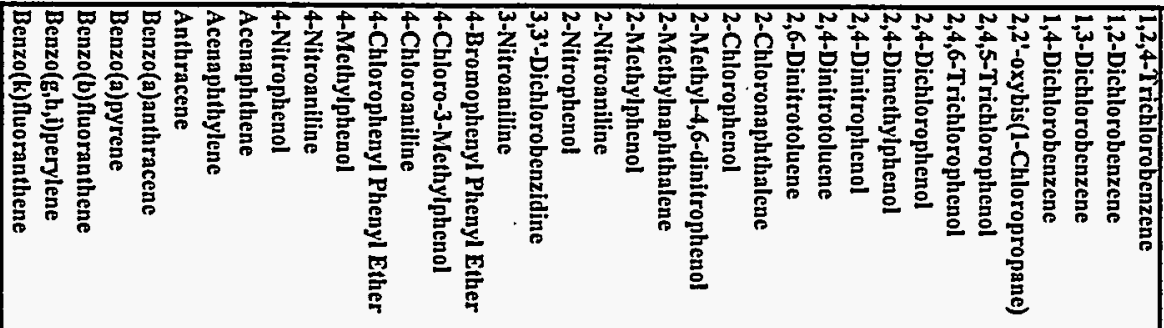 & 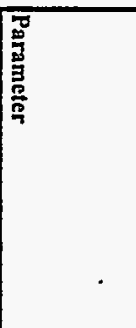 \\
\hline 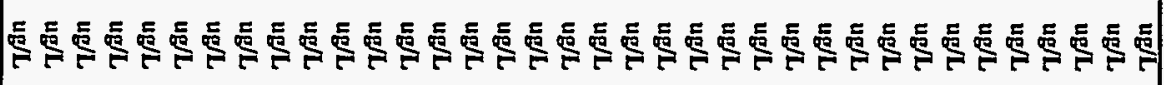 & 突 \\
\hline 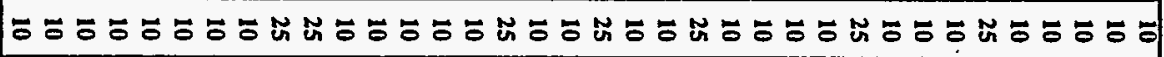 & 电 \\
\hline 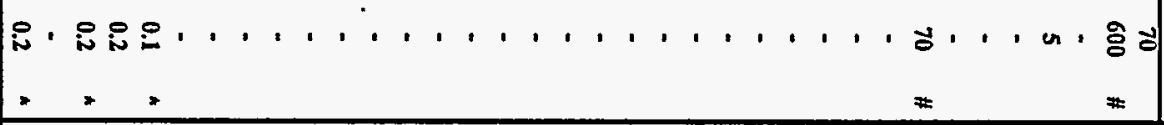 & ?ำ \\
\hline 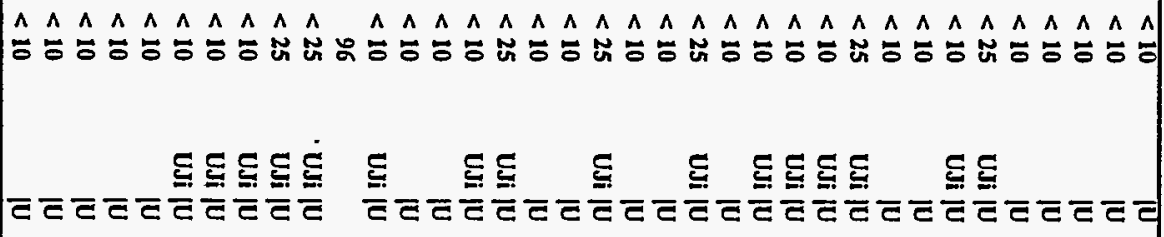 & 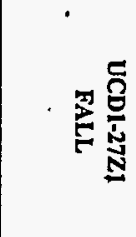 \\
\hline 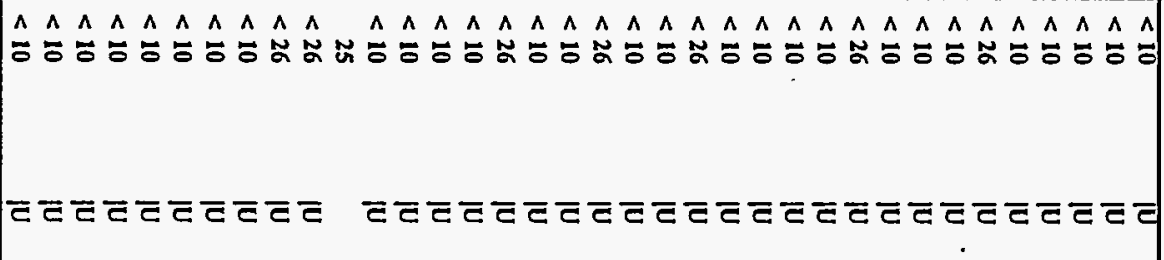 & $\sum_{\substack{3 \\
\frac{\pi}{n}}}^{\stackrel{n}{n}}$ \\
\hline 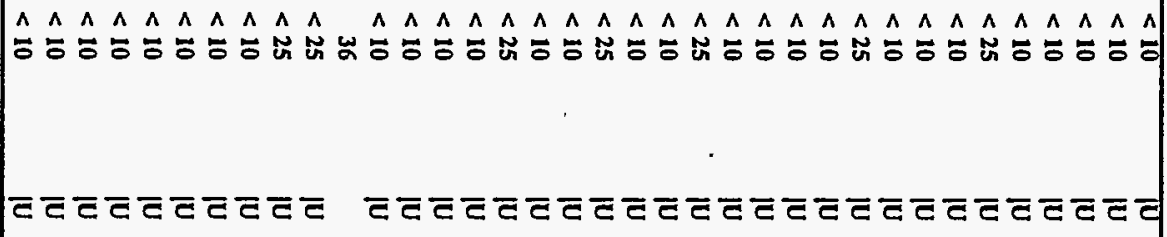 & 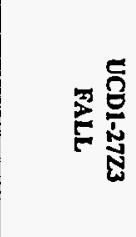 \\
\hline 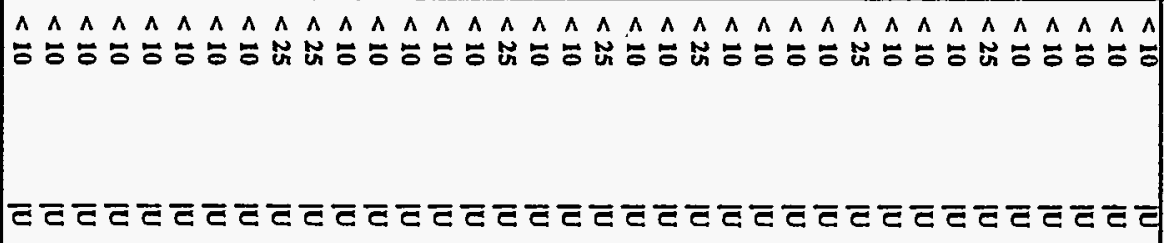 & 疍题 \\
\hline 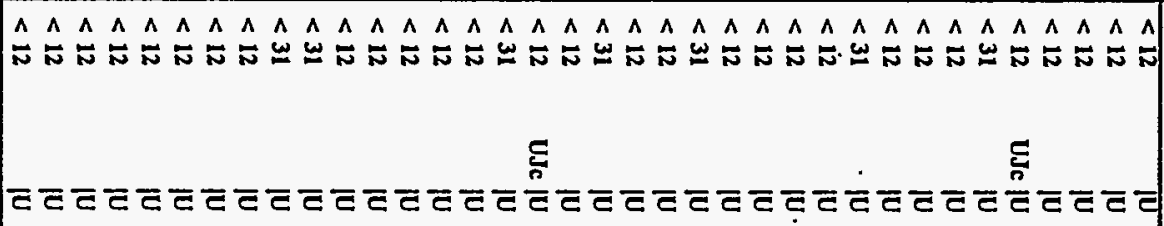 & 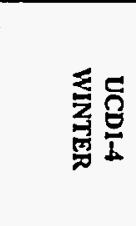 \\
\hline 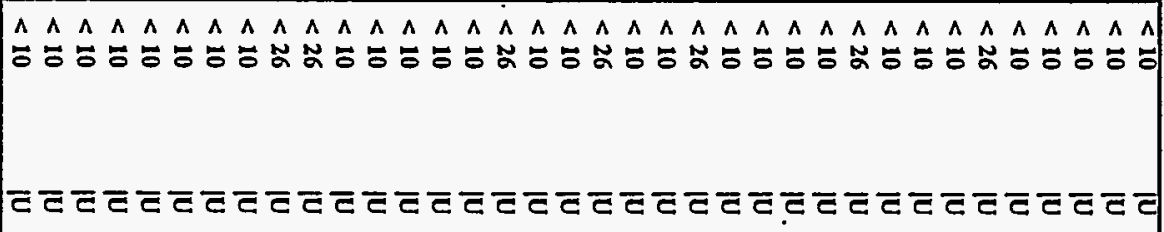 & 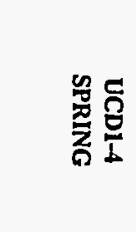 \\
\hline 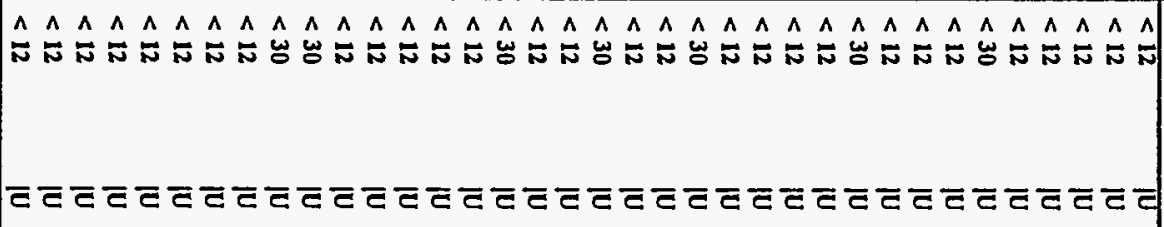 & 吾号 \\
\hline
\end{tabular}


TABLE A.2

ANALYTICAL RESULTS FOR GROUNDWATER 1995

SEMIVOLATILE ORGANIC.COMPOUNDS

1995 ANNUAL WATER MONITORING REPORT

LEHR ENVIRONMENTAL RESTORATION, DAVIS, CALIFORNIA

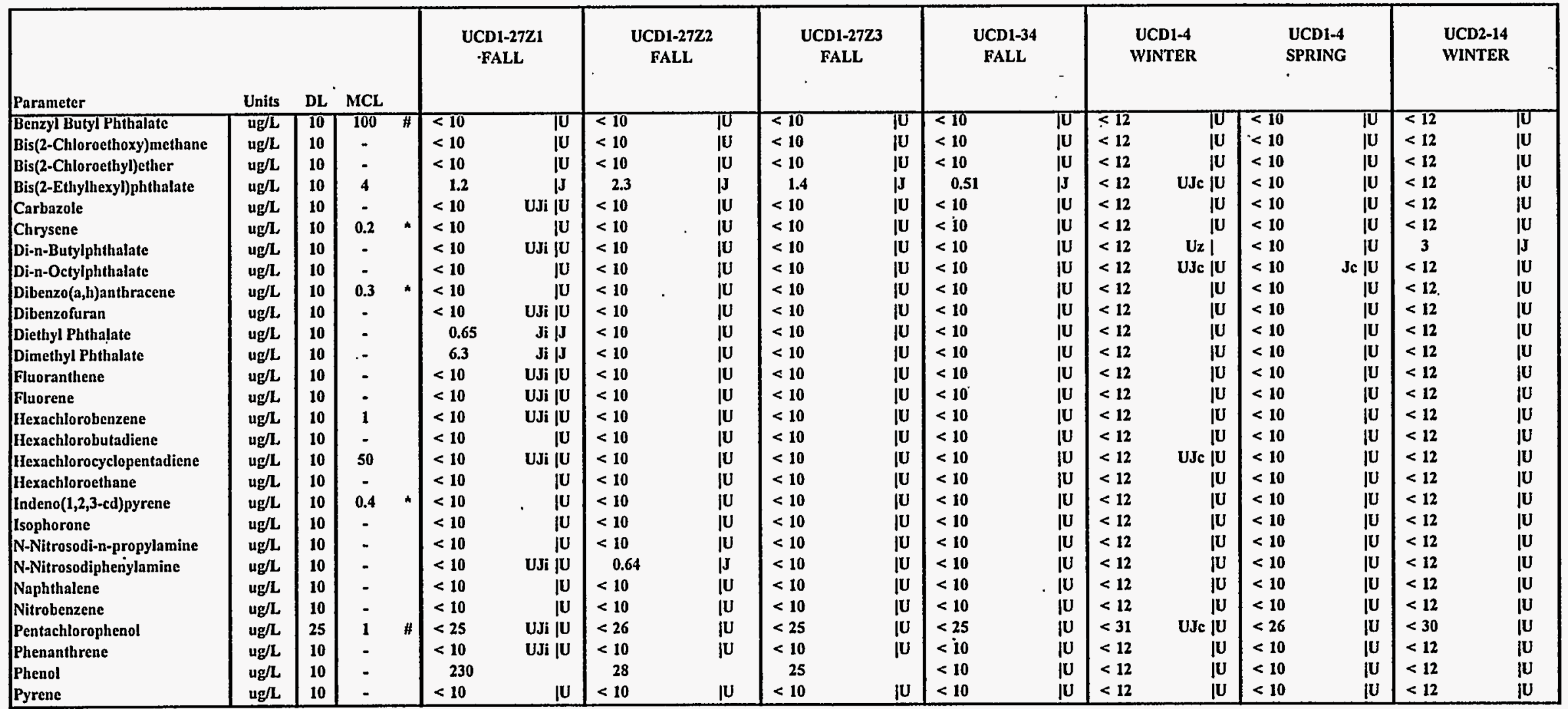


TABLE A.2

ANALYTICAL RESULTS FOR GROUNDWATER 1995

SEMIVOLATILE ORGANIC COMPOUNDS

1995 ANNUAL WATER MONITORING REPORT

LEHR ENVIRONMENTAL RESTORATION, DAVIS; CALIFORNIA

\begin{tabular}{|c|c|c|c|c|c|c|c|c|c|c|c|c|c|c|c|c|c|c|c|}
\hline Parameter & Units & DL & \multicolumn{2}{|l|}{ MCL } & \multicolumn{2}{|c|}{$\begin{array}{l}\text { UCD2-14 } \\
\text { SPRING }\end{array}$} & \multicolumn{2}{|c|}{$\begin{array}{l}\text { UCD2-15 } \\
\text { WINTER }\end{array}$} & \multicolumn{2}{|c|}{$\begin{array}{l}\text { UCD2-15 } \\
\text { SPRING } \\
\text {. }\end{array}$} & \multicolumn{2}{|c|}{$\begin{array}{l}\text { UCD2-16 } \\
\text { WINTER }\end{array}$} & \multicolumn{2}{|c|}{$\begin{array}{l}\text { UCD2-16 } \\
\text { SPRING }\end{array}$} & \multicolumn{2}{|c|}{$\begin{array}{l}\text { UCD2-17 } \\
\text { WINTER }\end{array}$} & \multicolumn{3}{|c|}{$\begin{array}{l}\text { UCD2-17 } \\
\text { SPRING }\end{array}$} \\
\hline 1,2,4-Trichlorobenzene & $\mathrm{ug} / \mathrm{L}$ & 10 & 70 & & $<11$ & Ju & $<12$ & TU & $<11$ & TU & $<12$ & ju & $<11$ & ju & $<12$ & Tu & $<10$ & & Tu \\
\hline 1,2-Dichlorobenzene & ug/L & 10 & 600 & & $<\boldsymbol{I 1}$ & נן & $<12$ & U & $<11$ & [U & $<12$ & ju & $<11$ & ju & $<12$ & ju & $<10$ & & |U \\
\hline 1,3-Dichlorobenzene & ug/L & 10 & - & & $<11$ & ju & $<12$ & ju & $<11$ & ןU & $<12$ & jU & $<11$ & ju & $<12$ & ju & $<10$ & & jU \\
\hline 1,4-Dichlorobenzenc & ug/L & 10 & 5 & & $<11$ & U & $<12$ & ju & $<11$ & ן & $<12$ & jU & $<11$ & ju & $<12$ & ju & $<10$ & & |U \\
\hline 2,2'-oxybis(1-Chloropropane) & ug/L. & 10 & - & & $<11$ & jU & $<12$ & UJe $\boldsymbol{j}$ & $<11$ & |U & $<12$ & UJc ju & $<11$ & ju & $<12$ & jU & $<10$ & & ju \\
\hline 2,4,5-Trichlorophenol & ug/L $/ \mathbf{L}$ & 25 & - & & $<27$ & U & $<31$ & ju & $<27$ & ju & $<\mathbf{3 1}$ & $\mathbf{j}$ & $<27$ & | & $<31$ & |U & $<25$ & . & jU \\
\hline 2,4,6-Trichlorophenol & ug/L & 10 & - & & $<11$ & U & $<12$ & ן $\mathbf{U}$ & $<11$ & |U & $<12$ & jU & $<11$ & ju & $<12$ & jU & $<10$ & & ju \\
\hline 2,4-Dichlorophenol & ug/L & 10 & 70 & $\#$ & $<11$ & ju & $<12$ & jU & $<11$ & jU & $<12$ & ju & $<11$. & ju & $<12$ & U & $<10$ & & U \\
\hline 2,4-Dimethylphenol & ug/L & 10 & - & & $<11$ & ju & $<12$ & ן & $<11$ & ןU & $<12$ & jU & $<11$ & ju & $<12$ & ju & $<10$ & & ן \\
\hline 2,4-Dinitrophenol & ug/L & 25 & - & & $<27$ & ju & $<31$ & jU & $<27$ & ju & $<\mathbf{3 1}$ & $\mathbf{j}$ & $<27$ & $\mathbf{U}$ & $<31$ & $\mathbf{U}$ & $<25$ & & J \\
\hline 2,4-Dinitrotoluene & $u g / L$ & 10 & - & & $<11$ & jU & $<12$ & ן $\mathbf{U}$ & $<11$ & jU & $<12$ & jU & $<11$ & ju & $<12$ & ju & $<10$ & 、 & jU \\
\hline 2,6-Dinitrotoluene & ug/L & 10 & - & & $<11$ & jU & $<12$ & |U & $<11$ & ןU & $<12$ & ju & $<11$ & J & $<12$ & ju & $<10$ & & ju \\
\hline 2-Chloronaphthalene & $u g / L$ & 10 & - & & $<11$ & ן & $<12$ & |U & $<11$ & ju & $<12$ & ןU & $<11$ & ju & $<12$ & ju & $<10$ & & jU \\
\hline 2-Chlorophenol & ug/L & 10 & - & & $<11$ & ju & $<12$ & jU & $<11$ & jU & $<12$ & ju & $<11$ & ju & $<12$ & U & $<10$. & & jU \\
\hline 2-Methyl-4,6-dinitrophenol & ug/L & 25 & - & & $<27$ & ןU & $<31$ & |U & $<27$ & |U & $<31$ & ju & $<27$ & ju & $<31$ & jU & $<25$ & & ju \\
\hline 2-Methylnaphthalene & ug/h & 10 & - & & $<11$ & ju & $<12$ & jU & $<11$ & ju & $<12$ & ju & $<11$ & jU & $<12$ & ju & $<10$ & & jU \\
\hline 2-Methylphenol & ugh & 10 & - & & $<11$ & ן & $<12$ & ן & $<11$ & ju & $<12$ & jU & $<11$ & ן & $<12$ & ju & $<10$ & & ju \\
\hline 2-Nitroaniline & ug/L & 25 & - & & $<27$ & ן U & $<\mathbf{3 1}$ & ju & $<27$ & ju & $<\mathbf{3 1}$ & ju & $<27$ & ju & $<\mathbf{3 1}$ & ju & $<25$ & & $\mathbf{U}$ \\
\hline 2-Nitrophenol & ug/L & 10 & .- & & $<11$ & ןU & $<12$ & jU & $<11$ & ן & $<12$ & jU &.$<11$ & ן & $<12$ & jU & $<10$ & & ju \\
\hline 3,3'-Dichlorobenzidine & $u g / L$. & 10 & - & & $<11$ & jU & $<12$ & UJe ju & $<11$ & ju & $<12$ & UJc ju & $<11$ & ju & $<12$ & ju & $<10$ & & ju \\
\hline 3-Nitroaniline & $u g / L$ & 25 & - & & $<27$ & ן $\mathbf{U}$ & $<31$ & $\cdot \boldsymbol{| U}$ & $<27$ & ןU & $<31$ & |U & $<27$ & U & $<31$ & jU & $<25$ & & ju \\
\hline 4-Bromophenyl Phenyl Ether & ug/h & 10 & - & & $<11$ & ju & $<12$ & ju & $<11$ & ju & $<12$ & jU & $<11$ & ju & $<12$ & ju & $<10$ & & |U \\
\hline 4-Chloro-3-Methylphenol & $u g / L$ & 10 & - & & $<11$ & (U & $<12$ & ju & $<11$ & ju & $<12$ & jU & $<11$ & ju & $<12$ & j & $<10$ & & ju \\
\hline 4-Chloroaniline & ug/L & 10 & - & & $<\mathbf{1 1}$ & ju & $<12$ & ju & $<11$ & ju & $<12$ & jU & $<11$. & ן & $<12$ & ju & $<10$ & & ju \\
\hline 4-Chlorophenyl Phenyl Ether & $u g / L$ & 10 & - & & $<11$ & ן U & $<12$ & ju & $<11$ & jU & $<12$ & ןU & $<11$ & ju & $<12$ & jU & $<10$ & & ju \\
\hline 4-Methylphenol & ug/L & 10 & - & & $<11$ & ju & $<12$ & ju & $<11$ & jU & $<12$ & jU & $<\mathbb{1 1}$ & ju & $<12$ & J & $<10$ & & JU \\
\hline 4-Nitroaniline & ug/L & 25 & - & & $<27$ & jU & $<31$ & ju & $<27$ & ju & $<31$ & jU & $<27$ & ju & $<\mathbf{3 1}$ & |U & $<25$ & & ju \\
\hline 4-Nitrophenol & $\mathrm{ug} / \mathrm{L}$ & 25 & - & & $<27$ & ju & $<\mathbf{3 1}$ & ju & $<27$ & jU & $<31$ & ju & $<27$ & [U & $<\mathbf{3 1}$ & ju & $<25$ & & ju \\
\hline Acenaphthene & ug/L & 10 & - & & $<11$ & ן & $<12$ & ןU & $<11$ & jU & $<12$ & ju & $<11$ & ןU & $<12$ & jU & $<10$ & & jU \\
\hline Acenaphthylene & ug/L & 10 & - & & $<11$ & |U & $<12$ & $\mathbf{U}$ & $<11$ & |U & $<12$ & jU & $<11$ & |U & $<12$ & ju & $<10$ & & U \\
\hline Anthracene & ug/L & 10 & - & & $<11$ & ןU & $<12$ & jU & $<11$ & jU & $<12$ & ju & $<11$ & ju & $<12$ & ju. & $<10$ & & 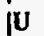 \\
\hline Benzo(a)anthracene & ug/L & 10 & 0.1 & " & $<11$ & ןU & $<12$ & jU & $<11$ & ן & $<12$ & jU & $<11$ & jU & $<12$ & ju & $<10$ & & ju \\
\hline Benzo(a)pyrene & $\mu g / L$ & 10 & 0.2 & & $<11$ & jU & $<12$ & jU & $<11$ & ju & $<12$ & jU & $<11$ & ju & $<12$ & ju & $<10$ & & ju \\
\hline Benzo(b)fluoranthene & ug/L & 10 & 0.2 & * & $<11$ & ןU & $<12$ & ju & $<11$ & $\mathbf{j} \mathbf{U}$ & $<12$ & ju & $<\dot{11}$ & jU & $<12$ & ju & $<10$ & & ju \\
\hline Benzo(g,h,i)perylene & ug/L & 10 & - & & $<\mathbf{1 1}$ & ju & $<12$ & ju & $<11$ & ju & $<12$ & jU & $<11$ & ju & $<12$ & U & $<10$ & & jU \\
\hline Benzo(k)nuoranthene & $u g / L$ & 10 & 0.2 & ^ & $<11$ & ju & $<12$ & jU & $<11$ & ןU & $<12$ & jU & $<11$ & jU & $<12$ & ju & $<10$ & & ju \\
\hline
\end{tabular}




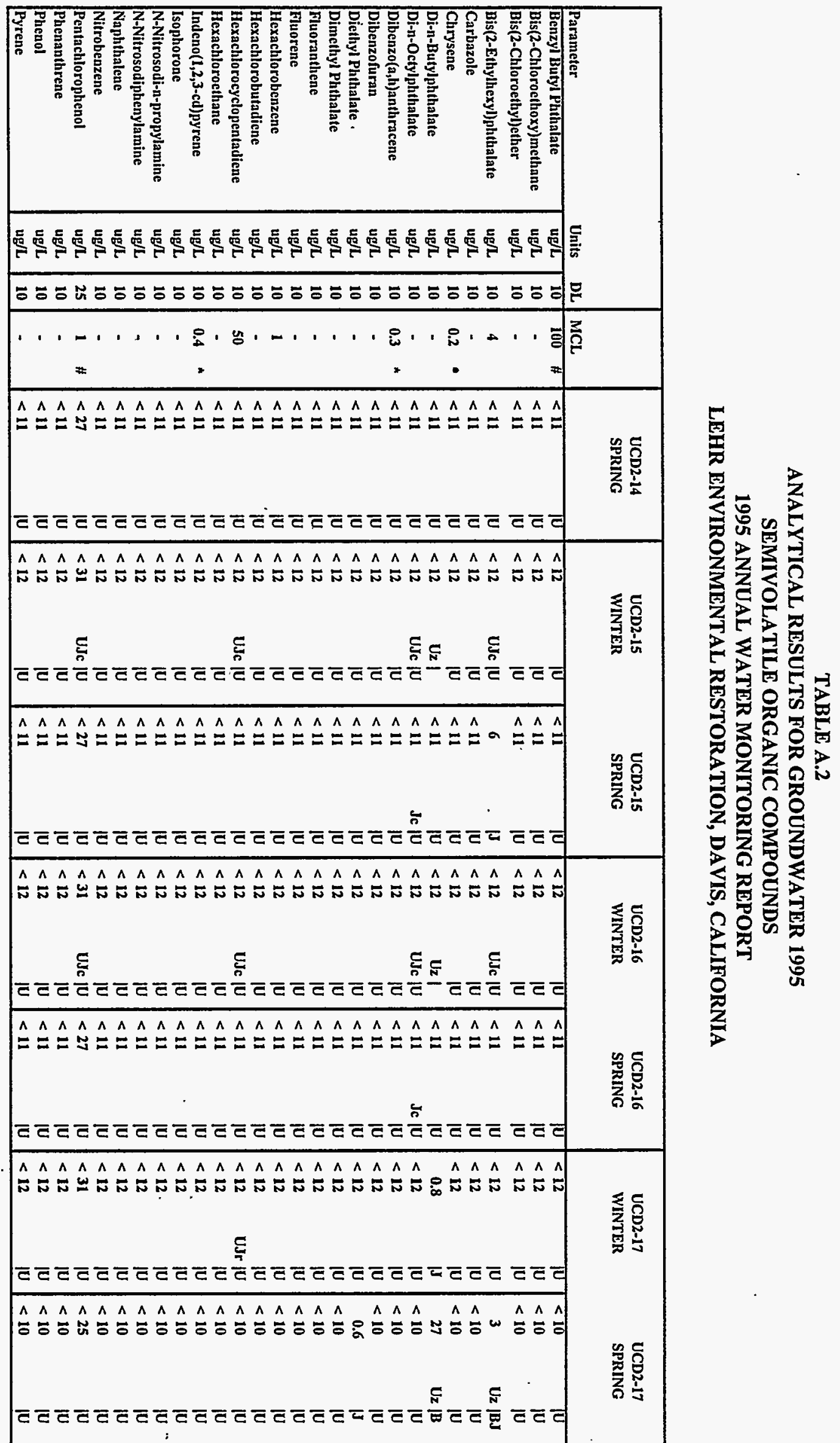




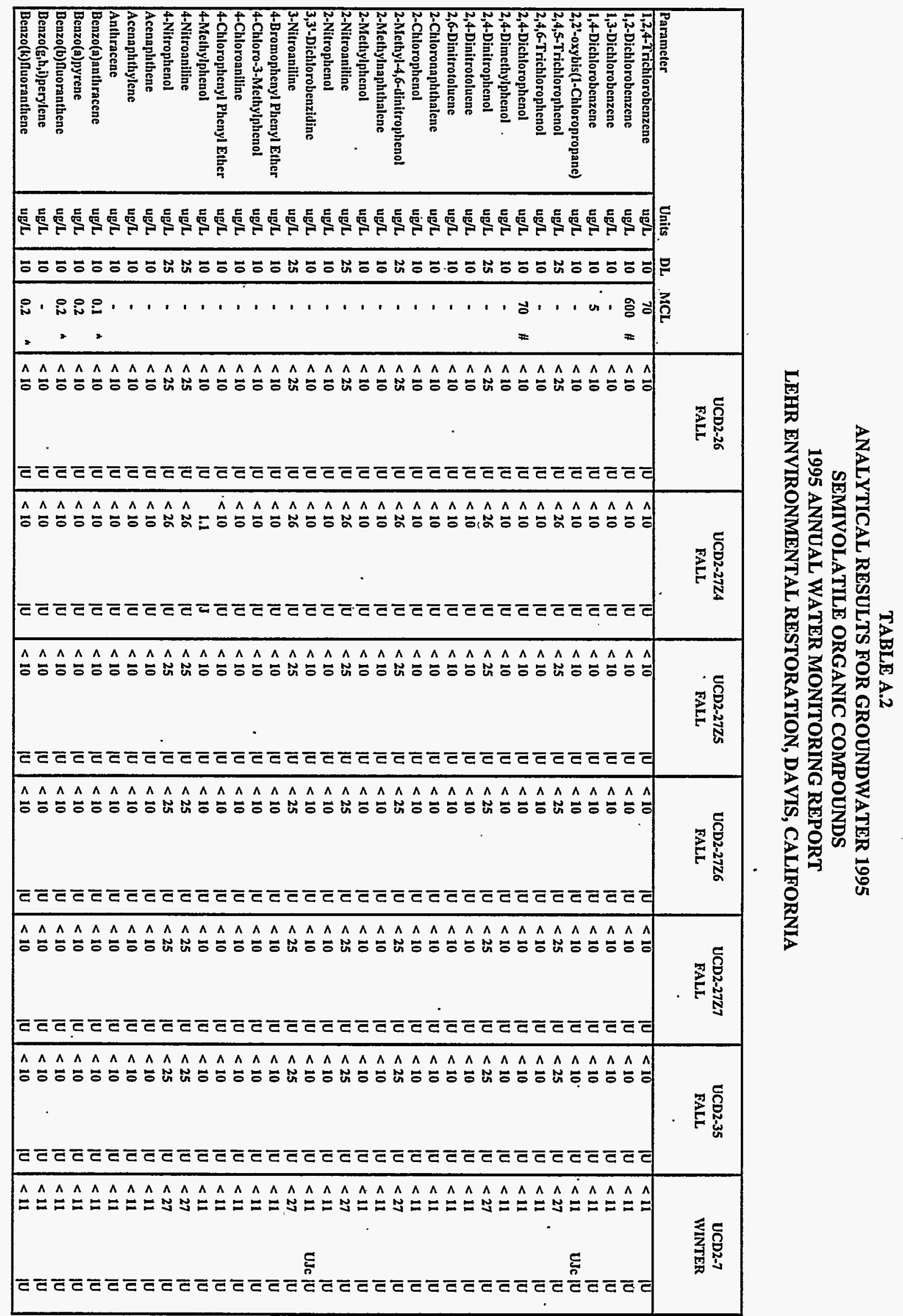




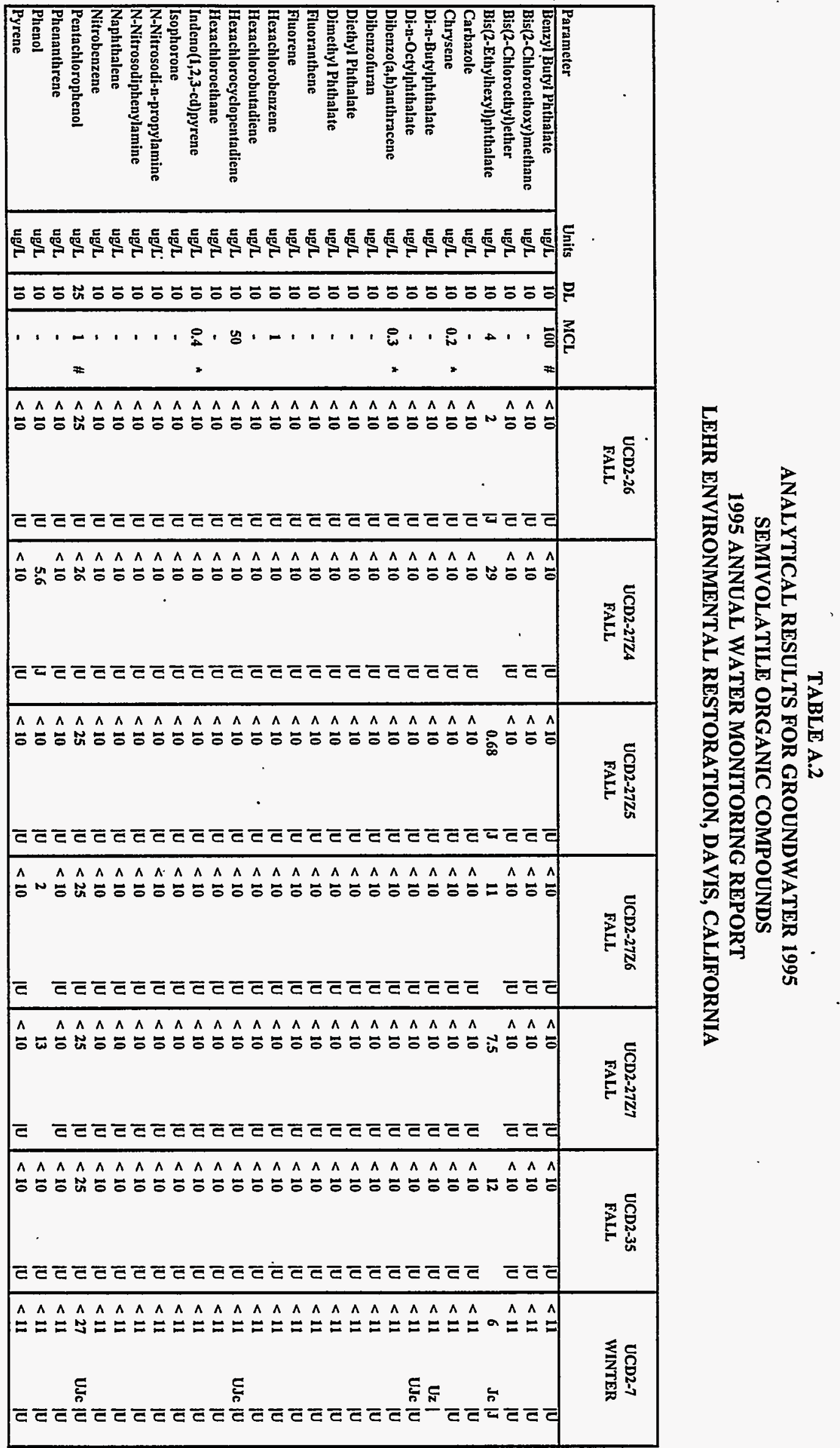




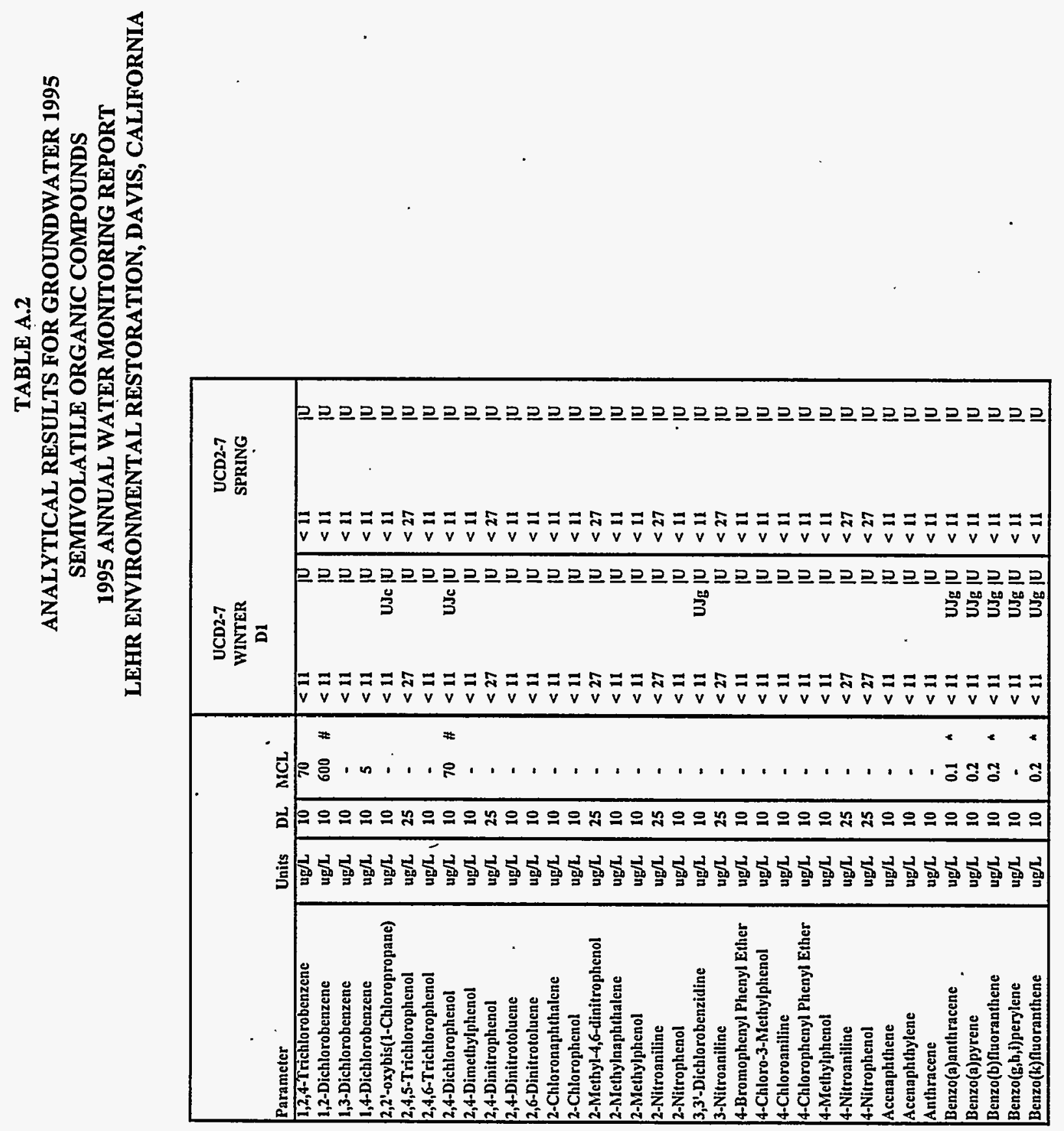


TABLE A.2

ANALYTICAL RESULTS FOR GROUNDWATER 1995

SEMIVOLATILE ORGANIC COMPOUNDS

1995 ANNUAL WATER MONITORING REPORT

LEHR ENVIRONMENTAL RESTORATION, DAVIS, CALIFORNIA

\begin{tabular}{|c|c|c|c|c|c|c|c|c|}
\hline Parameter & Units & DL. & \multicolumn{2}{|l|}{ MCL } & \multicolumn{2}{|c|}{$\begin{array}{c}\text { UCD2-7 } \\
\text { WINTER } \\
\text { D1 }\end{array}$} & \multicolumn{2}{|c|}{$\begin{array}{r}\text { UCD2-7 } \\
\text { SPRING }\end{array}$} \\
\hline Benzyl Butyl Phthalate & $\mathrm{ug} / \mathrm{L}$ & 10 & 100 & A & <II & UJg $\mid U$ & $<11$ & TU \\
\hline Bis(2-Chloroethoxy)methane & ug/L. & 10 & - & & $<11$ & ju & $<11$ & ju \\
\hline Bis(2-Chlorocthyl)ether & $\mathbf{u g} / \mathbf{L}$ & 10 & - & & $<11$ & ju & $<11$ & j \\
\hline Bis(2-Ethylhexyl)phthalate & ug/L & 10 & 4 & & 2 & $\mathbf{U J}_{\mathbf{g}} \mid \mathbf{J}$ & $<\mathbf{1 1}$ & ju \\
\hline Carbazole & ug/L & 10 & - & & $<11$ & jU & $<11$ & jU \\
\hline Chrysene & $\mathrm{ug} / \mathrm{L}$ & 10 & 0.2 & * & $<11$ & UJg $\mathbf{U}$ & $<11$ & ן $\mathbf{U}$ \\
\hline Di-n-Butylphthalate & ug/L & 10 & - & & $<11$ & $\mathbf{U z} \mid$ & $<11$ & $\mathrm{j} \mathrm{U}$ \\
\hline Di-n-Octylphthalate & ug/L. & 10 & - & & $<11$ & UJg & $<\mathbf{1 1}$ & Jc ju \\
\hline Dibenzo $(\mathrm{a}, \mathrm{h})$ anthracene & ug/L & 10 & & * & $<11$ & UJg & $<11$ & jU \\
\hline Dibenzofuran & $u g / L$. & 10 & - & & $<11$ & jU & $<11$ & jU \\
\hline Diethyl Phthalate & $\mathrm{ug} / \mathrm{L}$ & 10 & - & & $<11$ & ju & $<11$ & ןU \\
\hline Dimethyl Phthalate & ug/L & 10 & - & & $<11$ & jU & $<11$ & ן \\
\hline Fluoranthene & $\mathrm{ug} / \mathrm{L}$ & 10 & - & & $<11$ & UJg ju & $<11$ & $\mathrm{U}$ \\
\hline Fluorene & ug/L & 10 & - & & $<11$ & |U & $<\mathbf{1 1}$ & | \\
\hline Hexachlorobenzene & ug/L & 10 & 1 & & $<11$ & ju & $<11$ & ןU \\
\hline Hexachlorobutadiene & $u g / \mathbf{L}$ & 10 & - & & $<11$ & $\mathbf{I U}$ & $<11$ & $\mathbf{U}$ \\
\hline Hexachlorocyclopentadiene & ug/L & 10 & $\mathbf{5 0}$ & & $<11$ & UJc ju & $<11$ & ju \\
\hline Hexachloroethane & ug/L & 10 & - & & $<11$ & ju & $<11$ & U \\
\hline Indeno $(1,2,3+c d)$ pyrene & ug/L & 10 & 0.4 & ^ & $<11$ & $\mathbf{U J g} \boldsymbol{U}$ & $<11$ & ן $\mathbf{U}$ \\
\hline Isophorone & $\mathrm{ug} / \mathrm{L}$ & 10 & - & & $<11$ & [U & $<11$ & ן \\
\hline N-Nitrosodi-n-propylamine & $u g / L$ & 10 & - & & $<11$ & ju & $<11$ & ן \\
\hline N-Nitrosodiphenylamine & ug/L & 10 & - & & $<11$ & ju & $<11$ & ju \\
\hline Naphthalene & ug $/ \mathrm{L}$ & 10 & - & & $<11$ & ju & $<11$ & U \\
\hline Nitrobenzene & ug/L & 10 & - & & $<11$ & ju & $<11$ & ju \\
\hline Pentachlorophenol & ug/L & 25 & & $\#$ & $<27$ & UJe ju & $<27$ & ju \\
\hline Phenanthrene & $u g / L$ & 10 & - & & $<11$ & $\mathbf{U}$ & $<11$ & ju \\
\hline Phenol ` & ug/L & 10 & - & & $<11$ & jU & $<11$ & ן \\
\hline Pyrene & $\mathrm{ug} / \mathrm{L}$ & 10 & - & & $<11$ & UJg $\mathbf{U}$ & $<11$ & ju \\
\hline
\end{tabular}


TABLE A.2

ANALYTICAL RESULTS FOR GROUNDWATER 1995

SEMIVOLATILE ORGANIC COMPOUNDS

1995 ANNUAL WATER MONITORING REPORT

LEHR ENVIRONMENTAL RESTORATION, DAVIS, CALIFORNIA

D1 = Duplicate Sample

- = Parameter not analyzed or no MCL

DL $=$ Detection Limit

$<=$ Constituent below detection limit. Detection limits may vary depending on interference by other sample constituents.

MCL = Maximum Contaminant Level (primary), Title 22 Division 4 Chapter 15.

* = Proposed USEPA MCL

\# = USEPA MCL

Sec Appendix A for explanation of data qualifiers. 
TABLE A.3

ANALYTICAL RESULTS FOR GROUNDWATER 1995

PESTICIDES AND PCBS

1995 ANNUAL WATER MONITORING REPORT

LEHR ENVIRONMENTAL RESTORATION, DAVIS, CALIFORNIA

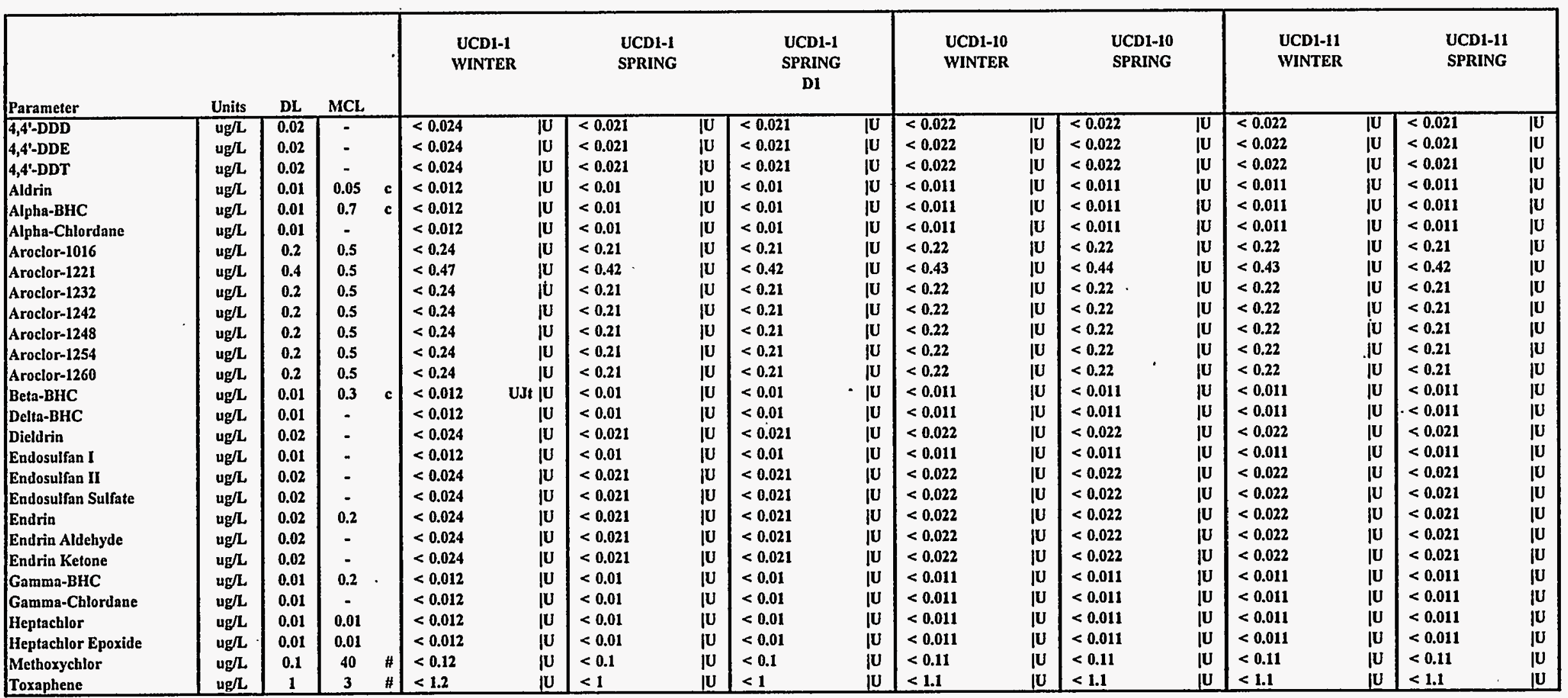




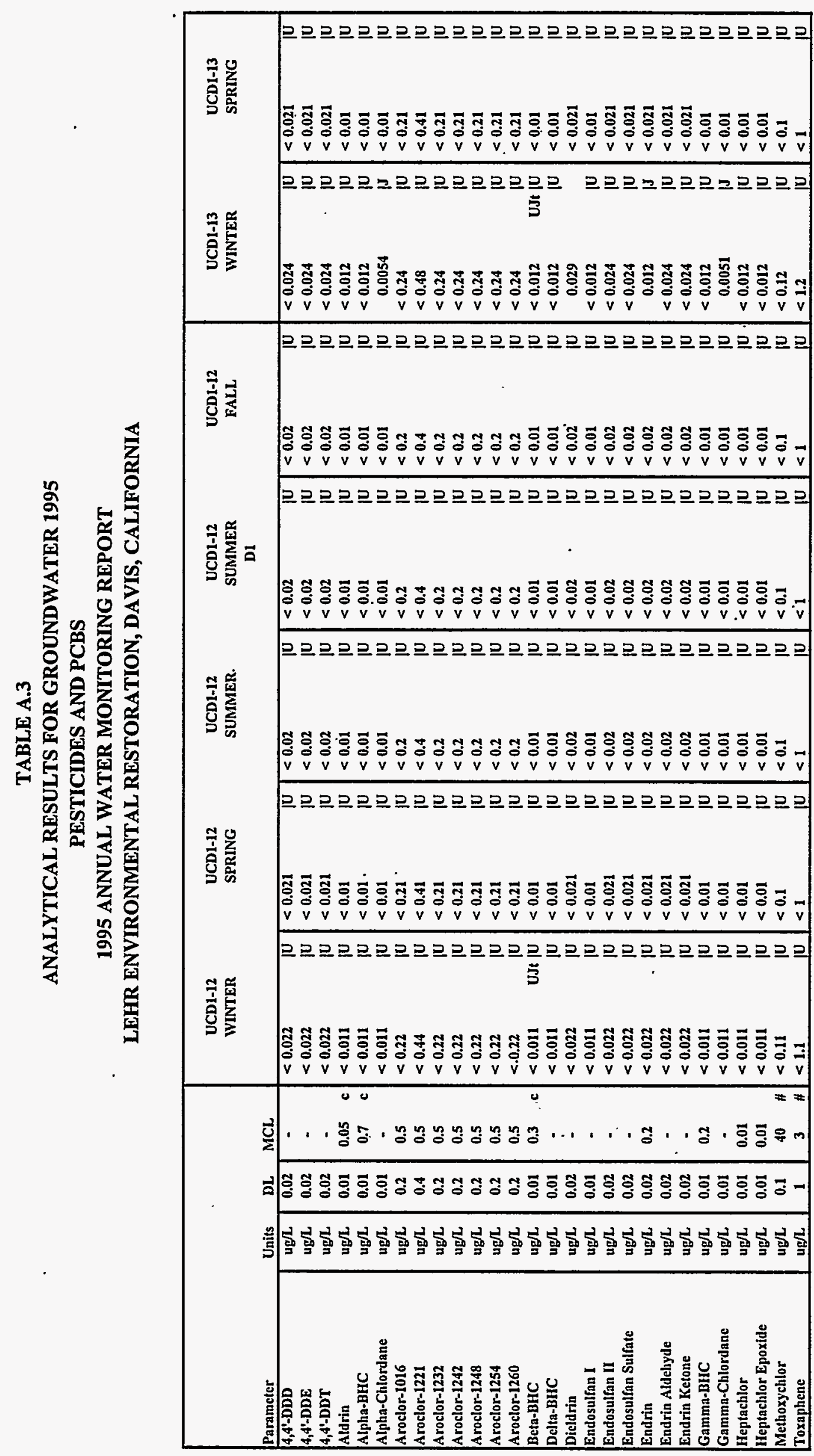


TABLE A.3

ANALYTICAL RESULTS FOR GROUNDWATER 1995

PESTICIDES AND PCBS

1995 ANNUAL WATER MONITORING REPORT LEHR ENVIRONMENTAL RESTORATION, DAVIS, CALIFORNIA

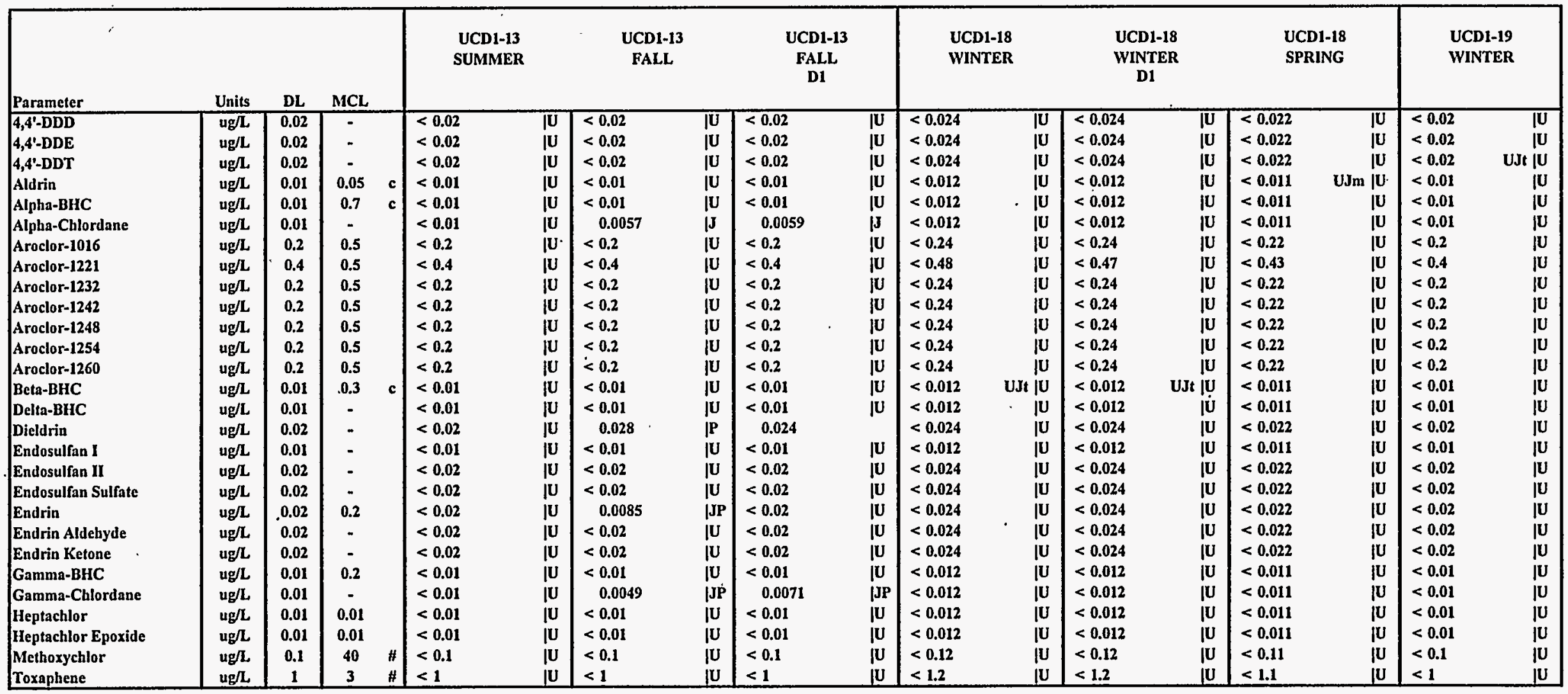


TABLE A.3

ANALYTICAL RESULTS FOR GROUNDWATER 1995

PESTICIDES AND PCBS

1995 ANNUAL WATER MONITORING REPORT

LEHR ENVIRONMENTAL RESTORATION, DAVIS, CALIFORNIA

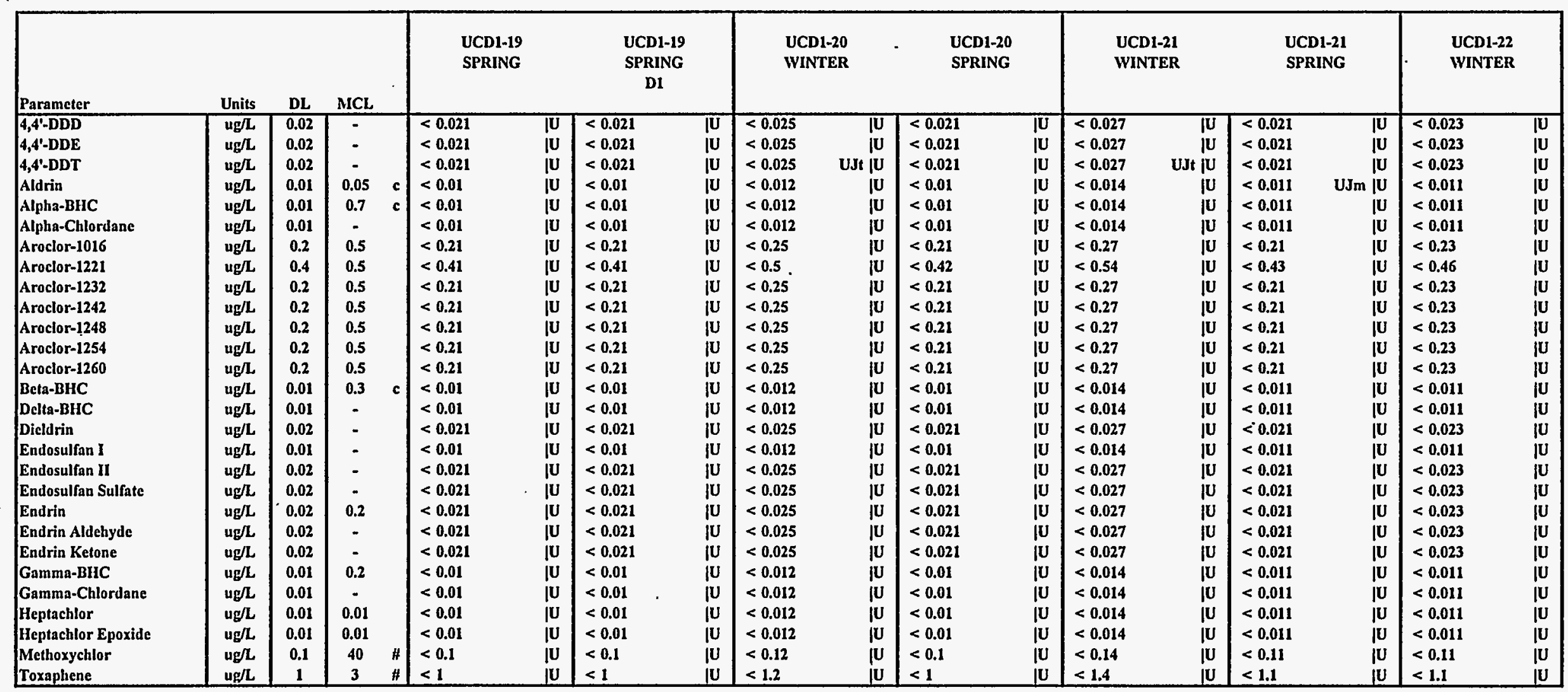


TABLE A.3

ANALYTICAL RESULTS FOR GROUNDWATER 1995

PESTICIDES AND PCBS

1995 ANNUAL WATER MONITORING REPORT

LEHR ENVIRONMENTAL RESTORATION, DAVIS, CALIFORNIA

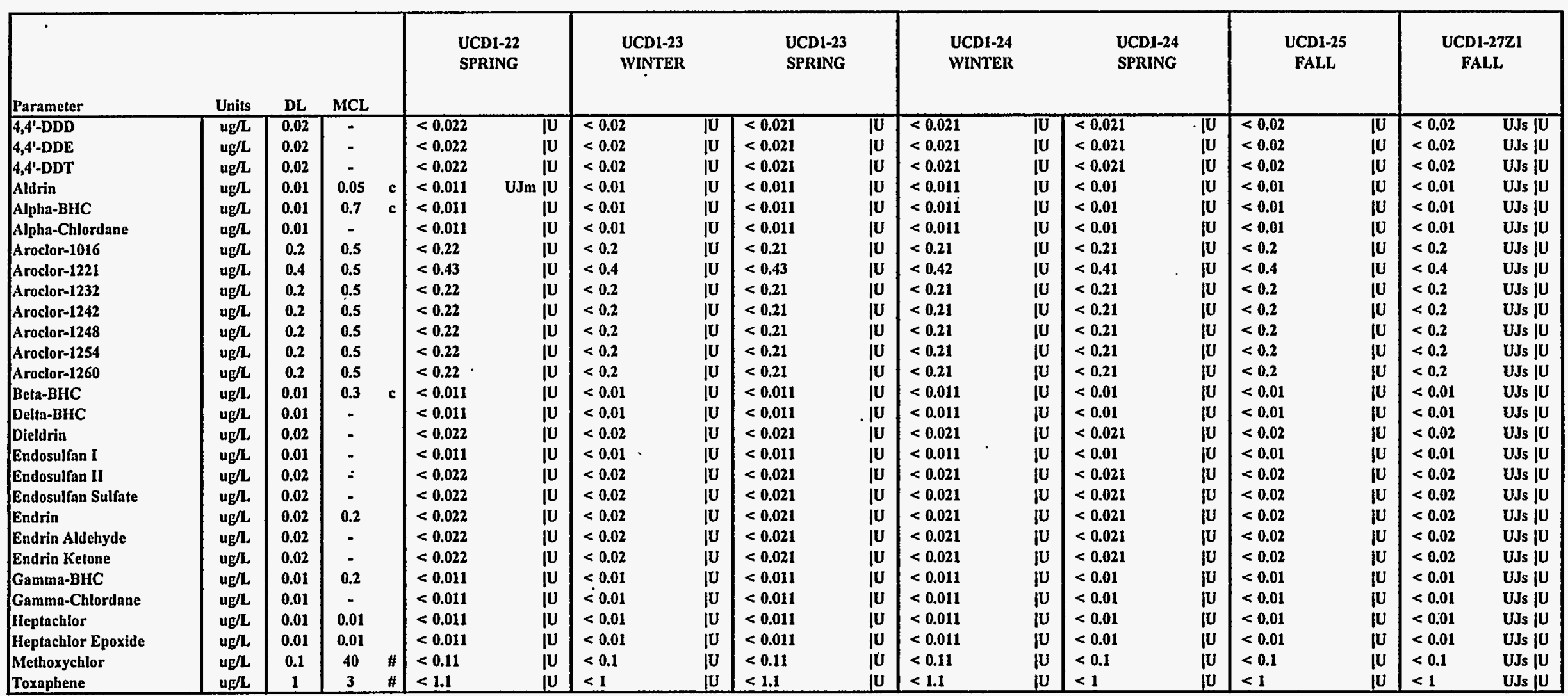


TABLE A.3

ANALYTICAL RESULTS FOR GROUNDWATER 1995

PESTICIDES AND PCBS

1995 ANNUAL WATER MONITORING REPORT

LEHR ENVIRONMENTAL RESTORATION, DAVIS, CALIFORNIA

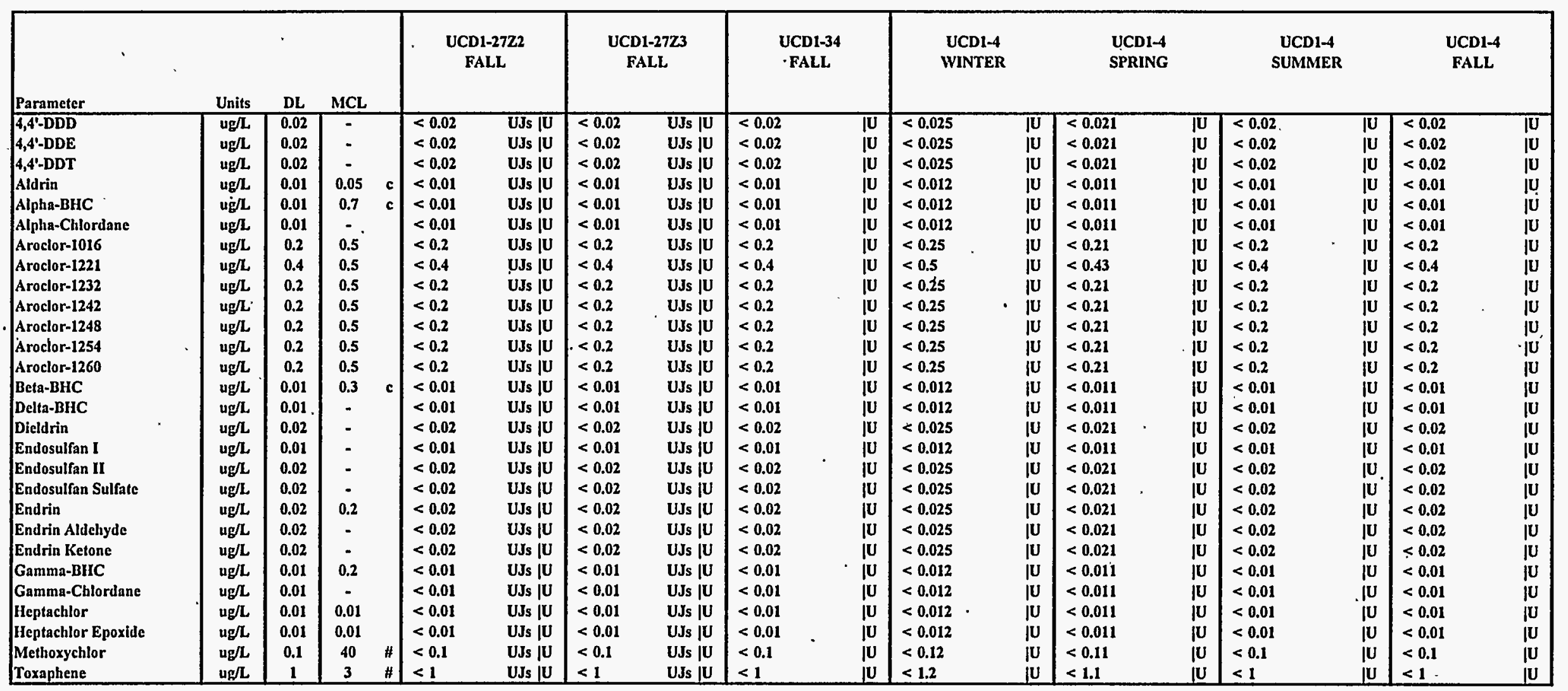


TABLE A.3

ANALYTICAL RESULTS FOR GROUNDWATER 1995

PESTICIDES AND PCBS

1995 ANNUAL WATER MONITORING REPORT

LEHR ENVIRONMENTAL RESTORATION, DAVIS, CALIFORNIA

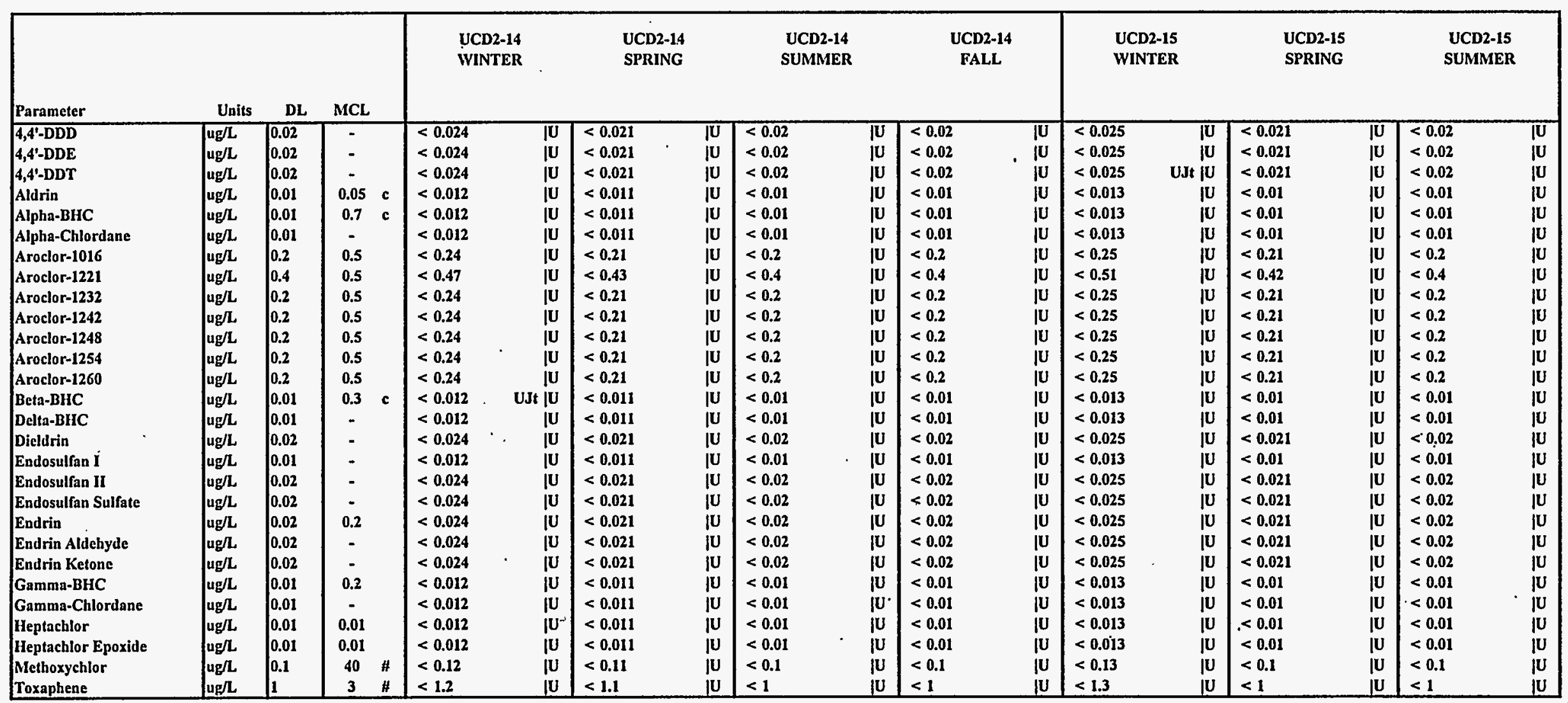


TABLE A.3

ANALYTICAL RESULTS FOR GROUNDWATER 1995

PESTICIDES AND PCBS

1995 ANNUAL WATER MONITORING REPORT LEHR ENVIRONMENTAL RESTORATION, DAVIS, CALIFORNIA

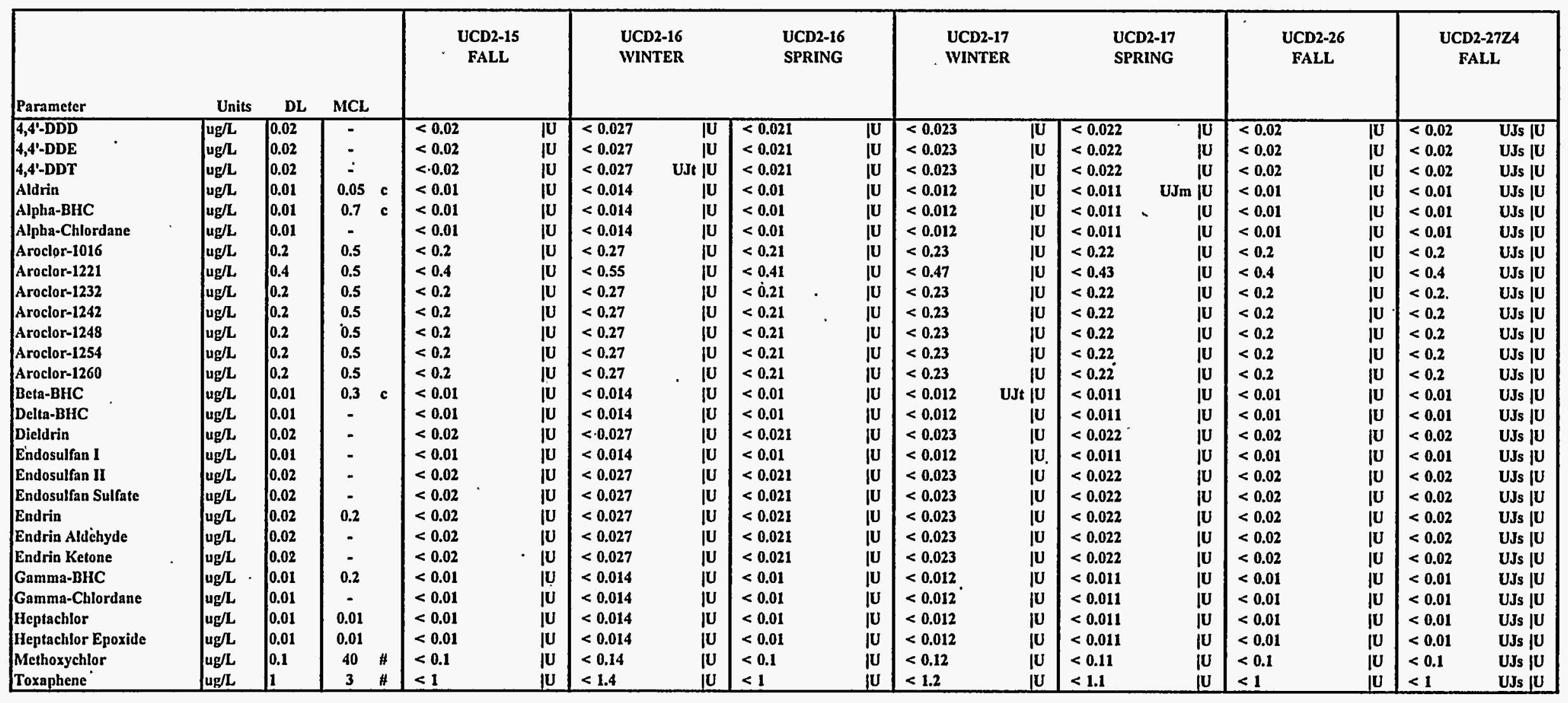


TABLE A.3

ANALYTICAL RESULTS FOR GROUNDWATER 1995

PESTICIDES AND PCBS

1995 ANNUAL WATER MONITORING REPORT

LEHR ENVIRONMENTAL RESTORATION, DAVIS, CALIFORNIA

\begin{tabular}{|c|c|c|c|c|c|c|c|c|c|c|c|c|c|c|c|c|c|c|}
\hline Parameter & Units & DL & \multicolumn{2}{|l|}{ MCL } & \multicolumn{2}{|c|}{$\begin{array}{c}\text { UCD2-2725 } \\
\text { FALL }\end{array}$} & \multicolumn{2}{|c|}{$\begin{array}{l}\text { UCD2-2726 } \\
\text { FALL. }\end{array}$} & \multicolumn{2}{|c|}{$\begin{array}{l}\text { UCD2-27Z7 } \\
\text { FALL }\end{array}$} & \multicolumn{2}{|c|}{$\begin{array}{c}\text { UCD2-35 } \\
\text { FALL }\end{array}$} & \multicolumn{2}{|c|}{$\begin{array}{c}\text { UCD2-7 } \\
\text { WINTER }\end{array}$} & \multicolumn{2}{|l|}{$\begin{array}{c}\text { UCD2-7 } \\
\text { WINTER } \\
\text { D1 }\end{array}$} & \multicolumn{2}{|c|}{$\begin{array}{l}\text { UCD2-7 } \\
\text { SPRING }\end{array}$} \\
\hline 4,4'-DDD & Jug/L & 0.02 & - & & $<0.02$ & |U & $<0.02$ & $\mid \mathbf{U}$ & $<0.021$ & $\mathrm{JU}$ & $<0.02$ & & $<0.022$ & $\mathbf{U}$ & $<0.022$ & $\mid \mathbf{U}$ & $<0.021$ & ju \\
\hline $4,4-\mathrm{DDE}$ & $\mathrm{ug} / \mathrm{L}$ & 0.02 & - & & $<0.02$ & jU & $<0.02$ & ju & $<0.021$ & |U & $<0.02$ & & $<0.022$ & $\mathbf{U}$ & $<0.022$ & ju & $<0.021$ & ן \\
\hline 4,4'-DDT & ug/L & 0.02 &. & & $<0.02$ & $\mid \mathbf{U}$ & $<0.02$ & $\mathbf{U}$ & $<0.021$ & jU & $<0.02$ & & $<0.022$ & |U & $<0.022$ & $\mathbf{j}$ & $<0.021$ & |U \\
\hline Aldrin & ug $/ L$ & 0.01 & 0.05 & c & $<0.01$ & $\mathbf{j} \mathbf{U}$ & $<0.01$ & $\mid \mathbf{U}$ & $<0.01$ & ju & $<0.01$ & & $<0.011$ & $\mid \mathbf{U}$ & $<0.011$ & $\mathbf{U}$ & $<0.01$ & ן \\
\hline Alpha-BHC & |ug/L & 0.01 & 0.7 & c & $<0.01$ & $\boldsymbol{U} \mathbf{U}$ & $<0.01$ & IU & $<0.01$ & ju & $<0.01$ & & $<0.011$ & [U & $<0.011$ & ju & $<0.01$ & ju \\
\hline Alpha-Chlordane & ug/L & 0.01 & - & & $<0.01$ & jU & $<0.01$ & $\mathbf{j}$ & $<0.01$ & |U & $<0.01$ & & $<0.011$ & $\boldsymbol{U} \mathbf{U}$ & $<0.011$ & |U & $<0.01$ & ן $\mathbf{U}$ \\
\hline Aroclor-1016 & |ug/L & 0.2 & 0.5 & & $<0.2$ & . $\mathbf{U}$ & $<0.2$ & ju & $<0.21$ & ju & $<0.2$ & & $<0.22$ & ju & $<0.22$ & ju & $<0.21$ & ju \\
\hline Aroclor-i221 & $\mathrm{ug} / \mathrm{L}$ & 0.4 & 0.5 & & $<0.4$ & ju & $<0.41$ & fU & $<0.41$ & ju & $<0.4$ & & $<0.43$ & ju & $<0.43$ & ju & $<0.42$ & ju \\
\hline Aroclor-1232 & $\mathrm{ug} / \mathrm{L}$ & 0.2 & 0.5 & & $<0.2$ & ju & $<0.2$ & ju & $<0.21$ & ju & $<0.2$ & & $<0.22$ & ju & $<0.22$ & ju & $<0.21$ & ju \\
\hline Aroclor-1242 & ug/L & 0.2 & 0.5 & & $<0.2$ & $\boldsymbol{U} \mathrm{U}$ & $<0.2$ & $\mid \mathbf{U}$ & $<0.21$ & |U & $<0.2$ & & $<0.22$ & $\mid \mathbf{U}$ & $<0.22$ & $\mathbf{U}$ & $<0.21$ & |U \\
\hline Aroclor-1248 & |ug/L & 0.2 & 0.5 & & $<0.2$ & ju & $<0.2$ & ju & $<0.21$ & ju & $<0.2$ & & $<0.22$ & $\boldsymbol{j} \mathbf{U}$ & $<0.22$ & ju & $<0.21$ & ju \\
\hline Aroclor-1254 & ug/L & 0.2 & 0.5 & & $<0.2$ & $\boldsymbol{j}$ & $<0.2$ & ju & $<0.21$ & ןU & $<0.2$ & & $<0.22$ & ju & $<0.22$ & $\mathbf{j}$ & $<0.21$ & ן \\
\hline Aroclor-1260 & ug/L & 0.2 & 0.5 & & $<0.2$ & $\boldsymbol{U}$ & $<0.2$ & $\boldsymbol{j} \mathbf{U}$ & $<0.21$ & ju & $<0.2$ & & $<0.22$ & ju & $<0.22$ & $\mathbf{j} \mathbf{U}$ & $<0.21$ & ju \\
\hline Beta-BHC & ug/L & 0.01 & 0.3 & c & $<0.01$ & $\boldsymbol{j} \mathbf{U}$ & $<0.01$ & $\boldsymbol{j} \mathbf{U}$ & $<0.01$ & $\mid \mathbf{U}$ & $<0.01$ & & $<0.011$ & $\boldsymbol{j} \mathbf{U}$ & $<0.011$ & $\mathbf{U}$ & $<0.01$ & |U \\
\hline Delta-BHC & $\mathrm{ug} / \mathrm{L}$ & 0.01 & - & & $<0.01$ & $\boldsymbol{j} \mathbf{U}$ & $<0.01$ & $\mathbf{j u}$ & $<0.01$ & $\mathbf{U}$ & $<0.01$ & & $<0.011$ & $\mathbf{j u}$ & $<0.011$ & ju & $<0.01$ & ן $\mathbf{U}$ \\
\hline Dicldrin & ug/L & 0.02 & - & & $<0.02$ & 伡 & $<0.02$ & |U & $<0.021$ & $\boldsymbol{j} \mathbf{U}$ & $<0.02$ & & $<0.022$ & $\boldsymbol{j} \mathbf{U}$ & $<0.022$ & $\boldsymbol{j} \mathbf{U}$ & $<0.021$ & ju \\
\hline Endosulfan I & ug/L & 0.01 & - & & $<0.01$ & $\boldsymbol{j}$ & $<0.01$ & |U & $<0.01$ & ju & $<0.01$ & & $<0.011$ & ju & $<0.011$ & ju & $<0.01$ & ju \\
\hline Endosulfan II & ug/L & 0.02 & - & & $<0.02$ & ju & $<0.02$ & $\mathbf{U}$ & $<0.021$ & $\boldsymbol{j} \mathbf{U}$ & $<0.02$ & & $<0.022$ & $\mathbf{j}$ & $<0.022$ & $\boldsymbol{U} \mathbf{U}$ & $<0.021$ & ן $\mathbf{U}$ \\
\hline Endosulfan Sulfate & ug/L & 0.02 & - & & $<0.02$ & ju & $<0.02$ & $\mathbf{U}$ & $<0.021$ & $\mathbf{j u}$ & $<0.02$ & & $<0.022$ & $\mathbf{j}$ & $<0.022$ & ju & $<0.021$ & ju \\
\hline Endrin & ug/L & 0.02 & 0.2 & & $<0.02$ & ן & $<0.02$ & |U & $<0.021$ & ju & $<0.02$ & & $<0.022$ & $\mathbf{j u}$ & $<0.022$ & ju & $<0.021$ & ju \\
\hline Endrin Aldehyde & $\mathrm{ug} / \mathrm{L}$ & 0.02 & - & & $<0.02$ & ju & $<0.02$ & ju & $<0.021$ & ju & $<0.02$ & & $<0.022$ & ju & $<0.022$ & ju & $<0.021$ & ju \\
\hline Endrin Ketone & ug/L & 0.02 & - & & $<0.02$ & |U & $<0.02$ & U & $<0.021$ & $\boldsymbol{U}$ & $<0.02$ & & $<0.022$ & $\mathbf{U}$ & $<0.022$ & IU & $<0.021$ & ן \\
\hline Gamma-BHC & |ug/L & 0.01 & 0.2 & & $<0.01$ & jU & $<0.01$ & $\boldsymbol{U}$ & $<0.01$ & $\boldsymbol{j}$ & $<0.01$ & & $<0.011$ & ju & $<0.011$ & ju & $<0.01$ & ן \\
\hline Gamma-Chlordane & ug/L & 0.01 & - & & $<0.01$ & ju & $<0.01$ & ju & $<0.01$ & ju & $<0.01$ & & $<0.011$ & $\mathbf{j u}$ & $<0.011$ & ju & $<0.01$ & ju \\
\hline Heptachlor & ug/L & 0.01 & 0.01 & & $<0.01$ & ןU & $<0.01$ & ju & $<0.01$ & ju & $<0.01$ & & $<0.011$ & $\mathbf{j}$ & $<0.011$ & $\boldsymbol{U}$ & $<0.01$ & ju \\
\hline Heptachlor Epoxide & |ug/L & 0.01 & 0.01 & & $<0.01$ & jU & $<0.01$ & UU & $<0.01$ & $\lceil\mathbf{U}$ & $<0.01$ & & $<0.011$ & $\boldsymbol{U} \mathbf{U}$ & $<0.011$ & ju & $<0.01$ & ן \\
\hline Methoxychlor & ug/L & 0.1 & 40 & $\#$ & $<0.1$ & ]U & $<0.1$ & ju & $<0.1$ & ju & $<0.1$ & & $<0.11$ & $\mathbf{j}$ & $<0.11$ & $\boldsymbol{U}$ & $<0.1$ & ju \\
\hline Toxaphene & $\mathrm{ug} / \mathrm{L}$ & 1 & 3 & \# & $<1$ & $\boldsymbol{U}$ & $<1$ & jU & $<1$ & $\mathbf{U}$ & $<1$ & & $<1.1^{\circ}$ & $\mathrm{U}$ & $<1.1$ & $\mathrm{U}$ & $<1$ & $\mathbf{U}$ \\
\hline
\end{tabular}


TABLE A.3

ANALYTICAL RESULTS FOR GROUNDWATER 1995

PESTICIDES AND PCBS

1995 ANNUAL WATER MONITORING REPORT

LEHR ENVIRONMENTAL RESTORATION, DAVIS, CALIFORNIA

D1 = Duplicate Sample

- = Parameter not analyzed or no MCL

DL = Detection Limit ${ }^{\cdot}$

$<$ - Constituent below detection limit. Detection linits may vary depending on interference by other sample constituents.

MCL = Maximum Contaminant Level (primary), Title 22 Division 4 Chapter 15.

\# = USEPA MCL

c = California State Action Level, Department of Health Services.

Sec Appendix A for explanation of data qualifiers. 
TABLE A.4

ANALYTICAL RESULTS FOR GROUNDWATER 1995

METALS

1995 ANNUAL WATER MONITORING REPORT

LEHR ENVIRONMENTAL RESTORATION, DAVIS, CALIFORNIA

\begin{tabular}{|c|c|c|c|c|c|c|c|c|c|c|c|c|c|c|c|c|}
\hline Parameter & Units & DL & \multicolumn{2}{|l|}{ MCL } & \multicolumn{2}{|c|}{$\begin{array}{c}\text { UCD1-1 } \\
\text { WINTER }\end{array}$} & \multicolumn{2}{|c|}{$\begin{array}{l}\text { UCD1-1 } \\
\text { SPRING }\end{array}$} & \multicolumn{2}{|c|}{$\begin{array}{c}\text { UCDI-1 } \\
\text { SPRING } \\
\text { D1 }\end{array}$} & $\begin{array}{l}\text { UCD1-1 } \\
\text { SUMMER }\end{array}$ & \multicolumn{2}{|c|}{$\begin{array}{r}\text { UCD1-10 } \\
\text { WINTER }\end{array}$} & \multicolumn{2}{|c|}{$\begin{array}{l}\text { UCD1-10 } \\
\text { SPRING }\end{array}$} & $\begin{array}{r}\text { UCD1-10 } \\
\text { SUMMER }\end{array}$ \\
\hline Antimony & ug/L & 5 & 6 & & $<5$ & IU & $<5$ & 10 & $<5$ & IU & - & $<5$ & 10 & $<5$ & III & - \\
\hline Arsenic & $\mathrm{ug} / \mathrm{L}$ & 2 & 50 & & 4 & Up i & $<2$ & ju & & ju & - & $3.7^{\circ}$ & & 2.5 & & - \\
\hline Barium & ug/L & 20 & 1000 & & 219 & & 237 & & 248 & & - & 25.7 & & 30.1 & & - \\
\hline Beryllium & ug/L & 1 & 4 & & $<1$ & ן U & $<1$ & U & $<1$ & |U & - & $<1$ & |U & $<1$ & |U & : \\
\hline Cadmium & $\mathbf{u g} / \mathbf{L}$ & 1 & 5 & $\#$ & $<1$ & ju & $<1$ & ju & $<1$ & ju & - & $<1$ & ju & $<1$ & ju & - \\
\hline Chromium & ug/L & 10 & 50 & & 32.8 & & 29.2 & & 30.1 & & - & 67.2 & & 74.9 & & - \\
\hline Chromium, Hexavalent (+6) & $\mathrm{ug} / \mathrm{L}$ & 10 & $\mathbf{5 0}$ & & 29 & & 18. & & 19 & & 32 & 69 & & 73 & & 74 \\
\hline Cobalt & ug/L & 10 & - & & 1.4 & |B & $<10$ & ן $\mathbf{U}$ & $<10$ & |U & - & $<10$ & $\boldsymbol{U} \mathbf{U}$ & $<10$ & $\mathbf{U}$ & - \\
\hline Copper & ug/L & 10 & 1000 & $\mathbf{s}$ & 1.9 & ןB & $<10$ & ju & $<10$ & jU & - & 2.6 & EB & $<10$ & |U & - \\
\hline Iron & $\mathbf{u g} / \mathbf{L}$ & 100 & 300 & $\mathbf{s}$ & $<100$ & ju & 18.8 & |B & 15.7 & |B & - & 24.9 & |B & 67.3 & |B & - \\
\hline Lead & ug/L & 2 & 15 & & $<2$ & ju & $<2$ & ju & $<2$ & ju & - & $<2$ & ju & $<2$ & |U & - \\
\hline Mercury & $\mathrm{ug} / \mathrm{L}$ & 0.2 & 2 & & $<0.2$ & ju & $<0.2$ & j & $<0.2$ & ju & - & $<0.2$ & ju & $<0.2$ & ju & - \\
\hline Molybdenum & ug/L & 10 & - & & $<10$ & ju & 2.2 & |B & 1.9 & |B & - & $<10$ & ju & 2.4 & |B & - \\
\hline Nickel & ug $/ \mathrm{L}$ & 20 & 100 & & 1 & |B & 1.7 & |B & 1.8 & [B & - & 1.1 & |B & 1.8 & |B & - \\
\hline Selenium & ug/L & 3 & 5 & $\#$ & $<3$ & ju & $<3$ & |u & $<3$ & |U & - & 23 & & 21.2 & & - \\
\hline Silver & ug/L & 10 & 50 & & $<10$ & ן & $<10$ & |U & $<10$. & |U & - & $<10$ & $\mathbf{U}$ & $<10$ & $\mid \mathbf{U}$ & - \\
\hline Thallium & ug/L & 10 & 2 & & $<10$ & |U & $<10$ & ju & $<10^{\circ}$ & ju & - & $<10$ & ju & $<10$ & ju & - \\
\hline Vanadium & ug/L & 10 & - & & 9.5 & [B & 4.9 & |B & 4.6 & |B & - & 10.3 & & 5.1 & |B & - \\
\hline Zinc & $\mathrm{ug} / \mathrm{L}$ & 20 & 5000 & $\mathbf{s}$ & 2.7 & B B & $<20$ & $\mathbf{U}$ & $<20$ & IU & - & 6.5 & |B & $<20$ & $\mathbf{U}$ & - \\
\hline
\end{tabular}


TABLE A.4

ANALYTICAL RESULTS FOR GROUNDWATER 1995

METALS

1995 ANNUAL WATER MONITORING REPORT

LEHR ENVIRONMENTAL RESTORATION, DAVIS, CALIFORNIA

\begin{tabular}{|c|c|c|c|c|c|c|c|c|c|c|c|c|c|c|c|}
\hline Parameter & Units & DL & \multicolumn{2}{|l|}{$\mathrm{MCL}$} & $\begin{array}{l}\text { UCD1-10 } \\
\text { FALL }\end{array}$ & \multicolumn{2}{|c|}{$\begin{array}{l}\text { UCD1-11 } \\
\text { WINTER }\end{array}$} & \multicolumn{2}{|c|}{$\begin{array}{l}\text { UCD1-11 } \\
\text { SPRING }\end{array}$} & $\begin{array}{l}\text { UCD1-11 } \\
\text { SUMMER }\end{array}$ & \multicolumn{2}{|c|}{$\begin{array}{l}\text { UCD1-12 } \\
\text { WINTER }\end{array}$} & \multicolumn{2}{|c|}{$\begin{array}{l}\text { UCDI-12 } \\
\text { SPRING }\end{array}$} & $\begin{array}{c}\text { UCD1-12 } \\
\text { SUMMIER }\end{array}$ \\
\hline & $u g / \mathrm{L}$ & 5 & 6 & & - & $<5$ & $\mathbf{U}$ & $<5$ & $\mathbf{I U}$ & - & $<5$ & $\mathbf{U}$ & $<5$ & IU & - \\
\hline Arsenic & ug/L & 2 & 50 & & - & 3.9 & $\mathrm{Jk}_{\mathrm{k}}$ & 2.8 & & - & 4.9 & Up i & $<2$ & iv & - \\
\hline Barium & ug/L & 20 & 1000 & & . & 262 & & 325 & & - & 340 & & 355 & & - \\
\hline Beryllium & $\mathrm{ug} / \mathrm{L}$ & 1 & 4 & & - & $<1$ & $\mathbf{U}$ & $<1$ & $\mathbf{U}$ & - & $<1$ & ןu & $<1$ & ju & - \\
\hline Cadmium & $u g / L$ & 1 & 5 & $\#$ & - & $<1$ & ן $\mathbf{U}$ & $<1$ & ju & - & $<1$ & ן & $<1$ & ju & - \\
\hline Chromium & $u g / h$ & 10 & 50 & & - & 266 & & 279 & & - & 191 & & 187 & & - \\
\hline Chromium, Hexavalent (+6) & ug/L & 10 & 50 & & 64 & 290 & & 270 & & 270 & 170 & & 140 & & 190 \\
\hline Cobalt & ug/L & 10 & - & & - & $<10$ & |U & $<10$ & |U & - & 1.9 & |B & $<10$ & jU & - \\
\hline Copper & ug/L & 10 & 1000 & $\mathbf{s}$ & - & 1 & jB & $<10$ & ju & - & 2 & |B & $<10$ & ju & - \\
\hline Iron & ug/L & 100 & 300 & $\mathbf{s}$ & - & 20 & |B & 34.8 & |B & - & $<100$ & jU & $<100$ & jU & - \\
\hline Lead & ug/ & 2 & 15 & & - & $<2$ & ju & $<2$ & ju & - & $<2$ & ju & $<2$ & UU & - \\
\hline Mercury & ug/L & 0.2 & 2 & - & - & 0.23 & & $<0.2$ & |U & - & $<0.2$ & j & $<0.2$ & jU & - \\
\hline Molybdenum & ug/L & 10 & - & & - & $<10$ & |U & 2.3 & |B & - & $<10$ & ju & $<10$ & ju & - \\
\hline Nickel & ug/L & 20 & 100 & & - & $<20$ & ן & 1.3 & |B & - & 5.7 & |B & 4.2 & jB & - \\
\hline Selenium & $\mathrm{ug} / \mathrm{L}$ & 3 & & $\#$ & - & 11.7 & & 9.9 & & - & 11.6 & & 11.6 & & - \\
\hline Silver & ug/L & 10 & 50 & & - & $<10$ & ju & $<10$ & |U & - & $<10$ & ןU & $<10$ & ju & - \\
\hline Thallium & ug/L & 10 & 2 & & - & $<10$ & ju & $<10$ & ju & - & $<10$ & ן & $<10$ & jU & - \\
\hline Vanadium & ug/L & 10 & - & & - & 4 & |B & $<10$ & ן & - & 8.7 & |B & 4.9 & jB & - \\
\hline Zinc & ug/L. & 20 & 5000 & $\mathrm{~s}$ & - & 6.1 & [B & $<20$ & $\mathbf{U}$ & - & 2.6 & B & $<20$ & ju & - \\
\hline
\end{tabular}


TABLE A.4

ANALYTICAL RESULTS FOR GROUNDWATER 1995

METALS

1995 ANNUAL WATER MONITORING REPORT

LEHR ENVIRONMENTAL RESTORATION, DAVIS, CALIFORNIA

\begin{tabular}{|c|c|c|c|c|c|c|c|c|c|c|c|c|c|c|}
\hline Parameter & Units & DL & \multicolumn{2}{|l|}{ MCL } & $\begin{array}{c}\text { UCD1-12 } \\
\text { FALL }\end{array}$ & \multicolumn{2}{|c|}{$\begin{array}{l}\text { UCD1-13 } \\
\text { WINTER }\end{array}$} & \multicolumn{2}{|c|}{$\begin{array}{l}\text { UCD1-13 } \\
\text { SPRING }\end{array}$} & $\begin{array}{c}\text { UCD1-13 } \\
\text { SUMMER }\end{array}$ & $\begin{array}{l}\text { UCD1-13 } \\
\text { FALL }\end{array}$ & $\begin{array}{c}\text { UCD1-13 } \\
\text { FALL } \\
\text { D1 }\end{array}$ & \multicolumn{2}{|c|}{$\begin{array}{l}\text { UCD1-18 } \\
\text { WINTER }\end{array}$} \\
\hline & ug/L & 5 & 6 & & - & $<5$ & $\boldsymbol{U}$ & $<5$ & |U & - & - & $=$ & $<5$ & U \\
\hline Arsenic & ug/L & 2 & 50 & & . & 3.3 & Upi & $<2$ & ju & - & - & - & 4.9 & Up 1 \\
\hline Barium & ug/L & 20 & 1000 & & - & 327 & & 352 & & - & - & - & 152 & \\
\hline Beryllium & ug/L & 1 & 4 & & - & $<1$ & $\boldsymbol{U} \mathbf{U}$ & $<1$ & ן & - & - & - & $<1$ & |U \\
\hline Cadmium & $\mathrm{ug} / \mathrm{L}$ & 1 & & $\#$ & - & $<1$ & $\boldsymbol{U} \mathbf{U}$ & $<1$ & ן & - & - & - & $<1$ & ju \\
\hline Chromium & ug/L & 10 & $\mathbf{5 0}$ & & $\cdot$ & 94.6 & & 90.6 & & - & - & - & 14.6 & \\
\hline Chromium, Hexavalent $(+6)$ & ug/L & 10 & 50 & & 170 & 86 & & 61 & & 85 & 76 & 75 & $<10$ & \\
\hline Cobalt & ug/L & 10 & - & & - & 1.5 & |B & $<10$ & ןU & - & - & - & 1.5 & |B \\
\hline Copper & ug/L & 10 & 1000 & $\mathbf{S}$ & - & 1.1 & |B & $<10$ & |U & - & - & - & 1.4 & |B \\
\hline Iron & ug/L. & 100 & 300 & $\mathbf{s}$ & - & $<100$ & |U & $<100$ & ן $\mathbf{U}$ & - & - & - & $<100$ & ju \\
\hline Lead & ug/L & 2 & 15 & & - & $<2$ & ju & $<2$ & ju & - & - & - & $<2$ & ju \\
\hline Mercury & ug/L & 0.2 & 2 & & - & $<0.2$ & ju & $<0.2$ & ן & - & - & - & $<0.2$ & |U \\
\hline Molybdenum & ug/L & 10 & - & & - & $<10$ & |U & $<10$ & |U & - & - & - & $<10$ & U \\
\hline Nickel & ug/L & 20 & 100 & & - & 1.5 & |B & 1.5 & |B & - & - & - & 65.1 & \\
\hline Sclenium & $\mathrm{ug} / \mathrm{L}$ & 3 & & \# & - & 4.1 & JQI & $<3$ & ן & - & - & - & $<3$. & ןU \\
\hline Silver & ug/L & 10 & 50 & & - & $<10$ & $\mid \mathbf{U}$ & $<10$ & $\mathbf{j}$ & - & - & - & $<10$ & |U \\
\hline Thallium & ug/L & 10 & 2 & & - & $<10$ & $\mathbf{U}$ & $<10$ & |U & - & - & - & $<10$ & ju \\
\hline Vanadium & ug/L & 10 & - & & - & 11.7 & & 7.7 & |B & - & - & - & 10.6 & \\
\hline Zinc & ug/L & 20 & 5000 & $\mathbf{s}$ & - & 2.4 & BB & $<20$ & $\mathrm{jU}$ & - & - & - & 1.8 & 吕 \\
\hline
\end{tabular}


TABLE A.4

ANALYTICAL RESULTS FOR GROUNDWATER 1995

METALS

1995 ANNUAL WATER MONITORING REPORT

LEHR ENVIRONMENTAL RESTORATION, DAVIS, CALIFORNIA

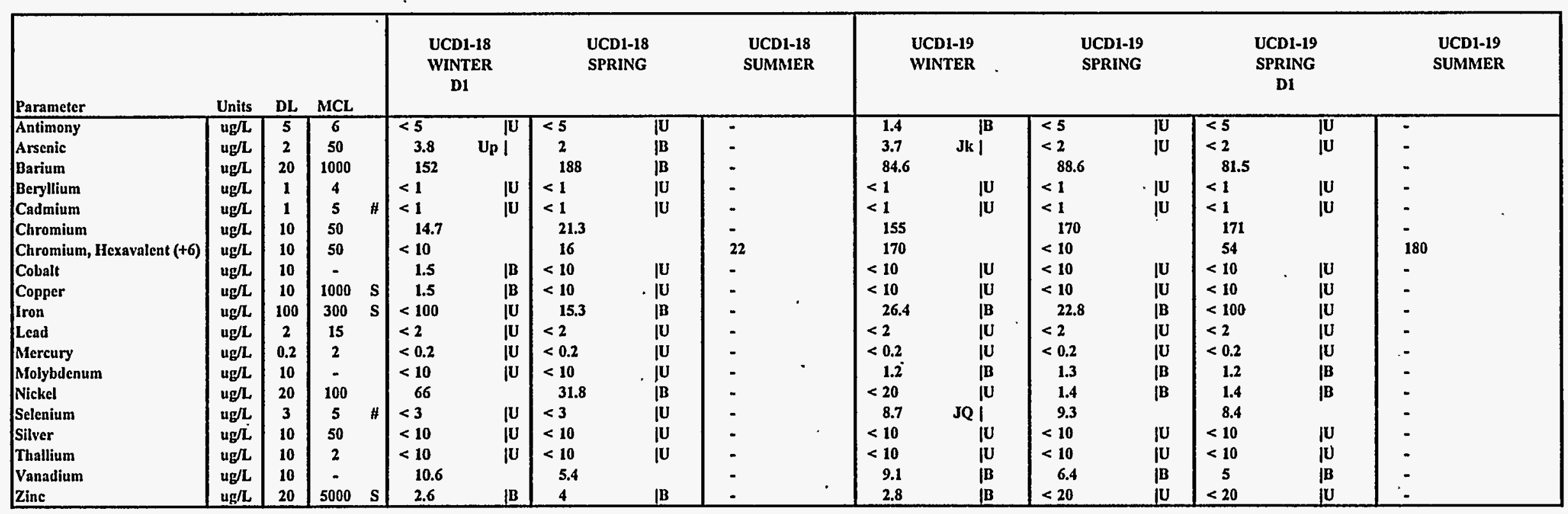


TABLE A.4

ANALYTICAL RESULTS FOR GROUNDWATER 1995

METALS

1995 ANNUAL WATER MONITORING REPORT

LEHR ENVIRONMENTAL RESTORATION, DAVIS, CALIFORNIA

\begin{tabular}{|c|c|c|c|c|c|c|c|c|c|c|c|c|c|c|c|c|}
\hline Parameter & Units & DL & \multicolumn{2}{|l|}{ MCL } & $\begin{array}{c}\text { UCD1-19 } \\
\text { FALL }\end{array}$ & \multicolumn{2}{|c|}{$\begin{array}{l}\text { UCD1-20 } \\
\text { WINTER }\end{array}$} & \multicolumn{2}{|c|}{$\begin{array}{l}\text { UCD1-20 } \\
\text { SPRING }\end{array}$} & $\begin{array}{l}\text { UCD1-20 } \\
\text { SUMMER }\end{array}$ & \multicolumn{2}{|c|}{$\begin{array}{l}\text { UCDI-21 } \\
\text { WINTER }\end{array}$} & \multicolumn{2}{|c|}{$\begin{array}{l}\text { UCD1-21 } \\
\text { SPRING }\end{array}$} & \multicolumn{2}{|c|}{$\begin{array}{l}\text { UCD1-22 } \\
\text { WINTER }\end{array}$} \\
\hline Antimony & $u g / L$ & $\mathbf{5}$ & 6 & & - & $<5$ & |U & $<5$ & ן & - & 1.1 & |B & $<5$ & $\mid \mathbf{U}$ & $<5$ & $\mid \mathbf{U}$ \\
\hline Arsenic & $\mathrm{ug} / \mathrm{L}$ & 2 & 50 & & & 4 & i & 2.1 & & & 2.6 & Jk | & $<2$ & |U & 3 & Jk I \\
\hline Barium & ug/L & 20 & 1000 & & - & 81.3 & & 97.8 & & - & 140 & & 140 & |B & 78.8 & \\
\hline Beryllium & $\mathrm{ug} / \mathrm{L}$ & 1 & 4 & & - & $<1$ & U & $<1$ & J & - & $<1$ & |U & $<1$ & |U & $<1$ & $\mathbf{U}$ \\
\hline Cadmium & ug/L & 1 & 5 & '\# & - & & ju & & ju & - & $<1$ & jU & $<1$ & jU & $<1$ & ju \\
\hline Chromium & $\mathrm{ug} / \mathrm{L}$ & 10 & 50 & & - & 27.7 & · & 28.6 & & - & 54.2 & & 58.2 & & 4.9 & ¡B \\
\hline Chromium, Hexavalent (+6) & ug/L & 10 & 50 & & 170 & 28 & & 29 & & 31 & 57 & & 58 & & $<10$ & \\
\hline Cobalt & ug/L & 10 & - & & $=$ & $<10$ & |U & $<10$ & |U & - & $<10$ & $\mathbf{U}$ & $<10$ & |U & $<10$ & |U \\
\hline Copper & ug/L & 10 & 1000 & $\mathbf{s}$ & - & $<10$ & |U & $<10$ & ju & - & $<10$ & ju & $<10$ & ju & $<10$ & ju \\
\hline Iron & ug/L & 100 & 300 & $\mathbf{s}$ & - & $<100$ & ןU & $<100$ & ju & - & 25.4 & jB & 28.3 & jB & $<100$ & ju \\
\hline Lead & ug/L. & 2 & 15 & & - & $<2$ & ן & $<2$ & ju & - & $<2$ & jU & $<2$ & ju & $<2$ & ju \\
\hline Mercury & ug/L & 0.2 & 2 & & - & $<0.2$ & ju & $<0.2$ & |U & - & $<0.2$ & ן & $<0.2$ & |U & $<0.2$ & |U \\
\hline Molybdenum & ug/L & 10 & - & & - & $<10$ & ju & $<10$ & ju & - & 1.5 & |B & $<10$ & U & 1.7 & jB \\
\hline Nickel & $\mathrm{ug} / \mathrm{L}$ & 20 & 100 & & - & $<20$ & |U & $<20$ & ju & - & $<20$ & $\mathbf{j} \mathbf{U}$ & $<20$ & ju & 8.3 & jB \\
\hline Selenium & ug/L & 3 & & $\#$ & - & $<3$ & jU & $<3$ & ju & - & 3.7 & JQ i & $<3$ & ju & $<3$ & jU \\
\hline Silver & ug/L & 10 & 50 & & - & $<10$ & ju & $<10$ & ju & - & $<10$ & |U & $<10$ & ju & $<10$ & |U \\
\hline Thallium & $\mathrm{ug} / \mathrm{L}$ & 10 & 2 & & - & $<10$ & ju & $<10$ & ju & - & $<10$ & jU & $<10$ & ju & $<10$ & ןU \\
\hline Vanadium & $\mathrm{ug} / \mathrm{L}$ & 10 & - & & - & 11.5 & & 10.1 & & - & 8.6 & |B & 8.1 & & 8.3 & [B \\
\hline Zinc & ug/L & 20 & 5000 & $\mathbf{s}$ & - & 2.3 & B B & $<20$ & $\mathbf{U}$ & - & 3.9 & |B & 2.7 & B & 2.7 & EB \\
\hline
\end{tabular}


TABLE A.4

ANALYTICAL RESULTS FOR GROUNDWATER 1995

METALS

1995 ANNUAL WATER MONITORING REPORT

LEHR ENVIRONMENTAL RESTORATION, DAVIS, CALIFORNIA

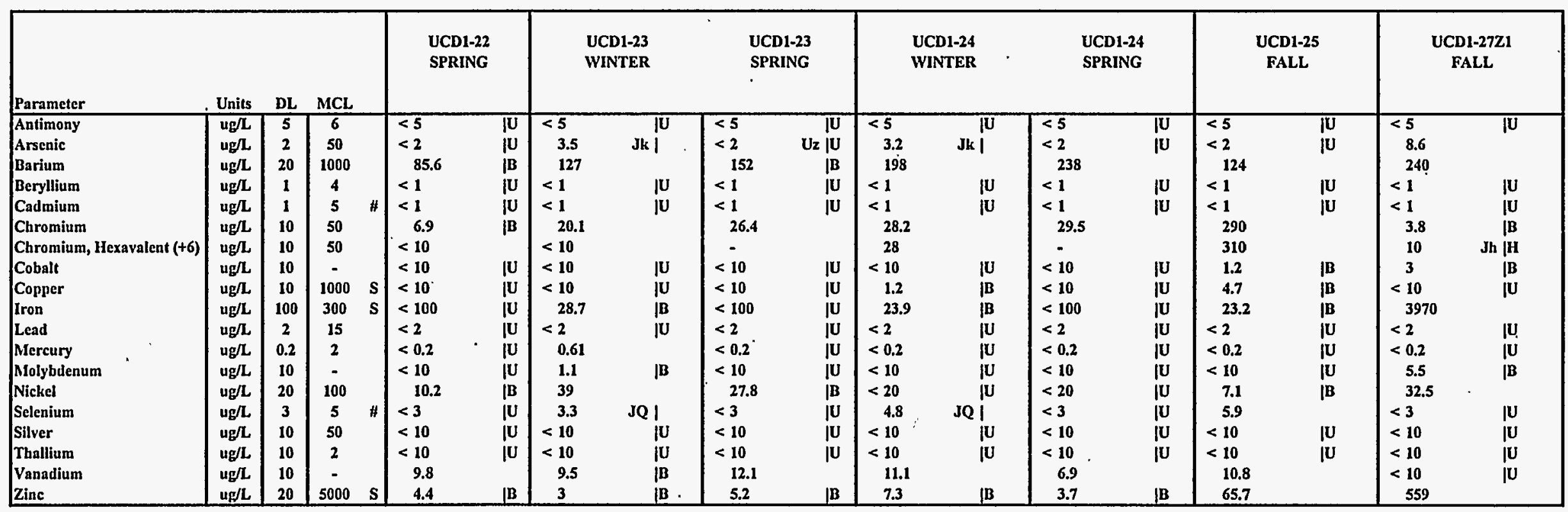


TABLE A.4

ANALYTICAL RESULTS FOR GROUNDWATER 1995

METALS

1995 ANNUAL WATER MONITORING REPORT

LEHR ENVIRONMENTAL RESTORATION, DAVIS, CALIFORNIA

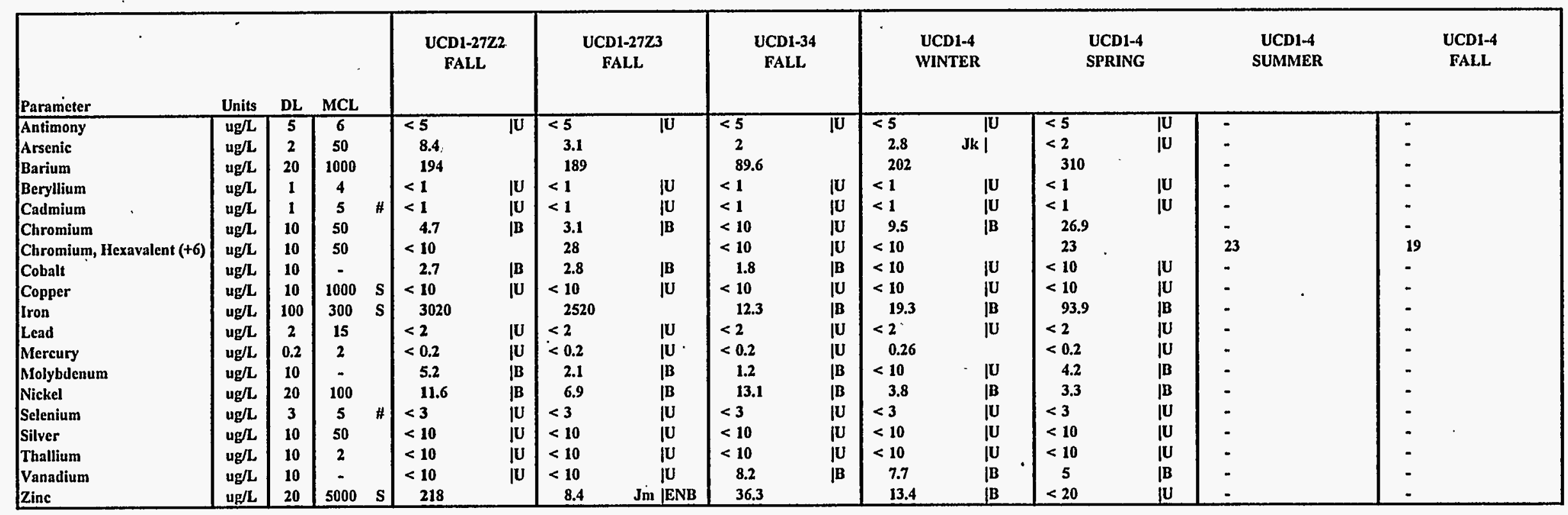


TABLE A.4

ANALYTICAL RESULTS FOR GROUNDWATER 1995

METALS

1995 ANNUAL WATER MONITORING REPORंT

LEHR ENVIRONMENTAL RESTORATION, DAVIS, CALIFORNIA

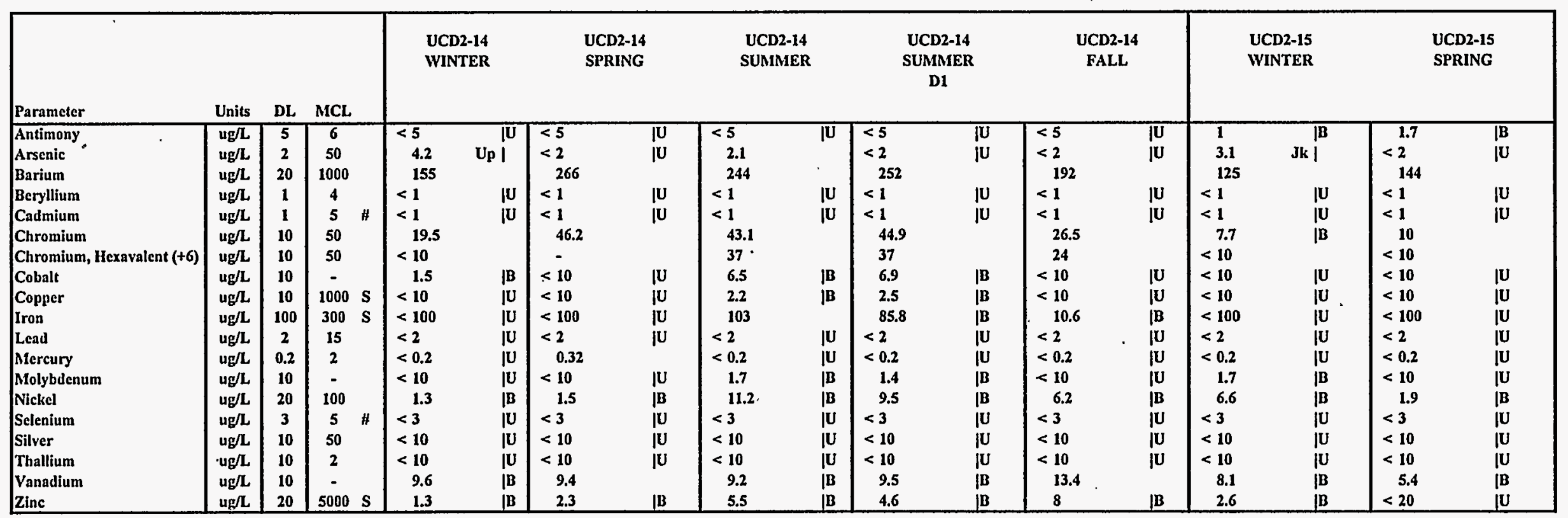


TABLE A.4

ANALYTICAL RESULTS FOR GROUNDWATER 1995

METALS

1995 ANNUAL WATER MONITORING REPORT

LEHR ENVIRONMENTAL RESTORATION, DAVIS, CALIFORNIA

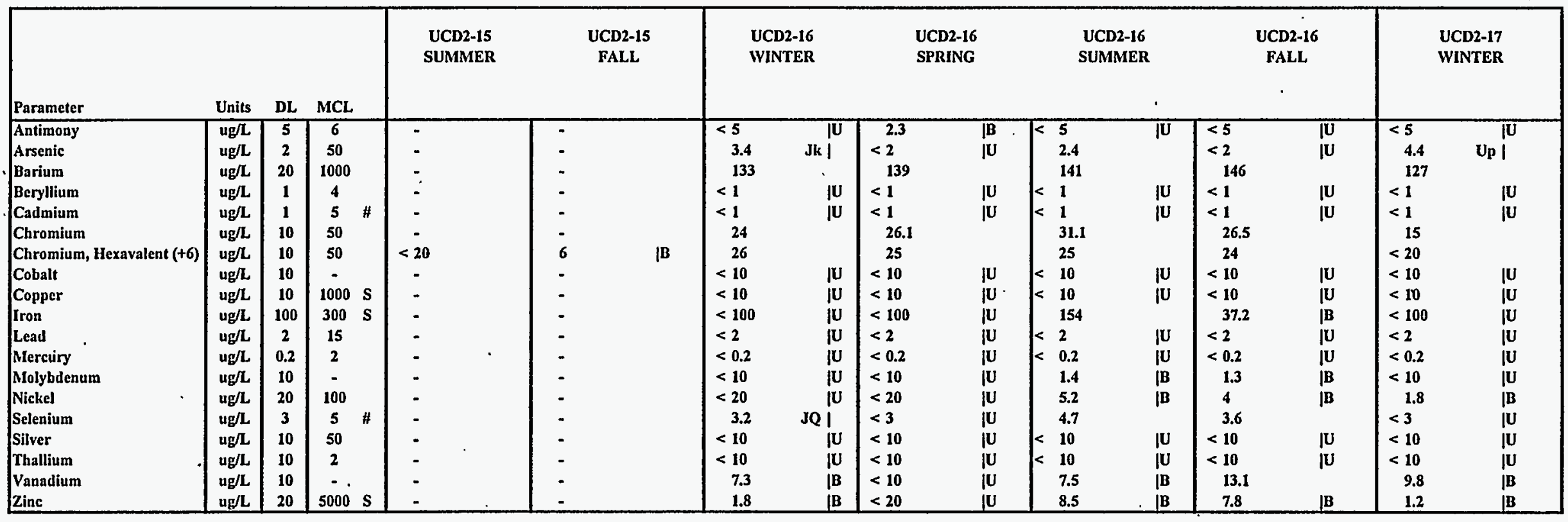


TABLE A.4

ANALYTICAL RESULTS FOR GROUNDWATER 1995

METALS

1995 ANNUAL WATER MONITORING REPORT

LEHR ENVIRONMENTAL RESTORATION, DAVIS, CALIFORNIA

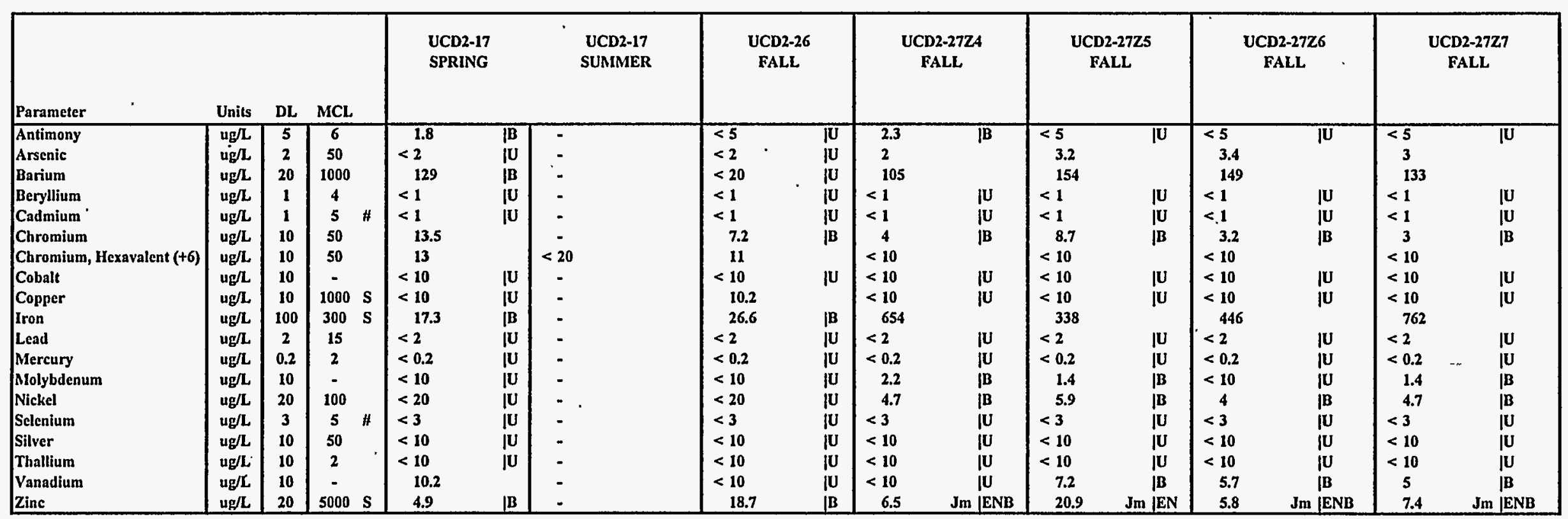


TABLE A.4

ANALYTICAL RESULTS FOR GROUNDWATER 1995

METALS

1995 ANNUAL WATER MONITORING REPORT

LEHR ENVIRONMENTAL RESTORATION, DAVIS, CALIFORNIA

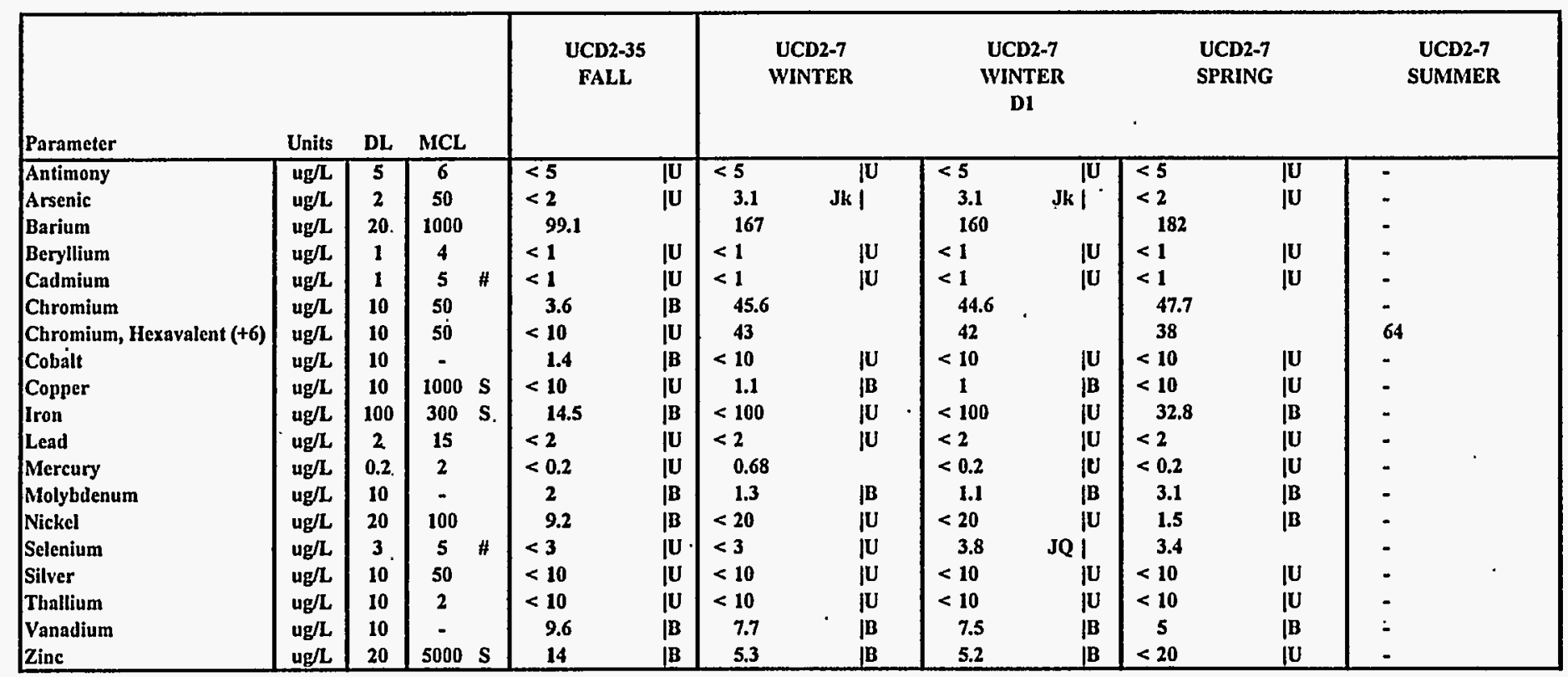


TABLE A.4

ANALYTICAL RESULTS FOR GROUNDWATER 1995

METALS

1995 ANNUAL WATER MONITORING REPORT

LEHR ENVIRONMENTAL RESTORATION, DAVIS, CALIFORNIA

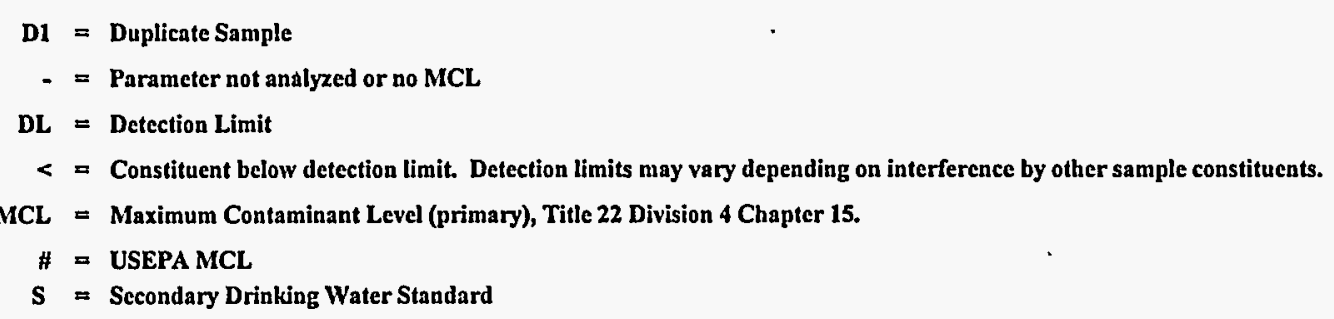

Sec Appendix A for explanation of data qualifiers. 
TABLE A.5

ANALYTICAL RESULTS FOR GROUNDWATER, 1995

RADIONUCLIDES

1995 WATER MONITORING REPORT

LEHR ENVIRONMENTAL RESTORATION, DAVIS, CALIFORNIA

\begin{tabular}{|c|c|c|c|c|c|c|c|c|c|c|c|c|c|c|c|c|c|c|}
\hline \multirow{2}{*}{ PARAMETER } & \multirow[t]{2}{*}{ METHOD } & \multirow[t]{2}{*}{$\begin{array}{l}\mathrm{MCL} \\
\mathrm{pCi} / \mathrm{L}\end{array}$} & \multicolumn{4}{|c|}{$\begin{array}{c}\text { UCD1-1 } \\
\text { WINTER }\end{array}$} & \multicolumn{4}{|c|}{$\begin{array}{l}\text { UCD1-1 } \\
\text { SPRING }\end{array}$} & \multicolumn{4}{|c|}{$\begin{array}{c}\text { UCD1-I } \\
\text { SPRING } \\
\text { D1 }\end{array}$} & \multicolumn{4}{|c|}{$\begin{array}{r}\text { UCD1-10 } \\
\text { WINTER }\end{array}$} \\
\hline & & & Value & & Error & MDA & Value & & Error & MDA & Value & & Error & MDA & Value & & Error & MDA \\
\hline Actinium-228 & 901.1 & - & 18 & & 22 & 30 & -6.1 & & 6.3 & 15 & 20 & & 22 & 32 & 1 & & 21 & 37 \\
\hline Bismuth-212 & 901.1 & - & 5 & & 43 & 60 & 5 & & 18 & 25 & 42 & & 42 & 43 & 37 & & 52 & 67 \\
\hline Bismuth-214 & 901.1 & - & 48 & & 18 & 20 & 12.8 & & 6.1 & 8.2 & 9 & & 14 & 19 & 8 & & 13 & 18 \\
\hline Cesium-134 & 901.1 & - & - & & - & - & - & & - & - & - & & - & - & - & & - & - \\
\hline Cesium-137 & 901.1 & - & 0.4 & & 4.9 & 8.9 & 0.4 & & 2.1 & 2.7 & 0 & & 0 & 8.6 & -5.4 & & 3.5 & 11 \\
\hline Cobalt-57 & 901.1 & - & - & & - & - & - & & - & - & - & & - & - & - & & - & - \\
\hline Cobalt-60 & $901 . i$ & - & 2.3 & & 3.4 & 9.3 & 0.8 & & 1.6 & 2.6 & -2.4 & & 2.9 & 11 & -0.3 & & 2.7 & 11 \\
\hline Lead-210 & 901.1 & - & -40 & & 110 & 170 & 0 & & 290 & 420 & 10 & & 110 & 170 & -10 & & 110 & 180 \\
\hline Lead-212 & $901 . i$ & - & 11 & & 10 & 14 & -4.5 & & 5 & 8.1 & 4.4 & & 9.8 & 14 & 0.7 & & 9.5 & 14 \\
\hline Lead-214 & 901.1 & $\because$ & 23 & & 14 & 21 & 12.2 & $\cdot$ & 5.7 & 7.9 & 16 & & 12 & 16 & 1 & & 10 & 18 \\
\hline Potassium-40 & 901.1 & - & 19 & & 73 & 110 & -8 & & 26 & 46 & -14 & & 67 & 110 & -5 & & 63 & 110 \\
\hline Ra-223 & 901.1 & - & - & & - & - & - & & - & - & - & & - & - & - & & - & - \\
\hline Radium-226 & 901.1 & $\mathbf{5}$ & -490 & & 130 & 200 & 43 & . & 60 & 85 & -70 & & 120 & 180 & -110 & & 110 & 180 \\
\hline Thallium-208 & 901.1 & - & 3.6 & & 7.6 & 11 & 0.6 & & 2.8 & 4.2 & -1 & & 6.7 & 10 & -5.8 & & 3.1 & 11 \\
\hline Thorium-234 & 901.1 & - & -21 & & 68 & 160 & -8 & & 46 & 150 & 24 & & 66 & 150 & 18 & & 67 & 150 \\
\hline Uranium-235 & 901.1 & - & -13 & & 15 & 44 & -5 & & 13 & 24. & -4 & & 27 & 42 & -2 & & 27 & 42 \\
\hline Radium-226 & $903.1 \mathrm{M}$ & 5 & 0.17 & & 0.18 & 0.29 & 0.3 & & 0.13 & 0.1 & 0.15 & & 0.11 & 0.14 & 0.019 & & 0.083 & 0.18 \\
\hline Strontium-90 & 905 & 8 & -0.3 & & 1.1 & 1 & 0.66 & & 0.51 & 0.84 & 0.7 & & 0.47 & 0.76 & -0.7 & & 3 & 1.5 \\
\hline Tritium & 906 & 20000 * & -220 & & 150 & 260 & -10 & & 220 & 260 & -50 & & 210 & 260 & 0 & . & 220 & 300 \\
\hline Gross Alpha & 9310 & 15 & 1.1 & IC & 2.5 & 4.8 & 0.1 & IC & 3.8 & 8.5 & 0.4 & |C & 3.8 & 8.4 & 1.9 & IC & 5.8 & 12 \\
\hline Gross Beta & 9310 & 50 & 7 & $\mathbf{J d} \mid \mathbf{C}$ & 3.7 & 5.8 & 2.2 & IC & 5.4 & 9.4 & -3.1 & IC & 5.1 & 9.4 & -2.8 & IC & 6.9 & 13 \\
\hline Americium-241 & LAS108 & - & -0.001 & $\mathbf{J m} \mid$ & 0.029 & 0.048 & -0.006 & & 0.044 & 0.071 & -0.049 & & 0.066 & 0.11 & -0.002 & Jm I & 0.03 & 0.058 \\
\hline Plutonium-241 & LAS178 & - & -0.1 & & 1.3 & 1.6 & -0.5 & $\mathbf{J z} \mathbf{I}$ & 1.8 & 1.4 & 0.3 & $\mathbf{J z} \mid$ & 1.8 & 1.1 & -0.2 & & 1.5 & 1.7 \\
\hline Carbon-14 & LSC & - & -9 & & 82 & 110 & 3 & & 83 & 110 & -19 & & 81 & 110 & -14 & & 78 & 100 \\
\hline
\end{tabular}


TABLE A.5

ANALYTICAL RESULTS FOR GROUNDWATER, 1995

RADIONUCLIDES

1995 WATER MONITORING REPORT

LEHR ENVIRONMENTAL RESTORATION, DAVIS, CALIFORNIA

\begin{tabular}{|c|c|c|c|c|c|c|c|c|c|c|c|c|c|c|c|c|c|c|}
\hline \multirow[t]{2}{*}{ PARAMETER } & \multirow[t]{2}{*}{ METHOD } & \multirow[t]{2}{*}{$\begin{array}{l}\text { MCL } \\
\mathrm{pCi} / L\end{array}$} & \multicolumn{4}{|c|}{$\begin{array}{l}\text { UCDI-10 } \\
\text { SPRING }\end{array}$} & \multicolumn{4}{|c|}{$\begin{array}{c}\text { UCD1-11 } \\
\text { WINTER }\end{array}$} & \multicolumn{4}{|c|}{$\begin{array}{l}\text { UCDI-11 } \\
\text { SPRING }\end{array}$} & \multicolumn{4}{|c|}{$\begin{array}{l}\text { UCD1-12 } \\
\text { WINTER }\end{array}$} \\
\hline & & & Value & & Error & MDA & Value & & Error & MDA & Value & & Error & MIDA & Value & & Error & MDA \\
\hline Actinium-228 & 901.1 & - & 2 & & 21 & 38 & 6 & & 21 & 37 & -9 & & 12 & 39 & 4.9 & & 8 & 14 \\
\hline Bismuth-212 & 901.1 & - & -21 & & 27 & 79 & 5 & & 35 & 58 & -43 & & 30 & 87 & -5 & & 17 & 26 \\
\hline Bismuth-214 & 901.1 & - & 19 & " & 14 & 18 & -2 & & 13 & 20 & 25 & & 16 & 19 & 17.5 & & 6.5 & 7.5 \\
\hline Cesium-134 & 901.1 & - & - & & - & - & - & & - & - & - & & - & - & - & & - & $\because$ \\
\hline Cesium-137 & 901.1 & - & 2.9 & & 5.9 & 7.7 & 0.5 & & 6.3 & 8.5 & -0.3 & & 6.3 & 9.1 & -0.1 & & 1.6 & 2.9 \\
\hline Cobalt-57 & 901.1 & - & - & & - & - & - & & - & - & - & & - & - & - & & - & - \\
\hline Cobalt-60 & 901.1 & - & -2.5 & & 1.5 & 9.8 & 1.2 & & 3.4 & 8.2 & -0.7 & & 4.9 & 11 & -0.33 & & 0.92 & 3.3 \\
\hline Lcad-210 & 901.1 & - & 10 & & 110 & 170 & -50 & & 100 & 170 & -20 & & 110 & 170 & -70 & & 290 & 420 \\
\hline Lead-212 & 901.1 & - & 6.2 & - & 9.7 & 14 & 7 & & 9.3 & 13 & 3.7 & & 9.8 & 14 & 8.5 & & 6 & 8.4 \\
\hline Lead-214 & 901.1 & - & 21 & & 12 & 16 & -11.4 & & 8.2 & 18 & 33 & & 13 & 16 & 12.3 & & 5.7 & 8.4 \\
\hline Potassium-40 & 901.1 & - & -13 & & 63 & 110 & 33 & & 74 & 110 & 3 & & 59 & 97 & -5 & & 26 & 44 \\
\hline Ra-223 & 901.1 & - & - & & - & - & - & & - & - & - & & - & - & - & & - & - \\
\hline Radium-226 & 901.1 & 5 & 40 & & 120 & 180 & -280 & & 120 & 180 & 80 & & 120 & 180 & -27 & & 57 & 84 \\
\hline Thallium-208 & 901.1 & - & -1.8 & · & 7.1 & 11 & -1.1 & & 6 & 9 & -0.7 & & 7 & 11 & 4 & & 3.1 & 4.2 \\
\hline Thorium-234 & 901.1 & - & -106 & & 67 & 160 & -37 & & 65 & 160 & 5 & & 67 & 160 & 6 & & 48 & 150 \\
\hline Uranium-235 & 901.1 & - & -8 & & 27 & 42 & 26 & & 26 & 35 & -4 & & 26 & 40 & -16 & & 9.2 & 26 \\
\hline Radium-226 & 903.1M & 5 & 0.24 & & 0.15 & 0.18 & 0.19 & & 0.18 & 0.28 & 0.093 & & 0.086 & 0.12 & 0.26 & & 0.19 & 0.099 \\
\hline Strontium-90 & 905 & 8 & 0.7 & & 0.47 & 0.77 & -0.9 & & 3.3 & 1.6 & 0.12 & & 0.46 & 0.79 & -0.9 & & 2.9 & 1.4 \\
\hline Tritium & 906 & 20000 & 30 & & 220 & 260 & -140 & & 200 & 310 & -40 & & 220 & 260 & -100 & & 170 & 260 \\
\hline Gross Alpha & 9310 & 15 & 0 & IC & 6 & 13 & 2.7 & |C & 5.2 & 9.4 & 0.8 & |C & 3.7 & 7.5 & 2.4 & |C & 8.4 & 17 \\
\hline Gross Beta & 9310 & 50 & 1.1 & ic & 6.8 & 12 & 1.4 & jc & 4.6 & 8 & 4.6 & jc & 5.3 & 8.8 & 6.4 & Jd $\mid C$ & 9.5 & 16 \\
\hline Americium-241 & LAS108 & - & 0.106 & & 0.062 & 0.069 & 0.006 & $\mathbf{J m} \mid$ & 0.058 & 0.099 & -0.004 & & 0.053 & 0.081 & 0.007 & $\mathbf{J m}$ & 0.039 & 0.063 \\
\hline Plutonium-241 & LAS178 & - & 1 & $\mathbf{J z} \mid$ & 1.8 & 1.2 & -0.9 & & 1.6 & 2.2 & 0 & $\mathbf{J z} \mid$ & 1.8 & 1.3 & -0.2 & & 1.4 & 1.9 \\
\hline Carbon-14 & LSC & - & -5 & & 82 & 110 & 2 & & 80 & 110 & 14 & & 84 & 110 & 119 & & 93 & 110 \\
\hline
\end{tabular}


TABLE A.5

ANALYTICAL RESULTS FOR GROUNDWATER, 1995

RADIONUCLIDES

1995 WATER MONITORING REPORT

LEHR ENVIRONMENTAL RESTORATION, DAVIS, CALIFORNIA

\begin{tabular}{|c|c|c|c|c|c|c|c|c|c|c|c|c|c|c|c|c|c|c|}
\hline \multirow{2}{*}{ PARAMETER } & \multirow{2}{*}{ METHOD } & \multirow{2}{*}{$\begin{array}{l}\text { MCL } \\
\mathrm{pCi} / \mathrm{L}\end{array}$} & \multicolumn{4}{|c|}{$\begin{array}{l}\text { UCD1-12 } \\
\text { SPRING }\end{array}$} & \multicolumn{4}{|c|}{$\begin{array}{l}\text { UCD1-12 } \\
\text { SUMMER }\end{array}$} & \multicolumn{4}{|c|}{$\begin{array}{l}\text { UCD1-12 } \\
\text { FALL }\end{array}$} & \multicolumn{4}{|c|}{$\begin{array}{l}\text { UCD1-13 } \\
\text { WINTER }\end{array}$} \\
\hline & & & Value & & Error & MDA & Value & & Error & MDA & Value & & Error & MDA & Value & & Error & MDA \\
\hline Actinium-228 & 901.1 & - & 7.2 & & 7.6 & 13 & -15 & & 12 & 42 & 7.9 & & 7.6 & 13 & 0.1 & & 7.8 & 14 \\
\hline Bismuth-212 & 901.1 & - & 3 & & 16 & 22 & - & & - & - & 3 & & 17 & 23 & 9 & & 18 & 25 \\
\hline Bismuth-214 & 901.1 & - & 12.3 & & 5.7 & 7.5 & 5 & & 12 & 18 & 14.4 & & 6.4 & 8.5 & 20.2 & & 6.9 & 8.1 \\
\hline Cesium-134 & 901.1 & - & - & & - & - & -1.7 & & 2.1 & 7.2 & - & & - & - & - & & - & - \\
\hline Cesium-137 & 901.1 & - & -1.2 & & 1.2 & 3.5 & 2.7 & & 5 & 7.8 & 0.4 & & 1.7 & 2.8 & -1.2 & & 1.9 & 3.6 \\
\hline Cobalt-57 & 901.1 & - & - & & - & - & 1.6 & & 3.4 & 4.4 & - & & - & - & - & & - & - \\
\hline Cobalt-60 & 901.1 & - & -0.1 & & 1.4 & 2.9 & -1 & & 1.6 & 11 & -0.2 & & 1.6 & 3.5 & 0.9 & & 1.4 & 2.4 \\
\hline Lead-210 & 901.1 & - & 30 & & 280 & 390 & - & & - & - & 80 & & 290 & 410 & -100 & & 290 & 420 \\
\hline Lead-212 & 901.1 & - & -1.2 & & 5.1 & 7.9 & 3 & & 9.7 & 14 & 6.2 & & 5.4 & 7.6 & 0.6 & & 5.1 & 7.9 \\
\hline Lead-214 & 901.1 & - & 9.2 & & 5.4 & 7.6 & -2 & Je! & 10 & 18 & 7.2 & & 5.1 & 7.7 & 23.1 & & 6.4 & 8.2 \\
\hline Potassium-40 & 901.1 & - & -14 & & 27 & 48 & 8 & & 68 & 100 & 5 & & 27 & 44 & 14 & & 29 & 45 \\
\hline $\mathrm{Ra}-223$ & 901.1 & - & - & & - & - & - & & - & - & -8 & & 20 & 61 & - & & - & - \\
\hline Radium-226 & 901.1 & 5 & -154 & & 58 & 84 & - & & - & - & 24 & & 56 & 82 & 7 & & 58 & 87 \\
\hline Thallium-208 & 901.1 & - & 0.7 & & 2.8 & 4.3 & -1.5 & & 6.5 & 9.5 & -1.7 & & 2.8 & 4.5 & -1.4 & & 2.8 & 4.5 \\
\hline Thorium-234 & 901.1 & - & 12 & & 48 & 150 & -51 & & 64 & 150 & -11 & & 47 & 140 & -19 & & 47 & 150 \\
\hline Uranium-235 & 901.1 & - & 8 & & 15 & 23 & -11 & & 26 & 41 & -9 & & 12 & 23 & -10 & & 13 & .23 \\
\hline Radium-226 & $903.1 \mathrm{M}$ & 5 & 0.19 & & 0.12 & 0.14 & 0.12 & & 0.13 & 0.21 & 0.55 & & 0.29 & 0.25 & 0.22 & & 0.2 & 0.29 \\
\hline Strontium-90 & 905 & 8 & 0.32 & & 0.5 & 0.84 & 0.09 & & 0.47 & 0.8 & 0.84 & & 0.65 & 1.1 & -0.6 & & 1.5 & 0.99 \\
\hline Tritium & 906 & 20000 & -100 & & 210 & 250 & -30 & & 220 & 300 & -40 & & 170 & 240 & 17600 & & 1100 & 250 \\
\hline Gross Alpha & 9310 & 15 & -2.8 & IC & 4.9 & 14 & 11 & IC & 11 & 17 & 6 & IC & 10 & 17 & 2.7 & |C & 5.7 & 11 \\
\hline Gross Beta & 9310 & 50 & 4.6 & jC & 7.4 & 13 & 0 & ic & 10 & 18 & 1.9 & ic & 6.6 & 12 & 15 & $\mathrm{Jd} \mid \mathrm{C}$ & 8 & 12 \\
\hline Americium-241 & LAS108 & - & -0.014 & & 0.049 & 0.077 & - & & - & - & - & & - & - & -0.026 & $\mathbf{J m} \mathbf{i}$ & 0.03 & 0.071 \\
\hline Plutonium-241 & LAS178 & - & -0.4 & $\mathbf{J z} \mid$ & 1.8 & 1.1 & - & & - & - & - & & - & - & 0.2 & & 1.8 & 2.8 \\
\hline Carbon-14 & LSC & - & 199 & & 98 & 110 & 208 & & 95 & $\cdot 100$ & 171 & & 96 & 100 & 1169 & $\mathrm{Jd} /$ & 92 & 41 \\
\hline
\end{tabular}


TABLE A.5

ANALYTICAL RESULTS FOR GROUNDWATER, 1995

RADIONUCLIDES

1995 WATER MONITORING REPORT

LEHR ENVIRONMENTAL RESTORATION, DAVIS, CALIFORNIA

\begin{tabular}{|c|c|c|c|c|c|c|c|c|c|c|c|c|c|c|c|c|c|c|}
\hline \multirow[t]{2}{*}{ PARAMETER } & \multirow[t]{2}{*}{ METHOD } & \multirow[t]{2}{*}{$\begin{array}{l}\text { MCL } \\
\mathrm{pCi} / \mathrm{L}\end{array}$} & \multicolumn{4}{|c|}{$\begin{array}{l}\text { UCD1-13 } \\
\text { SPRING }\end{array}$} & \multicolumn{4}{|c|}{$\begin{array}{c}\text { UCD1-13 } \\
\text { SUMMER }\end{array}$} & \multicolumn{4}{|c|}{$\begin{array}{c}\text { UCD1-13 } \\
\text { FALL }\end{array}$} & \multicolumn{4}{|c|}{$\begin{array}{c}\text { UCD1-13 } \\
\text { FALL } \\
\text { D1 }\end{array}$} \\
\hline & & & Value & & Error & MDA & Value & & Error & MDA & Value & & Error & MDA & Value & & Error & MDA \\
\hline Actinium-228 & 901.1 & $=$ & 0.1 & & 7.7 & 14 & -4 & & 21 & 40 & -1.1 & & 7.2 & 12 & 4.7 & & 7.4 & 13 \\
\hline Bismuth-212 & 901.1 & - & -3 & & 18 & 26 & - & & - & - & -1 & & 15 & 21 & 9 & & 18 & 23 \\
\hline Bismuth-214 & 901.1 & - & 14.2 & & 6.2 & 8.2 & 4 & & 13 & 19 & 2.1 & & 5.2 & 7.7 & 8.2 & & 5.5 & 7.6 \\
\hline Cesium-134 & 901.1 & - & - & & - & - & -3 & & 3.5 & 6.2 & - & & - & - & - & & - & $:$ \\
\hline Cesium-137 & 901.1 & - & -0.9 & & 1.2 & 3.5 & 2.4 & & 4.8 & 7.6 & -1 & . & 1.2 & 3.5 & -1.9 & & 1.4 & 4 \\
\hline Cobalt-57 & 901.1 & - & - & & - & - & 0.6 & & 3.3 & 4.3 & - & & - & - & - & & - & - \\
\hline Cobalt-60 & 901.1 & - & -0.93 & & 0.82 & 2.8 & 4.1 & & 4.6 & 7.5 & 1.2 & & 1.6 & 2.7 & -0.95 & & 0.85 & 3.3 \\
\hline Lead-210 & 901.1 & - & -130 & & 300 & 450 & - & & - & - & -60 & & 270 & 400 & -130 & & 280 & 420 \\
\hline Lead-212 & 901.1 & - & 5.5 & & 5.4 & 7.8 & 3 & - & 10 & 15 & 1.4 & & 4.8 & 7.2 & 0.2 & & 5.2 & 7.8 \\
\hline Lead-214 & 901.1 & - & 11.5 & & 5.4 & 7.5 & -1 & Je I & 11 & 18 & 1.7 . & & 4.7 & 7.5 & 5.8 & & 5.3 & 8.1 \\
\hline Potassium-40 & 901.1 & - & -2 & & 26 & 45 & 3 & & 66 & 100 & -15 & & 26 & 47 & 0 & & 25 & 43 \\
\hline Ra-223 & 901.1 & - & - & & - & - & - & & - & - & -13 & & 14 & 64 & -19 & & 14 & 56 \\
\hline Radium-226 & 901.1 & 5 & 19 & & 54 & 79 & - & & $\cdot$ & - & -173 & & 58 & 84 & 6 & & 56 & 83 \\
\hline Thallium-208 & 901.1 & - & 2.3 & & 2.8 & 4 & 5.3 & & 6.6 & 8.3 & 0.6 & . & 2.6 & 3.9 & 0 & & 2.7 & 4.2 \\
\hline Thorium-234 & 901.1 & - & 30 & & 47 & 130 & -24 & & 98 & 150 & -12 & & 48 & 140 & -8 & & 47 & 140 \\
\hline Uranium-235 & 901.1 & - & -2 & & 15 & 24 & -21 & & 13 & 42 & 7 & & 15 & 21 & -2 & & 12 & 22 \\
\hline Radium-226 & 903.1M & 5 & 0.23 & & 0.14 & 0.17 & 0.1 & & 0.15 & 0.26 & 0.25 & & 0.13 & 0.16 & 0.012 & & 0.068 & 0.14 \\
\hline Strontium-90 & 905 & 8 & 0.26 & & 0.47 & 0.81 & -0.17 & & 0.53 & 0.92 & -0.19 & & 0.57 & 1 & 0.4 & & 0.64 & 1.1 \\
\hline Tritium & 906 & 20000 * & a & & - & - & 19600 & . & 1200 & 300 & 16480 & & 960 & 210 & 15190 & & 910 & 210 \\
\hline Gross Alpha & 9310 & 15 & 3.4 & |C & 5.4 & 9.5 & 4.4 & |C & 7.6 & 13 & 0.8 & IC & 5.3 & 11 & 3.6 & IC & 6.6 & 12 \\
\hline Gross Beta & 9310 & 50 & 4 & jc & 6.6 & 11 & -3.8 & jc & 6.5 & 12 & 1.3 & jc & 4.1 & 7.1 & 3.8 & ic & 5 & 8.3 \\
\hline Americium-241 & LAS108 & . & 0.032 & & 0.058 & 0.081 & - & & - & - & - & & - & - & - & & . & $\because$ \\
\hline Plutonium-241 & LAS178 & . - & 0.1 & $\mathrm{Jz}_{1}$ & 1.9 & 1.6 & - & & . & - & - & & - & - & - & & - & - \\
\hline Carbon-14 & LSC & - & 900 & & 150 & 130 & 1750 & & 180 & 100 & 1900 & & 180 & 92 & 1770 & & 170 & 92 \\
\hline
\end{tabular}


TABLE A.5

ANALYTICAL RESULTS FOR GROUNDWATER, 1995

RADIONUCLIDES

1995 WATER MONITORING REPORT

LEHR ENVIRONMENTAL RESTORATION, DAVIS, CALIFORNIA

\begin{tabular}{|c|c|c|c|c|c|c|c|c|c|c|c|c|c|c|c|c|c|c|}
\hline \multirow{2}{*}{ PARAMETER } & \multirow{2}{*}{ METHOD } & \multirow{2}{*}{$\begin{array}{l}\text { MCL } \\
\text { pCi/L }\end{array}$} & \multicolumn{4}{|c|}{$\begin{array}{l}\text { UCDI-18 } \\
\text { WINTER }\end{array}$} & \multicolumn{4}{|c|}{$\begin{array}{c}\text { UCD1-18 } \\
\text { WINTER } \\
\text { D1 }\end{array}$} & \multicolumn{4}{|c|}{$\begin{array}{l}\text { UCDI-18 } \\
\text { SPRING }\end{array}$} & \multicolumn{4}{|c|}{$\begin{array}{l}\text { UCD1-19 } \\
\text { WINTER }\end{array}$} \\
\hline & & & Value & & Error & MDA & Value & & Error & MDA & Value & & Error & MDA & Value & & Error & MDA \\
\hline Actinium-228 & 901.1 & - & -19 & & 10 & 42 & -9.2 & & 6.7 & 36 & 6 & & 21 & 36 & -2.2 & & 7.6 & 14 \\
\hline Bismuth-212 & 901.1 & - & -4 & & 45 & 66 & -7 & & 50 & 74 & 9 & & $49^{\circ}$ & 68 & 14 & & 18 & 22 \\
\hline Bismuth-214 & 901.1 & - & 30 & & 16 & 21 & 9 & & 14 & 21 & 11 & & 13 & 19 & 0.2 & & 5.2 & 8.3 \\
\hline Cesium-134 & 901.1 & - & - & & - & - & - & & - & - & - & & - & - & - & & - & - \\
\hline Cesium-137 & 901.1 & - & 2.5 & & 5.2 & 8.9 & -2.3 & & 4.4 & 8.6 & -1.6 & & 4.9 & 9.2 & -1.3 & & 1.1 & 3.3 \\
\hline Cobalt-57 & 901.1 & - & - & & - & - & - & & - & - & - & & - & - & - & . & - & - \\
\hline Cobalt-60 & 901.1 & - & -3.2 & & 3 & 12 & 3.5 & & 4.1 & 9.6 & -0.1 & & 1.8 & 9.2 & -0.3 & & 1.3 & 3.5 \\
\hline Lead-210 & 901.1 & - & -40 & & 100 & 170 & 10 & & 100 & 160 & -40 & & 110 & 170 & 60 & & 290 & 410 \\
\hline Lead-212 & 901.1 & - & 2 & & 10 & 15 & 9 & & 10 & 14 & 1.8 & & 9.6 & 14 & 9 & & 5.3 & 7.1 \\
\hline Lead-214 & 901.1 & - & 18 & & 13 & 20 & 12 & & 12 & 18 & 5 & & 12 & 19 & 2.3 & & 4.7 & 7.4 \\
\hline Potassium-40 & 901.1 & - & 3 & & 62 & 100 & -8 & & 68 & 110 & -26 & & 76 & 130 & 6 & & 26 & 42 \\
\hline $\mathrm{Ra}-223$ & 901.1 & - & - & & - & - & - & & - & - & - & & - & - & - & & - & - \\
\hline Radium-226 & 901.1 & 5 & -140 & & 120 & 190 & -350 & & 120 & 180 & 30 & & 120 & 180 & -133 & & 57 & 85 \\
\hline Thallium-208 & 901.1 & - & 3 & & 7 & 9.9 & -0.2 & & 7.1 & 11 & 2.4 & & 7.1 & 10 & -0.8 & & 2.6 & 4.1 \\
\hline Thorium-234 & 901.1 & - & -41 & & 69 & 170 & -3 & & 66 & 150 & 8 & & 66 & 160 & 33 & & 47 & 140 \\
\hline Uranium-235 & 901.1 & - & 7 & & 27 & 41 & 10 & & 27 & 41 & -16 & & 15 & 42 & 11 & & 15 & 20 \\
\hline Radium-226 & $903.1 \mathrm{M}$ & 5 & 0.33 & & 0.23 & 0.24 & 0.4 & & 0.25 & 0.28 & 0.27 & & 0.15 & 0.056 & 0.18 & & 0.13 & 0.15 \\
\hline Strontium-90 & 905 & 8 & -0.6 & & 2.7 & 1.6 & -0.4 & & 2.1 & 1.4 & 0.52 & $\mathrm{Jm} \mid$ & 0.52 & 0.86 & -0.8 & & 3.5 & 1.7 \\
\hline Tritium & 906 & 20000 & 90 & & 190 & 260 & -270 & & 140 & 270 & -130 & & 160 & 220 & -110 & & 200 & 310 \\
\hline Gross Alpha & 9310 & 15 & 0.1 & IC & 3.3 & 6.9 & -1 & IC & 2.7 & 6.2 & 2.1 & $\mathrm{Jm} \mid \mathrm{C}$ & 3.1 & 5.4 & 5.7 & IC & 6.9 & 11 \\
\hline Gross Beta & 9310 & $\mathbf{5 0}$ & 3 & $\mathrm{Jd} \mathbf{j C}$ & 3.5 & 5.9 & 0.9 & Jd $\mid \mathbf{C}$ & 3.5 & 6 & 1.3 & ic & 3.3 & 5.6 & 4.3 & ic & 6.8 & 11 \\
\hline Americium-241 & LAS108 & - & 0.015 & $\mathrm{Jm} \mid$ & 0.084 & 0.14 & 0.002 & $\mathbf{J m} \mathbf{i}$ & 0.052 & 0.085 & 0.004 & & 0.014 & 0.031 & -0.019 & $\mathrm{Jm} \mathbf{i}$ & 0.05 & 0.087 \\
\hline Plutonium-241 & LAS178 & - & -0.7 & & 1.6 & 1.9 & 0 & & 1.3 & 1.6 & -0.1 & Jc.l & 2.3 & 3 & 0.1 & & 1.6 & 2 \\
\hline Carbon-14 & LSC & - & 42 & & 87 & 110 & -1 & & 83 & 110 & -1 & & 84 & 110 & -41 & & 77 & 110 \\
\hline
\end{tabular}


TABLE A.5

ANALYTICAL RESULTS FOR GROUNDWATER, 1995

RADIONUCLIDES

1995 WATER MONITORINंG REPORT

LEHR ENVIRONMENTAL RESTORATION, DAVIS, CALIFORNIA

\begin{tabular}{|c|c|c|c|c|c|c|c|c|c|c|c|c|c|c|c|c|c|c|}
\hline \multirow{2}{*}{ PARAMETER } & \multirow{2}{*}{ METHOD } & \multirow{2}{*}{$\begin{array}{l}\text { MCL } \\
\mathrm{pCi} / \mathrm{L}\end{array}$} & \multicolumn{4}{|c|}{$\begin{array}{l}\text { UCDI-19 } \\
\text { SPRING }\end{array}$} & \multicolumn{4}{|c|}{$\begin{array}{c}\text { UCD1-19 } \\
\text { SPRING } \\
\text { D1 }\end{array}$} & \multicolumn{4}{|c|}{$\begin{array}{l}\text { UCD1-20 } \\
\text { WINTER }\end{array}$} & \multicolumn{4}{|c|}{$\begin{array}{l}\text { UCD1-20 } \\
\text { SPRING }\end{array}$} \\
\hline & & & Valuc & & Error & MDA & Value & & Error & MDA & Value & & Error & $\overline{\text { MDA }}$ & Value & & Error & MDA \\
\hline Actinium-228 & 901.1 & - & 5.9 & & 7.7 & 14 & -3.2 & & 7.5 & 15 & -20 & & 130 & 220 & 7 & & 22 & 35 \\
\hline Bismuth-212 & 901.1 & - & -2 & & 16 & 24 & 6 & & 17 & 23 & -280 & & 230 & 430 & 0 & & 46 & 64 \\
\hline Bismuth-214 & 901.1 & - & 21.9 & & 6.8 & 8.1 & 19 & & 6.7 & 7.9 & -59 & & 54 & 110 & 26 & & 16 & 21 \\
\hline Cesium-134 & 901.1 & - & - & & - & - & - & & - & - & - & & - & - & - & & - & - \\
\hline Cesium-137 & 901.1 & - & -0.1 & & 2.3 & 3.2 & -0.3 & & 1.1 & 3.2 & 7 & & 41 & 52 & -1.5 & & 4.8 & 8.9 \\
\hline Cobalt-57 & 901.1 & - & - & . & - & - & - & & - & - & - & & - & - & - & & - & - \\
\hline Cobalt-60 & 901.1 & - & -0.1 & & 1.4 & 2.9 & -0.9 & & 1.1 & 3.8 & -11 & & 15 & 46 & -4 & & 3.5 & 11 \\
\hline Lead-210 & 901.1 & - & 100 & & 300 & 420 & 0 & & 280 & 400 & -400 & & 1300 & 2000 & -70 & & 100 & 170 \\
\hline Lead-212 & 901.1 & - & 1.2 & & 5.1 & 7.7 & -4.4 & & 5.2 & 8.3 & -14 & & 54 & 76 & -12 & & 9.5 & 15 \\
\hline Lead-214 & 901.1 & - & 24 & & 6.4 & 7.9 & 19.5 & & 6.2 & 7.7 & -67 & & 46 & 97 & 31 & & 13 & 17 \\
\hline Potassium-40 & 901.1 & - & 8 & & 28 & 45 & 11 & & 28 & 44 & -110 & & 400 & 620 & 51 & & 74 & 100 \\
\hline $\mathrm{Ra}-223$ & 901.1 & - & - & & - & - & - & & - & - & - & & - & - & - & & - & - \\
\hline Radium-226 & 901.1 & 5 & 0 & & 57 & 83 & 54 & & 57 & 80 & -580 & & 630 & 930 & -270 & & 120 & 180 \\
\hline Thallium-208 & 901.1 & - & 1.7 & & 3 & 4.4 & 0.9 & & 2.8 & 4.1 & 0 & & 42 & 57 & 0.4 & & 6.7 & 9.8 \\
\hline Thorium-234 & 901.1 & - & 8 & & 48 & 150 & 8 & & 49 & 150 & -260 & & 340 & 750 & -18 & & 65 & 160 \\
\hline Uranium-235 & 901.1 & - & 0 & & 16 & 24 & -3 & & 15 & 24 & 40 & & 150 & 210 & 14 & & 26 & 38 \\
\hline Radium-226 & 903.1M & 5 & 0.069 & & 0.1 & 0.18 & 0.112 & & 0.1 & 0.15 & 0.17 & & 0.11 & 0.051 & 0.103 & & 0.087 & 0.11 \\
\hline Strontium-90 & 905 & 8 & 0.75 & & 0.5 & 0.81 & 0.27 & & 0.46 & 0.79 & -0.6 & & 3 & 1.6 & 0.5 & & 0.48 & 0.8 \\
\hline Tritium & 906 & 20000 & -30 & & 220 & 260 & 20 & & 220 & 250 & -50 & & 220 & 310 & b & & - & - \\
\hline Gross Alpha & 9310 & 15 & 7.9 & |c & 6 & 8 & $\mathbf{0}$ & IC & 5 & 11 & 1.5 & IC & 2.3 & 3.9 & 1 & IC & 2.3 & 4.2 \\
\hline Gross Beta & 9310 & 50 & 3 & jc & 5.9 & 10 & $\mathbf{0}$ & ic & 6.1 & 11 & 0.8 & & 2.3 & 4 & 1.8 & iC & 2.6 & 4.4 \\
\hline Americium-241 & LAS108 & - & 0.01 & & 0.051 & 0.076 & 0.019 & & 0.053 & 0.078 & 0.018 & $\mathrm{Jm} \mid$ & 0.082 & 0.13 & -0.027 & & 0.041 & 0.07 \\
\hline Plutonium-241 & LAS178 & - & 0 & $\mathbf{J z}$ & 1.8 & 1.4 & 2.7 & $\mathbf{J z} \mid$ & 1.9 & 1.7 & -0.1 & & 1.6 & 2 & -0.3 & $\mathbf{J z} \mid$ & 1.9 & 1.9 \\
\hline Carbon-14 & LSC & - & -8 & & 82 & 110 & -12 & & 81 & 110 & 2 & & 80 & 110 & -12 & & 81 & 110 \\
\hline
\end{tabular}


TABLE A.5

ANALYTICAL RESULTS FOR GROUNDWATER, 1995

RADIONUCLIDES

1995 WATER MONITORING REPORT

LEHR ENVIRONMENTAL RESTORATION, DAVIS, CALIFORNIA

\begin{tabular}{|c|c|c|c|c|c|c|c|c|c|c|c|c|c|c|c|c|c|}
\hline \multirow{2}{*}{ PARAMETER } & \multirow{2}{*}{ METHOD } & \multirow{2}{*}{$\begin{array}{l}\text { MCL } \\
\text { pCi/L }\end{array}$} & \multicolumn{4}{|c|}{$\begin{array}{l}\text { UCD1-20 } \\
\text { SUMMER }\end{array}$} & \multicolumn{3}{|c|}{$\begin{array}{c}\text { UCDI-20 } \\
\text { FALL }\end{array}$} & \multicolumn{4}{|c|}{$\begin{array}{l}\text { UCD1-21 } \\
\text { WINTER }\end{array}$} & \multicolumn{4}{|c|}{$\begin{array}{l}\text { UCDI-21 } \\
\text { SPRING }\end{array}$} \\
\hline & & & Value & & Error & MDA & Value & Error & MDA & Value & & Error & $\overline{M D A}$ & Value & & Error & $\overline{\mathrm{MDA}}$ \\
\hline Actinium-228 & 901.1 & - & 2 & & 21 & 40 & - & - & - & -45 & & 46 & 230 & 0 & & 17 & 36 \\
\hline Bismuth-212 & 901.1 & - & - & & - & - & - & - & - & 0 & & 210 & 360 & -5 & & 42 & 64 \\
\hline Bismuth-214 & 901.1 & - & 3 & $\mathbf{J} \mathbf{z} \mid$ & 14 & 20 & - & - & - & 3 & & 78 & 110 & 33 & & 15 & 18 \\
\hline Cesium-134 & 901.1 & - & -0.9 & & 2.7 & 8.5 & - & - & - & - & & - & - & - & & - & - \\
\hline Cesium-137 & 901.1 & - & 2.1 & & 5.2 & 8.9 & - & - & - & 8 & & 43 & 54 & 4 & & 6.2 & 7.3 \\
\hline Cobalt-57 & 901.1 & - & -0.5 & & 2.6 & 4.6 & - & - & - & - & & - & - & - & & - & - \\
\hline Cobalt-60 & 901.1 & - & 2.4 & & 4.6 & 10 & - & - & - & 23 & & 20 & 36 & 3 & & 4.9 & 8.4 \\
\hline Lead-210 & 901.1 & - & - & & - & - & - & - & - & 0 & & 1300 & 1900 & -60 & & 100 & 170 \\
\hline Lead-212 & 901.1 & - & 2 & & 10 & 15 & - & - & - & -5 & & 56 & 77 & 5 & & 10 & 14 \\
\hline Lead-214 & 901.1 & - & 2 & $\mathbf{J z} \mid$ & 11 & 17 & - & - & - & -14 & & 63 & 99 & 7 & & 12 & 18 \\
\hline Potassium-40 & 901.1 & - & -21 & & 65 & 110 & - & - & - & -30 & & 390 & 590 & 10 & & 66 & 110 \\
\hline Ra-223 & 901.1 & - & - & & - & - & - & - & - & - & & - & - & - & & - & - \\
\hline Radium-226 & 901.1 & 5 & - & & - & - & - & - & - & -100 & & 650 & 950 & -290 & & 120 & 170 \\
\hline Thallium-208 & 901.1 & - & 0.8 & & 6.6 & 9.7 & - & - & - & -23 & & 40 & 58 & -5.5 & & 4 & 12 \\
\hline Thorium-234 & 901.1 & - & -28 & & 66 & 160 & - & - & - & -170 & & 340 & 770 & 15 & & 65 & 150 \\
\hline Uranium-235 & 901.1 & - & 9 & & 27 & 39 & - & - & - & -60 & & 150 & 230 & 15 & & 27 & 38 \\
\hline Radium-226 & 903.1M & 5 & 0.093 & & 0.086 & 0.11 & - & - & - & 0.14 & & 0.15 & 0.24 & 0.063 & & 0.092 & 0.15 \\
\hline Strontium-90 & 905 & 8 & -0.15 & & 0.26 & 0.47 & - & - & - & -1 & & 3.3 & 1.7 & 0.37 & $\mathbf{J m} \mid$ & 0.5 & 0.84 \\
\hline Tritium & 906 & 20000 * & -50 & & 220 & 290 & 70 & 170 & 220 & -100 & & 210 & 310 & 50 & & 180 & 220 \\
\hline Gross Alpha & 9310 & 15 & 0.6 & |C & 2.1 & 4 & - & - & - & -0.5 & |C & 4.6 & 11 & 0.3 & $\mathrm{Jm} \mid \mathrm{C}$ & 4 & 8.6 \\
\hline Gross Beta & 9310 & 50 & 0.7 & & 2.2 & 3.8 & - & - & - & 3.9 & iC & 6 & 10 & -1.5 & iC & 4.9 & 8.9 \\
\hline Americium-241 & LAS108 & - & - & & - & - & - & - & - & -0.09 & $\mathbf{J} \mathbf{m} \mid$ & 0.23 & 0.4 & 0.013 & & 0.029 & 0.048 \\
\hline Plutonium-241 & LAS178 & - & - & & . & - & - & - & - & 0.2 & & 1.8 & 2.4 & 1.7 & Je $\mid$ & 1.9 & 2.4 \\
\hline Carbon-14 & LSC & - & 102 & & 92 & 100 & - & - & - & -34 & & 77 & 100 & 35 & & 87 & 110 \\
\hline
\end{tabular}


TABLE A.5

ANALYTICAL RESULTS FOR GROUNDWATER, 1995

RADIONUCLIDES

1995 WATER MONITORING REPORT

LEHR ENVIRONMENTAL RESTORATION, DAVIS, CALIFORNIA

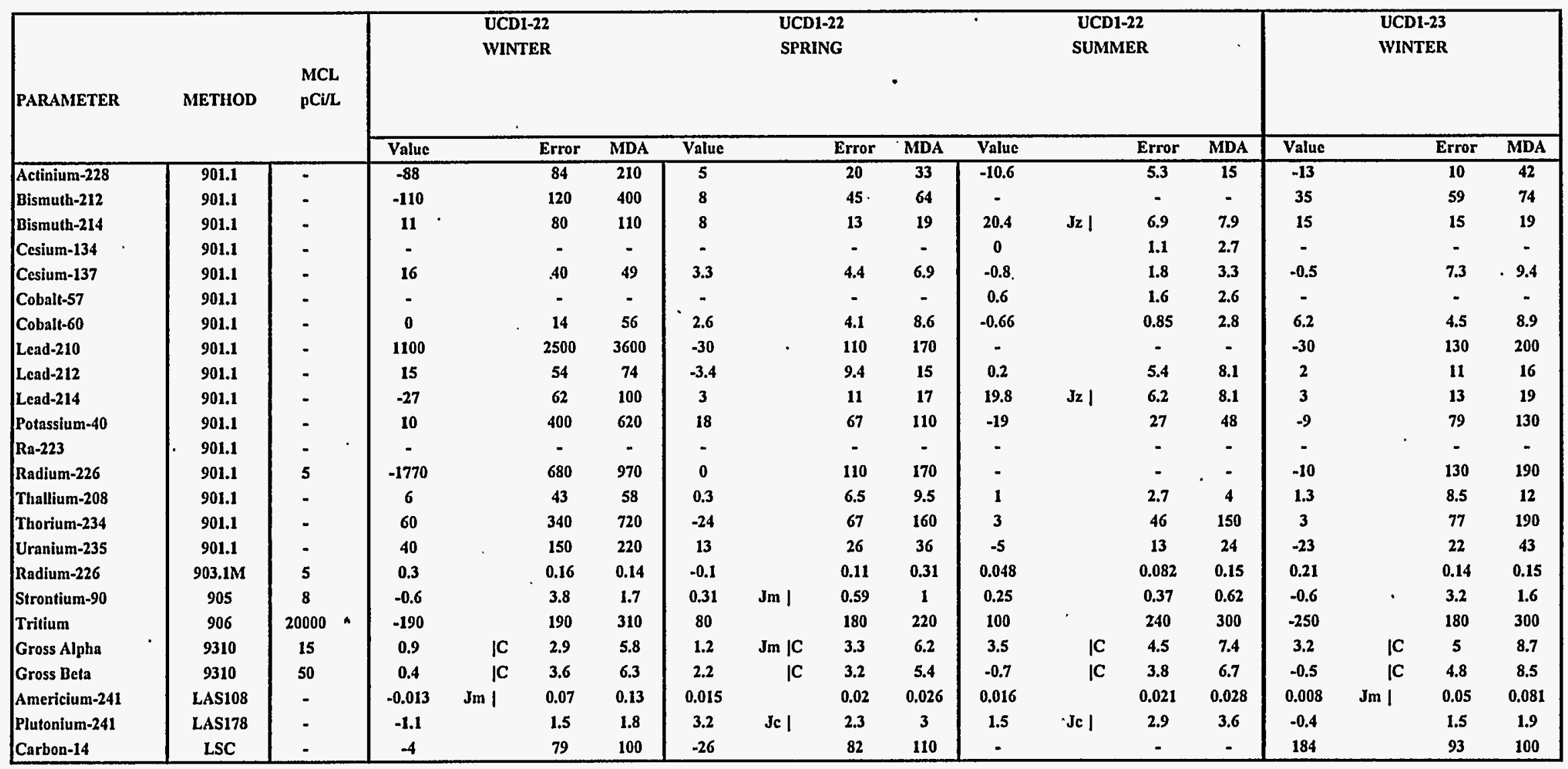


TABLE A.5

ANALYTICAL RESULTS FOR GROUNDWATER, 1995

RADIONUCLIDES

1995 WATER MONITORING REPORT

LEHR ENVIRONMENTAL RESTORATION, DAVIS, CALIFORNIA

\begin{tabular}{|c|c|c|c|c|c|c|c|c|c|c|c|c|c|c|c|c|c|c|}
\hline \multirow{2}{*}{ PARAMETER } & \multirow{2}{*}{ METHOD } & \multirow{2}{*}{$\begin{array}{l}\mathrm{MCL} \\
\mathrm{pCi} / \mathrm{L}\end{array}$} & \multicolumn{4}{|c|}{$\begin{array}{l}\text { UCD1-23 } \\
\text { SPRING }\end{array}$} & \multicolumn{4}{|c|}{$\begin{array}{r}\text { UCD1-23 } \\
\text { SUMMER }\end{array}$} & \multicolumn{4}{|c|}{$\begin{array}{l}\text { UCD1-24 } \\
\text { WINTER }\end{array}$} & \multicolumn{4}{|c|}{$\begin{array}{l}\text { UCD1-24 } \\
\text { SPRING }\end{array}$} \\
\hline & & & Value & & Error & MDA & Value & & Error & MDA & Value & & Error & MDA & Value & & Error & $\overline{\text { MDA }}$ \\
\hline Actinium-228 & 901.1 & - & 0.6 & & 7.7 & 14 & -3 & & 24 & 37 & -5 & & 22 & 40 & -11 & & 5 & 14 \\
\hline Bismuth-212 & 901.1 & - & 11 & & 16 & 19 & - & & - & - & 4 & & 47 & 67 & 0 & & 18 & 26 \\
\hline Bismuith-214 & 901.1 & - & 0.7 & & 5.1 & 7.8 & 11 & $\mathbf{J z}$ & 14 & 20 & -1 & & 13 & 20 & 8.5 & & 5.6 & 7.4 \\
\hline Cesium-134 & 901.1 & - &.- & & - & - & 1 & & 2.2 & 7.5 & - & & - & - & - & & - & - \\
\hline Cesium-137 & 901.1 & - & -0.2 & & 1.7 & 3.2 & 3.3 & & 5.2 & 8.7 & 2.1 & & 6.2 & 7.9 & -0.4 & & 1,7 & 3.1 \\
\hline Cobalt-57 & 901.1 & - & - & & - & - & 1.5 & & 2.4 & 4 & - & & - & - & - & & - & - \\
\hline Cobalt-60 & 901.1 & - & 0 & & 1.5 & 3.3 & 4.3 & & 4.4 & 7.9 & -0.1 & & 3.8 & 7.5 & 0.4 & & 1.4 & 2.7 \\
\hline Lead-210 & 901.1 & - & -20 & & 300 & 430 & - & & - & - & -120 & & 110 & 180 & 0 & & 280 & 400 \\
\hline Lead-212 & 901.1 & - & -2 & & 5.1 & 7.9 & -1.1 & & 9.4 & 14 & 0 & & 10 & 15 & -2.8 & & 5 & 7.9 \\
\hline Lead-214 & 901.1 & - & -0.2 & & 4.8 & 7.8 & 20 & $\mathbf{J z} \mid$ & 12 & 17 & -4.8 & & 8.7 & 19 & 2.9 & & 4.9 & 7.6 \\
\hline Potassium-40 & 901.1 & - & -20 & & 26 & 48 & -12 & & 73 & 120 & -43 & r & 64 & 120 & -23 & & 26 & 48 \\
\hline $\mathrm{Ra}-223$ & 901.1 & - & - & & - & - & - & & - & - & - & & - & - & - & & - & - \\
\hline Radium-226 & 901.1 & 5 & -188 & & 59 & 85 & - & & - & 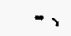 & -110 & & 110 & 180 & -55 & & 56 & 89 \\
\hline Thallium-208 & 901.1 & - & 1.4 & & 2.8 & 4.1 & -1.4 & & 6.2 & 9.6 & -3.8 & & 7.7 & 12 & 0.9 & & 2.8 & 4.1 \\
\hline Thorium-234 & 901.1 & - & -13 & & 48 & 150 & -17 & & 66 & 160 & -26 & & 66 & 150 & -12 & & 47 & 140 \\
\hline Uranium-235 & 901.1 & - & 3 & & 14 & 22 & 4 & & 27 & 42 & -2 & & 27 & 42 & 1 & & 12 & 22 \\
\hline Radium-226 & 903.1M & 5 & 0.15 & & 0.14 & 0.2 & 0.016 & & 0.1 & 0.21 & 0.09 & & 0.12 & 0.2 & 0.18 & - & 0.13 & 0.062 \\
\hline Strontium-90 & 905 & 8 & -0.15 & $\mathbf{J} \mathbf{m} \mid$ & 0.51 & 0.89 & -0.04 & & 0.25 & 0.44 & -0.6 & & 3.1 & 1.6 & 0.36 & $\mathbf{J} \mathbf{m} \mid$ & 0.49 & 0.83 \\
\hline Tritium & 906 & 20000 & 100 & & 180 & 220 & 120 & & 250 & 310 & 30 & & 220 & 310 & 20 & & 170 & 230 \\
\hline Gross Alpha & 9310 & 15 & 0.1 & $\mathrm{Jm} \mid \mathrm{C}$ & 2.7 & 6.1 & 1.2 & |C & 4 & 7.8 & -2 & |C & 3.5 & 9.5 & 2.8 & $\mathrm{Jm} \mid \mathrm{C}$ & 4.8 & 8.6 \\
\hline Gross Beta & 9310 & 50 & 0.3 & IC & 3.2 & 5.7 & 1.1 & IC & 4 & 6.9 & 2.2 & |C & 5.4 & 9.3 & 0 & IC & 4.6 & 8.1 \\
\hline Americium-241 & LAS108 & - & 0.006 & & 0.016 & 0.027 & - & & - & - & 0 & $\mathbf{J} \mathbf{m} \mid$ & 0.048 & 0.079 & 0.036 & & 0.028 & 0.016 \\
\hline Plutonium-241 & LAS178 & - & -0.5 & Jel & 1.5 & 1.9 & - & & - & - & 0 & & 1.5 & 1.8 & -1.1 & Jc I & 1.5 & 2.2 \\
\hline Carbon-14 & LSC & - & 230 & & 100 & 110 & 370 & & 110 & 100 & 45 & & 83 & 100 & 18 & & 86 & 110 \\
\hline
\end{tabular}


TABLE A.5

ANALYTICAL RESULTS FOR GROUNDWATER, 1995

RADIONUCLIDES

1995 WATER MONITORING REPORT

LEHR ENVIRONMENTAL RESTORATION, DAVIS, CALIFORNIA

\begin{tabular}{|c|c|c|c|c|c|c|c|c|c|c|c|c|c|c|c|c|c|c|}
\hline \multirow{2}{*}{ PARAMETER } & \multirow{2}{*}{ METHOD } & \multirow{2}{*}{$\begin{array}{l}\text { MCL } \\
\text { pCill }\end{array}$} & \multicolumn{4}{|c|}{$\begin{array}{l}\text { UCD1-25 } \\
\text { FALL } \\
\cdot\end{array}$} & \multicolumn{4}{|c|}{$\begin{array}{l}\text { UCD1-27Z1 } \\
\text { FALL }\end{array}$} & \multicolumn{4}{|c|}{$\begin{array}{l}\text { UCD1-27Z2 } \\
\text { FALL }\end{array}$} & \multicolumn{4}{|c|}{$\begin{array}{c}\text { UCD1-27Z3 } \\
\text { FALL }\end{array}$} \\
\hline & & & Value & & Error & MDA & Value & & Error & MDA & Value & & Error & MDA & Value & & Error & MDA \\
\hline Actinium-228 & 901.1 & - & 0.7 & & 7.6 & 13 & -6 & & 13 & 18 & 3 & & 13 & 15 & -2.2 & & 7.4 & 13 \\
\hline Bismuth-212 & 901.1 & - & -4 & & 11 & 28 & 4 & & 28 & 29 & 9 & & 27 & 26 & 6 & & 17 & 24 \\
\hline Bismuth-214 & 901.1 & - & 19.2 & & 6.8 & 8.4 & 13.4 & & 8.2 & 8.4 & 2 & & 7.5 & 8.7 & $9^{\circ}$ & & 6 & 8.5 \\
\hline Cesium-134 & 901.1 & - & - & & - & - & - & & - & - & - & & - & - & - & & - & - \\
\hline Cesium-137 & 901.1 & - & -1.3 & & 1.2 & 3.6 & 0.3 & & 2.2 & 3.7 & 2.7 & & 2.2 & 3.3 & -0.6 & & 1.1 & 3.3 \\
\hline Cobalt-57 & 901.1 & - & - & & - & - & - & & - & - & - & & - & - & - & & - & - \\
\hline Cobalt-60 & 901.1 & - & 1.1 & & 1.5 & 2.4 & -2.03 & & 0.52 & 4.6 & 0.4 & & 2.7 & 4.2 & -0.56 & & 0.84 & 3.8 \\
\hline Lead-210 & 901.1 & - & -160 & $\cdot$ & 310 & 470 & -4 & & 64 & 78 & 16 & & 59 & 69 & -60 & & 270 & 400 \\
\hline Lead-212 & 901.1 & - & 4.8 & & 5.3 & 7.5 & 2.8 & & 5.6 & 6.2 & -10.7 & & 5.1 & 6.1 & 2.5 & & 5 & 7.4 \\
\hline Lead-214 & 901.1 & . & 12.5 & & 5.6 & 8.1 & 6.7 & & 6.6 & 7.6 & 5.6 & & 6.5 & 7.4 & 7.9 & & 5.4 & 8.3 \\
\hline Potassium-40 & 901.1 & - & -6 & & 25 & 44 & 13 & & 43 & 50 & 31 & & 41 & 44 & -1 & & 27 & 46 \\
\hline Ra-223 & 901.1 & - & -8 & & 14 & 66 & -6.3 & & 6.3 & 64 & 7 & & 20 & 61 & -25 & & 13 & 69 \\
\hline Radium-226 & 901.1 & 5 & -4 & & 56 & 84 & -14 & & 67 & 76 & -173 & & 68 & 79 & -52 & & 56 & 87 \\
\hline Thallium-208 & 901.1 & - & 1.7 & & 2.9 & 4.1 & 1.7 & & 4 & 4.1 & 1.4 & & 4.1 & 4.4 & 0.9 & & 2.7 & 4 \\
\hline Thorium-234 & 901.1 & - & .24 & & 46 & 150 & 10 & & 39 & 70 & -19 & & 38 & 68 & -1 & & 47 & 150 \\
\hline Uranium-235 & 901.1 & - & -10.8 & & 7.8 & 24 & 1 & & 15 & 17 & 5 & & 15 & 17 & -2 & & 15 & 24 \\
\hline Radium-226 & $903.1 \mathrm{M}$ & 5 & 0.14 & & 0.11 & 0.16 & 0.085 & & 0.091 & 0.14 & 0.45 & & 0.16 & 0.15 & 0.29 & & 0.18 & 0.22 \\
\hline Strontium-90 & 905 & 8 & 0.38 & & 0.66 & 1.1 & 0.15 & & 0.68 & 1.2 & 0.32 & & 0.6 & 1 & 0.07 & & 0.46 & 0.8 \\
\hline Tritium & 906 & 20000 * & -80 & & 150 & 210 & -10 & & 170 & 250 & -90 & & 160 & 250 & -70 & & 170 & 250 \\
\hline Gross Alpha & 9310 & 15 & -4.1 & IC & 6.3 & 15 & -2.3 & |C & 3.3 & 8.4 & -0.5 & IC & 2.5 & 5.4 & 1.3 & |C & 2.5 & 4.6 \\
\hline Gross Beta & 9310 & 50 & 0.4 & ic & 10 & 5.8 & 0 & |C & 2.9 & 5.2 & 2 & ic & 2 & 3.3 & 1.9 & IC & 1.9 & 3 \\
\hline Americium-241 & LAS108 & - & 0.035 & & 0.037 & 0.044 & 0.01 & & 0.022 & 0.037 & 0,029 & & 0.028 & 0.02 & 0.012 & & 0.021 & 0.031 \\
\hline Plutonium-241 & LAS178 & - & 0.5 & Jl | & 3.5 & 5.2 & 0.2 & & 2.2 & 2.6 & -0.6 & & 2.2 & 2.7 & 1.5 & & 2.2 & 2.3 \\
\hline Carbon-14 & LSC & - & 19 & & 74 & 92 & 30 & & 85 & 100 & 19 & & 84 & 100 & -6 & & 82 & 100 \\
\hline
\end{tabular}


TABLE A.5

ANALYTICAL RESULTS FOR GROUNDWATER, 1995

RADIONUCLIDES

1995 WATER MONITORING REPORT

LEHR ENVIRONMENTAL RESTORATION, DAVIS, CALIFORNIA

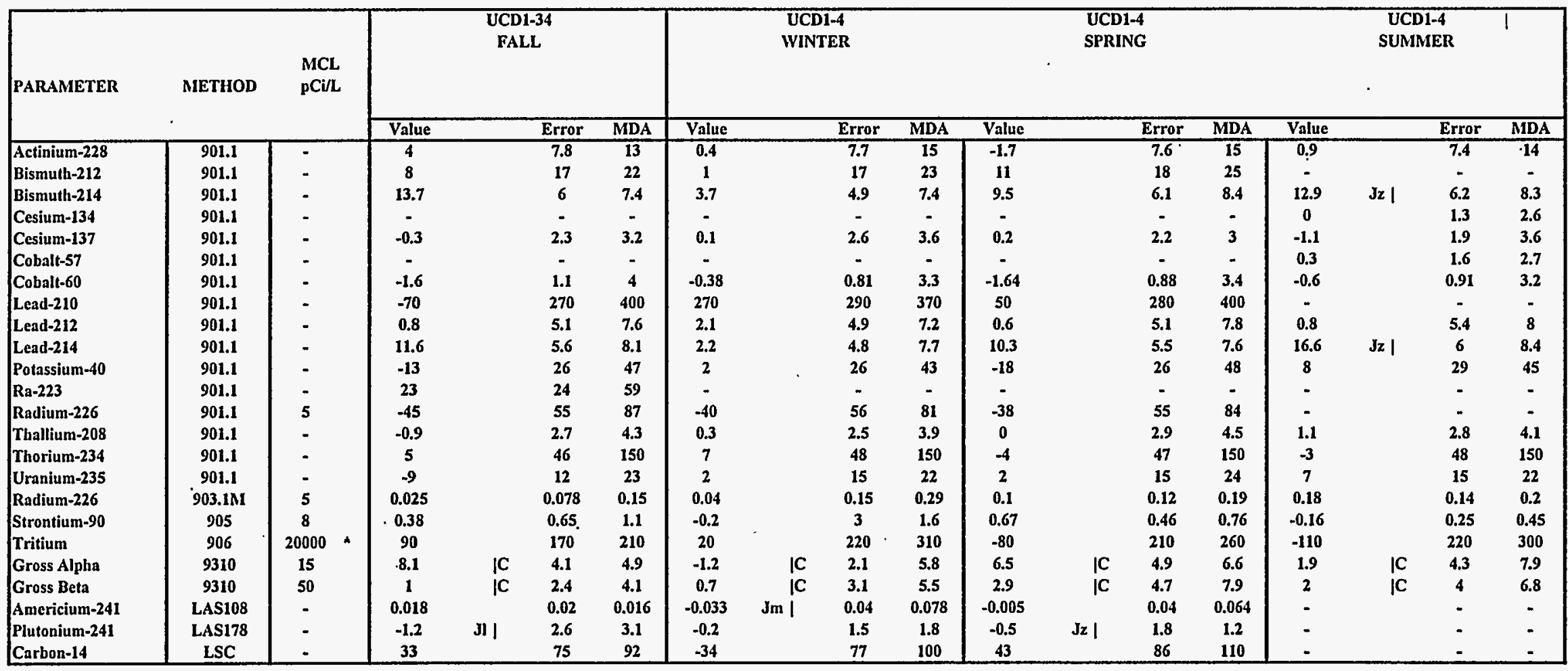


TABLE A.5

ANALYTICAL RESULTS FOR GROUNDWATER, 1995

RADIONUCLIDES

1995 WATER MONITORING REPORT

LEHR ENVIRONMENTAL RESTORATION, DAVIS, CALIFORNIA

\begin{tabular}{|c|c|c|c|c|c|c|c|c|c|c|c|c|c|c|c|c|c|c|}
\hline \multirow{2}{*}{ PARAMIETER } & \multirow{2}{*}{ METHOD } & \multirow{2}{*}{$\begin{array}{l}\text { MCL } \\
\mathrm{pCi} / \mathrm{L}\end{array}$} & \multicolumn{4}{|c|}{$\begin{array}{l}\text { UCD1-4 } \\
\text { FALL }\end{array}$} & \multicolumn{4}{|c|}{$\begin{array}{l}\text { UCD2-14 } \\
\text { WINTER }\end{array}$} & \multicolumn{4}{|c|}{$\begin{array}{l}\text { UCD2-14 } \\
\text { SPRING }\end{array}$} & \multicolumn{4}{|c|}{$\begin{array}{l}\text { UCD2-14 } \\
\text { SUMMER }\end{array}$} \\
\hline & & & Value & & Error & MDA & Value & & Error & MDA & Value & & Error & MDA & Valuc & & Error & MDA \\
\hline Actinium-228 & 901.1 & - & -0.1 & & 7.2 & 13 & -14 & & 11 & 41 & 29 & & 17 & 26 & 3 & & 18 & 31 \\
\hline Bismuth-212 & 901.1 & - & -5.5 & & 9.8 & 27 & 11 & & 46 & 63 & 18 & & 48 & 68 & - & & - & - \\
\hline Bismuth-214 & 901.1 & - & 10.6 & & 6.1 & 8 & 14 & & 13 & 18 & 2 & & 13 & 19 & 9 & & 14 & 20 \\
\hline Cesium-134 & 901.1 & - & - & & - & - & - & & - & - & - . & & - & - & -3.2 & & 2.7 & 6.8 \\
\hline Cesium-137 & 901.1 & - & -1 & & 1 & 3.3 & 3.6 & & 4.9 & 7.9 & 1.9 & & 6.6 & 8.7 & -0.6 & & 5.4 & 9.5 \\
\hline Cobalt-57 & 901.1 & - & - & & - & - & - & & - & - & - & & - & - & 2.5 & & 2.5 & 3.9 \\
\hline Cobalt-60 & 901.1 & - & -0.5 & & 1 & 3.6 & -1.3 & & 3.7 & 7.9 & -2.7 & & 3.4 & 12 & -2.5 & & 3.2 & 12 \\
\hline Lead-210 & 901.1 & - & 70 & & 290 & 400 & 0 & & 100 & 170 & 0 & & 100 & 160 & - & & - & - \\
\hline Lead-212 & 901.1 & - & 1.2 & & 4.9 & 7.4 & -4.7 & & 9.6 & 15 & 3 & & 10 & 14 & -2.9 & & 9.8 & 15 \\
\hline Lead-214 & 901.1 & - & 7.5 & & 5.3 & 8.3 & 7 & & 12 & 20 & -3 & & 11 & 18 & 23 & Je 1 & 12 & 17 \\
\hline Potassium-40 & 901.1 & - & -4 & & 25 & 44 & -8 & & 56 & 97 & -12 & & 66 & 110 & 16 & & 69 & 110 \\
\hline Ra-223 & 901.1 & - & -13 & & 13 & 66 & - & & - & - & - & & - & - & - & & - & - \\
\hline Radium-226 & 901.1 & 5 & -4 & & 54 & 82 & -350 & & 120 & 180 & -280 & ( & 120 & 170 & - & & - & - \\
\hline Thallium-208 & 901.1 & - & 1 & & 2.8 & 4.1 & 0.9 & & 7.5 & 11 & 2.2 & & 7 & 9.9 & -0.3 & & 7.1 & 11 \\
\hline Thorium-234 & 901.1 & - & -2 & & 48 & 160 & -26 & & 67 & 160 & -26 & & 64 & 150 & 40 & & 100 & 160 \\
\hline Uranium-235 & 901.1 & - & -3 & & 15 & 22 & 11 & & 27 & 41 & 15 & & 26 & 37 & 7 & & 28 & 44 \\
\hline Radium-226 & $903.1 \mathrm{M}$ & 5 & 0.133 & & 0.1 & 0.14 & 0.46 & & 0.26 & 0.29 & 0.11 & & 0.16 & 0.26 & 0.04 & & 0.13 & 0.24 \\
\hline Strontium-90 & 905 & 8 & 0.06 & & 0.66 & 1.1 & -0.6 & & 1.7 & 1 & -0.37 & $\mathbf{J} \cdot \mathbf{m} \mid$ & 0.49 & 0.87 & 1.06 & & 0.56 & 0.9 \\
\hline Tritium & 906 & 20000 & -30 & & 160 & 220 & 590 & & 240 & 260 & 5320 & & 500 & 220 & $\mathbf{5 5 9 0}$ & & 600 & 300 \\
\hline Gross Alpha & 9310 & 15 & 1 & IC & 3 & 5.7 & 2 & |C & 3.7 & 6.7 & 1.2 & $\mathrm{Jm} \mid C$ & 3.5 & 7 & 3.1 & IC & 4.9 & 8.5 \\
\hline Gross Beta & 9310 & 50 & 1.7 & ic & 2 & 3.4 & 2.7 & Jd jC & 3.5 & 5.9 & 1.9 & IC & 4.7 & 8 & 6.1 & ic & 4.6 & 7.3 \\
\hline Americium-241 & LAS108 & . & - & & - & - & 0.002 & $\mathbf{J m} \mathbf{i}$ & 0.031 & 0.054 & 0.014 & & 0.018 & 0.024 & 0.029 & & 0.035 & 0.046 \\
\hline Plutonium-241 & LAS178 & - & - & & - & - & -0.8 & & 1.4 & 1.9 & 0.6 & Jc 1 & 1.5 & 1.7 & 0.7 & Jc 1 & 2.8 & 3.5 \\
\hline Carbon-14 & LSC & - & - & & - & - & 64 & & 88 & 110 & 460 & & 130 & .130 & 510 & & 120 & 100 \\
\hline
\end{tabular}


TABLE A.5

ANALYTICAL RESULTS FOR GROUNDWATER, 1995

RADIONUCLIDES

1995 WATER MONITORING REPORT

LEHR ENVIRONMENTAL RESTORATION, DAVIS, CALIFORNIA

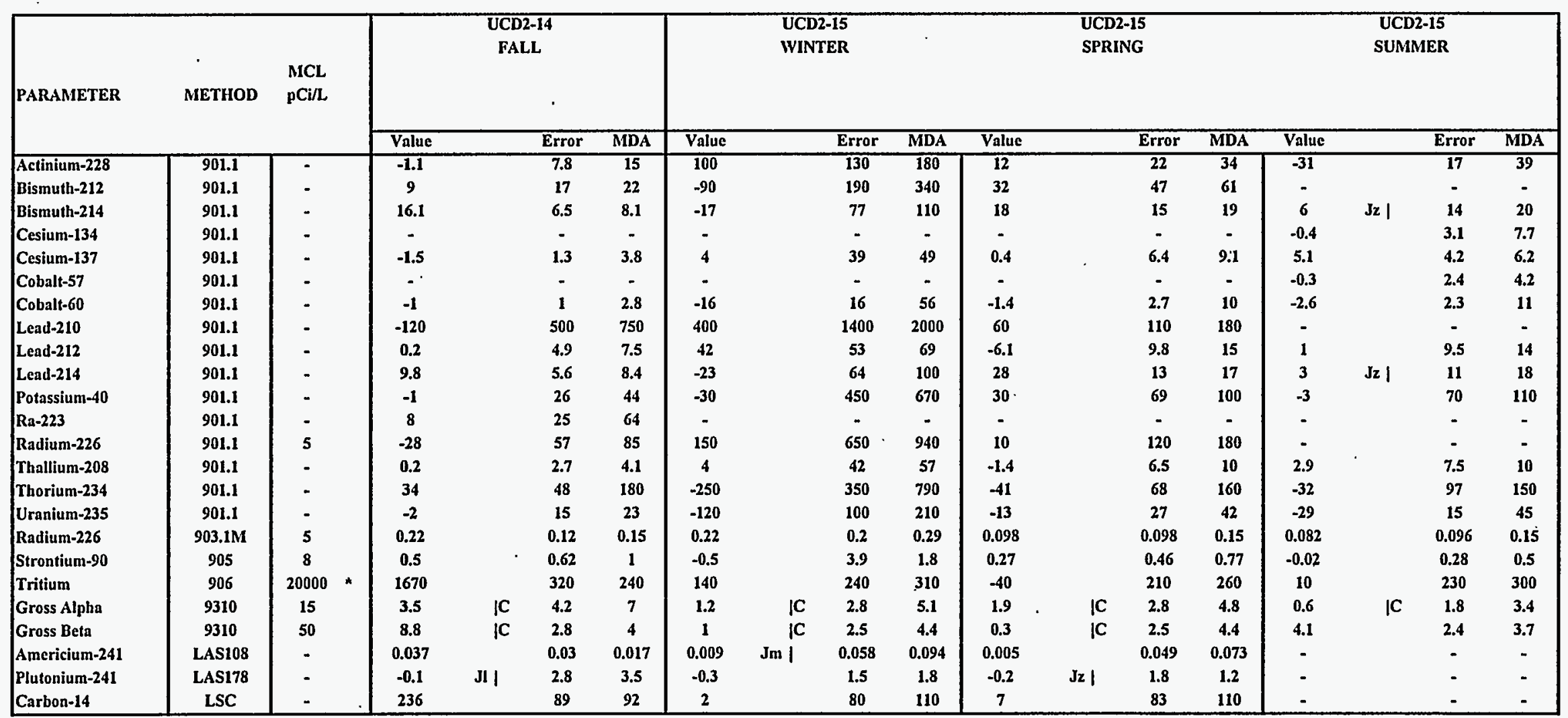


TABLE A.5

ANALYTICAL RESULTS FOR GROUNDWATER, 1995

RADIONUCLIDES

1995 WATER MONITORING REPORT

LEHR ENVIRONMENTAL RESTORATION, DAVIS, CALIFORNIA

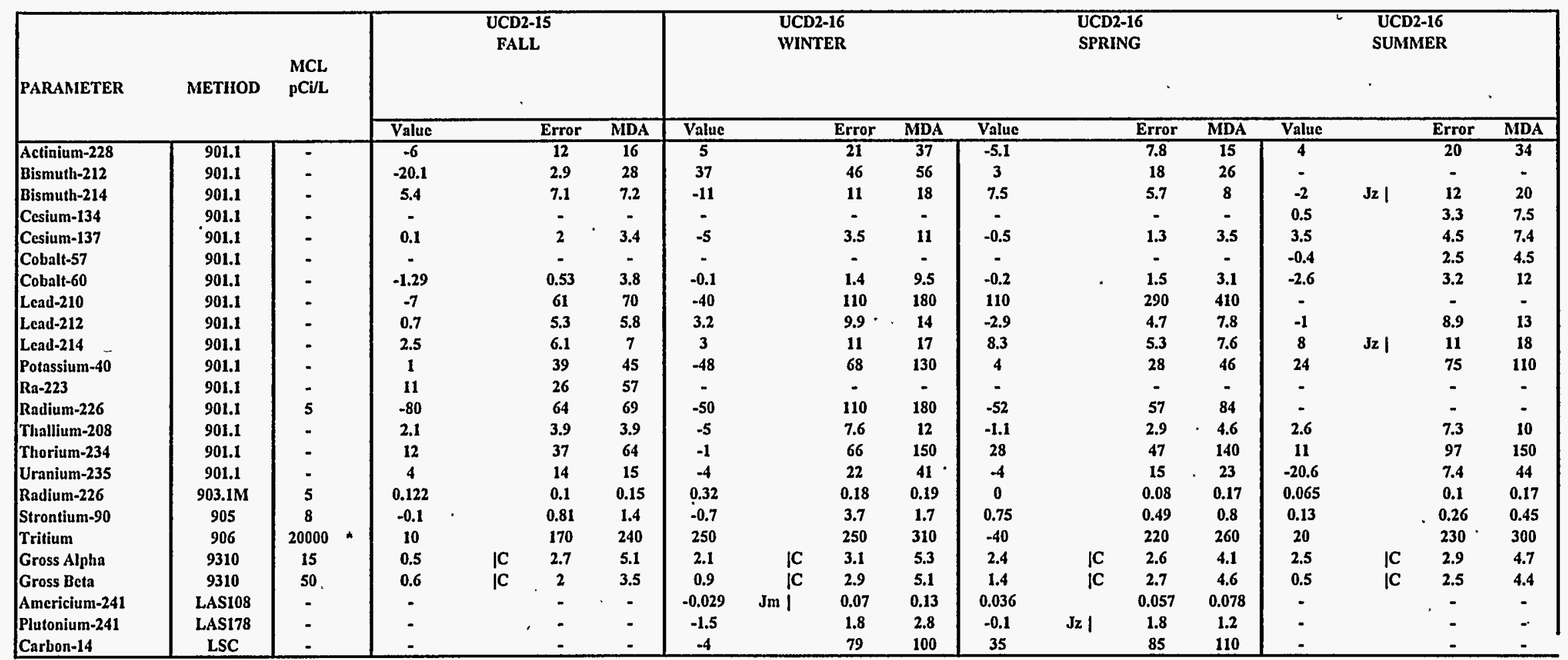


TABLE A.5

ANALYTICAL RESULTS FOR GROUNDWATER, 1995

RADIONUCLIDES

1995 WATER MONITORING REPORT

LEHR ENVIRONMENTAL RESTORATION, DAVIS, CALIFORNIA

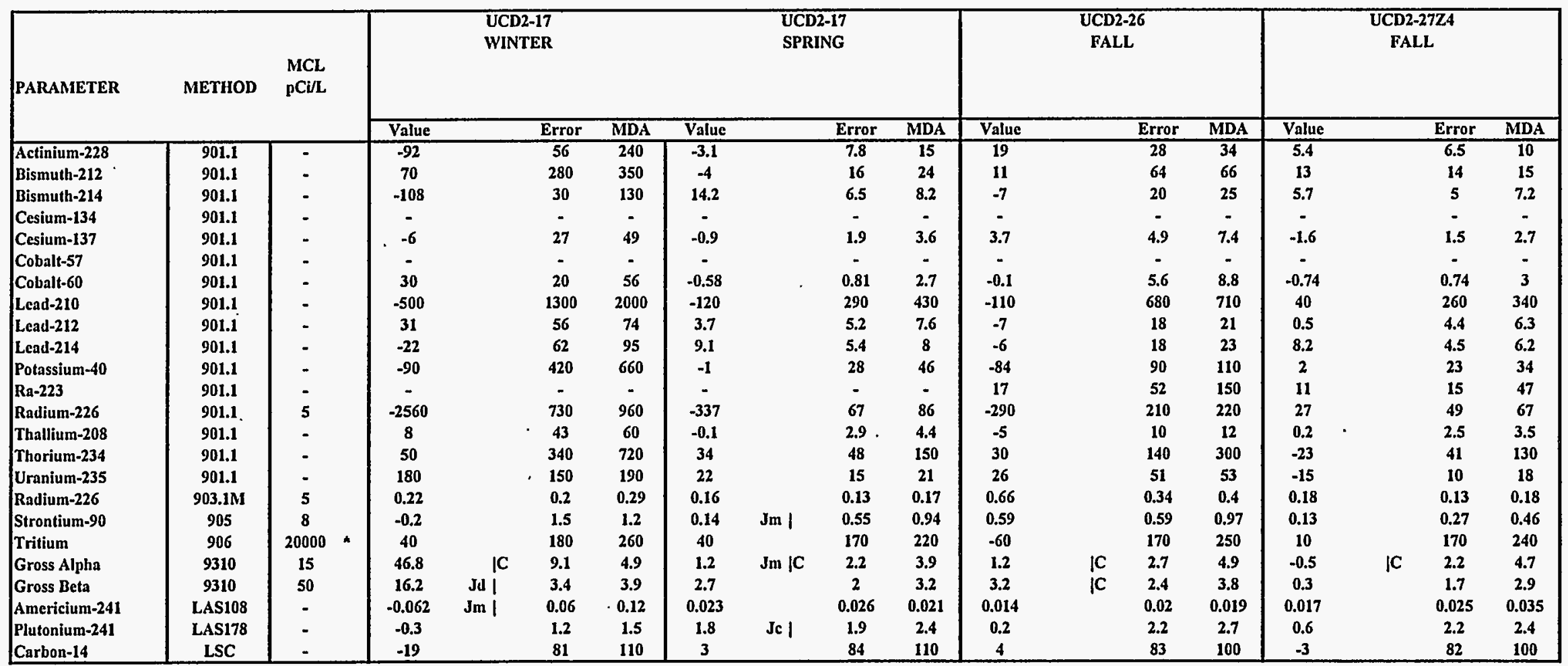


TABLE A.5

ANALYTICAL RESULTS FOR GROUNDWATER, 1995

RADIONUCLIDES

1995 WATER MONITORING REPORT

LEHR ENVIRONMENTAL RESTORATION, DAVIS, CALIFORNIA

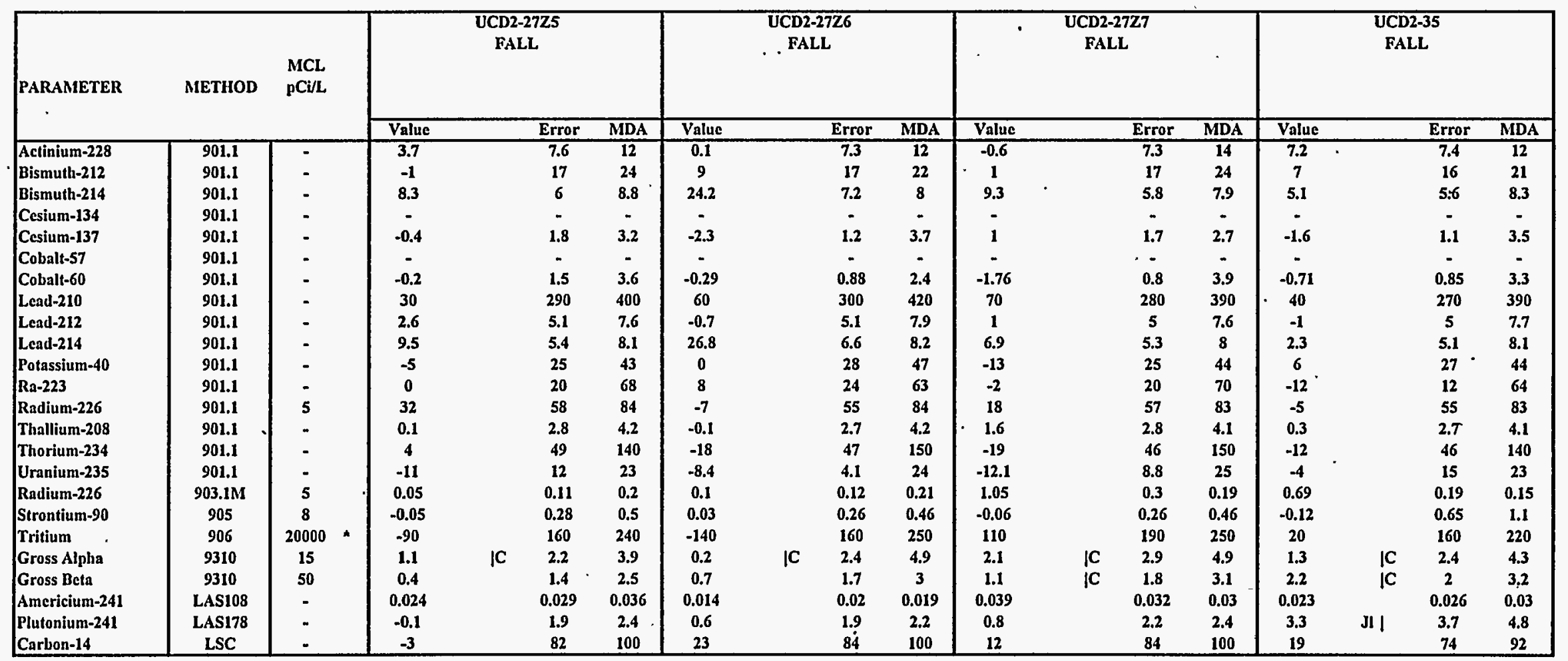


TABLE A.5

ANALYTICAL RESULTS FOR GROUNDWATER, 1995

RADIONUCLIDES

1995 WATER MONITORING REPORT

LEHR ENVIRONMENTAL RESTORATION, DAVIS, CALIFORNIA

\begin{tabular}{|c|c|c|c|c|c|c|c|c|c|c|c|c|c|c|c|c|c|c|}
\hline \multirow{2}{*}{ PARAMETER } & \multirow{2}{*}{ METHOD } & \multirow{2}{*}{$\begin{array}{l}\mathrm{MCL} \\
\mathrm{pCi} / \mathrm{L}\end{array}$} & \multicolumn{4}{|c|}{$\begin{array}{c}\text { UCD2-7 } \\
\text { WINTER }\end{array}$} & \multicolumn{4}{|c|}{$\begin{array}{c}\text { UCD2-7 } \\
\text { WINTER } \\
\text { D1 }\end{array}$} & \multicolumn{4}{|c|}{$\begin{array}{l}\text { UCD2-7 } \\
\text { SPRING }\end{array}$} & \multicolumn{4}{|c|}{$\begin{array}{c}\text { UCD2-7 } \\
\text { SUMMER }\end{array}$} \\
\hline & & & Value & & Error & MDA & Value & & Error & MDA & Value & & Error & MDA & Value & & Error & MDA \\
\hline Actinium-228 & 901.1 & - & -70 & & 120 & 220 & 8 & & 12 & 19 & -14 & & 15 & 46 & -3.4 & & 5.1 & 14 \\
\hline Bismuth-212 & 901.1 & - & 0 & & 270 & 460 & -10 & & 14 & 41 & 12 & & 51 & 69 & - & & - & - \\
\hline Bismuth-214 & 901.1 & - & -42 & & 73 & 130 & -0.6 & & 7.5 & 12 & 42 & & 17 & 19 & 25.2 & $\mathrm{Jz} \mathbf{I}$ & 7.3 & 8.1 \\
\hline Cesium-134 & $901.1^{\circ}$ & - & - & & - & - & - & & - & - & - & & - & - & 0.4 & & 1.3 & 2.7 \\
\hline Cesium-137 & 901.1 & - & 0 & & 45 & 63 & -2.2 & & 1.6 & 5 & -1.9 & & 5.1 & 9.5 & 0.2 & & 1.8 & 3.1 \\
\hline Cobalt-57 & 901.1 & - & - & & - & - & - & & - & - & - & & - & - & -0.8 & & 1.5 & 2.7 \\
\hline Cobalt-60 & 901.1 & - & -15 & & 34 & 94 & 1.3 & & 1.8 & 4 & -0.7 & & 3.3 & 11 & -0.28 & & 0.51 & 2.8 \\
\hline Lead-210 & 901.1 & - & -100 & & 1500 & 2400 & -40 & & 440 & 630 & 2 & & 99 & 160 & - & & - & - \\
\hline Lead-212 & 901.1 & - & -27 & & 66 & 100 & 2.7 & & 7.8 & 11 & 13 & & 10 & 14 & 2.3 & & 5.4 & 8 \\
\hline Lead-214 & 901.1 & - & -24 & & 57 & 120 & 2.9 & & 6.7 & 10 & 35 & & 13 & 17 & 26.1 & $\mathbf{J z} \mathbf{I}$ & 6.7 & 8.4 \\
\hline Potassium-40 & 901.1 & - & 400 & & $\mathbf{5 2 0}$ & 710 & 14 & & 37 & 58 & 73 & & 70 & 88 & 0 & & 26 & 44 \\
\hline $\mathbf{R n}_{\mathbf{n}}-223$ & 901.1 & - & - & & - & - & - & & - & - & - & & - & - & - & & - & - \\
\hline Radium-226 & 901.1 & 5 & -400 & & 750 & 1200 & -20 & & 83 & 130 & -80 & & 110 & 180 & - & & - & - \\
\hline Thallium-208 & 901.1 & - & 8 & & 46 & 66 & -3.5 & & 4.2 & 6.9 & 0.7 & & 7.2 & 10 & 0.4 & & 2.8 & 4.2 \\
\hline Thorium-234 & 901.1 & - & -310 & & 390 & 930 & 16 & & 68 & 210 & 5 & & 67 & 160 & -4 & & 48 & 150 \\
\hline Uranium-235 & 901.1 & - & 10 & & 170 & 250 & -3 & & 18 & 32 & -27 & & 17 & 47 & -3 & & 15 & 24 \\
\hline Radium-226 & $903.1 \mathrm{M}$ & 5 & 0.07 & & 0.13 & 0.24 & 0.24 & & 0.14 & 0.055 & 0.21 & & 0.14 & 0.18 & 0.111 & & 0.1 & 0.15 \\
\hline Strontium-90 & 905 & 8 & -0.8 & . & 3.8 & 1.8 & -0.7 & & 3.2 & 1.5 & 0.78 & & 0.51 & 0.83 & 0.17 & & 0.28 & 0.48 \\
\hline Tritium & 906 & 20000 * & 220 & & 250 & 310 & 360 & & 260 & 310 & 40 & & 220 & 260 & 0 & & 230 & 300 \\
\hline Gross Alpha & 9310 & 15 & 0.9 & IC & 2.5 & 5 & 0.8 & |C & 3 & 6 & 4.5 & |C & 3.8 & 5.5 & 4.6 & IC & 3.3 & 4.5 \\
\hline Gross Beta & 9310 & 50 & 1.9 & jc & 2.9 & 4.9 & 2 & iC & 3.2 & 5.4 & 1.8 & ic & 3.2 & 5.4 & 9.6 & jc & 3.7 & 5.5 \\
\hline Americium-241 & LAS108 & - & 0.036 & $\mathbf{J m} \mid$ & 0.09 & 0.14 & 0.025 & $\mathrm{Jm} \mid$ & 0.052 & 0.08 & -0.024 & & 0.042 & 0.072 & - & & - & - \\
\hline Plutonium-241 & LAS178 & - & 0.8 & & 1.6 & 1.9 & -0.3 & & 1.6 & 1.9 & 0.2 & $\mathbf{J z} \mid$ & 1.8 & 1.2 & - & & - & - \\
\hline Carbon-14 & LSC & - & -1 & & 80 & 110 & 19 & & 81 & 100 & -1 & & 82 & 110 & 85 & & 91 & 100 \\
\hline
\end{tabular}


TABLE A.5

ANALYTICAL RESULTS FOR GROUNDWATER 1995

RADIONUCLIDES

1995 ANNUAL WATER MONITORING REPORT

LEHR ENVIRONMENTAL RESTORATION, DAVIS, CALIFORNIA

D1 = Duplicate Sample

- = Parameter not analyzed or no MCL

DL = Detection Limit

MDA = Minimum Detectable Activity

* = Proposed MCL for Tritium is $60,000 \mathrm{pCi} / \mathrm{L}$

a = Reported tritium activity of $50 \mathrm{pCi} / \mathrm{L}$ is suspected to belong to UCD1-20. The tritium activity for UCD1-20 was reported as $16,600 \mathrm{pCi} / \mathrm{L}$ which is more consistent with historical trend for UCD1-13.

b = Reported tritium activity of $16,600 \mathrm{pCi} / \mathrm{L}$ is suspected to belong to UCD1-13. The tritium activity for UCD1-13 was reporied as $50 \mathrm{pCi} / \mathrm{L}$ which is more consistent with historical trend for UCD1-20.

Sec'Appendix A for explanation of data qualifiers. 
TABLE A.6

ANALYTICAL RESULTS FOR GROUNDWATER 1995

ANIONS AND CATIONS

1995 ANNUAL WATER MONITORING REPORT

LEHR ENVIRONMENTAL RESTORATION, DAVIS, CALIFORNIA

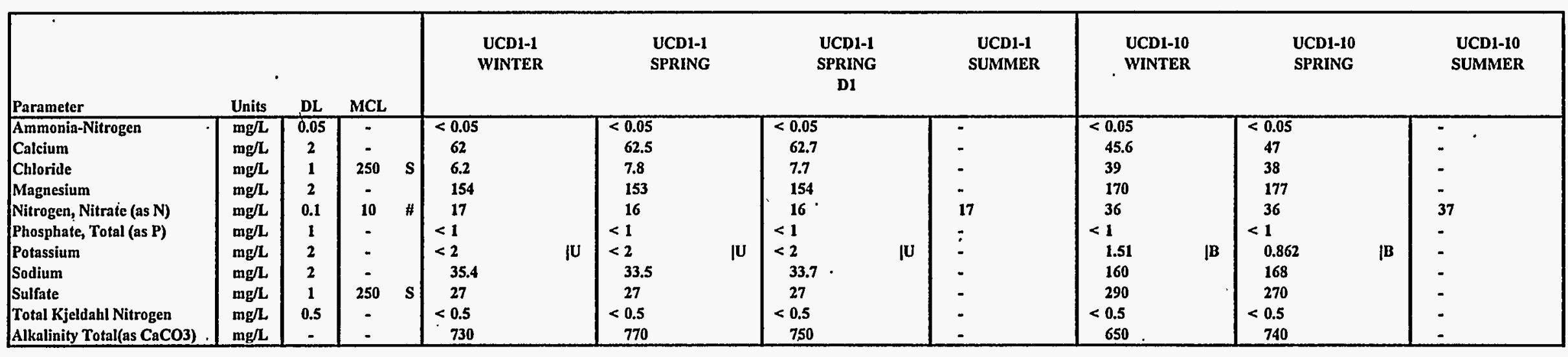


TABLE A.6

ANALYTICAL RESULTS FOR GROUNDWATER 1995

ANIONS AND CATIONS

1995 ANNUAL WATER MONITORING REPORT

LEHR ENVIRONMENTAL RESTORATION, DAVIS, CALIFORNIA

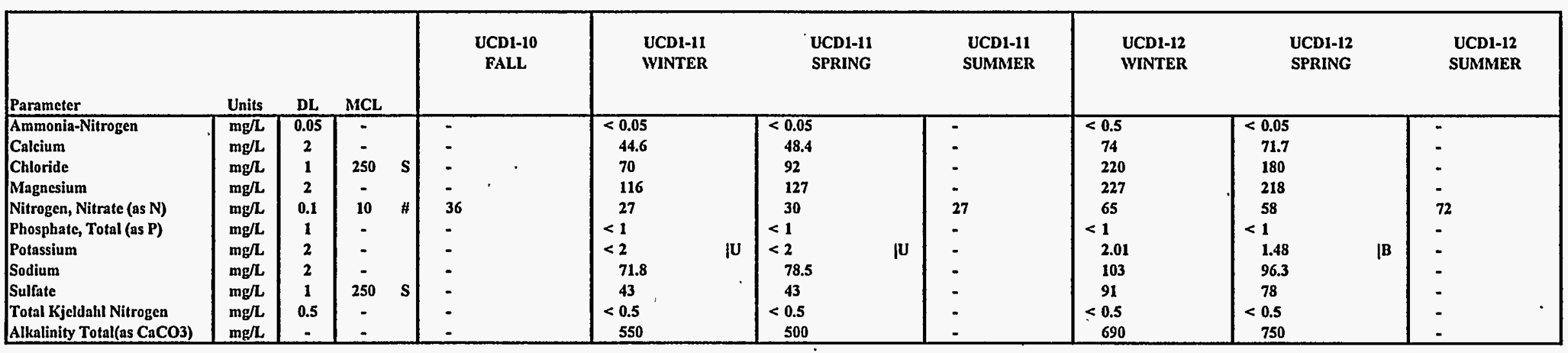


TABLE A.6

ANALYTICAL RESULTS FOR GROUNDWATER 1995

ANIONS AND CATIONS

1995 ANNUAL WATER MONITORING REPORT

LEHR ENVIRONMENTAL RESTORATION, DAVIS, CALIFORNIA

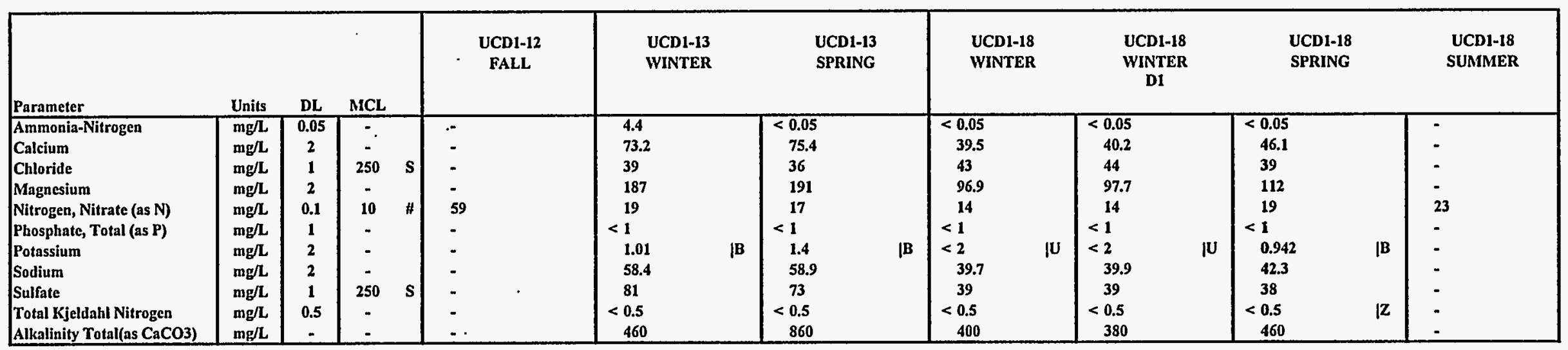


TABLE A.6

ANALYTICAL RESULTS FOR GROUNDWATER 1995

ANIONS AND CATIONS

1995 ANNUAL WATER MONITORING REPORT

LEHR ENVIRONMENTAL RESTORATION, DAVIS, CALIFORNIA

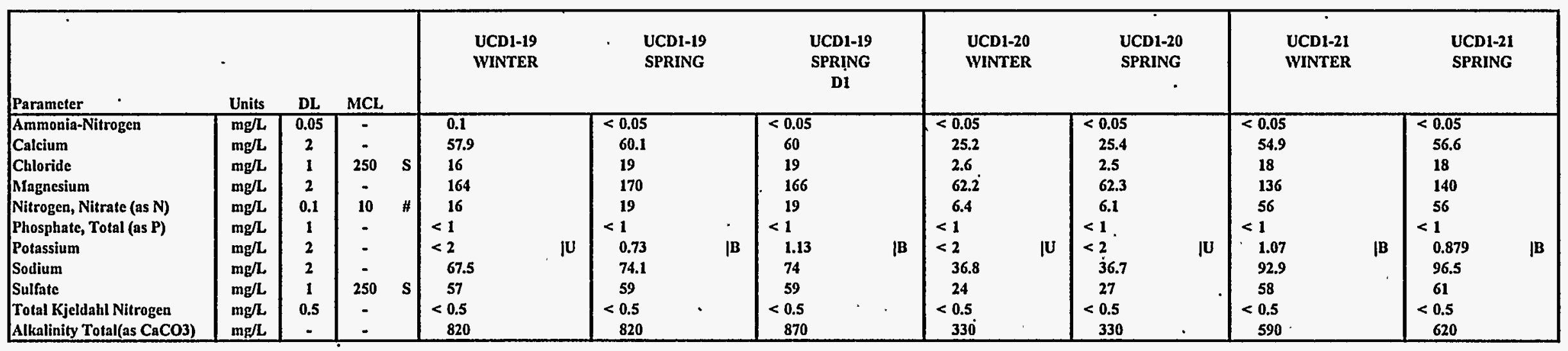


TABLE A.6

ANALYTICAL RESULTS FOR GROUNDWATER 1995

ANIONS AND CATIONS

1995 ANNUAL WATER MONITORING REPORT LEHR ENVIRONMENTAL RESTORATION, DAVIS, CALIFORNIA

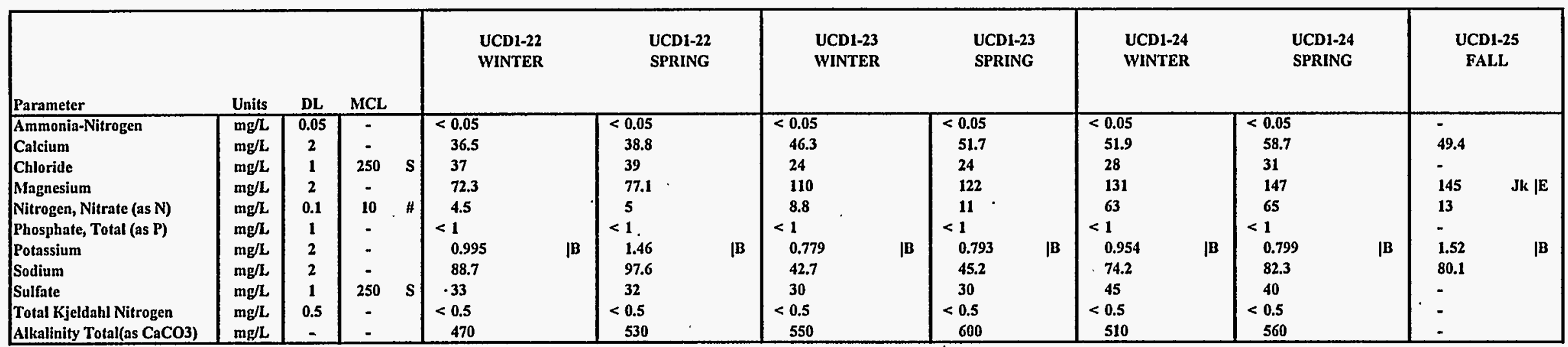


TABLE A.6

ANALYTICAL RESULTS FOR GROUNDWATER 1995

ANIONS AND CATIONS

1995 ANNUAL WATER MONITORING REPORT

LEHR ENVIRONMENTAL RESTORATION, DAVIS, CALIFORNIA

\begin{tabular}{|c|c|c|c|c|c|c|c|c|c|c|c|}
\hline Parameter & Units & DL & \multicolumn{2}{|l|}{ MCL } & $\begin{array}{l}\text { UCD1-27Z1 } \\
\text { FALL }\end{array}$ & $\begin{array}{l}\text { UCD1-27Z2 } \\
\text { FALL }\end{array}$ & $\begin{array}{c}\text { UCD1-27Z3 } \\
\text { FALL }\end{array}$ & $\begin{array}{c}\text { UCD1-34 } \\
\text { FALL }\end{array}$ & $\begin{array}{c}\text { UCD1-4 } \\
\text { WINTER }\end{array}$ & $\begin{array}{l}\text { UCD1-4 } \\
\text { SPRING }\end{array}$ & $\begin{array}{l}\text { UCDI-4 } \\
\text { SUMMER }\end{array}$ \\
\hline Ammonia-Nitrogen & $\mathrm{mg} / \mathrm{L}$ & 0.05 & - & & - & - & - & - & $<0.05$ & $<0.05$ & - \\
\hline Calcium & $\mathrm{mg} / \mathrm{h}$ & 2 & - & & 34.5 & 39 & 39.6 & 35.5 & 44 & 58.2 & - \\
\hline Chloride & $\mathrm{mg} / \mathrm{L}$ & 1 & 250 & $\mathbf{s}$ & - & - & - & . & 42 & 35 & - \\
\hline Magnesium & $\mathrm{mg} / \mathrm{L}$ & 2 & - & & 49.3 & $\dot{6} 4.9$ & 62.9 & Jk $\mid E$ & 75.5 & 111 & - \\
\hline Nitrogen, Nitrate (as N) & $\mathrm{mg} / \mathrm{L}$ & 0.1 & 10 & $\#$ & $<0.1$ & $<0.1$ & $<0.1$ & 0.16 & 1.2 & 6.1 & 7.3 \\
\hline Phosphate, Total (as P) & $\mathrm{mg} / \mathrm{L}$ & 1 & & & & & & & $<1$ & $<1$ & - \\
\hline Potassium & $\mathrm{mg} / \mathrm{L}$ & 2 &.- & & 2.25 & 1.26 & 1.48 & 1.79 & 0.791 & $<2$ & - \\
\hline Sodium & $\mathbf{m g} / \mathbf{L}$ & 2 & - & & 191 & 81.7 & 72.7 & 54.5 & 33.7 & 40.5 & - \\
\hline Sulfate & $\mathrm{mg} / \mathrm{L}$ & 1 & 250 & $\mathbf{s}$ & - & - & - & - & 34 & 40 & - \\
\hline Total Kjeldahl Nitrogen & $\mathrm{mg} / \mathrm{h}$ & 0.5 & - & & - & - & - & - & $<0.5$ & $<0.5$ & - \\
\hline Alkalinity Total(as $\mathrm{CaCO}$ ) & $\mathrm{mg} / \mathrm{L}$ & - & - & & - & - & - & . & 410 & $\mathbf{5 3 0}$ & - \\
\hline
\end{tabular}


TABLE A.6

ANALYTICAL RESULTS FOR GROUNDWATER 1995

ANIONS AND CATIONS

1995 ANNUAL WATER MONITORING REPORT

LEHR ENVIRONMENTAL RESTORATION, DAVIS, CALIFORNIA

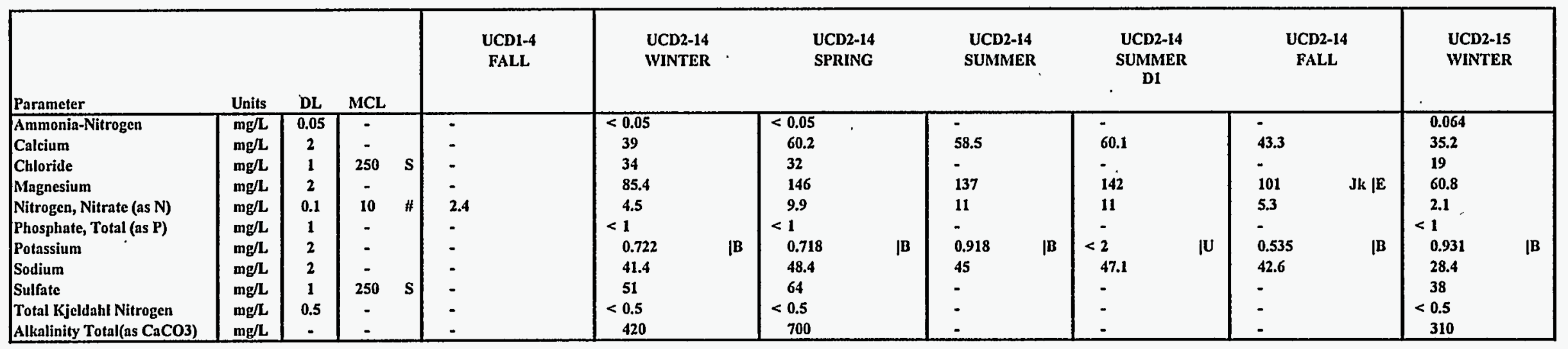


TABLE A.6

ANALYTICAL RESULTS FOR GROUNDWATER 1995

ANIONS AND CATIONS

1995 ANNUAL WATER MONITORING REPORT

LEHR ENVIRONMENTAL RESTORATION, DAVIS, CALIFORNIA

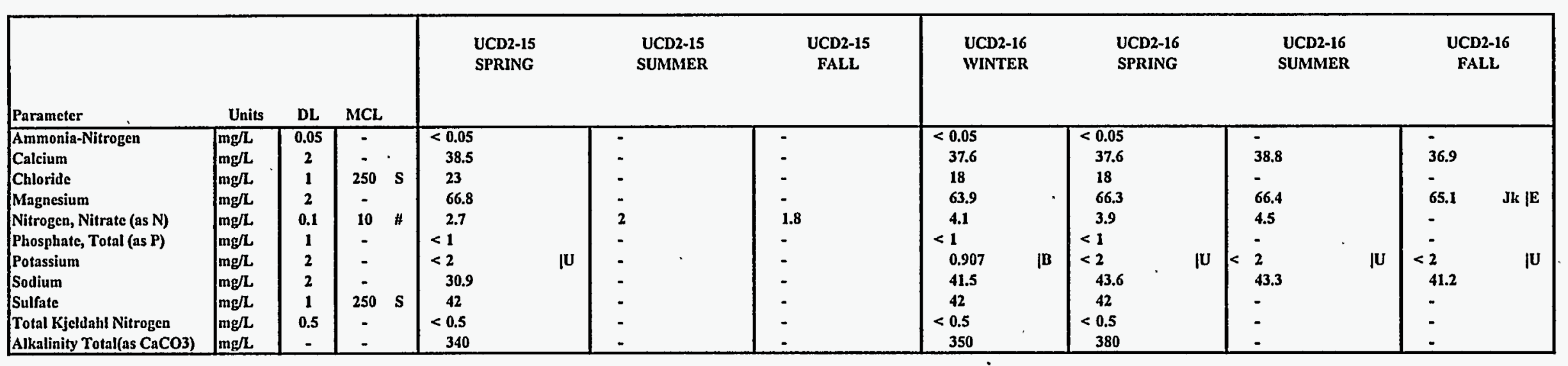


TABLE A.6

ANALYTICAL RESULTS FOR GROUNDWATER 1995

ANIONS AND CATIONS

1995 ANNUAL WATER MONITORING REPORT

LEHR ENVIRONMENTAL RESTORATION, DAVIS, CALIFORNIA

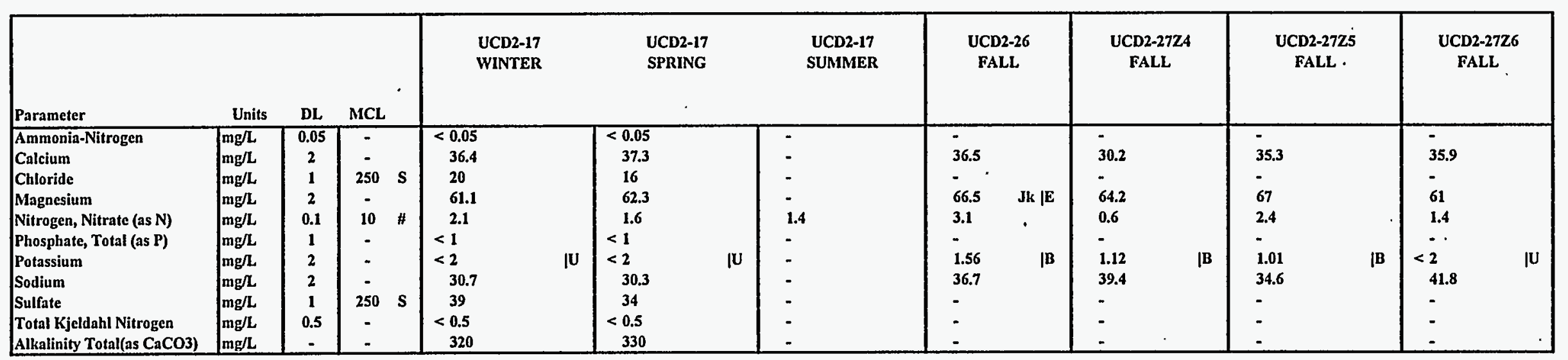


TABLE A.6

ANALYTICAL RESULTS FOR GROUNDWATER 1995 .

ANIONS AND CATIONS

1995 ANNUAL WATER MONITORING REPORT

LEHR ENVIRONMENTAL RESTORATION, DAVIS, CALIFORNIA.

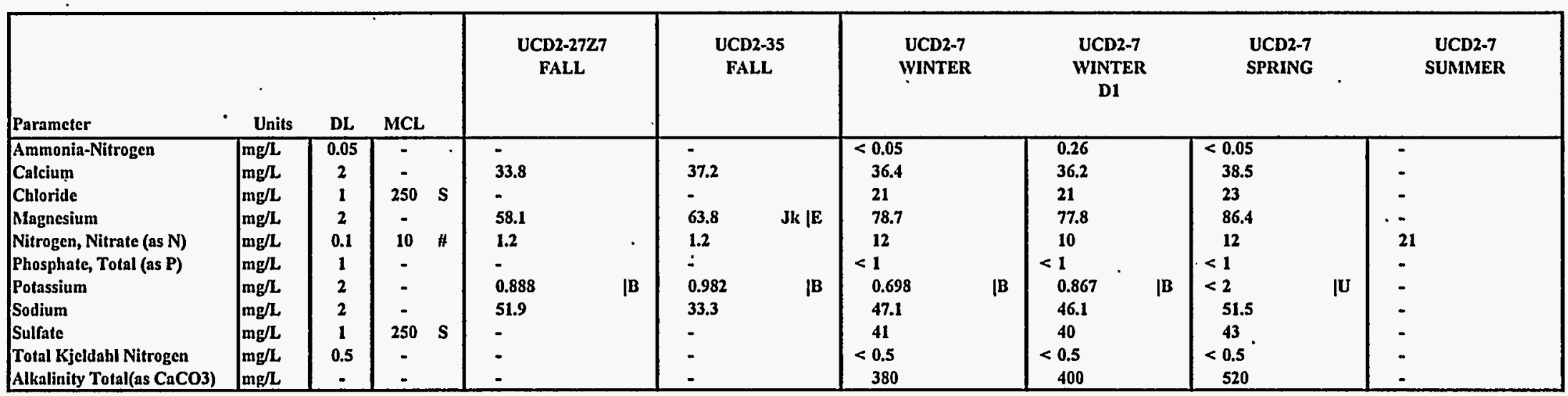


TABLE A.6

ANALYTICAL RESULTS FOR GROUNDWATER 1995

ANIONS AND CATIONS

1995 ANNUAL WATER MONITORING REPORT.

LEHR ENVIRONMENTAL RESTORATION, DAVIS, CALIFORNIA

D1 = Duplicate Sample

- = Parameter not analyzed or no MCL

DL $=$ Detection Limit

$<=$ Constituent below detection limit. Detection limits may vary depending on interference by other sample constituents.

MCL = Maximum Contaminant Level (primary), Title 22 Division 4 Chapter 15.

\# = USEPA MCL

$S=$ Secondary Drinking Water Standard

See Appendix A for explanation of data qualifiers. 
TABLE A.7

ANALYTICAL RESULTS FOR GROUNDWATER 1995

OTHER PARAMETERS

1995 ANNUAL WATER MONITORING REPORT

LEHR ENVIRONMENTAL RESTORATION, DAVIS, CALIFORNIA

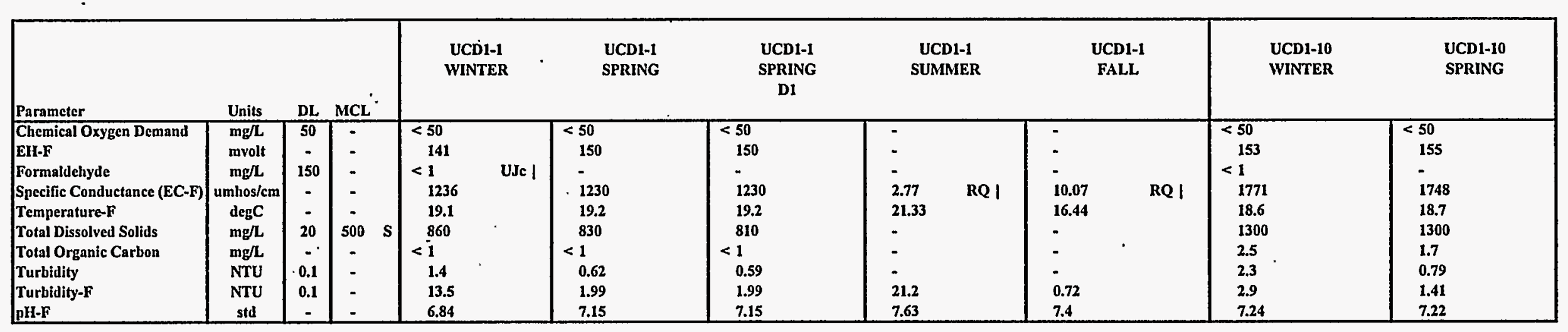


TABLE A.7

ANALYTICAL RESULTS FOR GROUNDWATER 1995

OTHER PARAMETERS

1995 ANNUAL WATER MONITORING REPORT

LEHR ENVIRONMENTAL RESTORATION, DAVIS, CALIFORNIA

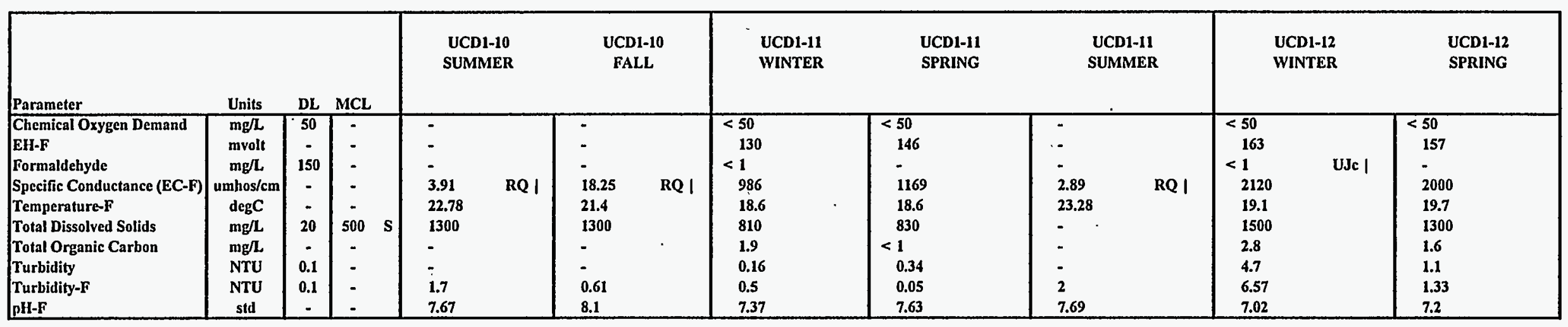


TABLE A.7

ANALYTICAL RESULTS FOR GROUNDWATER 1995

OTHER PARAMETERS

1995 ANNUAL WATER MONITORING REPORT

LEHR ENVIRONMENTAL RESTORATION, DAVIS, CALIFORNIA

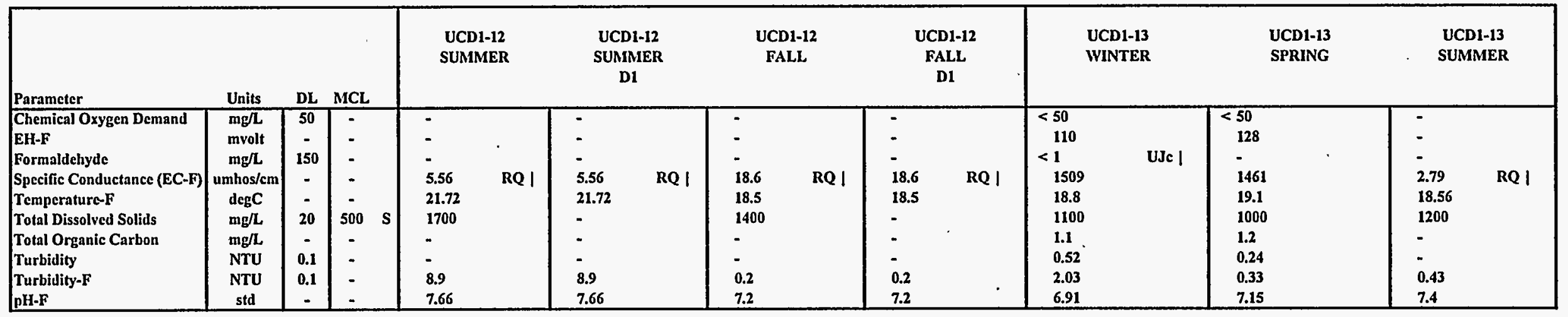


TABLE A.7

ANALYTICAL RESULTS FOR GROUNDWATER 1995

OTHER PARAMETERS

1995 ANNUAL WATER MONITORING REPORT

LEHR ENVIRONMENTAL RESTORATION, DAVIS, CALIFORNIA

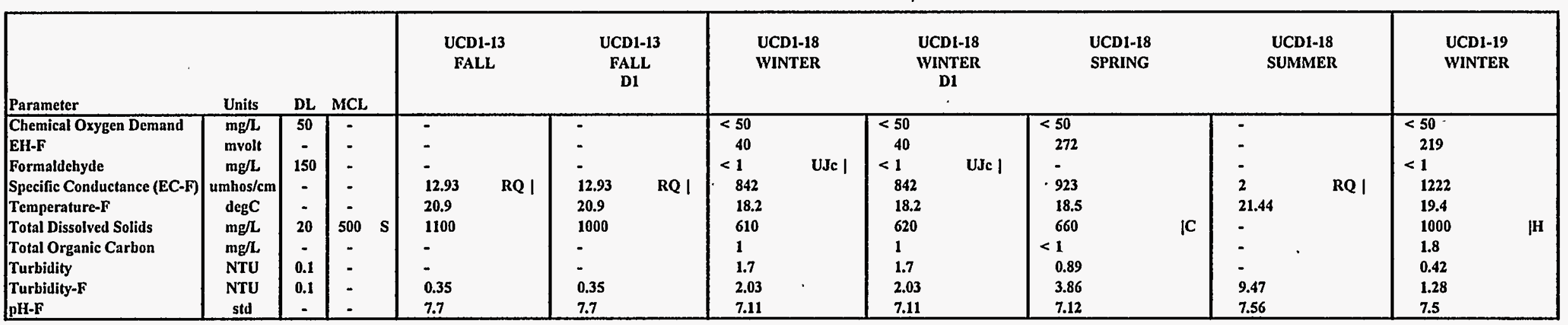


TABLE A.7

ANALYTICAL RESULTS FOR GROUNDWATER 1995

OTHER PARAMETERS

1995 ANNUAL WATER MONITORING REPORT

LEHR ENVIRONMENTAL RESTORATION, DAVIS, CALIFORNIA

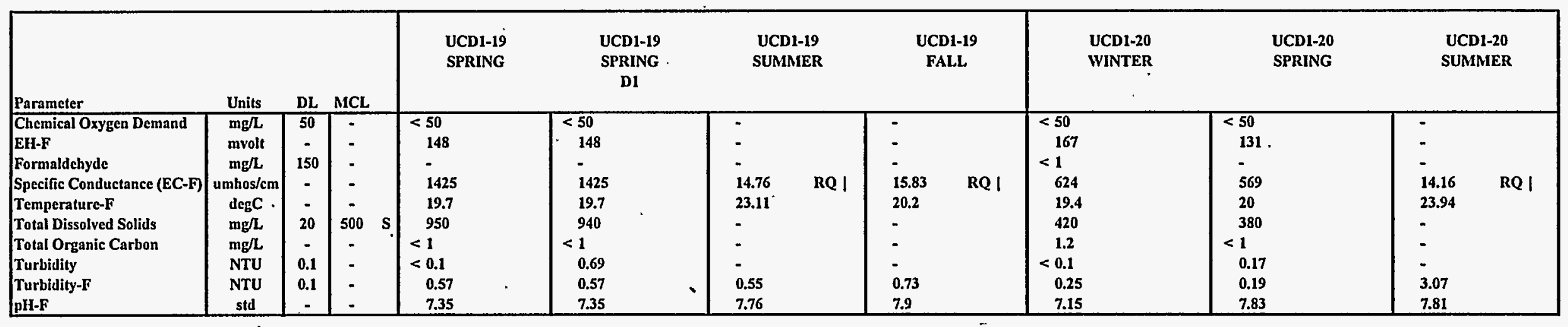


TABLE A.7

ANALYTICAL RESULTS FOR GROUNDWATER 1995

OTHER PARAMETERS

1995 ANNUAL WATER MONITORING REPORT

LEHR ENVIRONMENTAL RESTORATION, DAVIS, CALIFORNIA

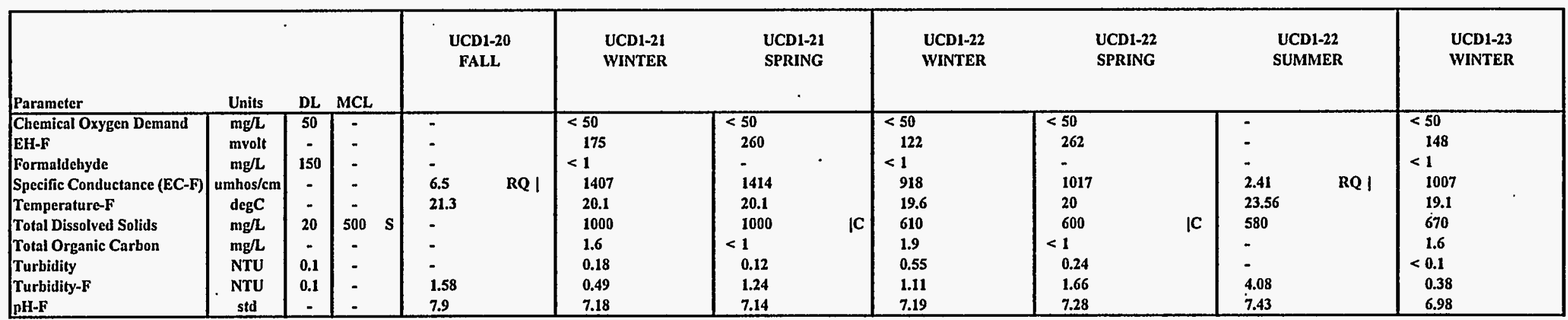


TABLE A.7

ANALYTICAL RESULTS FOR GROUNDWATER 1995

OTHER PARAMETERS

1995 ANNUAL WATER MONITORING REPORT

LEHR ENVIRONMENTAL RESTORATION, DAVIS, CALIFORNIA

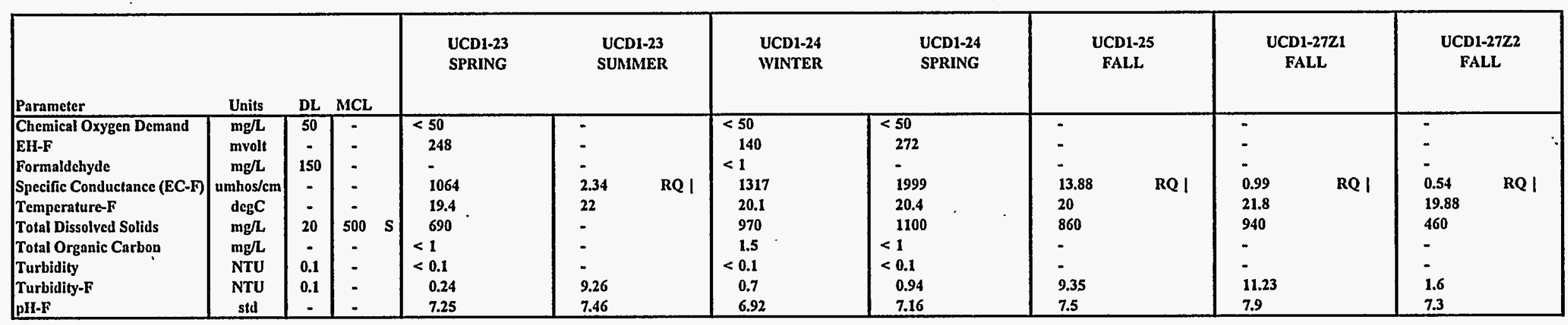


TABLE A.7

ANALYTICAL RESULTS FOR GROUNDWATER 1995

OTHER PARAMETERS

1995 ANNUAL WATER MONITORING REPORT

LEHR ENVIRONMENTAL RESTORATION, DAVIS, CALIFORNIA

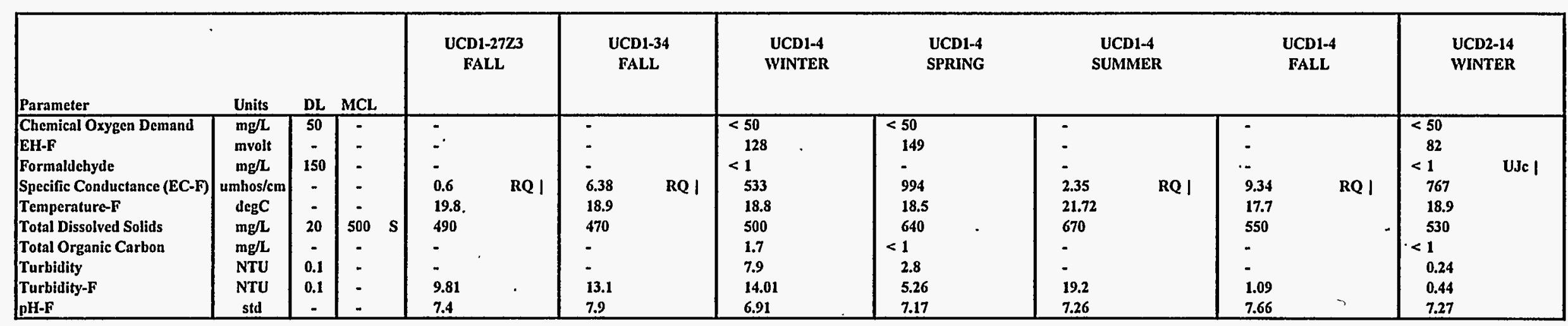


TABLE A.7

ANALYTICAL RESULTS FOR GROUNDWATER 1995

OTHER PARAMETERS

1995 ANNUAL WATER MONITORING REPORT

LEHR ENVIRONMENTAL RESTORATION, DAVIS, CALIFORNIA

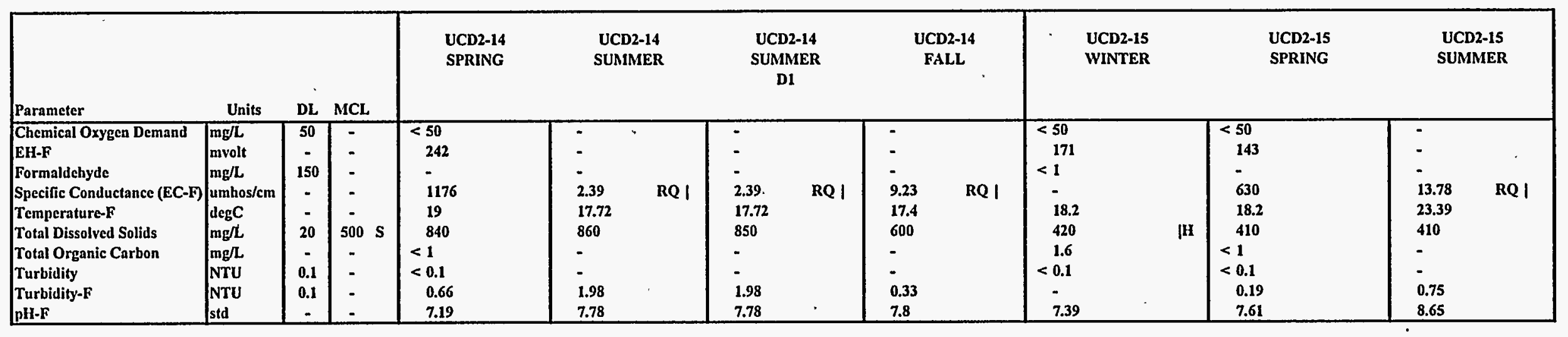


TABLE A.7

ANALYTICAL RESULTS FOR GROUNDWATER 1995

OTHER PARAMETERS

1995 ANNUAL WATER MONITORING REPORT

LEHR ENVIRONMENTAL RESTORATION, DAVIS, CALIFORNIA

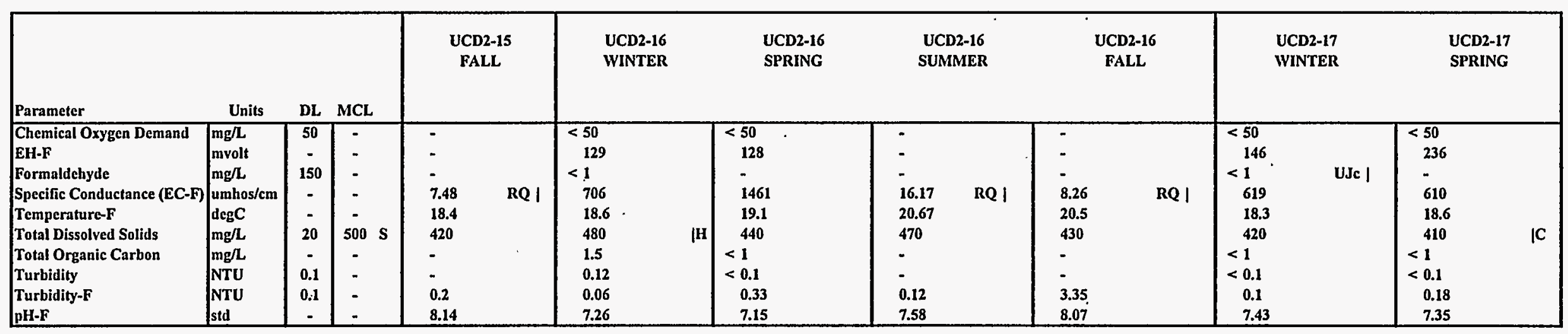


TABLE A.7

ANALYTICAL RESULTS FOR GROUNDWATER 1995

OTHER PARAMETERS

1995 ANNUAL WATER MONITORING REPORT

LEHR ENVIRONMENTAL RESTORATION, DAVIS, CALIFORNIA

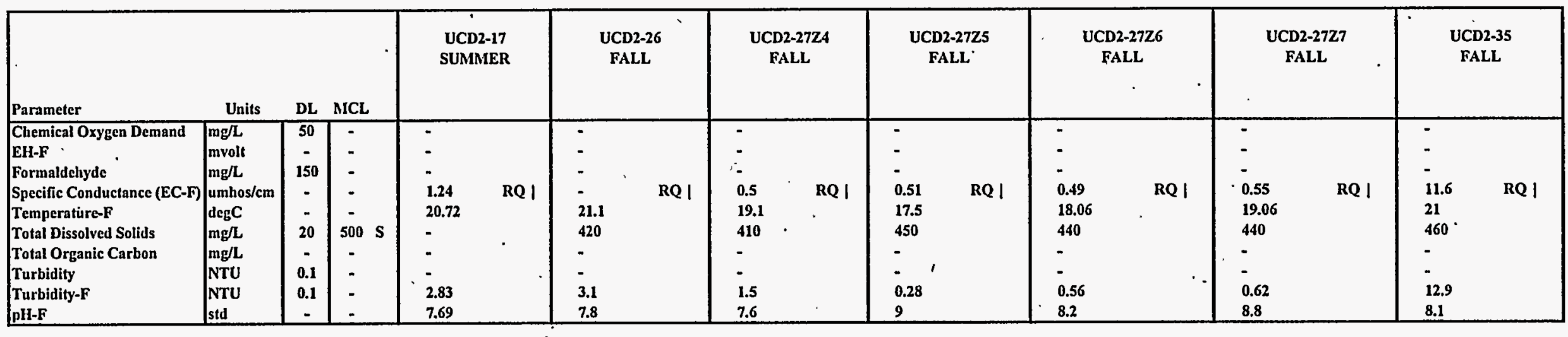


TABLE A.7

ANALYTICAL RESULTS FOR GROUNDWATER 1995

OTHER PARAMETERS

1995 ANNUAL WATER MONITORING REPORT

LEHR ENVIRONMENTAL RESTORATION, DAVIS, CALIFORNIA

\begin{tabular}{|c|c|c|c|c|c|c|c|c|}
\hline Parameter & \multicolumn{3}{|l|}{. } & $\begin{array}{c}\text { UCD2-7 } \\
\text { WINTER }\end{array}$ & $\begin{array}{c}\text { UCD2-7 } \\
\text { WINTER } \\
\text { D1 }\end{array}$ & $\begin{array}{r}\text { UCD2-7 } \\
\text { SPRING }\end{array}$ & \multicolumn{2}{|c|}{$\begin{array}{c}\text { UCD2-7 } \\
\text { SUMMER }\end{array}$} \\
\hline Chemical Oxygen Demand & $m g / L$ & 50 & - & $<50$ & $<50$ & $<50$ & - & \\
\hline EH-F & mvolt & - & . & 99 & 99 & 195 & - & \\
\hline Formaldehyde & mg/L & 150 & - & $<1$ & $<1$ & - & - & \\
\hline Specific Conductance (EC-F) & umhos/cm & - & - & 747 & 747 & 818 & 1.85 & RQI \\
\hline Temperature-F & $\operatorname{deg} C$ & - & - & 19 & 19 & 18.7 & 21.17 & \\
\hline Total Dissolved Solids & $\mathrm{mg} / \mathrm{L}$ & 20 & $500 \mathrm{~S}$ & 550 & 530 & $\mathbf{5 5 0}$ & & \\
\hline Total Organic Carbon & $\mathrm{mg} / \mathrm{L}$ & - & - & 2.1 & 1.4 & $<1$ & - & \\
\hline Turbidity & NTU & 0.1 & - & $<0.1$ & $<0.1$ & 0.14 & - & \\
\hline Turbidity-F & NTU & 0.1 & - & 0.33 & 0.33 & 0.12 & 0.68 & \\
\hline pH-F & std & - & - & 7.22 & 7.22 & 7.55 & 7.61 & . \\
\hline
\end{tabular}


TABLE A.7

ANALYTICAL RESULTS FOR GROUNDWATER 1995

OTHER PARAMETERS

1995 ANNUAL WATER MONITORING REPORT

LEHR ENVIRONMENTAL RESTORATION, DAVIS, CALIFORNIA

D1 = Duplicate Sample

- = Parameter not analyzed or no MCL

DL = Detection Limit

$<=$ Constituent below detection limit. Detection limits may vary depending on interference by other sample constituents.

MCL = Maximum Contaminant Level (primary), Title 22 Division 4 Chapter 15.

$S=$ Secondary Drinking Water Standard

See Appendix A for explanation of data qualifiers. 
TABLE A.8

ANALYTICAL RESULTS FOR TRIP BLANKS 1995

VOLATILE ORGANIC COMPOUNDS

1995 ANNUAL WATER MONITORING REPORT

LEHR ENVIRONMENTAL RESTORATION, DAVIS, CALIFORNIA

\begin{tabular}{|c|c|c|c|c|c|c|c|c|c|c|c|c|c|c|c|c|c|c|c|c|}
\hline Parameter & Units & DL & \multicolumn{2}{|l|}{ MCL } & \multicolumn{2}{|c|}{$\begin{array}{c}\text { TBGW0016 } \\
\text { WINTER }\end{array}$} & \multicolumn{2}{|c|}{$\begin{array}{l}\text { TBGW0017 } \\
\text { WINTER }\end{array}$} & \multicolumn{2}{|c|}{$\begin{array}{l}\text { TBGW0018 } \\
\text { WINTER }\end{array}$} & \multicolumn{2}{|c|}{$\begin{array}{l}\text { TBGW0020 } \\
\text { WINTER }\end{array}$} & \multicolumn{2}{|c|}{$\begin{array}{l}\text { TBSW0002 } \\
\text { WINTER }\end{array}$} & \multicolumn{2}{|c|}{$\begin{array}{l}\text { TBSW0003 } \\
\text { WINTER }\end{array}$} & \multicolumn{2}{|c|}{$\begin{array}{l}\text { TBGW0023 } \\
\text { SPRING }\end{array}$} & \multicolumn{2}{|c|}{$\begin{array}{l}\text { TBGW0024 } \\
\text { SPRING }\end{array}$} \\
\hline 1,1,1-Trichlorocthane & ug/L & 1 & 200 & & $<1$ & TU & $<1$ & TU & $<1$ & Tu & $<1$ & Tu & $<1$ & 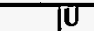 & $<2$ & TU & $<1$ & ए & $<1$ & Ju \\
\hline 1,1,2,2-Tetrachloroethane & $\mathrm{ug} / \mathrm{L}$ & 1 & 1 & & $<1$ & ju & $<1$ & jU & $<1$ & ju & $<1$ & ju & $<1$ & jU & $<2$ & ju & $<1$ & ju & $<1$ & ju \\
\hline 1,1,2-Trichloroethane & ug/L & 1 & 5 & \#) & $<1$ & ju & $<1$ & jU & $<1$ & U & $<1$ & ju & $<1$ & jU & $<2$ & ju & $<1$ & j & $<1$ & ju \\
\hline 1,1-Dichloroethane & $\mathrm{ug} / \mathrm{L}$ & 1 & 5 & & $<1$ & jU & $<1$ & jU & $<1$ & ju & $<1$ & ju & $<1$ & jU & $<2$ & ju & $<1$ & ju & $<1$ & ju \\
\hline 1,1-Dichloroethene & ug/L & 1 & 6 & & $<1$ & Uu & $<1$ & $\mathbf{U}$ & $<1$ & $\mathbf{U}$ & $<1$ & ju & $<1$ & $\mathbf{U}$ & $<2$ & jU & $<1$ & U & $<1$ & ju \\
\hline 1,2-Dibromo-3-chloropropane & $\mathrm{ug} / \mathrm{L}$ & 1 & - & & $<1$ & $\mathbf{R c} \boldsymbol{j} U$ & $<1$ & $\mathbf{R c} \mid \underline{U}$ & $<1$ & $\operatorname{Re} \mathbf{U}$ & $<1$ & $\mathrm{Rc} \boldsymbol{j}$ & $<1$ & $\mathbf{R c} \mid \mathbf{U}$ & $<2$ & $\operatorname{Re} \boldsymbol{U}$ & $<1$ & $R e j u$ & $<1$ & $\mathbf{R e} \mathbf{U}$ \\
\hline 1,2-Dibromoethane & ug/L & 1 & - & & $<1$ & Uu & $<1$ & ju & $<1$ & ju & $<1$ & UU & $<1$ & U & $<2$ & U & $<1$ & ju & $<1$ & ju \\
\hline 1,2-Dichlorobenzene & ug/L & 10 & 600 & \# & $<1$ & ju & $<1$ & ju & $<1$ & ju & $<1$ & U & $<1$ & ju & $<2$ & jU & $<1$ & ju & $<1$ & ju \\
\hline 1,2-Dichloroethane & $\mathrm{ug} / \mathrm{L}$ & 1 & 0.5 & & $<1$ & |U & $<1$ & $\mathrm{U}$ & $<1$ & IU & $<1$ & U & $<1$ & ju & $<2$ & $\mathbf{U}$ & $<1$ & ju & $<1$ & $\mathrm{U}$ \\
\hline 1,2-Dichloropropane & $\mathrm{ug} / \mathrm{L}$ & 1 & 5 & & $<1$ & ןU & $<1$ & ju & $<1$ & ju & $<1$ & jU & $<1$ & ju & $<2$ & ju & $<1$ & ju & $<1$ & J \\
\hline 1,3-Dichlorobenzene & $\mathrm{ug} / \mathrm{L}$ & 10 & - & & $<1$ & ju & $<1$ & $\mathrm{U}$ & $<1$ & U & $<1$ & $\mathbf{U}$ & $<\overline{1}$ & jU & $<2$ & U & $<1$ & $\mathrm{U}$ & $<1$ & $\mathrm{u}$ \\
\hline 1,4-Dichlorobenzene & $\mathrm{ug} / \mathrm{L}$ & 10 & 5 & & $<1$ & $\mathbf{j} \mathbf{U}$ & $<1$ & $\mathrm{ju}$ & $<1$ & ju & $<1$ & ju & $<1$ & ju & $<2$ & ju & $<1$ & ju & $<1$ & ju \\
\hline 2-Butanone & ug/L & 5 & - & & $<5$ & $R c j U$ & $<5$ & $\operatorname{Re} \mathbf{U}$ & $<5$ & 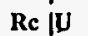 & $<5$ & $\operatorname{Re} \mathbf{U}$ & $<5$ & $\operatorname{Re} j$ & $<10$ & $\mathbf{R c j}$ & $<5$ & ju & $<5$ & U U \\
\hline 2-Hexanone & ug/L & 5 & - & & $<5$ & ju & $<5$ & $\mathbf{j}$ & $<5$ & jU & $<5$ & ju & $<5$ & ju & $<10$ & ju & $<5$ & ju & $<5$ & ju \\
\hline 4-Methyl-2-Pentanone & $\mathrm{ug} / \mathrm{L}$ & 5 & - & & $<5$ & ju & $<5$ & ju & $<5$ & $\mathbf{U}$ & $<5$ & jU & $<5$ & jU & $<10$ & ju & $<5$ & U & $<5$ & U \\
\hline Acctone & ugl & 5 & - & & $<5$ & $R c j u$ & $<5$ & $R_{c} \mid \mathbf{U}$ & $<5$ & Rcj|U & $<5$ & $\mathbf{R e} \boldsymbol{j} \mathbf{U}$ & $<5$ & $\mathbf{R c} \boldsymbol{j} \mathbf{U}$ & $<10$ & $\mathbf{R c}$ jU & 2.3 & Jc [BJ & $<5$ & $\mathbf{R c} \mid \mathbf{U}$ \\
\hline Benzene & $\mathrm{ug} / \mathrm{L}$ & 1 & 1 & & $<1$ & ju & $<1$ & UU & $<1$ & ju & $<1$ & Ju & $<1$ & ju & $<2$ & ju & $<1$ & jU • & $<1$ & jU \\
\hline Bromochloromethane & $\mathrm{ug} / \mathrm{L}$ & 1 & - & & $<1$ & ju & $<1$ & ןU & $<1$ & ju & $<1$ & jU & $<1$ & ju & $<2$ & ju & $<1$ & [U & $<1$ & ju \\
\hline Bromodichloromethane & ug/L & 1 & 100 & a & $<1$ & $\mathbf{U}$ & $<1$ & ju & $<1$ & ju & $<1$ & $\mathrm{U}$ & $<1$ & ju & $<2$ & ju & $<1$ & ju & $<1$ & ju \\
\hline Bromoform & ug/L & 1 & 100 & a & $<1$ & ju & $<1$ & jU & $<1$ & ju & $<1$ & ju & $<1$ & ju & $<2$ & ju & $<1$ & ju & $<1$ & ju \\
\hline Bromomethane & ug/L & 1 & - & & $<1$ & $\mathrm{Uz}$ & $<1$ & U & $<1$ & jU & $<1$ & ju & $<1$ & $\mathbf{U}$ & $<2$ & $\mathbf{U}$ & $<1$ & ju & $<1$ & ju \\
\hline Carbon Disulfide & ug/L & 1 & - & & $<1$ & $\mathbf{j U}$ & $<1$ & ju & $<1$ & ju & $<1$ & ju & $<1$ & ju & $<2$ & ju & $<1$ & ju & $<1$ & ju \\
\hline Carbon Tetrachloride & ug/L & 1 & 0.5 & & $<1$ & jU & $<1$ & jU & $<1$ & ju & $<1$ & ju & $<1$ & ju & $<2$ & $\mathbf{U}$ & $<1$ & $\mathbf{U}$ & $<1$ & $\mathbf{U}$ \\
\hline Chlorobenzene & $\mathrm{ug} / \mathrm{h}$ & 1 & 30 & & $<1$ & ן & $<1$ & ju & $<1$ & ju & $<1$ & ju & $<1$ & ju & $<2$ & ju & $<1$ & ju & $<1$ & ju \\
\hline Chlorocthane & ug/L & 1 & - & & $<1$ & ju & $<1$ & ju & $<1$ & $\mathbf{U}$ & $<1$ & U & $<1$ & ju & $<2$ & ן & $<1$ & jU & $<1$ & $\mathbf{U}$ \\
\hline Chloroform & ug/L & 1 & 100 & a & $<1$ & ju & $<1$ & jU & $<1$ & jU & $<1$ & ju & $<1$ & $\mathrm{j}$ & $<2$ & ju & $<1$ & ju & $<1$ & ju \\
\hline Chloromethane & ug/L & 1 & - & & $<\dot{1}$ & U & $<1$ & [U & $<1$ & jU & $<1$ & ju & $<1$ & ju & $<2$ & ju & $<1$ & ju & $<1$ & ju \\
\hline Dibromochloromethane & ug/L & 1 & 100 & a & $<1$ & ju & $<1$ & jU & $<1$ & jU & $<i$ & |U & $<1$ & ju & $<2$ & ju & $<1$ & ju & $<1$ & ju \\
\hline Ethyl Benzene & ug/L & 1 & 680 & & $<1$ & ju & $<1$ & ju & $<1$ & ju & $<1$ & jU & $<1$ & ju & $<2$ & Ju & $<1$ & ju & $<1$ & $\mathbf{U}$ \\
\hline Methylene Chloride & ug/L & 2 & 5 & & $<2$ & jU & $<2$ & ju & $<2$ & ju & $<2$ & jU & $<2$ & ן & $<4$ & ju & $<2$ & ju & $<2$ & ju \\
\hline Styrene & ug/L & 1 & $100 t$ & \# & $<1$ & ju & $<1$ & jU & $<1$ & [U & $<1$ & ju & $<1$ & ju & $<2$ & ju & $<1$ & ju & $<1$ & $\cdot \mathbf{j u}$ \\
\hline Tetrachloroethene & ug/L & 1 & 5 & & $<1$ & ju & $<1$ & ju & $<1$ & ju & $<1$ & ju & $<1$ & ju & $<2$ & ju & $<1$ & ju & $<1$ & $\mathbf{U}$ \\
\hline Iuene & ug/L & 1 & 1000 & \# & $<1$ & ju & $<1$ & ןU & $<1$ & jU & $<1$ & ju & $<1$ & ju & $<2$ & ju & $<1$ & jU & $<1$ & [U \\
\hline Trichloroethene & ug/L & 1 & 5 & & $<1$ & ju & $<1$ & ju & $<1$ & ju & $<1$ & ju & $<1$ & ju & $<2$ & U & $<1$ & IU & $<1$ & UU \\
\hline Vinyl Chloride & $\mathrm{ug} / \mathrm{L}$ & 1 & 0.5 & & $<1$ & ן & $<1$ & ןU & $<1$ & ju & $<1$ & ju & $<1$ & ju & $<2$ & ju & $<1$ & ןU & $<1$ & jU \\
\hline Xylenes (Totai) & ug/L & 1 & $1750^{\circ}$ & & 1.3 & & $<1$ & jU & $<1$ & ju & $<1$ & ju & $<1$ & ju & $<2$ & U & $<1$ & $\mathbf{U}$ & $<1$ & ju \\
\hline cis-1,2-Dichloroethene & ug/L & 1 & 6 & & $<1$ & $\mathrm{U}$ & $<1$ & ju & $<1$ & ju & $<1$ & jU & $<1$ & ju & $<2$ & ju & $<1$ & jU & $<1$ & ju \\
\hline -1,3-Dichloropropene & ug/L & 1 & - & & $<1$ & ju & $<1$ & ju & $<1$ & ju & $<1$ & ju & $<1$ & ju & $<2$ & J & $<1$ & jU & $<1$ & U \\
\hline trans-1,2-Dichloroethene & ug/L & 1 & 10 & & $<1$ & ju & $<1$ & ju & $<1$ & jU & $<1$ & ju & $<1$ & ju & $<2$ & ju & $<1$ & ju & $<1$ & ju \\
\hline trans-1,3-Dichloropropene & ug/L & 1 & - & & $<1$ & ן & $<1$ & ju & $<1$ & ju & $<1$ & ju & $<1$ & ju & $<2$ & ju & $<1$ & jU & $<\mathbf{i}$ & ju \\
\hline
\end{tabular}




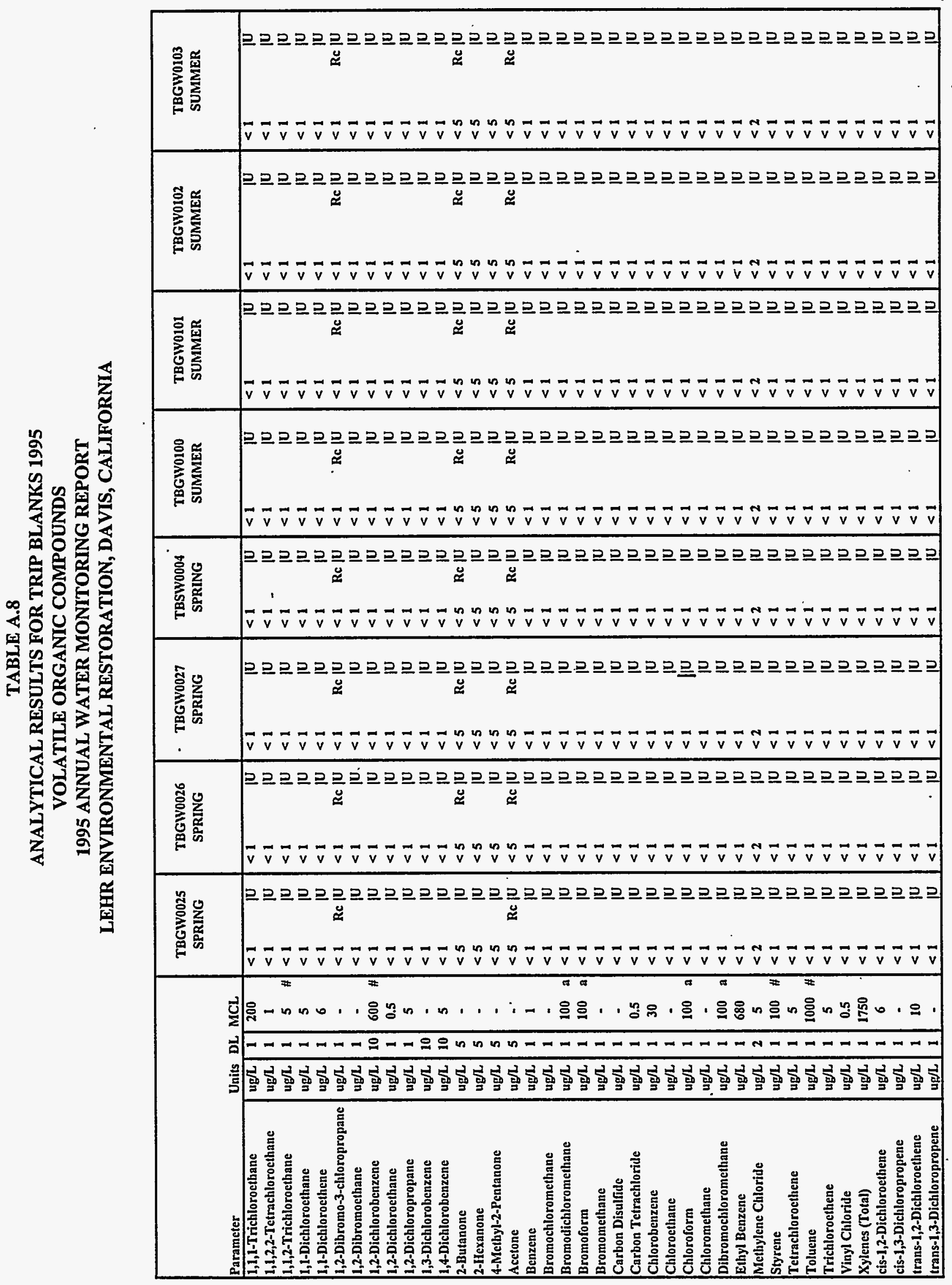


TABLE A.8

ANALYTICAL RESULTS FOR TRIP BLANKS 1995

VOLATILE ORGANIC COMPOUNDS

1995 ANNUAL WATER MONITORING REPORT

LEHR ENVIRONMENTAL RESTORATION, DAVIS, CALIFORNIA

\begin{tabular}{|c|c|c|c|c|c|c|c|c|c|c|c|c|c|c|c|c|c|c|c|}
\hline Parameter & Units & DL & MCL & \multicolumn{2}{|c|}{$\begin{array}{l}\text { TBGW0104 } \\
\text { SUMMER }\end{array}$} & \multicolumn{2}{|c|}{$\begin{array}{l}\text { TBGW0105 } \\
\text { SUMMER }\end{array}$} & \multicolumn{2}{|c|}{$\begin{array}{l}\text { TBGWO113 } \\
\text { FALL }\end{array}$} & \multicolumn{2}{|c|}{$\begin{array}{l}\text { TBGWV114 } \\
\text { FALL }\end{array}$} & \multicolumn{2}{|c|}{$\begin{array}{l}\text { TBGW0115 } \\
\text { FALL }\end{array}$} & \multicolumn{2}{|c|}{$\begin{array}{l}\text { TBGW0122 } \\
\text { FALL }\end{array}$} & \multicolumn{2}{|c|}{$\begin{array}{c}\text { TBGW0123 } \\
\text { FALL }\end{array}$} & \multicolumn{2}{|c|}{$\begin{array}{l}\text { TBGW0124 } \\
\text { FALL }\end{array}$} \\
\hline 1,1,1-Trichloroethane & ug/h & T] & 200 & $<1$ & & $<1$ & |U & $<1$ & JU & $<1$ & & $<1$ & $\bar{U}$ & $<1$ & IU & $<1$ & $\overline{\mathrm{IU}}$ & $<1$ & \\
\hline 1,1,2,2-Tetrachloroethane & ug/L & 1 & 1 & $<1$ & ju & $<1$ & ju & $<1$ & ju & $<1$ & ju & $<1$ & ju & $<1$ & ju & $<1$ & ju & $<1$ & ju \\
\hline 1,1,2-Trichlorocthane & ug $/ \mathbf{L}$ & 1 & 5 & $<1$ & ju & $<1$ & jU & $<1$ & ju & $<1$ & U & $<1$ & ju & $<1$ & $\mathbf{U}$ & $<1$ & ju & $<1$ & (U) \\
\hline 1,1-Dichloroethane & ug/L & 1 & 5 & $<1$ & ju & $<1$ & ju & $<1$ & ju & $<1$ & ju & $<1$ & ju & $<1$ & ju & $<1$ & jU & $<1$ & ju \\
\hline 1,1-Dichloroethene & $\mathrm{ug} / \mathbf{L}$ & 1 & 6 & $<1$ & ju & $<1$ & U & $<1$ & ju & $<1$ & נן & $<1$ & ju & $<1$ & ju & $<1$ & ju & $<1$ & U \\
\hline 1,2-Dibromo-3-chloropropane & ug/L & 1 & - & $<1$ & ju & $<1$ & Rc jU & $<1$ & ju & $<1$ & ju & $<1$ & ju & $<1$ & ju & $<1$ & [U & $<1$ & ju \\
\hline 1,2-Dibromoethane & $\mathbf{u g} / \mathbf{L}$ & 1 & - & $<1$ & ju & $<1$ & jU & $<1$ & jU & $<1$ & ju & $<1$ & ju & $<1$ & ju & $<1$ & jU & $<1$ & ju \\
\hline 1,2-Dichlorobenzene & ug/h & 10 & $600 \#$ & $<1$ & ju & $<1$ & ju & $<1$ & ju & $<1$ & ju & $<1$ & ju & $<1$ & ju & $<1$ & UU & $<1$ & ju \\
\hline 1,2-Dichloroethane & $\mathrm{ug} / \mathrm{L}$ & 1 & 0.5 & $<1$ & ju & $<1$ & ju & $<1$ & |U & $<1$ & ju & $<1$ & ן $\mathbf{U}$ & $<1$ & |U & $<1$ & |U & $<1$ & [U \\
\hline 1,2-Dichloropropane & ug/L & 1 & 5 & $<1$ & jU & $<1$ & ju & $<1$ & ju & $<1$ & ju & $<1$ & ju & $<1$ & jU & $<1$ & |U & $<1$ & ju \\
\hline 1,3-Dichlorobenzene & ug/L & 10 & - & $<1$ & ju & $<1$ & jU & $<1$ & jU & $<1$ & ju & $<1$ & ju & $<1$ & ju & $<1$ & ju & $<1$ & ju \\
\hline 1,4-Dichlorobenzene & ug/L & 10 & 5 & $<1$ & ju & $<1$ & ju & $<1$ & jU & $<1$ & ju & $<1$ & ju & $<1$ & ju & $<1$ & ju & $<1$ & ju \\
\hline 2-Butanone & ug/L & 5 & - & $<5$ & U & $<5$ & $\operatorname{Rc} \mathbf{U}$ & $<5$ & Rc jU & $<5$ & $\mathbf{R e} \mathbf{U}$ & $<5$ & $\operatorname{Re} \mathbf{U}$ & $<5$ & $\operatorname{Re} \mathbf{U}$ & $<5$ & 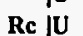 & $<5$ & $\operatorname{Re} U$ \\
\hline 2-Hexanone & ug/L & 5 & - & $<5$ & ju & $<5$ & jU & $<5$ & ju & $<5$ & ju & $<5$ & $\mathrm{ju}$ & $<5$ & UU & $<5$ & ju & $<5$ & U \\
\hline 4-Methyl-2-Pentanone & $\mathrm{ug} / \mathrm{L}$ & 5 & - & $<5$ & ju & $<5$ & $\mathbf{U}$ & $<5$ & ju & $<5$ & ju & $<5$ & ju & $<5$ & $\mathbf{U}$ & $<5$ & $\mathbf{U}$ & $<5$ & $\mathbf{U}$ \\
\hline Acctone & $\mathrm{ug} / \mathrm{L}$ & 5 & - & $<5$ & ju & $<5$ & $R c j U$ & 2.3 & $\mathbf{J c}$ [J & 2.5 & Je J J & $<5$ & $\operatorname{Re} \mathbf{U}$ & $<5$ & Re $\mathbf{U}$ & $<5$ & Rc $\mathbf{U}$ & $<5$ & $\operatorname{Re} \mathbf{U}$ \\
\hline Benzene & ug/L & 1 & 1 & $<1$ & ju & $<1$ & UU & $<1$ & U & $<1$ & ju & $<1$ & ju & $<1$ & U & $<1$ & $\mathrm{U}$ & $<1$ & $\mathbf{U}$ \\
\hline Bromochloromethane & ug/L & 1 & - & $<1$ & ju & $<1$ & jU & $<1$ & jU & $<1$ & ju & $<1$ & ju & $<1$ & ju & $<1$ & ju & $<1$ & ju \\
\hline Bromodichloromethane & ug/l & 1 & $100 a$ & $<1$ & ju & $<1$ & jU & $<1$ & $\mathbf{U}$ & $<1$ & ju & $<1$ & |U & $<1$ & ju & $<1$ & jU & $<1$ & ju \\
\hline Bromoform & ug/L & $i$ & $100 \mathrm{a}$ & $<1$ & ju & $<1$ & U & $<1$ & ju & $<1$ & U & $<1$ & |U & $<1$ & ju & $<1$ & ju & $<1$ & ju \\
\hline Bromomethane & ug/L & 1 & - & $<1$ & ju & $<1$ & $\mathbf{U}$ & $<1$ & ju & $<1$ & ju & $<1$ & J & $<1$ & ju & $<1$ & ju & $<1$ & J \\
\hline Carbon Disulfide & $\mathrm{ug} / \mathrm{L}$ & 1 & - & $<1$ & jU & $<1$ & ן & $<1$ & ju & $<1$ & ju & $<1$ & ju & $<1$ & ju & $<1$ & $\mathrm{ju}$ & $<1$ & jU \\
\hline Carbon Tetrachloride & $\mathrm{ug} / \mathrm{L}$ & 1 & 0.5 & $<1$ & jU & $<1$ & jU & $<1$ & ju & $<1$ & ju & $<1$ & $\mathrm{jU}$ & $<1$ & |U & $<1$ & ju & $<1$ & ju \\
\hline Chlorobenzene & ug/L & 1 & 30 & $<1$ & ju & $<1$ & j & $<1$ & jU & $<1$ & ju & $<1$ & ju & $<1$ & ju & $<1$ & jU & $<1$ & jU \\
\hline Chlorocthane & ug/L & 1 & - & $<1$ & J & $<1$ & ju & $<1$ & $\mathbf{U}$ & $<1$ & ju & $<1$ & ju & $<1$ & $\mathbf{U}$ & $<1$ & U & $<1$ & U \\
\hline Chloroform & ug/L & 1 & $100 a$ & $<1$ & ju & $<1$ & ju & $<1$ & ןU & $<1$ & ju & $<1$ & jU & $<1$ & ju & $<1$ & ju & $<1$ & jU \\
\hline Chloromethane & $\mathrm{ug} / \mathrm{L}$ & 1 & - & $<1$ & ju & $<1$ & ju & $<1$ & |U & $<1$ & ju & $<1$ & ןU & $<1$ & jU & $<1$ & ju & $<1$ & ju \\
\hline Dibromochloromethane & $\mathrm{ug} / \mathrm{h}$ & 1 & $100 \mathrm{a}$ & $<1$ & ju & $<1$ & ju & $<1$ & ju & $<1$ & jU & $<1$ & ju & $<1$ & ju & $<1$ & ju & $<1$ & ju \\
\hline Ethyl Benzene & ug/L & 1 & 680 & $<1$ & ju & $<1$ & ju & $<1$ & $\mathbf{U}$ & $<1$ & ju & $<1$ & U & $<1$ & $\mathbf{U}$ & $<1$ & ju & $<1$ & IU \\
\hline Methylene Chloride & $\mathrm{ug} / \mathrm{L}$ & 2 & 5 & $<2$ & ju & $<2$ & jU & $<2$ & ju & $<2$ & ju & $<2$ & ju & $<2$ & ju & $<2$ & ju & $<2$ & $\mathbf{U}$ \\
\hline Styrene & ug/L & 1 & $100 \#$ & $<1$ & |U & $<1$ & ן $\mathbf{U}$ & $<1$ & jU & $<1$ & jU & $<1$ & ju & $<1$ & jU & $<1$ & jU & $<1$ & ju \\
\hline Tetrachloroethene & ug/L & 1 & 5 & $<1$ & ju & $<1$ & ju & $<1$ & ju & $<1$ & ju & $<1$ & ju & $<1$ & ju & $<1$ & |ं & $<1$ & ju \\
\hline Toluene & ug/L & 1 & $1000 \#$ & $<1$ & ju & $<1$ & ן & $<1$ & |U & $<1$ & ju & $<1$ & ju & $<1$ & jU & $<1$ & ju & $<1$ & jU \\
\hline Trichloroethene & $\mathrm{ug} / \mathrm{L}$ & 1 & 5 & $<1$ & ju & $<1$ & ju & $<1$ & |U & $<1$ & ju & $<1$ & [U & $<1$ & ju & $<1$ & ju & $<1$ & IU \\
\hline Vinyl Chloride & ug/L & 1 & 0.5 & $<1$ & ju & $<1$ & $\mathrm{j}^{*}$ & $<1$ & [U & $<1$ & ju & $<1$ & ju & $<1$ & jU & $<1$ & ju & $<1$ & IU \\
\hline Xylenes (Total) & ug/L & 1 & 1750 & $<1$ & ju & $<1$ & ju & $<1$ & ju & $<1$ & ju & $<1$ & ju & $<1$ & [U & $<1$ & U & $<1$ & ju \\
\hline cis-1,2-Dichloroethene & ug/L & 1 & 6 & $<1$ & |U & $<1$ & $\mathrm{U}$ & $<1$ & ןU & $<1$ & jU & $<1$ & |U & $<1$ & jU & $<1$ & [U & $<1$ & ju \\
\hline cis-1,3-Dichloropropene & og $/ \mathbf{L}$ & 1 & - & $<1$ & Ju & $<1$ & ju & $<1$ & $\mathbf{u}$ & $<1$ & jU & $<1$ & ju & $<1$ & UU & $<1$ & ju & $<1$ & J \\
\hline trans-1,2-Dichloroethene & ug/L & 1 & 10 & $<1$ & jU & $<1$ & jU & $<1$ & jU & $<1$ & ju & $<1$ & ju & $<1$ & jU & $<1$ & ju & $<1$ & ju \\
\hline trans-1,3-Dichloropropene & ug/L & 1 & - & $<1$ & jU & $<1$ & jU & $<1$ & jU & $<1$ & ju & $<1$ & ju & $<1$ & jU & $<1$ & ju & $<1$ & JU \\
\hline
\end{tabular}


TABLE A.8

ANALYTICAL RESULTS FOR TRIP BLANKS 1995

VOLATILE ORGANIC COMPOUNDS

1995 ANNUAL WATER MONITORING REPORT

LEHR ENVIRONMENTAL RESTORATION, DAVIS, CALIFORNIA

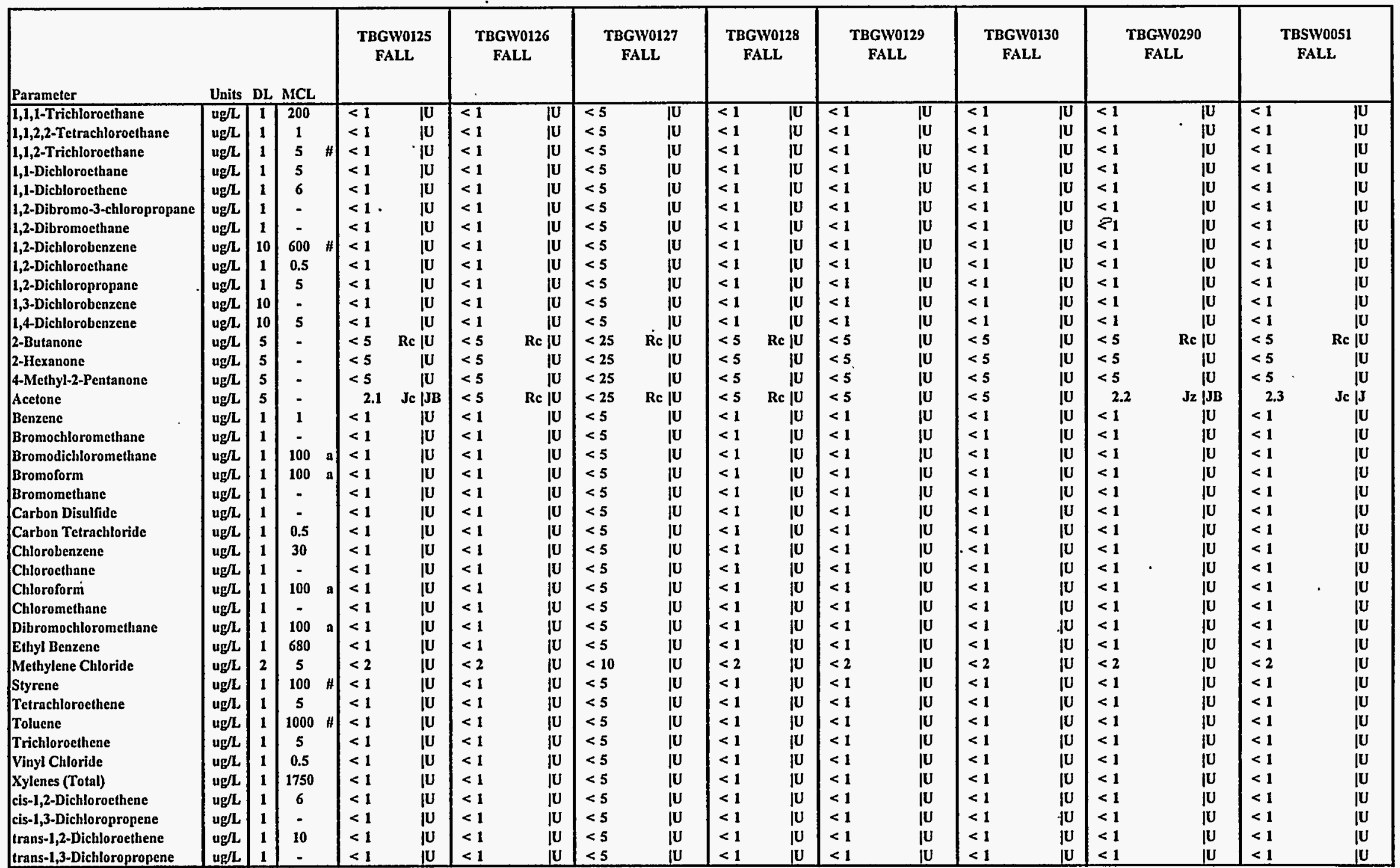


TABLE A.8

ANALYTICAL RESULTS FOR TRIP BLANKS 1995

VOLATILE ORGANIC COMPOUNDS

1995 ANNUAL WATER MONITORING REPORT

LEHR ENVIRONMENTAL RESTORATION, DAVIS, CALIFORNIA

D1 = Duplicate Sample

- = Parameter not analyzed or no MCL

DL = Detection Limit

$<$ Constituent below detection limit. Detection limits may vary depending on interference by other sample constituents.

MCL = Maximum Contaminant Level (primary), Title 22 Division 4 Chapter 15.

\# = USEPA MCL

a = MCL for total trihalomethanes, EPA, Region 9, PRG for specific trihalomethanes is less than this value.

See Appendix A for explanation of data qualifiers. 


\section{Appendix B}

Surface Water and Storm Water Analytical Results 



\section{Appendix B}

\section{Surface Water and Storm Water Analytical Results}

\section{How to Read Appendix B Data Qualifiers}

The key below defines the relationship between a given result and the associated data qualifiers, as presented in the tables of analytical results in Appendix B.

\begin{tabular}{|c|c|c|c|c||}
\hline Key $\rightarrow$ & A & B & C & D \\
\hline \hline Example Data $\rightarrow$ & $<$ & 5.200 & Uz & JB \\
\hline \hline A < Signifies that analyte level is less than indicated value. \\
B Indicated value in units as noted in table footnote. \\
C Data qualifier(s) ${ }^{*}$ assigned by Consultant's data review team. \\
D Data qualifier(s) ${ }^{*}$ assigned by reporting laboratory. \\
\hline
\end{tabular}

In the example above, the chemical was analyzed for, but was qualified as not detected due to the bias introduced by contamination in the method blank associated with this analysis. Presence of the analyte in the method blank is noted by the laboratory qualifier, "B." The Consultants qualifier, "Uz," indicates that the analyte concentration is considered to be below the adjusted detection limit (quantitation limit) due to the level of contamination in the method blank. 


\section{DATA VALIDATION QUALIFIERS \\ Assigned by Consultants Data Review Team}

The following definitions provide brief explanations of the qualifiers assigned in the data review process. Validation reason codes for GC/MS organics, GC organics, and metals are recorded by the data reviewers and retained in review support documentation for the data review process.

\begin{tabular}{|c|c|}
\hline Flag & Data Qualifier Definition \\
\hline$U$ & $\begin{array}{l}\text { The analyte was analyzed for, but was not detected above the reported sample } \\
\text { quantitation limit. }\end{array}$ \\
\hline $\mathrm{J}$ & $\begin{array}{l}\text { The analyte was positively identified; the associated numerical value is the approximate } \\
\text { concentration of the analyte in the sample. }\end{array}$ \\
\hline$N$ & $\begin{array}{l}\text { The analysis indicates the presence of an analyte for which there is presumptive } \\
\text { evidence to make a "tentative identification." }\end{array}$ \\
\hline NJ & $\begin{array}{l}\text { The analysis indicates the presence of an analyte that has been "tentatively identified" } \\
\text { and the associated numerical value represents its approximate concentration. }\end{array}$ \\
\hline UJ & $\begin{array}{l}\text { The analyte was not detected above the reported sample quantitation limit. However, } \\
\text { the reported.quantitation limit is approximate and may or may not represent the actual } \\
\text { limit of quantitation necessary to accurately and precisely measure the analyte in the } \\
\text { sample. }\end{array}$ \\
\hline $\mathbf{R}$ & $\begin{array}{l}\text { The sample results are rejected due to serious deficiencies in the ability to analyze the } \\
\text { sample and meet quality control criteria. The presence or absence of the analyte cannot } \\
\text { be verified. }\end{array}$ \\
\hline c & Calibration failure; poor or unstable response. \\
\hline d & Laboratory duplicate imprecision. \\
\hline$\dot{\mathrm{e}}$ & Laboratory duplicate control sample imprecision. \\
\hline$f$ & Field duplicate imprecision. \\
\hline h & Holding time violation. \\
\hline k & Serial dilution imprecision. \\
\hline 1 & Laboratory control sample recovery failure. \\
\hline $\mathbf{m}$ & Matrix spike/matrix spike duplicate recovery failure. \\
\hline ○ & Calibration blank contamination (metals/inorganics only). \\
\hline p & Preparation blank contamination (metals/inorganics only). \\
\hline
\end{tabular}




\section{Lockheed Analytical Services \\ Data Qualifiers for Organic Analyses \\ (Revised 01/19/1994)}

\begin{tabular}{|c|c|}
\hline \multicolumn{2}{|r|}{ For Use On the Analytical Data Reporting Forms } \\
\hline A & For CLP analyses only - The TIC is a suspected aldol-condensation product. \\
\hline B & $\begin{array}{l}\text { Any constituent that was also detected in the associated blank whose } \\
\text { concentration was greater than the practical or reporting detection limit (PQL or } \\
\text { RDL). }\end{array}$ \\
\hline C & Constituent confirmed by GS/MS analysis [pesticide/PCB analyses on/y]. \\
\hline D & $\begin{array}{l}\text { Constituent detected in the diluted sample. It also indicates that an accurate } \\
\text { quantitation is not possible due to surrogates being diluted out of the samples } \\
\text { during the course of the analysis. }\end{array}$ \\
\hline $\mathbf{E}$ & Constituent concentration exceeded the calibration range. \\
\hline $\mathbf{G}$ & $\begin{array}{l}\text { The quantitation is not gasoline or diesel but believed to be some other } \\
\text { combination of hydrocarbons. }\end{array}$ \\
\hline $\mathbf{H}$ & $\begin{array}{l}\text { Sample analysis performed outside of method- or client-specified maximum } \\
\text { holding time requirement. }\end{array}$ \\
\hline $\mathbf{J}$ & $\begin{array}{l}\text { Estimated value - (1) constituent detected at a level less than the RDL or PQL } \\
\text { and greater than or equal to the MDL; (2) estimated concentration for TICs (for } \\
\text { CLP reporting only) }\end{array}$ \\
\hline $\mathbf{N}$ & $\begin{array}{l}\text { For CLP reporting only - Tentatively identified constituents (TICs) identified } \\
\text { based on mass spectral library search. }\end{array}$ \\
\hline $\mathbf{P}$ & $\begin{array}{l}\text { For CLP reporting only - The percent difference between the concentrations } \\
\text { detected on both GC columns was greater than } 25 \text { percent [pesticide/PCB } \\
\text { analyses only]. }\end{array}$ \\
\hline $\mathbf{U}$ & $\begin{array}{l}\text { For CLP reporting only - Constituent was analyzed for but not detected } \\
\text { (sample quantitation must be corrected for dilution and percent moisture). }\end{array}$ \\
\hline $\mathrm{X}, \mathrm{Y}$, or $\mathrm{Z}$ & Analyst-defined qualifier. \\
\hline $\begin{array}{c}\text { N/A } \\
\text { (\% Moisture) }\end{array}$ & $\begin{array}{l}\text { N/A in the } \% \text { moisture cell indicates that data are reported on an "as received" } \\
\text { basis. A value in the \% moisture cell indicates that data are reported based on } \\
\text { a "dry weight" basis. For non-CLP work, RDLs are not adjusted for \% moisture } \\
\text { even when data are reported on a "dry weight" basis. }\end{array}$ \\
\hline \multicolumn{2}{|r|}{ For Use On the QC Data Reporting Forms } \\
\hline * & $\begin{array}{l}\text { QC data (i.e., percent recovery data for matrix spike, matrix spike duplicate, } \\
\text { laboratory control standard, or surrogates; and RPD for matrix spike duplicate } \\
\text { or unspiked duplicate) exceeded acceptance limits. }\end{array}$ \\
\hline
\end{tabular}




\section{Lockheed Analytical Services}

Data Qualifiers for Organic Analyses (contd)

\begin{tabular}{||c|l||}
\hline$a^{1}$ & $\begin{array}{l}\text { The spike recovery and/or RPD for matrix spike and matrix spike duplicates } \\
\text { cannot be evaluated due to insufficient spiking level compared to the elevated } \\
\text { sample analyte concentration. }\end{array}$ \\
\hline$b^{1}$ & $\begin{array}{l}\text { The RPD cannot be computed because the sample and/or duplicate } \\
\text { concentration was below the RDL. }\end{array}$ \\
\hline
\end{tabular}


Lockheed Analytical Services

Data Qualifiers for Inorganic Analyes

\begin{tabular}{|c|c|}
\hline \multicolumn{2}{|r|}{ For Use On the Analytical Data Reporting Forms } \\
\hline B & $\begin{array}{l}\text { For CLP analyses only - Reported value is less than the contract required } \\
\text { detection limit (CRDL) but greater than or equal to the instrument detection } \\
\text { limit (IDL). }\end{array}$ \\
\hline C & $\begin{array}{l}\text { For Routine, Non-CLP analyses only - Any constituent that was also detected } \\
\text { in the associated blank whose concentration was greater than the reporting } \\
\text { detection limit (RDL). }\end{array}$ \\
\hline D & $\begin{array}{l}\text { Presence of high levels of interfering constituents required dilution of sample } \\
\text { which increased the RDL by the dilution factor. }\end{array}$ \\
\hline $\mathbf{E}$ & Estimated value due to presence of interference. \\
\hline $\mathbf{H}$ & $\begin{array}{l}\text { Sample analysis performed outside of method- or client-specified maximum } \\
\text { holding time requirement. }\end{array}$ \\
\hline M & For CLP analyses only - Duplicate injection precision criterion was not met. \\
\hline $\mathbf{N}$ & Matrix spike recovery exceeded acceptance limits. \\
\hline $\mathbf{S}$ & Reported value was determined from the method of standard addition. \\
\hline $\mathbf{U}$ & $\begin{array}{l}\text { For CLP reporting only - Constituent was analyzed for but not detected } \\
\text { (sample quantitation must be corrected for dilution and percent moisture). }\end{array}$ \\
\hline $\mathbf{w}$ & $\begin{array}{l}\text { For AAS only - Post-digestion spike for Furnace AAS did not meet acceptance } \\
\text { criteria and sample absorbance is less than } 50 \% \text { of spike absorbance. }\end{array}$ \\
\hline$X, Y$, or $Z$ & Analyst-defined qualifier. \\
\hline * & $\begin{array}{l}\text { Relative Percent difference (RPD) for duplicate analysis exceeded acceptance } \\
\text { limits. }\end{array}$ \\
\hline+ & Correlation coefficient ( $r$ ) for the MSA is less than 0.995 . \\
\hline \multicolumn{2}{|r|}{ For Use On the QC Data Reporting Forms. } \\
\hline$a^{1}$ & $\begin{array}{l}\text { The spike recovery and/or RPD for matrix spike and matrix spike duplicates } \\
\text { cannot be evaluated due to insufficient spiking level compared to the elevated } \\
\text { sample analyte concentration. }\end{array}$ \\
\hline $\mathbf{b}^{1}$ & $\begin{array}{l}\text { The RPD cannot be computed because the sample and/or duplicate } \\
\text { concentration was below the RDL. }\end{array}$ \\
\hline
\end{tabular}




\begin{tabular}{||l|l||}
\hline \multicolumn{2}{|c|}{ For Use On the Analytical Data Reporting Forms } \\
\hline B & $\begin{array}{l}\text { Any constituent that was also detected in the associated blank whose } \\
\text { concentration was greater than the reporting detection limit (RDL) and/or } \\
\text { minimum detectable activity (MDA). }\end{array}$ \\
\hline C & $\begin{array}{l}\text { Presence of high TDS in sample required reduction of sample size which } \\
\text { increased the MDA. }\end{array}$ \\
\hline D & Constituent detected in the diluted sample. \\
\hline E & Constituent concentration exceeded the calibration or attenuation curve range. \\
\hline F & For Alpha Spectrometry Only - FWHM exceed acceptance limits. \\
\hline H & $\begin{array}{l}\text { Sample analysis performed outside of method-specified maximum holding time } \\
\text { requirement. }\end{array}$ \\
\hline Y & Chemical yield exceeded acceptance limits. \\
\hline$*$ & $\begin{array}{l}\text { FCor Use on the QC Data Reporting Forms } \\
\text { spike; and RPD for replicate analyses) exceeded acceptance limits. }\end{array}$ \\
\hline $\mathbf{a}^{1}$ & $\begin{array}{l}\text { The spike recovery and/or RPD for matrix spike and duplicates cannot be } \\
\text { evaluated due to insufficient spiking level compared to the elevated sample } \\
\text { analyte concentration. }\end{array}$ \\
\hline $\mathbf{b}^{1}$ & $\begin{array}{l}\text { The RPD cannot be computed because the sample and/or duplicate } \\
\text { concentration was below the MDA. }\end{array}$ \\
\hline
\end{tabular}




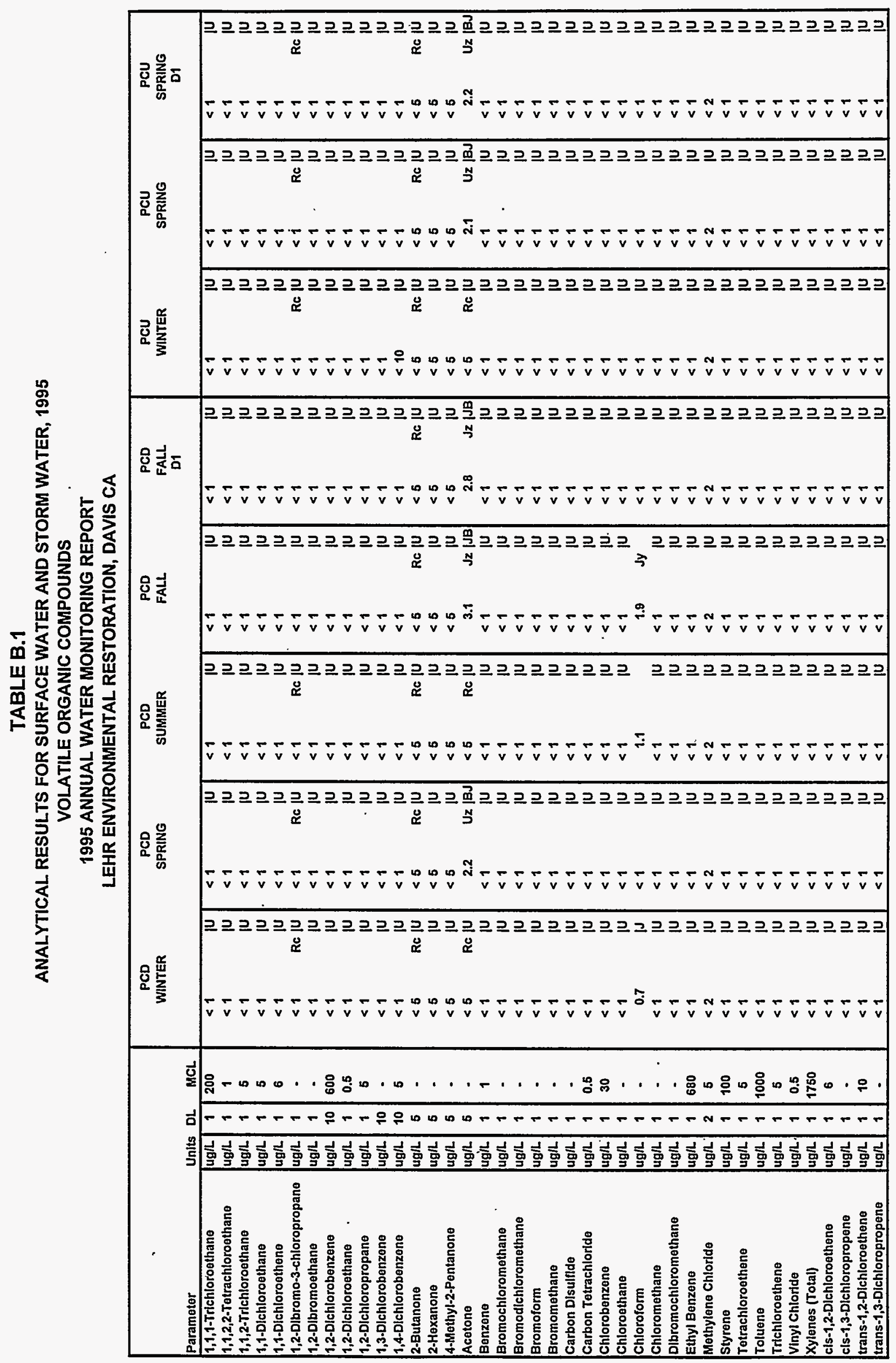


TABLE B.1

ANALYTICAL RESULTS FOR SURFACE WATER AND STORM WATER, 1995

VOLATILE ORGANIC COMPOUNDS

1995 ANNUAL WATER MONITORING REPORT

LEHR ENVIRONMENTAL RESTORATION, DAVIS CA

\begin{tabular}{|c|c|c|c|c|c|c|c|c|c|c|c|c|c|c|c|c|c|c|c|}
\hline Parameter & Units & DL & MCL & \multicolumn{2}{|c|}{$\begin{array}{c}\text { PCU } \\
\text { SUMMER }\end{array}$} & \multicolumn{2}{|c|}{$\begin{array}{c}\text { PCU } \\
\text { SUMMER } \\
\text { D1 }\end{array}$} & \multicolumn{2}{|c|}{$\begin{array}{l}\text { PCU } \\
\text { FALL }\end{array}$} & \multicolumn{2}{|c|}{$\begin{array}{c}\text { STPO } \\
\text { WINTER }\end{array}$} & \multicolumn{2}{|c|}{$\begin{array}{c}\text { STPO } \\
\text { WINTER } \\
\text { D1 }\end{array}$} & \multicolumn{2}{|c|}{$\begin{array}{c}\text { STPO } \\
\text { SPRING }\end{array}$} & \multicolumn{2}{|c|}{$\begin{array}{c}\text { STPO } \\
\text { SUMMER }\end{array}$} & \multicolumn{2}{|c|}{$\begin{array}{l}\text { STPO } \\
\text { FALL. }\end{array}$} \\
\hline 1,1,1-Trichloroethane & |ugh | & \begin{tabular}{l|l}
1 &
\end{tabular} & 200 & $<1$ & ןU & $<1$ & ju & $<1$ & ju & $<1$ & ju & $<1$ & IU & $<1$ & $\mathbf{U}$ & $<1$ & Ju & $<1$ & ju \\
\hline $1,1,2,2$-Tetrachloroethane & ugh & 1 & 1 & $<1$ & ju & $<1$ & ju & $<1$ & ju & $<1$ & ju & $<1$ & iu & $<1$ & ju & $<1$ & ju & $<1$ & ju \\
\hline 1,1,2-Trichloroethane & ugh & 1 & 5 & $<1$ & ju & $<1$ & ju & $<1$ & ju & $<1$ & ju & $<1$ & ju & $<1$ & ju & $<1$ & ju & $<1$ & ju \\
\hline 1,1-Dlchloroethane & uggh & 1 & 5 & $<1$ & ju & $<1$ & ju & $<1$ & ju & $<1$ & ju & $<1$ & ju & $<1$ & ju & $<1$ & ju & $<1$ & ju \\
\hline 1,1-Dlchloroethene & ugg $/ \mathrm{L}$ & 1 & 6 & $<1$ & IU & $<1$ & ju & $<1$ & U & $<1$ & ju & $<1$ & $\boldsymbol{U} \mathbf{U}$ & $<1$ & $\boldsymbol{U}$ & $<1$ & $\boldsymbol{U}$ & $<1$ & ju \\
\hline 1,2-Dlbromo-3-chloropropane & ug/L & 1 & - & $<1$ & $R \in \operatorname{Uu}$ & $<1$ & $R \in j U$ & $<1$ & ju & $<1$ & $R \in \operatorname{U}$ & $<1$ & $\operatorname{Re} \boldsymbol{U}$ & $<1$ & $\mathbf{R C} \boldsymbol{U} \mathbf{U}$ & $<1$ & $R \in \boldsymbol{U}$ & $<1$ & U \\
\hline 1,2-Dlbromoothane & ugh & 1 & - & $<1$ & ju & $<1$ & ju & $<1$ & ju & $<1$ & ju & $<1$ & ju & $<1$ & ju & $<1$ & ju & $<1$ & ju \\
\hline 1,2-Dlchlorobenzene & ugh & 10 & 600 & $<1$ & U & $<1$ & ju & $<1$ & ju & $<1$ & $\boldsymbol{U}$ & $<1$ & $\mathbf{U}$ & $<1$ & $\mathbf{u}$ & $<1$ & $\boldsymbol{U}$ & $<1$ & ju \\
\hline 1,2-Dichloroethane & ugh & 1 & 0.6 & $<1$ & ju & $<1$ & ju & $<1$ & ju & $<1$ & ju & $<1$ & ju & $<1$ & ju & $<1$ & ju & $<1$ & U \\
\hline 1,2-Dichloropropane & ugh & 1 & 5 & $<1$ & ju & $<1$ & ju & $<1$ & ju & $<1$ & ju & $<1$ & ju & $<1$ & ju & $<1$ & ju & $<1$ & ju. \\
\hline 1,3-Dlchlorobenzene & ughth & 10 & - & $<1$ & ju & $<1$ & U & $<1$ & ju & $<1$ & ju & $<1$ & ju & $<1$ & 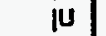 & $<1$ & ju & $<1$ & ן \\
\hline 1,4-Dlchlorobenzene & ug/L & 10 & 5 & $<1$ & $\boldsymbol{I U}$ & $<1$ & $\boldsymbol{U} \mathbf{U}$ & $<1$ & ఏU & $<1$ & $\boldsymbol{U}$ & $<1$ & $\boldsymbol{U}$ & $<1$ & Uu & $<1$ & $\boldsymbol{U} \mathbf{U}$ & $<1$ & JU \\
\hline 2-Butanone & ug $/ \mathrm{L}$ & 5 & - & $<5$ & $R \in$ JU & $<5$ & $\mathbf{R c} \boldsymbol{U}$ & $<5$ & $\operatorname{Rc} \boldsymbol{j} U$ & $<5$ & $R c \mid U$ & $<5$ & $R \in$ e U & $<5$ & $\mathbf{R c} \boldsymbol{U}$ & $<6$ & $\mathbf{R c} \boldsymbol{U} U$ & $<5$ & $\mathbf{R e} \boldsymbol{U} \mathbf{U}$ \\
\hline 2-Hexanone & ug $/ \mathrm{L}$ & 5 & - & $<5$ & ju & $<5$ & ju & $<5$ & ju & $<5$ & ju & $<5$ & ju & $<5$ & ju & $<5$ & ju & $<5$ & ju \\
\hline 4-Methy1-2-Pentanone & |ug/h & 5 & - & $<5$ & ju & $<5$ & ju & $<5$ & iU & $<5$ & ju & $<5$ & ju & $<5$ & $\boldsymbol{u}$ & $<6$ & iu & $<5$ & ju \\
\hline Acotone & ug/L & 5 & - & $<5$ & $R \in$ JU & $<5$ & $\mathbf{R c} \boldsymbol{U}$ & 2.8 & $\mathrm{Jz}$ |J $\mathrm{JB}$ & 2.2 & Je jJ & $<5$ & Reju & 3.1 & $\mathrm{Uz}|\mathrm{BJ}|$ & $<5$ & RG ju & 7.1 & $\mathrm{Jz}$ |B \\
\hline Benzene & ug/L & 1 & 1 & $<1$ & ju & $<1$ & ju & $<1$ & |i் | & $<1$ & $\boldsymbol{U} \mathbf{U}$ & $<1$ & IU & $<1$ & |u & $<1$ & IU & $<1$ & JU \\
\hline Bromochloromethane & ug $/ \mathrm{L}$ & 1 & - & $<1$ & ju & $<1$ & ju & $<1$ & ן & $<1$ & ju & $<1$ & ju & $<1$ & ju & $<1$ & ju & $<1$ & ju \\
\hline Bromodichloromethane & ug/L & 1 & - & $<1$ & ן & $<1$ & ju & $<1$. & ן & 1 & & 1.1 & & 2.6 & Jy & 1.1 & & 4.5 & \\
\hline Bromotorm & ug $/ \mathrm{L}$ & 1 & $:$ & $<1$ & ju & $<1$ & ju & $<1$ & ן & $<1$ & jU & $<1$ & U & 1.8 & & $<1$ & J & 2.9 & \\
\hline Bromomathane & |ug/L & 1 & - & $<1$ & ן & $<1$ & ju & $<1$ & ן & $<1$ & ju & $<1$ & ju & $<1$ & ן & $<1$ & ju & $<1$ & $\boldsymbol{U}$ \\
\hline Carbon Disulfido & $\operatorname{ug} / \mathrm{L}$ & 1 & - & $<1$ & ju & $<1$ & ju & $<1$ & ן & $<1$ & ju & $<1$ & $\boldsymbol{U}$ & $<1$ & U & $<1$ & ן & $<1$ & $\boldsymbol{U}$ \\
\hline Carbon Tetrachlorldo & $\mathrm{ug} / \mathrm{L}$ & 1 & 0.6 & $<1$ & ju & $<1$ & ju & $<1$ & ju & $<1$ & ju & $<1$ & ju & $<1$ & ju & $<1$ & ju & $<1$ & $\mathrm{U}$ \\
\hline Chlorobenzene & ug $/ L$ & 1 & 30 & $<1$ & ju & $<1$ & ju & $<1$ & ןU & $<1$ & ju & $<1$ & ju & $<1$ & ju & $<1$ & ju & $<1$ & U \\
\hline Chloroothane & |ug/L & 1 & - & $<1$ & ן & $<1$ & 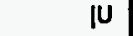 & $<1$ & ju & $<1$ & $\boldsymbol{U}$ & $<1$ & IU & $<1$ & $\boldsymbol{U}$ & $<1$ & Ju & $<1$ & IU \\
\hline Chloroform & |ug/L & 1 & - & $<1$ & ju & $<1$ & ju & $<1$ & ן & 3.7 & & 3.4 & & 2.2 & Jy & 5.3 & & 2.5 & Jy \\
\hline Chloromethane & ug $/ L$ & 1 & - & $<1$ & U & $<1$ & $\mathbf{u}$ & $<1$ & ju & $<1$ & ן $\mathbf{U}$ & $<1$ & IU & $<1$ & $\boldsymbol{U} \mathbf{U}$ & $<1$ & ן U & $<1$ & ju \\
\hline Dlbromochloromethane & ug/L & 1 & - & $<1$ & ju & $<1$ & ju & $<1$ & ju & $<1$ & ju & $<1$ & ן & 3.2 & & $<1$ & U & 6.4 & \\
\hline Ethyl Benzene & ug $/ \mathrm{L}$ & 1 & 680 & $<1$ & ju & $<1$ & ju & $<1$ & ju & $<1$ & ju & $<1$ & ju & $<1$ & ju & $<1$ & ju & $<1$ & U \\
\hline Methylene Chloride & ugh $/ \mathrm{h}$ & 2 & 6 & $<2$ & U & $<2$ & ju & $<2$ & נU & 7.4 & & 7.1 & & $<2$ & ju & 40 & $\mathrm{Jz}$ & 1.6 & J J \\
\hline Styrene & ug/L & 1 & 100 & $<1$ & |U & $<1$ & ju & $<1$ & ju & $<1$ & U & $<1$ & U & $<1$ & $\boldsymbol{U}$ & $<1$ & IU & $<1$ & ju \\
\hline Tetrachloroethene & ugh & 1 & 5 & $<1$ & U & $<1$ & ju & $<1$ & ju & $<1$ & U & $<1$ & ן & $<1$ & ן & $<1$ & ju & $<1$ & 伡 \\
\hline Toluenio & ugh $/ \mathrm{L}$ & 1 & 1000 & $<1$ & ן & $<1$ & ju & $<1$ & ju & $<1$ & $\mathbf{u}$ & $<1$ & ju & $<1$ & $\boldsymbol{u}$ & $<1$ & U & $<1$ & ju \\
\hline Trichloroethene & ug $/ \mathrm{L}$ & 1 & 5 & $<1$ & U & $<1$ & ju & $<1$ & ן & $<1$ & $\boldsymbol{u} \mathbf{u}$ & $<1$ & ן. & $<1$ & $\boldsymbol{U}$ & $<1$ & U & $<1$ & 伡 \\
\hline Vinyl Chlorlde & ugh $h$ & 1 & 0.5 & $<1$ & ן & $<1$ & ju & $<1$ & ן & $<1$ & IU & $<1$ & ju & $<1$ & 跑 & $<1$ & ן & $<1$ & ju \\
\hline Xylones (Total) & ug/h & 1 & 1750 & $<1$ & ן & $<1$ & ן & $<1$ & ן & $<1$ & ju & $<1$ & ju & $<1$ & U & $<1$ & נu & $<1$ & נן \\
\hline cls-1,2-Dichioroethene & ugh & 1 & 6 & $<1$ & ju & $<1$ & ju & $<1$ & ןU & $<1$ & ju & $<1$ & ju & $<1$ & u & $<1$ & U & $<1$ & U \\
\hline cls-1,3-Dlchloropropene & ug/L & 1 & - & $<1$ & ju & $<1$ & ju & $<1$ & ן & $<1$ & ן U & $<1$ & ju & $<1$ & IU & $<1$ & ju & $<1$ & נU \\
\hline trans-1,2-Dichloroethene & $\operatorname{ugg} / \mathrm{L}$ & 1 & 10 & $<1$ & ju & $<1$ & ju & $<1$ & ן & $<1$ & U & $<1$ & U & $<1$ & ן & $<1$ & !U & $<1$ & IU \\
\hline trans-1,3.Dlchloropropene & Lugh & 1 & $\cdot$ & $<1$ & IU & $<1$ & IU & $<1$ & ju & $<1$ & IU & $<1$ & Iu & $<1$ & ju & $<1$ & IU & $<1$ & IU \\
\hline
\end{tabular}


TABLE B.1

ANALYTICAL RESULTS FOR SURFACE WATER AND STORM WATER, 1995 VOLATILE ORGANIC COMPOUNDS

1995 ANNUAL WATER MONITORING REPORT LEHR ENVIRONMENTAL RESTORATION, DAVIS CA

\begin{tabular}{|c|c|c|c|c|c|c|c|c|c|}
\hline Parameter & Units & DL & $M C L$ & \multicolumn{2}{|c|}{$\begin{array}{c}\text { SWL00001 } \\
\text { WINTER }\end{array}$} & \multicolumn{2}{|c|}{$\begin{array}{c}\text { SWL00002 } \\
\text { WINTER }\end{array}$} & \multicolumn{2}{|c|}{$\begin{array}{c}\text { SWL00002 } \\
\text { WINTER } \\
\text { D1 }\end{array}$} \\
\hline 1,1,1-Trichloroethane & ugh/L & 1 & 200 & $<1$ & IU & $<1$ & IU & $<1$ & IU \\
\hline $1,1,2,2$-Tetrachloroethane & ugh & 1 & 1 & $<1$ & ju & $<1$ & ' & $\leqslant 1$ & ju \\
\hline 1,1,2-Trlehloroethane & ug/L & 1 & 5 & $<1$ & ju & $<1$ & ju & $<1$ & ju \\
\hline 1,1-Dlchloroethane & ugh/L & 1 & 5 & $<1$ & ju & $<1$ & ju & $<1$ & ju \\
\hline 1,1.Dichloroethene & ug/L & 1 & 6 & $<1$ & ju & $<1$ & ju & $<1$ & ju \\
\hline 1,2-Dlbromo-3-chloropropane & |ug/L & 1 & - & $<1$ & $\mathbf{R} \boldsymbol{e} \boldsymbol{U}$ & $<1$ & $R \in \boldsymbol{U} U$ & $<1$ & Rc JU \\
\hline 1,2-Dibromoethane & ug/L & 1 & - & $<1$ & ju & $<1$ & JU & $<1$ & IU \\
\hline 1,2-Dlchlorobenzene & ug/L & 10 & 600 & $<1$ & ju & $<1$ & U. & $<1$ & ju \\
\hline 1,2-Dichloroethane & |ug/L & 1 & 0.5 & $<1$ & ju & $<1$ & ן & $<1$ & ן \\
\hline 1,2-Dlchloropropane & |ugh & 1 & 5 & $<1$ & ju & $<1$ & U & $<1$ & ן U \\
\hline 1,3-Dlchlorobenzene & uggh & 10 & - & $<1$ & ju & $<1$ & $\boldsymbol{U}$ & $<1$ & $\boldsymbol{U}$ \\
\hline 1,4-Dlchlorobenzene & ug/L & 10 & 5 & $<1$ & ju & $<1$ & $\boldsymbol{U}$ & $<1$ & ju \\
\hline 2-Butanone & ug/L & 6 & - & $<6$ & $\operatorname{Re} \boldsymbol{U}$ & $<5$ & $\mathbf{R c} \boldsymbol{U}$ & $<5$ & $\mathbf{R} \mathbf{g} \boldsymbol{U}$ \\
\hline 2-Hexanone & ug/L & 5 & - & $<5$ & ju & $<5$ & ju & $<5$ & ju \\
\hline 4-Methyl-2-Pentanone & ugh & 5 & - & $<5$ & ju & $<5$ & ju & $<5$ & ן \\
\hline Acetone & ug/L & $\dot{5}$ & - & 6 & Je & 4.6 & UJG 昍J & 8.8 & Je \\
\hline Benzene & ugh & 1 & 1 & $<1$ & Ju & $<1$ & ju & $<1$ & IU \\
\hline Bromochloromethane & ugh & 1 & - & $<1$ & ju & $<1$ & U & $<1$ & ju \\
\hline Bromodlchloromethane & ug $/ \mathrm{L}$ & 1 & - & $<1$ & ן & $<1$ & ju & $<1$ & IU \\
\hline Bromóform & |ugh & 1 & - & $<1$ & ju & $<1$ & ju & $<1$ & ju \\
\hline Bromomethane & ug/L & 1 & - & $<1$ & ju & $<1$ & ju & $<1$ & ju \\
\hline Carbon DIsulflde & ugh $/ \mathrm{L}$ & 1 & - & $<1$ & ju & $<1$ & ju & $<1$ & ן \\
\hline Carbon Tetrachloride & |ugh & 1 & 0.6 & $<1$ & ן & $<1$ & ju & $<1$ & ju \\
\hline Chlorobenzene & ugh & 1 & 30 & $<1$ & ןU & $<1$ & ן & $<1$ & ju \\
\hline Chloroothane & ug $/ \mathrm{L}$ & 1 & - & $<1$ & UU & $<1$ & U & $<1$ & IU \\
\hline Chloroform & |ug/h & 1 & - & $<1$ & ju & $<1$ & $\mathbf{u}$ & $<1$ & ן \\
\hline Chloromethane & ugh & 1 & - & $<1$ & jU & $<1$ & U & $<1$ & ן U \\
\hline Dlbromochloromethane & ugg/L & 1 & - & $<1$ & ju & $<1$ & ju & $<1$ & Uاן \\
\hline Ethyl Benzene & ug/L & 1 & 680 & $<1$ & ju & $<1$ & $\boldsymbol{U}$ & $<1$ & !U \\
\hline Methylene Chloride & ugh & 2 & 5 & $<2$ & ju & $<2$ & U & $<2$ & ן \\
\hline Styrene & ug/ & 1 & 100 & $<1$ & ju & $<1$ & ju & $<1$ & ju \\
\hline Tetrachloroethene & ugh & 1 & 5 & $<1$ & ju & $<1$ & ju & $<1$ & ju \\
\hline Toluene & |ug/L & 1 & 1000 & 0.99 & IJ & $<1$ & $\boldsymbol{U}$ & $<1$ & U \\
\hline Trichloroethene & ug/L & 1 & 5 & $<1$ & ju & $<1$ & U & $<1$ & ju \\
\hline Vlnyl Chlorlde & ug/L & 1 & 0.5 & $<1$ & ju & $<1$ & ן & $<1$ & ju \\
\hline Xylenes (Total) & ugh & 1 & 1750 & $<1$ & . & $<1$ & U & $<1$ & ju \\
\hline cis-1,2-Dichloroethene & jugh & 1 & 6 & $<1$ & ju & $<1$ & ju & $<1$ & ju \\
\hline cls-1,3-Dlchloropropene & |ugh & 1 & - & $<1$ & ju & $<1$ & ju & $<1$ & ju \\
\hline trans-1,2-Dlchloroethene & ugh & 1 & 10 & $<1$ & נU & $<1$ & U & $<1$ & ju \\
\hline trans-1,3-Dlchloropropene & ugh & 1 & $\dot{-}$ & $<1$ & ju & $<1$ & ju & $<1$ & ju \\
\hline
\end{tabular}


TABLE B.1

ANALYTICAL RESULTS FOR SURFACE WATER AND STORM WATER, 1995

VOLATILE ORGANIC COMPOUNDS

ANNUAL WATER MONITORING REPORT

LEHR ENVIRONMENTAL RESTORATION, DAVIS CA

$\mathrm{DI}=$ Dupllcate sample.

. = Parameter not analyzed. Storm water runoff avallable for sampllng In March, 1995 only.

$<=$ Constituent below detectlon IImit. Detection limits may vary depending on interference by other sample constituents.

MCL = MaxImum Contaminant Lovel (prlmary), Titlo 22 Division 4 Chapter 15.

$-=$ Proposed USEPA MCL.

\# = USEPA MCL.

See Appendlx $B$ for explanation of data quallflers.

$A=M C L$ for total trihalomethanes is $100 \mathrm{ug} / \mathrm{L}$. Chloroform is the predominant tr/halomethane detected at LEHR. 
TABLE B.2

ANALYTICAL RESULTS FOR SURFACE WATER AND STORM WATER, 1995

SEMIVOLATILE ORGANIC COMPOUNDS

1995 ANNUAL WATER MONITORING REPORT

LEHR ENVIRONMENTAL RESTORATION, DAVIS CA

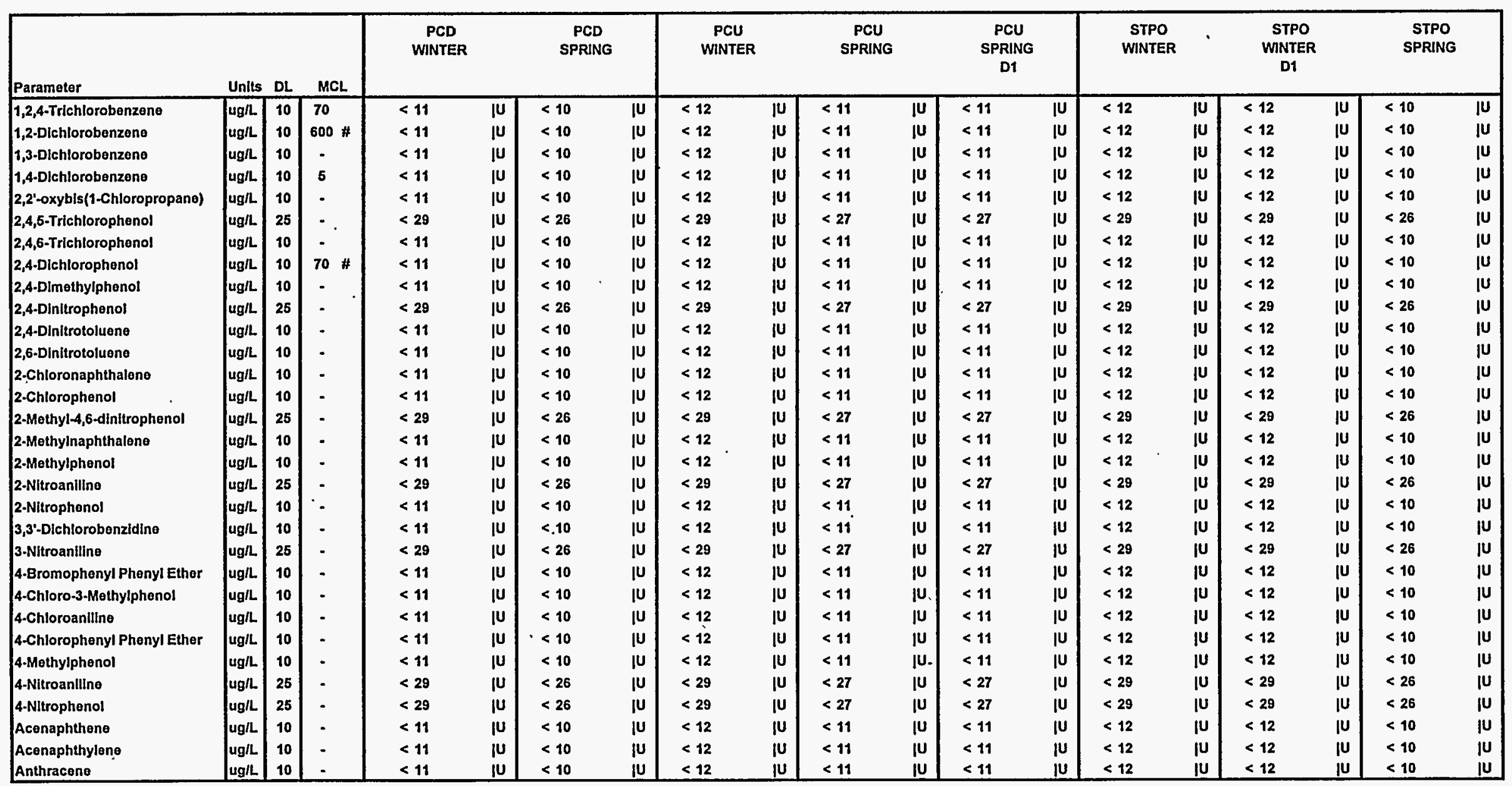


TABLE B.2

ANALYTICAL RESULTS FOR SURFACE WATER AND STORM WATER, 1995

SEMIVOLATILE ORGANIC COMPOUNDS

1995 ANNUAL WATER MONITORING REPORT

LEHR ENVIRONMENTAL RESTORATION, DAVIS CA

\begin{tabular}{|c|c|c|c|c|c|c|c|c|c|c|c|c|c|c|c|c|c|c|c|}
\hline \multirow{2}{*}{$\frac{\text { Parameter }}{\text { Benzo(a)anthracene }}$} & Units & $D L$ & $\mathrm{MCL}$ & \multicolumn{2}{|c|}{$\begin{array}{c}\text { PCD } \\
\text { WNTER }\end{array}$} & \multicolumn{2}{|c|}{$\begin{array}{c}\text { PCD } \\
\text { SPRING }\end{array}$} & \multicolumn{2}{|c|}{$\begin{array}{c}\text { PCU } \\
\text { WINTER }\end{array}$} & \multicolumn{2}{|c|}{$\begin{array}{l}\text { PCU } \\
\text { SPRING }\end{array}$} & \multicolumn{2}{|c|}{$\begin{array}{l}\text { PCU } \\
\text { SPRING } \\
\text { D1 }\end{array}$} & \multicolumn{2}{|c|}{$\begin{array}{l}\text { STPO } \\
\text { WINTER }\end{array}$} & \multicolumn{2}{|c|}{$\begin{array}{c}\text { STPO } \\
\text { WINTER } \\
\text { D1 }\end{array}$} & \multicolumn{2}{|c|}{$\begin{array}{c}\text { STPO } \\
\text { SPRING }\end{array}$} \\
\hline & ug $/ \mathrm{h}$ & 10 & $0.1 \cdot$ & $<11$ & ju & $<10$ & Ju & $<12$ & ju & $<11$ & jU & $<11$ & ju & $<12$ & UU & $<12$ & ju & $<10$ & jU \\
\hline Benzo(a)pyrene & ug $/ L$ & 10 & 0.2 & $<11$ & ju & $<10$ & ju & $<12$ & ju & $<11$. & ju & $<11$ & ju & $<12$ & ju & $<12$ & jU & $<10$ & ju \\
\hline Benzo(b)fluoranthene & ugg $/ L$ & 10 & 0.2 • & $<11$ & ju & $<10$ & ט & $<12$ & ju & $<11$ & ju & $<11$ & נן & $<12$ & ju & $<12$ & ju & $<10$ & ju \\
\hline Benzo(g,h,i)perylene & ugg $/ L$ & 10 & - & $<11$ & ju & $<10$ & Uן & $<12$ & ju & $<11$ & ט & $<11$ & ju & $<12$ & ju & $<12$ & ju & $<10$ & $\boldsymbol{u}$ \\
\hline Benzo(k)fluoranthene & ugih & 10 & 0.2 • & $<11$ & ju & $<10$ & $\mu$ & $<12$ & ju & $<11$ & $\boldsymbol{u}$ & $<11$ & ju & $<12$ & ju & $<12$ & ju & $<10$ & ju \\
\hline Benzyl Butyl Phthalate & ug/h & 10 & $100 \#$ & $<11$ & ju & $<10$ & ju & $<12$ & ju & $<11$ & ju & $<11$ & ju & $<12$ & ju & $<12$ & ju & $<10$ & $\boldsymbol{u}$ \\
\hline Bis(2-Chloroethoxy)methane & ug $/ \mathrm{L}$. & 10 & - & $<11$ & ju & $<10$ & ju & $<12$ & ן & $<11$ & ju & $<11$ & ju & $<12$ & ju & $<12$ & ju & $<10$ & ju \\
\hline Bis(2-Chloroethyl)ether & ug/L & 10 & - & $<11$ & ju & $<10$ & ju & $<12$ & ju & $<11$ & ju & $<11$ & ju & $<12$ & ju & $<12$ & ju & $<10$ & ju \\
\hline Bis(2-Ethylhexyl)phthalate & $\operatorname{ug} / \mathrm{L}$ & 10 & 4 & $<11$ & ju & 37 & $\mathrm{Uz}$ 百 & 0.7 & jJ & 2 & Uz |BJ & 10 & $\mathrm{Uz} \mid \mathrm{B} \mathrm{J}$ & 3 & j & 3 & jJ & 2 & Uz |BJ \\
\hline Carbazole & ugh & 10 & $\cdot$ & $<11$ & ju & $<10^{\circ}$ & ju & $<12$ & ju & $<11$ & ju & $<11$ & iu & $<12$ & ju & $<12$ & ju & $<10$ & ju \\
\hline Chrysene & ugg/L & 10 & 0.2 • & $<11$ & $\boldsymbol{u}$ & $<10$ & ju & $<12$ & ju & $<11$ & ju & $<11$ & ju & $<12$ & ju & $<12$ & ju & $<10$ & ju \\
\hline DI-n-Butylphthalato & ug/L & 10 & - & 3 & j & 59 & IB & $<12$ & ju & 130 & Jf $\mid \mathrm{B}$ & 38 & Jt & 3 & is & 2 & ju & 23 & 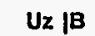 \\
\hline DI-n.Octylphthalate & $\mathrm{ug} / \mathrm{L}$ & 10 & - & $<11$ & ju & $<10$ & UJc ju & $<12$ & ju & $<11$ & UJe ju & $<11$ & UJe ju & $<12$ & ju & $<12$ & ju & $<10$ & UJc ju \\
\hline Dibenzo $(a, h)$ anthracene & ug/L & 10 & $0.3 \cdot$ & $<11$ & ju & $<10$ & ju & $<12$ & ju & $<11$ & ju & $<11$ & ju & $<12$ & ju & $<12$ & ju & $<10$ & $\boldsymbol{j u}$ \\
\hline Dlbenzofuran & ug/L & 10 & - & $<11$ & ju & $<10$ & ju & $<12$ & ju & $<11$ & ju & $<11$ & ju & $<12$ & ju & $<12$ & ju & $<10$ & $\boldsymbol{u}$ \\
\hline Dlothyl Phthalate & $u g / L$ & 10 & - & $<11$ & ju & 1. & ju & $<12$ & ju & 2 & is & 1 & jJ & $<12$ & ju & $<12$ & ju & 1 & js \\
\hline Dimethyl Phthalate & ug $/ \mathrm{L}$ & 10 & - & $<11$ & ju & $<10$ & ju & $<12$ & ju & $<11$ & ju & $<11$ & נU. & $<12$ & ju & $<12$ & ju & $<10$ & $\boldsymbol{U}$ \\
\hline Fluorantheno & ug/h & 10 & - & $<11$ & ju & $<10$ & ju & $<12$ & ju & $<11$ & ju & $<11$ & ju & $<12$ & IU & $<12$ & ju & $<10$ & $\mathbf{u}$ \\
\hline Fluorene & ug/h & 10 & - & $<11$ & ju & $<10$ & ju & $<12$ & ju & $<11$ & ju & $<11$ & טן & $<12$ & ן & $<12$ & ju & $<10$ & ju \\
\hline Hexachlorobenzene & ugh & 10 & 1 & $<11$ & u & $<10$ & ju & $<12$ & ju & $<11$ & ju & $<11$ & ju & $<12$ & ju & $<12$ & ju & $<10$ & ju \\
\hline Hexachlorobutadiene & |ugh & 10 & . & $<11$ & ju & $<10$ & ju & $<12$ & ju & $<11$ & ju & $<11$ & U & $<12$ & ן & $<12$ & ju & $<10$ & jU \\
\hline Hexachlorocyclopentadlene & ug/L & 10 & 60 & $<11$ & UJr $\mathbf{U}$ & $<10$ & ju & $<12$ & UJr ju & $<11$ & ju & $<11$ & ט & $<12$ & UJr ju & $<12$ & UJr U U & $<10$ & U \\
\hline Hexachloroethane & ug/L. & 10 & - & $<11$ & ju & $<10$ & ju & $<12$ & ju & $<11$ & U & $<11$ & ju & $<12$ & ju & $<12$ & UU & $<10$ & ju \\
\hline Indeno(1,2,3-cd)pyrene & ugh & 10 & 0.4 • & $<11$ & $\boldsymbol{u}$ & $<10$ & ju & $<12$ & ju & $<11$ & ju & $<11$ & ju & $<12$ & U & $<12$ & ju & $<10$ & ju \\
\hline Isophorone & ugih & 10 & - & $<11$ & ju & $<10$ & ju & $<12$ & ju & $<11$ & ju & $<11$ & ju & $<12$ & ju & $<12$ & iu & $<10$ & ju \\
\hline N-Nitrosodl-n-propylamino & ug/L & 10 & . & $<11$ & ju & $<10$ & ju & $<12$ & iu & $<11$ & U & $<11$ & ju & $<12$ & ju & $<12$ & ju & $<10$ & ju \\
\hline N-Nitrosodlphenylamlne & ug/L & 10 & . & $<11$ & ju & $<10$ & ju & $<12$ & ju & $<11$ & ju & $<11$ & ju & $<12$ & ju & $<12$ & ju & $<10$ & i ju \\
\hline Naphthalene & $\mathrm{ug} / \mathrm{L}$ & 10 & - & $<11$ & ju & $<10$ & ju & $<12$ & ju & $<11$ & ju & $<11$ & ju & $<12$ & ju & $<12$ & UU & $<10$ & $\boldsymbol{U}$ \\
\hline Nitrobenzono & $\mathrm{ug} / \mathrm{L}$ & 10 & - & $<11$ & iu & $<10$ & ju & $<12$ & ju & $<11$ & ן & $<11$ & ju & $<12$ & ju & $<12$ & ju & $<10$ & $\boldsymbol{U}$ \\
\hline Pentachlorophenol & $\mathrm{ug} / \mathrm{L}$ & 25 & $1 \#$ & $<29$ & ju & $<26$ & ju & $<29$ & ju & $<27$ & ju & $<27$ & ju & $<29$ & ju & $<29$ & ju & $<26$ & $\boldsymbol{U}$ \\
\hline Phenanthrene & $\mathrm{ug} / \mathrm{L}$ & 10 & - & $<11$ & UU & $<10$ & ju & $<12$ & ju & $<11$ & ןu & $<11$ & iu & $<12$ & ju & $<12$ & ju & $<10$ & ju \\
\hline Phenol & ug/L & 10 & - & $<11$ & ju & $<10$ & ju & $<12$ & ju & $<11$ & ju & $<11$ & ju & $<12$ & ju & $<12$ & Uן & $<10$ & ju \\
\hline Pyrene & $u g / L$ & 10 & - & $<11$ & [U & $<10$ & ju & $<12$ & ju & $<11$ & ju & $<11$ & ju & $<12$ & ju & $<12$ & ju & $<10$ & ju \\
\hline
\end{tabular}


TABLE B.2

ANALYTICAL RESULTS FOR SURFACE WATER AND STORM WATER, 1995

SEMIVOLATILE ORGANIC COMPOUNDS

1995 ANNUAL WATER MONITORING REPORT

LEHR ENVIRONMENTAL RESTORATION, DAVIS CA

\begin{tabular}{|c|c|c|c|c|c|c|c|c|c|}
\hline Parameter & Units & \multicolumn{2}{|l|}{. } & \multicolumn{2}{|c|}{$\begin{array}{c}\text { SWL00001 } \\
\text { WINTER }\end{array}$} & \multicolumn{2}{|c|}{$\begin{array}{c}\text { SWL00002 } \\
\text { WINTER }\end{array}$} & \multicolumn{2}{|c|}{$\begin{array}{c}\text { SWL00002 } \\
\text { WINTER } \\
\text { D1 }\end{array}$} \\
\hline 1,2,4-Trichlorobenzene & ug/h & 10 & 70 & $<10$ & נן & $<10$ & ju & $<10$ & IU \\
\hline 1,2-Dichlorobenzene & ug/L & 10 & $600 \#$ & $<10$ & ju & $<10$ & ju & $<10$ & ן \\
\hline 1,3-Dichlorobenzene & ugll & 10 & - & $<10$ & ju & $<10$ & ju & $<10$ & ju \\
\hline 1,4-Dlchlorobenzene & ug/L & 10 & 5 & $<10$ & ju & $<10$ & ju & $<10$ & ju \\
\hline 2,2'-oxybls(1-Chloropropane) & ug/L & 10 & - & $<10$ & ju & $<10$ & ju & $<10$ & u \\
\hline 2,4,5-Trichlorophenol & ug/L & 25 & - & $<25$ & ן & $<26$ & ןU & $<26$ & ן \\
\hline 2,4,6-Trichlorophenol & $u g / L$ & 10 & - & $<10$ & ju & $<10$ & ju & $<10$ & ju \\
\hline 2,4-Dichlorophenol & ug/L & 10 & $70 \#$ & $<10$ & ju & $<10$ & $\boldsymbol{U}$ & $<10$ & ju \\
\hline 2,4-DImethylphenol & ug/L & 10 & $\cdot$ & $<10$ & ju & $<10$ & ju & $<10$ & ju \\
\hline 2,4-DInltrophenol & ug/L & 25 & - & $<25$ & ju & $<26$ & ju & $<26$ & ju \\
\hline 2,4-Dinitrotoluene & ug/L & 10 & - & $<10$ & ju & $<10$ & ju & $<10$ & ju \\
\hline 2,6-Dinitrotoluene & $\mathrm{ug} / \mathrm{L}$ & 10 & - & $<10$ & ju & $<10$ & ju & $<10$ & j \\
\hline 2.Chtoronaphthatene & ug/L & 10 & - & $<10$ & j & $<10$ & ju & $<10$ & נן \\
\hline 2-Chtorophenol & ug/L & 10 & - & $<10$ & ן & $<10$ & ju & $<10$ & ju \\
\hline 2-Methyl-4,6-dinitrophenol & ug/L & 25 & - & $<25$ & ju & $<26$ & iu & $<26$ & ju \\
\hline 2-Methylnaphthalene & ug/L & 10 & - & $<10$ & ןU & $<10$ & ju & $<10$ & ju \\
\hline 2.Methylphenol & ug/L & 10 & - & $<10$ & iu & $<10$ & ju & $<10$ & ju \\
\hline 2-Nitroanillne & ug/L & 25 & - & $<25$ & ן & $<26$ & IU & $<26$ & ju \\
\hline 2-Nitrophenol & ug/L & 10 & - & $<10$ & ju & $<10$ & IU & $<10$ & ju \\
\hline 3,3'-Dichlorobenzldine & ug/L & 10 & - & $<10$ & ןU & $<10$ & ju & $<10$ & iu \\
\hline 3-Nitroanlline & ug/L & 25 & - & $<26$ & ju & $<26$ & ju & $<26$ & ju \\
\hline 4-Bromophenyl Phenyl Ether & ugh/L. & 10 & - & $<10$ & ju & $<10$ & ן & $<10$ & IU \\
\hline 4-Chloro-3-Methylphenol & ug/L & 10 & - & $<10$ & $\boldsymbol{U}$ & $<10$ & ju & $<10$ & ju \\
\hline 4-Chloroaniline & |ug $/ L$ & 10 & - & $<10$ & Ju & $<10$ & ju & $<10$ & ju \\
\hline 4-Chlorophenyl Phenyt Ether & ug $/ L$ & 10 & - & $<10$ & ן & $<10$ & UU & $<10$ & ju \\
\hline 4-Methylphenol & ug $/ \mathrm{L}$ & 10 & - & $<10$ & ןU & $<10$ & IU & $<10$ & U \\
\hline 4-Nitroanilline & ug $/ \mathrm{L}$ & 25 & - & $<25$ & ju & $<26$ & ju & $<26$ & ju \\
\hline 4-Nitrophenol & ug $/ L$ & 25 & - & $<25$ & e & $<26$ & ju & $<26$ & ju \\
\hline Acenaphthene & ug $/ L$ & 10 & - & $<10$ & ju & $<10$ & ju & $<10$ & ju \\
\hline Aconaphthylene & ugh & 10 & - & $<10$ & $\boldsymbol{U}$ & $<10$ & ju & $<10$ & ju \\
\hline Anthracene & |ugh & 10 & - & $<10$ & ju & $<10$ & ju & $<10$ & IU \\
\hline
\end{tabular}




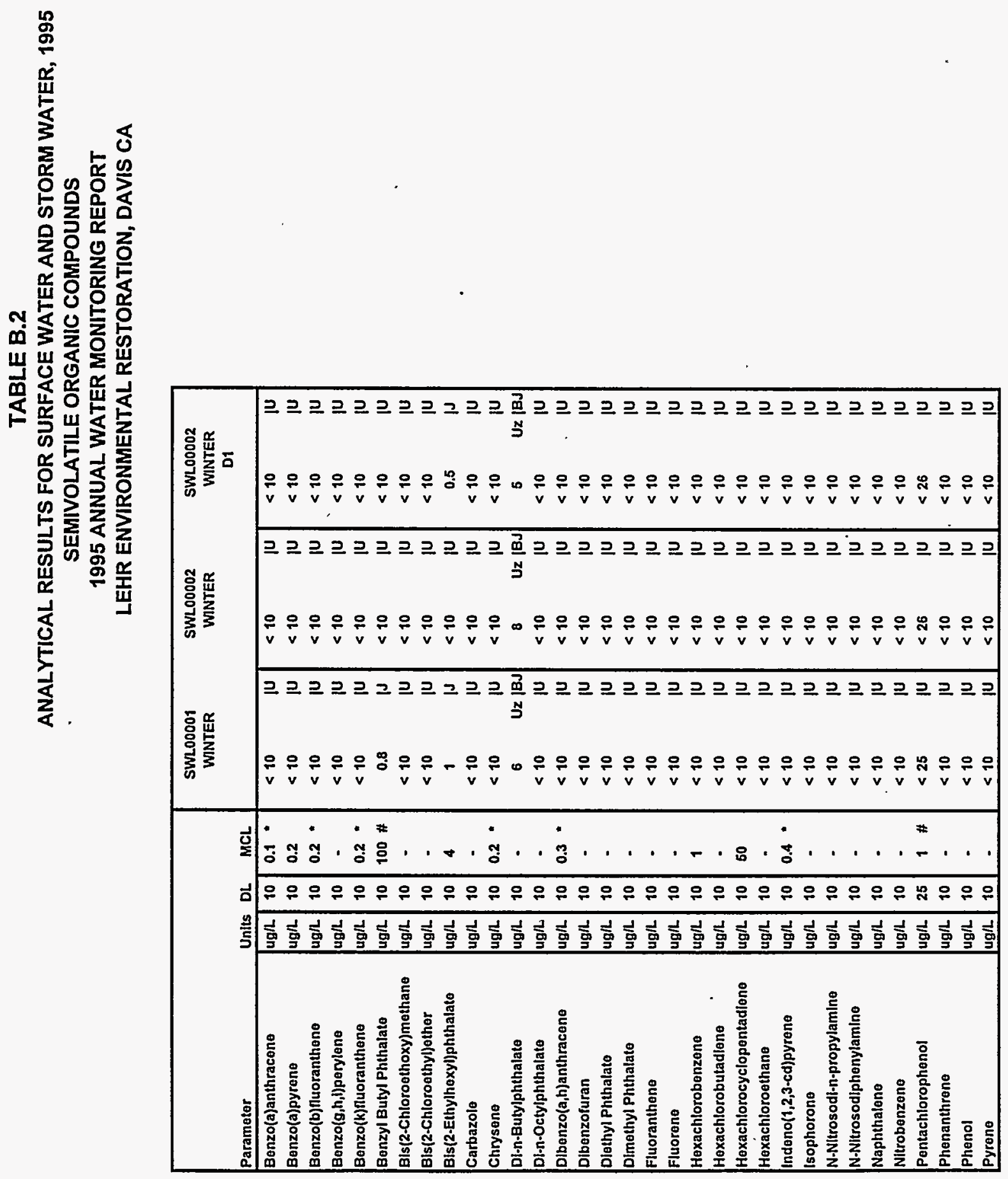


TABLE B.2

ANALYTICAL RESULTS FOR SURFACE WATER AND STORM WATER, 1995 SEMIVOLATILE ORGANIC COMPOUNDS

1995 ANNUAL WATER MONITORING REPORT LEHR ENVIRONMENTAL RESTORATION, DAVIS CA

DI = Dupllcate sample.

. = Parameter not analyzed. Storm water runoff avallable for sampling in March, 1995 only.

$<=$ Constituent below detection IImlt. Detection IImlts may vary depending on Interference by other sample constltuents.

$M C L=$ Maximum ContamInant Level (primairy), Titte 22 Division 4 Chapter 15.

= Proposed USEPA MCL.

\# = USEPA MCL.

See Appendlx $B$ for explanation of data qualifiers. 
TABLE B.3

ANALYTICAL RESULTS FOR SURFACE WATER AND STORM WATER, 1995

PESTICIDES AND PCBS

1995 ANNUAL WATER MONITORING REPORT

LEHR ENVIRONMENTAL RESTORATION, DAVIS CA

\begin{tabular}{|c|c|c|c|c|c|c|c|c|c|c|c|c|c|c|c|c|c|c|c|c|}
\hline \multirow{2}{*}{$\begin{array}{l}\text { Parameter } \\
44^{4}-\mathrm{DDD}\end{array}$} & Units & \multirow{2}{*}{$\frac{D L^{-}}{0.02}$} & $\mathrm{MCL}$ & \multicolumn{2}{|c|}{$\begin{array}{c}\text { PCD } \\
\text { WINTER }\end{array}$} & \multicolumn{2}{|c|}{$\begin{array}{c}\text { PCD } \\
\text { SPRING }\end{array}$} & \multicolumn{2}{|c|}{$\begin{array}{c}\text { PCD } \\
\text { SUMMER }\end{array}$} & \multicolumn{2}{|c|}{$\begin{array}{l}\text { PCD } \\
\text { FALL }\end{array}$} & \multicolumn{2}{|c|}{$\begin{array}{c}\text { PCD } \\
\text { FALL } \\
\text { D1 }\end{array}$} & \multicolumn{2}{|c|}{$\begin{array}{c}\text { PCU } \\
\text { WINTER }\end{array}$} & \multicolumn{2}{|c|}{$\begin{array}{c}\text { PCU } \\
\text { SPRING }\end{array}$} & \multicolumn{3}{|c|}{$\begin{array}{c}\text { PCU } \\
\text { SPRING } \\
\text { D1 }\end{array}$} \\
\hline & ugh & & $\cdot$ & $<0.022$ & IU & $<0.022$ & UJs $ן$ & $<0.02$ & UJs jU & $<0.02$ & UJs $\mid U$ & $<0.02$ & UJs $\mid U$ & $<0.024$ & $\boldsymbol{U} \mathbf{U}$ & $<0.022$ & UJs |U & $<0.021$ & & IU \\
\hline 4,4'DDE & ugg/L & 0.02 & - & $<0.022$ & ju & $<0.022$ & USs & $<0.02$ & UJs $\boldsymbol{U}$ & $<0.02$ & UJs ju & $<0.02$ & UJs $\mathbf{j}$ & $<0.024$ & ju & $<0.022$ & UJs & $<0.021$ & & 伡 \\
\hline 4,4'-DDT & ugh & 0.02 & - & $<0.022$ & ju & $<0.022$ & USs ju & $<0.02$ & UJs & $<0.02$ & UJs ju & $<0.02$ & UJs & $<0.024$ & ju & $<0.022$ & UJs $\mathbf{U}$ & $<0.021$ & & ju \\
\hline Aldrin & ug/L & 0.01 & $0.05 \mathrm{c}$ & $<0.011$ & ju & $<0.011$ & Uss ju & $<0.01$ & UJs $\mid \mathrm{U}$ & $<0.01$ & UJs ju & $<0.01$ & UJs $\boldsymbol{U}$ & $<0.012$ & ju & $<0.011$ & Ujs $\boldsymbol{U}$ & $<0.01$ & UJm & U \\
\hline Alpha-BHC & ug/L & 0.01 & $0.7 \mathrm{c}$ & $<0.011$ & ju & $<0.011$ & UJs ju & $<0.01$ & UJs ju & $<0.01$ & UJs ju & $<0.01$ & UJs ju & $<0.012$ & ju & $<0.011$ & Uss $j$ & $<0.01$ & & ju \\
\hline Alpha-Chlordane & ug $/ L$ & 0.01 & - & $<0.011$ & ju & $<0.011$ & Uss ju & $<0.01$ & UJs ju & $<0.01$ & USs ju & $<0.01$ & Uss ju & $<0.012$ & $\boldsymbol{U}$ & $<0.011$ & UJs $j$ & $<0.01$ & & ju \\
\hline Aroclor-1016 & ug/L & 0.2 & 0.5 & $<0.22$ & ju & $<0.22$ & UJs ju & $<0.2$ & USs ju & $<0.2$ & UJs ju & $<0.2$ & UJs ju & $<0.24$ & ju & $<0.22$ & UJs ju & $<0.21$ & & ju \\
\hline Aroclor-1221 & ug $/ \mathrm{L}$ & 0.4 & 0.5 & $<0.44$ & ju & $<0.43$ & UJs & $<0.4$ & UJs ju & $<0.4$ & Uss ju & $<0.41$ & UJs ju & $<0.47$ & ן & $<0.43$ & UJs ju & $<0.42$ & & ju \\
\hline Aroclor-1232 & ug $/ \mathrm{L}$ & 0.2 & 0.5 & $<0.22$ & ju & $<0.22$ & UJs & $<0.2$ & UJs ju & $<0.2$ & UJs ju & $<0.2$ & UJs ju & $<0.24$ & ju & $<0.22$ & UJs ju & $<0.21$ & & ju \\
\hline Aroclor-1242 & ug $/ L$ & 0.2 & 0.5 & $<0.22$ & ju & $<0.22$ & UJs $j$ & $<0.2$ & UJs ju & $<0.2$ & UJs ju & $<0.2$ & UJs $\boldsymbol{j}$ & $<0.24$ & ju & $<0.22$ & UJs ju & $<0.21$ & & ju \\
\hline Aroclor-1248 & ug/L. & 0.2 & 0.5 & $<0.22$ & ju & $<0.22$ & UJs $j$ & $<0.2$ & UJs $\boldsymbol{U}$ & $<0.2$ & UJs ju & $<0.2$ & UJs $j$ & $<0.24$ & ju & $<0.22$ & UJs ju & $<0.21$ & & ju \\
\hline Aroclor-1254 & ug $/ L$ & 0.2 & 0.5 & $<0.22$ & ju & $<0.22$ & UJs ju & $<0.2$ & UJs $\mid U$ & $<0.2^{\circ}$ & UJs ju & $<0.2$ & UJs $j u$ & $<0.24$ & ju & $<0.22$ & UJs ju & $<0.21$ & & ju \\
\hline Arocior-1260 & $\mathrm{ug} / \mathrm{L}$ & 0.2 & 0.5 & $<0.22$ & iu & $<0.22$ & UJs ju & $<0.2$ & UJs ju & $<0.2$ & UJs ju & $<0.2$ & UJs & $<0.24$ & iU & $<0.22$ & UJs & $<0.21$ & & ju \\
\hline Beta-BHC & ug/L. & 0.01 & $0.3 . c$ & $<0.011$ & UJt ju & $<0.011$ & UJs ju & $<0.01$ & UJs ju & $<0.01$ & UJs ju & $<0.01$ & UJs ju & $<0.012$ & UJt ju & $<0.011$ & UJs ju & $<0.01$ & & ju \\
\hline Delta-BHC & $\mathrm{ug} / \mathrm{L}$ & 0.01 & - & $<0.011$ & u & $<0.011$ & UJs & $<0.01$ & UJs ju & $<0.01$ & UJs ju & $<0.01$ & UJs & $<0.012$ & UU & $<0.011$ & UJs ju & $<0.01$ & & iv \\
\hline Dleldrin & ug/L & 0.02 & - & $<0.022$ & ju & $<0.022$ & UJs ju & $<0.02$ & UJs ju & $<0.02$ & UJs ju & $<0.02$ & UJs ju & $<0.024$ & ju & $<0.022$ & UJs ju & $<0.021$ & & ju \\
\hline Endosulfan I & ug/L & 0.01 & - & $<0.011$ & ju & $<0.011$ & UJs ju & $<0.01$ & UJs' ju & $<0.01$ & UJs ju & $<0.01$ & UJs ju & $<0.012$ & ju & $<0.011$ & UJs ju & $<0.01$ & & ju \\
\hline Endosulfan II V & ug/L & 0.02 & '- & $<0.022$ & ju & $<0.022$ & UJs $\omega^{U}$ & $<0.02^{\circ}$ & UJs $\boldsymbol{U}$ & $<0.02$ & UJs ju & $<0.02$ & UJs Ju & $<0.024$ & ju & $<0.022$ & UJs ju & $<0.021$ & & טן \\
\hline Endosulfan Sulfate & ug/L & 0.02 & - & $<0.022$ & ju & $<0.022$ & UJs ju & $<0.02$ & UJs $\boldsymbol{\mu}$ & $<0.02$ & UJs ju & $<0.02$ & UJs & $<0.024$ & ju & $<0.022$ & UJs & $<0.021$ & & U \\
\hline Endrin & |ug/L & 0.02 & 0.2 & $<0.022$ & |U & $<0.022$ & UJs ju & $<0.02$ & UJs $\boldsymbol{U}$ & $<0.02$ & UJs $\mid U$ & $<0.02$ & UJs & $<0.024$ & U & $<0.022$ & UJs $\mid U$ & $<0.021$ & & U \\
\hline Endrin Aldehyde & ugg $/ L$ & 0.02 & $\cdot$ & $<0.022$ & IU & $<0.022$ & UJs $\mathbf{U}$ & $<0.02$ & UJs $\boldsymbol{U}$ & $<0.02$ & UJs ju & $<0.02$ & UJs $\boldsymbol{U}$ & $<0.024$ & $\boldsymbol{U}$ & $<0.022$ & UJs $\boldsymbol{U}$ & $<0.021$ & & ju \\
\hline Endrin Kotone & ug/L. & 0.02 & - & $<0.022$ & ן & $<0.022$ & UJs & $<0.02$ & UJs $\boldsymbol{U}$ & $<0.02$ & UJs ju & $<0.02$ & UJs & $<0.024$ & ju & $<0.022$ & UJs $\boldsymbol{U}$ & $<0.021$ & & U \\
\hline Gamma-BHC & ug $/ \mathrm{L}$ & 0.01 & 0.2 & $<0.011$ & ן & $<0.011$ & UJs ju & $<0.01$ & UJs $\boldsymbol{U}$ & $<0.01$ & UJs ju & $<0.01$ & UJs ju & $<0.012$ & ju & $<0.011$ & UJs $j u$ & $<0.01$ & & ju \\
\hline Gamma-Chlordano & ug/L & 0.01 & - & $<0.011$ & ju & $<0.011$ & UJs ju & $<0.01$ & UJs $j$ & $<0.01$ & UJs ju & $<0.01$ & UJs ju & $<0.012$ & ju & $<0.011$ & UJs ju & $<0.01$ & & ju \\
\hline Heptachlor & ugh & 0.01 & 0.01 & $<0.011$ & ju & $<0.011$ & UJs ju & $<0.01$ & UJs ju & $<0.01$ & UJs ju & $<0.01$ & UJs ju & $<0.012$ & U & $<0.011$ & UJs ju & $<0.01$ & & ju \\
\hline Heptachlor Epoxlde & ug/L & 0.01 & 0.01 & $<0.011$ & ju & $<0.011$ & UJs U & $<0.01$ & UJs jU & $<0.01$ & UJs ju & $<0.01$ & UJs ju & $<0.012$ & ju & $<0.011$ & Uss ju & $<0.01$ & & ju \\
\hline Methoxychlor & ug/L & 0.1 & $40 \#$ & $<0.11$ & iu & $<0.11$. & UJs ju & $<0.1$ & UJs & $<0.1$ & UJs ju & $<0.1$ & UJs ju & $<0.12$ & ju & $<0.11$ & UJs ju & $<0.1$ & & ju \\
\hline Toxaphene & ug/L & 1 & $3 \#$ & $<1.1$ & ju & $<1.1$ & UJs ju & $<1$ & UJs jU & $<1$ & UJs IU & $<1$ & UJs jU & $<1.2$ & IU & $<1.1$ & UJs IU & $<1$ & & iu \\
\hline
\end{tabular}


TABLE B.3

ANALYTICAL RESULTS FOR SURFACE WATER AND STORM WATER, 1995

PESTICIDES AND PCBS

1995 ANNUAL WATER MONITORING REPORT

LEHR ENVIRONMENTAL RESTORATION, DAVIS CA

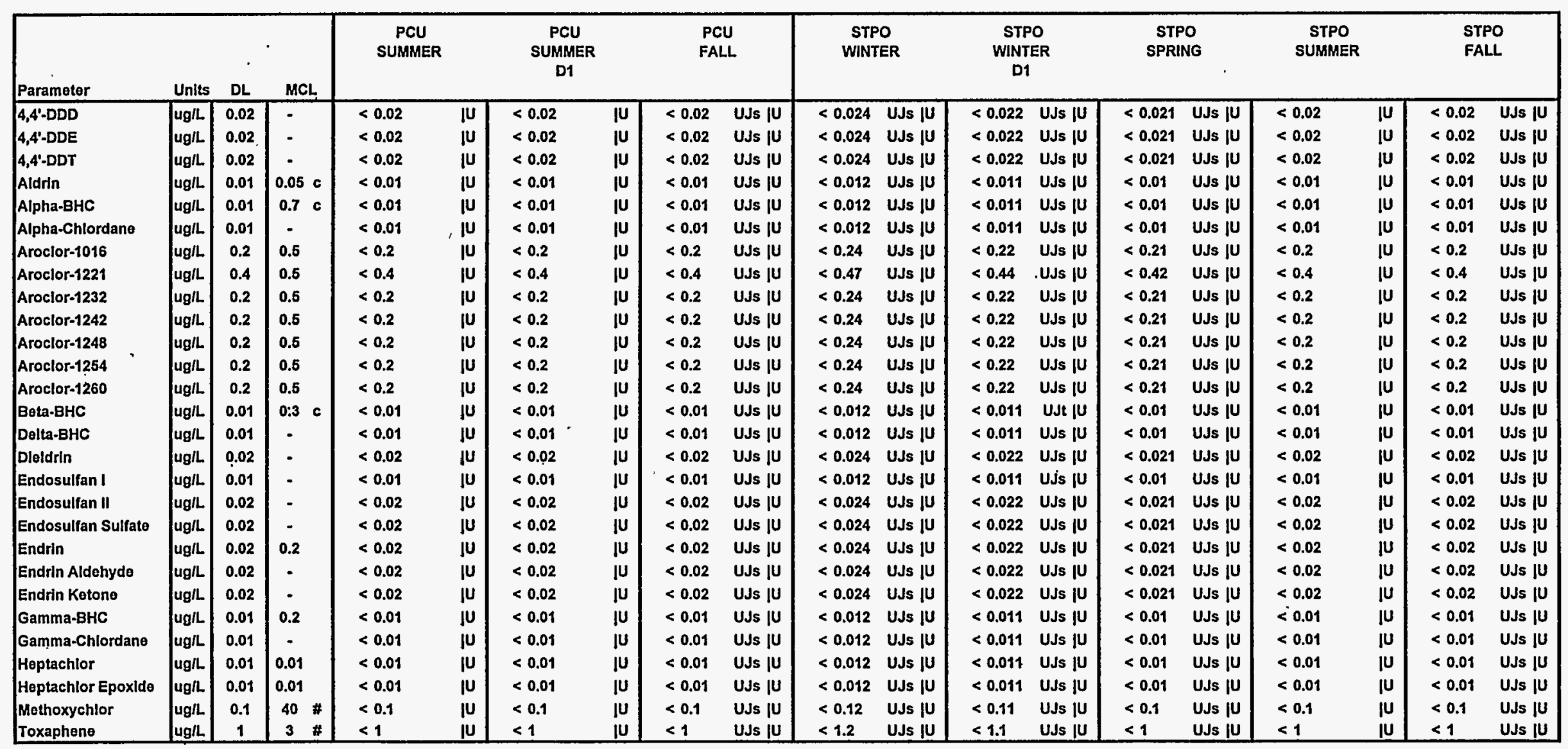


TABLE B.3

ANALYTICAL RESULTS FOR SURFACE WATER AND STORM WATER, 1995

PESTICIDES AND PCBS

1995 ANNUAL WATER MONITORING REPORT

LEHR ENVIRONMENTAL RESTORATION, DAVIS CA

\begin{tabular}{|c|c|c|c|c|c|c|c|c|c|}
\hline Parameter & Units & DL & $M C L$ & \multicolumn{2}{|c|}{$\begin{array}{c}\text { SWL00001 } \\
\text { WINTER }\end{array}$} & \multicolumn{2}{|c|}{$\begin{array}{c}\text { SWL00002 } \\
\text { WINTER }\end{array}$} & \multicolumn{2}{|c|}{$\begin{array}{c}\text { SWL00002 } \\
\text { WINTER } \\
\text { D1 }\end{array}$} \\
\hline 4,4'-DDD & ug/L & 0.02 & - & $<0.02$ & UJs $\mu^{U}$ & $<0.021$ & UJs jU & $<0.021$ & UJs 10 \\
\hline $4,4^{\prime}-\mathrm{DDE}$ & ugh/L & 0.02 & - & $<0.02$ & UJs & $<0.021$ & UJs iU & $<0.021$ & Uss iU \\
\hline 4,4'-DDT & |ugh & 0.02 & - & $<0.02$ & UJs $\mid U$ & $<0.021$ & UJs & $<0.021$ & UJs $\mid U$ \\
\hline Aldrin & ugih. & 0.01 & $0.05 \mathrm{c}$ & $<0.01$ & UJs & $<0.01$ & UJs $\boldsymbol{U}$ & $<0.01$ & UJs IU \\
\hline Alpha-BHC & |ugh/L & 0.01 & $0.7 \mathrm{c}$ & $<0.01$ & UJs $\boldsymbol{U}$ & $<0.01$ & UJs $\boldsymbol{U}$ & $<0.01$ & UJs IU \\
\hline Alpha-Chlordane & ugilL. & 0.01 & $\cdot$ & 0.015 & Js $\mathbb{P}$ & $<0.01$. & UJs & $\cdot<0.01$ & UJs $\mu$ \\
\hline Aroclor-1016 & ugil & 0.2 & 0.5 & $<0.2$ & UJs $\boldsymbol{U}$ & $<0.21$ & UJs $\boldsymbol{U}$ & $<0.21$ & UJs $j u$ \\
\hline Aroclor-1221 & |ugh/L & 0.4 & 0.5 & $<0.4$ & UJs & $<0.41$ & UJs ju & $<0.41$ & UJs ju \\
\hline Aroclor-1232 & |ug/L & 0.2 & 0.5 & $<0.2$ & UJs $\boldsymbol{U}$ & $<0.21$ & UJs ju & $<0.21$ & UJs IU \\
\hline Aroclor-1242 & |ug/L & 0.2 & 0.5 & $<0.2$ & UJs $\boldsymbol{U}$ & $<0.21$ & UJs ju & $<0.21$ & UJs $1 U$ \\
\hline Aroclor-1248 & ug/L & 0.2 & 0.5 & $<0.2$ & UJs ju & $<0.21$ & UJs $\boldsymbol{U}$ & $<0.21$ & UJs ju \\
\hline Aroclor-1254 & ug/L & 0.2 & 0.6 & $<0.2$ & UJs ju & $<0.21$ & UJs $\boldsymbol{U}$ & $<0.21$ & UJs ju \\
\hline Aroclor-1260 & ug/L & 0.2 & 0.6 & $<0.2$ & UJs $\mid U$ & $<0.21$ & UJs $\boldsymbol{U}$ & $<0.21$ & UJs ju \\
\hline Beta-BHC & ug $/ \mathrm{L}$ & 0.01 & $0.3 \mathrm{c}$ & $<0.01$ & UJs $[U$ & $<0.01$ & UJs & $<0.01$ & UJs ju \\
\hline Delta-BHC & ugih. & 0.01 & $\cdot$ & $<0.01$ & UJs $\boldsymbol{U}$ & $<0.01$ & UJs $\boldsymbol{\mu}$ & $<0.01$ & UJs iu \\
\hline Dleldrin & ugh & 0.02 & . & $<0.02$ & UJs $\boldsymbol{U}$ & $<0.021$ & UJs $\boldsymbol{U}$ & $<0.021$ & UJs ju \\
\hline Endosulfan I & ug/L. & 0.01 & - & $<0.01$ & UJs $\boldsymbol{U}$ & $<0.01$ & UJs $\boldsymbol{U}$ & $<0.01$ & UJs $\mu^{\prime}$ \\
\hline Endosulfan II & |ught & 0.02 & - & $<0.02$ & UJs & $<0.021$ & UJs $\boldsymbol{U}$ & $<0.021$ & UJs $1 U$ \\
\hline Endosulfan Sulfato & ugh & 0.02 & - & $<0.02$ & UJṣ ju & $<0.021$ & UJs $\boldsymbol{U}$ & $<0.021$ & UJs $1 U$ \\
\hline Endrin & lugh & 0.02 & 0.2 & $<0.02$ & UJs $j u$ & $<0.021$ & UJs $\boldsymbol{U}$ & $<0.021$ & UJs ju \\
\hline Endrin Aldehyde & ugil & 0.02 & - & $<0.02$ & UJs ju & $<0.021$ & UJs & $<0.021$ & UJs IU \\
\hline Endrin Ketone & ughl & 0.02 & - & $<0.02$ & UJs ju & $<0.021$ & UJs ju & $<0.021$ & ujs iu \\
\hline Gamma-BHC & ugh & 0.01 & 0.2 & $<0.01$ & UJs ju & $<0.01$ & UJs ju & $<0.01$ & Uss iu \\
\hline Gamma.Chlordane & ug/L & 0.01 & - & 0.013 & Js IP & $<0.01$ & UJs & $<0.01$ & UJs ju \\
\hline Heptachlor & ug/L & 0.01 & 0.01 & $<0.01$ & UJs & $<0.01$ & UJs $\boldsymbol{U}$ & $<0.01$ & UJs IU \\
\hline Hoptachlor Epoxlde & ug/L & 0.01 & 0.01 & $<0.01$ & UJs $\boldsymbol{U}_{\mathrm{U}}$ & $<0.01$ & UJs $\boldsymbol{U}$ & $<0.01$ & UJs \\
\hline Methoxychlor & ugh & 0.1 & $40 \#$ & $<0.1$ & UJs ju & $<0.1$ & UJs ju & $<0.1$ & UJs iu \\
\hline Toxaphene & ug $/ \mathrm{L}$ & 1 & & $<1$ & UJs $\mid U$ & $<1$ & UJs $\boldsymbol{U}$ & $<1$ & UJs $[\mathrm{U}$ \\
\hline
\end{tabular}


TABLE B.3

ANALYTICAL RESULTS FOR SURFACE WATER AND STORM WATER, 1995

PESTICIDES AND PCBS

1995 ANNUAL WATER MONITORING REPORT

LEHR ENVIRONMENTAL RESTORATION, DAVIS CA

DI = Dupllcate sample.

- = Parameter not analyzed. Storm water runoff avallable for sampling in March, 1995 only.

$<=$ Constituent below detectlon limit. Detection limits may vary depending on Interference by other sample constituents.

$M C L=$ Maxlmum Contaminant Level (primary), Title 22 Division 4 Chapter 16.

$C=$ Callfornla State Actlon Level, Department of Health Services.

\# = USEPA MCL.

See Appendlx B for explanation of data quallfiers. 
TABLE B.4

ANALYTICAL RESULTS FOR SURFACE WATER AND STORM WATER, 1995

METALS

1995 ANNUAL WATER MONITORING REPORT

LEHR ENVIRONMENTAL RESTORATION, DAVIS CA

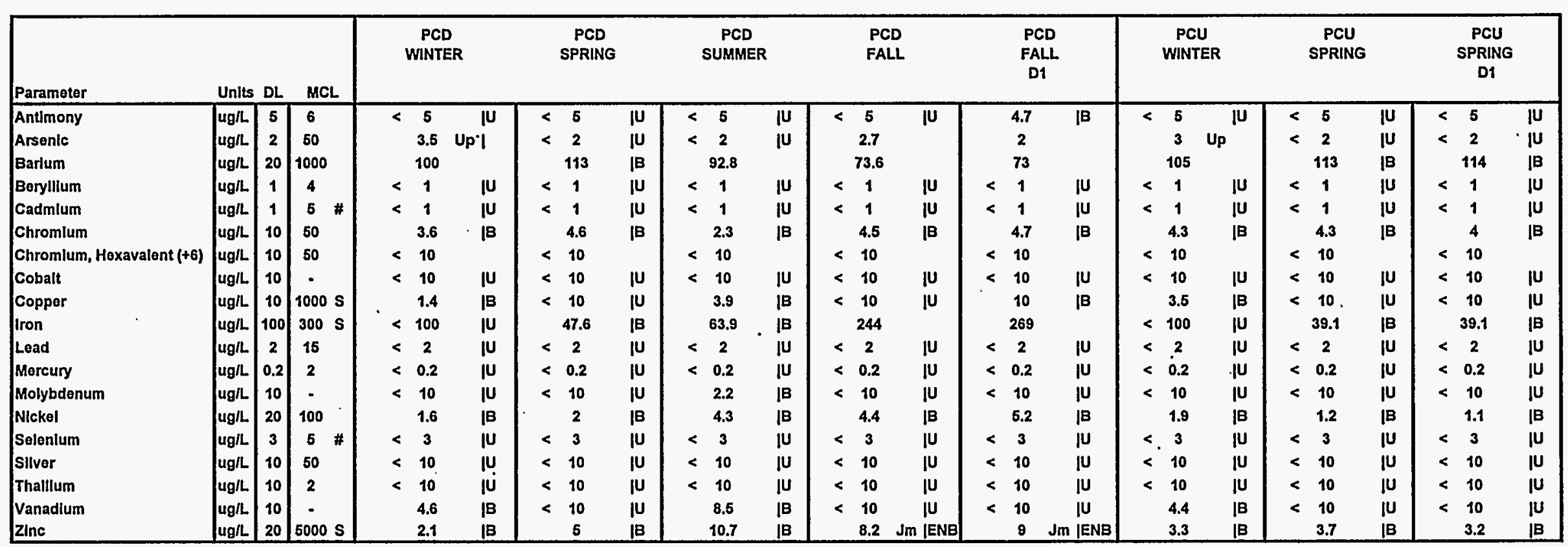


TABLE B.4

ANALYTICAL RESULTS FOR SURFACE WATER AND STORM WATER, 1995

METALS

1995 ANNUAL WATER MONITORING REPORT

LEHR ENVIRONMENTAL RESTORATION, DAVIS CA

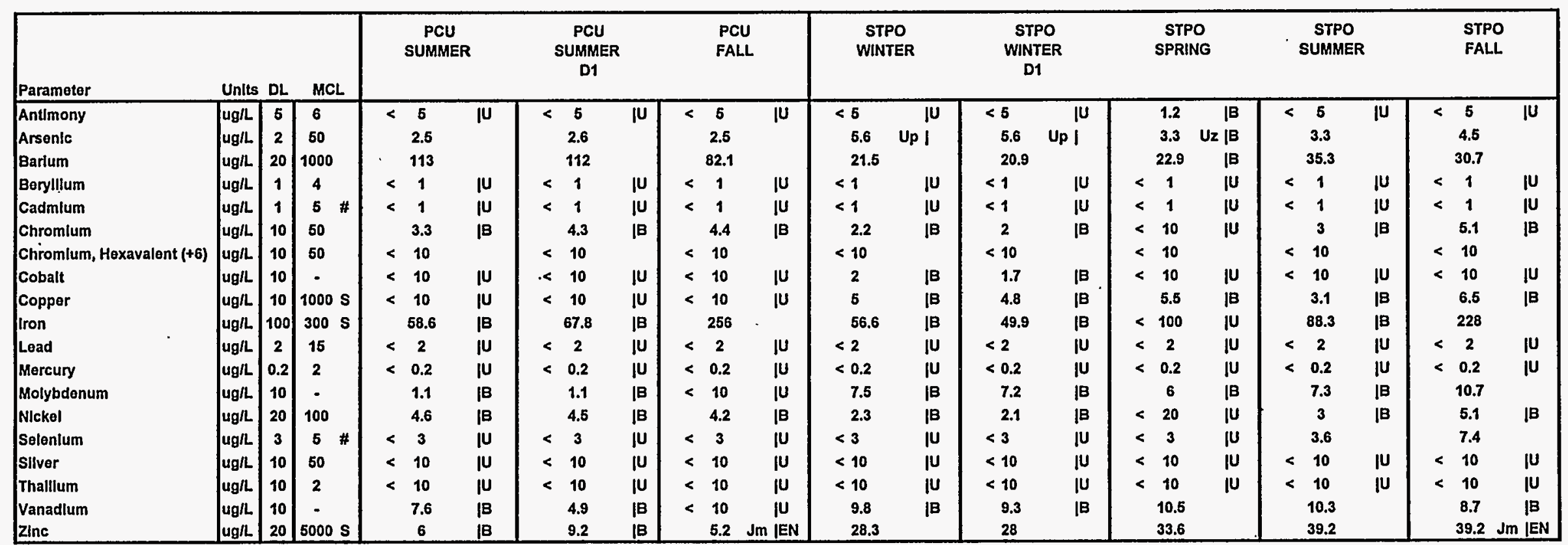


TABLE B.4

ANALYTICAL RESULTS FOR SURFACE WATER AND STORM WATER, 1995 METALS

1995 ANNUAL WATER MONITORING REPORT LEHR ENVIRONMENTAL RESTORATION, DAVIS CA

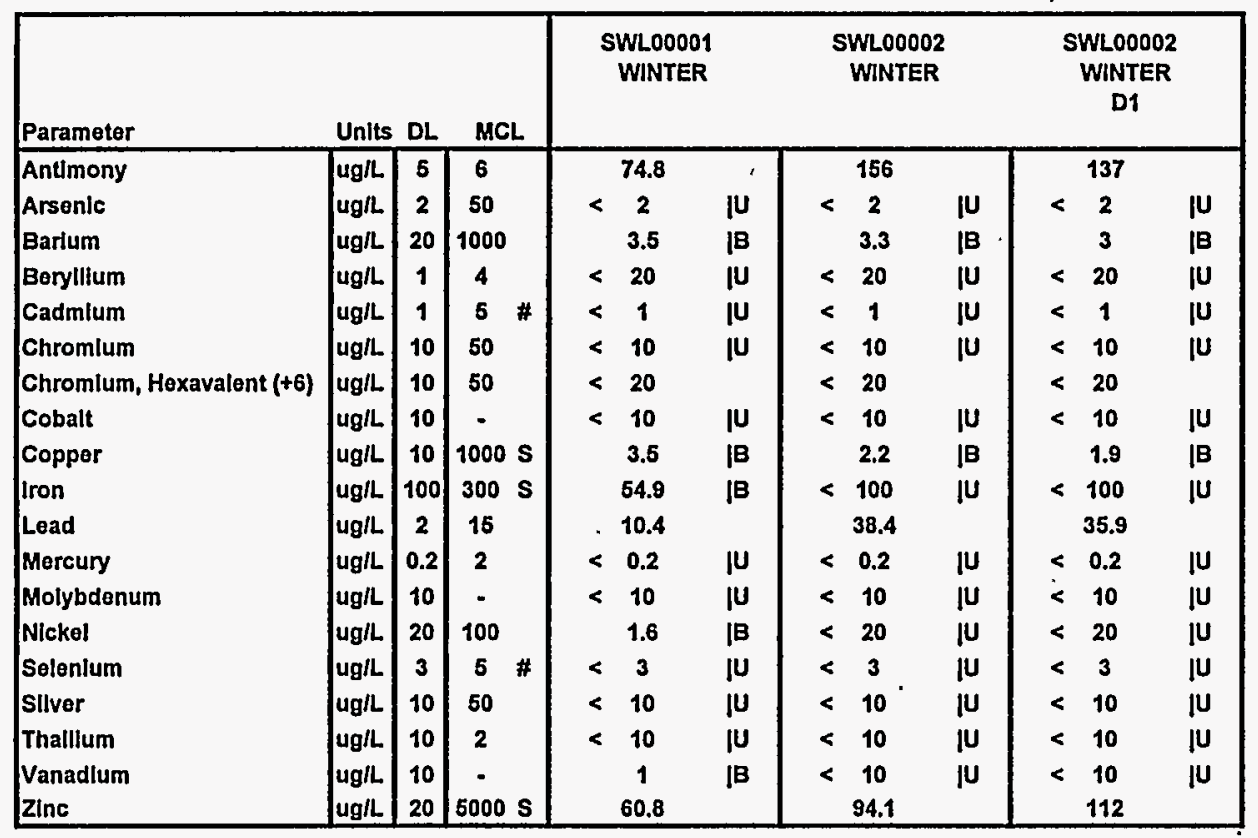


TABLE B.4

ANALYTICAL RESULTS FOR SURFACE WATER AND STORM WATER, 1995

METALS

1995 ANNUAL WATER MONITORING REPORT

LEHR ENVIRONMENTAL RESTORATION, DAVIS CA

\section{$D I=$ Dupllcato sample.}

= Parameter not analyzed. Storm water runoff avallable for sampling In March, 1995 only.

$<=$ Constituent below detection limlt. Detectlon limlts may vary depending on interference by other sample constituents.

$M C L=$ MaxImum Contaminant Lovel (primary), Title 22 Division 4 Chapter 15.

\# = USEPA MCL.

Seo Append $\mathrm{B}$ for explanation of data qualiflers.

$S=$ Secondary Drinking Water Standard 
TABLE B.5

ANALYTICAL RESULTS FOR SURFACE AND STORM WATER, 1995

RADIONUCLIDES

1995 WATER MONITORING REPORT

LEHR ENVIRONMENTAL RESTORATION, DAVIS, CALIFORNIA

\begin{tabular}{|c|c|c|c|c|c|c|c|c|c|c|c|c|c|c|c|c|c|c|c|}
\hline \multirow{2}{*}{ PARAMETER } & \multirow{2}{*}{ METHOD } & \multirow{2}{*}{\multicolumn{2}{|c|}{$\begin{array}{l}\text { MCL } \\
\text { pCi/L }\end{array}$}} & \multicolumn{4}{|c|}{$\begin{array}{c}\text { PCD } \\
\text { WINTER }\end{array}$} & \multicolumn{4}{|c|}{$\begin{array}{c}\text { PCD } \\
\text { SPRING }\end{array}$} & \multicolumn{4}{|c|}{$\begin{array}{c}\text { PCD } \\
\text { SUMMER }\end{array}$} & \multicolumn{4}{|c|}{$\begin{array}{c}\text { PCD } \\
\text { FALL }\end{array}$} \\
\hline & & & & Value & & Error & MDA & Value & & Error & MDA & Value & & Error & MDA & Value & & Error & MDA \\
\hline Actinium-228 & 901.1 & - & & -14.5 & & 9.7 & 40 & -34 & & 17 & 37 & 8 & & 72 & 120 & -1 & & 6.7 & 13 \\
\hline Bismuth-212 & 901.1 & - & & -8 & & 49 & 73 & 9 & & 42 & 58 & & & & & 6 & & 16 & 21 \\
\hline Bismuth-214 & 901.1 & - & & 8 & & 13 & 19 & 17 & & 13 & 17 & 33 & & 47 & 63 & 6.2 & & 6 & 9.1 \\
\hline Cesium-134 & 901.1 & - & & & & & & & & & & 2 & & 15 & 25 & & & . & \\
\hline Cesium-137 & 901.1 & - & & -0.8 & & 4.9 & 9.1 & 2.2 & & 5.5 & 9.2 & -2 & & 21 & 27 & 0.3 & & 2 & 3.3 \\
\hline Cobalt-57 & 901.1 & - & & & & & & & & & & 6.2 & & 9.9 & 16 & & & & \\
\hline Cobalt-60 & 901.1 & - & & 2.4 & & 3.7 & 9.3 & -1.6 & & 2.4 & 9.8 & 0 & & 15 & 30 & -0.33 & & 0.51 & 4.1 \\
\hline Lead-210 & 901.1 & - & & -40 & & 110 & 170 & 30 & & 110 & 170 & & & & & 190 & & 290 & 390 \\
\hline Lead-212 & 901.1 & - & & $\mathbf{0}$ & & 9.1 & 14 & 12 & & 10 & 14 & 33 & & 40 & 55 & 0.9 & & 5 & 7.5 \\
\hline Lead-214 & 901.1 & - & & -4 & & 11 & 20 & 8 & & 11 & 17 & 2 & Je 1 & 41 & 62 & 4.3 & & 5.2 & 8.3 \\
\hline Potassium-40 & 901.1 & - & & -41 & & 31 & 120 & 1 & & 66 & 110 & -60 & & 250 & 390 & 18 & & 30 & 46 \\
\hline Ra-223 & 901.1 & - & & & & & & & & & & & & & & 5 & & 19 & 60 \\
\hline Radium-226 & 901.1 & 5 & & 0 & & 120 & 180 & -160 & & 110 & 180 & & & & & -54 & & 57 & 85 \\
\hline Thallium-208 & 901.1 & - & & -2.3 & & 7.1 & 11 & 4.7 & & 7.7 & 11 & 16 & & 25 & 34 & 0.6 & & 3 & 4.4 \\
\hline Thorium-234 & 901.1 & - & & 0 & & 69 & 160 & -19 & & 66 & 160 & 330 & & 310 & 740 & -12 & & 49 & 150 \\
\hline Uranium-235 & 901.1 & - & & -11 & & 26 & 42 & 10 & & 27 & 38 & 40 & & 110 & 160 & 0 & & 16 & 24 \\
\hline Radium-226 & $903.1 \mathrm{M}$ & 5 & & 0.22 & & 0.23 & 0.36 & 0.18 & & 0.18 & 0.29 & 0.15 & & 0.17 & 0.27 & 0.12 & & 0.13 & 0.2 \\
\hline Strontium-90 & 905 & 8 & & -0.3 & & 1.5 & 1.2 & 0.06 & $\mathbf{J m} \mid$ & 0.5 & 0.86 & 0.7 & & 0.56 & 0.91 & 0.2 & & 0.29 & 0.49 \\
\hline Tritium & 906 & 20000 & * & $\mathbf{0}$ & & 180 & 260 & 10 & & 170 & 220 & -70 & & 220 & 300 & -70 & & 180 & 250 \\
\hline GrossAlpha & 9310 & 15 & & 1.7 & IC & 2.7 & 4.8 & 1.1 & $\mathrm{Jm} \mid \mathrm{C}$ & 1.8 & 3.2 & 2.3 & IC & 2.4 & 3.8 & 1.3 & IC & 1.5 & 2.5 \\
\hline GrossBeta & 9310 & $\mathbf{5 0}$ & & 3.9 & Jd I & 2.3 & 3.7 & 2 & & 1.8 & 3 & 7.3 & & 2.2 & 3.1 & 2.8 & & 1.4 & 2.2 \\
\hline Americium-241 & LAS108 & - & & -0.014 & $\mathbf{J m} \mathbf{i}$ & 0.04 & 0.085 & 0.024 & & 0.026 & 0.031 & 0.034 & & 0.027 & 0.015 & -0.0021 & & 0.0042 & 0.027 \\
\hline Plutonium-241 & LAS178 & - & & o & & 1.3 & 1.7 & 0.3 & $\mathrm{Jc} \mid$ & 1.5 & 1.8 & 0.4 & $\mathbf{J e} \mid$ & 2.8 & 3.7 & -0.3 & & 1.9 & 2.4 \\
\hline Carbon-14 & LSC & - & & -19 & & 81 & 110 & -23 & & 82 & 110 & 15 & $\mathrm{Jm} i$ & 11 & 12 & 1 & & 83 & 100 \\
\hline
\end{tabular}


TABLE B.5

ANALYTICAL RESULTS FOR SURFACE AND STORM WATER, 1995

RADIONUCLIDES

1995 WATER MONITORING REPORT

LEHR ENVIRONMENTAL RESTORATION, DAVIS, CALIIFORNIA

\begin{tabular}{|c|c|c|c|c|c|c|c|c|c|c|c|c|c|c|c|c|c|c|}
\hline \multirow{2}{*}{ PARAMETER } & \multirow{2}{*}{ METHOD } & \multirow{2}{*}{$\begin{array}{l}\text { MCL } \\
\text { pCi/L }\end{array}$} & \multicolumn{4}{|c|}{$\begin{array}{c}\text { PCD } \\
\text { FALL } \\
\text { D1 }\end{array}$} & \multicolumn{4}{|c|}{$\begin{array}{c}\text { PCU } \\
\text { WINTER }\end{array}$} & \multicolumn{4}{|c|}{$\begin{array}{c}\text { PCU } \\
\text { SPRING }\end{array}$} & \multicolumn{4}{|c|}{$\begin{array}{c}\text { PCU } \\
\text { SPING } \\
\text { D1 }\end{array}$} \\
\hline & & & Value & & Error & MDA & Value & & Error & MDA & Value & & Error & MDA & Value & & Error & $\overline{\mathrm{MDA}}$ \\
\hline Actinium-228 & 901.1 & - & 2.8 & & 8.2 & 15 & 0 & & 84 & 160 & 6 & & 21 & 38 & -1.3 & & 8 & 15 \\
\hline Bismuth-212 & 901.1 & - & -4 & & 18 & 26 & -161 & & 87 & 300 & -18 & & 21 & 71 & 4 & & 16 & 21 \\
\hline Bismuth-214 & 901.1 & - & 9.8 & & 6.2 & 8.7 & 5 & & 58 & 89 & 21 & & 14 & 18 & -1.9 & & 5.3 & 8.8 \\
\hline Cesium-134 & 901.1 & - & & & & & & & & & & & & & & & & \\
\hline Cesium-137 & 901.1 & - & -2 & & 1.9 & 3.5 & -5 & & 17 & 31 & -0.4 & & 4.8 & 8.7 & 0.2 & & 1.7 & 3 \\
\hline Cobalt-57 & 901.1 & - & & & & & & & & & & & & & & & & \\
\hline Cobalt-60 & 901.1 & - & 0.1 & & 1.6 & 3.2 & 7 & & .17 & 37 & 1.1 & & 4.1 & 7.9 & 1 & & 1.5 & 3 \\
\hline Lead-210 & 901.1 & - & -110 & & 280 & 410 & -100 & & 7800 & 11000 & 0 & & 110 & 170 & 110 & & 290 & 410 \\
\hline Lead-212 & 901.1 & - & 0.3 & & 5 & 7.6 & 16 & & 47 & 71 & $\mathbf{3}$ & & 10 & 15 & 2.6 & & 5.3 & 7.9 \\
\hline Lead-214 & 901.1 & - & 8.3 & & 5.7 & 8.4 & 2 & & 47 & 80 & 1 & & 11 & 19 & 0.1 & & 4.7 & 7.9 \\
\hline Potassium-40 & 901.1 & - & 10 & & 30 & 47 & 320 & & 330 & 470 & 23 & & 61 & 95 & 0 & & 28 & 47 \\
\hline $\mathrm{Ra}-223$ & 901.1 & - & 33 & & 20 & 60 & & & & & & & & & & & & \\
\hline Radium-226 & 901.1 & 5 & -3 & & 57 & 83 & -1110 & & 510 & 770 & 10 & . & 120 & 180 & -18 & & 59 & 91 \\
\hline Thallium-208 & 901.1 & - & 1.1 & & 2.8 & 4.2 & 22 & & 32 & 46 & 5.4 & & 7.3 & 9.7 & -2 & & 3 & 4.9 \\
\hline Thorium-234 & 901.1 & - & -36 & & 46 & 150 & -320 & & 350 & 1000 & -32 & & 66 & 160 & -24 & & 47 & 160 \\
\hline Uranium-235 & 901.1 & - & 0 & . & 16 & 24 & 10 & & 130 & 200 & 12 & & 27 & 39 & -6 & & 15 & 24 \\
\hline Radium-226 & 903.1M & 5 & 0.86 & & 0.24 & 0.16 & 0.07 & & 0.14 & 0.25 & 0.2 & & 0.15 & 0.2 & 0.05 & & 0.14 & 0.27 \\
\hline Strontium-90 & 905 & 8 & 0.25 & & 0.28 & 0.47 & -0.3 & & 1.1 & 1 & 0.21 & $\mathbf{J} \mathbf{m} \mid$ & 0.53 & 0.9 & -0.05 & $\mathbf{J} \mathbf{m} \mid$ & 0.52 & 0.9 \\
\hline Tritium & 906 & 20000 & -50 & & 180 & 250 & 0 & & 180 & 260 & 30 & & 170 & 220 & -50 & & 170 & 220 \\
\hline GrossAlpha & 9310 & 15 & 1.1 & IC & 1.8 & 3.2 & 1.5 & |C & 1.8 & 3 & 0.5 & $\mathrm{Jm} \mid \mathrm{C}$ & 1.8 & 3.4 & -0.1 & $\mathrm{Jm} \mid \mathrm{C}$ & 1.5 & 3.4 \\
\hline GrossBeta & 9310 & 50 & 3.4 & & 1.5 & 2.2 & 8.2 & $\mathrm{Jd} i$ & 2 & 2.6 & 1.6 & & 2.1 & 3.6 & 0.6 & & 1.7 & 3 \\
\hline Americium-241 & LAS108 & - & 0.035 & & 0.034 & 0.032 & 0.004 & $\mathrm{Jm} i$ & 0.031 & 0.055 & 0 & & 0 & 0.012 & 0.009 & & 0.019 & 0.031 \\
\hline Plutonium-241 & LAS178 & - & -0.4 & & 1.9 & 2.6 & 0.5 & . & 1.4 & 1.6 & -0.3 & Je 1 & 1.7 & 2.4 & -1.3 & Je 1 & 1.7 & 2.5 \\
\hline Carbon-14 & LSC & - & 52 & & 87 & 100 & 6 & & 84 & 110 & 18 & & 86 & 110 & 25 & & 86 & 110 \\
\hline
\end{tabular}


TABLE B.5

ANALYTICAL RESULTS FOR SURFACE AND STORM WATER, 1995

RADIONUCLIDES

1995 WATER MONITORING REPORT

LEHR ENVIRONMENTAL RESTORATION, DAVIS, CALIFORNIA

\begin{tabular}{|c|c|c|c|c|c|c|c|c|c|c|c|c|c|c|c|c|c|c|}
\hline \multirow{2}{*}{ PARAMETER } & \multirow{2}{*}{ METHOD } & \multirow{2}{*}{\multicolumn{2}{|c|}{$\begin{array}{l}\text { MCL } \\
\mathrm{pCi} / \mathrm{L}\end{array}$}} & \multicolumn{3}{|c|}{$\begin{array}{c}\text { PCU } \\
\text { SUMMER }\end{array}$} & \multicolumn{4}{|c|}{$\begin{array}{c}\text { PCU } \\
\text { SUMMER } \\
\text { DI }\end{array}$} & \multicolumn{4}{|c|}{$\begin{array}{r}\text { PCU } \\
\text { FALL }\end{array}$} & \multicolumn{4}{|c|}{$\begin{array}{c}\text { STPO } \\
\text { WINTER }\end{array}$} \\
\hline & & & & Value & Error & MDA & Valuc & & Error & MDA & Value & & Error & MDA & Value & & Error & MDA \\
\hline Actinium-228 & 901.1 & - & & - & - & - & 12 & & 22 & 39 & -17.1 & & 3 & 18 & 2.8 & & 7.5 & 12 \\
\hline Bismuth-212 & 901.1 & - & & - & - & - & - & & - & - & 6 & & 17 & 28 & 0 & & 16 & 24 \\
\hline Bismuth-214 & 901.1 & - & & - & - & - & 0 & & 13 & 20 & 9.3 & & 7.2 & 8.2 & 0.5 & & 4.9 & 7.5 \\
\hline Cesium-134 & 901.1 & - & & - & - & - & -0.4 & & 2.5 & 7.6 & - & & - & - & - & & - & - \\
\hline Cesium-137 & 901.1 & - & & - & - & - & -1.7 & & 4.6 & 8.3 & 0.2 & & 2.4 & 4.1 & 1.3 & & 1.6 & 2.4 \\
\hline Cobalt-57 & 901.1 & - & & - & - & - & -1 & & 1.7 & 4.6 & - & & - & - & - & & - & - \\
\hline Cobalt-60 & 901.1 & - & & - & - & - & -2.8 & & 2.9 & 12 & 0 & & 2 & 4 & -0.65 & & 0.98 & 3.6 \\
\hline Lead-210 & 901.1 & - & & - & - & - & - & & . & - & -30 & & 65 & 79 & -60 & & 270 & 400 \\
\hline Lead-212 & 901.1 & - & & - & - & - & -3.6 & & 9.6 & 15 & 4.1 & & 5.9 & 6.5 & -3.3 & & 4.9 & 7.9 \\
\hline Lead-214 & 901.1 & - & & - & - & - & -1 & Je I & 11 & 18 & 2.7 & & 6.7 . & 8.1 & -4.3 & 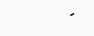 & 4.6 & 8.2 \\
\hline Potassium-40 & 901.1 & - & & - & - & - & 1 & & 71 & 110 & 19 & & 41 & 45 & 19 & & 27 & 40 \\
\hline Ra-223 & 901.1 & - & & - & - & - & - & & - & - & -28 & & 7.1 & 66 & - & & - & - \\
\hline Radium-226 & 901.1 & 5 & & - & - & - & - & . & - & - & -81 & & 68 & 78 & 31 & & 56 & 81 \\
\hline Thallium-208 & 901.1 & - & & - & - & - & 2.7 & & 7 & 9.5 & 1.4 & & 4.1 & 4.3 & -1.2 & & 2.7 & 4.3 \\
\hline Thorium-234 & 901.1 & - & & - & - & - & -77 & & 65 & 150 & -19 & & 39 & 71 & -24 & & 49 & 150 \\
\hline Uranium-235 & 901.1 & - & & - & - & - & -8 & & 28 & 43 & -9 & & 16 & 19 & -1.9 & & 7.4 & 22 \\
\hline Radium-226 & $903.1 \mathrm{M}$ & 5 & & - & - & - & 0.089 & & 0.086 & 0.06 & 0.26 & & 0.13 & 0.13 & 0 & & 0.15 & 0.32 \\
\hline Strontium-90 & 905 & 8 & & - & - & - & 0.21 & & 0.42 & 0.72 & -0.06 & & 0.37 & 0.66 & -0.2 & & 1.4 & 1.1 \\
\hline Tritium & 906 & 20000 & * & -70 & 220 & 300 & 60 & & 230 & 290 & 20 & & 190 & 250 & 60 & & 190 & 260 \\
\hline GrossAlpha & 9310 & 15 & & - & - & - & 0.6 & & 1.7 & 3.2 & 0.4 & IC & 1.7 & 3.1 & -0.4 & IC & 2.1 & 4.9 \\
\hline GrossBeta & 9310 & 50 & & - & - & - & 1.4 & & 1.9 & 3.2 & 4.6 & & 1.7 & 2.4 & 17.2 & Jd $\mathbf{~} \mathbf{C}$ & 3.6 & 4.5 \\
\hline Americium-241 & LAS108 & - & & - & - & - & 0.032 & & 0.028 & 0.018 & 0.02 & & 0.022 & 0.018 & 0.027 & $J_{\mathbf{m}} \mid$ & 0.039 & 0.063 \\
\hline Plutonium-241 & LAS178 & - & & - & - & - & 2.4 & & 2.4 & 2.6 & 0.6 & & 2.1 & 2.9 & .0 .8 & & 1.4 & 1.6 \\
\hline Carbon-14 & LSC & - & & -14 & 76 & 100 & 4 & & 78 & 100 & 34 & & 85 & 100 & 28 & & 85 & 110 \\
\hline
\end{tabular}


TABLE B.5

ANALYTICAL RESULTS FOR SURFACE AND STORM WATER, 1995

RADIONUCLIDES

1995 WATER MONITORING REPORT

LEHR ENVIRONMENTAL RESTORATION, DAVIS, CALIFORNIA

\begin{tabular}{|c|c|c|c|c|c|c|c|c|c|c|c|c|c|c|c|c|c|c|}
\hline \multirow{2}{*}{ PARAMETER } & \multirow{2}{*}{ METHOD } & \multirow{2}{*}{$\begin{array}{l}\mathrm{MCL} \\
\mathrm{pCi} / \mathrm{L}\end{array}$} & \multicolumn{4}{|c|}{$\begin{array}{c}\text { STPO } \\
\text { WINTER } \\
\text { D1 }\end{array}$} & \multicolumn{4}{|c|}{$\begin{array}{c}\text { STPO } \\
\text { SPRING }\end{array}$} & \multicolumn{4}{|c|}{$\begin{array}{c}\text { STPO } \\
\text { SUMMER }\end{array}$} & \multicolumn{4}{|c|}{$\begin{array}{l}\text { STPO } \\
\text { FALL }\end{array}$} \\
\hline & & & Value & & Error & MDA & Valuc & & Error & MDA & Value & & Error & MDA & Value & & Error & $M D_{A}$ \\
\hline Actinium-228 & 901.1 & - & 3.8 & & 7.4 & 12 & 6 & & 14 & 26 & -1 & & 20 & 38 & -0.4 & & 6.9 & 12 \\
\hline Bismuth-212 & 901.1 & - & -6 & & $\cdot 17$ & 26 & -8 & & 46 & 71 & - & & - & - & -1 & & 19 & 27 \\
\hline Bismuth-214 & 901.1 & - & 6.4 & & 5.3 & 7.2 & -4 & & 10 & 16 & 4 & & 13 & 18 & 6.5 & & 5.5 & 8 \\
\hline Cesium-134 & 901.1 & - & - & & - & - & - & & - & $\cdot$ & 0.8 & & 3.6 & 6.6 & - & & - & - \\
\hline Cesium-137 & 901.1 & - & -0.3 & & 1.7 & 3.1 & -2 & & 3.4 & 9.7 & -4.9 & & 5.4 & 10 & 2.5 & & 1.9 & 2.5 \\
\hline Cobalt-57 & 901.1 & - & - & & $\cdot$ & - & - & & $\cdot$ & - & -1.6 & & 2.5 & 4.4 & - & & - & - \\
\hline Cobalt -60 & 901.1 & $\because$ & 0 & & 1.6 & 3.3 & 5.9 & & 4.8 & 6 & -1.4 & & 1.5 & 7.6 & -0.5 & & 1.1 & 3.7 \\
\hline Lead-210 & 901.1 & - & -40 & & 280 & 410 & 0 & & 110 & 170 & - & & - & - & 90 & & 280 & 390 \\
\hline Lead-212 & 901.1 & - & 2.7 & & 5.1 & 7.7 & 2.3 & & 9.5 & 14 & -2 & & 10 & 16 & 1.9 & & 5 & 7.4 \\
\hline Lead-214 & 901.1 & - & 4.3 & & 5 & 7.8 & -6 & & 11 & 19 & 3 & Je I & 11 & 18 & 4.4 & & 5.1 & 8.1 \\
\hline Potassium-40 & 901.1 & - & 11 & & 27 & 43 & 10 & & 66 & 110 & 42 & & 77 & 110 & 5 & & 27 & 44 \\
\hline $\mathrm{Ra}-223$ & 901.1 & - & - & & - & - & - & & - & - & - & & - & - & 13 & & 19 & 55 \\
\hline Radium-226 & 901.1 & 5 & -34 & & 54 & 84 & -480 & & 120 & 170 & - & & - & - & 5 & & 55 & 82 \\
\hline Thallium-208 & 901.1 & - & 1.7 & & 3 & 4.4 & 0.9 & & 7.1 & 10 & 6.3 & & 7.1 & 9.2 & -0.2 & & 2.7 & 4.1 \\
\hline Thorium-234 & 901.1 & - & -48 & & 46 & 160 & 4 & & 65 & 150 & 40 & & 100 & 160 & -30 & & 47 & 150 \\
\hline Uranium-235 & 901.1 & - & -3 & & 14 & 22 & 17 & & 25 & 36 & 4 & & 27 & 41 & -16 & & 13 & 23 \\
\hline Radium-226 & 903.1M & 5 & 0.17 & & 0.17 & 0.26 & 0.46 & & 0.24 & 0.21 & 0.12 & & 0.16 & 0.27 & 0.8 & & 0.29 & 0.26 \\
\hline Strontium-90 & 905 & 8 & -0.5 & & 8.7 & 2.9 & -0.06 & $\mathrm{Jm} \mid$ & 0.56 & 0.97 & 0.53 & & 0.49 & 0.82 & 0.04 & & 0.26 & 0.46 \\
\hline Tritium & 906 & 20000 * & 50 & & 190 & 260 & 0 & & 170 & 230 & 0 & & 190 & 260 & 0 & & 180 & 240 \\
\hline GrossAlpha & 9310 & 15 & 3.2 & $\mid \mathbf{C}$ & 3 & 4.5 & 0 & $\mathbf{J m} \mid \mathrm{C}$ & 1.8 & 4 & 1 & |C & 2.8 & 5.4 & 1.7 & |C & 3.3 & 5.9 \\
\hline GrossBeta & 9310 & 50 & 29.5 & $\mathrm{Jd} \mid \mathrm{C}$ & 4.1 & 4.1 & 11.4 & IC & 3.3 & 4.6 & 19.3 & |C & 3.8 & 4.6 & 16.1 & IC & 3.5 & 4.4 \\
\hline Americium-241 & LAS108 & - & 0.097 & $\mathbf{J} \mathbf{m} \mid \mathbf{Y}$ & 0.075 & 0.1 & 0.02 & & 0.02 & 0.014 & 0.026 & & 0.032 & 0.042 & 0.012 & & 0.022 & 0.033 \\
\hline Plutonium-241 & LAS178 & - & -0.4 & & 1.2 & 1.4 & 0.6 & Je I & 1.7 & 2.2 & -1 & Jc I & 2.9 & 4.3 & 0.3 & & 2 & 2.7 \\
\hline Carbon-14 & LSC & - & -16 & & 82 & 110 & 14 & & 85 & 110 & 6 & $\mathbf{J} \mathbf{m} \mid$ & 10 & 12 & 37 & & 86 & 100 \\
\hline
\end{tabular}


TABLE B.5

ANALYTICAL RESULTS FOR SURFACE AND STORM WATER, 1995

RADIONUCLIDES

1995 WATER MONITORING REPORT

LEHR ENVIRONMENTAL RESTORATION, DAVIS, CALIFORNIA

\begin{tabular}{|c|c|c|c|c|c|c|c|c|c|c|c|c|c|c|}
\hline \multirow{2}{*}{ PARAMETER } & \multirow{2}{*}{ METHOD } & \multirow{2}{*}{$\begin{array}{l}\text { MCL } \\
\text { pCi/L }\end{array}$} & \multicolumn{4}{|c|}{$\begin{array}{c}\text { SWL00001 } \\
\text { WINTER } \\
\cdot\end{array}$} & \multicolumn{4}{|c|}{$\begin{array}{c}\text { SWL00002 } \\
\text { WINTER }\end{array}$} & \multicolumn{4}{|c|}{$\begin{array}{c}\text { SWL00002 } \\
\text { WINTER } \\
\text { D1 }\end{array}$} \\
\hline & & & Value & & Error & MDA & Valuc & & Error & MDA & Value & & Error & MDA \\
\hline Actinium-228 & 901.1 & - & -1.2 & & 7.4 & 0 & 2 & & 21 & 0 & 0.7 & & 7.7 & o \\
\hline Bismuth-212 & 901.1 & - & $\mathbf{0}$ & & 17 & 0 & 33 & & 49 & $\mathbf{0}$ & 3 & & 18 & 0 \\
\hline Cesium-134 & 901.1 & - & - & & - & - & - & & - & - & - & & $\cdot$ & - \\
\hline Cesium-137 & 901.1 & - & 1.4 & & 1.7 & 0 & 1.9 & & 7 & 0 & -0.5 & & 1.8 & 0 \\
\hline Cobalt-57 & 901.1 & - & - & & - & - & - & & - & - & - & & - & - \\
\hline Cobalt-60 & 901.1 & - & -0.21 & & 0.52 & 0 & -4.4 & & 3.8 & 0 & -0.3 & & 1.2 & 0 \\
\hline Lead-210 & 901.1 & - & 100 & & 290 & 0 & 70 & & 110 & 0 & -10 & & 270 & 0 \\
\hline Lead-212 & 901.1 & - & 2.3 & & 5.1 & 0 & 2 & & 10 & 0 & -3.1 & & 5.1 & 0 \\
\hline Thallium-208 & 901.1 & - & 0.3 & & 2.8 & 0 & 7.4 & ' & 7.6 & 0 & 0.5 & & 2.7 & 0 \\
\hline Thorium-234 & 901.1 & - & 14 & & 46 & 0 & -4 & & 65 & 0 & 24 & & 47 & 0 \\
\hline Uranium-235 & 901.1 & - & 6 & & 14 & .0 & -6 & & 28 & 0 & 0 & & 14 & 0 \\
\hline Radium-226 & $903.1 \mathrm{M}$ & 5 & 0 & & 0.13 & 0 & -0.039 & & 0.093 & 0 & 0.2 & & 0.17 & 0 \\
\hline Strontium-90 & 905 & 8 & -0.03 & & 0.77 & 0 & 0.06 & & 0.77 & 0 & $-0.17^{*}$ & & 0.81 & 0 \\
\hline Tritium & 906 & 20000 & -40 & $\mathbf{J m} \mid$ & 200 & 0 & -60 & $\mathrm{Jm} \mid$ & 200 & 0 & 150 & $\mathbf{J m} \mid$ & 220 & 0 \\
\hline GrossAlpha & 9310 & 15 & 0.14 & & 0.56 & 0 & 0.29 & & 0.54 & 0 & -0.06 & & 0.46 & 0 \\
\hline GrossBeta & 9310 & 50 & 1.7 & & 1.3 & 0 & 0.5 & & 1.2 & 0 & 0.7 & & 1.2 & 0 \\
\hline Americium-241 & LAS108 & - & 0.022 & & 0.024 & 0 & 0.012 & & 0.031 & 0 & 0.011 & & 0.021 & 0 \\
\hline Plutonium-241 & LAS178 & - & -0.5 & - & 1.4 & 0 & 0.3 & & 1.6 & 0 & -1.1 & & 2.4 & 0 \\
\hline Carbon-14 & LSC & - & 16 & & 83 & 0 & -3 & & 81 & 0 & -57 & & 76 & 0 \\
\hline
\end{tabular}


TABLE B.5

ANALYTICAL RESULTS FOR SURFACE AND STORM WATER, 1995

RADIONUCLIDES

1995 WATER MONITORING REPORT

\section{LEHR ENVIRONMENTAL RESTORATION, DAVIS, CALIFORNIA}

D1 = Duplicate Sample

- - Parameter not analyzed or no MCL Note: Only the result for Tritium was reported for PCU-Summer due to insufficient volume to analyze other constituents.

MDA = Method Detectable Activity

MCL = Maximum Contaminant Level (primary), Title 22 Division 4 Chapter 15.

- = Proposed MCL for Tritium is $60,000 \mathrm{pCi} / \mathrm{L}$

See Appendix B for explanation of data qualifiers. 
TABLE B.6

ANALYTICAL RESULTS FOR SURFACE WATER AND STORM WATER, 1995

ANIONS AND CATIONS

1995 ANNUAL WATER MONITORING REPORT

LEHR ENVIRONMENTAL RESTORATION, DAVIS CA

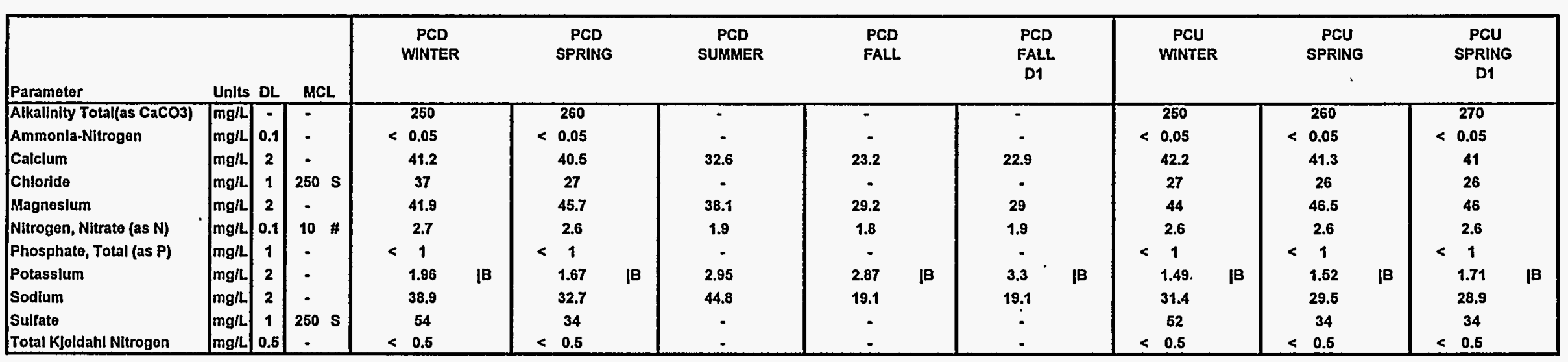


TABLE B.6

ANALYTICAL RESULTS FOR SURFACE WATER AND STORM WATER, 1995

ANIONS AND CATIONS

1995 ANNUAL WATER MONITORING REPORT

LEHR ENVIRONMENTAL RESTORATION, DAVIS CA

\begin{tabular}{|c|c|c|c|c|c|c|c|c|c|c|c|}
\hline Parameter & Units & DL & $\mathrm{MCL}$ & $\begin{array}{c}\text { PCU } \\
\text { SUMMER }\end{array}$ & $\begin{array}{c}\text { PCU } \\
\text { SUMMER } \\
\text { D1 }\end{array}$ & $\begin{array}{l}\text { PCU } \\
\text { FALL }\end{array}$ & $\begin{array}{l}\text { STPO } \\
\text { WINTER }\end{array}$ & $\begin{array}{c}\text { STPO } \\
\text { WINTER } \\
\text { D1 }\end{array}$ & $\begin{array}{l}\text { STPO } \\
\text { SPRING }\end{array}$ & $\begin{array}{c}\text { STPO } \\
\text { SUMMER }\end{array}$ & $\begin{array}{l}\text { STPO } \\
\text { FALL }\end{array}$ \\
\hline Alkalinlty Total(as CaCO3) & $\mathrm{mg} / \mathrm{L}$ & -1 & - & - & - & - & 180 & 180 & 180 & $\cdot$ & - \\
\hline Ammonla-Nitrogen & $\mathrm{mg} / \mathrm{L}$ & 0.1 & - & - & - & $\cdot$ & 3.2 & 3.2 & 1.4 & $\cdot$ & $\cdot$ \\
\hline Calclum & mg/L & 2 & - & 35.2 & 33.1 & 22.9 & 18.4 & 18 & 19.1 & 22.1 & 19.2 \\
\hline Chlorlde & mg/L & 1 & $250 \mathrm{~S}$ & - & - & - & 120 & 120 & 110 & - & - \\
\hline Magnesium & mg/L & 2 & - & 41.3 & 38.5 & 29.5 & 18.4 & 18.1 & 18.2 & 23.1 & 22 \\
\hline Nitrogen, Nitrate (as N) & mg/L & 0.1 & $10 \#$ & 1.5 & 1.5 & 1.8 & 7.5 & 7.4 & 9.4 & 1.5 & 3 \\
\hline Phosphate, Total (as P) & mghl & 1 & - & - & . & - & 2 & $<2$ & $<$ & - & - \\
\hline Potasslum & $\mathrm{mg} / \mathrm{L}$ & 2 & - & 1.67 & 1.92 & 2.5 & 10 & 9.87 & 9.09 & 8.69 & 14.6 \\
\hline Sodium & $\mathrm{mg} / \mathrm{L}$ & 2 & & 30.5 & 28.1 & 17.2 & 141 & 140 & 137 & 153 & 122 \\
\hline Sulfate & $\mathrm{mg} / \mathrm{L}$ & 1 & $250 \mathrm{~s}$ & - & - & - & 63 & 63 & 54 & - & - \\
\hline Total KJeldahI Nitrogen & mg/L & 0.5 & - & - & - & - & 3.1 & 3.1 & 2.5 & - & - \\
\hline
\end{tabular}


TABLE B.6

ANALYTICAL RESULTS FOR SURFACE WATER AND STORM WATER, 1995 ANIONS AND CATIONS

1995 ANNUAL WATER MONITORING REPORT LEHR ENVIRONMENTAL RESTORATION, DAVIS CA

\begin{tabular}{|c|c|c|c|c|c|c|c|c|c|}
\hline Parameter & Units & DL & MCL & $\begin{array}{r}\text { SWL0000 } \\
\text { WINTER }\end{array}$ & & $\begin{array}{c}\text { SWL00002 } \\
\text { WINTER }\end{array}$ & • & $\begin{array}{c}\text { SWLO0000 } \\
\text { WINTER } \\
\text { D1 }\end{array}$ & \\
\hline Alkalinity Total(as CaCO3) & mg/L & $\cdot$ & $\cdot$ & 15 & & 14 & & 14 & \\
\hline Ammonla-Nitrogen & mg/L & 0.1 & - & - & & - & & - & \\
\hline Calclum & |mg/L & 2 & - & 1.35 & 㫜 & 0.57 & IB & 0.6 & BB \\
\hline Chlorlde & $\mathrm{mgh}$ & 1 & $250 \mathrm{~S}$ & 2 & & $<1$ & & $<1$ & \\
\hline Magneslum & $\mathrm{mg} / \mathrm{h} \mid$ & 2 & - & 0.46 & J日 & 0.13 & IB & 0.13 & 旧 \\
\hline Nitrogen, Nitrate (as N) & mg/h & 0.1 & $10 \#$ & 0.24 & & 0.13 & & $<0.1$ & \\
\hline Phosphate, Total (as P) & mg/L & 1 & - & - & & - & & - & \\
\hline Potasslum & mg/h & 2 & - & 1.3 & 旧 & 0.89 & IB & $<$ & U \\
\hline Sodium & $\mathrm{mg} / \mathrm{h}$ & 2 & - & 3.66 & & 0.29 & |B & 0.27 & IB \\
\hline Sulfate & $\mathrm{mg} / \mathrm{L}$ & 1 & $250 \mathrm{~s}$ & 1.2 & & $<1$ & & $<1$ & \\
\hline Total KJoldah! Nitrogen & mg/L & 0.5 & - & - & & - & & - & \\
\hline
\end{tabular}


TABLE B.6

ANALYTICAL RESULTS FOR SURFACE WATER AND STORM WATER, 1995

ANIONS AND CATIONS

1995 ANNUAL WATER MONITORING REPORT

LEHR ENVIRONMENTAL RESTORATION, DAVIS CA

DI = Dupllcate sample.

- = Parameter not analyzed. Storm water runoff available for sampling in March, 1995 only.

$<=$ Constituent below detection limit. Detection IImits may vary depending on interference by other sample constituents.

$M C L=$ Maximum Contaminant Lovel (primary), Title 22 Divislon 4 Chapter 15.

\# = USEPA MCL.

See Appendix $B$ for explanation of data quallfiers.

$\mathbf{S}=$ Secondary Drinking Water Standard 
TABLE B.7

ANALYTICAL RESULTS FOR SURFACE WATER AND STORM WATER, 1995

OTHER PARAMETERS

1995 ANNUAL WATER MONITORING REPORT

LEHR ENVIRONMENTAL RESTORATION, DAVIS CA

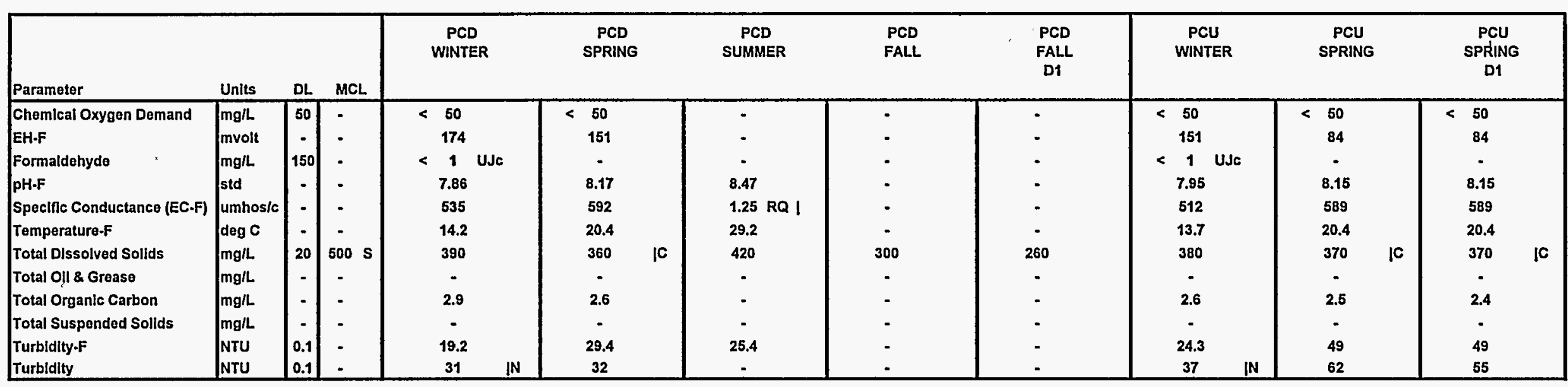


TABLE B.7

ANALYTICAL RESULTS FOR SURFACE WATER AND STORM WATER, 1995 OTHER PARAMETERS

1995 ANNUAL WATER MONITORING REPORT

LEHR ENVIRONMENTAL RESTORATION, DAVIS CA

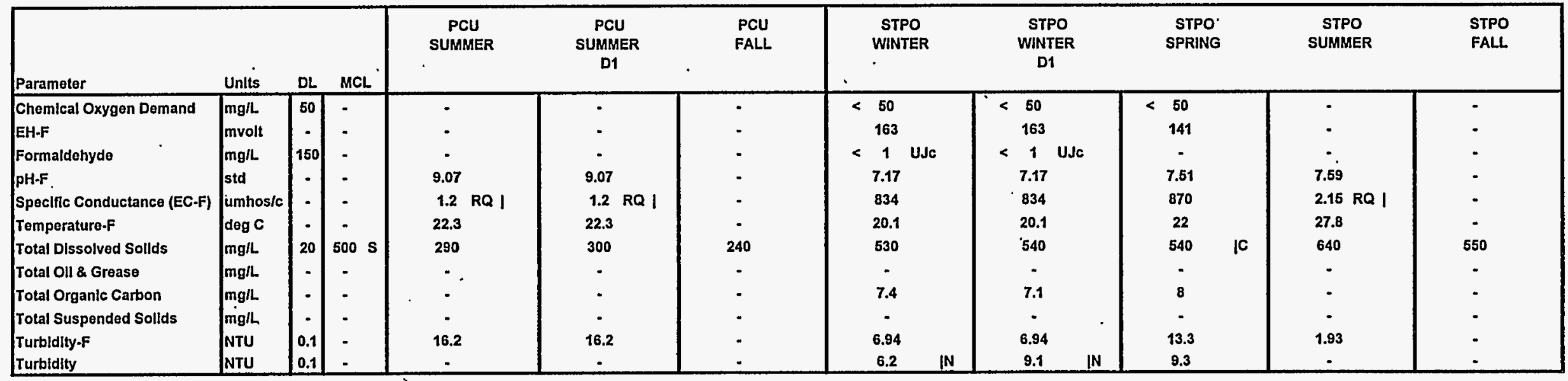


TABLE B.7

ANALYTICAL RESULTS FOR SURFACE WATER AND STORM WATER, 1995 OTHER PARAMETERS

1995 ANNUAL WATER MONITORING REPORT LEHR ENVIRONMENTAL RESTORATION, DAVIS CA

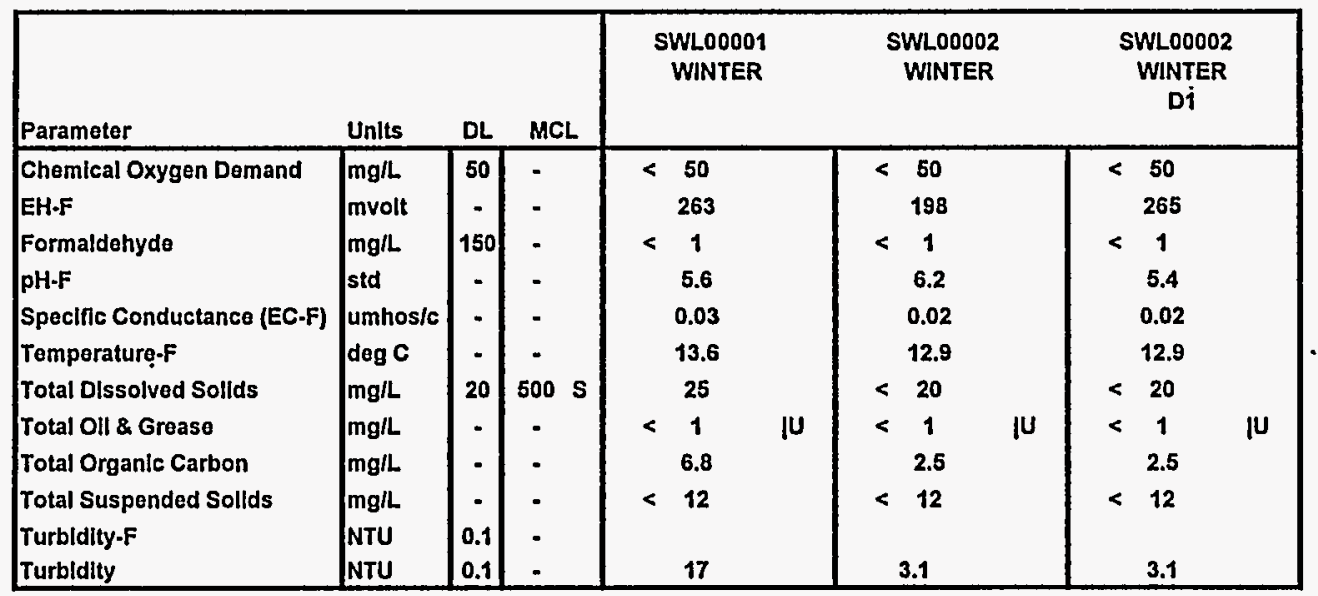


TABLE B.7

ANALYTICAL RESULTS FOR SURFACE WATER AND STORM WATER, 1995

OTHER PARAMETERS

1995 ANNUAL WATER MONITORING REPORT

LEHR ENVIRONMENTAL RESTORATION, DAVIS CA

$D \mid=$ Dupilcate sample.

. = Parameter not analyzed. Storm water runoff avallablo for sampling in March, 1995 only.

$<=$ Constituent below detection IImIt. Detection IImlts may vary depending on Interference by other sample constituents.

$M C L=$ Maximum Contaminant Level (primary), Title 22 DIvIsion 4 Chapter 15.

See Appendix $B$ for explanatlon of data qualifiers.

$S=$ Secondary Drinkling Water Standard 


\section{Appendix C}

Time Series Tables 

Groundwater Analytical Results 

Table C.1. Analytical Results for Groundwater, Fall 1990 Through Fall 1995, Volatile Organic Compounds

\section{Chloroform}

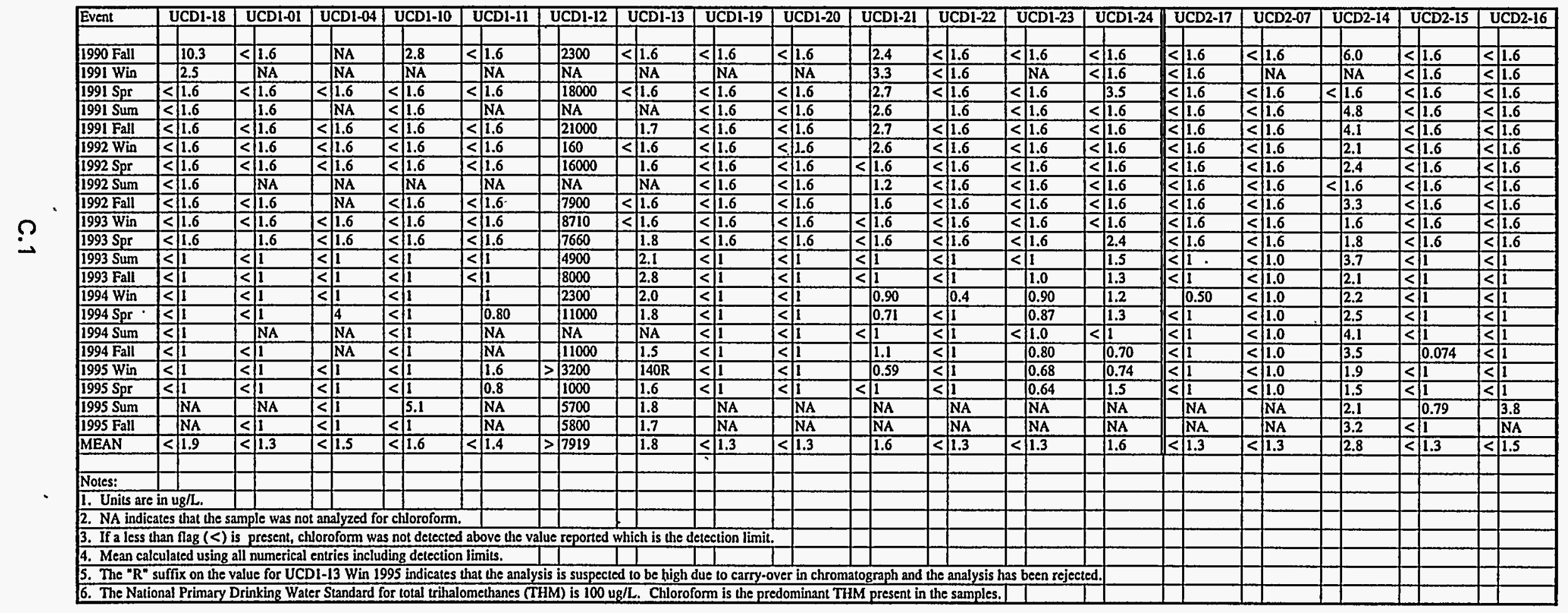


Table C.1. Analytical Results for Groundwater, Fall 1990 Through Fall 1995, Volatile Organic Compounds

\section{Bromodichloromethane}

\begin{tabular}{|c|c|c|c|c|c|c|c|c|c|c|c|c|c|c|c|c|c|c|c|c|c|c|}
\hline Event & UCDI-18 & UCDI-01 & $\mathrm{UCD1.04}$ & UCDI-10 & UCD1-11 & UCD1-12 & UCD1-13 & UCD1-19 & UCD1-20 & UCD1-21 & UCD1-22 & UCD1-23 & UCD1-24 & UCD2-17 & & CD2-07 & & SD2-14 & & $\overline{\mathrm{CD} 2-15}$ & & $\mathrm{CD} 2-16$ \\
\hline & & & & & & & & & & & & & & & & & & & & & & \\
\hline 1990 Fall & $<\longdiv { 2 . 2 }$ & $<\longdiv { 2 . 2 }$ & NA & $<\overline{2.2}$ & $<\longdiv { 2 . 2 }$ & $<\mid 2.2$ & $<\longdiv { 2 . 2 }$ & $<\longdiv { 2 . 2 }$ & $<\longdiv { 2 . 2 }$ & $<\longdiv { 2 . 2 }$ & $<\longdiv { 2 . 2 }$ & $<2.2$ & $<\longdiv { 2 . 2 }$ & $<\longdiv { 2 . 2 }$ & $</ 2$. & 2.2 & & 2.2 & & 2.2 & & 2.2 \\
\hline 1991 Win & \begin{tabular}{|l|l|}
$<2.2$ \\
\end{tabular} & NA & NA & NA & NA & NA & NA & \begin{tabular}{|l}
$\mathrm{NA}$ \\
\end{tabular} & NA & \begin{tabular}{l|l|}
$<2.2$ \\
\end{tabular} & \begin{tabular}{|l|l|}
$<2.2$ \\
\end{tabular} & NA & $<2.2$ & $<2.2$ & & $\sqrt{\mathrm{NA}}$ & & NA & & 2.2 & & 2.2 \\
\hline $1991 \mathrm{Spr}$ & $<\mid 2.2$ & \begin{tabular}{l|l|}
$<2.2$ \\
\end{tabular} & $<2.2$ & $<2.2$ & $<2.2$ & $<2.2$ & $<2.2$ & \begin{tabular}{l|l}
$<2.2$ \\
\end{tabular} & $<2.2$ & \begin{tabular}{|l|l|}
$<2.2$ \\
\end{tabular} & $<2$ & $<2.2$ & $<2.2$ & $<2$ & $<2$. & 2.2 & & 2.2 & & 2.2 & & 2.2 \\
\hline 1991 Sum & $<2$ & NA & NA & $<2.2$ & $\mathrm{NA}$ & NA & NA & $<2.2$ & $<2.2$ & $<2.2$ & $<2.2$ & $<2$ & \begin{tabular}{|l|l|}
$<2.2$ \\
\end{tabular} & \begin{tabular}{|l|l|}
$<2.2$ \\
\end{tabular} & & 2.2 & & 2.2 & & 2.2 & & 2.2 \\
\hline 1991 Fall & \begin{tabular}{|l|l|}
$<2.2$ \\
\end{tabular} & \begin{tabular}{|l|l|} 
& 2.2 \\
\end{tabular} & $<2.2$ & $<2.2$ & \begin{tabular}{l|l|}
$<\mid 2.2$ \\
\end{tabular} & 2.4 & $<2.2$ & $<2.2$ & $<2.2$ & $<<2.2$ & \begin{tabular}{|l|l|}
$<2.2$ \\
\end{tabular} & $<2.2$ & $<2.2$ & $<2$ & & 2.2 & & 2.2 & & 2.2 & & 2.2 \\
\hline 1992 Win & \begin{tabular}{l|l|}
2.2 \\
$<$
\end{tabular} & \begin{tabular}{|l|l|} 
& 2.2 \\
\end{tabular} & \begin{tabular}{|l|l|}
$<2.2$ \\
\end{tabular} & \begin{tabular}{l|l}
$<2.2$ \\
\end{tabular} & $<2$ & $\begin{array}{l}<2.2 \\
\end{array}$ & $<2.2$ & \begin{tabular}{|l|l|}
$<2.2$ \\
\end{tabular} & $<2.2$ & $<2.2$ & $<2.2$ & $<2.2$ & \begin{tabular}{|l|l|}
$<<2.2$ \\
\end{tabular} & $<2.2$ & & 2.2 & & 2.2 & & 2.2 & & 2.2 \\
\hline $1992 \mathrm{Spr}$ & \begin{tabular}{|l|l|}
$<2.2$ \\
\end{tabular} & $<2.2$ & $<2.2$ & $\begin{array}{l}<2.2 \\
\end{array}$ & $<\longdiv { 2 . 2 }$ & $<2.2$ & $<2.2$ & $<2.2$ & $<2.2$ & $<\longdiv { 2 . 2 }$ & $<2$ & $<2.2$ & \begin{tabular}{|l|l|}
$<2.2$ \\
\end{tabular} & $<\longdiv { 2 . 2 }$ & $<2$ & 2.2 & & 2.2 & & 2.2 & & 2.2 \\
\hline 1992 Sum & \begin{tabular}{l|l|}
$<2.2$ \\
\end{tabular} & NA & NA & NA & NA & NA & NA & \begin{tabular}{|l|l|}
$<<2.2$ \\
\end{tabular} & \begin{tabular}{|l|l|}
$<2.2$ \\
\end{tabular} & \begin{tabular}{l|l|}
$<$ & 2.2 \\
\end{tabular} & $<2.2$ & \begin{tabular}{|l|l|} 
& 2.2 \\
\end{tabular} & $<2.2$ & \begin{tabular}{|l|l|}
$<2.2$ \\
\end{tabular} & & 2.2 & & 2.2 & & 2.2 & & 2.2 \\
\hline 1992 Fall & \begin{tabular}{|l|l|}
$<2.2$ \\
\end{tabular} & \begin{tabular}{|l|}
2.2 \\
\end{tabular} & NA & $<2.2$ & $<\longdiv { 2 . 2 }$ & $<\mid 2.2$ & \begin{tabular}{|l|l|}
$<2,2$ \\
\end{tabular} & $<2.2$ & \begin{tabular}{l|l|}
$<<2.2$ \\
\end{tabular} & $<2.2$ & $<2.2$ & \begin{tabular}{|l|l|}
$<2.2$ \\
\end{tabular} & \begin{tabular}{|l|l|}
$<2.2$ \\
\end{tabular} & \begin{tabular}{|l|l|}
$<2.2$ \\
\end{tabular} & $<\sqrt{2}$ & 2.2 & & 2.2 & & 2.2 & & 2.2 \\
\hline 1993 Win & $<2.2$ & $<2.2$ & $<2.2$ & \begin{tabular}{|l|l|}
$<2.2$ \\
\end{tabular} & \begin{tabular}{l|l|}
$<<2.2$ \\
\end{tabular} & $<2$ & \begin{tabular}{|l|l|}
$<2.2$ \\
\end{tabular} & \begin{tabular}{l|l|}
$<<2.2$ \\
\end{tabular} & $<\longdiv { 2 . 2 }$ & $<2$ & \begin{tabular}{|l|l|}
$<$ & 2.2 \\
\end{tabular} & \begin{tabular}{|l|l|}
2.2 \\
\end{tabular} & \begin{tabular}{|l|l|}
$<2.2$ \\
\end{tabular} & $<2$ & & 2.2 & & 2.2 & & 2.2 & & 2.2 \\
\hline $1993 \mathrm{Spr}$ & \begin{tabular}{|l|l|}
$<2.2$ \\
\end{tabular} & \begin{tabular}{|l|l|}
$<2.2$ \\
\end{tabular} & \begin{tabular}{l|l|}
$<$ & 2.2 \\
\end{tabular} & $<2.2$ & $<2.2$ & \begin{tabular}{|l|l|} 
& 2.2 \\
\end{tabular} & \begin{tabular}{|l|l|}
$<2.2$ \\
\end{tabular} & \begin{tabular}{|l|l|}
$<2.2$ \\
\end{tabular} & \begin{tabular}{|l|l|} 
& 2.2 \\
\end{tabular} & $<2.2$ & \begin{tabular}{|l|l|} 
& 2.2 \\
\end{tabular} & $<\longdiv { 2 . 2 }$ & $<\longdiv { 2 . 2 }$ & $\leq 2.2$ & & 2.2 & & 2.2 & & 2.2 & & 2.2 \\
\hline 1993 Sum & $<1$ & $<1$ & $<1$ & $<\longdiv { 1 }$ & \begin{tabular}{|l|l|}
$<1$ \\
\end{tabular} & \begin{tabular}{|l|l|}
1.4 \\
\end{tabular} & $<\longdiv { 1 }$ & $\begin{array}{ll}<1 \\
\end{array}$ & \begin{tabular}{|l|l}
$<1$ \\
\end{tabular} & 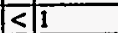 & $<\mid 1$ & \begin{tabular}{|l|l|} 
& 1 \\
\end{tabular} & \begin{tabular}{|l|l|}
$<$ & 1 \\
\end{tabular} & $<1$ & \begin{tabular}{l|l}
$<$ & 1 \\
\end{tabular} & & $<1$ & 1 & $<$ & 1 & $<1$ & \\
\hline 1993 Fall & $<1$ & \begin{tabular}{|l|l|}
$<1$ \\
\end{tabular} & $<i$ & \begin{tabular}{|l|l|}
$<$ & 1 \\
\end{tabular} & $<1$ & 1.2 & $\begin{array}{ll} & 1 \\
\end{array}$ & $\leq 1$ & $<1$ & $<1$ & $<1$ & \begin{tabular}{l|l} 
\\
\end{tabular} & $<1$ & $\leq 1$ & \begin{tabular}{l|l}
$<<1$ \\
\end{tabular} & & $<$ & 1 & $<1$ & $I$ & $<$ & \\
\hline 1994 Win & \begin{tabular}{|l|l|}
$<$ & 1 \\
\end{tabular} & \begin{tabular}{|l|l|}
$<1$ \\
\end{tabular} & $<1$ & $<1$ & \begin{tabular}{l|l}
$<1$ \\
\end{tabular} & 1.6 & \begin{tabular}{l|l} 
\\
\end{tabular} & $<1$ & $<1$ & \begin{tabular}{|l|l}
$<1$ \\
\end{tabular} & $<1$ & \begin{tabular}{|l|l|}
$<$ & 1 \\
\end{tabular} & \begin{tabular}{l|l}
$<1$ \\
\end{tabular} & $<1$ & \begin{tabular}{l|l|}
$<$ \\
\end{tabular} & & & 1 & & 1 & $<$ & \\
\hline $1994 \mathrm{Spr}$ & $<1$ & \begin{tabular}{|l|l|l|}
$<1$ \\
\end{tabular} & $<1$ & \begin{tabular}{|l|l|}
$<1$ \\
\end{tabular} & $<\mid \frac{1}{<}$ & 2.6 & \begin{tabular}{l|l}
$<1$ \\
\end{tabular} & $<1$ & \begin{tabular}{l|l}
$<$ & 1
\end{tabular} & $<1$ & \begin{tabular}{|l|l}
$<$ & 1 \\
\end{tabular} & $<1$ & $<1$ & $<1$ & \begin{tabular}{|l|l|}
$<1$ \\
\end{tabular} & & $<$ & I & $<$ & & $<$ & \\
\hline 1994 Sum & \begin{tabular}{|l|l|}
$<$ & 1 \\
\end{tabular} & NA & NA & \begin{tabular}{|l|l}
$<$ \\
\end{tabular} & \begin{tabular}{|l|} 
NA \\
\end{tabular} & $\overline{\mathrm{NA}}$ & NA & \begin{tabular}{|l|}
$<\mid 1$ \\
\end{tabular} & $<1$ & \begin{tabular}{|l|l|}
$<1$ \\
\end{tabular} & $<1$ & \begin{tabular}{|l|l|} 
& \\
\end{tabular} & $<1$ & $<1$ & \begin{tabular}{|l|l|}
$<$ \\
\end{tabular} & & $<$ & 1 & $<$ & & $<$ & \\
\hline 1994 Fall & $<1$ & $<\frac{1}{1}$ & \begin{tabular}{|l|} 
NA \\
\end{tabular} & \begin{tabular}{l|l}
$<1$ \\
\end{tabular} & \begin{tabular}{|l|l|}
$<$ & 1 \\
\end{tabular} & 1.3 & $<\mid 1$ & \begin{tabular}{l|l}
$<1$ \\
\end{tabular} & $<1$ & $<1$ & $<1$ & $<i$ & $<1$ & $<1$ & $<1$ & & $<$ & 1 & $<$ & & $<$ & \\
\hline 1995 Win & \begin{tabular}{|l|l|}
$<1$ \\
\end{tabular} & $<1$ & $<1$ & \begin{tabular}{|l|l|}
$<$ \\
\end{tabular} & \begin{tabular}{l|l}
$<<1$ \\
\end{tabular} & 1.2 & \begin{tabular}{|l|l|}
$<$ & 1 \\
\end{tabular} & $<1$ & $<1$ & $\begin{array}{ll}<1 \\
\end{array}$ & $\begin{array}{ll}<1 \\
\end{array}$ & $\begin{array}{ll}<1 \\
\end{array}$ & $<1$ & $<1$ & \begin{tabular}{l|l|l|}
$<$ & 1 \\
\end{tabular} & & $<1$ & & $<$ & & $<$ & \\
\hline $1995 \mathrm{Spr}$ & $<i$ & $<1$ & \begin{tabular}{l|l|}
$<$ & 1 \\
\end{tabular} & \begin{tabular}{l|l|l} 
&
\end{tabular} & $<1$ & $<\mid 1$ & $<1$ & $<1$ & $<\sqrt{1}$ & $<1$ & \begin{tabular}{|l|l|} 
& 1 \\
\end{tabular} & \begin{tabular}{|l|l|}
$<$ & 1
\end{tabular} & 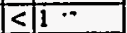 & \begin{tabular}{|l|l|}
$<1$ \\
\end{tabular} & $<1$ & & $<1$ & 1 & $<$ & & $<$ & \\
\hline 1995 Sum & NA & NA & $\frac{1}{<1}$ & 䜤 & NA & 0.82 & $<1$ & NA & NA & \begin{tabular}{|l|} 
NA \\
\end{tabular} & NA & NA & \begin{tabular}{|l|} 
NA \\
\end{tabular} & $\overline{N A}$ & & NA & $<1$ & & $<$ & & $<$ & 1 \\
\hline 1995 Fall & NA & $<1$ & $<1$ & $<1$ & $\mathrm{NA}$ & 0.63 & \begin{tabular}{|l|l|}
$<1$ \\
\end{tabular} & $\overline{\mathrm{NA}}$ & $\mathrm{NA}$ & NA & NA & NA & NA & NA & & NA & $<$ & 1 & 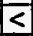 & & & $\mathrm{NA}$ \\
\hline MEAN & $\begin{array}{l}<1 \\
\end{array}$ & $<1$ & \begin{tabular}{|l|l|}
$<$ & 1 \\
\end{tabular} & $\begin{array}{ll}<1 \\
\end{array}$ & $<1$ & 1.3 & \begin{tabular}{l|l}
$<1$ \\
\end{tabular} & $<1$ & $<1$ & \begin{tabular}{l|l}
$<$ & 1 \\
\end{tabular} & \begin{tabular}{|l|l|}
$<$ & 1 \\
\end{tabular} & \begin{tabular}{|l|l|}
$<$ & 1 \\
\end{tabular} & \begin{tabular}{|l|l|}
$<1$ \\
\end{tabular} & $<1$ & $<1$ & & $\leq$ & 1 & $<$ & 1 & $<1$ & $I$ \\
\hline & & & & & & & & & & & & & & & & & & & & & & \\
\hline Notes: & & & & & & & & & & & & & & & & & & & & & & \\
\hline 1. Units an & in ug $/ \mathrm{L}$. & & & & & & & & & & & & & & & & & & & & & \\
\hline 2. NA ind & ales that the sa & imple was not & analyzed fo & r bromodich & oromethane. & & & & & & & & & & & & & & & & & \\
\hline 3. If a less & han flag $(<)$ & is present, br & omodichloro & methane was & not detected a & bove the valt & ue reported w & aich is the det & lection limit. & & & & & & & & & & & & & \\
\hline 4. Mean y & ues were calc & lated for rest & Itts from the & summer of 1 & 993 and latter & & 1 & $\square$ & & & & & & & & & & & & & & \\
\hline & & rinking Wat & & & & & & & & & & & & & & & & & & & & \\
\hline
\end{tabular}


Table C.1. Analytical Results for Groundwater, Fall 1990 Through Fall 1995, Volatile Organic Compounds

\section{1,1-dichloroethane}

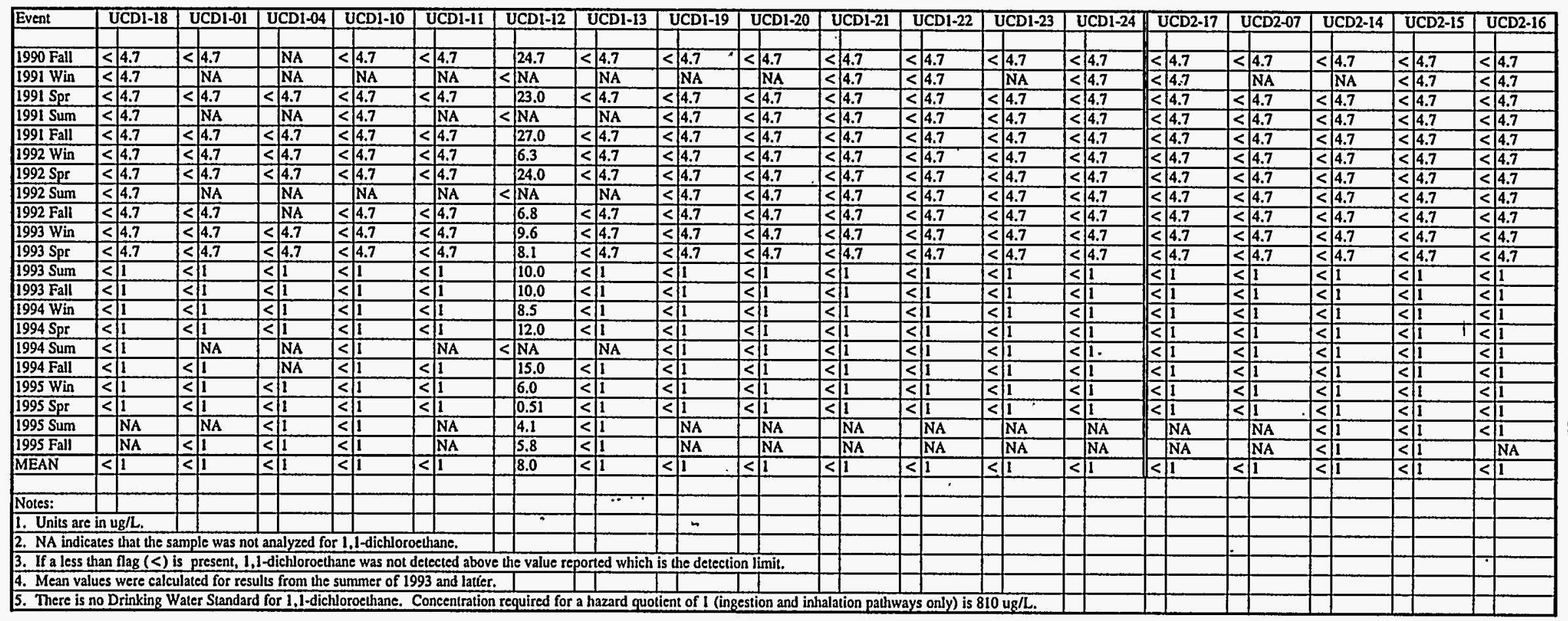


Table C.1. Analytical Results for Groundwater, Fall 1990 Through Fall 1995, Volatile Organic Compounds

\section{1,1-dichloroethene}

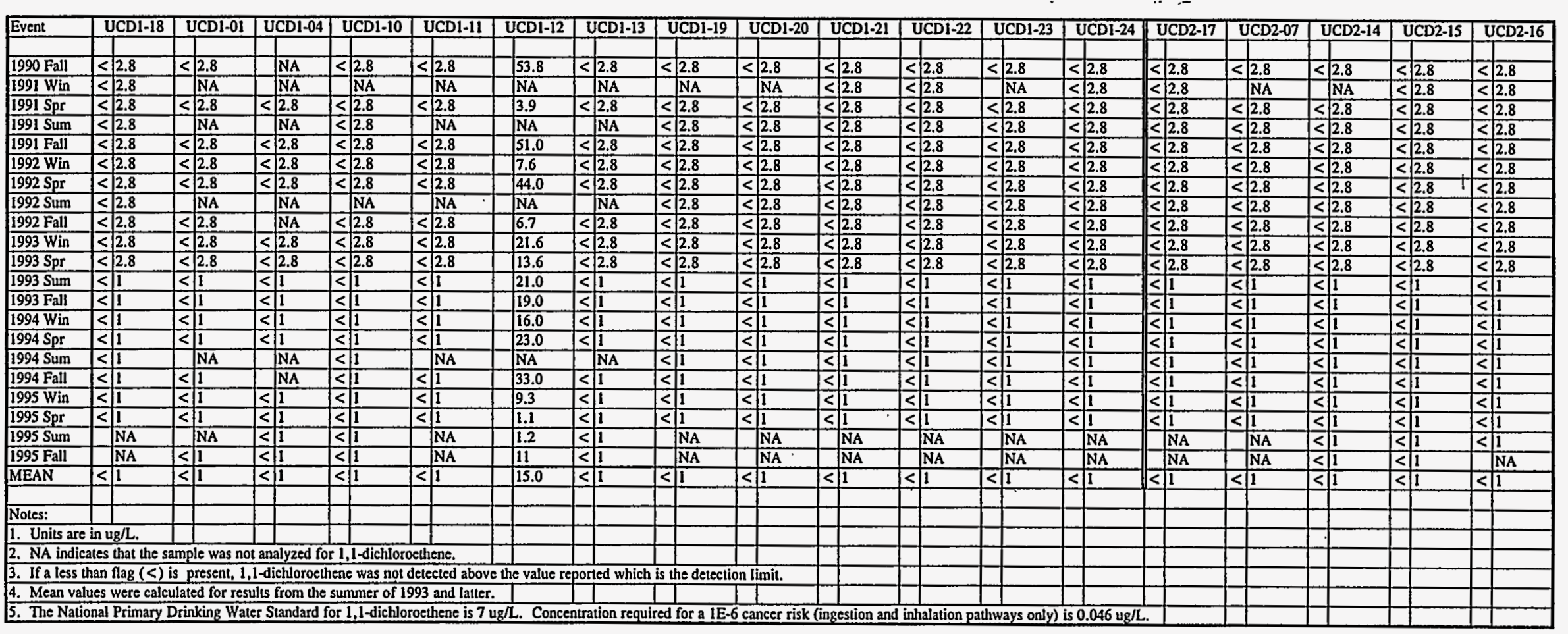


Table C.1. Analytical Results for Groundwater, Fall 1990 Through Fall 1995,

Volatile Organic Compounds

\section{1,2-dichloroethane}

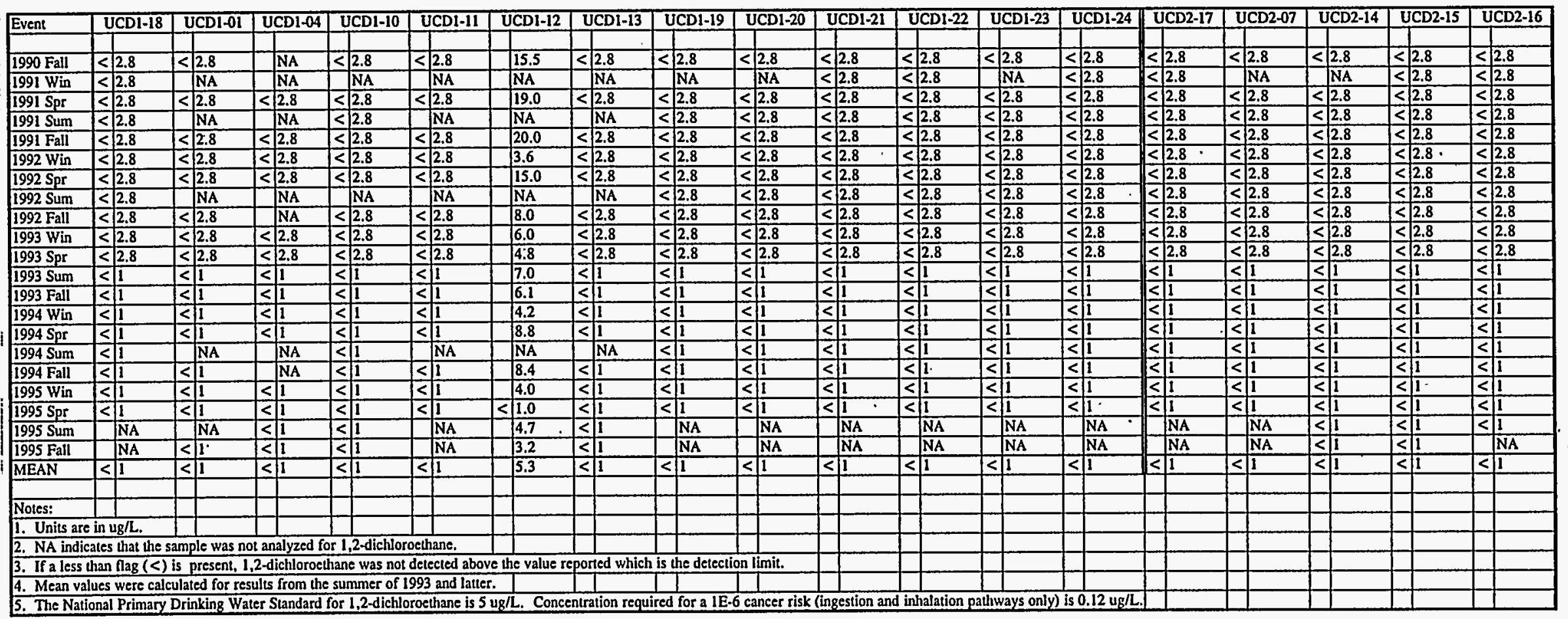


Table C.1. Analytical Results for Groundwater, Fall 1990 Through Fall 1995, Volatile Organic Compounds

\section{1,1,2-trichloroethane}

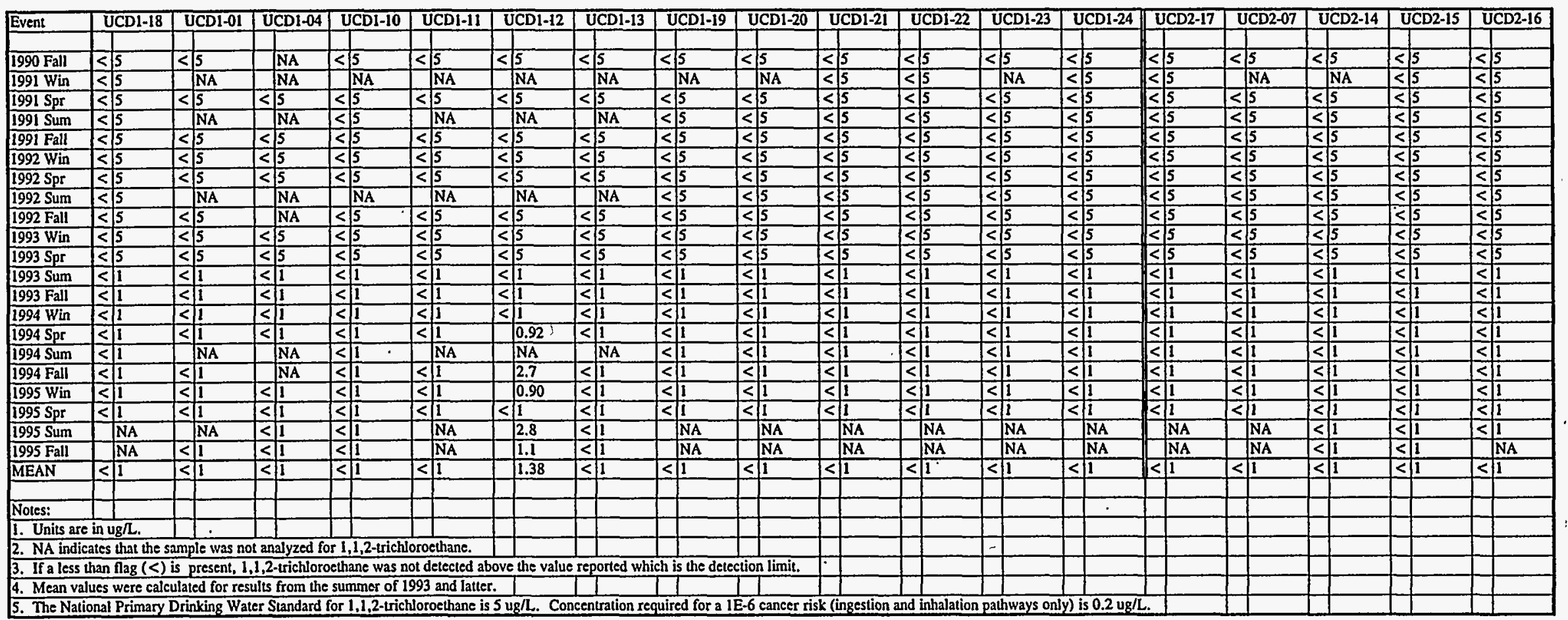


Table C.2. Analytical Results for Groundwater, Fall 1990 Through Fall 1995, Pesticides and PCBs

\section{Alpha Chlordane}

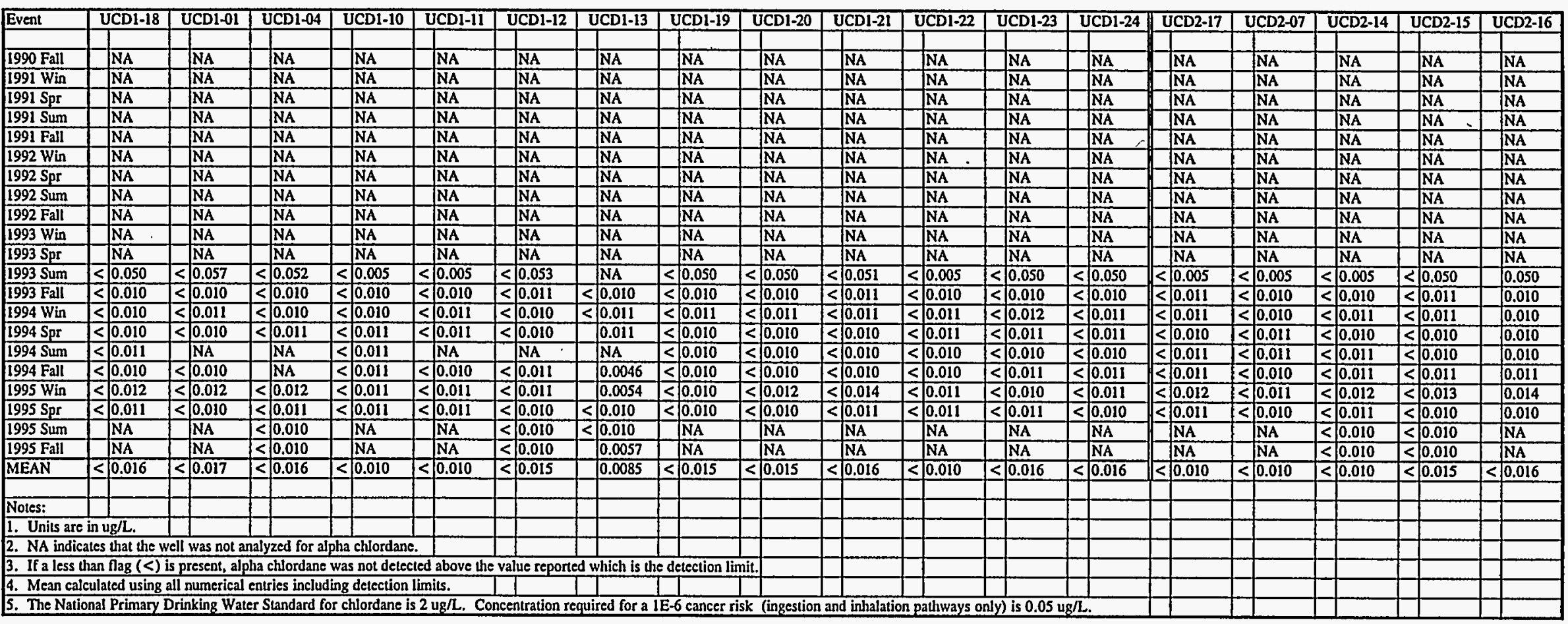


Table C.2. Analytical Results for Groundwater, Fall 1990 Through Fall 1995, Pesticides and PCBs

\section{Gamma Chlordane}

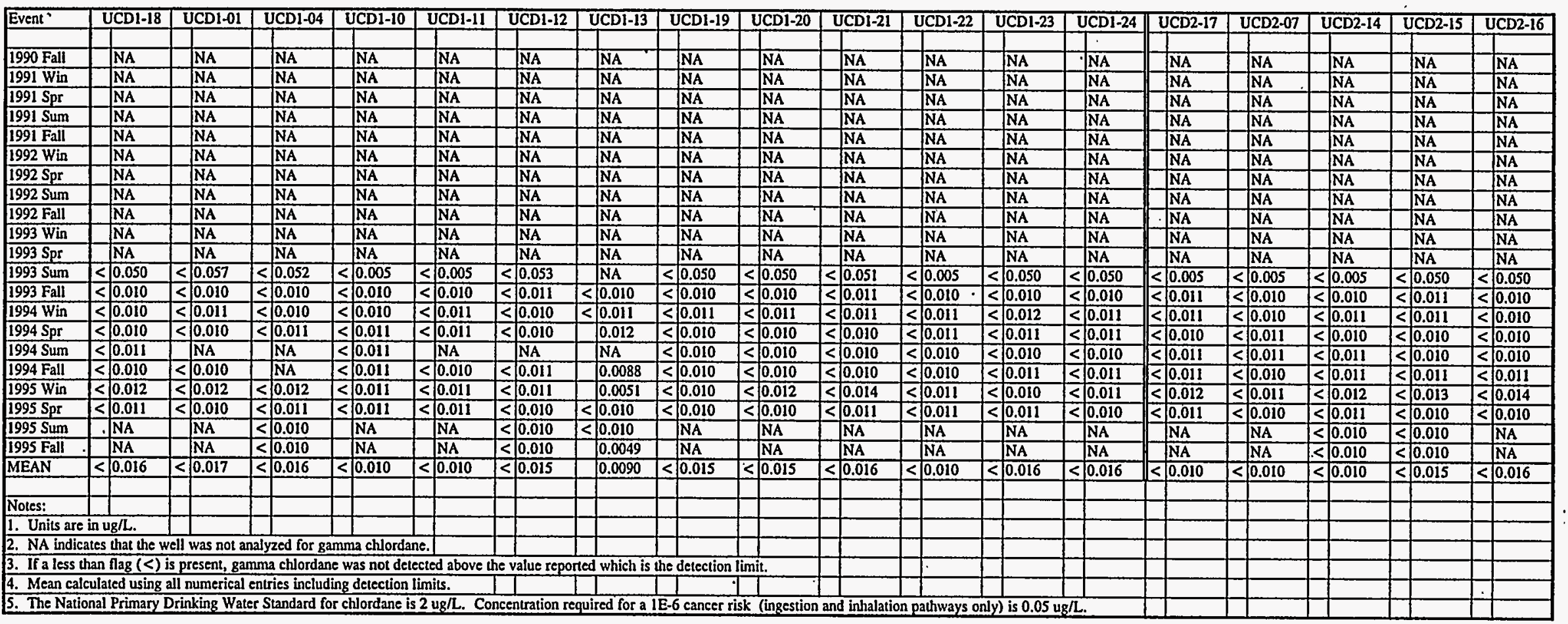


Table C.2. Analytical Results for Groundwater, Fall 1990 Through Fall 1995, Pesticides and PCBs

\section{Dieldrin}

\begin{tabular}{|c|c|c|c|c|c|c|c|c|c|c|c|c|c|c|c|c|c|c|c|}
\hline Event & UCD1-18 & UCDI-01 & \begin{tabular}{|l} 
UCD1-04 \\
\end{tabular} & UCD1-10 & UCDI-11 & UCDI-12 & UCDI-13 & UCDI-19 & UCDI-20 & UCDI-21 & UCD1-22 & UCD1-23 & UCD1-24 & UCD2-17 & UCD2-07 & & JCD2-14 & UCD2-15 & UCD2-16 \\
\hline & & & & & & & & & & & & & & & & & & & \\
\hline$\frac{1990 \text { Fall }}{1991 \text { Win }}$ & $\begin{array}{l}<\mid 0.002 \\
<0.002\end{array}$ & \begin{tabular}{c|c|c|}
$\leq 0.002$ \\
NA
\end{tabular} & $\frac{\mathrm{NA}}{\mathrm{NA}}$ & \begin{tabular}{|l|l}
$<<$ & 0.002 \\
$N A$
\end{tabular} & $\leq 0.002$ & $<0.002$ & 0.021 & \begin{tabular}{l|l|}
$<$ & 0.002 \\
\end{tabular} & $<0,0.002$ & $<\longdiv { 0 . 0 0 2 }$ & \begin{tabular}{l|l|}
$<$ & 0.002 \\
\end{tabular} & $<0.002$ & $<\mid 0.002$ & $<0.002$ & \begin{tabular}{l|l|}
$<$ & 0.002 \\
\end{tabular} & & 0.002 & \begin{tabular}{l|l|}
$<$ & 0.002 \\
\end{tabular} & \begin{tabular}{l|l|} 
& 0.002 \\
\end{tabular} \\
\hline$\frac{1991 \mathrm{Win}}{1991 \mathrm{Spr}}$ & $<0.002$ & $<0.002$ & \begin{tabular}{|l|l} 
& NA \\
$<0.002$
\end{tabular} & NA & NA & NA & 0.025 & NA & NA & \begin{tabular}{l|l|} 
& 0.002 \\
\end{tabular} & \begin{tabular}{l|l|}
$<$ & 0.002 \\
\end{tabular} & \begin{tabular}{|l|l|}
$<\mathrm{NA}$ \\
\end{tabular} & \begin{tabular}{|l|l|}
$<$ & 0.002 \\
\end{tabular} & $<0.002$ & NA & & NA & $\begin{array}{l}<<0.002 \\
\end{array}$ & $\begin{array}{l}0.002 \\
\end{array}$ \\
\hline$\frac{1991 \mathrm{Spr}}{1991 \mathrm{Sum}}$ & & $\leq \mid 0.002$ & \begin{tabular}{|l|l|} 
& 0.002 \\
\end{tabular} & $\leq \quad 0.002$ & $\leq 0.002$ & \begin{tabular}{l|l}
$<$ & 0.002 \\
\end{tabular} & $\leq 0.002$ & $<0.002$ & $<0.002$ & $\begin{array}{l}0.002 \\
\end{array}$ & \begin{tabular}{|l|l|}
$<$ & 0.002 \\
\end{tabular} & \begin{tabular}{|l|l|}
$<$ & 0.002 \\
\end{tabular} & \begin{tabular}{l|l|}
$<$ & 0.002 \\
\end{tabular} & $<0.002$ & $<0.002$ & & 0.002 & $<\longdiv { 0 . 0 0 2 }$ & \begin{tabular}{|c|}
0.002 \\
\end{tabular} \\
\hline 1991 Sum & $\leq \quad 0.002$ & NA & NA & NA & NA & NA & NA & $<0.002$ & $<\overline{0.002}$ & $\begin{array}{l} \\
\end{array}$ & \begin{tabular}{|l|l|}
$<$ & 0.002 \\
\end{tabular} & \begin{tabular}{l|l|}
$<0.002$ \\
\end{tabular} & \begin{tabular}{l|l|}
$<$ & 0.002 \\
\end{tabular} & 0.002 & $\begin{array}{l}<0.002 \\
\end{array}$ & & 0.002 & \begin{tabular}{|l|l|}
$<$ & 0.002 \\
\end{tabular} & \begin{tabular}{l|l|}
$<<0.002$ \\
\end{tabular} \\
\hline 1991 Fall & $<\quad 0.002$ & \begin{tabular}{l|l}
$<$ & 0.002 \\
\end{tabular} & \begin{tabular}{l|l|}
$<$ & 0.002 \\
\end{tabular} & \begin{tabular}{l|l|}
$\leq$ & 0.002 \\
\end{tabular} & \begin{tabular}{l|l|}
$<$ & 0.002 \\
\end{tabular} & \begin{tabular}{|l|l|} 
& 0.000 \\
\end{tabular} & 0.030 & \begin{tabular}{|l|l|}
$<$ & 0.002 \\
\end{tabular} & $<0.002$ & $\begin{array}{l}<0.002 \\
\end{array}$ & \begin{tabular}{|l|l|}
$<$ & 0.002 \\
\end{tabular} & \begin{tabular}{l|l|}
$<$ & 0.002 \\
\end{tabular} & \begin{tabular}{|l|l|}
0.002 \\
\end{tabular} & $\begin{array}{l} \\
\end{array}$ & $<0.002$ & $<$ & 0.002 & $\begin{array}{l}0.002 \\
\end{array}$ & \begin{tabular}{l|l|}
$<$ & 0.002 \\
\end{tabular} \\
\hline 1992 Win & $<0.002$ & \begin{tabular}{l|l|}
$<$ & 0.002 \\
\end{tabular} & \begin{tabular}{l|l}
$<\leq 002$ \\
\end{tabular} & $<0.002$ & \begin{tabular}{|l|l|}
$<$ & 0.002 \\
\end{tabular} & \begin{tabular}{|l|l|}
$<0.002$ \\
\end{tabular} & \begin{tabular}{|l|l|}
$<0.002$ \\
\end{tabular} & $\begin{array}{l}0.002 \\
\end{array}$ & \begin{tabular}{l|l|}
$<0.002$ \\
\end{tabular} & $\leq 0.002$ & \begin{tabular}{|l|l|}
$<0.002$ \\
\end{tabular} & \begin{tabular}{|l|l|}
$<$ & 0.002 \\
\end{tabular} & $<0.002$ & $\leq 0.002$ & $<0.002$ & & 0.002 & $<0.002$ & \begin{tabular}{|l|l|}
$<0.002$ \\
\end{tabular} \\
\hline $1992 \mathrm{Spr}$ & $\leq 0.002$ & $\leq 0.002$ & $\leq 0.002$ & $\leq 0.002$ & \begin{tabular}{|l|l|} 
& 0.002 \\
\end{tabular} & \begin{tabular}{|l|l|}
$<$ & 0.002 \\
\end{tabular} & 0.060 & \begin{tabular}{|l|l|}
$<$ & 0.002 \\
\end{tabular} & $<0.002$ & $<0.002$ & $\leq 0.002$ & $<\longdiv { 0 . 0 0 2 }$ & $<0.002$ & $\leq 0.002$ & \begin{tabular}{|l|l|}
$<.002$ \\
\end{tabular} & & 0.002 & $<0.002$ & \begin{tabular}{l|l|}
$<0.002$ \\
\end{tabular} \\
\hline $1992 \mathrm{Sum}$ & \begin{tabular}{|l|l|}
$<0.002$ \\
\end{tabular} & NA & NA & NA & NA & \begin{tabular}{|l|} 
NA \\
\end{tabular} & NA & \begin{tabular}{l|l}
$<0.002$ \\
\end{tabular} & \begin{tabular}{l|l}
$\leq 0.002$ \\
\end{tabular} & \begin{tabular}{|l|l|}
$<$ & 0.002 \\
\end{tabular} & $<0.002$ & \begin{tabular}{|l|l|}
$\leq 0.002$ \\
\end{tabular} & \begin{tabular}{l|l|l|} 
& 0.002 \\
\end{tabular} & $<0.002$ & \begin{tabular}{|l|l|} 
& 0.002 \\
\end{tabular} & & 0.002 & \begin{tabular}{l|l|} 
& 0.002 \\
\end{tabular} & $<\overline{0.002}$ \\
\hline 1992 Fall & $<0.002$ & $<0.002$ & NA & $<\overline{0.002}$ & $<0.002$ & \begin{tabular}{l|l|}
$<$ & 0.002 \\
\end{tabular} & 0.085 & $<0.002$ & $<\longdiv { 0 . 0 0 2 }$ & $\begin{array}{l}<0.002 \\
\end{array}$ & $<0.002$ & $<\longdiv { 0 . 0 0 2 }$ & $<\longdiv { 0 . 0 0 2 }$ & $<0.002$ & $<0.002$ & & 0.002 & \begin{tabular}{l|l|}
$<0.002$ \\
\end{tabular} & $\begin{array}{ll}<<002 \\
\end{array}$ \\
\hline 1993 Win & $<0.002$ & $<0.002$ & $<0.002$ & $<0.002$ & $<0.002$ & \begin{tabular}{|l|l|}
$<0.002$ \\
\end{tabular} & 0.030 & \begin{tabular}{l|l}
$<0.002$ \\
\end{tabular} & \begin{tabular}{l|l|}
$\leq 0.002$ \\
\end{tabular} & \begin{tabular}{l|l|}
$<0.002$ \\
\end{tabular} & $<\longdiv { 0 . 0 0 2 }$ & $\begin{array}{ll}<0.002 \\
\end{array}$ & \begin{tabular}{|l|l|}
$\leq 0.002$ \\
\end{tabular} & $<\longdiv { 0 . 0 0 2 }$ & $<\overline{0.002}$ & & 0.000 & NA & \begin{tabular}{l|l|}
$<002$ \\
\end{tabular} \\
\hline $1993 \mathrm{Spr}$. & $<0.002$ & $<0.002$ & $<0.002$ & $\begin{array}{l}<0.002 \\
\end{array}$ & $<0.002$ & $<0.002$ & 0.070 & $<0.002$ & $<\longdiv { 0 . 0 0 2 }$ & \begin{tabular}{l|l|}
$<$ & 0.002 \\
\end{tabular} & \begin{tabular}{l|l}
$<$ & 0.002 \\
\end{tabular} & $<0.002$ & $<\longdiv { 0 . 0 0 2 }$ & $<\longdiv { 0 . 0 0 2 }$ & $<0.002$ & & $\overline{0.002}$ & $<00.000$ & \begin{tabular}{|l|l}
$<0.002$ \\
\end{tabular} \\
\hline $1993 \mathrm{Sum}$ & $<0.100$ & \begin{tabular}{l|l|}
$<$ & 0.110 \\
\end{tabular} & \begin{tabular}{|l|l|} 
& 0.100 \\
\end{tabular} & $<0.010$ & \begin{tabular}{|l|l|}
$<$ & 0.010 \\
\end{tabular} & $<\overline{0.110}$ & $\overline{\mathrm{NA}}$ & \begin{tabular}{|l|l|l|l|} 
& 0.100 \\
\end{tabular} & \begin{tabular}{l|l}
$\leq$ & 0.100 \\
\end{tabular} & $<0.100$ & $<0.010$ & $\begin{array}{l}<.100 \\
\end{array}$ & $<0.100$ & 0.010 & $<0.010$ & $<$ & 0.010 & $<0.100$ & \begin{tabular}{l|l}
$<100$ \\
\end{tabular} \\
\hline 1993 Fall & \begin{tabular}{l|l|}
$<$ & 0.021 \\
\end{tabular} & \begin{tabular}{l|l|}
$<021$ \\
\end{tabular} & \begin{tabular}{|l|l|}
$<$ & 0.021 \\
\end{tabular} & $\begin{array}{l}0.020 \\
\end{array}$ & \begin{tabular}{|l|l}
$<0.020$ \\
\end{tabular} & $<0.021$ & 0.059 & $<\overline{0.021}$ & $\begin{array}{lll} & 0.021\end{array}$ & \begin{tabular}{|l|l|} 
& 0.021 \\
\end{tabular} & $<\mid 0.021$ & \begin{tabular}{l|l}
$<0.021$ \\
\end{tabular} & \begin{tabular}{l|l}
$<$ & 0.021
\end{tabular} & \begin{tabular}{l|l} 
& 0.021
\end{tabular} & \begin{tabular}{l|l}
0.021 \\
\end{tabular} & & 0.020 & $<0.021$ & \begin{tabular}{l|l}
$<0.021$ \\
\end{tabular} \\
\hline $1994 \mathrm{Win}$ & $<0,021$ & \begin{tabular}{|l|l|}
$<$ & 0.021 \\
\end{tabular} & \begin{tabular}{|l|l|}
$<0.021$ \\
\end{tabular} & \begin{tabular}{|l|l|}
$<0220$ \\
\end{tabular} & \begin{tabular}{|l|l|l|}
$<.0222$ \\
\end{tabular} & $<0.021$ & 0.048 & \begin{tabular}{l|l} 
& 0.022 \\
\end{tabular} & $\leq 0.022$ & $<0.023$ & $\begin{array}{ll}<.022 \\
\end{array}$ & \begin{tabular}{l|l}
$<$ & 0.024 \\
\end{tabular} & \begin{tabular}{l|l}
$\leq 0.022$ \\
\end{tabular} & \begin{tabular}{|ll} 
& 0.021 \\
\end{tabular} & \begin{tabular}{|l|l|l|} 
& 0.021 \\
\end{tabular} & $<$ & 0.021 & $<0.022$ & \begin{tabular}{l|l} 
& 0.021 \\
\end{tabular} \\
\hline $1994 \mathrm{Spr}$ & $<\longdiv { 0 . 0 2 0 }$ & $<0,0.021$ & \begin{tabular}{l|l|l|} 
& 0.021
\end{tabular} & $<\mid 0.021$ & $<\longdiv { 0 . 0 2 1 }$ & $<\quad 0.020$ & 0.076 & $<\overline{0.020}$ & $<0.021$ & 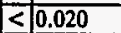 & \begin{tabular}{l|l}
$<$ & 0.021
\end{tabular} & $<\longdiv { 0 . 0 2 2 }$ & $<\overline{0.021}$ & \begin{tabular}{|l|l|}
$<020$
\end{tabular} & $\begin{array}{ll}< & 0.021\end{array}$ & $\overline{<}$ & 0.021 & $<0.021$ & $<0.021$ \\
\hline $1994 \mathrm{Sum}$ & $<\overline{0.022}$ & \begin{tabular}{|l}
$\mathrm{NA}$ \\
\end{tabular} & ZNA & \begin{tabular}{|l|l|l|}
$<$ & 0.021 \\
\end{tabular} & NA & NA & NA & \begin{tabular}{l|l} 
& 0.020 \\
\end{tabular} & $\begin{array}{l}0.020 \\
\end{array}$ & 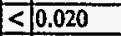 & $<0.020$ & $<\longdiv { 0 . 0 2 0 }$ & \begin{tabular}{l|l} 
& 0.020 \\
\end{tabular} & \begin{tabular}{|l|l|}
$<$ & 0.021 \\
\end{tabular} & \begin{tabular}{l|l|} 
& 0.021 \\
\end{tabular} & $\overline{<}$ & 0.022 & 0.020 & 0.020 \\
\hline 1994 Fall & \begin{tabular}{l|l|} 
\\
\end{tabular} & \begin{tabular}{|l|l|}
$\leq 020$ \\
\end{tabular} & $\overline{\text { NA }}$ & $<0.022$ & $<00.021$ & \begin{tabular}{l|l|}
$<$ & 0.022 \\
\end{tabular} & $\overline{0.094}$ & \begin{tabular}{l|l}
$<021$ \\
\end{tabular} & $<0.021$ & \begin{tabular}{l|l}
$<$ & 0.021
\end{tabular} & $<0.021$ & $<0.021$ & $<\longdiv { 0 . 0 2 2 }$ & 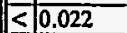 & $\begin{array}{lll} & 0.020\end{array}$ & & 0.022 & $\begin{array}{l}<021 \\
\end{array}$ & $<\frac{0.022}{<}$ \\
\hline $1995 \mathrm{Win}$ & \begin{tabular}{|l|l|}
$<$ & 0.024 \\
\end{tabular} & \begin{tabular}{l|l|}
$<0.024$ \\
\end{tabular} & \begin{tabular}{l|l}
$<$ & 0.025 \\
\end{tabular} & $<0.022$ & $\leq 0.022$ & \begin{tabular}{l|l}
$<$ & 0.022 \\
\end{tabular} & 0.029 & \begin{tabular}{l|l}
$<0.020$ \\
\end{tabular} & $<\longdiv { 0 . 0 2 5 }$ & $\begin{array}{l}\leq .027 \\
\end{array}$ & $<0.023$ & \begin{tabular}{l|l}
$\leq 0.020$ \\
\end{tabular} & $\leq 0.021$ & \begin{tabular}{l|l}
$<0.023$ \\
\end{tabular} & \begin{tabular}{l|l|}
$<022$ \\
\end{tabular} & & 0.024 & $<0.025$ & $<0.027$ \\
\hline $1995 \mathrm{Spr}$ & \begin{tabular}{|l|l|}
$<0.022$ \\
\end{tabular} & \begin{tabular}{l|l|} 
& 0.021 \\
\end{tabular} & \begin{tabular}{l|l|l|} 
& 0.021 \\
\end{tabular} & \begin{tabular}{|l|l|} 
& 0.0222 \\
\end{tabular} & $<\overline{0.021}$ & $<\overline{0.021}$ & \begin{tabular}{|l|l|l}
$<$ & 0.021 \\
\end{tabular} & \begin{tabular}{|l|l|}
$<0.021$ \\
\end{tabular} & $<0.021$ & \begin{tabular}{l|l}
$<021$ \\
\end{tabular} & \begin{tabular}{|l|l}
$<022$ \\
\end{tabular} & $\begin{array}{ll} & 0.021\end{array}$ & \begin{tabular}{l|l} 
& 0.021
\end{tabular} & $<0.022$ & \begin{tabular}{l|l}
$<0.021$ \\
\end{tabular} & & 0.021 & $\begin{array}{ll}<0.021 \\
\end{array}$ & $<0.021$ \\
\hline $1995 \mathrm{Sum}$ & NA & NA & \begin{tabular}{|l|l|l|l} 
& 0.020 \\
\end{tabular} & \begin{tabular}{|l|}
$\mathrm{NA}$ \\
\end{tabular} & NA & $<0.020$ & \begin{tabular}{|l|l|l|} 
& 0.020 \\
\end{tabular} & NA & NA & NA & NA & NA & NA & NA & NA & & 0.020 & \begin{tabular}{l|l|l}
$<0.020$ \\
\end{tabular} & NA \\
\hline 1995 Fall & NA & $\begin{array}{l}\mathrm{NA} \\
\end{array}$ & \begin{tabular}{|l|l|}
$<020$ \\
\end{tabular} & NA & NA & $<\quad 0.020$ & 0.028 & NA & NA & NA & \begin{tabular}{|l|} 
NA \\
\end{tabular} & $\overline{\mathrm{NA}}$ & NA & NA & NA & & 0.020 & \begin{tabular}{l|l}
$<0.020$ \\
\end{tabular} & NA \\
\hline \multirow[t]{2}{*}{ MEAN } & $<0.014$ & $<0.017$ & \begin{tabular}{l|l}
$<$ & 0.019 \\
\end{tabular} & $\leq 0.011$ & $\leq 0.010$ & $<0.017$ & 0.041 & $<0.015$ & $<0.015$ & $<\mid 0.014$ & \begin{tabular}{|l|l|}
$\leq 0.010$ \\
\end{tabular} & $\begin{array}{ll}\leq 015 \\
\end{array}$ & $<0.014$ & $<0.010$ & $<0.010$ & $<<$ & 0.011 & \begin{tabular}{l|l}
$<$ & 0.015 \\
\end{tabular} & \begin{tabular}{l|l}
$<014$ \\
\end{tabular} \\
\hline \multirow{2}{*}{\multicolumn{20}{|c|}{ Noles: }} \\
\hline & & & & & & & & & & & & & & & & & & & \\
\hline \multirow{2}{*}{\multicolumn{20}{|c|}{ 1. Units are in ug/L. }} \\
\hline & & & & & & & & & & & & & & & & & & & \\
\hline \multirow{2}{*}{\multicolumn{20}{|c|}{$\begin{array}{l}\text { the value reported which is the detection limit. } \\
\text { mits. }\end{array}$}} \\
\hline & & & & & & & & & & & & & & & & & & & \\
\hline D MUte & & & & & & & & & & & & & & & & & & & \\
\hline
\end{tabular}


Table C.2. Analytical Results for Groundwater, Fall 1990 Through Fall 1995, Pesticides and PCBs

\section{Endrin}

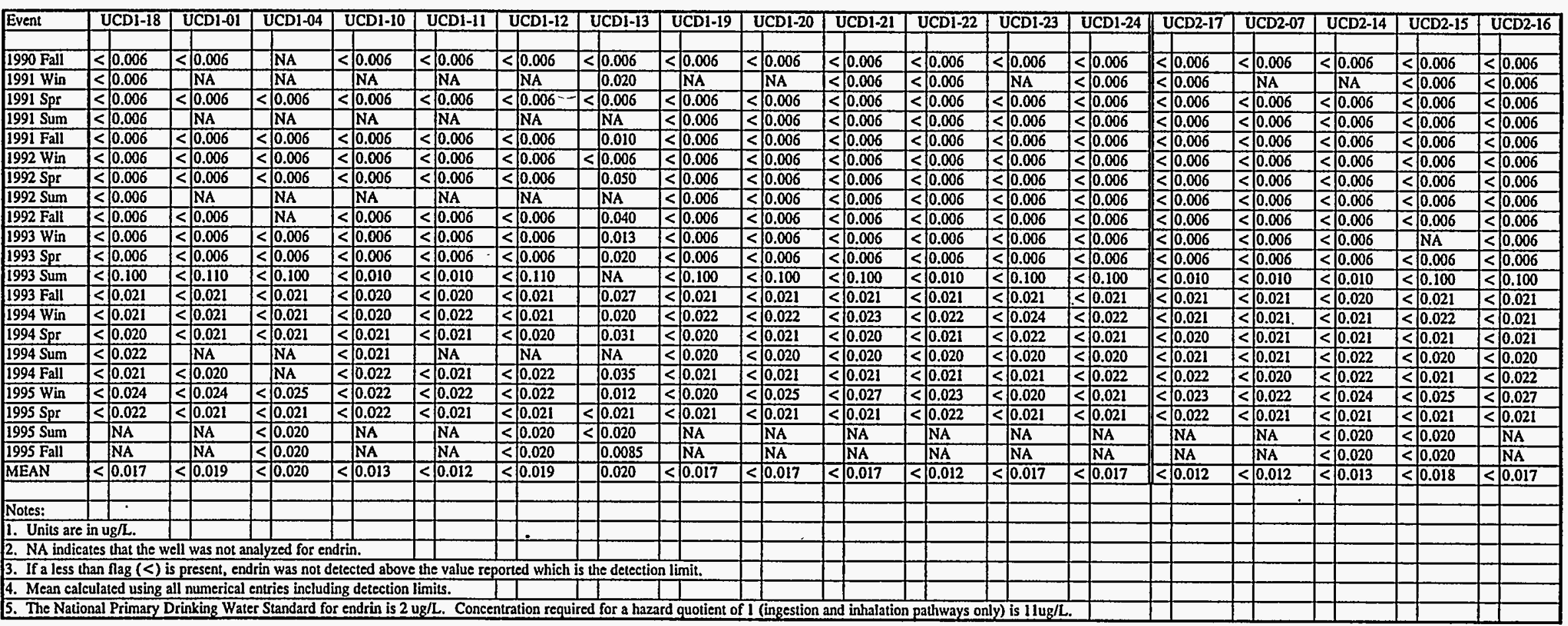


Table C.3. Analytical Results for Groundwater, Fall 1990 Through Fall 1995, Metals

\section{Antimony}

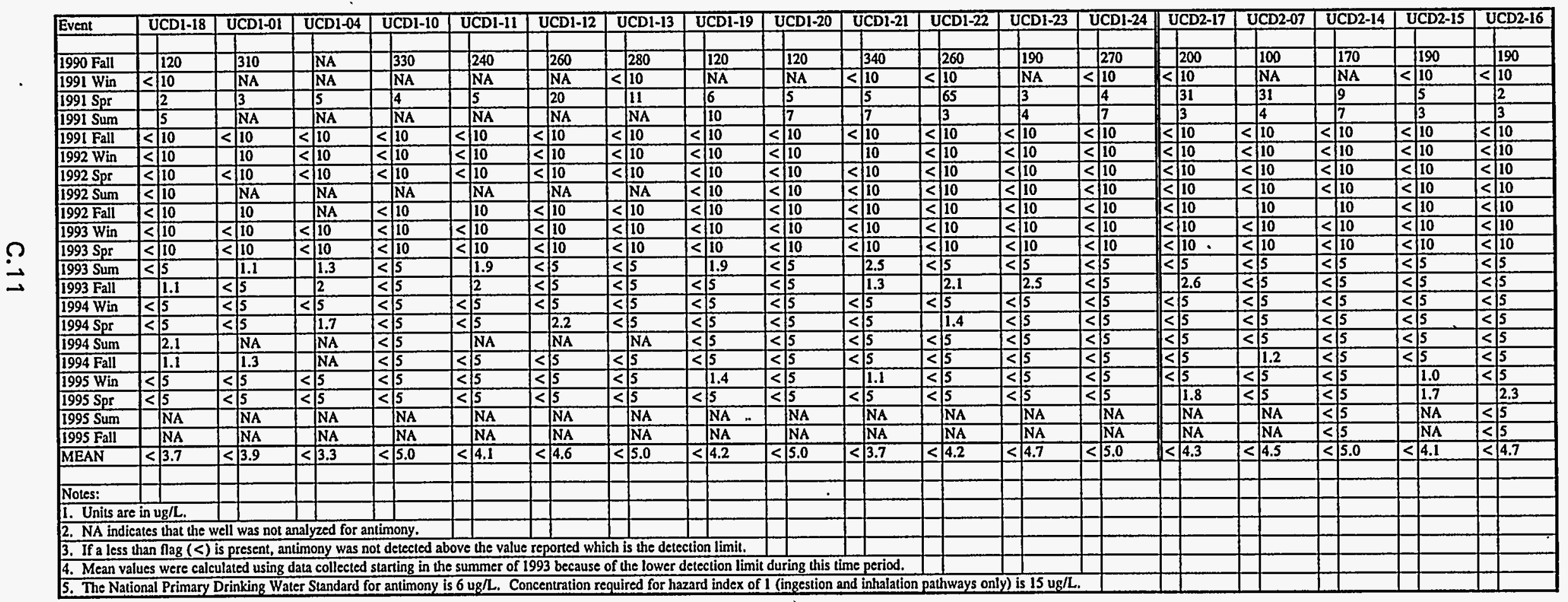


Table C.3. Analytical Results for Groundwater, Fall 1990 Through Fall 1995, Metals

Arsenic

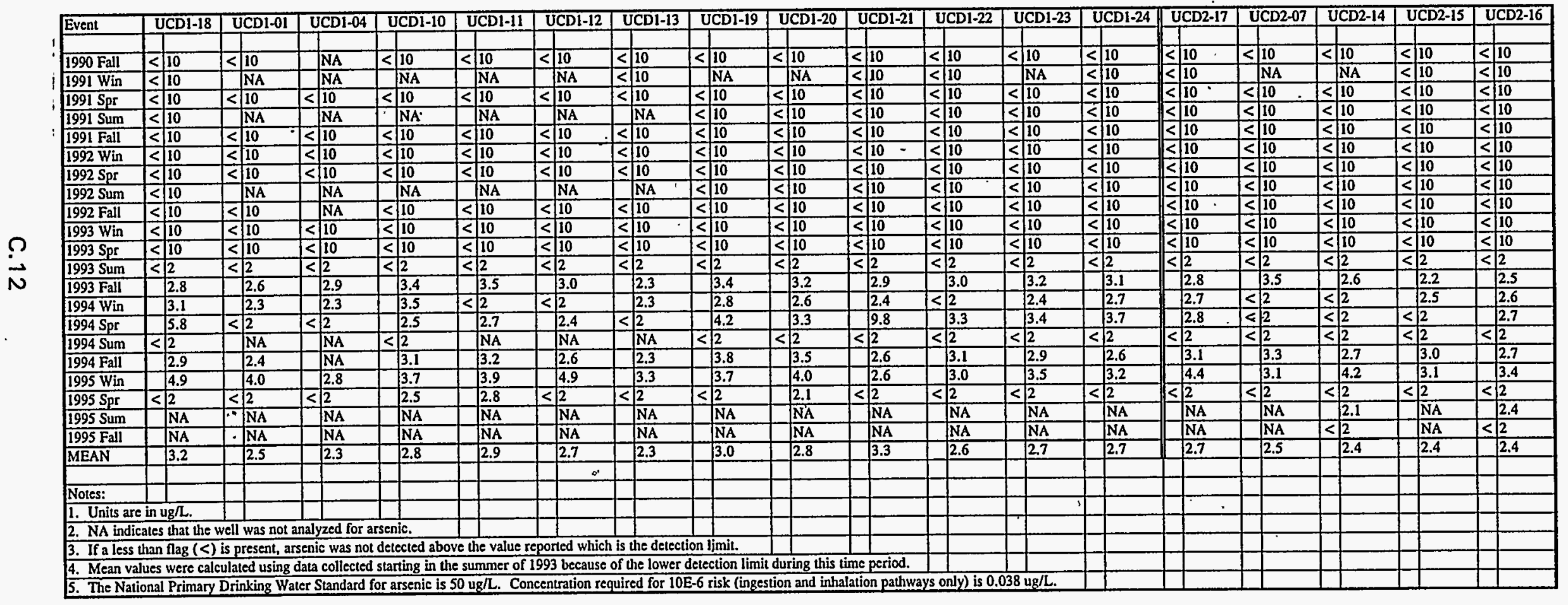


Table C.3. Analytical Results for Groundwater, Fall 1990 Through Fall 1995, Metals

\section{Barium}

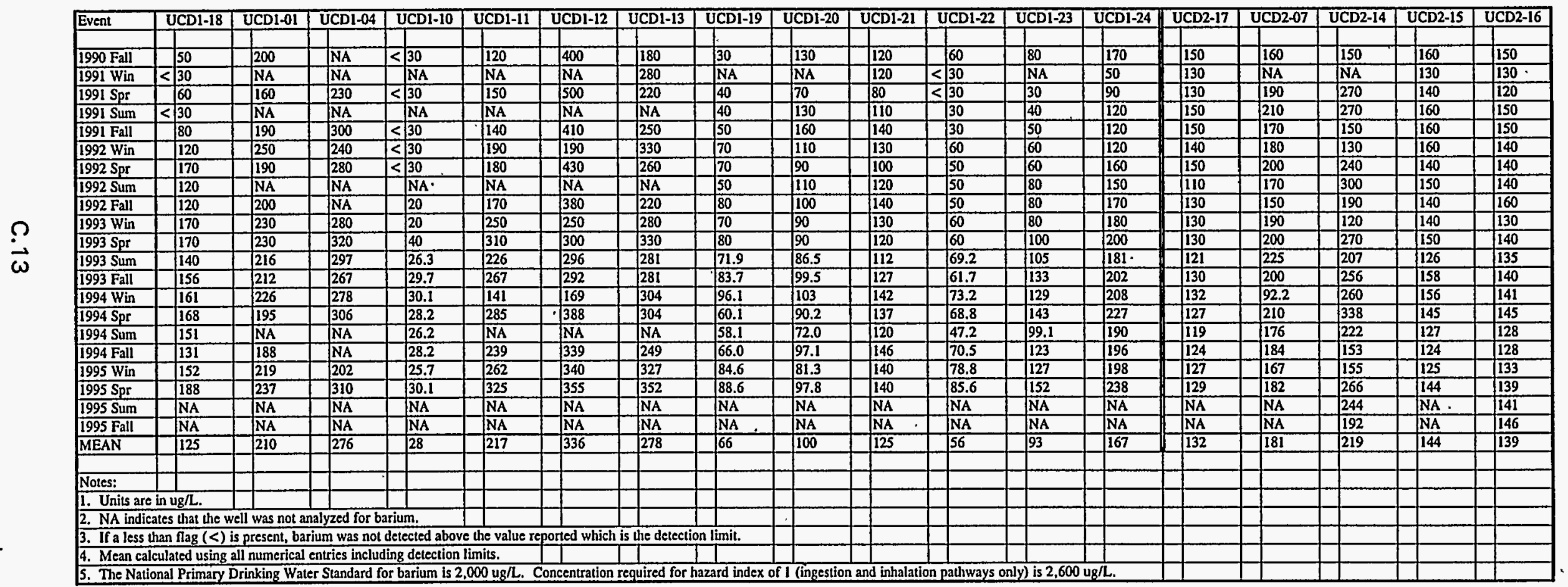

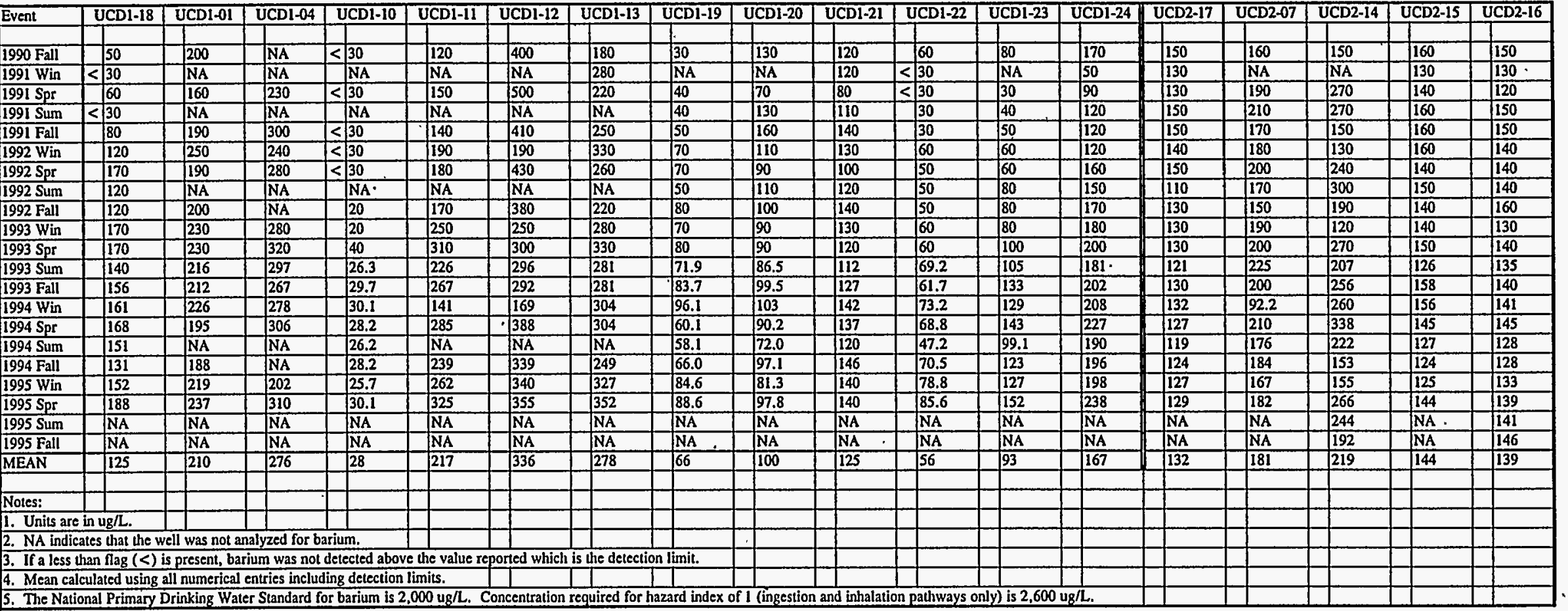


Table C.3. Analytical Results for Groundwater, Fall 1990 Through Fall 1995, Metals

\section{Beryllium}

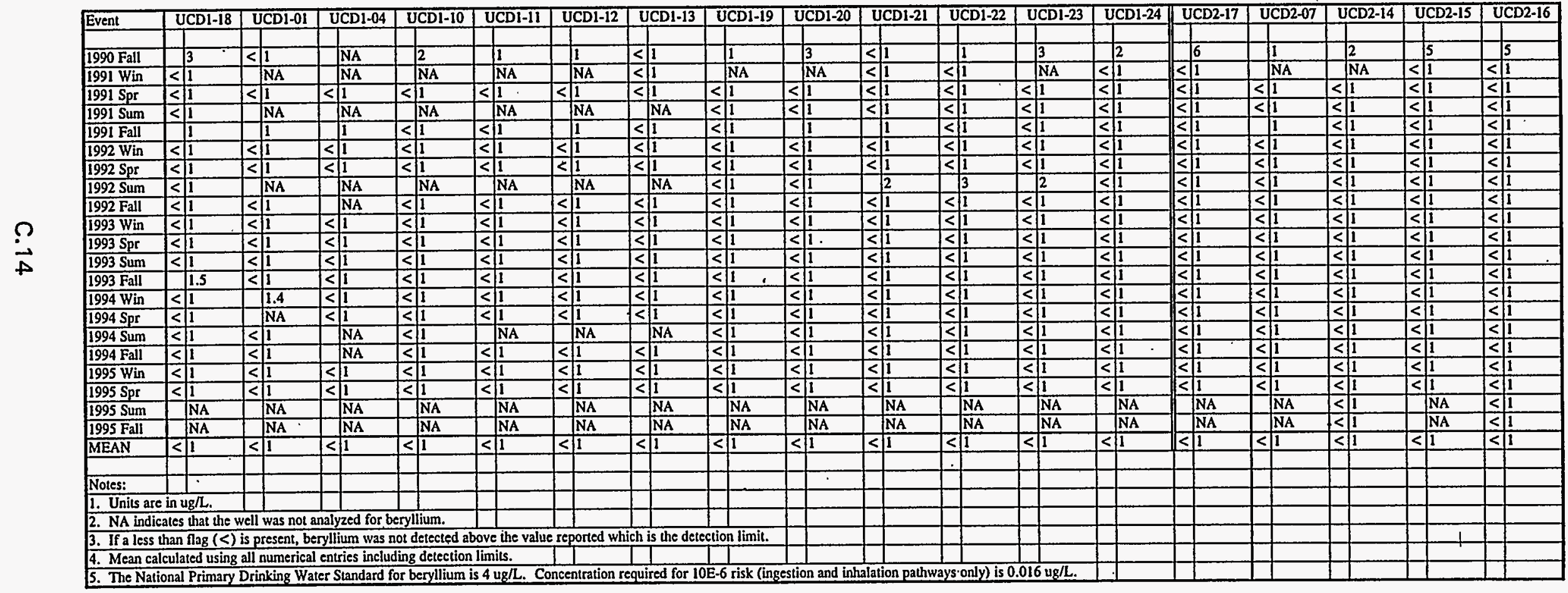


Table C.3. Analytical Results for Groundwater, Fall 1990 Through Fall 1995, Metals

\section{Cadmium}

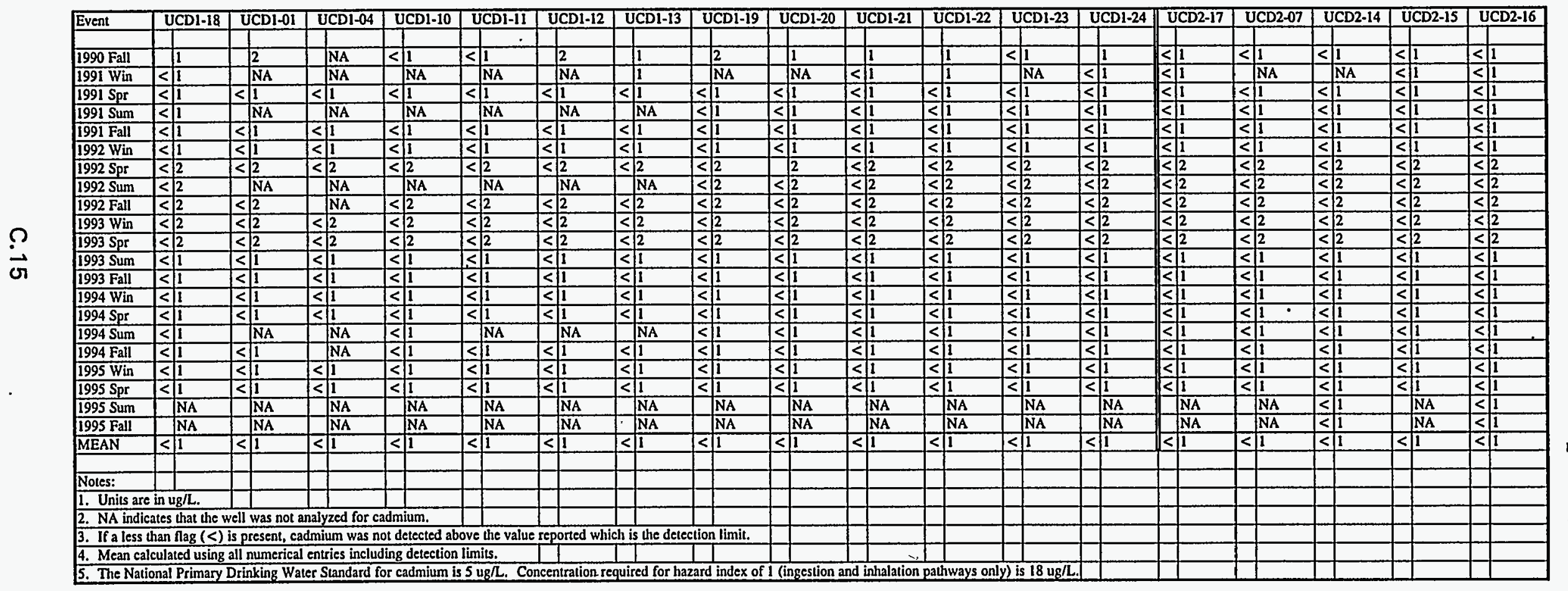


Table C.3. Analytical Results for Groundwater, Fall 1990 Through Fall 1995, Metals

\section{Cobalt}

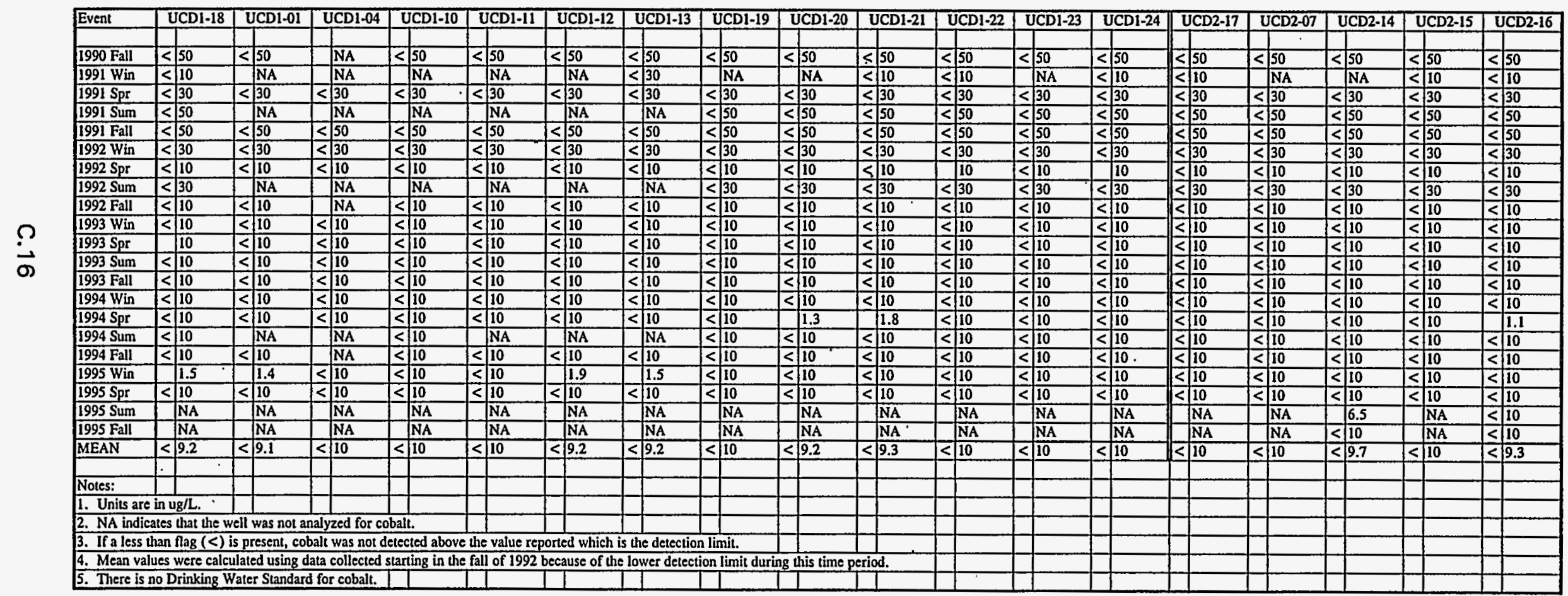


Table C.3. Analytical Results for Groundwater, Fall 1990 Through Fall 1995, Metals

Total Chromium

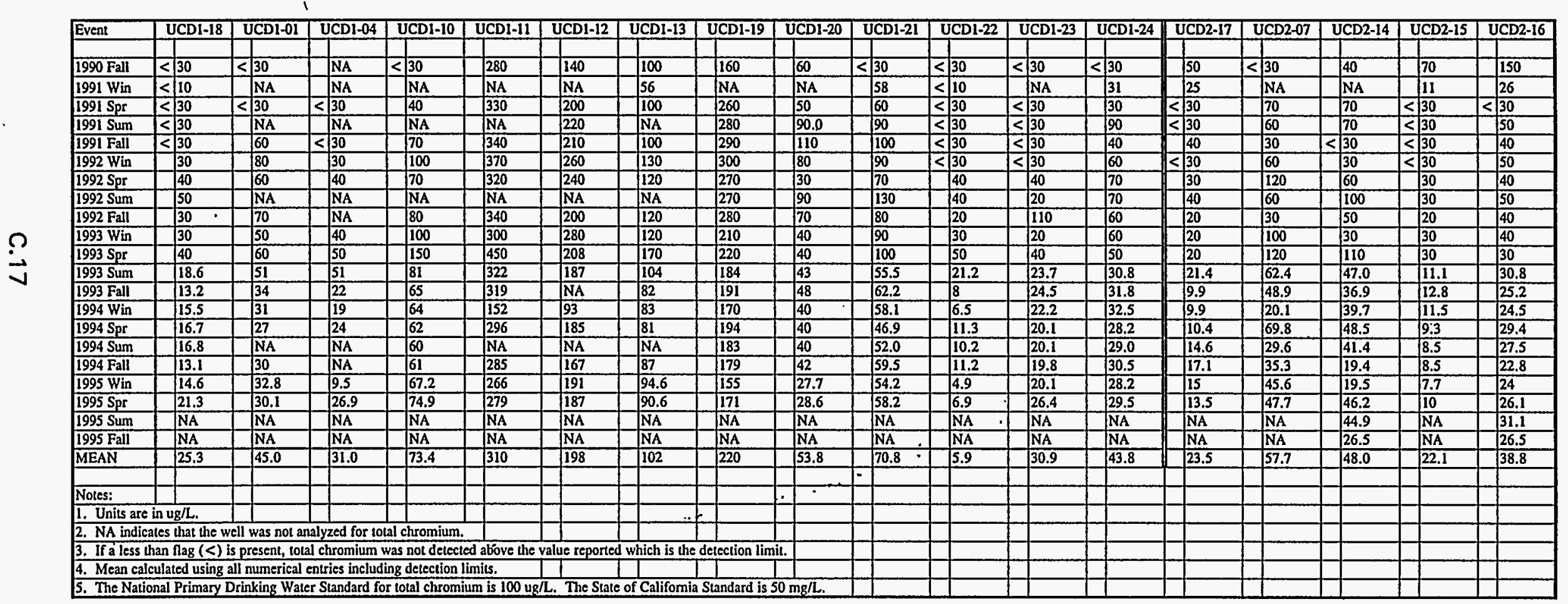


Table C.3. Analytical Results for Groundwater, Fall 1990 Through Fall 1995, Metals

\section{Hexavalent Chromium}

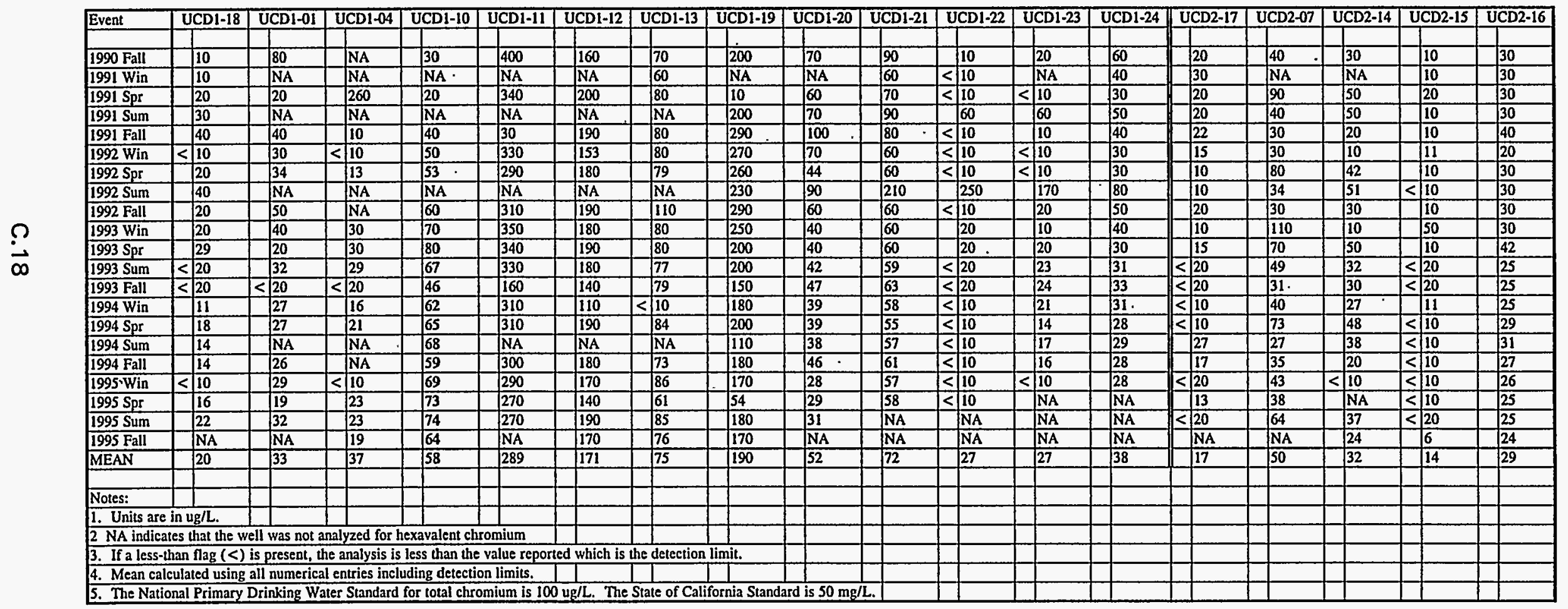


Table C.3. Analytical Results for Groundwater, Fall 1990 Through Fall 1995, Metals

\section{Copper}

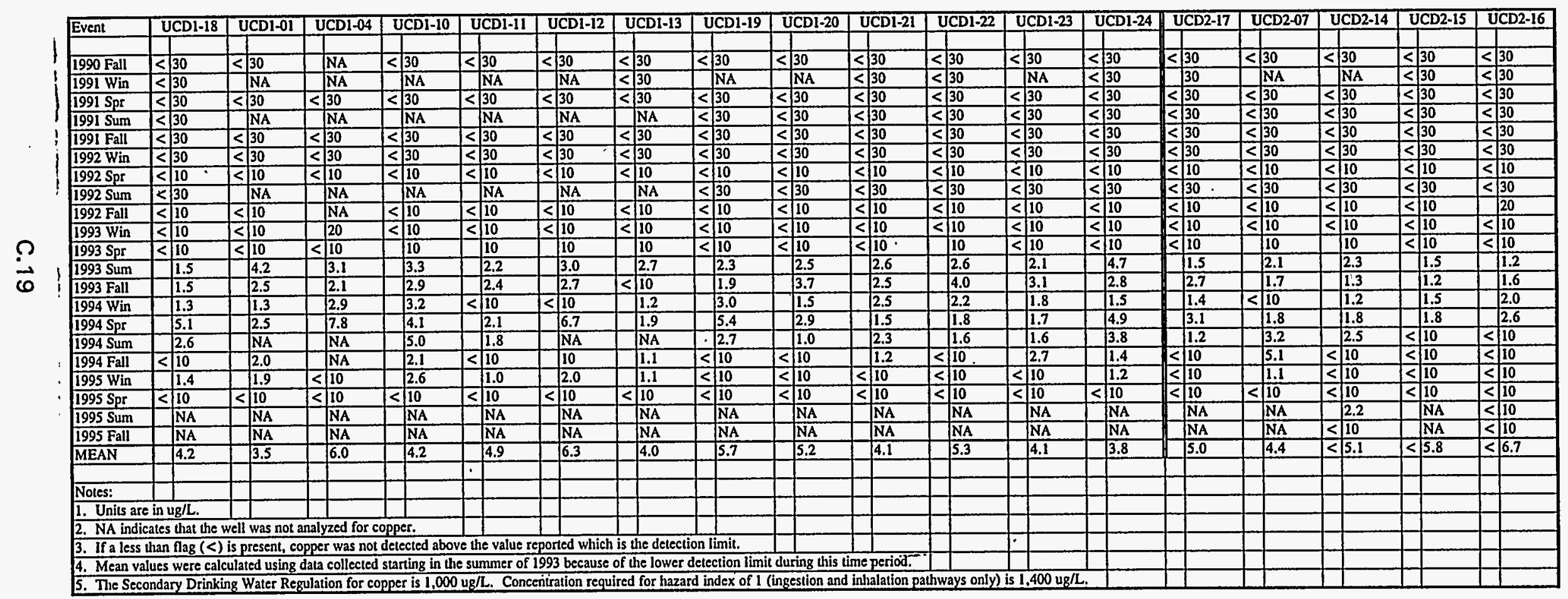


Table C.3. Analytical Results for Groundwater, Fall 1990 Through Fall 1995, Metals

Iron

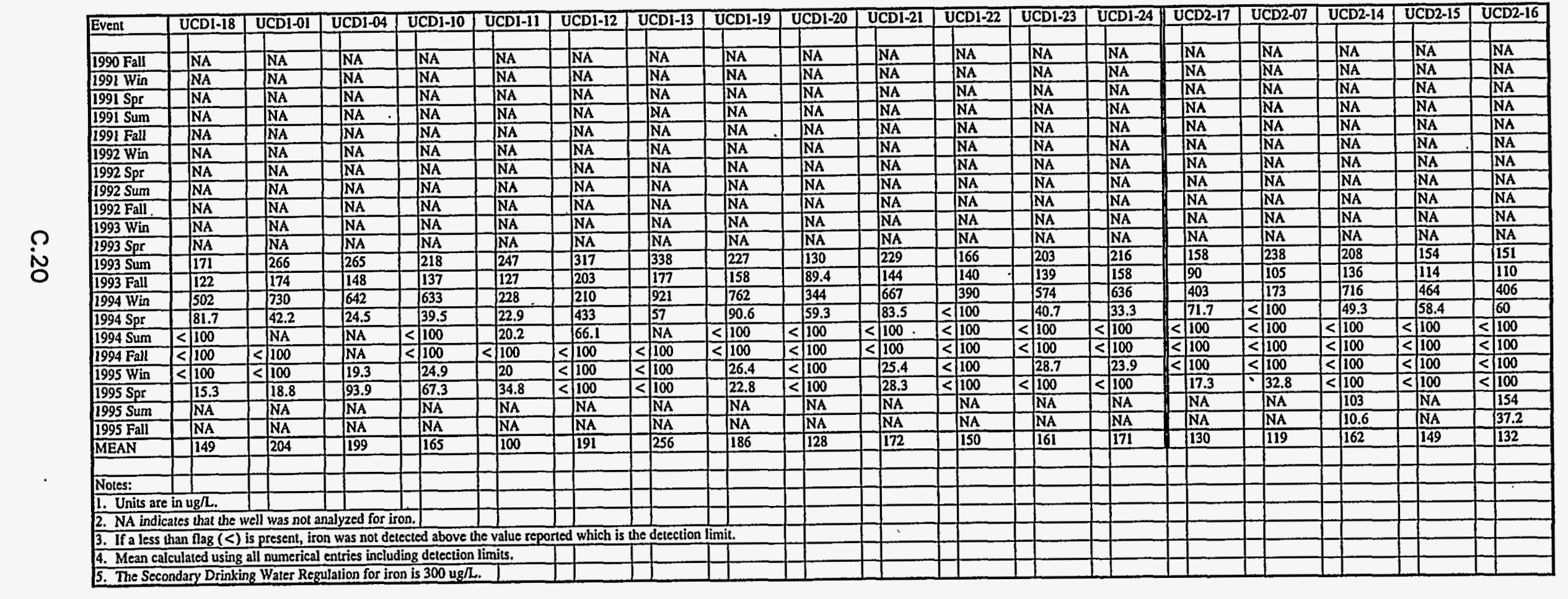


Table C.3. Analytical Results for Groundwater, Fall 1990 Through Fall 1995, Metals

\section{Lead}

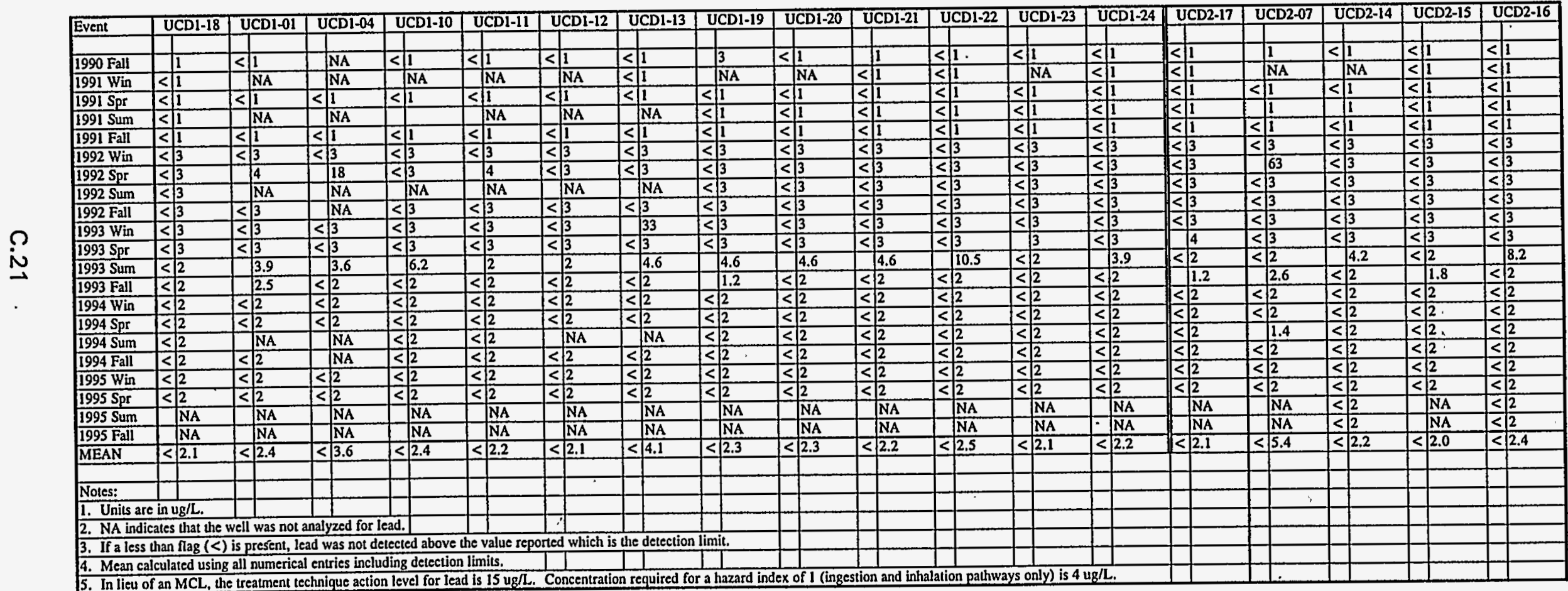


Table C.3. Analytical Results for Groundwater, Fall 1990 Through Fall 1995, Metals

\section{Manganese}

\begin{tabular}{|c|c|c|c|c|c|c|c|c|c|c|c|c|c|c|c|c|c|c|}
\hline Event & UCDI-18 & UCDI-01 & UCD1-04 & UCD1-10 & UCDI-11 & UCDI-12 & UCDI-13 & UCD1-19 & UCDI-20 & UCD1-21 & UCD1-22 & UCD1-23 & UCDI-24 & UCD2-17. & UCD2-07 & UCD2-14 & UCD2-15 & UCD2-16 \\
\hline & & & & & & & & & & & & & & & & & & \\
\hline 1990 Fall & NA & $\overline{\mathrm{NA}}$ & $\overline{\mathrm{NA}}$ & NA & $\overline{\mathrm{NA}}$ & $\sqrt{\mathrm{NA}}$ & NA & NA & $\overline{\mathrm{NA}}$ & $\sqrt{\mathrm{NA}}$ & $\sqrt{\mathrm{NA}}$ & NA & NA & $\sqrt{N A}$ & $\sqrt{\mathrm{NA}}$ & NA & NA & $\overline{\mathrm{NA}}$ \\
\hline $1991 \mathrm{Win}$ & NA & $\mathrm{NA}$ & $\sqrt{N A}$ & $\overline{\mathrm{NA}}$ & NA & NA & NA & NA & NA & $\overline{\mathrm{NA}}$ & NA & $\overline{\mathrm{NA}}$ & NA & NA & NA & NA & NA & NA \\
\hline $1991 \mathrm{Spr}$ & NA & $\mathrm{NA}$ & NA & NA & NA & NA & NA. & NA & NA & NA & NA & NA & NA & NA & NA & NA & NA & NA \\
\hline $1991 \mathrm{Sum}$ & NA & $\overline{\mathrm{NA}}$ & $\overline{\mathrm{NA}}$ & NA & $\overline{\mathrm{NA}}$ & NA & $\overline{N A}$ & $\sqrt{\mathrm{NA}}$ & NA & NA & NA & NA & $\sqrt{\mathrm{NA}}$ & $\overline{\text { NA }}$ & NA & NA & NA & NA \\
\hline 1991 Fall & NA & NA & NA & NA & $\mathrm{NA}$ & $\overline{N A}$ & NA & NA & $\widehat{\mathrm{NA}}$ & NA & NA & $\overline{\mathrm{NA}}$ & NA & NA & NA & NA & NA & NA \\
\hline $1992 \mathrm{Win}$ & $\mathrm{NA}$ & NA & NA & NA & NA & NA & NA & NA & $\mathrm{NA}$ & NA & NA & NA & NA & NA & NA & NA & NA & NA \\
\hline $1992 \mathrm{Spr}$ & NA & NA & NA & NA & $\overline{\mathbf{N A}}$ & $\sqrt{\mathrm{NA}}$ & $\overline{\mathrm{NA}}$ & NA & $\overline{\mathrm{NA}}$ & NA & NA & $\mathrm{NA}$ & NA & NA & NA & NA & $\mathrm{NA}$ & NA \\
\hline 1992 Sum & NA & $\mathrm{NA}$ & NA & NA & NA & NA & NA & NA & NA & NA & NA & NA & NA & NA & NA & NA & NA & NA \\
\hline 1992 Fall & $\overline{\mathrm{NA}}$ & $\overline{\mathrm{NA}}$ & $\overline{\mathrm{NA}}$ & NA & $\widehat{\mathrm{NA}}$ & NA & $\overline{\mathrm{NA}}$ & $\overline{\mathrm{NA}}$ & NA & $\overline{N A}$ & NA & NA & NA & NA & $\mathrm{NA}$ & NA & NA & NA \\
\hline 1993 Win & NA & NA & NA & NA & NA & NA & NA & NA & NA & NA & NA & NA & NA & NA & NA & NA & NA & NA \\
\hline $1993 \mathrm{Spr}$ & NA & NA & NA & NA & $\overline{N A}$ & NA & $\overline{\mathrm{NA}}$ & NA & NA & NA & NA & NA & NA & NA & NA & NA & NA & NA \\
\hline $1993 \mathrm{Sum}$ & NA & NA & $\mathrm{NA}$ & NA & NA & NA & NA & $\overline{\mathrm{NA}}$ & $\overline{\mathrm{NA}}$ & $\sqrt{\mathrm{NA}}$ & NA & $\sqrt{N A}$ & NA & $\mathrm{NA}$ & NA & $\overline{\mathrm{NA}}$ & NA & NA \\
\hline 1993 Fall & NA & NA & NA & NA & NA & NA & NA & NA & $\sqrt{\mathrm{NA}}$ & NA & NA & NA & NA & NA & NA & NA & NA & NA \\
\hline $1994 \mathrm{Win}$ & \begin{tabular}{|l}
$\mathrm{NA}$ \\
\end{tabular} & NA & $\overline{N A}$ & \begin{tabular}{|l|}
$\mathrm{NA}$ \\
\end{tabular} & NA & \begin{tabular}{|l|}
$\mathrm{NA}$ \\
\end{tabular} & \begin{tabular}{|l}
$\mathrm{NA}$ \\
\end{tabular} & NA & NA & NA & NA & NA & \begin{tabular}{|l|}
$\mathrm{NA}$ \\
\end{tabular} & NA & NA & \begin{tabular}{|l|} 
NA \\
\end{tabular} & NA & NA \\
\hline $1994 \mathrm{Spr}$ & $<\mid 10$ & 1.1 & 1 & $<10$ & $<10$ & \begin{tabular}{l|l|}
$<$ & 10 \\
\end{tabular} & \begin{tabular}{|l|l|}
$<$ & 10 \\
\end{tabular} & \begin{tabular}{|l|l|}
$<10$ \\
\end{tabular} & \begin{tabular}{|l|l|}
$<10$ \\
\end{tabular} & \begin{tabular}{l|l|}
$<$ & 10
\end{tabular} & \begin{tabular}{|l|l|}
$<$ & 10 \\
\end{tabular} & 12.4 & \begin{tabular}{l|l|} 
& 10 \\
\end{tabular} & \begin{tabular}{|l|l|} 
& 10
\end{tabular} & $<\longdiv { 1 0 }$ & \begin{tabular}{|l|l|} 
& 10 \\
\end{tabular} & $<10$ & \begin{tabular}{l|l|}
$<$ & 10 \\
\end{tabular} \\
\hline 1994 Sum & 9.9 & $\widehat{\mathrm{NA}}$ & $\mathrm{NA}$ & \begin{tabular}{|l|l|}
$<10$ \\
\end{tabular} & NA & NA & NA & $<10$ & \begin{tabular}{l|l|} 
& 10 \\
\end{tabular} & $\leq \quad 10$ & \begin{tabular}{l|l|}
$\leq 10$ \\
\end{tabular} & 1.7 & \begin{tabular}{l|l|l|}
10 \\
\end{tabular} & $<10$ & \begin{tabular}{|l|l|}
$<10$ \\
\end{tabular} & $\begin{array}{lll}<10 \\
\end{array}$ & \begin{tabular}{|l|l|}
$\leq 10$ \\
\end{tabular} & \begin{tabular}{|l|l|}
$<$ & 10 \\
\end{tabular} \\
\hline 1994 Fall & NA & NA & NA & NA & $\mathrm{NA}$ & NA & NA & NA & NA & \begin{tabular}{|l|}
$\mathrm{NA}$ \\
\end{tabular} & NA & NA & NA & NA & NA & NA & NA & NA \\
\hline 1995 Win & $\overline{N A}$ & NA & NA & $\mathrm{NA}$ & NA & NA & NA & NA & NA & $\mathrm{NA}$ & NA & NA & NA & $\hat{N A}$ & NA & NA & NA & NA \\
\hline $1995 \mathrm{Spr}$ & $\mathrm{NA}$ & NA & NA & $\overline{\mathrm{NA}}$ & $\sqrt{\mathrm{NA}}$ & NA & $\overline{N A}$ & $\sqrt{\mathrm{NA}}$ & $\sqrt{\mathrm{NA}}$ & NA & NA & NA & NA & NA & $\sqrt{\mathrm{NA}}$ & NA & NA & NA \\
\hline $1995 \mathrm{Sum}$ & NA & $\overline{\mathrm{NA}}$ & $\overline{\mathrm{NA}}$ & NA & NA & $\overline{\mathrm{NA}}$ & NA & $\sqrt{\mathrm{NA}}$ & NA & NA & NA & NA & NA & NA & NA & NA & NA & NA \\
\hline 1995 Fall & NA & $\mathrm{NA}$ & $\overline{\mathrm{NA}}$ & $\sqrt{\mathrm{NA}}$ & $\sqrt{\mathrm{NA}}$ & NA & $\sqrt{\mathrm{NA}}$ & $\widehat{N A}$ & NA & NA & $\overline{\mathrm{NA}}$ & NA & NA & NA & NA & NA & NA & NA \\
\hline & & & & & & & & & & & & & & & & & & \\
\hline & & & & & & & & & & & & & & & & & & \\
\hline Notes: & & & & & & & & & & & & & & & & & & \\
\hline 1. Units ar & in ug $/ \mathrm{L}$. & & & & & & & & & & & & & & & & & \\
\hline 2. NA ind & tes that the $\mathrm{w}$ & 11 was not at & alyzed for $M$ & anganese. & & & & & & & & & & & & & & \\
\hline 3. If a less & an flag (<) & & (1) & got detecter & ve the & to & his th & tion limit & & & & & & & & & & \\
\hline & & & & & & & & & & and in & & only) is & /L. & & & & & \\
\hline
\end{tabular}


Table C.3. Analytical Results for Groundwater, Fall 1990 Through Fall 1995, Metals

\section{Mercury}

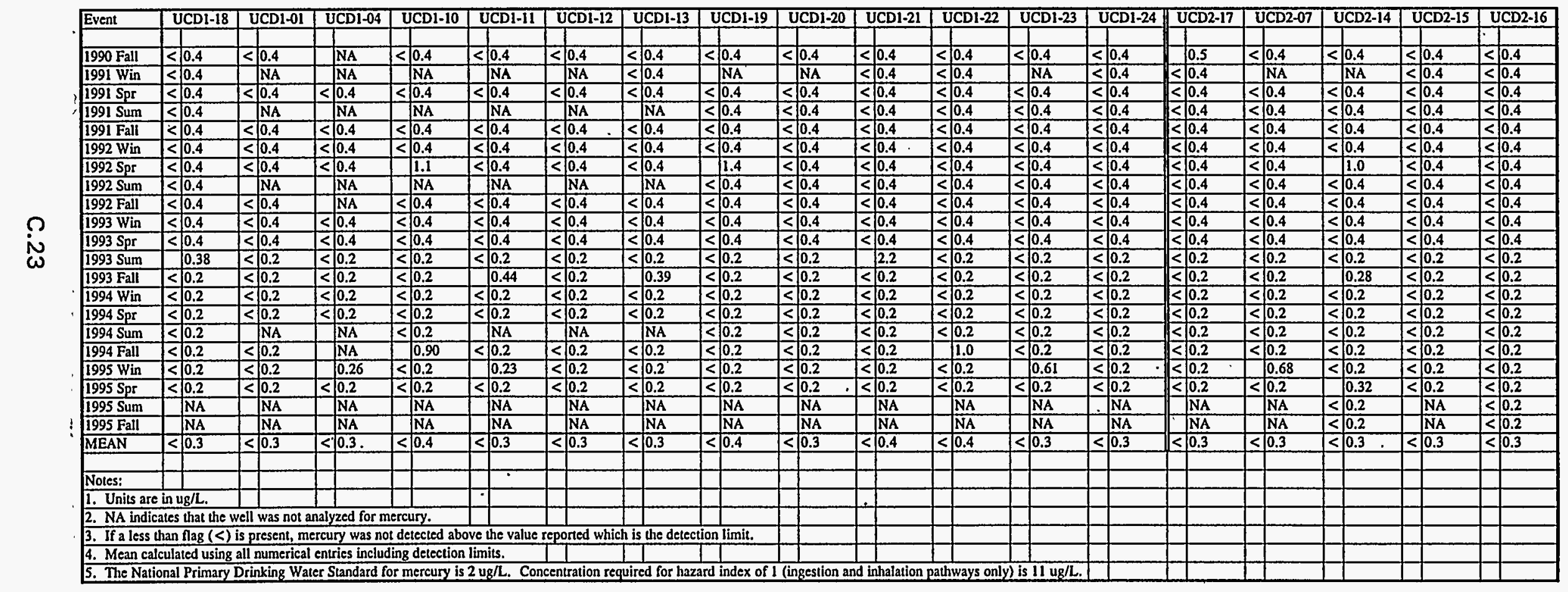


Table C.3. Analytical Results for Groundwater, Fall 1990 Through Fall 1995, Metals

\section{Molybdenum}

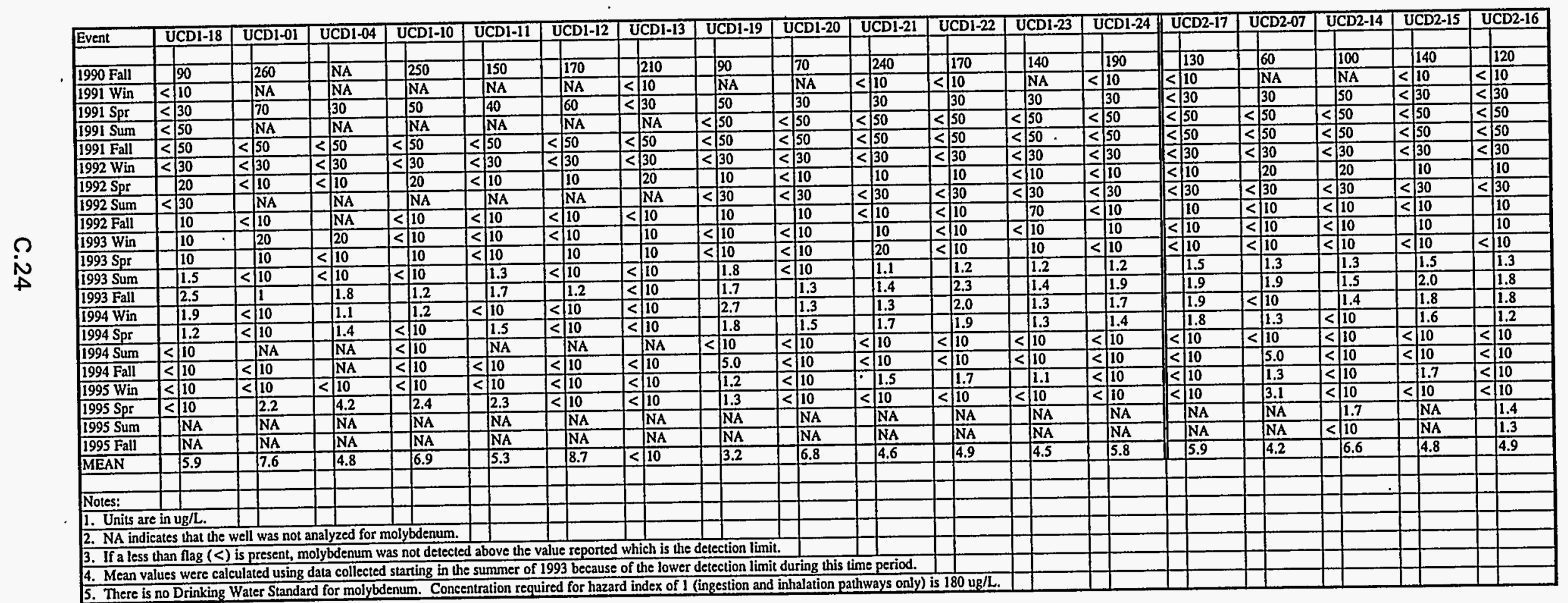


Table C.3. Analytical Results for Groundwater, Fall 1990 Through Fall 1995, Metals

\section{Nickel}

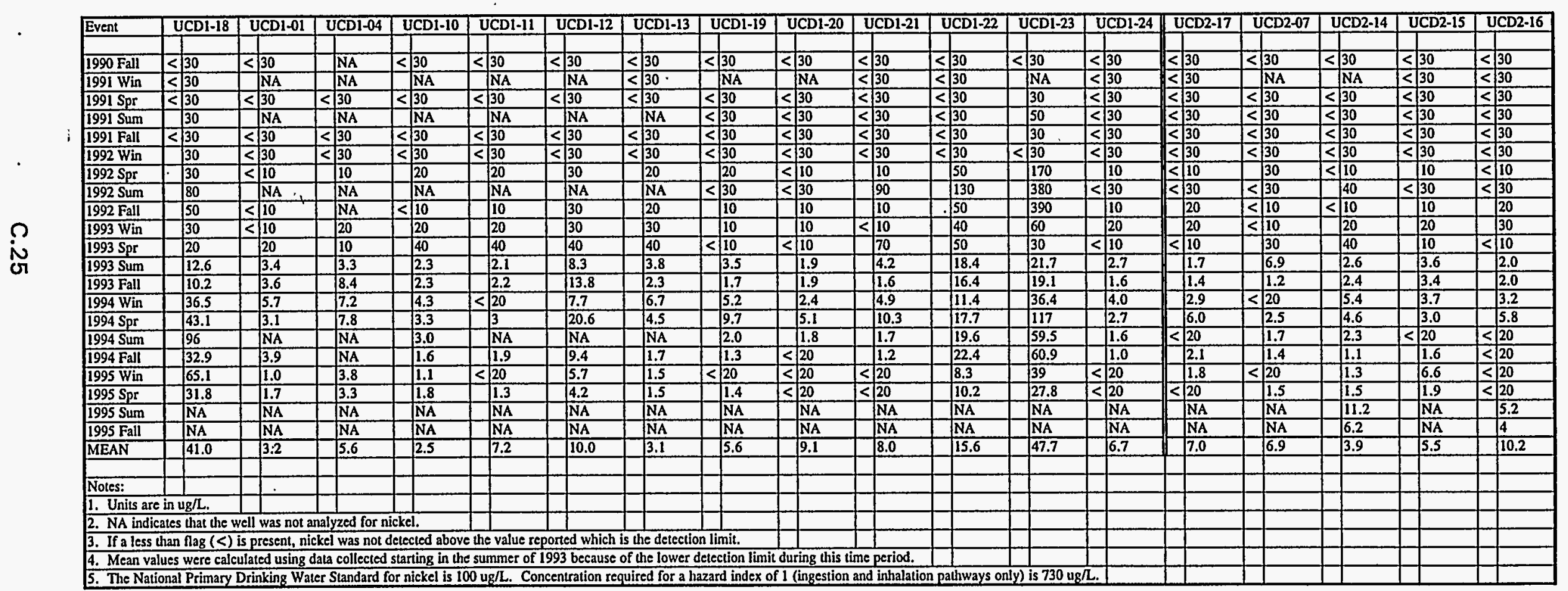


Table C.3. Analytical Results for Groundwater, Fall 1990 Through Fall 1995, Metals

\section{Selenium}

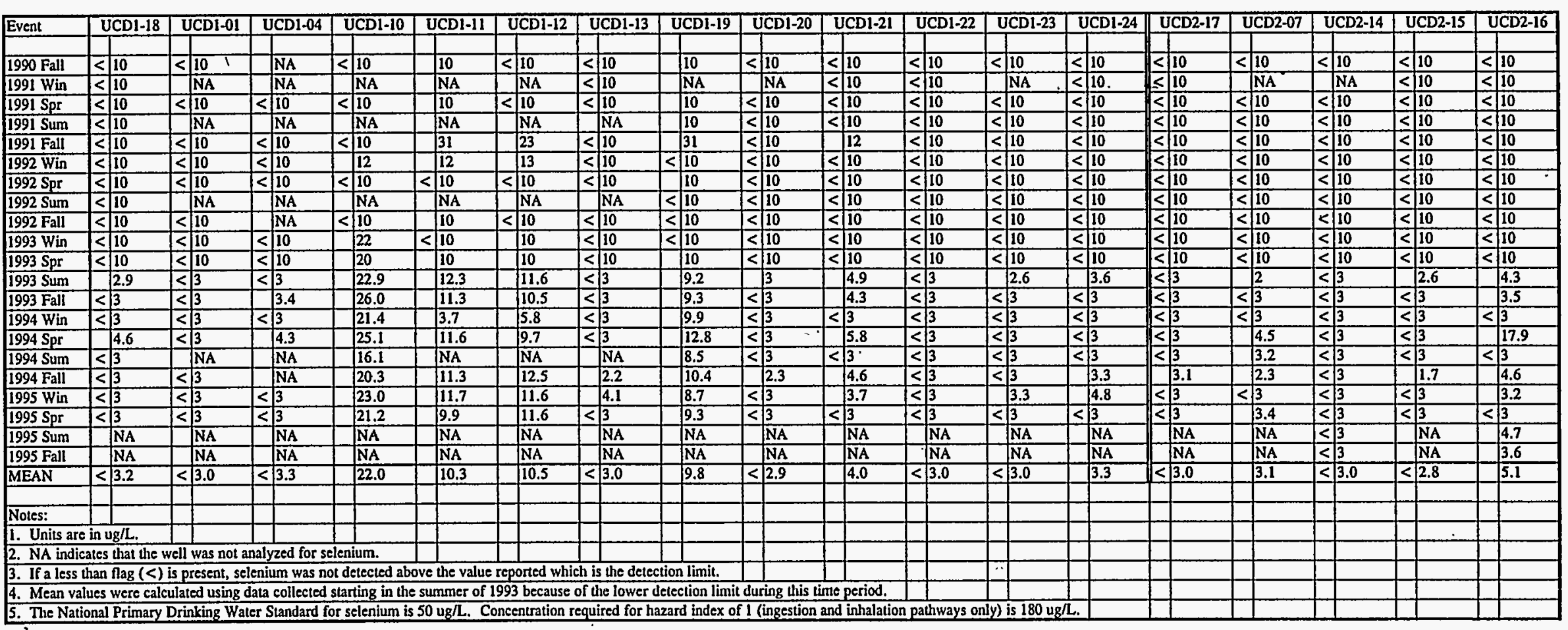


Table C.3. Analytical Results for Groundwater, Fall 1990 Through Fall 1995, Metals .

\section{Silver}

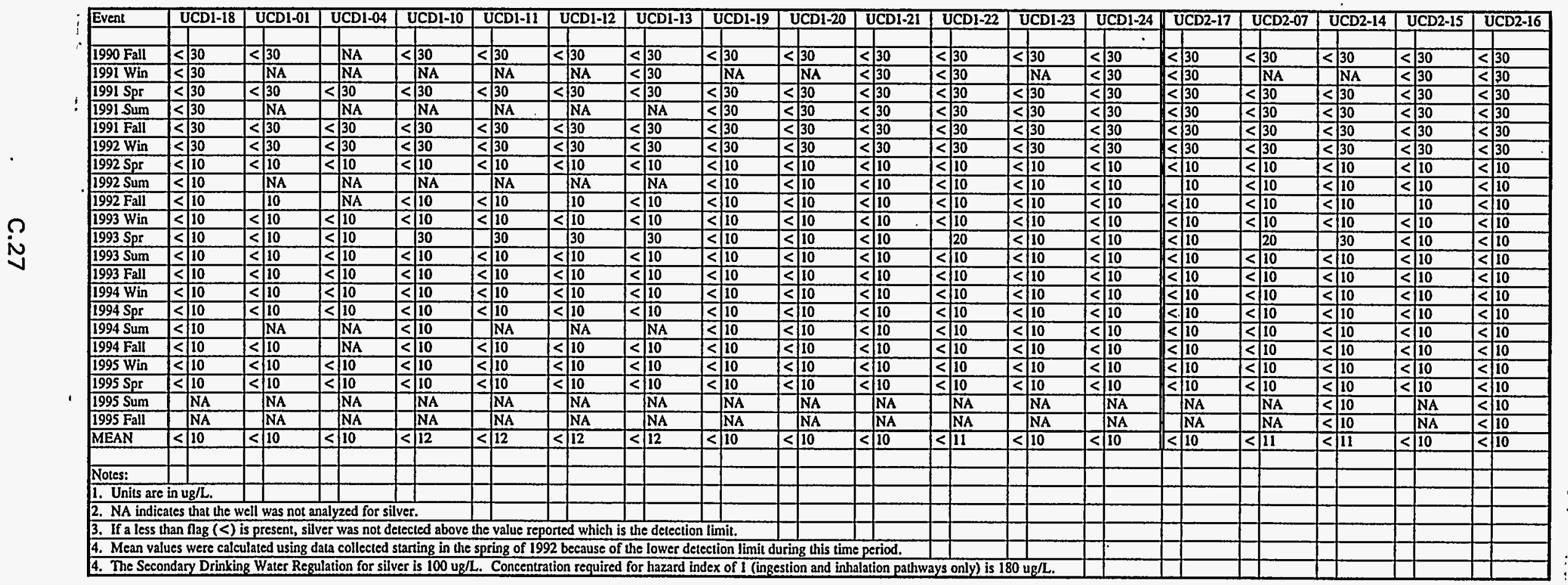


Table C.3. Analytical Results for Groundwater, Fall 1990 Through Fall 1995, Metals

\section{Thallium}

\begin{tabular}{|c|c|c|c|c|c|c|c|c|c|c|c|c|c|c|c|c|c|c|c|c|c|c|}
\hline \multirow{2}{*}{ Event } & \multirow{2}{*}{ UCDI-18 } & \multirow{2}{*}{\begin{tabular}{|l|} 
UCD1-01 \\
\end{tabular}} & \multirow{2}{*}{ UCD1-04 } & \multirow{2}{*}{ UCD1-10 } & \multirow{2}{*}{ UCDI-11 } & \multirow{2}{*}{ UCDI-12 } & \multirow{2}{*}{ UCDI-13 } & \multirow{2}{*}{ UCD1-19 } & \multirow{2}{*}{ UCD1-20 } & \multirow{2}{*}{ UCD1-21 } & \multirow{2}{*}{ UCDI-22 } & \multirow{2}{*}{ UCD1-23 } & \multirow[t]{2}{*}{ UCD1-24 } & \multirow[t]{2}{*}{\begin{tabular}{||l|l}
$U C D 2-17$ \\
\end{tabular}} & \multicolumn{2}{|c|}{ UCD2-07 } & \multicolumn{2}{|c|}{ UCD2-14 } & \multicolumn{2}{|c|}{ UCD2-15 } & \multicolumn{2}{|c|}{ UCD2-16 } \\
\hline & & & & & & & & & & & & & & & & & & & & & & \\
\hline & 120 & 270 & $\mathrm{NA}$ & 250 & 180 & 200 & 220 & 110 & 110 & 280 & 200 & 150 & 210 & 1150 & & 80 & & 150 & & 140 & & 150 \\
\hline $1991 \mathrm{Win}$ & $<30$ & NA & NA & NA & $\mathrm{NA}$ & NA & 80 & NA & NA & $<30$ & $<30$ & NA & $<30$ & $<30$ & & NA & & NA & & 30 & & 30 \\
\hline $1991 \mathrm{Spr}$ & 5 & 2 & 7 & 6 & 9 & 31 & 14 & 11 & 8 & 口i1 & 6 & 7 & 10 & 6 & & 11 & & 12 & & 5 & & 7 \\
\hline 1991 Sum & 6 & NA & $\overline{\mathrm{NA}}$ & NA & $\overline{\mathrm{NA}}$ & NA & NA & 8 & 10 & 10 & 5 & 5 & 8 & 74 & 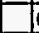 & 6 & & 9 & & 5 & & 5 \\
\hline 1991 Fall & 5 & 8 & 6 & 13 & $<5$ & 23 & 13 & 9 & 12 & 7 & $<\longdiv { 5 }$ & 7 & 8 & $<5$ & 7 & 7 & $<$ & & & 9 & & 6 \\
\hline 1992 Win & $\leq 10$ & 20 & $<10$ & 30 & 130 & 130 & 100 & 40 & 20 & 110 & $<10$ & $\leq 10$ & 10 & $<\quad 10$ & & 30 & & 10 & & 10 & & 20 \\
\hline $1992 \mathrm{Spr}$ & $<10$ & $<10$ & $<10$ & $<\mid 10$ & \begin{tabular}{l|l|}
$<$ & 10 \\
\end{tabular} & $<10$ & $<10$ & $<10$ & $<10$ & $<10$ & $<10$ & \begin{tabular}{l|l|}
$<10$ \\
\end{tabular} & $<10$ & \begin{tabular}{|l|l|}
$<10$ \\
\end{tabular} & & 10 & & 10 & & 10 & & 10 \\
\hline 1992 Sum & $<10$ & NA & NA & NA & $\mathrm{NA}$ & $\overline{\mathrm{NA}}$ & NA & $<10$ & $\leq 10$ & $\leq 10$ & $<10$ & \begin{tabular}{l|l}
$<$ & 10 \\
\end{tabular} & $<10$ & \begin{tabular}{|l|l|} 
& 10 \\
\end{tabular} & $<1$ & 10 & & 10 & & 10 & & 10 \\
\hline 1992 Fall & $<5$ & $<5$ & NA & $<5$ & $<5$ & $<5$ & \begin{tabular}{|l|l|} 
& 5 \\
\end{tabular} & $<5$ & $<\mid 5$ & $<5$ & $<5$ & $<5$ & $\begin{array}{l}5 \\
\end{array}$ & $<5$ & $<5$ & & $<$ & & & 5 & 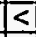 & 5 \\
\hline 1993 Win & $<10$ & $<10$ & \begin{tabular}{|l|l|}
$<10$ \\
\end{tabular} & $<10$ & $<10$ & $\leq 10$ & $\leq 10$ & \begin{tabular}{|l|l|}
$<$ & 10 \\
\end{tabular} & $\leq 10$ & $<10$ & \begin{tabular}{|l|l|}
$<$ & 10 \\
\end{tabular} & $<\longdiv { 1 0 }$ & $\begin{array}{l}<10 \\
\end{array}$ & $<10$ & $<1$ & 10 & & 10 & & 10 & & 10 \\
\hline $1993 \mathrm{Spr}$ & $<10$ & $<10$ & $<10$ & $<10$ & $<10$ & $<10$ & $<10$ & \begin{tabular}{|l|l|}
$<$ & 10 \\
\end{tabular} & \begin{tabular}{l|l}
$<10$ \\
\end{tabular} & \begin{tabular}{|l|l|}
$<$ & 10 \\
\end{tabular} & \begin{tabular}{|l|l|}
$<$ & 10 \\
\end{tabular} & \begin{tabular}{l|l|}
$<10$ \\
\end{tabular} & \begin{tabular}{l|l|} 
& 10 \\
\end{tabular} & $<10$ & & 10 & & 10 & & 10 & & 10 \\
\hline $1993 \mathrm{Sum}$ & 2.4 & $<10$ & \begin{tabular}{l|l|}
$<$ & 10 \\
\end{tabular} & I & \begin{tabular}{l|l}
$\leq 10$ \\
\end{tabular} & \begin{tabular}{|l|l|}
$\leq 10$ \\
\end{tabular} & $<>10$ & \begin{tabular}{|l|l|}
4.9 \\
\end{tabular} & 3 & 3 & \begin{tabular}{|l|l}
1.1 \\
\end{tabular} & 2.9 & 3.1 & \begin{tabular}{|l|l|}
1.2 \\
\end{tabular} & & 1.1 & & 10 & & 2.7 & & 7.7 \\
\hline 1993 Fall & \begin{tabular}{|l|l|}
$<$ & 10 \\
\end{tabular} & \begin{tabular}{|l|l|} 
& 10 \\
\end{tabular} & \begin{tabular}{l|l|} 
& 10 \\
\end{tabular} & \begin{tabular}{l|l|}
$<10$ \\
\end{tabular} & $<10$ & $<10$ & \begin{tabular}{l|l|}
$<<10$ \\
\end{tabular} & \begin{tabular}{|l|l|}
$<$ & 10 \\
\end{tabular} & $<10$ & \begin{tabular}{|l|l|}
$<$ & 10 \\
\end{tabular} & \begin{tabular}{|l|l|}
$<$ & 10 \\
\end{tabular} & \begin{tabular}{l|l|}
$<<10$ \\
\end{tabular} & \begin{tabular}{|l|l|}
$<$ & 10 \\
\end{tabular} & $<10$ & $<1$ & 10 & & 10 & & 10 & & 10 \\
\hline 1994 Win & $<10$ & \begin{tabular}{l|l|} 
& 10 \\
\end{tabular} & $<10$ & \begin{tabular}{|l|l|} 
& 10 \\
\end{tabular} & $<\longdiv { 1 0 }$ & $<10$ & $<10$ & \begin{tabular}{l|l|}
$<<10$ \\
\end{tabular} & \begin{tabular}{|l|l|}
$<$ & 10 \\
\end{tabular} & \begin{tabular}{l|l|}
$<<10$ \\
\end{tabular} & \begin{tabular}{l|l|}
$<<10$ \\
\end{tabular} & \begin{tabular}{|l|l|}
$<10$ \\
\end{tabular} & $<10$ & \begin{tabular}{|l|l|} 
& 10 \\
\end{tabular} & & 10 & & 10 & & $\frac{10}{10}$ & & 10 \\
\hline $1994 \mathrm{Spr}$ & \begin{tabular}{l|l|l|} 
& 10 \\
\end{tabular} & \begin{tabular}{|l|l|}
$<$ & 10 \\
\end{tabular} & \begin{tabular}{|l|l|}
$<$ & 10 \\
\end{tabular} & \begin{tabular}{|l|l|}
$<$ & 10 \\
\end{tabular} & $<10$ & $<10$ & \begin{tabular}{|l|}
10 \\
\end{tabular} & \begin{tabular}{l|l|} 
& 10 \\
\end{tabular} & $<10$ & \begin{tabular}{|l|l|}
$<10$. \\
\end{tabular} & \begin{tabular}{|l|l|}
$<$ & 10 \\
\end{tabular} & $<110$ & \begin{tabular}{l|l|l|} 
& 10 \\
\end{tabular} & $<10$ & & 10 & $<$ & 10 & & 10 & & 10 \\
\hline 1994 Sum & $<\mid 10$ & NA & NA & $<10$ & NA & NA & NA & $<10$ & $<10$ & $\leq 10$ & \begin{tabular}{|l|l|} 
& 10 \\
\end{tabular} & $<10$ & \begin{tabular}{|l|l|}
10 \\
\end{tabular} & \begin{tabular}{|l|l|}
$<10$ \\
\end{tabular} & & 10 & & 10 & & 10 & & 10 \\
\hline 1994 Fall & \begin{tabular}{l|l|}
$<<$ & 10 \\
\end{tabular} & $\begin{array}{l}<\longdiv { 1 0 } \\
\end{array}$ & NA & $<10$ & $<10$ & \begin{tabular}{l|l|}
$<10$ \\
\end{tabular} & $\begin{array}{ll}10 \\
\end{array}$ & \begin{tabular}{l|l}
$<$ & 10 \\
\end{tabular} & $\leq 10$ & $<10$ & $<110$ & \begin{tabular}{l|l} 
& 10 \\
\end{tabular} & \begin{tabular}{|l|l|}
$<10$ \\
\end{tabular} & $<10$ & & 10 & & 10 & & 10 & & 10 \\
\hline 1995 Win & $<10$ & $<10$ & \begin{tabular}{l|l|l|}
$<$ & 10 \\
\end{tabular} & $<10$ & $<\longdiv { 1 0 }$ & $<10$ & $<10$ & $<10$ & $\leq 10$ & $<1 \overline{10}$ & \begin{tabular}{|l|l|}
$<$ & 10 \\
\end{tabular} & $<10$ & \begin{tabular}{l|l|} 
& 10 \\
\end{tabular} & \begin{tabular}{l|l} 
& 10 \\
\end{tabular} & & 10 & & 10 & & 10 & & 10 \\
\hline $1995 \mathrm{Spr}$ & \begin{tabular}{l|l|}
$<<$ & 10 \\
\end{tabular} & \begin{tabular}{|l|l|}
10 \\
\end{tabular} & \begin{tabular}{|l|l|}
$<$ & 10 \\
\end{tabular} & $<10$ & $<10$ & $<\frac{10}{10}$ & \begin{tabular}{l|l|l|}
10 \\
\end{tabular} & \begin{tabular}{l|l|l|}
$<$ & 10 \\
\end{tabular} & \begin{tabular}{l|l|} 
& 10 \\
\end{tabular} & \begin{tabular}{l|l|}
$<$ & 10 \\
\end{tabular} & \begin{tabular}{|l|l|}
$<$ & 10 \\
\end{tabular} & \begin{tabular}{l|l|}
$<<10$ \\
\end{tabular} & \begin{tabular}{l|l|}
$<10$ \\
\end{tabular} & \begin{tabular}{l|l}
$<$ & 10 \\
\end{tabular} & & 10 & & 10 & & 10 & & 10 \\
\hline 1995 Sum & $\mid \overline{N A}$ & NA & NA & NA & NA & NA & $\overline{\mathrm{NA}}$ & \begin{tabular}{|l|l} 
NA \\
\end{tabular} & NA & NA & \begin{tabular}{|l|l|} 
NA \\
\end{tabular} & NA & $\overline{\mathrm{NA}}$ & \begin{tabular}{|l|} 
NA \\
\end{tabular} & & NA & & 10 & & $\overline{\text { NA }}$ & & 10 \\
\hline 1995 Fall & NA & NA & $\overline{\mathrm{NA}}$ & NA & $\overline{\mathrm{NA}}$ & NA & $\overline{N A}$ & NA & NA & NA & NA & NA & NA & $\overline{\mathrm{NA}}$ & & $\overline{\mathrm{NA}}$ & & 10 & & NA & & 10 \\
\hline MEAN & $<9.1$ & $<10.0$ & \begin{tabular}{l|l} 
& 10.0 \\
\end{tabular} & \begin{tabular}{|l|l|} 
& 8.9 \\
\end{tabular} & $<\quad 10.0$ & $\leq 10.0$ & $<\quad 10.0$ & \begin{tabular}{l|l|}
$<9.4$ \\
\end{tabular} & \begin{tabular}{l|l|}
$<9.1$ \\
\end{tabular} & \begin{tabular}{|l|l|} 
& 9.2 \\
\end{tabular} & \begin{tabular}{|l|l|}
$<8.9$ \\
\end{tabular} & \begin{tabular}{l|l|} 
\\
\end{tabular} & \begin{tabular}{|l|l|}
$<9.1$ \\
\end{tabular} & $<8.9$ & & 8.9 & & 10.0 & & 9.1 & & 9.8 \\
\hline & & & & & & & & & & & & & & & & & & & & & & \\
\hline Notes: & & & & & & & & & & & & & & & & & & & & & & \\
\hline 1. Units an & in ug/L. & & & & & & & & & & & & & & & & & & & & & \\
\hline 2. NA indi & tes that th & rell was not & alyzed for & allium. & & & & & & & & & & & & & & & & & & \\
\hline $2 \sin$ & & & & & & ted $\mathrm{W}$ & $h$ is the detec & ion limit. & & & & & & & & & & & & & & \\
\hline 4. Mean ve & Jes were ca & ylated usin & ta collecte & starting in & summer & 993. & & & & & & & & & & & & & & & & \\
\hline 5. The Nat & serat & Drinking Wat & S Standard fo & or thallium & & & & & & & & & & & & & & & & & & \\
\hline
\end{tabular}


Table C.3. Analytical Results for Groundwater, Fall 1990 Through Fall 1995, Metals

\section{Vanadium}

\begin{tabular}{|c|c|c|c|c|c|c|c|c|c|c|c|c|c|c|c|c|c|c|}
\hline Event & UCDI-18 & UCD1-01 & UCD1-04 & UCDI-10 & UCD1-11 & UCDI-12 & UCDI-13 & UCDI-19 & UCDI-20 & UCD1-21 & UCD1-22 & UCD1-23 & UCD1-24 & UCD2-17 & UCD2-07 & UCD2-14 & UCD2-15 & UCD2-16 \\
\hline & & & & & & & & & & & & & & & & & & \\
\hline 1990 Fall & \begin{tabular}{l|l}
$<50$ \\
$<5$
\end{tabular} & $\begin{array}{l}<50 \\
\end{array}$ & NA & \begin{tabular}{|l|l|}
50 \\
\end{tabular} & \begin{tabular}{|l|l|}
$<50$ \\
\end{tabular} & \begin{tabular}{|l|l|}
$<50$ \\
\end{tabular} & $<50$ & $\begin{array}{l}<50 \\
\end{array}$ & \begin{tabular}{l|l|}
$<50$ \\
\end{tabular} & $<50$ & $<\longdiv { 5 0 }$ & $<50$ & $<\longdiv { 5 0 }$ & \begin{tabular}{|l|l|}
$\leq 50$ \\
\end{tabular} & $<50$ & $<50$ & $<50$ & $<50$ \\
\hline 1991 Win & \begin{tabular}{|l|l|}
$<<100$ \\
\end{tabular} & NA & $\mathrm{NA}$ & $\mathrm{NA}$ & NA & NA & \begin{tabular}{|l|l|}
$<100$ \\
\end{tabular} & NA & NA & 100 & \begin{tabular}{|l|l|}
$<100$ \\
\end{tabular} & $\overline{\mathrm{NA}}$ & $<100$ & $<\quad 100$ & NA & NA & $<<100$ & \begin{tabular}{|l|l|}
$<100$ \\
\end{tabular} \\
\hline $1991 \mathrm{Spr}$ & $<30$ & $<3$ & $<30$ & $<30$ & \begin{tabular}{|l|l|}
$<30$ \\
\end{tabular} & $<3$ & $<30$ & \begin{tabular}{|l|l|} 
& 30 \\
\end{tabular} & $\begin{array}{l}30 \\
\end{array}$ & $<30$ & $<30$ & $<30$ & $<30$ & \begin{tabular}{|l|l}
30 \\
\end{tabular} & $\leq 30$ & $<30$ & $\begin{array}{l}<30 \\
\end{array}$ & \begin{tabular}{|l|l|}
$<30$ \\
\end{tabular} \\
\hline 1991 Sum & $<50$ & NA & NA & NA & NA & NA & NA & $<50$ & $\begin{array}{l}<50 \\
\end{array}$ & $<50$ & $<50$ & \begin{tabular}{|l|l|}
$<50$ \\
\end{tabular} & $<50$ & $\leq 50$ & $<50$ & \begin{tabular}{|l|l|} 
& 50 \\
\end{tabular} & $<50$ & $<50$ \\
\hline 1991 Fall & $<50$ & $<50$ & \begin{tabular}{|l|l|}
$<50$ \\
\end{tabular} & $<5$ & $<50$ & \begin{tabular}{l|l|} 
& 50 \\
\end{tabular} & $<\longdiv { 5 0 }$ & $<50$ & $<50$ & $<50$ & $\leq 50$ & $<50$ & $\begin{array}{l}<50 \\
\end{array}$ & \begin{tabular}{|l|l|}
$\leq 50$ \\
\end{tabular} & $<50$ & $<50$ & $<50$ & $<50$ \\
\hline 1992 Win & $<30$ & $<30$ & $<30$ & $<30$ & $<30$ & $<\longdiv { 3 0 }$ & $<30$ & $<30$ & $<30$ & $<30$ & $<30$ & $<30$ & $<30$ & $<30$ & $<30$ & $<30$ & $<\longdiv { 3 0 }$ & $<30$ \\
\hline $1992 \mathrm{Spr}$ & 10 & 20 & 10 & 20 & \begin{tabular}{|l|l|}
$<$ & 10 \\
\end{tabular} & 30 & 20 & 20 & 10 & 10 & $<10$ & \begin{tabular}{|l|l|}
$<10$ \\
\end{tabular} & 10 & 10 & 10 & 10 & 10 & $<10$ \\
\hline 1992 Sum & $<30$ & NA & $\sqrt{\mathrm{NA}}$ & NA & NA & $\overline{\mathrm{NA}}$ & NA & $<3$ & $<3$ & $<30$ & $<30$ & $<30$ & $<30$ & $<30$ & $<330$ & $<30$ & $<<30$ & $<30$ \\
\hline 1992 Fall & 20 & 10 & NA & 10 & 10 & 20 & 10 & 20 & 20 & 20 & 10 & 20 & 20 & 10 & \begin{tabular}{|l|l|}
$<$ & 10 \\
\end{tabular} & $<10$ & $<10$ & 20 \\
\hline $1993 \mathrm{Win}$ & 20 & 30 & 40 & 30 & 10 & 20 & 20 & 20 & 20 & 20 & 20 & 20 & 30 & 20 & \begin{tabular}{|l|l|}
$<10$ \\
\end{tabular} & $<10$ & 20 & 20 \\
\hline $1993 \mathrm{Spr}$ & 20 & 20 & 20 & 20 & 20 & 30 & 30 & 20 & 20 & 10 & 10 & 20 & 20 & 20 & \begin{tabular}{|l|l|}
10 \\
\end{tabular} & 20 & 10 & 10 \\
\hline 1993 Sum & 10 & 3.2 & 2.5 & 2.8 & 2.3 & 3.5 & 3.8 & 9.1 & 11.7 & 8.8 & 4.9 & 10 & 11.6 & 5.0 & 4.2 & 3.2 & 9.1 & 7.4 \\
\hline 1993 Fall & 10.5 & 9.4 & 9.1 & 12.0 & 8.2 & 8.8 & 10.8 & 11.4 & 11.4 & 10.4 & 9.8 & 11.7 & 12.0 & 9.1 & 9.5 & 9.7 & 9.4 & 9.4 \\
\hline $1994 \mathrm{Win}$ & 10.6 & 9.4 & 9.1 & 11.3 & 3.1 & 4.0 & 10.6 & 12.1 & 12.1 & 10.8 & 9.3 & 10.8 & 12.6 & 9.3 & 4.3 & 10.1 & 9.6 & 9.2 \\
\hline $1994 \mathrm{Spr}$ & 8.5 & 8.8 & 9.0 & 8.9 & 6.2 & 5.4 & 9.9 & 2.5 & 10.7 & 6.9 & 8.4 & 10.3 & 12.0 & 9.5 & 8.1 & 10.6 & 9.0 & 6.7 \\
\hline 1994 Sum & 9.0 & NA & NA & 10.7 & NA & NA & NA & 8.5 & 10.9 & 8.3 & 6.5 & 8.7 & 10.5 & 7.8 & $\overline{8.6}$ & 8.9 & 7.9 & 7.5 \\
\hline 1994 Fall & 9.5 & 8.8 & NA & 9.6 & $\frac{1.3}{4.3}$ & 6.1 & 8.5 & 8.3 & 10.6 & 8.4 & 7.9 & 8.8 & 10.3 & 8.8 & 8.2 & 8.5 & 7.9 & 7.7 \\
\hline 1995 Win & 10.6 & 9.5 & 7.7 & 10.3 & \begin{tabular}{|l|l|}
4.0 \\
\end{tabular} & 8.7 & 11.7 & 9.1 & 11.5 & 8.6 & 8.3 & 9.5 & 11.1 & 9.8 & 7.7 & 9.6 & 8.1 & \begin{tabular}{|l|l|}
7.3 \\
\end{tabular} \\
\hline $1995 \mathrm{Spr}$ & 5.4 & 4.9 & 5.0 & 5.1 & $<\frac{10}{10}$ & 4.9 & 7.7 & 6.4 & 10.1 & 8.1 & 9.8 & 12.1 & 6.9 & 10.2 & 5.0 & 9.4 & 5.4 & $<10$ \\
\hline $1995 \mathrm{Sum}$ & NA & NA & $\mathrm{NA}$ & NA & NA & NA & NA & $\overline{\mathrm{NA}}$ & NA & NA & NA & $N A$ & NA & NA & NA & 9.2 & NA & 7.5 \\
\hline 1995 Fall & NA & NA & NA & NA & $\overline{\mathrm{NA}}$ & $\sqrt{\mathrm{NA}}$ & NA. & $\mathrm{NA}$ & NA & NA & NA & NA & NA & NA & NA & 13.4 & $\overline{\mathrm{NA}}$ & 13.1 \\
\hline MEAN & 9.3 & 7.7 & 7.1 & 8.8 & 5.4 & 5.9 & 9.0 & 8.4 & 11.1 & 8.8 & 8.1 & 10.2 & 10.9 & 8.7 & 7.0 & 9.3 & 8.3 & 8.6 \\
\hline & & & & & & & & & & & & & & & & & & \\
\hline Notes: & & & & & & & & & & & & & & & & & & \\
\hline 1. Units a & in ug/L. & & & & & & & & & & & & & & & & & \\
\hline 2. NA ind & ates that the & cll was not a & nalyzed for $v$ & nadium. & & & & & & & & & & & & & & \\
\hline 3. If a less & han flag (<) & s present, va & nadium was & ot detected al & bove the val & reported wl & ch is the dete & ction limit. & & & & & & & & & & \\
\hline 4. Mean v & ues were cal & lated using 0 & lata collected & starting in the & summer o & 1993 because & of the lower d & etection limi & during this ti & ime period. & & & & & & & & \\
\hline 5. There i & no Drinking & /ater Standar & d for vanadiu & n. Concents: & ation requirec & for hazard i & dex of 1 (inge & stion and inl & alation pathw & ays only) is & $60 \mathrm{ug} / \mathrm{L}$. & & & & & & & \\
\hline
\end{tabular}


Table C.3. Analytical Results for Groundwater, Fall 1990 Through Fall 1995, Metals

Zinc

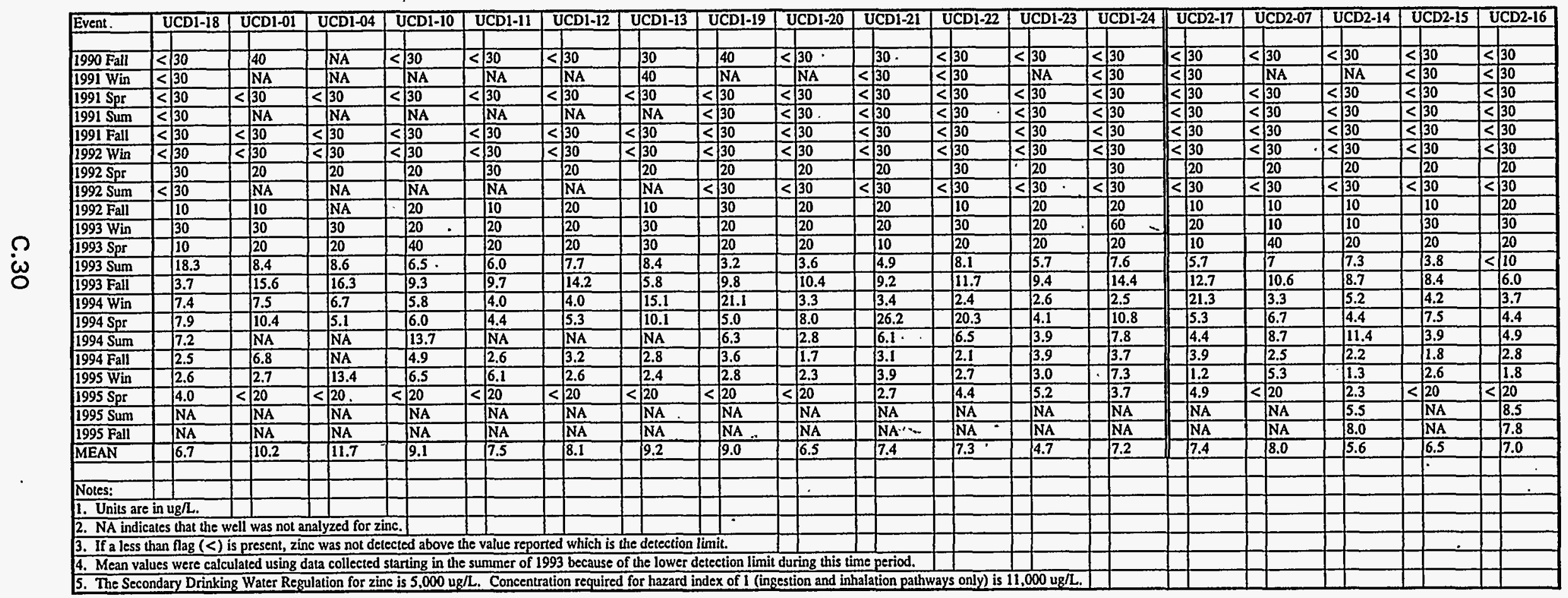


Table C.4. Analytical Results for Groundwater, Fall 1990 Through Fall 1995, Radionuclides

Tritium

\begin{tabular}{|c|c|c|c|c|c|c|c|c|c|c|c|c|c|c|c|c|c|c|c|}
\hline Event & UCDI-18 & UCD1-01 & UCD1-04 & UCDI-10 & UCD1-11 & UCDI-12 & UCDI-13 & UCDI-19 & UCD1-20 & UCD1-21 & UCDI-22 & UCD1-23 & UCD1-24 & UCD2-17 & & $\overline{I C D 2-07}$ & UCD2-14 & UCD2-15 & UCD2-16 \\
\hline & & & & & & & & & & & & & & & & & & & \\
\hline $1990 \mathrm{Fall}$ & 1618 & \begin{tabular}{l|l|}
$<$ & 500 \\
\end{tabular} & NA & $\begin{array}{l}<500 \\
\end{array}$ & 1382 & 1172 & 29066 & 1100 & 1573 & $<\mid 500^{\circ}$ & $<5$ & $<500$ & $\longdiv { 1 2 3 1 }$ & $<1500$ & $<5$ & 500 & 3864 & $<500$ & $\begin{array}{l}<500 \\
\end{array}$ \\
\hline $1991 \mathrm{~W}$ in & \begin{tabular}{l|l|} 
& 500 \\
\end{tabular} & \begin{tabular}{|l|} 
NA \\
\end{tabular} & NA & \begin{tabular}{|l|}
$\mathrm{NA}$ \\
\end{tabular} & NA & NA & NA & NA & NA & \begin{tabular}{|l|l|}
$<500$ \\
\end{tabular} & $<500$ & NA & $<500$ & $<500$ & & NA & NA & $<500$ & \begin{tabular}{|l|l|}
$<500$ \\
\end{tabular} \\
\hline $1991 \mathrm{Spr}$ & \begin{tabular}{l|l|l} 
& 500 \\
\end{tabular} & \begin{tabular}{|l|l|} 
& 500 \\
\end{tabular} & \begin{tabular}{l|l}
500 \\
\end{tabular} & \begin{tabular}{|l|l|}
$<500$ \\
\end{tabular} & \begin{tabular}{l|l|}
500 \\
\end{tabular} & $<500$ & 26186 & $\begin{array}{l}<500 \\
\end{array}$ & $<500$ & $\begin{array}{l}<500 \\
\end{array}$ & \begin{tabular}{l|l}
$<500$ \\
\end{tabular} & $<5500$ & $\leq 500$ & $<500$ & $<5$ & 500 & 7728 & $<500$ & $\begin{array}{l}<500 \\
\end{array}$ \\
\hline 1991 Sum & $<500$ & NA & NA & $<500$ & NA & NA & NA & $\begin{array}{l}500 \\
\end{array}$ & \begin{tabular}{l|l|l|}
$<500$ \\
\end{tabular} & \begin{tabular}{l|l|}
$<000$ \\
\end{tabular} & \begin{tabular}{|l|l|} 
& 500 \\
\end{tabular} & \begin{tabular}{l|l|} 
& 500 \\
\end{tabular} & \begin{tabular}{|l|l|}
500 \\
\end{tabular} & $<5$ & $<5$ & 500 & 5482 & $<1<500$ & \begin{tabular}{l|l}
500 \\
$<$
\end{tabular} \\
\hline 1991 Fall & \begin{tabular}{|l|l|}
$<500$ \\
\end{tabular} & \begin{tabular}{l|l|} 
& 500 \\
\end{tabular} & $<500$ & \begin{tabular}{l|l|}
$<$ & 500 \\
\end{tabular} & $<500$ & $<500$ & 24576 & \begin{tabular}{|l|l|}
$<500$ \\
\end{tabular} & $<\mid 500$ & 9752 & \begin{tabular}{|l|l|}
$<500$ \\
\end{tabular} & \begin{tabular}{l|l|l|} 
& 500 \\
\end{tabular} & $<500$ & $<500$ & $<5$ & 500 & 652 & $<5$ & $<500$ \\
\hline 1992 Win & \begin{tabular}{|l|l|}
$<$ & 500 \\
\end{tabular} & $<500$ & $<500$ & \begin{tabular}{|l|l|}
$<500$ \\
\end{tabular} & $<500$ & $<500$ & 22594 & \begin{tabular}{l|l|}
$<500$ \\
\end{tabular} & \begin{tabular}{l|l|l|} 
& 500 \\
\end{tabular} & $<<500$ & \begin{tabular}{|l|l|}
$<000$ \\
\end{tabular} & $<500$ & $<500$ & $<500$ & $<5$ & 500 & $<500$ & \begin{tabular}{|l|l|}
$<500$ \\
\end{tabular} & $<500$ \\
\hline $1992 \mathrm{Spr}$ & \begin{tabular}{l|l|}
$<$ & 500 \\
\end{tabular} & \begin{tabular}{|l|l|}
$<$ & 500 \\
\end{tabular} & \begin{tabular}{l|l|}
$<500$ \\
\end{tabular} & \begin{tabular}{l|l|}
$<<00$ \\
\end{tabular} & $<500$ & $<500$ & 20589 & \begin{tabular}{|l|l|}
500 \\
\end{tabular} & $<500$ & $<500$ & $<500$ & 2081 & \begin{tabular}{|l|l}
$<00$ \\
\end{tabular} & $<500$ & $<5$ & 500 & 3848 & $<500$ & $\begin{array}{l}<00 \\
<00\end{array}$ \\
\hline 1992 Sum & 9965 & \begin{tabular}{|l|}
$\mathrm{NA}$ \\
\end{tabular} & $\mathrm{NA}$ & \begin{tabular}{|l|}
$\mathrm{NA}$ \\
\end{tabular} & \begin{tabular}{|l|}
$\mathrm{NA}$ \\
\end{tabular} & NA & NA & \begin{tabular}{l|l|}
$<500$ \\
\end{tabular} & $<500$ & \begin{tabular}{|l|l|}
$<500$ \\
\end{tabular} & \begin{tabular}{l|l}
$<500$ \\
\end{tabular} & $<500$ & \begin{tabular}{|l|l|}
$<<500$ \\
\end{tabular} & $<5$ & & 691 & 5525 & $<500$ & $<500$ \\
\hline $1992 \mathrm{Fall}$ & \begin{tabular}{|l|l|}
614 \\
\end{tabular} & \begin{tabular}{|l|l|} 
& 500 \\
\end{tabular} & $\mathrm{NA}$ & \begin{tabular}{|l|l|} 
& 500 \\
\end{tabular} & $<500$ & $<500$ & \begin{tabular}{|l|}
29838 \\
\end{tabular} & \begin{tabular}{|l|l|}
$<500$ \\
\end{tabular} & $<500$ & \begin{tabular}{|l|l|}
509 \\
\end{tabular} & \begin{tabular}{l|l}
$<00$ \\
\end{tabular} & \begin{tabular}{l|l}
$<$ & 500 \\
\end{tabular} & \begin{tabular}{l|l}
$<<500$ \\
\end{tabular} & $<500$ & $<$ & 500 & 1204 & $<1500$ & 1200 \\
\hline 1993 Win & \begin{tabular}{|l|l|} 
& 500 \\
\end{tabular} & 625 & $<500$ & $<500$ & 1024 & 9921 & 20705 & 1640 & 1550 & \begin{tabular}{|l|l|}
500 \\
\end{tabular} & \begin{tabular}{|l|l|}
$<500$ \\
\end{tabular} & \begin{tabular}{|l|l|}
500 \\
\end{tabular} & \begin{tabular}{|l|l}
965 \\
\end{tabular} & $<500$ & $<$ & 500 & 908 & $<500$ & $<<500$ \\
\hline $1993 \mathrm{Spr}$ & \begin{tabular}{|l|l|} 
& 500 \\
\end{tabular} & 2211 & 2022 & $<500$ & $<500$ & $<500$ & 20917 & $<500$ & $<500$ & \begin{tabular}{|l|l|}
811 \\
\end{tabular} & \begin{tabular}{|l|l|}
$<00$ \\
\end{tabular} & 7334 & \begin{tabular}{|l|l|}
500 \\
\end{tabular} & $\begin{array}{l}<500 \\
\end{array}$ & $<$ & 500 & 5949 & 1524 & \begin{tabular}{l|l}
$<500$ \\
\end{tabular} \\
\hline $1993 \mathrm{Sum}$ & $<220$ & $<230$ & $<230$ & $<230$ & $<230$ & $<230$ & 19620 & $<2220$ & $\begin{array}{l}<<220 \\
\end{array}$ & \begin{tabular}{|l|l|} 
& 220 \\
\end{tabular} & $<230$ & $<220$ & $<220$ & $<230$ & $<$ & 220 & 3810 & $<\frac{220}{220}$ & $<220$ \\
\hline 1993 Fall & \begin{tabular}{|l|l|}
$<<240$ \\
\end{tabular} & \begin{tabular}{l|l}
$<<240$ \\
\end{tabular} & $<240$ & $<240$ & $<240$ & $<240$ & 20590 & $<240$ & $<240$ & $<240$ & $<240$ & $<240$ & $<240$ & $<240$ & $\frac{1}{<1}$ & $\frac{20}{240}$ & $\frac{7760}{4760}$ & $<\frac{240}{240}$ & $\begin{array}{l}<<250 \\
<25\end{array}$ \\
\hline 1994 Win & \begin{tabular}{l|l|}
$<<260$ \\
\end{tabular} & $<260$ & $<260$ & $<260$ & $<260$ & $<260$ & 21490 & $<230$ & $\begin{array}{l}<240 \\
\end{array}$ & $\begin{array}{l}<240 \\
\end{array}$ & \begin{tabular}{|l|l|}
$<240$ \\
\end{tabular} & \begin{tabular}{|l|l|}
$<240$ \\
\end{tabular} & $<240$ & \begin{tabular}{l|l}
$<240$ \\
\end{tabular} & $<$ & 260 & 5440 & $<240$ & \begin{tabular}{l|l}
$<<240$ \\
\end{tabular} \\
\hline $1994 \mathrm{Spr}$ & $<250$ & $<250$ & $<240$ & \begin{tabular}{|l|l|l|} 
& 250 \\
\end{tabular} & $<250$ & $<2250$ & 19250 & \begin{tabular}{|l|l|}
$<240$ \\
\end{tabular} & $<240$ & $<250$ & $<250$ & $<2250$ & $<240$ & \begin{tabular}{l|l}
$<240$ \\
\end{tabular} & $<$ & 250 & 8320 & $|<| \frac{140}{240}$ & $<240$ \\
\hline 1994 Sum & $<250$ & NA & $\mathrm{NA}$ & \begin{tabular}{|l|l|}
$<$ & 260 \\
\end{tabular} & $\overline{\mathrm{NA}}$ & $\mathrm{NA}$ & NA & $<2270$ & $<260$ & $<260$ & \begin{tabular}{|l|l|}
$<270$ \\
\end{tabular} & $<270$ & $<<270$ & $<260$ & $<$ & 260 & 2500 & $<<\frac{110}{270}$ & $<1270$ \\
\hline 1994 Fall & $<240$ & $<240$ & NA & \begin{tabular}{l|l|}
$<<240$ \\
\end{tabular} & $<240$ & $<240$ & 450 & $<240$ & $\begin{array}{l}<<0 \\
\end{array}$ & $<240$ & $<240$ & $<2$ & \begin{tabular}{|l|l|}
$<240$ \\
$<1$
\end{tabular} & $\begin{array}{c}<\mid 240 \\
<240\end{array}$ & < & $\frac{200}{240}$ & $\frac{200}{17900}$ & $\mid \frac{10}{240}$ & $\begin{array}{l}<<20 \\
240\end{array}$ \\
\hline $1995 \mathrm{Win}$ & $\begin{array}{l}<270 \\
\end{array}$ & $<2$ & $<310$ & $<300$ & $\begin{array}{l}310 \\
\end{array}$ & $<260$ & 17600 & $<310$ & $<310$ & $<310$ & $<310$ & $<300$ & $<310$ & $<260$ & & 310 & 590 & $<310$ & $<310$ \\
\hline $1995 \mathrm{Spr}$ & $<220$ & $<260$ & $<260$ & $<260$ & $<260$ & $<250$ & \begin{tabular}{l|l|}
$<<260 R$ \\
\end{tabular} & $<260$ & $16600 \mathrm{R}$ & $<220$ & $<<220$ & $<220$ & $<230$ & $<220$ & $<\mid$ & 260 & 5320 & $<260$ & $<220$ \\
\hline 1995 Sum & $\mathrm{NA}$ & \begin{tabular}{|l|}
$\mathrm{NA}$ \\
\end{tabular} & $\leq 300$ & NA & NA & $\leq 300$ & 19600 & $\mathrm{NA}$ & $<290$ & NA & $<300$ & $<310$ & NA & NA & $<$ & 300 & 5590 & $<300$ & $<300$ \\
\hline 1995 Fall & NA & NA & $<220$ & NA & $\mathrm{NA}$ & $<240$ & 16480 & \begin{tabular}{|l|l} 
NA \\
\end{tabular} & $<220$ & NA & NA & NA & $\mid \frac{\mathrm{NA}}{\mathrm{NA}}$ & $-\frac{n}{N A}$ & & $\mathrm{NA}$ & $\frac{5700}{1670}$ & $<240$ & $\frac{1}{N A}$ \\
\hline MEAN & \begin{tabular}{|l|l|}
$<$ & 480 \\
\end{tabular} & $<5500$ & $<470$ & $<390$ & $<480$ & $<430$ & 20597 & $<490$ & $<494$ & $<900$ & $<390$ & $<480$ & $<\frac{460}{460}$ & $<390$ & <ا & $\frac{10 n}{400}$ & $\frac{1000}{4580}$ & $<\frac{140}{430}$ & $<\frac{10 \pi}{430}$ \\
\hline & & & & & & & & & & & & & & & & & & & \\
\hline Notes: & & & & & & & & & & & & & & & & & & & \\
\hline 1. Units as & $\mathrm{pCi/L}$ & & & & & & & & & & & & & & & & & & \\
\hline 2. NA ind & s that the w & II was not ana & yzed for triti & & & & & & & & & & & & & & & & \\
\hline 3. A less I & nag ( $<)$ ind & cates that the & ritum activit & was less than & the MDA re & $\frac{1}{1}$ & & & & & & & & & & & & & \\
\hline 4. Rejecter & alyses $(R)$ in & the spring que & ter oflags f & r wells UCD & -13 and UCD & $1-20$ are th & it to Lave t & in switched. & $\frac{1}{\text { hese results }}$ & e not inclu & $d$ in the mean. & & & & & & & & \\
\hline 5. Mean C. & lated using a & 1 numerical en & ries includin & MDA values & & & & & & & & & & & & & & & \\
\hline 6. The Nat & If Primary D & inking Water & Standard for & ritium is 20.0 & $\frac{1}{0 \mathrm{pCi} / \mathrm{L}}$ & & & & & & & & & & & & t. & & \\
\hline
\end{tabular}


Table C.4. Analytical Results for Groundwater, Fall 1990 Through Fall 1995, Radionuclides

Carbon-14.

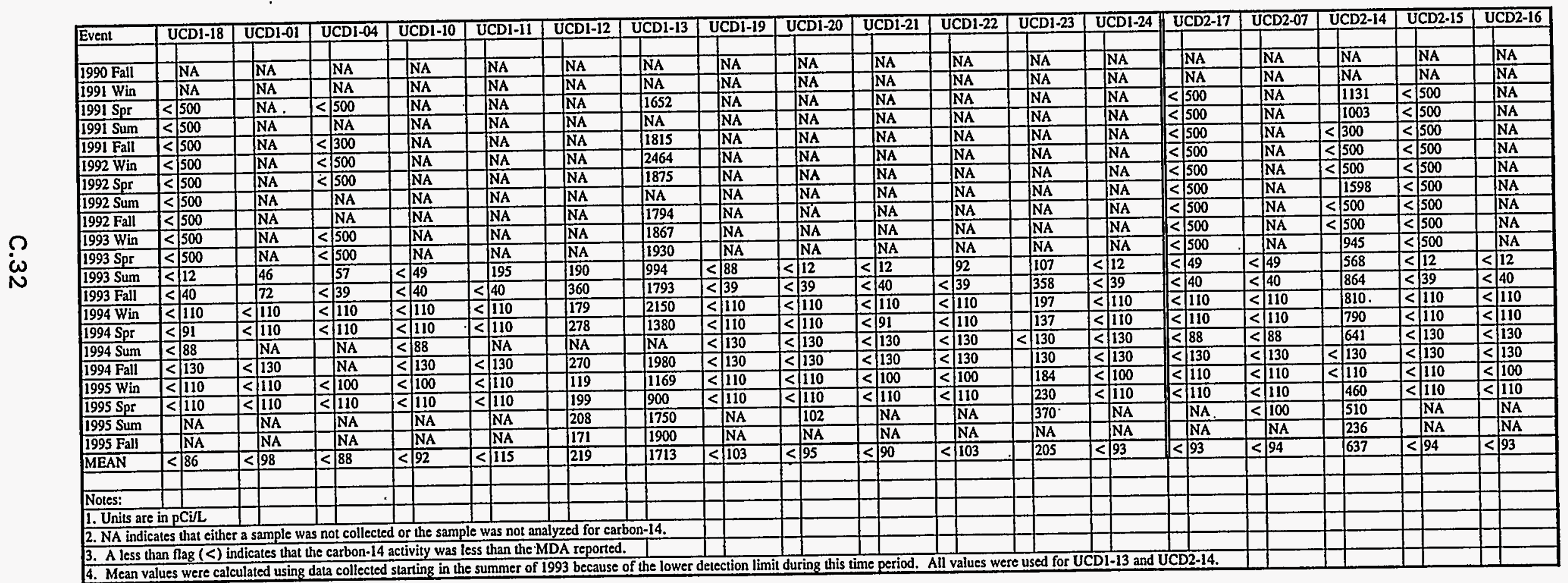


Table C.4. Analytical Results for Groundwater, Fall 1990 Through Fall 1995, Radionuclides

\section{Radium-226}

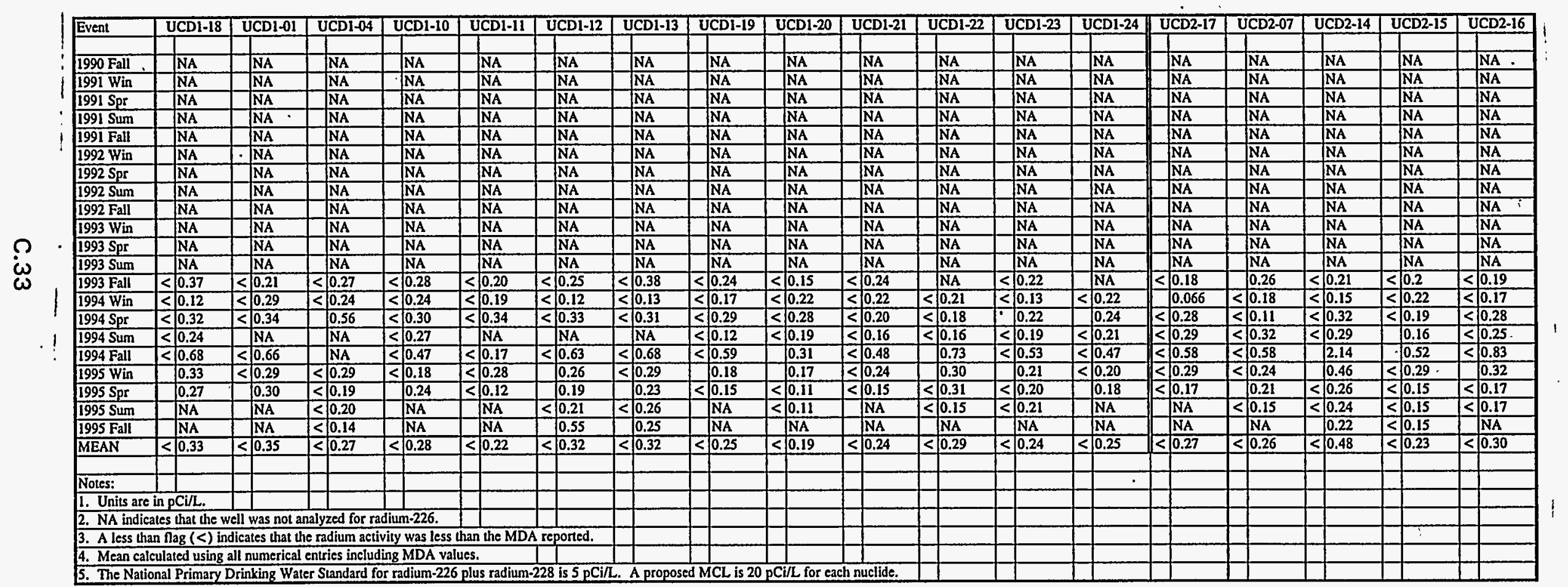


Table C.4. Analytical Results for Groundwater, Fall 1990 Through Fall 1995, Radionuclides

Strontium-90

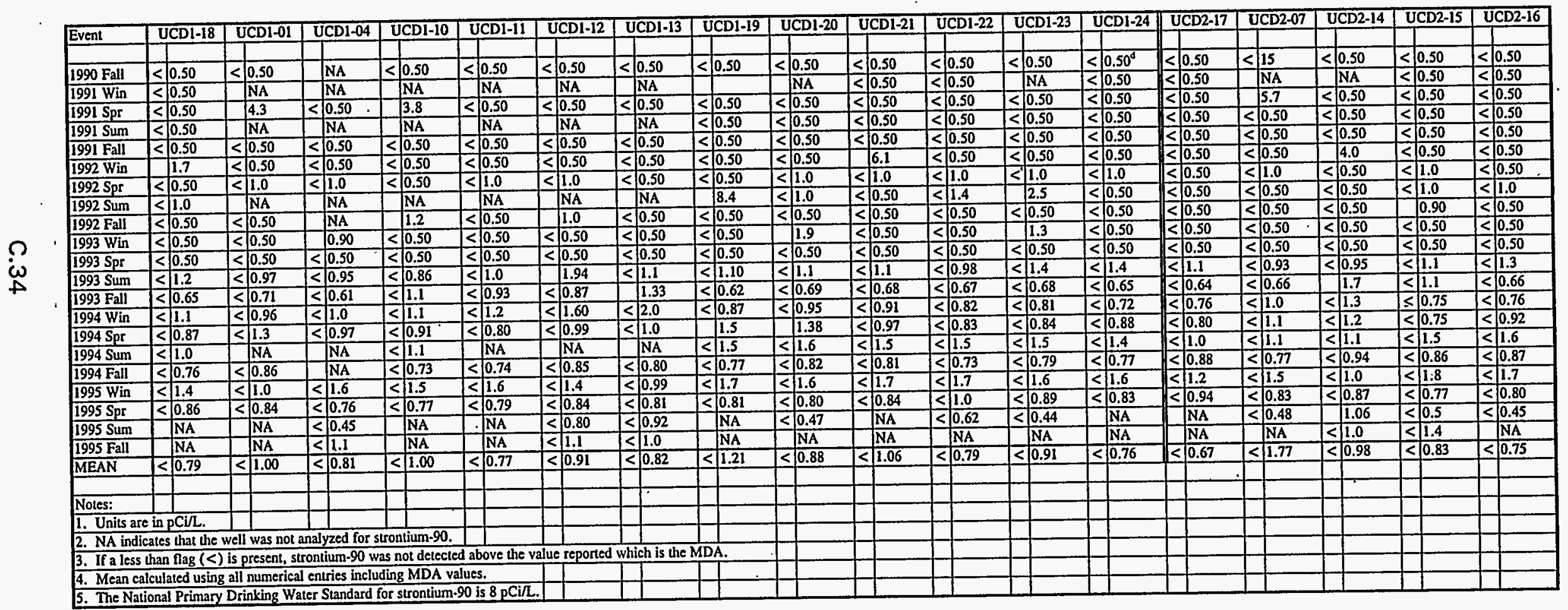


Table C.4. Analytical Results for Groundwater, Fall 1990 Through Fall 1995, Radionuclides .

\section{Cesium-137}

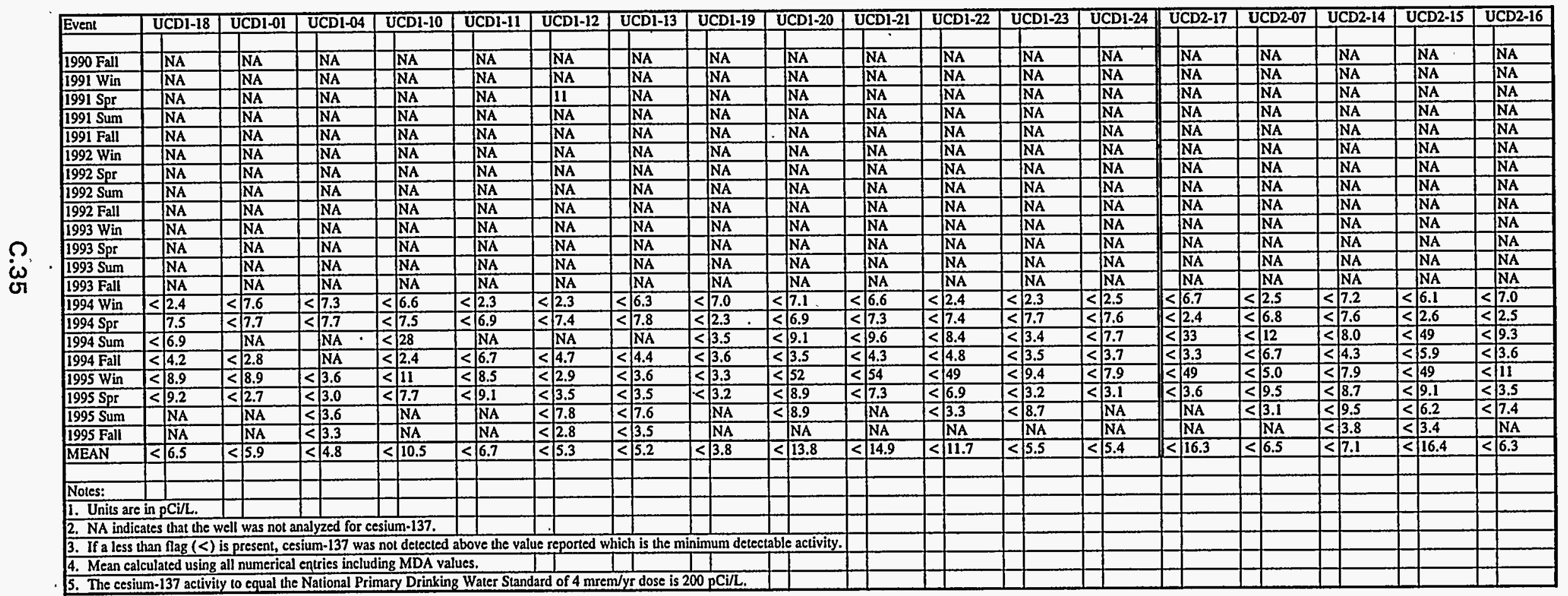


Table C.4. Analytical Results for Groundwater, Fall 1990 Through Fall 1995, Radionuclides

Plutonium-241

\begin{tabular}{|c|c|c|c|c|c|c|c|c|c|c|c|c|c|c|c|c|c|c|}
\hline Event & UCDI-18 & UCD1-01 & UCDI-04 & UCDI-10 & UCD1-11 & UCD1-12 & UCDI-13 & UCDI-19 & UCDI-20 & UCD1-21 & UCDI-22 & UCD1-23 & UCD1-24 & UCD2-17 & UCD2-07 & UCD2-14 & UCD2-15 & UCD2-16 \\
\hline & & & & & & & & & & & & & & & & & & \\
\hline 1990 Fall & NA & $\overline{\mathrm{NA}}$ & NA & NA & NA & NA & NA & NA & NA & NA & NA & NA & NA & NA & NA & NA & NA & NA \\
\hline 1991 Win & NA & NA & NA & NA & NA & NA & NA & NA & NA & NA & NA & NA & NA & NA & NA & NA & NA & NA \\
\hline $1991 \mathrm{Spr}$ & NA & NA & NA & NA & NA & NA & NA & NA & NA & NA & NA & NA & NA & NA & NA & NA & $\sqrt{\mathrm{NA}}$ & NA \\
\hline $1991 \mathrm{sum}$ & NA & NA & NA & NA & NA & NA & NA & NA & $\overline{\mathrm{NA}}$ & NA & $\sqrt{\mathrm{NA}}$ & NA & $\sqrt{\mathrm{NA}}$ & NA & $\mathrm{NA}$ & NA & NA & NA \\
\hline 1991 Fall & NA & NA & NA & NA & NA & NA & $\overline{\mathrm{NA}}$ & NA & NA & NA & NA & NA & NA & NA & $\overline{\mathrm{NA}}$ & $\overline{\mathrm{NA}}$ & $\overline{\mathrm{NA}}$ & $\overline{\text { NA }}$ \\
\hline 1992 Win & NA & NA & NA & NA & NA & $\widehat{\mathrm{NA}}$ & NA & NA & NA & NA & $\overline{\mathrm{NA}}$ & $\widehat{N A}$ & $\overline{\mathrm{NA}}$ & $\overline{\mathrm{NA}}$ & $\overline{\mathrm{NA}}$ & $\overline{N A}$ & $\overline{N A}$ & NA \\
\hline $1992 \mathrm{Spr}$ & NA & NA & NA & NA & NA & NA & $\overline{\mathrm{NA}}$ & $\overline{\mathrm{NA}}$ & $\sqrt{\mathrm{NA}}$ & $\overline{\mathrm{NA}}$ & NA & NA & $\sqrt{\mathrm{NA}}$ & NA & NA & NA & NA & NA \\
\hline 1992 Sum & NA & NA & NA & NA & NA & NA & $\overline{\mathrm{NA}}$ & NA & NA & NA & NA & NA & NA & NA & NA & NA & $\overline{\mathrm{NA}}$ & NA \\
\hline 1992 Fall & NA & NA & NA & NA & NA & $\mathrm{NA}$ & NA & NA & NA & NA & NA & NA & NA & NA & NA & NA & NA & NA \\
\hline $1993 \mathrm{Win}$ & $\mathrm{NA}$ & NA & NA & NA & NA & NA & NA & NA & NA & NA & NA & NA & NA & NA & NA & NA & NA & NA \\
\hline $1993 \mathrm{Spr}$ & NA & NA & NA & NA & NA & NA & NA & NA & NA & NA & NA & NA & NA & NA & NA & NA & NA & NA \\
\hline 1993 Sum & NA & NA & NA & NA & NA & NA & NA & $\overline{\mathrm{NA}}$ & $\overline{\mathrm{NA}}$ & $\overline{\mathrm{NA}}$ & $\overline{\mathrm{NA}}$ & NA & $\overline{N A}$ & $\mathrm{NA}$ & NA & NA & NA & NA \\
\hline 1993 Fall & NA & NA & NA & NA & NA & NA & NA & NA & $\overline{N A}$ & NA & NA & NA & NA & $\widehat{N A}$ & $\sqrt{\mathrm{NA}}$ & NA & NA & $\overline{\mathrm{NA}}$ \\
\hline $1994 \mathrm{~W}$ in & NA & NA & NA & NA & NA & NA & NA & NA & NA & NA & NA & NA & NA & NA & $\sqrt{\mathrm{NA}}$ & NA & NA & NA \\
\hline $1994 \mathrm{Spr}$ & NA & NA & NA & NA & NA & NA & NA & NA & \begin{tabular}{|l|}
$\mathrm{NA}$ \\
\end{tabular} & NA & \begin{tabular}{|l|} 
NA \\
\end{tabular} & \begin{tabular}{|l|}
$\mathrm{NA}$ \\
\end{tabular} & \begin{tabular}{|l|}
$\mathrm{NA}$ \\
\end{tabular} & $\overline{\mathrm{NA}}$ & \begin{tabular}{|l}
$\mathrm{NA}$ \\
\end{tabular} & NA & $\overline{\mathrm{NA}}$ & NA \\
\hline 1994 Sum & $<3$ & NA & NA & \begin{tabular}{|l|l|} 
& 2.6 \\
\end{tabular} & NA & NA & NA & \begin{tabular}{|l|l|}
$<2.9$ \\
\end{tabular} & \begin{tabular}{|l|l|} 
& 3.3 \\
\end{tabular} & \begin{tabular}{|l|l|}
$<7.8$ \\
\end{tabular} & $<\sqrt{3}$ & \begin{tabular}{|l|l|}
$<2.9$ \\
\end{tabular} & \begin{tabular}{|c|c|}
3.8 \\
\end{tabular} & \begin{tabular}{|l|l|}
$<2.3$ \\
\end{tabular} & \begin{tabular}{|l|l|}
$<3.2$ \\
\end{tabular} & \begin{tabular}{|l|l|} 
& 2.3 \\
\end{tabular} & \begin{tabular}{l|l|}
$<2.8$ \\
\end{tabular} & \begin{tabular}{l|l}
$<$ & 3 \\
\end{tabular} \\
\hline 1994 Fall & \begin{tabular}{l|l|}
1.7 \\
\end{tabular} & \begin{tabular}{|l|l|}
$<$ & 1.6 \\
\end{tabular} & NA & $<1.7$ & $<1.0$ & $<2.0$ & \begin{tabular}{|l|l|}
$<1.8$ \\
\end{tabular} & \begin{tabular}{|l|l|}
$<$ & 1.5 \\
\end{tabular} & \begin{tabular}{|l|l|}
$<$ & 1.5 \\
\end{tabular} & \begin{tabular}{|l|l|}
$<$ & 1.6 \\
\end{tabular} & \begin{tabular}{l|l|}
$<1.6$ \\
\end{tabular} & \begin{tabular}{l|l|} 
& 1.4 \\
\end{tabular} & $\begin{array}{ll}<1.5 \\
\end{array}$ & $\begin{array}{l}<1.8 \\
\end{array}$ & $\begin{array}{l}<1.2 \\
\end{array}$ & $\begin{array}{l}<.7 \\
\end{array}$ & $<2$ & $<2$ \\
\hline 1995 Win & \begin{tabular}{l|l}
$<1.6$ \\
\end{tabular} & \begin{tabular}{|l|l|}
$<$ & 1.6 \\
\end{tabular} & \begin{tabular}{|l|l|}
$<1.8$ \\
\end{tabular} & \begin{tabular}{|l|l|}
$<1.7$ \\
\end{tabular} & $<\mid 2.2$ & \begin{tabular}{|l|l|}
$<$ & 1.9 \\
\end{tabular} & \begin{tabular}{|l|l|}
$<<2.8$ \\
\end{tabular} & $<2.0$ & $<2.0$ & $\begin{array}{l}<2.4 \\
\end{array}$ & $<1.8$ & $<1.9$ & \begin{tabular}{|l|l|} 
& 1.8 \\
\end{tabular} & \begin{tabular}{l|l|}
$<$ & 1.5 \\
\end{tabular} & \begin{tabular}{|l|l|}
$<<1.9$ \\
\end{tabular} & \begin{tabular}{l|l}
$<1.9$ \\
\end{tabular} & \begin{tabular}{l|l|}
$<$ & 1.8 \\
\end{tabular} & \begin{tabular}{l|l|}
$<2.8$ \\
\end{tabular} \\
\hline $1995 \mathrm{Spr}$ & $<3$ & $<1.4$ & \begin{tabular}{|l|l|}
$<1.2$ \\
\end{tabular} & $\begin{array}{ll}<1.2 \\
\end{array}$ & $<1.3$ & \begin{tabular}{|l|l|}
$<1.1$ \\
\end{tabular} & \begin{tabular}{|l|l|}
$<1.6$ \\
\end{tabular} & \begin{tabular}{l|l|}
$<1.4$ \\
\end{tabular} & $<1.9$ & $<2.4$ & 3.2 & \begin{tabular}{|l|l|} 
& 1.9 \\
\end{tabular} & $<2.2$ & \begin{tabular}{|l|l|}
$<2.4$ \\
\end{tabular} & \begin{tabular}{|l|l|}
$<1.2$ \\
\end{tabular} & \begin{tabular}{|l|l|} 
& 1.7 \\
\end{tabular} & \begin{tabular}{|l|l|}
$<1.2$ \\
\end{tabular} & \begin{tabular}{|l|l|}
$<1.2$ \\
\end{tabular} \\
\hline $1995 \mathrm{Sum}$ & NA & NA & NA & NA & NA & NA & $\mathrm{NA}$ & NA & $\mathrm{NA}^{\circ}$ & NA & \begin{tabular}{|l|l|}
$<3.6$ \\
\end{tabular} & NA & NA & NA & NA & $<3.5$ & NA & NA \\
\hline 1995 Fall & NA & $\overline{\mathrm{NA}}$ & NA & NA & NA & NA & $\overline{N A}$ & DA & $\sqrt{\mathrm{NA}}$ & NA & NA & $\mathrm{NA}$ & NA & NA & NA & $<2.8$ & NA & NA \\
\hline \multirow[t]{2}{*}{ MEAN } & $\begin{array}{l}<2.5 \\
\end{array}$ & $<1.5$ & $<1.5$ & $<1.8$ & \begin{tabular}{|l|l|}
$<$ & 1.5 \\
\end{tabular} & $<1.7$ & $<2.1$ & $<2.0$ & $<2.2$ & $<3.6$ & $\begin{array}{l}<2.6 \\
\end{array}$ & $<<2.0$ & $<2.3$ & $<2.0$ & $<1.9$ & $<2.3$ & \begin{tabular}{|l|l|}
2.0 \\
\end{tabular} & $<\longdiv { 2 . 4 }$ \\
\hline & & 4 & & & & & & & & & & & & & & & & \\
\hline \multicolumn{19}{|c|}{\begin{tabular}{|l|l|} 
Notes: & \\
\end{tabular}} \\
\hline \multicolumn{19}{|c|}{\begin{tabular}{|l|l|l|l|l|l|l|l|l|l|l|l|l} 
1. Units are in $\mathrm{pC} / \mathrm{L}$. & & & & \\
\end{tabular}} \\
\hline \multirow{2}{*}{\multicolumn{19}{|c|}{$\begin{array}{l}\text { 2. NA indicates that the well was not analyzed for plutonium-24l. } \\
\text { 3. A less than flag }(<) \text { indicates that the plutonium activity was less than the MDA reported }\end{array}$}} \\
\hline & & & & & & & & & & & & & & & & & & \\
\hline 4. Mean c & ulated using & lil numerical & entries includ & ing MDA val & ues. & & & & & & & & & & & & & \\
\hline
\end{tabular}


Table C.4. Analytical Results for Groundwater, Fall 1990 Through Fall 1995, Radionuclides

Americium-241

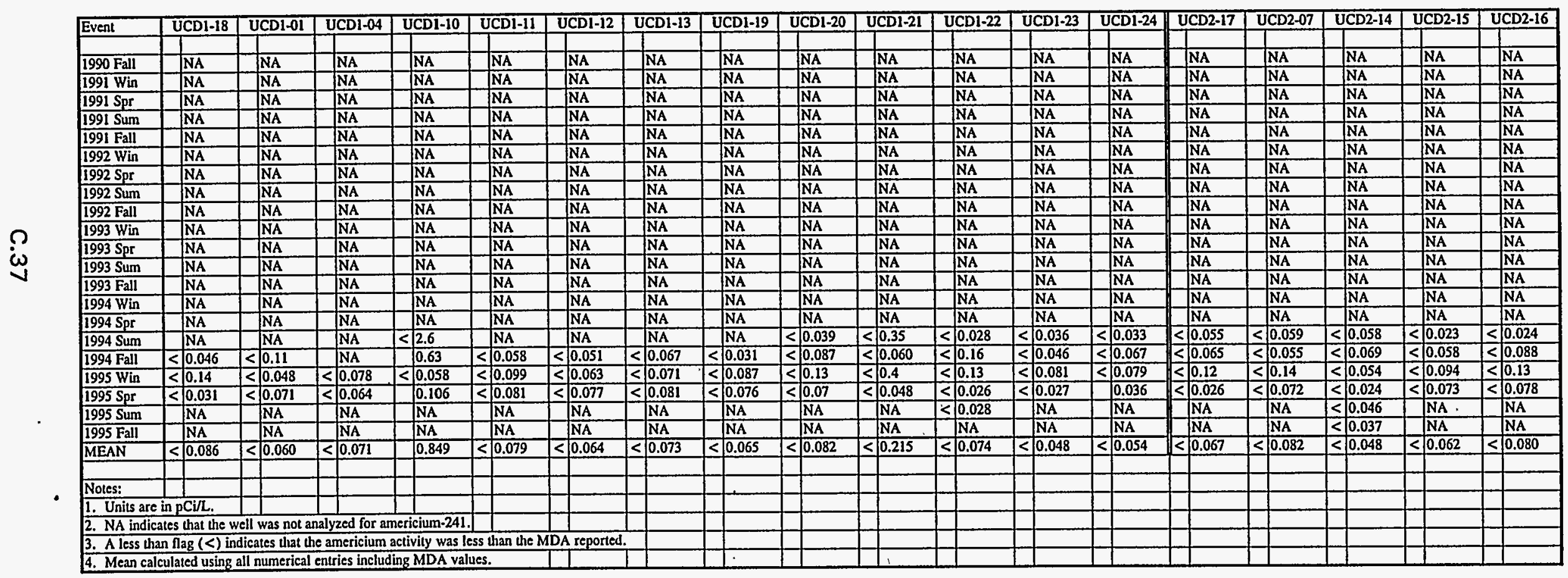


Table C.4. Analytical Results for Groundwater, Fall 1990 Through Fall 1995, Radionuclides

\section{Gross Alpha}

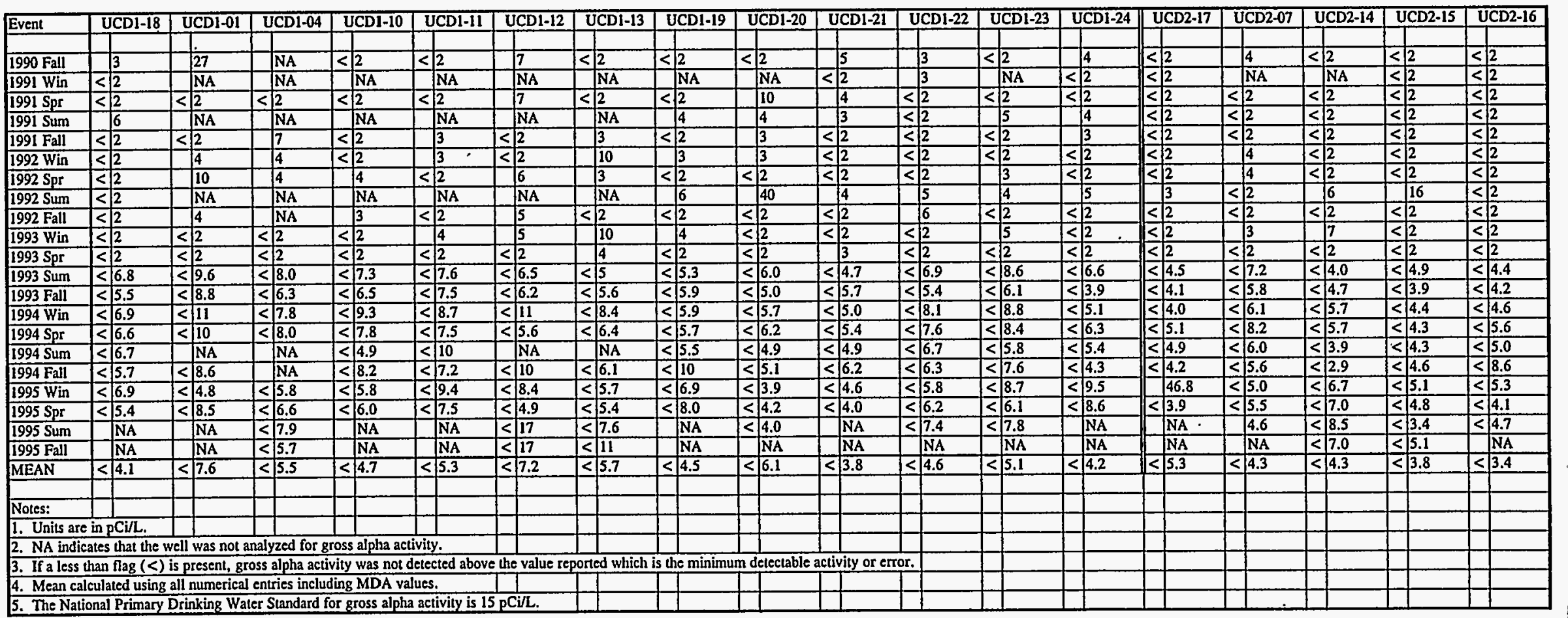


Table C.4. Analytical Results for Groundwater, Fall 1990 Through Fall 1995, Radionuclides

\section{Gross Beta}

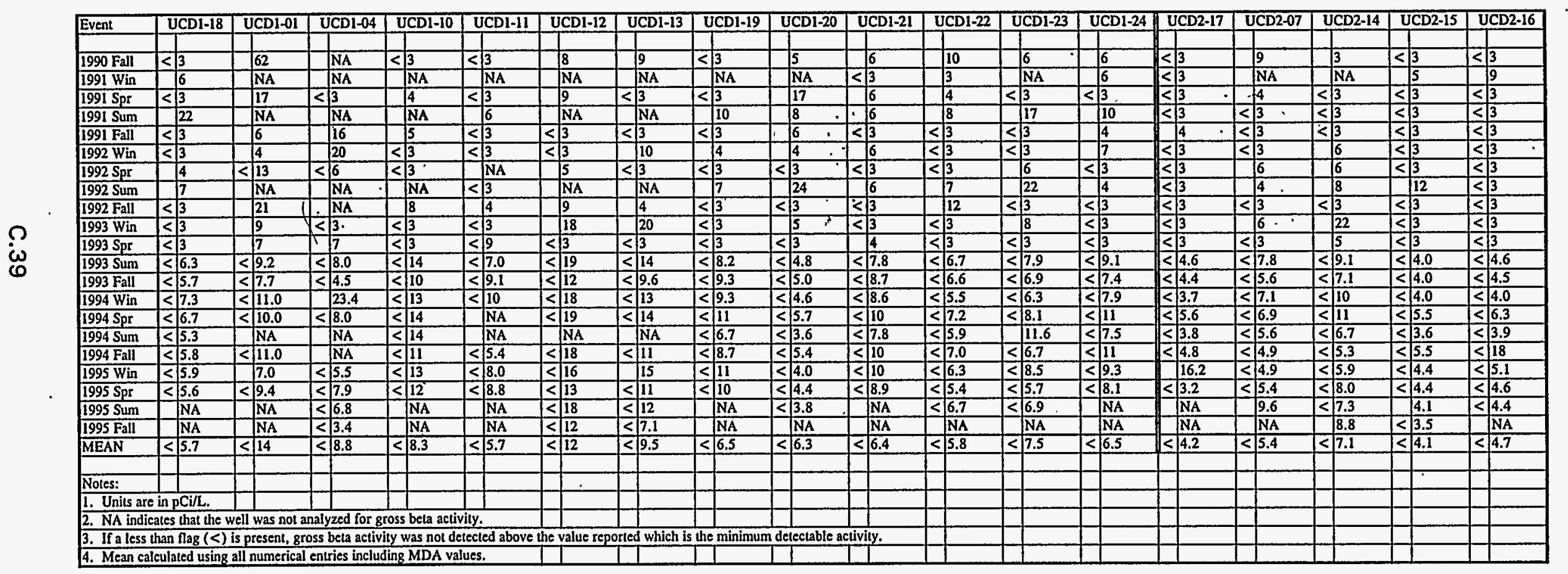


Table C.5. Analytical Results for Groundwater, Fall 1990 Through Fall 1995, Cations and Anions

Calcium

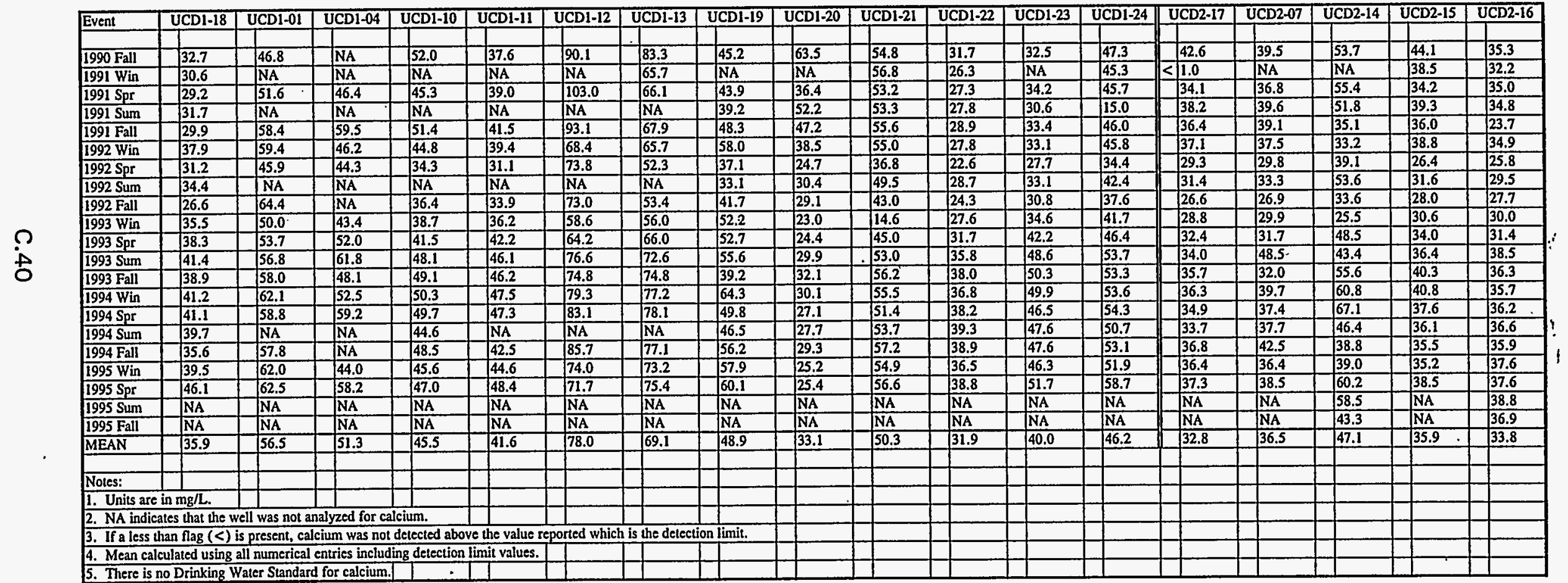

\begin{tabular}{|c|c|c|c|c|c|c|c|c|c|c|c|c|c|c|c|c|c|c|}
\hline Event & UCDI-18 & UCD1-01 & UCD1-04 & UCD1-10 & UCD1-11 & UCD1-12 & UCDI-13 & UCDI-19 & UCD1-20 & UCDI-2I & UCD1-22 & UCD1-23 & UCD1-24 & UCD2-17 & UCD2-07 & UCD2-14 & UCD2-15 & UCD2-16 \\
\hline & & & & & & & & & & & & & & & & & & \\
\hline 1990 Fall & 32.7 & 46.8 & $\mathrm{NA}$ & 52.0 & 37.6 & 90.1 & 83.3 & 45.2 & 63.5 & 54.8 & 31.7 & 32.5 & 47.3 & 42.6 & 39.5 & 53.7 & 44.1 & 35.3 \\
\hline $1991 \mathrm{Win}$ & 30.6 & NA & NA & $\overline{\mathrm{NA}}$ & $\overline{N A}$ & NA & 65.7 & $\overline{\mathrm{NA}}$ & NA & 56.8 & 26.3 & NA & 45.3 & $\leq 1.0$ & NA & NA & 38.5 & 32.2 \\
\hline $1991 \mathrm{Spr}$ & 29.2 & 51.6 & 46.4 & 45.3 & 39.0 & 103.0 & 66.1 & 43.9 & 36.4 & 53.2 & 27.3 & 34.2 & 45.7 & 34.1 & 36.8 & 55.4 & 34.2 & 35.0 \\
\hline 1991 Sum & 31.7 & NA & NA & NA & NA & NA & NA & 39.2 & 52.2 & 53.3 & 27.8 & 30.6 & 15.0 & 38.2 & 39.6 & 51.8 & 39.3 & 34.8 \\
\hline 1991 Fall & 29.9 & 58.4 & 59.5 & 51.4 & 41.5 & 93.1 & 67.9 & 48.3 & 47.2 & 55.6 & 28.9 & 33.4 & 46.0 & 36.4 & 39.1 & 35.1 & 36.0 & 23.7 \\
\hline $1992 \mathrm{Win}$ & 37.9 & 59.4 & 46.2 & 44.8 & 39.4 & 68.4 & 65.7 & 58.0 & 38.5 & 55.0 & 27.8 & 33.1 & 45.8 & 37.1 & 37.5 & 33.2 & 38.8 & 34.9 \\
\hline $1992 \mathrm{Spr}$ & 31.2 & 45.9 & $\overline{44.3}$ & 34.3 & 31.1 & 73.8 & 52.3 & 37.1 & 24.7 & 36.8 & 22.6 & 27.7 & 34.4 & 29.3 & 29.8 & 39.1 & 26.4 & 25.8 \\
\hline $1992 \mathrm{Sum}$ & 34.4 & NA & NA & $\overline{\mathrm{NA}}$ & $\overline{\mathrm{NA}}$ & $\mathrm{NA}$ & NA & 33.1 & 30.4 & 49.5 & 28.7 & 33.1 & 42.4 & 31.4 & 33.3 & 53.6 & 31.6 & 29.5 \\
\hline 1992 Fall & 26.6 & 64.4 & NA & 36.4 & 33.9 & 73.0 & 53.4 & 41.7 & 29.1 & 43.0 & 24.3 & 30.8 & 37.6 & 26.6 & 26.9 & 33.6 & 28.0 & 27.7 \\
\hline $1993 \mathrm{Win}$ & 35.5 & 50.0 & 43.4 & 38.7 & 36.2 & 58.6 & 56.0 & 52.2 & 23.0 & 14.6 & 27.6 & 34.6 & 41.7 & 28.8 & 29.9 & 25.5 & 30.6 & 30.0 \\
\hline $1993 \mathrm{Spr}$ & 38.3 & 53.7 & 52.0 & 41.5 & 42.2 & 64.2 & 66.0 & 52.7 & 24.4 & 45.0 & 31.7 & 42.2 & 46.4 & 32.4 & 31.7 & 48.5 & 34.0 & 31.4 \\
\hline $1993 \mathrm{Sum}$ & 41.4 & 56.8 & 61.8 & 48.1 & 46.1 & 76.6 & 72.6 & 55.6 & 29.9 & 53.0 & 35.8 & 48.6 & 53.7 & 34.0 & 48.5 & 43.4 & 36.4 & 38.5 \\
\hline 1993 Fall & 38.9 & 58.0 & 48.1 & 49.1 & 46.2 & 74.8 & 74.8 & 39.2 & 32.1 & 56.2 & 38.0 & 50.3 & 53.3 & 35.7 & 32.0 & 55.6 & 40.3 & 36.3 \\
\hline $1994 \mathrm{Win}$ & 41.2 & 62.1 & 52.5 & 50.3 & 47.5 & 79.3 & 777.2 & 64.3 & 30.1 & 55.5 & 36.8 & 49.9 & 53.6 & 36.3 & 39.7 & 60.8 & 40.8 & 35.7 \\
\hline $1994 \mathrm{Spr}$ & 41.1 & 58.8 & 59.2 & 49.7 & 47.3 & 83.1 & 78.1 & 49.8 & 27.1 & 51.4 & 38.2 & 46.5 & 54.3 & 34.9 & 37.4 & 67.1 & 37.6 & 36.2 \\
\hline $1994 \mathrm{Sum}$ & 39.7 & NA & $\mathrm{NA}$ & 44.6 & $\mathrm{NA}$ & NA & $\overline{\mathrm{NA}}$ & 46.5 & 27.7 & 53.7 & 39.3 & 47.6 & 50.7 & 33.7 & 37.7 & 46.4 & 36.1 & 36.6 \\
\hline 1994 Fall & 35.6 & 57.8 & $\overline{\mathrm{NA}}$ & 48.5 & 42.5 & 85.7 & 77.1 & 56.2 & 29.3 & 57.2 & 38.9 & 47.6 & 53.1 & 36.8 & 42.5 & 38.8 & 35.5 & 35.9 \\
\hline $1995 \mathrm{Win}$ & 39.5 & 62.0 & 44.0 & 45.6 & 44.6 & 74.0 & 73.2 & 57.9 & 25.2 & 54.9 & 36.5 & 46.3 & 51.9 & 36.4 & 36.4 & 39.0 & 35.2 & 37.6 \\
\hline $1995 \mathrm{Spr}$ & 46.1 & 62.5 & 58.2 & 47.0 & 48.4 & 71.7 & 75.4 & 60.1 & 25.4 & 56.6 & 38.8 & 51.7 & 58.7 & 37.3 & 38.5 & 60.2 & 38.5 & 37.6 \\
\hline $1995 \mathrm{Sum}$ & NA & NA & $\overline{N A}$ & NA & NA & NA & NA & NA & NA & INA & $\mathrm{NA}$ & NA & NA & NA & NA & 58.5 & NA & 38.8 \\
\hline 1995 Fall & $\overline{\mathrm{NA}}$ & NA & NA & NA & NA & NA & NA & NA & NA & NA & NA & INA & NA & NA & NA & 43.3 & $\mathrm{NA}$ & 36.9 \\
\hline MEAN & 35.9 & 56.5 & 51.3 & 45.5 & 41.6 & 78.0 & 69.1 & 48.9 & 33.1 & 50.3 & 31.9 & 40.0 & 46.2 & 32.8 & 36.5 & 47.1 & 35.9 & 33.8 \\
\hline & & & & & & & & & & & & & & & & & & \\
\hline Notes: & & & & & & & & & & & & & & & & & & \\
\hline 1. Units are & $\mathrm{n} \mathrm{mg/L}$. & & & & & & & & & & & & & & & & & \\
\hline 2. NA indic & Ies that the $x$ & Il was not & yzed for $c$ & cium. & & & & & & & & & & & & & & \\
\hline & flag (<) & esentis, & & delected a & the value & red w & is the dele & a limit. & & & & & & & & & & \\
\hline 4. Mean cal & ulated using & 11 numerical & ntries includ & ig detection & imit values. & & & & & & & & & & & & & \\
\hline & & & & & & & & & & & & & & & & & & \\
\hline
\end{tabular}


Table C.5. Analytical Results for Groundwater, Fall 1990 Through Fall 1995, Cations and Anions

\section{Magnesium}

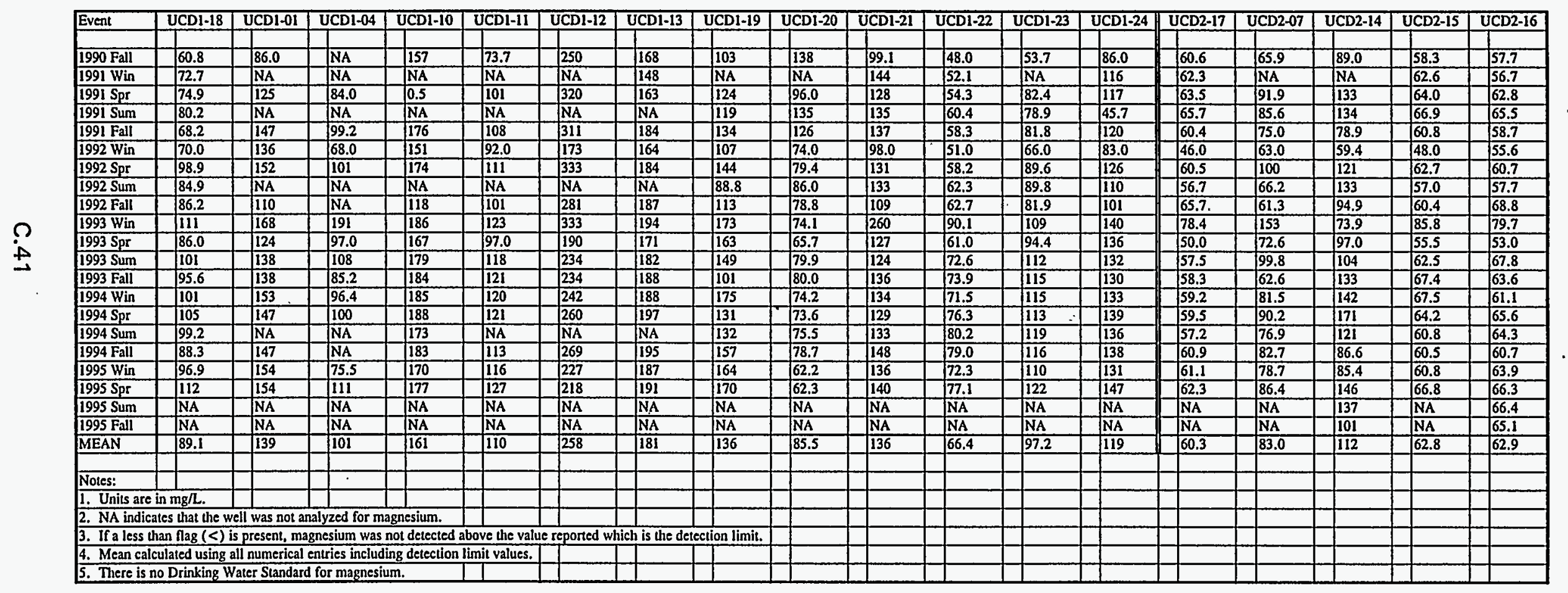


Table C.5. Analytical Results for Groundwater, Fall 1990 Through Fall 1995, Cations and Anions

Sodium

\begin{tabular}{|c|c|c|c|c|c|c|c|c|c|c|c|c|c|c|c|c|c|c|}
\hline Event & UCD1-18 & UCDI-01 & UCD1-04 & UCD1-10 & UCDI-II & UCD1-12 & UCD1-13 & UCDI-19 & UCDI-20 & UCD1-21 & UCD1-22 & UCD1-23 & UCDI-24 & UCD2-17 & UCD2-07 & UCD2-14 & UCD2-15 & UCD2-16 \\
\hline & & & & & & & & & & & & & & & & & & \\
\hline 1990 Fall & 42.9 & 39.1 & NA & 157 & 67.9 & 115 & 57.7 & 77.0 & 63.9 & 94.3 & 92.1 & 39.1 & 73.6 & 10.4 & 50.7 & 46.3 & 27.4 & 21.7 \\
\hline 1991 Win & 32.4 & NA & NA & NA & NA & NA & 53.3 & NA & NA & 88.4 & 71.6 & NA & 70.1 & 30.4 & NA & NA & 27.5 & 46.6 \\
\hline $1991 \mathrm{Spr}$ & 43.9 & 40.7 & 39.4 & 166 & 77.8 & 130 & 60.8 & 76.1 & 59.5 & 108 & 88.0 & 43.8 & 79.3 & 37.7 & 64.7 & 51.2 & 34.4 & 47.5 \\
\hline 1991 Sum & 23.7 & NA & NA & NA & NA & NA & NA & 51.7 & 39.8 & 85.9 & 54.7 & 28.6 & 58.6 & 19.2 & 34.0 & 30.8 & 20.7 & 34.5 \\
\hline 1991 Fall & 29.9 & 29.8 & 32.5 & 102 & 48.6 & 86.0 & 36.2 & 44.3 & 39.5 & 65.9 & 46.2 & 27.5 & 50.2 & 19.2 & 27.0 & 51.6 & 19.4 & 28.8 \\
\hline 1992 Win & 35.3 & 30.2 & 29.8 & 153 & 57.0 & 73.0 & 54.6 & 60.5 & 45.5 & 77.1 & 57.1 & 34.9 & 63.4 & 26.0 & 42.6 & 32.4. & 25.6 & 48.1 \\
\hline $1992 \mathrm{Spr}$ & $\frac{10}{40.1}$ & \begin{tabular}{|l|}
36.7 \\
\end{tabular} & 37.9 & 128 & 81.9 & 102 & 48.9 & 61.8 & 47.2 & 86.3 & 69.0 & 37.1 & 69.7 & 25.8 & 52.6 & 39.4 & 41.0 & 37.4 \\
\hline 1992 Sum & 35.5 & NA & NA & NA & NA & NA & NA & 67.3 & 45.3 & 98.2 & 80.2 & 42.1 & 72.6 & 28.0 & 44.6 & 47.9 & 27.3 & 41.0 \\
\hline 1992 Fall & 38.8 & 36.1 & $\overline{\mathrm{NA}}$ & 151 & 72.9 & 108 & 54.1 & 66.9 & 44.4 & 84.9 & 70.1 & 40.8 & 69.7 & 21.5 & 35.9 & 40.8 & 27.9 & 42.6 \\
\hline 1993 Win & 39.9 & 38.1 & 36 & 166 & 77.6 & 93.0 & 61.9 & 75.5 & 42.3 & 103 & 85.2 & 42.3 & 75.2 & 27.0 & 62.0 & 36.4 & 25.6 & 43.4 \\
\hline $1993 \mathrm{Spr}$ & 42.8 & 36.2 & 42.8 & 190 & 75.8 & 11.1 & 63.3 & 73.2 & 40.2 & 88.4 & 104 & 44.1 & 63.2 & 29.6 & 54.7 & 44.1 & 30.9 & 42.1 \\
\hline $1993 \mathrm{Sum}$ & 38.3 & 32.5 & 37.6 & 168 & 77.4 & 95.7 & 58.6 & 71.2 & 40.3 & $99.6^{\circ}$ & 90.1 & 43.6 & 79.0 & 28.5 & 60.6 & 39.6 & 28.9 & 46.4 \\
\hline 1993 Fall & 40.8 & 32.5 & 32.3 & 170 & 76.0 & 102 & 57.7 & 43.0 & 39.4 & 94.4 & 102 & 43.1 & 73.8 & 28.4 & 41.1 & 46.4 & 29.9 & 45.1 \\
\hline 1994 Win & 39.2 & 33.8 & 35.3 & 169 & 74.3 & 102 & 56.3 & 73.0 & 40.2 & 98.2 & 89.7 & 44.9 & 77.4 & 29.5 & 53.4 & 47.2 & 31.1 & 42.7 \\
\hline $1994 \mathrm{Spr}$ & 41.4 & 35.7 & 37.8 & 175 & 79.5 & 103 & 64.3 & $\overline{71.2}$ & $\longdiv { 3 8 . 2 }$ & 93.7 & 98.3 & 45.6 & 82.3 & 29.0 & 59.9 & 53.3 & 28.9 & 46.9 \\
\hline 1994 Sum & 35.9 & NA & NA & 159 & NA & NA & $\overline{\mathrm{NA}}$ & 68.8 & 38.0 & 102 & 95.0 & 47.3 & 77.3 & 28.9 & 40.2 & 43.2 & 29.1 & 46.0 \\
\hline 1994 Fall & 39.5 & 37.4 & NA & 173 & 73.0 & 103 & 59.1 & 75.8 & 40.0 & 99.2 & 97.1 & 47.1 & 80.8 & 29.9 & 41.0 & 43.2 & 29.0 & 41.5 \\
\hline 1995 Win & 39.7 & 35.4 & 33.7 & 160 & 71.8 & 103 & 58.4 & 67.5 & 36.8 & 92.9 & 88.7 & 42.7 & 74.2 & 30.7 & 47.1 & 41.4 & 28.4 & 41.5 \\
\hline $1995 \mathrm{Spr}$ & 42.3 & 33.5 & 40.5 & 168 & 78.5 & 96.3 & 58.9 & 74.1 & 36.7 & 96.5 & 97.6 & 45.2 & 82.3 & 30.3 & 51.5 & 48.4 & 30.9 & 43.6 \\
\hline $1995 \mathrm{Sum}$ & NA & NA & NA & NA & NA & NA & NA & NA & NA & NA & NA & NA & NA & NA & NA & 45.0 & NA & 43.3 \\
\hline 1995 Fall & NA & NA & NA & NA & NA & NA & NA & NA & NA & NA & NA & NA & NA & NA & NA & 42.6 & NA & 41.2 \\
\hline MEAN & 38.0 & 35.2 & 36.3 & 159.7 & 72.7 & 94.9 & 56.5 & 66.6 & 43.2 & 92.5 & 83.0 & 41.1 & 72.2 & 26.8 & 48.0 & 43.6 & 28.6 & 41.5 \\
\hline & & & & & & & & & & & & & & & & & & \\
\hline Notes: & & & & & & & & & & & & & & & & . & & \\
\hline 1. Units a & in $\mathrm{mg} / \mathrm{L}$. & & & & 1 & & & & & & & & & & & & & \\
\hline 2. NA ind & tes that the w & dil was not a & valyzed for so & fium. & & & & & & & & & & & & & & \\
\hline 3. If a less & $\operatorname{lan}$ flag $(<)$ & present, so & lium was not & letected abov & e the value re & orted which & is the detecti & In limit. & & & & & & & & & & \\
\hline 4. Mean c & ulated using & II numerical & entries includ & ing detection & limit values. & & & & & & & & & & & & & \\
\hline 5. There I & 0 Drinking Y & ater Standar & for sodium. & & 口 & & & & & & & & & & & & & \\
\hline
\end{tabular}


Table C.5. Analytical Results for Groundwater, Fall 1990 Through Fall 1995, Cations and Anions

\section{Potassium}

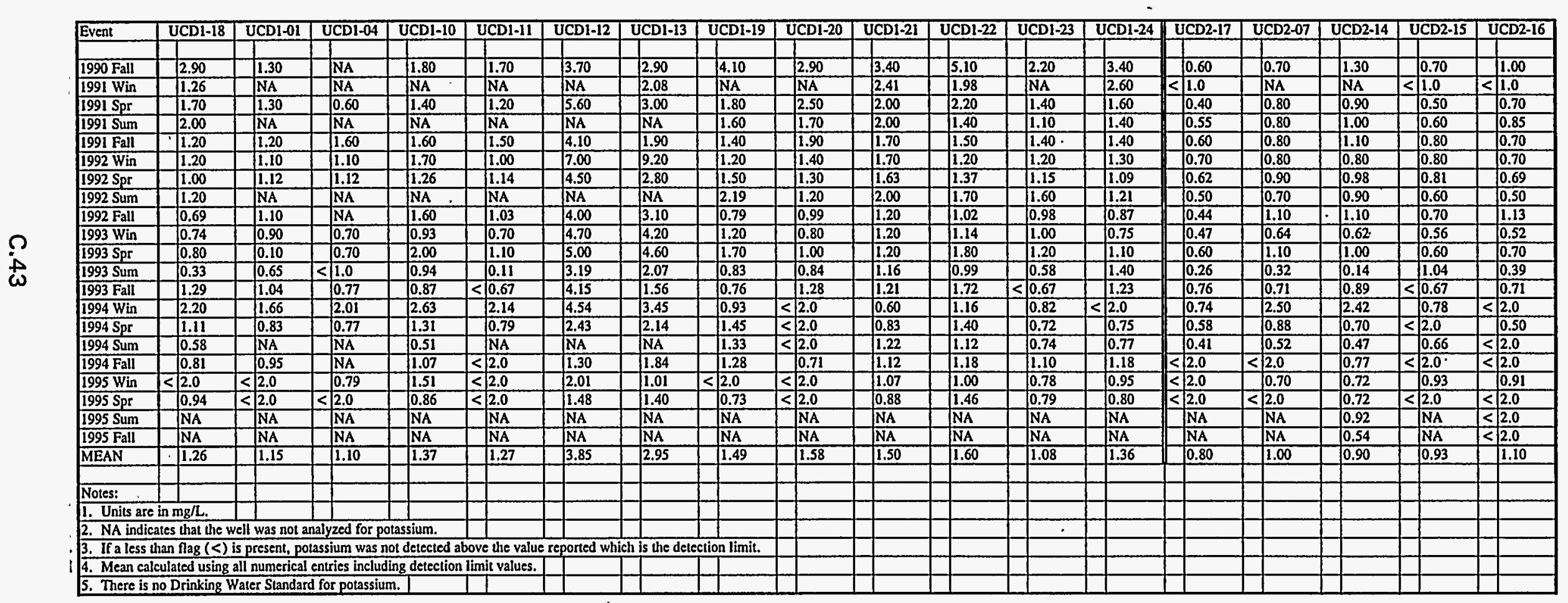


Table C.5. Analytical Results for Groundwater, Fall 1990 Through Fall 1995, Cations and Anions

\section{Ammonia}

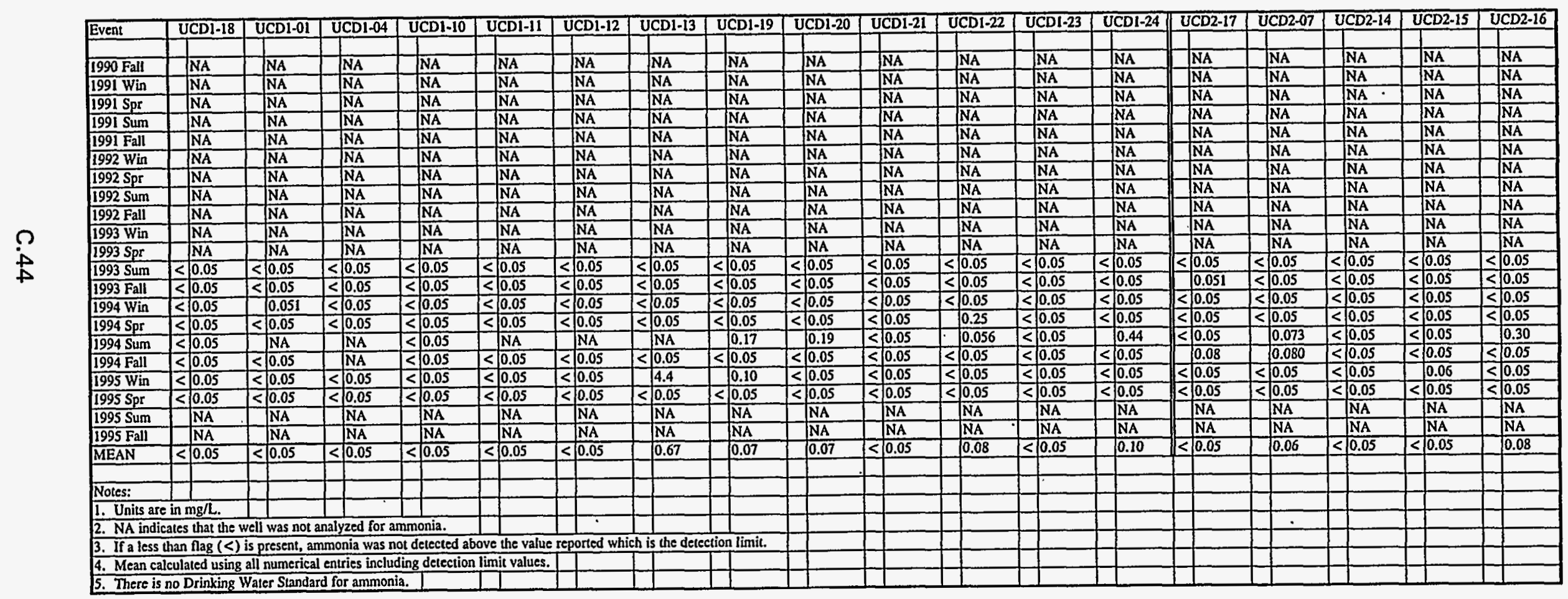


Table C.5. Analytical Results for Groundwater, Fall 1990 Through Fall 1995, Cations and Anions

\section{Nitrate}

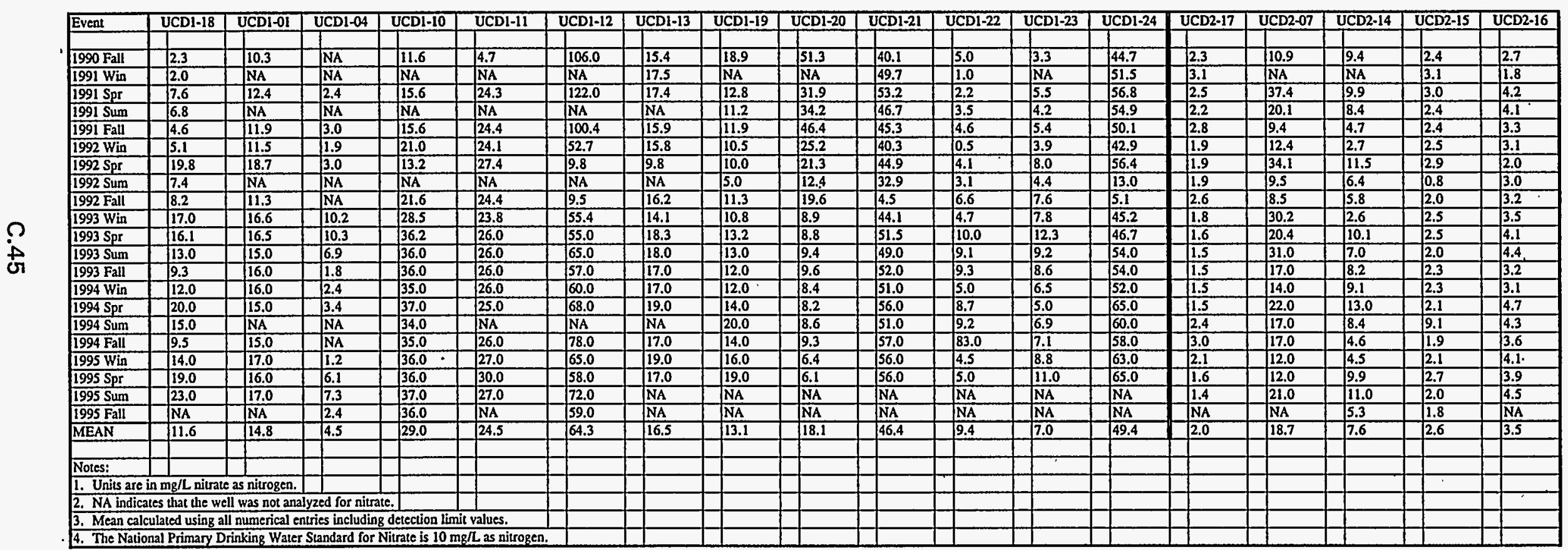


Table C.5. Analytical Results for Groundwater, Fall 1990 Through Fall 1995, Cations and Anions

Alkalinity

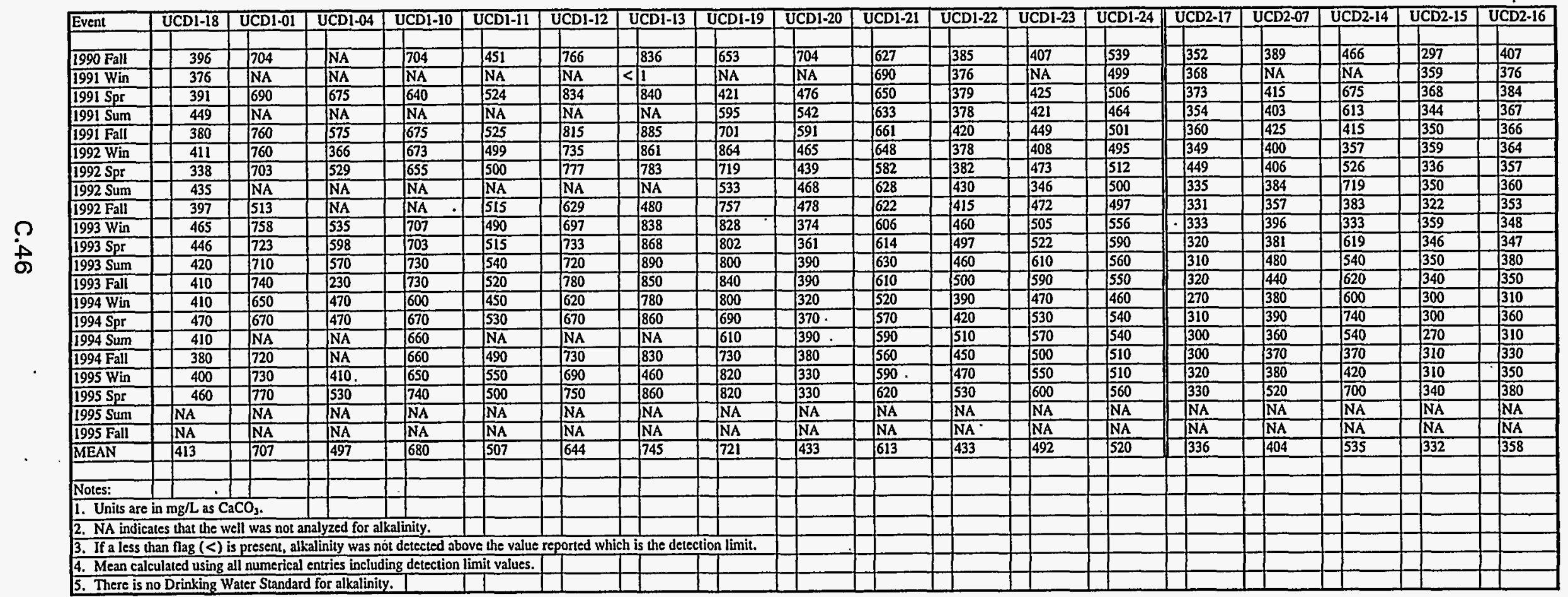


Table C.5. Analytical Results for Groundwater, Fall 1990 Through Fall 1995, Cations and Anions

\section{Sulfate}

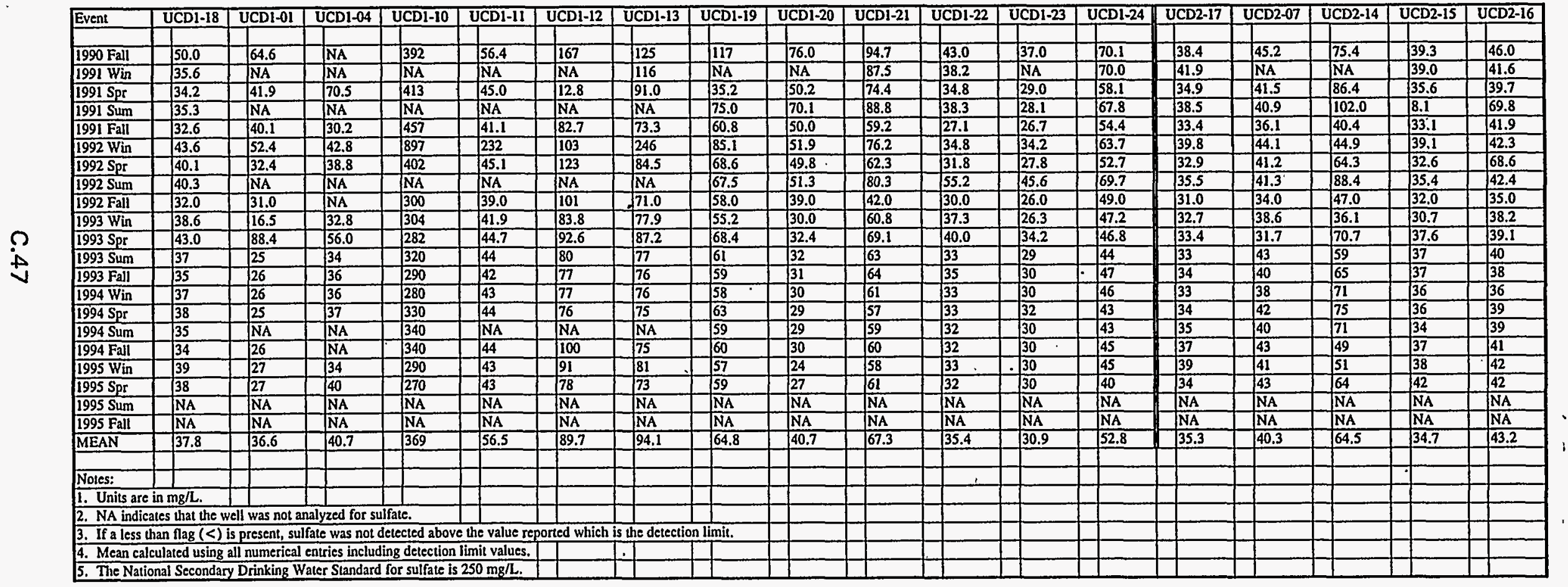


Table C.5. Analytical Results for Groundwater, Fall 1990 Through Fall 1995, Cations and Anions

\section{Chloride}

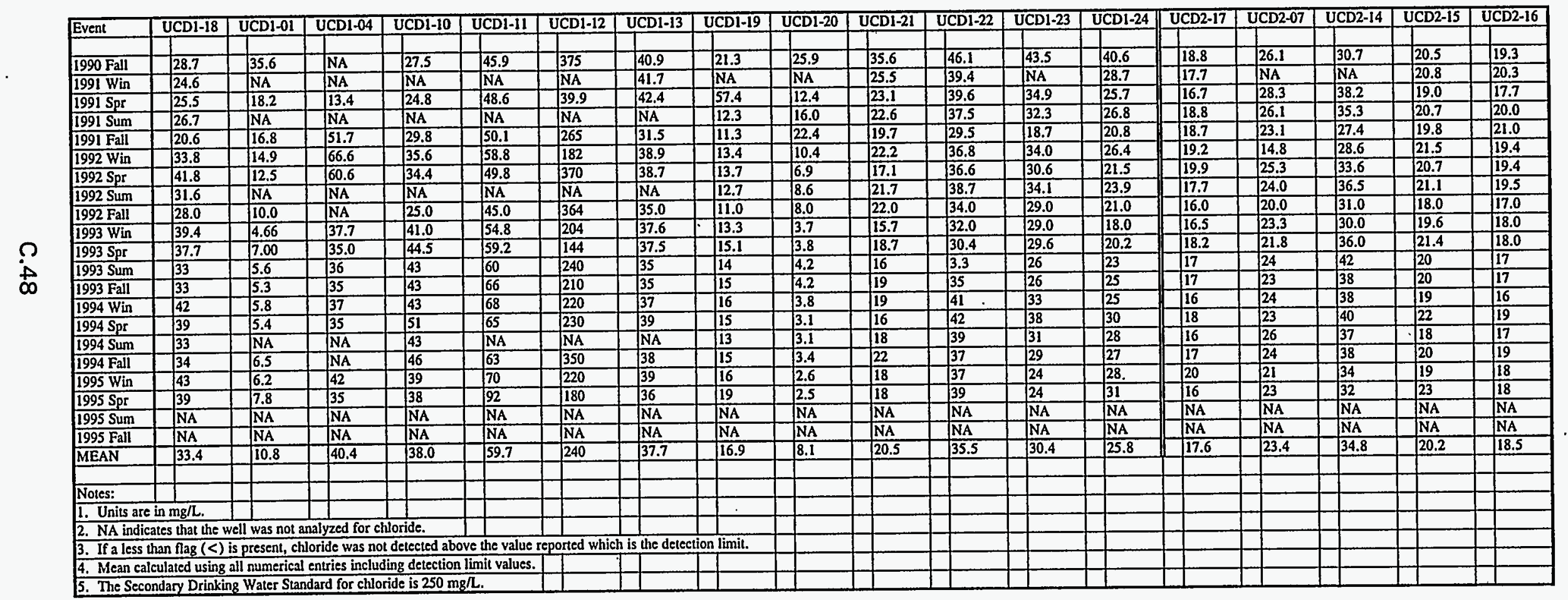


Table C.5. Analytical Results for Groundwater, Fall 1990 Through Fall 1995, Cations and Anions

\section{Phosphate}

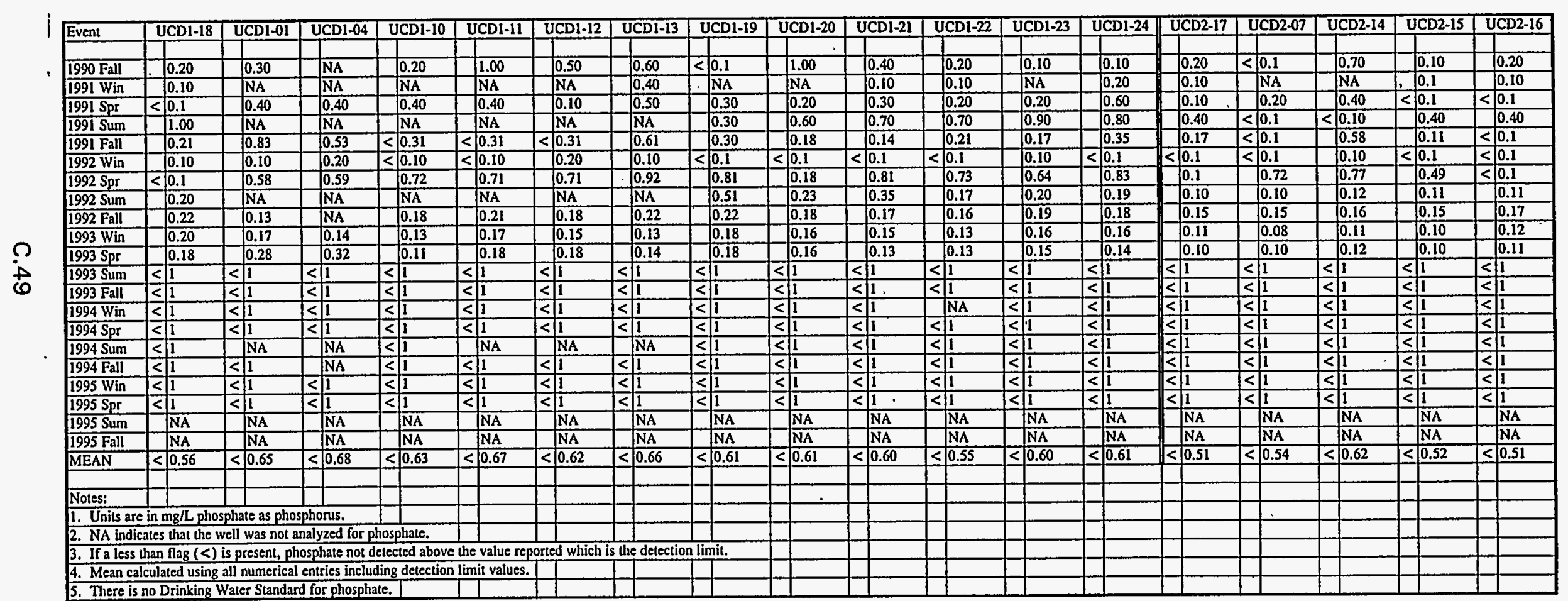


Table C.6. Analytical Results for Groundwater, Fall 1990 Through Fall 1995, Other Parameters

$\mathrm{pH}$

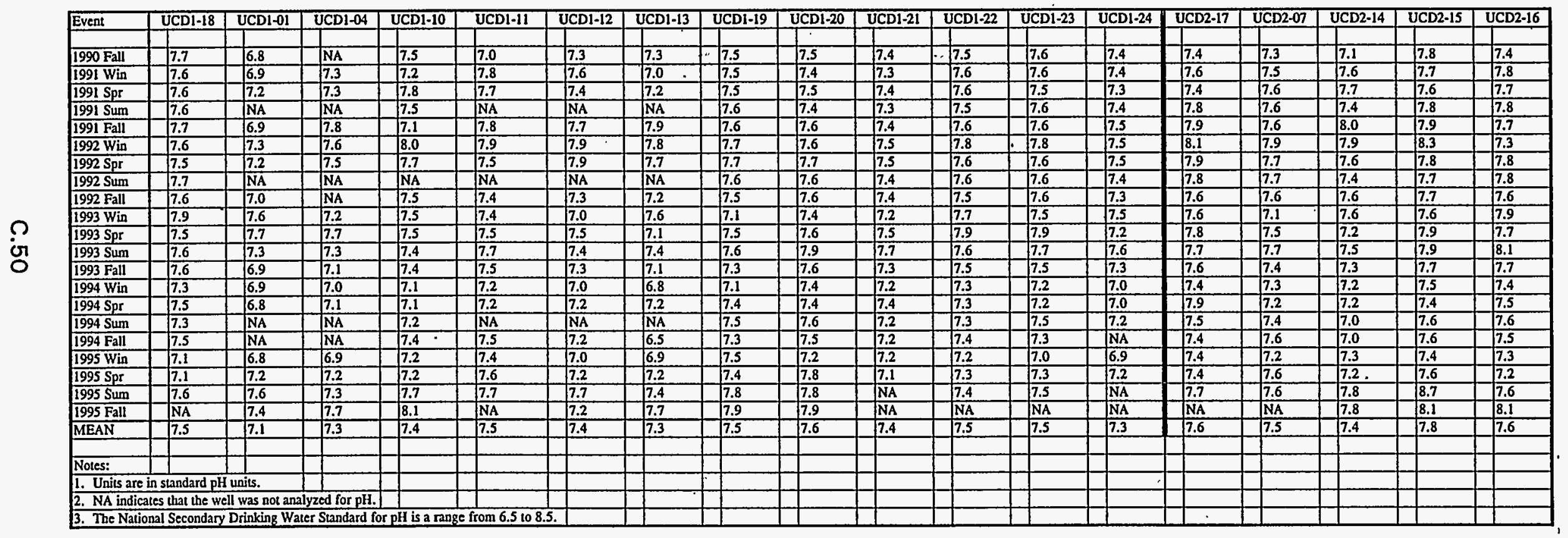


Table C.6. Analytical Results for Groundwater, Fall 1990 Through Fall 1995, Other Parameters

Temperature

\begin{tabular}{|c|c|c|c|c|c|c|c|c|c|c|c|c|c|c|c|c|c|c|c|}
\hline & Event & UCDI-18 & UCDI-01 & UCDI-04 & UCDI-10 & UCDI-11 & $\mathrm{UCDI-12}$ & UCDI-13 & UCDI-19 & UCD1-20 & UCDI-21 & UCD1-22 & UCD1-23 & UCD1-24 & UCD2-17 & UCD2-07 & UCD2-14 & UCD2-15 & UCD2-16 \\
\hline$!$ & & & & & & & & & & & & & & & & & & & \\
\hline & 1990 Fall & 19.2 & 18.1 & NA & 18.0 & 18.5 & 18.7 & 18.5 & 20.3 & 19.5 & 20.5 & 20.0 & 20.6 & 20.1 & 18.8 & 19.1 & 19.0 & 18.3 & 18.4 \\
\hline & $1991 \mathrm{Win}$ & 16.6 & 17.7 & 16.9 & 16.7 & 16.5 & 17.6 & 18.7 & 18.1 & 16.4 & 18.2 & 19.0 & 18.2 & 18.4 & 16.7 & 18.2 & 16.8 & 16.1 & 15.0 \\
\hline & $1991 \mathrm{Spr}$ & 18.0 & 18.6 & 19.6 & 18.7 & 18.5 & 19.2 & 19.1 & 19.6 & 19.2 & 20.1 & 19.8 & 20.3 & 21.1 & 18.0 & 18.7 & 18.5 & 17.9 & 18.2 \\
\hline & 1991 Sum & 19.7 & NA & NA & 21.4 & NA & $\overline{\mathrm{NA}}$ & NA & 20.3 & 21.0 & 21.3 & 21.3 & 22.6 & 20.6 & 18.7 & 19.3 & 19.5 & 19.3 & 18.7 \\
\hline & 1991 Fall & 18.7 & 16.9 & 16.9 & 17.1 & 17.5 & 17.6 & 17.1 & 18.4 & 19.4 & 19.5 & 19.3 & 19.9 & 17.9 & 17.8 & 17.7 & 16.6 & 16.6 & 18.2 \\
\hline & 1992 Win & 17.8 & 17.9 & 17.8 & 17.5 & 17.3 & 17.9 & 18.1 & 18.9 & 18.7 & 19.8 & 19.5 & 19.8 & 19.3 & 17.6 & 17.8 & 17.4 & 17.2 & 17.5 \\
\hline & $1992 \mathrm{Spr}$ & 18.9 & 18.4 & 18.9 & 18.6 & 18.0 & 18.7 & 17.8 & 19.7 & 19.0 & 20.2 & 19.8 & 21.2 & 19.9 & 18.3 & 17.7 & 17.4 & 18.7 & 18.9 \\
\hline ! & 1992 Sum & 19.2 & NA & NA & NA & NA & NA & NA & 23.6 & 21.2 & 22.1 & 20.7 & 24.9 & 20.8 & 18.8 & 19.7 & 18.9 & 19.1 & 18.7 \\
\hline & 1992 Fall & 18.1 & 16.1 & NA & 16.5 & 17.6 & 17.3 & 15.6 & 18.2 & 19.0 & 19.7 & 18.9 & 18.3 & 19.2 & 17.9 & 15.6 & 15.6 & 17.2 & 18.2 \\
\hline & $1993 \mathrm{Win}$ & 16.4 & 17.0 & 17.2 & 15.2 & 16.3 & 16.2 & 17.0 & 16.9 & 17.3 & 18.8 & 17.2 & 16.7 & 16.1 & 16.1 & 16.1 & 16.8 & 19.3 & 16.9 \\
\hline & $1993 \mathrm{Spr}$ & 19.5 & 20.8 & 20.2 & 18.3 & 18.8 & 19.4 & 19.3 & 20.1 & 20.2 & 19.5 & 20.3 & 19.8 & 19.4 & 19.6 & 19.8 & 18.3 & 18.3 & 19.5 \\
\hline & $1993 \mathrm{Sum}$ & 19.3 & 21.1 & 19.4 & 21.1 & 19.7 & 21.2 & 20.6 & 18.9 & 19.2 & 19.7 & 20.2 & 19.4 & 19.2 & 18.3 & 20.5 & 20.3 & 18.8 & 19.1 \\
\hline & 1993 Fall & 18.5 & 19.8 & 19.0 & $\overline{18.5}$ & 18.6 & 19.2 & 19.1 & 19.7 & 19.5 & 20.1 & 19.5 & 19.1 & 20.0 & 18.3 & 19.1 & 18.8 & 17.9 & 18.5 \\
\hline & $1994 \mathrm{Win}$ & 18.1 & 19.9 & 19.2 & 18.8 & 18.9 & 19.4 & 19.3 & 19.9 & 20.0 & 20.9 & 19.8 & 20.1 & 20.0 & 18.6 & 19.2 & 18.8 & 18.5 & 19.2 \\
\hline & $1994 \mathrm{Spr}$ & 18.6 & 20.5 & 19.4 & 18.8 & 18.8 & 19.3 & 19.1 & 19.7 & 19.8 & 20.3 & 20.4 & 19.8 & 20.5 & 18.5 & 19.1 & 19.5 & 18.5 & 18.7 \\
\hline & 1994 Sum & 18.7 & $\overline{N A}$ & $\overline{N A}$ & 20.1 & NA & NA & NA & 19.9 & 21.3 & 20.5 & 20.8 & 20.7 & 21.3 & 19.2 & 19.2 & 19.4 & 18.5 & 18.8 \\
\hline 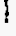 & 1994 Fall & 18.6 & NA & $\sqrt{N A}$ & 19.1 & 18.8 & 19.7 & 19.2 & 19.6 & 19.6 & 20.0 & 19.8 & 19.2 & NA & 18.4 & 18.7 & 18.8 & 18.4 & 18.4 \\
\hline & $1995 \mathrm{Win}$ & 18.2 & 19.1 & 18.8 & 18.6 & 18.6 & 19.1 & 18.8 & 19.4 & 19.4 & 20.1 & 19.6 & 19.1 & 20.1 & 18.3 & 19.0 & 18.9 & $\overline{18.2}$ & 18.6 \\
\hline & $1995 \mathrm{Spr}$ & 18.5 & 19.2 & 18.5 & 18.7 & 18.6 & 19.7 & 19.1 & 19.7 & 20.0 & 20.1 & 20.0 & 19.4 & 20.4 & 18.6 & 18.7 & 19.0 & 18.2 & 19.1 \\
\hline & $1995 \mathrm{Sum}$ & 21.4 & 21.3 & 21.7 & 22.8 & 23.3 & 21.7 & 18.6 & 23.1 & 23.9 & NA & 23.6 & 22.0 & NA & 20.7 & 21.2 & 17.7 & 23.4 & 20.7 \\
\hline & 1995 Fall & $\overline{\mathrm{NA}}$ & 16.4 & 17.7 & $\overline{21.4}$ & INA & 18.5 & 20.9 & 20.2 & 21.3 & $\overline{\mathrm{NA}}$ & $\overline{\mathrm{NA}}$ & NA & NA & NA & NA & 17.4 & 18.4 & 20.5 \\
\hline & MEAN & 18.6 & 18.8 & 18.8 & 18.8 & 18.5 & 18.9 & 18.7 & 19.7 & 19.8 & 20.1 & 20.0 & 20.1 & 19.7 & 18.4 & 18.7 & 18.3 & 18.4 & 18.6 \\
\hline & & & & & & & & & & & & & & & & & & & \\
\hline & Notes: & & & & & & & & & & & & & & & & & & \\
\hline & 1. Units ar & ${ }^{\circ} \mathrm{C}$. & & & & & & & & & & & & & & & & & \\
\hline & 2. NA ind & tes that th & 1 was no & alyzed fo & perature. & & & & & & & & & & & & & & \\
\hline
\end{tabular}


Table C.6. Analytical Results for Groundwater, Fall 1990 Through Fall 1995, Other Parameters

\section{Eh}

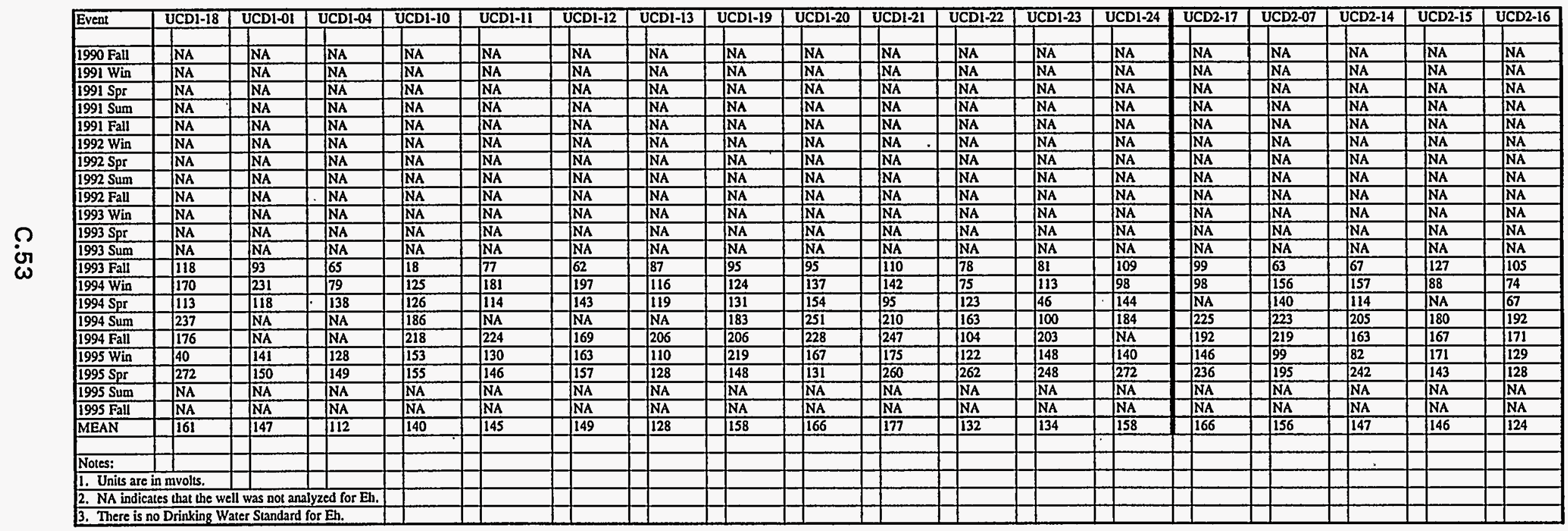


Table C.6. Analytical Results for Groundwater, Fall 1990 Through Fall 1995, Other Parameters

Turbidity

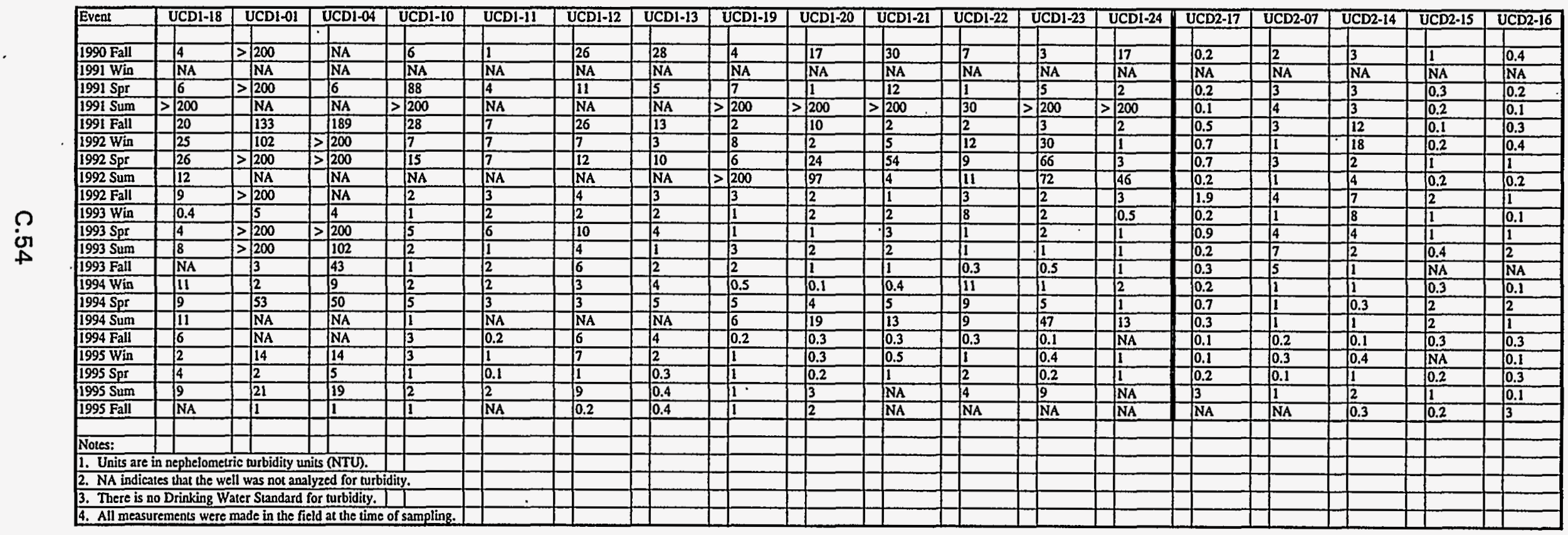


Table C.6. Analytical Results for Groundwater, Fall 1990 Through Fall 1995, Other Parameters

Total Dissolved Solids

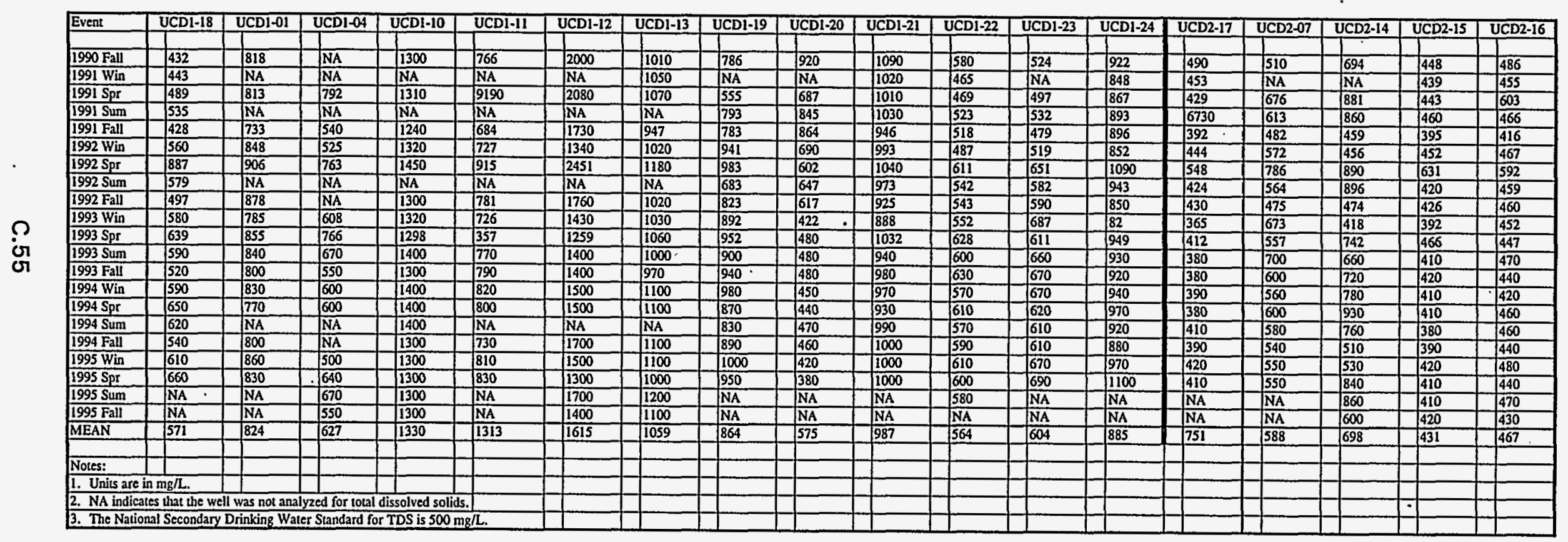




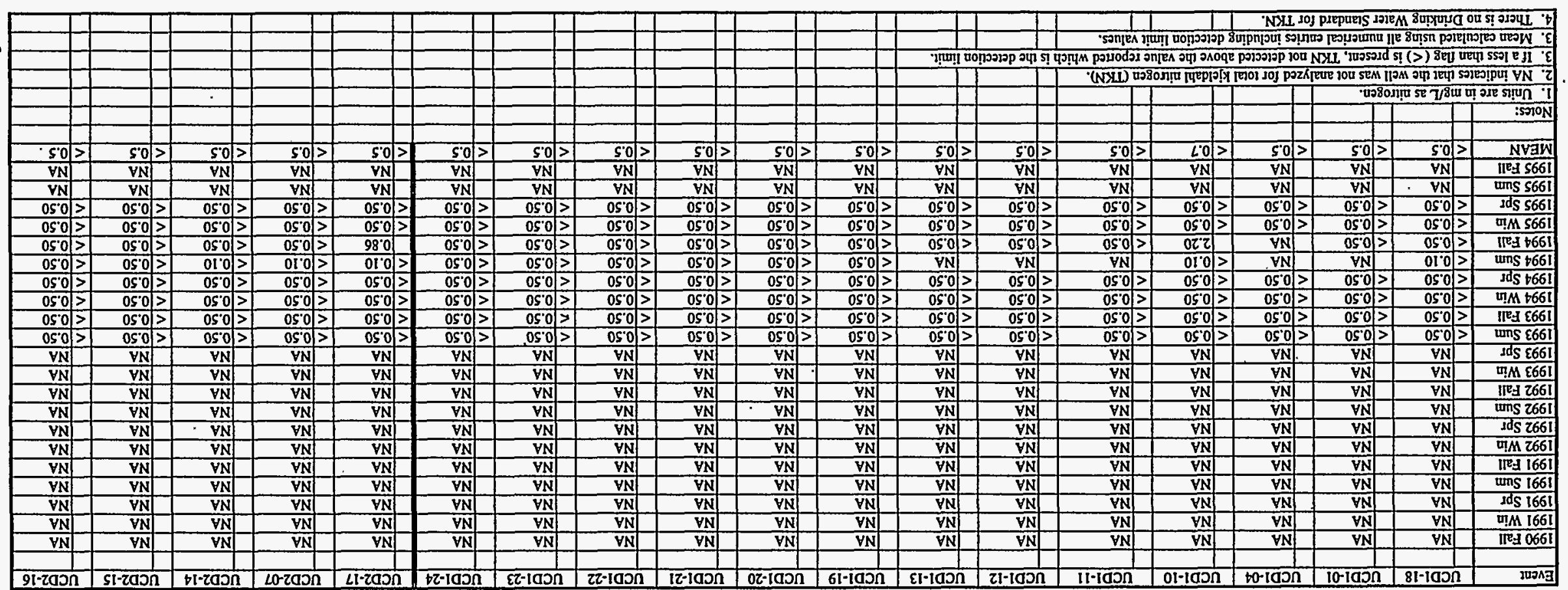

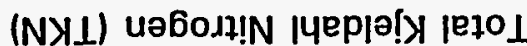

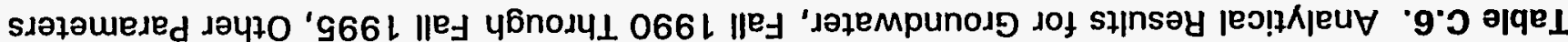


Table C.6. Analytical Results for Groundwater, Fall 1990 Through Fall 1995, Other Parameters

Total Organic Carbon (TOC)

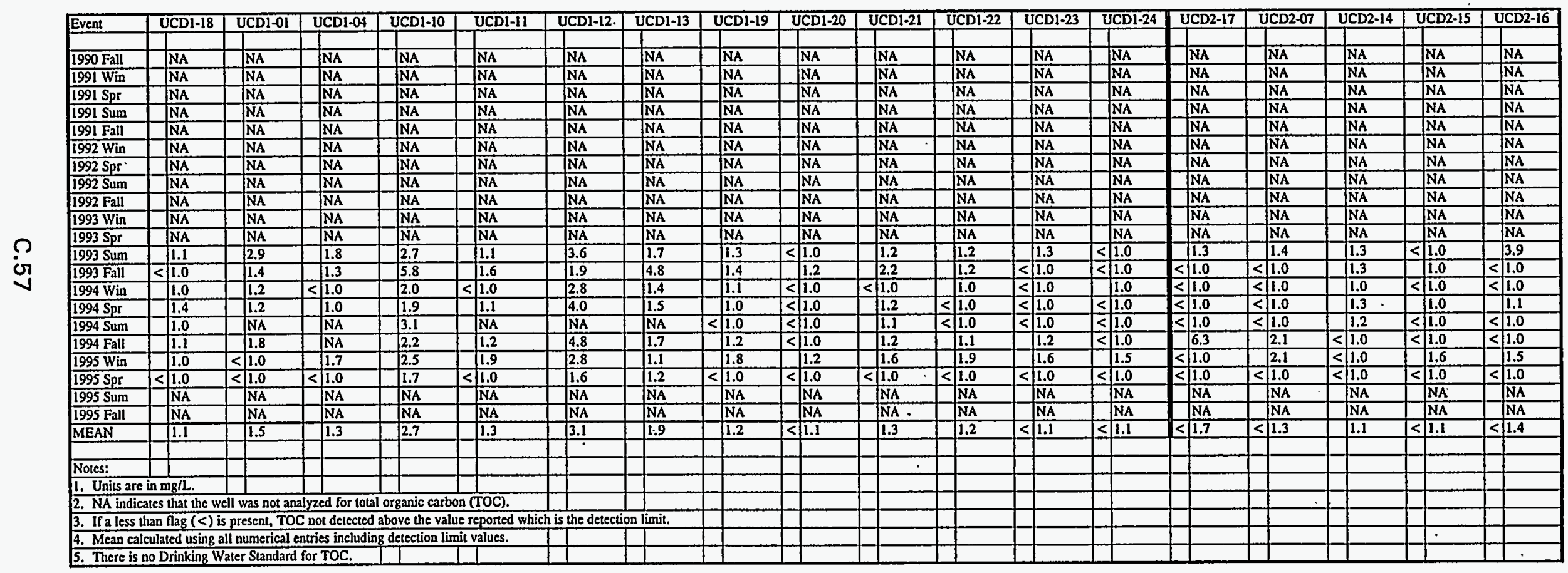


Table C.6. Analytical Results for Groundwater, Fall 1990 Through Fall 1995, Other Parameters

Chemical Oxygen Demand (COD)

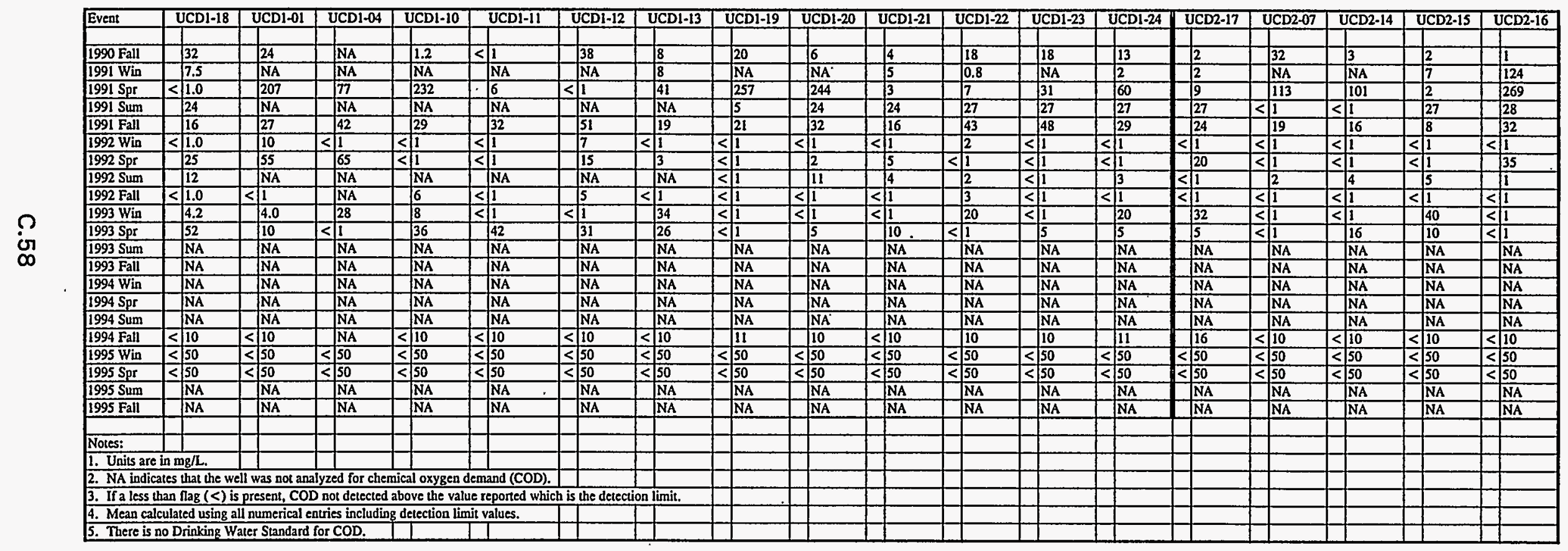


Surface Water Analytical Results 
Table C.7. Analytical Results for Surface Water, Fall 1990 Through Fall 1995, Volatile Organic Compounds

\section{Chloroform (ug/L)}

\begin{tabular}{|c|c|c|c|}
\hline Event & PCU & STPO & PCD \\
\hline 1990 Fall & 4.9 & 3.4 & 10 \\
\hline 1991 Win & $<1.6$ & 7.4 & $<1.6$ \\
\hline $1991 \mathrm{Spr}$ & $<1.6$ & 3.1 & 10 \\
\hline 1991 Sum & $<1.6$ & 5.7 & 14 \\
\hline 1991 Fall & 2.0 & 7.2 & $<1.6$ \\
\hline 1992 Win & $<1.6$ & 4.7 & $<1.6$ \\
\hline $1992 \mathrm{Spr}$ & $<1.6$ & 33 & 4.7 \\
\hline 1992 Sum & 19 & 5.5 & 8.9 \\
\hline 1992 Fall & 3.6 & 5.8 & 4.7 \\
\hline 1993 Win & $<1.6$ & 5.0 & $<1.6$ \\
\hline $1993 \mathrm{Spr}$ & $<1.6$ & 7.9 & $<1.6$ \\
\hline 1993 Sum & $<1.0$ & 16 & . 1.2 \\
\hline 1993 Fall & $<1.0$ & 14 & $<1.0$ \\
\hline 1994 Win & $<1.0$ & 3.9 & 0.5 \\
\hline 1994 Spr & $<1.0$ & 5.6 & 8.4 \\
\hline 1994 Sum & 2.2 & 3.7 & 4.4 \\
\hline 1994 Fall & $<1.0$ & 3.8 & 2.8 \\
\hline 1995 Win & $<1.0$ & 3.7 & 0.7 \\
\hline $1995 \mathrm{Spr}$ & $<1.0$ & 2.2 & $<1.0$ \\
\hline 1995 Sum & $<1.0$ & 5.3 & 1.1 \\
\hline 1995 Fall & $<1.0$ & 2.5 & 1.9 \\
\hline
\end{tabular}

Bromodichloromethane (ug/L)

\begin{tabular}{l|ccc} 
Event & PCU & STPO & PCD \\
\hline 1990 Fall & $<2.2$ & $<2.2$ & $<2.2$ \\
\hline 1991 Win & $<2.2$ & $<2.2$ & $<2.2$ \\
1991 Spr & $<2.2$ & 2.2 & 4.1 \\
1991 Sum & $<2.2$ & $<2.2$ & 5.8 \\
1991 Fall & $<2.2$ & $<2.2$ & $<2.2$ \\
\hline 1992 Win & $<2.2$ & $<2.2$ & $<2.2$ \\
1992 Spr & $<2.2$ & 13 & $<2.2$ \\
1992 Sum & 7.1 & 1.3 & 2.8 \\
1992 Fall & $<2.2$ & $<2.2$ & $<2.2$ \\
\hline 1993 Win & $<2.2$ & $<2.2$ & $<2.2$ \\
1993 Spr & $<2.2$ & $<2.2$ & $<2.2$ \\
1993 Sum & $<1.0$ & $<1.0$ & $<1.0$ \\
1993 Fall & $<1.0$ & $<1.0$ & $<1.0$ \\
\hline 1994 Win & $<1.0$ & 1.1 & $<1.0$ \\
1994 Spr & $<1.0$ & 0.9 & 1.6 \\
1994 Sum & $<1.0$ & $<1.0$ & 1.1 \\
1994 Fall & $<1.0$ & $<1.0$ & $<1.0$ \\
\hline 1995 Win & $<1.0$ & 1.0 & $<1.0$ \\
1995 Spr & $<1.0$ & 2.6 & $<1.0$ \\
1995 Sum & $<1.0$ & 1.1 & $<1.0$ \\
1995 Fall & $<1.0$ & 4.5 & $<1.0$ \\
\hline
\end{tabular}


Table C.7. Analytical Results for Surface Water, Fall 1990 Through Fall 1995, Volatile Organic Compounds

Dibromochloromethane (ug/L)

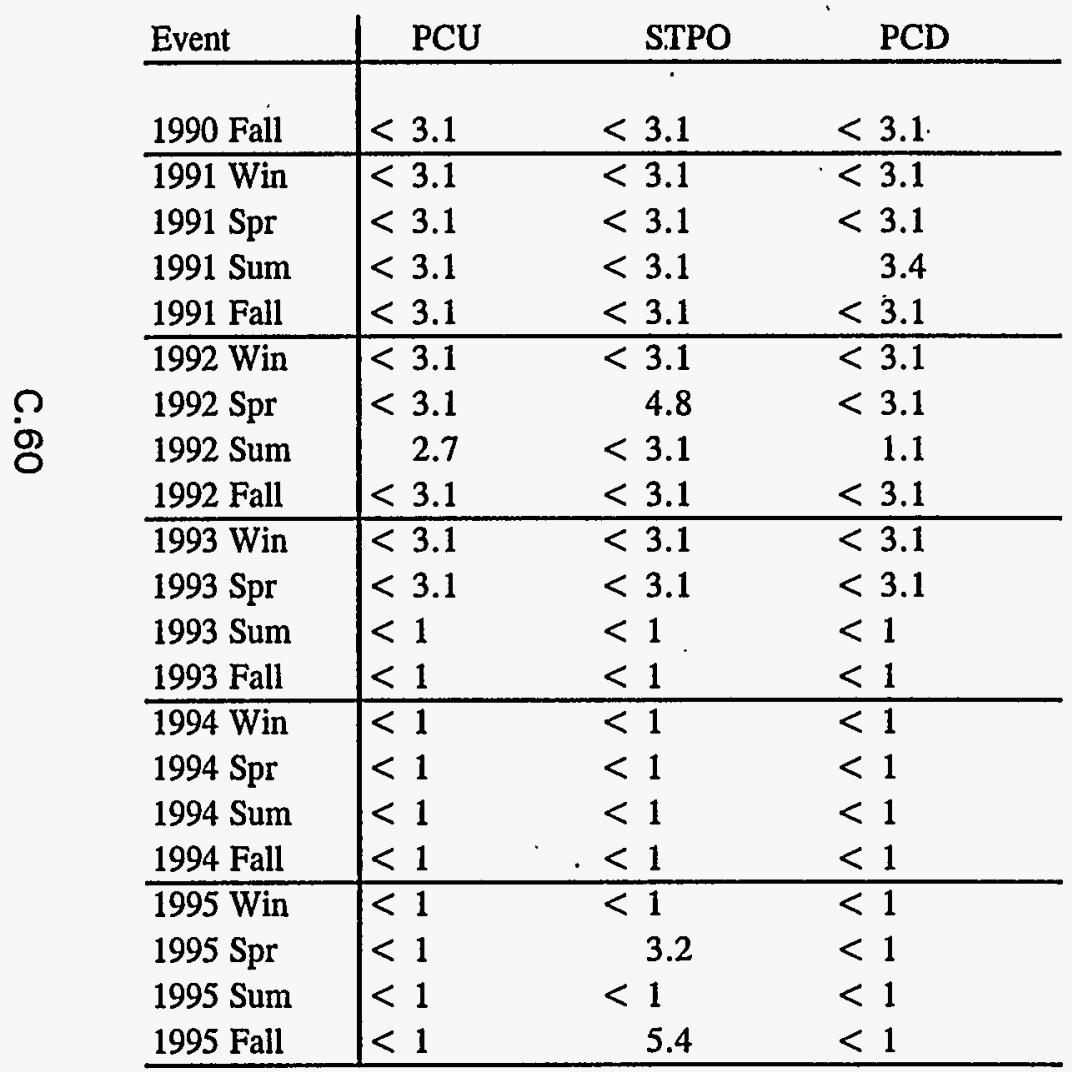

Bromoform (ug/L)

\begin{tabular}{l|ccc} 
Event & \multicolumn{1}{c}{ PCU } & STPO & PCD \\
\hline 1990 Fall & $<4.7$ & $<4.7$ & $<4.7$ \\
\hline 1991 Win & $<4.7$ & $<4.7$ & $<4.7$ \\
1991 Spr & $<4.7$ & $<4.7$ & $<4.7$ \\
1991 Sum & $<4.7$ & $<4.7$ & $<4.7$ \\
1991 Fall & $<4.7$ & $<4.7$ & $<4.7$ \\
\hline 1992 Win & $<4.7$ & $<4.7$ & $<4.7$ \\
1992 Spr & $<4.7$ & $<4.7$ & $<4.7$ \\
1992 Sum & $<4.7$ & $<4.7$ & $<4.7$ \\
1992 Fall & $<4.7$ & $<4.7$ & $<4.7$ \\
\hline 1993 Win & $<4.7$ & $<4.7$ & $<4.7$ \\
1993 Spr & $<4.7$ & $<4.7$ & $<4.7$ \\
1993 Sum & $<1$ & $<1$ & $<1$ \\
1993 Fall & $<1$ & $<1$ & $<1$ \\
\hline 1994 Win & $<1$ & $<1$ & $<1$ \\
1994 Spr & $<1$ & $<1$ & $<1$ \\
1994 Sum & $<1$ & $<1$ & $<1$ \\
1994 Fall & $<1$ & $<1$ & $<1$ \\
\hline 1995 Win & $<1$ & $<1$ & $<1$ \\
1995 Spr & $<1$ & 1.8 & $<1$ \\
1995 Sum & $<1$ & $<1$ & $<1$ \\
1995 Fall & $<1$ & 2.9 & $<1$ \\
\hline
\end{tabular}


Table C.7. Analytical Results for Surface Water, Fall 1990 Through Fall 1995, Volatile Organic Compounds

Methylène Chloride (ug/L)

\begin{tabular}{|c|c|c|c|}
\hline Event & PCU & STPO & PCD \\
\hline 1990 Fall & $<2.8$ & $<2.8$ & $<2.8$ \\
\hline 1991 Win & $<2.8$ & $<2.8$ & $<2.8$ \\
\hline $1991 \mathrm{Spr}$ & $<2.8$ & 5.4 & $<2.8$ \\
\hline 1991 Sum & $<2.8$ & $<2.8$ & $<2.8$ \\
\hline 1991 Fall & $<2.8$ & $<2.8$ & $<2.8$ \\
\hline 1992 Win & $<2.8$ & 4.1 & 3.0 \\
\hline $1992 \mathrm{Spr}$ & $<2.8$ & $<2.8$ & $<2.8$ \\
\hline 1992 Sum & $<2.8$ & 5.2 & $<2.8$ \\
\hline 1992 Fall & $<2.8$ & $<2.8$ & $<2.8$ \\
\hline 1993 Win & $<2.8$ & $<2.8$ & $<2.8$ \\
\hline $1993 \mathrm{Spr}$ & $<2.8$ & 3.8 & $<2.8$ \\
\hline 1993 Sum & $\mid<2$ & 3.9 & 1.8 \\
\hline 1993 Fall & $<2$ & 5.7 & 0.90 \\
\hline 1994 Win & $<2$ & $\overline{6.8}$ & 1.3 \\
\hline $1994 \mathrm{Spr}$ & $<2$ & 2.5 & 0.76 \\
\hline 1994 Sum & $<2$ & 13 & $<2$ \\
\hline 1994 Fall & $<2$ & 5.3 & $<2$ \\
\hline 1995 Win & $<2$ & 7.4 & $<2$ \\
\hline 1995 Spr & $<2$ & $<2$ & $<2$ \\
\hline 1995 Sum & $\mid<2$ & 40 & $<2$ \\
\hline 1995 Fall & $<2$ & 1.6 & $<2$ \\
\hline
\end{tabular}

Acetone (ug/L)

\begin{tabular}{l|ccc} 
Event & PCU & STPO & PCD \\
\hline 1990 Fall & NA & NA & NA \\
\hline 1991 Win & NA & NA & NA \\
1991 Spr & NA & NA & NA \\
1991 Sum & NA & NA & NA \\
1991 Fall & NA & NA & NA \\
\hline 1992 Win & NA & NA & NA \\
1992 Spr & NA & NA & NA \\
1992 Sum & NA & NA & NA \\
1992 Fall & NA & NA & NA \\
\hline 1993 Win & NA & NA & NA \\
1993 Spr & NA & NA & NA \\
1993 Sum & $<5$ & 36 & 9.2 \\
1993 Fall & $<5$ & $<5$ & $<5$ \\
\hline 1994 Win & $<5$ & 4.3 & 18 \\
1994 Spr & 3.9 & 4.9 & 5.7 \\
1994 Sum & 3.3 & $<5$ & 2.9 \\
1994 Fall & $<5$ & $<5$ & 5.0 \\
\hline 1995 Win & $<5$ & 2.2 & $<5$ \\
1995 Spr & 2.1 & 3.1 & 2.2 \\
1995 Sum & $<5$ & $<5$ & $<5$ \\
1995 Fall & 2.8 & 7.1 & 3.1 \\
\hline
\end{tabular}

Notes:

1. NA indicates that no sample was analyzed for this constituent. 
Table C.7. Analytical Results for Surface Water, Fall 1990 Through Fall 1995, Volatile Organic Compounds

\section{Total Trihalomethanes (ug/L)}

\begin{tabular}{l|lll} 
Event & \multicolumn{1}{c}{ PCU } & STPO & PCD \\
\hline & & & 20 \\
1990 Fall & $<15$ & 13 & $<12$ \\
\hline 1991 Win & $<12$ & 17 & 22 \\
1991 Spr & $<12$ & 13 & 28 \\
1991 Sum & $<12$ & 16 & $<12$ \\
1991 Fall & $<12$ & 17 & $<12$ \\
\hline 1992 Win & $<12$ & 15 & 15 \\
1992 Spr & $<12$ & 56 & 18 \\
1992 Sum & 34 & 15 & 15 \\
1992 Fall & $<14$ & 16 & $<12$ \\
\hline 1993 Win & $<12$ & 15 & $<12$ \\
1993 Spr & $<12$ & 18 & 4 \\
1993 Sum & $<4$ & 19 & $<4$ \\
1993 Fall & $<4$ & 17 & 4 \\
\hline 1994 Win & $<4$ & 7 & 12 \\
1994 Spr & $<4$ & 9 & 8 \\
1994 Sum & $<5$ & 7 & 6 \\
1994 Fall & $<4$ & 7 & 4 \\
\hline 1995 Win & $<20$ & 7 & 4 \\
1995 Spr & $<4$ & 10 & 5 \\
1995 Sum & $<4$ & 15 & 4 \\
1995 Fall & $<4$ & & 4 \\
\hline
\end{tabular}

Notes:

1. Total Trihalomethanes is the sum of conentrations of chloroform, bromodichloromethane, dibromochloromethane, and bromoform. 
Table C.8. Analytical Results for Surface Water, Fall 1990 Through Fall 1995,

Pesticides and PCBs

Chlordane (ug/L)

\begin{tabular}{|c|c|c|c|}
\hline Event & PCU & STPO & PCD \\
\hline 1990 Fall & $<0.014$ & $<0.014$ & $<0.014$ \\
\hline 1991 Win & $<0.014$ & $<0.014$ & $<0.014$ \\
\hline $1991 \mathrm{Spr}$ & $<0.014$ & $<0.014$ & $<0.014$ \\
\hline 1991 Sum & $<0.014$ & $<0.014$ & $<0.014$ \\
\hline 1991 Fall & $<0.014$ & $<0.014$ & $<0.014$ \\
\hline 1992 Win & $<0.014$ & $<0.014$ & $<0.014$ \\
\hline $1992 \mathrm{Spr}$ & $<0.014$ & $<0.014$ & $<0.014$ \\
\hline 1992 Sum & $<0.014$ & $<0.014$ & $<0.014$ \\
\hline 1992 Fall & $<0.014$ & $<0.014$ & $<0.014$ \\
\hline 1993 Win & $<0.014$ & $<0.014$ & $<0.014$ \\
\hline $1993 \mathrm{Spr}$ & $<0.014$ & $<0.014$ & $<0.014$ \\
\hline 1993 Sum & $\mathrm{NA}$ & NA & NA \\
\hline 1993 Fall & NA & NA & NA \\
\hline 1994 Win & $\mathrm{NA}$ & $\overline{\mathrm{NA}}$ & NA \\
\hline $1994 \mathrm{Spr}$ & NA & NA & NA \\
\hline 1994 Sum & NA & $\mathrm{NA}$ & NA \\
\hline 1994 Fall & NA & $\mathrm{NA}$ & $\mathrm{NA}$ \\
\hline 1995 Win & NA & $\mathrm{NA}$ & $\mathrm{NA}$ \\
\hline $1995 \mathrm{Spr}$ & NA & $\mathrm{NA}$ & NA \\
\hline 1995 Sum & NA & NA & NA \\
\hline 1995 Fall & $\mathrm{NA}$ & $\mathrm{NA}$ & NA \\
\hline
\end{tabular}

Dieldrin (ug/L)

\begin{tabular}{l|rrr} 
Event & PCU & STPO & PCD \\
\hline 1990 Fall & $<0.002$ & $<0.002$ & $<0.002$ \\
\hline 1991 Win & 0.015 & 0.007 & 0.012 \\
1991 Spr & $<0.002$ & $<0.002$ & $<0.002$ \\
1991 Sum & $<0.002$ & $<0.002$ & $<0.002$ \\
1991 Fall & $<0.002$ & $<0.002$ & $<0.002$ \\
\hline 1992 Win & $<0.002$ & $<0.002$ & $<0.002$ \\
1992 Spr & $<0.002$ & $<0.002$ & $<0.002$ \\
1992 Sum & $<0.002$ & $<0.002$ & $<0.002$ \\
1992 Fall & $<0.002$ & $<0.002$ & 0.043 \\
\hline 1993 Win & $<0.002$ & 0.006 & $<0.002$ \\
1993 Spr & $<0.002$ & $<0.002$ & $<0.002$ \\
1993 Sum & $<0.100$ & $<0.100$ & $<0.100$ \\
1993 Fall & $<0.020$ & $<0.021$ & $<0.020$ \\
\hline 1994 Win & $<0.021$ & $<0.021$ & $<0.021$ \\
1994 Spr & $<0.021$ & $<0.020$ & $<0.020$ \\
1994 Sum & $<0.021$ & $<0.021$ & $<0.021$ \\
1994 Fall & $<0.022$ & $<0.022$ & $<0.022$ \\
\hline 1995 Win & $<0.024$ & $<0.024$ & $<0.022$ \\
1995 Spr & $<0.022$ & $<0.021$ & $<0.022$ \\
1995 Sum & $<0.020$ & $<0.020$ & $<0.020$ \\
1995 Fall & $<0.020$ & $<0.020$ & $<0.020$ \\
\hline
\end{tabular}

Notes:

1. NA indicates that no sample was analyzed for this constituent. 
Table C.8. Analytical Results for Surface Water, Fall 1990 Through Fall 1995, Pesticides and PCBs

Delta-BHC (ug/L)

\begin{tabular}{|c|c|c|c|}
\hline Event & PCU & STPO & PCD \\
\hline 1990 Fall & 0.023 & 0.027 & 0.012 \\
\hline 1991 Win & $<0.009$ & $<0.009$ & $<0.009$ \\
\hline $1991 \mathrm{Spr}$ & $<0.009$ & $<0.009$ & $<0.009$ \\
\hline 1991 Sum & $<0.009$ & 0.033 & $<0.009$ \\
\hline 1991 Fall & $<0.009$ & $<0.009$ & $<0.009$ \\
\hline 1992 Win & $<0.009$ & $<0.009$ & $<0.009$ \\
\hline $1992 \mathrm{Spr}$ & $<0.009$ & $<0.009$ & $<0.009$ \\
\hline 1992 Sum & $<0.009$ & 0.048 & $<0.009$ \\
\hline 1992 Fall & 0.055 & 0.095 & $<0.009$ \\
\hline 1993 Win & $<0.009$ & $<0.009$ & $<0.009$ \\
\hline $1993 \mathrm{Spr}$ & $<0.009$ & $<0.009$ & $<0.009$ \\
\hline 1993 Sum & $<0.050$ & $<0.050$ & $<0.051$ \\
\hline 1993 Fall & $<0.010$ & $<0.010$ & $<0.010$ \\
\hline 1994 Win & $<0.010$ & $<0.011$ & $<0.010$ \\
\hline 1994 Spr & $<0.010$ & $<0.010$ & $<0.010$ \\
\hline 1994 Sum & $<0.010$ & $<0.011$ & $<0.011$ \\
\hline 1994 Fall & $<0.011$ & $<0.011$ & $<0.011$ \\
\hline 1995 Win & $<0.012$ & $<0.012$ & $<0.011$ \\
\hline $1995 \mathrm{Spr}$ & $<0.011$ & $<0.010$ & $<0.011$ \\
\hline 1995 Sum & $<0.010$ & $<0.010$ & $<0.010$ \\
\hline 1995 Fall & $<0.010$ & $<0.010$ & $<0.010$ \\
\hline
\end{tabular}

Gamma-BHC (ug/L)

\begin{tabular}{l|rrr} 
Event & PCU & STPO & PCD \\
\hline 1990 Fall & $<0.004$ & $<0.004$ & $<0.004$ \\
\hline 1991 Win & 0.006 & $<0.004$ & $<0.004$ \\
1991 Spr & $<0.004$ & $<0.004$ & $<0.004$ \\
1991 Sum & $<0.004$ & $<0.004$ & $<0.004$ \\
1991 Fall & $<0.004$ & $<0.004$ & $<0.004$ \\
\hline 1992 Win & 0.004 & $<0.004$ & $<0.004$ \\
1992 Spr & $<0.004$ & $<0.004$ & $<0.004$ \\
1992 Sum & $<0.004$ & 0.052 & $<0.004$ \\
1992 Fall & $<0.004$ & $<0.004$ & 0.019 \\
\hline 1993 Win & $<0.004$ & $<0.004$ & $<0.004$ \\
1993 Spr & $<0.004$ & 0.009 & $<0.004$ \\
1993 Sum & $<0.050$ & $<0.050$ & $<0.051$ \\
1993 Fall & $<0.010$ & $<0.010$ & $<0.010$ \\
\hline 1994 Win & 0.010 & 0.020 & 0.011 \\
1994 Spr & $<0.010$ & $<0.010$ & $<0.010$ \\
1994 Sum & 0.026 & 0.066 & $<0.011$ \\
1994 Fall & $<0.011$ & 0.013 & $<0.011$ \\
\hline 1995 Win & $<0.012$ & $<0.012$ & $<0.011$ \\
1995 Spr & $<0.011$ & $<0.010$ & $<0.011$ \\
1995 Sum & $<0.010$ & $<0.010$ & $<0.010$ \\
1995 Fall & $<0.010$ & $<0.010$ & $<0.010$ \\
\hline
\end{tabular}


Table C.9. Analytical Results for Surface Water, Fall 1990 Through Fall 1995, Metals

Antimony (ug/L)

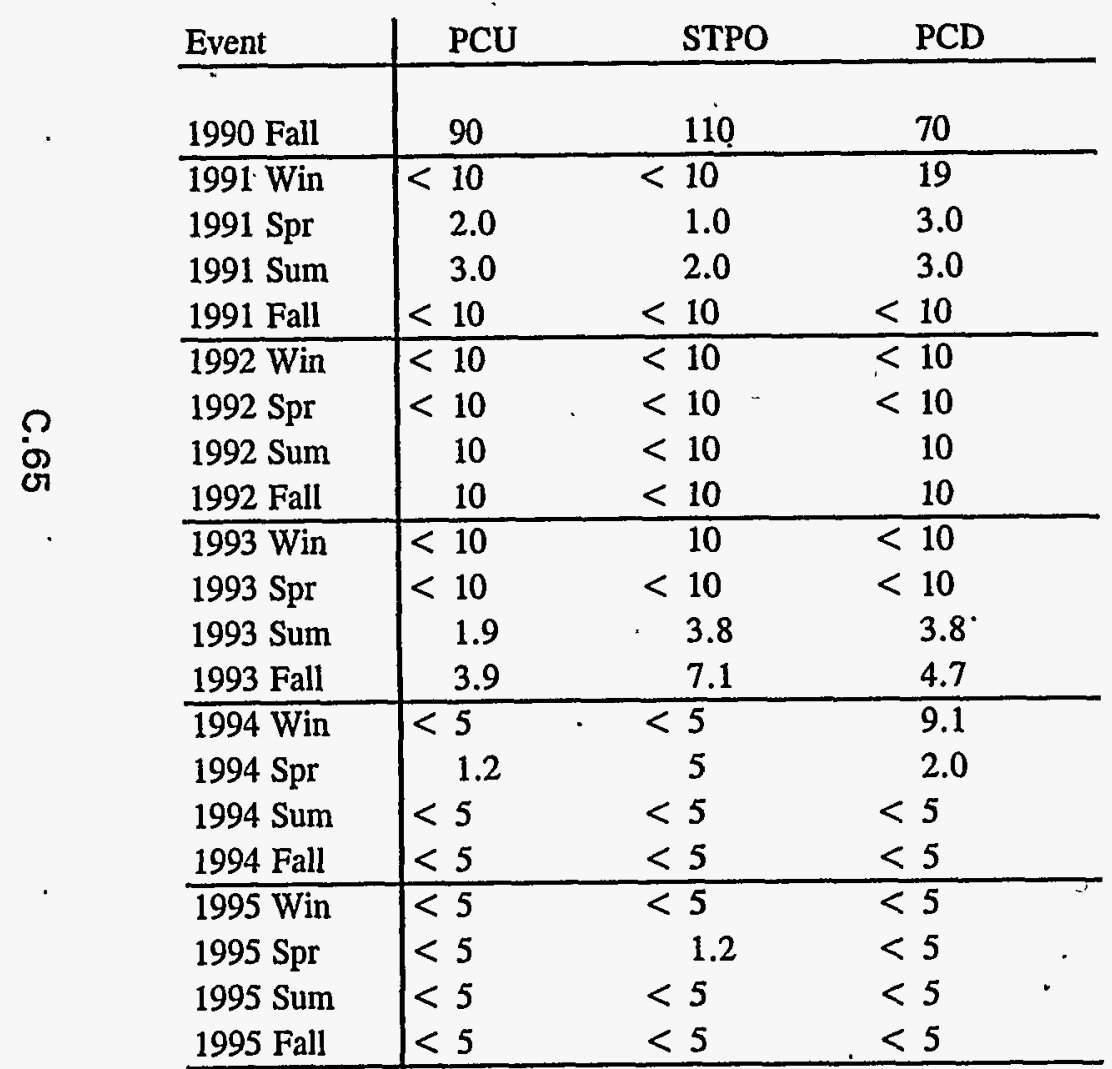

Arsenic (ug/L)

\begin{tabular}{l|ccc} 
Event & PCU & STPO & PCD \\
\hline 1990 Fall & $<10$ & $<10$ & $<10$ \\
\hline 1991 Win & $<10$ & $<10$ & $<10$ \\
1991 Spr & $<10$ & $<10$ & $<10$ \\
1991 Sum & $<10$ & $<10$ & $<10$ \\
1991 Fall & $<10$ & $<10$ & $<10$ \\
\hline 1992 Win & $<10$ & $<10$ & $<10$ \\
1992 Spr & $<10$ & $<10$ & $<10$ \\
1992 Sum & $<10$ & $<10$ & $<10$ \\
1992 Fall & $<10$ & $<10$ & $<10$ \\
\hline 1993 Win & $<10$ & $<10$ & $<10$ \\
1993 Spr & $<10$ & $<10$ & $<10$ \\
1993 Sum & $<2.0$ & 2.1 & 1.0 \\
1993 Fall & 2.2 & 5.0 & 2.7 \\
\hline 1994 Win & $<2.0$ & 4.4 & $<2.0$ \\
1994 Spr & 3.5 & 7.4 & 7.4 \\
1994 Sum & 6.5 & 4.0 & 5.3 \\
1994 Fall & 1.5 & 5.0 & 2.6 \\
\hline 1995 Win & 3.0 & 5.6 & 3.5 \\
1995 Spr & $<2.0$ & 3.3 & $<2.0$ \\
1995 Sum & 2.6 & 3.3 & $<2.0$ \\
1995 Fall & 2.5 & 4.5 & 2.7 \\
\hline
\end{tabular}


Table C.9. Analytical Results for Surface Water, Fall 1990 Through Fall 1995, Metals

Barium (ug/L)

\begin{tabular}{|c|c|c|c|}
\hline Event & PCU & STPO & PCD \\
\hline 1990 Fall & 40 & 40 & 40 \\
\hline 1991 Win & 40 & $<30$ & 70 \\
\hline $1991 \mathrm{Spr}$ & 80 & $<30$ & 70 \\
\hline 1991 Sum & 110 & 60 & 120 \\
\hline 1991 Fall & 80 & 30 & 60 \\
\hline 1992 Win & 60 & $<30$ & 60 \\
\hline $1992 \mathrm{Spr}$ & 80 & 30 & 70 \\
\hline 1992 Sum & 50 & 30 & 50 \\
\hline 1992 Fall & 30 & 20 & 30 \\
\hline 1993 Win & 50 & 20 & 50 \\
\hline $1993 \mathrm{Spr}$ & 80 & 40 & 80 \\
\hline 1993 Sum & 59 & 23.1 & 78.3 \\
\hline 1993 Fall & 80.1 & 23.9 & 73.3 \\
\hline 1994 Win & 62.5 & 26.1 & 60.3 \\
\hline $1994 \mathrm{Spr}$ & 82.4 & 20.6 & 55.0 \\
\hline 1994 Sum & 55.4 & 23.8 & 36.7 \\
\hline 1994 Fall & 67.5 & 25.2 & 62.0 \\
\hline 1995 Win & 105 & 21.5 & 100 \\
\hline $1995 \mathrm{Spr}$ & 113 & 22.9 & 113 \\
\hline 1995 Sum & 112 & 35.3 & 92.8 \\
\hline 1995 Fall & 82.1 & 30.7 & 73.6 \\
\hline
\end{tabular}

Copper (ug/L)

\begin{tabular}{l|ccc} 
Event & PCU & STPO & PCD \\
\hline 1990 Fall & $<30$ & $<30$ & $<30$ \\
\hline 1991 Win & 30 & 30 & $<30$ \\
1991 Spr & $<30$ & $<30$ & $<30$ \\
1991 Sum & $<30$ & $<30$ & 50 \\
1991 Fall & $<30$ & $<30$ & $<30$ \\
\hline 1992 Win & $<30$ & $<30$ & $<30$ \\
1992 Spr & $<10$ & $<10$ & $<10$ \\
1992 Sum & $<30$ & $<30$ & $<30$ \\
1992 Fall & $<10$ & $<10$ & $<10$ \\
\hline 1993 Win & 40 & 10 & $<10$ \\
1993 Spr & $<10$ & 10 & $<10$ \\
1993 Sum & 3.9 & 3.4 & 3.7 \\
1993 Fall & 4.9 & 9.8 & 4.3 \\
\hline 1994 Win & 3.5 & 9.6 & 6.6 \\
1994 Spr & 4.0 & 10.1 & 6.9 \\
1994 Sum & 3.3 & 6.6 & 2.7 \\
1994 Fall & 1.9 & 4.8 & 2.5 \\
\hline 1995 Win & 3.5 & 5.0 & 1.4 \\
1995 Spr & $<10$ & 5.5 & $<10$ \\
1995 Sum & $<10$ & 3.1 & 3.9 \\
1995 Fall & $<10$ & 6.5 & $<10$ \\
\hline
\end{tabular}


Table C.9. Analytical Results for Surface Water, Fall 1990 Through Fall 1995, Metals

Chromium - Hexavalent (ug/L)

\begin{tabular}{|c|c|c|c|}
\hline Event & $\mathrm{PCU}$ & STPO & PCD \\
\hline 1990 Fall. & $<10$ & 10 & $<10$ \\
\hline 1991 Win & $<10$ & $<10$ & $<10$ \\
\hline $1991 \mathrm{Spr}$ & 18 & 11 & 19 \\
\hline 1991 Sum & 10 & 10 & 20 \\
\hline 1991 Fall & $<10$ & $<10$ & $<10$ \\
\hline 1992 Win & $<10$ & $<10$ & $<10$ \\
\hline $1992 \mathrm{Spr}$ & 10 & $<10$ & $<10$ \\
\hline 1992 Sum & 43 & 45 & 42 \\
\hline 1992 Fall & 10 & 10 & $<10$ \\
\hline 1993 Win & $<10$ & 10 & $<10$ \\
\hline $1993 \mathrm{Spr}$ & $<10$ & $<10$ & $<10$ \\
\hline 1993 Sum & $<20$ & $<20$ & $<20$ \\
\hline 1993 Fall & $<20$ & $<20$ & $<20$ \\
\hline 1994 Win & $<10$ & $<10$ & $<10$ \\
\hline $1994 \mathrm{Spr}$ & $<10$ & $<10$ & $<10$ \\
\hline 1994 Sum & $<10$ & $<10$ & $<10$ \\
\hline 1994 Fall & $<10$ & $<10$ & $<10$ \\
\hline 1995 Win & $<10$ & $<10$ & $<10$ \\
\hline $1995 \mathrm{Spr}$ & $<10$ & $<10$ & $<10$ \\
\hline 1995 Sum & $<10$ & $<10$ & $<10$ \\
\hline 1995 Fall & $<10$ & $<10$ & $\cdot<10$ \\
\hline
\end{tabular}

Chromium - Total (ug/L)

\begin{tabular}{l|ccc} 
Event & PCU & STPO & PCD \\
\hline 1990 Fall & 60 & 110 & $<30$ \\
\hline 1991 Win & $<10$ & $<10$ & 11 \\
1991 Spr & $<30$ & $<30$ & $<30$ \\
1991 Sum & $<30$ & $<30$ & $<30$ \\
1991 Fall & $<30$ & $<30$ & $<30$ \\
\hline 1992 Win & $<30$ & $<30$ & $<30$ \\
1992 Spr & $<10$ & $<10$ & 10 \\
1992 Sum & 30 & 10 & 10 \\
1992 Fall & 20 & 10 & $<10$ \\
\hline 1993 Win & $<10$ & $<10$ & 80 \\
1993 Spr & $<10$ & $<10$ & $<10$ \\
1993 Sum & 1.7 & $<10$ & $<10$ \\
1993 Fall & 3.3 & 2.8 & 2.7 \\
\hline 1994 Win & 2.1 & 3.9 & 2.1 \\
1994 Spr & $<10$ & 4.2 & 2.8 \\
1994 Sum & $<10$ & 2.7 & 1.2 \\
1994 Fall & 1.3 & 3.2 & 1.8 \\
\hline 1995 Win & 4.3 & 2.2 & 3.6 \\
1995 Spr & 4.3 & $<10$ & 4.6 \\
1995 Sum & 4.3 & 3.0 & 2.3 \\
1995 Fall & 4.4 & 5.1 & 4.5 \\
\hline
\end{tabular}


Table C.9. Analytical Results for Surface Water, Fall 1990 Through Fall 1995, Metals

Lead (ug/L)

\begin{tabular}{|c|c|c|c|}
\hline Event & $\mathrm{PCU}$ & STPO & PCD \\
\hline 1990 Fall & 2 & 1 & 2 \\
\hline 1991 Win & 4 & $<1$ & 4 \\
\hline $1991 \mathrm{Spr}$ & $<1$ & 1 & 1 \\
\hline 1991 Sum & 3 & 7 & 7 \\
\hline 1991 Fall & $<1$ & 1 & $<1$ \\
\hline 1992 Win & $<3$ & $<3$ & $<3$ \\
\hline $1992 \mathrm{Spr}$ & $<3$ & $<3$ & $<3$ \\
\hline 1992 Sum & $<3$ & $<3$ & $<3$ \\
\hline 1992 Fall & $<3$ & $<3$ & 10 \\
\hline 1993 Win & $<3$ & $<3$ & $<3$ \\
\hline $1993 \mathrm{Spr}$ & $<3$ & $<3$ & $<3$ \\
\hline 1993 Sum & 7.3 & 11.2 & 6 \\
\hline 1993 Fall & $<2$ & 1.7 & $<2$ \\
\hline 1994 Win & $<2$ & $<2$ & $<2$ \\
\hline 1994 Spr & $<2$ & 1.2 & $<2$ \\
\hline 1994 Sum & $<2$ & $<2$ & $<2$ \\
\hline 1994 Fall & $<2$ & $<2$ & $<2$ \\
\hline 1995 Win & $<2$ & $<2$ & $\overline{<2}$ \\
\hline $1995 \mathrm{Spr}$ & $<2$ & $<2$ & $<2$ \\
\hline 1995 Sum & $<2$ & $<2$ & $<2$ \\
\hline 1995 Fall & $<2$ & $<2$ & $<2$ \\
\hline
\end{tabular}

Manganese (ug/L)

\begin{tabular}{|c|c|c|c|}
\hline Event & PCU & STPO & PCD \\
\hline 1990 Fall & NA & NA & NA \\
\hline 1991 Win & NA & $\overline{\mathrm{NA}}$ & $\overline{\mathrm{NA}}$ \\
\hline 1991 Spr & NA & NA & NA \\
\hline 1991 Sum & NA & NA & NA \\
\hline 1991 Fall & NA & NA & NA \\
\hline 1992 Win & NA & $\mathrm{NA}$ & NA \\
\hline 1992 Spr & NA & NA & NA \\
\hline 1992 Sum & $\mathrm{NA}$ & NA & $\mathrm{NA}$ \\
\hline 1992 Fall & NA & NA & NA \\
\hline 1993 Win & $\mathrm{NA}$ & NA & NA \\
\hline 1993 Spr & NA & NA & NA \\
\hline 1993 Sum & NA & NA & NA \\
\hline 1993 Fall & NA & NA & NA \\
\hline 1994 Win & $\overline{\mathrm{NA}}$ & NA & NA \\
\hline 1994 Spr & 1.5 & 8.1 & 2.0 \\
\hline 1994 Sum & 6.4 & 21.8 & 8.0 \\
\hline 1994 Fall & NA & NA & NA \\
\hline 1995 Win & NA & $\mathrm{NA}$ & NA \\
\hline 1995 Spr & NA & NA & NA \\
\hline 1995 Sum & NA & NA & NA \\
\hline 1995 Fall & NA & NA & NA \\
\hline
\end{tabular}

Notes:

1. NA indicates that no sample was analyzed for this constituent. 
Table C.9. Analytical Results for Surface Water, Fall 1990 Through Fall 1995, Metals

Mercury (ug/L)

\begin{tabular}{l|lll} 
Event & PCU & STPO & PCD \\
\hline & & & \\
1990 Fall & $<0.4$ & $<0.4$ & $<0.4$ \\
\hline 1991 Win & $<0.4$ & $<0.4$ & $<0.4$ \\
1991 Spr & $<0.4$ & $<0.4$ & $<0.4$ \\
1991 Sum & $<0.4$ & $<0.4$ & $<0.4$ \\
1991 Fall & $<0.4$ & $<0.4$ & $<0.4$ \\
\hline 1992 Win & $<0.4$ & $<0.4$ & $<0.4$ \\
1992 Spr & 0.4 & $<0.4$ & $<0.4$ \\
1992 Sum & $<0.4$ & $<0.4$ & $<0.4$ \\
1992 Fall & $<0.4$ & $<0.4$ & $<0.4$ \\
\hline 1993 Win & $<0.4$ & $<0.4$ & $<0.4$ \\
1993 Spr & $<0.4$ & $<0.4$ & $<0.4$ \\
1993 Sum & $<0.2$ & 0.63 & 0.27 \\
1993 Fall & $<0.2$ & 0.25 & $<0.2$ \\
\hline 1994 Win & $<0.2$ & $<0.2$ & $<0.2$ \\
1994 Spr & $<0.2$ & $<0.2$ & $<0.2$ \\
1994 Sum & $<0.2$ & $<0.2$ & $<0.2$ \\
1994 Fall & $<0.2$ & $<0.2$ & $<0.2$ \\
\hline 1995 Win & $<0.2$ & $<0.2$ & $<0.2$ \\
1995 Spr & $<0.2$ & $<0.2$ & $<0.2$ \\
1995 Sum & $<0.2$ & $<0.2$ & $<0.2$ \\
1995 Fall & $<0.2$ & $<0.2$ & $<0.2$ \\
\hline
\end{tabular}

Molybdenum (ug/L)

\begin{tabular}{l|ccc} 
Event & PCU & STPO & PCD \\
\hline 1990 Fall & 60 & 70 & 50 \\
\hline 1991 Win & $<10$ & $<10$ & $<10$ \\
1991 Spr & $<30$ & $<30$ & $<30$ \\
1991 Sum & $<50$ & $<50$ & $<50$ \\
1991 Fall & $<50$ & 50 & $<50$ \\
\hline 1992 Win & $<30$ & $<30$ & $<30$ \\
1992 Spr & $<10$ & $<10$ & $<10$ \\
1992 Sum & $<30$ & $<30$ & $<30$ \\
1992 Fall & $<10$ & $<10$ & $<10$ \\
\hline 1993 Win & $<10$ & $<10$ & 10 \\
1993 Spr & $<10$ & 110 & 10 \\
1993 Sum & 3.5 & 57.9 & 4.6 \\
1993 Fall & 1.1 & 61.3 & 9.2 \\
\hline 1994 Win & $<10$ & 56.1 & 5.7 \\
1994 Spr & 2.9 & 34.9 & 6.3 \\
1994 Sum & 39.9 & 28.3 & 27.6 \\
1994 Fall & $<10$ & 14.3 & 6.0 \\
\hline 1995 Win & $<10$ & 7.5 & $<10$ \\
1995 Spr & $<10$ & 6.0 & $<10$ \\
1995 Sum & 1.1 & 7.3 & 2.2 \\
1995 Fall & $<10$ & 10.7 & $<10$ \\
\hline
\end{tabular}


Table C.9. Analytical Results for Surface Water, Fall 1990 Through Fall 1995, Metals

Nickel (ug/L)

\begin{tabular}{l|ccc} 
Event & PCU & STPO & PCD \\
\hline 1990 Fall & $<30$ & $<30$ & $<30$ \\
\hline 1991 Win & 50 & $<30$ & $<30$ \\
1991 Spr & $<30$ & $<30$ & $<30$ \\
1991 Sum & $<30$ & $<30$ & $<30$ \\
1991 Fall & $<30$ & $<30$ & $<30$ \\
\hline 1992 Win & $<30$ & $<30$ & $<30$ \\
1992 Spr & $<10$ & $<10$ & $<10$ \\
1992 Sum & 50 & $<30$ & $<30$ \\
1992 Fall & $<10$ & $<10$ & $<10$ \\
\hline 1993 Win & 10 & $<10$ & 310 \\
1993 Spr & $<10$ & $<10$ & $<10$ \\
1993 Sum & 2.6 & 2.3 & 2.4 \\
1993 Fall & 2.7 & 2.2 & 2.5 \\
\hline 1994 Win & 4.9 & 3.3 & 5.6 \\
1994 Spr & 11.1 & 11.9 & 11.1 \\
1994 Sum & 2.5 & $<20.0$ & 1.9 \\
1994 Fall & 1.7 & 1.6 & 1.8 \\
\hline 1995 Win & 1.9 & 2.3 & 1.6 \\
1995 Spr & 1.2 & $<20.0$ & 2.0 \\
1995 Sum & 4.5 & 3.0 & .4 .3 \\
1995 Fall & 4.2 & 5.1 & 4.4
\end{tabular}

Selenium (ug/L)

\begin{tabular}{l|ccc} 
Event & PCU & STPO & PCD \\
\hline 1990 Fall & $<10$ & $<10$ & $<10$ \\
\hline 1991 Win & $<10$ & $<10$ & $<10$ \\
1991 Spr & $<10$ & $<10$ & $<10$ \\
1991 Sum & $<10$ & $<10$ & $<10$ \\
1991 Fall & $<10$ & 39 & $<10$ \\
\hline 1992 Win & $<10$ & $<10$ & $<10$ \\
1992 Spr & $<10$ & $<10$ & $<10$ \\
1992 Sum & $<10$ & $<10$ & $<10$ \\
1992 Fall & $<10$ & $<10$ & $<10$ \\
\hline 1993 Win & $<10$ & $<10$ & $<10$ \\
1993 Spr & $<10$ & $<10$ & $<10$ \\
1993 Sum & $<3$ & $<3.0$ & $<3$ \\
1993 Fall & $<3$ & $<3.0$ & $<3$ \\
\hline 1994 Win & $<3$ & $<3.0$ & $<3$ \\
1994 Spr & $<3$ & 4.3 & 3.8 \\
1994 Sum & $<3$ & $<3.0$ & $<3$ \\
1994 Fall & $<3$ & $<3.0$ & $<3$ \\
\hline 1995 Win & $<3$ & $<3.0$ & $<3$ \\
1995 Spr & $<3$ & $<3.0$ & $<3$ \\
1995 Sum & $<3$ & 3.6 & $<3$ \\
1995 Fall & $<3$ & 7.4 & $<3$ \\
\hline
\end{tabular}


Table C.9. Analytical Results for Surfảce Water, Fall 1990 Through Fall 1995, Metals

Silver (ug/L)

\begin{tabular}{|c|c|c|c|}
\hline Event & PCU & STPO & PCD \\
\hline 1990 Fall & $<30$ & $<30$ & $<30$ \\
\hline 1991 Win & $<40$ & $<30$ & $<30$ \\
\hline $1991 \mathrm{Spr}$ & $<30$ & $<30$ & $<30$ \\
\hline 1991 Sum & $<30$ & 30 & $<30$ \\
\hline 1991 Fall & $<30$ & $<30$ & $<30$ \\
\hline 1992 Win & $<30$ & $<30$ & $<30$ \\
\hline 1992 Spr & $<10$ & $<30$ & $<10$ \\
\hline 1992 Sum & $<10$ & $<10$ & $<10$ \\
\hline 1992 Fall & $<10$ & $<10$ & $<10$ \\
\hline 1993 Win & $<10$ & $<10$ & $<10$ \\
\hline $1993 \mathrm{Spr}$ & $<10$ & $<10$ & $<10$ \\
\hline 1993 Sum & $<10$ & $<10$ & $<10$ \\
\hline 1993 Fall & $<10$ & $<10$ & $<10$ \\
\hline 1994 Win & $<10$ & $<10$ & $<10$ \\
\hline $1994 \mathrm{Spr}$ & $<10$ & $<10$ & $<10$ \\
\hline 1994 Sum & $<10$ & $<10$ & $<10$ \\
\hline 1994 Fall & $<10$ & $<10$ & $<10$ \\
\hline 1995 Win & $<10$ & $<10$ & $<10$ \\
\hline $1995 \mathrm{Spr}$ & $<10$ & $<10$ & $<10$ \\
\hline 1995 Sum & $<10$ & $<.10$ & $<10$ \\
\hline 1995 Fall & $<10$ & $<10$ & $<10$ \\
\hline
\end{tabular}

Thallium (ug/L)

\begin{tabular}{l|ccc} 
Event & PCU & STPO & PCD \\
\hline 1990 Fall & 90 & 100 & 80 \\
\hline 1991 Win & $<30$ & $<30$ & $<30$ \\
1991 Spr & 4.0 & 1.0 & 1.0 \\
1991 Sum & 2.0 & $<1$ & 1.0 \\
1991 Fall & $<5$ & $<5$ & $<5$ \\
\hline 1992 Win & 50 & 60 & $<10$ \\
1992 Spr & $<10$ & $<10$ & $<10$ \\
1992 Sum & $<10$ & $<10$ & $<10$ \\
1992 Fall & $<5$ & $<5$ & $<5$ \\
\hline 1993 Win & $<10$ & $<10$ & $<10$ \\
1993 Spr & $<10$ & $<10$ & $<10$ \\
1993 Sum & 2.9 & 4.7 & 2.4 \\
1993 Fall & $<10$ & $<10$ & $<10$ \\
\hline 1994 Win & $<10$ & $<10$ & $<10$ \\
1994 Spr & $<10$ & $<10$ & $<10$ \\
1994 Sum & $<10$ & $<10$ & $<10$ \\
1994 Fall & $<10$ & $<10$ & $<10$ \\
\hline 1995 Win & $<10$ & $<10$ & $<10$ \\
1995 Spr & $<10$ & $<10$ & $<10$ \\
1995 Sum & $<10$ & $<10$ & $<10$ \\
1995 Fall & $<10$ & $<10$ & $<10$ \\
\hline
\end{tabular}


Table C.9. Analytical Results for Surface Water, Fall 1990 Through Fall 1995, Metals

Vanadium (ug/L)

\begin{tabular}{|c|c|c|c|}
\hline Event & PCU & STPO & PCD \\
\hline 1990 Fall & $<50$ & $<50$ & $<50$ \\
\hline 1991 Win & $<100$ & $<100$ & $<100$ \\
\hline $1991 \mathrm{Spr}$ & $<30$ & $<30$ & $<30$ \\
\hline 1991 Sum & $<50$ & $<50$ & $<50$ \\
\hline 1991 Fall & $<50$ & $<50$ & $<50$ \\
\hline 1992 Win & $<30$ & $<30^{\circ}$ & $<30$ \\
\hline $1992 \mathrm{Spr}$ & $<10$ & 10 & 20 \\
\hline 1992 Sum & $<30$ & $<30$ & $<30$ \\
\hline 1992 Fall & $<10$ & $<10$ & $<10$ \\
\hline 1993 Win & $<10$ & $<10$ & $<10$ \\
\hline $1993 \mathrm{Spr}$ & $<10$ & $<10$ & $<10$ \\
\hline 1993 Sum & 5.8 & 8.1 & 8.0 \\
\hline 1993 Fall & 3.4 & 9.7 & 5.0 \\
\hline 1994 Win & 2.8 & 12.3 & 4.0 \\
\hline $1994 \mathrm{Spr}$ & 8.8 & 12.0 & 12 \\
\hline 1994 Sum & 13.4 & 5.6 & 8.8 \\
\hline 1994 Fall & 2.6 & 9.6 & 5.9 \\
\hline 1995 Win & 4.4 & 9.8 . & 4.6 \\
\hline $1995 \mathrm{Spr}$ & $<10$ & 10.5 & $<10$ \\
\hline 1995 Sum & 4.9 & 10.3 & 8.5 \\
\hline 1995 Fall & $<10$ & 8.7 & $<10$ \\
\hline
\end{tabular}

Zinc (ug/L)

\begin{tabular}{l|ccl} 
Event & PCU & STPO & PCD \\
\hline 1990 Fall & 39 & $<30$ & 1190 \\
\hline 1991 Win & 50 & 40 & 50 \\
1991 Spr & $<30$ & 30 & 30 \\
1991 Sum & 30 & 100 & 40 \\
1991 Fall & $<30$ & 30 & $<30$ \\
\hline 1992 Win & $<30$ & 30 & $<30$ \\
1992 Spr & 30 & 40 & 10 \\
1992 Sum & $<30$ & 30 & $<30$ \\
1992 Fall & 30 & 30 & 20 \\
\hline 1993 Win & 20 & 40 & 30 \\
1993 Spr & 10 & 60 & 10 \\
1993 Sum & 5.0 & 21.1 & 5.5 \\
1993 Fall & 6.5 & 32.6 & 10.0 \\
\hline 1994 Win & 4.3 & 33.1 & 6.0 \\
1994 Spr & 3.9 & 23.4 & 12.8 \\
1994 Sum & 17.7 & 16.2 & 14.6 \\
1994 Fall & 1.6 & 41.0 & 10.0 \\
\hline 1995 Win & 3.3 & 28.3 & 2.1 \\
1995 Spr & 3.7 & 33.6 & 5.0 \\
1995 Sum & 9.2 & 39.2 & 10.7 \\
1995 Fall & 5.2 & 39.2 & 8.2 \\
\hline
\end{tabular}


Table C.10. Analytical Results for Surface Water, Fall 1990 Through Fall 1995, Radionuclides

Tritium (pCi/)

\begin{tabular}{l|ccc} 
Event & PCU & STPO & PCD \\
\hline 1990 Fall & \multicolumn{1}{c}{1341} & 704 & 1313 \\
\hline 1991 Win & $<500$ & $<500$ & $<500$ \\
1991 Spr & $<500$ & $<500$ & $<500$ \\
1991 Sum & $<500$ & $<500$ & $<500$ \\
1991 Fall & $<500$ & $<500$ & $<500$ \\
\hline 1992 Win & $<500$ & 752 & 754 \\
1992 Spr & $<500$ & $<500$ & $<500$ \\
1992 Sum & 650 & $<500$ & 800 \\
1992 Fall & $<500$ & 1724 & 851 \\
\hline 1993 Win & $<500$ & $<500$ & $<500$ \\
1993 Spr & $<500$ & $<500$ & $<500$ \\
1993 Sum & $<220$ & 300 & $<220$ \\
1993 Fall & $<240$ & $<240$ & $<240$ \\
\hline 1994 Win & $<260$ & $<260$ & $<260$ \\
1994 Spr & $<250$ & 320 & 2030 \\
1994 Sum & $<270$ & $<260$ & 570 \\
1994 Fall & $<240$ & 480 & $<240$ \\
\hline 1995 Win & $<260$ & $<260$ & $<260$ \\
1995 Spr & $<220$ & $<230$ & $<220$ \\
1995 Sum & $<290$ & $<260$ & $<300$ \\
1995 Fall & $<250$ & $<240$ & $<250$ \\
\hline
\end{tabular}

Carbon-14 (pCi/L)

\begin{tabular}{l|ccc} 
Event & PCU & STPO & PCD \\
\hline 1990 Fall & NA & NA & NA \\
\hline 1991 Win & NA & NA & NA \\
1991 Spr & NA & NA & NA \\
1991 Sum & NA & NA & NA \\
1991 Fall & NA & NA & NA \\
\hline 1992 Win & NA & NA & NA \\
1992 Spr & NA & NA & NA \\
1992 Sum & NA & NA & NA \\
1992 Fall & NA & NA & NA \\
\hline 1993 Win & NA & NA & NA \\
1993 Spr & NA & NA & NA \\
1993 Sum & 36 & $<12$ & $<12$ \\
1993 Fall & 122 & 65 & $<40$ \\
\hline 1994 Win & $<110$ & $<110$ & $<110$ \\
1994 Spr & $<91$ & $<91$ & $<91$ \\
1994 Sum & $<130$ & $<130$ & $<130$ \\
1994 Fall & $<130$ & $<130$ & $<130$ \\
\hline 1995 Win & $<110$ & $<110$ & $<110$ \\
1995 Spr & $<110$ & $<110$ & $<110$ \\
1995 Sum & $<100$ & $<12$. & 15 \\
1995 Fall & $<100$ & $<100$ & $<100$ \\
\hline
\end{tabular}

Notes:

1. NA indicates that no sample was analyzed for this constituent. 
Table C.10. Analytical Results for Surface Water, Fall 1990 Through Fall 1995, Radionuclides

Strontium-90 (pCi/L)

\begin{tabular}{|c|c|c|c|}
\hline Event & PCU & STPO & PCD \\
\hline 1990 Fall & 2.6 & 5.4 & 4.2 \\
\hline 1991 Win & $<0.5$ & 0.8 & $<0.5$ \\
\hline $1991 \mathrm{Spr}$ & $<0.5$ & $<0.5$ & $<0.5$ \\
\hline 1991 Sum & $<0.5$ & $<0.5$ & 1.8 \\
\hline 1991 Fall & $<0.5$ & $<0.5$ & $<0.5$ \\
\hline 1992 Win & $<0.5$ & $<0.5$ & $<0.5$ \\
\hline $1992 \mathrm{Spr}$ & $<1.0$ & $<1$ & $<1.0$ \\
\hline 1992 Sum & 6.9 & 1.5 & 2.5 \\
\hline 1992 Fall & 0.90 & $<0.5$ & $<0.5$ \\
\hline 1993 Win & $<0.5$ & 1.0 & $<0.5$ \\
\hline $1993 \mathrm{Spr}$ & $<0.5$ & $<0.5$ & $<0.5$ \\
\hline 1993 Sum & $<1.3$ & $<1.2$ & $<1.2$ \\
\hline 1993 Fall & $<0.79$ & $<1.2$ & $<0.89$ \\
\hline 1994 Win & $<1.1$ & $<1.2$ & $<1.2$ \\
\hline $1994 \mathrm{Spr}$ & $<1.0$ & $<1.6$ & $<1.0$ \\
\hline 1994 Sum & $<1.7$ & $<1.7$ & 2.1 \\
\hline 1994 Fall & $<0.69$ & $<0.97$ & $<0.78$ \\
\hline 1995 Win & $<1.0$ & $<1.1$ & $<1.2$ \\
\hline $1995 \mathrm{Spr}$ & $<0.90$ & $<0.97$ & $<0.86$ \\
\hline 1995 Sum & $<0.72$ & $<0.82$ & $<0.91$ \\
\hline 1995 Fall & $<0.66$ & $<0.46$ & $<0.49$ \\
\hline
\end{tabular}

Radium-226 (pCi/L)

\begin{tabular}{l|ccc} 
Event & PCU & STPO & PCD \\
\hline 1990 Fall & NA & NA & NA \\
\hline 1991 Win & NA & NA & NA \\
1991 Spr & NA & NA & NA \\
1991 Sum & NA & NA & NA \\
1991 Fall & NA & NA & NA \\
\hline 1992 Win & NA & NA & NA \\
1992 Spr & NA & NA & NA \\
1992 Sum & NA & NA & NA \\
1992 Fall & NA & NA & NA \\
\hline 1993 Win & NA & NA & NA \\
1993 Spr & NA & NA & NA \\
1993 Sum & NA & NA & NA \\
1993 Fall & $<0.28$ & $<0.38$ & 0.29 \\
\hline 1994 Win & $<0.20$ & $<0.12$ & $<0.25$ \\
1994 Spr & $<0.33$ & $<0.10$ & $<0.33$ \\
1994 Sum & $<0.20$ & $<0.15$ & $<0.18$ \\
1994 Fall & $<0.58$ & $<0.32$ & $<0.43$ \\
\hline 1995 Win & $<0.25$ & $<0.32$ & $<0.36$ \\
1995 Spr & $<0.20$ & 0.46 & $<0.29$ \\
1995 Sum & 0.089 & $<0.27$ & $<0.27$ \\
1995 Fall & 0.26 & 0.80 & $<0.20$ \\
\hline
\end{tabular}

Notes:

1. NA indicates that no sample was analyzed for this constituent. 
Table C.10. Analytical Results for Surface Water, Fall 1990 Through Fall 1995, Radionuclides

Plutonium-241 (pCi/L)

\begin{tabular}{|c|c|c|c|}
\hline Event & PCU & STPO & $\mathrm{PCD}$ \\
\hline 1990 Fall & NA & NA & NA \\
\hline 1991 Win & $\mathrm{NA}$ & $\mathrm{NA}$ & NA \\
\hline $1991 \mathrm{Spr}$ & NA & NA & NA \\
\hline 1991 Sum & NA & NA & NA \\
\hline 1991 Fall & NA & $\mathrm{NA}$ & NA \\
\hline 1992 Win & $\overline{\mathrm{NA}}$ & $\overline{\mathrm{NA}}$ & $\overline{\mathrm{NA}}$ \\
\hline $1992 \mathrm{Spr}$ & NA & NA & $\mathrm{NA}$ \\
\hline 1992 Sum & $\mathrm{NA}$ & $\mathrm{NA}$ & NA \\
\hline 1992 Fall & $\mathrm{NA}$ & $\mathrm{NA}$ & NA \\
\hline 1993 Win & $\mathrm{NA}$ & $\mathrm{NA}$ & $\mathrm{NA}$ \\
\hline $1993 \mathrm{Spr}$ & $\mathrm{NA}$ & $\mathrm{NA}$ & NA \\
\hline 1993 Sum & NA & $\mathrm{NA}$ & NA \\
\hline 1993 Fall & NA & $\mathrm{NA}$ & NA \\
\hline 1994 Win & NA & $\overline{\mathrm{NA}}$ & NA \\
\hline 1994 Spr & NA & $\mathrm{NA}$ & NA \\
\hline 1994 Sum & $<2.7$ & $<3.2$ & $<4.2$ \\
\hline 1994 Fall & $<2.2$ & $<1.7$ & $<1.6$ \\
\hline 1995 Win & $<1.6$ & $<1.6$ & $<1.7$ \\
\hline $1995 \mathrm{Spr}$ & $<2.4$ & $<2.2$ & $<1.8$ \\
\hline 1995 Sum & $<2.6$ & $<4.3$ & $<3.7$ \\
\hline 1995 Fall & $<2.9$ & $<2.7$ & $<2.4$ \\
\hline
\end{tabular}

Notes:

1. NA indicates that no sample was analyzed for this constituent.
Americium-241 (pCi/L)

\begin{tabular}{l|ccc} 
Event & PCU & STPO & PCD \\
\hline 1990 Fall & NA & NA & NA \\
\hline 1991 Win & NA & NA & NA \\
1991 Spr & NA & NA & NA \\
1991 Sum & NA & NA & NA \\
1991 Fall & NA & NA & NA \\
\hline 1992 Win & NA & NA & NA \\
1992 Spr & NA & NA & NA \\
1992 Sum & NA & NA & NA \\
1992 Fall & NA & NA & NA \\
\hline 1993 Win & NA & NA & NA \\
1993 Spr & NA & NA & NA \\
1993 Sum & NA & NA & NA \\
1993 Fall & NA & NA & NA \\
\hline 1994 Win & NA & NA & NA \\
1994 Spr & NA & NA & NA \\
1994 Sum & $<0.047$ & $<0.026$ & $<0.026$ \\
1994 Fall & $<0.079$ & $<0.100$ & $<0.047$ \\
\hline 1995 Win & $<0.055$ & $<0.063$ & $<0.085$ \\
1995 Spr & $<0.012$ & $<0.020$ & $<0.031$ \\
1995 Sum & 0.032 & $<0.042$ & 0.034 \\
1995 Fall & $<0.022$ & $<0.033$ & $<0.027$ \\
\hline
\end{tabular}

Notes:

1. NA indicates that no sample was analyzed for this constituent. 
Table C.10. Analytical Results. for Surface Water, Fall 1990 Through Fall 1995, Radionuclides

Bismuth-214 (pCi/L)

\begin{tabular}{|c|c|c|c|}
\hline Event & PCU & STPO & PCD \\
\hline 1990 Fall & NA & NA & NA \\
\hline 1991 Win & $\overline{N A}$ & $\overline{N A}$ & NA \\
\hline $1991 \mathrm{Spr}$ & NA & NA & $\mathbf{N A}$ \\
\hline 1991 Sum & $\mathrm{NA}$ & NA & $\mathbf{N A}$ \\
\hline 1991 Fall & NA & NA & NA \\
\hline 1992 Win & NA & $\overline{\text { NA }}$ & NA \\
\hline $1992 \mathrm{Spr}$ & $\mathrm{NA}$ & NA & NA \\
\hline 1992 Sum & $\mathrm{NA}$ & NA & NA \\
\hline 1992 Fall & NA & NA & NA \\
\hline 1993 Win & $\overline{\mathrm{NA}}$ & NA & . NA \\
\hline $1993 \cdot S p r$ & NA & NA & NA \\
\hline 1993 Sum & NA & NA & NA \\
\hline 1993 Fall & NA & NA & NA \\
\hline 1994 Win & $<8.4$ & 17.2 & . $\quad 25.4$ \\
\hline 1994 Spr & 20 & $<7.9$ & $<16$ \\
\hline 1994 Sum & $<8.6$ & $<21$ & $<19$ \\
\hline 1994 Fall & $<20$ & $<9$ & $<19$ \\
\hline 1995 Win & $<89$ & $<7.5$ & $<19$ \\
\hline $1995 \mathrm{Spr}$ & 21 & $<16$ & $<17$ \\
\hline 1995 Sum & $<20$. & $<18$ & $<63$ \\
\hline 1995 Fall & 9.3 & $<8$ &.$<9.1$ \\
\hline
\end{tabular}

Notes:

1. NA indicates that no sample was analyzed for this constituent.
Thallium-208 (pCi/L)

\begin{tabular}{l|ccc} 
Event & PCU & STPO & PCD \\
\hline & & & NA \\
1990 Fall & NA & NA & NA \\
\hline 1991 Win & NA & NA & NA \\
1991 Spr & NA & NA & NA \\
1991 Sum & NA & NA & NA \\
1991 Fall & NA & NA & NA \\
\hline 1992 Win & NA & NA & NA \\
1992 Spr & NA & NA & NA \\
1992 Sum & NA & NA & NA \\
1992 Fall & NA & NA & NA \\
\hline 1993 Win & NA & NA & NA \\
1993 Spr & NA & NA & NA \\
1993 Sum & NA & NA & NA \\
1993 Fall & NA & $<8.8$ & $<9.8$ \\
\hline 1994 Win & $<4.0$ & $<4.1$ & $<8.7$ \\
1994 Spr & 6.3 & $<12$ & $<9.3$ \\
1994 Sum & $<4.9$ & $<4.6$ & $<10$ \\
1994 Fall & $<9.0$ & $<4.3$ & $<11$ \\
\hline 1995 Win & $<46$ & $<10$ & $<11$ \\
1995 Spr & $<9.7$ & $<9.2$ & $<34$ \\
1995 Sum & $<9.5$ & $<4.1$ & $<4.4$ \\
1995 Fall & $<4.3$ & &
\end{tabular}

Notes:

1. NA indicates that no sample was analyzed for this constituent. 
Table C.10. Analytical Results for Surface Water, Fall 1990 Through Fall 1995, Radionuclides

Gross Alpha (pCi/L)

\begin{tabular}{|c|c|c|c|}
\hline Event & PCU & STPO & PCD \\
\hline 1990 Fall & $<2$ & $<2$ & $<2$ \\
\hline 1991 Win & 3 & 7 & $<2$ \\
\hline $1991 \mathrm{Spr}$ & 3 & $<2$ & $<2$ \\
\hline 1991 Sum & $<2$ & $<2$ & 3 \\
\hline 1991 Fall & $<2$ & $<2$ & 25 \\
\hline 1992 Win & $<2$ & 3 & $<2$ \\
\hline 1992 Spr & $<2$ & $<2$ & $<2$ \\
\hline 1992 Sum & 3 & $<2$. & 3 \\
\hline 1992 Fall & 8 & 12 & 9 \\
\hline 1993 Win & $<2$ & 3 & $<2$ \\
\hline $1993 \mathrm{Spr}$ & 4 & 3 & 3 \\
\hline 1993 Sum & $<2.7$ & $<5.1$ & $<4$ \\
\hline 1993 Fall & $<3.1$ & $<5.7$ & $<3.3$ \\
\hline 1994 Win & $<1.8$ & $<4.8$ & $<2.5$ \\
\hline 1994 Spr & $<2.8$ & $<4.5$ & $<4.7$ \\
\hline 1994 Sum & $<4.5$ & $<3.4$ & $<3.6$ \\
\hline 1994 Fall & $<2.2$ & 13.9 & $<3.4$ \\
\hline 1995 Win & $<3.0$ & $<4.9$ & $<4.8$ \\
\hline $1995 \mathrm{Spr}$ & $<3.4$ & $<4: 0$ & $<3.2$ \\
\hline 1995 Sum & $<3.2$ & $<5.4$ & $<3.8$ \\
\hline 1995 Fall & $<3.1$ & $<5.9$ & $<2.5$ \\
\hline
\end{tabular}

Gross Beta (pCi/L)

\begin{tabular}{l|lll} 
Event & PCU & STPO & PCD \\
\hline 1990 Fall & 17 & 20 & 8 \\
\hline 1991 Win & 7 & 34 & 13 \\
1991 Spr & 9 & 12 & 7 \\
1991 Sum & 3 & 11 & 9 \\
1991 Fall & $<3$ & 4 & 46 \\
\hline 1992 Win & 7 & 15 & 4 \\
1992 Spr & 4 & 6 & 10 \\
1992 Sum & 15 & 7 & 16 \\
1992 Fall & 29 & 37 & 34 \\
\hline 1993 Win & 5 & 9 & $<3$ \\
1993 Spr & 10 & 9 & 7 \\
1993 Sum & 2.7 & 27.3 & 4.3 \\
1993 Fall & $<2.7$ & 41.0 & 5.5 \\
\hline 1994 Win & $<2.4$ & 17.2 & 3.3 \\
1994 Spr & $<2.3$ & 11.9 & 8.8 \\
1994 Sum & 13.8 & 14.5 & 22 \\
1994 Fall & $<2.8$ & 17.1 & 3.9 \\
\hline 1995 Win & 8.2 & 17.2 & 3.9 \\
1995 Spr & $<3.6$ & 11.4 & $<3$ \\
1995 Sum & $<3.2$ & 19.3 & 7.3 \\
1995 Fall & 4.6 & 16.1 & 2.8 \\
\hline
\end{tabular}


Table C.11. Analytical Results for Surface Water, Fall 1990 Through Fall 1995, Cations and Anions

Calcium (mg/L)

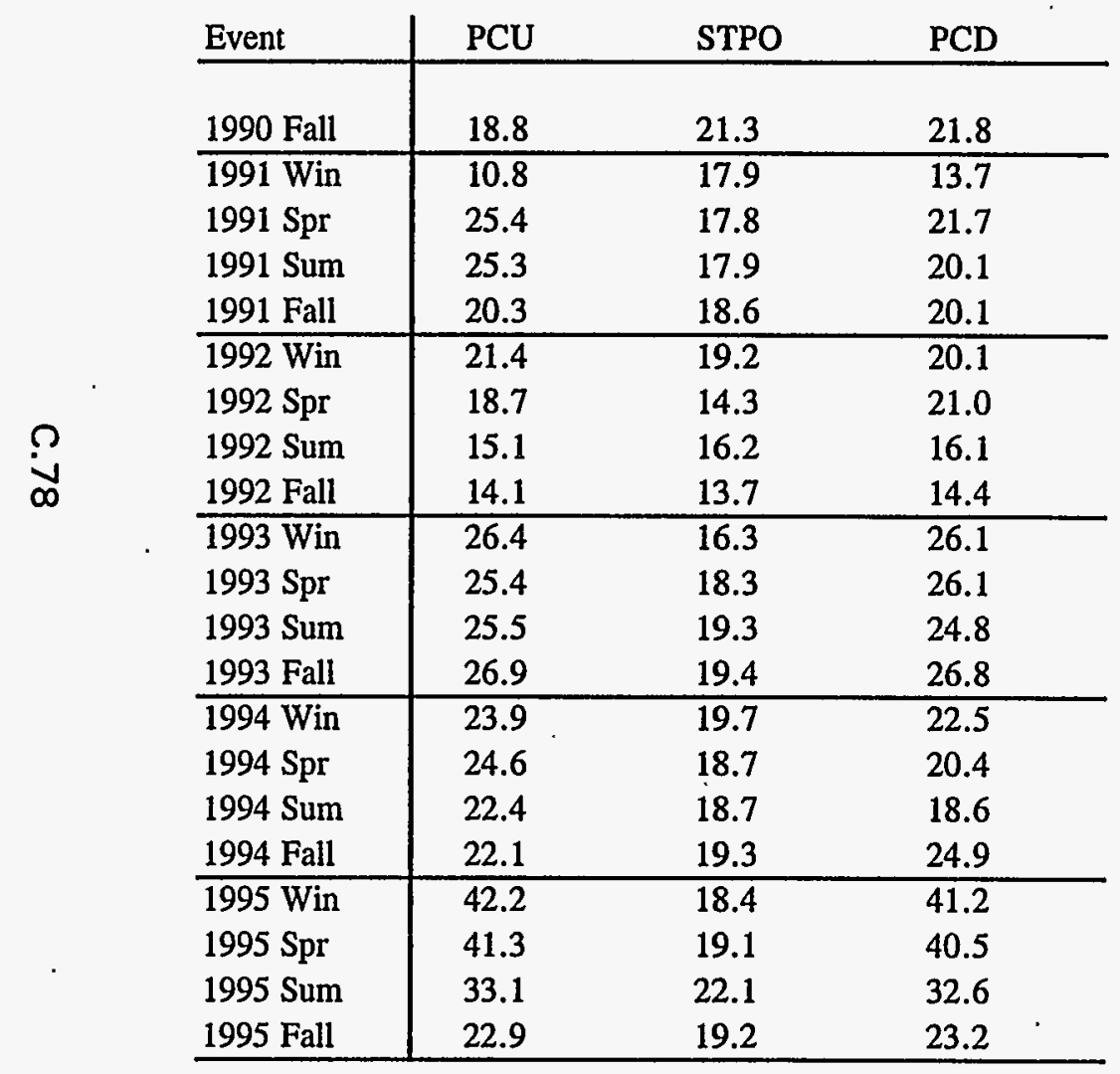

Magnesium (mg/L)

\begin{tabular}{l|lll} 
Event & PCU & STPO & PCD \\
\hline 1990 Fall & 21.5 & 20.8 & 21.0 \\
\hline 1991 Win & 6.0 & 17.7 & 7.4 \\
1991 Spr & 35.3 & 21.4 & 27.6 \\
1991 Sum & 36.7 & 20.4 & 25.3 \\
1991 Fall & 30.8 & 22.8 & 28.5 \\
\hline 1992 Win & 19.0 & 21.7 & 18.6 \\
1992 Spr & 36.1 & 20.7 & 28.2 \\
1992 Sum & 21.4 & 22.0 & 22.0 \\
1992 Fall & 23.9 & 23.7 & 22.9 \\
\hline 1993 Win & 20.2 & 23.0 & 20.5 \\
1993 Spr & 34.4 & 21.4 & 35.3 \\
1993 Sum & 35.8 & 21.6 & 33.2 \\
1993 Fall & 35.8 & 20.3 & 35.0 \\
\hline 1994 Win & 24.0 & 21.1 & 22.5 \\
1994 Spr & 38.6 & 21.6 & 28.5 \\
1994 Sum & 26.3 & 19.9 & 20.7 \\
1994 Fall & 32.0 & 19.5 & 33.4 \\
\hline 1995 Win & 44.0 & 18.4 & 41.9 \\
1995 Spr & 46.5 & 18.2 & 45.7 \\
1995 Sum & 38.5 & 23.1 & 38.1 \\
1995 Fall & 29.5 & 22.0 & 29.2 \\
\hline
\end{tabular}




\begin{tabular}{|c|c|c|c|}
\hline$\angle 8^{\circ} Z$ & $9+I$ & $0 S^{\prime} z$ & II $e_{4}$ S66I \\
\hline$\varsigma 6 \cdot \tau$ & 69.8 & $26^{\circ} \mathrm{I}$ & uns S66I \\
\hline$\angle 9^{\circ} I$ & $60^{\circ} 6$ & $\tau \varsigma^{\circ} I$ & IdS S66I \\
\hline $96 . I$ & $0.0 \mathrm{I}$ & $60^{\circ} 1$ & u!M $\ 66 \mathrm{I}$ \\
\hline $0 \tau^{\circ} \varsigma$ & $\varepsilon \cdot Z I$ & $29^{\circ} 1$ & IIPH $766 \mathrm{I}$ \\
\hline$t \tau * t$ & $L L S$ & $6 L \cdot 9$ & uns $766 I$ \\
\hline $80^{\circ} \mathrm{s}$ & 0.01 & $0 L^{\prime} I$ & IdS $766 I$ \\
\hline$t s^{\circ} t$ & $\varepsilon+l$ & IS $\varepsilon$ & u!M $\nabla 66 \mathrm{I}$ \\
\hline$t S^{\prime} Z$ & $I^{\prime} \varepsilon I$ & $20^{\circ} 1$ & II $e_{G} \varepsilon 66 I$ \\
\hline $0 z \tau$ & +1.8 & SS I I & uns \&66I \\
\hline $0 S^{\circ} \tau$ & $t^{\prime} s Z$ & $00^{\circ} z$ & JdS E66I \\
\hline$\angle E^{\prime} Z$ & $\tau \cdot \varepsilon I$ & [9. I & u!M \&66I \\
\hline Z.II & $0^{\circ} \mathrm{SI}$ & ह.9I & II $\mathbb{E}_{\mathrm{H}} \mathrm{Z66I}$ \\
\hline $06^{\circ} \mathrm{L}$ & 6.95 & $0 z: L$ & uns $266 I$ \\
\hline$t I^{\circ} 9$ & $\angle E^{\prime} 8$ & $68^{\circ} \mathrm{I}$ & IdS Z66I \\
\hline $06^{\circ}$ & $6 . \mathrm{II}$ & $08 \cdot z$ & U!M Z66I \\
\hline $09^{\circ} t$ & $6 \cdot 2 I$ & $0 L^{\prime} I$ & II $P_{\mathrm{H}} \mathrm{I} 66 \mathrm{I}$ \\
\hline $0 z^{\circ} 9$ & $0 \varepsilon^{\cdot} 8$ & $00^{\circ} \varepsilon$ & uns I66I \\
\hline $00^{\circ} 8$ & $6^{\circ} \mathrm{ZI}$ & $06^{\circ} \mathrm{I}$ & IdS I66I \\
\hline I. II & $\varepsilon^{\cdot} \varepsilon I$ & 619 & u!M I66I \\
\hline$\varepsilon^{\prime} 0 \mathrm{I}$ & I.OI & $t^{\circ} 0 I$ & II $\mathbb{R}_{H}$ 066I \\
\hline$\overline{\alpha \supset d}$ & OdIS & กगd & JuวAG \\
\hline
\end{tabular}

(T/8̂u) un!ssplod

\begin{tabular}{|c|c|c|c|}
\hline l'6I & $Z Z I$ & $\tau \cdot L I$ & II \\
\hline 8.60 & $\varepsilon \$ l$ & I.82 & wns $\$ 66 I$ \\
\hline 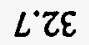 & $\angle E I$ & s.6z & IdS S66I \\
\hline $6.8 \varepsilon$ & $I t I$ & †'IE & u! $M S 66 \mathrm{I}$ \\
\hline $9: 65$ & t9I & 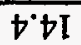 & IIP \\
\hline$\downarrow \varepsilon I$ & 9SI & $0 \phi I$ & uns $t 66 \mathrm{I}$ \\
\hline$\varepsilon<8$ & 6ZI & 6.12 & IdS $t 66 I$ \\
\hline $9^{\circ} 0 \varepsilon$ & $\downarrow 9 I$ & $L \cdot 8 I$ & u!M \\
\hline $9 \cdot 8 \varepsilon$ & t9I & s.6I & IIPG E66I \\
\hline$\dagger^{\dagger} I \varepsilon$ & ObI & $\angle \cdot 6 I$ & uns E66I \\
\hline $9^{\circ} \angle Z$ & $\tau " \tau \varepsilon$ & $L^{\prime} \varepsilon Z$ & JdS E66I \\
\hline $9 \cdot \varepsilon Z$ & IEI & $\tau \cdot \varepsilon z$ & u!M E66I \\
\hline ZEI & 9EI & $9 \varepsilon I$ & IIE] Z66I \\
\hline$\varepsilon b \mathrm{I}$ & $6 \varepsilon I$ & $\angle D I$ & uns $266 \mathrm{I}$ \\
\hline †.88 & โદા & {$[\cdot \tau z$} & Ids $266 \mathrm{I}$ \\
\hline I'It & EOI & $\tau \cdot \mathrm{sI}$ & u!M $\tau 66 \mathrm{I}$ \\
\hline $0.9 \varepsilon$ & 982 & $8^{\prime} \varepsilon Z$ & IIP] I66I \\
\hline LOI & $62 I$ & $8^{\circ} 6 t$ & uns I66I \\
\hline EII & $09 I$ & $6.6 I$ & IdS I66I \\
\hline L'89 & IZI & $8^{\circ} \mathcal{S E}$ & u!M I66I \\
\hline IOI & tOI & t0I & $\overline{\text { IIE⿹ 066I }}$ \\
\hline बगd & OdIS & กวd & 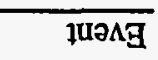 \\
\hline
\end{tabular}

(T/8ิtu) um!̣pos

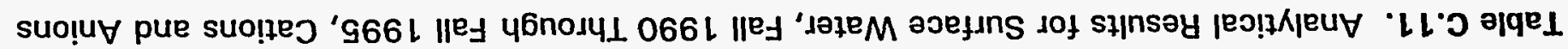


Table C.11. Analytical Results for Surface Water, Fall 1990 Through Fall 1995, Cations and Anions

Alkalinity (mg/L as $\mathrm{CaCO}_{3}$ )

\begin{tabular}{l|lll} 
Event & PCU & STPO & PCD \\
\hline & & & \\
1990 Fall & 198 & 204 & 187 \\
\hline 1991 Win & 115 & 194 & 138 \\
1991 Spr & 184 & 220 & 193 \\
1991 Sum & 246 & 217 & 194 \\
1991 Fall & 185 & 183 & 200 \\
\hline 1992 Win & 133 & 155 & 140 \\
1992 Spr & 219 & 174 & 200 \\
1992 Sum & 190 & 224 & 194 \\
1992 Fall & 185 & 216 & 160 \\
\hline 1993 Win & 146 & 172 & 141 \\
1993 Spr & 228 & 218 & 218 \\
1993 Sum & 210 & 210 & 200 \\
1993 Fall & 190 & 210 & 200 \\
\hline 1994 Win & 100 & 200 & 130 \\
1994 Spr & 210 & 200 & 160 \\
1994 Sum & 230 & 200 & 190 \\
1994 Fall & 170 & 180 & 190 \\
\hline 1995 Win & 250 & 180 & 250 \\
1995 Spr & 260 & 180 & 260 \\
1995 Sum & NA & NA & NA \\
1995 Fall & NA & NA & NA \\
\hline
\end{tabular}

Notes:

1. NA indicates that no sample was analyzed for this constituent.
Sulfate (mg/L)

\begin{tabular}{l|lll} 
Event & PCU & STPO & PCD \\
\hline 1990 Fall & 75.7 & 73.6 & 77.6 \\
\hline 1991 Win & 36.2 & 66.2 & 51.6 \\
1991 Spr & 26.8 & 64.8 & 54.6 \\
1991 Sum & 84.6 & 64.1 & 55.7 \\
1991 Fall & 22.4 & 50.4 & 30.6 \\
\hline 1992 Win & 24.4 & 41.0 & 26.6 \\
1992 Spr & 29.4 & 54.8 & 44.1 \\
1992 Sum & 61.9 & 52.5 & 66.6 \\
1992 Fall & 60.0 & 65.0 & 78.0 \\
\hline 1993 Win & 52.0 & 130 & 50.2 \\
1993 Spr & 34.5 & 62.8 & 38.1 \\
1993 Sum & 38.0 & 59.0 & 30.0 \\
1993 Fall & 25.0 & 58.0 & 29.0 \\
\hline 1994 Win & 28.0 & 56.0 & 30.0 \\
1994 Spr & 28.0 & 58.0 & 47.0 \\
1994 Sum & 93.0 & 56.0 & 68.0 \\
1994 Fall & 24.0 & 65.0 & 38.0 \\
\hline 1995 Win & 52.0 & 63.0 & 54.0 \\
1995 Spr & 34.0 & 54.0 & 34.0 \\
1995 Sum & NA & NA & NA \\
1995 Fall & NA & NA & NA \\
\hline
\end{tabular}

\section{Notes:}

1. NA indicates that no sample was analyzed for this constituent. 
Table C.11. Analytical Results for Surface Water, Fall 1990 Through Fall 1995, Cations and Anions

Total Phosphorus as P (mg/L)

\begin{tabular}{|c|c|c|c|}
\hline Event & PCU & STPO & PCD \\
\hline 1990 Fall & 4.5 & 4.40 & 3.2 \\
\hline 1991 Win & 1.0 & 4.70 & 2.8 \\
\hline $1991 \mathrm{Spr}$ & $<0.1$ & 3.60 & 1.6 \\
\hline 1991 Sum & $<0.1$ & 3.30 & 1.5 \\
\hline 1991 Fall & $<0.31$ & 11.7 & 3.2 \\
\hline 1992 Win & $<0.1$ & 4.70 & 0.90 \\
\hline $1992 \mathrm{Spr}$ & 0.22 & 2.50 & 1.1 \\
\hline 1992 Sum & 1.73 & 2.22 & 1.49 \\
\hline 1992 Fall & 4.07 & 4.78 & 3.44 \\
\hline 1993 Win & 0.09 & 2.91 & 0.12 \\
\hline $1993 \mathrm{Spr}$ & $<0.05$ & 2.94 & .0 .16 \\
\hline 1993 Sum & $<1$ & $<10$ & $<1$ \\
\hline 1993 Fall & $<1$ & $<10$ & $<1$ \\
\hline 1994 Win & $<1$ & $<2$ & $<1$ \\
\hline $1994 \mathrm{Spr}$ & $<1$ & 2.90 & $<1$ \\
\hline 1994 Sum & $<2$ & $<2$ & $<2$ \\
\hline 1994 Fall & $<1$ & $<4$ & $<1$ \\
\hline 1995 Win & $<1$ & $<2$ & $<1$ \\
\hline $1995 \mathrm{Spr}$ & $<1$ & $<5$ & $<1$ \\
\hline 1995 Sum & NA & NA & NA \\
\hline 1995 Fall & NA & NA & NA \\
\hline
\end{tabular}

Notes:

1. NA indicates that no sample was analyzed for this constituent.
Chloride (mg/L)

\begin{tabular}{l|lll} 
Event & PCU & STPO & PCD \\
\hline 1990 Fall & 156 & 139 & 156 \\
\hline 1991 Win & 29 & 142 & 63 \\
1991 Spr & 13 & 76 & 65 \\
1991 Sum & 33 & 156 & 146 \\
1991 Fall & 8.5 & 106 & 57 \\
\hline 1992 Win & 12 & 181 & 49 \\
1992 Spr & 19 & 147 & 93 \\
1992 Sum & 160 & 148 & 139 \\
1992 Fall & 147 & 124 & 165 \\
\hline 1993 Win & 13 & 121 & 13 \\
1993 Spr & 19 & 128 & 25 \\
1993 Sum & 16 & 130 & 26 \\
1993 Fall & 15 & 150 & 32 \\
\hline 1994 Win & 12 & 150 & 23 \\
1994 Spr & 17 & 100 & 85 \\
1994 Sum & 100 & 130 & 120 \\
1994 Fall & 12 & 160 & 57 \\
\hline 1995 Win & 27 & 120 & 37 \\
1995 Spr & 26 & 110 & 27 \\
1995 Sum & NA & NA & NA \\
1995 Fall & NA & NA & NA \\
\hline
\end{tabular}

Notes:

1. NA indicates that no sample was analyzed for this constituent. 
Table C.11. Analytical Results for Surface Water, Fall 1990 Through Fall 1995, Cations and Anions

Nitrate-Nitrogen (ug/L)

\begin{tabular}{|c|c|c|c|c|}
\hline & Event & PCU & STPO & PCD \\
\hline & 1990 Fall & 7300 & 6500 & 5700 \\
\hline & $1991 \mathrm{Win}$ & 400 & 11900 & 2000 \\
\hline & $1991 \mathrm{Spr}$ & $<100$ & 6500 & 5700 \\
\hline & 1991 Sum & $<100$ & 5220 & 3910 \\
\hline & 1991 Fall & $<100$ & 10940 & 3470 \\
\hline & 1992 Win & 300 & 8750 & 2340 \\
\hline & 1992 Spr & $<100$ & 11100 & 3510 \\
\hline & 1992 Sum & 5980 & 5720 & 3960 \\
\hline & 1992 Fall & 3960 & 2200 & 4090 \\
\hline$\infty$ & 1993 Win & 230 & 8950 & 280 \\
\hline & $1993 \mathrm{Spr}$ & 1210 & 4700 & 1080 \\
\hline & 1993 Sum & 540 & 3100 & 690 \\
\hline & 1993 Fall & 1000 & 3100 & 1700 \\
\hline & 1994 Win & 4300 & 6700 & 1600 \\
\hline & 1994 Spr & $<100$ & 6100 & 3700 \\
\hline & 1994 Sum & 1100 & 890 & 1500 \\
\hline & 1994 Fall & 290 & 3600 & 3600 \\
\hline 3 & 1995 Win & 2600 & 7500 & 2700 \\
\hline & $1995 \mathrm{Spr}$ & 2600 & 9400 & 2600 \\
\hline & 1995 Sum & 1500 & 1500 & 1900 \\
\hline & 1995 Fall & 1800 & 3000 & 1800 \\
\hline
\end{tabular}

Ammonia-Nitrogen (ug/L)

\begin{tabular}{l|lll} 
Event & PCU & STPO & PCD \\
\hline & & & \\
1990 Fall & NA & NA & NA \\
\hline 1991 Win & NA & NA & NA \\
1991 Spr & NA & NA & NA \\
1991 Sum & NA & NA & NA \\
1991 Fall & NA & NA & NA \\
\hline 1992 Win & NA & NA & NA \\
1992 Spr & NA & NA & NA \\
1992 Sum & NA & NA & NA \\
1992 Fall & NA & NA & NA \\
\hline 1993 Win & NA & NA & NA \\
1993 Spr & NA & NA & NA \\
1993 Sum & 67 & 3900 & 560 \\
1993 Fall & $<50$ & 8300 & 530 \\
\hline 1994 Win & 97 & 4900 & 290 \\
1994 Spr & 68 & 4800 & 600 \\
1994 Sum & 1500 & 3300 & 77 \\
1994 Fall & $<50$ & 5800 & 350 \\
\hline 1995 Win & $<50$ & 3200 & $<50$ \\
1995 Spr & $<50$ & 1400 & $<50$ \\
1995 Sum & NA & NA & NA \\
1995 Fall & NA & NA & NA \\
\hline & & &
\end{tabular}

Notes:

1. NA indicates that no sample was analyzed for this constituent. 
Table C.12. Analytical Results for Surface Water, Fall 1990 Through Fall 1995, Other Parameters

pH (st'd units)

\begin{tabular}{ll|lll} 
Event & PCU & STPO & PCD \\
\hline & & & \\
1990 Fall & 7.2 & 7.3 & 7.4 \\
\hline 1991 Win & 8.3 & 7.3 & 7.6 \\
1991 Spr & 8.8 & 7.3 & 8.2 \\
1991 Sum & 9.2 & 7.3 & 8.8 \\
1991 Fall & 8.4 & 7.4 & 8.6 \\
\hline 1992 Win & 8.4 & 7.3 & 8.0 \\
1992 Spr & 8.9 & 7.2 & 8.3 \\
1992 Sum & 7.8 & 7.5 & 7.8 \\
1992 Fall & 7.5 & 7.5 & 7.7 \\
\hline 1993 Win & NA & NA & NA \\
1993 Spr & 7.6 & 8.0 & 7.7 \\
1993 Sum & 8.1 & 7.5 & 7.1 \\
1993 Fall & 8.1 & 7.4 & 7.8 \\
\hline 1994 Win & 7.9 & 7.0 & 7.7 \\
1994 Spr & 8.4 & 7.2 & 7.7 \\
1994 Sum & 7.6 & 7.1 & 6.9 \\
1994 Fall & 8.2 & 7.0 & 7.5 \\
\hline 1995 Win & 8.0 & 7.2 & 7.9 \\
1995 Spr & 8.2 & 7.5 & 8.2 \\
1995 Sum & 9.1 & 7.6 & 8.5 \\
1995 Fall & - & - & - \\
\hline
\end{tabular}

Notes:
1. All measurement were made in the field.
2. Field data for Fall 1995 are unavailable.

Field record forms lost by sampling team.

3. NA indicates that no sample was analyzed for this constituent.
Temperature $\left({ }^{\circ} \mathrm{C}\right)$

\begin{tabular}{l|lll} 
Event & PCU & STPO & PCD \\
\hline 1990 Fall & 16.5 & 22.8 & 16.0 \\
\hline 1991 Win & 14.1 & 17.9 & 16.6 \\
1991 Spr & 20.7 & 23.9 & 23.2 \\
1991 Sum & 25.6 & 27.2 & 28.9 \\
1991 Fall & 10.5 & 20.0 & 16.0 \\
\hline 1992 Win & 14.4 & 23.6 & 19.7 \\
1992 Spr & 22.4 & 24.7 & 27.7 \\
1992 Sum & 20.8 & 28.8 & 25.8 \\
1992 Fall & 14.1 & 20.8 & 14.1 \\
\hline 1993 Win & 9.4 & 17.1 & 9.2 \\
1993 Spr & 19.9 & 20.7 & 19.8 \\
1993 Sum & 23.3 & 27.1 & 24.0 \\
1993 Fall & 10.2 & 19.3 & 11.2 \\
\hline 1994 Win & 10.9 & 20.2 & 14.1 \\
1994 Spr & 19.8 & 25.2 & 25.4 \\
1994 Sum & 22.0 & 25.9 & 21.0 \\
1994 Fall & 6.8 & 22.2 & 9.2 \\
\hline 1995 Win & 13.7 & 20.1 & 14.2 \\
1995 Spr & 20.1 & 22.0 & 20.4 \\
1995 Sum & 22.3 & 27.8 & 29.2 \\
1995 Fall & - & - & - \\
\hline
\end{tabular}

Notes:

1. All measurement were made in the field.

2. Field data for Fall 1995 are unavailable.

Field record forms lost by sampling team. 
Table C.12. Analytical Results for Surface Water, Fall 1990 Through Fall 1995, Other Parameters

Turbidity - Field

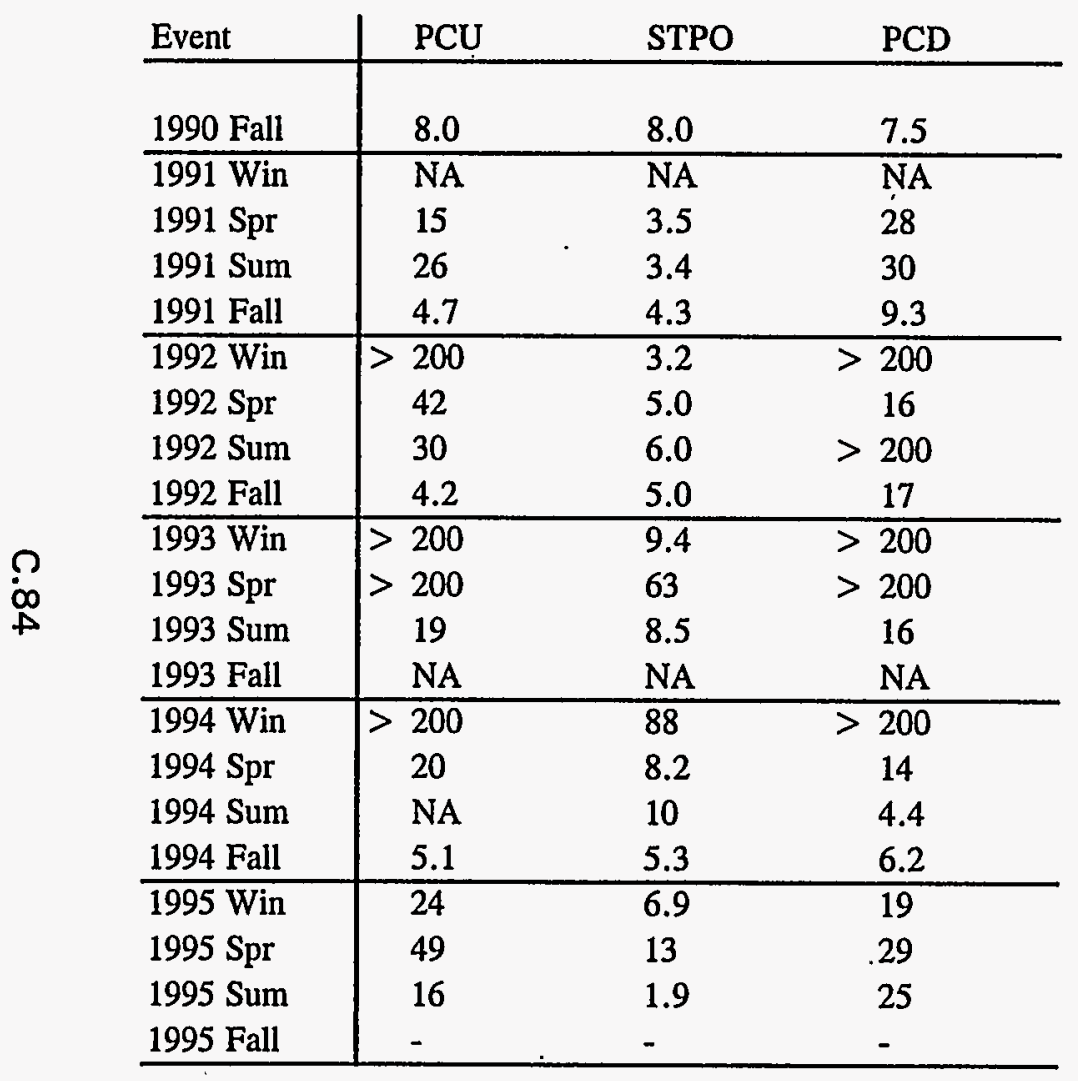

Notes:

1. All measurement were made in the field.

2. Field data for Fall 1995 are unavailable.

Field record forms lost by sampling team.

3. NA indicates that no sample was analyzed for this constituent.
Turbidity - Lab

\begin{tabular}{l|lll} 
Event & PCU & STPO & PCD \\
\hline 1990 Fall & 6.5 & 9.0 & 8.4 \\
\hline 1991 Win & 52 & 6.4 & 37 \\
1991 Spr & 39 & 4.5 & 25 \\
1991 Sum & 29 & 7.3 & 26 \\
1991 Fall & 8.5 & 8.6 & 8.7 \\
\hline 1992 Win & 110 & 2.6 & 77 \\
1992 Spr & 31 & 2.8 & 14 \\
1992 Sum & 8.1 & 1.8 & 8.6 \\
1992 Fall & 3.9 & 3.5 & 18 \\
\hline 1993 Win & 128 & 5.9 & 112 \\
1993 Spr & 10 & 16 & 25 \\
1993 Sum & 19 & 7.1 & 16 \\
1993 Fall & 4.8 & 4.7 & 10 \\
\hline 1994 Win & 130 & 5.5 & 120 \\
1994 Spr & 18 & 6.9 & 17 \\
1994 Sum & 59 & 6.9 & 2.8 \\
1994 Fall & 10 & 4.6 & 4.7 \\
\hline 1995 Win & 37 & 6.2 & 31 \\
1995 Spr & 62 & 9.3 & 32 \\
1995 Sum & NA & NA & NA \\
1995 Fall & NA & NA & NA \\
\hline
\end{tabular}

Notes:

1. NA indicates that no sample was analyzed for this constituent. 
Table C.12. Analytical Results for Surface Water, Fall 1990 Through Fall 1995, Other Parameters

Specific Conductance (umhos/cm)

\begin{tabular}{ll|lll} 
& Event & PCU & STPO & PCD \\
\hline & 1990 Fall & 1080 & 1237 & 1017 \\
\hline 1991 Win & 365 & 1223 & 622 \\
1991 Spr & 381 & 982 & 680 \\
1991 Sum & 691 & 1166 & 985 \\
1991 Fall & 373 & 1110 & 569 \\
\hline 1992 Win & 333 & 1111 & 518 \\
1992 Spr & 487 & 953 & 728 \\
1992 Sum & 840 & 770 & 1390 \\
& 1992 Fall & 967 & 1042 & 894 \\
\hline 1993 Win & 453 & 1190 & 404 \\
o & 1993 Spr & 670 & 1310 & 749 \\
& 1993 Sum & 488 & 1101 & 566 \\
1993 Fall & 332 & 979 & 410 \\
\hline 1994 Win & 263 & 506 & 349 \\
1994 Spr & 446 & 917 & 766 \\
1994 Sum & 933 & 577 & 882 \\
1994 Fall & 283 & 1001 & 409 \\
\hline 1995 Win & 512 & 834 & 535 \\
1995 Spr & 589 & 870 & 592 \\
1995 Sum & $1.2 \mathrm{R}$ & $2.15 \mathrm{R}$ & $1.25 \mathrm{R}$ \\
& 1995 Fall & - & - & - \\
\hline
\end{tabular}

Total Dissolved Solids (mg/L)

\begin{tabular}{l|lll} 
Event & PCU & STPO & PCU \\
\hline 1990 Fall & 642 & 602 & 644 \\
\hline 1991 Win & 202 & 596 & 326 \\
1991 Spr & 277 & 558 & 459 \\
1991 Sum & 430 & 632 & 568 \\
1991 Fall & 196 & 605 & 306 \\
\hline 1992 Win & 210 & 625 & 296 \\
1992 Spr & 400 & 619 & 507 \\
1992 Sum & 572 & 575 & 562 \\
1992 Fall & 582 & 515 & 593 \\
\hline 1993 Win & 225 & 514 & 236 \\
1993 Spr & 289 & 595 & 298 \\
1993 Sum & 290 & 560 & 290 \\
1993 Fall & 260 & 590 & 280 \\
\hline 1994 Win & 220 & 600 & 250 \\
1994 Spr & 260 & 500 & 410 \\
1994 Sum & 570 & 560 & 540 \\
1994 Fall & 220 & 590 & 330 \\
\hline 1995 Win & 380 & 530 & 390 \\
1995 Spr & 370 & 540 & 360 \\
1995 Sum & 290 & 640 & 420 \\
1995 Fall & 240 & 550 & 300 \\
\hline
\end{tabular}

Notes:

1. All measurements were made in the field.

2. Summer 1995 data rejected - no calibration records

3. Field data for Fall 1995 are unavailable.

Field record forms lost by sampling team. 
Table C.12. Analytical Results for Surface Water, Fall 1990 Through Fall 1995, Other Parameters

Total Kjeldahl Nitrogen (ug/L)

\begin{tabular}{|c|c|c|c|}
\hline Event & PCU & STPO & PCD \\
\hline 1990 Fall & NA & NA & NA \\
\hline 1991 Win & $\mathrm{NA}$ & $\mathrm{NA}$ & $\mathrm{NA}$ \\
\hline $1991 \mathrm{Spr}$ & NA & NA & NA \\
\hline 1991 Sum & NA & NA & NA \\
\hline 1991 Fall & NA & NA & NA \\
\hline 1992 Win & $\overline{N A}$ & $\overline{\mathrm{NA}}$ & NA \\
\hline $1992 \mathrm{Spr}$ & NA & NA & NA \\
\hline 1992 Sum & NA & NA & NA \\
\hline 1992 Fall & $\mathrm{NA}$ & NA & $\mathrm{NA}$ \\
\hline 1993 Win & $\overline{N A}$ & $\mathrm{NA}$ & $\overline{\mathrm{NA}}$ \\
\hline $1993 \mathrm{Spr}$ & $\mathrm{NA}$ & NA & NA \\
\hline 1993 Sum & $<500$ & 4000 & 670 \\
\hline 1993 Fall & $<500$ & 9200 & 1300 \\
\hline 1994 Win & $<500$ & 6200 & 930 \\
\hline $1994 \mathrm{Spr}$ & $<500$ & 6700 & 1500 \\
\hline 1994 Sum & 2200 & 3500 & 660 \\
\hline 1994 Fall & $<500$ & 6100 & 720 \\
\hline 1995 Win & $<500$ & 3100 & $<500$ \\
\hline $1995 \mathrm{Spr}$ & $<500$ & 2500 & $<500$ \\
\hline 1995 Sum & NA & $\mathrm{NA}$ & NA \\
\hline 1995 Fall & $\mathrm{NA}$ & NA: & $\mathrm{NA}$ \\
\hline
\end{tabular}

Notes:

1. NA indicates that no sample was analyzed for this constituent.
Total Organic Carbon (mg/L)

\begin{tabular}{l|lll} 
Event & PCU & STPO & PCD \\
\hline & & & \\
1990 Fall & NA & NA & NA \\
\hline 1991 Win & NA & NA & NA \\
1991 Spr & NA & NA & NA \\
1991 Sum & NA & NA & NA \\
1991 Fall & NA & NA & NA \\
\hline 1992 Win & NA & NA & NA \\
1992 Spr & NA & NA & NA \\
1992 Sum & NA & NA & NA \\
1992 Fall & NA & NA & NA \\
\hline 1993 Win & NA & NA & NA \\
1993 Spr & NA & NA & NA \\
1993 Sum & 11 & 7.7 & 11 \\
1993 Fall & 3.6 & 7.1 & 4.3 \\
\hline 1994 Win & 9.4 & 8.4 & 6.5 \\
1994 Spr & 5.1 & 8.7 & 6.7 \\
1994 Sum & 6.0 & 4.2 & 4.8 \\
1994 Fall & 3.7 & 6.5 & 4.6 \\
\hline 1995 Win & 2.6 & 7.4 & 2.9 \\
1995 Spr & 2.5 & 8.0 & 2.6 \\
1995 Sum & NA & NA & NA \\
1995 Fall & NA & NA & NA \\
\hline & & &
\end{tabular}

Notes:

1. NA indicates that no sample was analyzed for this constituent. 
Table C.12. Analytical Results for Surface Water, Fall 1990 Through Fall 1995, Other Parameters

\begin{tabular}{|c|c|c|c|}
\hline Event & PCU & STPO & PCD \\
\hline 1990 Fall & $\mathrm{NA}$ & NA & $\mathrm{NA}$ \\
\hline 1991 Win & $\mathrm{NA}$ & $\mathrm{NA}$ & $\mathrm{NA}$ \\
\hline $1991 \mathrm{Spr}$ & NA & NA & NA \\
\hline 1991 Sum & NA & NA & NA \\
\hline 1991 Fall & NA & NA & $\mathrm{NA}$ \\
\hline 1992 Win & $\mathrm{NA}$ & $\overline{\mathrm{NA}}$ & $\mathrm{NA}$ \\
\hline $1992 \mathrm{Spr}$ & NA & NA & NA \\
\hline 1992 Sum & NA & NA & NA \\
\hline 1992 Fall & $\mathrm{NA}$ & $\mathrm{NA}$ & $\mathrm{NA}$ \\
\hline 1993 Win & $\overline{\mathrm{NA}}$ & NA & $\mathrm{NA}$ \\
\hline $1993 \mathrm{Spr}$ & NA & NA & NA \\
\hline 1993 Sum & NA & NA & NA \\
\hline 1993 Fall & 39 & 52 & 41 \\
\hline 1994 Win & 127 & 132 & 133 \\
\hline $1994 \mathrm{Spr}$ & 108 & 88 & 81 \\
\hline 1994 Sum & 164 & 192 & 167 \\
\hline 1994 Fall & 148 & 174 & 181 \\
\hline 1995 Win & 151 & 163 & 174 \\
\hline $1995 \mathrm{Spr}$ & 84 & 141 & 151 \\
\hline 1995 Sum & NA & $\mathrm{NA}$ & $\mathrm{NA}$ \\
\hline 1995 Fall & NA & NA & NA \\
\hline
\end{tabular}

Chemical Oxygen Demand (mg/L)

\section{Notes:}

1. All measurements were made in the field.

2. NA indicates that no sample was analyzed for this constituent. -

\begin{tabular}{l|lll} 
Event & PCU & STPO & PCD \\
\hline 1990 Fall & 23.3 & 32.9 & 32.9 \\
\hline 1991 Win & 90.8 & 8.0 & 151 \\
1991 Spr & 496 & 279 & 49.4 \\
1991 Sum & 23.6 & 23.8 & 25.7 \\
1991 Fall & 27.2 & 46.4 & 40.0 \\
\hline 1992 Win & 10.8 & 20.2 & 10.4 \\
1992 Spr & 5.0 & $<1$ & 10.0 \\
1992 Sum & $<$ & $<1$ & $<1.0$ \\
1992 Fall & 16.6 & 27.5 & 20.2 \\
\hline 1993 Win & 27.9 & 55.8 & 4.0 \\
1993 Spr & 5.2 & 31.2 & 36.3 \\
1993 Sum & NA & NA & NA \\
1993 Fall & NA & NA & NA \\
\hline 1994 Win & NA & NA & NA \\
1994 Spr & NA & NA & NA \\
1994 Sum & NA & NA & NA \\
1994 Fall & 19.0 & 25.0 & 19.0 \\
\hline 1995 Win & $<50$ & $<50$ & $<50$ \\
1995 Spr & $<50$ & $<50$ & $<50$ \\
1995 Sum & NA & NA & NA \\
1995 Fall & NA & NA & NA \\
\hline
\end{tabular}

Notes:

1. NA indicates that no sample was analyzed for this constituent. 

Appendix D

Fall Quarter 1995 Water Monitoring 



\section{Appendix D}

\section{Fall Quarter 1995 Water Monitoring}

This Appendix summarizes activities associated with fall quarter 1995 water monitoring. Sampling and shipping procedures, quality assurance activities, and data validation for the fall quarter are discussed. Fall quarter data are presented along with other 1995 results in the main body of this report.

\section{Sampling and Shipping Procedures}

Twenty-three groundwater samples (including two duplicate samples) were collected from 21 monitoring wells, and four surface water samples (including one duplicate sample) were collected from three surface water locations between November 28 and

December 12, 1995. Groundwater and surface water samples were collected and handled according to procedures described in the Water Monitoring Plan (PNL 1994). Storm water samples were collected and handled according to procedures described in the Final Draft RI/FS Work Plan (DOE 1994a). Groundwater monitoring well and surface water sample locations are presented in Figures 2.2, 2.3, and 2.4, in Section 2 of the main report.

Groundwater samples were collected by purging and sampling monitoring wells with dedicated electric submersible pumps. Duplicate samples were collected from monitoring wells UCD1-13 and UCD1-12.

Surface water samples were collected from three locations along Putah Creek: the UC Davis Wastewater Treatment Plant outfall tributary (designated STPO); upstream of the LEHR facility (designated PCU); and downstream of the LEHR facility (designated PCD) (Figure 2.4 in Section 2 of the report). A duplicate sample was collected at sample location PCD.

Requested chemical and radiological analyses of groundwater and surface water are listed in Tables 2.2, 2.3, 2.4, and 2.5. Samples were shipped daily via Federal Express for overnight delivery to Lockheed Analytical Services (Lockheed) in Las Vegas, Nevada. Immediately upon receipt of the sample shipment, Lockheed documented the condition of the sample containers on the chain-of-custody forms and faxed a copy of the chain-ofcustody to the task manager. Lockheed was given approval to begin analyses on all samples that arrived in acceptable condition. 


\section{Quality Assurance Activities}

On December 6 and 7, 1995, an audit of Lockheed Analytical Services was conducted by PNNL Quality Assurance and technical staff. Laboratory operations and quality assurance practices were reviewed to assess compliance with project controlling documents, including the PNNL Master Agreement, the Laboratory Services Agreement, the Quality Assurance Project Plan (QAPjP) (PNL 1994) and USEPA standard methods. Details of the audit results are discussed in Section 2.0 of this report.

\section{Data Validation}

The results of the data validation indicate that some sample results for the field and laboratory quality control data fell outside specified validation criteria: field duplicate imprecision for some radionuclides, contamination of some laboratory method blanks from acetone, calibration failures associated with volatile analyses, and some surrogate recoveries for pesticide results that fell below the acceptable ranges established in the QAPjP (the criteria for pesticide surrogates are advisory only, and the results did not impact the data quality). Data validation flags have been applied to those sample results (see Appendixes A and B). Field duplicates were collected from wells UCD1-12 and UCD113 , and surface water location PCD.

During the radiation process, some data points were identified as unreliable. Instrument calibration failures for volatile compounds were noted for acetone and 2-butanone. This qualification is based on validation guidance documents, which differ from technical requirements. For subject compounds, the method has no established criteria for . calibration. However, for the purpose of data validation, the criteria established for other compounds are presumed reliable for all compounds. Although qualified as unreliable, these calibration failures are not expected to impact the data significantly. Historically, these compounds have not been detected in groundwater monitoring samples at the LEHR site, until this fall when detected in well UCD1/2-27.

The presence of acetone, a common laboratory contaminant, in the associated laboratory method blanks was not significant. Some primary samples had reported detections of acetone at.concentrations similar to those reported in the method blanks. Sample results associated with these blanks have been qualified with " $\mathrm{J}$ " to indicate the bias introduced by the analytical process (see Tables 5.3 and 6.3).

The calculated relative percent difference between field duplicate samples collected and analyzed for fall quarter were within specified guidelines with two exceptions, radium-226 and bismuth-214 for well UCD1-13. The duplicate results for fall quarter monitoring indicate that samples were representative of field conditions.

Data validation results show that requested analyses were completed. The required holding time for each analytical method was met. More than $98 \%$ of the data were judged to be valid, according to criteria set forth in the QAPjP. The fall quarter 1995 data, which 
Data validation results show that requested analyses were completed. The required holding time for each analytical method was met. More than $98 \%$ of the data were judged to be valid, according to criteria set forth in the QAPjP. The fall quarter 1995 data, which include approximately 3,144 individual analytical results (both detected and nondetected), were deemed reliable for their intended purpose with the exception of 36 results. A detailed discussion of the data validation results is presented in Section 3 of this report. 

Appendix E

Groundwater Elevation Data 



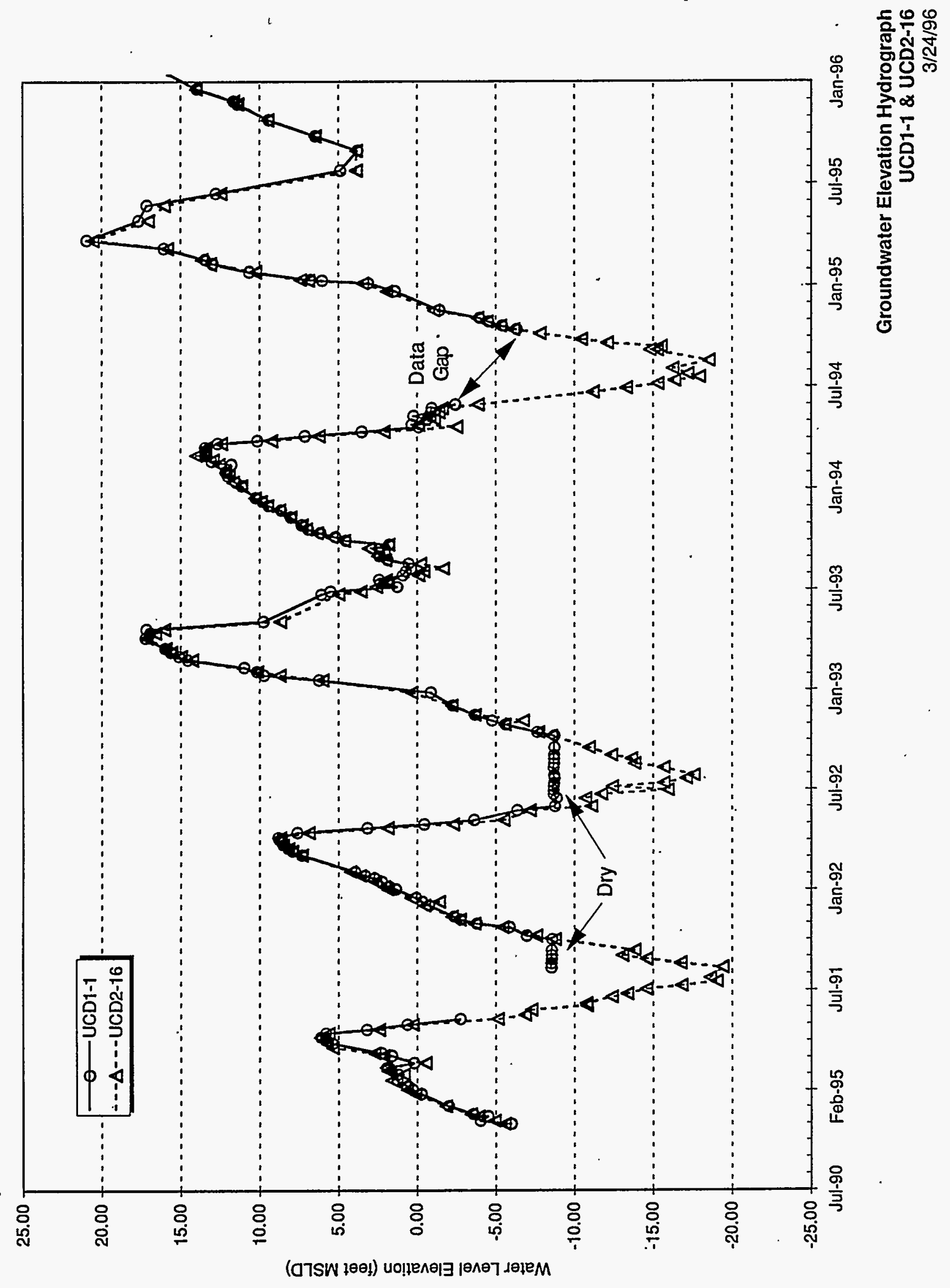

E.1 


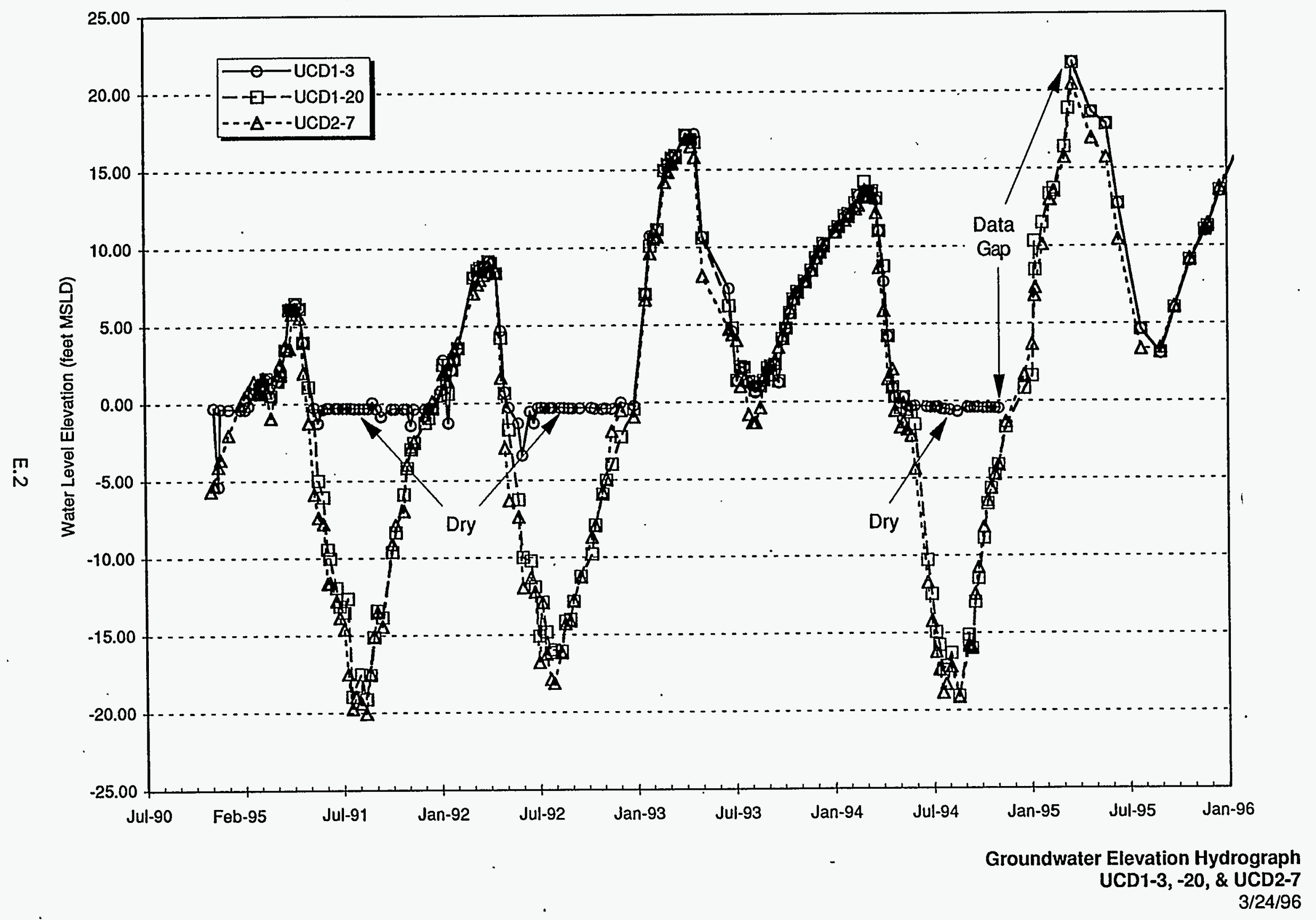




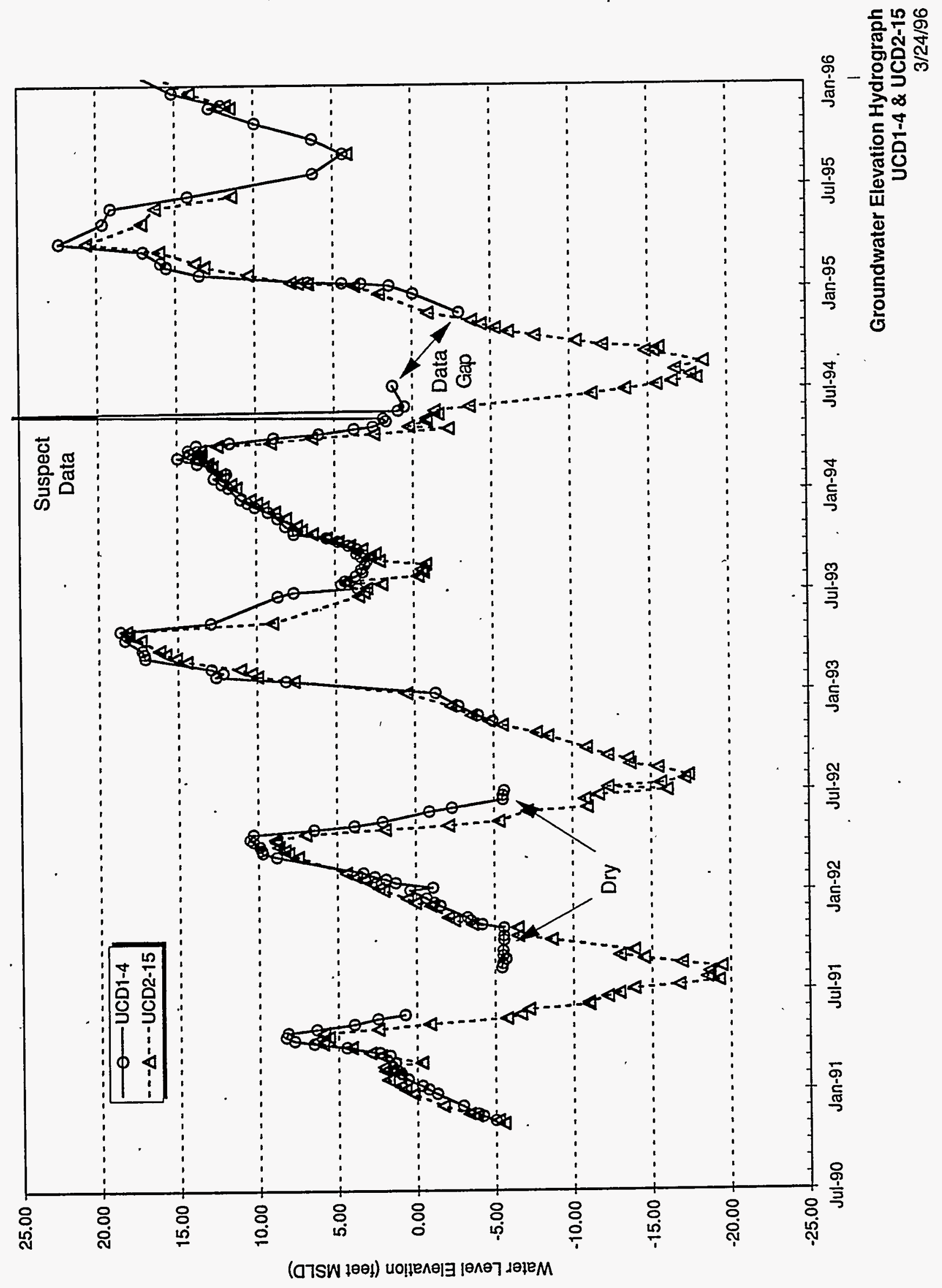

E. 3 


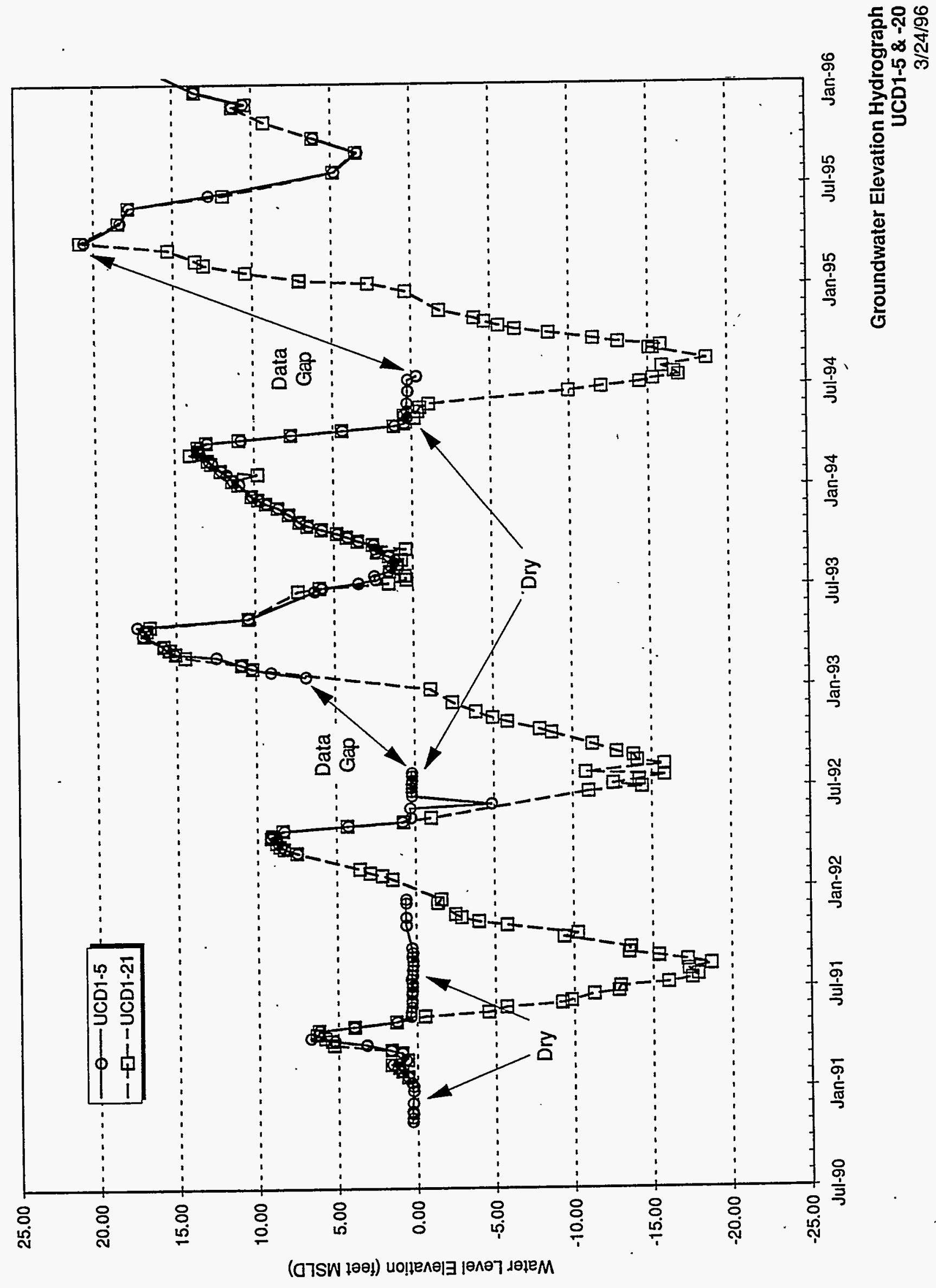

E. 4 


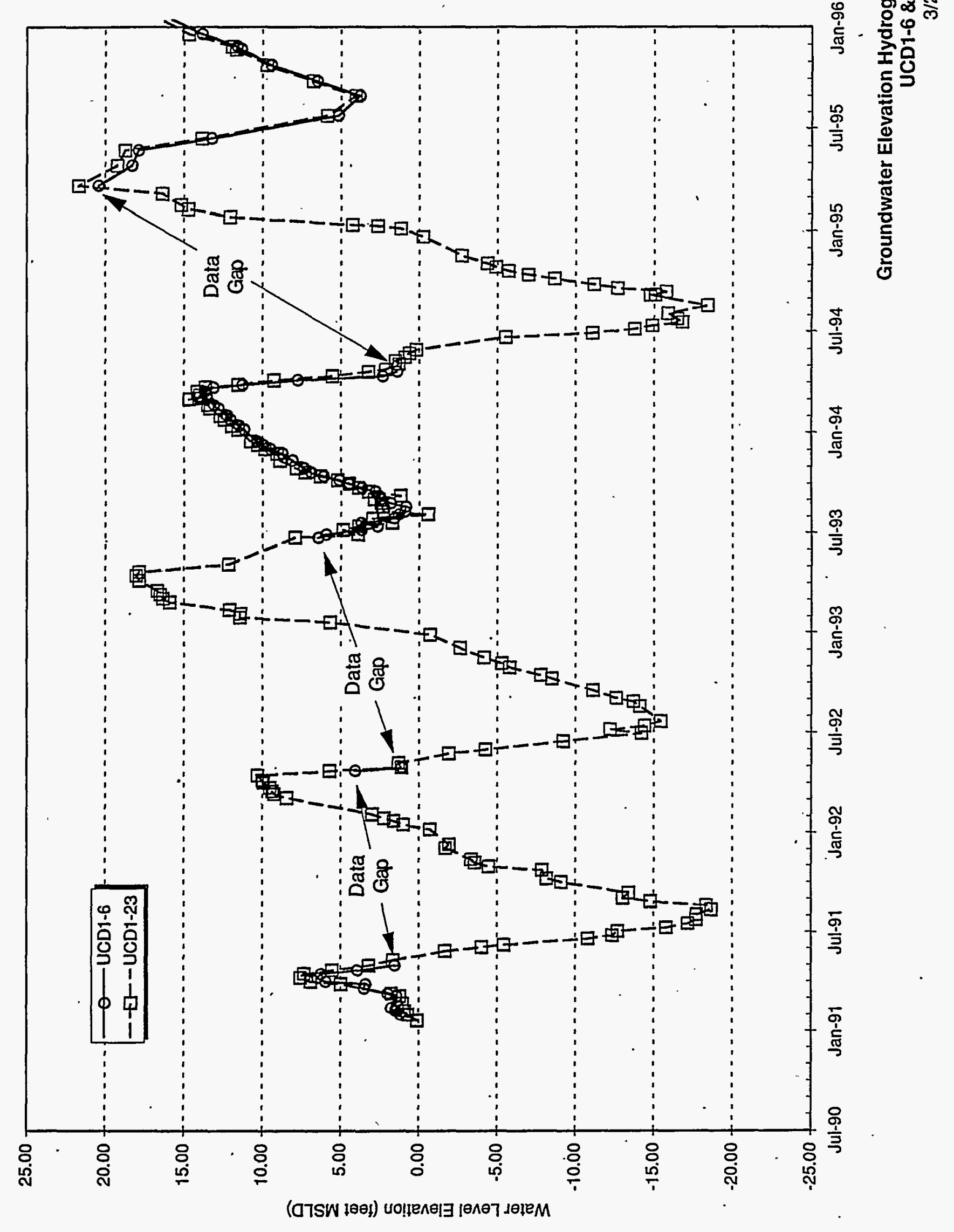

E. 5 


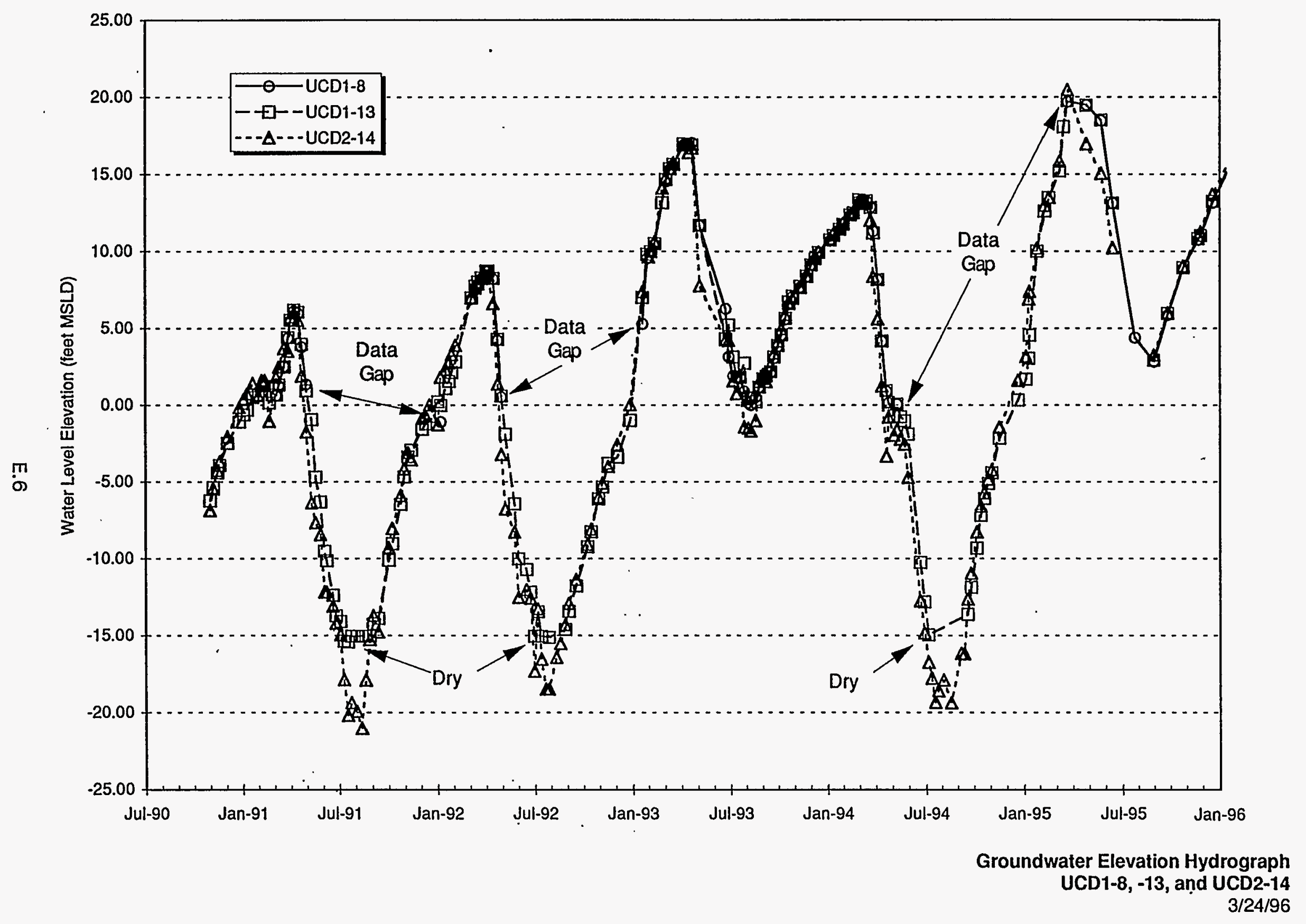




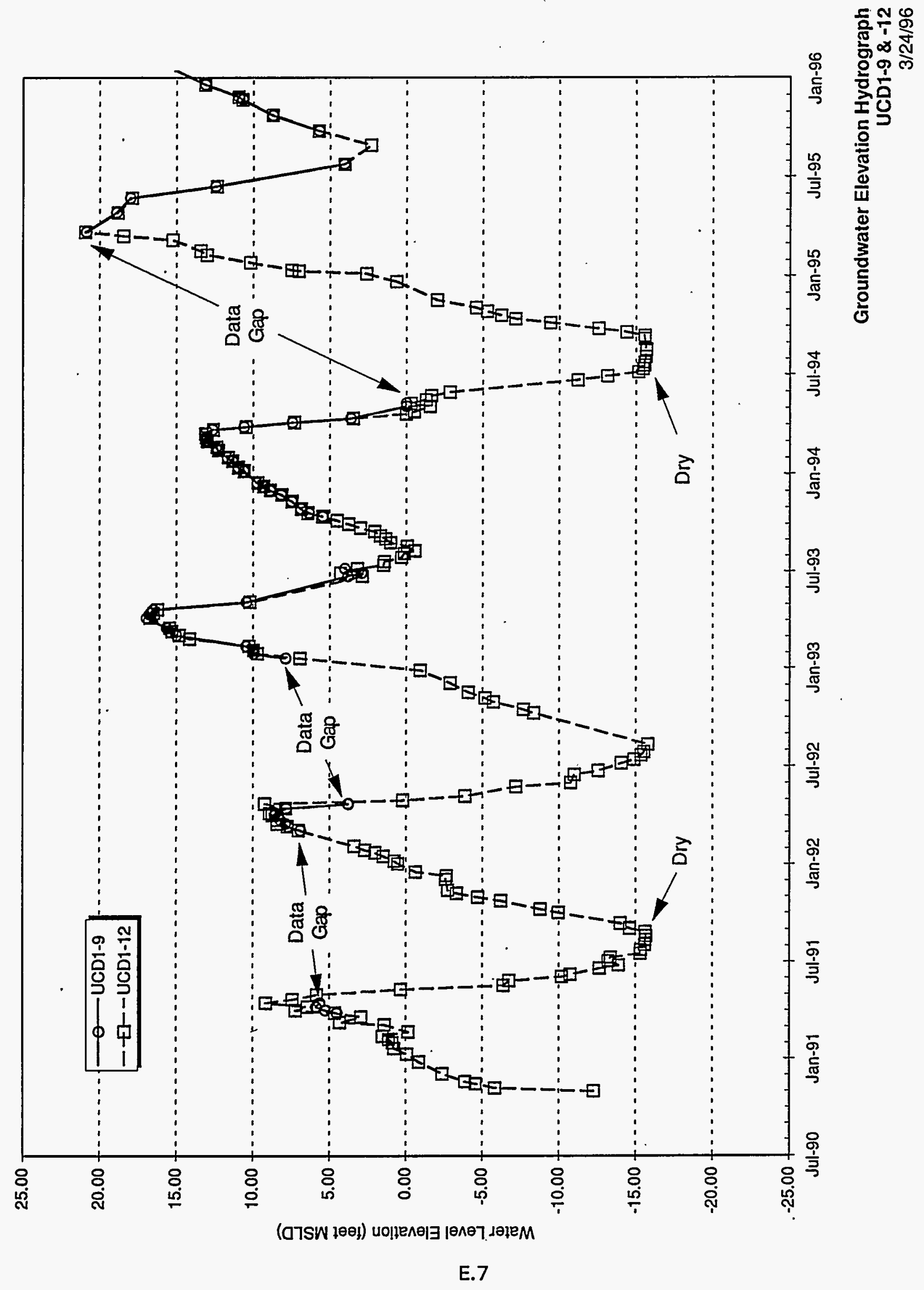




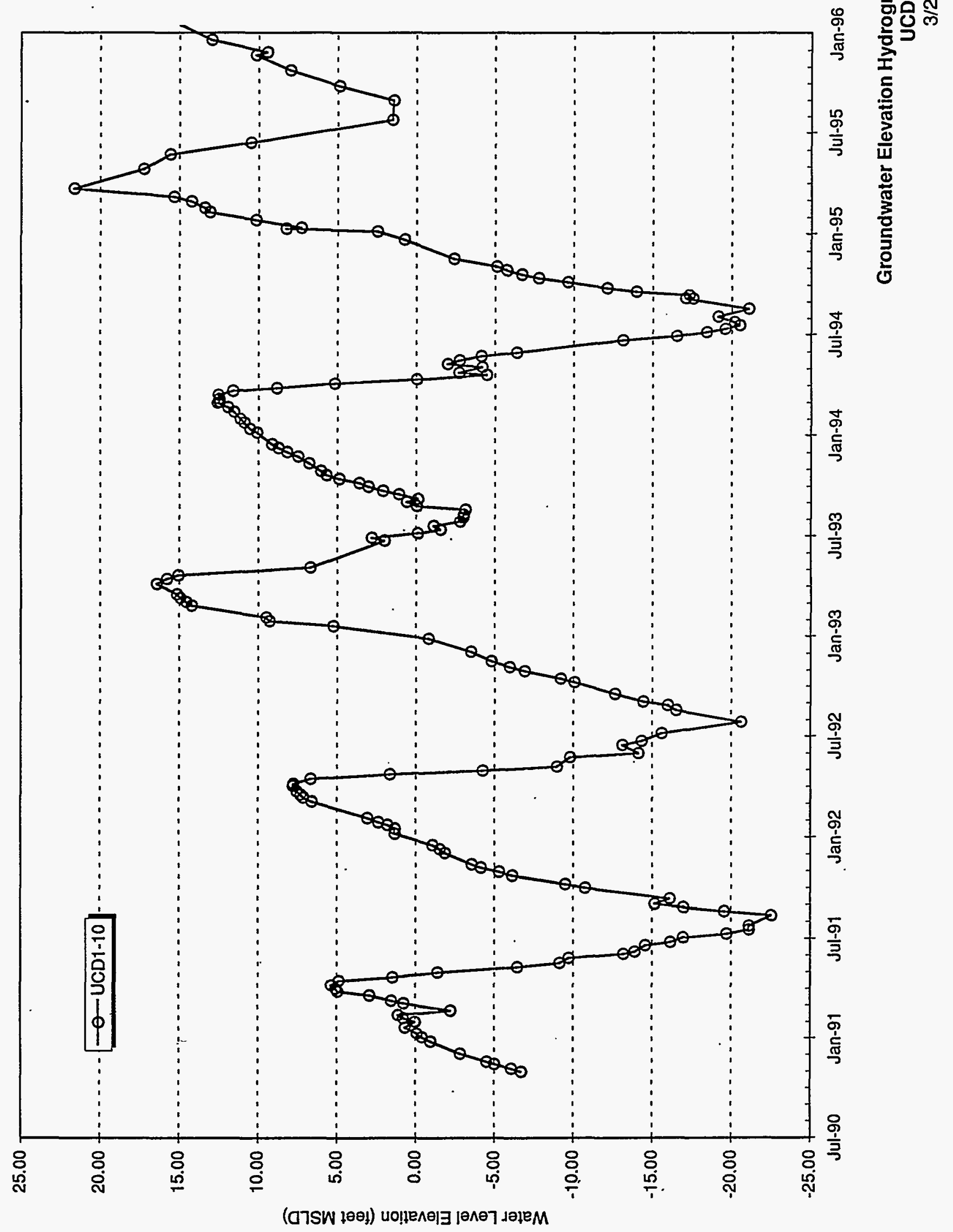

E.8 


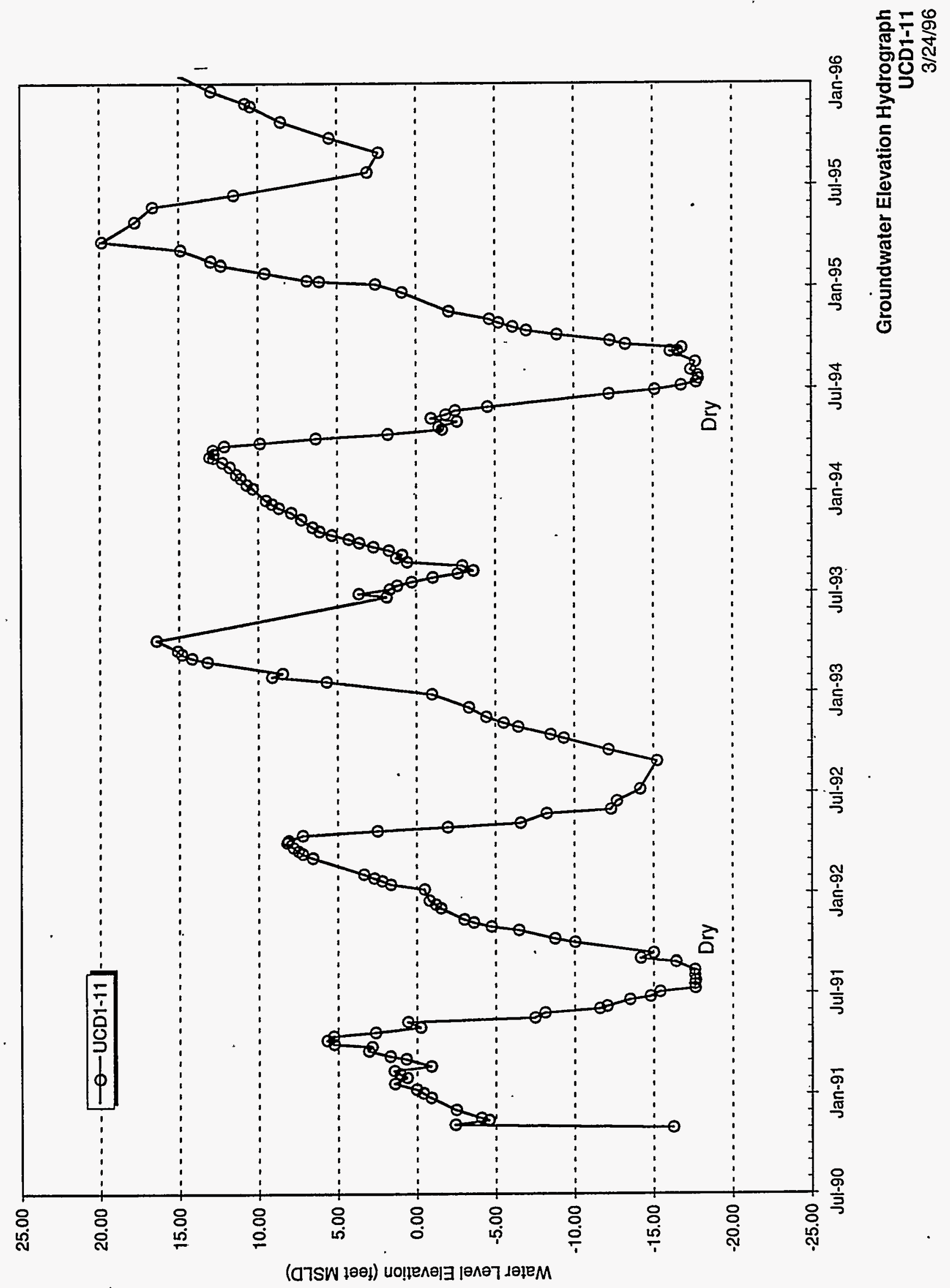

E. 9 


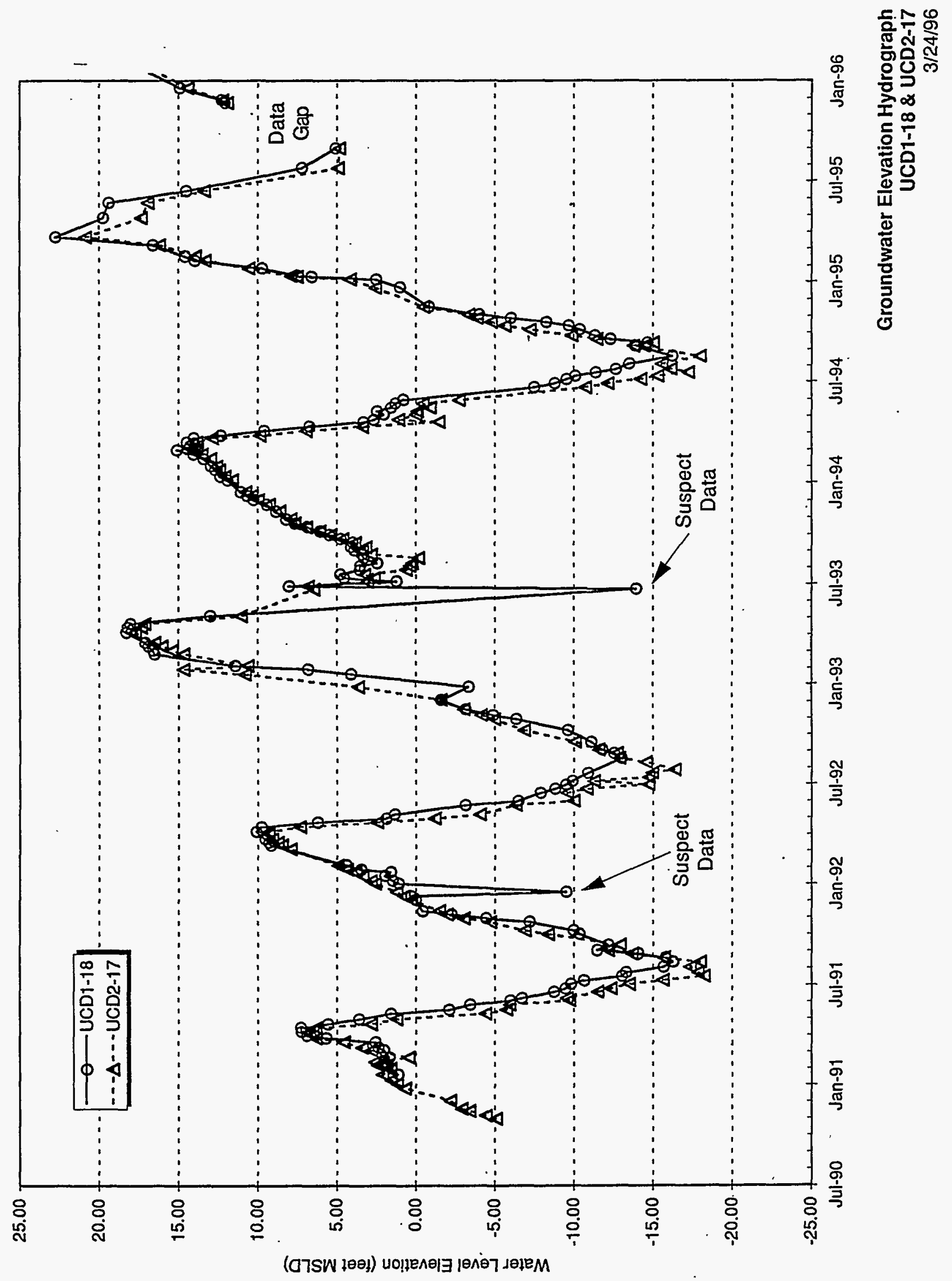

E. 10 


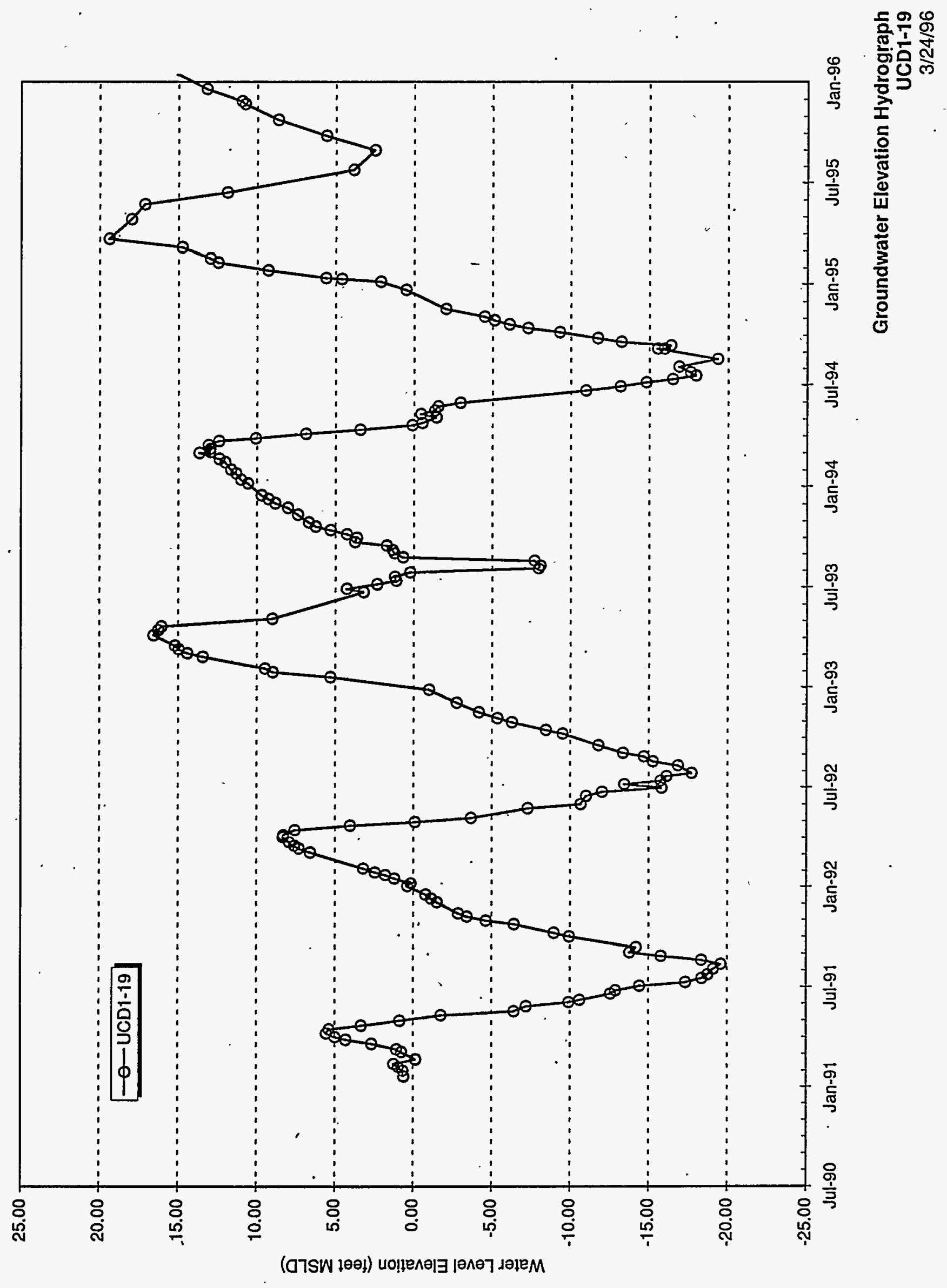

E. 11 


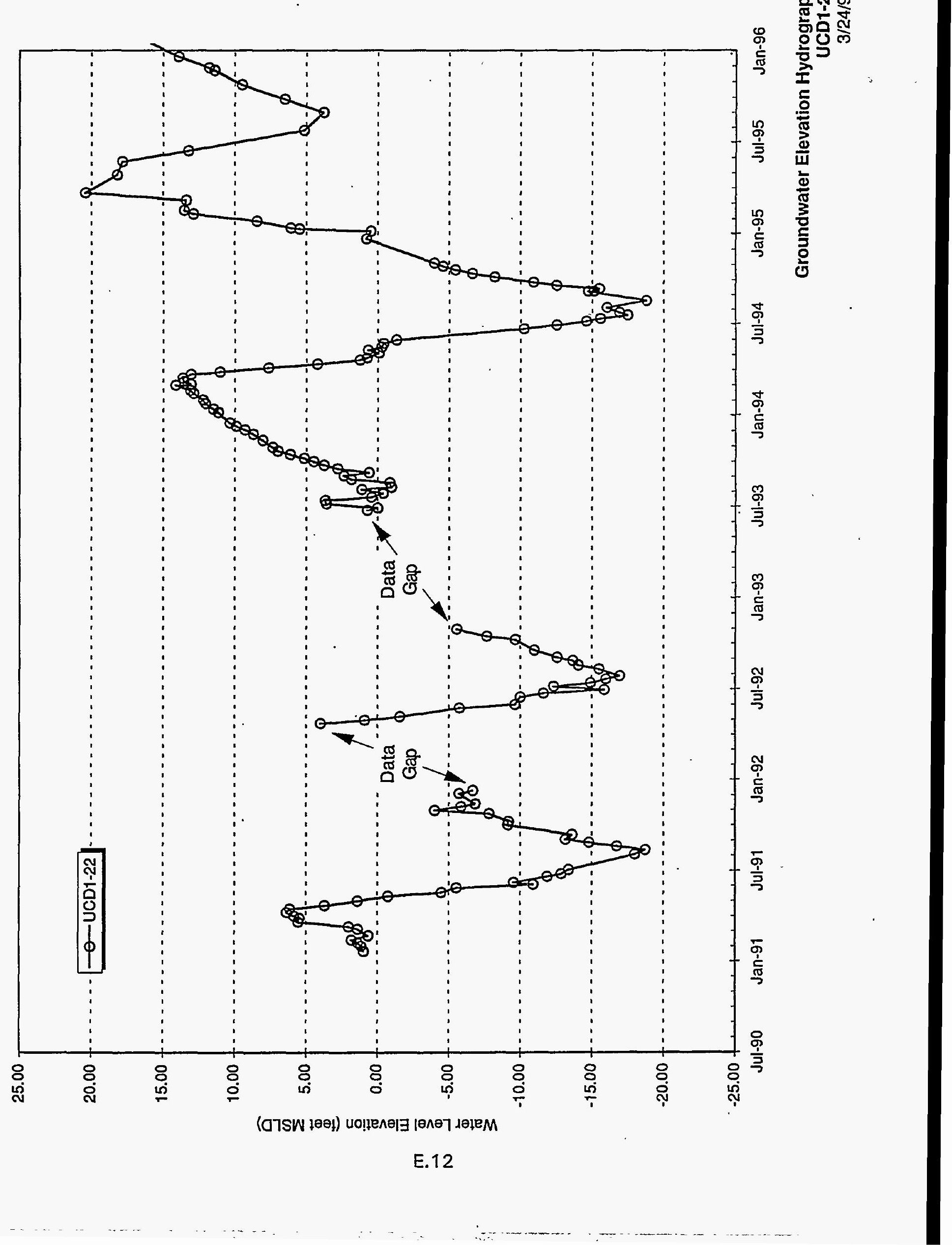




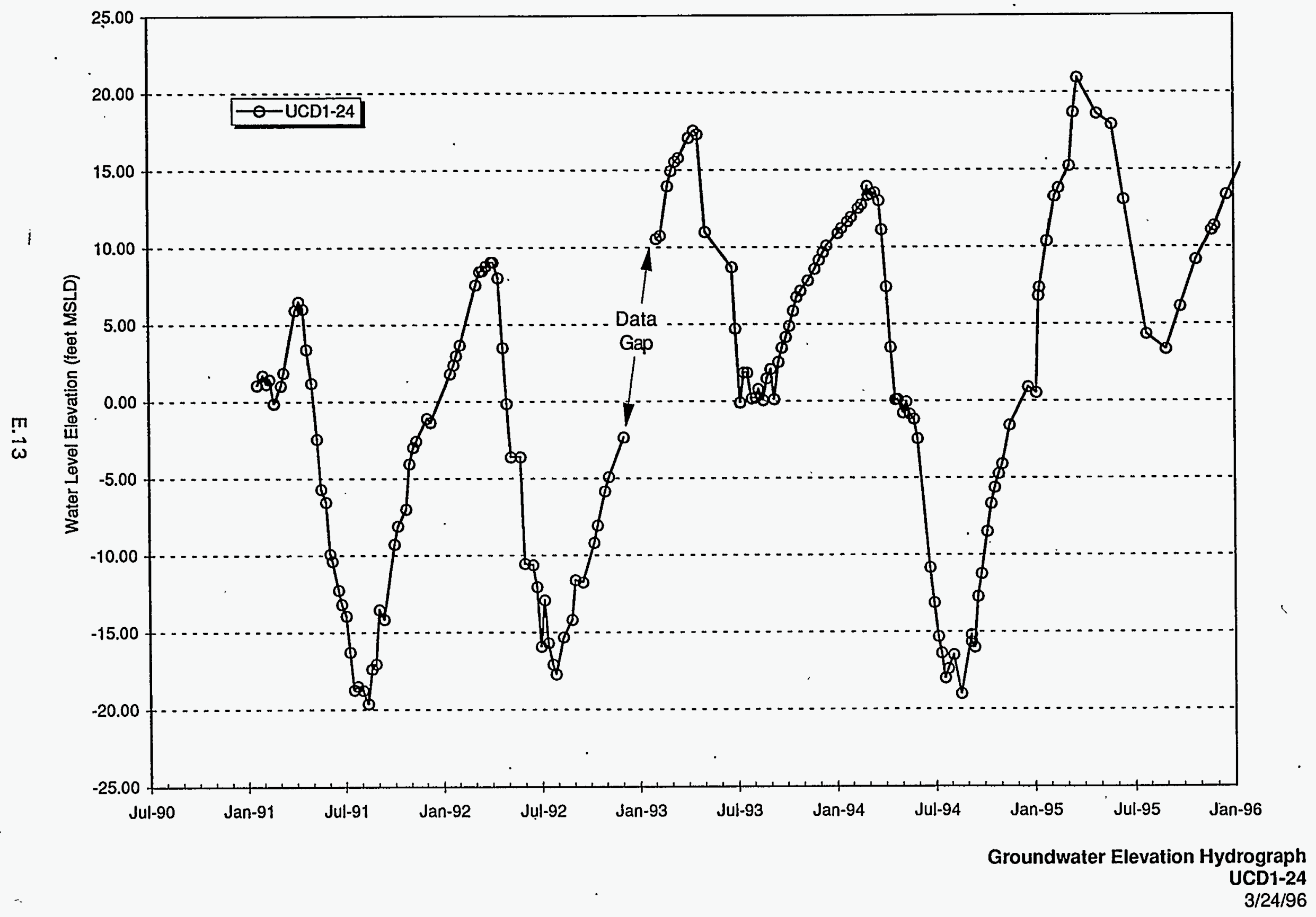


Table E.1. Cumulative Groundwater Elevation Data for HSU-1, 1990-1995

\begin{tabular}{|c|c|c|c|c|c|c|c|c|}
\hline \multicolumn{2}{|c|}{ WELL } & DATE & $\begin{array}{c}\text { PROTECTIVE } \\
\text { CASING } \\
\text { ELEVATION } \\
\text { (feet) }\end{array}$ & $\begin{array}{l}\text { DEPTH } \\
\text { TO } \\
\text { WATER } \\
\text { (feet) } \\
\end{array}$ & $\begin{array}{c}\text { GROUND- } \\
\text { WATER } \\
\text { ELEVATION } \\
\text { (feet) } \\
\end{array}$ & & COMMENTS & \\
\hline UCDY- & 1 & $11 / 2 / 90$ & 50.05 & 56.03 & -5.98 & & & \\
\hline UCD1. & 1 & $11 / 8 / 90$ & 50.05 & 54.08 & -4.03 & & & \\
\hline UCD1- & 1 & $11 / 16 / 90$ & 50.05 & 54.56 & -4.51 & & & \\
\hline UCD1- & 1 & $11 / 20 / 90$ & 50.05 & 53.60 & -3.55 & & & \\
\hline UCD1- & 1 & $12 / 5 / 90$ & 50.05 & 52.04 & -1.99 & & & \\
\hline UCD1- & 1 & $12 / 27 / 90$ & 50.05 & 50.30 & -0.25 & & & \\
\hline UCDY- & 1 & $1 / 4 / 91$ & 50.05 & 49.77 & 0.28 & & & \\
\hline UCD1-. & 1 & $1 / 11 / 91$ & 50.05 & 49.45 & 0.60 & & & \\
\hline UCDY- & 1 & $1 / 22 / 91$ & 50.05 & 48.77 & 1.28 & & & \\
\hline UCD1. & 1 & $2 / 1 / 91$ & 50.05 & 48.85 & 1.20 & & & \\
\hline UCD1- & 1 & $2 / 8 / 91$ & 50.05 & 48.42 & 1.63 & & & \\
\hline UCD1- & 1 & $2 / 14 / 91$ & 50.05 & 48.10 & 1.95 & & & \\
\hline UCD1- & 1 & 2/22/91 & 50.05 & 49.84 & 0.21 & & & \\
\hline UCD1- & 1 & $3 / 7 / 91$ & 50.05 & 48.44 & 1.61 & & & \\
\hline UCD1- & 1 & $3 / 12 / 91$ & 50.05 & 47.77 & 2.28 & & & \\
\hline UCD1- & 1 & $3 / 29 / 91$ & 50.05 & 44.67 & 5.38 & & & \\
\hline UCD1- & 1 & $4 / 3 / 91$ & 50.05 & 44.33 & 5.72 & & & \\
\hline UCD1- & 1 & $4 / 10 / 91$ & 50.05 & 43.91 & 6.14 & & & \\
\hline UCD1- & 1 & $4 / 17 / 91$ & 50.05 & 44.24 & 5.81 & & & \\
\hline UCD1- & $i$ & $4 / 24 / 91$ & 50.05 & 46.83 & 3.22 & & & - \\
\hline UCD1- & 1 & $5 / 3 / 91$ & 50.05 & 49.41 & 0.64 & & & \\
\hline UCD1- & 1 & $5 / 13 / 91$ & 50.05 & 52.79 & -2.74 & & & \\
\hline UCD1- & 1 & $8 / 14 / 91$ & 50.05 & 58.59 & -8.54 & dry & & \\
\hline UCD1- & 1 & $8 / 21 / 91$ & 50.05 & 58.59 & -8.54 & $d r y$ & & \\
\hline UCD1- & 1 & $8 / 29 / 91$ & 50.05 & 58.61 & -8.56 & dry & & \\
\hline UCD1- & 1 & $9 / 4 / 91$ & 50.05 & 58.60 & -8.55 & dry & & \\
\hline UCD1- & 1 & $9 / 13 / 91$ & 50.05 & 58.61 & -8.56 & dry & & \\
\hline UCD1- & 1 & $10 / 3 / 91$ & 50.05 & 58.63 & -8.58 & $d r y$ & & \\
\hline UCD1- & 1 & $10 / 10 / 91$ & 50.05 & 57.02 & -6.97 & & & \\
\hline UCD1- & 1 & $10 / 25 / 91$ & 50.05 & 55.93 & -5.88 & & & \\
\hline UCD1- & 1 & $11 / 1 / 91$ & 50.05 & 53.85 & -3.80 & & & \\
\hline UCD1- & 1 & $11 / 8 / 91$ & 50.05 & 52.79 & -2.74 & & & \\
\hline UCD1- & 1 & $11 / 14 / 91$ & 50.05 & 52.39 & -2.34 & & & \\
\hline UCD1- & 1 & $12 / 5 / 91$ & 50.05 & 50.81 & -0.76 & & & \\
\hline UCD1- & 1 & $12 / 11 / 91$ & 50.05 & 50.38 & -0.33 & & & \\
\hline UCD1- & 1 & $12 / 19 / 91$ & 50.05 & 49.95 & 0.10 & & & \\
\hline UCD1- & 1 & 1/3/92 & 50.05 & 48.70 & 1.35 & & & \\
\hline UCD1- & 1 & $1 / 8 / 92$ & 50.05 & 48.27 & 1.78 & & & \\
\hline UCD1- & 1 & $1 / 17 / 92$ & 50.05 & 47.80 & 2.25 & & & \\
\hline UCD1- & 1 & $1 / 24 / 92$ & 50.05 & 47.32 & 2.73 & & & \\
\hline UCD1- & 1 & $1 / 29 / 92$ & 50.05 & 46.73 & 3.32 & & & \\
\hline UCD1- & 1 & $2 / 5 / 92$ & 50.05 & 46.09 & 3.96 & & & \\
\hline UCD1- & 1 & $3 / 6 / 92$ & 50.05 & 42.72 & 7.33 & & & \\
\hline UCD1- & 1 & $3 / 13 / 92$ & 50.05 & 42.11 & 7.94 & & & \\
\hline UCD1- & 1 & $3 / 18 / 92$ & 50.05 & 41.83 & 8.22 & & & \\
\hline UCD1- & 1 & $3 / 25 / 92$ & 50.05 & 41.53 & 8.52 & & & \\
\hline UCD1- & 1 & 4/3/92 & 50.05 & 41.29 & 8.76 & & & \\
\hline UCD1- & 1 & $4 / 7 / 92$ & $50.05^{\circ}$ & 41.21 & 8.84 & & & \\
\hline UCD1- & 1 & $4 / 16 / 92$ & 50.05 & 42.43 & 7.62 & & & \\
\hline UCD1- & 1 & $4 / 24 / 92$ & 50.05 & 46.88 & 3.17 & & & \\
\hline UCD1- & 1 & $5 / 1 / 92$ & 50.05 & 50.49 & -0.44 & & & \\
\hline UCD1- & 1 & $5 / 8 / 92$ & 50.05 & 53.65 & -3.60 & & & \\
\hline UCD1- & 1 & $5 / 26 / 92$ & 50.05 & 56.41 & -6.36 & & & \\
\hline UCD1- & 1 & $6 / 2 / 92$ & 50.05 & 58.80 & -8.75 & & • & \\
\hline UCD1- & 1 & $6 / 17 / 92$ & 50.05 & 58.91 & -8.86 & & & . \\
\hline UCD1- & 1 & $6 / 24 / 92$ & 50.05 & 58.73 & -8.68 & & & \\
\hline UCD1- & 1 & $7 / 2 / 92$ & 50.05 & 58.72 & -8.67 & & & \\
\hline
\end{tabular}


Table E.1. Cumulative Groundwater Elevation Data for HSSU-1, 1990-1995

\begin{tabular}{|c|c|c|c|c|c|c|c|}
\hline WELL & & DATE & $\begin{array}{l}\text { PROTECTIVE } \\
\text { CASING } \\
\text { 'ELEVATION } \\
\text { (feot) }\end{array}$ & $\begin{array}{l}\text { DEPTH } \\
\text { TO } \\
\text { WATER } \\
\text { (feot) }\end{array}$ & $\begin{array}{c}\text { GROUND- } \\
\text { WATER } \\
\text { ELEVATION } \\
\text { (feet) }\end{array}$ & COMMENTS & \\
\hline UCD1- & 1 & $7 / 8 / 92$ & 50.05 & 58.73 & -8.68 & - & \\
\hline UCD1- & 1 & $7 / 15 / 92$ & 50.05 & 58.74 & -8.69 & & \\
\hline UCD1. & 1 & $7 / 23 / 92$ & 50.05 & 58.79 & -8.74 & & \\
\hline UCD1. & 1 & $7 / 29 / 92$ & 50.05 & 58.74 & -8.69 & & \\
\hline UCD1. & 1 & $8 / 12 / 92$ & 50.05 & 58.72 & -8.67 & & \\
\hline UCD1- & 1 & $8 / 19 / 92$ & 50.05 & 58.76 & -8.71 & & \\
\hline UCD1. & 1 & $8 / 28 / 92$ & 50.05 & 58.75 & -8.70 & & \\
\hline UCD1- & 1 & $9 / 4 / 92$ & 50.05 & 58.74 & -8.69 & & \\
\hline UCD1- & 1 & $9 / 18 / 92$ & 50.05 & 58.77 & -8.72 & & \\
\hline UCD1. & 1 & 10/9/92 & 50.05 & 58.79 & -8.74 & & \\
\hline UCD1. & 1 & $10 / 16 / 92$ & 50.05 & 57.69 & -7.64 & & \\
\hline UCD1- & 1 & $10 / 30 / 92$ & 50.05 & 55.67 & -5.62 & & \\
\hline UCD1- & 1 & $11 / 6 / 92$ & 50.05 & 54.81 & -4.76 & & \\
\hline UCD1- & 1 & $11 / 17 / 92$ & 50.05 & 53.72 & -3.67 & & \\
\hline UCD1- & 1 & $12 / 4 / 92$ & 50.05 & 52.30 & -2.25 & & \\
\hline UCD1- & 1 & $12 / 28 / 92$ & 50.05 & 50.93 & -0.88 & & \\
\hline UCD1. & 1 & $1 / 20 / 93$ & 50.05 & 43.82 & 6.23 & & \\
\hline UCD1- & 1 & $1 / 29 / 93$ & 50.05 & 40.31 & 9.74 & & \\
\hline UCD1- & 1 & $2 / 5 / 93$ & 50.05 & 39.88 & 10.17 & - & \\
\hline UCD1- & 1 & $2 / 12 / 93$ & 50.05 & 39.06 & 10.99 & & \\
\hline UCD1- & 1 & $2 / 26 / 93$ & 50.05 & 35.48 & 14.57 & & \\
\hline UCD1- & 1 & $3 / 5 / 93$ & 50.05 & 34.94 & 15.11 & & \\
\hline UCD1- & 1 & $3 / 12 / 93$ & 50.05 & 34.41 & 15.64 & & \\
\hline UCD1- & 1 & $3 / 19 / 93$ & 50.05 & 34.07 & 15.98 & & • \\
\hline UCD1- & 1 & $4 / 7 / 93$ & 50.05 & 32.82 & 17.23 & & \\
\hline UCD1- & 1 & $4 / 16 / 93$ & 50.05 & 33.10 & 16.95 & & \\
\hline UCD1- & 1 & $4 / 23 / 93$ & 50.05 & 32.87 & 17.18 & & \\
\hline UCD1- & 1 & $5 / 7 / 93$ & 50.05 & 40.28 & 9.77 & & \\
\hline UCD1- & 1 & $6 / 24 / 93$ & 50.05 & 43.95 & 6.10 & & \\
\hline UCD1. & 1 & $6 / 30 / 93$ & 50.05 & 44.52 & 5.53 & & \\
\hline UCD1- & 1 & 7/8/93 & 50.05 & 48.77 & 1.28 & & \\
\hline UCD1- & 1 & $7 / 15 / 93$ & 50.05 & 47.70 & 2.35 & & \\
\hline UCD1. & 1 & $7 / 21 / 93$ & 50.05 & 47.62 & 2.43 & & \\
\hline UCD1- & 1 & $7 / 30 / 93$ & 50.05 & 49.12 & 0.93 & & \\
\hline UCD1- & 1 & $8 / 6 / 93$ & 50.05 & 49.33 & ' 0.72 & , & \\
\hline UCD1- & 1 & $8 / 11 / 93$ & 50.05 & 49.68 & 0.37 & & \\
\hline UCD1- & 1 & $8 / 20 / 93$ & 50.05 & 49.51 & 0.54 & & \\
\hline UCD1- & 1 & $8 / 27 / 93$ & 50.05 & 48.17 & 1.88 & & \\
\hline UCD1- & 1 & $9 / 3 / 93$ & 50.05 & 47.62 & 2.43 & & 、 \\
\hline UCD1- & 1 & $9 / 9 / 93$ & 50.05 & 47.64 & 2.41 & . & \\
\hline UCD1- & 1 & $9 / 17 / 93$ & 50.05 & 47.19 & 2.86 & & \\
\hline UCD1- & 1 & $9 / 24 / 93$ & 50.05 & 48.26 & 1.79 & & \\
\hline UCD1- & 1 & $10 / 1 / 93$ & 50.05 & 45.50 & 4.55 & & \\
\hline UCD1- & 1 & $10 / 8 / 93$ & 50.05 & 44.85 & 5.20 & & \\
\hline UCDi- & 1 & $10 / 15 / 93$ & 50.05 & 43.88 & 6.17 & & \\
\hline UCD1- & 1 & $10 / 22 / 93$ & 50.05 & 43.11 & 6.94 & & \\
\hline UCD1. & 1 & $10 / 29 / 93$ & 50.05 & 42.71 & 7.34 & & \\
\hline UCD1- & 1 & $11 / 12 / 93$ & 50.05 & 42.01 & 8.04 & & * \\
\hline UCD1. & 1 & $11 / 24 / 93$ & 50.05 & 41.41 & 8.64 & & \\
\hline UCD1- & 1 & $12 / 3 / 93$ & 50.05 & 40.60 & 9.45 & . & \\
\hline UCD1- & 1 & $12 / 10 / 93$ & 50.05 & 40.21 & 9.84 & & \\
\hline UCD1- & 1 & $12 / 17 / 93$ & 50.05 & 39.79 & 10.26 & & \\
\hline UCD1- & 1 & $1 / 7 / 94$ & 50.05 & 38.92 & 11.13 & • & \\
\hline UCD1- & 1 & $1 / 14 / 94$ & 50.05 & 38.55 & 11.50 & & \\
\hline UCD1- & 1 & $1 / 25 / 94$ & 50.05 & 38.04 & 12.01 & & \\
\hline UCD1- & 1 & $2 / 1 / 94$ & 50.05 & 37.89 & 12.16 & & • \\
\hline UCD1- & 1 & $2 / 14 / 94$ & 50.05 & 38.27 & 11.78 & & \\
\hline
\end{tabular}


Table E.1. Cumulative Groundwater Elevation Data for HSU-1, 1990-1995

\begin{tabular}{|c|c|c|c|c|c|c|}
\hline WEL & & DATE & $\begin{array}{l}\text { PROTECTIVE } \\
\text { CASING } \\
\text { ELEVATION } \\
\text { (feet) }\end{array}$ & $\begin{array}{l}\text { DEPTH } \\
\text { TO } \\
\text { WATER } \\
\text { (feet) }\end{array}$ & $\begin{array}{c}\text { GROUND- } \\
\text { WATER } \\
\text { ELEVATION } \\
\text { (feet) }\end{array}$ & COMMENTS \\
\hline UCD1- & 1 & $2 / 20 / 94$ & 50.05 & 37.02 & 13.03 & \\
\hline UCD1- & 1 & $3 / 2 / 94$ & 50.05 & 36.57 & 13.48 & \\
\hline UCD1- & 1 & $3 / 4 / 94$ & 50.05 & 36.64 & 13.41 & \\
\hline UCD1- & 1 & $3 / 10 / 94$ & 50.05 & 36.60 & 13.45 & \\
\hline UCD1- & -1 & $3 / 16 / 94$ & 50.05 & 36.58 & 13.47 & \\
\hline UCD1- & 1 & $3 / 24 / 94$ & 50.05 & 37.39 & 12.66 & \\
\hline UCD1- & 1 & $3 / 29 / 94$ & 50.05 & 39.91 & 10.14 & \\
\hline UCD1- & 1 & $4 / 6 / 94$ & 50.05 & 42.92 & 7.13 & \\
\hline UCD1- & 1 & $4 / 13 / 94$ & 50.05 & 46.52 & 3.53 & \\
\hline UCD1- & 1 & $4 / 22 / 94$ & 50.05 & 50.14 & -0.09 & \\
\hline UCD1- & 1 & $4 / 26 / 94$ & 50.05 & 49.71 & 0.34 & . \\
\hline UCD1- & 1 & $5 / 6 / 94$ & 50.05 & 50.71 & -0.66 & \\
\hline UCD1- & 1 & $5 / 11 / 94$ & 50.05 & 49.83 & 0.22 & \\
\hline UCD1- & 1 & $5 / 18 / 94$ & 50.05 & 50.91 & -0.86 & \\
\hline UCD1- & 1 & $5 / 25 / 94$ & 50.05 & 50.97 & -0.92 & \\
\hline UCD1- & 1 & $6 / 1 / 94$ & 50.05 & 52.47 & -2.42 & \\
\hline UCD1- & 1 & $10 / 14 / 94$ & 50.05 & 56.38 & -6.33 & dry previous week \\
\hline UCD1- & 1 & $10 / 21 / 94$ & 50.05 & 55.46 & -5.41 & \\
\hline UCD1- & $i$ & $10 / 28 / 94$ & 50.05 & 54.58 & -4.53 & $\cdot$ \\
\hline UCD1- & 1 & $11 / 4 / 94$ & 50.05 & 54.01 & -3.96 & \\
\hline UCD1. & 1 . & $11 / 18 / 94$ & 50.05 & 51.48 & -1.43 & \\
\hline UCD1- & 1 & $12 / 22 / 94$ & 50.05 & 48.62 & 1.43 & \\
\hline UCD1- & 1 & $1 / 6 / 95$ & 50.05 & 46.93 & 3.12 & \\
\hline UCD1- & 1 & $1 / 11 / 95$ & 50.05 & 44.02 & 6.03 & . \\
\hline UCO1- & 1 & $1 / 13 / 95$ & 50.05 & 43.29 & 6.76 & . \\
\hline UCD1- & 1 & $1 / 27 / 95$ & 50.05 & 39.38 & 10.67 & \\
\hline UCDI- & 1 & $2 / 10 / 95$ & 50.05 & 37.08 & 12.97 & \\
\hline UCD1- & 1 & $2 / 17 / 95$ & 50.05 & 36.57 & 13.48 & \\
\hline UCDI- & 1 & $3 / 9 / 95$ & 50.05 & 33.97 & 16.08 & \\
\hline UCD1- & 1 & $3 / 24 / 95$ & 50.05 & 29.09 & 20.96 & \\
\hline UCD1- & 1 & $4 / 28 / 95$ & 50.05 & 32.38 & 17.67 & \\
\hline UCD1- & 1 & $5 / 25 / 95$ & 50.05 & 32.92 & 17.13 & \\
\hline UCD1- & 1 & $6 / 16 / 95$ & 50.05 & 37.28 & 12.77 & \\
\hline UCD1- & $i$ & $7 / 26 / 95$ & 50.05 & $45.18 \mathrm{a}$ & $4.87 \mathrm{a}$ & \\
\hline UCD1- & 1 & $8 / 31 / 95$ & 50.05 & $46.27 \mathrm{a}$ & $3.78 \mathrm{a}$ & \\
\hline UCD1- & 1 & $9 / 26 / 95$ & 50.05 & $43.59 \mathrm{a}$ & $6.46 \mathrm{a}$ & \\
\hline UCD1- & 1 & $10 / 25 / 95$ & 50.05 & $40.62 \mathrm{a}$ & $9.43 \mathrm{a}$ & \\
\hline UCD1. & 1 & $11 / 22 / 95$ & 50.05 & $38.62 \mathrm{a}$ & $11.43 \mathrm{a}$ & \\
\hline UCD1- & 1 & $11 / 27 / 95$ & 50.05 & 38.40 & 11.65 & \\
\hline UCD1. & 1 & $12 / 19 / 95$ & 50.05 & 36.07 & 13.98 & . \\
\hline UCD1- & 3 & $11 / 8 / 90$ & 50.05 & 50.34 & -0.29 & \\
\hline UCD1. & 3 & $11 / 16 / 90$ & 50.05 & 55.43 & -5.38 & \\
\hline UCD1. & 3 & $11 / 20 / 90$ & 50.05 & 50.39 & -0.34 & \\
\hline UCD1- & 3 & $12 / 5 / 90$ & 50.05 & 50.44 & -0.39 & \\
\hline UCD1- & 3 & $12 / 27 / 90$ & 50.05 & 50.43 & -0.38 & \\
\hline UCD1- & 3 & $1 / 4 / 91$ & 50.05 & 50.44 & -0.39 & \\
\hline UCD1- & 3 & $1 / 11 / 91$ & 50.05 & 50.20 & -0.15 & \\
\hline UCD1- & 3 & $1 / 22 / 91$ & 50.05 & 49.41 & 0.64 & \\
\hline UCD1- & 3 & $2 / 1 / 91$ & 50.05 & 49.10 & 0.95 & \\
\hline UCD1- & 3 & $2 / 8 / 91$ & 50.05 & 48.81 & 1.24 & \\
\hline UCD1- & 3 & $2 / 14 / 91$ & 50.05 & 48.49 & 1.56 & • \\
\hline UCD1- & 3 & $2 / 22 / 91$ & 50.05 & 49.55 & 0.50 & \\
\hline UCD1- & 3 & $3 / 7 / 91$ & 50.05 & 48.58 & 1.47 & \\
\hline UCD1. & 3 & $3 / 12 / 91$ & 50.05 & 48.22 & 1.83 & - \\
\hline UCD1- & 3 & $3 / 22 / 91$ & 50.05 & 46.58 & 3.47 & \\
\hline UCD1- & 3 & $3 / 29 / 91$ & 50.05 & 43.93 & 6.12 & \\
\hline
\end{tabular}


Table E.1. Cumulative Groundwater Elevation Data for HSU-1, 1990-1995

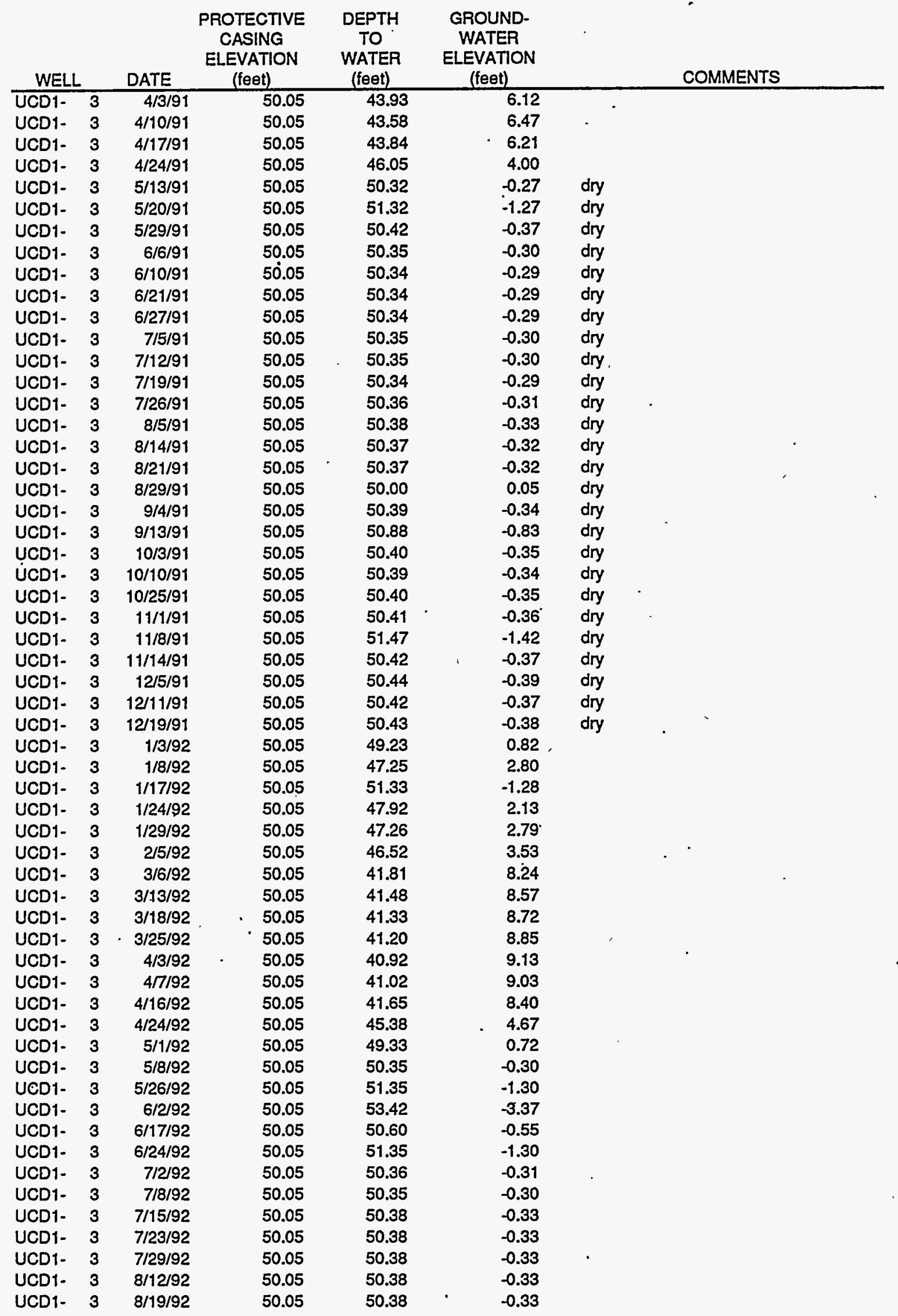


Table E.1. Cumulative Groundwater Elevation Data for HSU-1, 1990-1995

\begin{tabular}{|c|c|c|c|c|c|c|}
\hline WELL & & DATE & $\begin{array}{l}\text { PROTECTIVE } \\
\text { CASING } \\
\text { ELEVATION } \\
\text { (feet) }\end{array}$ & $\begin{array}{l}\text { DEPTH } \\
\text { TO } \\
\text { WATER } \\
\text { (feet) }\end{array}$ & $\begin{array}{l}\text { GROUND- } \\
\text { WATER } \\
\text { ELEVATION } \\
\text { (feet) }\end{array}$ & COMMENTS \\
\hline UCD1- & 3 & $8 / 28 / 92$ & 50.05 & 50.40 & -0.35 & \\
\hline UCD1- & 3 & $9 / 4 / 92$ & 50.05 & 50.40 & -0.35 & . \\
\hline UCD1- & 3 & $9 / 18 / 92$ & 50.05 & 50.39 & -0.34 & \\
\hline UCD1- & 3 & 10/9/92 & 50.05 & 50.38 & -0.33 & \\
\hline UCD1- & 3 & $10 / 16 / 92$ & 50.05 & 50.42 & -0.37 & \\
\hline UCD1- & 3 & $10 / 30 / 92$ & 50.05 & 50.45 & -0.40 & \\
\hline UCD1- & 3 & $11 / 6 / 92$ & 50.05 & 50.43 & -0.38 & \\
\hline UCD1- & 3 & $11 / 17 / 92$ & 50.05 & 50.45 & -0.40 & \\
\hline UCD1- & 3 & $12 / 4 / 92$ & 50.05 & 50.05 & 0.00 & \\
\hline UCD1- & 3 & $12 / 28 / 92$ & 50.05 & 50.26 & -0.21 & \\
\hline UCD1- & 3 & $1 / 20 / 93$ & 50.05 & 43.04 & 7.01 & \\
\hline UCD1- & 3 & $1 / 29 / 93$ & 50.05 & 39.30 & 10.75 & \\
\hline UCD1- & 3 & $2 / 5 / 93$ & 50.05 & 39.54 & 10.51 & \\
\hline UCD1- & 3 & $2 / 12 / 93$ & 50.05 & 38.88 & 11.17 & \\
\hline UCD1- & 3 & $2 / 26 / 93$ & 50.05 & 34.98 & 15.07 & \\
\hline UCD1- & 3 & $3 / 5 / 93$ & 50.05 & 34.64 & 15.41 & \\
\hline UCD1- & 3 & $3 / 12 / 93$ & 50.05 & 34.27 & 15.78 & \\
\hline UCD1. & 3 & $3 / 19 / 93$ & 50.05 & 34.07 & 15.98 & \\
\hline UCD1- & 3 & $4 / 7 / 93$ & 50.05 & 32.79 & 17.26 & \\
\hline UCD1- & 3 & $4 / 16 / 93$ & 50.05 & 33.06 & 16.99 & \\
\hline UCD1- & 3 & $4 / 23 / 93$ & 50.05 & 32.74 & 17.31 & \\
\hline UCD1- & 3 & $5 / 7 / 93$ & 50.05 & 39.36 & 10.69 & \\
\hline UCD1- & 3 & $6 / 24 / 93$ & 50.05 & 42.70 & 7.35 & • \\
\hline UCD1- & 3 & $6 / 30 / 93$ & 50.05 & 45.47 & 4.58 & \\
\hline UCD1- & 3 & $7 / 8 / 93$ & 50.05 & 48.68 & 1.37 & \\
\hline UCD1- & 3 & $7 / 15 / 93$ & 50.05 & 47.85 & 2.20 & $\cdot$ \\
\hline UCD1- & 3 & $7 / 21 / 93$ & 50.05 & 47.81 & 2.24 & • \\
\hline UCD1. & 3 & $7 / 30 / 93$ & 50.05 & 48.81 & 1.24 & \\
\hline UCD1- & 3 & $8 / 6 / 93$ & 50.05 & 49.00 & 1.05 & \\
\hline UCD1- & 3 & $8 / 11 / 93$ & 50.05 & 49.43 & 0.62 & \\
\hline UCD1- & 3 & $8 / 20 / 93$ & 50.05 & 49.02 & 1.03 & . \\
\hline UCD1- & 3 & $8 / 27 / 93$ & 50.05 & 48.71 & 1.34 & \\
\hline UCD1- & 3 & $9 / 3 / 93$ & 50.05 & 47.99 & 2.06 & \\
\hline UCD1- & 3 & 9/9/93 & 50.05 & 47.79 & 2.26 & \\
\hline UCD1- & 3 & $9 / 17 / 93$ & 50.05 & 47.69 & 2.36 & \\
\hline UCD1- & 3 & $9 / 24 / 93$ & 50.05 & 48.81 & 1.24 & \\
\hline UCD1- & 3 & $10 / 1 / 93$ & 50.05 & 46.03 & 4.02 & \\
\hline UCD1- & 3 & $10 / 8 / 93$ & 50.05 & 45.35 & 4.70 & \\
\hline UCD1- & 3 & $10 / 15 / 93$ & 50.05 & 44.35 & 5.70 & \\
\hline UCD1- & 3 & $10 / 22 / 93$ & 50.05 & 43.47 & 6.58 & \\
\hline UCD1:- & 3 & $10 / 29 / 93$ & 50.05 & 42.96 & 7.09 & \\
\hline UCD1- & 3 & $11 / 12 / 93$ & 50.05 & 42.30 & 7.75 & \\
\hline UCD1- & 3 & $11 / 24 / 93$ & 50.05 & 41.63 & 8.42 & \\
\hline UCD1- & 3 & 12/3/93 & 50.05 & 40.85 & 9.20 & \\
\hline UCD1- & 3 & $12 / 10 / 93$ & 50.05 & 40.40 & 9.65 & \\
\hline UCD1- & 3 & $12 / 17 / 93$ & 50.05 & 39.89 & 10.16 & \\
\hline UCD1- & 3 & $1 / 7 / 94$ & 50.05 & 39.16 & 10.89 & \\
\hline UCD1- . & 3 & $1 / 14 / 94$ & 50.05 & 38.79 & 11.26 & \\
\hline UCD1- & 3 & $1 / 25 / 94$ & 50.05 & 38.13 & 11.92 & \\
\hline UCD1- & 3 & $2 / 1 / 94$ & 50.05 & 37.95 & 12.10 & \\
\hline UCD1- & 3 & $2 / 14 / 94$ & 50.05 & 37.53 & 12.52 & \\
\hline UCD1- & 3 & $2 / 20 / 94$ & 50.05 & 36.76 & 13.29 & \\
\hline UCD1- & 3 & $3 / 4 / 94$ & 50.05 & 36.54 & 13.51 & \\
\hline UCD1- & 3 & $3 / 10 / 94$ & 50.05 & 36.51 & 13.54 & \\
\hline UCD1- & 3 & $3 / 16 / 94$ & 50.05 & 36.49 . & 13.56 & \\
\hline UCD1- & 3 & $3 / 24 / 94$ & 50.05 & $37.03^{\circ}$ & 13.02 & \\
\hline UCD1- & 3 & $3 / 29 / 94$ & 50.05 & 39.03 & 11.02 & \\
\hline
\end{tabular}


Table E.1. Cumulative Groundwater Elevation Data for HSU-1, 1990-1995

\begin{tabular}{|c|c|c|c|c|c|c|}
\hline WELL & ' & DATE & $\begin{array}{l}\text { PROTECTIVE } \\
\text { CASING } \\
\text { ELEVATION } \\
\text { (feet) }\end{array}$ & $\begin{array}{l}\text { DEPTH } \\
\text { TO } \\
\text { WATER } \\
\text { (feet) }\end{array}$ & $\begin{array}{l}\text { GROUND- } \\
\text { WATER } \\
\text { ELEVATION } \\
\text { (feet) }\end{array}$ & COMMENTS \\
\hline UCD1- & 3 & $4 / 6 / 94$ & 50.05 & 42.30 & 7.75 & \\
\hline UCD1- & 3 & $4 / 13 / 94$ & 50.05 & 45.84 & 4.21 & \\
\hline UंCD1- & 3 & $4 / 22 / 94$ & 50.05 & 49.14 & 0.91 & \\
\hline UCD1- & 3 & $4 / 26 / 94$ & 50.05 & 49.82 & 0.23 & \\
\hline UCD1- & 3 & $5 / 6 / 94$ & 50.05 & 50.20 & -0.15 & . \\
\hline UCD1- & 3 & $5 / 1.1 / 94$ & 50.05 & 49.79 & 0.26 & \\
\hline UCD1- & 3 & $5 / 18 / 94$ & 50.05 & 50.36 & -0.31 & \\
\hline UCD1- & 3 & $5 / 25 / 94$ & 50.05 & 50.38 & -0.33 & \\
\hline UCD1- & 3 & $6 / 1 / 94$ & 50.05 & 50.35 & -0.30 & \\
\hline UCD1- & 3 & $6 / 23 / 94$ & 50.05 & 50.41 & -0.36 & \\
\hline UCD1- & 3 & 7/1/94 & 50.05 & 50.43 & -0.38 & unclear meter response \\
\hline UCD1- & 3 & $7 / 8 / 94$ & 50.05 & 50.42 & -0.37 & \\
\hline UCD1- & 3 & $7 / 14 / 94$ & 50.05 & 50.39 & -0.34 & - \\
\hline UCD1. & 3 & $7 / 20 / 94$ & 50.05 & 50.55 & -0.50 & . \\
\hline UCD1- & 3 & $7 / 26 / 94$ & 50.05 & 50.56 & -0.51 & \\
\hline UCD1- & 3 & $8 / 5 / 94$ & 50.05 & 50.61 & -0.56 & \\
\hline UCD1- & 3 & $8 / 19 / 94$ & 50.05 & 50.71 & -0.66 & \\
\hline UCD1- & 3 & $9 / 7 / 94$ & 50.05 & 50.44 & -0.39 & \\
\hline UCD1- & 3 & $9 / 7 / 94$ & 50.05 & 50.45 & -0.40 & . \\
\hline UCD1- & 3 & $9 / 13 / 94$ & 50.05 & 50.45 & -0.40 & \\
\hline UCD1- & 3 & $9 / 19 / 94$ & 50.05 & 50.45 & -0.40 & \\
\hline UCD1- & 3 & $9 / 26 / 94$ & 50.05 & 50.46 & -0.41 & \\
\hline UCD1- & 3 & $10 / 7 / 94$ & 50.05 & 50.47 & -0.42 & \\
\hline UCD1- & 3 & $10 / 14 / 94$ & 50.05 & 50.48 & -0.43 & \\
\hline UCD1- & 3 & $10 / 21 / 94$ & 50.05 & 50.49 & -0.44 & \\
\hline UCD1- & 3 & $10 / 28 / 94$ & 50.05 & 50.49 & -0.44 & \\
\hline UCD1- & 3 & $11 / 4 / 94$ & 50.05 & 50.50 & -0.45 & \\
\hline UCD1- & 3 & $12 / 22 / 94$ & 50.05 & & NA & 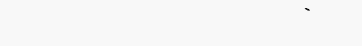 \\
\hline UCD1- & 3 & $3 / 24 / 95$ & 50.05 & 28.21 & 21.84 & \\
\hline UCD1- & 3 & $4 / 28 / 95$ & 50.05 & 31.41 & 18.64 & \\
\hline UCD1- & 3 & $5 / 25 / 95$ & 50.05 & 32.15 & 17.90 & \\
\hline UCD1- & 3 & $6 / 16 / 95$ & 50.05 & 37.31 & 12.74 & \\
\hline UCD1- & 3 & $7 / 26 / 95$ & 50.05 & $45.42 \mathrm{a}$ & $4.63 a$ & \\
\hline UCD1- & 3 & $8 / 31 / 95$ & 50.05 & $46.99 a$ & $3.06 \mathrm{a}$ & \\
\hline UCD1- & 3 & 9/26/95 & 50.05 & $44.07 \mathrm{a}$ & $5.98 \mathrm{a}$ & \\
\hline UCD1- & 3 & $10 / 25 / 95$ & 50.05 & $41.02 \cdot a$ & $9.03 \mathrm{a}$ & ' \\
\hline UCD1- & 3 & $11 / 22 / 95$ & 50.05 & $39.09 \mathrm{a}$ & $10.96 \mathrm{a}$ & \\
\hline UCD1- & 3 & $11 / 27 / 95$ & 50.05 & 38.82 & 11.23 & no pump \\
\hline UCD1- & 3 & $12 / 19 / 95$ & 50.05 & 36.55 & 13.50 & \\
\hline UCD1. & 4 & $11 / 8 / 90$ & 51.69 & 56.68 & -4.99 & \\
\hline UCD1- & 4 & $11 / 16 / 90$ & 51.69 & 55.88 & -4.19 & \\
\hline UCD1- & 4 & $11 / 20 / 90$ & 51.69 & 55.56 & -3.87 & \\
\hline UCD1- & 4 & $12 / 5 / 90$ & 51.69 & 54.60 & -2.91 & $\cdot$ \\
\hline UCD1- & 4 & $12 / 27 / 90$ & 51.69 & 52.95 & -1.26 & . \\
\hline UCD1- & 4 & $1 / 4 / 91$ & 51.69 & 52.42 & -0.73 & \\
\hline UCD1- & 4 & $1 / 11 / 91$ & 51.69 & 52.01 & -0.32 & \\
\hline UCD1- & 4 & $1 / 22 / 91$ & 51.69 & 51.10 & 0.59 & \\
\hline UCD1- & 4 & $2 / 1 / 91$ & 51.69 & 50.69 & 1.00 & \\
\hline UCD1- & 4 & $2 / 8 / 91$ & 51.69 & 50.41 & 1.28 & \\
\hline UCD1- & 4 & $2 / 14 / 91$ & 51.69 & 50.14 & 1.55 & \\
\hline UCD1- & 4 & $2 / 22 / 91$ & 51.69 & 50.29 & 1.40 & \\
\hline UCD1- & 4 & $3 / 7 / 91$ & 51.69 & 49.97 & 1.72 & \\
\hline UCD1- & 4 & $3 / 12 / 91$ & 51.69 & 49.31 & 2.38 & . \\
\hline UCD1- & 4 & $3 / 22 / 91$ & 51.69 & 47.22 & 4.47 & \\
\hline UCD1- & 4 & $3 / 29 / 91$ & 51.69 & 45.13 & 6.56 & \\
\hline UCD1- & 4 & $4 / 3 / 91$ & 51.69 & 43.90 & 7.79 & \\
\hline
\end{tabular}


Table E.1. Cumulative Groundwater Elevation Data for HSU-1, 1990-1995

\begin{tabular}{|c|c|c|c|c|c|c|c|c|c|}
\hline \multicolumn{2}{|c|}{ WELL } & DATE & $\begin{array}{l}\text { PROTECTIVE } \\
\text { CASING } \\
\text { ELEVATION } \\
\text { (feet) }\end{array}$ & $\begin{array}{l}\text { DEPTH } \\
\text { TO } \\
\text { WATER } \\
\text { (feet) }\end{array}$ & $\begin{array}{l}\text { GROUND- } \\
\text { WATER } \\
\text { ELEVATION } \\
\text { (feet) }\end{array}$ & & COMMENTS & & \\
\hline UCD1- & 4 & $4 / 10 / 91$ & 51.69 & 43.36 & 8.33 & & & & . \\
\hline UCD1- & 4 & $4 / 17 / 91$ & 51.69 & 43.49 & 8.20 & & & & \\
\hline UCD1. & 4 & $4 / 24 / 91$ & 51.69 & 45.30 & 6.39 & & & & \\
\hline UCD1- & 4 & $5 / 3 / 91$ & 51.69 & 47.69 & 4.00 & & & & \\
\hline UCD1- & 4 & $5 / 13 / 91$ & 51.69 & 49.19 & 2.50 & & & & \\
\hline UCD1- & 4 & $5 / 20 / 91$ & $51: 69$ & 50.93 & 0.76 & & & & \\
\hline UCD1- & 4 & $8 / 14 / 91$ & 51.69 & 57.12 & -5.43 & dry & & & \\
\hline UCD1- & 4 & $8 / 21 / 91$ & 51.69 & 57.17 & -5.48 & dry & & & \\
\hline UCD1- & 4 & $8 / 29 / 91$ & 51.69 & 57.39 & -5.70 & dry & & & \\
\hline UCD1- & 4 & $9 / 4 / 91$ & 51.69 & 57.20 & -5.51 & dry & & & \\
\hline UCD1- & 4 & $9 / 13 / 91$ & 51.69 & 57.21 & -5.52 & dry & & & \\
\hline UCD1- & 4 & $10 / 3 / 91$ & 51.69 & 57.26 & -5.57 & dry & & - & \\
\hline UCD1- & 4 & $10 / 10 / 91$ & 51.69 & 57.27 & -5.58 & dry & & . & \\
\hline UCD1- & 4 & $10 / 25 / 91$ & 51.69 & 57.27 & -5.58 & dry & & & \\
\hline UCD1- & 4 & $11 / 1 / 91$ & 51.69 & 55.89 & -4.20 & & & & \\
\hline UCD1- & 4 & $11 / 8 / 91$ & 51.69 & 55.17 & -3.48 & & & & \\
\hline UCD1- & 4 & $11 / 14 / 91$ & 51.69 & 54.95 & -3.26 & & & & \\
\hline UCD1- & 4 & $12 / 5 / 91$ & 51.69 & 53.21 & -1.52 & & & & \\
\hline UCD1- & 4 & $12 / 11 / 91$ & 51.69 & 52.84 & -1.15 & & & & \\
\hline UCD1- & 4 & $12 / 19 / 91$ & 51.69 & 52.35 & -0.66 & & - & & \\
\hline UCD1.- & 4 & $1 / 3 / 92$ & 51.69 & 51.31 & 0.38 & & & & \\
\hline UCD1- & 4 & $1 / 8 / 92$ & 51.69 & 52.76 & -1.07 & & & & \\
\hline UCD1. & 4 & $1 / 17 / 92$ & 51.69 & 50.39 & 1.30 & & & & . \\
\hline UCD1- & 4 & $1 / 24 / 92$ & 51.69 & 49.77 & 1.92 & & & . & \\
\hline UCD1- & 4 & $1 / 29 / 92$ & 51.69 & 49.06 & 2.63 & & & $\cdot$ & \\
\hline UCD1- & 4 & $2 / 5 / 92$ & 51.69 & 48.33 & 3.36 & & & & \\
\hline UCD1- & 4 & $3 / 6 / 92$ & 51.69 & 42.85 & 8.84 & & & & \\
\hline UCD1- & 4 & $3 / 13 / 92$ & 51.69 & 41.96 & 9.73 & & & & \\
\hline UCD1- & 4 & $3 / 18 / 92$ & 51.69 & 41.90 & 9.79 & & & & \\
\hline UCD1- & 4 & $3 / 25 / 92$ & 51.69 & 41.77 & 9.92 & & & & \\
\hline UCD1- & 4 & $4 / 3 / 92$ & 51.69 & 41.38 & 10.31 & & & & \\
\hline UCD1. & 4 & $4 / 7 / 92$ & 51.69 & 41.16 & 10.53 & & & & \\
\hline UCD1- & 4 & $4 / 16 / 92$ & 51.69 & 41.38 & 10.31 & & & & \\
\hline UCD1- & 4 & $4 / 24 / 92$ & 51.69 & 45.23 & 6.46 & & . & & \\
\hline UCD1- & 4 & $5 / 1 / 92$ & 51.69 & 47.78 & 3.91 & & & & \\
\hline UCD1- & 4 & $5 / 8 / 92$ & 51.69 & 49.59 & 2.10 & & & & \\
\hline UCD1- & 4 & $5 / 26 / 92$ & 51.69 & 52.56 & -0.87 & & & & \\
\hline UCD1- & 4 & $6 / 2 / 92$ & 51.69 & 54.01 & -2.32 & & & & \\
\hline UCD1- & 4 & $6 / 17 / 92$ & 51.69 & 57.24 & -5.55 & & & & \\
\hline UCD1- & 4 & $6 / 24 / 92$ & 51.69 & 57.23 & -5.54 & & & & \\
\hline UCD1- & 4 & $7 / 2 / 92$ & 51.69 & 57.33 & -5.64 & & & & \\
\hline UCD1- & 4 & $11 / 6 / 92$ & 51.69 & 56.62 & -4.93 & & & & \\
\hline UCD1- & 4 & $11 / 17 / 92$ & 51.69 & 55.70 & -4.01 & & & & \\
\hline UCD1- & 4 & $12 / 4 / 92$ & 51.69 & 54.46 & -2.77 & & & & \\
\hline UCD1- & 4 & $12 / 28 / 92$ & 51.69 & 53.02 & -1.33 & & & & \\
\hline UCD1. & 4 & $1 / 20 / 93$ & 51.69 & 43.51 & 8.18 & & & & \\
\hline UCD1- & 4 & $1 / 29 / 93$ & 51.69 & 39.10 & 12.59 & & & & \\
\hline UCD1- & 4 & $2 / 5 / 93$ & 51.69 & 39.55 & 12.14 & & & & \\
\hline UCD1- & 4 & $2 / 12 / 93$ & 51.69 & 38.81 & 12.88 & & & & \\
\hline UCD1- & 4 & $3 / 5 / 93$ & 51.69 & 34.62 & 17.07 & & & & \\
\hline UCD1- & 4 & $3 / 12 / 93$ & 51.69 & 34.56 & 17.13 & & & & \\
\hline UCD1- & 4 & $3 / 19 / 93$ & 51.69 & 34.45 & 17.24 & & & & \\
\hline UCD1- & 4 & $4 / 7 / 93$ & 51.69 & 33.35 & 18.34 & & & & \\
\hline UCD1- & 4 & $4 / 16 / 93$ & 51.69 & 33.42 & 18.27 & & & & \\
\hline UCD1- & 4 & $4 / 23 / 93$ & 51.69 & 33.05 & 18.64 & & & & \\
\hline UCD1- & 4 & $5 / 7 / 93$ & 51.69 & 38.77 & 12.92 & & & & \\
\hline UCD1- & 4 & $6 / 24 / 93$ & 51.69 & 43.02 & 8.67 & & & & \\
\hline
\end{tabular}


Table E.1. Cumulative Groundwater Elevation Data for HSU-1, 1990-1995

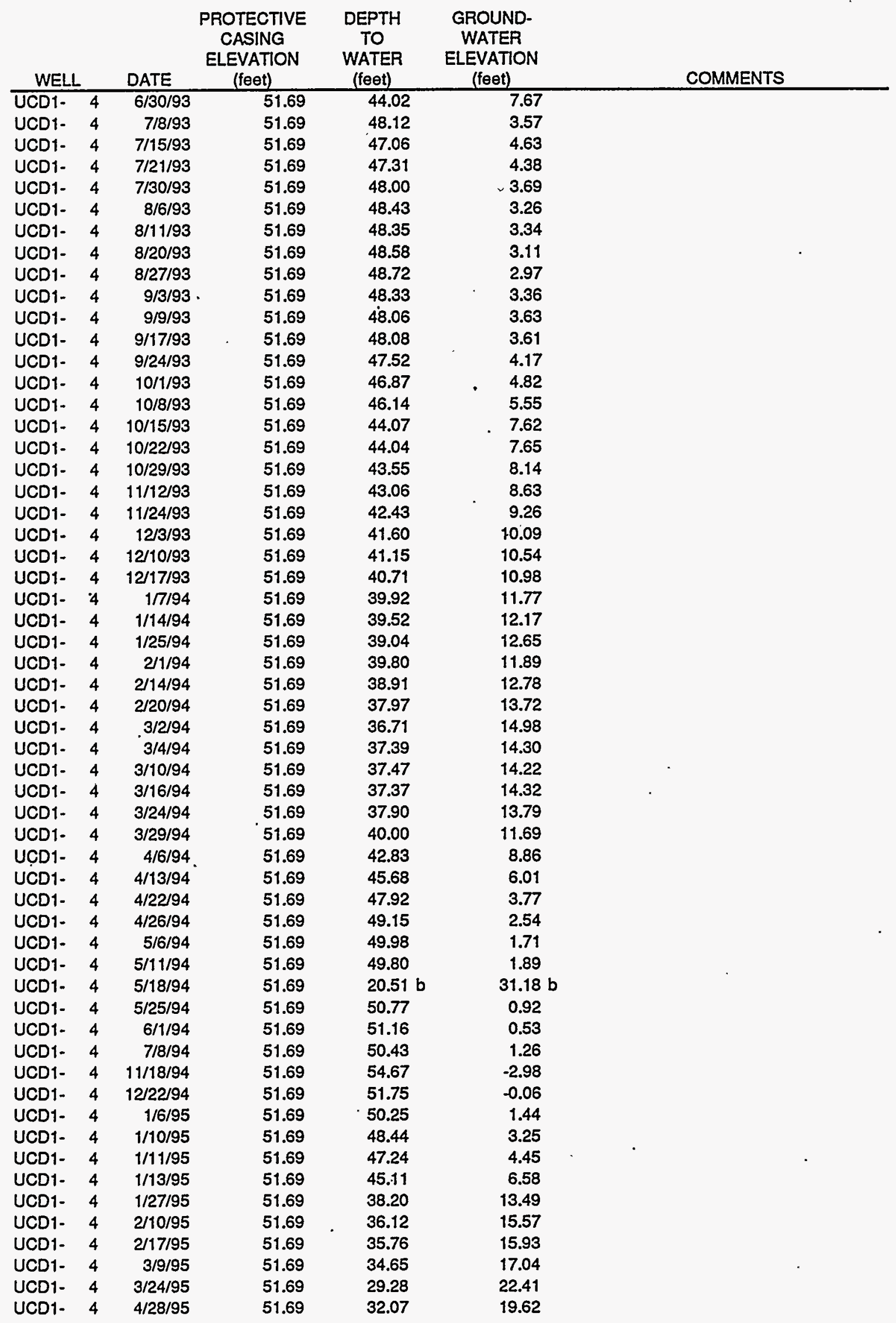


Table E.1. Cumulative Groundwater Elevation Data for HSU-1, 1990-1995

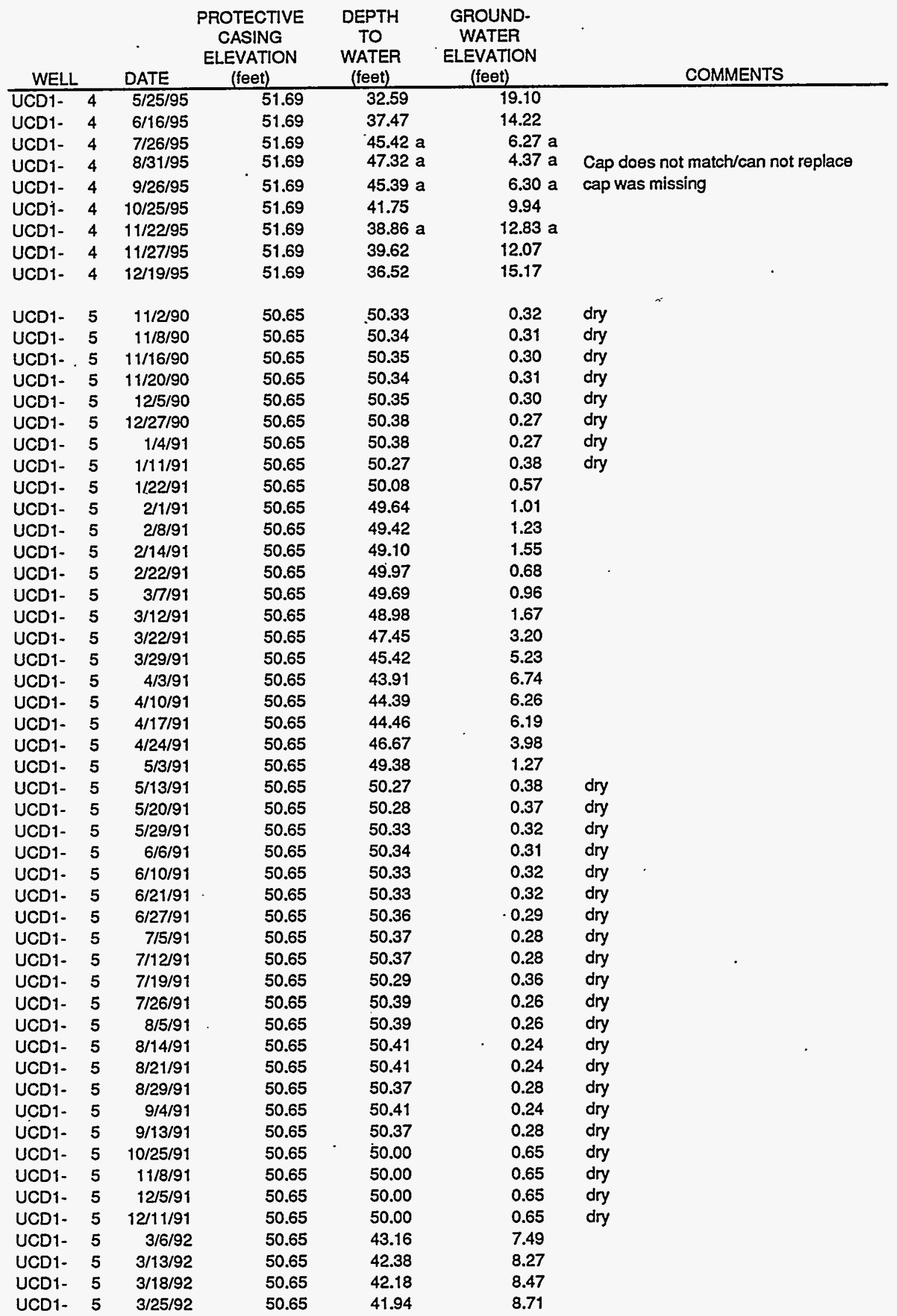


Table E.1. Cumulative Groundwater Elevation Data for HSU-1, 1990-1995

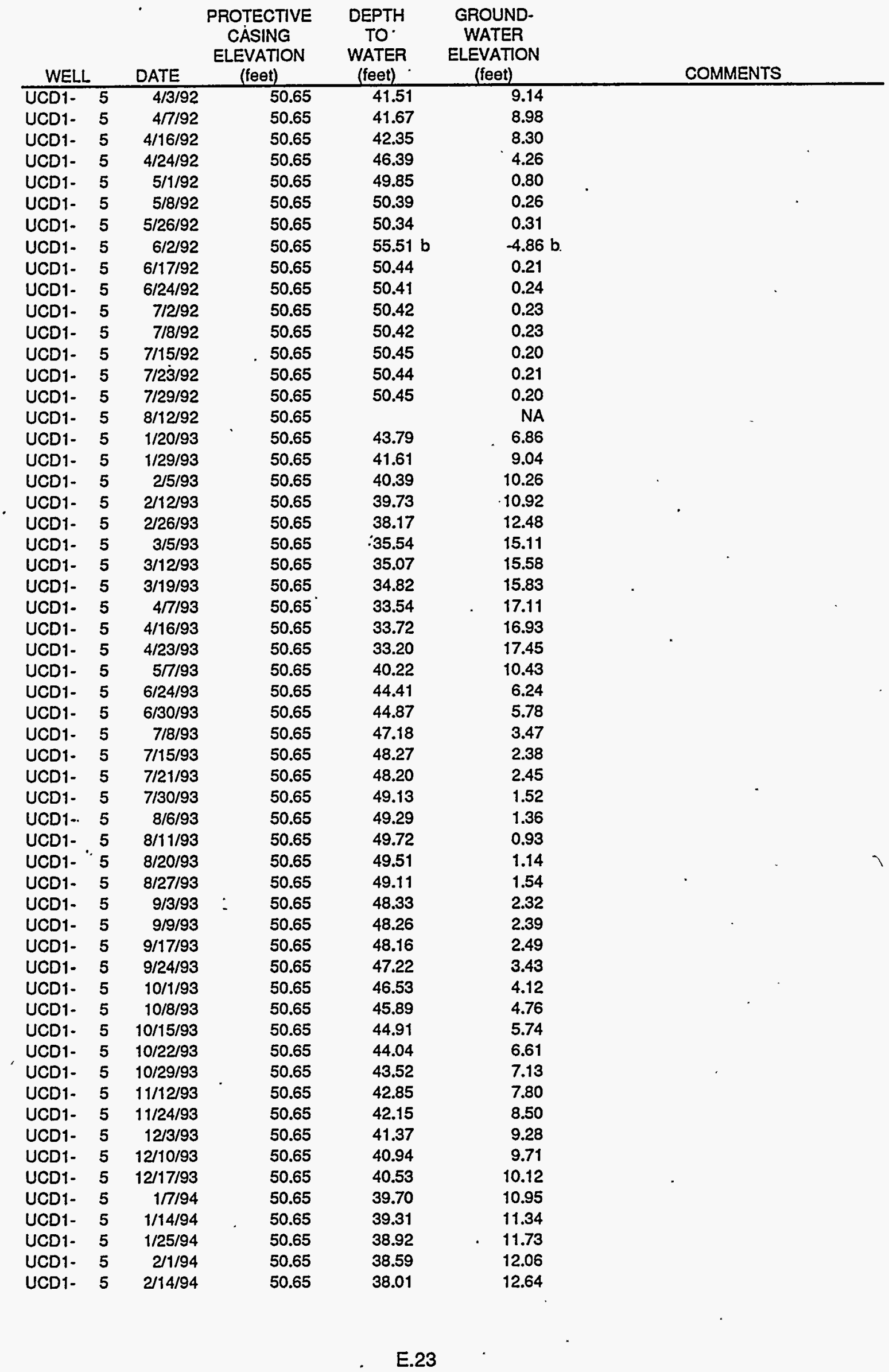


Table E.1. Cumulative Groundwater Elevation Data for HSU-1, 1990-1995

\begin{tabular}{|c|c|c|c|c|c|c|}
\hline WELL & & DATE & $\begin{array}{l}\text { PROTECTIVE } \\
\text { CASING } \\
\text { ELEVATION } \\
\text { (feet) } \\
\end{array}$ & $\begin{array}{l}\text { DEPTH } \\
\text { TO } \\
\text { WATER } \\
\text { (feet) }\end{array}$ & $\begin{array}{l}\text { GROUND- } \\
\text { WATER } \\
\text { ELEVATION } \\
\text { (feet) }\end{array}$ & COMMENTS \\
\hline UCD1- & 5 & $2 / 20 / 94$ & 50.65 & 37.78 & 12.87 & \\
\hline UCD1- & 5 & $3 / 4 / 94$ & 50.65 & 37.24 & 13.41 & \\
\hline UCD1- & 5 & $3 / 10 / 94$ & 50.65 & 37.18 & 13.47 & \\
\hline UCD1- & 5 & $3 / 16 / 94$ & 50.65 & 37.16 & 13.49 & \\
\hline UCD1- & 5 & $3 / 24 / 94$ & 50.65 & 37.69 & 12.96 & \\
\hline UCD1- & 5 & $3 / 29 / 94$ & 50.65 & 39.85 & 10.80 & \\
\hline UCD1- & 5 & $4 / 6 / 94$ & 50.65 & 43.02 & 7.63 & \\
\hline UCD1- & 5 & $4 / 13 / 94$ & 50.65 & 46.27 & 4.38 & \\
\hline UCD1- & 5 & $4 / 22 / 94$ & 50.65 & 49.57 & 1.08 & \\
\hline UCD1: & 5 & $4 / 26 / 94$ & 50.65 & 50.19 & 0.46 & \\
\hline UCD1- & 5 & $5 / 6 / 94$ & 50.65 & 50.36 & 0.29 & \\
\hline UCD1- & 5 & $5 / 11 / 94$ & 50.65 & 50.15 & 0.50 & \\
\hline UCD1- & 5 & $5 / 18 / 94$ & 50.65 & 50.36 & 0.29 & \\
\hline UCD1- & 5 & $6 / 1 / 94$ & 50.65 & 50.35 & 0.30 & \\
\hline UCD1- & 5 & $6 / 23 / 94$ & 50.65 & 50.43 & 0.22 & \\
\hline UCD1- & 5 & $7 / 14 / 94$ & 50.65 & 50.43 & 0.22 & \\
\hline UCD1- & 5 & $7 / 20 / 94$ & 50.65 & 50.96 & -0.31 & \\
\hline UCD1- & 5 & $7 / 26 / 94$ & 50.65 & & NA & \\
\hline UCD1- & 5 & $3 / 24 / 95$ & 50.65 & 30.05 & 20.60 & \\
\hline UCD1- & 5 & $4 / 28 / 95$ & 50.65 & 32.32 & 18.33 & \\
\hline UCD1- & 5 & $5 / 25 / 95$ & 50.65 & 32.91 & 17.74 & \\
\hline UCD1- & 5 & $6 / 16 / 95$ & 50.65 & 37.89 & 12.76 & \\
\hline UCD1- & 5 & $7 / 26 / 95$ & 50.65 & $45.84 \mathrm{a}$ & $4.81 \mathrm{a}$ & \\
\hline UCD1- & 5 & $8 / 31 / 95$ & 50.65 & 47.32 a & $3.33 \mathrm{a}$ & \\
\hline UCD1- & 5 & 9/26/95 & 50.65 & $44.54 \mathrm{a}$ & $6.11 \mathrm{a}$ & \\
\hline UCD1- & 5 & $10 / 25 / 95$ & $48.44^{\bullet}$ & & NA & well damaged \\
\hline UCD1- & 5 & $11 / 22 / 95$ & $48.44^{\bullet}$ & 37.24 a & $11.20 \mathrm{a}$ & Regrouted new elevation/added silicone \\
\hline UCD1- & 5 & $11 / 27 / 95$ & $48.44^{*}$ & 37.92 & 10.52 & no pump \\
\hline UCD1- & 5 & $12 / 19 / 95$ & $48.44^{\bullet}$ & 34.82 & 13.62 & \\
\hline UCD1- & 6 & $2 / 1 / 91$ & 50.94 & 49.75 & 1.19 & \\
\hline UCD1- & 6 & $2 / 8 / 91$ & 50.94 & 49.48 & 1.46 & \\
\hline UCD1- & 6 & $2 / 14 / 91$ & 50.94 & 49.18 & 1.76 & \\
\hline UCD1- & 6 & $3 / 7 / 91$ & 50.94 & 49.51 & 1.43 & \\
\hline UCD1- & 6 & $3 / 12 / 91$ & 50.94 & 48.94 & 2.00 & \\
\hline UCD1- & 6 & $3 / 22 / 91$ & 50.94 & 47.43 & 3.51 & \\
\hline UCD1- & 6 & $3 / 29 / 91$ & 50.94 & 47.54 & 3.40 & . \\
\hline UCD1- & 6 & $4 / 3 / 91$ & 50.94 & 44.99 & 5.95 & \\
\hline UCD1- & 6 & $4 / 10 / 91$ & 50.94 & 44.47 & 6.47 & . \\
\hline UCD1- & 6 & $4 / 17 / 91$ & 50.94 & 44.70 & 6.24 & \\
\hline UCD1- & 6 & $4 / 24 / 91$ & 50.94 & 47.02 & 3.92 & \\
\hline UCD1- & 6 & $5 / 3 / 91$ & 50.94 & 49.42 & 1.52 & \\
\hline UCD1- & 6 & $4 / 24 / 92$ & 50.94 & 46.87 & 4.07 & \\
\hline UCD1- & 6 & $5 / 1 / 92$ & 50.94 & 49.83 & 1.11 & \\
\hline UCD1- & 6 & $6 / 24 / 93$ & 50.94 & 44.49 & 6.45 & \\
\hline UCD1- & 6 & $6 / 30 / 93$ & 50.94 & 45.00 & 5.94 & \\
\hline UCD1- & 6 & $7 / 8 / 93$ & 50.94 & 47.24 & 3.70 & \\
\hline UCD1- & 6 & $7 / 15 / 93$ & 50.94 & 48.27 & 2.67 & \\
\hline UCD1- & 6 & $7 / 21 / 93$ & 50.94 & 47.25 & 3.69 & \\
\hline UCD1- & 6 & $7 / 30 / 93$ & 50.94 & 49.35 & 1.59 & \\
\hline UCD1. & 6 & $8 / 6 / 93$ & 50.94 & 49.63 & 1.31 & \\
\hline UCD1- & 6 & $8 / 11 / 93$ & 50.94 & 50.01 & 0.93 & \\
\hline UCD1- & 6 & $8 / 20 / 93$ & 50.94 & 50.08 & 0.86 & \\
\hline UCD1- & 6 & $8 / 27 / 93$ & 50.94 & 49.11 & 1.83 & \\
\hline UCDi- & 6 & 9/3/93 & 50.94 & 48.48 & 2.46 & \\
\hline UCD1- & 6 & 9/9/93 & 50.94 & 48.34 & 2.60 & \\
\hline UCD1- & 6 & $9 / 17 / 93$ & 50.94 & 48.13 & 2.81 & \\
\hline
\end{tabular}


Table E.1. Cumulative Groundwater Elevation Data for HSU-1, 1990-1995

\begin{tabular}{|c|c|c|c|c|c|c|c|}
\hline \multicolumn{2}{|c|}{ WELL } & DATE & $\begin{array}{c}\text { PROTECTIVE } \\
\text { CASING } \\
\text { ELEVATION } \\
\text { (feet) }\end{array}$ & $\begin{array}{l}\text { DEPTH } \\
\text { TO } \\
\text { WATER } \\
\text { (feet) }\end{array}$ & $\begin{array}{c}\text { GROUND- } \\
\text { WATER } \\
\text { ELEVATION } \\
\text { (feet) }\end{array}$ & & COMMENTS \\
\hline UCD1- & 6 & $9 / 24 / 93$ & 50.94 & 47.27 & 3.67 & & \\
\hline UCD1- & 6 & $10 / 1 / 93$ & 50.94 & 46.47 & 4.47 & & \\
\hline UCD1- & 6 & $10 / 8 / 93$ & 50.94 & 45.83 & 5.11 & & \\
\hline UCD1- & 6 & $10 / 15 / 93$ & 50.94 & 44.83 & 6.11 & & \\
\hline UCD1- & 6 & $10 / 22 / 93$ & 50.94 & 44.02 & 6.92 & & \\
\hline UCD1- & 6 & $10 / 29 / 93$ & 50.94 & 43.56 & 7.38 & & \\
\hline UCD1- & 6 & $11 / 12 / 93$ & 50.94 & 42.85 & 8.09 & & \\
\hline UCD1- & 6 & $11 / 24 / 93$ & 50.94 & 42.20 & 8.74 & & \\
\hline UCD1- & 6 & $12 / 3 / 93$ & 50.94 & 41.47 & 9.47 & & \\
\hline UCD1- & 6 & $12 / 10 / 93$ & 50.94 & 41.00 & 9.94 & & \\
\hline UCD1- & 6 & $12 / 17 / 93$ & 50.94 & 40.59 & 10.35 & & \\
\hline UCD1- & 6 & $1 / 7 / 94$ & 50.94 & 39.79 & 11.15 & & \\
\hline UCD1- & 6 & $1 / 14 / 94$ & 50.94 & 39.47 & 11.47 & & 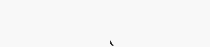 \\
\hline UCD1- & 6 & $1 / 25 / 94$ & 50.94 & 38.91 & 12.03 & & \\
\hline UCD1- & 6 & $2 / 1 / 94$ & 50.94 & 38.72 & 12.22 & & . \\
\hline UCD1- & 6 & $2 / 14 / 94$ & 50.94 & 38.15 & 12.79 & & \\
\hline UCD1- & 6 & $2 / 20 / 94$ & 50.94 & 37.84 & 13.10 & & \\
\hline UCD1. & 6 & $3 / 4 / 94$ & 50.94 & 37.42 & 13.52 & & \\
\hline UCD1- & 6 & $3 / 10 / 94$ & 50.94 & 37.39 & 13.55 & & \\
\hline UCD1- & 6 & $3 / 16 / 94$ & 50.94 & 37.27 & 13.67 & & \\
\hline UCD1- & 6 & $3 / 24 / 94$ & 50.94 & 37.81 & 13.13 & & \\
\hline UCD1- & 6 & $3 / 29 / 94$ & 50.94 & 39.68 . & 11.26 & & \\
\hline UCD1- & 6 & $4 / 6 / 94$ & 50.94 & 43.20 & 7.74 & & \\
\hline UCD1. & 6 & $4 / 13 / 94$ & 50.94 & 48.64 & 2.30 & & \\
\hline UCD1- & 6 & $4 / 22 / 94$ & 50.94 & 49.55 & 1.39 & & \\
\hline UCD1- & 6 & $5 / 6 / 94$ & 50.94 & $0.00 \mathrm{~b}$ & NA & & \\
\hline UCD1- & 6 & $5 / 11 / 94$ & 50.94 & $0.00 \mathrm{~b}$ & NA & & \\
\hline UCD1- & 6 & $3 / 24 / 95$ & 50.94 & 30.52 & 20.42 & & \\
\hline UCD1- & 6 & $4 / 28 / 95$ & 50.94 & 32.65 & 18.29 & & \\
\hline UCD1. & 6 & $5 / 25 / 95$ & 50.94 & 33.06 & 17.88 & & \\
\hline UCD1- & 6 & $6 / 16 / 95$ & 50.94 & 37.71 & 13.23 & & \\
\hline UCD1- & 6 & $7 / 26 / 95$ & 50.94 & $45.77 \mathrm{a}$ & 5.17 & & \\
\hline UCD1- & 6 & $8 / 31 / 95$ & 50.94 & $47.14 a$ & 3.80 & & \\
\hline UCD1- & 6 & $9 / 26 / 95$ & 50.94 & $44.45 \mathrm{a}$ & 6.49 & & \\
\hline UCD1. & 6 & $10 / 25 / 95$ & 50.94 & $41.50 \mathrm{a}$ & 9.44 & & \\
\hline UCD1- & 6 & $11 / 22 / 95$ & 50.94 & $39.59 \mathrm{a}$ & 11.35 & & - \\
\hline UCD1- & 6 & $11 / 27 / 95$ & 50.94 & 39.32 & 11.62 & no pump & \\
\hline UCD1- & 6 & $12 / 19 / 95$ & 50.94 & 37.10 & 13.84 & & \\
\hline UCD1- & 8 & $2 / 8 / 91$ & 51.51 & 50.63 & 0.88 & & \\
\hline UCD1- & 8 & $2 / 14 / 91$ & 51.51 & 50.30 & 1.21 & & \\
\hline UCD1- & 8 & $3 / 7 / 91$ & 51.51 & 50.89 & 0.62 & & \\
\hline UCD1- & 8 & $3 / 12 / 91$ & 51.51 & 50.23 & 1.28 & & \\
\hline UCD1. & 8 & $3 / 22 / 91$ & 51.51 & 49.00 & 2.51 & & \\
\hline UCD1. & 8 & $3 / 29 / 91$ & 51.51 & 47.14 & 4.37 & & \\
\hline UCD1- & 8 & $4 / 3 / 91$ & 51.51 & 46.03 & 5.48 & & \\
\hline UCD1- & 8 & $4 / 10 / 91$ & 51.51 & 45.38 & 6.13 & & \\
\hline UCD1- & 8 & $4 / 17 / 91$ & 51.51 & 45.53 & 5.98 & & \\
\hline UCD1- & 8 & $4 / 24 / 91$ & 51.51 & 47.71 & 3.80 - & & \\
\hline UCD1- & 8 & $5 / 3 / 91$ & 51.51 & 50.16 & 1.35 & & \\
\hline UCD1- & 8 & $1 / 8 / 92$ & 51.51 & 52.62 & -1.11 & & . \\
\hline UCD1- & 8 & $3 / 6 / 92$ & 51.51 & 44.60 & 6.91 & & \\
\hline UCD1- & 8 & $3 / 13 / 92$ & 51.51 & 43.84 & 7.67 & . & . \\
\hline UCD1- & 8 & $3 / 18 / 92$ & 51.51 & 43.62 & 7.89 & & \\
\hline UCD1- & 8 & $3 / 25 / 92$ & 51.51 & 43.28 & 8.23 & & \\
\hline UCD1- & 8 & $4 / 3 / 92$ & 51.51 & 42.84 & 8.67 & & \\
\hline UCD1- & 8 & $4 / 7 / 92$ & 51.51 & 42.82 & 8.69 & & \\
\hline
\end{tabular}


Table E.1. Cumulative Groundwater Elevation Data for HSU-1, 1990-1995

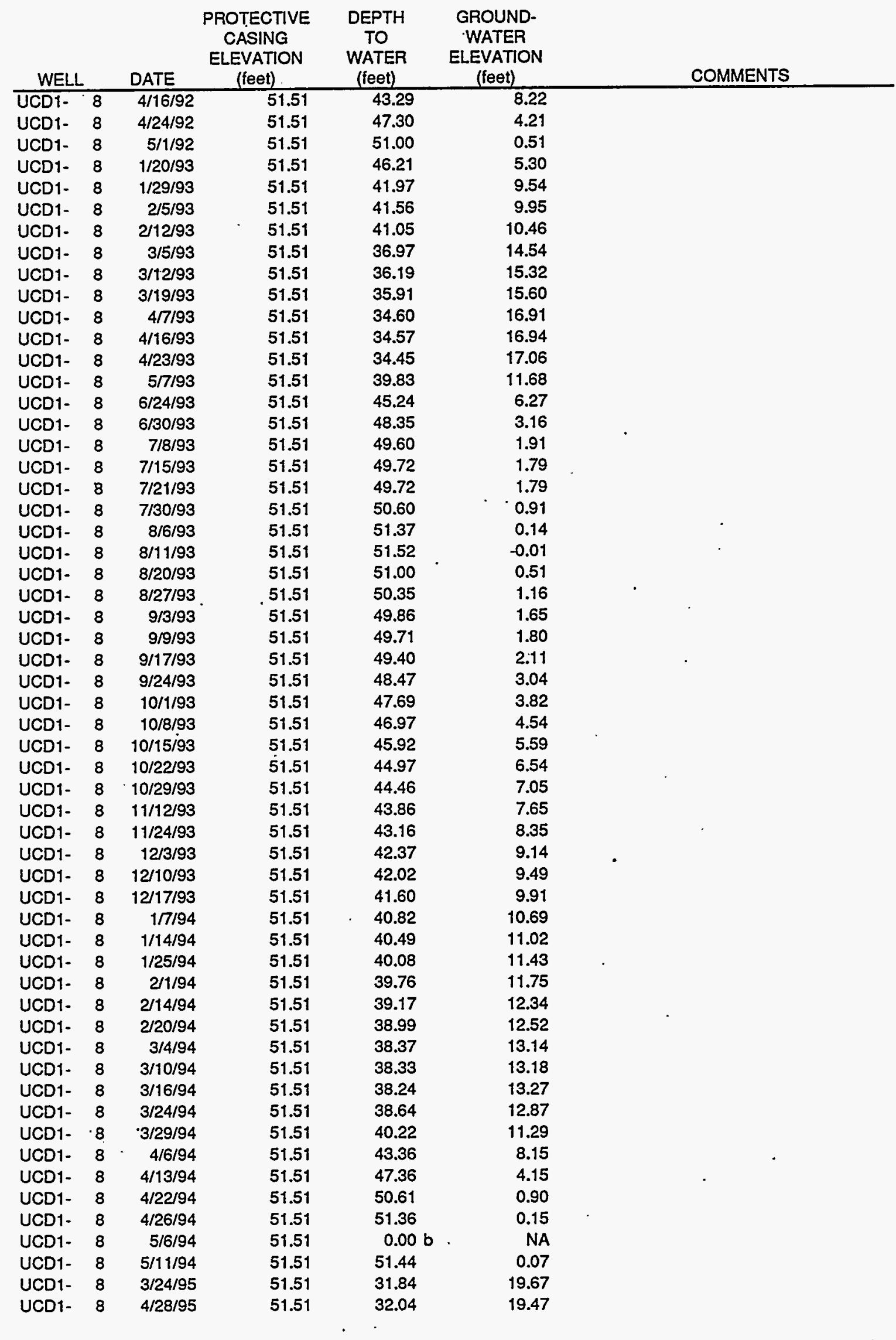


Table E.1. Cumulative Groundwater Elevation Data for HSU-1, 1990-1995

\begin{tabular}{|c|c|c|c|c|c|c|c|c|}
\hline \multicolumn{2}{|c|}{ WELL } & DATE & $\begin{array}{c}\text { PROTECTIVE } \\
\text { CASING } \\
\text { ELEVATION } \\
\text { (feet) }\end{array}$ & $\begin{array}{l}\text { DEPTH } \\
\text { TO } \\
\text { WATER } \\
\text { (feet) }\end{array}$ & $\begin{array}{l}\text { GROUND- } \\
\text { WATER } \\
\text { ELEVATION } \\
\text { (feet) }\end{array}$ & & COMMENTS & \\
\hline UCD1- & 8 & $5 / 25 / 95$ & 51.51 & 33.05 & 18.46 & & & \\
\hline UCD1- & 8 & $6 / 16 / 95$ & 51.51 & 38.40 & 13.11 & . & & \\
\hline UCD1- & 8 & $7 / 26 / 95$ & 51.51 & 47.12 a & $4.39 \mathrm{a}$ & & & \\
\hline UCD1- & 8 & $8 / 31 / 95$ & 51.51 & $48.67 \mathrm{a}$ & $2.84 \mathrm{a}$ & & & \\
\hline UCD1- & 8 & $9 / 26 / 95$ & 51.51 & $45.64 \mathrm{a}$ & $5.87 a$ & no caps & & \\
\hline UCD1- & 8 & $10 / 25 / 95$ & 51.51 & $42.60 \mathrm{a}$ & $8.91 \mathrm{a}$ & & & \\
\hline UCD1- & 8 & $11 / 22 / 95$ & 51.51 & 40.77 a & $10.74 \mathrm{a}$ & & & \\
\hline UCD1- & 8 & $11 / 27 / 95$ & 51.51 & 40.54 & 10.97 & no pump & & \\
\hline UCD1- & 8 & $12 / 19 / 95$ & 51.51 & 38.36 & 13.15 & & & \\
\hline UCD1- & 9 & $3 / 29 / 91$ & 51.38 & 46.87 & 4.51 & & & \\
\hline UCD1- & 9 & $4 / 3 / 91$ & 51.38 & 46.13 & 5.25 & & & \\
\hline UCD1- & 9 & $4 / 10 / 91$ & 51.38 & 45.52 & 5.86 & & & \\
\hline UCD1- & 9 & $4 / 17 / 91$ & 51.38 & 45.74 & 5.64 & & & \\
\hline UCD1- & 9 & $3 / 6 / 92$ & 51.38 & 44.48 & 6.90 & $\cdot$ & & \\
\hline UCD1- & 9 & $3 / 13 / 92$ & 51.38 & 43.65 & 7.73 & & & • \\
\hline UCD1- & 9 & $3 / 18 / 92$ & 51.38 & 43.48 & 7.90 & & & \\
\hline UCD1- & 9 & $3 / 25 / 92$ & 51.38 & 43.18 & 8.20 & & & \\
\hline UCD1. & 9 & $4 / 3 / 92$ & 51.38 & 42.79 & 8.59 & & & \\
\hline UCD1- & 9 & $4 / 7 / 92$ & 51.38 & 42.86 & 8.52 & & & \\
\hline UCD1- & 9 & $4 / 16 / 92$ & 51.38 & 43.55 & 7.83 & & & \\
\hline UCD1. & 9 & $4 / 24 / 92$ & 51.38 & 47.59 & 3.79 & & & \\
\hline UCD1- & 9 & $1 / 20 / 93$ & 51.38 & 43.50 & 7.88 & & & \\
\hline UCD1- & 9 & $1 / 29 / 93$ & 51.38 & 41.42 & 9.96 & & & \\
\hline UCD1. & 9 & $2 / 5 / 93$ & 51.38 & 41.41 & 9.97 & & & \\
\hline UCD1- & 9 & $2 / 12 / 93$ & 51.38 & 40.92 & 10.46 & & & \\
\hline UCD1- & 9 & $3 / 5 / 93$ & 51.38 & 36.38 & 15.00 & & & \\
\hline UCD1- & 9 & $3 / 12 / 93$ & 51.38 & 35.91 & 15.47 & & & \\
\hline UCD1- & 9 & $3 / 19 / 93$ & 51.38 & 35.73 & 15.65 & & & \\
\hline UCD1- & 9 & $4 / 7 / 93$ & 51.38 & 34.45 & 16.93 & & & \\
\hline UCD1- & 9 & $4 / 16 / 93$ & 51.38 & 34.68 & 16.70 & & & \\
\hline UCD1- & 9 & $4 / 23 / 93$ & 51.38 & 34.89 & 16.49 & . & - & \\
\hline UCD1- & 9 & $5 / 7 / 93$ & 51.38 & 40.94 & 10.44 & & . & \\
\hline UCD1- & 9 & $6 / 24 / 93$ & 51.38 & $4 \dot{7} .56$ & 3.82 & & & \\
\hline UCD1- & 9 & $6 / 30 / 93$ & 51.38 & 48.47 & 2.91 & & & \\
\hline UCD1- & 9 & $7 / 8 / 93$ & 51.38 & 47.35 & 4.03 & & & \\
\hline UCD1- & 9 & $10 / 15 / 93$ & 51.38 & 45.91 & 5.47 & & & \\
\hline UCD1- & 9 & $10 / 22 / 93$ & 51.38 & 45.02 & 6.36 & & & \\
\hline UCD1- & 9 & $10 / 29 / 93$ & 51.38 & 44.51 & 6.87 & & & \\
\hline UCD1- & 9 & $11 / 12 / 93$ & 51.38 & 43.89 & 7.49 & & & \\
\hline UCD1. & 9 & $11 / 24 / 93$ & 51.38 & 43.20 & 8.18 & & & \\
\hline UCD1- & 9 & 12/3/93 & 51.38 & 42.47 & 8.91 & & & \\
\hline UCD1- & 9 & $12 / 10 / 93$ & 51.38 & 42.04 & 9.34 & & & \\
\hline UCD1- & 9 & $12 / 17 / 93$ & 51.38 & 41.64 & 9.74 & & & \\
\hline UCD1- & 9 & $1 / 7 / 94$ & 51.38 & 40.78 & 10.60 & & & \\
\hline UCD1- & 9 & $1 / 14 / 94$ & 51.38 & 40.41 & 10.97 & $\cdot$ & & \\
\hline UCD1- & 9 & $1 / 25 / 94$ & 51.38 & 40.03 & 11.35 & & & \\
\hline UCD1- & 9 & $2 / 1 / 94$ & 51.38 & 39.69 & 11.69 & & & - \\
\hline UCD1- & 9 & $2 / 14 / 94$ & 51.38 & 39.09 & 12.29 & & & \\
\hline UCD1- & 9 & $2 / 20 / 94$ & 51.38 & 38.96 & 12.42 & & & \\
\hline UCD1- & 9 & $3 / 4 / 94$ & 51.38 & 38.30 & 13.08 & & & \\
\hline UCD1. & 9 & $3 / 10 / 94$ & 51.38 & 38.29 & 13.09 & & & \\
\hline UCD1. & 9 & $3 / 16 / 94$ & 51.38 & 38.19 & 13.19 & & & \\
\hline UCD1- & 9 & $3 / 24 / 94$ & 51.38 & 38.72 & 12.66 & & & \\
\hline UCD1- & 9 & $3 / 29 / 94$ & 51.38 & 40.77 & 10.61 & & & \\
\hline UCD1- & 9 & $4 / 6 / 94$ & 51.38 & 43.93 & 7.45 & & & \\
\hline UCD1- & 9 & $4 / 13 / 94$ & 51.38 & 47.75 & 3.63 & & & \\
\hline
\end{tabular}


Table E.1. Cumulative Groundwater Elevation Data for HSU-1, 1990-1995

\begin{tabular}{|c|c|c|c|c|c|}
\hline WELL & DATE & $\begin{array}{l}\text { PROTECTIVE } \\
\text { CASING } \\
\text { ELEVATION } \\
\text { (feet) }\end{array}$ & $\begin{array}{l}\text { DEPTH } \\
\text { TO } \\
\text { WATER } \\
\text { (feet) }\end{array}$ & $\begin{array}{c}\text { GROUND- } \\
\text { WATER } \\
\text { EL:EVATION } \\
\text { (feet) }\end{array}$ & COMMENTS \\
\hline UCD1- 9 & $5 / 6 / 94$ & 51.38 & $0.00 \mathrm{~b}$ & NA & \\
\hline UCD1- & $5 / 11 / 94$ & 51.38 & $0.00 \mathrm{~b}$ & NA & \\
\hline UCDY. & $3 / 24 / 95$ & 51.38 & 30.52 & 20.86 & \\
\hline UCDY- & $4 / 28 / 95$ & 51.38 & 32.47 & 18.91 & \\
\hline UCD1- & $5 / 25 / 95$ & 51.38 & 33.38 & 18.00 & \\
\hline UCD1- & $6 / 16 / 95$ & 51.38 & 38.91 & 12.47 & \\
\hline UCDi- & $7 / 26 / 95$ & 51.38 & $47.30 \mathrm{a}$ & $4.08 \mathrm{a}$ & \\
\hline UCD1- & $8 / 31 / 95$ & 51.38 & & NA & dry; Caps are missing \\
\hline UCDY- & $9 / 26 / 95$ & 51.38 & $45.67 \mathrm{a}$ & $5.71 \mathrm{a}$ & caps missing \\
\hline UCD1- & $10 / 25 / 95$ & 51.38 & $42.65 \mathrm{a}$ & $8.73 a$ & \\
\hline UCD1- & $11 / 22 / 95$ & 51.38 & $40.70 \mathrm{a}$ & $10.68 \mathrm{a}$ & \\
\hline UCD1- & $11 / 27 / 95$ & 51.38 & 40.42 & 10.96 & \\
\hline UCD1- & $12 / 19 / 95$ & 51.38 & 38.26 & 13.12 & \\
\hline UCD1- 10 & $11 / 2 / 90$ & 49.87 & 56.58 & -6.71 & \\
\hline UCD1- 10 & $11 / 8 / 90$ & 49.87 & 55.93 & -6.06 & \\
\hline UCD1- 10 & $11 / 16 / 90$ & 49.87 & 54.86 & -4.99 & \\
\hline UCD1- 10 & $11 / 20 / 90$ & 49.87 & 54.38 & -4.51 & \\
\hline UCD1- 10 & $12 / 5 / 90$ & 49.87 & 52.71 & -2.84 & \\
\hline UCD1- 10 & $12 / 27 / 90$ & 49.87 & 50.82 & -0.95 & \\
\hline UCD1- 10 & $1 / 4 / 91$ & 49.87 & 50.27 & -0.40 & . \\
\hline UCD1- 10 & $1 / 11 / 91$ & 49.87 & 49.97 & -0.10 & - \\
\hline UCD1- 10 & $1 / 22 / 91$ & 49.87 & 49.20 & 0.67 & \\
\hline UCD1- 10 & $2 / 1 / 91$ & 49.87 & 49.85 & 0.02 & \\
\hline UCD1- 10 & $2 / 8 / 91$ & 49.87 & 49.10 & 0.77 & \\
\hline UCD1- 10 & $2 / 14 / 91$ & 49.87 & 48.78 & 1.09 & \\
\hline UCD 1- 10 & $2 / 22 / 91$ & 49.87 & 52.10 & -2.23 & \\
\hline UCD1- 10 & $3 / 7 / 91$ & 49.87 & 49.13 & 0.74 & . \\
\hline UCD1- 10 & $3 / 12 / 91$ & 49.87 & 48.35 & 1.52 & \\
\hline UCD1- 10 & $3 / 22 / 91$ & 49.87 & 46.96 & 2.91 & \\
\hline UCD1- 10 & $3 / 29 / 91$ & 49.87 & 44.92 & 4.95 & \\
\hline UCD1- 10 & $4 / 3 / 91$ & 49.87 & 44.81 & 5.06 & \\
\hline UCD1- 10 & $4 / 10 / 91$ & 49.87 & 44.51 & 5.36 & \\
\hline UCDi- 10 & $4 / 17 / 91$ & 49.87 & 44.99 & 4.88 & - \\
\hline UCD1- 10 & $4 / 24 / 91$ & 49.87 & 48.39 & 1.48 & \\
\hline UCD1- 10 & $5 / 3 / 91$ & 49.87 & 51.26 & -1.39 & \\
\hline UCD1- 10 & $5 / 13 / 91$ & 49.87 & 56.32 & -6.45 & \\
\hline UCD $1-10$ & $5 / 20 / 91$ & 49.87 & 59.01 & -9.14 & \\
\hline UCD1- 10 & $5 / 29 / 91$ & 49.87 & 59.58 & -9.71 & \\
\hline UCD1- 10 & $6 / 6 / 91$ & 49.87 & 63.06 & -13.19 & \\
\hline UCD1- 10 & $6 / 10 / 91$ & 49.87 & 63.76 & -13.89 & \\
\hline UCD1- 10 & $6 / 21 / 91$ & 49.87 & 64.43 & -14.56 & \\
\hline UCD1- 10 & $6 / 27 / 91$ & 49.87 & 66.03 & -16.16 & \\
\hline UCD1- 10 & $7 / 5 / 91$ & 49.87 & 66.83 & -16.96 & \\
\hline UCD1- 10 & $7 / 12 / 91$ & 49.87 & 69.59 & -19.72 & \\
\hline UCD1- 10 & $7 / 19 / 91$ & 49.87 & 71.00 & -21.13 & \\
\hline UCD1- 10 & $7 / 26 / 91$ & 49.87 & 71.00 & -21.13 & \\
\hline UCD1- 10 & $8 / 14 / 91$ & 49.87. & 72.41 & -22.54 & - \\
\hline UCD1- 10 & $8 / 21 / 91$ & 49.87 & 69.45 & -19.58 & \\
\hline UCD1- 10 & $8 / 29 / 91$ & 49.87 & 66.85 & -16.98 & \\
\hline UCD1- 10 & $9 / 4 / 91$ & 49.87 & 65.04 & -15.17 & \\
\hline UCD1- 10 & $9 / 13 / 91$ & 49.87 & 66.00 & -16.13 & \\
\hline UCD1- 10 & $10 / 3 / 91$ & 49.87 & 60.63 & -10.76 & \\
\hline UCD1- 10 & $10 / 10 / 91$ & 49.87 & 59.36 & -9.49 & \\
\hline UCD1- 10 & $10 / 25 / 91$ & 49.87 & 56.00 & -6.13 & \\
\hline UCD1- 10 & $11 / 1 / 91$ & 49.87 & 55.17 & -5.30 & \\
\hline UCD1- 10 & $11 / 8 / 91$ & 49.87 & 54.00 & -4.13 & \\
\hline
\end{tabular}


Table E.1. Cumulative Groundwater Elevation Data for HSU-1, 1990-1995

\begin{tabular}{|c|c|c|c|c|c|c|}
\hline W'ELL & DATE & $\begin{array}{c}\text { PROTECTIVE } \\
\text { CASING } \\
\text { ELEVATION } \\
\text { (feet) }\end{array}$ & $\begin{array}{l}\text { DEPTH } \\
\text { TO } \\
\text { WATER } \\
\text { (feet) }\end{array}$ & $\begin{array}{c}\text { GROUND- } \\
\text { WATER } \\
\text { ELEVATION } \\
\text { (feet) }\end{array}$ & & COMMENTS \\
\hline UCD1- 10 & $11 / 14 / 91$ & 49.87 & 53.42 & -3.55 & & \\
\hline UCD1- 10 & $12 / 5 / 91$ & 49.87 & 51.71 & -1.84 & & \\
\hline UCD1- 10 & $12 / 11 / 91$ & -49.87 & 51.41 & -1.54 & & \\
\hline UCD1- 10 & $12 / 19 / 91$ & 49.87 & 50.93 & -1.06 & & \\
\hline UCD1- 10 & 1/8/92 & 49.87 & 48.54 & 1.33 & & \\
\hline UCD1- 10 & $1 / 17 / 92$ & 49.87 & 48.55 & 1.32 & & \\
\hline UCD1- 10 & $1 / 24 / 92$ & 49.87 & 48.08 & 1.79 & & \\
\hline UCD1- 10 & $1 / 29 / 92$ & 49.87 & 47.49 & 2.38 & & \\
\hline UCD1- 10 & $2 / 5 / 92$ & 49.87 & 46.81 & 3.06 & & \\
\hline UCD1- 10 & $3 / 6 / 92$ & 49.87 & 43.27 & 6.60 & & \\
\hline UCD1- 10 & $3 / 13 / 92$ & 49.87 & 42.72 & 7.15 & & \\
\hline UCD1- 10 & $3 / 18 / 92$ & 49.87 & 42.57 & 7.30 & & \\
\hline UCD1- 10 & $3 / 25 / 92$ & 49.87 & 42.31 & 7.56 & & \\
\hline UCD1- 10 & $4 / 3 / 92$ & 49.87 & 42.06 & 7.81 & & \\
\hline UCD1- 10 & $4 / 7 / 92$ & 49.87 & 42.09 & 7.78 & $\cdot$ & \\
\hline UCD1- 10 & $4 / 16 / 92$ & 49.87 & 43.19 & 6.68 & & \\
\hline UCD1- 10 & $4 / 24 / 92$ & 49.87 & 48.21 & 1.66 & & $\cdot$ \\
\hline UCD1- 10 & $5 / 1 / 92$ & 49.87 & 54.13 & -4.26 & & \\
\hline UCD1- 10 & 5/8/9?2 & 49.87 & 58.83 & -8.96 & & \\
\hline UCD1- 10 & $5 / 26 / 92$ & 49.87 & 59.66 & -9.79 & & \\
\hline UCD1- 10 & $6 / 2 / 92$ & 49.87 & 64.01 & -14.14 & & . \\
\hline UCD1- 10 & $6 / 17 / 92$ & 49.87 & 63.00 & -13.13 & & \\
\hline UCD1- 10 & $6 / 24 / 92$ & 49.87 & 64.20 & -14.33 & & \\
\hline UCD1- 10 & $7 / 8 / 92$ & 49.87 & 65.47 & -15.60 & & \\
\hline UCD1- 10 & $7 / 29 / 92$ & 49.87 & 70.51 & -20.64 & & \\
\hline UCD1- 10 & $8 / 19 / 92$ & 49.87 & 66.39 & -16.52 & & \\
\hline UCD1- 10 & $8 / 28 / 92$ & 49.87 & 65.85 & -15.98 & & \\
\hline UCD1- 10 & $9 / 4 / 92$ & 49.87 & 64.29 & -14.42 & & \\
\hline UCD1- 10 & $9 / 18 / 92$ & 49.87 & 62.51 & -12.64 & & \\
\hline UCD1- 10 & $10 / 9 / 92$ & 49.87 & 59.92 & -10.05 & & \\
\hline UCD1- 10 & $10 / 16 / 92$ & 49.87 & 59.06 & -9.19 & & \\
\hline UCD1- 10 & $10 / 30 / 92$ & 49.87 & 56.77 & -6.90 & & \\
\hline UCD1- 10 & $11 / 6 / 92$ & 49.87 & 55.83 & -5.96 & & \\
\hline UCD1- 10 & $11 / 17 / 92$ & 49.87 & 54.68 & -4.81 & & \\
\hline UCD1- 10 & $12 / 4 / 92$ & 49.87 & 53.36 & -3.49 & & \\
\hline UCD1- 10 & $12 / 28 / 92$ & 49.87 & 50.68 & -0.81 & & \\
\hline UCD1- 10 & $1 / 20 / 93$ & 49.87 & 44.63 & 5.24 & & \\
\hline UCD1- 10 & $1 / 29 / 93$ & 49.87 & 40.60 & 9.27 & & \\
\hline UCD1- 10 & $2 / 5 / 93$ & 49.87 & 40.38 & 9.49 & & \\
\hline UCD1- 10 & $2 / 26 / 93$ & 49.87 & 35.66 & 14.21 & & \\
\hline UCD1- 10 & $3 / 5 / 93$ & 49.87 & 35.30 & 14.57 & & \\
\hline UCD1- 10 & $3 / 12 / 93$ & 49.87 & 34.92 & 14.95 & & \\
\hline UCD1- 10 & $3 / 19 / 93$ & 49.87 & 34.73 & 15.14 & & \\
\hline UCD1- 10 & $4 / 7 / 93$ & 49.87 & 33.46 & 16.41 & & \\
\hline UCD1- 10 & $4 / 16 / 93$ & 49.87 & 34.09 & 15.78 & , & \\
\hline UCD1- 10 & $4 / 23 / 93$ & 49.87 & 34.80 & 15.07 & & \\
\hline UCD1- 10 & $5 / 7 / 93$ & 49.87 & 43.17 & 6.70 & & \\
\hline UCD1- 10 & $6 / 25 / 93$ & 49.87 & 47.88 & 1.99 & & \\
\hline UCD1- 10 & $6 / 30 / 93$ & 49.87 & 47.07 & 2.80 & & \\
\hline UCD1- 10 & $7 / 8 / 93$ & 49.87 & 49.98 & -0.11 & & \\
\hline UCD1- 10 & $7 / 15 / 93$ & 49.87 & 51.44 & -1.57 & & \\
\hline UCD1- 10 & $7 / 21 / 93$ & 49.87 & 51.00 & -1.13 & . & . \\
\hline UCD1- 10 & $7 / 30 / 93$ & 49.87 & 52.68 & -2.81 & & \\
\hline UCD1- 10 & $8 / 6 / 93$ & 49.87 & 52.87 & -3.00 & & \\
\hline UCD1- 10 & $8 / 11 / 93$ & 49.87 & 52.87 & -3.00 & & \\
\hline UCD1- 10 & $8 / 20 / 93$ & 49.87 & 53.00 & -3.13 & & \\
\hline UCD1- 10 & $8 / 27 / 93$ & 49.87 & 49.90 & -0.03 & & \\
\hline
\end{tabular}


Table E.1. Cumulative Groundwater Elevation Data for HSU-1, 1990-1995

\begin{tabular}{|c|c|c|c|c|c|c|c|c|}
\hline WELL & & DATE & $\begin{array}{l}\text { PROTECTIVE } \\
\text { CASING } \\
\text { ELEVATION } \\
\text { (feet) }\end{array}$ & $\begin{array}{c}\text { DEPTH } \\
\text { TO } \\
\text { WATER } \\
\text { (feet) }\end{array}$ & $\begin{array}{c}\text { GROUND- } \\
\text { WATER } \\
\text { ELEVATION } \\
\text { (feet) }\end{array}$ & - & COMMENTS & \\
\hline UCD1- & 10 & $9 / 3 / 93$ & 49.87 & 49.25 & 0.62 & & & \\
\hline UCD1- & 10 & 9/9/93 & 49.87 & 50.00 & -0.13 & & & \\
\hline UCD1- & 10 & $9 / 17 / 93$ & 49.87 & 48.78 & 1.09 & & & \\
\hline UCD1- & 10 & $9 / 24 / 93$ & 49.87 & 47.75 & 2.12 & & & \\
\hline UCD1. & 10 & $10 / 1 / 93$ & 49.87 & 46.83 & 3.04 & & & \\
\hline UCD1- & 10 & $10 / 8 / 93$ & 49.87 & 46.25 & 3.62 & & & \\
\hline UCD1- & 10 & $10 / 15 / 93$ & 49.87 & 44.99 & 4.88 & & & \\
\hline UCD1- & 10 & $10 / 22 / 93$ & 49.87 & 44.17 & 5.70 & & & \\
\hline UCD1- & 10 & $10 / 29 / 93$ & 49.87 & 43.83 & 6.04 & & & \\
\hline UCD1- & 10 & $11 / 12 / 93$ & 49.87 & 43.08 & 6.79 & & & \\
\hline UCD1- & 10 & $11 / 24 / 93$ & 49.87 & 42.39 & 7.48 & & & \\
\hline UCD1- & 10 & $12 / 3 / 93$ & 49.87 & 41.69 & 8.18 & & & \\
\hline UCD1. & 10 & $12 / 10 / 93$ & 49.87 & 41.14 & 8.73 & & & \\
\hline UCD1- & 10 & $12 / 17 / 93$ & 49.87 & 40.73 & 9.14 & & & \\
\hline UCD1- & 10 & $1 / 7 / 94$ & 49.87 & 39.78 & 10.09 & & & \\
\hline UCD1- & 10 & $1 / 14 / 94$ & 49.87 & 39.35 & 10.52 & & & \\
\hline UCD1- & 10 & $1 / 25 / 94$ & 49.87 & 38.96 & 10.91 & & & \\
\hline UCD1- & 10 & (.) $2 / 1 / 94$ & 49.87 & 38.72 & 11.15 & & & \\
\hline UCD1- & 10 & $2 / 14 / 94$ & 49.87 & 38.31 & 11.56 & & & \\
\hline UCD1- & 10 & $2 / 22 / 94$ & 49.87 & 37.94 & 11.93 & & & \\
\hline UCD1- & 10 & $3 / 2 / 94$ & 49.87 & 37.27 & 12.60 & & & \\
\hline UCD1- & 10 & $3 / 4 / 94$ & 49.87 & 37.41 & 12.46 & & & \\
\hline UCD1- & 10 & $3 / 10 / 94$ & 49.87 & 37.38 & 12.49 & & & \\
\hline UCD1- & 10 & $3 / 16 / 94$ & 49.87 & 37.33 & 12.54 & . & & \\
\hline UCD1- & 10 & $3 / 24 / 94$ & 49.87 & 38.25 & 11.62 & & & \\
\hline UCD1- & 10 & $3 / 29 / 94$ & 49.87 & 41.04 & 8.83 & & & \\
\hline UCD1- & 10 & $4 / 6 / 94$ & 49.87 & 44.70 & 5.17 & & & \\
\hline UCD1- & 10 & $4 / 13 / 94$ & 49.87 & 49.90 & -0.03 & & & \\
\hline UCD1- & 10 & $4 / 22 / 94$ & 49.87 & 54.36 & -4.49 & & & \\
\hline UCD1- & 10 & $4 / 26 / 94$ & 49.87 & 52.59 & -2.72 & & & \\
\hline UCD1- & 10 & $5 / 6 / 94$ & 49.87 & 54.06 & -4.19 & & & \\
\hline UCD1- & 10 & $5 / 11 / 94$ & 49.87 & 51.87 & -2.00 & & & \\
\hline UCD1- & 10 & $5 / 18 / 94$ & 49.87 & 52.63 & -2.76 & & & \\
\hline UCD1- & 10 & $5 / 25 / 94$ & 49.87 & 54.00 & -4.13 & & & \\
\hline UCD1- & 10 & $6 / 1 / 94$ & 49.87 & 56.27 & -6.40 & & & \\
\hline UCD1- & 10 & $6 / 23 / 94$ & 49.87 & 62.98 & -13.11 & & & \\
\hline UCD1- & 10 & $7 / 1 / 94$ & 49.87 & 66.40 & -16.53 & & & \\
\hline UCD1- & 10 & $7 / 8 / 94$ & 49.87 & 68.28 & -18.41 & & & \\
\hline UCD1- & 10 & $7 / 14 / 94$ & 49.87 & 69.45 & -19.58 & & & \\
\hline UCD1- & 10 & $7 / 20 / 94$ & 49.87 & 70.37 & -20.50 & & & \\
\hline UCD1- & 10 & $7 / 26 / 94$ & 49.87 & 70.02 & -20.15 & & & \\
\hline UCD1- & 10 & $8 / 5 / 94$ & 49.87 & 69.00 & -19.13 & & & \\
\hline UCD1- & 10 & $8 / 19 / 94$ & 49.87 & 70.95 & -21.08 & & & \\
\hline UCD1- & 10 & $9 / 7 / 94$ & 49.87 & 66.95 & -17.08 & & & \\
\hline UCD1- & 10 & $9 / 7 / 94$ & 49.87 & 67.40 & -17.53 & & & \\
\hline UCD1- & 10 & $9 / 13 / 94$ & 49.87 & 67.19 & -17.32 & & & \\
\hline UCD1. & 10 & $9 / 19 / 94$ & 49.87 & 63.82 & -13.95 & & & \\
\hline UCD1- & 10 & $9 / 26 / 94$ & 49.87 & 61.99 & -12.12 & & & \\
\hline UCD1- & 10 & $10 / 7 / 94$ & 49.87 & 59.50 & -9.63 & & & \\
\hline UCD1- & 10 & $10 / 14 / 94$ & 49.87 & 57.65 & -7.78 & & & \\
\hline UCD1- & 10 & $10 / 21 / 94$ & 49.87 & 56.58 & -6.71 & & . & \\
\hline UCD1. & 10 & $10 / 28 / 94$ & 49.87 & 55.62 & -5.75 & & & \\
\hline UCD1- & 10 & $11 / 4 / 94$ & 49.87 & 54.99 & -5.12 & & & \\
\hline UCD1- & 10 & $11 / 18 / 94$ & 49.87 & 52.26 & -2.39 & & & \\
\hline UCD1- & 10 & $12 / 22 / 94$ & 49.87 & 49.13 & 0.74 & & & \\
\hline UCD1- & 10 & $1 / 6 / 95$ & 49.87 & 47.38 & 2.49 & & & \\
\hline UCD1- & 10 & $1 / 11 / 95$ & 49.87 & 41.61 & 8.26 & & & \\
\hline
\end{tabular}


Table E.1. Cumulative Groundwater Elevation Data for HSU-1, 1990-1995

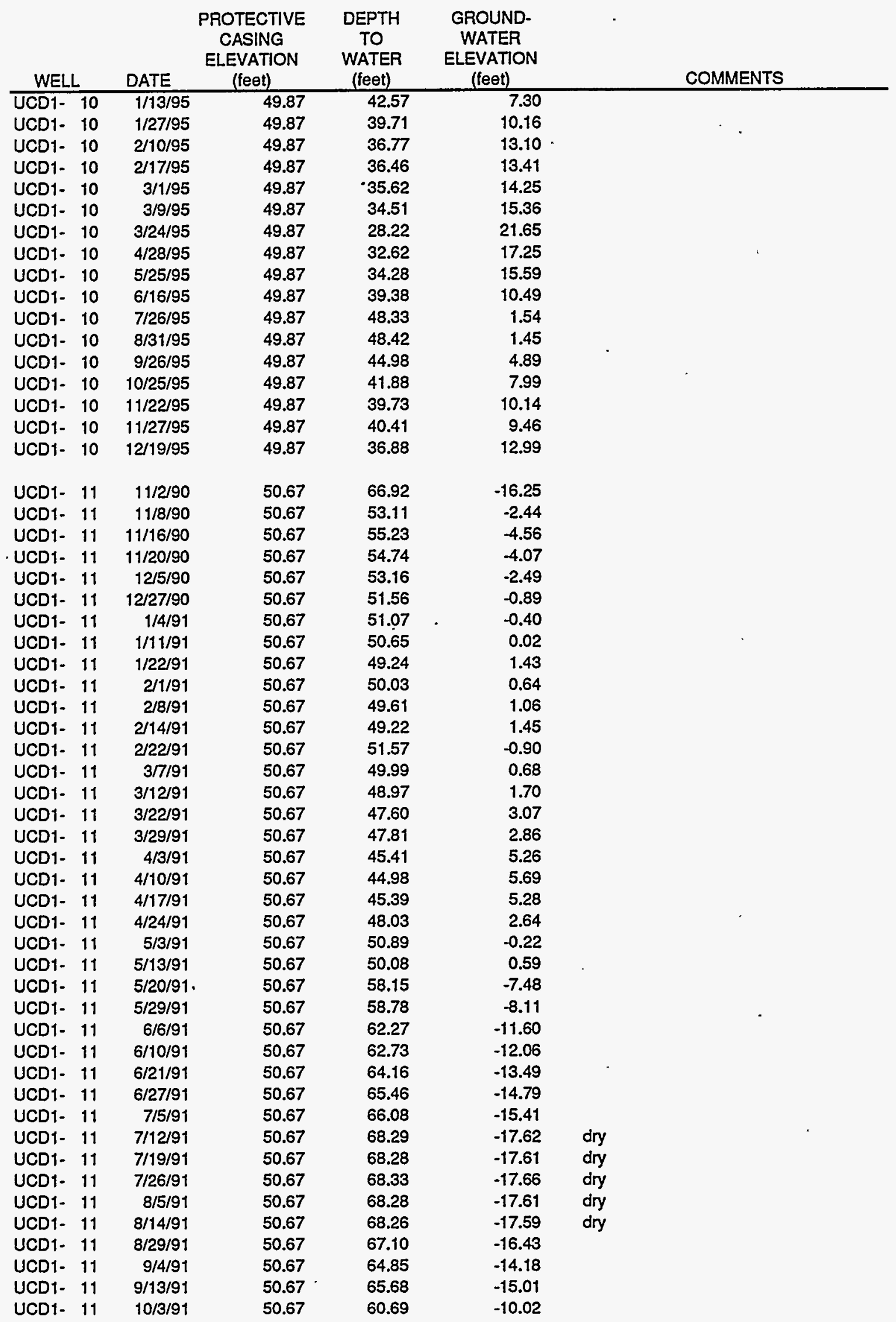


Table E.1. Cumulative Groundwater Elevation Data for HSU-1, 1990-1995

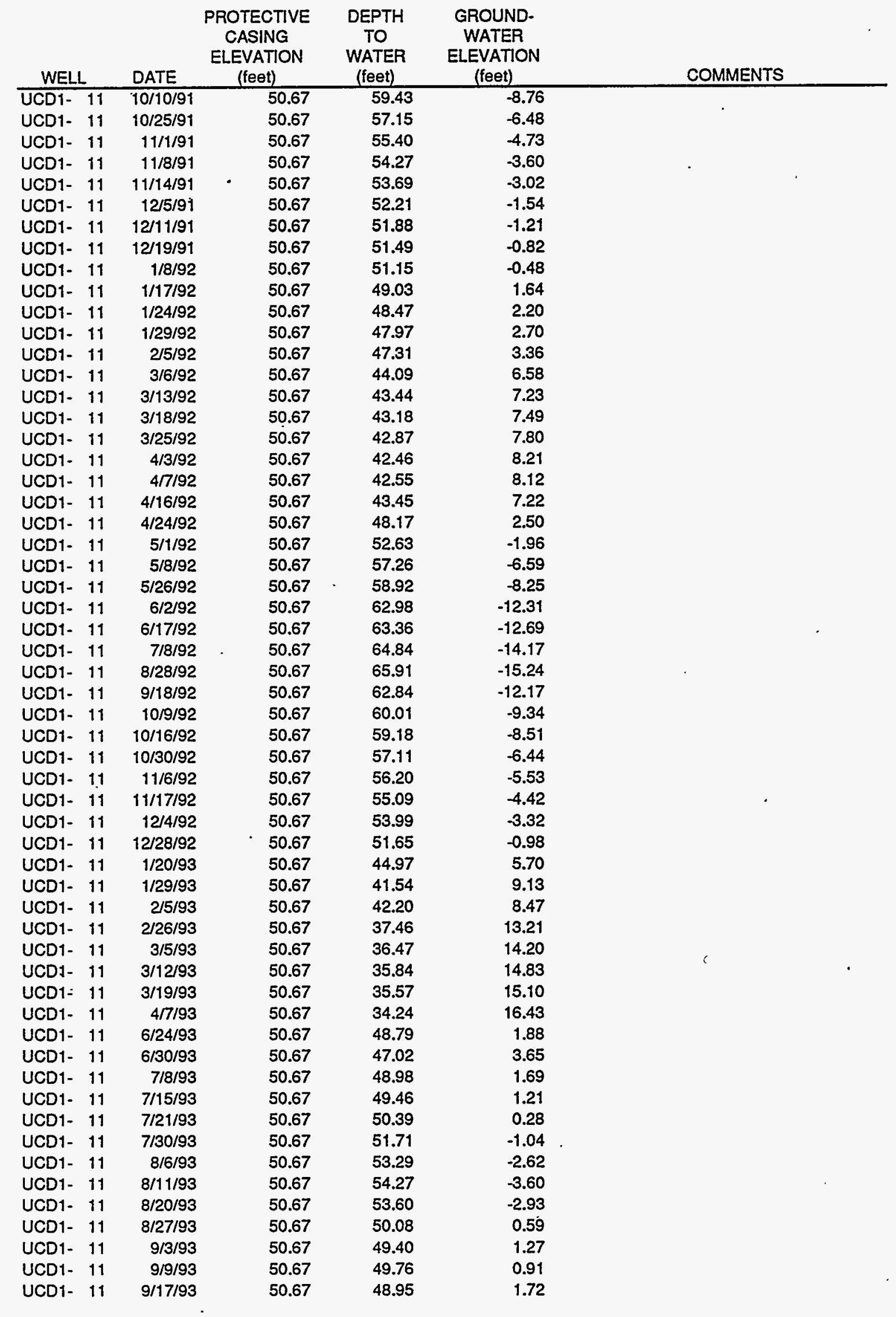


Table E.1. Cumulative Groundwater Elevation Data for HSU-1, 1990-1995

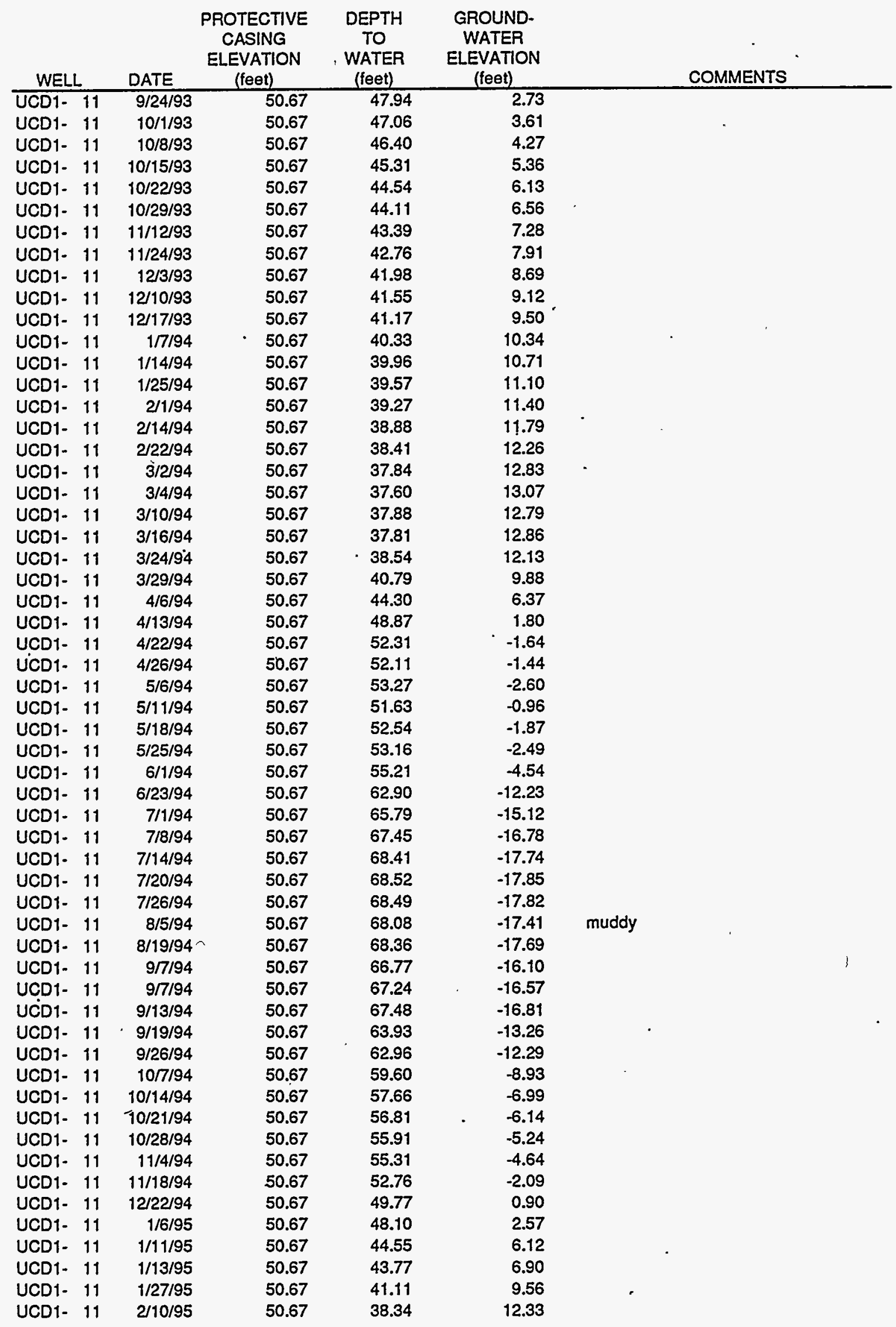


Table E.1. Cumulative Groundwater Elevation Data for HSU-1, 1990-1995

\begin{tabular}{|c|c|c|c|c|c|c|c|}
\hline WEL & & DATE & $\begin{array}{c}\text { PROTECTIVE } \\
\text { CASING } \\
\text { ELEVATION } \\
\text { (feet) }\end{array}$ & $\begin{array}{l}\text { DEPTH } \\
\text { TO } \\
\text { WATER } \\
\text { (feet) }\end{array}$ & $\begin{array}{c}\text { GROUND- } \\
\text { WATER } \\
\text { ELEVATION } \\
\text { (feet) }\end{array}$ & \multicolumn{2}{|l|}{ COMMENTS } \\
\hline UCD1- & $\overline{11}$ & $2 / 17 / 95$ & 50.67 & 37.70 & 12.97 & & \\
\hline UCD1- & 11 & $3 / 9 / 95$ & 50.67 & 35.80 & 14.87 & & \\
\hline UCD1- & 11 & $3 / 24 / 95$ & 50.67 & 30.81 & 19.86 & & \\
\hline UCD1- & 11 & $4 / 28 / 95$ & 50.67 & 32.89 & 17.78 & & \\
\hline UCD1- & 11 & $5 / 25 / 95$ & 50.67 & 34.01 & 16.66 & & · \\
\hline UCD1. & 11 & $6 / 16 / 95$ & 50.67 & 39.13 & 11.54 & & \\
\hline UCD1- & 11 & $7 / 26 / 95$ & 50.67 & 47.59 & 3.08 & Cap was missing. & \\
\hline UCD1- & 11 & $8 / 31 / 95$ & 50.67 & 48.30 & 2.37 & & \\
\hline UCD1- & 11 & $9 / 26 / 95$ & 50.67 & 45.18 & 5.49 & caps missing & \\
\hline UCD1- & 11 & $10 / 25 / 95$ & 50.67 & 42.12 & 8.55 & missing electrical cap & \\
\hline UCD1- & 11 & $11 / 22 / 95$ & 50.67 & 40.21 & 10.46 & & \\
\hline UCD1- & 11 & $11 / 27 / 95$ & 50.67 & 39.86 & 10.81 & & \\
\hline UCD1- & 11 & $12 / 19 / 95$ & 50.67 & 37.72 & 12.95 & & \\
\hline UCD1- & 12 & $11 / 2 / 90$ & 51.76 & 64.05 & -12.29 & & \\
\hline UCD1- & 12 & $11 / 8 / 90$ & 51.76 & 57.59 & -5.83 & & \\
\hline UCD1- & 12 & $11 / 16 / 90$ & 51.76 & 56.34 & -4.58 & & \\
\hline UCD1- & 12 & $11 / 20 / 90$ & 51.76 & 55.67 & -3.91 & & \\
\hline UCD1- & 12 & $12 / 5 / 90$ & 51.76 & 54.15 & -2.39 & . & \\
\hline UCD1- & 12 & $12 / 27 / 90$ & 51.76 & 52.63 & -0.87 & & \\
\hline UCD1- & 12 & $1 / 11 / 91$ & 51.76 & 51.85 & -0.09 & & \\
\hline UCD1- & 12 & $1 / 22 / 91$ & 51.76 & 51.01 & 0.75 & & \\
\hline UCD1- & 12 & $2 / 1 / 91$ & 51.76 & 50.88 & 0.88 & & \\
\hline UCD1- & 12 & $2 / 8 / 91$ & 51.76 & 50.67 & 1.09 & & \\
\hline UCD1- & 12 & $2 / 14 / 91$ & 51.76 & 50.26 & 1.50 & & \\
\hline UCD1- & 12 & $2 / 22 / 91$ & 51.76 & 51.92 & -0.16 & & \\
\hline UCDT- & 12 & $3 / 7 / 91$ & 51.76 & 50.38 & 1.38 & & \\
\hline UCD1- & 12 & $3 / 12 / 91$ & 51.76 & 47.46 & 4.30 & & \\
\hline UCD1- & 12 & $3 / 22 / 91$ & 51.76 & 48.85 & 2.91 & • & \\
\hline UCD1- & 12 & $3 / 29 / 91$ & 51.76 & 47.15 & 4.61 & . & \\
\hline UCD1- & 12 & $4 / 3 / 91$ & 51.76 & 44.55 & 7.21 & & \\
\hline UCD1- & 12 & $4 / 10 / 91$ & 51.76 & 45.35 & 6.41 & & \\
\hline UCD1- & 12 & $4 / 17 / 91$ & 51.76 & 42.63 & 9.13 & & \\
\hline UCD1- & 12 & $4 / 24 / 91$ & 51.76 & 44.37 & 7.39 & & \\
\hline UCD1- & 12 & $5 / 3 / 91$ & 51.76 & 45.94 & 5.82 & & \\
\hline UCD1- & 12 & $5 / 13 / 91$ & 51.76 & 51.44 & 0.32 & & \\
\hline UCD1- & 12 & $5 / 20 / 91$ & 51.76 & 58.15 & -6.39 & & \\
\hline UCD1- & 12 & $5 / 29 / 91$ & 51.76 & 58.52 & -6.76 & & \\
\hline UCD1- & 12 & $6 / 6 / 91$ & 51.76 & 61.96 & -10.20 & & \\
\hline UCD1- & 12 & $6 / 10 / 91$ & 51.76 & 62.52 & -10.76 & & \\
\hline UCD1- & 12 & $6 / 21 / 91$ & 51.76 & 64.41 & -12.65 & & \\
\hline UCD1- & 12 & $6 / 27 / 91$ & 51.76 & 65.67 & -13.91 & & \\
\hline UCD1- & 12 & $7 / 5 / 91$ & 51.76 & 65.00 & -13.24 & & \\
\hline UCD1- & 12 & $7 / 12 / 91$ & 51.76 & 65.13 & -13.37 & & \\
\hline UCD1- & 12 & $7 / 19 / 91$ & 51.76 & 67.08 & -15.32 & dry & \\
\hline UCD1- & 12 & $7 / 26 / 91$ & 51.76 & 67.10 & -15.34 & $d r y$ & \\
\hline UCD1- & 12 & $8 / 5 / 91$ & 51.76 & 67.36 & -15.60 & $d r y$ & \\
\hline UCD1- & 12 & $8 / 14 / 91$ & 51.76 & 67.40 & -15.64 & dry & \\
\hline UCD1- & 12 & $8 / 21 / 91$ & 51.76 & 67.45 & -15.69 & dry & \\
\hline UCD1- & 12 & $8 / 29 / 91$ & 51.76 & 67.40 & -15.64 & & \\
\hline UCD1- & 12 & $9 / 4 / 91$ & 51.76 & 66.38 & -14.62 & & \\
\hline UCD1- & 12 & $9 / 13 / 91$ & 51.76 & 65.77 & -14.01 & & \\
\hline UCD1- & 12 & $10 / 3 / 91$ & 51.76 & 61.73 & -9.97 & & \\
\hline UCD1- & 12 & $10 / 10 / 91$ & 51.76 & 60.55 & -8.79 & & \\
\hline UCD1. & 12 & $10 / 25 / 91$ & 51.76 & 57.96 & -6.20 & & \\
\hline UCD1- & 12 & $11 / 1 / 91$ & 51.76 & 56.46 & -4.70 & & \\
\hline UCD1- & 12 & $11 / 8 / 91$ & 51.76 & 55.08 & -3.32 & & \\
\hline
\end{tabular}


Table E.1. Cumulative Groundwater Elevation Data for HSU-1, 1990-1995

\begin{tabular}{|c|c|c|c|c|c|c|c|}
\hline WELL & DATE & $\begin{array}{c}\text { PROTECTIVE } \\
\text { CASING } \\
\text { ELEVATION } \\
\text { (feet) }\end{array}$ & $\begin{array}{l}\text { DEPTH } \\
\text { TO } \\
\text { WATER } \\
\text { (feet) }\end{array}$ & $\begin{array}{c}\text { GROUND- } \\
\text { WATER } \\
\text { ELEVATION } \\
\text { (feet) }\end{array}$ & & COMMENTS & \\
\hline UCD1- 12 & $11 / 14 / 91$ & 51.76 & 54,50 & -2.74 & & & \\
\hline UCD1- 12 & $12 / 5 / 91$ & 51.76 & 54.36 & -2.60 & & & \\
\hline UCD1- 12 & $12 / 11 / 91$ & 51.76 & 54.41 & -2.65 & & & \\
\hline UCD1- 12 & $12 / 19 / 91$ & 51.76 & 52.40 & -0.64 & & & \\
\hline UCD1- 12 & 1/3/92 & 51.76 & 51.25 & 0.51 & & & . \\
\hline UCD1- 12 & $1 / 8 / 92$ & 51.76 & 51.00 & 0.76 & & & \\
\hline UCD1- 12 & $1 / 17 / 92$ & 51.76 & 50.28 & 1.48 & & & \\
\hline UCD1- 12 & $1 / 24 / 92$ & 51.76 & 49.73 & 2.03 & & & \\
\hline UCD1- 12 & $1 / 29 / 92$ & 51.76 & 49.07 & 2.69 & & & \\
\hline UCD1- 12 & 2/5/92 & 51.76 & 48.38 & 3.38 & & & \\
\hline UCD1- 12 & $3 / 6 / 92$ & 51.76 & 44.75 & 7.01 & & & \\
\hline UCD1. 12 & $3 / 13 / 92$ & 51.76 & 44.00 & 7.76 & & & \\
\hline UCD1- 12 & $3 / 18 / 92$ & 51.76 & 43.35 & 8.41 & & & \\
\hline UCD1- 12 & $3 / 25 / 92$ & 51.76 & 43.35 & 8.41 & & & \\
\hline UCD1- 12 & $4 / 3 / 92$ & 51.76 & 43.02 & 8.74 & & & \\
\hline UCD1- 12 & $4 / 7 / 92$ & 51.76 & 42.86 & 8.90 & & & \\
\hline UCD1- 12 & $4 / 16 / 92$ & 51.76 & 43.90 & 7.86 & & & \\
\hline UCD1- 12 & $4 / 24 / 92$ & 51.76 & 42.54 & 9.22 & & & \\
\hline UCD1- 12 & $5 / 1 / 92$ & 51.76 & 51.56 & 0.20 & & & \\
\hline UCD1- 12 & $5 / 8 / 92$ & 51.76 & 55.63 & -3.87 & & & \\
\hline UCD1- 12 & $5 / 26 / 92$ & 51.76 & 58.97 & -7.21 & & & \\
\hline UCD1- 12 & $6 / 2 / 92$ & 51.76 & 62.55 & -10.79 & & & \\
\hline UCD1- 12 & $6 / 17 / 92$ & 51.76 & 62.76 & -11.00 & & & \\
\hline UCD1- 12 & $6 / 24 / 92$ & 51.76 & 64.33 & -12.57 & & & \\
\hline UCD1- 12 & $7 / 8 / 92$ & 51.76 & 65.84 & -14.08 & & . & \\
\hline UCD1- 12 & $7 / 15 / 92$ & 51.76 & 66.66 & -14.90 & & & . \\
\hline UCD1- 12 & $7 / 23 / 92$ & 51.76 & 67.15 & -15.39 & & & \\
\hline UCD1- 12 & $7 / 29 / 92$ & 51.76 & 67.30 & -15.54 & & & \\
\hline UCD1- 12 & $8 / 12 / 92$ & 51.76 & 67.57 & -15.81 & & & \\
\hline UCD1- 12 & $10 / 9 / 92$ & 51.76 & 60.10 & -8.34 & & & . \\
\hline UCD1- 12 & $10 / 16 / 92$ & 51.76 & 59.45 & -7.69 & & & \\
\hline UCD1- 12 & $10 / 30 / 92$ & 51.76 & 57.49 & -5.73 & & & \\
\hline UCD1- 12 & $11 / 6 / 92$ & 51.76 & 56.93 & -5.17 & . & & \\
\hline UCD1- 12 & $11 / 17 / 92$ & 51.76 & 55.83 & -4.07 & & & \\
\hline UCD1- 12 & $12 / 4 / 92$ & 51.76 & 54.65 & -2.89 & & & \\
\hline UCD1- 12 & $12 / 28 / 92$ & 51.76 & 52.68 & -0.92 & & & \\
\hline UCD1- 12 & $1 / 20 / 93$ & 51.76 & 44.83 & 6.93 & & & \\
\hline UCD1- 12 & $1 / 29 / 93$ & 51.76 & 42.06 & 9.70 & & & \\
\hline UCD1- 12 & $2 / 5 / 93$ & 51.76 & 41.80 & 9.96 & & & \\
\hline UCD1- 12 & $2 / 12 / 93$ & 51.76 & 41.49 & 10.27 & & & \\
\hline UCD1- 12 & $2 / 26 / 93$ & 51.76 & 37.64 & 14.12 & & & $\cdot$ \\
\hline UCD1- 12 & $3 / 5 / 93$ & 51.76 & 36.96 & 14.80 & & & \\
\hline UCD1- 12 & $3 / 12 / 93$ & 51.76 & 36.48 & 15.28 & & & \\
\hline UCD1- 12 & $3 / 19 / 93$ & 51.76 & 36.34 & 15.42 & & & \\
\hline UCD1- 12 & $4 / 7 / 93$ & 51.76 & 35.06 & 16.70 & & & \\
\hline UCD1- 12 & $4 / 16 / 93$ & 51.76 & 35.29 & 16.47 & & & - \\
\hline UCD1- 12 & $4 / 23 / 93$ & 51.76 & 35.54 & 16.22 & & & \\
\hline UCD1. 12 & $5 / 7 / 93$ & 51.76 & 41.57 & 10.19 & & & \\
\hline UCD1- 12 & $6 / 24 / 93$ & 51.76 & 48.90 & 2.86 & & & \\
\hline UCD $1-12$ & $6 / 30 / 93$ & 51.76 & 47.48 & 4.28 & & & \\
\hline UCD1- 12 & $7 / 8 / 93$ & 51.76 & 48.58 & 3.18 & & . & \\
\hline UCDI- 12 & $7 / 15 / 93$ & 51.76 & 50.29 & 1.47 & & & \\
\hline UCD1- 12 & $7 / 21 / 93$ & 51.76 & 50.33 & 1.43 & . & & \\
\hline UCD -12 & $7 / 30 / 93$ & 51.76 & 51.46 & 0.30 & & & \\
\hline UCD1- 12 & $8 / 6 / 93$ & 51.76 & 51.63 & 0.13 & & - & \\
\hline UCD1- 12 & $8 / 11 / 93$ & 51.76 & 52.35 & -0.59 & & . & \\
\hline UCD1- 12 & $8 / 20 / 93$ & 51.76 & 51.85 & -0.09 & & & \\
\hline
\end{tabular}


Table E.1. Cumulative Groundwater Elevation Data for HSU-1, 1990-1995

\begin{tabular}{|c|c|c|c|c|c|c|}
\hline WELL & DATE & $\begin{array}{c}\text { PROTECTIVE } \\
\text { CASING } \\
\text { ELEVATION } \\
\text { (feet) }\end{array}$ & $\begin{array}{l}\text { DEPTH } \\
\text { TO } \\
\text { WATER } \\
\text { (feet) }\end{array}$ & $\begin{array}{c}\text { GROUND- } \\
\text { WATER } \\
\text { ELEVATION } \\
\text { (feet) }\end{array}$ & COMMENTS & \\
\hline UCD1- 12 & $8 / 27 / 93$ & 51.76 & 50.74 & 1.02 & & \\
\hline UCD1-. 12 & $9 / 3 / 93$ & 51.76 & 50.42 & 1.34 & & \\
\hline UCD1- 12 & 9/9/93 & 51.76 & 50.08 & 1.68 & & \\
\hline UCD1- 12 & $9 / 17 / 93$ & 51.76 & 49.71 & 2.05 & & \\
\hline UCD1- 12 & $9 / 24 / 93$ & 51.76 & 48.77 & 2.99 & & \\
\hline UCD1- 12 & $10 / 1 / 93$ & 51.76 & 47.99 & 3.77 & & \\
\hline UCD1- 12 & $10 / 8 / 93$ & 51.76 & 47.25 & 4.51 & & \\
\hline UCD1- 12 & $10 / 15 / 93$ & 51.76 & 46.30 & 5.46 & & \\
\hline UCD1- 12 & $10 / 22 / 93$ & 51.76 & 45.33 & 6.43 & & \\
\hline UCD1- 12 & $10 / 29 / 93$ & 51.76 & 44.92 & 6.84 & & \\
\hline UCD1- 12 & $11 / 12 / 93$ & 51.76 & 44.30 & 7.46 & & \\
\hline UCD1- 12 & $11 / 24 / 93$ & 51.76 & 43.64 & 8.12 & & \\
\hline UCD1- 12 & $12 / 3 / 93$ & 51.76 & 42.88 & 8.88 & & \\
\hline UCD1- 12 & $12 / 10 / 93$ & 51.76 & 42.46 & 9.30 & & \\
\hline UCD1- 12 & $12 / 17 / 93$ & 51.76 & 42.07 & 9.69 & & \\
\hline UCD1- 12 & $1 / 7 / 94$ & 51.76 & 41.19 & 10.57 & & \\
\hline UCD1- 12 & $1 / 14 / 94$ & 51.76 & 40.82 & 10.94 & & - \\
\hline UCD1- 12 & $1 / 25 / 94$ & 51.76 & 40.44 & 11.32 & & \\
\hline UCD1- 12 & $2 / 1 / 94$ & 51.76 & 40.13 & 11.63 & & \\
\hline UCD1- 12 & $2 / 14 / 94$ & 51.76 & 39.51 & 12.25 & - & \\
\hline UCD1- 12 & $2 / 20 / 94$ & 51.76 & 39.40 & 12.36 & - & \\
\hline UCD1- 12 & $3 / 2 / 94$ & 51.76 & 38.78 & 12.98 & & \\
\hline UCD1- 12 & $3 / 4 / 94$ & 51.76 & 38.74 & 13.02 & & \\
\hline UCD1- 12 & $3 / 10 / 94$ & 51.76 & 38.71 & 13.05 & & \\
\hline UCD1- 12 & $3 / 16 / 94$ & 51.76 & 38.65 & 13.11 & & \\
\hline UCD1- 12 & $3 / 24 / 94$ & 51.76 & 39.16 & 12.60 & & \\
\hline UCD1- 12 & $3 / 29 / 94$ & 51.76 & 41.29 & 10.47 & & \\
\hline UCD1- 12 & $4 / 6 / 94$ & 51.76 & 44.44 & 7.32 & & \\
\hline UCD1- 12 & $4 / 13 / 94$ & 51.76 & 48.30 & 3.46 & & \\
\hline UCD1- 12 & $4 / 22 / 94$ & 51.76 & 51.77 & -0.01 & & \\
\hline UCD1- 12 & $4 / 26 / 94$ & 51.76 & 52.28 & -0.52 & & \\
\hline UCD1- 12. & $5 / 6 / 94$ & 51.76 & 53.32 & -1.56 & & \\
\hline UCD1- 12 & $5 / 11 / 94$ & 51.76 & 52.08 & -0.32 & & \\
\hline UCD1- 12 & $5 / 18 / 94$ & 51.76 & 53.07 & -1.31 & & \\
\hline UCD1- 12 & $5 / 25 / 94$ & 51.76 & 53.40 & -1.64 & & \\
\hline UCD1- 12 & $6 / 1 / 94$ & 51.76 & 54.63 & -2.87 & & \\
\hline UCD1- 12 & $6 / 23 / 94$ & 51.76 & 62.98 & -11.22 & & \\
\hline UCD1- 12. & $7 / 1 / 94$ & 51.76 & 64.92 & -13.16 & unclear meter response & \\
\hline UCD1- 12 & $7 / 8 / 94$ & 51.76 & 66.92 & -15.16 & & \\
\hline UCD1- 12 & $7 / 14 / 94$ & 51.76 & 67.22 & -15.46 & & \\
\hline UCD1- 12 & $7 / 20 / 94$ & 51.76 & 67.34 & -15.58 & & \\
\hline UCD1- 12 & $7 / 26 / 94$ & 51.76 & 67.30 & -15.54 & • & \\
\hline UCD1- 12 & $8 / 5 / 94$ & 51.76 & 67.43 & -15.67 & & \\
\hline UCD1- 12 & $8 / 19 / 94$ & 51.76 & 67.45 & -15.69 & & \\
\hline UCD1- 12 & $9 / 7 / 94$ & 51.76 & 67.33 & -15.57 & & \\
\hline UCD1- 12 & $9 / 7 / 94$ & 51.76 & 67.33 & -15.57 & & \\
\hline UCD1- 12 & $9 / 13 / 94$ & 51.76 & 67.34 & -15.58 & & \\
\hline UCD1- 12 & $9 / 19 / 94$ & 51.76 & 66.16 & -14.40 & & \\
\hline UCD1- 12 & $9 / 26 / 94$ & 51.76 & 64.33 & -12.57 & lid found open & \\
\hline UCD1- 12 & $10 / 7 / 94$ & 51.76 & 61.18 & -9.42 & & \\
\hline UCD1- 12 & $10 / 14 / 94$ & 51.76 & 58.91 & -7.15 & & \\
\hline UCD1- 12 & $10 / 21 / 94$ & 51.76 & 57.98 & -6.22 & & . \\
\hline UCD1- 12 & $10 / 28 / 94$ & 51.76 & 57.05 & -5.29 & & . \\
\hline UCD1- 12 & $11 / 4 / 94$ & 51.76 & 56.33 & -4.57 & & \\
\hline UCD1- 12 & $11 / 18 / 94$ & 51.76 & 53.79 & -2.03 & & \\
\hline UCD1- 12 & $12 / 22 / 94$ & 51.76 & 51.12 & 0.64 & & \\
\hline UCD1- 12 & $1 / 6 / 95$ & 51.76 & 49.15 & 2.61 & & \\
\hline
\end{tabular}


Table E.1. Cumulative Groundwater Elevation Data for HSU-1, 1990-1995

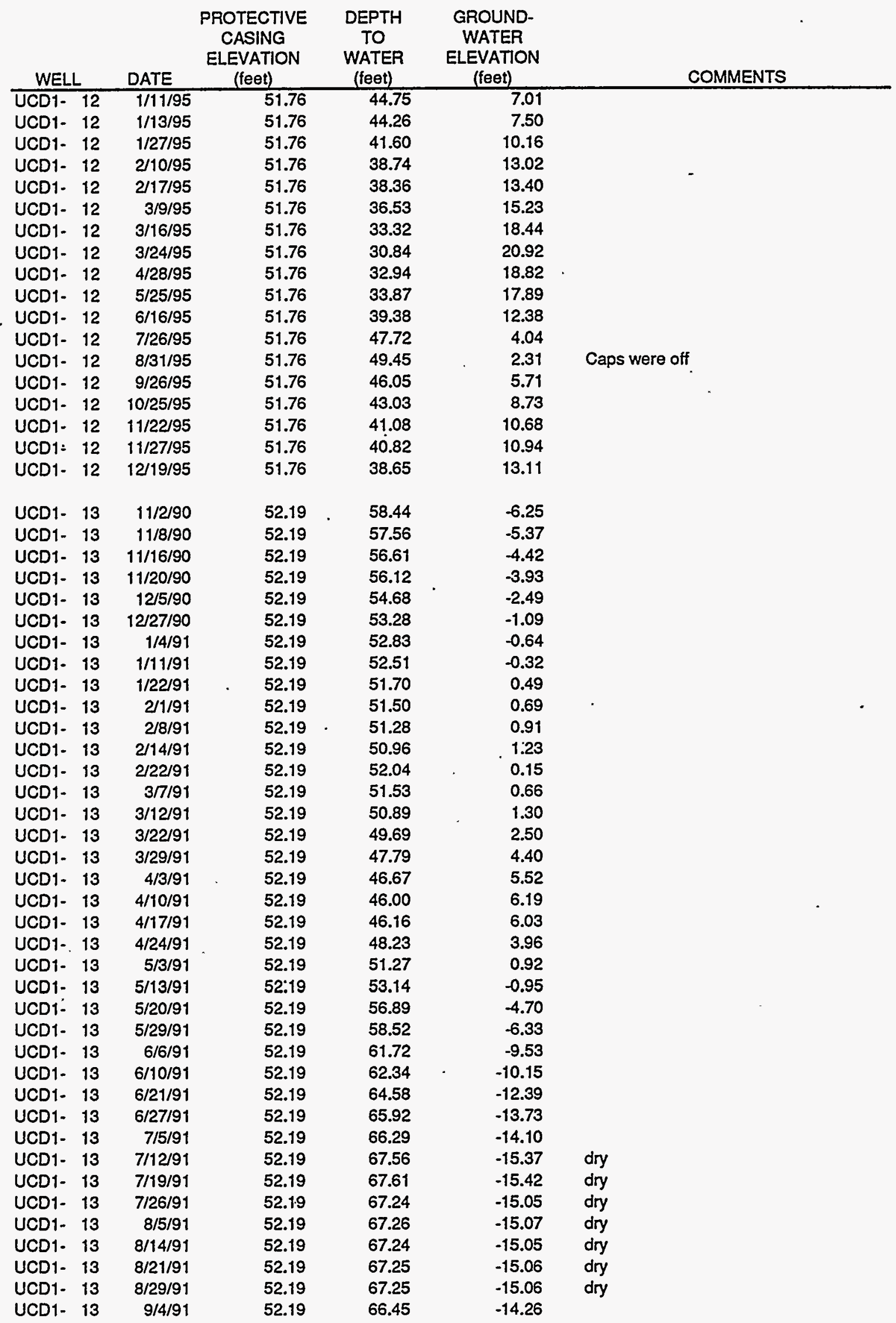


Table E.1. Cumulative Groundwater Elevation Data for HSU-1, 1990-1995

\begin{tabular}{|c|c|c|c|c|c|}
\hline WELL & DATE & $\begin{array}{c}\text { PROTECTIVE } \\
\text { CASING } \\
\text { ELEVATION } \\
\text { (feet) }\end{array}$ & $\begin{array}{l}\text { DEPTH } \\
\text { TO } \\
\text { WATER } \\
\text { (feet) }\end{array}$ & $\begin{array}{c}\text { GROUND- } \\
\text { WATER } \\
\text { ELEVATION } \\
\text { (feet) }\end{array}$ & COMMENTS \\
\hline UCD1- 13 & $9 / 13 / 91$ & 52.19 & 66.09 & -13.90 & \\
\hline UCD1- 13 & $10 / 3 / 91$ & 52.19 & 62.31 & -10.12 & \\
\hline UCD1- 13 & $10 / 10 / 91$ & 52.19 & 61.24 & $-9: 05$ & \\
\hline UCD1- 13 & $10 / 25 / 91$ & 52.19 & 58.65 & -6.46 & \\
\hline UCD1- 13 & $11 / 1 / 91$ & 52.19 & 56.88 & -4.69 & \\
\hline UCD1- 13 & $11 / 8 / 91$ & 52.19 & 55.56 & -3.37 & \\
\hline UCD1- 13 & $11 / 14 / 91$ & 52.19 & 55.13 & -2.94 & \\
\hline UCD1- 13 & $12 / 5 / 91$ & 52.19 & 53.78 & -1.59 & \\
\hline UCD1- 13 & $12 / 11 / 91$ & 52.19 & 53.44 & -1.25 & $\cdot$ \\
\hline UCD1- 13 & $12 / 19 / 91$ & 52.19 & 52.93 & -0.74 & \\
\hline UCD1- 13 & 1/3/92 & 52.19 & 52.00 & 0.19 & \\
\hline UCD1- 13 & $1 / 8 / 92$ & 52.19 & 52.23 & -0.04 & \\
\hline UCD1- 13 & $1 / 17 / 92$ & 52.19 & 51.12 & 1.07 & \\
\hline UCD1- 13 & $1 / 24 / 92$ & 52.19 & 50.66 & 1.53 & \\
\hline UCD1- 13 & $1 / 29 / 92$ & 52.19 & 50.08 & 2.11 & \\
\hline UCDi- 13 & 2/5/92 & 52.19 & 49.38 & 2.81 & \\
\hline UCD1- 13 & $3 / 6 / 92$ & 52.19 & 45.22 & 6.97 & . \\
\hline UCD1- 13 & $3 / 13 / 92$ & 52.19 & 44.45 & 7.74 & . \\
\hline UCD1- 13 & $3 / 18 / 92$ & 52.19 & 44.19 & 8.00 & \\
\hline UCD1- 13 & $3 / 25 / 92$ & 52.19 & 43.92 & 8.27 & \\
\hline UCD1- 13 & $4 / 3 / 92$ & 52.19 & 43.47 & 8.72 & \\
\hline UCD1- 13 & $4 / 7 / 92$ & 52.19 & 43.45 & 8.74 & \\
\hline UCD1- 13 & $4 / 16 / 92$ & 52.19 & 43.94 & 8.25 & \\
\hline UCD1- 13 & $4 / 24 / 92$ & 52.19 & 47.92 & 4.27 & \\
\hline UCD1- 13 & $5 / 1 / 92$ & 52.19 & 51.60 & 0.59 & \\
\hline UCD1- 13 & $5 / 8 / 92$ & 52.19 & 54.11 & -1.92 & \\
\hline UCD1- 13 & $5 / 26 / 92$ & 52.19 & 58.64 & -6.45 & $\cdot$ \\
\hline UCD1- 13 & $6 / 2 / 92$ & 52.19 & 62.22 & -10.03 & \\
\hline UCD1- 13 & $6 / 17 / 92$ & 52.19 & 62.91 & -10.72 & \\
\hline UCD1- 13 & $6 / 24 / 92$ & 52.19 & 64.40 & -12.21 & \\
\hline UCD1- 13 & 7/2/92 & 52.19 & 67.24 & -15.05 & \\
\hline UCD1- 13 & $7 / 8 / 92$ & 52.19 & 65.67 & -13.48 & \\
\hline UCD1- 13 & $7 / 15 / 92$ & 52.19 & 67.25 & -15.06 & \\
\hline UCD1- 13 & $7 / 29 / 92$ & 52.19 & 67.34 & -15.15 & \\
\hline UCD1- 13 & $8 / 28 / 92$ & 52.19 & 66.82 & -14.63 & \\
\hline UCD1- 13 & $9 / 4 / 92$ & 52.19 & 65.63 & -13.44 & \\
\hline UCD1- 13 & $9 / 18 / 92$ & 52.19 & 63.98 & -11.79 & \\
\hline UCD1- 13 & $10 / 9 / 92$ & 52.19 & 61.45 & -9.26 & . \\
\hline UCD1- 13 & $10 / 16 / 92$ & 52.19 & 60.47 & -8.28 & \\
\hline UCD1- 13 & $10 / 30 / 92$ & 52.19 & 58.31 & -6.12 & \\
\hline UCD1- 13 & $11 / 6 / 92$ & 52.19 & 57.53 & -5.34 & \\
\hline UCD1- 13 & $11 / 17 / 92$ & 52.19 & 56.00 & -3.81 & \\
\hline UCD1- 13 & $12 / 4 / 92$ & 52.19 & 55.59 & -3.40 & \\
\hline UCD1- 13 & $12 / 28 / 92$ & 52.19 & 53.21 & -1.02 & \\
\hline UCD1- 13 & $1 / 20 / 93$ & 52.19 & 45.21 & 6.98 & \\
\hline UCD1- 13 & $1 / 29 / 93$ & 52.19 & 42.38 & 9.81 & \\
\hline UCD1- 13 & $2 / 5 / 93$ & 52.19 & 42.22 & 9.97 & \\
\hline UCD1- 13 & 2/12/93 & 52.19 & 41.69 & 10.50 & \\
\hline UCD1- 13 & $2 / 26 / 93$ & 52.19 & 39.05 & 13.14 & \\
\hline UCD1- 13 & $3 / 5 / 93$ & 52.19 & 37.55 & 14.64 & \\
\hline UCD1- 13 & $3 / 12 / 93$ & 52.19 & 36.84 & 15.35 & \\
\hline UCD1- 13 & $3 / 19 / 93$ & 52.19 & 36.54 & 15.65 & \\
\hline UCD1- 13 & $4 / 7 / 93$ & 52.19 & 35.23 & 16.96 & \\
\hline UCD1- 13 & $4 / 16 / 93$ & 52.19 & 35.23 & 16.96 & \\
\hline UCD1- 13 & $4 / 23 / 93$ & 52.19 & 35.28 & 16.91 & \\
\hline UCD1- 13 & $5 / 7 / 93$ & 52.19 & 40.51 & 11.68 & \\
\hline UCD1- 13 & $6 / 24 / 93$ & 52.19 & 47.88 & 4.31 & \\
\hline
\end{tabular}


Table E.1. Cumulative Groundwater Elevation Data for HSU-1, 1990-1995

\begin{tabular}{|c|c|c|c|c|c|}
\hline WELL & DATE & $\begin{array}{c}\text { PROTECTIVE } \\
\text { CASING } \\
\text { ELEVATION } \\
\text { (feet) }\end{array}$ & $\begin{array}{l}\text { DEPTH } \\
\text { TO } \\
\text { WATER } \\
\text { (feet) } \\
\end{array}$ & $\begin{array}{c}\text { GROUND- } \\
\text { WATER } \\
\text { ELEVATION } \\
\text { (feet) }\end{array}$ & COMMENTS \\
\hline UCD1- 13 & $6 / 30 / 93$ & 52.19 & 47.00 & 5.19 & \\
\hline UCD1- 13 & $7 / 8 / 93$ & 52.19 & 49.08 & 3.11 & \\
\hline UCD1- 13 & $7 / 15 / 93$ & 52.19 & 50.33 & 1.86 & \\
\hline UCD1- 13 & $7 / 21 / 93$ & 52.19 & 50.40 & $\cdot 1.79$ & \\
\hline UCD1- 13 & $7 / 30 / 93$ & 52.19 & 49.47 & 2.72 & \\
\hline UCD1- 13 & $8 / 6 / 93$ & 52.19 & 51.77 & 0.42 & . \\
\hline UCD1- 13 & $8 / 11 / 93$ & 52.19 & 51.70 & 0.49 & \\
\hline UCD1- 13 & $8 / 20 / 93$ & 52.19 & 52.00 & 0.19 & \\
\hline UCD1- 13 & $8 / 27 / 93$ & 52.19 & 51.02 & 1.17 & \\
\hline UCD1- 13 & $9 / 3 / 93$ & 52.19 & 50.48 & 1.71 & \\
\hline UCD1- 13 & 9/9/93 & 52.19 & 50.31 & 1.88 & \\
\hline UCD1- 13 & $9 / 17 / 93$ & 52.19 & 50.02 & 2.17 & \\
\hline UCD1- 13 & $9 / 24 / 93$ & 52.19 & 49.06 & 3.13 & \\
\hline UCD1- 13 & $10 / 1 / 93$ & 52.19 & 48.32 & 3.87 & \\
\hline UCD1- 13 & $10 / 8 / 93$ & 52.19 & 47.63 & 4.56 & 3 \\
\hline UCD1- 13 & $10 / 15 / 93$ & 52.19 & 46.56 & 5.63 & \\
\hline UCD1- 13 & $10 / 22 / 93$ & 52.19 & 45.48 & 6.71 & \\
\hline UCD1- 13 & $10 / 29 / 93$ & 52.19 & 45.11 & 7.08 & \\
\hline UCD1- 13 & $11 / 12 / 93$ & 52.19 & 44.48 & 7.71 & \\
\hline UCD1- 13 & $11 / 24 / 93$ & 52.19 & 43.81 & 8.38 & \\
\hline UCD1- 13 & $12 / 3 / 93$ & 52.19 & 43.08 & 9.11 & \\
\hline UCD1- 13 & $12 / 10 / 93$ & 52.19 & 42.68 & 9.51 & \\
\hline UCD1- 13 & $12 / 17 / 93$ & 52.19 & 42.28 & 9.91 & \\
\hline UCD1- 13 & $1 / 7 / 94$ & 52.19 & 41.47 & 10.72 & \\
\hline UCD1- 13 & $1 / 14 / 94$ & 52.19 & 41.18 & 11.01 & \\
\hline UCD1- 13 & $1 / 25 / 94$ & 52.19 & 40.78 & 11.41 & \\
\hline UCD1- 13 & $2 / 1 / 94$ & 52.19 & 40.45 & 11.74 & \\
\hline UCD1- 13 & $2 / 14 / 94$ & 52.19 & 39.84 & 12.35 & \\
\hline UCD1- 13 & $2 / 20 / 94$ & 52.19 & 39.69 & 12.50 & \\
\hline UCD1- 13 & $3 / 2 / 94$ & 52.19 & 38.87 & 13.32 & \\
\hline UCD1- 13 & $3 / 4 / 94$ & 52.19 & 39.06 & 13.13 & \\
\hline UCD1- 13 & $3 / 10 / 94$ & 52.19 & 39.01 & 13.18 & \\
\hline UCD1- 13 & $3 / 16 / 94$ & 52.19 & 38.92 & 13.27 & \\
\hline UCD1- 13 & $3 / 24 / 94$ & 52.19 & 39.35 & 12.84 & \\
\hline UCD1- 13 & $3 / 29 / 94$ & 52.19 & 41.00 & 11.19 & \\
\hline UCD1- 13 & $4 / 6 / 94$ & 52.19 & 44.03 & 8.16 & \\
\hline UCD1- 13 & $4 / 13 / 94$ & 52.19 & 48.04 & 4.15 & \\
\hline UCD1- 13 & $4 / 22 / 94$ & 52.19 & 51.26 & 0.93 & \\
\hline UCD1- 13 & $4 / 26 / 94$ & 52.19 & 52.01 & 0.18 & \\
\hline UCD1- 13 & $5 / 6 / 94$ & 52.19 & 52.90 & -0.71 & \\
\hline UCD1- 13 & $5 / 11 / 94$ & 52.19 & 52.14 & 0.05 & \\
\hline UCD1- 13 & $5 / 18 / 94$ & 52.19 & 52.96 & -0.77 & \\
\hline UCD1- 13 & $5 / 25 / 94$ & 52.19 & 53.23 & -1.04 & \\
\hline UCD1- 13 & $6 / 1 / 94$ & 52.19 & 54.10 & -1.91 & \\
\hline UCD1- 13 & $6 / 23 / 94$ & 52.19 & 62.46 & -10.27 & \\
\hline UCD1- 13 & $7 / 1 / 94$ & 52.19 & 65.02 & -12.83 & \\
\hline UCD1- 13 & $7 / 8 / 94$ & 52.19 & 67.17 & -14.98 & \\
\hline UCD1- 13 & $9 / 19 / 94$ & 52.19 & 65.84 & -13.65 & dry previous week \\
\hline UCD1- 13 & $9 / 26 / 94$ & 52.19 & 64.06 & -11.87 & \\
\hline UCD1- 13 & $10 / 7 / 94$ & 52.19 & 61.52 & -9.33 & \\
\hline UCD1- 13 & $10 / 14 / 94$ & 52.19 & 59.38 & -7.19 & \\
\hline UCD1- 13 & $10 / 21 / 94$ & 52.19 & 58.29 & -6.10 & \\
\hline UCD1- 13 & $10 / 28 / 94$ & 52.19 & 57.31 & -5.12 & \\
\hline UCD1- 13 & $11 / 4 / 94$ & 52.19 & 56.60 & -4.41 & \\
\hline UCD1- 13 & $11 / 18 / 94$ & 52.19 & 54.35 & -2.16 & \\
\hline UCD1- 13 & $12 / 22 / 94$ & 52.19 & 51.88 & 0.31 & \\
\hline UCD1- 13 & $1 / 6 / 95$ & 52.19 & 50.50 & 1.69 & \\
\hline
\end{tabular}


Table E.1. Cumulative Groundwater Elevation Data for HSU-1, 1990-1995

\begin{tabular}{|c|c|c|c|c|c|}
\hline WELL & DATE & $\begin{array}{c}\text { PROTECTIVE } \\
\text { CASING } \\
\text { ELEVATION } \\
\text { (feet) }\end{array}$ & $\begin{array}{l}\text { DEPTH } \\
\text { TO } \\
\text { WATER } \\
\text { (feet) }\end{array}$ & $\begin{array}{c}\text { GROUND- } \\
\text { WATER } \\
\text { ELEVATION } \\
\text { (feet) }\end{array}$ & COMMENTS \\
\hline UCD1- 13 & $1 / 11 / 95$ & 52.19 & 49.15 & 3.04 & \\
\hline UCD1- 13 & $1 / 13 / 95$ & 52.19 & 47.65 & 4.54 & \\
\hline UCD1- 13 & $1 / 27 / 95$ & 52.19 & 42.18 & 10.01 & \\
\hline UCD1- .13 & $2 / 10 / 95$ & 52.19 & 39.58 & 12.61 & \\
\hline UCD1- 13 & $2 / 17 / 95$ & 52.19 & 38.71 & 13.48 & \\
\hline UCD1- 13 & $3 / 9 / 95$ & 52.19 & 36.98 & 15.21 & \\
\hline UCD1- 13 & $3 / 16 / 95$ & 52.19 & 34.12 & 18.07 & \\
\hline UCD1- 13 & $3 / 24 / 95$ & 52.19 & 32.45 & 19.74 & \\
\hline UCD1- 13 & $4 / 28 / 95$ & 52.19 & 32.73 & 19.46 & \\
\hline UCD1- 13 & $5 / 25 / 95$ & 52.19 & 33.70 & 18.49 & \\
\hline UCD1- 13 & $6 / 16 / 95$ & 52.19 & 39.06 & 13.13 & 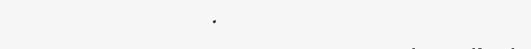 \\
\hline UCD1- 13 & $7 / 26 / 95$ & 52.19 & & NA & Continuous well level indicator installed. \\
\hline UCD1- 13 & $8 / 31 / 95$ & 52.19 & 49.28 & 2.91 & Electrical cap is off \\
\hline UCD1- 13 & $9 / 26 / 95$ & 52.19 & 46.24 & 5.95 & \\
\hline UCD1- 13 & $10 / 25 / 95$ & 52.19 & 43.25 & 8.94 & \\
\hline UCD1- 13 & $11 / 22 / 95$ & 52.19 & 41.38 & 10.81 & \\
\hline UCD1- 13 & $11 / 27 / 95$ & 52.19 & 41.18 & 11.01 & \\
\hline UCD1- 13 & $12 / 19 / 95$ & 52.19 & 38.95 & 13.24 & \\
\hline UCD1- 18 & $1 / 22 / 91$ & 48.56 & 47.43 & 1.13 & \\
\hline UCD1- 18 & $2 / 1 / 91$ & 48.56 & 47.01 & 1.55 & \\
\hline UCD1- 18 & $2 / 8 / 91$ & 48.56 & 46.80 & 1.76 & \\
\hline UCD1- 18 & $2 / 14 / 91$ & 48.56 & 46.52 & 2.04 & \\
\hline UCD1- 18 & $2 / 22 / 91$ & 48.56 & 46.89 & 1.67 & \\
\hline UCD1- 18 & $3 / 7 / 91$ & 48.56 & 46.54 & 2.02 & \\
\hline UCDt- 18 & $3 / 12 / 91$ & 48.56 & 46.19 & 2.37 & . \\
\hline UCD1- 18 & $3 / 22 / 91$ & 48.56 & $46: 00$ & 2.56 & \\
\hline UCD1- 18 & $3 / 29 / 91$ & 48.56 & 42.88 & 5.68 & \\
\hline UCD1- 18 & $4 / 3 / 91$ & 48.56 & 41.66 & 6.90 & \\
\hline UCD1- 18 & $4 / 10 / 91$ & 48.56 & 41.32 & 7.24 & \\
\hline UCD1- 18 & $4 / 17 / 91$ & 48.56 & 41.29 & 7.27 & . \\
\hline UCD1- 18 & $4 / 24 / 91$ & 48.56 & 42.99 & 5.57 & \\
\hline UCD1- 18 & $5 / 3 / 91$ & 48.56 & 44.95 & 3.61 & \\
\hline UCD1- 18 & $5 / 13 / 91$ & 48.56 & 46.96 & 1.60 & \\
\hline UCD1- 18 & $5 / 20 / 91$ & 48.56 & 50.68 & -2.12 & \\
\hline UCD1- 18 & $5 / 29 / 91$ & 48.56 & 51.99 & -3.43 & \\
\hline UCD1- 18 & $6 / 6 / 91$ & 48.56 & 54.54 & -5.98 & \\
\hline UCD1- 18 & $6 / 10 / 91$ & 48.56 & 55.27 & -6.71 & \\
\hline UCD1- 18 & $6 / 21 / 91$ & 48.56 & 57.28 & -8.72 & \\
\hline UCD1- 18 & $6 / 27 / 91$ & 48.56 & 58.02 & -9.46 & \\
\hline UCD1- 18 & 7/5/91 & 48.56 & 58.35 & -9.79 & \\
\hline UCD1- 18 & $7 / 12 / 91$ & 48.56 & 59.19 & -10.63 & \\
\hline UCD1- 18 & $7 / 19 / 91$ & 48.56 & 61.60 & -13.04 & \\
\hline UCD1- 18 & $7 / 26 / 91$ & 48.56 & 61.85 & -13.29 & \\
\hline UCD1- 18 & $8 / 5 / 91$ & 48.56 & 64.22 & -15.66 & \\
\hline UCD1- 18 & $8 / 14 / 91$ & 48.56 & 64.83 & -16.27 & \\
\hline UCD1- 18 & $8 / 21 / 91$ & 48.56 & 64.37 & -15.81 & \\
\hline UCD1- 18 & $8 / 29 / 91$ & 48.56 & 62.60 & -14.04 & \\
\hline UCD1- 18 & 9/4/91 & 48.56 & 60.02 & -11.46 & \\
\hline UCD1- 18 & $9 / 13 / 91$ & 48.56 & 60.74 & -12.18 & \\
\hline UCD1- 18 & $10 / 3 / 91$ & 48.56 & 58.92 & -10.36 & \\
\hline UCD1- 18 & $10 / 10 / 91$ & 48.56 & $58.54^{\circ}$ & -9.98 & \\
\hline UCD1- 18 & $10 / 25 / 91$ & 48.56 & 55.77 & -7.21 & \\
\hline UCD1- 18 & $11 / 1 / 91$ & 48.56 & 53.02 & -4.46 & \\
\hline UCD1- 18 & $11 / 8 / 91$ & 48.56 & 50.81 & -2.25 & \\
\hline UCDi- 18 & $11 / 14 / 91$ & 48.56 & 49.00 & -0.44 & \\
\hline UCD1- 18 & $12 / 5 / 91$ & 48.56 & 48.52 & 0.04 & \\
\hline
\end{tabular}


Table.E.1. Cumulative Groundwater Elevation Data for HSU-1, 1990-1995

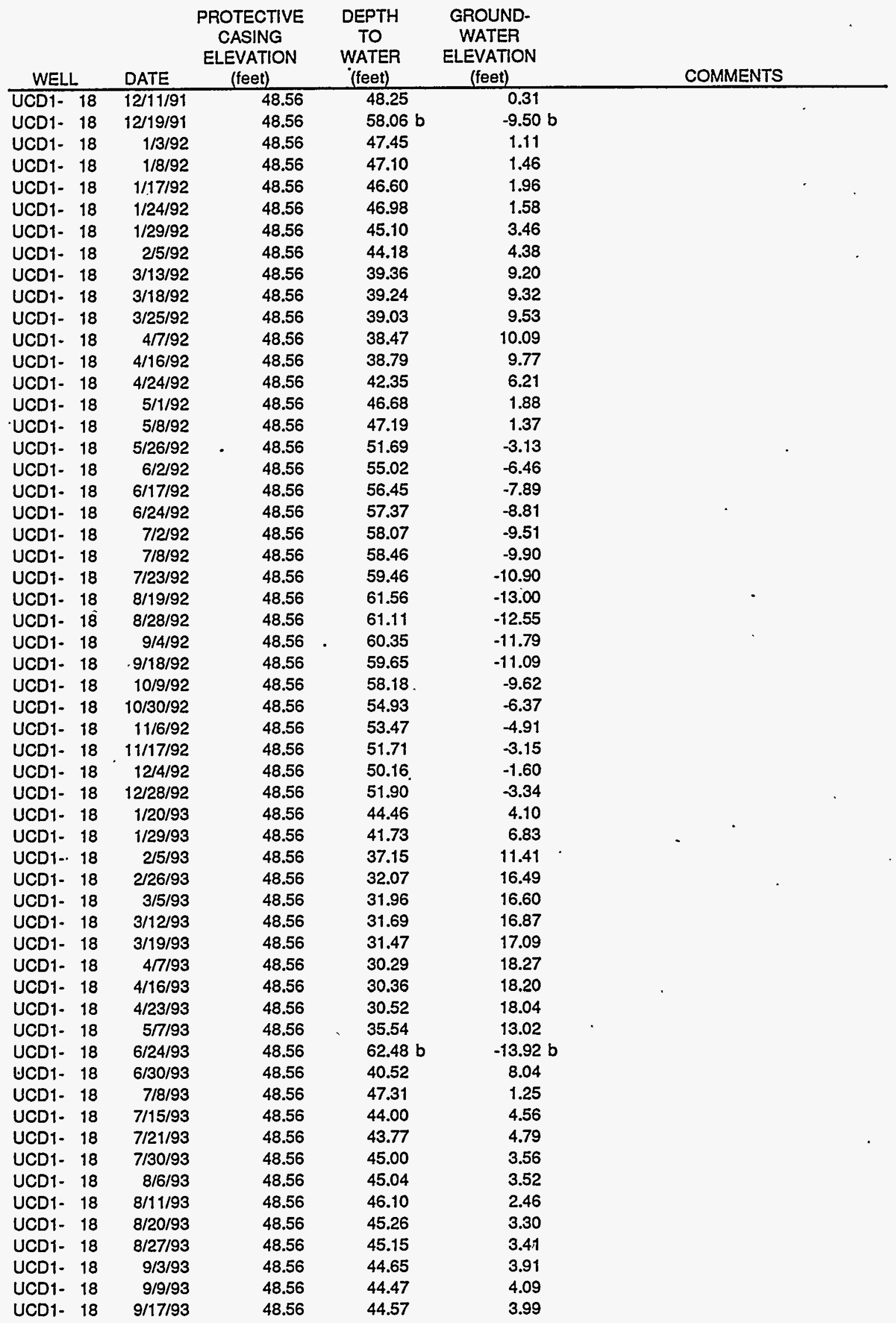


Table E.1. Cumulative Groundwater Elevation Data for HSU-1, 1990-1995

\begin{tabular}{|c|c|c|c|c|c|}
\hline WELL & DATE & $\begin{array}{c}\text { PROTECTIVE } \\
\text { CASING } \\
\text { ELEVATION } \\
\text { (feet) }\end{array}$ & $\begin{array}{l}\text { DEPTH } \\
\text { TO } \\
\text { WATER } \\
\text { (feet) }\end{array}$ & $\begin{array}{c}\text { GROUND- } \\
\text { WATER } \\
\text { ELEVATION } \\
\text { (feet) }\end{array}$ & COMMENTS \\
\hline UCD1- 18 & $9 / 24 / 93$ & 48.56 & 43.79 & 4.77 & \\
\hline UCD1- 18 & $10 / 1 / 93$ & 48.56 & 43.07 & 5.49 & \\
\hline UCD1- 18 & $10 / 8 / 93$ & 48.56 & 42.50 & 6.06 & \\
\hline UCD1- 18 & $10 / 15 / 93$ & 48.56 & 41.68 & 6.88 & \\
\hline UCD1- 18 & $10 / 22 / 93$ & 48.56 & 40.90 & 7.66 & \\
\hline UCD1- 18 & $10 / 29 / 93$ & 48.56 & 40.36 & 8.20 & \\
\hline UCD1- 18 & $11 / 12 / 93$ & 48.56 & 39.72 & 8.84 & \\
\hline UCD1- 18 & $11 / 24 / 93$ & 48.56 & 39.13 & 9.43 & \\
\hline UCD1- 18 & $12 / 3 / 93$ & 48.56 & 38.29 & 10.27 & \\
\hline UCD1- 18 & $12 / 10 / 93$ & 48.56 & 37.89 & 10.67 & \\
\hline UCD1- 18 & $12 / 17 / 93$ & 48.56 & 37.48 & 11.08 & . \\
\hline UCD1- 18 & $1 / 7 / 94$ & 48.56 & 36.66 & 11.90 & \\
\hline UCD1- 18 & $1 / 14 / 94$ & 48.56 & 36.20 & 12.36 & \\
\hline UCD1- 18 & $1 / 25 / 94$ & 48.56 & 35.86 & 12.70 & \\
\hline UCD1- 18 & $2 / 1 / 94$ & 48.56 & 35.61 & 12.95 & \\
\hline UCD1- 18 & $2 / 14 / 94$ & 48.56 & 35.14 & 13.42 & \\
\hline UCD1- 18 & $2 / 22 / 94$ & 48.56 & 34.52 & 14.04 & \\
\hline UCD1- 18 & $3 / 2 / 94$ & 48.56 & . 33.48 & 15.08 & \\
\hline UCD1- 18 & $3 / 4 / 94$ & 48.56 & 34.18 & 14.38 & \\
\hline UCD1- 18 & $3 / 10 / 94$ & 48.56 & 34.27 & 14.29 & \\
\hline UCD1- 18 & $3 / 16 / 94$ & 48.56 & 34.09 & 14.47 & \\
\hline UCD1- 18 & $3 / 24 / 94$ & 48.56 & 34.51 & 14.05 & \\
\hline UCD1- 18 & $3 / 29 / 94$ & 48.56 & 36.23 & 12.33 & \\
\hline UCD1- 18 & $4 / 6 / 94$ & 48.56 & 38.97 & 9.59 & - \\
\hline UCD1- 18 & $4 / 13 / 94$ & 48.56 & 41.82 & 6.74 & \\
\hline UCD1- 18 & $4 / 22 / 94$ & 48.56 & 45.23 & 3.33 & \\
\hline UCD1- 18 & $4 / 26 / 94$ & 48.56 & 45.89 & 2.67 & . \\
\hline UCD1- 18 & $5 / 6 / 94$ & 48.56 & 46.54 & 2.02 & \\
\hline UCD1- 18 & $5 / 11 / 94$ & 48.56 & 46.09 & 2.47 & \\
\hline UCD $1-18$ & $5 / 18 / 94$ & 48.56 & 47.01 & 1.55 & \\
\hline UCD1- 18 & $5 / 25 / 94$ & 48.56 & 47.27 & 1.29 & \\
\hline UCD1- 18 & $6 / 1 / 94$ & 48.56 & 47.76 & 0.80 & \\
\hline UCD $1-18$ & $6 / 23 / 94$ & 48.56 & 56.04 & -7.48 & \\
\hline UCD 1.18 & $7 / 1 / 94$ & 48.56 & 57.35 & -8.79 & $\cdot$ \\
\hline UCD1- 18 & $7 / 8 / 94$ & 48.56 & 58.10 . & -9.54 & unclear meter response \\
\hline UCD1- 18 & $7 / 14 / 94$ & 48.56 & 58.66 & -10.10 & \\
\hline UCD1- 18 & $7 / 20 / 94$ & 48.56 & 59.94 & -11.38 & \\
\hline UCD1- 18 & $7 / 26 / 94$ & 48.56 & 61.21 & -12.65 & \\
\hline UCD1- 18 & $8 / 5 / 94$ & 48.56 & 62.06 & -13.50 & \\
\hline UCD1- 18 & $8 / 19 / 94$ & 48.56 & 64.77 & -16.21 & \\
\hline UCD1- 18 & $9 / 7 / 94$ & 48.56 & 62.56 & -14.00 & \\
\hline UCD1- 18 & $9 / 7 / 94$ & 48.56 & 62.40 & -13.84 & \\
\hline UCD1- 18 & $9 / 13 / 94$ & 48.56 & 63.20 & -14.64 & \\
\hline UCD1- 18 & $9 / 19 / 94$ & 48.56 & 60.86 & -12.30 & \\
\hline UCD1- 18 & $9 / 26 / 94$ & 48.56 & 59.89 & -11.33 & \\
\hline UCD1- 18 & $10 / 7 / 94$ & 48.56 & 58.95 & -10.39 & \\
\hline UCD1- 18 & $10 / 14 / 94$ & 48.56 & 58.23 & -9.67 & \\
\hline UCD1- 18 & $10 / 21 / 94$ & 48.56 & 56.84 & -8.28 & . \\
\hline UCD1- 18 & $10 / 28 / 94$ & 48.56 & 54.56 & -6.00 & \\
\hline UCD1- 18 & $11 / 4 / 94$ & 48.56 & 52.55 & -3.99 & \\
\hline UCD1- 18 & $11 / 18 / 94$ & 48.56 & 49.36 & -0.80 & \\
\hline UCD1- 18 & $12 / 22 / 94$ & 48.56 & 47.55 & 1.01 & \\
\hline UCD1- 18 & $1 / 6 / 95$ & 48.56 & 46.05 & 2.51 & \\
\hline UCD1- 18 & $1 / 11 / 95$ & 48.56 & 41.97 & 6.59 & . \\
\hline UCD1- 18 & $1 / 13 / 95$ & 48.56 & 41.13 & 7.43 & \\
\hline UCD1- 18 & $1 / 27 / 95$ & 48.56 & 38.83 & 9.73 & \\
\hline UCD1- 18 & $2 / 10 / 95$ & 48.56 & 34.58 & 13.98 & \\
\hline
\end{tabular}


Table E.1. Cumulative Groundwater Elevation Data for HSU-1, 1990-1995

\begin{tabular}{|c|c|c|c|c|c|}
\hline WELL & DATE & $\begin{array}{c}\text { PROTECTIVE } \\
\text { CASING } \\
\text { ELEVATION } \\
\text { (feel) }\end{array}$ & $\begin{array}{l}\text { DEPTH } \\
\text { TO } \\
\text { WATER } \\
\text { (feet) }\end{array}$ & $\begin{array}{c}\text { GROUND-- } \\
\text {. WATER } \\
\text { ELEVATION } \\
\text { (feet) }\end{array}$ & COMMENTS \\
\hline UCD1- 18 & $2 / 17 / 95$ & 48.56 & 33.97 & 14.59 & \\
\hline UCD1- 18 & $3 / 9 / 95$ & 48.56 & 31.94 & 16.62 & . \\
\hline UCD1- 18 & $3 / 24 / 95$ & 48.56 & 25.82 & 22.74 & \\
\hline UCD1- 18 & $4 / 28 / 95$ & 48.56 & 28.78 & 19.78 & . \\
\hline UCD1- 18 & $5 / 25 / 95$ & 48.56 & 29.17 & 19.39 & \\
\hline UCD1- 18 & $6 / 16 / 95$ & 48.56 & 34.04 & 14.52 & \\
\hline UCD1- 18 & $7 / 26 / 95$ & 48.56 & 41.33 & 7.23 & Cap was off/inner tube is gone. \\
\hline UCD1- 18 & $8 / 31 / 95$ & 48.56 & 43.48 & 5.08 & \\
\hline UCD1- 18 & $9 / 26 / 95$ & 48.56 & & NA & \\
\hline UCD1- 18 & $10 / 25 / 95$ & 48.56 & & NA & Continuous monitoring equipment \\
\hline UCD1- 18 & $11 / 22 / 95$ & 48.56 & 36.49 & 12.07 & \\
\hline UCD1- 18 & $11 / 27 / 95$ & 48.56 & 36.28 & 12.28 & no pump \\
\hline UCD1- 18 & $12 / 19 / 95$ & 48.56 & 33.66 & 14.90 & Pump not installed \\
\hline UCD1- 19 & $1 / 22 / 91$ & 51.33 & 50.73 & 0.60 & \\
\hline UCD1- 19 & $2 / 1 / 91$ & 51.33 & 50.66 & 0.67 & \\
\hline UCD1- 19 & $2 / 8 / 91$ & 51.33 & 50.38 & 0.95 & \\
\hline UCD1- 19 & $2 / 14 / 91$ & 51.33 & 50.10 & 1.23 & \\
\hline UCD1- 19 & $2 / 22 / 91$ & 51.33 & 51.48 & -0.15 & \\
\hline UCD1- 19 & $3 / 7 / 91$ & 51.33 & 50.58 & 0.75 & \\
\hline UCD1- 19 & $3 / 12 / 91$ & 51.33 & 50.26 & 1.07 & \\
\hline UCD1- 19 & $3 / 22 / 91$ & 51.33 & 48.68 & 2.65 & \\
\hline UCD1- 19 & $3 / 29 / 91$ & 51.33 & 47.02 & 4.31 & \\
\hline UCD1- 19 & $4 / 3 / 91$ & 51.33 & 46.32 & 5.01 & . \\
\hline UCD1- 19 & $4 / 10 / 91$ & 51.33 & 45.77 & 5.56 & \\
\hline UCD1- 19 & $4 / 17 / 91$ & 51.33 & 45.95 & 5.38 & \\
\hline UCD1- 19 & $4 / 24 / 91$ & 51.33 & 48.02 & 3.31 & \\
\hline UCD1- 19 & $5 / 3 / 91$ & 51.33 & 50.47 & 0.86 & \\
\hline UCD1- 19 & $5 / 13 / 91$ & 51.33 & 53.08 & -1.75 & . \\
\hline UCD1- 19 & $5 / 20 / 91$ & 51.33 & 57.74 & -6.41 & \\
\hline UCD1- 19 & $5 / 29 / 91$ & 51.33 & 58.54 & -7.21 & \\
\hline UCD1- 19 & $6 / 6 / 91$ & 51.33 & 61.23 & -9.90 & . \\
\hline UCD1- 19 & $6 / 10 / 91$ & 51.33 & 61.93 & -10.60 & \\
\hline UCD1- 19 & $6 / 21 / 91$ & 51.33 & 63.89 & -12.56 & \\
\hline UCD1- 19 & $6 / 27 / 91$ & 51.33 & 64.23 & -12.90 & \\
\hline UCD1- 19 & $7 / 5 / 91$ & 51.33 & 65.76 & -14.43 & \\
\hline UCD1- 19 & $7 / 12 / 91$ & 51.33 & 68.68 & -17.35 & \\
\hline UCD1- 19 & $7 / 19 / 91$ & 51.33 & 69.71 & -18.38 & \\
\hline UCD1- 19 & $7 / 26 / 91$ & 51.33 & 70.08 & -18.75 & \\
\hline UCD1- 19 & $8 / 5 / 91$ & 51.33 & 70.42 & -19.09 & \\
\hline UCD1- 19 & $8 / 14 / 91$ & 51.33 & 70.93 & -19.60 & \\
\hline UCD1- 19 & $8 / 21 / 91$ & 51.33 & 69.68 & -18.35 & . \\
\hline UCD1- 19 & $8 / 29 / 91$ & 51.33 & 67.10 & -15.77 & \\
\hline UCD1- 19 & $9 / 4 / 91$ & 51.33 & 65.12 & -13.79 & \\
\hline UCD1- 19 & $9 / 13 / 91$ & 51.33 & 65.52 & -14.19 & \\
\hline UCD1- 19 & $10 / 3 / 91$ & 51.33 & 61.26 & -9.93 & \\
\hline UCD1- 19 & $10 / 10 / 91$ & 51.33 & 60.29 & -8.96 & \\
\hline UCD1- 19 & $10 / 25 / 91$ & 51.33 & 57.75 & -6.42 & \\
\hline UCD1- 19 & $11 / 1 / 91$ & 51.33 & 55.96 & -4.63 & \\
\hline UCD1- 19 & $11 / 8 / 91$ & 51.33 & 54.75 & -3.42 & \\
\hline UCD1- 19 & $11 / 14 / 91$ & 51.33 & 54.19 & -2.86 & \\
\hline UCD1- 19 & $12 / 5 / 91$ & 51.33 & 52.82 & -1.49 & \\
\hline UCD1- 19 & $12 / 11 / 91$ & 51.33 & 52.46 & -1.13 & \\
\hline UCD1- 19 & $12 / 19 / 91$ & 51.33 & 52.10 & -0.77 & \\
\hline UCD1- 19 & $1 / 3 / 92$ & 51.33 & 50.96 & 0.37 & \\
\hline UCD1- 19 & $1 / 8 / 92$ & 51.33 & 51.17 & 0.16 & \\
\hline UCD1- 19 & $1 / 17 / 92$ & 51.33 & 50.11 & 1.22 & \\
\hline
\end{tabular}


Table E.1. Cumulative Groundwater Elevation Data for HSU-1, 1990-1995

\begin{tabular}{|c|c|c|c|c|c|c|}
\hline WELL & DATE & $\begin{array}{l}\text { PROTECTIVE } \\
\text { CASING } \\
\text { ELEVATION } \\
\text { (feet) }\end{array}$ & $\begin{array}{l}\text { DEPTH } \\
\text { TO } \\
\text { WATER } \\
\text { (feet) }\end{array}$ & $\begin{array}{c}\text { GROUND- } \\
\text { WATER } \\
\text { ELEVATION } \\
\text { (feet) }\end{array}$ & $\dot{ }$ & COMMENTS \\
\hline UCD1- 19 & $1 / 24 / 92$ & 51.33 & 49.55 & 1.78 & & \\
\hline UCD1- 19 & $1 / 29 / 92$ & 51.33 & 48.87 & 2.46 & & \\
\hline UCD1- 19 & $2 / 5 / 92$ & 51.33 & 48.11 & 3.22 & & \\
\hline UCD1- 19 & $3 / 6 / 92$ & 51.33 & 44.73 & 6.60 & & \\
\hline UCD1- 19 & $3 / 13 / 92$ & 51.33 & 44.02 & 7.31 & & \\
\hline UCD1- 19 & $3 / 18 / 92$ & 51.33 & 43.72 & 7.61 & & \\
\hline UCD1- 19 & $3 / 25 / 92$ & 51.33 & 43.40 & 7.93 & & \\
\hline UCD1- 19 & 4/3/92 & 51.33 & 43.00 & 8.33 & & \\
\hline UCD1- 19 & $4 / 7 / 92$ & 51.33 & 43.04 & 8.29 & & \\
\hline UCD1- 19 & $4 / 16 / 92$ & 51.33 & 43.75 & 7.58 & & \\
\hline UCD1- 19 & $4 / 24 / 92$ & 51.33 & 47.27 & 4.06 & & \\
\hline UCD1- 19 & $5 / 1 / 92$ & 51.33 & 51.41 & -0.08 & & \\
\hline UCD1- 19 & $5 / 8 / 92$ & 51.33 & 55.00 & -3.67 & & \\
\hline UCD1- 19 & $5 / 26 / 92$ & 51.33 & 58.61 & -7.28 & & \\
\hline UCD1- 19 & $6 / 2 / 92$ & 51.33 & 62.00 & -10.67 & & \\
\hline UCD1- 19 & $6 / 17 / 92$ & 51.33 & 62.33 & -11.00 & & \\
\hline UCD1- 19 & $6 / 24 / 92$ & 51.33 & 63.34 & -12.01 & & \\
\hline UCD1- 19 & $7 / 2 / 92$ & 51.33 & 67.14 & -15.81 & & \\
\hline UCD1- 19 & $7 / 8 / 92$ & 51.33 & 64.76 & -13.43 & & \\
\hline UCD1- 19 & $7 / 15 / 92$ & 51.33 & 67.09 & -15.76 & & \\
\hline UCD1- 19 & $7 / 23 / 92$ & 51.33 & 67.45 & -16.12 & & \\
\hline UCD1- 19 & $7 / 29 / 92$ & 51.33 & 69.04 & -17.71 & & $\cdot$ \\
\hline UCD1- 19 & $8 / 12 / 92$ & 51.33 & 68.18 & -16.85 & & \\
\hline UCD1- 19 & $8 / 19 / 92$ & 51.33 & 66.60 & -15.27 & & \\
\hline UCD1- 19 & $8 / 28 / 92$ & 51.33 & 66.01 & -14.68 & & \\
\hline UCD1- 19 & $9 / 4 / 92$ & 51.33 & 64.69 & -13.36 & & \\
\hline UCD1- 19 & $9 / 18 / 92$ & 51.33 & 63.13 & -11.80 & & \\
\hline UCD1- 19 & 10/9/92 & 51.33 & 60.84 & -9.51 & & \\
\hline UCD1- 19 & $10 / 16 / 92$ & $51.33^{\circ}$ & 59.76 & -8.43 & & \\
\hline UCD1- 19 & $10 / 30 / 92$ & 51.33 & 57.61 & -6.28 & & \\
\hline UCD1- 19 & $11 / 6 / 92$ & 51.33 & 56.68 & -5.35 & & \\
\hline UCD1- 19 & $11 / 17 / 92$ & 51.33 & 55.48 & -4.15 & & \\
\hline UCD1- 19 & $12 / 4 / 92$ & 51.33 & 54.08 & -2.75 & & \\
\hline UCD1- 19 & $12 / 28 / 92$ & 51.33 & 52.32 & -0.99 & & \\
\hline UCD1- 19 & 1/20/93 & 51.33 & 46.01 & 5.32 & & \\
\hline UCD1- 19 & $1 / 29 / 93$ & 51.33 & 42.35 & 8.98 & & \\
\hline UCD1- 19 & $2 / 5 / 93$ & 51.33 & 41.86 & 9.47 & & \\
\hline UCD1- 19 & $2 / 26 / 93$ & 51.33 & 37.90 & 13.43 & $\cdot$ & \\
\hline UCD1- 19 & $3 / 5 / 93$ & 51.33 & 36.90 & 14.43 & & \\
\hline UCD1- 19 & $3 / 12 / 93$ & 51.33 & 36.36 & 14.97 & & \\
\hline UCD1- 19 & $3 / 19 / 93$ & 51.33 & 36.14 & 15.19 & & \\
\hline UCD1- 19 & $4 / 7 / 93$ & 51.33 & 34.76 & 16.57 & & \\
\hline UCD1- 19 & $4 / 16 / 93$ & 51.33 & 35.07 & 16.26 & & \\
\hline UCD1- 19 & $4 / 23 / 93$ & 51.33 & 35.27 & 16.06 & & \\
\hline UCD1- 19 & $5 / 7 / 93$ & 51.33 & 42.32 & 9.01 & & \\
\hline UCO1- 19 & $6 / 24 / 93$ & 51.33 & 48.13 & 3.20 & & \\
\hline UCD1- 19 & $6 / 30 / 93$ & 51.33 & 47.04 & 4.29 & & \\
\hline UCD1- 19 & $7 / 8 / 93$ & 51.33 & 49.00 & 2.33 & & \\
\hline UCD1- 19 & $7 / 15 / 93$ & 51.33 & 50.19 & 1.14 & & \\
\hline UCD1- 19 & $7 / 21 / 93$ & 51.33 & 50.13 & 1.20 & . & \\
\hline UCD1- 19 & $7 / 30 / 93$ & 51.33 & 51.10 & 0.23 & & \\
\hline UCD1- 19 & $8 / 6 / 93$ & 51.33 & 59.29 & -7.96 & & \\
\hline UCD1- 19 & $8 / 11 / 93$ & 51.33 & 59.38 & -8.05 & & \\
\hline UCD1- 19 & $8 / 20 / 93$ & 51.33 & 59.01 & -7.68 & & \\
\hline UCD1- 19 & $8 / 27 / 93$ & 51.33 & 50.63 & 0.70 & & \\
\hline UCD1- 19 & $9 / 3 / 93$ & 51.33 & 50.09 & 1.24 & . & \\
\hline UCD1- 19 & $9 / 9 / 93$ & 51.33 & 49.97 & 1.36 & & \\
\hline
\end{tabular}


Table E.1. Cumulative Groundwater Elevation Data for HSU-1, 1990-1995

\begin{tabular}{|c|c|c|c|c|c|}
\hline WELL & DATE & $\begin{array}{c}\text { PROTECTIVE } \\
\text { CASING } \\
\text { ELEVATION } \\
\text { (feet) }\end{array}$ & $\begin{array}{l}\text { DEPTH } \\
\text { TO } \\
\text { WATER } \\
\text { (feet) }\end{array}$ & $\begin{array}{c}\text { GROUND- } \\
\text { WATER } \\
\text { ELEVATION } \\
\text { (feet) } \\
\end{array}$ & COMMENTS \\
\hline UCD1- 19 & $9 / 17 / 93$ & 51.33 & 49.59 & 1.74 & \\
\hline UCD1- 19 & $9 / 24 / 93$ & 51.33 & 47.56 & 3.77 & \\
\hline UCD1- 19 & $10 / 1 / 93$ & 51.33 & 47.69 & 3.64 & \\
\hline UCD1- 19 & $10 / 8 / 93$ & 51.33 & 47.04 & 4.29 & 1 \\
\hline UCD1- 19 & $10 / 15 / 93$ & 51.33 & 46.00 & 5.33 & \\
\hline UCD1- 19 & $10 / 22 / 93$ & 51.33 & 45.06 & 6.27 & \\
\hline UCD1- 19 & $10 / 29 / 93$ & 51.33 & 44.63 & 6.70 & $\cdot$ \\
\hline UCD1- 19 & $11 / 12 / 93$ & 51.33 & 43.94 & 7.39 & \\
\hline UCD1- 19 & $11 / 24 / 93$ & 51.33 & 43.29 & 8.04 & \\
\hline UCD1- 19 & $12 / 3 / 93$ & 51.33 & 42.50 & 8.83 & v \\
\hline UCD1- 19 & $12 / 10 / 93$ & 51.33 & 42.05 & 9.28 & \\
\hline UCD1- 19 & $12 / 17 / 93$ & 51.33 & 41.63 & 9.70 & \\
\hline UCD1- 19 & $1 / 7 / 94$ & 51.33 & 40.74 & 10.59 & \\
\hline UCD1- 19 & $1 / 14 / 94$ & 51.33 & 40.31 & 11.02 & \\
\hline UCD1- 19 & $1 / 25 / 94$ & 51.33 & 40.00 & 11.33 & \\
\hline UCD1- 19 & $2 / 1 / 94$ & 51.33 & 39.69 & 11.64 & \\
\hline UCD1- 19 & $2 / 14 / 94$ & 51.33 & 39.32 & 12.01 & \\
\hline UCD1- 19 & $2 / 20 / 94$ & 51.33 & 38.92 & 12.41 & \\
\hline UCD1- 19 & $3 / 2 / 94$ & 51.33 & 37.66 & 13.67 & \\
\hline UCD1- 19 & $3 / 4 / 94$ & 51.33 & 38.35 & 12.98 & \\
\hline UCD1- 19 & $3 / 10 / 94$ & 51.33 & 38.34 & 12.99 & \\
\hline UCD1- 19 & $3 / 16 / 94$ & 51.33 & 38.23 & 13.10 & \\
\hline UCD1- 19 & $3 / 24 / 94$ & 51.33 & 38.91 & 12.42 & \\
\hline UCD1- 19 & $3 / 29 / 94$ & 51.33 & 41.24 & 10.09 & \\
\hline UCD1- 19 & $4 / 6 / 94$ & 51.33 & 44.43 & 6.90 & \\
\hline UCD1- 19 & $4 / 13 / 94$ & 51.33 & 47.88 & 3.45 & \\
\hline UCD1- 19 & $4 / 22 / 94$ & 51.33 & 51.20 & 0.13 & \\
\hline UCD1- 19 & $4 / 26 / 94$ & 51.33 & 51.85 & -0.52 & \\
\hline UCD1: 19 & $5 / 6 / 94$ & 51.33 & 52.76 & -1.43 & \\
\hline UCD1- 19 & $5 / 11 / 94$ & 51.33 & 51.76 & -0.43 & \\
\hline UCD1- 19 & $5 / 18 / 94$ & 51.33 & 52.65 & -1.32 & \\
\hline UCD1- 19 & $5 / 25 / 94$ & 51.33 & 52.86 & -1.53 & \\
\hline UCD1- 19 & $6 / 1 / 94$ & 51.33 & 54.28 & -2.95 & \\
\hline UCD1. 19 & $6 / 23 / 94$ & 51.33 & 62.27 & -10.94 & \\
\hline UCD1- 19 & $7 / 1 / 94$ & 51.33 & 64.48 & -13.15 & \\
\hline UCD1- 19 & $7 / 8 / 94$ & 51.33 & 66.14 & -14.81 & unclear meter response \\
\hline UCD1- 19 & $7 / 14 / 94$ & 51.33 & 67.79 & -16.46 & \\
\hline UCD1- 19 & $7 / 20 / 94$ & 51.33 & 69.29 & -17.96 & \\
\hline UCD1- 19 & $7 / 26 / 94$ & 51.33 & 68.92 & -17.59 & \\
\hline UCD1- 19 & $8 / 5 / 94$ & 51.33 & 68.20 & -16.87 & \\
\hline UCD1- 19 & $8 / 19 / 94$ & 51.33 & 70.66 & -19.33 & \\
\hline UCD1- 19 & $9 / 7 / 94$ & 51.33 & 66.85 & -15.52 & \\
\hline UCD1- 19 & $9 / 7 / 94$ & 51.33 & 67.27 & -15.94 & \\
\hline UCD1- 19 & $9 / 13 / 94$ & 51.33 & 67.70 & -16.37 & \\
\hline UCD1- 19 & $9 / 19 / 94$ & 51.33 & 64.53 & -13.20 & \\
\hline UCD1- 19 & $9 / 26 / 94$ & 51.33 & 63.04 & -11.71 & \\
\hline UCD1- 19 & $10 / 7 / 94$ & 51.33 & 60.61 & -9.28 & \\
\hline UCD1- 19 & $10 / 14 / 94$ & 51.33 & 58.58 & -7.25 & \\
\hline UCD1- 19 & $10 / 21 / 94$ & 51.33 & 57.40 & -6.07 & \\
\hline UCD1- 19 & $10 / 28 / 94$ & 51.33 & 56.44 & -5.11 & \\
\hline UCD1- 19 & $11 / 4 / 94$ & 51.33 & 55.80 & -4.47 & \\
\hline UCD1- 19 & $11 / 18 / 94$ & 51.33 & 53.35 & -2.02 & \\
\hline UCD1- 19 & $12 / 22 / 94$ & 51.33 & 50.80 & 0.53 & \\
\hline UCD1- 19 & $1 / 6 / 95$ & 51.33 & 49.20 & 2.13 & \\
\hline UCD1- 19 & $1 / 11 / 95$ & 51.33 & 46.70 & 4.63 & \\
\hline UCD1- 19 & $1 / 13 / 95$ & 51.33 & 45.70 & 5.63 & \\
\hline UCD1- 19 & $1 / 27 / 95$ & 51.33 & 42.02 & 9.31 & \\
\hline
\end{tabular}


Table E.1. Cumulative Groundwater Elevation Data for HSU-1, 1990-1995

\begin{tabular}{|c|c|c|c|c|c|c|}
\hline WELL & DATE & $\begin{array}{c}\text { PRÓTECTIVE } \\
\text { CASING } \\
\text { ELEVATION } \\
\text { (feet) } \\
\end{array}$ & $\begin{array}{l}\text { DEPTH } \\
\text { TO } \\
\text { WATER } \\
\text { (feet) }\end{array}$ & $\begin{array}{c}\text { GROUND- } \\
\text { WATER } \\
\text { ELEVATION } \\
\text { (feet) } \\
\end{array}$ & - & COMMENTS \\
\hline UCD1- 19 & $2 / 10 / 95$ & .51 .33 & 38.83 & 12.50 & & \\
\hline UCD1- 19 & $2 / 17 / 95$ & 51.33 & 38.34 & 12.99 & & \\
\hline UCD1- 19 & $3 / 9 / 95$ & 51.33 & 36.57 & 14.76 & & \\
\hline UCD1- 19 & $3 / 24 / 95$ & 51.33 & 31.93 & 19.40 & & \\
\hline UCD1- 19 & $4 / 28 / 95$ & 51.33 & 33.35 & 17.98 & & \\
\hline UCD1- 19 & $5 / 25 / 95$ & 51.33 & 34.20 & 17.13 & & • \\
\hline UCD1- 19 & $6 / 16 / 95$ & 51.33 & 39.42 & 11.91 & & \\
\hline UCD1- 19 & $7 / 26 / 95$ & 51.33 & 47.45 & 3.88 & & \\
\hline UCD1- 19 & $8 / 31 / 95$ & 51.33 & 48.81 & 2.52 & & \\
\hline UCD1- 19 . & $9 / 26 / 95$ & 51.33 & 45.71 & 5.62 & & \\
\hline UCD1- 19 & $10 / 25 / 95$ & 51.33 & 42.65 & 8.68 & & \\
\hline UCD1- 19 & $11 / 22 / 95$ & 51.33 & 40.54 & 10.79 & & \\
\hline UCD1- 19 & $11 / 27 / 95$ & 51.33 & 40.34 & 10.99 & & \\
\hline UCD1- 19 & $12 / 19 / 95$ & 51.33 & 38.11 & 13.22 & & \\
\hline UCD1- 20 & $1 / 22 / 91$ & 49.78 & 49.11 & 0.67 & & \\
\hline UCD1- 20 & $2 / 1 / 91$ & 49.78 & 48.81 & 0.97 & & \\
\hline UCD1- 20 & $2 / 8 / 91$ & 49.78 & 48.48 & 1.30 & & \\
\hline UCD1. 20 & $2 / 14 / 91$ & 49.78 & 48.16 & 1.62 & & \\
\hline UCD1- 20 & $2 / 22 / 91$ & 49.78 & 49.30 & 0.48 & & \\
\hline UCD1- 20 & $3 / 7 / 91$ & 49.78 & 48.34 & 1.44 & & \\
\hline UCD1- 20 & $3 / 12 / 91$ & 49.78 & 47.95 & 1.83 & & \\
\hline UCD1- 20 & $3 / 22 / 91$ & 49.78 & 46.30 & 3.48 & & \\
\hline UCD1- 20 & $3 / 29 / 91$ & 49.78 & 43.71 & 6.07 & & \\
\hline UCD1- 20 & $4 / 3 / 91$ & 49.78 & 43.64 & 6.14 & & \\
\hline UCD1- 20 & $4 / 10 / 91$ & 49.78 & 43.30 & 6.48 & & \\
\hline UCD1- 20 & $4 / 17 / 91$ & 49.78 & 43.61 & 6.17 & & \\
\hline UCD1- 20 & $4 / 24 / 91$ & 49.78 & 45.83 & 3.95 & & \\
\hline UCD1- 20 & $5 / 3 / 91$ & 49.78 & 48.70 & 1.08 & & \\
\hline UCD1- 20 & $5 / 13 / 91$ & 49.78 & 50.50 & -0.72 & & \\
\hline UCD1- 20 & $5 / 20 / 91$ & 49.78 & 54.78 & -5.00 & & \\
\hline UCD1- 20 & $5 / 29 / 91$ & 49.78 & 55.85 & -6.07 & & \\
\hline UCD1- 20 & $6 / 6 / 91$ & 49.78 & 59.22 & -9.44 & & \\
\hline UCD1- 20 & $6 / 10 / 91$ & 49.78 & 59.82 & -10.04 & & \\
\hline UCD1- 20 & $6 / 21 / 91$ & 49.78 & 61.73 & -11.95 & · & \\
\hline UCD1- 20 & $6 / 27 / 91$ & 49.78 & 62.91 & -13.13 & & \\
\hline UCD1- 20 & $7 / 5 / 91$ & 49.78 & 63.32 & -13.54 & & \\
\hline UCD1- 20 & $7 / 12 / 91$ & 49.78 & 62.40 & -12.62 & & \\
\hline UCD1- 20 & $7 / 19 / 91$ & 49.78 & 68.73 & -18.95 & & . \\
\hline UCD1- 20 & $7 / 26 / 91$ & 49.78 & 68.05 & -18.27 & & \\
\hline UCD1- 20 & $8 / 5 / 91$ & 49.78 & 67.30 & -17.52 & & \\
\hline UCD1- 20 & $8 / 14 / 91$ & 49.78 & 68.90 & -19.12 & & \\
\hline UCD1- 20 & $8 / 21 / 91$ & 49.78 & 67.34 & -17.56 & & \\
\hline UCD1- 20 & $8 / 29 / 91$ & 49.78 & 64.91 & -15.13 & & \\
\hline UCD1- 20 & $9 / 4 / 91$ & 49.78 & .63 .19 & -13.41 & & \\
\hline UCD1- 20 & $9 / 13 / 91$ & 49.78 & 63.61 & -13.83 & . & \\
\hline UCD1- 20 & $10 / 3 / 91$ & 49.78 & 59.40 & -9.62 & & \\
\hline UCD1- 20 & $10 / 10 / 91$ & 49.78 & 58.13 & -8.35 & & \\
\hline UCD1- 20 & $10 / 25 / 91$ & 49.78 & 55.68 & -5.90 & & \\
\hline UCD1- 20 & $11 / 1 / 91$ & 49.78 & 53.96 & -4.18 & & \\
\hline UCD1- 20 & $11 / 8 / 91$ & 49.78 & 52.75 & -2.97 & & \\
\hline UCD1- 20 & $11 / 14 / 91$ & 49.78 & 52.35 & -2.57 & & \\
\hline UCD1- 20 & $12 / 5 / 91$ & 49.78 & 51.04 & -1.26 & & \\
\hline UCD1- 20 & $12 / 11 / 91$ & 49.78 & 50.72 & -0.94 & & \\
\hline UCD1- 20 & $12 / 19 / 91$ & 49.78 & 50.13 & -0.35 & & \\
\hline UCD1- 20 & $1 / 3 / 92$ & 49.78 & 49.28 & 0.50 & & \\
\hline UCD1- 20 & $1 / 8 / 92$ & 49.78 & 47.28 & 2.50 & & \\
\hline
\end{tabular}


Table E.1. Cumulative Groundwater Elevation Data for HSU-1, 1990-1995

\begin{tabular}{|c|c|c|c|c|c|c|c|c|}
\hline WELL & DATE & $\begin{array}{c}\text { PROTECTIVE } \\
\text { CASING } \\
\text { ELEVATION } \\
\text { (feet) }\end{array}$ & $\begin{array}{l}\text { DEPTH } \\
\text { TO } \\
\text { WATER } \\
\text { (feet) }\end{array}$ & $\begin{array}{l}\text { GROUND- } \\
\text { WATER } \\
\text { ELEVATION } \\
\text { (feet) } \\
\end{array}$ & - & & COMMENTS & \\
\hline UCD1- 20 & $1 / 17 / 92$ & 49.78 & 49.17 & 0.61 & & - & & \\
\hline UCD1- 20 & $1 / 24 / 92$ & 49.78 & 47.61 & 2.17 . & & & & \\
\hline UCD1- 20 & $1 / 29 / 92$ & 49.78 & 46.94 & $2.84^{\circ}$ & & & & \\
\hline UCD1- 20 & $2 / 5 / 92$ & 49.78 & 46.22 & 3.56 & & & & \\
\hline UCD1- 20 & $3 / 6 / 92$ & 49.78 & 41.65 & 8.13 & & & $\cdot$ & \\
\hline UCD1- 20 & $3 / 13 / 92$ & 49.78 & 41.20 & 8.58 & & & . & \\
\hline UCD 1 - 20 & $3 / 18 / 92$ & 49.78 & 41.05 & 8.73 & & & & \\
\hline UCD1- 20 & $3 / 25 / 92$ & 49.78 & 40.94 & 8.84 & & & & \\
\hline UCD1- 20 & $4 / 3 / 92$ & 49.78 & 40.62 & 9.16 & & & & \\
\hline UCD1- 20 & $4 / 7 / 92$ & 49.78 & 40.72 & 9.06 & - & & & \\
\hline UCD1- 20 & $4 / 16 / 92$ & 49.78 & 41.41 & 8.37 & & & $\because$ & \\
\hline UCD1- 20 & $4 / 24 / 92$ & 49.78 & 45.56 & 4.22 & & & & \\
\hline UCDi- 20 & $5 / 1 / 92$ & 49.78 & 49.15 & 0.63 & & & & \\
\hline UCD1- 20 & $5 / 8 / 92$ & 49.78 & 51.50 & -1.72 & & & & \\
\hline UCD1- 20 & $5 / 26 / 92$ & 49.78 & 56.06 & -6.28 & & & & \\
\hline UCD1- 20 & $6 / 2 / 92$ & 49.78 & 59.78 & -10.00 & . & & & \\
\hline UCD1- 20 & $6 / 17 / 92$ & 49.78 & 60.00 & -10.22 & & & & \\
\hline UCD1- 20 & $6 / 24 / 92$ & 49.78 & 61.69 & -11.91 & & & & \\
\hline UCD1- 20 & $7 / 2 / 92$ & 49.78 & 64.88 & -15.10 & & & · & \\
\hline UCD1- 20 & $7 / 8 / 92$ & 49.78 & 62.68 & -12.90 & & & & \\
\hline UCD1- 20 & $7 / 15 / 92$ & 49.78 & 64.60 & -14.82 & & & & \\
\hline UCD1- 20 & - 7/23/92 & 49.78 & 65.94 & -16.16 & & & & \\
\hline UCD1- 20 & $7 / 29 / 92$ & 49.78 & 65.75 & -15.97 & & & & \\
\hline UCD1- 20 & $8 / 12 / 92$ & 49.78 & 65.86 & -16.08 & & & & \\
\hline UCD1- 20 & $8 / 19 / 92$ & 49.78 & 63.90 & -14.12 & & & & . \\
\hline UCD1- 20 & $8 / 28 / 92$ & 49.78 & 63.80 & -14.02 & & & & \\
\hline UCDi- 20 & $9 / 4 / 92$ & 49.78 & 62.65 & -12.87 & & & & \\
\hline UCD1- 20 & $9 / 18 / 92$ & 49.78 & 61.02 & -11.24 & & . & & \\
\hline UCD1- 20 & $10 / 9 / 92$ & 49.78 & 59.59 & -9.81 & & & & - \\
\hline UCD1- 20 & $10 / 16 / 92$ & 49.78 & 57.69 & -7.91 & & & & \\
\hline UCD1- 20 & $10 / 30 / 92$ & 49.78 & 55.70 & -5.92 & & & & \\
\hline UCD1- 20 & $11 / 6 / 92$ & 49.78 & 54.81 & -5.03 & & & & \\
\hline UCD1- 20 & $11 / 17 / 92$ & 49.78 & 53.78 & -4.00 & & & & \\
\hline UCD1- 20 & $12 / 4 / 92$ & 49.78 & 52.01 & -2.23 & & & & \\
\hline UCD1- 20 & $12 / 28 / 92$ & 49.78 & 50.21 & -0.43 & & & & \\
\hline UCD1- 20 & $1 / 20 / 93$ & 49.78 & 42.79 & 6.99 & & & & \\
\hline UCD1- 20 & $1 / 29 / 93$ & 49.78 & 39.68 & 10.10 & & & & \\
\hline UCD1- 20 & $2 / 5 / 93$ & 49.78 & 39.26 & 10.52 & & & & \\
\hline UCD1- 20 & $2 / 12 / 93$ & 49.78 & 38.63 & 11.15 & & & & \\
\hline UCD1- 20 & $2 / 26 / 93$ & 49.78 & 34.80 & 14.98 & & & & \\
\hline UCD1- 20 & $3 / 5 / 93$ & 49.78 & 34.43 & 15.35 & & & & \\
\hline UCD1- 20 & $3 / 12 / 93$ & 49.78 & 34.04 & 15.74 & & & & \\
\hline UCD1- 20 & $3 / 19 / 93$ & 49.78 & .33 .85 & 15.93 & & & & \\
\hline UCD1- 20 & $4 / 7 / 93$ & 49.78 & 32.55 & 17.23 & & & & \\
\hline UCD1- 20 & $4 / 16 / 93$ & 49.78 & 32.83 & 16.95 & & & & \\
\hline UCD1- 20 & $4 / 23 / 93$ & 49.78 & 33.01 & 16.77 & & & & \\
\hline UCD1- 20 & $5 / 7 / 93$ & 49.78 & 39.17 & 10.61 & & & & \\
\hline UCD1- 20 & $6 / 24 / 93$ & 49.78 & 43.56 & 6.22 & & & & \\
\hline UCD1- 20 & $6 / 30 / 93$ & -49.78 & 45.00 & 4.78 & & & & \\
\hline UCD1- 20 & $7 / 8 / 93$ & 49.78 & 48.38 & 1.40 & & & & \\
\hline UCD1- 20 & $7 / 15 / 93$ & 49.78 & 47.50 & 2.28 & & & & \\
\hline UCD1- 20 & $7 / 21 / 93$ & 49.78 & 47.56 & 2.22 & & & & \\
\hline UCD1- 20 & $7 / 30 / 93$ & 49.78 & 48.46 & 1.32 & & & & \\
\hline UCD1- 20 & $8 / 6 / 93$ & 49.78 & 48.54 & 1.24 & & & & \\
\hline UCD1- 20 & $8 / 11 / 93$ & 49.78 & 49.02 & 0.76 & & & & \\
\hline UCD1- 20 & $8 / 20 / 93$ & 49.78 & 48.76 & 1.02 & & & & \\
\hline UCD1- 20 & $8 / 27 / 93$ & 49.78 & 48.40 & - $\quad 1.38$ & & & & \\
\hline
\end{tabular}


Table E.1. Cumulative Groundwater Elevation Data for HSU-1, 1990-1995

\begin{tabular}{|c|c|c|c|c|c|}
\hline WELL & DATE & $\begin{array}{c}\text { PROTECTIVE } \\
\text { CASING } \\
\text { ELEVATION } \\
\text { (feet) }\end{array}$ & $\begin{array}{l}\text { DEPTH } \\
\text { TO } \\
\text { WATER } \\
\text { (feet) }\end{array}$ & $\begin{array}{c}\text { GROUND- } \\
\text { WATER } \\
\text { ELEVATION } \\
\text { (feet) }\end{array}$ & COMMENTS \\
\hline UCD1- 20 & $9 / 3 / 93$ & 49.78 & 47.58 & 2.20 & \\
\hline UCD1- 20 & 9/9/93 & 49.78 & 47.48 & 2.30 & \\
\hline UCD1- 20 & $9 / 17 / 93$ & 49.78 & 47.36 & 2.42 & \\
\hline UCD1- 20 & $9 / 24 / 93$ & 49.78 & 48.44 & 1.34 & \\
\hline UCD1- 20 & $10 / 1 / 93$ & 49.78 & 45.71 & 4.07 & \\
\hline UCD1- 20 & $10 / 8 / 93$ & 49.78 & 45.04 & 4.74 & \\
\hline UCD1- 20 & $10 / 15 / 93$ & 49.78 & 44.03 & 5.75 & \\
\hline UCD1- 20 & $10 / 22 / 93$ & 49.78 & 43.15 & 6.63 & \\
\hline UCD1- 20 & $10 / 29 / 93$ & 49.78 & 42.67 & 7.11 & \\
\hline UCD1- 20 & $11 / 12 / 93$ & 49.78 & 42.00 & 7.78 & \\
\hline UCD1- 20 & $11 / 24 / 93$ & 49.78 & 41.32 & 8.46 & \\
\hline UCD1- 20 & $12 / 3 / 93$ & 49.78 & 40.50 & 9.28 & \\
\hline UCD1- 20 & $12 / 10 / 93$ & 49.78 & 40.11 & 9.67 & \\
\hline UCD1- 20 & $12 / 17 / 93$ & 49.78 & 39.59 & 10.19 & \\
\hline UCD1- 20 & $1 / 7 / 94$ & 49.78 & 38.85 & 10.93 & \\
\hline UCD1- 20 & $1 / 14 / 94$ & 49.78 & 38.51 & 11.27 & \\
\hline UCD1- 20 & $1 / 25 / 94$ & 49.78 & 37.82 & 11.96 & \\
\hline UCD1- 20 & $2 / 1 / 94$ & 49.78 & 37.66 & 12.12 & \\
\hline UCD1- 20 & $2 / 14 / 94$ & 49.78 & 36.99 & 12.79 & \\
\hline UCD1- 20 & $2 / 20 / 94$ & 49.78 & 36.49 & 13.29 & \\
\hline UCD1- 20 & $3 / 2 / 94$ & 49.78 & 35.61 & 14.17 & \\
\hline UCD1- 20 & $3 / 4 / 94$ & 49.78 & 36.24 & 13.54 & \\
\hline UCD1- 20 & $3 / 10 / 94$ & 49.78 & 36.23 & 13.55 & \\
\hline UCD1- 20 & $3 / 16 / 94$ & 49.78 & 36.19 & 13.59 & \\
\hline UCD1- 20 & $3 / 24 / 94$ & 49.78 & 36.70 & 13.08 & \\
\hline UCD1- 20 & $3 / 29 / 94$ & 49.78 & 38.76 & 11.02 & \\
\hline UCD1- 20 & $4 / 6 / 94$ & 49.78 & 41.06 & 8.72 & \\
\hline UCD1- 20 & $4 / 13 / 94$ & 49.78 & 45.56 & 4.22 & \\
\hline UCD1- 20 & $4 / 22 / 94$ & 49.78 & 48.87 & 0.91 & • \\
\hline UCD1- 20 & $4 / 26 / 94$ & 49.78 & 49.49 & 0.29 & \\
\hline UCD1- 20 & $5 / 6 / 94$ & 49.78 & 50.23 & -0.45 & \\
\hline UCD1- 20 & $5 / 11 / 94$ & 49.78 & 49.53 & 0.25 & \\
\hline UCD1- 20 & $5 / 18 / 94$ & 49.78 & 50.36 & -0.58 & \\
\hline UCD1- 20 & $5 / 25 / 94$ & 49.78 & 50.53 & -0.75 & \\
\hline UCD1- 20 & $6 / 1 / 94$ & 49.78 & 51.27 & -1.49 & \\
\hline UCD1- 20 & $6 / 23 / 94$ & 49.78 & 60.08 & -10.30 & \\
\hline UCD1- 20 & $7 / 1 / 94$ & 49.78 & 62.27 & -12.49 & \\
\hline UCD1- 20 & $7 / 8 / 94$ & 49.78 & 64.73 & -14.95 & unclear meter response \\
\hline UCD1- 20 & $7 / 14 / 94$ & 49.78 & 65.57 & -15.79 & \\
\hline UCD1- 20 & $7 / 20 / 94$ & 49.78 & 67.18 & -17.40 & \\
\hline UCD1- 20 & $7 / 26 / 94$ & 49.78 & 66.93 & -17.15 & \\
\hline UCD1- 20 & $8 / 5 / 94$ & 49.78 & 66.08 & -16.30 & \\
\hline UCD1- 20 & $8 / 19 / 94$ & 49.78 & 68.85 & -19.07 & \\
\hline UCD1- 20 & $9 / 7 / 94$ & 49.78 & 64.92 & -15.14 & missing vent cap \\
\hline UCD1- 20 & $9 / 7 / 94$ & 49.78 & 65.28 & -15.50 & \\
\hline UCD1- 20 & $9 / 13 / 94$ & 49.78 & 65.80 & -16.02 & \\
\hline UCD1- 20 & $9 / 19 / 94$ & 49.78 & 62.78 & -13.00 & \\
\hline UCD1- 20 & $9 / 26 / 94$ & 49.78 & 61.26 & -11.48 & \\
\hline UCD1- 20 & $10 / 7 / 94$ & 49.78 & 58.65 & -8.87 & $\cdot$ \\
\hline UCD1- 20 & $10 / 14 / 94$ & 49.78 & 56.44 & -6.66 & \\
\hline UCD1- 20 & $10 / 21 / 94$ & 49.78 & 55.45 & -5.67 & $\cdot$ \\
\hline UCD1- 20 & $10 / 28 / 94$ & 49.78 & 54.56 & -4.78 & \\
\hline UCO1- 20 & $11 / 4 / 94$ & 49.78 & 53.92 & -4.14 & \\
\hline UCD1- 20 & $11 / 18 / 94$ & 49.78 & 51.45 & -1.67 & \\
\hline UCD1- 20 & $12 / 22 / 94$ & 49.78 & 48.96 & 0.82 & \\
\hline UCD1- 20 & $1 / 6 / 95$ & 49.78 & 48.16 & 1.62 & \\
\hline UCD1- 20 & $1 / 11 / 95$ & 49.78 & 39.45 & 10.33 & \\
\hline
\end{tabular}


Table E.1. Cumulative Groundwater Elevation Data for HSU-1, 1990-1995

\begin{tabular}{|c|c|c|c|c|c|}
\hline WELL & DATE & $\begin{array}{c}\text { PROTECTIVE } \\
\text { CASING } \\
\text { ELEVATION } \\
\text { (feet) } \\
\end{array}$ & $\begin{array}{l}\text { DEPTH } \\
\text { TO } \\
\text { WATER } \\
\text { (feet) }\end{array}$ & $\begin{array}{l}\text { GROUND- } \\
\text { WATER } \\
\text { ELEVATION } \\
\text { (feet) }\end{array}$ & COMMENTS \\
\hline UCD1- 20 & $1 / 13 / 95$ & 49.78 & 41.31 & 8.47 & \\
\hline UCD1- 20 & $1 / 27 / 95$ & 49.78 & 38.27 & 11.51 & \\
\hline UCD1- 20 & $2 / 10 / 95$ & 49.78 & 36.42 & 13.36 & \\
\hline UCD1- 20 & $2 / 17 / 95$ & 49.78 & 36.07 & 13.71 & \\
\hline UCD1- 20 & $3 / 9 / 95$ & 49.78 & 33.40 & 16.38 & \\
\hline UCD1- 20 & $3 / 16 / 95$ & 49.78 & 30.90 & 18.88 & \\
\hline UCD1- 20 & $3 / 24 / 95$ & 49.78 & 27.99 & 21.79 & \\
\hline UCD1- 20 & $4 / 28 / 95$ & 49.78 & 31.17 & 18.61 & \\
\hline UCD1- 20 & $5 / 25 / 95$ & 49.78 & 31.91 & 17.87 & \\
\hline UCD1- 20 & $6 / 16 / 95$ & 49.78 & 37.04 & 12.74 & \\
\hline UCD1- 20 & $7 / 26 / 95$ & 49.78 & 45.15 & 4.63 & \\
\hline UCD1- 20 & $8 / 31 / 95$ & 49.78 & 46.67 & 3.11 & Cap is missing \\
\hline UCD1- 20 & $9 / 26 / 95$ & 49.78 & 43.76 & 6.02 & caps missing \\
\hline UCD1- 20 & $10 / 25 / 95$ & 49.78 & 40.71 & 9.07 & \\
\hline UCD1- 20 & $11 / 22 / 95$ & 49.78 & 38.77 & 11.01 & \\
\hline UCD1- 20 & $11 / 27 / 95$ & 49.78 & 38.55 & 11.23 & \\
\hline UCD1- 20 & $12 / 19 / 95$ & 49.78 & 36.22 & 13.56 & \\
\hline UCD1-' 21 & $1 / 22 / 91$ & 48.73 & 48.16 & 0.57 & \\
\hline UCD1- 21 & $2 / 1 / 91$ & 48.73 & 47.79 & 0.94 & \\
\hline UCD1- 21 & 2/8/91 & 48.73 & 47.56 & 1.17 & \\
\hline UCD1- 21 & $2 / 14 / 91$ & 48.73 & 47.12 & 1.61 & \\
\hline UCD1- 21 & $2 / 22 / 91$ & 48.73 & 48.13 & 0.60 & \\
\hline UCD1- 21 & $3 / 7 / 91$ & 48.73 & 47.79 & 0.94 & \\
\hline UCD1- 21 & $3 / 12 / 91$ & 48.73 & 47.10 & 1.63 & $\cdot$ \\
\hline UCD1- 21 & $3 / 22 / 91$ & 48.73 & 43.46 & 5.27 & \\
\hline UCD1- 21 & $3 / 29 / 91$ & 48.73 & 43.33 & 5,40 & \\
\hline UCD1- 21 & $4 / 3 / 91$ & 48.73 & 42.93 & 5.80 & \\
\hline UCD1- 21 & $4 / 10 / 91$ & 48.73 & 42.36 & 6.37 & \\
\hline UCD1- 21 & $4 / 17 / 91$ & 48.73 & 42.52 & 6.21 & \\
\hline UCD1- 21 & $4 / 24 / 91$ & 48.73 & 44.77 & 3.96 & \\
\hline UCD1- 21 & $5 / 3 / 91$ & 48.73 & 47.44 & 1.29 & . \\
\hline UCD1- 21 & $5 / 13 / 91$ & 48.73 & 49.25 & -0.52 & \\
\hline UCD1- 21 & $5 / 20 / 91$ & 48.73 & 53.30 & -4.57 & • \\
\hline UCD1- 21 & $5 / 29 / 91$ & 48.73 & 54.44 & -5.71 & \\
\hline UCD1- 21 & $6 / 6 / 91$ & 48.73 & 57.97 & -9.24 & \\
\hline UCD1- 21 & $6 / 10 / 91$ & 48.73 & 58.55 & -9.82 & \\
\hline UCD1- 21 & $6 / 21 / 91$ & 48.73 & 60.00 & -11.27 & 1 \\
\hline UCD1- 21 & $6 / 27 / 91$ & 48.73 & 61.56 & -12.83 & \\
\hline UCD1- 21 & $7 / 5 / 91$ & 48.73 & 61.67 & -12.94 & \\
\hline UCD1- 21 & $7 / 12 / 91$ & 48.73 & 64.71 & -15.98 & \\
\hline UCD1- 21 & $7 / 19 / 91$ & 48.73 & 66.21 & -17.48 & \\
\hline UCD1- 21 & $7 / 26 / 91$ & 48.73 & 66.59 & -17.86 & \\
\hline UCD1- 21 & $8 / 5 / 91$ & .48 .73 & 66.00 & -17.27 & \\
\hline UCD1- 21 & $8 / 14 / 91$ & 48.73 & 67.43 & -18.70 & \\
\hline UCD1- 21 & $8 / 21 / 91$ & 48.73 & 65.92 & -17.19 & \\
\hline UCD1- 21 & $8 / 29 / 91$ & 48.73 & 64.10 & -15.37 & \\
\hline UCD1- 21 & 9/4/91 & 48.73 & 62.23 & -13.50 & \\
\hline UCD1- 21 & $9 / 13 / 91$ & 48.73 & 62.35 & -13.62 & \\
\hline UCD1- 21 & $10 / 3 / 91$ & 48.73 & 58.13 & -9.40 & \\
\hline UCD1- 21 & $10 / 10 / 91$ & 48.73 & 58.99 & -10.26 & $\cdot$ \\
\hline UCD1- 21 & $10 / 25 / 91$ & 48.73 & 54.51 & -5.78 & \\
\hline UCD1- 21 & $11 / 1 / 91$ & 48.73 & 52.71 & -3.98 & 一 \\
\hline UCD1- 21 & $11 / 8 / 91$ & 48.73 & 51.63 & -2.90 & \\
\hline UCD1- 21 & $11 / 14 / 91$ & 48.73 & 51.23 & -2.50 & \\
\hline UCD1- 21 & $12 / 5 / 91$ & 48.73 & 50.12 & -1.39 & \\
\hline UCD1- 21 & $12 / 11 / 91$ & 48.73 & 50.34 & -1.61 & \\
\hline
\end{tabular}


Table E.1. Cumulative Groundwater Elevation Data for HSU-1, 1990-1995

\begin{tabular}{|c|c|c|c|c|c|c|}
\hline WELL & DATE & $\begin{array}{c}\text { PROTECTIVE } \\
\text { CASING } \\
\text { ELEVATION } \\
\text { (feet) }\end{array}$ & $\begin{array}{c}\text { DEPTH } \\
\text { TO } \\
\text { WATER } \\
\text { (feet) }\end{array}$ & $\begin{array}{l}\text { GROUND- } \\
\text { WATER } \\
\text { ELEVATION } \\
\text { (feet) } \\
\end{array}$ & & COMMENTS \\
\hline UCD1- 21 & $1 / 17 / 92$ & 48.73 & 47.25 & 1.48 & & \\
\hline UCD1- 21 & $1 / 24 / 92$ & 48.73 & 46.62 & 2.11 & · & \\
\hline UCD1- 21 & $1 / 29 / 92$ & 48.73 & 45.85 & 2.88 & & . \\
\hline UCD1- 21 & 2/5/92 & 48.73 & 45.20 & 3.53 & & \\
\hline UCD1- 21 & $3 / 6 / 92$ & 48.73 & 41.21 & 7.52 & & \\
\hline UCD1- 21 & $3 / 13 / 92$ & 48.73 & 40.40 & 8.33 & & \\
\hline UCD1- 21 & $3 / 18 / 92$ & 48.73 & 40.15 & 8.58 & & \\
\hline UCD1- 21 & $3 / 25 / 92$ & 48.73 & 39.94 & 8.79 & & \\
\hline UCD1- 21 & 4/3/92 & 48.73 & 39.60 & 9.13 & & - \\
\hline UCD1- 21 & $4 / 7 / 92$ & 48.73 & 39.69 & 9.04 & & \\
\hline UCD1- 21 & $4 / 16 / 92$ & 48.73 & 40.35 & 8.38 & & \\
\hline UCD1- 21 & $4 / 24 / 92$ & 48.73 & 44.41 & 4.32 & & \\
\hline UCD1- 21 & $5 / 1 / 92$ & 48.73 & 47.99 & 0.74 & & \\
\hline UCD1- 21 & $5 / 8 / 92$ & 48.73 & 49.73 & -1.00 & & \\
\hline UCD1- 21 & $6 / 24 / 92$ & 48.73 & 59.75 & -11.02 & & \\
\hline UCD1- 21 & $7 / 2 / 92$ & 48.73 & 63.12 & -14.39 & & \\
\hline UCD1- 21 & $7 / 8 / 92$ & 48.73 & 61.29 & -12.56 & & \\
\hline UCD1- 21 & $7 / 15 / 92$ & 48.73 & 62.96 & -14.23 & & \\
\hline UCD1- 21 & $7 / 23 / 92$ & 48.73 & 64.55 & -15.82 & & \\
\hline UCD1- 21 & $7 / 29 / 92$ & 48.73 & 59.57 & -10.84 & & \\
\hline UCD1- 21 & $8 / 12 / 92$ & 48.73 & 64.55 & -15.82 & & \\
\hline UCD1- 21 & $8 / 19 / 92$ & 48.73 & 62.82 & -14.09 & & \\
\hline UCD1- 21 & $8 / 28 / 92$ & 48.73 & 62.61 & -13.88 & & \\
\hline UCD1- 21 & $9 / 4 / 92$ & 48.73 & 61.52 & -12.79 & & \\
\hline UCD1- 21 & $9 / 18 / 92$ & 48.73 & 60.01 & -11.28 & & \\
\hline UCD1- 21 & 10/9/92 & 48.73 & 57.44 & -8.71 & & \\
\hline UCD1- 21 & $10 / 16 / 92$ & 48.73 & 56.67 & -7.94 & & \\
\hline UCD1- 21 & $10 / 30 / 92$ & 48.73 & 54.64 & -5.91 & & \\
\hline UCD1- 21 & $11 / 6 / 92$ & 48.73 & 53.69 & -4.96 & & \\
\hline UCD1- 21 & $11 / 17 / 92$ & 48.73 & 52.62 & -3.89 & & \\
\hline UCD1- 21 & $12 / 4 / 92$ & 48.73 & 51.17 & -2.44 & & \\
\hline UCD1- 21 & $12 / 28 / 92$ & 48.73 & 49.77 & -1.04 & & \\
\hline UCD1- 21 & $2 / 5 / 93$ & 48.73 & 38.52 & 10.21 & & \\
\hline UCD1- 21 & $2 / 12 / 93$ & 48.73 & 37.83 & 10.90 & $\cdot$ & \\
\hline UCD1- 21 & $2 / 26 / 93$ & 48.73 & 34.29 & 14.44 & & \\
\hline UCD1- 21 & $3 / 5 / 93$ & 48.73 & 33.68 & 15.05 & & \\
\hline UCD1- 21 & $3 / 12 / 93$ & 48.73 & 33.26 & 15.47 & & \\
\hline UCD1- 21 & $3 / 19 / 93$ & 48.73 & 32.96 & 15.77 & & \\
\hline UCD1- 21 & $4 / 7 / 93$ & 48.73 & 31.70 & 17.03 & & \\
\hline UCD1- 21 & $4 / 16 / 93$ & 48.73 & 31.88 & 16.85 & & \\
\hline UCD1- 21 & $4 / 23 / 93$ & 48.73 & 32.08 & 16.65 & & \\
\hline UCD1- 21 & $5 / 7 / 93$ & 48.73 & 38.29 & 10.44 & & \\
\hline UCD1- 21 & $6 / 24 / 93$ & 48.73 & 41.46 & 7.27 & & \\
\hline UCD1- 21 & $6 / 30 / 93$ & 48.73 & 42.81 & 5.92 & & \\
\hline UCD1- 21 & $7 / 8 / 93$ & 48.73 & 47.19 & 1.54 & & \\
\hline UCD1- 21 & $7 / 15 / 93$ & 48.73 & 48.33 & 0.40 & & \\
\hline UCD1- 21 & $7 / 21 / 93$ & 48.73 & 48.25 & 0.48 & & \\
\hline UCD1- 21 & $7 / 30 / 93$ & 48.73 & 47.27 & 1.46 & & \\
\hline UCD1- 21 & $8 / 6 / 93$ & 48.73 & 47.70 & 1.03 & & \\
\hline UCD1- 21 & $8 / 11 / 93$ & 48.73 & 47.77 & 0.96 & & \\
\hline UCD1- 21 & $8 / 20 / 93$ & 48.73 & 48.00 & 0.73 & & \\
\hline UCD1- 21 & $8 / 27 / 93$ & 48.73 & 47.20 & 1.53 & & \\
\hline UCD1- 21 & 9/3/93 & 48.73 & 46.44 & 2.29 & & \\
\hline UCD1- 21 & 9/9/93 & 48.73 & 48.29 & 0.44 & & \\
\hline UCD1- 21 & $9 / 17 / 93$ & 48.73 & 46.19 & 2.54 & & \\
\hline UCD1- 21 & $9 / 24 / 93$ & 48.73 & 45.23 & 3.50 & & \\
\hline UCO1- 21 & $10 / 1 / 93$ & 48.73 & 44.55 & 4.18 & & \\
\hline
\end{tabular}


Table E.1. Cumulative Groundwater Elevation Data for HSU-1, 1990-1995

\begin{tabular}{|c|c|c|c|c|c|c|}
\hline WELL & DATE & $\begin{array}{c}\text { PROTECTIVE } \\
\text { CASING } \\
\text { ELEVATION } \\
\text { (feet) }\end{array}$ & $\begin{array}{l}\text { DEPTH } \\
\text { TO } \\
\text { WATER } \\
\text { (feet) }\end{array}$ & $\begin{array}{c}\text { GROUND- } \\
\text { WATER } \\
\text { ELEVATION } \\
\text { (feet) }\end{array}$ & COMMENTS & \\
\hline UCD1- 21 & $10 / 8 / 93$ & 48.73 & 43.92 & 4.81 & & \\
\hline UCD1- 21 & $10 / 15 / 93$ & 48.73 & 42.94 & 5.79 & & \\
\hline UCD1- 21 & $10 / 22 / 93$ & 48.73 & 42.06 & 6.67 & & \\
\hline UCD1- 21 & $10 / 29 / 93$ & 48.73 & 41.55 & 7.18 & & \\
\hline UCD1- 21 & $11 / 12 / 93$ & 48.73 & 40.89 & 7.84 & & \\
\hline UCD1- 21 & $11 / 24 / 93$ & 48.73 & 40.20 & 8.53 & & \\
\hline UCD1. 21 & $12 / 3 / 93$ & 48.73 & 39.46 & 9.27 & & \\
\hline UCD1- 21 & $12 / 10 / 93$ & 48.73 & 38.96 & 9.77 & & \\
\hline UCD1- 21 & $12 / 17 / 93$ & 48.73 & 38.55 & 10.18 & & \\
\hline UCD1- 21 & $1 / 7 / 94$ & 48.73 & 37.72 & 11.01 & & \\
\hline UCD1- 21 & $1 / 14 / 94$ & 48.73 & 37.35 & 11.38 & & \\
\hline UCD1- 21 & $1 / 25 / 94$ & 48.73 & 38.96 & 9.77 & & \\
\hline UCD1- 21 & 2/1/94 & 48.73 & 36.63 & 12.10 & & \\
\hline UCD1- 21 & $2 / 14 / 94$ & 48.73 & 36.02 & 12.71 & & \\
\hline UCD1- 21 & $2 / 20 / 94$ & 48.73 & 35.80 & 12.93 & & \\
\hline UCD1- 21 & $3 / 2 / 94$ & 48.73 & 34.67 & 14.06 & & \\
\hline UCD1- 21 & $3 / 4 / 94$ & 48.73 & 35.27 & 13.46 & & \\
\hline UCD1- 21 & $3 / 10 / 94$ & 48.73 & 35.19 & 13.54 & & \\
\hline UCD1- 21 & $3 / 16 / 94$ & 48.73 & 35.17 & 13.56 & & \\
\hline UCD1- 21 & $3 / 24 / 94$ & 48.73 & 35.71 & 13.02 & & \\
\hline UCD1- 21 & $3 / 29 / 94$ & 48.73 & 37.78 & 10.95 & & \\
\hline UCD1- 21 & $4 / 6 / 94$ & 48.73 & 41.06 & 7.67 & . & \\
\hline UCD1- 21 & $4 / 13 / 94$ & 48.73 & 44.32 & 4.41 & & \\
\hline UCD1- 21 & $4722 / 94$ & 48.73 & 47.61 & 1.12 & - & \\
\hline UCD1- 21 & $4 / 26 / 94$ & 48.73 & 48.24 & 0.49 & & \\
\hline UCD1- 21 & $5 / 6 / 94$ & 48.73 & 48.91 & -0.18 & * & \\
\hline UCD1- 21 & $5 / 11 / 94$ & 48.73 & 48.23 & 0.50 & & \\
\hline UCD1- 21 & $5 / 18 / 94$ & 48.73 & 49.13 & -0.40 & & \\
\hline UCD1- 21 & $5 / 25 / 94$ & 48.73 & 49.27 & -0.54 & & \\
\hline UCD1- 21 & $6 / 1 / 94$ & 48.73 & 49.84 & -1.11 & & \\
\hline UCD1- 21 & $6 / 23 / 94$ & 48.73 & 58.69 & -9.96 & & \\
\hline UCD1- 21 & $7 / 1 / 94$ & 48.73 & 60.77 & -12.04 & unclear meter response & \\
\hline UCD1. 21 & $7 / 8 / 94$ & 48.73 & 63.20 & -14.47 & unclear meter response & \\
\hline UCD1- 21 & $7 / 14 / 94$ & 48.73 & 64.06 & -15.33 & & \\
\hline UCD1- 21 & $7 / 20 / 94$ & 48.73 & 65.67 & -16.94 & & \\
\hline UCD1- 21 & $7 / 26 / 94$ & 48.73 & 65.40 & -16.67 & & \\
\hline UCD1- 21 & $8 / 5 / 94$ & 48.73 & 64.63 & -15.90 & & \\
\hline UCD1- 21 & $8 / 19 / 94$ & 48.73 & 67.43 & -18.70 & & \\
\hline UCD1- 21 & $9 / 7 / 94$ & 48.73 & 63.81 & -15.08 & & \\
\hline UCD1- 21 & $9 / 7 / 94$ & 48.73 & 64.04 & -15.31 & & \\
\hline UCD1- 21 & $9 / 13 / 94$ & 48.73 & 64.55 & -15.82 & & \\
\hline UCD1. 21 & $.9 / 19 / 94$ & 48.73 & 61.86 & -13.13 & & . \\
\hline UCD1- 21 & $9 / 26 / 94$ & 48.73 & 60.25 & -11.52 & & \\
\hline UCD1- 21 & $10 / 7 / 94$ & 48.73 & 57.45 & -8.72 & & \\
\hline UCD1- 21 & $10 / 14 / 94$ & 48.73 & 55.31 & -6.58 & & \\
\hline UCD1- 21 & $10 / 21 / 94$ & 48.73 & 54.29 & -5.56 & & \\
\hline UCD1- 21 & $10 / 28 / 94$ & 48.73 & 53.37 & -4.64 & & \\
\hline UCD1- 21 & $11 / 4 / 94$ & 48.73 & 52.75 & -4.02 & & \\
\hline UCD1- 21 & $11 / 18 / 94$ & 48.73 & 50.51 & -1.78 & & \\
\hline UCD1- 21 & $12 / 22 / 94$ & 48.73 & 48.37 & 0.36 & & \\
\hline UCD1- 21 & $1 / 6 / 95$ & 48.73 & 46.01 & 2.72 & & \\
\hline UCD1- 21 & $1 / 11 / 95$ & 48.73 & 41.67 & 7.06 & $\cdot$ & \\
\hline UCD1- 21 & $1 / 13 / 95$ & 48.73 & 41.72 & 7.01 & & \\
\hline UCD1- 21 & $1 / 27 / 95$ & 48.73 & 38.31 & 10.42 & . & \\
\hline UCD1- 21 & $2 / 10 / 95$ & 48.73 & 35.66 & 13.07 & & \\
\hline UCD1- 21 & $2 / 17 / 95$ & 48.73 & 35.16 & 13.57 & & \\
\hline UCD1- 21 & $3 / 9 / 95$ & 48.73 & 33.42 & 15.31 & & \\
\hline
\end{tabular}


Table E.1. Cumulative Groundwater. Elevation Data for HSU-1, 1990-1995

\begin{tabular}{|c|c|c|c|c|c|}
\hline WELL & DATE & $\begin{array}{c}\text { PROTECTIVE } \\
\text { CASING } \\
\text { ELEVATION } \\
\text { (feet) }\end{array}$ & $\begin{array}{l}\text { DEPTH } \\
\text {-TO } \\
\text { WATER } \\
\text { (feet) }\end{array}$ & $\begin{array}{c}\text { GROUND- } \\
\text { WATER } \\
\text { ELEVATION } \\
\text { (feet) }\end{array}$ & COMMENTS \\
\hline UCD1- 21 & $3 / 24 / 95$ & 48.73 & 27.86 & 20.87 & \\
\hline UCD1- 21 & $4 / 28 / 95$ & 48.73 & 30.30 & 18.43 & \\
\hline UCD1- 21 & $5 / 25 / 95$ & 48.73 & 30.94 & 17.79 & \\
\hline UCD1- 21 & $6 / 16 / 95$ & 48.73 & 36.91 & 11.82 & \\
\hline UCD1- 21 & $7 / 26 / 95$ & 48.73 & 43.87 & 4.86 & \\
\hline UCD1- 21 & $8 / 31 / 95$ & 48.73 & 45.33 & 3.40 & \\
\hline UCD1- 21 & $9 / 26 / 95$ & 48.73 & 42.56 & 6.17 & \\
\hline UCD1- 21 & $10 / 25 / 95$ & 48.73 & 39.51 & 9.22 & \\
\hline UCD1- 21 & $11 / 22 / 95$ & $48.77^{\bullet}$ & 37.57 & 11.20 & Regrouted - new elevation \\
\hline UCD1- 21 & $11 / 27 / 95$ & $48.77^{\bullet}$ & 38.36 & 10.41 & \\
\hline UCD1- 21 & $12 / 19 / 95$ & $48.77^{\bullet}$ & 35.19 & 13.58 & . \\
\hline UCD1- 22 & $1 / 22 / 91$ & 48.99 & 48.04 & 0.95 & . \\
\hline UCD1- 22 & $2 / 1 / 91$ & 48.99 & 47.85 & 1.14 & ${ }^{\circ}$ \\
\hline UCD1- 22 & $2 / 8 / 91$ & 48.99 & 47.64 & 1.35 & \\
\hline UCD1- 22 & $2 / 14 / 91$. & 48.99 & 47.21 & 1.78 & \\
\hline UCD1- 22 & $2 / 22 / 91$ & 48.99 & 48.36 & 0.63 & \\
\hline UCD1- 22 & $3 / 7 / 91$ & 48.99 & 47.59 & 1.40 & \\
\hline UCD1- 22 & $3 / 12 / 91$ & 48.99 & 46.96 & 2.03 & \\
\hline UCD1- 22 & $3 / 22 / 91$ & 48.99 & 43.44 & 5.55 & \\
\hline UCD1- 22 & $3 / 29 / 91$ & 48.99 & 43.54 & 5.45 & \\
\hline UCD1- 22 & $4 / 3 / 91$ & 48.99 & 43.14 & 5.85 & \\
\hline UCD1- 22 & $4 / 10 / 91$ & 48.99 & 42.63 & 6.36 & \\
\hline UCD1- 22 & $4 / 17 / 91$ & 48.99 & 42.85 & 6.14 & \\
\hline UCD1- 22 & $4 / 24 / 91$ & 48.99 & 45.28 & 3.71 & \\
\hline UCD1- 22 & $5 / 3 / 91$ & 48.99 & 47.61 & 1.38 & \\
\hline UCD1- 22 & $5 / 13 / 91$ & 48.99 & 49.73 & -0.74 & \\
\hline UCD1- 22 & $5 / 20 / 91$ & 48.99 & 53.47 & -4.48 & \\
\hline UCD1- 22 & $5 / 29 / 91$ & 48.99 & 54.52 & -5.53 & \\
\hline UCD1- 22 & $6 / 6 / 91$ & 48.99 & 59.88 & -10.89 & \\
\hline UCD1- 22 & $6 / 10 / 91$ & 48.99 & 58.53 & -9.54 & \\
\hline UCD1- 22 & $6 / 21 / 91$ & 48.99 & 60.88 & -11.89 & \\
\hline UCD1- 22 & $6 / 27 / 91$ & 48.99 & 61.85 & -12.86 & - \\
\hline UCD1- 22 & $7 / 5 / 91$ & 48.99 & 62.36 & -13.37 & \\
\hline UCD1- 22 & $8 / 5 / 91$ & 48.99 & 66.99 & -18.00 & \\
\hline UCD1- 22 & $8 / 14 / 91$ & 48.99 & 67.73 & -18.74 & \\
\hline UCD1- 22 & $8 / 21 / 91$ & 48.99 & 65.73 & -16.74 & \\
\hline UCD1- 22 & $8 / 29 / 91$ & 48.99 & 63.79 & -14.80 & \\
\hline UCD1- 22 & $9 / 4 / 91$ & 48.99 & 62.13 & -13.14 & \\
\hline UCD1- 22 & $9 / 13 / 91$ & 48.99 & 62.59 & -13.60 & \\
\hline UCD 1- 22 & $10 / 3 / 91$ & 48.99 & 58.11 & -9.12 & . \\
\hline UCD1- 22 & $10 / 10 / 91$ & 48.99 & 58.17 & -9.18 & . \\
\hline UCD1- 22 & $10 / 25 / 91$ & 48.99 & 56.83 & -7.84 & \\
\hline UCD1- 22 & $11 / 1 / 91$ & 48.99 & 53.00 & -4.01 & \\
\hline UCD1- 22 & $11 / 8 / 91$ & 48.99 & 54.86 & -5.87 & ? \\
\hline UCD1- 22 & $11 / 14 / 91$ & 48.99 & 55.83 & -6.84 & \\
\hline UCD1- 22 & $12 / 5 / 91$ & 48.99 & 54.70 & -5.71 & \\
\hline UCD 1- 22 & $12 / 11 / 91$ & 48.99 & 55.67 & -6.68 & \\
\hline UCD1- 22 & $4 / 24 / 92$ & 48.99 & 45.01 & 3.98 & \\
\hline UCD1- 22 & $5 / 1 / 92$ & 48.99 & 48.08 & 0.91 & \\
\hline UCD1- 22 & $5 / 8 / 92$ & 48.99 & 50.54 & -1.55 & \\
\hline UCD1- 22 & $5 / 26 / 92$ & 48.99 & 54.72 & -5.73 & \\
\hline UCD1- 22 & $6 / 2 / 92$ & 48.99 & 58.61 & -9.62 & \\
\hline UCD1- 22 & $6 / 17 / 92$ & 48.99 & 59.00 & -10.01 & \\
\hline UCD1- 22 & $6 / 24 / 92$ & 48.99 & 60.60 & -11.61 & \\
\hline UCD1- 22 & 7/2/92 & 48.99 & 64.85 & -15.86 & \\
\hline UCD1- 22 & $7 / 8 / 92$ & 48.99 & 61.32 & -12.33 & \\
\hline
\end{tabular}


Table E.1. Cumulative Groundwater Elevation Data for HSU-1, 1990-1995

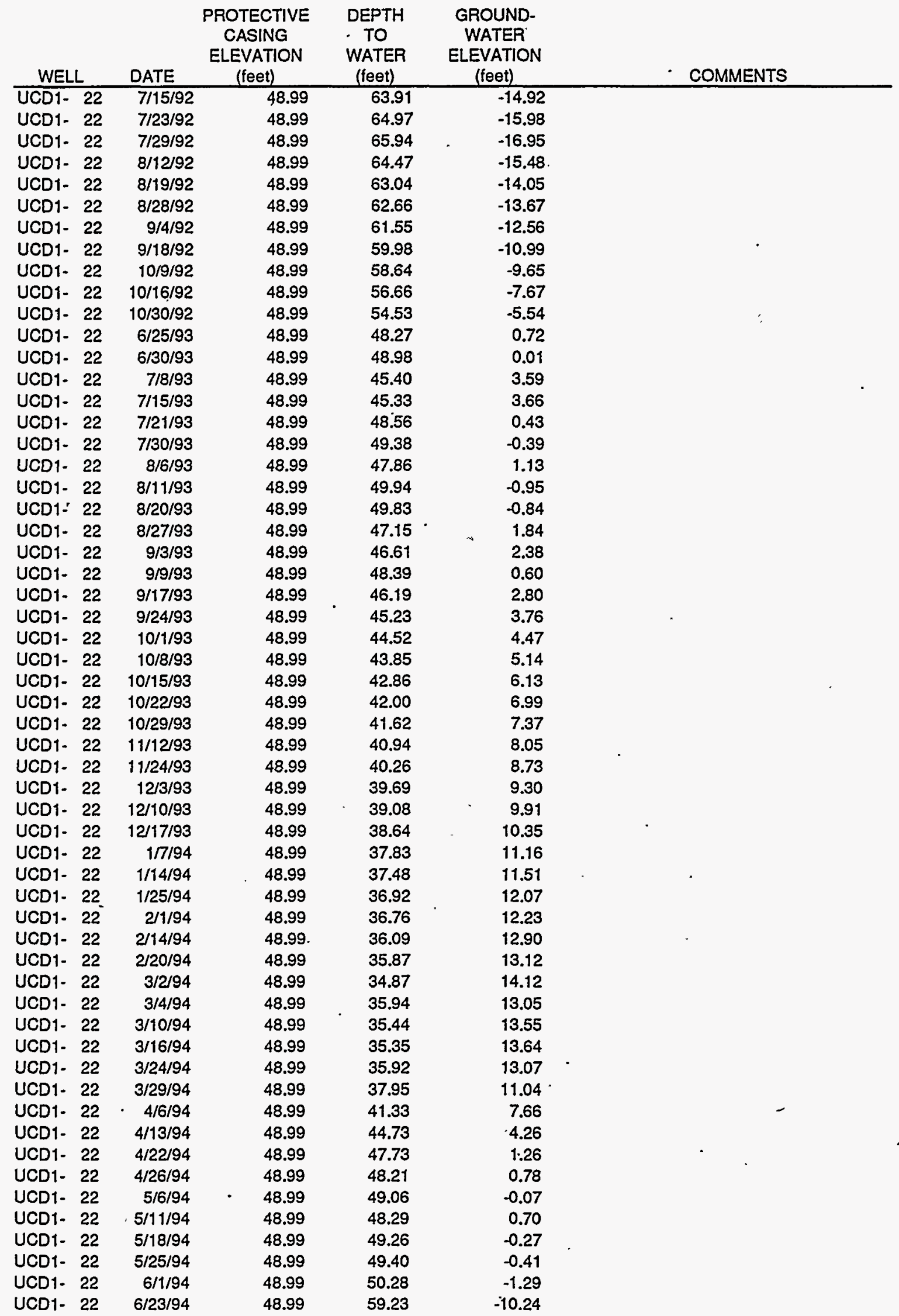


Table E.1. Cumulative Groundwater Elevation Data for HSU-1, 1990-1995

\begin{tabular}{|c|c|c|c|c|c|}
\hline WELL & DATE & $\begin{array}{c}\text { PROTECTIVE } \\
\text { CASING } \\
\text { ELEVATION } \\
\text { (feet) }\end{array}$ & $\begin{array}{l}\text { DEPTH } \\
\text { TO } \\
\text { WATER } \\
\text { (feet) }\end{array}$ & $\begin{array}{c}\text { GROUND- } \\
\text { WATER } \\
\text { ELEVATION } \\
\text { (feet) }\end{array}$ & COMMENTS \\
\hline UCD1- 22 & $7 / 1 / 94$ & 48.99 & 61.51 & -12.52 & \\
\hline UCD1- 22 & $7 / 8 / 94$ & 48.99 & 63.55 & -14.56 & \\
\hline UCD1- 22 & $7 / 14 / 94$ & 48.99 & 64.55 & -15.56 & \\
\hline UCD1- 22 & $7 / 20 / 94$ & 48.99 & 66.46 & -17.47 & \\
\hline UCD1- 22 & $7 / 26 / 94$ & 48.99 & 65.86 & -16.87 & \\
\hline UCD1- 22 & $8 / 5 / 94$ & 48.99 & 65.00 & -16.01 & \\
\hline UCD1- 22 & $8 / 19 / 94$ & 48.99 & 67.78 & -18.79 & \\
\hline UCD1- 22 & $9 / 7 / 94$ & 48.99 & 63.70 & -14.71 & \\
\hline UCD1- 22 & $9 / 7 / 94$ & 48.99 & 64.10 & -15.11 & \\
\hline UCD1- 22 & $9 / 13 / 94$ & 48.99 & 64.47 & -15.48 & \\
\hline UCD1- 22 & $9 / 19 / 94$ & 48.99 & 61.49 & -12.50 & \\
\hline UCD1- 22 & $9 / 26 / 94$ & 48.99 & 59.88 & -10.89 & \\
\hline UCD1- 22 & $10 / 7 / 94$ & 48.99 & 57.17 & -8.18 & \\
\hline UCD1- 22 & $10 / 14 / 94$ & 48.99 & 55.60 & -6.61 & \\
\hline UCD1- 22 & $10 / 21 / 94$ & 48.99 & 54.41 & -5.42 & \\
\hline UCD1- 22 & $10 / 28 / 94$ & 48.99 & 53.56 & -4.57 & \\
\hline UCD1- 22 & $11 / 4 / 94$ & 48.99 & 52.96 & -3.97 & \\
\hline UCD1- 22 & $12 / 22 / 94$ & 48.99 & 48.21 & 0.78 & \\
\hline UCD1- 22 & $1 / 6 / 95$ & 48.99 & 48.50 & 0.49 & \\
\hline UCD1- 22 & $1 / 11 / 95$ & 48.99 & 43.50 & 5.49 & \\
\hline UCD1- 22 & $1 / 13 / 95$ & 48.99 & 42.89 & 6.10 & \\
\hline UCD1- 22 & $1 / 27 / 95$ & 48.99 & 40.52 & 8.47 & \\
\hline UCD1- 22 & $2 / 10 / 95$ & 48.99 & 36.09 & 12.90 & \\
\hline UCD1- 22 & $2 / 17 / 95$ & 48.99 & 35.47 & 13.52 & \\
\hline UCD1- 22 & $3 / 9 / 95$ & 48.99 & 35.61 & 13.38 & • \\
\hline UCD1- 22 & $3 / 24 / 95$ & 48.99 & 28.58 & 20.41 & \\
\hline UCD1- 22 & $4 / 28 / 95$ & 48.99 & 30.78 & 18.21 & \\
\hline UCD1- 22 & $5 / 25 / 95$ & 48.99 & 31.17 & 17.82 & \\
\hline UCD1- 22 & $6 / 16 / 95$ & 48.99 & 35.75 & 13.24 & \\
\hline UCD1- 22 & $7 / 26 / 95$ & 48.99 & 43.82 & 5.17 & \\
\hline UCD1- 22 & $8 / 31 / 95$ & 48.99 & 45.20 & 3.79 & \\
\hline UCD1- 22 & $9 / 26 / 95$ & 48.99 & 42.46 & 6.53 & \\
\hline UCD1- 22 & $10 / 25 / 95$ & 48.99 & 39.49 & 9.50 & \\
\hline UCD1- 22 & $11 / 22 / 95$ & $49.06^{\circ}$ & 37.64 & 11.42 & Regrouted - new elevation \\
\hline UCD1- 22 & $11 / 27 / 95$ & $49.06^{\circ}$ & 37.28 & 11.78 & \\
\hline UCD1- 22 & $12 / 19 / 95$ & $49.06^{\circ}$ & 35.13 & 13.93 & \\
\hline UCD1- 23 & $1 / 22 / 91$ & 49.29 & 49.19 & 0.10 & \\
\hline UCD1- 23 & 2/1/91 & 49.29 & 48.59 & 0.70 & \\
\hline UCD1- 23 & $2 / 8 / 91$ & 49.29 & 48.38 & 0.91 & \\
\hline UCD1- 23 & $2 / 14 / 91$ & 49.29 & 47.94 & 1.35 & , \\
\hline UCD1- 23 & $2 / 22 / 91$ & 49.29 & 48.24 & 1.05 & \\
\hline UCD1- 23 & $3 / 7 / 91$ & 49.29 & 48.08 & 1.21 & \\
\hline UCD1- 23 & $3 / 12 / 91$ & 49.29 & 47.56 & 1.73 & \\
\hline UCD1- 23 & $3 / 29 / 91$ & 49.29 & 44.31 & 4.98 & \\
\hline UCD1- 23 & $4 / 3 / 91$ & 49.29 & 42.39 & 6.90 & \\
\hline UCD1- 23 & $4 / 10 / 91$ & 49.29 & 41.74 & 7.55 & \\
\hline UCD1- 23 & $4 / 17 / 91$ & 49.29 & 41.98 & 7.31 & 一 \\
\hline UCD1- 23 & $4 / 24 / 91$ & 49.29 & 43.76 & 5.53 & \\
\hline UCD1- 23 & $5 / 3 / 91$ & 49.29 & 46.10 & 3.19 & \\
\hline UCD1- 23 & $5 / 13 / 91$ & 49.29 & 47.64 & 1.65 & \\
\hline UCD1- 23 & $5 / 29 / 91$ & 49.29 & 50.96 & -1.67 & \\
\hline UCD1- 23 & $6 / 6 / 91$ & 49.29 & 53.31 & -4.02 & \\
\hline UCD1- 23 & $6 / 10 / 91$ & 49.29 & 54.71 & -5.42 & \\
\hline UCD1- 23 & $6 / 21 / 91$ & 49.29 & 60.10 & -10.81 & \\
\hline UCD1- 23 & $6 / 27 / 91$ & 49.29 & 61.66 & -12.37 & \\
\hline UCD1- 23 & $7 / 5 / 91$ & 49.29 & 62.00 & -12.71 & \\
\hline
\end{tabular}


Table E.1. Cumulative Groundwater Elevation Data for HSU-1, 1990-1995

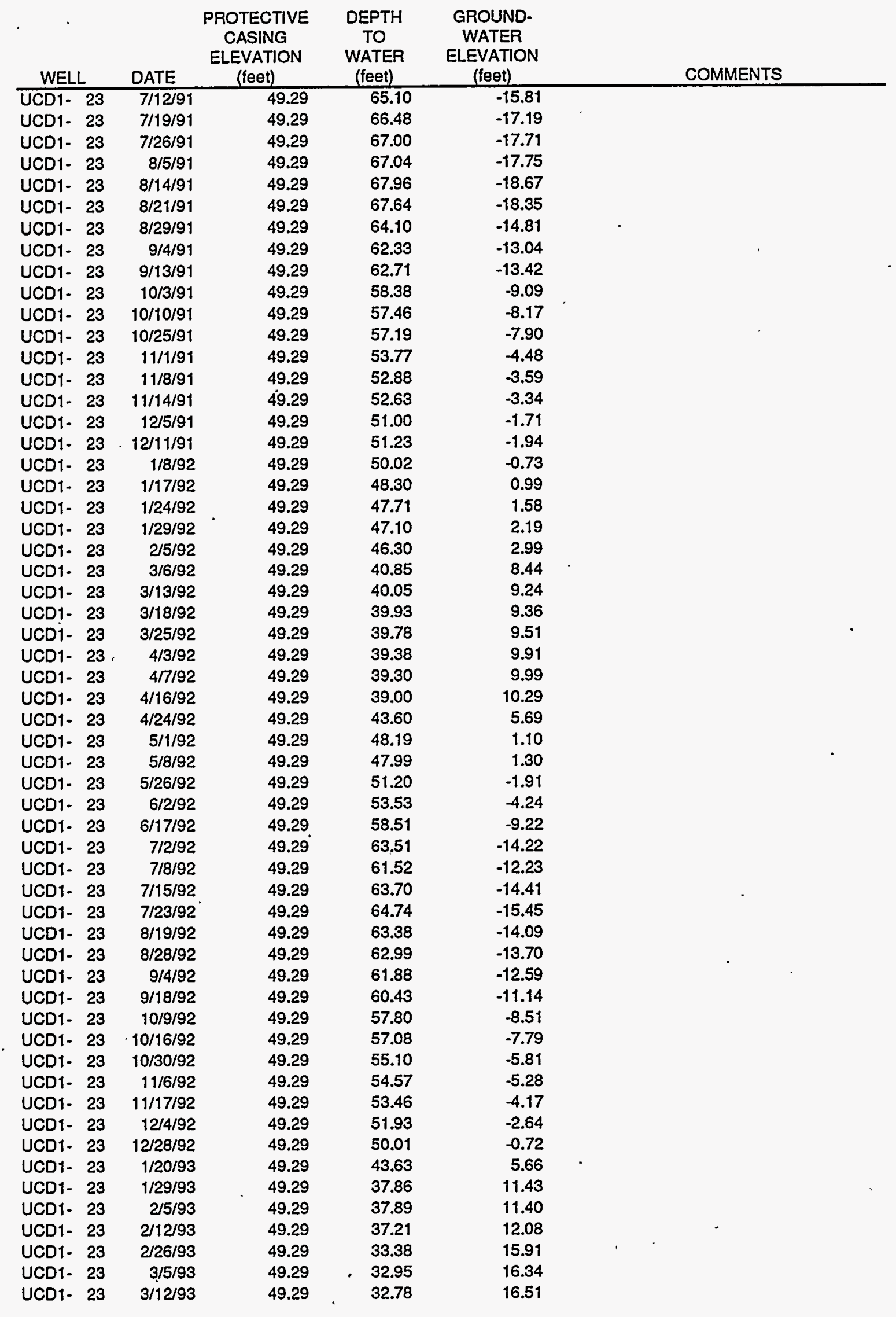


Table E.1. Cumulative Groundwater Elevation Data for HSU-1, 1990-1995

\begin{tabular}{|c|c|c|c|c|c|}
\hline WELL & DATE & $\begin{array}{c}\text { PROTECTIVE } \\
\text { CASING } \\
\text { ELEVATION } \\
\text { (feet) }\end{array}$ & $\begin{array}{l}\text { DEPTH } \\
\text { TO } \\
\text { WATER } \\
\text { (feet) } \\
\end{array}$ & $\begin{array}{c}\text { GROUND- } \\
\text { WATER } \\
\text { ELEVATION } \\
\text { (feet) }\end{array}$ & COMMENTS \\
\hline UCD1- 23 & $3 / 19 / 93$ & 49.29 & 32.61 & 16.68 & \\
\hline UCD1- 23 & $4 / 7 / 93$ & 49.29 & 31.47 & 17.82 & \\
\hline UCD1- 23 & $4 / 16 / 93$ & 49.29 & 31.27 & 18.02 & \\
\hline UCD1- 23 & $4 / 23 / 93$ & 49.29 & 31.47 & 17.82 & \\
\hline UCD1- 23 & $5 / 7 / 93$ & 49.29 & 37.15 & 12.14 & \\
\hline UCD1- 23 & $6 / 24 / 93$ & 49.29 & 41.38 & 7.91 & \\
\hline UCD1- 23 & $6 / 30 / 93$ & 49.29 & 45.38 & 3.91 & \\
\hline UCD1- 23 & $7 / 8 / 93$ & 49.29 & 44.44 & 4.85 & \\
\hline UCD1- 23 & $7 / 15 / 93$ & 49.29 & 45.44 & 3.85 & \\
\hline UCD1- 23 & $7 / 21 / 93$ & 49.29 & 47.58 & 1.71 & \\
\hline UCD1- 23 & $7 / 30 / 93$ & 49.29 & 46.33 & 2.96 & \\
\hline UCD1- 23 & $8 / 6 / 93$ & 49.29 & 49.86 & -0.57 & \\
\hline UCD1- 23 & $8 / 11 / 93$ & 49.29 & 47.02 & 2.27 & \\
\hline UCD1- 23 & $8 / 20 / 93$ & 49.29 & 46.93 & 2.36 & \\
\hline UCD1- 23 & $8 / 27 / 93$ & 49.29 & 46.83 & 2.46 & \\
\hline UCD1- 23 & $9 / 3 / 93$ & 49.29 & 46.42 & 2.87 & \\
\hline UCD1- 23 & 9/9/93 & 49.29 & 48.10 & 1.19 & \\
\hline UCD1- 23 & $9 / 17 / 93$ & 49.29 & 46.06 & 3.23 & \\
\hline UCD1- 23 & $9 / 24 / 93$ & 49.29 & 45.42 & 3.87 & \\
\hline UCD1- 23 & $10 / 1 / 93$ & 49.29 & 44.80 & 4.49 & \\
\hline UCD1- 23 & $10 / 8 / 93$ & 49.29 & $44.08^{\circ}$ & 5.21 & \\
\hline UCD1- 23 & $10 / 15 / 93$ & 49.29 & 43.00 & 6.29 & \\
\hline UCD1- 23 & $10 / 22 / 93$ & 49.29 & 42.00 & 7.29 & \\
\hline UCD1- 23 & $10 / 29 / 93$ & 49.29 & 41.45 & 7.84 & \\
\hline UCD1- 23 & $11 / 12 / 93$ & 49.29 & 40.43 & 8.86 & \\
\hline UCD1- 23 & $11 / 24 / 93$ & 49.29 & 40.24 & 9.05 & \\
\hline UCD1- 23 & $12 / 3 / 93$ & 49.29 & 39.46 & 9.83 & \\
\hline UCD1- 23 & $12 / 10 / 93$ & 49.29 & 39.01 & 10.28 & \\
\hline UCD1- 23 & $12 / 17 / 93$ & 49.29 & 38.58 & 10.71 & \\
\hline UCD1- 23 & $1 / 7 / 94$ & 49.29 & 37.76 & 11.53 & $\cdot$ \\
\hline UCD1- 23 & $1 / 14 / 94$ & 49.29 & 37.35 & 11.94 & \\
\hline UCD1- 23 & $1 / 25 / 94$ & 49.29 & 36.90 & 12.39 & \\
\hline UCD1- 23 & $2 / 1 / 94$ & 49.29 & 36.64 & 12.65 & \\
\hline UCD1- 23 & $2 / 14 / 94$ & 49.29 & 35.97 & 13.32 & \\
\hline UCD1- 23 & $2 / 20 / 94$ & 49.29 & 35.83 & 13.46 & \\
\hline UCD1- 23 & $3 / 2 / 94$ & 49.29 & 34.65 & 14.64 & purged $3 / 2 / 94$ \\
\hline UCD1- 23 & $3 / 4 / 94$ & 49.29 & 35.23 & 14.06 & \\
\hline UCD1- 23 & $3 / 10 / 94$ & 49.29 & 35.29 & 14.00 & \\
\hline UCD1- 23 & $3 / 16 / 94$ & 49.29 & 35.17 & 14.12 & \\
\hline UCD1- 23 & $3 / 24 / 94$ & 49.29 & 35.68 & 13.61 & \\
\hline UCD1- 23 & $3 / 29 / 94$ & 49.29 & 37.76 & 11.53 & $\cdot$ \\
\hline UCD1- 23 & $4 / 6 / 94$ & 49.29 & 40.04 & 9.25 & \\
\hline UCD1- 23 & $4 / 13 / 94$ & 49.29 & 43.77 & 5.52 & \\
\hline UCD1- 23 & $4 / 22 / 94$ & 49.29 & 46.07 & 3.22 & \\
\hline UCD1- 23 & $4 / 26 / 94$ & 49.29 & $47: 19$ & 2.10 & \\
\hline UCD1- 23 & $5 / 6 / 94$ & 49.29 & 48.01 & 1.28 & \\
\hline UCD1- 23 & $5 / 11 / 94$ & 49.29 & 47.79 & 1.50 & \\
\hline UCD1- 23 & $5 / 18 / 94$ & 49.29 & 48.38 & 0.91 & \\
\hline UCD1- 23 & $5 / 25 / 94$ & 49.29 & 48.69 & 0.60 & \\
\hline UCD1- 23 & $6 / 1 / 94$ & 49.29 & 49.13 & 0.16 & \\
\hline UCD1- 23 & $6 / 23 / 94$ & 49.29 & 54.84 & -5.55 & \\
\hline UCD1- 23 & $7 / 1 / 94$ & 49.29 & 60.39 & -11.10 & unclear meter respons $\theta$ \\
\hline UCD1- 23 & $7 / 8 / 94$ & 49.29 & 63.07 & -13.78 & unclear meter response \\
\hline UCD1- 23 & $7 / 14 / 94$ & 49.29 & 64.21 & -14.92 & \\
\hline UCD1- 23 & $7 / 20 / 94$ & 49.29 & 66.11 & -16.82 & \\
\hline UCD1- 23 & $7 / 26 / 94$ & 49.29 & 65.78 & -16.49 & \\
\hline UCD1- 23 & $8 / 5 / 94$ & 49.29 & 65.20 & -15.91 & \\
\hline
\end{tabular}


Table E.1. Cumulative Groundwater Elevation Data for HSU-1, 1990-1995

\begin{tabular}{|c|c|c|c|c|c|c|}
\hline WEL & - & DATE & $\begin{array}{c}\text { PROTECTIVE } \\
\text { CASING } \\
\text { ELEVATION } \\
\text { (feet) }\end{array}$ & $\begin{array}{l}\text { DEPTH } \\
\text { TO } \\
\text { WATER } \\
\text { (feet) }\end{array}$ & $\begin{array}{c}\text { GROUND- } \\
\text { WATER } \\
\text { ELEVATION } \\
\text { (feet) }\end{array}$ & COMMENTS \\
\hline UCD1- & 23 & $8 / 19 / 94$ & 49.29 & 67.70 & -18.41 & \\
\hline UCD1- & 23 & $9 / 7 / 94$ & 49.29 & 64.07 & -14.78 & \\
\hline UCD1- & 23 & $9 / 7 / 94$ & 49.29 & 64.38 & -15.09 & \\
\hline UCD1- & 23 & $9 / 13 / 94$ & 49.29 & 65.07 & -15.78 & \\
\hline UCD1- & 23 & $9 / 19 / 94$ & 49.29 & 61.98 & -12.69 & \\
\hline UCD1- & 23 & $9 / 26 / 94$ & 49.29 & 60.47 & -11.18 & \\
\hline UCD1- & 23 & $10 / 7 / 94$ & 49.29 & 57.96 & -8.67 & \\
\hline UCD1. & 23 & $10 / 14 / 94$ & 49.29 & 56.29 & -7.00 & • \\
\hline UCD1- & 23 & $10 / 21 / 94$ & 49.29 & 55.02 & -5.73 & \\
\hline UCD1- & 23 & $10 / 28 / 94$ & 49.29 & 54.21 & -4.92 & \\
\hline UCD1. & 23 & $11 / 4 / 94$ & 49.29 & 53.66 & -4.37 & \\
\hline UCD1- & 23 & $11 / 18 / 94$ & 49.29 & 52.02 & -2.73 & \\
\hline UCD1- & 23 & $12 / 22 / 94$ & 49.29 & 49.56 & -0.27 & \\
\hline UCD1- & 23 & $1 / 6 / 95$ & 49.29 & 48.14 & 1.15 & \\
\hline UCD1- & 23 & $1 / 11 / 95$ & 49.29 & 46.65 & 2.64 & . \\
\hline UCD1- & 23 & $1 / 13 / 95$ & 49.29 & 45.03 & 4.26 & \\
\hline UCD1- & 23 & $1 / 27 / 95$ & 49.29 & 37.23 & 12.06 & \\
\hline UCD 1- & 23 & $2 / 10 / 95$ & 49.29 & 34.57 & 14.72 & \\
\hline UCD1- & 23 & $2 / 17 / 95$ & 49.29 & 34.13 & 15.16 & \\
\hline UCD1- & 23 & $3 / 9 / 95$ & 49.29 & 32.92 & 16.37 & \\
\hline UCD1. & 23 & $3 / 24 / 95$ & 49.29 & 27.60 & 21.69 & \\
\hline UCD1- & 23 & $4 / 28 / 95$ & 49.29 & 30.07 & 19.22 & \\
\hline UCD1- & 23 & $5 / 25 / 95$ & 49.29 & 30.57 & 18.72 & . \\
\hline UCD1- & 23 & $6 / 16 / 95$ & 49.29 & 35.44 & 13.85 & \\
\hline UCD1- & 23 & $7 / 26 / 95$ & 49.29 & 43.41 & 5.88 & Cap Loose in well. Replaced. \\
\hline UCD1. & 23 & $8 / 31 / 95$ & 49.29 & 45.24 & 4.05 & \\
\hline UCD1. & 23 & $9 / 26 / 95$ & 49.29 & 42.52 & 6.77 & \\
\hline UCD1. & 23 & $10 / 25 / 95$ & 49.29 & 39.58 & 9.71 & \\
\hline UCD1- & 23 & $11 / 22 / 95$ & 49.39 • & 37.71 & 11.68 & Regrouted - new elevation \\
\hline UCD1- & 23 & $11 / 27 / 95$ & $49.39^{\circ}$ & 37.46 & 11.93 & \\
\hline UCD1- & 23 & $12 / 19 / 95$ & $49.399^{*}$ & 34.71 & 14.68 & \\
\hline UCD1. & 24 & $1 / 22 / 91$ & 48.84 & 47.78 & 1.06 & \\
\hline UCD1- & 24 & $2 / 1 / 91$ & 48.84 & 47.13 & 1.71 & \\
\hline UCD1- & 24 & $2 / 8 / 91$ & 48.84 & 47.69 & 1.15 & \\
\hline UCD1- & 24 & $2 / 14 / 91$ & 48.84 & 47.39 & 1.45 & \\
\hline UCDI. & 24 & $2 / 22 / 91$ & 48.84 & 48.96 & -0.12 & \\
\hline UCD1- & 24 & $3 / 7 / 91$ & 48.84 & 47.79 & 1.05 & \\
\hline UCD1- & 24 & $3 / 12 / 91$ & 48.84 & 46.96 & 1.88 & \\
\hline UCD1. & 24 & $4 / 3 / 91$ & 48.84 & 42.88 & 5.96 & \\
\hline UCD1- & 24 & $4 / 10 / 91$ & 48.84 & 42.34 & 6.50 & . \\
\hline UCD1- & 24 & $4 / 17 / 91$ & 48.84 & 42.79 & 6.05 & \\
\hline UCD1- & 24 & $4 / 24 / 91$ & 48.84 & 45.44 & 3.40 & \\
\hline UCD1- & 24 & $5 / 3 / 91$ & 48.84 & 47.64 & 1.20 & \\
\hline UCD1- & 24 & $5 / 13 / 91$ & 48,84 & 51.27 & -2.43 & . \\
\hline UCD1. & 24 & $5 / 20 / 91$ & 48.84 & 54.53 & -5.69 & \\
\hline UCO1- & 24 & $5 / 29 / 91$ & 48.84 & 55.38 & -6.54 & \\
\hline UCD1- & 24 & $6 / 6 / 91$ & 48.84 & 58.76 & -9.92 & . \\
\hline UCD1- & 24 & $6 / 10 / 91$ & 48.84 & 59.21 & -10.37 & . \\
\hline UCD1. & 24 & $6 / 21 / 91$ & 48.84 & 61.10 & -12.26 & \\
\hline UCD1- & 24 & $6 / 27 / 91$ & 48.84 & 62.01 & -13.17 & \\
\hline UCD1- & 24 & $7 / 5 / 91$ & 48.84 & 62.78 & -13.94 & \\
\hline UCD1. & 24 & $7 / 12 / 91$ & 48.84 & 65.10 & -16.26 & \\
\hline UCD1. & 24 & $7 / 19 / 91$ & 48.84 & 67.56 & -18.72 & \\
\hline UCD1- . & 24 & $7 / 26 / 91$ & 48.84 & 67.35 & -18.51 & \\
\hline UCD1- & 24 & $8 / 5 / 91$ & 48.84 & 67.60 & -18.76 & \\
\hline UCD1- & 24 & $8 / 14 / 91$ & 48.84 & 68.46 & -19.62 & \\
\hline
\end{tabular}


Table E.1. Cumulative Groundwater Elevation Data for HSU-1, 1990-1995

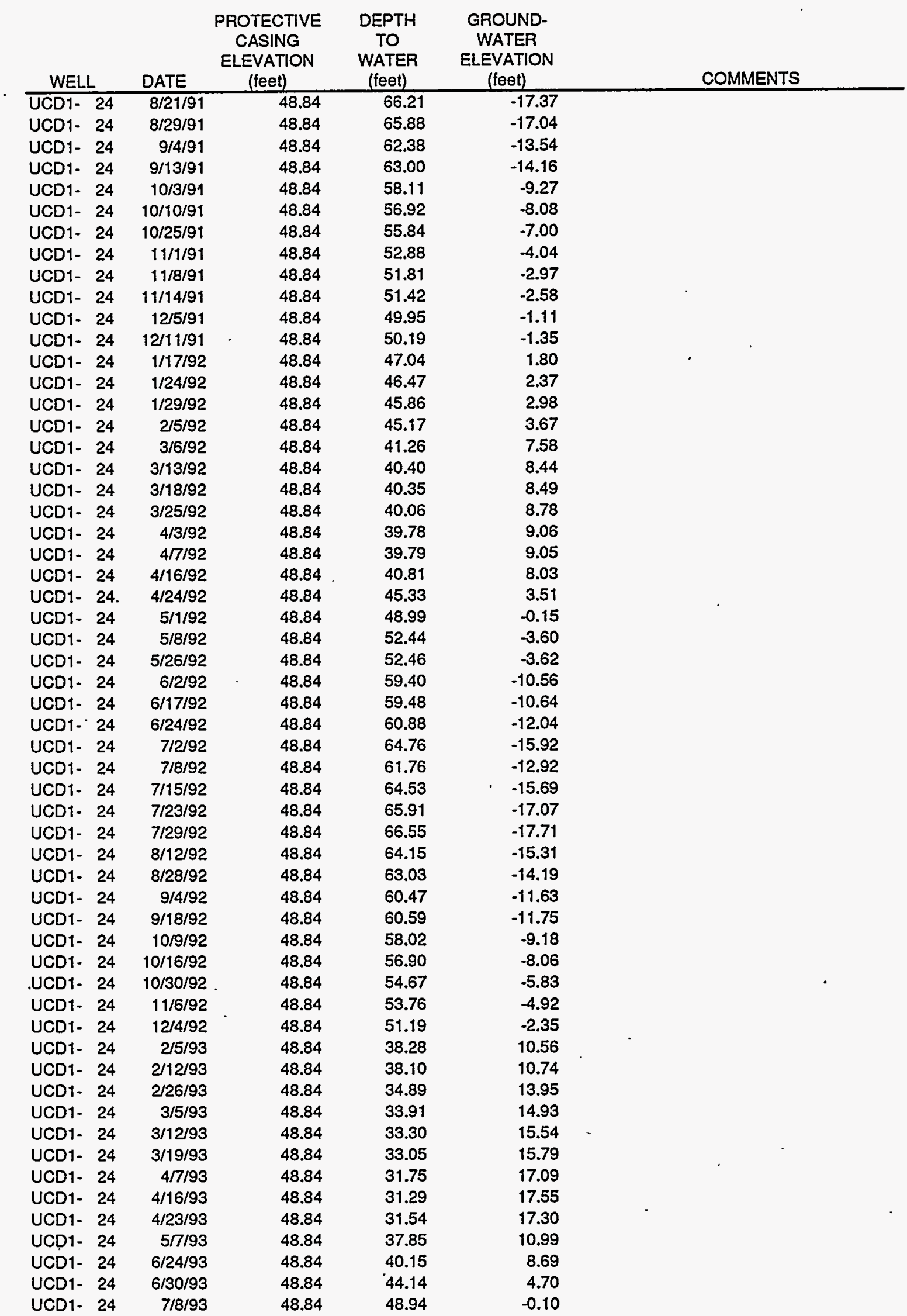


Table E.1. Cumulative Groundwater Elevation Data for HSU-1, 1990-1995

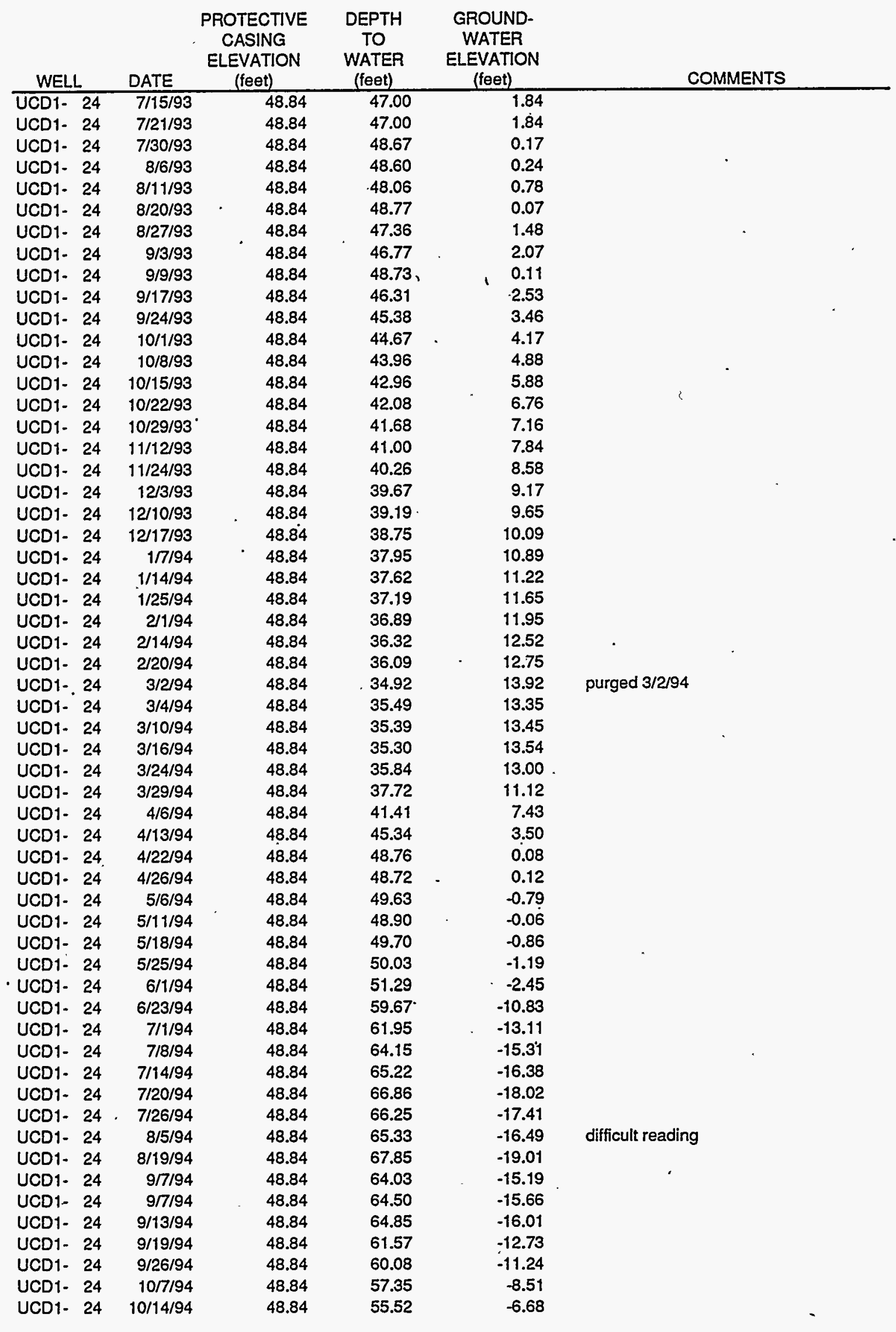


Table E.1. Cumulative Groundwater Elevation Data for HSU-1, 1990-1995

\begin{tabular}{|c|c|c|c|c|c|}
\hline WELL & DATE & $\begin{array}{l}\text { PROTECTIVE } \\
\text { CASING } \\
\text { ELEVATION } \\
\text { (feet) }\end{array}$ & $\begin{array}{c}\text { DEPTH } \\
\text { TO } \\
\text { WATER } \\
\text { (feet) }\end{array}$ & $\begin{array}{l}\text { GROUND- } \\
\text { WATER } \\
\text { ELEVATION } \\
\text { (feet) }\end{array}$ & COMMENTS \\
\hline UCD1- 24 & $10 / 21 / 94$ & 48.84 & 54.47 & -5.63 & \\
\hline UCD1- 24 & $10 / 28 / 94$ & 48.84 & 53.58 & -4.74 & \\
\hline UCD1- 24 & $11 / 4 / 94$ & 48.84 & 52.95 & -4.11 & \\
\hline UCD1- 24 & $11 / 18 / 94$ & 48.84 & 50.42 & -1.58 & \\
\hline UCD1- 24 & $12 / 22 / 94$ & 48.84 & 47.94 & 0.90 & \\
\hline UCD1- 24 & $1 / 6 / 95$ & 48.84 & 48.31 & 0.53 & \\
\hline UCD1- 24 & $1 / 11 / 95$ & 48.84 & 42.02 & 6.82 & \\
\hline UCD1- 24 & $1 / 13 / 95$ & 48.84 & 41.48 & 7.36 & \\
\hline UCD1- 24 & $1 / 27 / 95$ & 48.84 & 38.45 & 10.39 & \\
\hline UCD1- 24 & $2 / 10 / 95$ & 48.84 & 35.58 & $13.26^{l}$ & \\
\hline UCD1- 24 & $2 / 17 / 95$ & 48.84 & 35.02 & 13.82 & \\
\hline UCD1- 24 & $3 / 9 / 95$ & 48.84 & 33.62 & 15.22 & \\
\hline UCD1- 24 & $3 / 16 / 95$ & 48.84 & 30.14 & 18.70 & . \\
\hline UCD1- 24 & $3 / 24 / 95$ & 48.84 & 27.91 & 20.93 & \\
\hline UCD1- 24 & $4 / 28 / 95$ & 48.84 & 30.22 & 18.62 & \\
\hline UCD1- 24 & $5 / 25 / 95$ & 48.84 & 30.91 & 17.93 & \\
\hline UCD1- 24 & $6 / 16 / 95$ & 48.84 & 35.78 & 13.06 & \\
\hline UCD1- 24 & $7 / 26 / 95$ & 48.84 & 44.50 & 4.34 & \\
\hline UCD1- 24 & $8 / 31 / 95$ & 48.84 & 45.50 & 3.34 & \\
\hline UCD1- 24 & 9/26/95 & 48.84 & 42.70 & 6.14 & \\
\hline UCD1- 24 & $10 / 25 / 95$ & 48.84 & 39.68 & 9.16 & \\
\hline UCD1- 24 & $11 / 22 / 95$ & $48.91^{\circ}$ & 37.83 & 11.08 & Regrouted - new elevation \\
\hline UCD1- 24 & $11 / 27 / 95$ & $48.91^{\circ}$ & 37.58 & 11.33 & \\
\hline UCD1- 24 & $12 / 19 / 95$ & 48.91 & 35.55 & 13.36 & \\
\hline UCD1- 25 & $11 / 27 / 95$ & 48.80 & 38.30 & 10.50 & \\
\hline UCD1- 25 & $12 / 19 / 95$ & 48.80 & 35.88 & 12.92 & \\
\hline UCD1- 34 & $11 / 27 / 95$ & 55.70 & 43.72 & 11.98 & \\
\hline UCD1- 34 & $12 / 19 / 95$ & 55.70 & 40.36 & 15.34 & \\
\hline
\end{tabular}


Table E.2. Cumulative Groundwater Elevation Data for HSU-2, 1990-1995

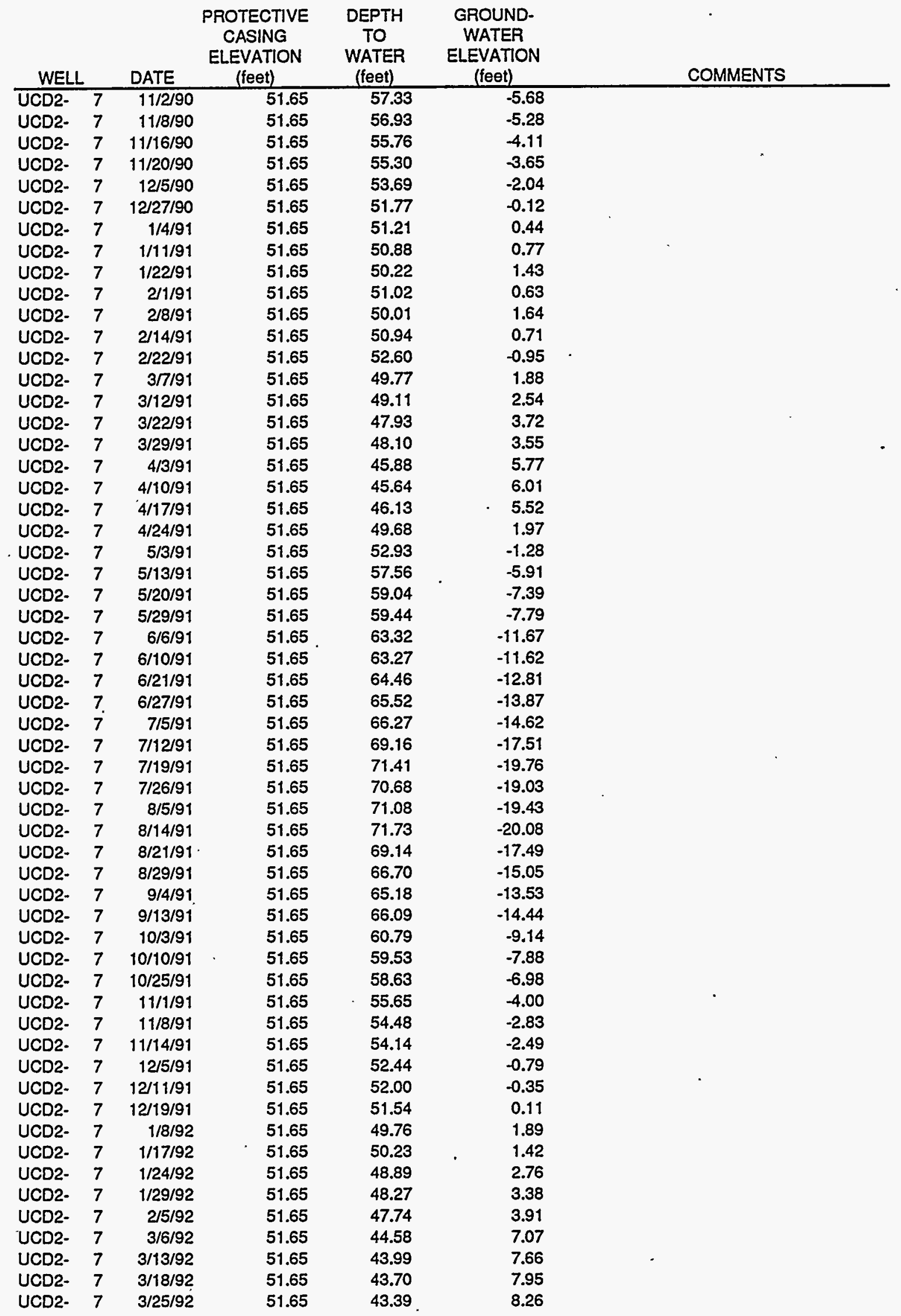


Table E.2. Cumulative Groundwater Elevation Data for HSU-2, 1990-1995

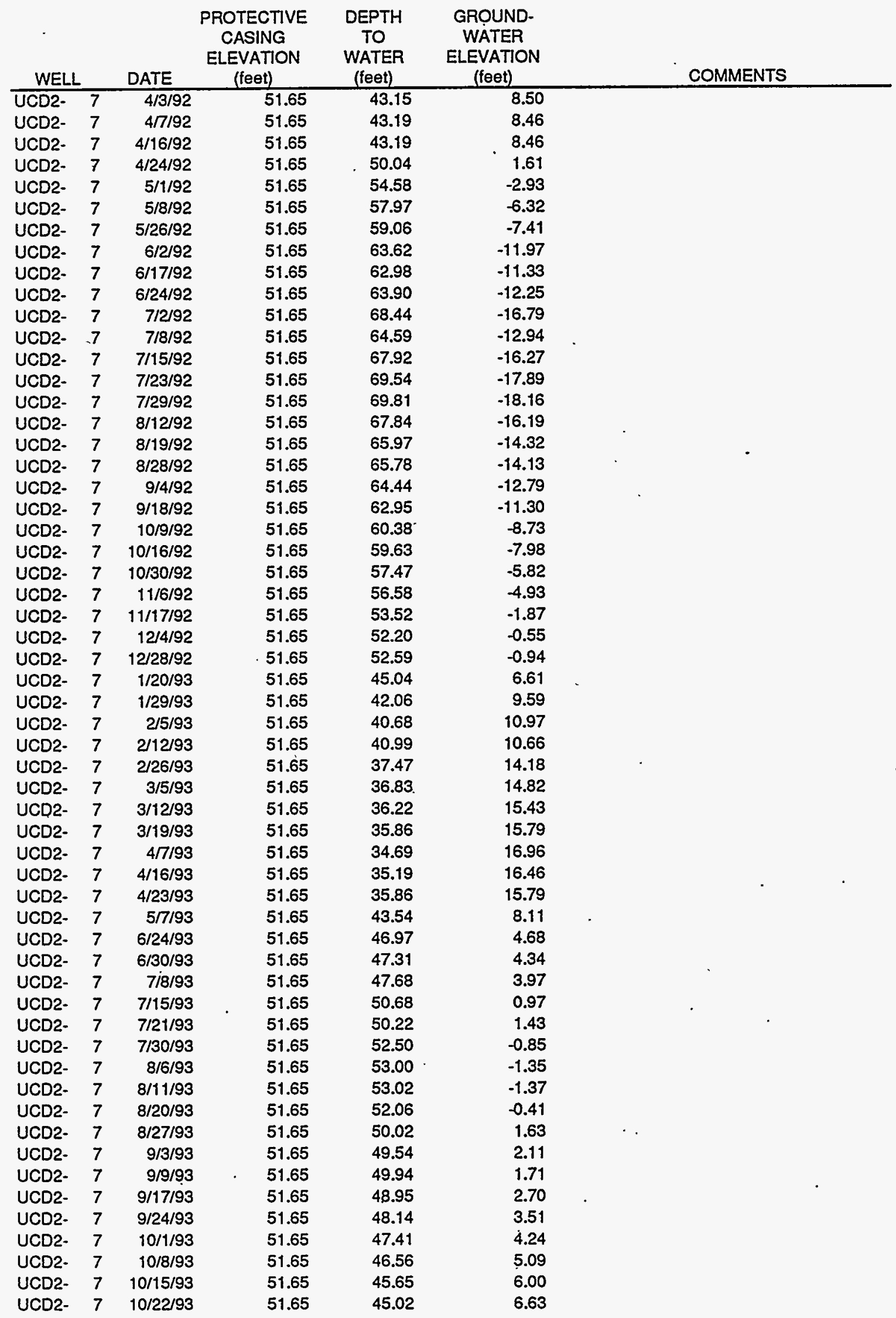


Table E.2. Cumulative Groundwater Elevation Data for HSU-2, 1990-1995

\begin{tabular}{|c|c|c|c|c|c|c|c|c|}
\hline WELL & & DATE & $\begin{array}{c}\text { PROTECTIVE } \\
\text { CASING } \\
\text { ELEVATION } \\
\text { (feet) }\end{array}$ & $\begin{array}{l}\text { DEPTH } \\
\text { TO } \\
\text { WATER } \\
\text { (feet) }\end{array}$ & $\begin{array}{c}\text { GROUND- } \\
\text { WATER } \\
\text { ELEVATION } \\
\text { (feet) }\end{array}$ & & COMMENTS & \\
\hline UCD2- & 7 & $10 / 29 / 93$ & 51.65 & 44.61 & 7.04 & & $x$ & \\
\hline UCD2. & 7 & $11 / 12 / 93$ & 51.65 & 43.92 & 7.73 & & & \\
\hline UCD2- & 7 & $11 / 24 / 93$ & 51.65 & 43.19 & 8.46 & & & \\
\hline UCD2- & 7 & $12 / 3 / 93$ & 51.65 & 42.41 & 9.24 & & & \\
\hline UCD2- & 7 & $12 / 10 / 93$ & 51.65 & 42.03 & 9.62 & & & \\
\hline UCD2- & 7 & $12 / 17 / 93$ & 51.65 & 41.61 & 10.04 & & & \\
\hline UCD2- & 7 & $1 / 7 / 94$ & 51.65 & 40.73 & 10.92 & & & \\
\hline UCD2. & 7 & $1 / 14 / 94$ & 51.65 & 40.38 & 11.27 & & & \\
\hline UCD2- & 7 & $1 / 25 / 94$ & 51.65 & 39.96 & 11.69 & & & \\
\hline UCD2- & 7 & $2 / 1 / 94$ & 51.65 & 39.73 & 11.92 & & & \\
\hline UCD2- & 7 & $2 / 14 / 94$ & 51.65 & 39.26 & 12.39 & & & \\
\hline UCD2- & 7 & $2 / 20 / 94$ & 51.65 & 39.01 & 12.64 & & & \\
\hline UCD2- & 7 & $3 / 2 / 94$ & 51.65 & 38.38 & 13.27 & & & \\
\hline UCD2- & 7 & $3 / 4 / 94$ & 51.65 & 38.50 & 13.15 & & & $\cdot$ \\
\hline UCD2- & 7 & $3 / 10 / 94$ & 51.65 & 38.45 & 13.20 & & & \\
\hline UCD2- & 7 & $3 / 16 / 94$ & 51.65 & 38.43 & 13.22 & & & \\
\hline UCD2- & 7 & $3 / 24 / 94$ & 51.65 & 39.49 & 12.16 & & & \\
\hline UCD2- & 7 & $3 / 29 / 94$ & 51.65 & 43.03 & 8.62 & & & \\
\hline UCD2- & 7 & $4 / 6 / 94$ & 51.65 & 45.81 & 5.84 & & & \\
\hline UCD2- & 7 & $4 / 13 / 94$ & 51.65 & 50.22 & 1.43 & & & \\
\hline UCD2- & 7 & $4 / 22 / 94$ & 51.65 & 49.60 & 2.05 & . & & \\
\hline UCD2- & 7 & $4 / 26 / 94$ & 51.65 & 52.28 & -0.63 & & & \\
\hline UCD2- & 7 & $5 / 6 / 94$ & 51.65 & 53.36 & -1.71 & & & \\
\hline UCD2- & 7 & $5 / 11 / 94$ & 51.65 & 53.04 & -1.39 & - & & \\
\hline UCD2- & 7 & $5 / 18 / 94$ & 51.65 & 53.51 & -1.86 & & $\cdot$ & \\
\hline UCD2. & 7 & $5 / 25 / 94$ & 51.65 & 53.89 & -2.24 & & & \\
\hline UCD2- & 7 & $6 / 1 / 94$ & 51.65 & 56.05 & -4.40 & & & \\
\hline UCD2- & 7 & $6 / 23 / 94$ & 51.65 & 63.40 & -11.75 & & & \\
\hline UCD2- & 7 & $7 / 1 / 94$ & 51.65 & 65.90 & -14.25 & & & \\
\hline UCD2- & 7 & $7 / 8 / 94$ & 51.65 & 67.88 & -16.23 & & & \\
\hline UCD2- & 7 & $7 / 14 / 94$ & 51.65 & 69.02 & -17.37 & & & \\
\hline UCD2- & 7 & $7 / 20 / 94$ & 51.65 & 70.51 & -18.86 & & & \\
\hline UCD2- & 7 & $7 / 26 / 94$ & 51.65 & 69.96 & -18.31 & & & \\
\hline UCD2- & 7 & $8 / 5 / 94$ & 51.65 & 68.85 & -17.20 & & & \\
\hline UCD2- & 7 & $8 / 19 / 94$ & 51.65 & 70.81 & -19.16 & & & \\
\hline UCD2- & 7 & $9 / 7 / 94$ & 51.65 & 67.41 & -15.76 & & & \\
\hline UCD2- & 7 & $9 / 7 / 94$ & 51.65 & 67.52 & -15.87 & & & \\
\hline UCD2- & 7 & $9 / 13 / 94$ & 51.65 & 67.64 & -15.99 & & & \\
\hline UCD2- & 7 & $9 / 19 / 94$ & 51.65 & 64.15 & -12.50 & & & \\
\hline UCD2- & 7 & $9 / 26 / 94$ & 51.65 & 62.46 & -10.81 & & & \\
\hline UCD2- & 7 & $10 / 7 / 94$ & 51.65 & 59.82 & -8.17 & & & \\
\hline UCD2- & 7 & $10 / 14 / 94$ & 51.65 & 58.13 & -6.48 & & & \\
\hline UCD2- & 7 & $10 / 21 / 94$ & 51.65 & 57.29 & -5.64 & & . & \\
\hline UCD2- & 7 & $10 / 28 / 94$ & 51.65 & 56.38 & -4.73 & & & \\
\hline UCD2- & 7 & $11 / 4 / 94$ & 51.65 & 55.81 & -4.16 & & & \\
\hline UCD2- & 7 & $11 / 18 / 94$ & 51.65 & 53.02 & -1.37 & & & \\
\hline UCD2- & 7 & $12 / 22 / 94$ & 51.65 & 49.96 & 1.69 & & & \\
\hline UCD2- & 7 & $1 / 6 / 95$ & 51.65 & 48.00 & 3.65 & & & \\
\hline UCD2- & 7 & $1 / 11 / 95$ & 51.65 & 44.86 & 6.79 & & & \\
\hline UCD2- & 7 & $1 / 13 / 95$ & 51.65 & 44.33 & 7.32 & & & \\
\hline UCD2- & 7 & $1 / 27 / 95$ & 51.65 & 41.55 & 10.10 & & & \\
\hline UCD2- & 7 & $2 / 10 / 95$ & 51.65 & 38.67 & 12.98 & & & \\
\hline UCD2- & 7 & $2 / 17 / 95$ & 51.65 & 38.11 & 13.54 & & & \\
\hline UCD2- & 7 & $3 / 9 / 95$ & 51.65 & 35.95 & 15.70 & & & \\
\hline UCD2- & 7 & $3 / 24 / 95$ & 51.65 & 31.24 & 20.41 & & & \\
\hline UCD2- & 7 & $4 / 28 / 95$ & 51.65 & 34.70 & 16.95 & & & \\
\hline UCD2- & 7 & $5 / 25 / 95$ & 51.65 & 35.95 & 15.70 & & & \\
\hline
\end{tabular}


Table E.2. Cumulative Groundwater Elevation Data for HSU-2, 1990-1995

\begin{tabular}{|c|c|c|c|c|c|c|}
\hline WELI & & DATE & $\begin{array}{c}\text { PROTECTIVE } \\
\text { CASING } \\
\text { ELEVATION } \\
\text { (feet) }\end{array}$ & $\begin{array}{l}\text { DEPTH } \\
\text { TO } \\
\text { WATER } \\
\text { (feet) }\end{array}$ & $\begin{array}{l}\text { GROUND- } \\
\text { WATER } \\
\text { ELEVATION } \\
\text { (feet) }\end{array}$ & COMMENTS \\
\hline UCD2- & 7 & $6 / 16 / 95$ & 51.65 & 41.25 & 10.40 & \\
\hline UCD2- & 7 & $7 / 26 / 95$ & 51.65 & $48.32 \mathrm{a}$ & $3.33 \mathrm{a}$ & \\
\hline UCD2- & 7 & $8 / 31 / 95$ & 51.65 & $48.24 a$ & $3.41 \mathrm{a}$ & Caps are off / loose \\
\hline UCD2- & 7 & $9 / 26 / 95$ & 51.65 & $45.54 \mathrm{a}$ & $6.11 \mathrm{a}$ & \\
\hline UCD2- & 7 & $10 / 25 / 95$ & 51.65 & $42.49 \mathrm{a}$ & $9.16 \mathrm{a}$ & \\
\hline UCD2- & 7 & $11 / 22 / 95$ & 51.65 & $40.52 \mathrm{a}$ & $11.13 \mathrm{a}$ & \\
\hline UCD2- & 7 & 11/27/95. & 51.65 & 40.24 & 11.41 & \\
\hline UCD2- & 7 & $12 / 19 / 95$ & 51.65 & 37.88 & 13.77 & \\
\hline UCD2- & 14 & $11 / 2 / 90$ & 51.72 & 58.60 & -6.88 & \\
\hline UCD2- & 14 & $11 / 8 / 90$ & 51.72 & 57.19 & -5.47 & $\cdot$ \\
\hline UCD2- & 14 & $11 / 16 / 90$ & 51.72 & 56.00 & -4.28 & - \\
\hline UCD2- & 14 & $11 / 20 / 90$ & 51.72 & 55.41 & -3.69 & \\
\hline UCD2- & 14 & $12 / 5 / 90$ & 51.72 & 53.81 & -2.09 & \\
\hline UCD2- & 14 & $12 / 27 / 90$ & 51.72 & 51.90 & -0.18 & \\
\hline UCD2- & 14 & $1 / 4 / 91$ & 51.72 & 51.37 & 0.35 & \\
\hline UCD2- & 14 & $1 / 11 / 91$ & 51.72 & 51.01 & 0.71 & \\
\hline UCD2- & 14 & $1 / 22 / 91$ & 51.72 & 50.34 & 1.38 & . \\
\hline UCD2- & 14 & $2 / 1 / 91$ & 51.72 & 51.11 & 0.61 & \\
\hline UCD2- & 14 & $2 / 8 / 91$ & 51.72 & 50.13 & 1.59 & \\
\hline UCD2- & 14 & $2 / 14 / 91$ & 51.72 & 50.12 & 1.60 & \\
\hline UCD2- & 14 & $2 / 22 / 91$ & 51.72 & 52.79 & -1.07 & \\
\hline UCD2- & 14 & $3 / 7 / 91$ & 51.72 & 49.94 & 1.78 & \\
\hline UCD2- & 14 & $3 / 12 / 91$ & 51.72 & 49.26 & 2.46 & \\
\hline UCD2- & 14 & $3 / 22 / 91$ & 51.72 & 48.04 & 3.68 & \\
\hline UCD2- & 14 & $3 / 29 / 91$ & 51.72 & 48.21 & 3.51 & \\
\hline UCD2- & 14 & 4/3/91 & 51.72 & 46.01 & 5.71 & \\
\hline UCD2- & 14 & $4 / 10 / 91$ & 51.72 & 45.75 & 5.97 & \\
\hline UCD2- & 14 & $4 / 17 / 91$ & 51.72 & 46.23 & 5.49 & \\
\hline UCD2- & 14 & $4 / 24 / 91$ & 51.72 & 49.84 & 1.88 & \\
\hline UCD2- & 14 & $5 / 3 / 91$ & 51.72 & 53.49 & -1.77 & \\
\hline UCD2- & 14 & $5 / 13 / 91$ & 51.72 & 58.11 & -6.39 & \\
\hline UCD2- & 14 & $5 / 20 / 91$ & 51.72 & 59.40 & -7.68 & \\
\hline UCD2- & 14 & $5 / 29 / 91$ & 51.72 & 60.19 & -8.47 & \\
\hline UCD2- & 14 & $6 / 6 / 91$ & 51.72 & 63.93 & -12.21 & \\
\hline UCD2- & 14 & $6 / 10 / 91$ & 51.72 & 63.92 & -12.20 & \\
\hline UCD2- & 14 & $6 / 21 / 91$ & 51.72 & 64.84 & -13.12 & \\
\hline UCD2- & 14 & $6 / 27 / 91$ & 51.72 & 65.95 & -14.23 & \\
\hline UCD2- & 14 & $7 / 5 / 91$ & 51.72 & 66.68 & -14.96 & \\
\hline UCD2- & 14 & $7 / 12 / 91$ & 51.72 & 69.61 & -17.89 & \\
\hline UCD2- & 14 & $7 / 19 / 91$ & 51.72 & 71.93 & -20.21 & \\
\hline UCD2- & 14 & $7 / 26 / 91$ & 51.72 & 71.10 & -19.38 & \\
\hline UCD2- & 14 & $8 / 5 / 91$ & 51.72 & 71.67 & -19.95 & \\
\hline UCD2- & 14 & $8 / 14 / 91$ & 51.72 & 72.75 & -21.03 & \\
\hline UCO2- & 14 & $8 / 21 / 91$ & 51.72 & 69.65 & -17.93 & \\
\hline UCD2- & 14 & $8 / 29 / 91$ & 51.72 & 67.02 & -15.30 & \\
\hline UCD2- & 14 & $9 / 4 / 91$ & 51.72 & 65.44 & -13.72 & \\
\hline UCD2- & 14 & $9 / 13 / 91$ & 51.72 & 66.51 & -14.79 & \\
\hline UCD2- & 14 & $10 / 3 / 91$ & 51.72 & 61.04 & -9.32 & \\
\hline UCD2- & 14 & $10 / 10 / 91$ & 51.72 & 59.79 & -8.07 & \\
\hline UCD2- & 14 & $10 / 25 / 91$ & 51.72 & 57.65 & -5.93 & . \\
\hline UCD2- & 14 & $11 / 1 / 91$ & 51.72 & 55.92 & -4.20 & \\
\hline UCD2- & 14 & $11 / 8 / 91$ & 51.72 & 54.85 & -3.13 & \\
\hline UCD2- & 14 & $11 / 14 / 91$ & 51.72 & 55.31 & -3.59 & \\
\hline UCD2- & 14 & $12 / 5 / 91$ & 51.72 & 52.62 & -0.90 & \\
\hline UCD2- & 14 & $12 / 11 / 91$ & 51.72 & .52 .44 & -0.72 & \\
\hline UCD2- & 14 & $12 / 19 / 91$ & 51.72 & 51.72 & 0.00 & \\
\hline
\end{tabular}


Table E.2. Cumulative Groundwater Elevation Data for HSU-2, 1990-1995

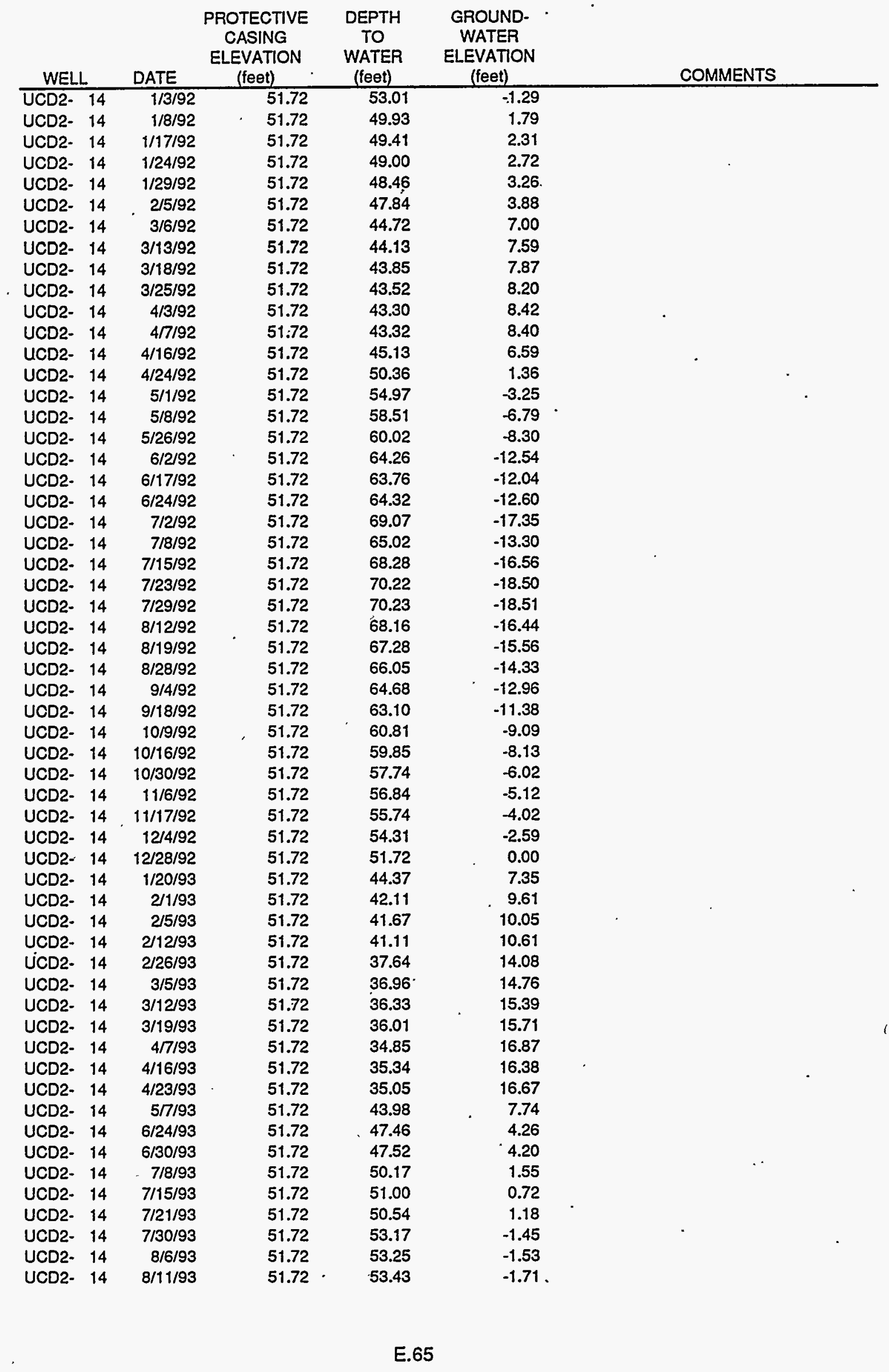


Table E.2. Cumulative Groundwater Elevation Data for HSU-2, 1990-1995

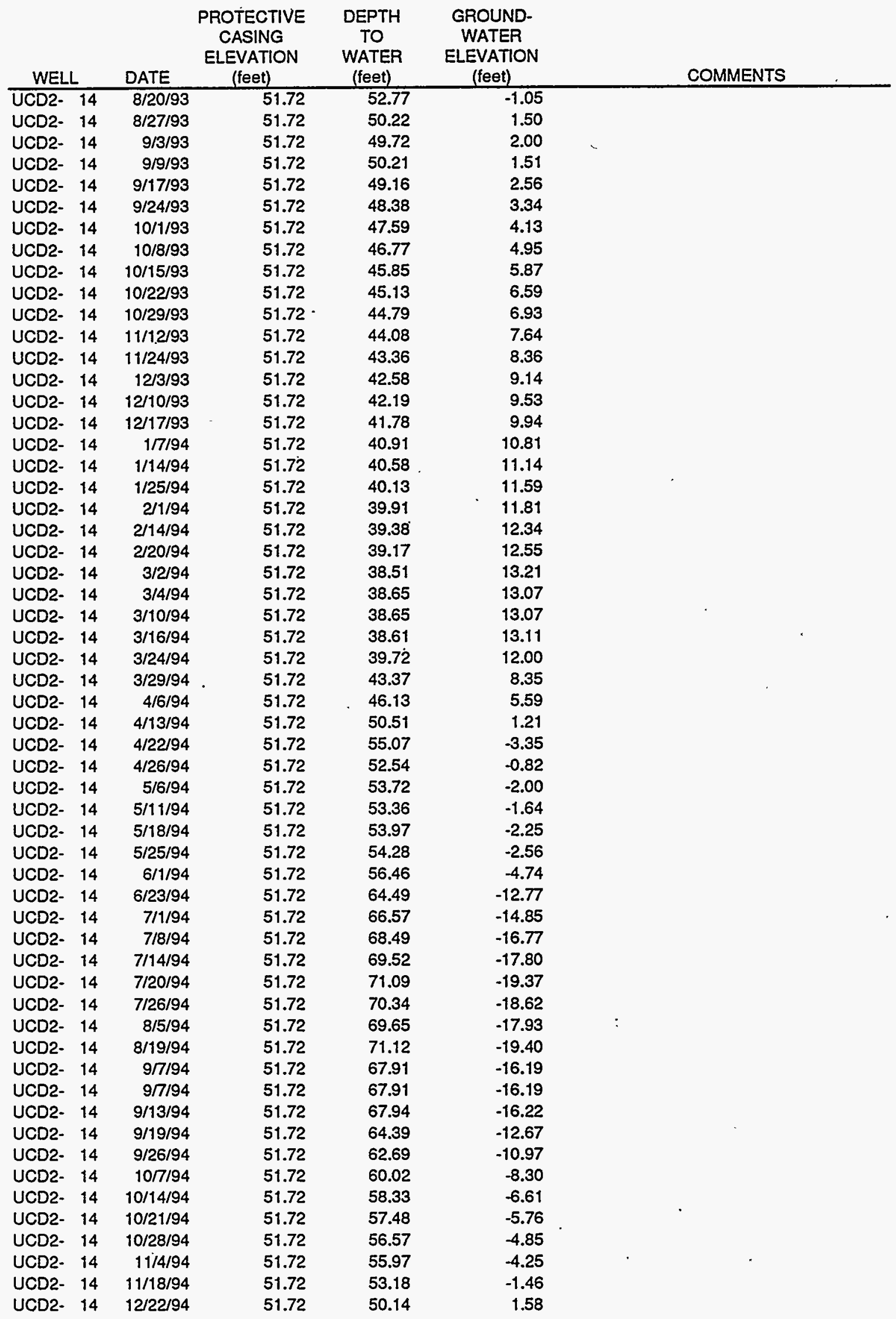


Table E.2. Cumulative Groundwater Elevation Data for HSU-2, 1990-1995

\begin{tabular}{|c|c|c|c|c|c|}
\hline WELL & DATE & $\begin{array}{c}\text { PROTECTIVE } \\
\text { CASING } \\
\text { ELEVATION } \\
\text { (feet) }\end{array}$ & $\begin{array}{l}\text { DEPTH } \\
\text { TO } \\
\text { WATER } \\
\text { (feet) }\end{array}$ & $\begin{array}{c}\text { GROUND- } \\
\text { WATER } \\
\text { ELEVATION } \\
\text { (feet) }\end{array}$ & COMMENTS \\
\hline UCD2- 14 & $1 / 6 / 95$ & 51.72 & 48.58 & 3.14 & \\
\hline UCD2. 14 & $1 / 11 / 95$ & 51.72 & 44.85 & 6.87 & \\
\hline UCD2- 14 & $1 / 13 / 95$ & 51.72 & 44.36 & 7.36 & - \\
\hline UCD2. 14 & $1 / 27 / 95$ & 51.72 & 41.56 & 10.16 & \\
\hline UCD2- 14 & $2 / 10 / 95$ & 51.72 & 38.77 & 12.95 & \\
\hline UCD2. 14 & $2 / 17 / 95$ & 51.72 & 38.24 & 13.48 & \\
\hline UCD2- 14 & $3 / 9 / 95$ & 51.72 & 35.91 & 15.81 & . \\
\hline UCD2- 14 & $3 / 24 / 95$ & 51.72 & 31.30 & 20.42 & \\
\hline UCD2- 14 & $4 / 28 / 95$ & 51.72 & 34.79 & 16.93 & \\
\hline UCD2. 14 & $5 / 25 / 95$ & 51.72 & 36.69 & 15.03 & \\
\hline UCD2. 14 & $6 / 16 / 95$ & 51.72 & 41.51 & 10.21 & \\
\hline UCD2- 14 & $7 / 26 / 95$ & 51.72 & & NA & Cont. water level indicator installed \\
\hline UCD2. 14 & $8 / 31 / 95$ & 51.72 & 48.49 & 3.23 & \\
\hline UCD2- 14 & $9 / 26 / 95$ & 51.72 & 45.74 & 5.98 & \\
\hline UCD2- 14 & $10 / 25 / 95$ & 51.72 & 42.69 & 9.03 & . \\
\hline UCD2- 14 & $11 / 22 / 95$ & 51.72 & 40.71 & 11.01 & \\
\hline UCD2- 14 & $11 / 27 / 95$ & 51.72 & 40.48 & 11.24 & \\
\hline UCD2- 14 & $12 / 19 / 95$ & 51.72 & 38.06 & 13.66 & \\
\hline UCD2- 15 & $11 / 2 / 90$ & 51.49 & 57.00 & -5.51 & \\
\hline UCD2- 15 & $11 / 8 / 90$ & 51.49 & 56.65 & -5.16 & \\
\hline UCD2. 15 & $11 / 16 / 90$ & 51.49 & 55.29 & -3.80 & \\
\hline UCD2. 15 & $11 / 20 / 90$ & 51.49 & 54.80 & -3.31 & \\
\hline UCD2. 15 & $12 / 5 / 90$ & 51.49 & 53.18 & -1.69 & \\
\hline UCD2- 15 & $12 / 27 / 90$ & 51.49 & 51.26 & 0.23 & \\
\hline UCD2- 15 & $1 / 4 / 91$ & 51.49 & 50.73 & 0.76 & \\
\hline UCD2. $\quad 15$ & $1 / 11 / 91$ & 51.49 & 50.42 & .1 .07 & \\
\hline UCD2- 15 & $1 / 22 / 91$ & 51.49 & 49.67 & 1.82 & \\
\hline UCD2- 15 & $2 / 1 / 91$ & 51.49 & 50.31 & 1.18 & \\
\hline UCD2- 15 & $2 / 8 / 91$ & 51.49 & 49.48 & 2.01 & \\
\hline UCD2- 15 & $2 / 14 / 91$ & 51.49 & 49.40 & 2.09 & \\
\hline UCD2- 15 & $2 / 22 / 91$ & 51.49 & 51.80 & -0.31 & \\
\hline UCD2- 15 & $3 / 7 / 91$ & 51.49 & 49.20 & 2.29 & - \\
\hline UCD2- 15 & $3 / 12 / 91$ & 51.49 & 48.51 & 2.98 & \\
\hline UCD2- $\quad 15$ & $3 / 22 / 91$ & 51.49 & 47.32 & 4.17 & \\
\hline UCD2- 15 & $3 / 29 / 91$ & 51.49 & 45.51 & 5.98 & \\
\hline UCD2- 15 & $4 / 3 / 91$ & 51.49 & 45.07 & 6.42 & \\
\hline UCD2- 15 & $4 / 10 / 91$ & 51.49 & 45.88 & 5.61 & \\
\hline UCD2- 15 & $4 / 17 / 91$ & 51.49 & 45.59 & 5.90 & \\
\hline UCD2- 15 & $4 / 24 / 91$ & 51.49 & 49.03 & 2.46 & \\
\hline UCD2. 15 & $5 / 3 / 91$ & 51.49 & 52.33 & -0.84 & . \\
\hline UCD2- 15 & $5 / 13 / 91$ & 51.49 & 57.23 & -5.74 & . \\
\hline UCD2- 15 & $5 / 20 / 91$ & 51.49 & 58.14 & -6.65 & \\
\hline UCD2- 15 & $5 / 29 / 91$ & 51.49 & 58.67 & -7.18 & . \\
\hline UCD2- $\quad 15$ & $6 / 6 / 91$ & 51.49 & 62.50 & -11.01 & \\
\hline UCD2- 15 & $6 / 10 / 91$ & 51.49 & 62.47 & -10.98 & \\
\hline UCD2- 15 & $6 / 21 / 91$ & 51.49 & 63.69 & -12.20 & \\
\hline UCD2- 15 & $6 / 27 / 91$ & 51.49 & 64.41 & -12.92 & . \\
\hline UCD2- $\quad 15$ & $7 / 5 / 91$ & 51.49 & 65.35 & -13.86 & \\
\hline UCD2- 15 & $7 / 12 / 91$ & 51.49 & 68.23 & -16.74 & \\
\hline UCD2- 15 & $7 / 19 / 91$ & 51.49 & 70.74 & -19.25 & \\
\hline UCD2- 15 & $7 / 26 / 91$ & 51.49 & 70.02 & -18.53 & . \\
\hline UCD2- $\quad 15$ & $8 / 5 / 91$ & 51.49 & 70.16 & -18.67 & \\
\hline UCD2- 15 & $8 / 14 / 91$ & 51.49 & 70.92 & -19.43 & \\
\hline UCD2- 15 & $8 / 21 / 91$ & 51.49 & 68.39 & -16.90 & \\
\hline UCD2- 15 & $8 / 29 / 91$ & 51.49 & 66.00 & -14.51 & \\
\hline UCD2- 15 & $9 / 4 / 91$ & 51.49 & 64.48 & -12.99 & \\
\hline
\end{tabular}


Table E.2. Cumulative Groundwater Elevation Data for HSU-2, 1990-1995

\begin{tabular}{|c|c|c|c|c|c|c|}
\hline \multicolumn{2}{|c|}{ WELL } & DATE & $\begin{array}{c}\text { PROTECTIVE } \\
\text { CASING } \\
\text { ELEVATION } \\
\text { (feet) }\end{array}$ & $\begin{array}{l}\text { DEPTH } \\
\text { TO } \\
\text { WATER } \\
\text { (feet) }\end{array}$ & $\begin{array}{c}\text { GROUND- } \\
\text { WATER } \\
\text { ELEVATION } \\
\text { (feet) }\end{array}$ & COMMENTS \\
\hline UCD2- & 15 & $9 / 13 / 91$ & 51.49 & 65.35 & -13.86 & \\
\hline UCD2- & 15 & $10 / 3 / 91$ & 51.49 & 60.13 & -8.64 & \\
\hline UCD2- & 15 & $10 / 10 / 91$ & 51.49 & 58.00 & -6.51 & \\
\hline UCD2- & 15 & $10 / 25 / 91$ & 51.49 & 57.96 & -6.47 & \\
\hline UCD2- & 15 & $11 / 1 / 91$ & 51.49 & 55.08 & -3.59 & \\
\hline UCD2. & 15 & $11 / 8 / 91$ & 51.49 & 53.92 & -2.43 & \\
\hline UCD2- & 15 & $11 / 14 / 91$ & 51.49 & 53.54 & -2.05 & \\
\hline UCD2- & 15 & $12 / 5 / 91$ & 51.49 & 52.45 & -0.96 & \\
\hline UCD2- & 15 & $12 / 11 / 91$ & 51.49 & 51.43 & 0.06 & \\
\hline UCD2- & 15 & $12 / 19 / 91$ & 51.49 & 50.93 & 0.56 & \\
\hline UCD2- & 15 & 1/3/92 & 51.49 & 49.44 & 2.05 & \\
\hline UCD2- & 15 & $1 / 8 / 92$ & 51.49 & 49.13 & 2.36 & \\
\hline UCD2- & 15 & $1 / 17 / 92$ & 51.49 & 48.63 & 2.86 & \\
\hline UCD2- & 15 & $1 / 24 / 92$ & 51.49 & 48.22 & 3.27 & \\
\hline UCD2- & 15 & $1 / 29 / 92$ & 51.49 & 47.69 & 3.80 & \\
\hline UCD2- & 15 & 2/5/92 & 51.49 & 47.06 & 4.43 & \\
\hline UCD2- & 15 & $3 / 6 / 92$ & 51.49 & 44.01 & 7.48 & \\
\hline UCD2- & 15 & $3 / 13 / 92$ & 51.49 & 43.40 & 8.09 & \\
\hline UCD2- & 15 & $3 / 18 / 92$ & 51.49 & 43.16 & 8.33 & \\
\hline UCD2- & 15 & $3 / 25 / 92$ & 51.49 & 42.80 & 8.69 & \\
\hline UCD2- & 15 & 4/3/92 & 51.49 & 42.64 & 8.85 & \\
\hline UCD2- & 15 & $4 / 7 / 92$ & 51.49 & 42.60 & 8.89 & \\
\hline UCD2- & 15 & $4 / 16 / 92$ & 51.49 & 44.51 & 6.98 & \\
\hline UCD2- & 15 & $4 / 24 / 92$ & 51.49 & 49.55 & 1.94 & \\
\hline UCD2- & 15 & $5 / 1 / 92$ & 51.49 & 53.60 & -2.11 & \\
\hline UCD2- & 15 & $5 / 8 / 92$ & 51.49 & 56.82 & -5.33 & \\
\hline UCD2- & 15 & $5 / 26 / 92$ & 51.49 & 58.64 & -7.15 & \\
\hline UCD2- & 15 & $6 / 2 / 92$ & 51.49 & 62.44 & -10.95 & \\
\hline UCD2- & 15 & $6 / 17 / 92$ & 51.49 & 62.31 & -10.82 & \\
\hline UCD2- & 15 & $6 / 24 / 92$ & 51.49 & 63.18 & -11.69 & \\
\hline UCD2- & 15 & $7 / 2 / 92$ & 51.49 & 67.52 & -16.03 & \\
\hline UCD2- & 15 & $7 / 8 / 92$ & 51.49 & 63.76 & -12.27 & \\
\hline UCD2- & 15 & $7 / 15 / 92$ & 51.49 & 67.14 & -15.65 & \\
\hline UCD2- & 15 & $7 / 23 / 92$ & 51.49 & 68.67 & -17.18 & \\
\hline UCD2- & 15 & $7 / 29 / 92$ & 51.49 & 68.89 & -17.40 & \\
\hline UCD2- & 15 & $8 / 12 / 92$ & 51.49 & 66.95 & -15.46 & \\
\hline UCD2- & 15 & $8 / 19 / 92$ & 51.49 & 65.20 & -13.71 & \\
\hline UCD2- & 15 & $8 / 28 / 92$ & 51.49 & 65.04 & -13.55 & \\
\hline UCD2- & 15 & 9/4/92 & 51.49 & 63.76 & -12.27 & \\
\hline UCD2. & 15 & $9 / 18 / 92$ & 51.49 & 62.41 & -10.92 & \\
\hline UCD2- & 15 & $10 / 9 / 92$ & 51.49 & 59.96 & -8.47 & \\
\hline UCD2: & 15 & $10 / 16 / 92$ & 51.49 & 59.23 & -7.74 & \\
\hline UCD2- & 15 & $10 / 30 / 92$ & 51.49 & 57.07 & -5.58 & \\
\hline UCD2- & 15 & $11 / 6 / 92$ & 51.49 & 56.24 & -4.75 & \\
\hline UCD2- & 15 & $11 / 17 / 92$ & 51.49 & 55.09 & -3.60 & . \\
\hline UCD2- & 15 & $12 / 4 / 92$ & 51.49 & 53.79 & -2.30 & \\
\hline UCD2- & 15 & $12 / 28 / 92$ & 51.49 & 51.00 & 0.49 & \\
\hline UCD2- & 15 & $1 / 20 / 93$ & 51.49 & 43.84 & 7.65 & \\
\hline UCD2. & 15 & $1 / 29 / 93$ & 51.49 & 41.52 & 9.97 & \\
\hline UCD2- & 15 & $2 / 5 / 93$ & 51.49 & 41.13 & 10.36 & \\
\hline UCD2- & 15 & $2 / 12 / 93$ & 51.49 & 40.46 & 11.03 & . \\
\hline UCD2- & 15 & $2 / 26 / 93$ & 51.49 & 37.04 & 14.45 & \\
\hline UCD2- & 15 & $3 / 5 / 93$ & 51.49 & 36.36 & 15.13 & \\
\hline UCD2- & 15. & $3 / 12 / 93$ & 51.49 & 35.72 & $15.77^{\circ}$ & \\
\hline UCD2. & 15 & $3 / 19 / 93$ & 51.49 & 35.34 & 16.15 & \\
\hline UCD2- & 15 & $4 / 7 / 93$ & 51.49 & 34.14 & 17.35 & \\
\hline UCD2. & 15 & $4 / 16 / 93$ & 51.49 & 33.39 & 18.10 & \\
\hline
\end{tabular}


Table E.2. Cumulative Groundwater Elevation Data for HSU-2, 1990-1995

\begin{tabular}{|c|c|c|c|c|c|c|}
\hline WELI & & DATE & $\begin{array}{c}\text { PROTECTIVE } \\
\text { CASING } \\
\text { ELEVATION } \\
\text { (feet) }\end{array}$ & $\begin{array}{l}\text { DEPTH } \\
\text { TO } \\
\text { WATER } \\
\text { (feet) }\end{array}$ & $\begin{array}{c}\text { GROUND- } \\
\text { WATER } \\
\text { ELEVATION } \\
\text { (feet) }\end{array}$ & COMMENTS \\
\hline UCD2- & 15 & $4 / 23 / 93$ & 51.49 & 33.24 & 18.25 & \\
\hline UCD2. & 15 & $5 / 7 / 93$ & 51.49 & 42.49 & 9.00 & \\
\hline UCD2- & 15 & $6 / 24 / 93$ & 51.49 & 47.98 & 3.51 & \\
\hline UCD2- & 15 & $6 / 30 / 93$ & 51.49 & 48.35 & 3.14 & \\
\hline UCD2- & 15 & $7 / 8 / 93$ & 51.49 & 48.52 & 2.97 & \\
\hline UCD2- & 15 & $7 / 15 / 93$ & 51.49 & 49.46 & 2.03 & \\
\hline UCD2- & 15 & $7 / 21 / 93$ & 51.49 & 47.17 & 4.32 & \\
\hline UCD2- & 15 & $7 / 30 / 93$ & 51.49 & 51.77 & -0.28 & \\
\hline UCD2- & 15 & $8 / 6 / 93$ & 51.49 & 52.12 & -0.63 & \\
\hline UCD2. & 15 & $8 / 11 / 93$ & 51.49 & 52.02 & -0.53 & \\
\hline UCD2- & 15 & $8 / 20 / 93$ & 51.49 & 52.26 & -0.77 & \\
\hline UCD2- & 15 & $8 / 27 / 93$ & 51.49 & 49.31 & 2.18 & \\
\hline UCD2- & 15. & 9/3/93 & 51.49 & 48.77 & 2.72 & \\
\hline UCD2- & 15 & 9/9/93 & 51.49 & 49.05 & 2.44 & \\
\hline UCD2- & 15 & $9 / 17 / 93$ & 51.49 & 48.22 & 3.27 & \\
\hline UCD2- & 15 & $9 / 24 / 93$ & 51.49 & 47.44 & 4.05 & \\
\hline UCD2- & 15 & $10 / 1 / 93$ & 51.49 & 46.72 & 4.77 & \\
\hline UCD2- & 15 & $10 / 8 / 93$ & 51.49 & 45.96 & 5.53 & \\
\hline UCD2- & 15 & $10 / 15 / 93$ & 51.49 & 45.07 & 6.42 & \\
\hline UCD2- & 15 & $10 / 22 / 93$ & 51.49 & 44.38 & 7.11 & \\
\hline UCD2- & 15 & $10 / 29 / 93$ & 51.49 & 44.06 & 7.43 & \\
\hline UCD2- & 15 & $11 / 12 / 93$ & 51.49 & 43.36 & 8.13 & \\
\hline UCD2- & 15 & $11 / 24 / 93$ & 51.49 & 42.68 & 8.81 & \\
\hline UCD2- & 15 & $12 / 3 / 93$ & 51.49 & 41.96 & 9.53 & \\
\hline UCD2. & 15 & $12 / 10 / 93$ & 51.49 & 41.56 & 9.93 & \\
\hline UCD2- & 15 & $12 / 17 / 93$ & 51.49 & 41.15 & 10.34 & \\
\hline UCD2- & 15 & $1 / 7 / 94$ & 51.49 & 40.26 & 11.23 & \\
\hline UCD2- & 15 & $1 / 14 / 94$ & 51.49 & 39.92 & 11.57 & \\
\hline UCD2- & 15 & $1 / 25 / 94$ & 51.49 & 39.45 & 12.04 & \\
\hline UCD2. & 15 & $2 / 1 / 94$ & 51.49 & 39.26 & 12.23 & \\
\hline UCD2- & 15 & $2 / 14 / 94$ & 51.49 & 38.73 & $12.76^{\circ}$ & \\
\hline UCD2. & 15 & $2 / 20 / 94$ & 51.49 & 38.48 & 13.01 & \\
\hline UCD2. & 15 & $3 / 2 / 94$ & 51.49 & 37.69 & 13.80 & \\
\hline UCD2- & 15 & $3 / 4 / 94$ & 51.49 & 38.04 & 13.45 & \\
\hline UCD2- & 15 & $3 / 10 / 94$ & 51.49 & 38.05 & 13.44 & \\
\hline UCD2- & 15 & $3 / 16 / 94$ & 51.49 & 37.96 & 13.53 & \\
\hline UCD2- & 15 & $3 / 24 / 94$ & 51.49 & 39.05 & 12.44 & \\
\hline UCD2. & 15 & $3 / 29 / 94$ & 51.49 & 42.45 & 9.04 & \\
\hline UCD2- & 15 & $4 / 6 / 94$ & 51.49 & 45.07 & 6.42 & \\
\hline UCD2- & 15 & $4 / 13 / 94$ & 51.49 & 49.04 & .2 .45 & \\
\hline UCD2- & 15 & $4 / 22 / 94$ & 51.49 & 53.76 & -2.27 & \\
\hline UCD2- & 15 & $4 / 26 / 94$ & 51.49 & 51.25 & 0.24 & \\
\hline UCD2- & 15 & $5 / 6 / 94$ & 51.49 & 52.40 & -0.91 & \\
\hline UCD2. & 15 & $5 / 11 / 94$ & 51.49 & 52.38 & -0.89 & \\
\hline UCD2- & 15 & $5 / 18 / 94$ & 51.49 & 53.15 & -1.66 & \\
\hline UCD2- & 15 & $5 / 25 / 94$ & 51.49 & 52.90 & -1.41 & \\
\hline UCD2- & 15 & $6 / 1 / 94$ & 51.49 & 55.14 & -3.65 & \\
\hline UCD2. & 15 & $6 / 23 / 94$ & 51.49 & 62.91 & -11.42 & \\
\hline UCD2. & 15 & $7 / 1 / 94$ & 51.49 & 65.04 & -13.55 & \\
\hline UCD2- & 15 & $7 / 8 / 94$ & 51.49 & 67.03 & -15.54 & \\
\hline UCD2- & 15 & $7 / 14 / 94$ & 51.49 & 68.01 & -16.52 & \\
\hline UCD2- & 15 & $7 / 20 / 94$ & 51.49 & 69.58 & -18.09 & \\
\hline UCD2- & 15 & $7 / 26 / 94$ & 51.49 & 69.21 & -17.72 & \\
\hline UCD2- & 15 & $8 / 5 / 94$ & 51.49 & 68.22 & -16.73 & \\
\hline UCD2- & 15 & $8 / 19 / 94$ & 51.49 & 70.00 & -18.51 & \\
\hline UCD2. & 15 & $9 / 7 / 94$ & 51.49 & 66.31 & -14.82 & \\
\hline UCD2. & 15 & $9 / 7 / 94$ & 51.49 & 66.87 & -15.38 & \\
\hline
\end{tabular}


Table E.2. Cumulative Groundwater Elevation Data for HSU-2, 1990-1995

\begin{tabular}{|c|c|c|c|c|c|c|}
\hline WELI & & DATE & $\begin{array}{c}\text { PROTECTIVE } \\
\text { CASING } \\
\text { ELEVATION } \\
\text { (feet) }\end{array}$ & $\begin{array}{c}\text { DEPTH } \\
\text { TO } \\
\text { WATER } \\
\text { (feet) } \\
\end{array}$ & $\begin{array}{c}\text { GROUND- } \\
\text { WATER } \\
\text { ELEVATION } \\
\text { (feet) }\end{array}$ & COMMENTS \\
\hline UCD2- & 15 & $9 / 13 / 94$ & 51.49 & 67.17 & -15.68 & \\
\hline UCD2- & 15 & $9 / 19 / 94$ & 51.49 & 63.60 & -12.11 & \\
\hline UCD2- & 15 & $9 / 26 / 94$ & 51.49 & 61.93 & -10.44 & \\
\hline UCD2- & 15 & $10 / 7 / 94$ & 51.49 & 59.29 & -7.80 & \\
\hline UCD2- & 15 & $10 / 14 / 94$ & 51.49 & 57.63 & -6.14 & \\
\hline UCD2- & 15 & $10 / 21 / 94$ & 51.49 & 56.78 & -5.29 & \\
\hline UCD2- & 15 & $10 / 28 / 94$ & 51.49 & 55.88 & -4.39 & \\
\hline UCD2- & 15 & $11 / 4 / 94$ & 51.49 & 55.29 & -3.80 & \\
\hline UCD2- & 15 & $11 / 18 / 94$ & 51.49 & 52.49 & -1.00 & \\
\hline UCD2- & 15 & $12 / 22 / 94$ & 51.49 & 49.42 & 2.07 & \\
\hline UCD2. & 15 & 1/6/95 & 51.49 & 47.85 & 3.64 & · \\
\hline UCD2- & 15 & $1 / 10 / 95$ & 51.49 & 44.90 & 6.59 & \\
\hline UCD2- & 15 & $1 / 11 / 95$ & 51.49 & 44.26 & 7.23 & \\
\hline UCD2- & 15 & $1 / 13 / 95$ & 51.49 & 43.81 & 7.68 & • \\
\hline UCD2- & 15 & $1 / 27 / 95$ & 51.49 & 41.12 & 10.37 & \\
\hline UCD2- & 15 & $2 / 10 / 95$ & 51.49 & 38.33 & 13.16 & \\
\hline UCD2- & 15 & $2 / 17 / 95$ & 51.49 & 37.76 & 13.73 & \\
\hline UCD2. & 15 & $3 / 9 / 95$ & 51.49 & 35.49 & 16.00 & \\
\hline UCD2. & 15 & $3 / 24 / 95$ & 51.49 & 30.86 & 20.63 & \\
\hline UCD2- & 15 & $4 / 28 / 95$ & 51.49 & 34.34 & 17.15 & \\
\hline UCD2- & 15 & $5 / 25 / 95$ & 51.49 & 35.21 & 16.28 & \\
\hline UCD2- & 15 & $6 / 16 / 95$ & 51.49 & 40.08 & 11.41 & \\
\hline UCD2- & 15 & $7 / 26 / 95$ & 51.49 & & NA & Cont. water level indicator installed \\
\hline UCD2- & 15 & $8 / 31 / 95$ & 51.49 & 47.42 & 4.07 & Caps are off \\
\hline UCD2- & 15 & $9 / 26 / 95$ & 51.49 & & NA & Continuous well level indicator \\
\hline UCD2- & 15 & $10 / 25 / 95$ & 51.49 & & NA & Continuous monitoring equipment \\
\hline UCD2- & 15 & $11 / 22 / 95$ & 51.49 & 40.01 & 11.48 & \\
\hline UCD2- & 15 & $11 / 27 / 95$ & 51.49 & 39.72 & 11.77 & no pump \\
\hline UCD2. & 15 & $12 / 19 / 95$ & 51.49 & 37.45 & 14.04 & \\
\hline UCD2- & 16 & $11 / 2 / 90$ & 49.95 & 55.52 & -5.57 & \\
\hline UCD2- & 16 & $11 / 8 / 90$ & 49.95 & 54.88 & 4.93 & \\
\hline UCD2- & 16 & $11 / 16 / 90$ & 49.95 & 53.88 & -3.93 & \\
\hline UCD2- & 16 & $11 / 20 / 90$ & 49.95 & 53.38 & -3.43 & \\
\hline UCD2- & 16 & $12 / 5 / 90$ & 49.95 & 51.79 & -1.84 & \\
\hline UCD2- & 16 & $12 / 27 / 90$ & 49.95 & 49.85 & 0.10 & \\
\hline UCD2- & 16 & $1 / 4 / 91$ & 49.95 & 49.34 & 0.61 & \\
\hline UCD2- & 16 & $1 / 11 / 91$ & 49.95 & 49.13 & 0.82 & \\
\hline UCD2- & 16 & $1 / 22 / 91$ & 49.95 & 48.37 & 1.58 & \\
\hline UCD2- & 16 & $2 / 1 / 91$ & 49.95 & 49.10 & 0.85 & \\
\hline UCD2. & 16 & $2 / 8 / 91$ & 49.95 & 48.10 & 1.85 & \\
\hline UCD2- & 16 & $2 / 14 / 91$ & 49.95 & 48.01 & 1.94 & . \\
\hline UCD2- & 16 & $2 / 22 / 91$ & 49.95 & 50.51 & -0.56 & \\
\hline UCD2- & 16 & $3 / 7 / 91$ & 49.95 & 47.83 & 2.12 & \\
\hline UCD2- & 16 & $3 / 12 / 91$ & 49.95 & 47.27 & 2.68 & \\
\hline UCD2- & 16 & $3 / 22 / 91$ & 49.95 & 44.60 & 5.35 & \\
\hline UCD2- & 16 & $3 / 29 / 91$ & 49.95 & 44.25 & 5.70 & \\
\hline UCD2- & 16 & $4 / 3 / 91$ & 49.95 & 44.07 & 5.88 & \\
\hline UCD2- & 16 & $4 / 10 / 91$ & 49.95 & 43.81 & 6.14 & \\
\hline UCD2- & 16 & $4 / 17 / 91$ & 49.95 & 44.33 & 5.62 & \\
\hline UCD2- & 16 & $4 / 24 / 91$ & 49.95 & 47.54 & 2.41 & \\
\hline UCD2- & 16 & $5 / 3 / 91$ & 49.95 & 49.68 & 0.27 & \\
\hline UCD2- & 16 & $5 / 13 / 91$ & 49.95 & 55.10 & -5.15 & \\
\hline UCD2- & 16 & $5 / 20 / 91$ & 49.95 & 56.84 & -6.89 & \\
\hline UCD2- & 16 & $5 / 29 / 91$ & 49.95 & 57.23 & -7.28 & \\
\hline UCD2- & 16 & $6 / 6 / 91$ & 49.95 & 60.76 & -10.81 & \\
\hline UCD2- & 16 & $6 / 10 / 91$ & 49.95 & 60.73 & -10.78 & \\
\hline
\end{tabular}


Table E.2. Cumulative Groundwater Elevation Data for HSU-2, 1990-1995

\begin{tabular}{|c|c|c|c|c|c|c|c|}
\hline WELL & DATE & $\begin{array}{l}\text { PROTECTIVE } \\
\text { CASING } \\
\text { ELEVATION } \\
\text { (feet) }\end{array}$ & $\begin{array}{l}\text { DEPTH } \\
\text { TO } \\
\text { WATER } \\
\text { (feet) }\end{array}$ & $\begin{array}{c}\text { GROUND- } \\
\text { WATER } \\
\text { ELEVATION } \\
\text { (feet) }\end{array}$ & & COMMENTS & \\
\hline UCD2- 16 & $6 / 21 / 91$ & 49.95 & 62.36 & -12.41 & & & \\
\hline UCD2. 16 & $6 / 27 / 91$ & 49.95 & 63.32 & -13.37 & & & \\
\hline UCD2- 16 & $7 / 5 / 91$ & 49.95 & 64.54 & -14.59 & & & \\
\hline UCD2- 16 & $7 / 12 / 91$ & 49.95 & 66.78 & -16.83 & & & \\
\hline UCD2. 16 & $7 / 19 / 91$ & 49.95 & 69.00 & -19.05 & & & \\
\hline UCD2- 16 & $7 / 26 / 91$ & 49.95 & 68.58 & -18.63 & & & \\
\hline UCD2- 16 & $8 / 14 / 91$ & 49.95 & 69.38 & -19.43 & & & \\
\hline UCD2- 16 & $8 / 21 / 91$ & 49.95 & 66.71 & -16.76 & & 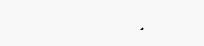 & \\
\hline UCD2- 16 & $8 / 29 / 91$ & 49.95 & 64.54 & -14.59 & & & \\
\hline UCD2- 16 & $9 / 4 / 91$ & 49.95 & 63.02 & $: 13.07$ & & & \\
\hline UCD2- 16 & $9 / 13 / 91$ & 49.95 & 63.80 & -13.85 & & & · \\
\hline UCD2- 16 & $10 / 3 / 91$ & 49.95 & 58.74 & -8.79 & & & \\
\hline UCD2. 16 & $10 / 10 / 91$ & 49.95 & 57.55 & -7.60 & • & & \\
\hline UCD2- 16 & $10 / 25 / 91$ & 49.95 & 55.40 & -5.45 & & & \\
\hline UCD2- 16 & $11 / 1 / 91$ & 49.95 & 53.61 & -3.66 & & & \\
\hline UCD2- 16 & $11 / 8 / 91$ & 49.95 & 52.69 & -2.74 & & & \\
\hline UCD2- 16 & $11 / 14 / 91$ & 49.95 & 52.17 & -2.22 & & $\cdot$ & \\
\hline UCD2- 16 & $12 / 5 / 91$ & 49.95 & 50.45 & -0.50 & & & \\
\hline UCD2: 16 & $12 / 11 / 91$ & 49.95 & 51.37 & -1.42 & & & \\
\hline UCD2- 16 & $12 / 19 / 91$ & 49.95 & 49.56 & 0.39 & $\cdot$ & & \\
\hline UCD2. 16 & $1 / 3 / 92$ & 49.95 & 48.09 & 1.86 & & & \\
\hline UCD2- 16 & $1 / 8 / 92$ & 49.95 & 47.88 & 2.07 & & & \\
\hline UCD2- 16 & $1 / 17 / 92$ & 49.95 & 47.32 & 2.63 & & . & \\
\hline UCD2. 16 & $1 / 24 / 92$ & 49.95 & 46.90 & 3.05 & & & \\
\hline UCD2- -16 & 1/29/92 & 49.95 & 46.35 & 3.60 & & & \\
\hline UCD2- 16 & $2 / 5 / 92$ & 49.95 & 45.73 & 4.22 & & & \\
\hline UCD2- 16 & $3 / 6 / 92$ & 49.95 & 42.65 & 7.30 & & & \\
\hline UCD2- 16 & $3 / 13 / 92$ & 49.95 & 42.04 & 7.91 & & & \\
\hline UCD2- 16 & $3 / 18 / 92$ & 49.95 & 41.79 & 8.16 & & & \\
\hline UCD2- 16 & $3 / 25 / 92$ & 49.95 & 41.44 & 8.51 & & & \\
\hline UCD2. 16 & 4/3/92 & 49.95 & 41.32 & 8.63 & & & \\
\hline UCD2- 16 & $4 / 7 / 92$ & 49.95 & 41.28 & 8.67 & & & \\
\hline UCD2- 16 & $4 / 16 / 92$ & 49.95 & 43.07 & 6.88 & & & \\
\hline UCD2- 16 & $4 / 24 / 92$ & 49.95 & 48.09 & 1.86 & & & \\
\hline UCD2- 16 & $5 / 1 / 92$ & 49.95 & 52.27 & -2.32 & & & \\
\hline UCD2: 16 & $5 / 8 / 92$ & 49.95 & 55.46 & -5.51 & & & \\
\hline UCD2- 16 & $5 / 26 / 92$ & 49.95 & 57.14 & -7.19 & - & & \\
\hline UCD2- 16 & 6/2/92 & 49.95 & 61.05 & -11.10 & & & - \\
\hline UCD2- 16 & $6 / 17 / 92$ & 49.95 & 60.67 & -10.72 & & & \\
\hline UCD2- 16 & $6 / 24 / 92$ & 49.95 & 61.69 & -11.74 & & & \\
\hline UCD2- 16 & 7/2/92 & 49.95 & 65.89 & -15.94 & . & & \\
\hline UCD2- 16 & $7 / 8 / 92$ & 49.95 & 62.39 & -12.44 & & & \\
\hline UCD2- 16 & $7 / 15 / 92$ & 49.95 & 65.63 & -15.68 & & & \\
\hline UCD2- 16 & $7 / 23 / 92$ & 49.95 & 67.07 & -17.12 & & & \\
\hline UCD2- 16 & $7 / 29 / 92$ & 49.95 & 67.54 & -17.59 & & & \\
\hline UCD2- 16 & $8 / 12 / 92$ & 49.95 & 65.63 & -15.68 & & & \\
\hline UCD2- 16 & $8 / 19 / 92$ & 49.95 & 63.80 & -13.85 & & & \\
\hline UCD2- 16 & $8 / 28 / 92$ & 49.95 & 63.59 & -13.64 & & & \\
\hline UCD2- 16 & $9 / 4 / 92$ & 49.95 & 62.32 & -12.37 & & & \\
\hline UCD2- 16 & $9 / 18 / 92$ & 49.95 & 60.90 & -10.95 & & & \\
\hline UCD2- 16 & 10/9/92 & 49.95 & 58.51 & -8.56 & & & \\
\hline UCD2- 16 & $10 / 16 / 92$ & 49.95 & 57.69 & -7.74 & & & \\
\hline UCD2: 16 & $10 / 30 / 92$ & 49.95 & 55.55 & -5.60 & & & \\
\hline UCD2- 16 & $11 / 6 / 92$ & 49.95 & 56.72 & -6.77 & & & \\
\hline UCD2- 16 & $11 / 17 / 92$ & 49.95 & 53.65 & -3.70 & & & \\
\hline UCD2- 16 & $12 / 4 / 92$ & 49.95 & 52.17 & -2.22 & & & \\
\hline UCD2- 16 & $12 / 28 / 92$ & 49.95 & 49.64 & 0.31 & & & \\
\hline
\end{tabular}


Table E.2. Cumulative Groundwater Elevation Data for HSU-2, 1990-1995

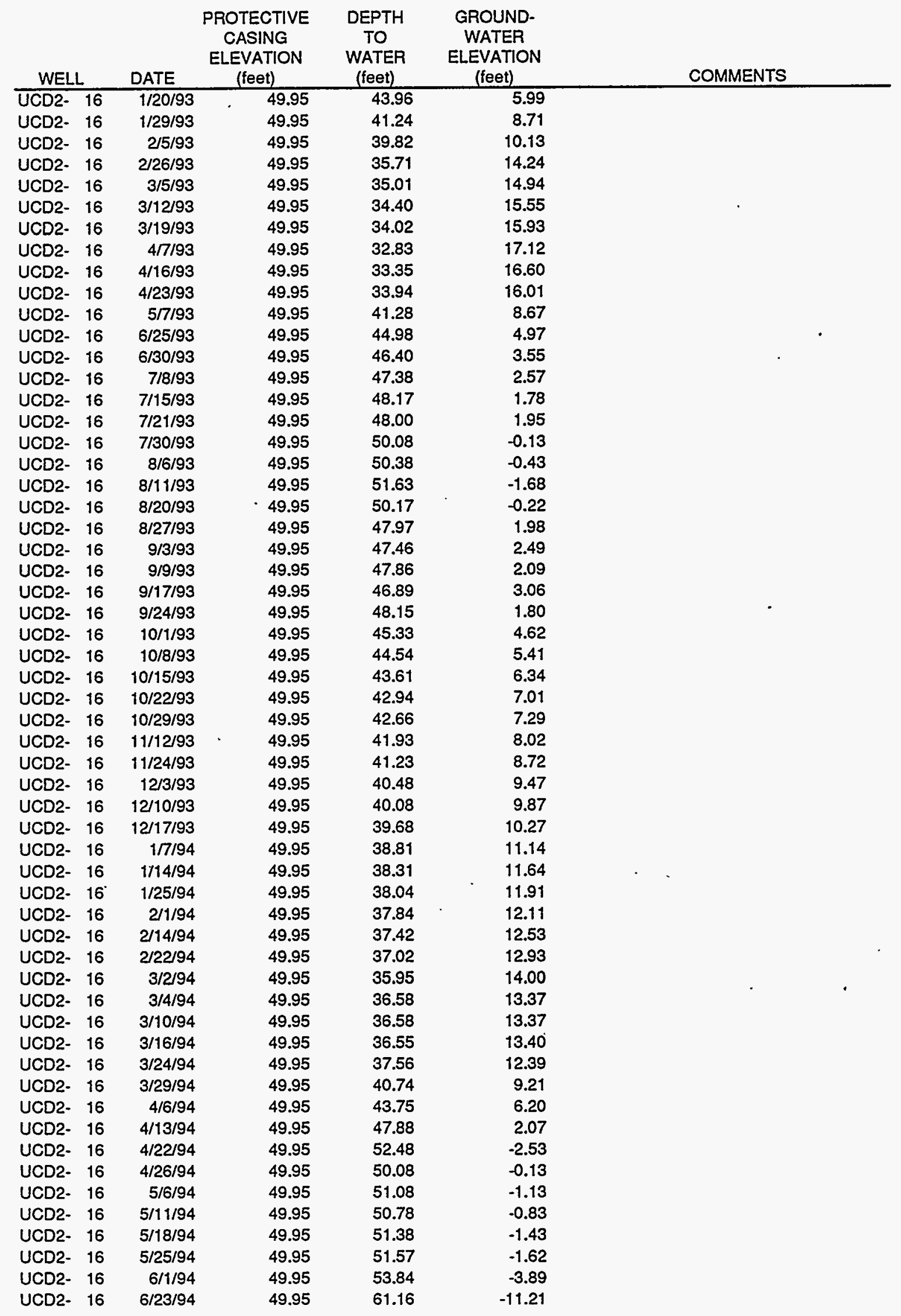


Table E.2. Cumulative Groundwater Elevation Data for HSU-2, 1990-1995

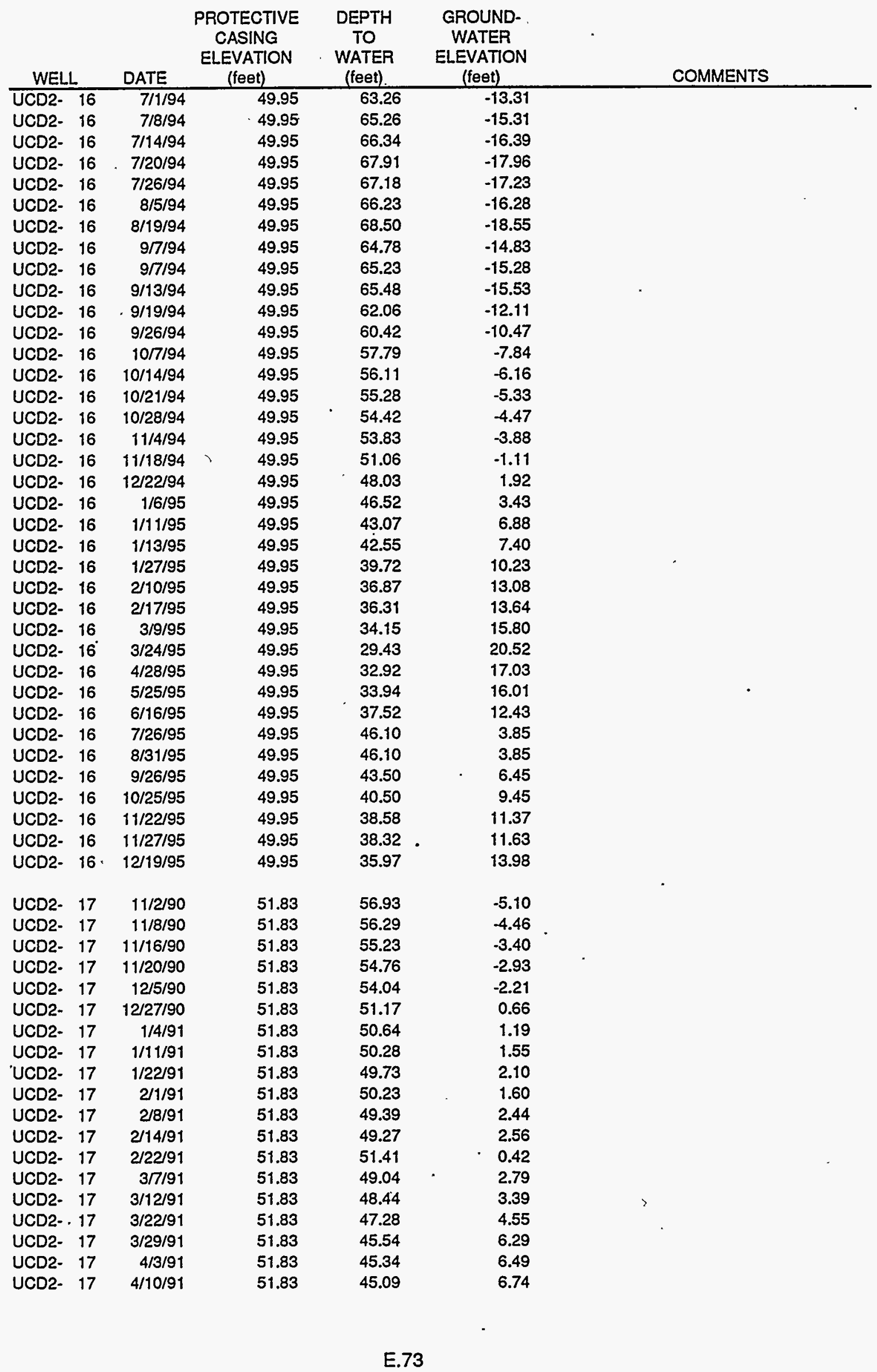


Table E.2. Cumulative Groundwater Elevation Data for HSU-2, 1990-1995

\begin{tabular}{|c|c|c|c|c|c|c|}
\hline WEL & & DATE & $\begin{array}{c}\text { PROTECTIVE } \\
\text { CASING } \\
\text { ELEVATION } \\
\text { (feet) }\end{array}$ & $\begin{array}{l}\text { DEPTH } \\
\text { TO } \\
\text { WATER } \\
\text { (feet) }\end{array}$ & $\begin{array}{c}\text { GROUND- } \\
\text { WATER } \\
\text { ELEVATION } \\
\text { (feet) }\end{array}$ & COMMENTS \\
\hline UCD2- & 17 & $4 / 17 / 91$ & 51.83 & 45.50 & 6.33 & \\
\hline UCD2- & 17 & $4 / 24 / 91$ & 51.83 & 49.00 & 2.83 & \\
\hline UCD2- & 17 & $5 / 3 / 91$ & 51.83 & 50.63 & 1.20 & \\
\hline UCD2- & 17 & $5 / 13 / 91$ & 51.83 & 56.25 & -4.42 & \\
\hline UCD2- & 17 & $5 / 20 / 91$ & 51.83 & 57.59 & -5.76 & \\
\hline UCD2- & 17 & $5 / 29 / 91$ & 51.83 & 57.79 & -5.96 & \\
\hline UCD2- & 17 & $6 / 6 / 91$ & 51.83 & 61.57 & -9.74 & \\
\hline UCD2- & 17 & $6 / 10 / 91$ & 51.83 & 61.41 & -9.58 & \\
\hline UCD2. & 17 & $6 / 21 / 91$ & 51.83 & 63.33 & -11.50 & \\
\hline UCD2. & 17 & $6 / 27 / 91$ & 51.83 & .64 .16 & -12.33 & \\
\hline UCD2. & 17 & $7 / 5 / 91$ & 51.83 & 65.33 & -13.50 & \\
\hline UCD2- & 17 & $7 / 12 / 91$ & 51.83 & 67.48 & -15.65 & \\
\hline UCD2- & 17 & $7 / 19 / 91$ & 51.83 & 70.06 & -18.23 & \\
\hline UCD2- & 17 & $7 / 26 / 91$ & 51.83 & 69.70 & -17.87 & \\
\hline UCD2- & 17 & $8 / 5 / 91$ & 51.83 & 69.20 & -17.37 & · \\
\hline UCD2- & 17 & $8 / 14 / 91$ & 51.83 & 69.84 & -18.01 & \\
\hline UCD2. & 17 & $8 / 21 / 91$ & 51.83 & 67.54 & -15.71 & \\
\hline UCD2. & 17 & $8 / 29 / 91$ & 51.83 & 65.53 & -13.70 & \\
\hline UCD2. & 17 & $9 / 4 / 91$ & 51.83 & 64.03 & -12.20 & \\
\hline UCD2- & 17 & $9 / 13 / 91$ & 51.83 & 64.74 & -12.91 & \\
\hline UCD2- & 17 & $10 / 3 / 91$ & 51.83 & 60.21 & -8.38 & \\
\hline UCD2- & 17 & $10 / 10 / 91$ & 51.83 & 58.74 & -6.91 & \\
\hline UCD2- & 17 & $10 / 25 / 91$ & 51.83 & 56.58 & -4.75 & \\
\hline UCD2. & 17 & $11 / 1 / 91$ & 51.83 & 54.90 & -3.07 & \\
\hline UCD2. & 17 & $11 / 8 / 91$ & 51.83 & 53.82 & -1.99 & \\
\hline UCD2. & 17 & $11 / 14 / 91$ & 51.83 & 53.34 & -1.51 & \\
\hline UCD2- & 17 & $12 / 5 / 91$ & 51.83 & 51.58 & 0.25 & \\
\hline UCD2- & 17 & $12 / 11 / 91$ & 51.83 & 51.09 & 0.74 & \\
\hline UCD2- & 17 & $12 / 19 / 91$ & 51.83 & 50.61 & 1.22 & : \\
\hline UCD2- & 17 & $1 / 3 / 92$ & 51.83 & 49.26 & 2.57 & \\
\hline UCD2- & 17 & $1 / 8 / 92$ & 51.83 & 49.01 & 2.82 & \\
\hline UCD2- & 17 & $1 / 17 / 92$ & 51.83 & 48.47 & 3.36 & \\
\hline UCD2- & 17 & $1 / 24 / 92$ & 51.83 & 48.04 & 3.79 & \\
\hline UCD2- & 17 & $1 / 29 / 92$ & 51.83 & 47.49 & 4.34 & \\
\hline UCD2- & 17 & $2 / 5 / 92$ & 51.83 & 46.91 & 4.92 & \\
\hline UCD2- & 17 & $3 / 6 / 92$ & 51.83 & 43.91 & 7.92 & \\
\hline UCD2- & 17 & $3 / 13 / 92$ & 51.83 & 43.37 & 8.46 & \\
\hline UCD2- & 17 & $3 / 18 / 92$ & 51.83 & 43.09 & 8.74 & \\
\hline UCD2- & 17 & $3 / 25 / 92$ & 51.83 & 42.76 & 9.07 & \\
\hline UCD2- & 17 & $4 / 3 / 92$ & 51.83 & 42.54 & 9.29 & \\
\hline UCD2- & 17 & $4 / 7 / 92$ & 51.83 & 42.53 & 9.30 & \\
\hline UCD2- & 17 & $4 / 16 / 92$ & 51.83 & 44.51 & 7.32 & \\
\hline UCD2- & 17 & $4 / 24 / 92$ & 51.83 & 49.39 & 2.44 & \\
\hline UCD2. & 17 & $5 / 1 / 92$ & 51.83 & 53.03 & -1.20 & \\
\hline UCD2. & 17 & $5 / 8 / 92$ & 51.83 & 55.91 & -4.08 & \\
\hline UCD2. & 17 & $5 / 26 / 92$ & 51.83 & 58.15 & $-6: 32$ & \\
\hline UCD2- & 17 & $6 / 2 / 92$ & 51.83 & 61.82 & -9.99 & \\
\hline UCD2. & 17 & $6 / 17 / 92$ & 51.83 & 61.43 & -9.60 & \\
\hline UCD2- & 17 & $6 / 24 / 92$ & 51.83 & 62.67 & -10.84 & \\
\hline UCD2- & 17 & 7/2/92 & 51.83 & 66.58 & -14.75 & \\
\hline UCD2. & 17 & $7 / 8 / 92$ & 51.83 & 63.12 & -11.29 & \\
\hline UCD2. & 17 & $7 / 15 / 92$ & 51.83 & 66.61 & -14.78 & \\
\hline UCD2- & 17 & $7 / 23 / 92$ & 51.83 & 66.79 & -14.96 & \\
\hline UCD2- & 17 & $7 / 29 / 92$ & 51.83 & 68.22 & -16.39 & \\
\hline UCD2- & 17 & $8 / 12 / 92$ & 51.83 & 66.40 & -14.57 & \\
\hline UCD2- & 17 & $8 / 19 / 92$ & 51.83 & 64.71 & -12.88 & \\
\hline UCD2. & 17 & $8 / 28 / 92$ & 51.83 & 64.58 & -12.75 & \\
\hline
\end{tabular}


Table E.2. Cumulative Groundwater Elevation Data for HSU-2, 1990-1995

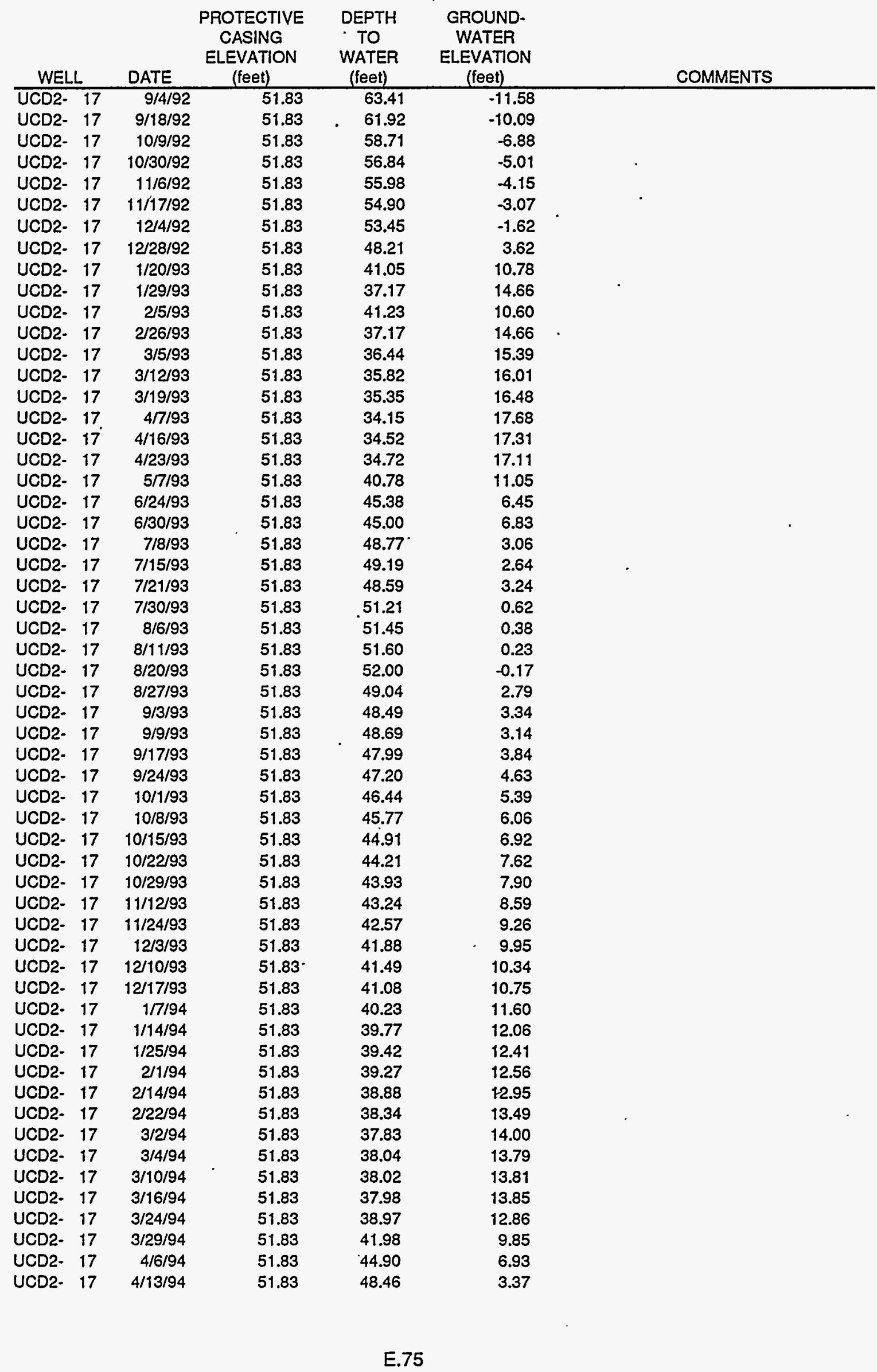


Table E.2. Cumulative Groundwater Elevation Data for HSU-2, 1990-1995

\begin{tabular}{|c|c|c|c|c|c|}
\hline WELL & DATE & $\begin{array}{c}\text { PROTECTIVE } \\
\text { CASING } \\
\text { ELEVATION } \\
\text { (feet) }\end{array}$ & $\begin{array}{l}\text { DEPTH } \\
\text { TO } \\
\text { WATER } \\
\text { (feet) }\end{array}$ & $\begin{array}{c}\text { GROUND- } \\
\text { WATER } \\
\text { ELEVATION } \\
\text { (feet) }\end{array}$ & COMMENTS \\
\hline UCD2- 17 & $4 / 22 / 94$ & 51.83 & 53.30 & -1.47 & \\
\hline UCD2- 17 & $4 / 26 / 94$ & 51.83 & 50.77 & 1.06 & \\
\hline UCD2- 17 & $5 / 6 / 94$ & 51.83 & 51.86 & -0.03 & \\
\hline UCD2- 17 & $5 / 11 / 94$ & 51.83 & 51.95 & -0.12 & \\
\hline UCD2- 17 & $5 / 18 / 94$ & 51.83 & 52.76 & -0.93 & \\
\hline UCD2- 17 & $5 / 25 / 94$ & 51.83 & 52.29 & -0.46 & \\
\hline UCD2- 17 & $6 / 1 / 94$ & 51.83 & 54.57 & -2.74 & \\
\hline UCD2- 17 & $6 / 23 / 94$ & 51.83 & 62.57 & -10.74 & \\
\hline UCD2- 17 & $7 / 1 / 94$ & 51.83 & 64.00 & -12.17 & \\
\hline UCO2- 17 & $7 / 8 / 94$ & 51.83 & 66.07 & -14.24 & \\
\hline UCD2- 17 & $7 / 14 / 94$ & 51.83 & 67.14 & -15.31 & \\
\hline UCD2- 17 & $7 / 20 / 94$ & 51.83 & 69.08 & -17.25 & . \\
\hline UCD2- 17 & $7 / 26 / 94$ & 51.83 & 68.00 & -16.17 & \\
\hline UCD2- 17 & $8 / 5 / 94$ & 51.83 & 67.41 & -15.58 & \\
\hline UCD2- 17 & $8 / 19 / 94$ & 51.83 & 69.81 & -17.98 & \\
\hline UCD2- 17 & $9 / 7 / 94$ & 51.83 & 65.62 & -13.79 & \\
\hline UCD2- 17 & $9 / 7 / 94$ & 51.83 & 66.29 & -14.46 & \\
\hline UCD2- 17 & $9 / 13 / 94$ & 51.83 & 66.85 & -15.02 & \\
\hline UCD2- 17 & $9 / 19 / 94$ & 51.83 & 63.30 & -11.47 & \\
\hline UCD2- 17 & $9 / 26 / 94$ & 51.83 & 61.72 & -9.89 & \\
\hline UCD2- 17 & $10 / 7 / 94$ & 51.83 & 59.08 & -7.25 & \\
\hline UCD2- 17 & $10 / 14 / 94$ & 51.83 & 57.49 & -5.66 & \\
\hline UCD2- 17 & $10 / 21 / 94$ & 51.83 & 56.62 & -4.79 & \\
\hline UCD2- 17 & $10 / 28 / 94$ & 51.83 & 55.73 & 3.90 & \\
\hline UCD2- 17 & $11 / 4 / 94$ & 51.83 & 55.17 & -3.34 & \\
\hline UCD2- 17 & $11 / 18 / 94$ & 51.83 & 52.32 & -0.49 & , \\
\hline UCD2- 17 & $12 / 22 / 94$ & 51.83 & 49.28 & 2.55 & \\
\hline UCD2- 17 & $1 / 6 / 95$ & 51.83 & 47.70 & 4.13 & \\
\hline UCD2- 17 & $1 / 11 / 95$ & 51.83 & 44.35 & 7.48 & . \\
\hline UCD2- 17 & $1 / 13 / 95$ & 51.83 & 43.94 & 7.89 & \\
\hline UCD2- 17 & $1 / 27 / 95$ & 51.83 & 41.29 & 10.54 & \\
\hline UCD2- 17 & $2 / 10 / 95$ & 51.83 & 38.50 & 13.33 & \\
\hline UCD2. 17 & $2 / 17 / 95$ & 51.83 & 37.90 & 13.93 & \\
\hline UCD2- 17 & 3/9/95 & 51.83 & 35.65 & 16.18 & \\
\hline UCD2- 17 & $3 / 24 / 95$ & 51.83 & 31.00 & 20.83 & \\
\hline UCO2. 17 & $4 / 28 / 95$ & 51.83 & 34.48 & 17.35 & . \\
\hline UCO2- 17 & $5 / 25 / 95$ & 51.83 & 34.93 & 16.90 & \\
\hline UCD2- 17 & $6 / 16 / 95$ & 51.83 & 38.48 & 13.35 & \\
\hline UCD2- 17 & $7 / 26 / 95$ & 51.83 & 46.92 & 4.91 & Lock missing \\
\hline UCD2- 17 & $8 / 31 / 95$ & 51.83 & 47.00 & 4.83 & \\
\hline UCD2. 17 & $9 / 26 / 95$ & 51.83 & & NA & Continuous well level indicator working \\
\hline UCD2- 17 & $10 / 25 / 95$ & 51.83 & & NA & Continuous monitoring equipment \\
\hline UCD2- 17 & $11 / 22 / 95$ & 51.83 & 39.93 & 11.90 & \\
\hline UCD2- 17 & $11 / 27 / 95$ & 51.83 & 39.64 & 12.19 & no pump \\
\hline UCD2- $17^{-}$ & $12 / 19 / 95$ & 51.83 & 37.44 & 14.39 & Pump not installed \\
\hline UCD2- 26 & $11 / 27 / 95$ & 48.79 & 38.00 & 10.79 & \\
\hline UCD2- 26 & $12 / 19 / 95$ & 48.79 & - & NA & Blue well cap not installed completely \\
\hline UCD2- 35 & $11 / 27 / 95$ & 55.71 & 44.36 & 11.35 & - \\
\hline UCD2. 35 & $12 / 19 / 95$ & 55.71 & 41.97 & 13.74 & \\
\hline
\end{tabular}

a - Includes a correction factor of $0.02 \mathrm{ft}$. (added) 